\title{
Irradiation of 20L LEU Uranyl Sulfate Solution for Production of Mo-99
}

Experimental Operations and Facilities Division 


\begin{abstract}
About Argonne National Laboratory
Argonne is a U.S. Department of Energy laboratory managed by UChicago Argonne, LLC under contract DE-AC02-06CH11357. The Laboratory's main facility is outside Chicago, at 9700 South Cass Avenue, Lemont, Illinois 60439. For information about Argonne and its pioneering science and technology programs, see www.anl.gov.
\end{abstract}

\title{
DOCUMENT AVAILABILITY
}

Online Access: U.S. Department of Energy (DOE) reports produced after 1991 and a growing number of pre-1991 documents are available free at OSTI.GOV (http://www.osti.gov/),

a service of the US Dept. of Energy's Office of Scientific and Technical Information.

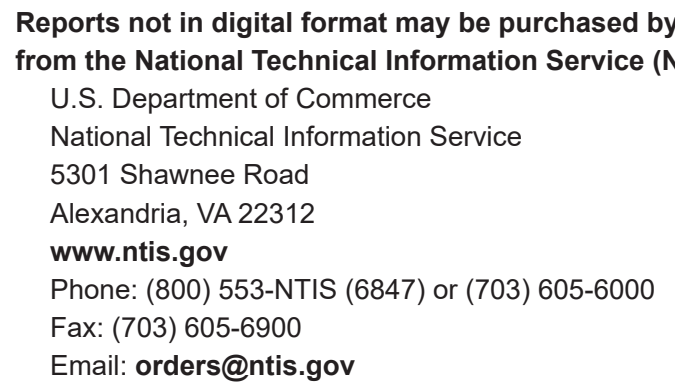

Reports not in digital format are available to DOE and DOE contractors from the Office of Scientific and Technical Information (OSTI):

U.S. Department of Energy

Office of Scientific and Technical Information

P.O. Box 62

Oak Ridge, TN 37831-0062

www.osti.gov

Phone: (865) 576-8401

Fax: (865) 576-5728

Email: reports@osti.gov

\begin{abstract}
Disclaimer
This report was prepared as an account of work sponsored by an agency of the United States Government. Neither the United States Government nor any agency thereof, nor UChicago Argonne, LLC, nor any of their employees or officers, makes any warranty, express or implied, or assumes any legal liability or responsibility for the accuracy, completeness, or usefulness of any information, apparatus, product, or process disclosed, or represents that its use would not infringe privately owned rights. Reference herein to any specific commercial product, process, or service by trade name, trademark, manufacturer, or otherwise, does not necessarily constitute or imply its endorsement, recommendation, or favoring by the United States Government or any agency thereof. The views and opinions of document authors expressed herein do not necessarily state or reflect those of the United States Government or any agency thereof, Argonne National Laboratory, or UChicago Argonne, LLC.
\end{abstract}


ANL-21/16

\section{Irradiation of 20L LEU Uranyl Sulfate Solution for Production of Mo-99}

by

S. D. Chemerisov¹, M. Kalensky², D. McLain ${ }^{3}$, P. Tkac², T. Brossard², K. Quigley ${ }^{1}$, J. Byrnes², D. A. Rotsch4, J. F. Krebs², R. Gromov¹, J. Bailey', B. Micklich5, J. Jerden², G. F. Vandegrift², A. J. Youker ${ }^{6}$, K. Alford ${ }^{1}$, K. Wesolowski ${ }^{1}$, C. Jonah ${ }^{1}$, A. Patapenka ${ }^{1}$, and A. Reavis ${ }^{1}$

${ }^{1}$ Experimental Operations and Facilities Division, Argonne National Laboratory ${ }^{2}$ Chemical and Fuel Cycle Technologies Division, Argonne National Laboratory ${ }^{3}$ Strategic Security Sciences Division, Argonne National Laboratory ${ }^{4}$ Physics Division, Argonne National Laboratory ${ }^{5}$ Photon Sciences Division, Argonne National Laboratory ${ }^{6}$ Operations and Business Management Division, Argonne National Laboratory

April 2021 



\section{CONTENTS}

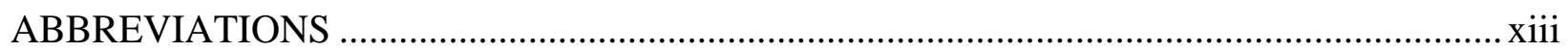

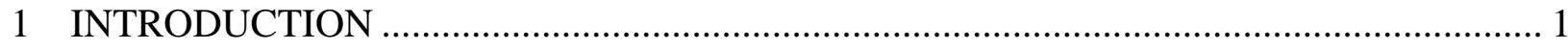

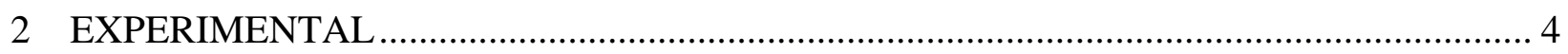

2.1 Linac Irradiation Hardware and Safety Considerations ............................................ 4

2.1.1 Linac and Beamline Configuration ........................................................... 4

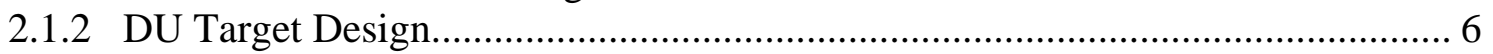

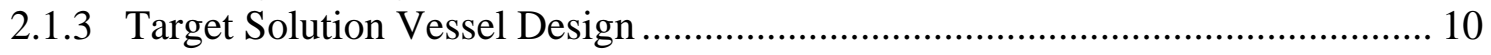

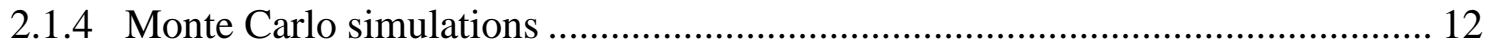

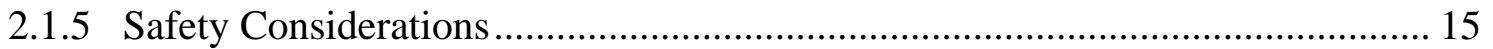

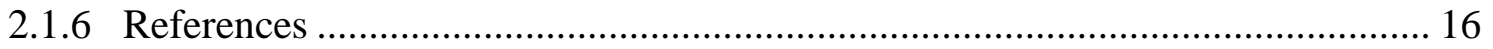

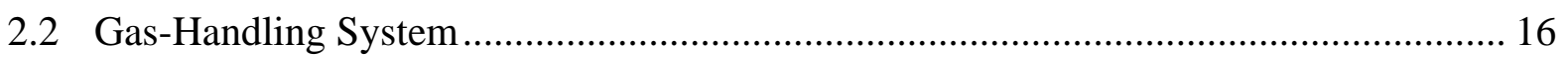

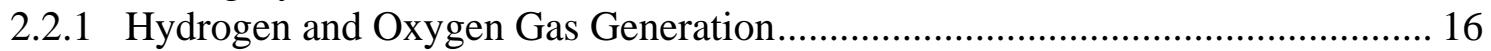

2.2.2 Gas-handling System Setup................................................................. 17

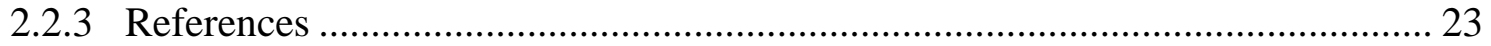

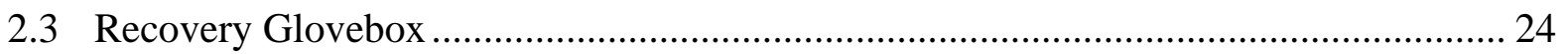

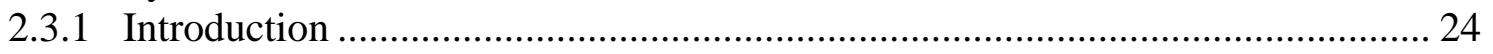

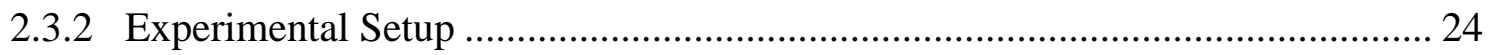

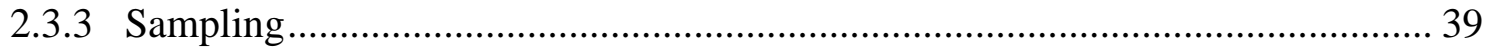

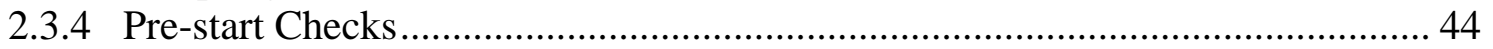

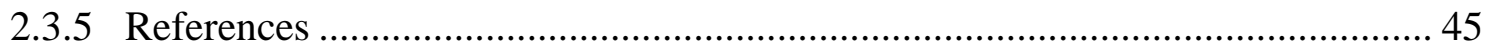

2.4 Hot Cell Processes ............................................................................................ 46

2.4.1 Concentration Column.......................................................................... 46

2.4.2 LEU-Modified Cintichem Process .............................................................. 53

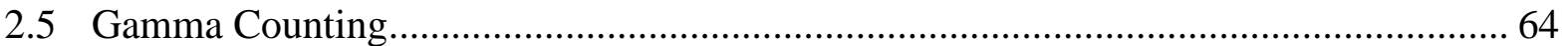

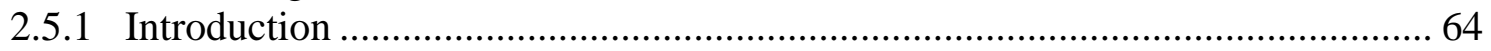

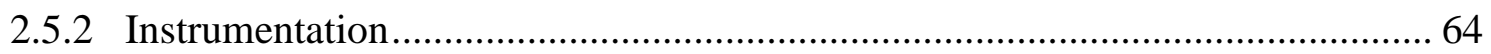

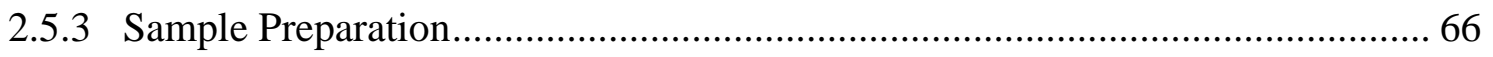

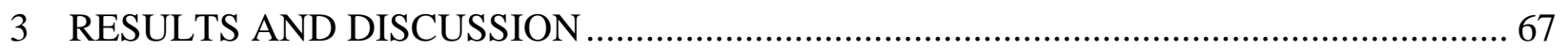

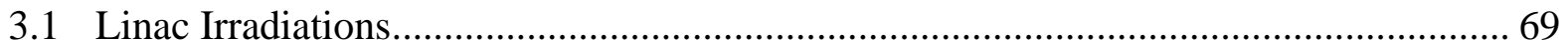

3.1.1 Irradiation \#0 with Accidental Rad Gas Release, 3/8/18 .............................. 69

3.1.2 Irradiation \#1, 10/1/19 ......................................................................... 73

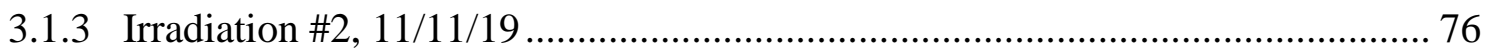

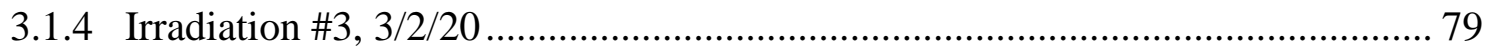

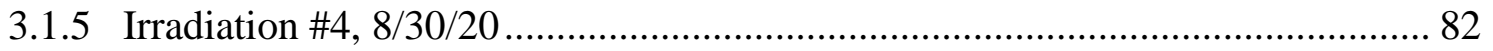

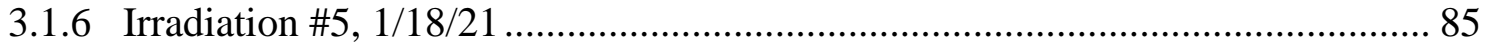

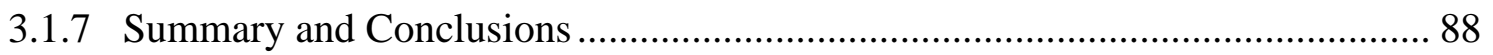

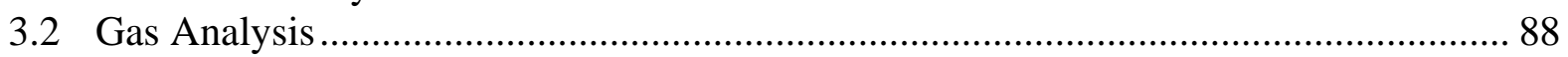

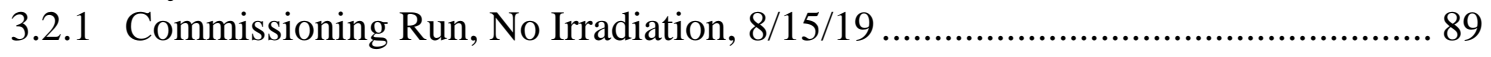

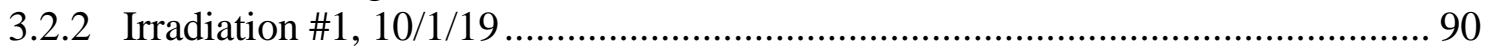

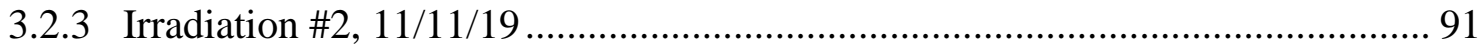

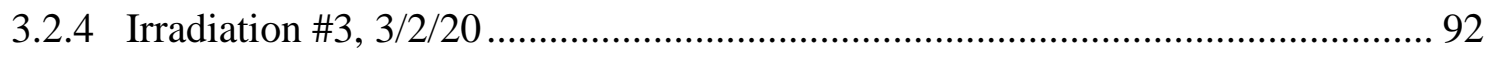




\section{CONTENTS (Cont.)}

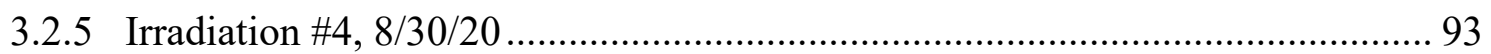

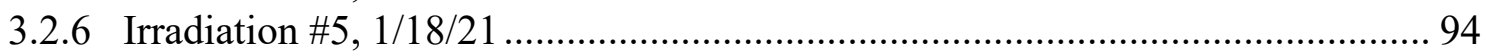

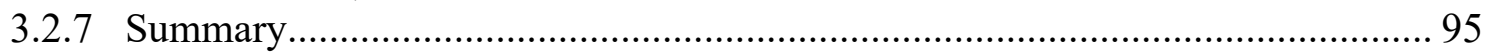

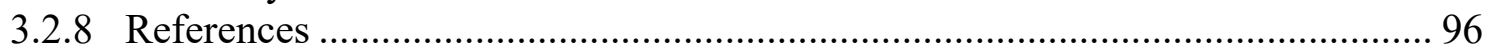

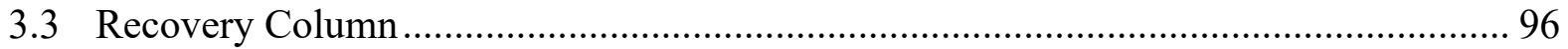

3.3.1 Commissioning Run, No Irradiation, 8/14/19 …............................................. 96

3.3.2 Irradiation \#1, 10/1/19 ............................................................................ 99

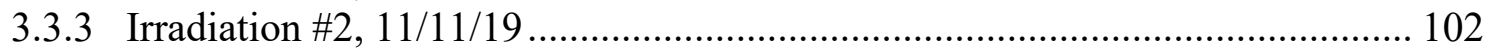

3.3.4 Irradiation \#3, 3/2/20 ........................................................................... 104

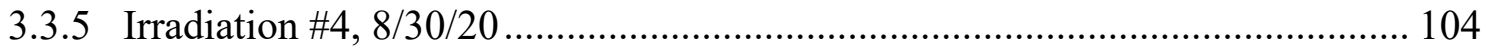

3.3.6 Irradiation \#5, 1/18/21 ............................................................................ 107

3.4 Hot Cell Purification Process ................................................................................ 109

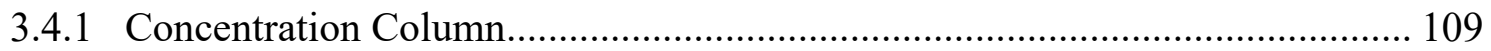

3.4.2 LEU Modified Cintichem Process.................................................................. 118

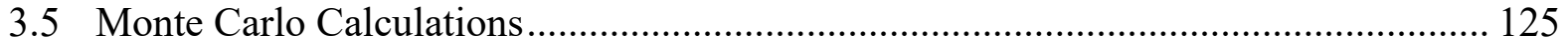

3.5.1 Scope of the Work ..................................................................................... 125

3.5.2 Simulation Procedure and Experimental Assembly ...................................... 125

3.5.3 Isotope Accumulation and Burnup Studies ................................................. 128

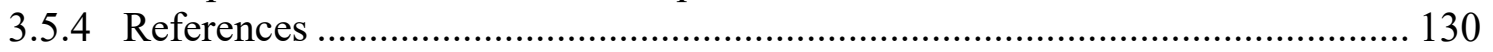

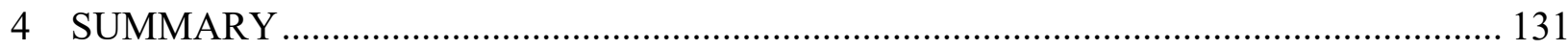

APPENDIX 1 Calculation Note NE-EO-2015-05: “Thermal/Hydraulic Analysis of DU

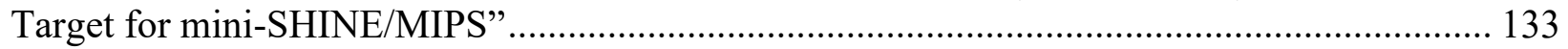

APPENDIX 2 Calculation Note NE-CALC-2015, ver. 1: “Thermal-Hydraulic Analysis of the Stoppage of Coolant Flow" .................................................................................... 148

APPENDIX 3 Calculation Note NE-CALC-2015-03: "Thermal-Hydraulic Analysis of the Overall Performance of the DU Target Cooling System"

APPENDIX 4 Calculation Note NE-CALC-2015-04: "Evaluation of the Structural Integrity of Zircaloy-4 Clad Containment for the DU Target Disks" .................................... 207

APPENDIX 5 Calculation Note NE-EO-2014-006: "DU Target Disk Clad Analysis”........... 222

APPENDIX 6 Calculation Note NE-EO-doc89: "Structural Analysis of Inconel Window for DU Target Assembly for ${ }^{99}$ Mo Production"

APPENDIX 7 Memo: "Radionuclide inventories and HazCat-3 sum-of-fraction for $35 \mathrm{MeV}$ mini-SHINE irradiations".

APPENDIX 8 LEAF-PROC-016, Rev. 3: AMORE Gas Handling Alarm and Interlock Checklist 267

APPENDIX 9 LEAF-PROC-017, Rev. 1: Monitoring the Gas Handling System during the AMORE Experiment. 


\section{CONTENTS (Cont.)}

APPENDIX 10 LEAF-PROC-018, Rev.3: AMORE Gas Handling Pre-Run Checklist 293

APPENDIX 11 LEAF-PROC-020, Rev. 2: Maintenance and Leak Testing in Catalyst Pump Enclosure 301

APPENDIX 12 LEAF-PROC-021, Rev. 2: Maintenance and Leak Testing in D-024 Analytical Enclosure 310

APPENDIX 13 LEAF-PROC-022, Rev. 2: Maintenance and Leak Testing in D-035 Gas Distribution Hub Enclosure 321

APPENDIX 14 LEAF-PROC-023, Rev. 2: Maintenance and Leak Testing of the Gas Collection System Enclosure 330

APPENDIX 15 Sampling Gas from AMORE Collection Cylinders 344

APPENDIX 16 Release of Gas from AMORE Collection Cylinders

APPENDIX 17 LEAF-PROC-024, Rev. 3: ${ }^{99}$ Mo PHASE II Production Tests - LabVIEW

${ }^{99}$ Mo Remote Recovery Data Acquisition and Control System: Complete Operations Abridged Version. 350

APPENDIX 18 Resin Washing Procedure 505

APPENDIX 19 LEAF-PROC-001, Rev. 1: 20L Tank Cooling System: Initial Startup, Ambient.

APPENDIX 20 LEAF-PROC-002, Rev. 1: 20L Tank Cooling System: Initial Startup, Elevated Temperature

APPENDIX 21 LEAF-PROC-003, Rev. 0: 20L Tank Cooling System: Routine Startup, Ambient. 526

APPENDIX 22 LEAF-PROC-004, Rev. 0: Chiller Cooling System: Initial and Routine Startup 533

APPENDIX 23 LEAF-PROC-006, Rev. 2: DU Target Cooling System: Initial Startup..... 538 APPENDIX 24 LEAF-PROC-007, Rev. 2: DU Target Cooling System: Routine Startup Procedure 545

APPENDIX 25 LEAF-PROC-027, Rev. 0: LEAF Linac General Operating Procedure 552 APPENDIX 26 LEAF-PROC-012, Rev. 0: AMORE Startup Checklist for Beam on Target... 563 APPENDIX 27 LEAF-PROC-011, Rev. 3: LEAF D-024 Hot Cell. 211/D-024 Hot Cell Operations AMORE. 569 


\section{FIGURES}

2.1.1.1 Average beam power versus beam energy for the linac. Measurements were taken at $20 \mathrm{~kW}$ RF power per modulator. The maximum average beam power, $25 \mathrm{~kW}$, is achieved at a $30-\mathrm{MeV}$ beam energy, $240-\mathrm{Hz}$ pulse rate, and $5.5-\mu \mathrm{s}$ pulse width. 5

2.1.1.2 Beamline configuration for AMORE irradiations...................................................... 5

2.1.2.1 Depleted-uranium target assembly ........................................................................... 7

2.1.2.2 Coolant flow through the depleted-uranium target .................................................... 7

2.1.2.3 Overall cooling system piping and instrumentation diagram. $\mathrm{CH} 1$ is the chiller that supplies cooling. Water for the DU target and the vessel is cooled by exchange with this chilled water through $\mathrm{Hx} 1$ and $\mathrm{Hx} 2$, respectively........................... 8

2.1.2.4 Cooling system for target solution vessel and DU target............................................ 9

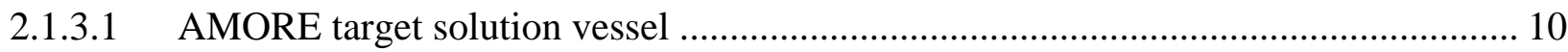

2.1.3.2 Target solution vessel cross-section inside the shielded box ....................................... 11

2.1.3.3 Target solution vessel and depleted-uranium target installed inside the shielded

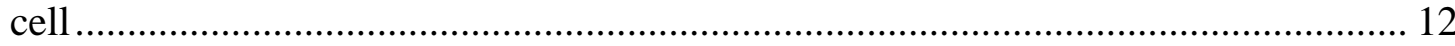

2.1.4.1 Buildup of ${ }^{99} \mathrm{Mo}$ in the 20-L uranyl nitrate solution .................................................. 13

2.1.4.2 (Top) HazCat-3 SOF for the entire assembly, the uranyl sulfate solution, the target assembly, and the balance of the system. (Bottom) HazCat-3 SOF for the entire assembly, the nuclides ${ }^{131} \mathrm{I}$ and ${ }^{133} \mathrm{I}$, and the remainder of the nuclides.

2.2.2.1 Diagram of the gas-handling system gas flow. All subsystems interconnect and lead to the GCS.

2.2.2.2 Catalytic recombiner design. (1) cylindrical part of the catalyst housing;

(2) conical reducers; (3) fiberglass layer; (4) catalyst................................................. 18

2.2.2.3 Condenser design ............................................................................................. 19

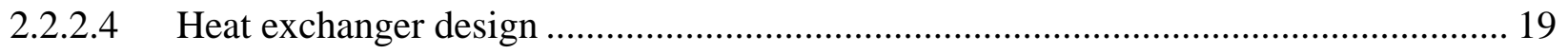

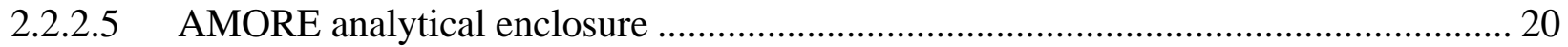

2.2.2.6 Diagram and function of the gas collection system ................................................ 21

2.2.2.7 Photograph of the gas collection system inside the enclosure .................................... 22

2.3.2.2.1 Image of the recovery glovebox with access port on the left. The three main windows and three cabinets discussed are to the right of the switch panel in the image.

2.3.2.2.2 Engineering diagram of the glovebox main cavity and cabinets below....................... 28 


\section{FIGURES (Cont.)}

2.3.2.2.3 Engineering diagram top view above cabinet \#2 and cabinet \#3, showing the locations of the surge tank and sample collection ladders 28

2.3.2.2.4 Piping and instrumentation diagram for the recovery glovebox 29

2.3.2.3.1 Images of the recovery column A) prior to assembly with frit and top collar removed and B) after filling and assembly, prior to installation in the recovery glovebox

2.3.2.3.2 Images of the recovery column (A) wrapped in heat tape and insulation and (B) installed in the lead-shielded pot

2.3.2.3.3 Recovery column in lead-shielded pot attached to the rest of the system, viewed (A) inside glovebox and (B) inside cabinet \#1

2.3.2.5.1 Image showing the relationship between the Dump Tank and TSV Hot Cell. These were connected via L-shaped transfer lines shielded by lead bricks. Image showing the relationship between the Dump Tank and TSV Hot Cell. These were connected via L-shaped transfer lines shielded by lead bricks. 33

2.3.2.6.1 Effluent cart with lid and valve manifold on top, in place inside cabinet \# 3 . 34

2.3.2.7.1 Drawing of the verification tank resting on its balance inside the shielded verification cart 35

2.3.2.8.1 Schematic diagram of a sample ladder rung with arrows showing the flow of solution during normal operation

2.3.2.8.2 (A) Diagram of the concentric needle system used to retrieve samples from sample ladders. (B) Needle system with sample vials in sample pots in the glovebox.

2.3.2.9.1 Main LabVIEW user interface for remote operation of the recovery glovebox 38

2.3.2.10.1 One of the leak sensors used in the glovebox system, composed of an adsorbent tube folded in half with one wire inserted in each side. The wires and adsorbent are held in place by zip-ties.

2.3.3.1.1 Diagram of sparge line connection used for sampling the target solution

2.3.3.2.1 LabVIEW operator interface for sample recovery after irradiation and processing....

2.3.3.2.2 Manifold used to direct atmosphere and vacuum to the appropriate sample ladder rung. White one-way check valves preventing backflow of gases into the glovebox are circled in green.

2.3.3.2.3 Alternate sample loop with manual valves used in later irradiations. 43 


\section{FIGURES (Cont.)}

2.3.3.3.1 Diagram of sampling setup for effluent bottles (A) while sampling with a syringe and (B) after cutting the PEEK tubing and allowing it to drop back into the bottle

2.4.1.1.1 The D-024 Hot Cell, where final purification of ${ }^{99}$ Mo is performed ........................ 46

2.4.1.1.2 Image of interior of the D-024 Hot Cell: concentration column (A); column control board (B); acidification vessel (C); balance (D); reagent vials (E); and valves for exterior solution line $(\mathrm{F})$.

2.4.1.1.3 Image of quick-connect system: compressed-gas connection (A); exhaust valve connecting to gas collection system (B); and water in/out (hidden behind the board)

2.4.1.1.4 Diagram of the 3-L, 5-neck vessel used for receiving and acidifying the ${ }^{99} \mathrm{Mo}$ product prior to loading on the concentration column ....

2.4.1.2.1 Schematic diagram of ${ }^{99}$ Mo concentration column

2.4.2.4.1 LMC glassware, plastic coated except for the fritted-glass column. From left to right: flat-bottom bottle, double-sided bottle, 51-mm fritted-glass column containing $20 \mathrm{~mL}$ of glass beads, $\mathrm{AgC} / \mathrm{ZrO} / \mathrm{AC}$ column, charcoal filter column. All glassware uses crimps to hold septa in place.

2.4.2.4.2 From left to right: double-sided needle with male-to-male Luer connector in the middle, one-way Luer check-valve needle, 40-mm 0.3- $\mu \mathrm{m}$ filter with needles, 40-mm 0.3- $\mu \mathrm{m}$ filter.

2.4.2.4.3 Various models of aluminum needle adapters ................................................... 58

2.4.2.4.4 D-type aluminum needle adapter with side port for vacuum line ............................. 58

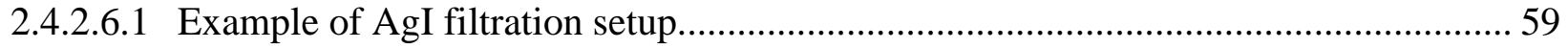

2.4.2.7.1 Experimental setup for filtration of Mo-ABO precipitate....................................... 60

2.4.2.8.1 Experimental setup for dissolution of Mo-ABO precipitate ................................... 61

2.4.2.9.1 Experimental setup for combination column ..................................................... 62

2.5.2.1 Coaxial HPGe cooled with $\mathrm{LN}_{2}$ and recycler (left) and the same detector with associated shielding and movable sample holder (right)

2.5.2.2 Mechanically cooled coaxial detector coupled to an autosampling unit with a shielded canyon for counting

3.1.1.1 Beam energy spectrum at $\sim 38 \mathrm{MeV}$ for Irradiation \#0 on 3/8/18. After tune-up, the injector current was lowered to shift the beam energy to $\sim 40 \mathrm{MeV}$

3.1.1.2 Beam profile on the target window for Irradiation \#0. Red circle outlines the target beam window boundary..... 


\section{FIGURES (Cont.)}

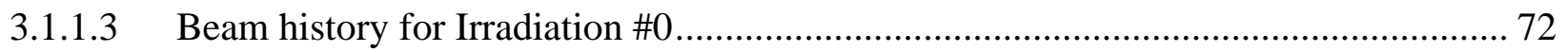

3.1.2.1 Beam-energy spectrum for Irradiation \#1 on 10/1/19. The energy spectrum was recorded at a lower energy than $40 \mathrm{MeV}$ because of spectrometer limitations. After initial tune-up, the beam peak current was reduced to adjust the peak energy to $40 \mathrm{MeV}$.

3.1.2.2 Beam profile on the target window for Irradiation \#1. Red circle outlines the

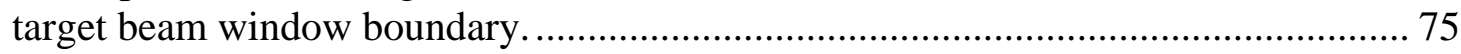

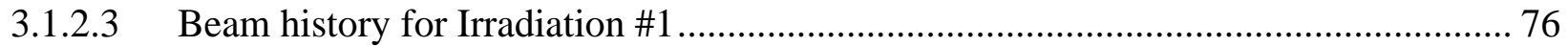

3.1.3.1 Beam energy spectrum for Irradiation \#2 on 11/11/19. The energy spectrum was recorded at a lower energy than $40 \mathrm{MeV}$ because of the thermal limitation of the spectrometer. After the initial tune-up, the beam peak current was reduced to adjust the peak energy to $40 \mathrm{MeV}$.

3.1.3.2 Beam profile on the target window for Irradiation \#2. Red circle outlines the target beam window boundary.

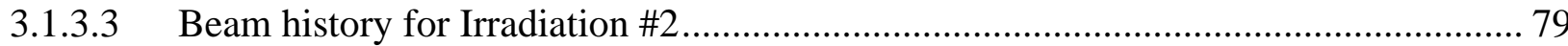

3.1.4.1 Beam energy spectrum for Irradiation \#3 on 3/1/20. The energy spectrum was recorded at a lower energy than $40 \mathrm{MeV}$ because of the thermal limitation of the spectrometer. After initial tune-up, beam peak current was reduced to adjust the peak energy to $40 \mathrm{MeV}$.

3.1.4.2 Beam profile on the target window for Irradiation \#3. Red circle outlines the target beam window boundary.

3.1.4.3 Beam history for Irradiation \#3

3.1.5.1 Beam energy spectrum for Irradiation \#4 on 8/30/20. The energy spectrum was recorded on August 28 at a lower energy than $40 \mathrm{MeV}$ because of the thermal limitation of the spectrometer. After initial tune-up, the beam peak current was reduced to adjust the peak energy to $40 \mathrm{MeV}$

3.1.5.2 Beam profile on the target window for Irradiation \#4. Red circle outlines the target beam window boundary.

3.1.5.3 Beam history for Irradiation \#4 ..................................................................... 85

3.1.6.1 Beam energy spectrum for Irradiation \#5 on 1/17/21. The energy spectrum was recorded on January 15 at a lower energy than $40 \mathrm{MeV}$ because of the thermal limitation of the spectrometer. After initial tune-up, the beam peak current was reduced to adjust the peak energy to $40 \mathrm{MeV}$.

3.1.6.2 Beam profile on the target window for Irradiation \#5. Red circle outlines the target beam window boundary. 


\section{FIGURES (Cont.)}

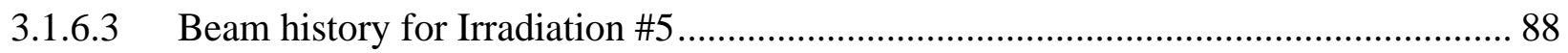

3.2.2.1 Gas concentration and linac beam power for Irradiation \#1 on 10/1/19 ...................... 91

3.2.3.1 Gas concentration and linac beam power for Irradiation \#2 on 11/11/19.................... 92

3.2.4.1 Gas concentration and linac beam power for Irradiation \#3 on 3/1/20 ........................ 93

3.2.5.1 Gas concentration and linac beam power for Irradiation \#4 on 8/30/20....................... 94

3.2.6.1 Gas concentration and linac beam power for Irradiation \#5 on 1/17/21 ...................... 95

3.3.1.1 Uranyl peroxide precipitate in target solution that was extracted from the system

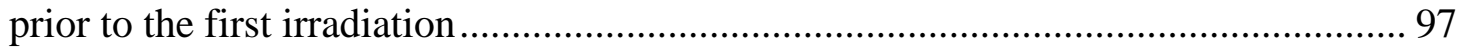

3.4.2.2.1 Photographs of major separation steps of LMC process: a) initial iodine precipitation; b) precipitation and filtration of Mo-ABO precipitate;

c) dissolution of Mo-ABO precipitate with heating using heat gun; d) final purification using iodine precipitation and the combination column $(\mathrm{Ag} / \mathrm{C}, \mathrm{HZO}, \mathrm{CC})$

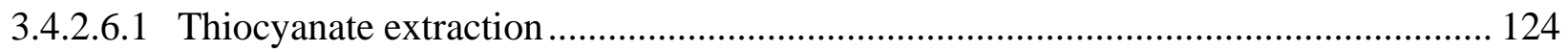

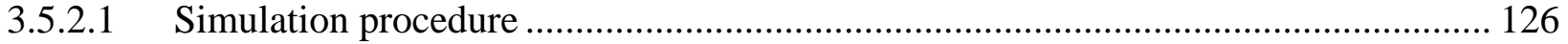

3.5.2.2 Radiation energy deposition: (top) energy deposition profile along the beam axis (averaged over the $\mathrm{X}, \mathrm{Y}$ directions) in the target; (bottom) 3D map of energy deposition

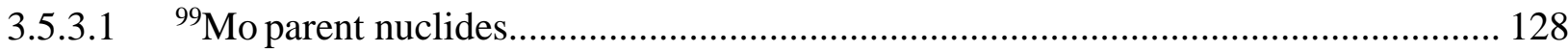

3.5.3.2 Irradiation beam-power profile normalized on maximum value …........................... 129

\section{TABLES}

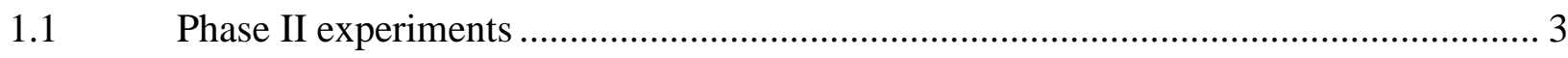

2.1.4.1 Activities of the radionuclides with highest $\mathrm{HC}-3 \mathrm{SOF}$ at the end of the fifth

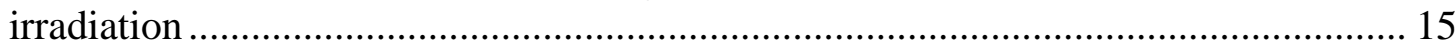

2.5.2.1 Isotopes and associated peaks analyzed during gamma spectroscopy .......................... 66

3.3.1.1 Processing samples taken and retrieved during the commissioning run....................... 98

3.3.1.2 Results from gamma analysis of samples recovered during the commissioning run

3.3.2.1 Samples taken and retrieved during processing for the first irradiation ...................... 99 


\section{TABLES (Cont.)}

3.3.2.2 Activities of isotopes in the various samples collected, with $1 \sigma$ uncertainty, for Irradiation \#1. Activities are listed as total $\mathrm{mCi}$ in the system

3.3.3.1 Samples taken and retrieved during processing for the second irradiation. 102

3.3.3.2 Activities of isotopes in the processed target solution sample, with $1 \sigma$ uncertainty, for Irradiation \#2. Activities are listed as total mCi in the system. .. 103

3.3.5.1 Activities of radionuclides in the target mixing sample, with $1 \sigma$ uncertainty, for Irradiation \#4. Activities are listed as total $\mathrm{mCi}$ in the irradiated target solution. ..... 105

3.3.5.2 Average activities of radionuclides in the effluent bottle samples, with $1 \sigma$ uncertainty, for Irradiation \#4. Activities are listed as total $\mu \mathrm{Ci}$ in the given effluent bottle.

3.3.6.1 Average activities of the various radionuclides in the irradiated target and processed target solutions, with $1 \sigma$ uncertainty, for Irradiation \#5. Activities are $\mathrm{mCi}$ present in the total target solution, which had roughly the same mass before and after irradiation.

3.3.6.2 Activities of radionuclides found in the effluent bottle samples, with $1 \sigma$ uncertainty, for Irradiation \#5. Activities are listed as total $\mu \mathrm{Ci}$ in the given effluent bottle.

3.4.1.1.1 Activities detected in the concentration-column fractions, decay corrected to the addition of the spike solution during the commissioning run

3.4.1.1.2 Relative distribution of ${ }^{99} \mathrm{Mo}$ in various fractions of the concentration column during the commissioning run.

3.4.1.2.1 Activities detected in the concentration column fractions, decay corrected to EOB during the first irradiation

3.4.1.2.2 Distribution of activity of various isotopes in the fractions collected from the concentration column during the first irradiation.

3.4.1.3.1 Activities detected in the concentration-column fractions, decay corrected to EOB during the second irradiation

3.4.1.3.2 Distribution of activity of various isotopes in the fractions collected from the concentration column during the second irradiation

3.4.1.5.1 Activities detected in the concentration column fractions, decay corrected to EOB during the fourth irradiation.

3.4.1.5.2 Distribution of activity of various isotopes in the fractions collected from the concentration column during the fourth irradiation.

3.4.1.6.1 Activities detected in the concentration column fractions, decay corrected to EOB during the fifth irradiation 


\section{TABLES (Cont.)}

3.4.1.6.2 Distribution of activity of various isotopes in the fractions collected from the concentration column during the fifth irradiation

3.4.2.1.1 Activities detected in LMC fractions

3.4.2.2.1 Activities detected in LMC fractions calculated at EOB .....

3.4.2.2.2 Distribution of various fission products and Mo in the RFW and ${ }^{99}$ Mo product as part of the LMC process................................................................................ 120

3.4.2.3.1 Activities detected in LMC fractions calculated at EOB ..................................... 121

3.4.2.3.2 Distribution of various fission products and Mo in the RFW and ${ }^{99}$ Mo product as

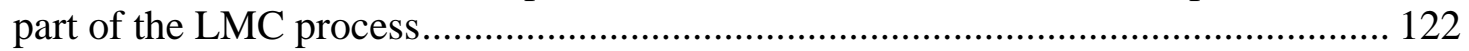

3.4.2.6.1 Activities detected in LMC fractions calculated at EOB ..................................... 123

3.4.2.6.2 Distribution of various fission products and Mo in the RFW and ${ }^{99}$ Mo product as part of the LMC process..

3.4.2.6.3 Radionuclidic purity in ${ }^{99}$ Mo product solution from the LMC process calculated at 36 hours after EOB.

3.5.3.1 Activities at EOB 130 


\section{ABBREVIATIONS}

\begin{tabular}{|c|c|}
\hline $\mathrm{ABO}$ & alpha-benzoin oxime \\
\hline $\mathrm{AC}$ & activated charcoal \\
\hline $\mathrm{Ag} / \mathrm{C}$ & silver-coated charcoal \\
\hline AMORE & Argonne Molybdenum Research Experiment \\
\hline ASE & Accelerator Safety Envelope \\
\hline DU & depleted uranium \\
\hline EOB & end of bombardment \\
\hline FEP & fluorinated ethylene propylene \\
\hline FMI & Fluid Metering, Inc. \\
\hline FWHM & Full Width at Half Maximum \\
\hline GCS & gas collection system \\
\hline GDH & gas distribution hub \\
\hline $\mathrm{HC}$ & hazard category \\
\hline HDPE & high-density polyethylene \\
\hline HPGe & high-purity germanium \\
\hline $\mathrm{HZO}$ & hydrous zirconia \\
\hline ICPMS & Inductively Coupled Plasma Mass Spectrometry \\
\hline LEAF & Low Energy Accelerator Facility \\
\hline LEU & low-enriched uranium \\
\hline linac & linear accelerator \\
\hline LMC & LEU-Modified Cintichem \\
\hline $\mathrm{LN}_{2}$ & liquid nitrogen \\
\hline MDA & minimum detectable activity \\
\hline $\mathrm{PE}$ & polyethylene \\
\hline PEEK & polyether ether ketone \\
\hline PP & polypropylene \\
\hline PSI & pounds per square inch \\
\hline RFW & raw fission waste \\
\hline RGA & Residual Gas Analyzer \\
\hline RRF & Relative Response Function \\
\hline SAD & Safety Assessment Document \\
\hline SOF & sum-of-fractions \\
\hline
\end{tabular}


TSV target solution vessel

VDG Van de Graaff

XRD X-ray diffraction 


\section{INTRODUCTION}

Argonne National Laboratory, with support from the National Nuclear Security Administration's Office of Material Management and Minimization, is assisting SHINE Medical Technologies in the development of accelerator-driven production of ${ }^{99}$ Mo using low-enriched uranium (LEU). Technetium-99m $\left({ }^{99 \mathrm{~m}} \mathrm{Tc}\right)$, a daughter of ${ }^{99} \mathrm{Mo}$, is a workhorse of nuclear medicine, used in approximately 40,000 medical testing procedures each day just in the U.S.

In 2015-2016, as part of its Phase I irradiation campaign, Argonne successfully demonstrated accelerator-driven subcritical fission of an aqueous LEU uranyl sulfate solution using an electron linac accelerator. In Phase I, a tantalum converter was used as the fast-neutron source with a maximum beam power of $10 \mathrm{~kW}$, and the solution volume was limited to $5 \mathrm{~L}$. This configuration generated a peak fission power density of $0.05 \mathrm{~kW} / \mathrm{L}$ and production of $60 \mathrm{mCi}$ ${ }^{99} \mathrm{Mo} / \mathrm{kWh}-\mathrm{kg}-{ }^{235} \mathrm{U}$. Separation methods applied to purify ${ }^{99} \mathrm{Mo}$ showed that the product met the British Pharmacopoeia purity specifications. $\sim 1 \mathrm{Ci}$ of ${ }^{99} \mathrm{Mo}$ was sent to GE Healthcare, where it was separately loaded onto a DRYTEC ${ }^{\text {TM }}{ }^{99}{ }^{9} \mathrm{Tc}$ Generator, and the ${ }^{99 \mathrm{~m}} \mathrm{Tc}$ product was tested using GE Healthcare ${ }^{99 \mathrm{~m}}$ Tc-based products: Myoview ${ }^{\mathrm{TM}}$ (kit for the preparation of ${ }^{99 \mathrm{~m}} \mathrm{Tc}$ Tetrofosmin for injection) and Ceretec ${ }^{\mathrm{TM}}$ (kit for the preparation of ${ }^{99 \mathrm{~m}} \mathrm{Tc}$ Exametazime for injection). Quality-control testing performed on the reconstituted kits indicated the viability of ${ }^{99 \mathrm{~m}} \mathrm{Tc}$ radiopharmaceuticals prepared using ${ }^{99}$ Mo product solution produced at Argonne [1, 2].

In the Phase II irradiation campaign, several major modifications were made to allow for increased production of ${ }^{99} \mathrm{Mo}$. Among these changes, the tantalum target was replaced by a depleted-uranium (DU) disc target, allowing an increase in power up to $20 \mathrm{~kW}$, while the volume of $140 \mathrm{~g}-\mathrm{U} / \mathrm{L}$ solution was increased up to $18 \mathrm{~L}$. These changes led to an increased peak fission power density of $0.3 \mathrm{~kW} / \mathrm{L}$, and to production of $\sim 87.5 \mathrm{mCi}-{ }^{99} \mathrm{Mo} / \mathrm{kWh}-\mathrm{kg}-{ }^{235} \mathrm{U}$.

Changes to the gas-handling system in Phase II included relocation of the gas analytical system from the main irradiation vault to an adjacent room, to minimize radiation damage to components and electronics. The relocation of the gas analytical system led to a 5-min delay in data analysis of hydrogen and oxygen production in the target solution vessel (TSV). Rapid changes in hydrogen production were mitigated by slowly ramping up the linac power to avoid spikes in hydrogen concentration. Monitoring of the analytical system was moved from a space adjacent to the linac vault to the linac control room to avoid the high radiation fields caused by the fission gas moving through the manifold in the analytical enclosure.

Further modifications were made to the target solution monitoring and ${ }^{99}$ Mo recovery gloveboxes to accommodate the increase in target-solution volume and ${ }^{99}$ Mo production. The solution-monitoring and ${ }^{99}$ Mo-recovery gloveboxes were replaced by a single large "recovery glovebox" that incorporated the functions of both gloveboxes from Phase I. The Phase II glovebox was built using 2-in.-thick carbon steel to provide extra shielding, and, wherever feasible, liquid lines were made of 1/4-in.-O.D. 316L stainless steel tubing to allow a higher flow rate than in Phase I. Shielding was also added to the effluent collection vessels, verification tank, and column. Several sensors from Phase I ( $\mathrm{pH}$, turbidity, conductivity) were removed since they did not provide usable measurements. Also, the Phase II glovebox recovery system split the 
solution-handling system into base-side and acid-side sections to eliminate uranium precipitation in the lines due to changes in $\mathrm{pH}$. Splitting the system into two sides also prevented crosscontamination of the feed and effluent and the unnecessary dilution/neutralization of the target solution. The recovery column size was increased to $40 \times 100 \mathrm{~mm}$. The increased column size was required to handle the larger volume of target solution used in Phase II.

For the hot-cell concentration and purification operations, changes were made to the concentration-column setup. The volumes and flow rates of the system were increased to accommodate the larger volumes of solution being processed, and the length of the concentration column was increased from $1 \mathrm{~cm}$ to $1.5 \mathrm{~cm}$, while the diameter stayed at $1 \mathrm{~cm}$. The column loading speed was increased from $16 \mathrm{~mL} / \mathrm{min}$ to $50 \mathrm{~mL} / \mathrm{min}$ and the column stripping was increased from $25 \mathrm{~mL}$ at $4 \mathrm{~mL} / \mathrm{min}$ to $66 \mathrm{~mL}$ at $11 \mathrm{~mL} / \mathrm{min}$. Additional safety features were added to the system, including a 1/4-in. gas collection line connected to the receiving vessel for $\mathrm{pH}$ adjustment and a liquid trap installed between the gas collection system (GCS) and the connections inside the hot cell. Further, a shielded effluent-storage system with a connection to the GCS was constructed under the hot cell to store the effluent from the concentration column for fission-product decay and long-term storage. No modifications were made to the LEUModified Cintichem (LMC) process between Phases I and II.

The processing steps following irradiation are briefly described here: irradiated LEU uranyl sulfate solution ( $18 \mathrm{~L}$ of $140 \mathrm{~g}$-U/L solution) at $\mathrm{pH}=1$ was mixed for several hours ${ }^{1}$ and then loaded on a titania $\left(\mathrm{TiO}_{2}\right)$ primary recovery column by remote operation in the recovery glovebox to separate ${ }^{99}$ Mo from uranyl sulfate solution. After the solution was loaded, the titania column was washed with $\mathrm{pH}=1$ sulfuric acid and water, and then ${ }^{99} \mathrm{Mo}$, along with reduced but not negligible amounts of other fission products, was stripped using $1 \mathrm{M} \mathrm{NaOH}$. The strip solution was transferred to hot-cell operations for concentration and further purification. Approximately $2 \mathrm{~L}$ of solution containing ${ }^{99} \mathrm{Mo}$ in $1 \mathrm{M} \mathrm{NaOH}$ was received in the hot cell and then acidified to $\mathrm{pH}=2$ using $\mathrm{HNO}_{3}$. The solution was then loaded onto a titania concentration column, and the column was washed with dilute acid and water. The ${ }^{99} \mathrm{Mo}$ was then stripped from the concentration column in $\sim 70 \mathrm{~mL}$ of $1 \mathrm{M} \mathrm{NaOH}$. The alkaline solution containing ${ }^{99} \mathrm{Mo}$ was then acidified to $\sim 1 \mathrm{M} \mathrm{HNO}_{3}$ solution, and the LMC process was used for final purification. The product from the LMC process contained purified ${ }^{99} \mathrm{Mo}$ in $\sim 50 \mathrm{~mL}$ of $0.2 \mathrm{M} \mathrm{NaOH}$.

Phase II experiments are summarized in Table 1.1, and experimental results obtained from these experiments are discussed later in this report.

Mixing was done to ensure a constant feed composition to the column. There was no mechanical mixing in the irradiation tank during irradiation. 
TABLE 1.1 Phase II experiments

\begin{tabular}{llc}
\hline \multicolumn{1}{c}{ Experiment } & \multicolumn{1}{c}{$\begin{array}{c}\text { End of } \\
\text { Bombardment } \\
\text { (date, time) }\end{array}$} & $\begin{array}{c}\text { Chemical } \\
\text { Processing }\end{array}$ \\
\hline Commissioning run, no irradiation & $8 / 14 / 19$ & Yes \\
Irradiation \#1 & $10 / 1 / 19,20: 00$ & Yes \\
Irradiation \#2 & $11 / 11 / 19,02: 00$ & Yes \\
Irradiation \#3 & $3 / 2 / 20$ & No \\
Irradiation \#4 & $8 / 30 / 20,07: 00$ & Yes \\
Irradiation \#5 & $1 / 18 / 21,8: 00$ & Yes \\
\hline
\end{tabular}

\section{References}

[1] Youker, A.J., Chemerisov, S.D., Tkac, P., Kalensky, M., Heltemes, T.A., Rotsch, D.A., Krebs, J.F., Makarashvili, V., Stepinski, D.C., Alford, K., Bailey, J., Byrnes, J., Gromov, R., Hafenrichter, L., Hebden, A., Jerden, J., Jonah, C., Micklich, B., Quigley, K., Schneider, J., Wesolowski, K., Vandegrift, G.F., and Sun, Z., Compendium of Phase-I Mini-SHINE Experiments, ANL/NE-16/39, Argonne National Laboratory, October 2016. Available at https://publications.anl.gov/anlpubs/2017/01/131828.pdf

[2] Youker, A.J., Chemerisov, S.D., Tkac, P., Kalensky, M., Heltemes, T.A., Rotsch, D.A., Vandegrift, G.F., Krebs, J.F., Makarashvili, V., and Stepinski, D.C.. Fission-Produced ${ }^{99}$ Mo Without a Nuclear Reactor. J. Nucl. Med. 2017; 58:514-517. 


\section{EXPERIMENTAL}

\subsection{LINAC IRRADIATION HARDWARE AND SAFETY CONSIDERATIONS}

Mini-SHINE experiments (5-L uranyl sulfate solution irradiation or Phase 1 irradiations) and the Argonne Molybdenum Research Experiment (AMORE or Phase 2 irradiations) were performed using the high-current electron linear accelerator (linac) at the Low Energy Accelerator Facility (LEAF) at Argonne. This linac can provide electron-beam energies of up to $50 \mathrm{MeV}$ and deposited power on a target of up to $30 \mathrm{~kW}$. Mini-SHINE experiments use an electron/X-ray/neutron converter to generate neutrons that produce fission in the target solution. In Phase 1, the target solution was 90-150 g-U/L LEU uranyl sulfate at $\mathrm{pH} 1$. The converter was a water-cooled solid tantalum slug; the maximum beam power on the converter was limited to $10 \mathrm{~kW}$, and the target solution volume was limited to $5 \mathrm{~L}$. This configuration generated a peak fission power density of $0.05 \mathrm{~W} / \mathrm{mL}$. In Phase 2, the solution was $145 \mathrm{~g}$-U/L LEU uranyl sulfate at $\mathrm{pH}$ 1. The X-ray converter/photo-neutron target was an array of water-cooled DU disks, with the maximum allowable power on the target limited to $20 \mathrm{~kW}$, and the solution volume was $18 \mathrm{~L}$. This configuration generated a fission power density of up to $0.3 \mathrm{~W} / \mathrm{mL}$. Several reports have been published on mini-SHINE developments for phases 1 and $2[1,2]$.

\subsubsection{Linac and Beamline Configuration}

AMORE irradiations were conducted at the Argonne linac, which is an L-band (1.3-GHz) RF accelerator operating at a maximum (no-load) energy of $53 \mathrm{MeV}$. The maximum average beam power for this machine can reach $25 \mathrm{~kW}$ at $30 \mathrm{MeV}$ beam energy. The average beam power as a function of beam energy for the linac at nominal operation parameters $(20 \mathrm{~kW}$ peak RF power per klystron) is shown in Figure 2.1.1.1. For AMORE irradiation, we have chosen 40-MeV beam energy, because at this energy we can achieve maximum neutron yield with heat deposition limitations imposed by the current design of the DU target.

In the AMORE experiment, the accelerated electron beam is delivered to a photo-neutron target through a beamline that consists of several beam elements. The shielded enclosure housing the AMORE experiment is located on the 10-degree beamline. To direct the beam to the target, it is first deflected by 10 degrees to the right, then 10 degrees to the left, creating an offset from the zero-degree beamline. This beamline has two pairs of quadrupole magnets (focusing elements) and a pair of correction dipole magnets. Drawings of the beamline and the location of the shielded box housing the target and the vessel are shown in Figure 2.1.1.2. 


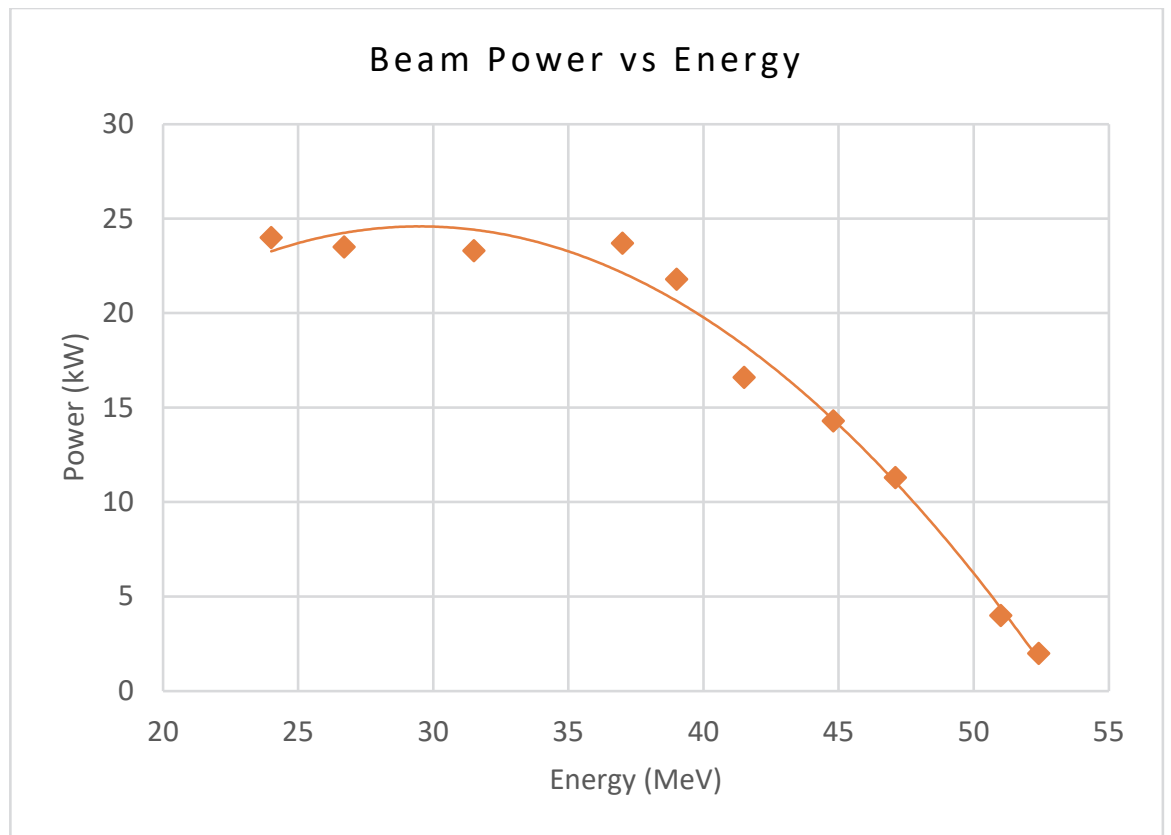

FIGURE 2.1.1.1 Average beam power versus beam energy for the linac. Measurements were taken at $20 \mathrm{~kW}$ RF power per modulator. The maximum average beam power, $25 \mathrm{~kW}$, is achieved at a $30-\mathrm{MeV}$ beam energy, $240-\mathrm{Hz}$ pulse rate, and $5.5-\mu$ s pulse width.

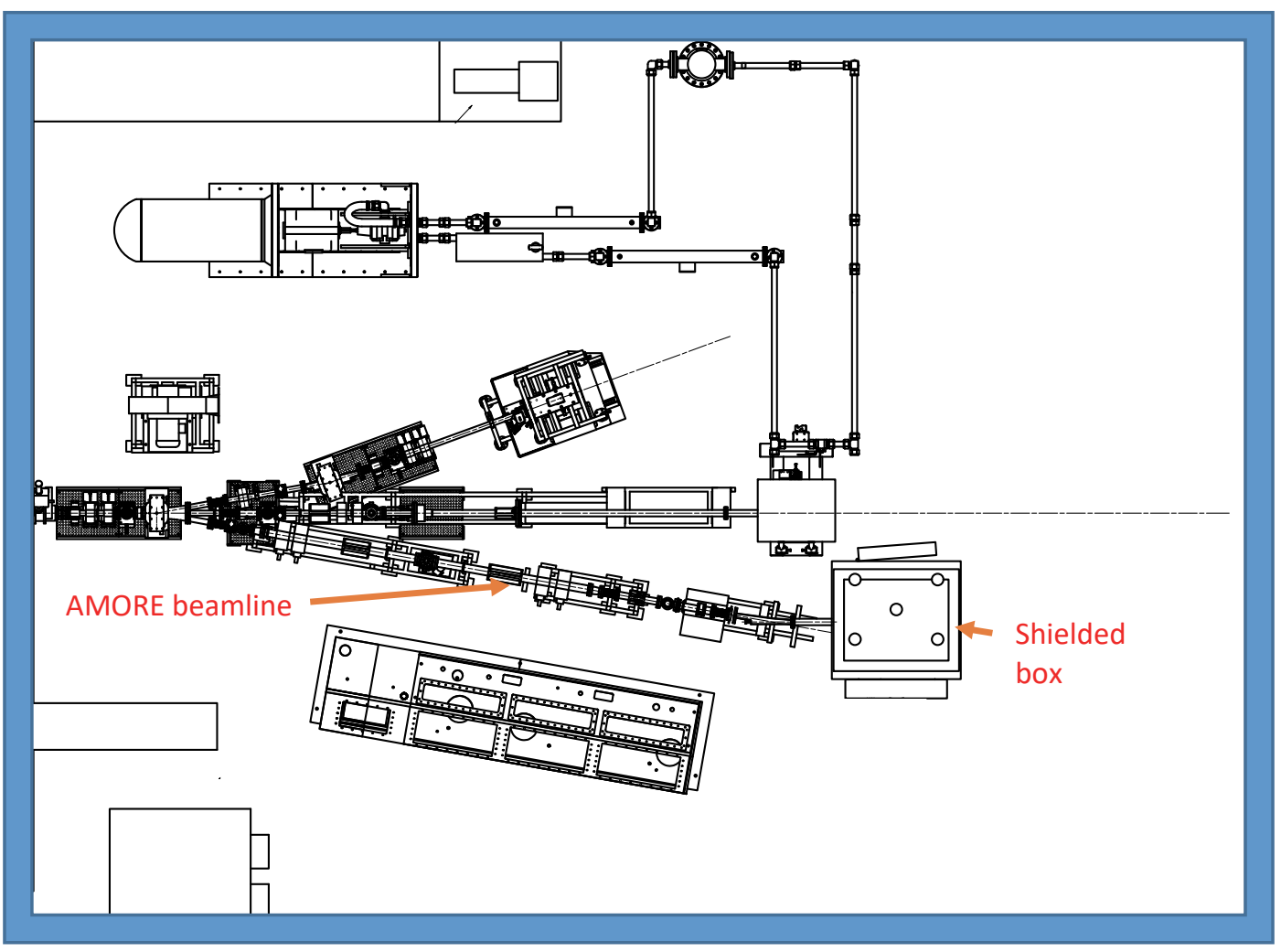

FIGURE 2.1.1.2 Beamline configuration for AMORE irradiations 
Before each irradiation, the beam energy was tuned to $40 \mathrm{MeV}$; then the electron beam was transported through the first 10-degree magnet to the second, and beam losses in the beamline were minimized. After that, the second 10-degree magnet was turned on and beam was placed on the target window. With the beam on the target window, transport was optimized to achieve minimum loss and desired beam size on the target. The beamline is equipped with current monitors from Bergoz that allow us to measure the beam current leaving the accelerator, passing through the first 10-degree magnet and arriving at the second 10-degree magnet. Also, for initial tune-up, the second 10-degree magnet chamber is equipped with a through port with a window so beam can be brought out and beam shape and current can be measured at that point. We used optical transition radiation detection to observe the beam position and shape on the target. Generally, the minimum acceptable size of the beam on the target is limited by peak heat generation in the target disks and the coolant's ability to remove the heat without boiling on the surface. The target was designed to accept $20-\mathrm{mm}$ by $20-\mathrm{mm}$ Full Width at Half Maximum (FWHM) at 20-kW beam power. Most of the irradiations were limited to lower power, because of high hydrogen gas generation, so we used a smaller beam size for most irradiations.

\subsubsection{DU Target Design}

The target assembly (Figure 2.1.2.1) contains the DU target. The target consists of 21 Zircaloy-4-clad uranium disks, $2.1 \mathrm{in}$. in diameter, arranged in an array as shown in Figure 2.1.2.1. Of these disks, 11 are 0.232 in. thick, and 10 are 0.074 in. thick. The Zircaloy-4 cladding on the faces of the disks is 0.010 in. thick. The Zircaloy is metallurgically bonded to the DU; this bonding is critical in providing efficient heat transfer from the uranium to the coolant. The DU disks are arranged in a horizontal stack, with alternating spacers and flow orifices to facilitate water flow from the target cooling loop across and around the target disks to optimize cooling (Figure 2.1.2.2). The "stack" of DU disks and spacers is held in place and compressed by a spring housing at the back of the target, which also allows for thermal expansion of the disks during irradiation. The overall dimensions of the target assembly are $2.75 \mathrm{in}$. in diameter by $6.92 \mathrm{in.} \mathrm{in} \mathrm{length.} \mathrm{The} \mathrm{total} \mathrm{quantity} \mathrm{of} \mathrm{DU} \mathrm{in} \mathrm{the} \mathrm{target} \mathrm{assembly} \mathrm{is} 6.01 \mathrm{lb}$. $(2.73 \mathrm{~kg})$. Several calculation notes on the target and cooling system designs are presented in Appendices 1-6.

The direction of coolant flow through the target assembly is shown in Figure 2.1.2.2. Coolant water enters at the bottom of the target housing from the cooling system at a design flow rate of nominally $40 \mathrm{GPM}$ at a temperature of $65^{\circ} \mathrm{F}$. The flow is directed across both faces of each disk by flow diverters, and disk spacing was designed to accommodate the calculated heat distribution throughout the target. The flow-channel gap between the disks is nominally 0.039 in. to provide a minimum average flow velocity of $22 \mathrm{ft} / \mathrm{s}$ in the gap for the worst-case (hottest) disk. This flow velocity is required to provide sufficient convective cooling at the full beam power of $20 \mathrm{~kW}$ while maintaining the temperature of the disk surfaces below the boiling point $\left(212^{\circ} \mathrm{F}\right)$ of the coolant. The required flow for each disk is controlled by the fixed-orifice resistance at the inlet to the individual intra-disk channels. The coolant water exits the flow channels into the outlet manifold and then flows out of the target assembly back to the cooling system. The total required heat removal from the target disks at full beam power is $16.4 \mathrm{~kW}$, which results in an $\sim 3^{\circ} \mathrm{F}$ temperature rise of the coolant from inlet to outlet of the target assembly. Under these flow conditions, the average static hydraulic pressure in the target 


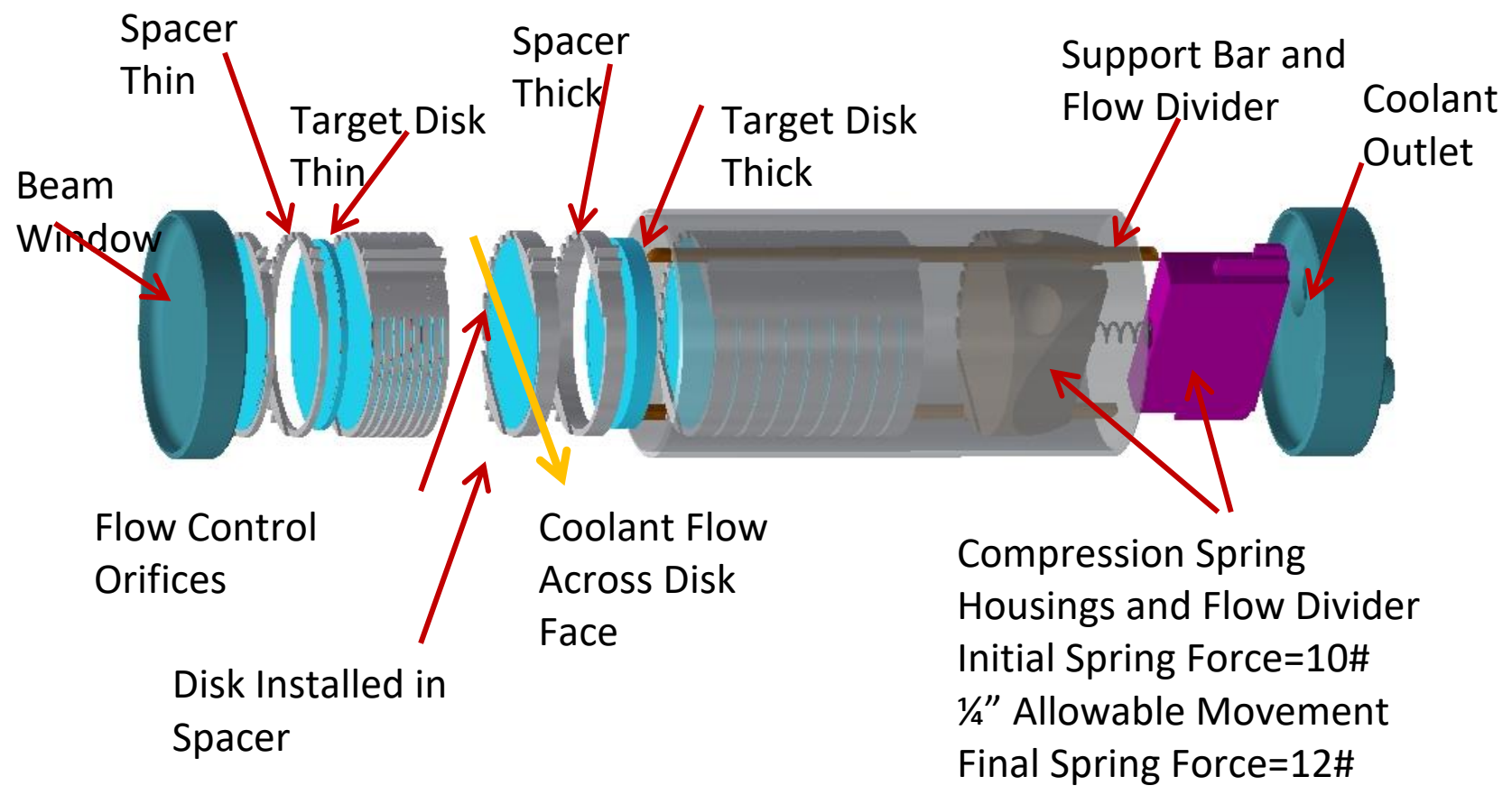

FIGURE 2.1.2.1 Depleted-uranium target assembly

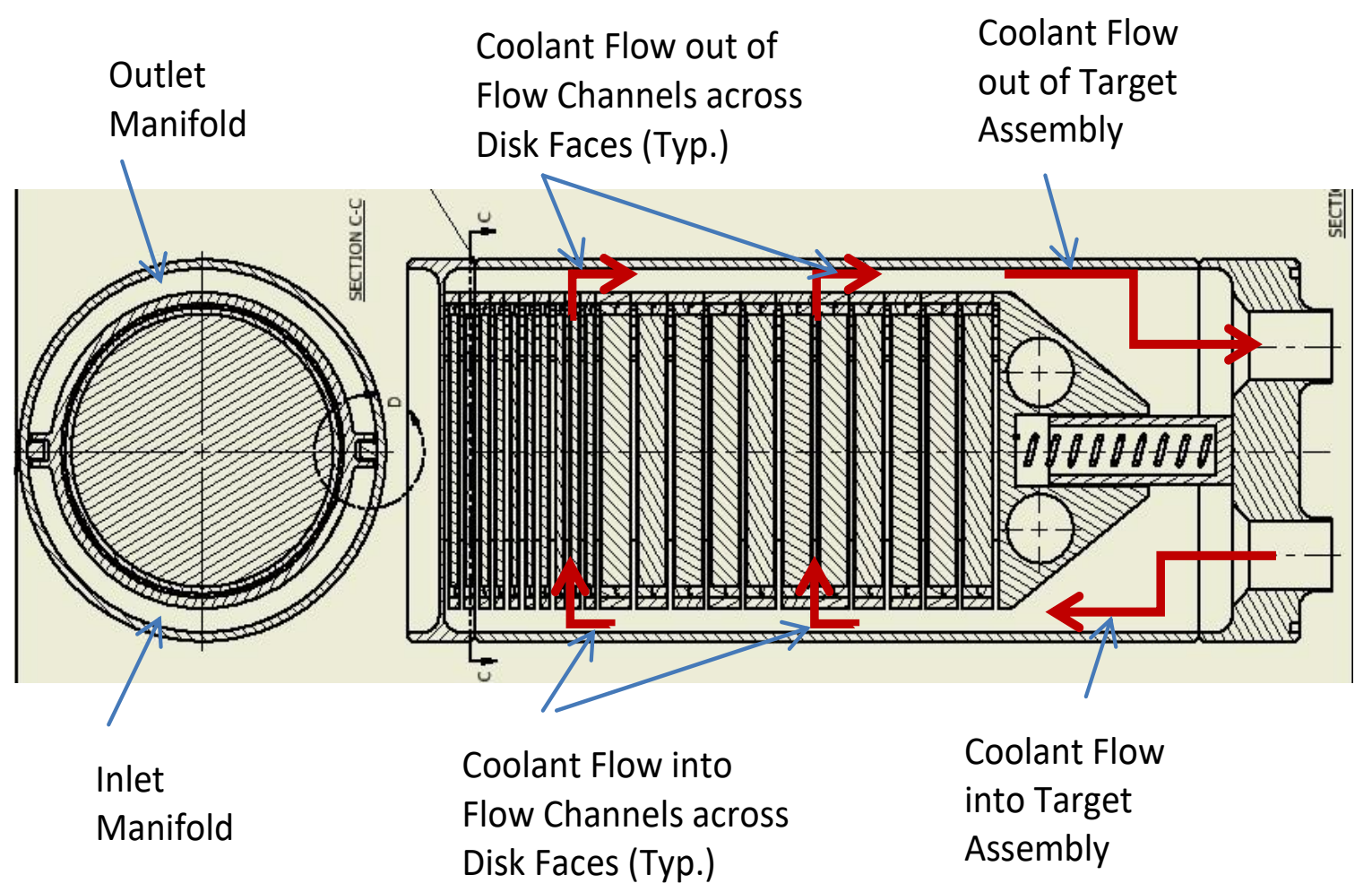

FIGURE 2.1.2.2 Coolant flow through the depleted-uranium target 
assembly is $22 \mathrm{psig}$, with a pressure differential between inlet and outlet of $16 \mathrm{psig}$. At the maximum allowed disk surface temperature of $212^{\circ} \mathrm{F}$, the maximum uranium temperature in the middle of the disks remains below $300^{\circ} \mathrm{F}$, which prevents both significant grain growth in the uranium and excessive thermal stresses in the bonded Zircaloy-4 cladding. The general coolingsystem piping and instrumentation diagram, which illustrates both the solution and target cooling loops, is shown in Figure 2.1.2.3.

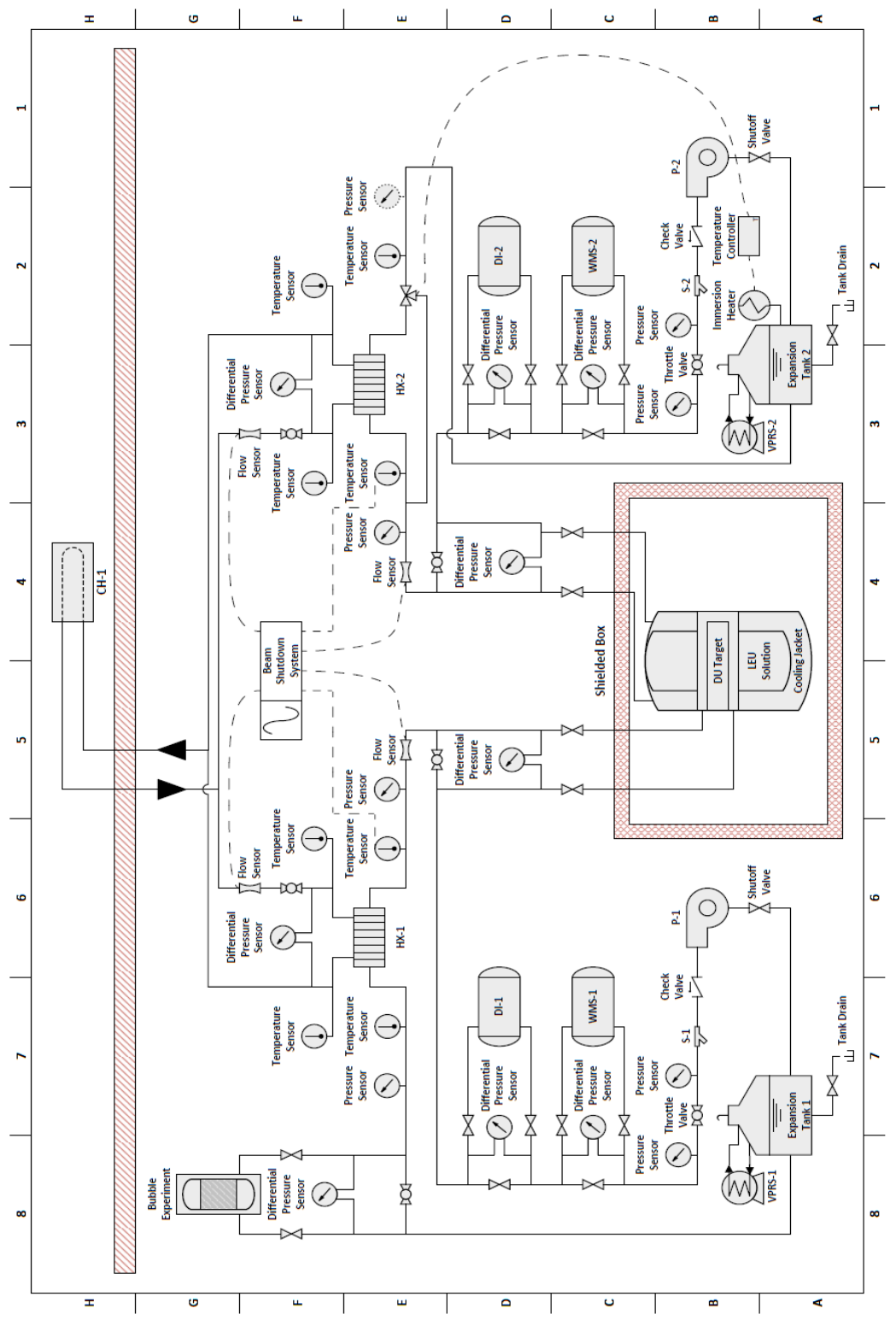

FIGURE 2.1.2.3 Overall cooling system piping and instrumentation diagram. $\mathrm{CH} 1$ is the chiller that supplies cooling. Water for the DU target and the vessel is cooled by exchange with this chilled water through $\mathrm{Hx} 1$ and $\mathrm{Hx} 2$, respectively 
The installed cooling system is shown in Figure 2.1.2.4. The cooling system is placed inside an air-tight enclosure because of the possibility of fission products entering the cooling water if the Zirconium cladding on the DU disks is breached. The enclosure is connected to a HEPA/Silver Zeolite filtered exhaust system to prevent radioactive particulates and iodine from escaping.

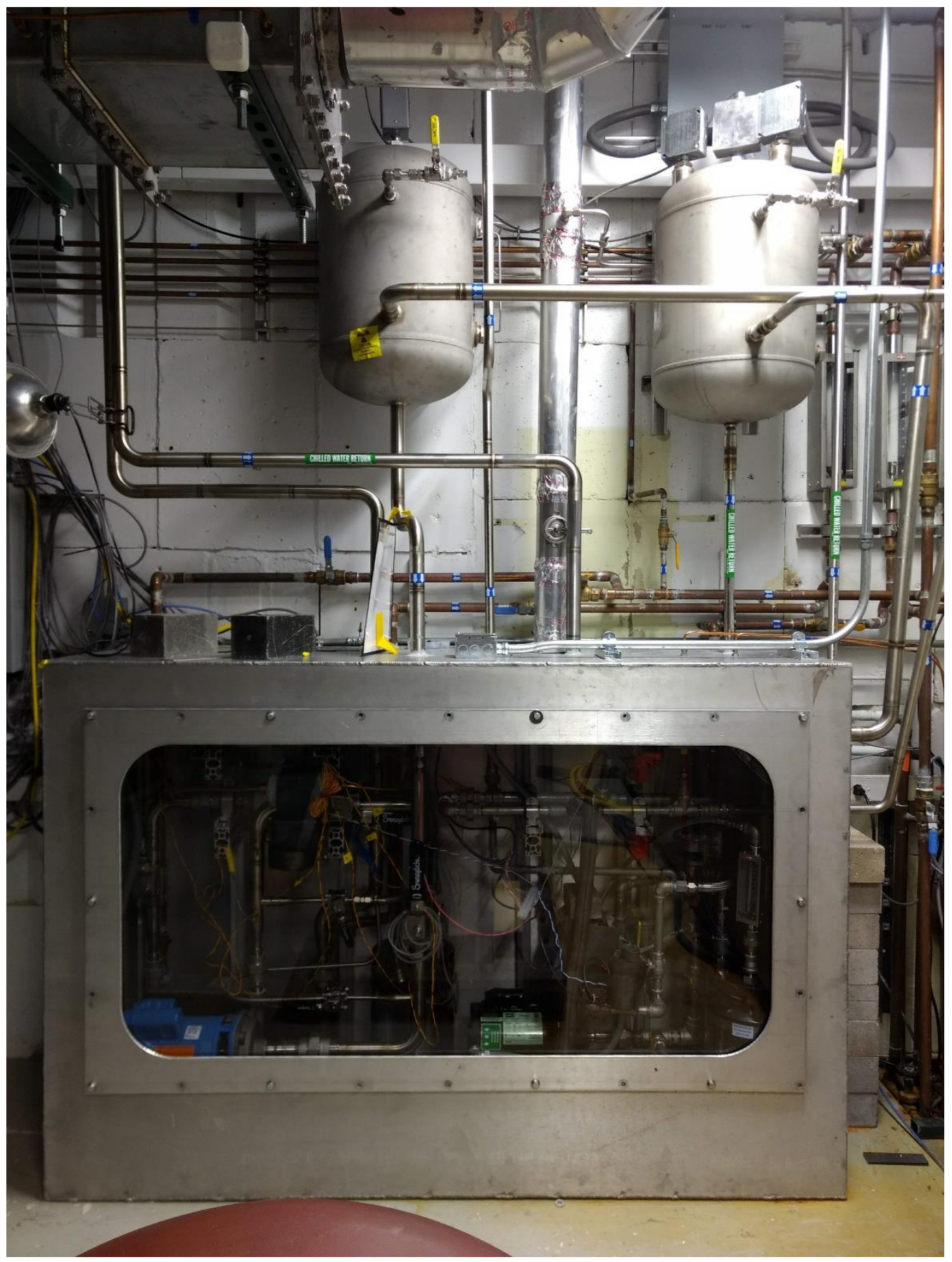

FIGURE 2.1.2.4 Cooling system for target solution vessel and DU target 


\subsubsection{Target Solution Vessel Design}

The TSV and all of its components are shown in Figure 2.1.3.1, and Figure 2.1.3.2 shows a cutaway view of the vessel and its position relative to the shielded box and DU target. Penetrations in the target vessel accommodate the incoming radiation beam, instrumentation (thermocouples), dry wells for inserting tubes containing smaller volumes of materials to be irradiated (mini-AMORE experiments), cooling loop connections, a drain tube extending to the bottom of the inner vessel for removal of the solution following irradiation, gas ports, and a 2-in. viewing port on top of the vessel. There are cooling loops for the target tube to remove the heat in the target generated by impingement of the accelerator beam, and for the outer water jacket. To reduce water losses during irradiation, gas ports inside the vessel are equipped with condensers. The vessel is positioned inside a hot cell. A helium cover gas is maintained over the LEU solution, and fission and radiolytic gases from the process are collected in a gas collection and analysis system connected to the vessel's gas ports. The completely installed TSV, DU target, and beamline are shown in Figure 2.1.3.3.

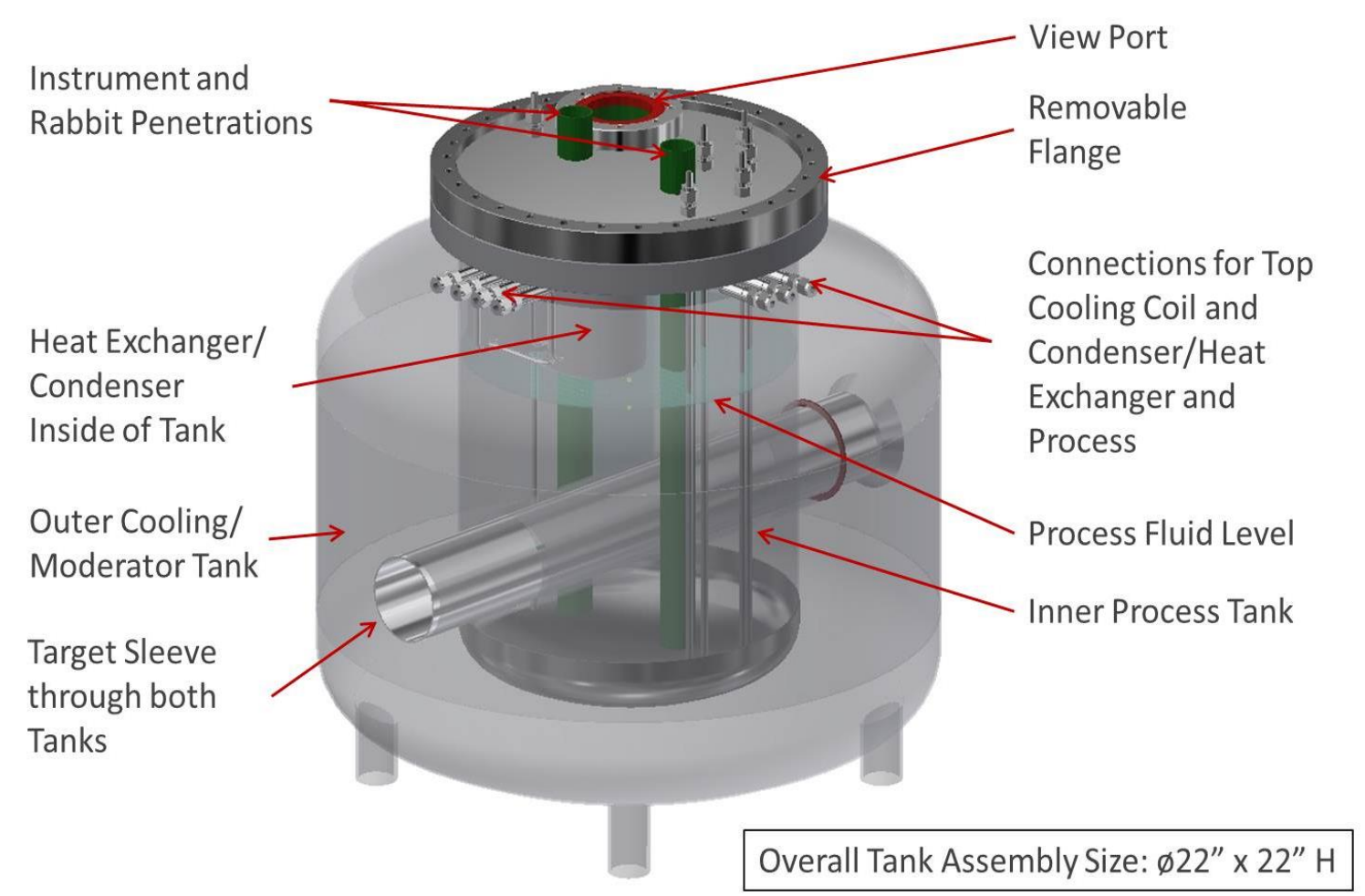

FIGURE 2.1.3.1 AMORE target solution vessel 


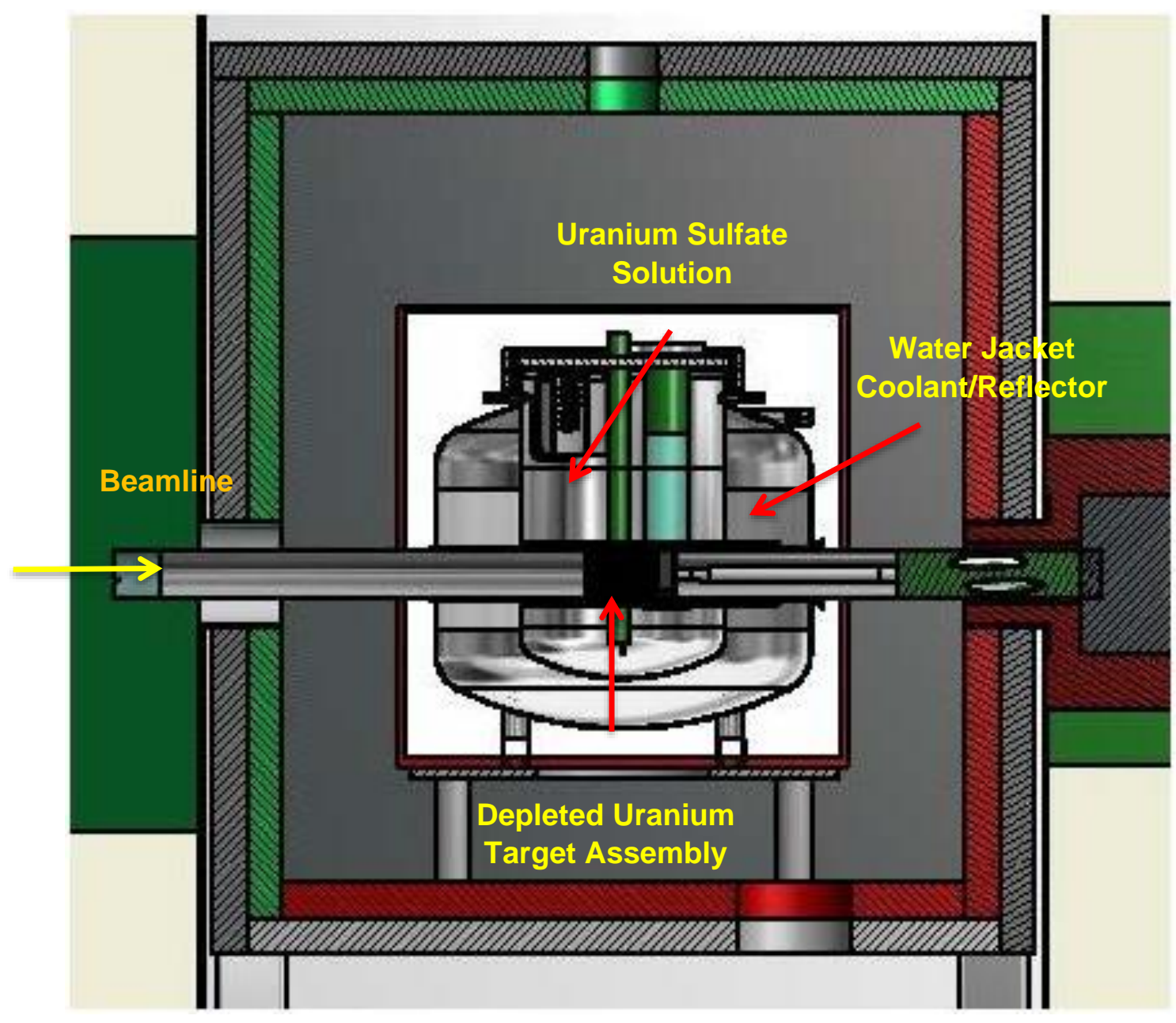

FIGURE 2.1.3.2 Target solution vessel cross-section inside the shielded box 


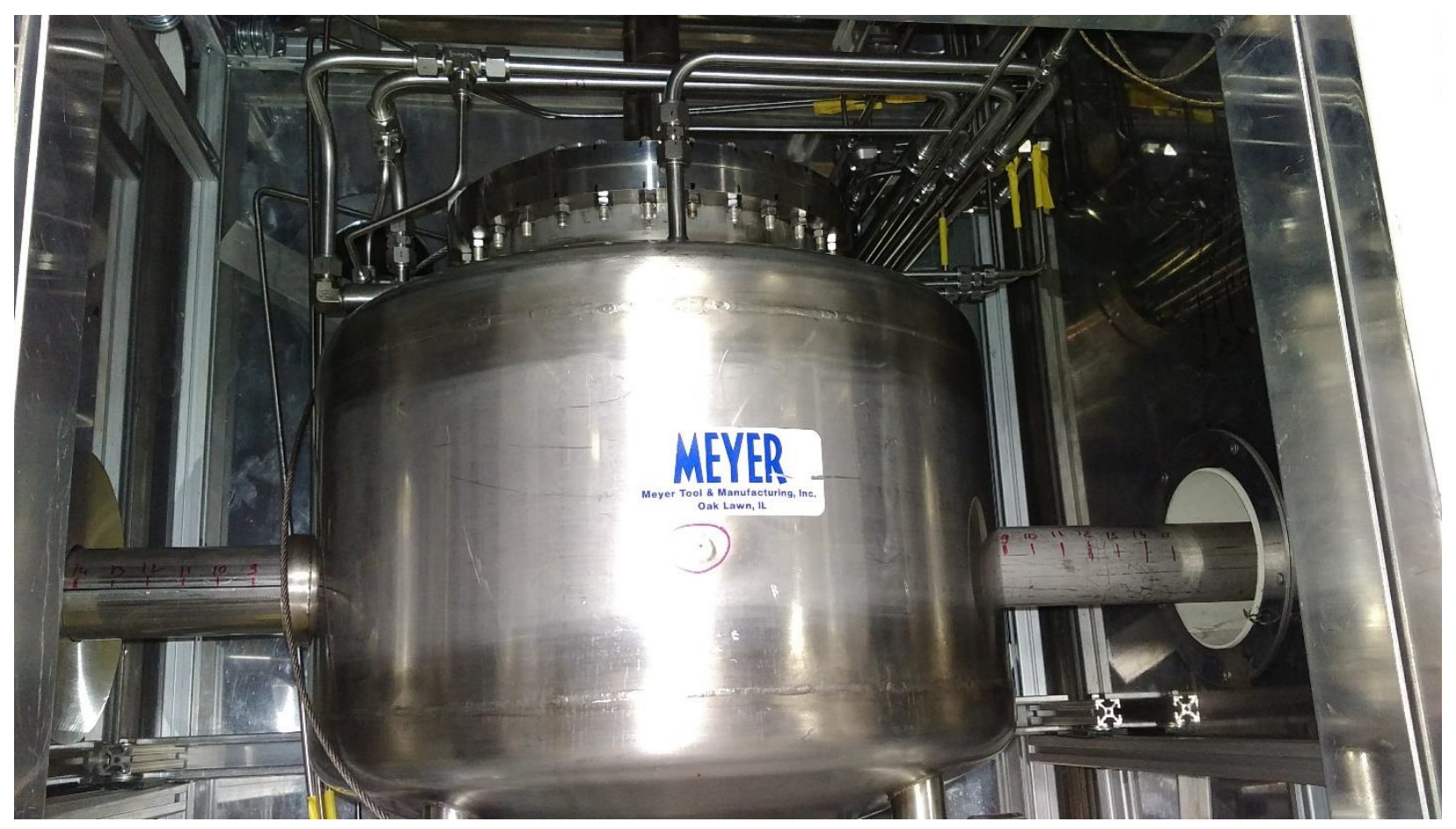

FIGURE 2.1.3.3 Target solution vessel and depleted-uranium target installed inside the shielded cell

\subsubsection{Monte Carlo simulations}

To predict accumulation of radioisotopes in the AMORE experiments, Monte Carlo simulations using the MCNP code were performed (see Section 3.5). A 35-MeV beam energy and $20-\mathrm{kW}$ beam power were used. It was predicted that one would need to irradiate $18 \mathrm{~L}$ of LEU uranyl sulfate solution for 19.3 hours to produce $20 \mathrm{Ci}$ of ${ }^{99} \mathrm{Mo}$ (Figure 2.1.4.1). The proposed irradiation campaign was to consist of a maximum of 5 irradiations, separated by 4 weeks of cool-down time. Radionuclide inventories were calculated at the shutdown of each irradiation and for decay times out to one year following the final irradiation (as well as at intermediate times during the irradiation process). More details of the simulation results are described in Reference [1] and Appendix 7. 


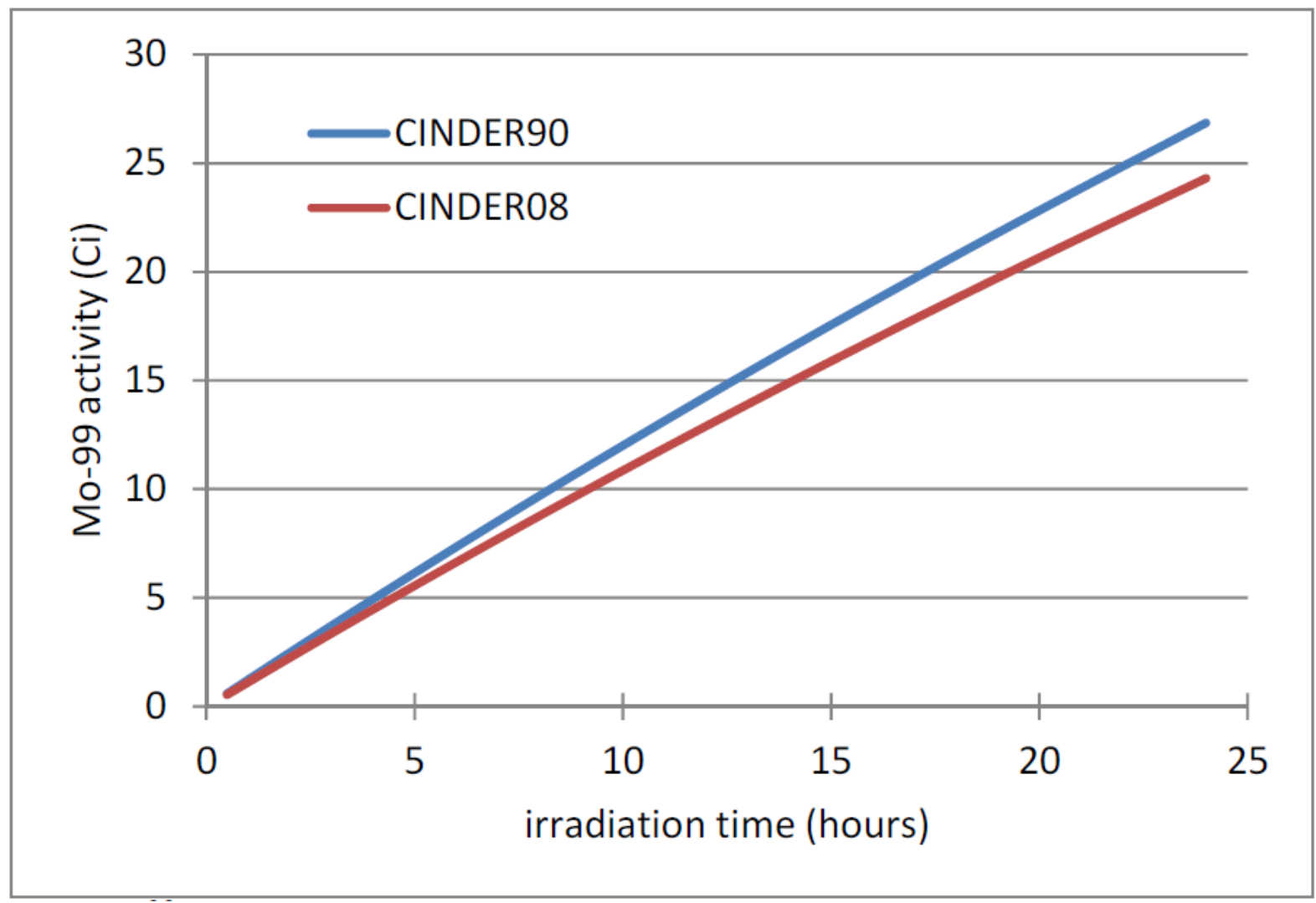

FIGURE 2.1.4.1 Buildup of ${ }^{99} \mathrm{Mo}$ in the 20-L uranyl nitrate solution

Figure 2.1.4.2 shows the hazard category 3 (HC-3) sum-of-fractions (SOF) in the entire irradiated volume, as well as in selected subsets, for the complete campaign of five 19.3-hour irradiations (to produce $20 \mathrm{Ci}$ of ${ }^{99} \mathrm{Mo}$ each), with 4-week breaks between successive irradiations. The percentage of the SOF for the solution is about $91-92 \%$ of the total.

Table 2.1.4.1 lists the top 41 contributors to the SOF, with their activities, at shutdown following the fifth irradiation. The contribution of the target varies between 8 and $9 \%$, and only a small contribution to the SOF comes from the box and vessels. The SOF is dominated by the fission products ${ }^{131} \mathrm{I}$ and ${ }^{133} \mathrm{I}$ out to several months following the last irradiation, by which time the entire SOF is only about 0.01 . 


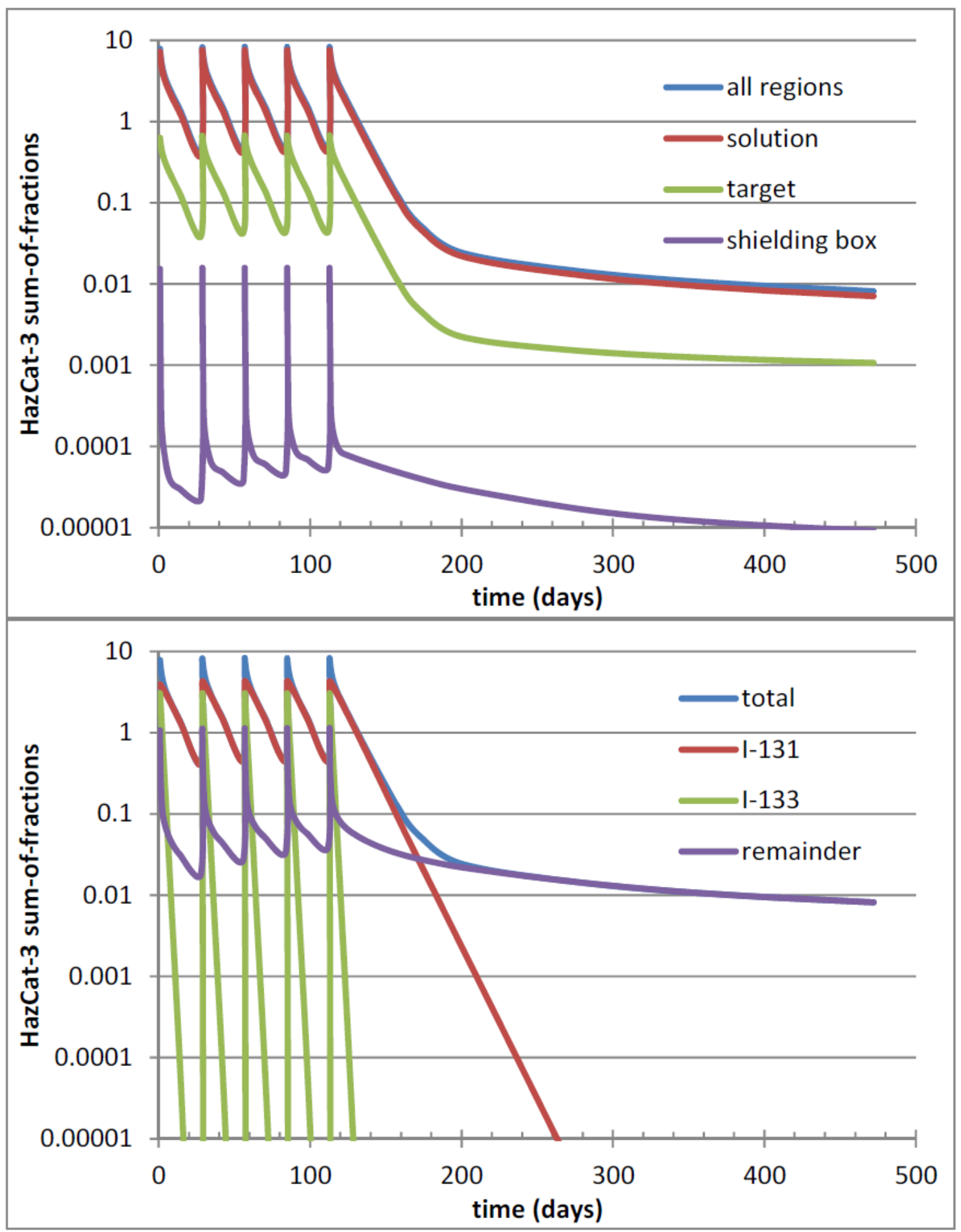

FIGURE 2.1.4.2 (Top) HazCat-3 SOF for the entire assembly, the uranyl sulfate solution, the target assembly, and the balance of the system. (Bottom) HazCat-3 SOF for the entire assembly, the nuclides ${ }^{131} I$ and ${ }^{133} I$, and the remainder of the nuclides. 
TABLE 2.1.4.1 Activities of the radionuclides with highest HC-3 SOF at the end of the fifth irradiation

\begin{tabular}{cccc}
\hline Nuclide & Activity in Ci & Nuclide & Activity in Ci \\
\hline${ }^{131} \mathrm{I}$ & $6.84 \mathrm{E}-01$ & ${ }^{143} \mathrm{Ce}$ & $3.66 \mathrm{E}+00$ \\
${ }^{133} \mathrm{I}$ & $5.71 \mathrm{E}+00$ & ${ }^{134} \mathrm{Te}$ & $1.79 \mathrm{E}+01$ \\
${ }^{135} \mathrm{I}$ & $1.20 \mathrm{E}+01$ & ${ }^{92} \mathrm{Y}$ & $1.21 \mathrm{E}+01$ \\
${ }^{88} \mathrm{Kr}$ & $8.59 \mathrm{E}+00$ & $95 \mathrm{Zr}$ & $6.04 \mathrm{E}-01$ \\
${ }^{138} \mathrm{Xe}$ & $1.61 \mathrm{E}+01$ & ${ }^{132} \mathrm{I}$ & $1.32 \mathrm{E}+00$ \\
${ }^{87} \mathrm{Kr}$ & $6.68 \mathrm{E}+00$ & ${ }^{141} \mathrm{Ce}$ & $7.95 \mathrm{E}-01$ \\
${ }^{91} \mathrm{Sr}$ & $1.47 \mathrm{E}+01$ & ${ }^{99} \mathrm{Mo}$ & $2.41 \mathrm{E}+00$ \\
${ }^{97} \mathrm{Zr}$ & $6.38 \mathrm{E}+00$ & ${ }^{239} \mathrm{~Np}$ & $5.46 \mathrm{E}+00$ \\
${ }^{92} \mathrm{Sr}$ & $1.47 \mathrm{E}+01$ & ${ }^{85} \mathrm{Kr} *$ & $2.66 \mathrm{E}+00$ \\
${ }^{134} \mathrm{I}$ & $2.01 \mathrm{E}+01$ & ${ }^{94} \mathrm{Y}$ & $1.65 \mathrm{E}+01$ \\
${ }^{142} \mathrm{La}$ & $1.49 \mathrm{E}+01$ & ${ }^{32} \mathrm{P}$ & $6.83 \mathrm{E}-03$ \\
${ }^{135} \mathrm{Xe}$ & $5.54 \mathrm{E}+00$ & ${ }^{129} \mathrm{Sb}$ & $1.23 \mathrm{E}+00$ \\
${ }^{140} \mathrm{La}$ & $1.09 \mathrm{E}+00$ & ${ }^{105} \mathrm{Ru}$ & $2.16 \mathrm{E}+00$ \\
${ }^{132} \mathrm{Te}$ & $1.55 \mathrm{E}+00$ & ${ }^{93} \mathrm{Y}$ & $9.45 \mathrm{E}+00$ \\
${ }^{140} \mathrm{Ba}$ & $1.39 \mathrm{E}+00$ & ${ }^{89} \mathrm{Rb}$ & $1.21 \mathrm{E}+01$ \\
${ }^{138} \mathrm{Cs}$ & $1.72 \mathrm{E}+01$ & ${ }^{147} \mathrm{Nd}$ & $5.21 \mathrm{E}-01$ \\
${ }^{89} \mathrm{Sr}$ & $5.20 \mathrm{E}-01$ & ${ }^{97} \mathrm{Nb}$ & $5.71 \mathrm{E}+00$ \\
${ }^{91} \mathrm{Y}$ & $5.18 \mathrm{E}-01$ & ${ }^{131} \mathrm{Te} *$ & $2.72 \mathrm{E}-01$ \\
${ }^{144} \mathrm{Ce}$ & $1.40 \mathrm{E}-01$ & ${ }^{130} \mathrm{Sb}$ & $2.01 \mathrm{E}+00$ \\
${ }^{135} \mathrm{Xe} *$ & $2.31 \mathrm{E}+00$ & ${ }^{131} \mathrm{Sb}$ & $6.54 \mathrm{E}+00$ \\
${ }^{143} \mathrm{Pr}$ & $1.03 \mathrm{E}+00$ & & \\
\hline
\end{tabular}

\subsubsection{Safety Considerations}

A comprehensive safety analysis for the linac facilities was conducted to evaluate the consequences of possible incidents due to the AMORE inventory. A full-facility fire was designated the "design basis" accident related to AMORE-generated radionuclides because it constitutes a credible, but extremely low-probability, event that produces the maximum set of consequences. The consequence determination was based on a maximum radionuclide inventory for the entirety of the AMORE irradiation campaign, resulting in maximum and "design-basis" consequences.

The calculated doses for this accident are documented in the consequence calculation.

Public consequence $($ at Argonne site boundary) $=\underline{\mathbf{0 . 3 2} \mathbf{~ m R e m} / \text { year }}$

Co-located worker $(100-\mathrm{m}$ distance $)=\underline{\mathbf{2 6} \mathbf{~ m R e m} / \text { year }}$. 


\subsubsection{References}

[1] Chemerisov, S., Bailey, J., Makarashvili, V., Micklich, B., and Vandegrift, G.F. Design of the Phase-2 Target for Mini-SHINE/MIPS Experiments. ANL/CSE-14/9, Argonne National Laboratory, 2012.

[2] Chemerisov, S., and Vandegrift, G.F. Mini-SHINE/MIPS Experiment. ANL/CSE-14/2, Argonne National Laboratory, 2011.

\subsection{GAS-HANDLING SYSTEM}

The purpose of the gas-handling system is to collect all radioactive fission gases and to keep hydrogen concentration in the AMORE system below the flammability limit (4\%). These goals are achieved in three ways: (1) Keep chemical processes under sub-atmospheric conditions, preventing the release of fission gas; (2) store fission gas for decay, to release at a later date; and (3) analyze for and recombine the hydrogen and oxygen generated from the radiolysis of water. The essential parts of the system are the GCS, the gas-distribution hub (GDH), the catalyst and pump, and the analytical system.

The GCS is the main feature, as it keeps all processes of the AMORE experiment subatmospheric and is used to store radioactive gases for decay. The GDH serves as a central connection point to the GCS. The catalyst pump recirculates the headspace gas of the TSV through a catalyst that recombines hydrogen and oxygen. The analytical system is used to monitor hydrogen and oxygen and includes safety interlocks that shut down the experiment at a $2 \%$ hydrogen level. It also generates an audible alarm at a $1 \%$ hydrogen level to notify the operator to reduce beam power by $50 \%$.

\subsubsection{Hydrogen and Oxygen Gas Generation}

Hydrogen, hydrogen peroxide $\left(\mathrm{H}_{2} \mathrm{O}_{2}\right)$, and oxygen are the molecular products generated by the radiolysis of water. The overall reactions are shown in Equations (1) and (2). The initial stage of the process is the formation of a solvated electron and the ionized and excited states of the water molecule created by incident radiation: $\mathrm{H}_{2} \mathrm{O}^{\bullet}, \mathrm{H}_{2} \mathrm{O}^{+}$, and $\mathrm{e}^{-}(\mathrm{aq})$. Collisions generate radical fragments: $\mathrm{H}+, \mathrm{H}^{\bullet}, \mathrm{OH}^{\bullet}, \mathrm{OH}^{-}$, and others. These fragments combine to form molecular species: $\mathrm{H}_{2}, \mathrm{H}_{2} \mathrm{O}_{2}$, or re-formed water [1]. Subsequent decomposition of $\mathrm{H}_{2} \mathrm{O}_{2}$ generates oxygen.

$$
\begin{aligned}
2 \mathrm{H}_{2} \mathrm{O}+\gamma & \rightarrow \mathrm{H}_{2}+\mathrm{H}_{2} \mathrm{O}_{2} \\
2 \mathrm{H}_{2} \mathrm{O}_{2}+\gamma & \rightarrow \mathrm{O}_{2}+2 \mathrm{H}_{2} \mathrm{O}
\end{aligned}
$$

A steady-state hydrogen and oxygen concentration can be maintained in the TSV because the rate of gas generation is linear with respect to the linac beam power [2]. Since the beam power for 99Mo production needs to be as high as possible, maintaining hydrogen concentration in the vessel depends on the rate of hydrogen/oxygen recombination in the catalyst. Therefore, 
the flow rate through the catalyst should be high enough to maintain hydrogen at a safe level. In these experiments, the length of the tubing to and from the pump and in the heat exchanger/condenser limited the flow of gas through the catalyst. This caused pressure in the return lines to increase, and the pressure in the supply lines and inside the TSV headspace to be reduced, thus increasing the differential pressure across the pump and reducing the gas flow through the catalyst. This result ultimately established an upper limit of approximately $18 \mathrm{~kW}$ of beam power with a KNF Neuberger Inc. NBR872 double-diaphragm pump, and $12 \mathrm{~kW}$ with a Senior Aerospace MB-151 Metal Bellows pump.

We had the ability to add oxygen to the TSV through a solenoid valve connected to a cylinder containing $40 \%$ oxygen. During previous experiments, the TSV was purged with helium before the irradiation [3]. Hydrogen is liberated into the headspace faster than oxygen in the initial stages of radiolysis. $\mathrm{H}_{2} \mathrm{O}_{2}$ is produced from the reaction of radicals and ions. Molecular oxygen is produced from the decomposition of $\mathrm{H}_{2} \mathrm{O}_{2}$ and has a greater solubility in the solution than hydrogen. The result is a delay in the occurrence of oxygen in the headspace gases. During the present set of experiments, the TSV was not purged prior to the irradiations.

\subsubsection{Gas-handling System Setup}

The uranyl sulfate solution resides inside the TSV, as shown in Figure 2.2.2.1. The headspace gas of the vessel was initially recirculated through a catalytic recombiner using a KNF Neuberger Inc. N186.1.2SN.12E double-diaphragm pump. After the March 2020 irradiation, that pump overheated, and it was replaced with the Senior Aerospace MB-151 Metal Bellows pump used in earlier irradiations. The catalyst, shown in Figure 2.2.2.2, is composed of platinum/palladium on alumina/cordierite and has a honeycomb configuration, which allows the gas to flow down the length of the bed. The catalytic material was commonly used to reduce emissions in diesel engine exhaust. Here, it is being used as a reactor to recombine the hydrogen and oxygen produced from the radiolysis of water. The purpose is to reduce the concentration of hydrogen in the headspace of the TSV to well below the flammability limit of 4\%. Part of the safety basis for these experiments was to keep the concentration of hydrogen to less than $1 \%$ (see Appendix 8). The catalyst is heated to $130^{\circ} \mathrm{C}$ to facilitate the removal of water produced in the catalyst bed during the recombination reaction. This operating temperature also prevents condensation of water vapor on the catalyst surface caused by humidity in the headspace gas. It was found during sodium sulfate irradiations in April 2014 that condensation on the catalyst inhibits the active sites of the catalyst, causing a buildup of hydrogen in the system.

A condenser (Figure 2.2.2.3) is located inside the TSV, upstream of the point where the headspace gas flows to the recirculating pump, and serves to decrease the humidity of the headspace gas prior to entering the catalyst. The gas passes around the outside of a water-cooled coil. At the catalyst exhaust, a heat exchanger (Figure 2.2.2.4) recondenses the water vapor generated in the catalyst. In the heat exchanger, gas passes through the interior of a water-cooled tubing coil. 


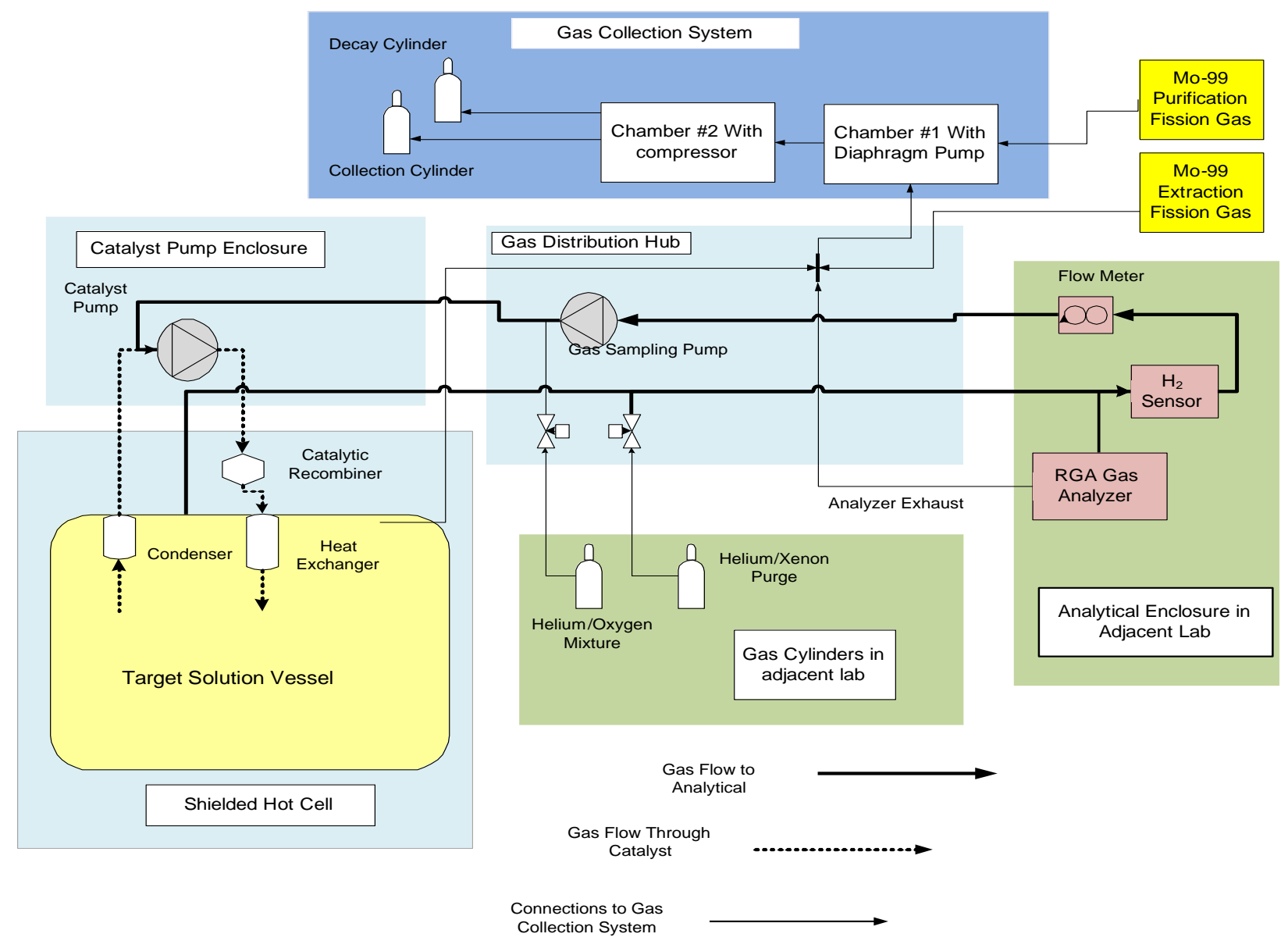

FIGURE 2.2.2.1 Diagram of the gas-handling system gas flow. All subsystems interconnect and lead to the GCS.

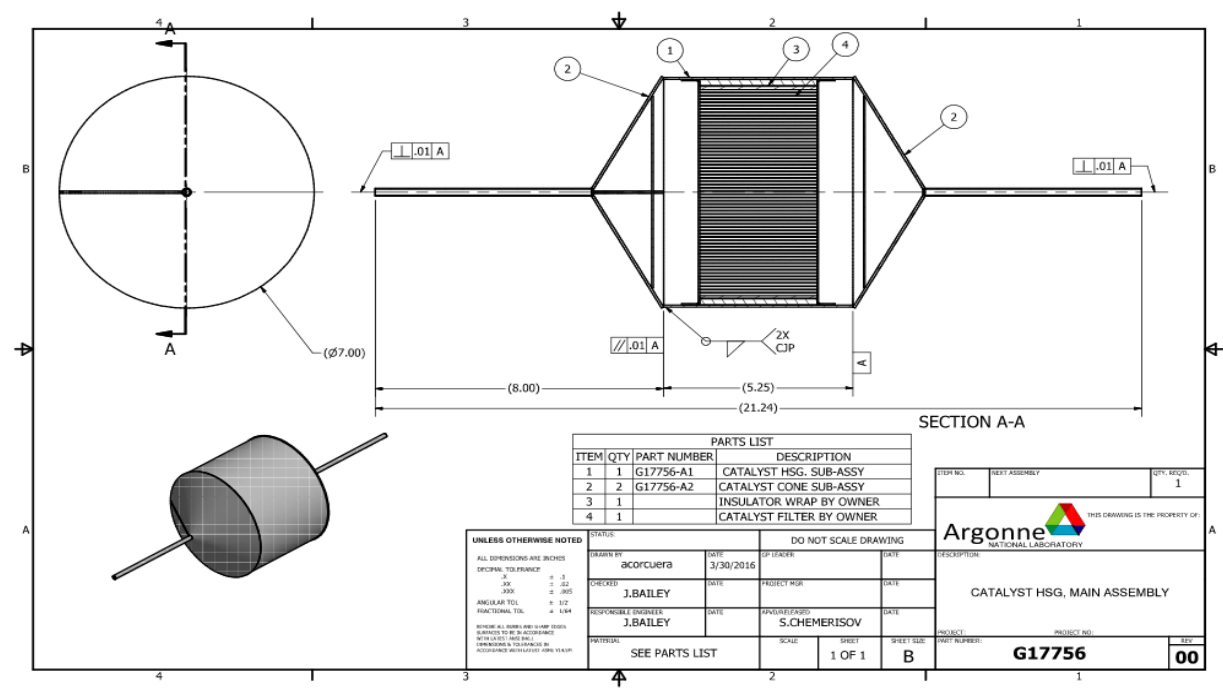

FIGURE 2.2.2.2 Catalytic recombiner design. (1) cylindrical part of the catalyst housing; (2) conical reducers; (3) fiberglass layer; (4) catalyst 


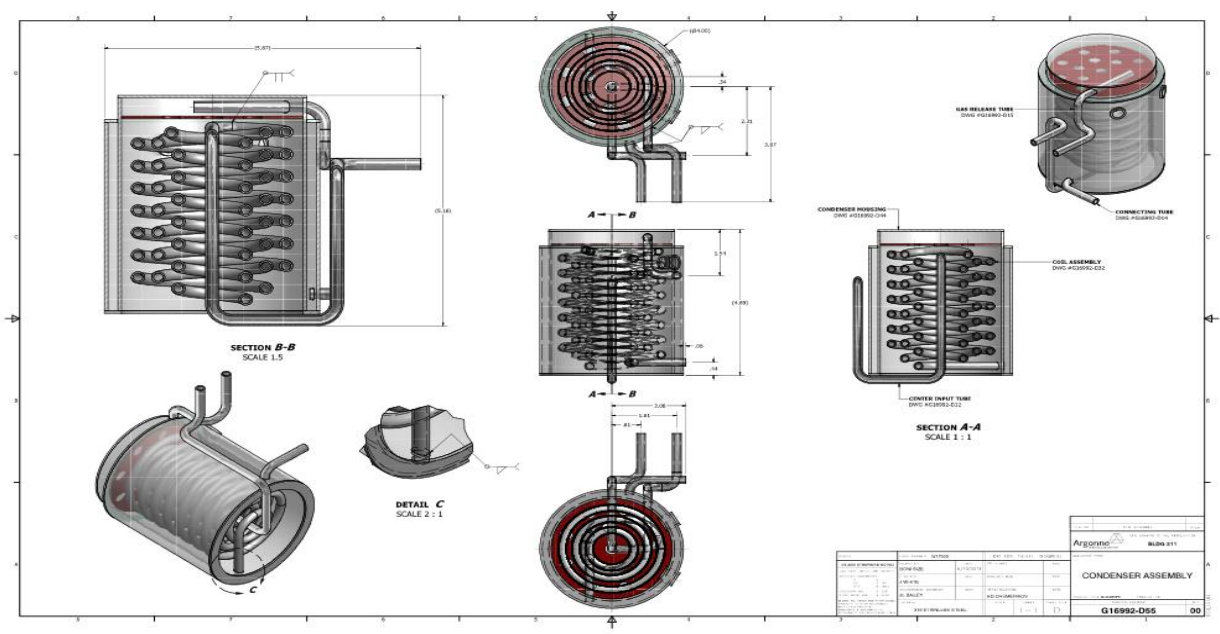

FIGURE 2.2.2.3 Condenser design

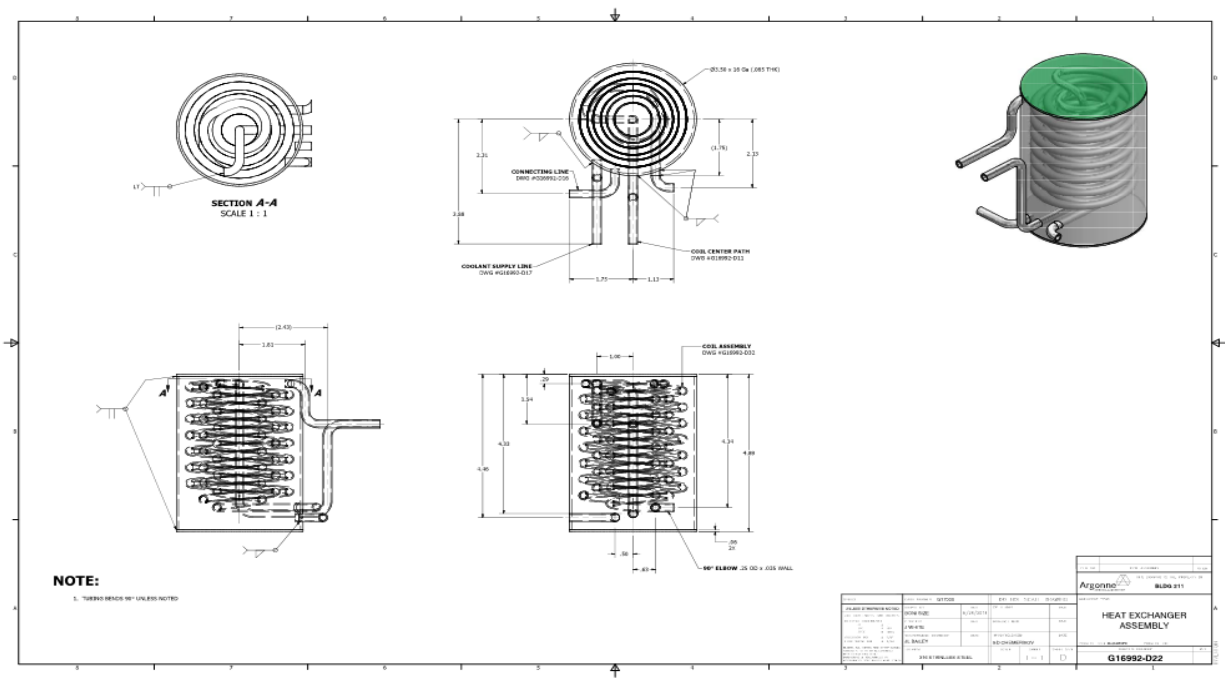

FIGURE 2.2.2.4 Heat exchanger design

Analytical instrumentation is connected to the vessel headspace by stainless steel tubing. The instruments are located inside an enclosure (Figure 2.2.2.5) in a room adjacent to the linac irradiation cell because the high radiation dose has been shown not only to interfere with gas measurement but also to cause total failure of any equipment containing sophisticated electronics. A metal bellows diaphragm pump (Senior Aerospace MB-41) is used to transport the gas from the headspace of the TSV to the analytical system. The gas is pulled from the headspace, analyzed, and then returned to the vessel. Headspace gases were analyzed using two instruments: an $\mathrm{H}_{2}$ Scan hydrogen sensor and a Pfeiffer Prisma Plus QMG 220 Residual Gas Analyzer (RGA), which is equipped with a quadrupole mass filter and a secondary electron multiplier as the detector. 


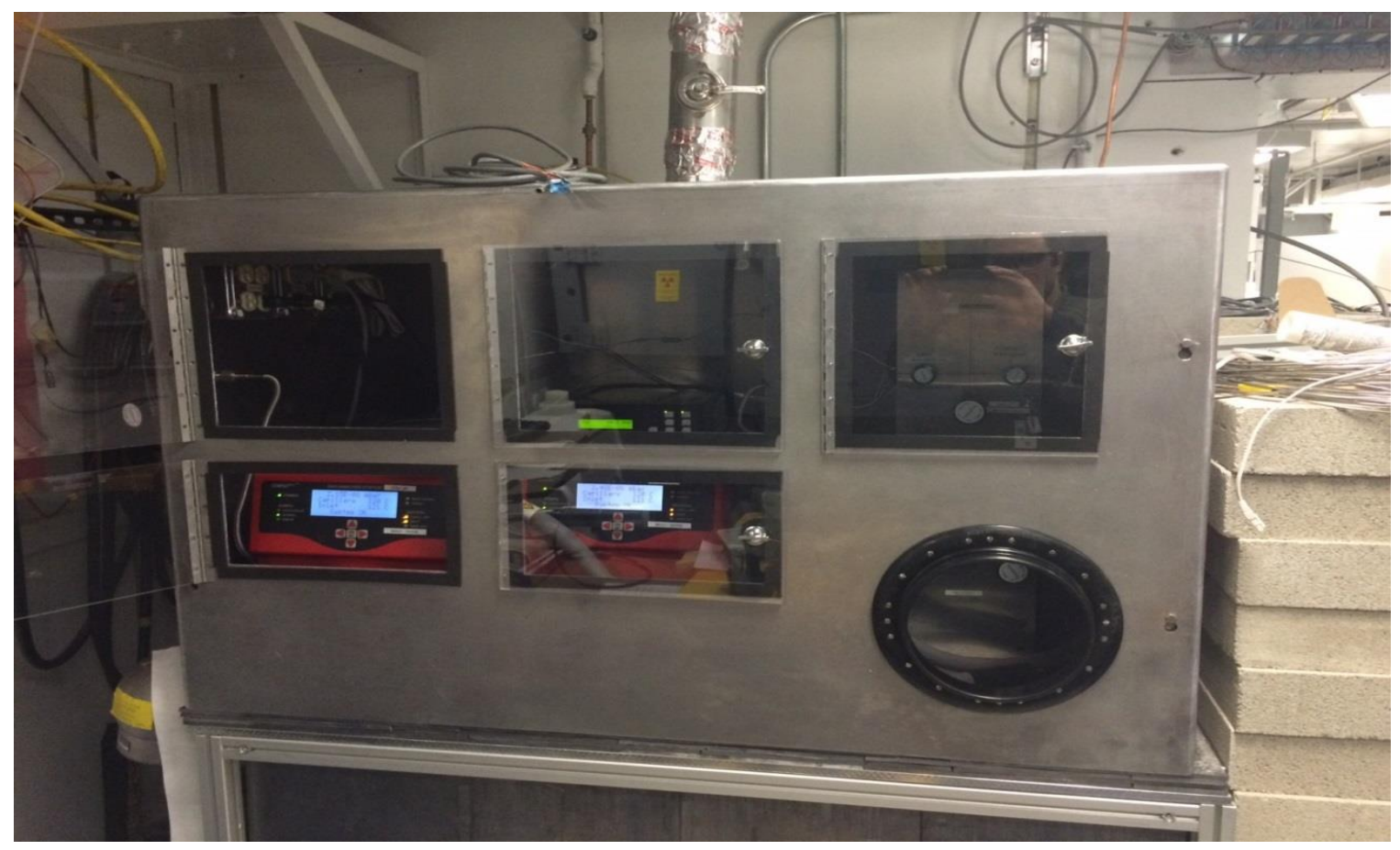

FIGURE 2.2.2.5 AMORE analytical enclosure

The GCS (Figures 2.2.2.6 and 2.2.2.7) connects to the headspace of the TSV by a single 1/4-in. stainless steel tube via the GDH. The GCS maintains the TSV at sub-atmospheric pressure, preventing the release of volatile isotopes of iodine and the fission gases xenon and krypton. It also provides an outlet for purge gas and prevents over-pressurization of the vessel. Gas from the analytical instruments, as well as purge gas and fission gas released from the ${ }^{99}$ Mo extraction process, exhaust to the GCS through the GDH. A single 1/4-in. stainless steel tube connects the ${ }^{99}$ Mo purification and recovery processes to collect fission gases released from those processes.

The GCS (Figure 2.2.2.6) consists of two chambers and two collection cylinders connected in series. Each chamber is equipped with a pressure transducer (OMEGA Engineering Model MMA030V10H3C0T3A6CE) to measure the pressure inside the chamber. The collection cylinders are also connected to a transducer (OMEGA Engineering Model

MMG5.0KV10P2C0T3A6CE) to measure their pressure. Check valves (Parker Hannifin 4ACAL-1/3-NE-SS) are installed between Chamber \#1 and Chamber \#2 and between Chamber \#2 and the collection cylinders. The check valves prevent the backflow of gas between chambers and from the collection cylinders to Chamber \#2. Later, we installed check valves with higher cracking pressure to reduce the rate of back-leak through the valves. The inlet of Chamber \#1 is connected to the TSV, the GDH, and the ${ }^{99}$ Mo purification process; the outlet connects to the inlet of Chamber \#2. Inside Chamber \#1, there is a diaphragm pump (GAST Manufacturing Corporation Model D0AP704AAEMD). The inlet of the pump is open to the chamber volume, and the outlet connects directly to Chamber \#2. Chamber \#2 has a compressor (NARDI Compressor Atlantic 100) inside. The compressor inlet is open to the chamber volume; the outlet is connected to the collection cylinders. At the inlet of the GCS, humidity is reduced by a 
water-cooled condenser. A catalyst bed further reduces the concentration of hydrogen. A cartridge containing silver-impregnated zeolite is used to trap iodine. There is a solenoid valve between Chamber \#2 and the collection cylinders. It is interlocked and automatically closes when a high pressure is detected in the chamber. A flow-limiting orifice is installed between Chamber \#2 and the collection cylinders in case of a system failure that could lead to release of the gases into the enclosure. This orifice limits the rate of gas released into the enclosure, ensuring that it can be handled by the enclosure ventilation system (50 SCFM). There is a port on the collection cylinders for sampling and releasing the collected gas. A relief valve on Chamber \#1 and a burst disc on Chamber \#2 prevent over-pressurization of the chambers. Water-cooled fans inside each chamber cool the equipment. Power relays to the diaphragm pump and compressor are controlled with OMEGA Engineering Cni8 controllers. The controllers also have relays connected to interlocks that will shut down the linac if an over-pressurization occurs. Multiple procedures address the periodic maintenance and replacement of parts in the GHS (see Appendices 9-12).

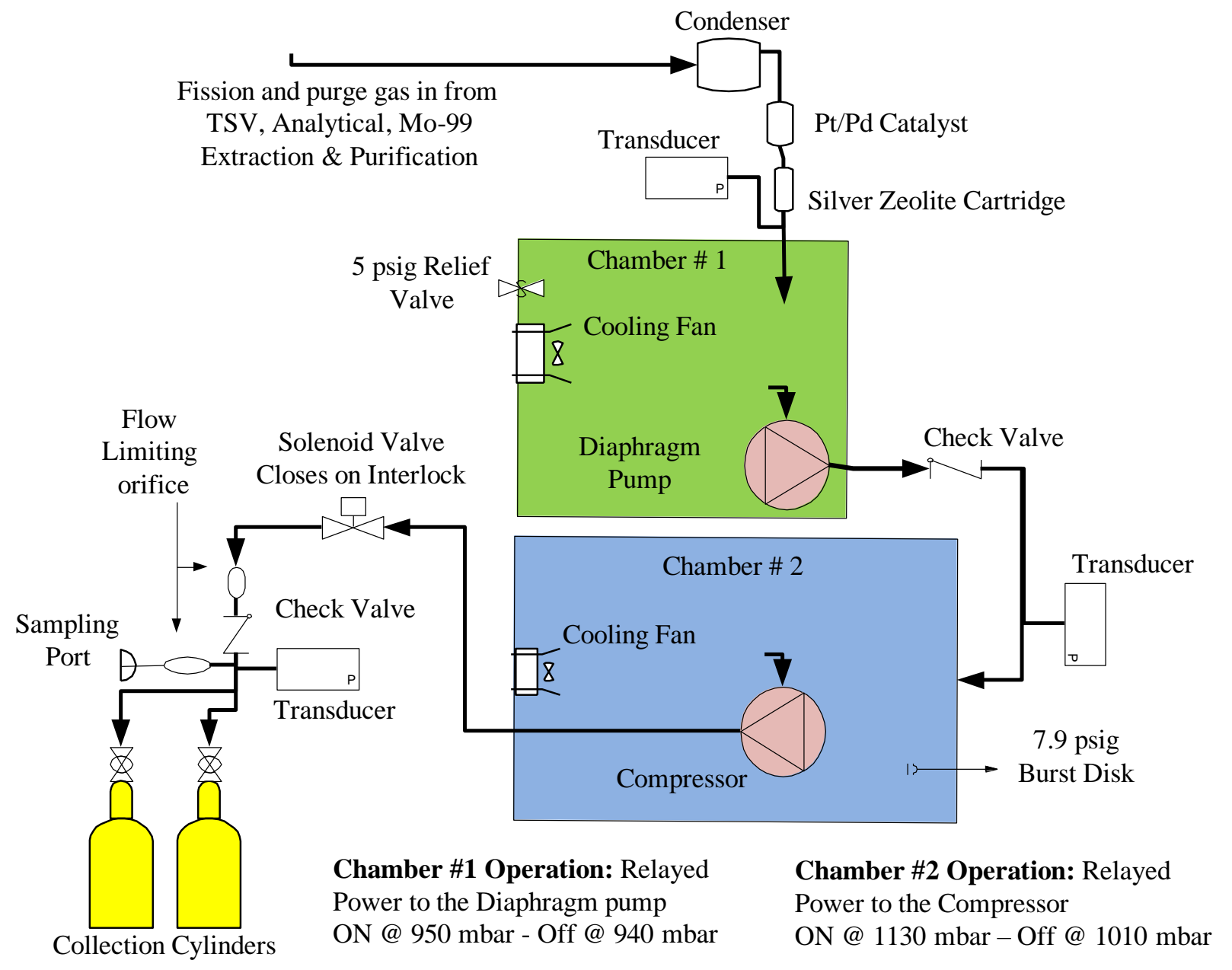

FIGURE 2.2.2.6 Diagram and function of the gas collection system 


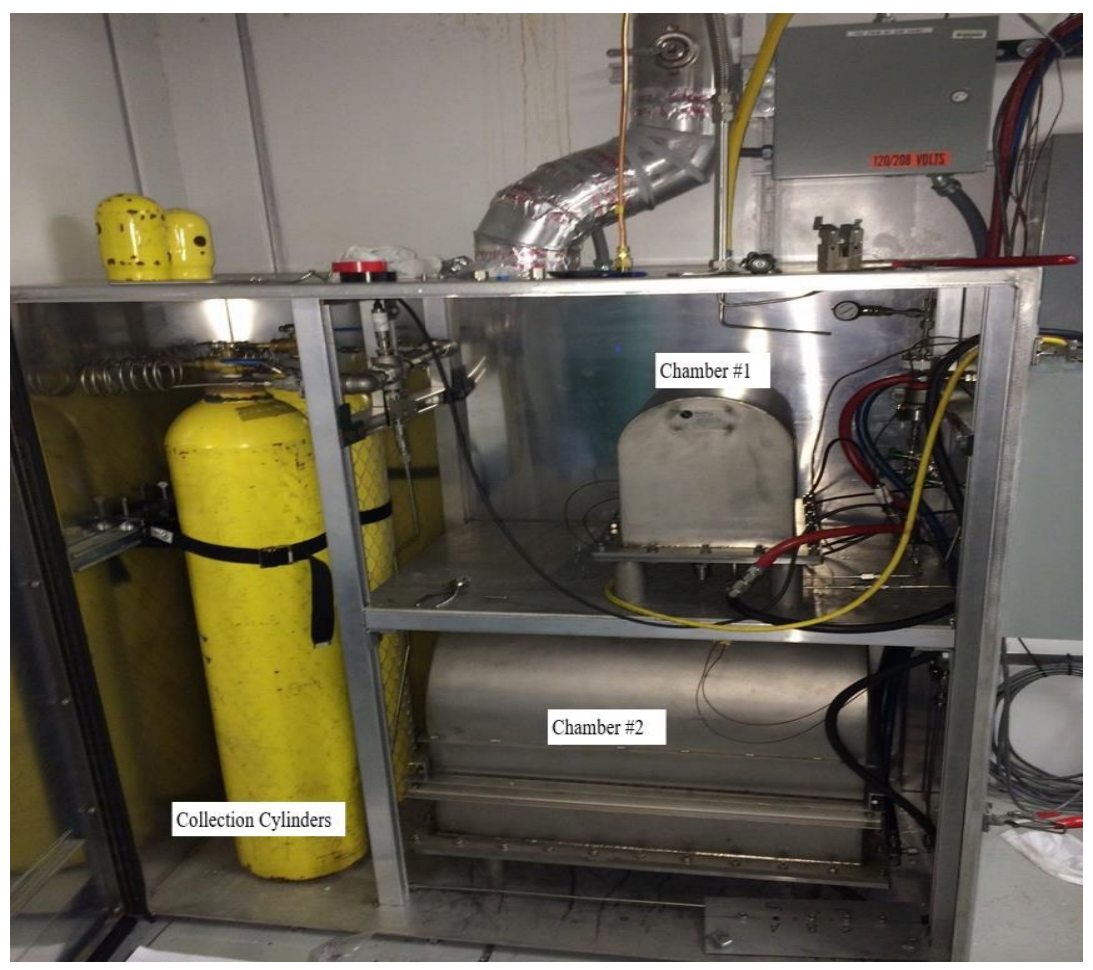

FIGURE 2.2.2.7 Photograph of the gas collection system inside the enclosure

The GCS functions to maintain all AMORE processes at sub-atmospheric pressure and to store fission gas for decay. As gas enters the AMORE system, the pressure rises in Chamber \#1. At 950 mbar, the diaphragm pump actuates and reduces the pressure in the system and pressurizes Chamber \#2. The pump turns off at 940 mbar. When the pressure inside Chamber \#2 reaches 1130 mbar, the compressor actuates and transfers gas to the collection cylinders. The compressor turns off at 1010 mbar. Check valves prevent backflow of the gas. This arrangement effectively maintains the entire AMORE system at sub-atmospheric pressure and within a narrow pressure range.

Before each experiment, the performance and configuration of the GHS is verified (see Appendices 13 and 14). The catalyst pump flow is verified, along with associated alarms and interlocks. A calibration check is performed on the analytical instruments. Interlocks and alarms associated with hydrogen concentration are verified. Instrument calibration is performed if necessary. The GCS is tested by adding helium into the system to ensure that the pumps are functioning and turn on and off at specified pressures. The alarms and interlocks associated with over-pressurization are also verified. 
During the AMORE experiment, gas is collected and stored in one of the collection cylinders while the other is kept closed. The makeup of the gas is predominantly helium, nitrogen and oxygen with small amounts of fission gas. Some of this fission gas is from a postirradiation purge of the analytical lines. The gas lines in the analytical enclosure are purged with helium immediately after each irradiation (see Appendix 8). Since the enclosure is located in a lab adjacent to the irradiation cell and has co-located AMORE activities, the lines are purged to reduce the radiation field to allow entry into the space. Specifically, the lines are purged of shortlived isotopes of xenon, which cause a significant radiation field $(>500 \mathrm{mR})$ in the lab. After an irradiation, chemical processing of the solution occurs. This step accounts for most of the atmospheric leakage into the GHS. Solution transfer, vacuum pump operation, and solution sampling all contribute to the gas collected in the GCS.

Inside the collection cylinders, the short-lived isotopes of xenon quickly decay, leaving

${ }^{133} \mathrm{Xe}$ (half-life, 5.25 days) and ${ }^{85} \mathrm{Kr}$ (half-life, 10.8 years). Approximately 60 days (10 half-lives of ${ }^{133} \mathrm{Xe}$ ) after a cylinder is filled, a sample is taken and analyzed by gamma spectroscopy to determine the concentration of radioactive isotopes (see Appendix 15). The gas is then carefully released to the atmosphere (see Appendix 16).

\subsubsection{References}

[1] Spinks, J.W.T., and Wood, R.J, An Introduction to Radiation Chemistry. John Wiley \& Sons, Inc., New York, 1990.

[2] Kalensky, M., Youker, A., Chemerisov, S., and Brossard, T. Analysis of Radiolytically Generated Gases in Mini-AMORE Experiment, ANL/CFC-18/3, Argonne National Laboratory,2018.

[3] Youker, A.J., Chemerisov, S.D., Tkac, P., Kalensky, M., Heltemes, T.A., Rotsch, D.A., Krebs, J.F., Makarashvili, V., Stepinski, D.C., Alford, K., Bailey, J., Byrnes, J., Gromov, R., Hafenrichter, L., Hebden, A., Jerden, J., Jonah, C., Micklich, B., Quigley, K., Schneider, J., Wesolowski, K., Vandegrift, G.F., and Sun, Z., Compendium of Phase-I Mini-SHINE Experiments, ANL/NE-16/39, Argonne National Laboratory, October 2016. Available at https://publications.anl.gov/anlpubs/2017/01/131828.pdf 


\subsection{RECOVERY GLOVEBOX}

\subsubsection{Introduction}

In Phase I of the AMORE project, initial processing of the irradiated target solution was carried out in the Target Solution Monitoring Glovebox and the Molybdenum Recovery Glovebox.[1] In Phase II, these two gloveboxes were combined into the "Recovery Glovebox" discussed here. The recovery glovebox was operated according to LEAF-PROC-024 (see Appendix 17). The primary purpose of recovery glovebox operations was to load freshly irradiated LEU uranyl sulfate solution (140 g-U/L) on a chromatographic column packed with titania sorbent and to carry out the initial separation of ${ }^{99}$ Mo from the bulk uranyl sulfate target solution containing fission products. Once the initial separation was completed, the ${ }^{99}$ Mo product was pumped directly to the hot cell, where it was further processed using the concentration column and LMC processes discussed in Sections 2.4.1 and 2.4.2 below.

In addition to combining the two gloveboxes from Phase I, the recovery glovebox implemented several changes based on observations and experience from operating the Phase I system and requirements from scaling up the operation. Owing to the inability to obtain measurements of $\mathrm{pH}$, turbidity, and conductivity during Phase I, these sensors were removed from the recovery glovebox. Another lesson learned from Phase I was that the system needed to be split so that acid and base solutions stayed in separate systems to prevent cross-contamination of the feed and effluent and to prevent unnecessary dilution/neutralization of the target solution. As a result, the Phase II system had an acid side and a base side, each with its own feed pump, sampling ladders, feed vessels, effluent vessels, and piping. The only point where the acid and base lines overlapped was the recovery column. Finally, owing to the much larger volume of solution being processed (20 L in Phase II vs $5 \mathrm{~L}$ in Phase I) and the much higher activities generated during these irradiations, the recovery glovebox was built using 2-in.-thick carbon steel, and liquid lines, wherever possible, were made of $1 / 4$ in. O.D. $316 \mathrm{~L}$ stainless steel tubing to provide extra shielding. In the areas where flexible tubing was required, fluorinated ethylene propylene (FEP) tubing of similar O.D. was used. The effluent collection vessels, verification tank, and column also required additional shielding because of the scale-up.

\subsubsection{Experimental Setup}

\subsubsection{Operation of the Glovebox}

The operation of the recovery glovebox is extremely complicated and is described in its entirety in LEAF-PROC-024 (see Appendix 17). A brief description of glovebox operations is included here to give an idea of the process steps involved. Several steps had to be undertaken before an irradiation could start. The first of these was removal of the spent column. During the previous irradiation and processing, fission and activation products built up in the column, so it was removed - inside its shielded pot—-from the system and stored until radiation levels abated. Once the column was removed, the verification tank was put in its place and the uranyl sulfate 
target solution was pumped to it to verify its mass and take a sample. By measuring the mass of the entire target solution when it was pumped into the verification tank and the mass of the target solution sample taken from the verification tan can determine the total volume of solution in the system via the density of the sample, which could be measured directly. This information was important, as the total volume of target solution was one of the parameters bound by the Accelerator Safety Envelope (ASE). After analyzing the sample taken from the verification tank, adjustments were made to the target solution to ensure that it adhered to all the ASE parameters (discussed in Section 2.3.4). This is also the time when stable Mo and Fe were introduced into the system. Stable Mo was required as a carrier for the LMC process (discussed in Section 2.4.2), and $\mathrm{Fe}$ was required to prevent the formation of uranyl peroxide precipitate during and after irradiation. Once the target solution was within the ASE parameters and the Mo and Fe had been added, the target solution was pumped into the TS, where it would stay until irradiation. Next, the verification tank was removed and replaced with a new packed, shielded column. At this point, the processing feed bottles were refilled with acid, water, and base solutions, and the effluent cart containing seven separate empty bottles to receive waste and raffinate was installed. Finally, the lines in the glovebox were primed with solution and the column was leak-checked before an irradiation could commence.

During irradiation, the system was monitored remotely. Initially, the target solution was circulated from the TSV through a mixing pathway that did not go through the column, then returned to the TSV during irradiation. This practice was terminated when it was realized that it would not be possible to re-engage the pump if it was stopped by a tripped leak sensor or pressure interlock until the linac was cleared of the radiation hazard (requiring several hours). Instead, solution was circulated for three hours after the irradiation was complete to ensure that a homogenous solution was loaded on the column. Some mixing was achieved during the irradiation through convection and bubble formation caused by radiolysis.[2]

Following irradiation and mixing, the circulation flow path was cleared of target solution by pumping it into the TSV while pulling in glovebox atmosphere from the surge vessel. Once this step was completed, the titania column was conditioned with $\mathrm{pH} 1 \mathrm{H}_{2} \mathrm{SO}_{4}$ and the heaters serving the acid line and column were turned on. Once the acid line and column were at temperature, the column was loaded by pumping the target solution from the TSV through the column with the column effluent directed to the dump tank, where it was stored for the remainder of the process. After loading, the column was washed with additional acid and then water to remove weakly retained contaminants, with both washes directed to their respective bottles (post-load acid wash and post-load water wash) in the effluent cart. Following these steps, the base-side lines were primed, the base line heater was turned on, and the acid line heater was turned off. At this point, the column was stripped using $1 \mathrm{M} \mathrm{NaOH}$. This solution was sent directly to the hot cell used for concentration column operations. The column-stripping step was followed by rinsing the column and base lines with water, then rinsing the acid lines with acid while bypassing the column. When these steps were complete, the glovebox portion of solution processing ended.

During any part of the irradiation, mixing, and processing steps, samples could be taken using in-line remote sampling ladders. Once the residual radiation in the irradiation cell had abated, these samples were retrieved from the glovebox and sent for analysis. Over the course of 
the project, these sample ladders began to malfunction, and an alternate sample loop was installed and used for the final irradiation (discussed in Section 2.3.3.2). Once samples were recovered, the target solution could remain in the dump tank or be pumped back up to the TSV for storage until its properties needed to be measured/verified prior to the next irradiation. After these steps were complete, the acid side of the system was washed with acid and the base side of the system was washed with water to ensure that any components of the irradiated solution were removed from the system. This step was followed by purging the system with $\mathrm{N}_{2}$ to ensure that all the acid and water from the rinse solutions had been removed from the system as well. Following the final wash and $\mathrm{N}_{2}$ purge, the glovebox system was considered reset and was left idle until preparations for the next irradiation began.

\subsubsection{Glovebox Description}

The glovebox as a whole, seen in FIGURE 2.3.2.2.1 Image of the recovery glovebox with access port on the left. The three main windows and three cabinets discussed are to the right of the switch panel in the image., is composed of a shielded access port with a small window on the far left, with an electric-motor-powered door on the inside separating the main glovebox cavity, which stretches across the remainder of the box (to the right of the switch panel in Figure 2.3.2.2.1. The main cavity of the glovebox can be roughly divided into thirds, according to the placement of the three large windows and cabinets. Figure 2.3.2.2.2 is a detailed schematic of the glovebox main cavity and the cabinets below it. Furthest to the left is cabinet \#1, which contains the recovery column or the verification tank, depending on the operational step (illustrated with column in place). In the glovebox above cabinet \#1 are the column pre-heaters, as well as the connections for the verification tank and for the column itself, along with associated solenoid valves. In the middle section is cabinet \#2, which contains the feed cart with all the feed solution containers and a balance to monitor solution levels. In the glovebox above cabinet \#2 are both Fluid Metering, Inc. (FMI) pumps used to move solution, pressure and flow sensors for each side of the system, and the surge tank (used to catch the target solution if the system malfunctioned). Furthest to the right is cabinet \#3, which houses the shielded effluent cart with all seven of the effluent containers and a balance to monitor solution levels. In the glovebox above cabinet \#3 are the inlet and outlet connections to the TSV, the outlets to the various effluent bottles, the outlet to the dump tank, the three sets of sample ladders (one each for target mixing, column loading, and column stripping), and all of the sample pots. The engineering drawing in Figure 2.3.2.2.2 does not illustrate the surge tank or the sample ladders, as these were behind a shielded lead curtain in this drawing. It was later determined that this shielding was not necessary, and it was therefore not installed. These structures are shown in the top-view engineering diagram found in Figure 2.3.2.2.3. A piping and instrumentation diagram illustrating how the entire system is connected is found in Figure 2.3.2.2.4. The shielded verification tank replaced the column in cabinet \#1 prior to each irradiation while the target solution parameters were obtained, as discussed above and in Section 2.3.4 The external nitrogen tank was connected to the system to purge the liquid lines during the washout process, as described above. When utilized, the external $\mathrm{N}_{2}$ tank was attached to the glovebox through valve V-2038. Pipe P-305 was then attached to either valve V-2001 or V-2033 to provide gas for purging the acid lines or base lines, respectively (see Figure 2.3.2.2.4). 


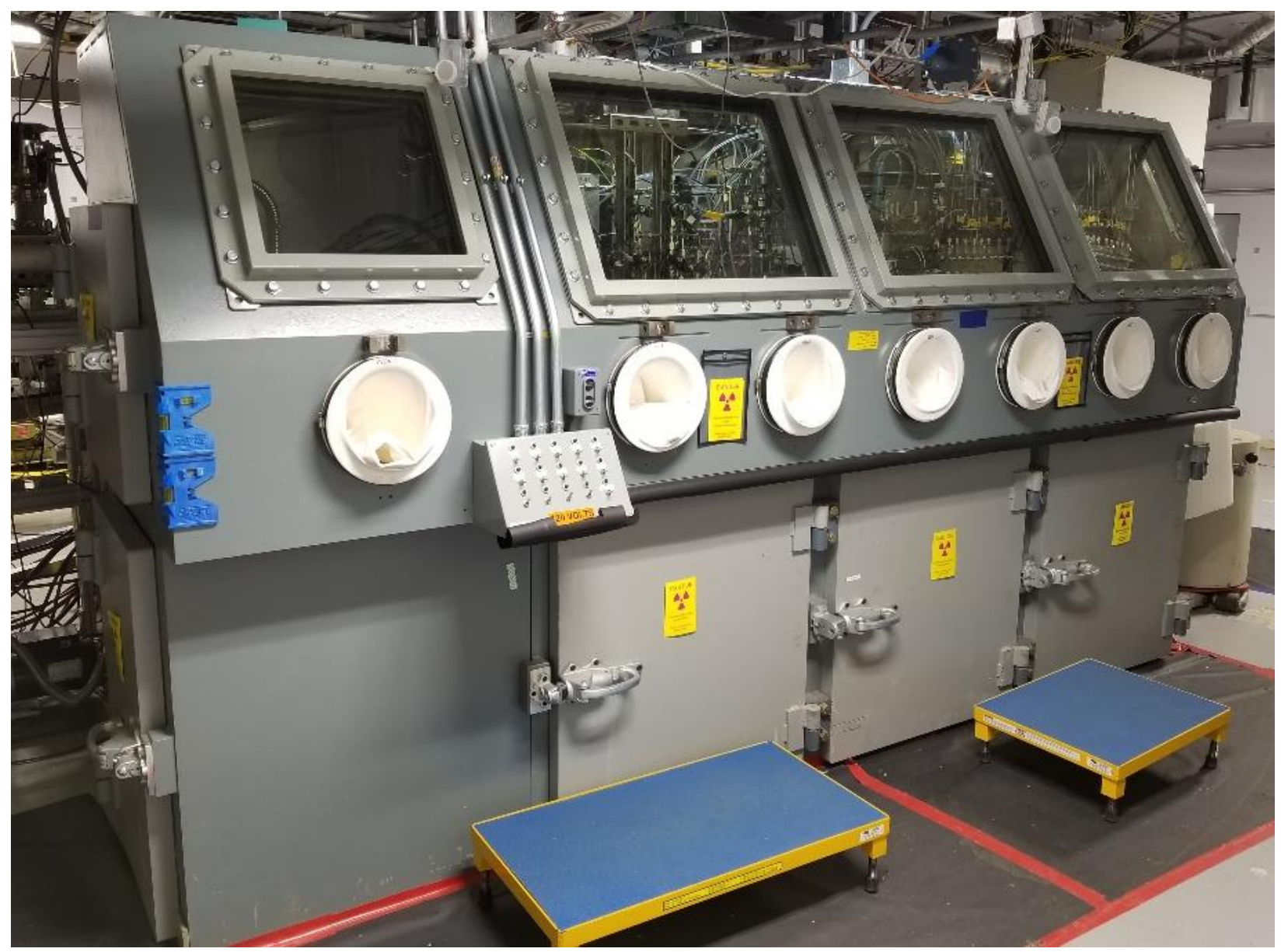

FIGURE 2.3.2.2.1 Image of the recovery glovebox with access port on the left. The three main windows and three cabinets discussed are to the right of the switch panel in the image. 


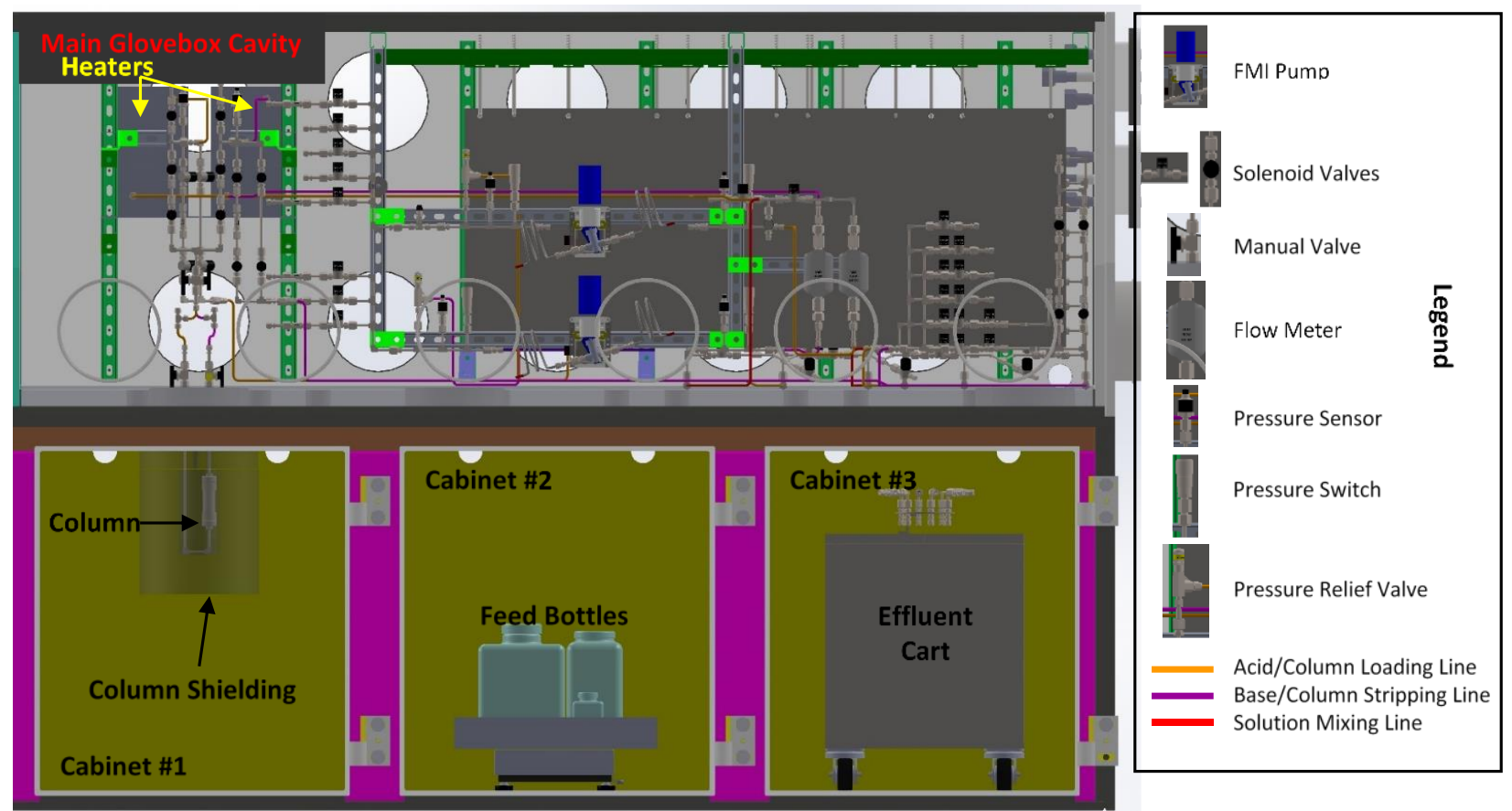

FIGURE 2.3.2.2.2 Engineering diagram of the glovebox main cavity and cabinets below

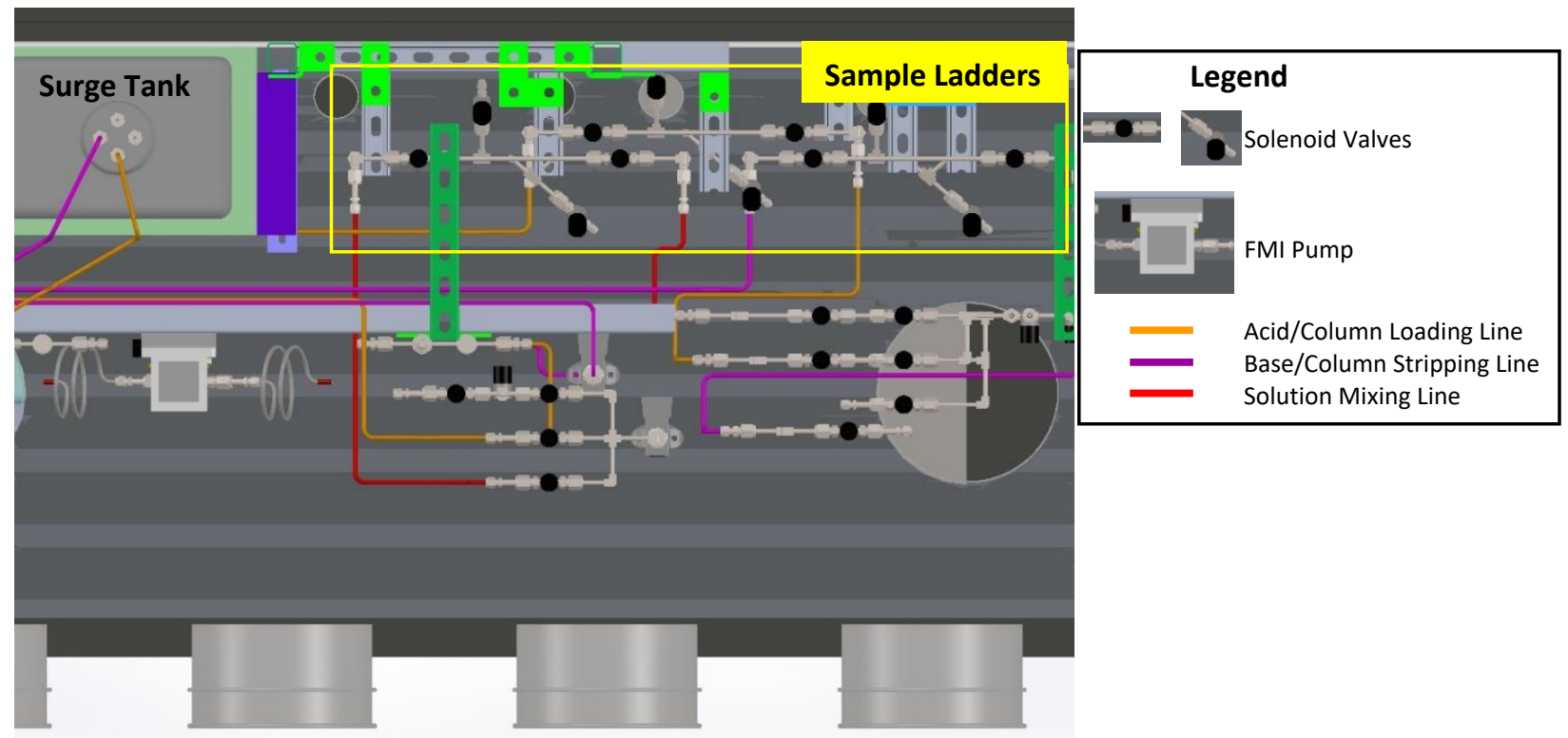

FIGURE 2.3.2.2.3 Engineering diagram top view above cabinet \#2 and cabinet \#3, showing the locations of the surge tank and sample collection ladders 


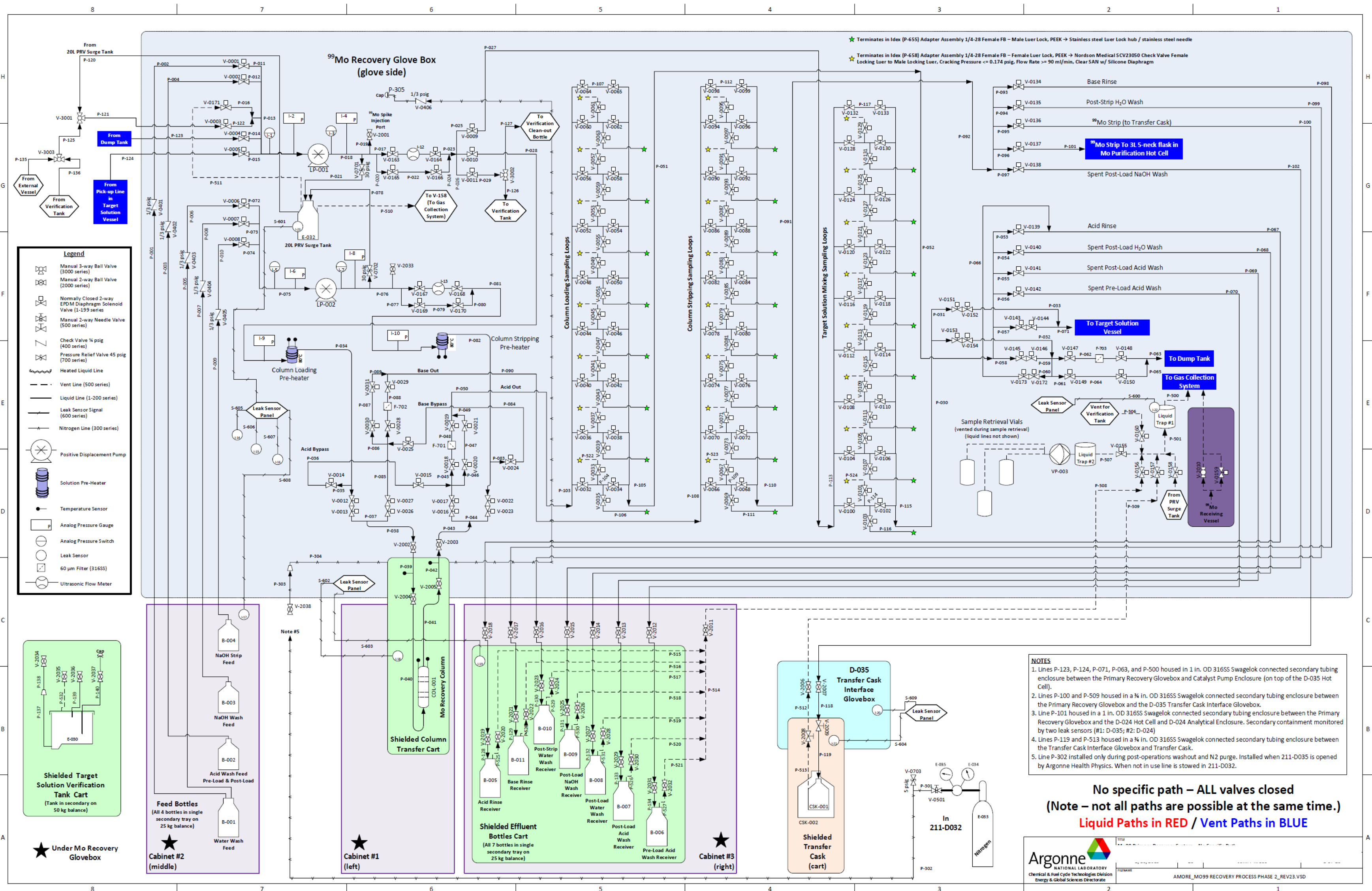

FIGURE 2.3.2.2.4 Piping and instrumentation diagram for the recovery glovebox 


\subsubsection{Recovery Column}

Like the rest of the recovery glovebox, the primary separation column itself was also scaled up to handle the larger volume of solution and additional ${ }^{99}$ Mo that needed to be retained.[2] The column is a $40 \mathrm{~mm}$ I.D. x $100 \mathrm{~mm}$ long Macherey-Nagel VarioPrep constructed of $316 \mathrm{SS}$ and using Viton O-rings and $40 \mu \mathrm{m} 316 \mathrm{SS}$ frits. The column was packed with titania resin from ZirChrom (Sachtopore-NP, $60 \AA$ A, $110 \mu \mathrm{m}$ size), which was acid washed with $\mathrm{Ph} 1$ $\mathrm{H}_{2} \mathrm{SO}_{4}$ at room temperature prior to use to remove fines and otherwise leachable contaminants, following the method developed in Section 5.5 of Reference [3].

To pack the column, the bottom half was assembled to the specified dimensions (see Appendix 18) and clamped in a ring stand. A beaker was placed under the column to collect water as it drained through, and a mark was placed on the inside of the column at the desired bed height (4.25 in. tall). The previously washed resin was slurried with water and poured into the column, allowing excess water to drain out the bottom while keeping a small head of water over the resin bed. Once the bed reached the desired bed height, the column bottom was capped and the top frit and collar were installed and hand-tightened. Once assembled, the column was moved to a vise and the top collar was further tightened using a pipe-wrench, ensuring that the top cap was removed to allow for water displacement and that the column inlet and outlet were pointing in the same direction during tightening. The overall length of the column from collar end to collar end when tightened was 6.75 in. Images of a fresh column are found in Figure 2.3.2.3.1. Once assembled, water was circulated through the column in each direction for 1-2 hours to ensure that any remaining fines were removed. As a leak check, the column was capped and the pressure was raised to approximately 35 PSI for 1 hour, checking periodically for pressure decreases and minor leaks. Once the column was determined to be leak-tight, the remaining piping, valves, thermocouples, heat tape, and insulation were added to the column prior to installation. A completely assembled column, ready for installation, is shown in Figure 2.3.2.3.2 along with the carbon-steel-encased lead-shielded pot it was placed in. 


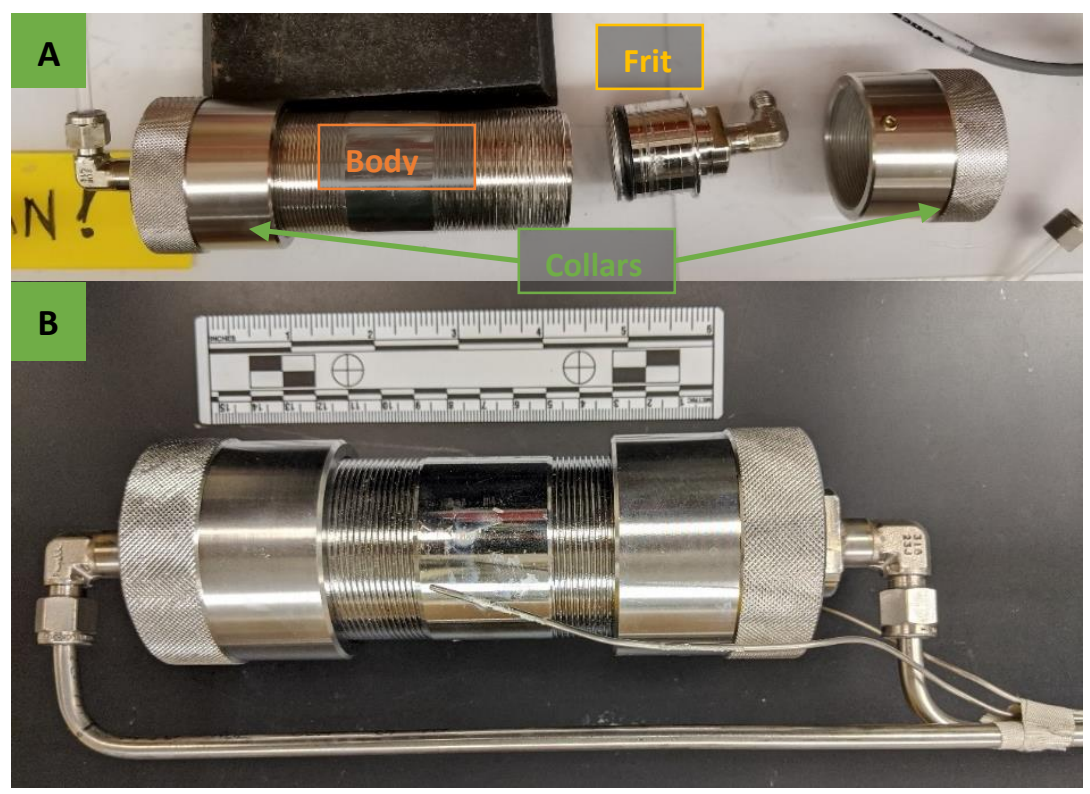

FIGURE 2.3.2.3.1 Images of the recovery column A) prior to assembly with frit and top collar removed and B) after filling and assembly, prior to installation in the recovery glovebox

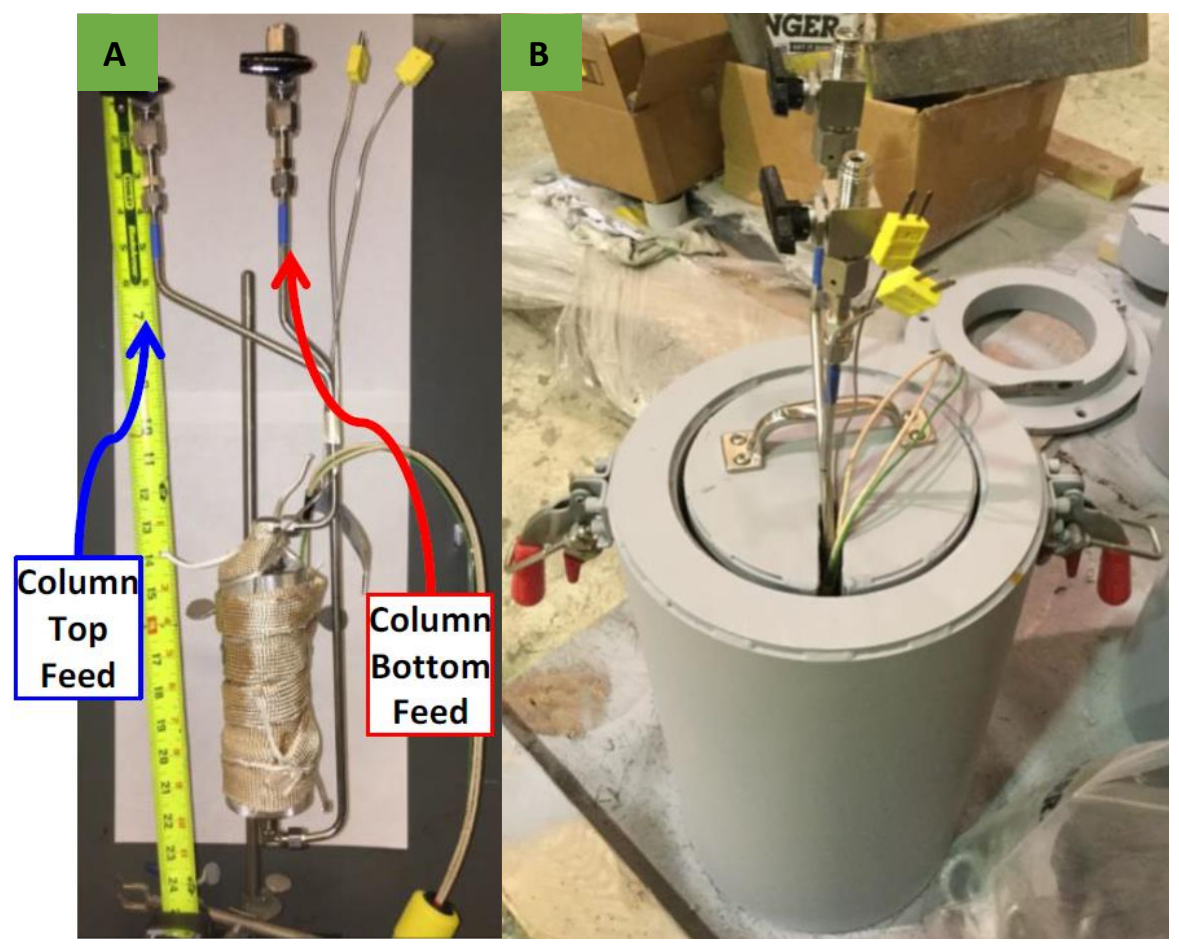

FIGURE 2.3.2.3.2 Images of the recovery column (A) wrapped in heat tape and insulation and $(B)$ installed in the lead-shielded pot 
The column and carbon-steel-encased lead-shielded pot were placed in shielded cabinet \#1 under the glovebox, where the column was coupled to the system through a port in the glovebox floor. Images of the column attached to the rest of the system can be seen in Figure 2.3.2.3.3. To ensure minimal delay between irradiations, multiple column pots were kept on hand so a spent column could be removed and fission/activation products allowed to decay in its pot while a new column was put in place. During normal operations, all column activities using the acid-side system (pre-load acid washing, column loading, post-load acid washing, and post-load water washing) were carried out in the upflow direction (solution flowing from bottom to top of the column bed), while all base-side system column activities (column stripping and post-strip water wash) were carried out in the downflow direction (from top to bottom of the column bed). All acid solutions were $\mathrm{pH} 1 \mathrm{H}_{2} \mathrm{SO}_{4}$ (including the uranyl sulfate target solution) and all base solutions were $1 \mathrm{M} \mathrm{NaOH}$. Adsorption of Mo on the titania column was performed at $80^{\circ} \mathrm{C}$ with the temperature monitored using K-type thermocouples at the inlet and outlet of the column, as well as on the column body itself. The optimal temperature in which the highest $\mathrm{K}_{\mathrm{d}}(\mathrm{Mo})$ values would occur was previously determined to be $80^{\circ} \mathrm{C}$.[4]

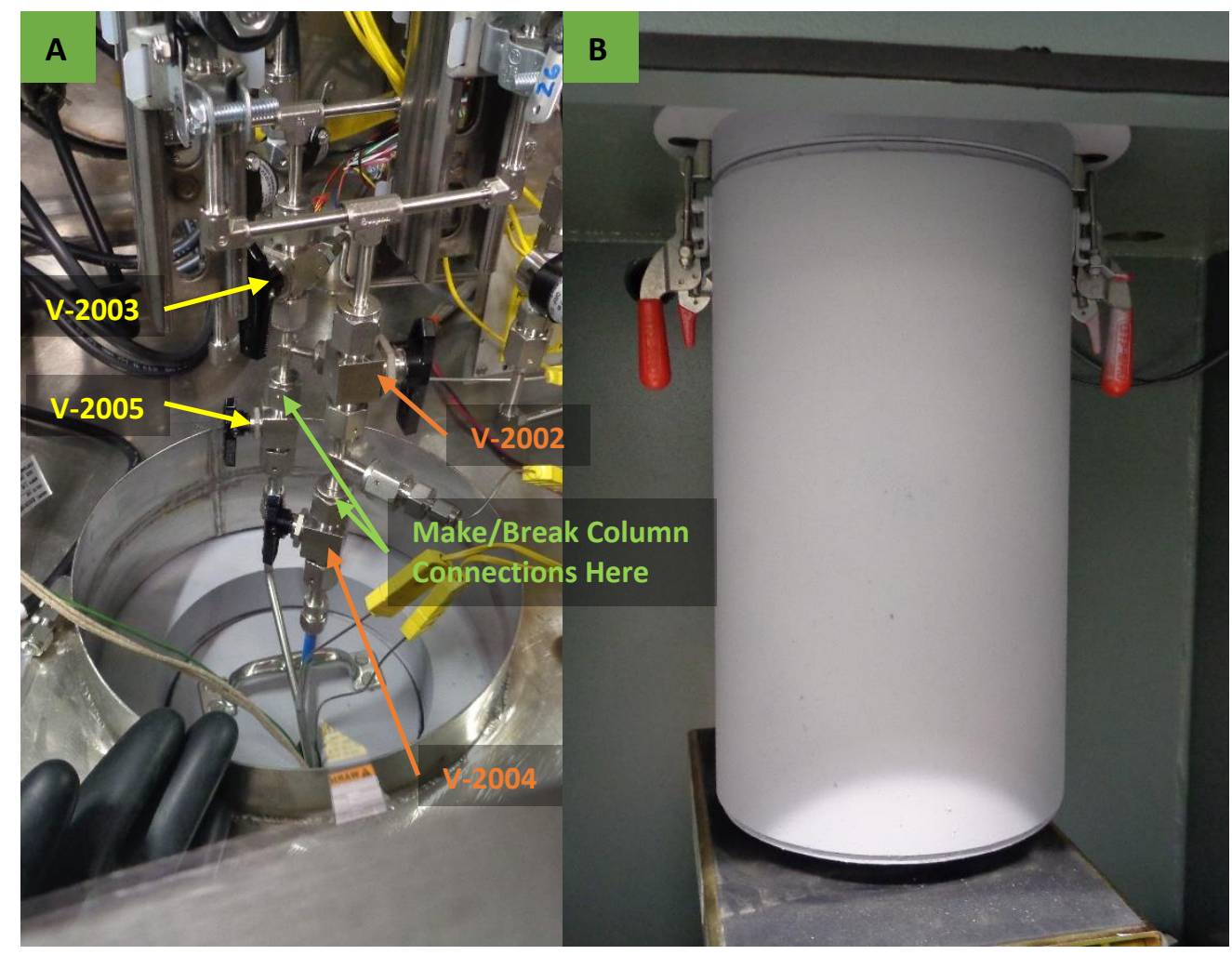

FIGURE 2.3.2.3.3 Recovery column in lead-shielded pot attached to the rest of the system, viewed (A) inside glovebox and (B) inside cabinet \#1 


\subsubsection{Fluid Handling}

Solutions introduced to the system were held in one of four feed bottles in Cabinet \#2 under the glovebox. The acid feed bottle contained $\mathrm{pH} 1 \mathrm{H}_{2} \mathrm{SO}_{4}$ used in conditioning the column, post-load acid washing of the column, and washing of the acid lines in the system after processing. The water-wash feed bottle contained $18 \mathrm{M} \Omega \cdot \mathrm{cm}$ deionized water, which was used in the post-load and post-strip water washes of the column and in washing the base lines after processing. The $\mathrm{NaOH}$ strip feed and $\mathrm{NaOH}$ wash feed bottles both contained $1 \mathrm{M} \mathrm{NaOH}$. The $\mathrm{NaOH}$ wash feed bottle would have been used during an optional post-load $\mathrm{NaOH}$ wash of the column, but this step was never opted for. The $\mathrm{NaOH}$ strip feed was used for priming the base lines and stripping Mo product from the column.

Solutions were transferred within the glovebox and between the glovebox and other components (TSV, dump tank, or hot cell) using positive displacement pumps (model QV-50) from FMI, which were fitted with ceramic-lined pump heads employing $3 / 8$ in. O.D. ceramic pistons in 316 SS housings (model Q2-CSC) and Rulon ${ }^{\circledR}$ AR (fluorocarbon filled PTFE) lip seals. The individual pumps for the acid and base sides of the system were controlled by independent FMI V-300 controllers. All liquid lines in the system were made of either $316 \mathrm{~L}$ stainless steel tubing or FEP tubing. The only lines in the system that were not $1 / 4$ in. O.D. were associated with the gas handling system and the sample retrieval system (discussed in

Sections 2.2.2 and 2.3.3, respectively).

\subsubsection{Dump Tank}

After the target solution was passed through the column, it was sent to the shielded dump tank, which was located directly below the TSV.

Figure 2.3.2.5.1 shows this arrangement, with lead bricks between the dump tank and TSV hot cell to shield workers from the lines connecting the two. These were arranged in an "L" shape to help reduce shine from either vessel. The TSV and dump tank were located outside the far-right end of the glovebox. The very top of the TSV hot cell can be seen on the right in Figure 2.3.2.2, behind the glovebox.

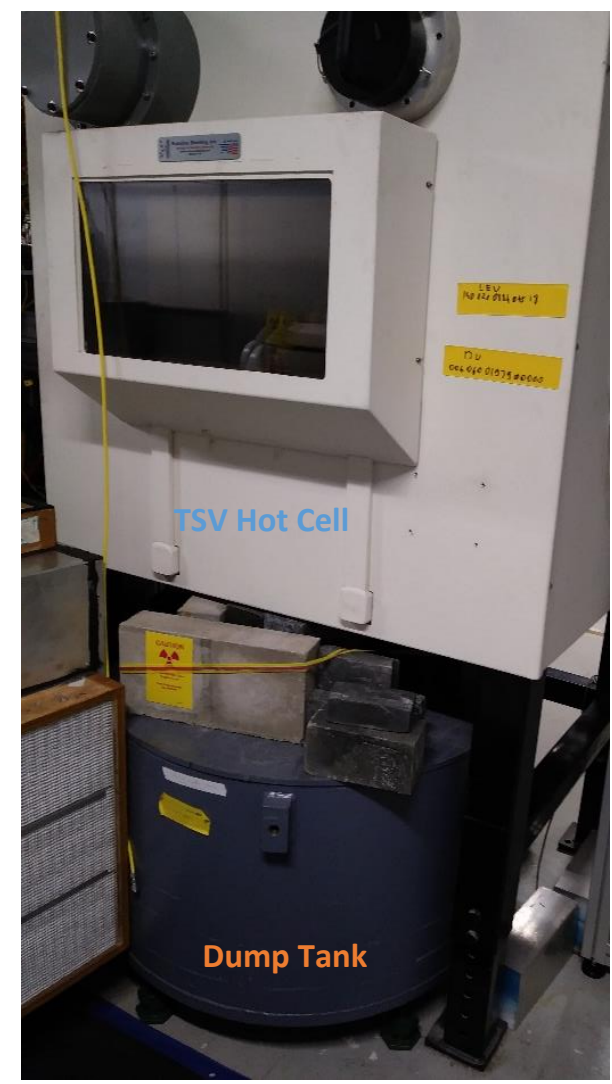

FIGURE 2.3.2.5.1 Image showing the relationship between the Dump Tank and TSV Hot Cell. These were connected via $L$-shaped transfer lines shielded by lead bricks (seen in picture). Image showing the relationship between the Dump Tank and TSV Hot Cell. These were connected via $L$-shaped transfer lines shielded by lead bricks (seen in picture). 


\subsubsection{Effluent Cart}

The effluent cart was a thick steel box (with 1-in.-thick sides) on wheels, which housed the seven effluent bottles that collected waste from processing the target solution. Four different sizes of bottles were used to collect effluent: $64 \mathrm{oz}, 1 \mathrm{gal}, 2 \mathrm{gal}$, and 5 gal. The three smaller bottles were made of polyethylene (PE) with silicone seals at the caps, while the 5-gal containers were composed of high-density polyethylene (HDPE). The seven effluent bottles were contained within a PE tray which was placed on a $25-\mathrm{kg}$ balance (Ohaus, model D25QRUS, $0.002 \mathrm{~kg}$ resolution) inside the cart. A rotating cam mechanism was installed in the bottom of the steel box to lift the effluent-bottle secondary tray off the balance during transport, when re-connecting the balance cables and establishing a connection between the balance and its indicator, and when taring the balance once the cart was positioned in its cabinet. Two scissor jacks were used to level the effluent cart once it was positioned in its cabinet, to ensure accurate balance readings. The lid of the steel shielding box was split, requiring only half the lid to be removed to replace effluent bottles. To the half of the lid that remained attached to the box, attached were two manifolds: one to connect the liquid lines from each effluent bottle to the associated effluent line in the glovebox and the second to connect the vent lines from each effluent bottle to the line running to the gas collection system (GCS). An image of the effluent cart with the manifolds in place in cabinet \#3 is shown in Figure 2.3.2.6.1. Two effluent carts were kept on hand so one could be used in processing while the other was emptied, to expedite the irradiation schedule.

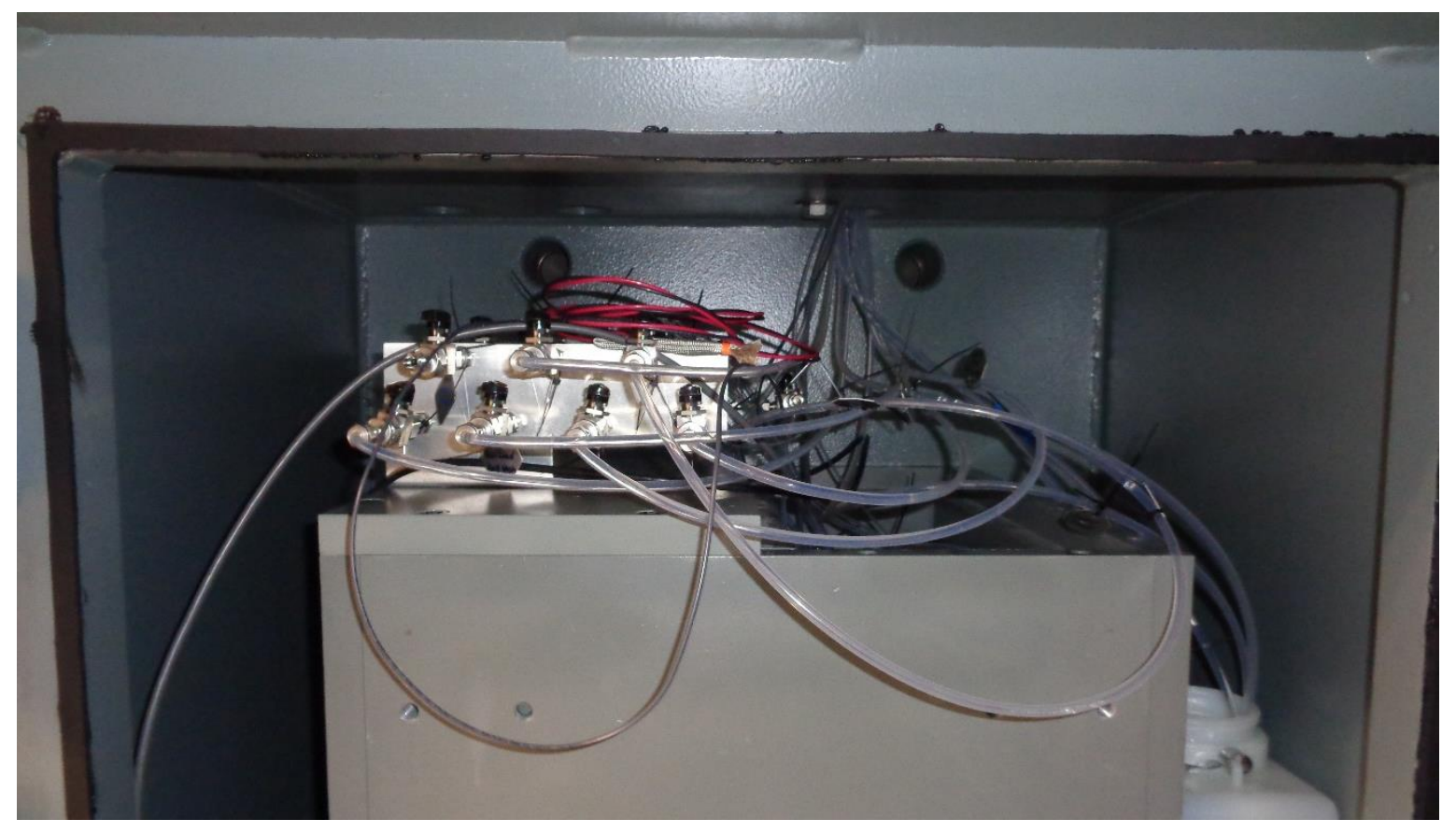

FIGURE 2.3.2.6.1 Effluent cart with lid and valve manifold on top, in place inside cabinet \# 3 


\subsubsection{Verification Tank}

The verification tank was used to determine the total mass of uranyl sulfate target solution and to take samples of the target solution to determine density, U concentration, and $\mathrm{pH}$. The determination of these parameters was critical, as the ASE specified the maximums of these parameters. This procedure is discussed further in Section 2.3.4. Verification also was one of the few processes that utilized clear FEP lines, through which the target solution could be directly viewed and inspected to ensure that it was free of precipitate. The verification tank itself was a 35 L stainless-steel vessel (Eagle Stainless, KTT-CTH-36-316L-J, slant bottom, 1.5 in. TriClamp with EPDM gasket bottom connection; the clamped lid seal was a platinum-cured silicone gasket, $10 \mathrm{Ra}$ finish) that resided on a 50-kg balance (Ohaus, model D50QLUS, $0.005 \mathrm{~kg}$ resolution) in a shielded cart with 1-in.-thick steel sides, a diagram of which is shown in Figure 2.3.2.7.1. As in the effluent cart design, a rotating cam mechanism was installed in the bottom of the steel box to lift the verification-tank secondary tray off the balance during transport, when re-connecting the balance cables and establishing a connection between the balance and its indicator, and when taring the balance once the cart was positioned in its cabinet. Two scissor jacks were used to level the verification tank cart once it was positioned in its cabinet to ensure accurate balance readings. When connected to the glovebox system, valves V-2034, V-2035, and V-2036 on the verification tank were connected to pipes P-136 (G8), P-506 (E2), and P-126 (G6) on the glovebox system, respectively. Valve V-2037 was not connected to the glovebox system, as it is the "sparge line."

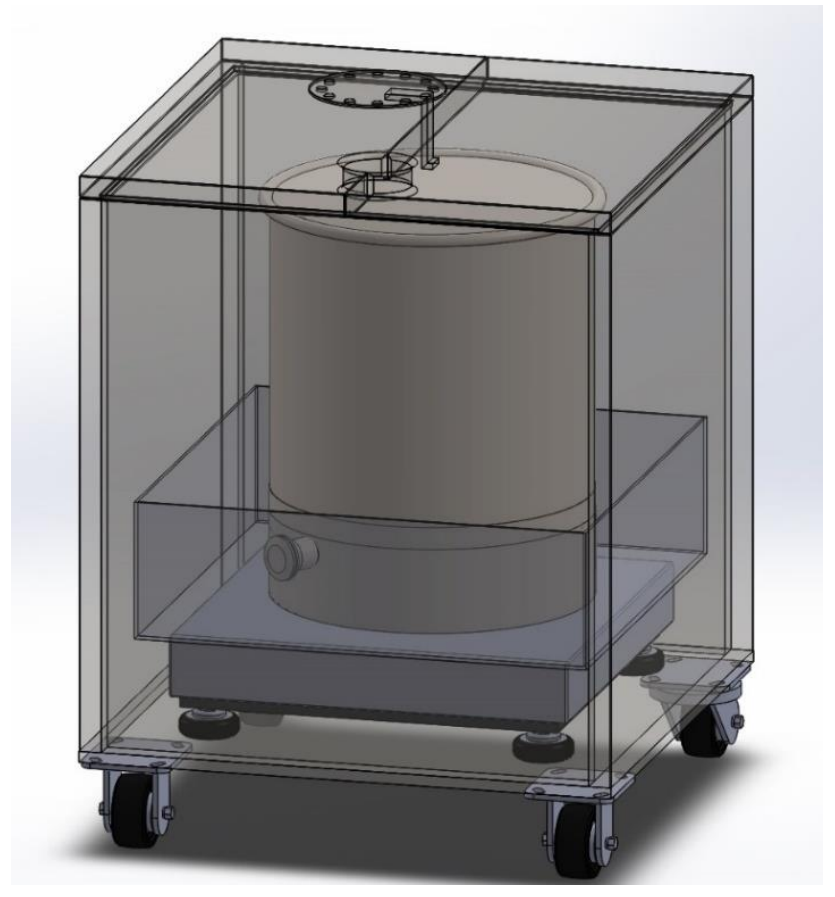

FIGURE 2.3.2.7.1 Drawing of the verification tank resting on its balance inside the shielded verification cart 


\subsubsection{In-line Sampling Ladders}

In addition to the samples taken from the verification tank prior to irradiation, it was often desirable to take samples at different points during irradiation, circulation, and processing. Owing to the high dose rates in the irradiation cell, this sampling had to be done remotely, and was accomplished using sample ladders located in the glovebox. These sample ladders were designed such that the target solution would pass through the sampling ladder "rung" until a sample was to be collected. Figure 2.3.2.8.1 illustrates the structure of an individual sample ladder rung with arrows indicating the flow path of solution. In the figure, valves V-0100 and V-0102 would be closed when a sample needed to be taken and solution would begin flowing to the next higher rung in the ladder. The other valves in the diagram, V-0101 and V-0103, were used when retrieving samples after the irradiation.

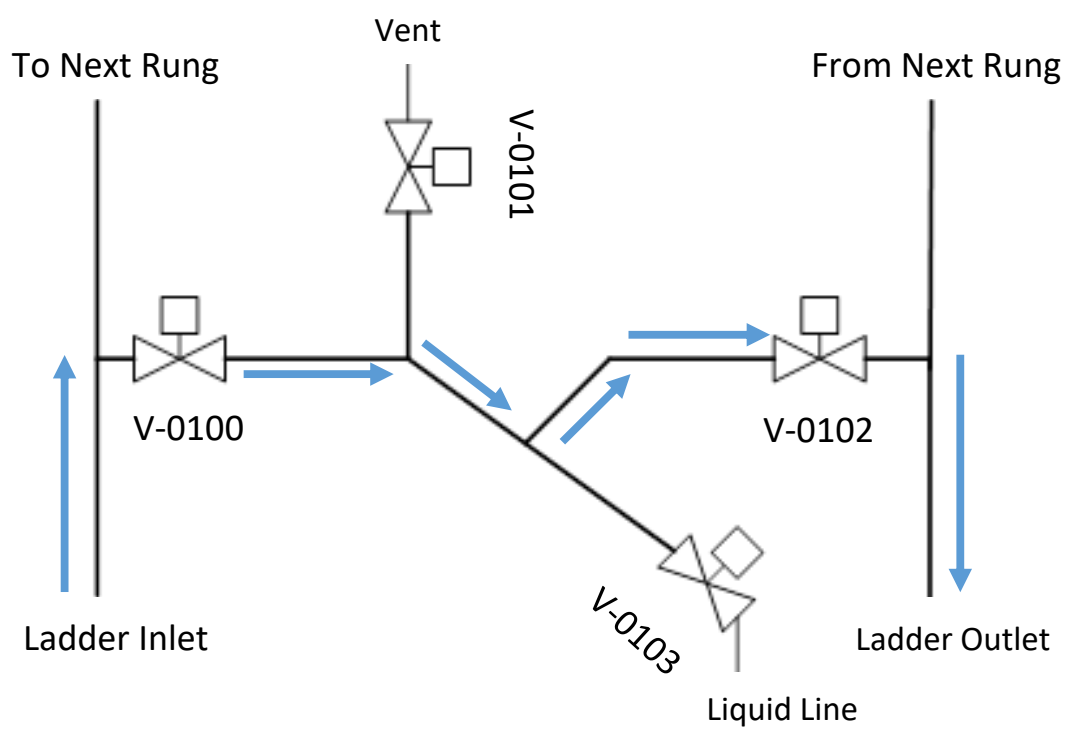

FIGURE 2.3.2.8.1 Schematic diagram of a sample ladder rung with arrows showing the flow of solution during normal operation

For the sample ladder depicted in Figure 2.3.2.8.1, V-0101 would be connected to the glovebox atmosphere through a one-way check valve (vent line), while V-0103 would be connected to a $1 / 16$ in. FEP liquid line running to the needle setup shown in Figure 2.3.2.8.2A. The concentric needles in Figure 2.3.2.8.2A penetrated through the septum of an evacuated glass vial, and the $1 / 8$ in. vent line attached to the outer syringe needle was run to a vacuum pump through a liquid trap. When a sample needed to be retrieved, valves V-0100 and V-0102 were closed while V-0101 and V-0103 (FIGURE 2.3.2.8.1 Schematic diagram of a sample ladder rung with arrows showing the flow of solution during normal operation 2.3.2.8.1) were opened and the vacuum pump was engaged to pull the sample into the evacuated vial. Each glass vial was also held in a stainless steel or tungsten sample pot for extra shielding, as samples could be highly radioactive. An image of several of the sample retrieval vials in sample pots with the concentric needles inserted can be seen in Figure 2.3.2.8.2B. 


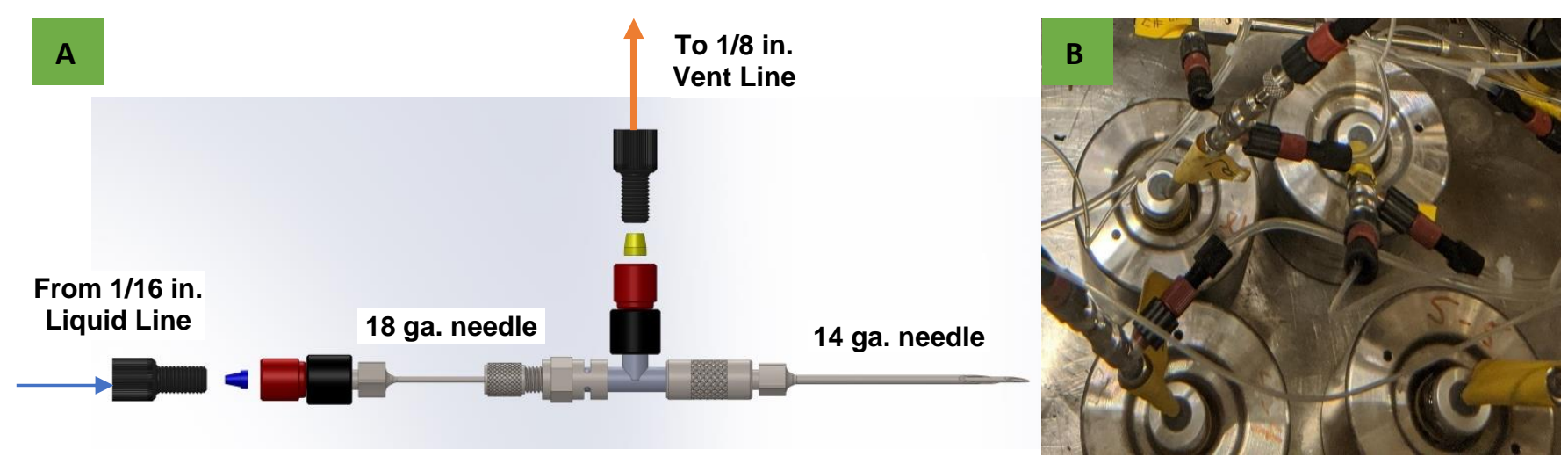

FIGURE 2.3.2.8.2 (A) Diagram of the concentric needle system used to retrieve samples from sample ladders. (B) Needle system with sample vials in sample pots in the glovebox.

\subsubsection{Remote-operation Software}

The radiation levels in the beamline cell (D035) during and directly after irradiation were much too high to operate the system manually. To give an idea of the situation, when $8 \mathrm{Ci}$ of ${ }^{99}$ Mo were produced, the dose rate at the face of the shielded glovebox three days after the end of bombardment (EOB) was $300 \mathrm{mR} / \mathrm{hr}$. This radiation was generated by target solution in the sampling loops and by activation of the glovebox components during irradiation, as the vast majority of the uranyl sulfate target solution was contained in the shielded dump tank at the time. To retrieve the produced ${ }^{99} \mathrm{Mo}$ as soon after target-solution irradiation as possible required that the primary recovery system be remotely controlled. A process control program, developed in National Instruments ${ }^{\mathrm{TM}}$ LabVIEW software, was used to remotely operate all solenoid valves and record process parameters. A total of 92 control signals were used to operate the 171 solenoid valves in the system. The LabVIEW -based process control system was designed to operate in either a manual or semi-automated mode. In manual mode, the operator had full control of all the liquid solenoid valves, while in semi-automated mode the operator simply pressed the NEXT STEP button and the appropriate solenoid valves would open and close to proceed with the next step of processing. Though the main user interface display appears complicated (Figure 2.3.2.9.1), the flow path of the liquid was highlighted in accordance with the solenoid valves that were open, making it easy for the operator to check that the appropriate step was being followed.

The program captures the temperature of acid- and base-line heaters, as well as the column temperature. The pressure is also measured at six different locations throughout the system, and data are collected on the masses of the feed bottles, effluent bottles, and verification cart (when installed), as well as on the pressures of the gas collection reservoirs and temperatures in the linac. Data are collected by the LabVIEW system every two seconds, and each data point is associated with a time stamp so data from irradiations could be reviewed later. 


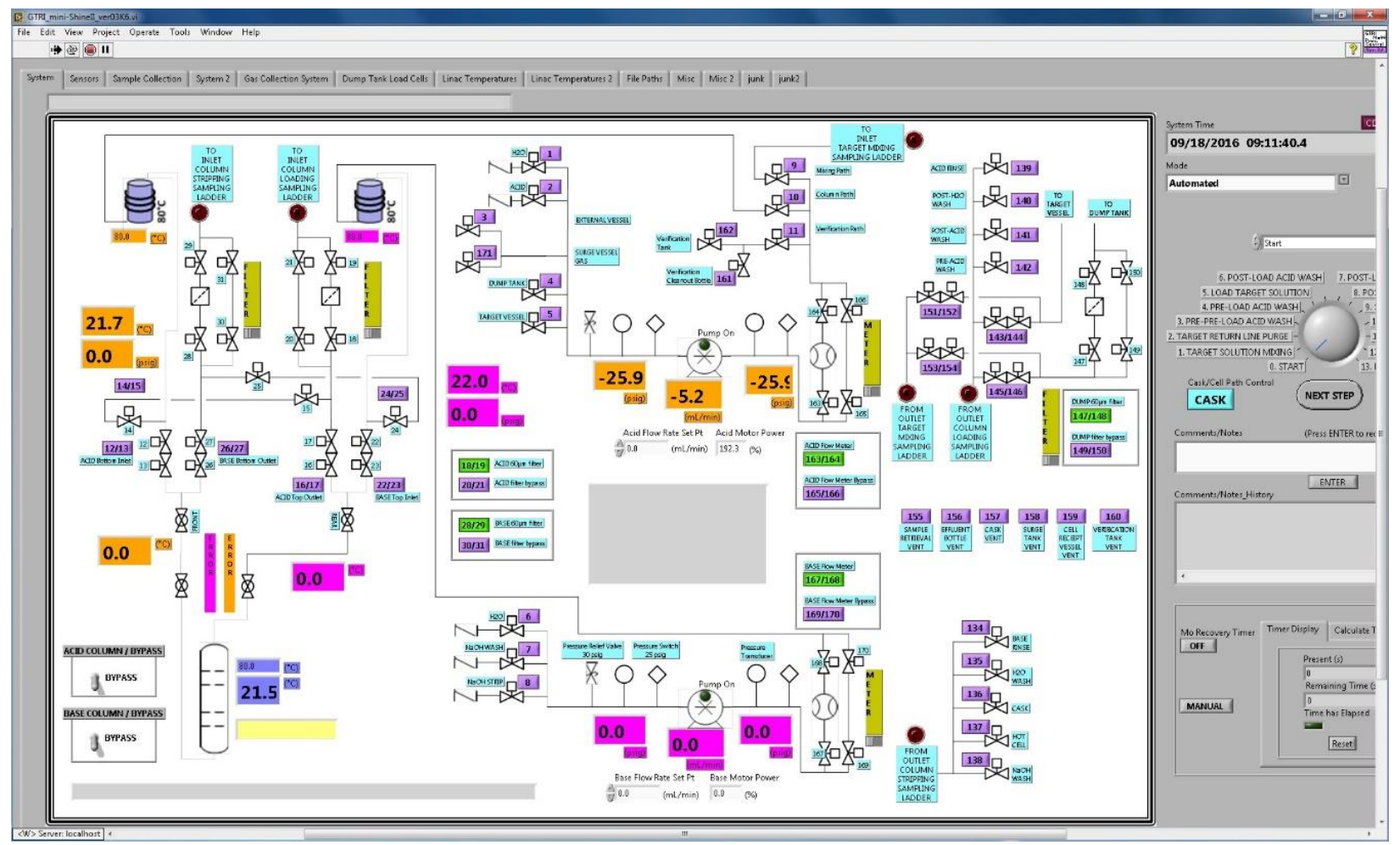

FIGURE 2.3.2.9.1 Main LabVIEW user interface for remote operation of the recovery glovebox

\subsubsection{Leak Sensors}

In addition to needing to pump solution around the system remotely, it was advantageous to be able to tell if the system was leaking at any point during processing. If a leak were occurring, processing would halt and the system put in a safe condition so that all the solution would not be pumped out into the glovebox cavity or wherever the leak was occurring. Leak sensors were distributed throughout the glovebox system. Locations included the dump tank, effluent-bottle secondary tray, feed-bottle secondary tray, surge-vessel secondary tray, and two on the floor of the glovebox. In addition, a sensor was placed in the column pot to detect possible leaks from the column. If moisture were detected by a leak sensor, a light on the alarm panel would illuminate and an audible alarm would sound until the leak sensor was dry.

The leak sensors themselves operated by having moisture complete a circuit. They were composed of two wires with approximately $1 / 2$ in. of bare wire exposed, separated by a moistureabsorbing material. The moisture-absorbing material also surrounded the wires to ensure that they did not short out, and everything was zip-tied in place. Figure 2.3.2.10.1 shows an image of one of these sensors. The other ends of the two wires were then connected to the alarm system. If moisture were present, it would be absorbed by the wire-surrounding material and complete the circuit, thus sounding the alarm. 


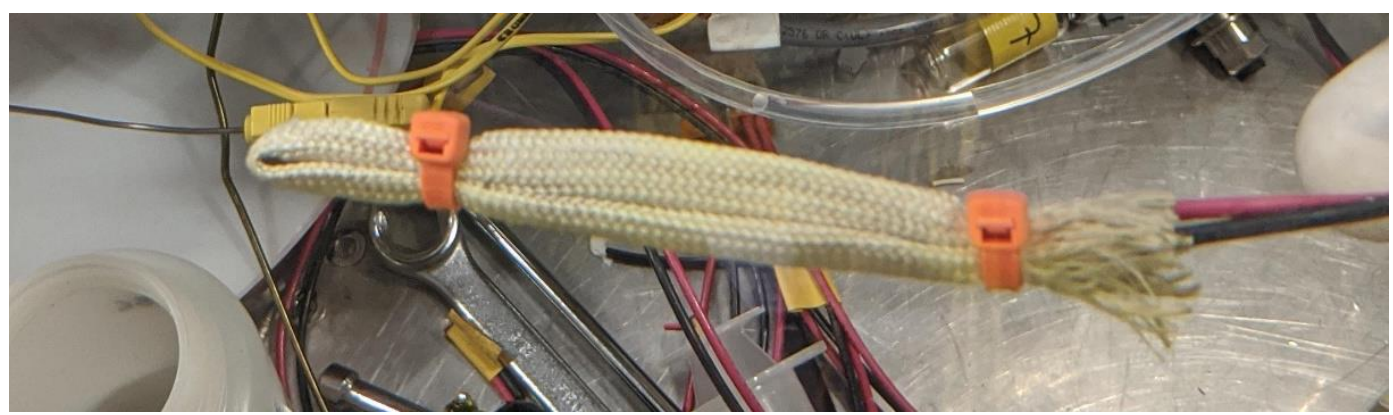

FIGURE 2.3.2.10.1 One of the leak sensors used in the glovebox system, composed of an adsorbent tube folded in half with one wire inserted in each side. The wires and adsorbent are held in place by zip-ties.

\subsubsection{Sampling}

All sampling was based on mass. In addition to recording the mass of all samples taken, the mass of the target solution was verified prior to each irradiation and the masses of the reagents used during processing were tracked at both the feed bottles and effluent bottles. The mass of solution used to strip the column was also verified by measuring the mass of solution transferred to the hot cell at the end of processing. This was done because it was possible to achieve lower relative uncertainty via mass than via volume. This lower uncertainty, in turn, minimized the uncertainty associated with parameters determined for the target solution and the numbers reported for production and recovery of ${ }^{99} \mathrm{Mo}$. It also allowed for the direct calculation of total product and contaminants in the various solutions from the samples.

\subsubsection{Target Solution}

Samples were taken to assess target solution parameters directly from the verification tank via the $1 / 8$ in. O.D. FEP sparge line, which had a manual valve attached to the end. To obtain these samples, a $60 \mathrm{~mL}$ syringe with a 3-way Luer valve was connected to the manual spargeline valve (V-2037), as shown in Figure 2.3.3.1.1. A small amount of solution was drawn into the syringe, then the 3-way valve was switched and the sample was dispensed into a sample bottle. Following dispensing, the syringe was used to inject air into the sparge line and force the remaining target solution back into the verification tank. 


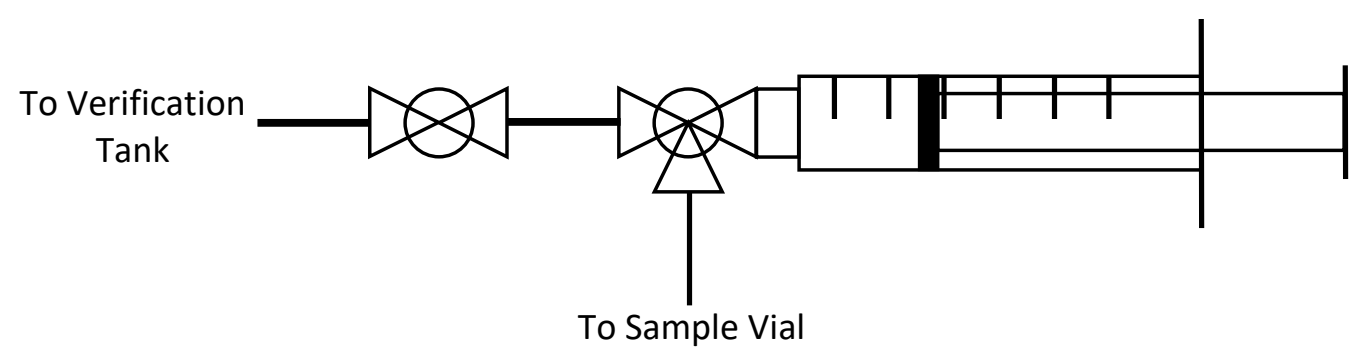

FIGURE 2.3.3.1.1 Diagram of sparge line connection used for sampling the target solution

If a sample of the target solution had to be taken when the verification tank was not installed for some reason, this could be done through valve V-2001 (G6 of Figure 2.3.2.2.4). To utilize this port, a small length of $1 / 4$ in. O.D. FEP tubing was attached to the valve using Swagelok fittings and fed through an HDPE Nalgene bottle lid. To execute the sampling, a bottle was screwed on to the lid attached to the port and the FMI pump was turned on to circulate the target solution through the system. When solution reached the open port, it would push solution into the waiting bottle. Once an adequate sample was retrieved, the FMI pump was reversed to pull any solution remaining in the FEP tubing back into the system; then the port was closed, and circulation could continue or the target solution could be returned to the TSV. After these steps, the bottle was unscrewed from the port attachment and capped before being removed from the glovebox.

\subsubsection{In-line Samples}

Following an irradiation and processing, samples taken using the in-line sampling ladders had to be retrieved. As mentioned in Section 2.3.2.9, high radiation levels prevented entry to the beamline cell for several days after the EOB. Once sufficient time had elapsed for the radiation levels to fall to reasonable levels (usually 3-4 days), entry was made and it was possible to retrieve samples from the sample ladders for gamma counting. A separate LabVIEW program was used for sample retrieval (Figure 2.3.3.2.1), and the vacuum pump used to ensure that samples moved to the evacuated vials in shielded pots had to be turned on manually at the glovebox for each retrieval. Each sample ladder was also connected to a manifold of manual valves that had to be manipulated to direct the vacuum to, and open to atmosphere, the appropriate sets of solenoid valves. An example of one of these manifolds is shown in Figure 2.3.3.2.2; the valves controlling which sample ladder rung is exposed to the glovebox atmosphere (through a one-way check valve) are along the top, and the corresponding valves controlling which sample vial and ladder rung are exposed to vacuum are along the bottom. 


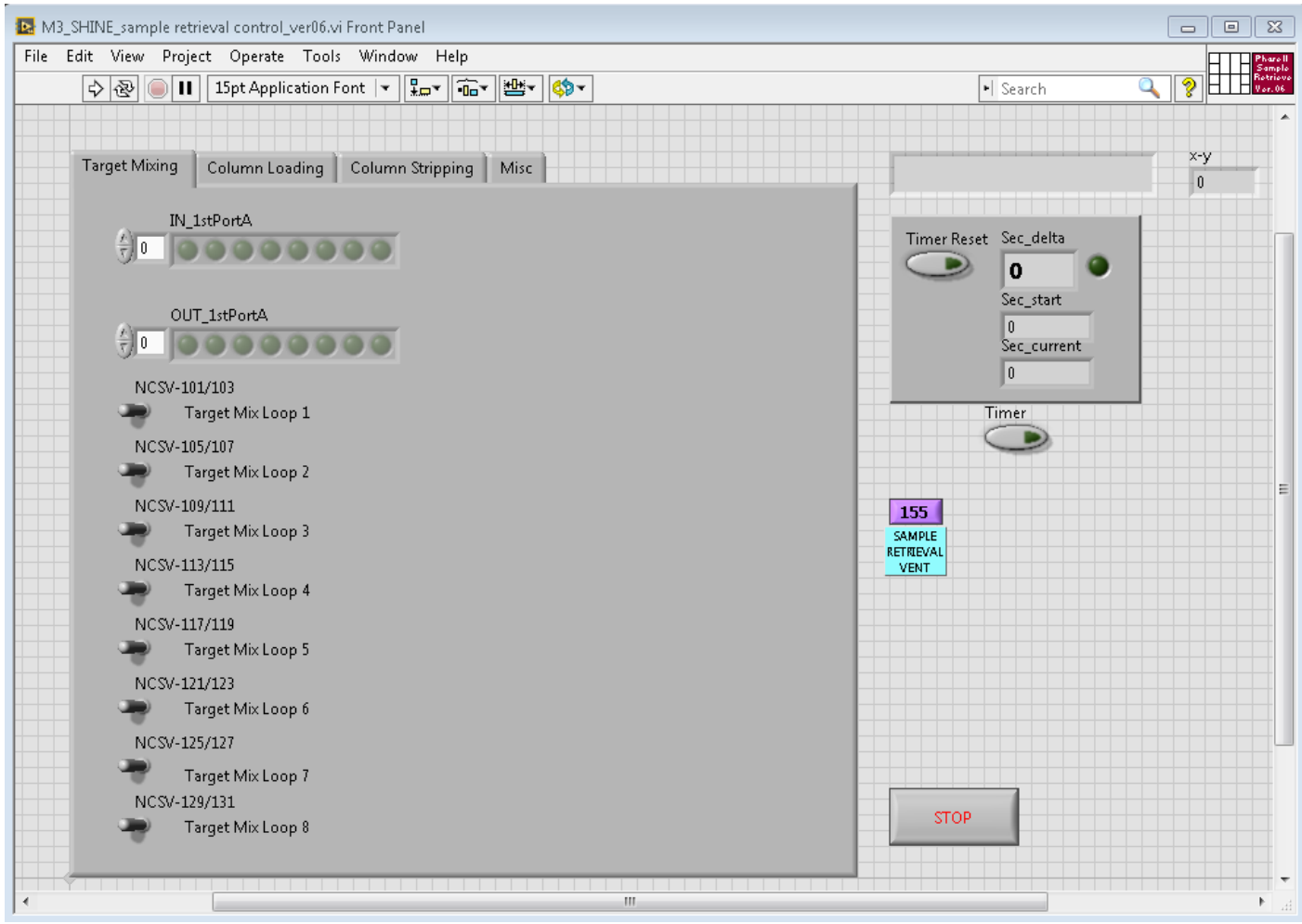

FIGURE 2.3.3.2.1 LabVIEW operator interface for sample recovery after irradiation and processing

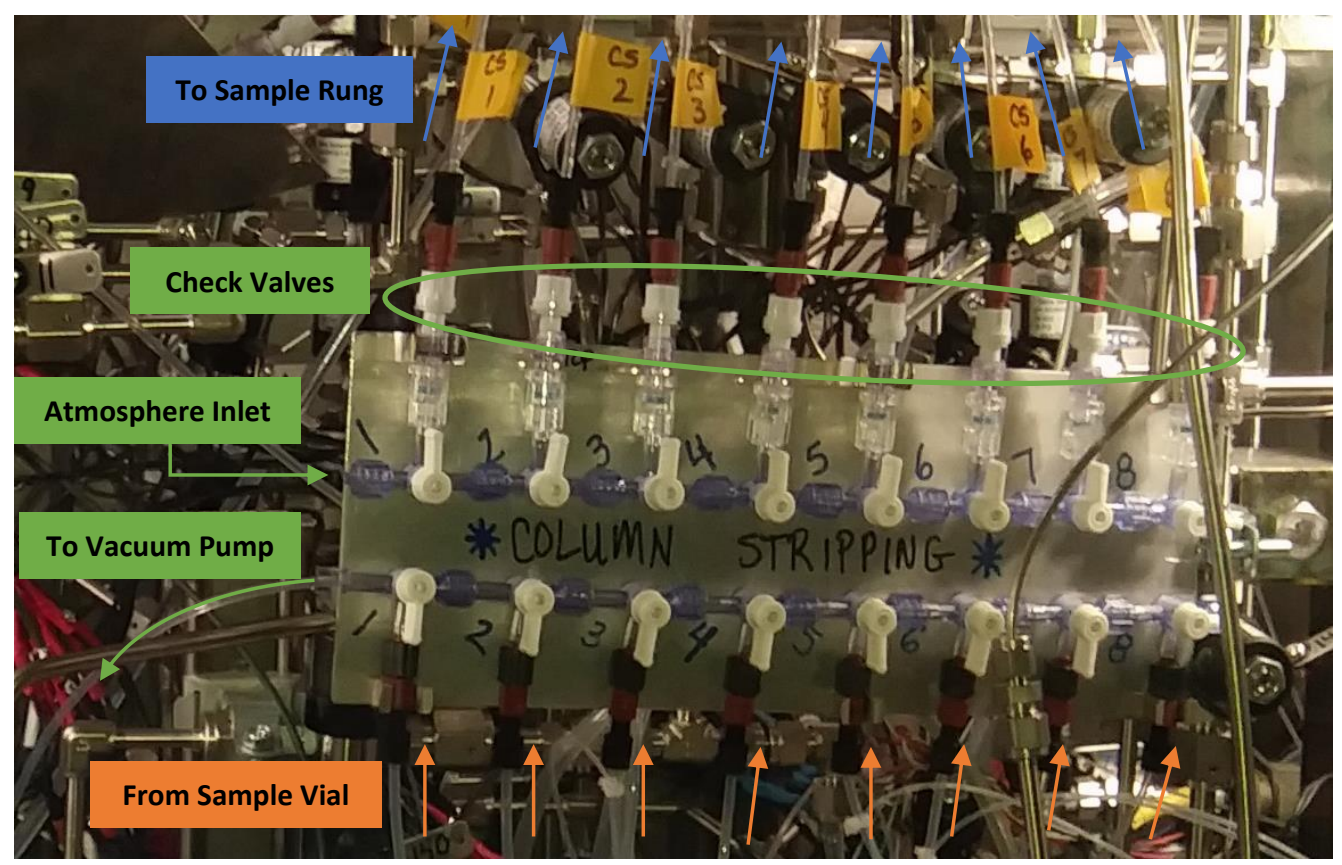

FIGURE 2.3.3.2.2 Manifold used to direct atmosphere and vacuum to the appropriate sample ladder rung. White one-way check valves preventing backflow of gases into the glovebox are circled in green. 
Over time, the sampling ladders began only working intermittently. Initially, it was thought that this was because the vacuum pump used to pull the samples from the sample loops was not working. The power supply of the pump had been damaged by several rounds of radiation exposure, and replacing it fixed the pump. Unfortunately, fixing the pump did not allow retrieval of most of the samples, so it was thought that the plastic pieces used in the manifolds were leaking. This seemed plausible, as they had not been replaced in several months and had also been exposed several times to high radiation levels, which can degrade the plastic. Upon replacement, however, it was found that many of the samples still could not be retrieved from the ladders. Finally, it was determined that one or both solenoid valves used for retrieving the samples from the ladders were not opening. It is still unclear whether this was happening because of radiation damage, buildup of precipitate, uranyl sulfate residue, or some other factor (see detailed discussion in Section 3.3).

While most of the sampling loops resulted in data that were informational but not necessarily consequential to the experiment, it was not urgent to find a solution until none of the samples from the target mixing sample ladder could be recovered. This is because the target mixing sample ladder was the only place in the system where a sample could be taken that was representative of the target solution at the EOB and would allow for the calculation of ${ }^{99} \mathrm{Mo}$ production and process yields. As more of the target mixing sample ladders stopped functioning, it was decided to install an alternate sample loop that used only manual valves so a sample of the target solution at the end of irradiation could be retrieved reliably. While the manual valves slightly increased the time when workers were required to be in the high radiation field for a single sample, only one sample was captured instead of up to 24 samples that would have been captured using the sample ladders, and therefore the total time a worker spent in the radiation field was decreased. The alternate sample loop was placed in the acid-side flow meter bypass loop between solenoid valves V-0165 and V-0166 (G6 of Figure 2.3.2.2.4). In Figure 2.3.3.2.3, the alternate sample loop is shown with the vent manual valve pointing left and the retrieval manual valve pointing towards the camera. The retrieval valve was connected to $1 / 8 \mathrm{in}$. FEP liquid line that led to the same concentric needle setup used with the sample ladders, replacing the $1 / 16$ in. liquid line. This alternate sample loop was operated in much the same way as the loops in the sample ladder, in that the solenoid valves on either side were closed while the vent and sample-line valves were opened and vacuum was pulled on the associated sample vial. When this sample loop was implemented, no other samples were collected in the normal sample ladders to minimize the dose to workers when they went to retrieve this sample. This sample loop could also be used as an alternate way to collect samples of the target solution if the verification tank was not installed. 


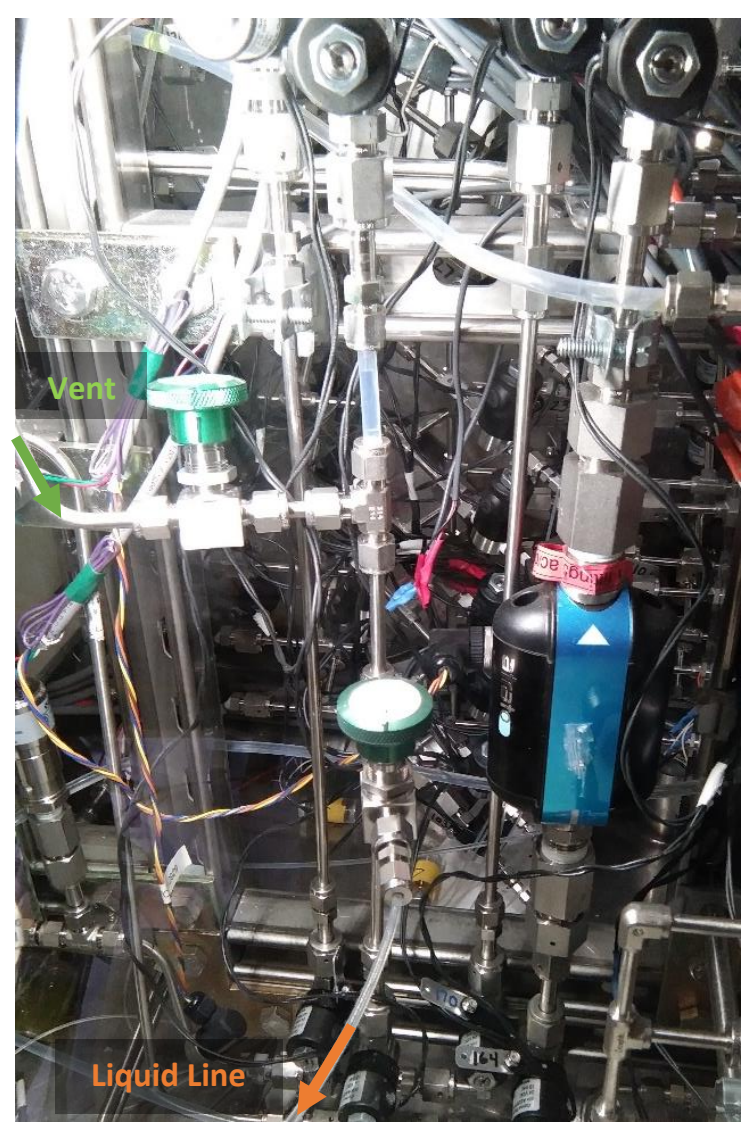

FIGURE 2.3.3.2.3 Alternate sample loop with manual valves used in later irradiations

After being retrieved from the glovebox, samples were taken to a laboratory and diluted by mass to prepare them for gamma spectroscopy. See Section 2.5 for details on gamma instrumentation and analysis methods.

\subsubsection{Effluent Bottles}

In certain instances later in the experimental sequence, it was also desired to take samples from the various effluent bottles. To do this, the $1 / 4$ in. O.D. FEP liquid lines feeding the effluent bottles were disconnected at the double-valve joint in the effluent-cart manifold and 1/16 in. O.D. polyether ether ketone (PEEK) tubing was threaded through the line into the bottles. A syringe was then attached to the PEEK tubing using a Luer valve fitting, and the solution was pulled into the syringe. Once the desired volume had been obtained, the Luer valve was closed and the PEEK line was cut off and allowed to drop back into the eluent bottle to minimize the chance of radiological contamination and release of fission gasses into the glovebox environment. Diagrams of the effluent bottle, tubes, valves, and syringe during sampling and after cutting off the $1 / 16$ in. PEEK line are shown in Figure 2.3.3.3.1A and B, respectively. 


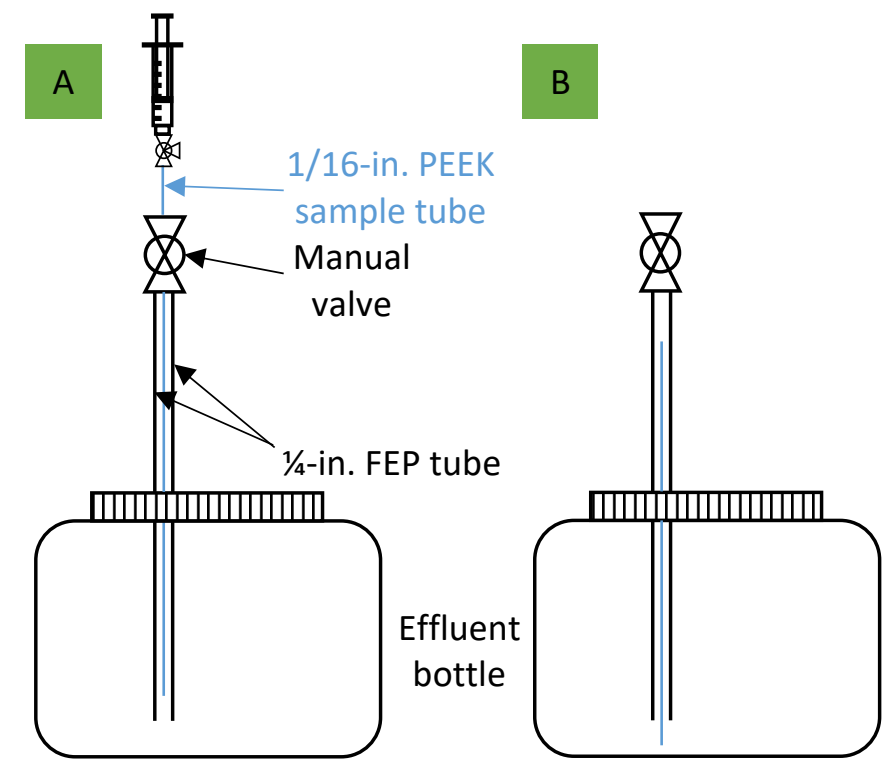

FIGURE 2.3.3.3.1 Diagram of sampling setup for effluent bottles (A) while sampling with a syringe and (B) after cutting the PEEK tubing and allowing it to drop back into the bottle

\subsubsection{Pre-start Checks}

Prior to irradiation, it was necessary to determine if the target solution was within acceptable specifications set by the ASE. The ASE specified the concentration of LEU at 120$140 \mathrm{~g} \mathrm{U} / \mathrm{L}$, and total volume of the target solution between 5 and $20 \mathrm{~L}$ at the beginning of the irradiation, to ensure safe operation of the target vessel. The $\mathrm{pH}$ of the target solution was also specified to be between 0.95 and 1.05 to ensure proper loading of Mo on the column. Often, after an irradiation and processing, it was found that the uranium concentration in the target solution was low. This was a result of small amounts of acid and water being held up in the glovebox system even after purging it with $\mathrm{N}_{2}$ after the previous irradiation and diluting the target solution as it passed through the system. This holdup also caused minor fluctuations in the $\mathrm{pH}$ of the target solution. In addition to the slight dilution from residual processing solutions, it was found that the irradiated target solution would precipitate uranyl peroxide upon standing unless Fe was added to the solution (this phenomenon is discussed in Reference [5]).

Samples of the target solution were retrieved directly from the verification tank when determining if ASE specifications were being met. This retrieval was carried out using the sparge line, as described in Section 2.3.3.1. If any of the ASE parameters were found to be out of specification, calculations were performed and makeup solution was added. As mentioned above, the most common issue was that the $\mathrm{U}$ concentration was found to be low. To correct this problem, the makeup solution was prepared by dissolving LEU from the same batch used to make the target solution in a small amount of $\mathrm{H}_{2} \mathrm{SO}_{4}$, resulting in a concentration of $636 \mathrm{~g} \mathrm{U} / \mathrm{L}$ at $\mathrm{pH}$ 1. It was also possible to add water or concentrated $\mathrm{H}_{2} \mathrm{SO}_{4}$ to adjust the $\mathrm{pH}$, although this adjustment was not required during these experiments. In addition to adjusting the uranium 
concentration and $\mathrm{pH}$, a small amount of stable Mo carrier was added prior to each irradiation to facilitate chemical processing post-irradiation and when Fe was added to prevent uranyl peroxide precipitation.

All of these solutions were added to the target solution directly through the same sparge line setup described for taking samples from the verification tank. To adjust the target solution, the make-up (and carrier) solutions were mixed in a bottle, then drawn into a $60 \mathrm{~mL}$ syringe through the 3-way valve connected to the verification-tank sparge-line valve. Once the syringe was full, the 3-way valve was switched, the manual sparge valve was opened, and the solution was injected into the verification tank. Once all the make-up solution was injected, air was injected using the same process to ensure that all the solution was forced into the verification tank from the sparge line before closing the manual sparge-line valve and disconnecting the syringe and 3-way valve.

To mix the target solution after any adjustments, the solution was pumped to the dump tank and returned to the verification tank. Upon returning the solution to the verification tank, sampling and analysis were repeated to ensure the solution was within the ASE boundary conditions.

\subsubsection{References}

[1] Youker, A.J., Chemerisov, S.D., Tkac, P., Kalensky, M., Heltemes, T.A., Rotsch, D.A., Krebs, J.F., Makarashvili, V., Stepinski, D.C., Alford, K., Bailey, J., Byrnes, J., Gromov, R., Hafenrichter, L., Hebden, A., Jerden, J., Jonah, C., Micklich, B., Quigley, K., Schneider, J., Wesolowski, K., Vandegrift, G.F., and Sun, Z., Compendium of Phase-I Mini-SHINE Experiments, ANL/NE-16/39, Argonne National Laboratory, October 2016. Available at https://publications.anl.gov/anlpubs/2017/01/131828.pdf

[2] Stepinski, D., and Vandegrift, G.F., SHINE and Mini-SHINE Column Designs for Recovery of Mo from $140 \mathrm{~g}$-U/L Uranyl Sulfate, ANL/NE-16/11, Argonne National Laboratory, 2016.

[3] Stepinski, D.C., Abdul, M., Youker, A.J., Rotsch, D.A., Tkac, P. Chemerisov, S., and Vandegrift, G.F., Optimization of SHINE Process: Design and Verification of Plant-Scale AG 1 Anion-Exchange Concentration Column and Titania Sorbent Pretreatment, ANL/NE16/7, Argonne National Laboratory, 2016.

[4] Youker, A.J., Stepinski, D.C., Ling, L., Chung, P.-L., and Vandegrift, G.F., Plant-Scale Column Designs for SHINE Target Solutions, ANL/CSE-14/24, Argonne National Laboratory, 2014.

[5] Kalensky, M., Chemerisov, S., Youker, A.J., Tkac, P., Krebs, J.F., Quigley, K., Lowers, R., Bakel, A., and Vandegridt, G.F., Means to Eliminate Uranyl Peroxide Precipitation in SHINE Target Solution, ANL/CSE-13/21, Argonne National Laboratory, 2013. 


\subsection{HOT CELL PROCESSES}

\subsubsection{Concentration Column}

\subsubsection{Experimental Design Features of the D-024 Hot Cell}

The ${ }^{99}$ Mo produced by subcritical fission of an aqueous solution of uranyl sulfate was recovered from the irradiated solution on a titania column, washed with acid and then water, and eluted with base. The alkaline strip, containing the desired ${ }^{99} \mathrm{Mo}$, was transferred directly to the D-024 Hot Cell (Figure 2.4.1.1.1) for final purification.

A solution transfer line for Phase II, which consists of 1/4-in.-diameter 304 SS tubing in 6 -in. lengths, welded end-to-end to form an 90-ft length, was installed during the installation of the Phase I line. Both lines are held inside the secondary containment. Prior to sealing the secondary containment, the lines were vacuum- and liquid-tested. Minimal solution holdup was observed while testing liquid transfers in the lines. Several liquid monitors were installed in the secondary containment for leak detection. Both lines were connected via swage lock fittings to valves within the D-024 Hot Cell.

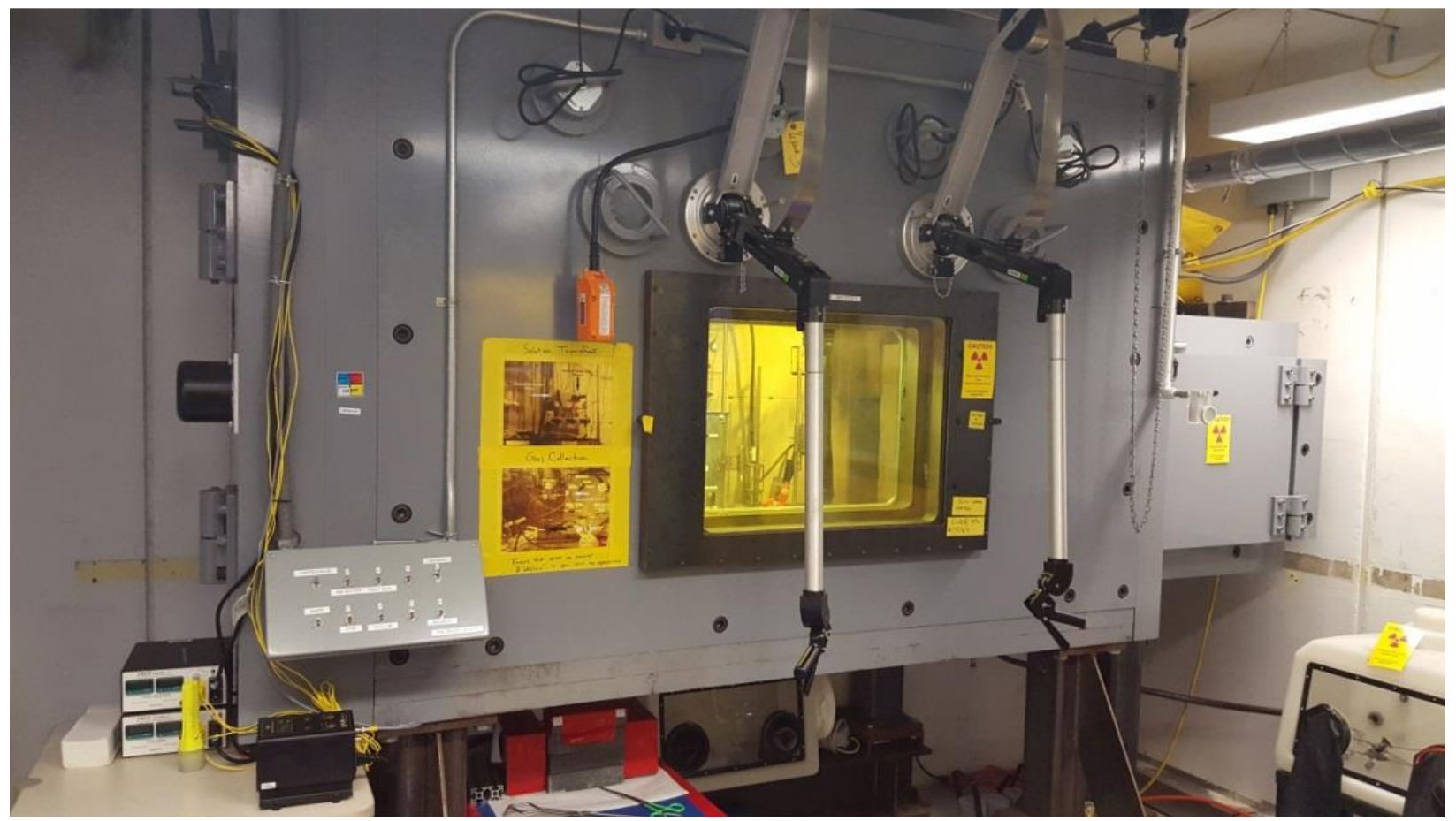

FIGURE 2.4.1.1.1 The D-024 Hot Cell, where final purification of ${ }^{99}$ Mo is performed 
The interior of the D-024 Hot Cell was designed to accommodate various aspects of the project and allow for adaptation to the research being performed. An image of the interior of the D-024 Hot Cell is shown in Figure 2.4.1.1.2; various features are pointed out in the image. A quick-connect system was installed that enables three vacuum connections for simultaneous use, water in and out, and the option for compressed gas (Figure 2.4.1.1.3). These features rest on scaffolding that was welded to the inner back wall of the hot cell to increase floor space and, thus, workspace. The scaffold supports the following:

- the quick-connect system

- vacuum line

- vacuum traps

- gas scrubbers

- pumps

- $\mathrm{pH}$ probes

- solution vessels

- columns

- the concentration-column valve board

- $\quad$ valves for the ${ }^{99}$ Mo transfer lines

- $\quad$ electrical wiring

It also serves as a barrier to protect and manage various lengths of tubing.

Electric controls for heaters, stirrers, balances, $\mathrm{pH}$ probe readout, lights, etc., were placed on the exterior of the hot cell. Consideration was given to the placement (interior or exterior of the hot cell) of systems that require routine replacement or recharging.

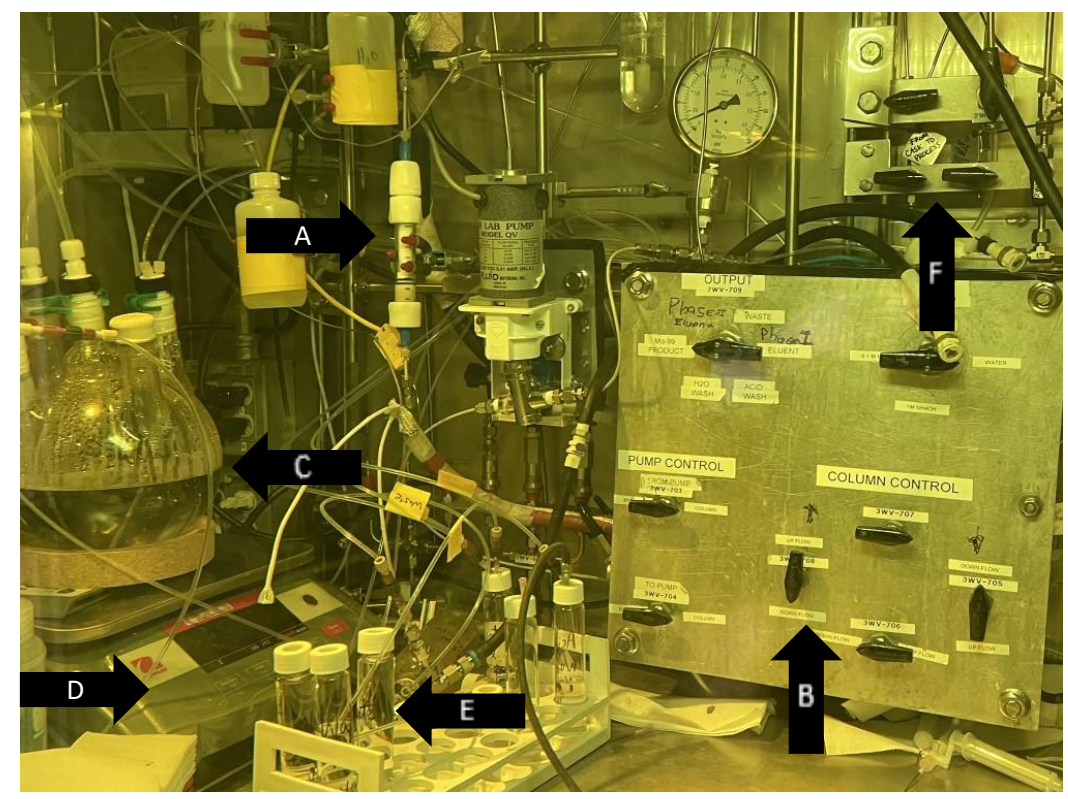

FIGURE 2.4.1.1.2 Image of interior of the D-024 Hot Cell: concentration column (A); column control board (B); acidification vessel $(C)$; balance $(D)$; reagent vials $(E)$; and valves for exterior solution line $(\mathrm{F})$ 


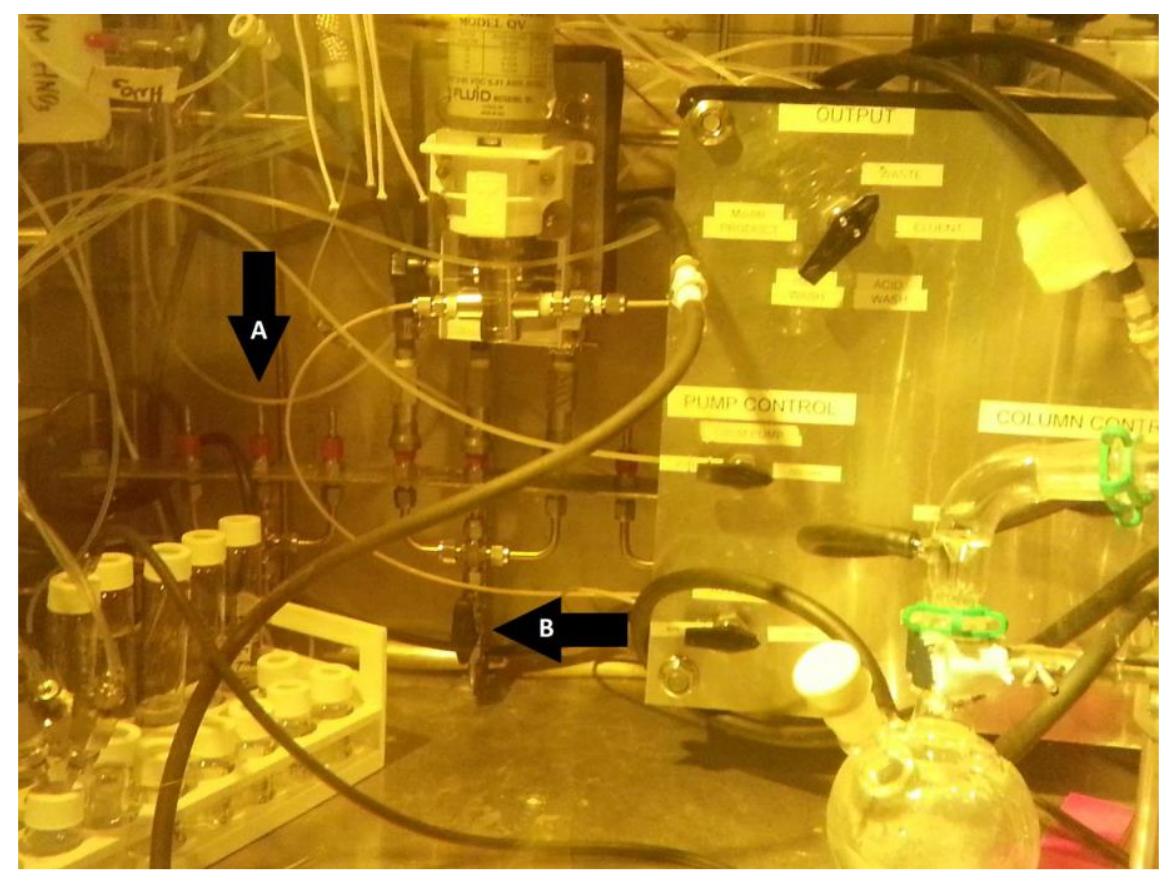

FIGURE 2.4.1.1.3 Image of quick-connect system: compressed-gas connection (A); exhaust valve connecting to gas collection system (B); and water in/out (hidden behind the board)

The new items installed for the Phase II experiments include a larger vessel-a 3-L, 5neck, plastic-coated flask (Figure 2.4.1.1.4) — to receive the solution from the primary recovery glovebox. An ultra-thin magnetic stirrer was placed between the top-loading balance and the flask. The pre-weighed flask was held in place by a lightweight cork ring. The concentration column, which used $0.8 \mathrm{~mL}$ of titania sorbent in Phase I, was updated to use $1.5 \mathrm{~mL}$ of sorbent for the Phase II experiments. A liquid trap was installed between the GCS and the connections inside the D-024 Hot Cell as an added safety feature. Also, a series of six 5-L vessels were installed beneath the hot cell inside a plastic secondary container. These vessels had their headspace connected to the GCS to vent any residual fission gases when the irradiated solutions were sent to the vessels for storage until their ultimate disposal. These vessels were all leakchecked before installation. Finally, before the last experiment, all the plastic lines and tubing were replaced. During one of the practice runs, a leak was observed coming from behind the valve board. When troubleshooting the leak, it was found that the valve had become extremely brittle and had developed a spiral crack when the needle on the line was being placed into the receiving container. Some of the other plastic lines were found to be in a similar condition, so the decision was made to replace all lines. A protocol was established for replacing all lines within 2 years of installation, to prevent future equipment failures of this type. 


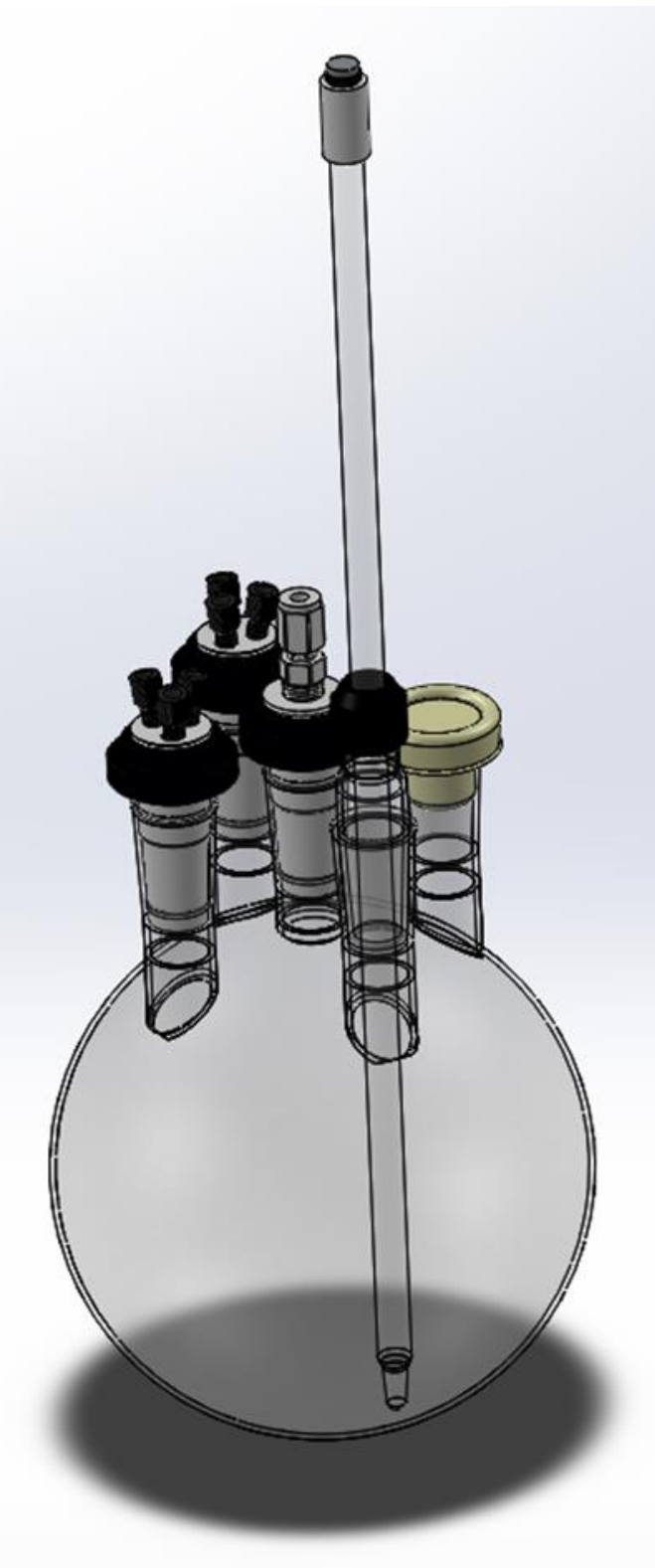

FIGURE 2.4.1.1.4 Diagram of the 3-L, 5-neck vessel used for receiving and acidifying the ${ }^{99}$ Mo product prior to loading on the concentration column
Before each experiment, a checklist was followed to ensure that the hot cell was prepared for the coming run. This checklist included collecting all sampling syringes, 60 -mL collection vessels, and $20-\mathrm{mL}$ scintillation vials with septum tops; refilling the solution reservoirs inside the hot cell with water, $1.0 \mathrm{M} \mathrm{NaOH}$, and $0.01 \mathrm{M} \mathrm{HNO}_{3}$; placing $8 \mathrm{M} \mathrm{HNO}_{3}$ inside the 3-L flask; and installing the concentration column. Before installation, the concentration column was packed with $1.5 \mathrm{~mL}$ of titania sorbent (S40, 40-micron particle size, 60-angstrom pore size). The sorbent was prepared in advance by putting the sorbent into purified water and shaking. Once the sorbent settled, the supernatant was removed using a pipette to remove the fine particles. This procedure was repeated multiple times, until the solution above the settled resin was clear and colorless. The sorbent was then packed into the column using the slurry method. The packed column was then attached to an FMI pump, and purified water was run through the column in the upflow and downflow directions for at least one hour in each direction. The effluents were then checked to make sure all fines had been removed. The column was then inspected for any channeling, and the column caps were tightened in case the resin had compressed during the flow tests. The $\mathrm{pH}$ probe was calibrated with $\mathrm{pH}$ standard solutions as close to the date of the experiment as possible, and the probe was then placed inside the 3-L flask. All ports were checked and confirmed to be sealed to the vessel as a final check before receiving any solution from the glovebox team.

Immediately before receiving the solution from the glovebox, a system interface step was performed, with a member of each team present to verify the conditions inside the D-024 Hot Cell. A member of each team signed off on the checklist in both sets of work instructions. The steps included ensuring that all the ports on the 5-neck vessel were properly sealed, the $\mathrm{pH}$ probe was in place, the balance was zeroed and the weight was recorded, and finally the proper valves were opened and connected to the GCS. 


\subsubsection{Concentration-Column Design and Operation}

The ${ }^{99} \mathrm{Mo}$ product from the recovery column, in $1.0 \mathrm{M} \mathrm{NaOH}$ solution, was received into the 5-neck, 3-L vessel within the D-024 Hot Cell. The ultra-thin magnetic stirrer was activated to mix and sample the solution prior to acidification and concentration. Sampling the solution provides an accurate account of the product strip from the recovery column, which allows for determinations of experiment and equipment performance. Approximately $1700 \mathrm{~g}$ of solution was transferred from the recovery glovebox to the flask in the D-024 Hot Cell. This volume is too large to be handled with the LMC process (maximum volume, $100 \mathrm{~mL}$ ). Therefore, the solution was concentrated using a second column. A schematic diagram depicts the system used during concentration-column operation (Figure 2.4.1.2.1).

The column separation system consists of an FMI positive-displacement pump, which allows for forward and reverse flow; Swagelok 3-, 4-, and 5-way switching valves; and Hamilton 3-port flow valves with a T-plug to direct the process streams. All the valves were mounted on a board, and this valve board was mounted on the previously mentioned scaffold. Reagent bottles were mounted on the scaffold and connected to the valve board using 1/8-in. HDPE tubing. Stainless steel tubing (1/8 in.) was used to connect the valves to each other and the preheater, and HDPE or PEEK tubing was used to connect the column, reagent, and collection bottles. The concentration-column system is equipped with a solution preheater, and the solution lines feeding into the column are wrapped with heating tape. During the experiment, any vessels or sample vials receiving solution were connected to the GCS described in Section 2.2.2. A liquid trap was installed between the D-024 Hot Cell and the GCS to guarantee that no liquid could be transferred into the GCS or the gas-collection lines. This trap was inspected and verified before and during the experiments and is located directly above the valve board inside the hot cell. The eluent from the feed and wash steps of the concentration column was collected in a 5-L storage vessel inside the hot cell; the headspace of this vessel was also plumbed to the GCS. The effluent was to be held in this vessel until the majority of the fission nuclides decayed. Once the radiation field had dropped sufficiently, the effluent solution was transferred to the effluent storage flasks underneath the hot cell. The effluent storage area was lined with multiple layers of lead brick and connected to the GCS. All storage vessels were leak-checked before use with actual columneffluent solution. The effluent and rinse solutions were transferred into these vessels for storage until the solutions were ready for disposal. 


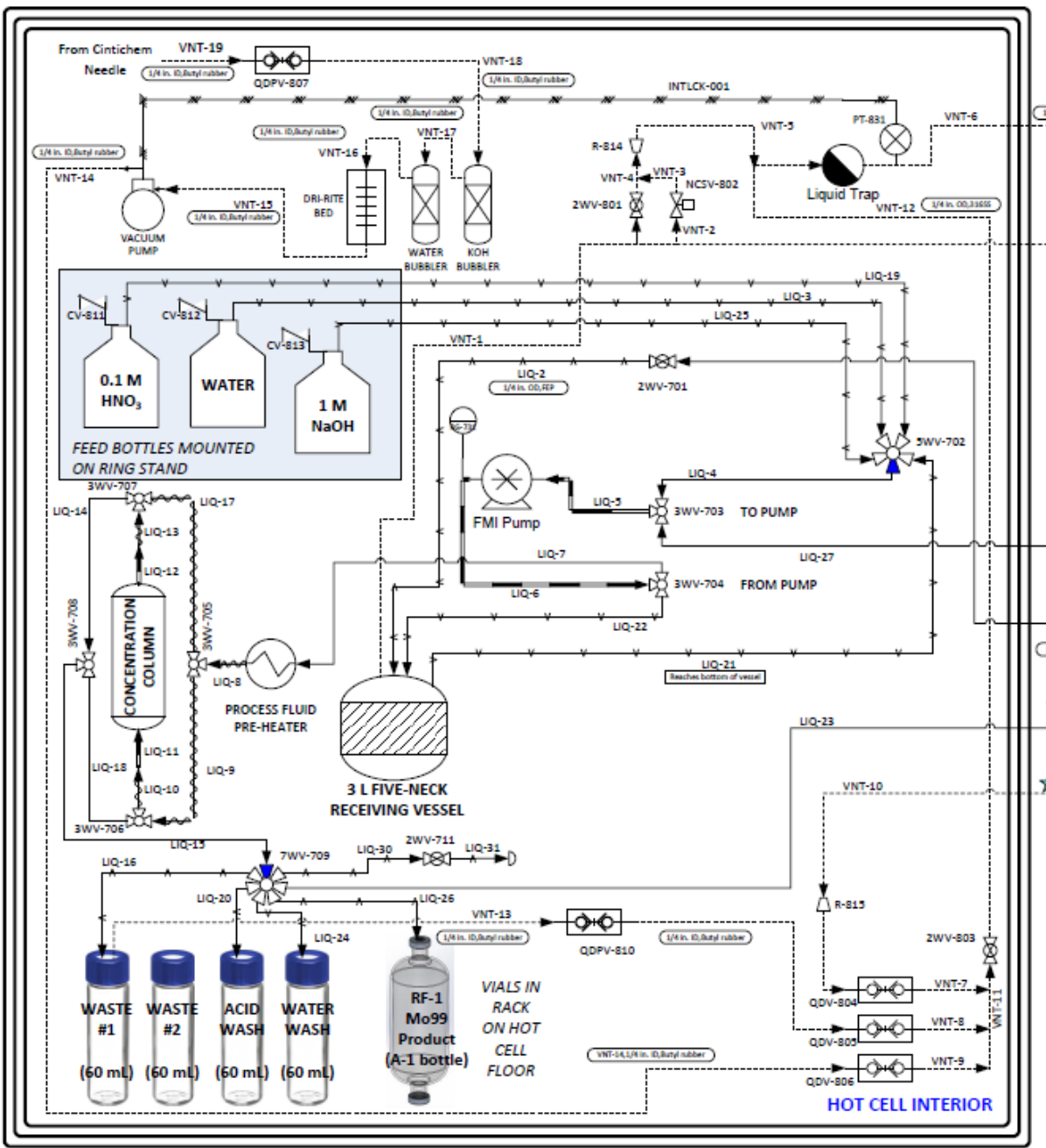

Stainless steel liquid line, $1 \%$ in. OD (unless noted otherwise)

Stainless steel vent line, $3 /$ i in. OD

(unless noted otherwise)

(unless noted otherwise)

Stainless steel heated liquid line, 16 in. OD

$\longrightarrow$ PEEK tubing, $1 \%$ in. OD

Low voltage interlock signal

Log Manual 3-way ball valive

ㄱ. Tubing reducer

员 Normally-closed solenoid valve

Polycarbonate, silicone diaphragm check valve Stainless steel valved quick-disconnect fitting

- 1 - Plastic valved quick-disconnect fitting

$\ominus$ Pressure gauge

Q Pressure Transduce (interiocked to vacuum pump)

Manual 5-port ball valve

(blue is common port)

38 Manual 7-port ball valve

S3 unes in seconasy containment

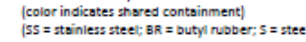

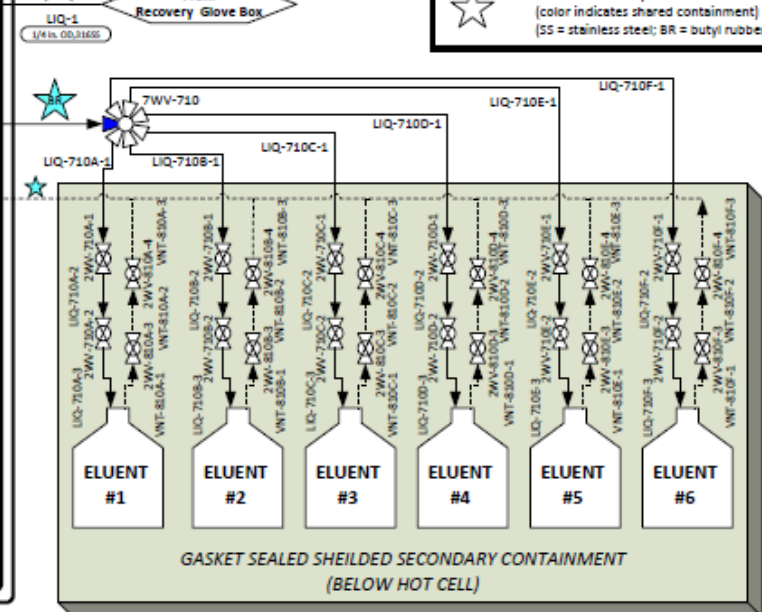

FIGURE 2.4.1.2.1 Schematic diagram of ${ }^{99}$ Mo concentration column 
After the ${ }^{99} \mathrm{Mo}$ solution was sampled, the $\mathrm{pH}$ of the $1.0 \mathrm{M} \mathrm{NaOH}$ solution in the $3-\mathrm{L}$ receiving vessel was adjusted with $8 \mathrm{M} \mathrm{HNO}_{3}$ to $\mathrm{pH}=2$ (monitored with a calibrated $\mathrm{pH}$ probe). The acidification was performed dropwise with a $60-\mathrm{mL}$ syringe fitted with a 1 -in. needle. The needle was inserted into the port fitted with the rubber septum. To simplify the acidification process, approximately 75 percent of the calculated value of $8 \mathrm{M} \mathrm{HNO}_{3}$ necessary to reach $\mathrm{pH} 2$ was placed in the 3-L flask when preparing the hot cell for the experiment. The stir plate underneath the flask was activated before final acidification to ensure proper mixing and uniformity of the solution. The system was then purged of air by passing water through the lines. The dead volume of the system $(15 \mathrm{~mL})$ was significant; hence, column pre-equilibration, wash, and strip volumes were increased from the volumes of previous experiments to ensure that an appropriate volume reaches the column and is collected. All equilibration, washing, and elution steps were timed steps, and therefore, a stopwatch was used by the operator working the pump. The operator working the manipulators was responsible for verifying that the solution was being transferred into the appropriate container at the desired flow rate. The pre-equilibration, loading, and washing steps were carried out at $80^{\circ} \mathrm{C}$. The FMI pump was kept at a power of $67.2 \%$ for these steps, which corresponded to a flow rate of $50 \mathrm{~mL} / \mathrm{min}$. The flow rate was verified during the installation and preparation of the hot cell. An Omnifit BenchMark column packed with S40 Sachtopore sorbent $(1.5 \times 1 \mathrm{~cm}, 1.5-\mathrm{mL}$ bed volume $)$ from ZirChrom Separations, Inc., Anoka, MN, was pre-equilibrated with $22 \mathrm{~mL}$ of $0.01 \mathrm{M} \mathrm{HNO}_{3}$ and subsequently loaded with 1.7 $\mathrm{L}$ of acidified ${ }^{99} \mathrm{Mo}$ solution flowing at $50 \mathrm{~mL} / \mathrm{min}$ in the upflow direction. Subsequently, the column was washed with $44 \mathrm{~mL}$ of $0.01 \mathrm{M} \mathrm{HNO}_{3}$ and $44 \mathrm{~mL}$ of water solution at $50 \mathrm{~mL} / \mathrm{min}$ in the upflow direction at $80^{\circ} \mathrm{C}$. During the product-stripping step, the initial $10 \mathrm{~mL}$ of strip solution was loaded, and eluent was collected in the water-wash collection flask (to remove the water present in the system dead volume). Subsequently, ${ }^{99} \mathrm{Mo}$ was stripped with $66 \mathrm{~mL}$ of $1 \mathrm{M}$ $\mathrm{NaOH}$ in the downflow direction at $11 \mathrm{~mL} / \mathrm{min}$ at $70^{\circ} \mathrm{C}$. For the stripping step, the FMI pump was set to a power of $14.8 \%$, which was also tested and verified during the pump installation. As the last step, lines were rinsed with $50 \mathrm{~mL}$ of water, which was collected as waste. Each process stream was collected in pre-marked and pre-weighed bottles. Samples of the eluent, acid wash, water wash, waste, and final product were collected for analysis.

Approximately one week after the experiment was completed, output lines and lines that had contained the ${ }^{99} \mathrm{Mo}$ effluent or product were flushed with water. These rinse solutions were directed into the effluent storage container inside the D-024 hot cell. The line between the primary recovery glovebox and the hot cell was also rinsed and blown dry with compressed helium. The helium was regulated at 4 psi during the process so as not to overwhelm the GCS, which was operational for all solution transfers. The rinse solutions were all held inside the hot cell for a period of time before being transferred to the 5-L flasks in the shielded solution-storage vessels below the hot cell for long-term storage while awaiting disposal.

When the final AMORE run was performed, the concentration-column operations proceeded as planned; however, there were some issues. The 3-L, 5-neck flask in the D-024 Hot Cell received $1699 \mathrm{~g}$ of solution from the primary recovery glovebox. The solution was adjusted to a $\mathrm{pH}$ of 2 with approximately $175 \mathrm{~g}$ of $8 \mathrm{M} \mathrm{HNO}_{3}$ with a counter-correction with $7.7 \mathrm{~g}$ of $10 \mathrm{M} \mathrm{NaOH}$, resulting in a total volume of $1881.7 \mathrm{~g}$. This solution was loaded onto the column with a set point of $50 \mathrm{~mL} / \mathrm{min}$, but it took approximately 1 hour to completely load the column. Following the wash steps, the column was then eluted into the appropriate vessel for the LMC 
process to proceed. The initial feed sample indicated that approximately $9 \mathrm{Ci}$ of ${ }^{99} \mathrm{Mo}$ was delivered to the D-024 Hot Cell. However, the sample taken from the RF-1 bottle before the LMC process had only $2 \mathrm{Ci}$ of activity in it. These numbers account for only a $22 \%$ recovery rate from the concentration-column process. Analysis of a sample of the concentration-column effluent demonstrated that there was breakthrough from the column, but only of $500 \mathrm{mCi}$. There are a few possibilities that could account for the percent recovery rate. First, there may have been a sampling error, i.e., the sample was not adequately mixed and/or a representative sample of the solution was not taken. It is possible that the remaining ${ }^{99} \mathrm{Mo}$ was fixed on the column and unable to be recovered. In an attempt to determine the root cause, the column was eluted a second time with $50 \mathrm{~mL}$ of $1 \mathrm{M} \mathrm{NaOH}$. This solution was collected, sampled, and analyzed via gamma spectroscopy. Unfortunately, because of issues with the GCS, this operation was performed on March 8, which was over 17 half-lives after the irradiation, so no ${ }^{99} \mathrm{Mo}$ or ${ }^{99 \mathrm{~m}} \mathrm{Tc}$ was detected in the sample. We concluded that there could have been a few reasons for the activity to remain on the column. The reasons include channeling in the packing of the column, which would account for some of the breakthrough and inefficient elution; incorrect $\mathrm{pH}$ of the concentration-column loading solution; or the need for a larger elution volume to fully elute the column to recover all the activity. The other samples taken did not show any significant amount of ${ }^{99} \mathrm{Mo}$, so we cannot definitively say what happened to the remaining activity.

Although the concentration column did not perform as intended during this experiment, the technology has been proven to be effective. The multiple experiments during Phase I and preliminary experiments of Phase II demonstrated a recovery of at least $90 \%$ for the ${ }^{99} \mathrm{Mo}$. Also, previous benchtop experiments performed with cold materials [1] found that the loaded ${ }^{99} \mathrm{Mo}$ from a 2-L solution should need approximately 16 bed volumes of $1.0 \mathrm{M} \mathrm{NaOH}$ to be sufficiently recovered. For these reasons, we believe there was another underlying issue during this particular experiment.

\subsubsection{References}

[1] Stepinski, D., and Vandegrift, G., SHINE and Mini-SHINE Column Designs for Recovery of Mo from 140 g-U/L Uranyl Sulfate, ANL/NE-16/11, Argonne National Laboratory, 2016.

\subsubsection{LEU-Modified Cintichem Process}

The LMC purification process is a small-scale process that accommodates selective precipitation of Mo from acidic conditions, where Mo is predominantly present as $\mathrm{MoO}_{2}{ }^{2+}$, using alpha-benzoin oxime (ABO). The complexation reaction between $\mathrm{Mo}$ and $\mathrm{ABO}$ can be expressed as follows:

$$
\mathrm{MoO}_{2}{ }^{2+}+2 \mathrm{C}_{6} \mathrm{H}_{5} \mathrm{CH}(\mathrm{OH}) \mathrm{C}(: \mathrm{NOH}) \mathrm{C}_{6} \mathrm{H}_{5} \rightarrow \mathrm{MoO}_{2}\left[\mathrm{C}_{6} \mathrm{H}_{5} \mathrm{CH}(\mathrm{O}) \mathrm{C}(: \mathrm{NOH}) \mathrm{C}_{6} \mathrm{H}_{5}\right]_{2}+2 \mathrm{H}^{+} \text {(Eq. 1) }
$$

It is important for Mo to be present in relevant chemical concentrations to form a precipitate with $\mathrm{ABO}$, so for trace levels of Mo (as ${ }^{99} \mathrm{Mo}$ ), Mo carrier needs to be added. The process was originally developed in 1974 for purification of ${ }^{99}$ Mo produced by irradiation of 
highly enriched uranium as $\mathrm{UO}_{2}$ at the Cintichem facility in New York [1]. The Cintichem process was later modified by Argonne to allow processing of irradiated LEU targets dissolved in nitric acid [2-6].

Although the LMC process was developed for purification of fission-made ${ }^{99}$ Mo from irradiated U metal or uranium oxide targets, it could also be utilized, with some minor modifications, for accelerator-driven production of ${ }^{99}$ Mo from uranyl sulfate solution. This process has been demonstrated and discussed previously [7]. In summary, after the strip solution is received from the concentration column in $1 \mathrm{M} \mathrm{NaOH}$, the solution is acidified by adding $10 \mathrm{M} \mathrm{HNO}_{3}$ to make $\sim 1.2 \mathrm{M} \mathrm{HNO}_{3}$. Then, radioactive iodide is co-precipitated with carrieradded $\mathrm{NaI}$ after mixing with $\mathrm{AgNO}_{3}$ solution. Because of the low solubility of $\mathrm{AgI}\left(8.3 \times 10^{-17}\right.$ mol.L $\mathrm{L}^{-1}[8]$ ) and fast isotopic exchange between iodine and iodide, the removal of radioactive iodine and iodide is considered near-quantitative. However, because of the low rate of iodateiodide isotopic exchange, this procedure does not completely remove radioiodine in the form of iodate. Next, Mo carrier is added, followed by Ru and Rh hold-back carriers to prevent their coprecipitation with the Mo-ABO complex. $\mathrm{KMnO}_{4}$ is added to keep Mo as $\mathrm{Mo}$ (VI) before complexation with $\mathrm{ABO}$. After addition of $\mathrm{ABO}$, the Mo-ABO precipitate is filtered and washed with $0.1 \mathrm{M} \mathrm{HNO}_{3}$. After repeated washing, the precipitate is redissolved in dilute $\mathrm{NaOH} / \mathrm{H}_{2} \mathrm{O}_{2}$ solution by applying heat. Precipitation of trace iodine is repeated by adding $\mathrm{NaI}$ and $\mathrm{AgNO}_{3}$, and the solution is then loaded onto a combination column that contains three sorbents: activated charcoal (AC), hydrous zirconia (HZO, which acts as a cation exchanger), and silver-coated charcoal $(\mathrm{Ag} / \mathrm{C})$. The $\mathrm{AgI}$ precipitate is collected on the combination column. The $\mathrm{I}_{2}$ does not coprecipitate with the silver ions, but can be removed by reaction with silver metal $(\mathrm{Ag} / \mathrm{C}$ resin) to form the insoluble AgI. The solubility of silver iodate is also limited, but is significantly larger $\left(3.1 \times 10^{-8}\right.$ mol.L $\left.{ }^{-1}\right)[8]$ than that of $\mathrm{AgI}$. It is likely that $\mathrm{AgIO}_{4}$ is even more soluble than $\mathrm{AgIO}_{3}$. Mo passes through the combination column, while organic residuals are retained on the AC. The purified ${ }^{99} \mathrm{Mo}$ effluent is recovered in $\sim 0.2 \mathrm{M} \mathrm{NaOH}$ solution.

\subsubsection{Reagents Used in LMC Process}

All chemical reagents used in this work were of analytical-reagent-grade purity and were used without further purification. All aqueous solutions were prepared with deionized water with a resistivity $\geq 18 \mathrm{M} \Omega \cdot \mathrm{cm}$.

\section{Silver-coated Charcoal (Ag/C)}

Twenty grams of AC (50-100 mesh) was mixed with water to form a slurry-like mixture, and $15 \mathrm{~mL}$ of $10 \% \mathrm{AgNO}_{3}$ in $0.1 \mathrm{M} \mathrm{HNO}_{3}$ was added. After $5 \mathrm{~min}$ of mixing. $4 \mathrm{~mL}$ of freshly prepared $5 \% \mathrm{Na}_{2} \mathrm{SO}_{3}$ was added and mixed in. Next, $40 \mathrm{~mL}$ of $0.1 \mathrm{M} \mathrm{NaOH}$ was added while mixing, and the mixture was heated at $80-90^{\circ} \mathrm{C}$ for $30 \mathrm{~min}$. After cooling, the material was washed several times with water until the wash solution was clear. 


\section{Hydrous Zirconia (HZO)}

$\mathrm{HZO}$ was prepared by dissolving $\mathrm{ZrOCl}_{2}$ in water and precipitated as zirconia by adding enough $\mathrm{NH}_{4} \mathrm{OH}$ to make a solution with $\mathrm{pH} \geq 8$. The slurry was covered and mixed for $20 \mathrm{~min}$, and the $\mathrm{pH}$ was checked again and adjusted with $\mathrm{NH}_{4} \mathrm{OH}$ to $\mathrm{pH} \geq 8$ if needed. The precipitate was allowed to settle overnight, then centrifuged and washed with water until the $\mathrm{pH}$ of the supernatant reached $\mathrm{pH}=5-7$. After the final water wash, the precipitate was filtered under vacuum and slowly dried at $\sim 40^{\circ} \mathrm{C}$ for $\sim 3$ days. After drying, the precipitate was added to water, and very fine particles were decanted using water. After decanting, the material was allowed to air-dry overnight and then sieved to a size range of 100-200 mesh. The HZO resin was washed again with water and $0.2 \mathrm{M} \mathrm{NaOH}$ and stored under $0.2 \mathrm{M} \mathrm{NaOH}$.

\section{Alpha Benzoin Oxime (2\% ABO in $0.4 \mathrm{M} \mathrm{NaOH})$}

ABO $(0.4 \mathrm{~g})$ was combined with $20 \mathrm{~mL}$ of $0.4 \mathrm{M} \mathrm{NaOH}$ and heated while stirring until fully dissolved. The solution was allowed to cool and was used the same day.

\section{Mo Carrier Solution (10 mg Mo/mL)}

$375 \mathrm{mg}$ of $\mathrm{MoO}_{3}$ was dissolved in $15 \mathrm{~mL}$ of $1 \mathrm{M} \mathrm{NaOH}$, neutralized with $5 \mathrm{~mL}$ of $4 \mathrm{M}$ $\mathrm{HNO}_{3}$, and diluted to $25 \mathrm{~mL}$ with $0.1 \mathrm{M} \mathrm{NaOH}$. The solution was prepared within seven days of actual processing.

\section{Rh Carrier Solution (8 mg Rh/mL)}

$500 \mathrm{mg}$ of $\mathrm{RhCl}_{3} \cdot 3 \mathrm{H}_{2} \mathrm{O}$ was transferred into a volumetric flask containing $15 \mathrm{~mL}$ of water, and then $1 \mathrm{~mL}$ of $70 \% \mathrm{HNO}_{3}$ was added and the solution was diluted to $25 \mathrm{~mL}$ with water. The solution was mixed until the $\mathrm{RhCl}_{3}$ was completely dissolved.

\section{Ru Carrier Solution (5 mg Ru/mL)}

$250 \mathrm{mg}$ of $\mathrm{K}_{3} \mathrm{RuCl}_{6}$ was transferred into a volumetric flask containing $11 \mathrm{~mL}$ of $70 \%$ $\mathrm{HNO}_{3}$ and mixed. The solution was diluted with water to $50 \mathrm{~mL}$.

\subsubsection{Reagents Used for Product-purity Analyses}

\section{Fission Product Carrier Solution}

$10 \mathrm{mg}$ of $\mathrm{RhCl}_{3} \cdot 3 \mathrm{H}_{2} \mathrm{O}$ was transferred into a flask containing $50 \mathrm{~mL}$ of water, acidified with $1 \mathrm{~mL}$ of $70 \% \mathrm{HNO}_{3}$, and mixed until fully dissolved.

$11 \mathrm{~mL}$ of $70 \% \mathrm{HNO}_{3}$ was added to a beaker containing $5 \mathrm{~mL}$ of water. $10 \mathrm{mg}$ of $\mathrm{K}_{3} \mathrm{RuCl}_{6}$ was added and mixed in until fully dissolved. 
$500 \mathrm{mg}$ of $\mathrm{MoO}_{3}$ was dissolved in $20 \mathrm{~mL}$ of $1 \mathrm{M} \mathrm{NaOH}$ and acidified with $2 \mathrm{~mL}$ of $4 \mathrm{M}$ $\mathrm{HNO}_{3}$. $\mathrm{Rh}, \mathrm{Ru}$ and Mo solutions were combined in a volumetric flask and diluted to $200 \mathrm{~mL}$ with water.

\section{Ethyl Acetate, Pre-equilibrated}

$0.33 \mathrm{~mL}$ of $0.1 \mathrm{M} \mathrm{NaOH}$ was combined with $3.33 \mathrm{~mL}$ of $10 \% \mathrm{H}_{2} \mathrm{SO}_{4}$ and mixed. $167 \mathrm{uL}$ of $\mathrm{Fe}_{2}\left(\mathrm{SO}_{4}\right)_{3}(10 \mathrm{mg} \mathrm{Fe} / \mathrm{mL})$ in $1 \% \mathrm{H}_{2} \mathrm{SO}_{4}$ was added and mixed in, followed by $0.33 \mathrm{~mL}$ of $50 \%$ $\mathrm{NH}_{4} \mathrm{SCN}$ in water and further mixing. Then $0.83 \mathrm{~mL}$ of $10 \% \mathrm{SnCl}_{2}$ in $10 \% \mathrm{HCl}$ was added and mixed in. The solution was combined with $20 \mathrm{~mL}$ of ethyl acetate and mixed for $1 \mathrm{~min}$ using a vortex mixer. The phases were separated by centrifugation and the ethyl acetate was used the same day.

\subsubsection{Mo-Product Analyses}

\section{Thiocyanate Extraction of Mo}

Extraction was performed in a 50-mL plastic vial. $0.1 \mathrm{~mL}$ of fission-product carrier solution was added into $10 \mathrm{~mL}$ of $10 \% \mathrm{H}_{2} \mathrm{SO}_{4}$ and the solution was swirled. Then $0.5 \mathrm{~mL}$ of $\mathrm{Fe}_{2}\left(\mathrm{SO}_{4}\right)_{3}(10 \mathrm{mg} \mathrm{Fe} / \mathrm{mL})$ in $1 \% \mathrm{H}_{2} \mathrm{SO}_{4}$ was added, followed by addition of $0.1 \mathrm{~mL}$ of ${ }^{99} \mathrm{Mo}$ product solution obtained after the LMC process, and the solution was swirled. Then $1 \mathrm{~mL}$ of $50 \% \mathrm{NH}_{4} \mathrm{SCN}$ in water was added and the solution was mixed. Next, $2.5 \mathrm{~mL}$ of $10 \% \mathrm{SnCl}_{2}$ in $10 \% \mathrm{HCl}$ was added and the solution was mixed. The aqueous solution was then combined with $12 \mathrm{~mL}$ of ethyl acetate (pre-equilibrated) and mixed for $2 \mathrm{~min}$. The aqueous phase was separated and combined with $5 \mathrm{~mL}$ of fresh ethyl acetate (pre-equilibrated) and mixed for another $2 \mathrm{~min}$. The aqueous phase was separated and used to determine the activity of the fission product via gamma counting.

\section{Iodine extraction using chloroform}

Extraction was performed in a 20-mL glass scintillation vial. One drop of $\mathrm{KI}$ in water $\left(10 \mathrm{mg} \mathrm{I} / \mathrm{mL}\right.$ ) was added to $10 \mathrm{~mL}$ of water, followed by addition of $0.1 \mathrm{~mL}$ of ${ }^{99}$ Mo product solution obtained after the $\mathrm{LMC}$ process. Then one drop of $8 \mathrm{M} \mathrm{HNO}_{3}$ was added and the solution was combined with $5 \mathrm{~mL}$ of $\mathrm{CHCl}_{3}$. Then two drops of $35 \% \mathrm{HCl}$ and 4 drops of $20 \%$ $\mathrm{NaNO}_{2}$ in water were added and mixed in for $1 \mathrm{~min}$. The phases were allowed to separate, and the organic phase (bottom phase) was transferred into a new glass vial. Then two drops of $20 \%$ $\mathrm{NaNO}_{2}$ in water were added to the aqueous phase, and the solution was combined with $5 \mathrm{~mL}$ of $\mathrm{CHCl}_{3}$ and mixed for $1 \mathrm{~min}$. The phases were allowed to separate, and the organic phase (bottom) was transferred and combined with $\mathrm{CHCl}_{3}$ from the first extraction step. The combined organic phase was used to determine the activity of ${ }^{131} \mathrm{I}$ using gamma counting. 


\subsubsection{Preparation for the LEU-Modified Cintichem Process}

Prior to performing each LMC process with irradiated solution, reagent solutions were prepared according to the descriptions above. The combination column (Figure 2.4.2.4.1) was prepared by placing glass wool on the bottom of the column, then loading slurries of $\mathrm{Ag} / \mathrm{C}$, $\mathrm{HZO}$, and $\mathrm{AC}$ in that order. Then glass wool was placed on top of the sorbents, and the column was sealed. The column was repeatedly washed with $0.2 \mathrm{M} \mathrm{NaOH}$ solution until the $\mathrm{pH}$ of the eluent was alkaline. The column was stored under $0.2 \mathrm{M} \mathrm{NaOH}$ solution and was washed again just before placing into the hot cell right before LMC processing. LMC glassware used for the LMC process is shown in Figure 2.4.2.4.1. Double-sided needles were prepared by combining two needles with male-to-male Luer-lock connectors. The 40-mm 0.3- $\mu \mathrm{m}$ PP filter (Zenpure) for filtration of AgI precipitate (Figure 2.4.2.4.2) was pre-wetted. Reagents used for the LMC process were loaded into syringes and transferred into the hot cell prior to processing.

One-way check valves with needles were used during vacuum transfer of solution between bottles, and only allowed air to enter the system. Normal needles were not used for venting, to minimize release of fission gasses. The needles and filter assembly are shown in Figure 2.4.2.4.2.

Needle guards used to interconnect bottles and needles are shown in Figures 2.4.2.4.3 and 2.4.2.4.4.

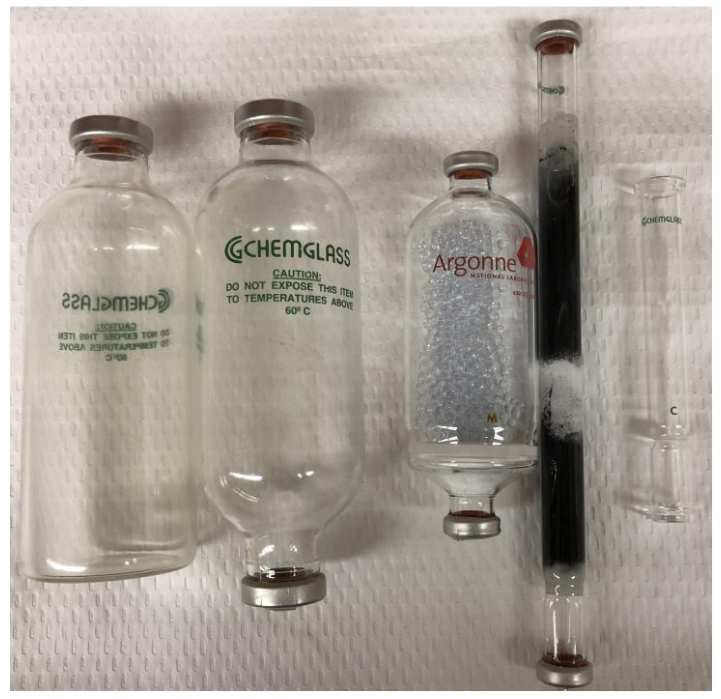

FIGURE 2.4.2.4.1 LMC glassware, plastic coated except for the fritted-glass column. From left to right: flat-bottom bottle, double-sided bottle, 51-mm fritted-glass column containing $\sim 20 \mathrm{~mL}$ of glass beads, $\mathrm{AgC/ZrO/AC} \mathrm{column,} \mathrm{charcoal} \mathrm{filter}$ column (shown empty). All glassware uses crimps to hold septa in place.

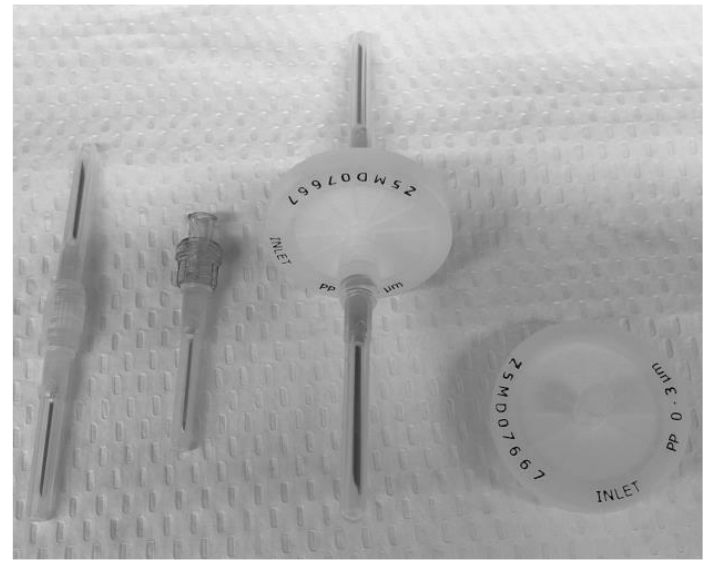

FIGURE 2.4.2.4.2 From left to right: double-sided needle with male-to-male Luer connector in the middle, one-way Luer check-valve needle, $40-\mathrm{mm}$ 0.3- $\mu \mathrm{m}$ filter with needles, $40-\mathrm{mm}$ 0.3- $\mu \mathrm{m}$ filter 


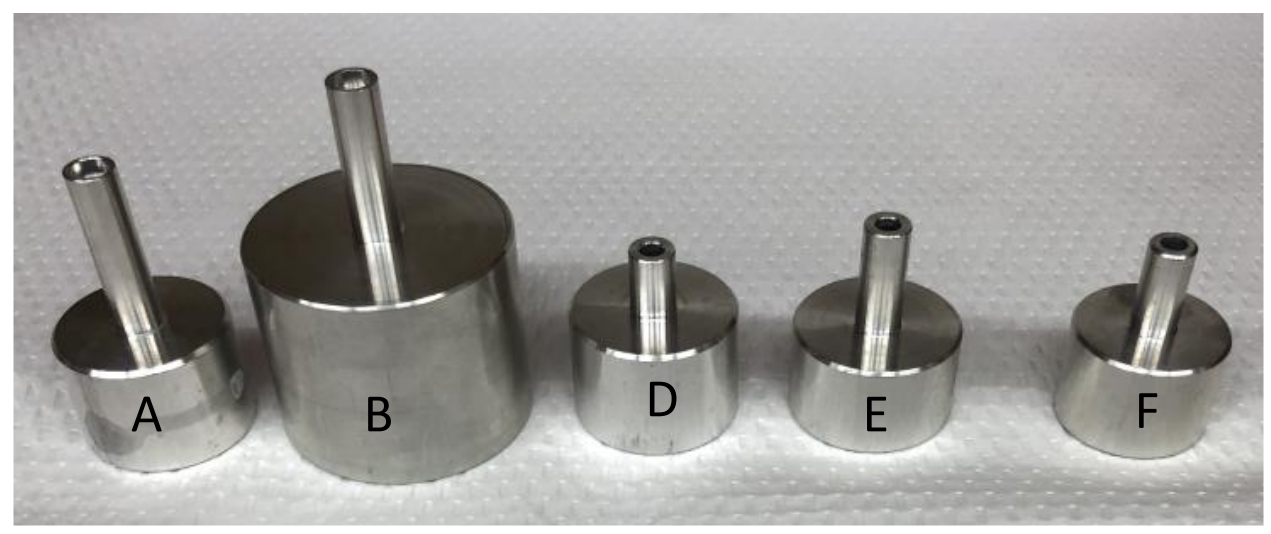

FIGURE 2.4.2.4.3 Various models of aluminum needle adapters

\subsubsection{Acidification of Strip Solution from Concentration Column}

The alkaline strip solution from the concentration column was received in the RF-1 bottle (RF denotes raw fission). The mass of solution in the RF-1 bottle was verified by weighing. An addition of $0.25 \mathrm{~mL}$ of $10 \mathrm{M}$ $\mathrm{HNO}_{3}$ solution was used per $1 \mathrm{~mL}$ of column strip solution. Before addition of an appropriate amount of $10 \mathrm{M} \mathrm{HNO}_{3}$ solution, the RF-1 bottle was connected to the GCS using a needle on top of the bottle. After acidification, the RF-1 bottle contents were mixed by shaking.

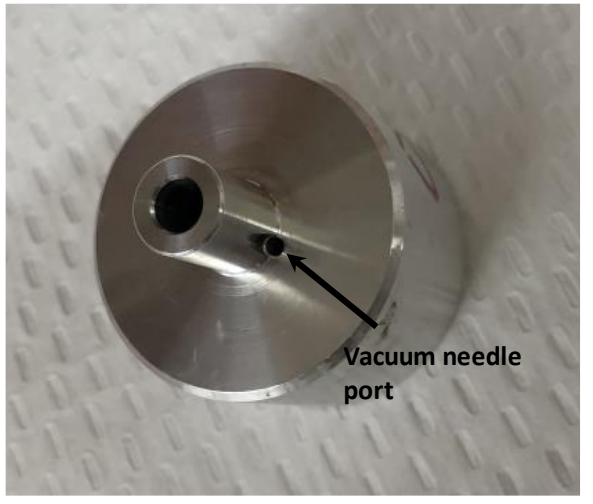

FIGURE 2.4.2.4.4 D-type aluminum needle adapter with side port for vacuum line

\subsubsection{Silver Iodide Precipitation}

After acidification, $4 \mathrm{mg}$ of iodide as NaI carrier was added, and the solution was mixed. Then $0.5 \mathrm{~mL}$ of $10 \% \mathrm{AgNO}_{3}$ was added, and a white precipitate formed. The bottle was mixed again. To remove excess silver, $1 \mathrm{~mL}$ of $1 \mathrm{M} \mathrm{HCl}$ was added, and additional precipitate formed. The solution was mixed and allowed to sit for $2 \mathrm{~min}$. The solution and precipitate from the RF-1 bottle were then transferred through the 40-mm 0.3- $\mu \mathrm{m}$ PP (Zenpure) filter by applying vacuum to the bottom bottle (RF-2). A typical setup for filtration of AgI precipitation is shown in Figure 2.4.2.6.1. The RF-1 bottle was then rinsed with $11 \mathrm{~mL}$ of $4 \mathrm{M} \mathrm{HNO}_{3}$, and the solution was passed through the filter. 


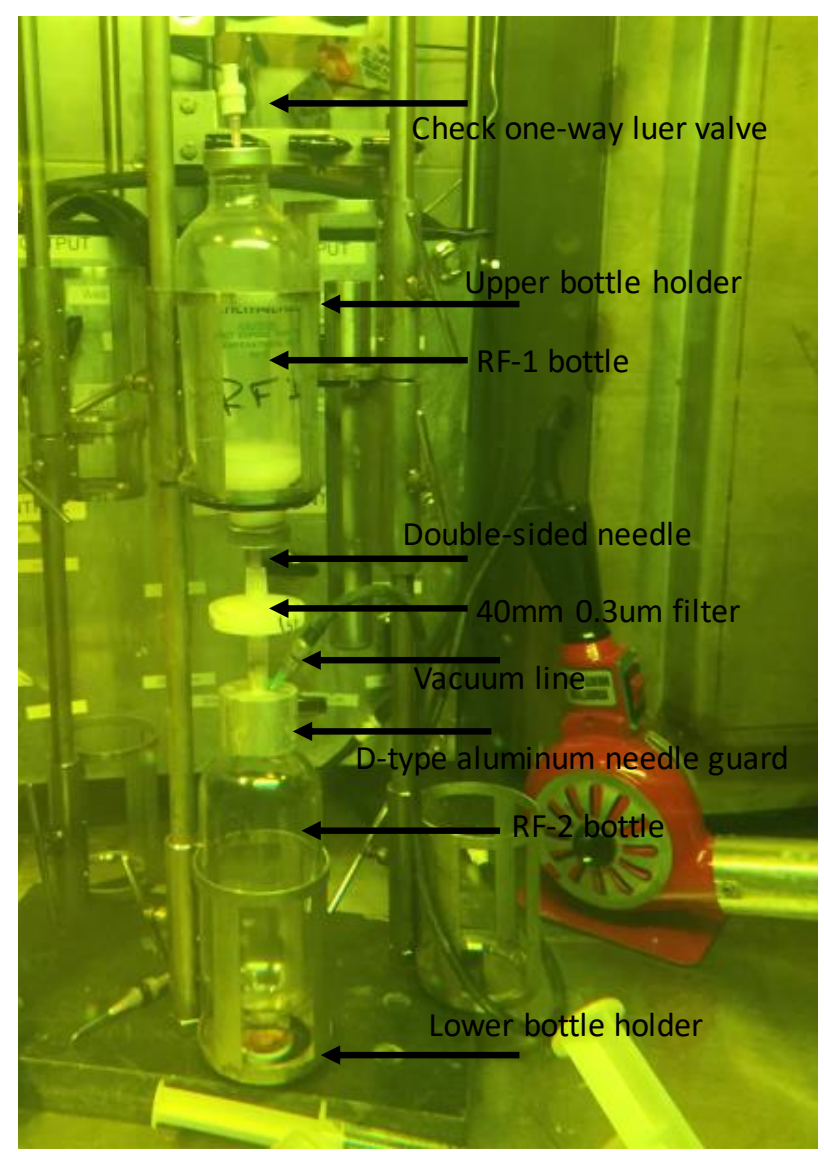

FIGURE 2.4.2.6.1 Example of AgI filtration setup

\subsubsection{Mo-ABO Precipitation}

Following the transfer of the RF-1 solution and wash into the RF-2 bottle, the bottle was evacuated, and $0.5 \mathrm{~mL}$ of Mo carrier solution was added $(10 \mathrm{mg} \mathrm{Mo} / \mathrm{mL})$. Then $2.5 \% \mathrm{KMnO}_{4}$ solution was added dropwise until a deep pink color persisted for $\sim 30 \mathrm{sec}$. This was followed by the addition of $1.5 \mathrm{~mL}$ of $\mathrm{Rh}(8 \mathrm{mg} \mathrm{Rh} / \mathrm{mL})$ and $2 \mathrm{~mL}$ of $\mathrm{Ru}(5 \mathrm{mg} \mathrm{Ru} / \mathrm{mL})$ carriers. The solution in the RF-2 bottle was mixed and allowed to sit for $1 \mathrm{~min}$. Then $20 \mathrm{~mL}$ of $2 \% \mathrm{ABO}$ in $0.4 \mathrm{M}$ $\mathrm{NaOH}$ was added, and the resulting slurry was mixed and allowed to sit for $1 \mathrm{~min}$. Upon addition of $\mathrm{ABO}$, a beige precipitate formed. The slurry containing the Mo-ABO precipitate was than filtered through a fritted bottle containing glass beads by applying vacuum to the RFW (raw fission waste) bottle. The experimental setup for filtration of Mo-ABO is shown in Figure 2.4.2.7.1. The RF-2 bottle on top contains Mo-ABO precipitate after addition of ABO, and Mo-ABO precipitate is transferred into the fritted-filter bottle, while the solution containing the majority of other radionuclides passes through the filter into the RFW bottle. The fritted-filter bottle contains glass beads that allow for better mixing during the washing steps for the Mo$\mathrm{ABO}$ precipitate and during the dissolution of the Mo-ABO precipitate. It should be noted that for these experiments, the fritted-filter bottle was modified from the original design (Figure 2.4.1.4.1) by the Argonne glass blower, who extended the height of the bottle to allow a better grip with remote manipulators. 


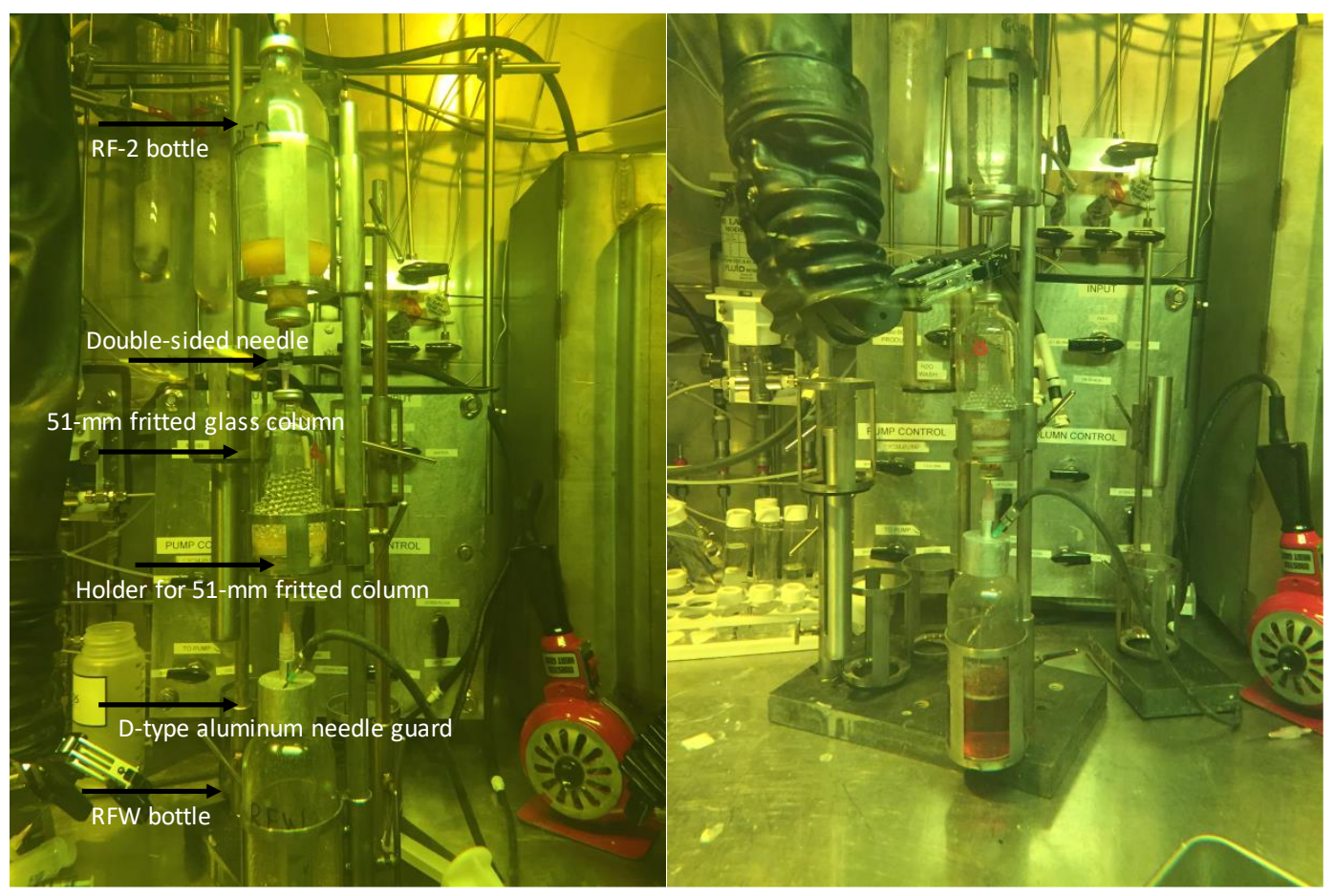

\section{FIGURE 2.4.2.7.1 Experimental setup for filtration of Mo-ABO precipitate}

The Mo-ABO precipitate was washed three times with $20 \mathrm{~mL}$ of $0.1 \mathrm{M} \mathrm{HNO}_{3}$, and five times with $10 \mathrm{~mL}$ of $0.1 \mathrm{M} \mathrm{HNO}_{3}$. The first three washes were performed by adding the $0.1 \mathrm{M}$ $\mathrm{HNO}_{3}$ solution to the RF-2 bottle, briefly mixing it, and then passing it through the frit containing the Mo-ABO precipitate. If no significant amount of precipitate was present in the RF-2 bottle, the next 2-3 washes were applied directly to the fritted-filter bottle. The last 2-3 washes with $10 \mathrm{~mL}$ of $0.1 \mathrm{M} \mathrm{HNO}_{3}$ were performed by loading the wash solution under the frit and pulling it through the frit by applying vacuum in the chamber above, which contains MoABO precipitate. Wash solution was then transferred by vacuum to the RFW bottle. The RFW bottle was weighed, and an aliquot was taken for gamma counting. The solution in the RFW bottle first appeared clear, but over time, formation of a precipitate was observed. This formation is due to an excess of $\mathrm{ABO}$ that did not precipitate with Mo but, owing to its limited solubility under acidic conditions, precipitated after the filtration step. It was confirmed experimentally that the amount of the Mo-ABO complex in the RFW bottle was very small and the presence of precipitate was caused by the limited solubility of excess ABO. 


\subsubsection{Dissolution of Mo-ABO Precipitate}

After complete washing of the Mo-ABO with $0.1 \mathrm{M} \mathrm{HNO}_{3}, 10 \mathrm{~mL}$ of $0.4 \mathrm{M} \mathrm{NaOH} / 1 \%$ $\mathrm{H}_{2} \mathrm{O}_{2}$ was added to the Mo-ABO precipitate and heat was applied by positioning a heat gun at the fritted-filter bottle. The bottle was vented through a charcoal filter (the small glass column shown in Figure 2.4.2.8.1, filled with AC) into the GCS. The experimental setup for dissolution of Mo-ABO precipitate is shown in Figure 2.4.2.8.1. Heat was applied until bubbles started to evolve; then the solution was allowed to cool for five minutes. The resulting solution was removed from the fritted-filter bottle by connecting into the 1-A bottle. The remaining undissolved precipitate was then dissolved in the same manner by adding $10 \mathrm{~mL}$ of $0.2 \mathrm{M}$ $\mathrm{NaOH} / 1 \% \mathrm{H}_{2} \mathrm{O}_{2}$ and heating. The fritted-filter bottle was then rinsed with $10 \mathrm{~mL}$ of $0.2 \mathrm{M} \mathrm{NaOH}$ and its contents collected into the 1-A bottle.

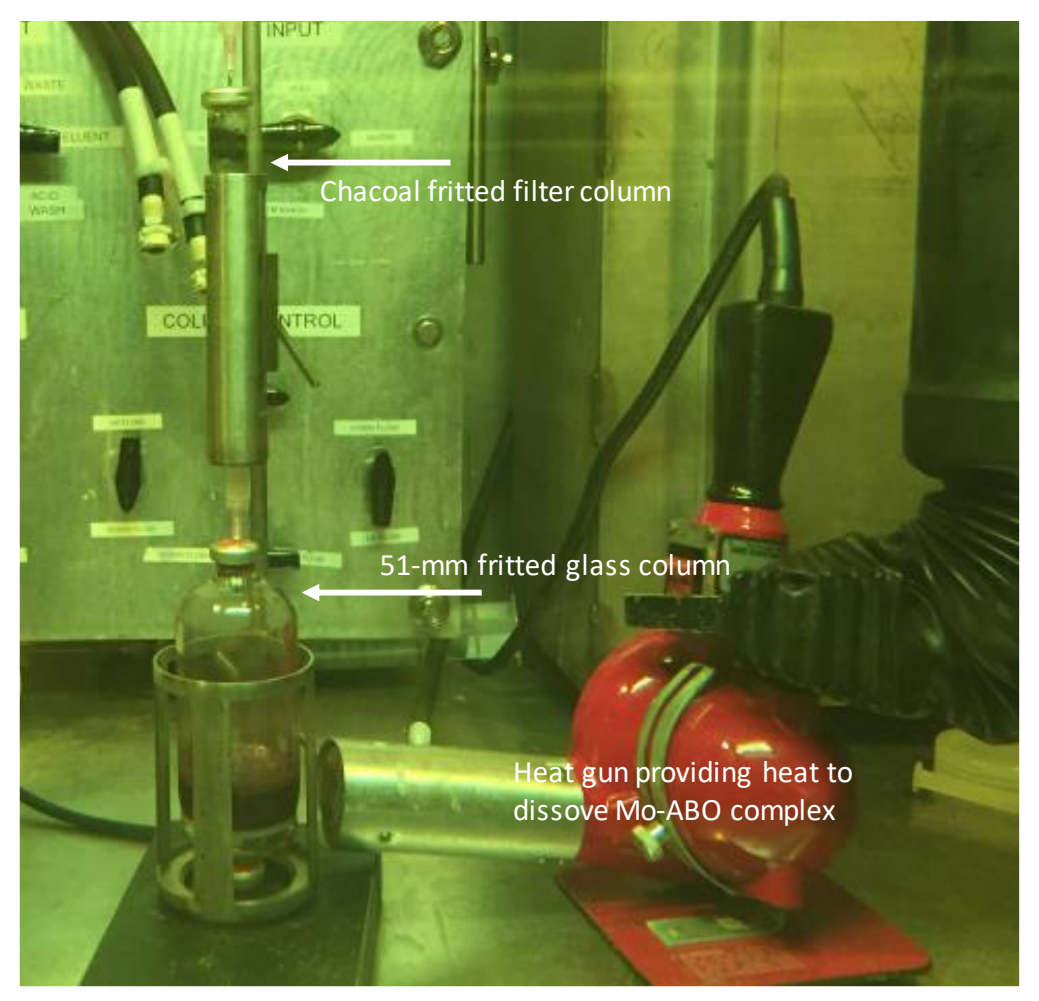

FIGURE 2.4.2.8.1 Experimental setup for dissolution of MoABO precipitate 


\subsubsection{Combination Column}

After dissolution of the Mo-ABO precipitate, a final purification was performed by using a combination column (AC-HZO-Ag/C). Before loading the solution from the 1-A bottle onto the column, another iodine precipitation step was performed. After adding $4 \mathrm{~mL}$ of NaI solution ( $1 \mathrm{mg}-\mathrm{I} / \mathrm{mL}$ ) to the $1-\mathrm{A}$ bottle and mixing, $0.5 \mathrm{~mL}$ of $10 \% \mathrm{AgNO}_{3}$ in $0.1 \mathrm{M} \mathrm{HNO}_{3}$ was added and mixed. A gray-black precipitate formed, and the solution was allowed to sit for five minutes. Then the slurry was loaded onto the column, and the effluent was collected in the 1-B bottle at the bottom. Figure 2.4.2.9.1 shows the 1-A bottle with black precipitate on top, the combination column in the middle, and the 1-B collection bottle for ${ }^{99}$ Mo product at the bottom. Elution was initiated by turning on a vacuum pump connected to the 1-B bottle until a steady flow of drops was observed exiting the column. Then the vacuum pump was turned off, and the column was eluted by gravity at $1-3 \mathrm{~mL}$ per minute. After the initial solution passed through the column, the 1-A bottle was rinsed with $10 \mathrm{~mL}$ of $0.2 \mathrm{M} \mathrm{NaOH}$, which was then run through the column. Once the additional $10 \mathrm{~mL}$ of solution passed through the column, a vacuum was applied to the 1-B bottle to recover all solution from the column. The resulting purified ${ }^{99}$ Mo product was recovered as a $\sim 0.2 \mathrm{M} \mathrm{NaOH}$ solution.

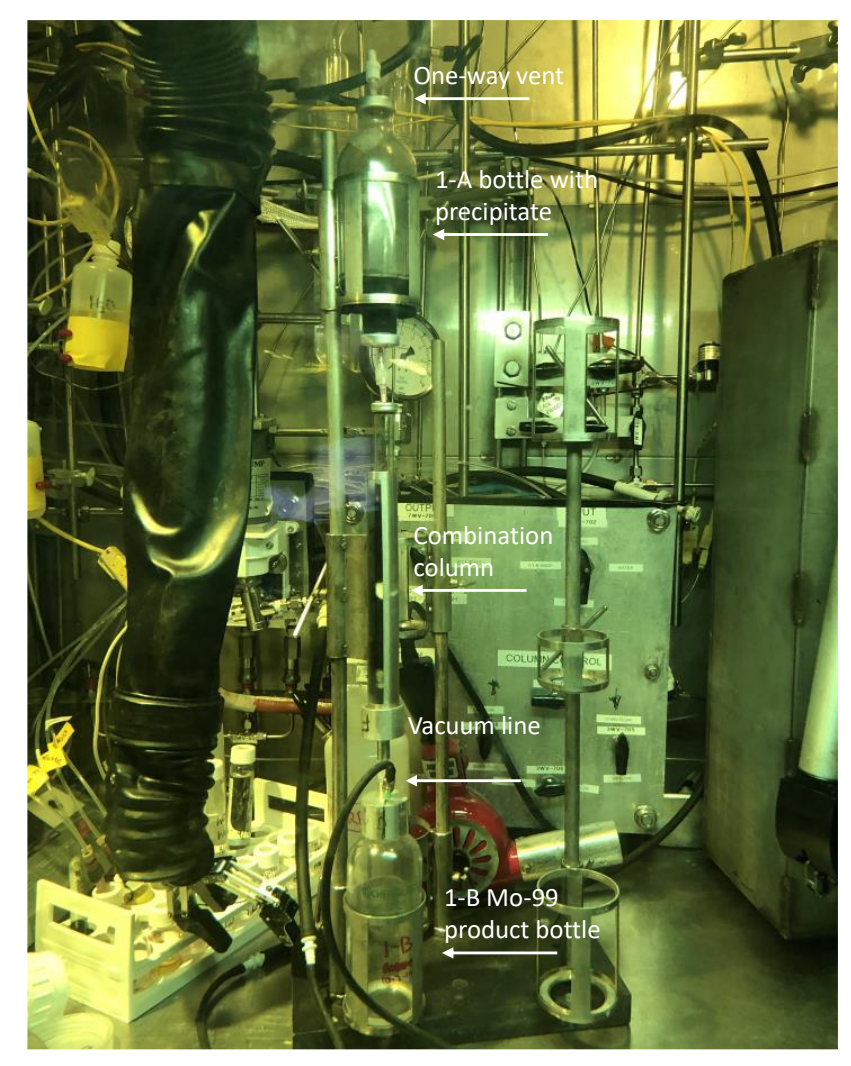

FIGURE 2.4.2.9.1 Experimental setup for combination column 


\subsubsection{References}

[1] A. Hirofumi, H. Kramer, J. McGovern, M. Thornton, and A. Thornton, Production of high purity fission product molybdenum-99, U.S. Patent 3,799,883, 1974.

[2] D. Wu, S. Landsberger, B. A. Buchholz, and G. F. Vandegrift, Processing of LEU Targets for Mo-99 Production-Testing and Modification of the Cintichem Process, 1995 International Meeting on Reduced Enrichment for Research and Test Reactors, Paris, France, September 18-21, 1994 (available at: http://www.rertr.anl.gov/MO99/WU95.pdf).

[3] Z. Aliludin, A. Mutalib, A. Sukmana, Kadarisman, A. H. Gunawan, G. F. Vandegrift. D. Wu, B. Srinivasan, and J. L. Snelgrove, Processing of LEU Targets for Mo-99 ProductionDemonstration of a Modified Cintichem Process, 1995 International Meeting on Reduced Enrichment for Research and Test Reactors, Paris, France, September 18-21, 1994 (available at: http://www.rertr.anl.gov/MO99/BATAN95.pdf).

[4] D. Wu, S. Landsberger, and G. F. Vandegrift, Progress in Chemical Treatment of LEU Targets by the Modified Cintichem Process, 1996 International Meeting on Reduced Enrichment for Research and Test Reactors, Seoul, Korea, October 7-10, 1996 (available at: http://www.rertr.anl.gov/99MO96/WU96.PDF).

[5] R. A. Leonard, L. Chen, C. J. Mertz, and G. F. Vandegrift, Progress in Dissolving Modified LEU Cintichem Targets, 1996 International Meeting on Reduced Enrichment for Research and Test Reactors, Seoul, Korea, October 7-10, 1996 (available at: http://www.rertr.anl.gov/99MO96/LEONAR96.PDF).

[6] A. Bakel., A. Leyva, T. Wiencek, A. Hebden, K. Quigley, J. Falkenberg, L. Hafenrichter, and G. Vandegrift, Overview of Progress Related to Implementation of the LEU-Modified Cintichem Process, 2008 International RERTR Meeting, Washington, D.C., October 5-9, 2008 (available at http://www.rertr.anl.gov/RERTR30/pdf/S8-1_Bakel.pdf).

[7] A. J. Youker, S. D. Chemerisov, P. Tkac, M. Kalensky, T. A. Heltemes, D. A. Rotsch, J. F. Krebs, V. Makarashvili, D. C. Stepinski, K. Alford, J. Bailey, J. Byrnes, R. Gromov, L. Hafenrichter, A. Hebden, J. Jerden, C. Jonah, B. Micklich, K. Quigley, J Schneider, K. Wesolowski, G. F. Vandegrift, and Z. Sun, Compendium of Phase-I Mini-SHINE Experiments, ANL/NE-16/39, Argonne National Laboratory, October 2016 (available at https://publications.anl.gov/anlpubs/2017/01/131828.pdf).

[8] D. A. Skoog and D. M. West, Fundamentals of Analytical Chemistry, Holt, Rinehart, and Winston, New York, 1963, p. 770. 


\subsection{GAMMA COUNTING}

\subsubsection{Introduction}

Gamma spectroscopy was performed on aqueous samples to determine the initial and produced activities of fission products at various points in solution processing. This information was then used as a way to quantify the recovery achieved by the various steps as well as the overall procedure, and to determine the points where several important isotopes/contaminants were removed from the final product.

\subsubsection{Instrumentation}

Gamma spectroscopy was performed using one of two high-purity germanium (HPGe) detectors, both of which were calibrated with an Eckert \& Ziegler mixed-isotope standard. Both HPGe detectors used were coaxial geometry connected to ORTEC DSPEC 50 digital analyzers and were calibrated at various distances to accommodate samples of different strengths. One of the instruments was cooled using $\mathrm{LN}_{2}$ with an ORTEC Möbius Recycler to extend the refilling interval, and was set up with simple shielding around the detector and a movable sample holder with set distances (seen in Figure 2.5.2.1). The other HPGe instrument was mechanically cooled using an ORTEC X-COOLER III and was coupled to an autosampling system with a shielded canyon where the sample resided for counting (see Figure 2.5.2.2).

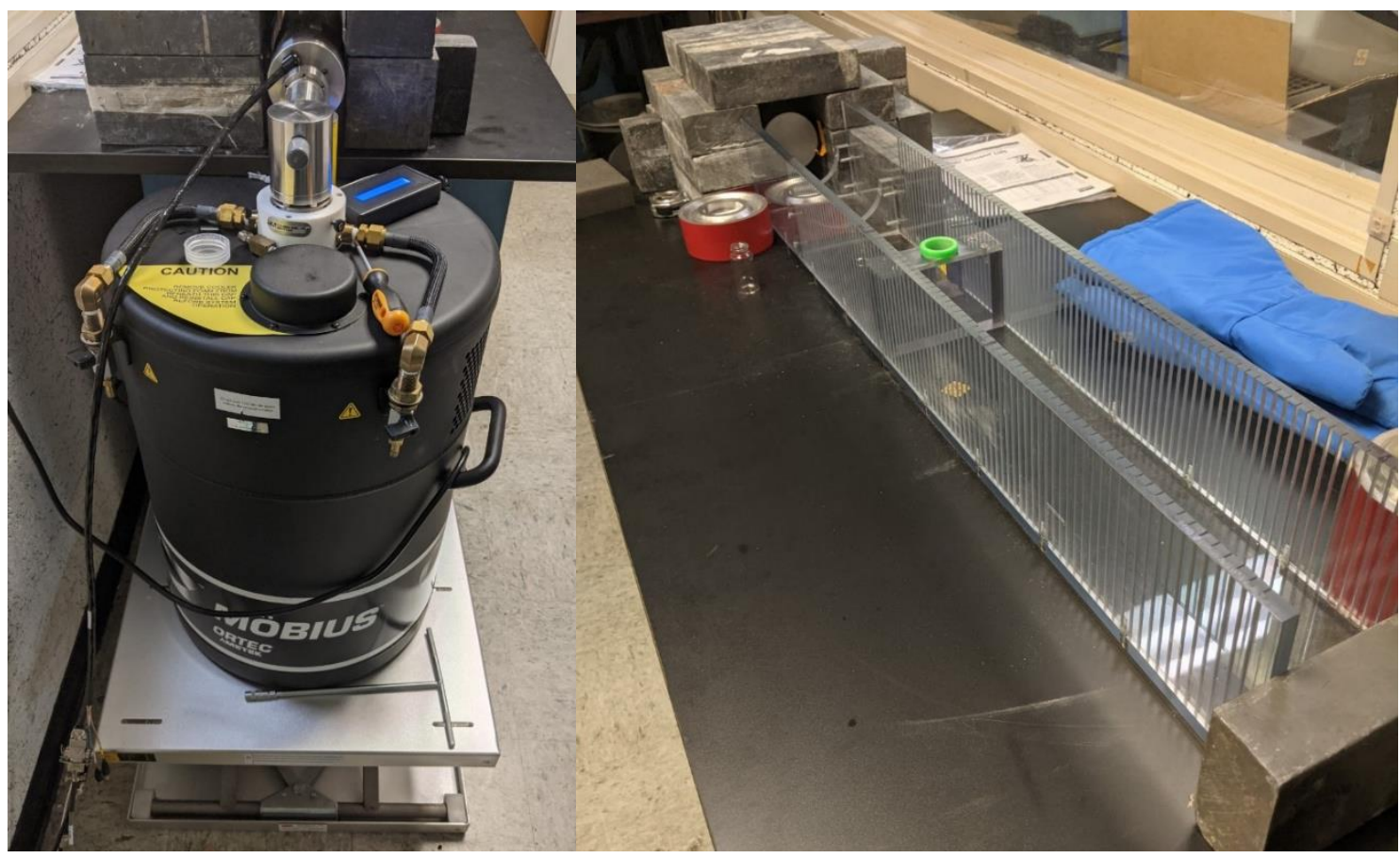

FIGURE 2.5.2.1 Coaxial HPGe cooled with $\mathrm{LN}_{2}$ and recycler (left) and the same detector with associated shielding and movable sample holder (right) 


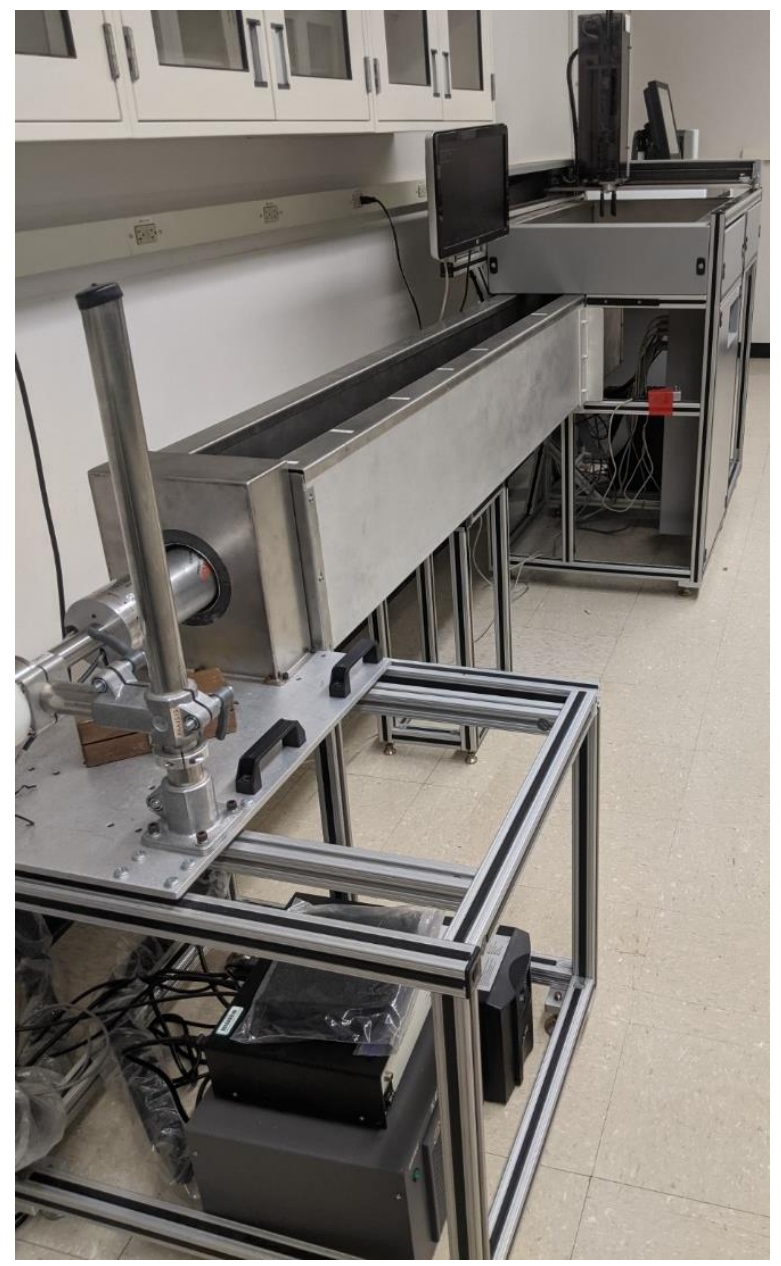

FIGURE 2.5.2.2 Mechanically cooled coaxial detector coupled to an autosampling unit with a shielded canyon for counting

Regardless of which instrument was used to collect the gamma spectra, resulting data for the different solutions were analyzed and decay-corrected with GammaVision software.

For each sample, multiple analyses were executed over time to ensure that the reported activities were not skewed by overlapping peaks. In most cases, only the 33 nuclides and associated peaks listed in Table 2.5.2.1 were quantified. In several cases, however, large increases in reported activities were observed when the same samples were counted a second or third time. This increase indicated the presence of overlapping peaks from unidentified constituents and was found to occur primarily in the target solution samples after irradiation. In these instances, a more complex library was used to subtract the minor peaks of the nuclides of interest from the spectrum, resulting in much better agreement. The only nuclides for which this approach did not work were ${ }^{105} \mathrm{Rh}$ and ${ }^{131 \mathrm{~m}} \mathrm{Xe}$. The interference on these peaks was caused by ${ }^{147} \mathrm{Nd}$ and ${ }^{140} \mathrm{Ba}$, respectively, which were only present in the target solution and therefore did not cause discrepancies in any samples taken after glovebox processing. 
It should be noted that high activity concentrations of ${ }^{131} \mathrm{I},{ }^{133} \mathrm{I},{ }^{135} \mathrm{I}$, and ${ }^{99}$ Mo in most of the samples following the initial glovebox processing meant that aliquots of even a few milligrams often resulted in significant detector dead time, which made the signal of some radionuclides with lower gamma branching ratios (such as ${ }^{125} \mathrm{Sn}$ ) difficult to identify. The iodine isotopes $\left({ }^{131} \mathrm{I},{ }^{132} \mathrm{I},{ }^{133} \mathrm{I}\right.$, and $\left.{ }^{135} \mathrm{I}\right)$ could all be easily identified, since their parents were either short-lived (half-life $<1 \mathrm{hr}$ ) or were removed by the recovery column in the glovebox $\left({ }^{131 \mathrm{~m}} \mathrm{Te}\right.$,

${ }^{132} \mathrm{Te}$ ). The recovery column also removed all ${ }^{237} \mathrm{U}$ and ${ }^{239} \mathrm{~Np}$ from the process stream.

TABLE 2.5.2.1 Isotopes and associated peaks analyzed during gamma spectroscopy

\begin{tabular}{cccc}
\hline Isotope & Peak Energy Used $(\mathrm{keV})$ & Isotope & Peak Energy Used $(\mathrm{keV})$ \\
\hline & & & \\
${ }^{95} \mathrm{Zr}$ & 756.7 & ${ }^{97} \mathrm{Zr}$ & 743.4 \\
${ }^{95} \mathrm{Nb}$ & 765.8 & ${ }^{92} \mathrm{Sr}$ & 1384.1 \\
${ }^{237} \mathrm{U}$ & 208.0 & ${ }^{99 \mathrm{~m}} \mathrm{Tc}$ & 140.5 \\
${ }^{156} \mathrm{Eu}$ & 1230.7 & ${ }^{131 \mathrm{~m}} \mathrm{Xe}$ & 163.9 \\
${ }^{137} \mathrm{Cs}$ & 661.7 & ${ }^{135} \mathrm{Xe}$ & 249.8 \\
${ }^{239} \mathrm{~Np}$ & 277.6 & ${ }^{133 \mathrm{~m}} \mathrm{Xe}$ & 233.2 \\
${ }^{99} \mathrm{Mo}$ & 181.1 & ${ }^{132} \mathrm{Te}$ & 49.8 \\
${ }^{103} \mathrm{Ru}$ & 497.1 & ${ }^{97} \mathrm{Nb}$ & 657.9 \\
${ }^{132} \mathrm{I}$ & 522.7 & ${ }^{105} \mathrm{Rh}$ & 318.9 \\
${ }^{131} \mathrm{Te}$ & 793.8 & ${ }^{127} \mathrm{Sn}$ & 1088.9 \\
${ }^{131} \mathrm{Sb}$ & 685.5 \\
${ }^{133} \mathrm{I}$ & 637.0 & ${ }^{91} \mathrm{Sr}$ & 555.56 \\
& 529.9 & & 1024.3 \\
${ }^{136} \mathrm{Cs}$ & 818.5 & ${ }^{147} \mathrm{Nd}$ & 91.1 \\
& 1048.1 & & 340.06 \\
${ }^{140} \mathrm{Ba}$ & 423.7 & ${ }^{151} \mathrm{Pm}$ & 266.9 \\
${ }^{156} \mathrm{Sm}$ & 165.7 & ${ }^{93} \mathrm{Y}$ & 1260.4 \\
${ }^{140} \mathrm{La}$ & 1596.2 & & \\
${ }^{143} \mathrm{Ce}$ & 293.3 & & \\
\hline
\end{tabular}

\subsubsection{Sample Preparation}

All solutions were prepared for gamma spectroscopy by mass-based serial dilution. Because the original solution volumes were also tracked by mass, this method reduced uncertainty in dilution factors and allowed very small samples to be correlated to large initial solutions with higher confidence. Small samples sizes were often necessary because of the highly radioactive nature of the parent solutions. 


\section{RESULTS AND DISCUSSION}

The first Phase II post-irradiation processing was performed on March 9, 2018. After successful recovery-column processing in the recovery glovebox, the solution containing ${ }^{99} \mathrm{Mo}$ and other fission products was transferred to the hot cell for further purification. At the beginning of concentration-column processing inside the hot cell, an elevated background reading occurred in the linac facility. Fission gases from the hot-cell stack were being recirculated into the building, and this caused elevated readings on the radiation detectors within the linac facility.

During the subsequent fact-finding process, a strong correlation was established between the increase in readings and the installation of a $\mathrm{pH}$ probe, which caused a 3 -liter processing vessel to be opened to the processing-hot-cell atmosphere. This incident was investigated by internal and external committees. Both committees arrived at very similar conclusions and recommendations. On the basis of the results of the investigations, multiple corrective actions were proposed and executed. The main parts of the corrective actions were as follows:

- Develop a process for screening of the experimental work against facility safety bases.

- Develop a configuration management program for the facility.

- Conduct a design review of the building exhaust and extend the exhaust stack for the hot cell to prevent the possibility of recirculation.

- Conduct design reviews for all systems connected to the GCS.

- Setup dedicated equipment for monitoring radiation levels in LEAF during processing.

- Modify the facility Safety Assessment Document (SAD) and ASE to include additional descriptions of the experiment and additional credited controls.

- After implementation of the corrective actions, conduct an Accelerator Readiness Review for the restart of experimental activities.

In accordance with the corrective actions identified, several modifications were implemented, and a Commissioning Plan was developed. Modified operational procedures were subsequently developed and are attached as multiple appendices to this report. The purpose of the Commissioning Plan was to check that all systems were operating as intended. The Commissioning Plan consisted of testing all the systems by performing the associated operations with a solution spiked with a small portion of ${ }^{99} \mathrm{Mo}$. The results of the test provided a baseline for separation process readiness, allowed improvements to the procedures and training, and provided integrated verification of the chemical separation, purification, and gas collection systems within the controls described in the SAD and ASE. Those two documents encompass all the safety aspects of Accelerator Facility operations: the SAD provides the bases for safe 
operations of the facility and the ASE lists the limits for the operation parameters and credited controls. Because the type of accident (gas release and recirculation) was not discussed in the SAD and controls were not established in the ASE, both documents had to go through significant rewriting and a vetting process with DOE ASO.

The Commissioning Plan consisted of the following sections:
a. Prerequisite Conditions (including GCS pre-checks);
b. GCS Commissioning;
c. Recovery Glove Box Processing Commissioning;
d. D-024 Hot Cell Commissioning; and
e. Radiation Monitoring Commissioning.

A commissioning run was carried out from August 13 to 15, 2019, with a ${ }^{99}$ Mo spike (no irradiation). Experimental conditions were verified prior to commencing the commissioning run, in accordance with updated SAD and ASE requirements:

- U concentration was $132 \mathrm{~g} / \mathrm{L}$ and was below the SAD limit of $<145 \mathrm{~g}-\mathrm{U} / \mathrm{L}$.

- No makeup solution was added, and ${ }^{235} \mathrm{U}$ enrichment remained at or below the original value of $19.86 \%{ }^{235} \mathrm{U}$, which is below the SAD limit of $<20 \%{ }^{235} \mathrm{U}$.

- The LEU solution volume was $\sim 17.6 \mathrm{~L}$, which is below the SAD limit of $<20 \mathrm{~L}$.

During mixing of the ${ }^{99} \mathrm{Mo}$ spike with the LEU solution, the presence of a precipitate was observed in the LEU solution. A sample of the precipitate was analyzed using powder X-ray diffraction analysis (XRD) and confirmed the presence of uranyl peroxide. From the results of the XRD analysis, we concluded that this precipitate had formed during the first Phase II irradiation in March 2018. Formation of uranyl peroxide under irradiation conditions is not unexpected and was confirmed during our small-scale irradiations of a similar uranyl sulfate solution at the Van de Graaff (VDG) generator. The formation of uranium precipitate is due to complexation of the uranyl ion with peroxide that forms during the radiolysis of water. This behavior is well understood and can be prevented by addition of a $\mathrm{Fe}^{2+} / \mathrm{Fe}^{3+}$ catalyst, as was confirmed by subsequent VDG irradiations [1]. The presence of precipitate did not cause any safety concerns; however, it is experimentally undesirable, as it may complicate solution transfer and sample collection. Prior to irradiation of the LEU solution, an attempt was made to remove the precipitate from the system by repeated pumping of the solution from the TSV to the verification tank through a high-capacity filter. In addition, an iron catalyst was added to the LEU solution to minimize the likelihood of future precipitation.

\section{References}

[1] Kalensky, M., Brossard, T., and Tkac, P., Low LET Irradiations of Uranyl Sulfate Solutions in the Absence and Presence of $\mathrm{Fe}^{+2}$ and $\mathrm{Fe}^{+3}$ Ions, ANL-19/44, Argonne National Laboratory, September 2019. 


\subsection{LINAC IRRADIATIONS}

Six irradiations were performed as part of the Phase II studies. Table 1.1 briefly describes these experiments. Each is discussed further in the text below.

\subsubsection{Irradiation \#0 with Accidental Rad Gas Release, 3/8/18}

The first irradiation of the uranyl sulfate solution in the study was conducted on March 8, 2018. For the first test with the beam, the time for irradiation was limited to a maximum of 4 hours at high power. This run duration was chosen for two reasons:

- One-fifth of the planned full-production scale allowed a good estimation of the activity produced at a full 20-hour irradiation, and

- Four hours would be enough to establish thermal equilibrium in the uranyl sulfate solution, as well as steady-state gas generation rates.

This irradiation provided us with fission-product production rates, which were compared with the expectation from our Monte Carlo (MCNPX) calculations.

Preparation for the irradiation started on the day prior to irradiation. Performance of the cooling system was verified using procedures LEAF-PROC-001, -002, -003, -004, -006, and 007 (see Appendices 19-24). Initial beam tuning was performed following procedure LEAFPROC-027 (Appendix 25).

On the day of irradiation, the beam parameters were verified. Readiness of all AMORE systems for irradiation was verified according to procedure LEAF-PROC-012 (Appendix 26). Owing to limitations for heat dissipation in the spectrometer magnet, the energy spectrum was measured at $38 \mathrm{MeV}$. The resulting energy spectrum is presented in Figure 3.1.1.1. After spectrum acquisition, the peak beam current was reduced to shift the beam energy peak to $40 \mathrm{MeV}$. After energy verification, the beam was placed on the target window at low power $(500 \mathrm{~W})$, and the beam shape was adjusted to produce a $16 \times 14.1-\mathrm{mm} \mathrm{FWHM}^{2}$ beam spot on the target face (Figure 3.1.1.2).

Irradiation started at 9:15 am with $\sim 500 \mathrm{~W}$ of beam power on the target. Over a period of $45 \mathrm{~min}$, the power was increased to $6 \mathrm{~kW}$, where it stayed for $1 \mathrm{~h}$. The power was then increased to $10 \mathrm{~kW}$, then to $12 \mathrm{~kW}$, and finally to $13.8 \mathrm{~kW}$. A beam power of $13.8 \mathrm{~kW}$ was the highest at which we could continuously perform irradiation while maintaining the hydrogen concentration below $1 \%$ in the vessel off-gas.

Full Width at Half Maximum. The beam profile has a Gaussian shape; measurement of the FWHM values for the beam intensity distribution is a well-established practice. 
To test the target performance at full beam power, we decreased the beam power to $10 \mathrm{~kW}$, waited until the hydrogen concentration dropped and stabilized, and then increased the power to $18 \mathrm{~kW}$ for a short period of time. During these short power increases, we monitored the temperature of the target cooling water. Figure 3.1.1.3 depicts the beam history for this irradiation. The irradiation was ended at $4 \mathrm{pm}$.

At the end of irradiation, the gas-analysis and -collection system was purged to reduce the amount of radioactive gas in the gas-analysis manifold. During irradiation, a significant amount of gaseous fission products is accumulated in the gas lines connected to the experiment. Those fission products generated a high radiation field in the rooms used by personnel for processing of the solution. Therefore, prior to the start of the solution processing, the gas manifolds have to be purged to reduce dose rates in the occupied areas. Processing of the irradiated solution started at $\sim 4 \mathrm{pm}$.

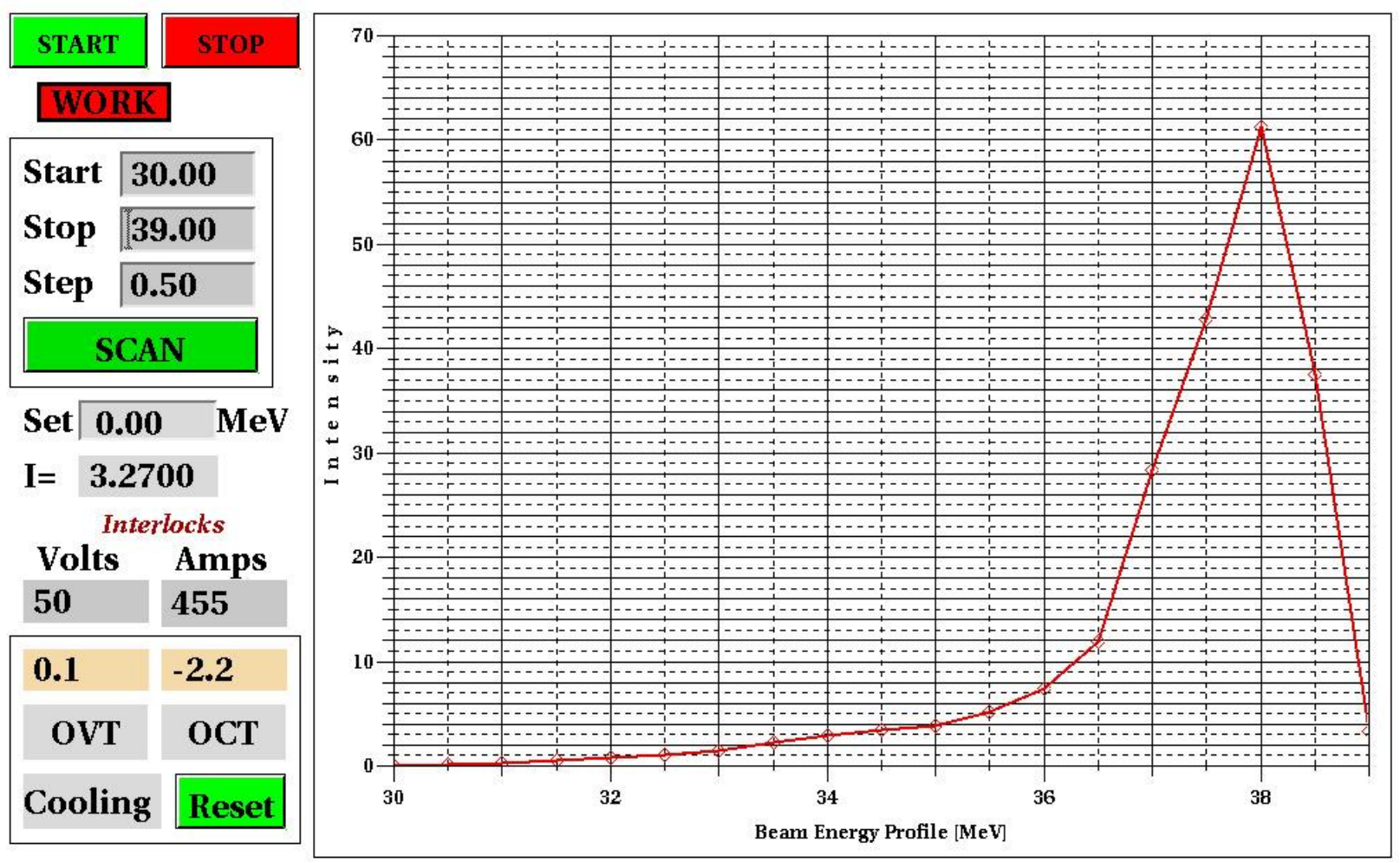

FIGURE 3.1.1.1 Beam energy spectrum at $\sim 38 \mathrm{MeV}$ for Irradiation \#0 on $3 / 8 / 18$. After tune-up, the injector current was lowered to shift the beam energy to $\sim 40 \mathrm{MeV}$. 


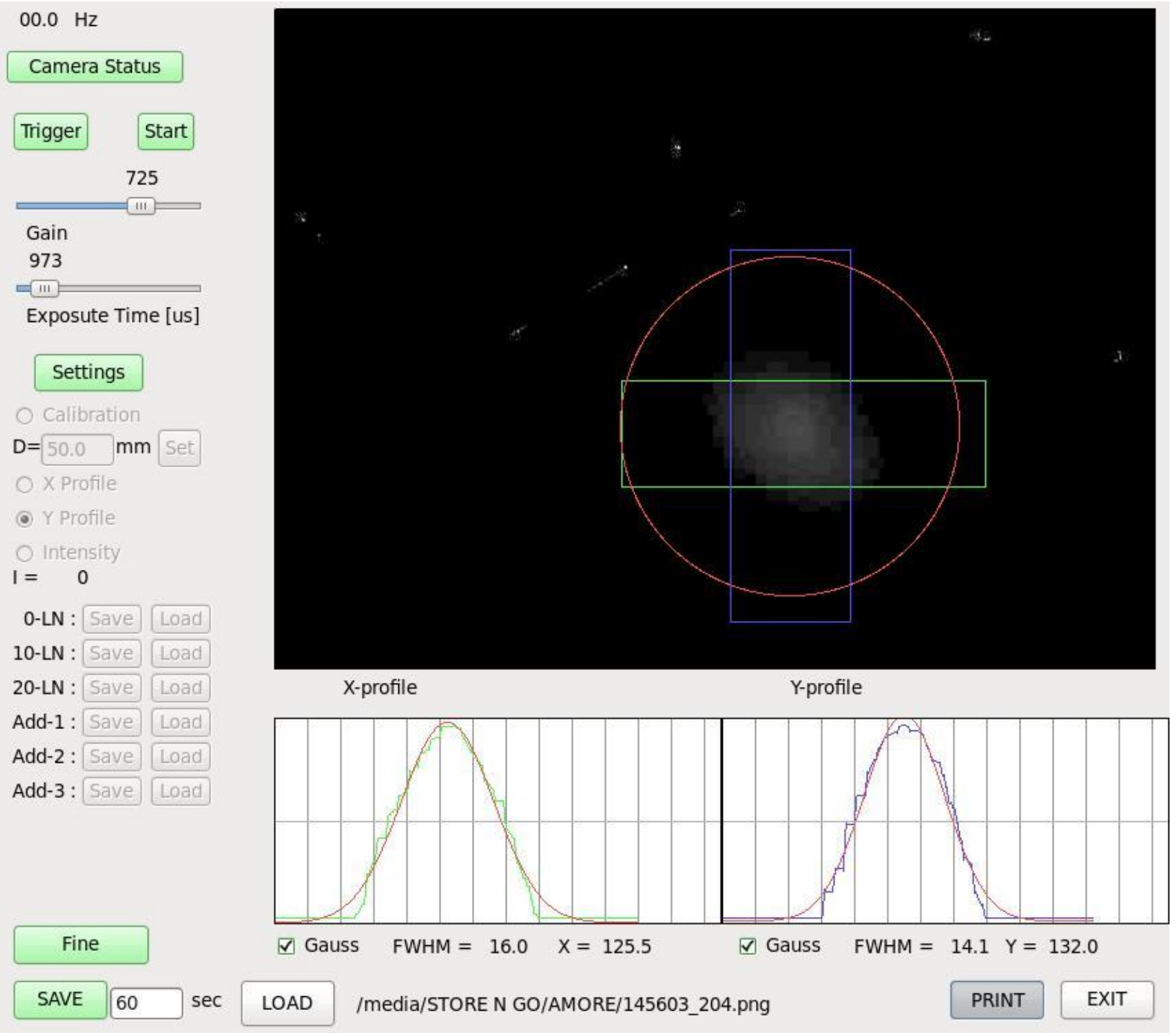

FIGURE 3.1.1.2 Beam profile on the target window for Irradiation \#0. Red circle outlines the target beam window boundary. 


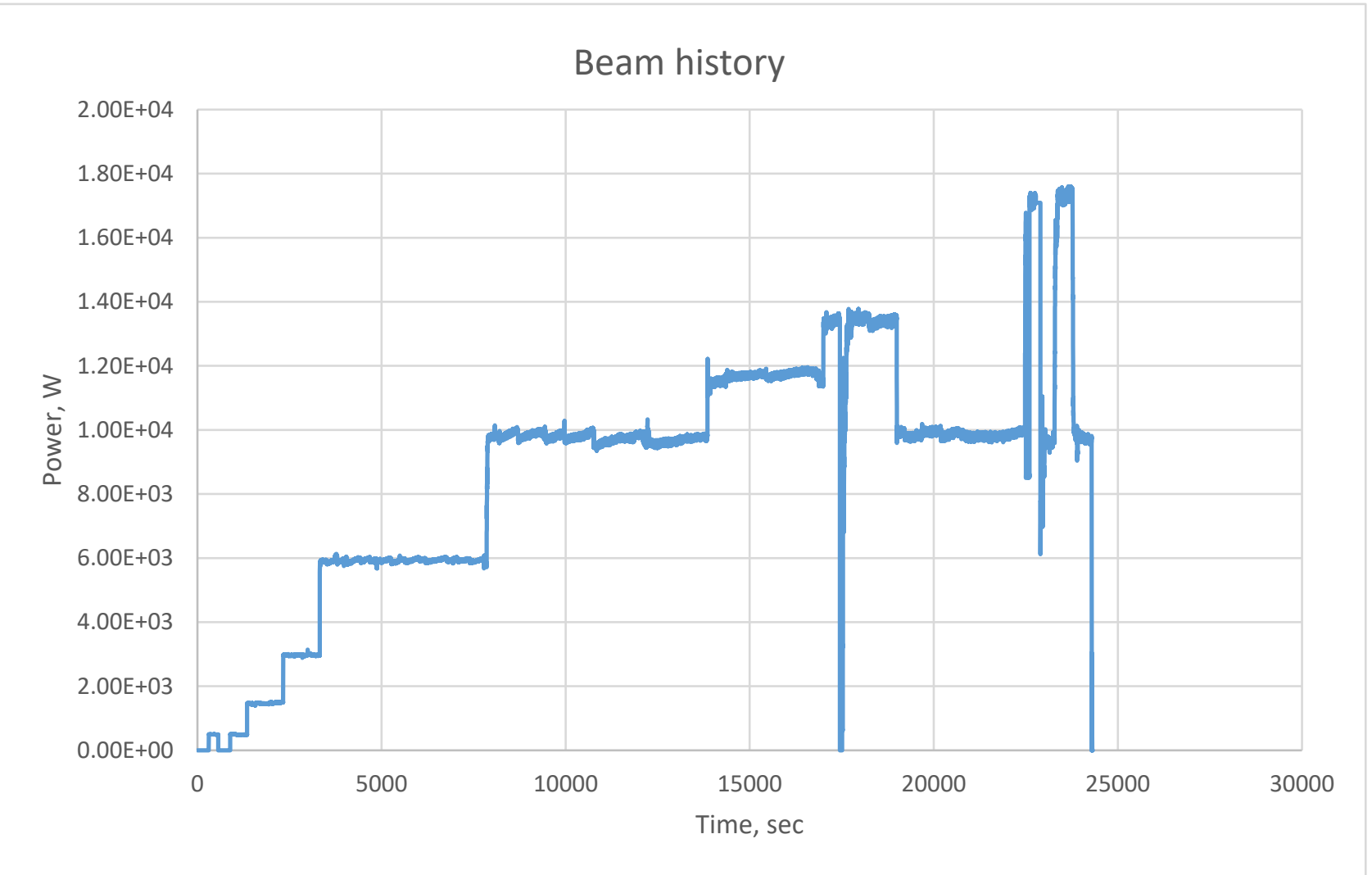

FIGURE 3.1.1.3 Beam history for Irradiation \#0

A commissioning run was carried out from August 13 to 15,2019 , with a ${ }^{99}$ Mo spike (no irradiation). Prior to performing the experimental work, all workers were trained and qualified (qualification cards were filled out and signed). A pre-job briefing was held on August 12 to confirm everyone's readiness to perform the work. All training documentation is stored in an online database. Experimental conditions were verified prior to commencing the commissioning run in accordance with updated SAD and ASE requirements:

- The U concentration was $132 \mathrm{~g} / \mathrm{L}$, which is below the SAD limit of $<145 \mathrm{~g}-\mathrm{U} / \mathrm{L}$;

- No makeup solution was added, and ${ }^{235} \mathrm{U}$ enrichment remained at or below the original value of $19.86 \%{ }^{235} \mathrm{U}$, which is below the SAD limit of $<20 \%{ }^{235} \mathrm{U}$; and

- The LEU solution volume was $~ 17.6 \mathrm{~L}$, which is below the SAD limit of $<20 \mathrm{~L}$. 
During mixing of the ${ }^{99} \mathrm{Mo}$ spike with the LEU solution, the presence of a precipitate was observed in the LEU solution. A sample of precipitate was analyzed using XRD, and confirmed the presence of uranyl peroxide. From the XRD analysis results, we conclude that this precipitate formed during the first Phase II irradiation in March 2018. The formation of uranyl peroxide under irradiation conditions is not unexpected, and was confirmed during our small-scale irradiations of a similar uranyl sulfate solution at the VDG generator. The formation of uranium precipitate is due to the complexation of the uranyl ion with peroxide that forms through radiolysis of water. This behavior is well understood and can be prevented by the addition of a $\mathrm{Fe}^{2+} / \mathrm{Fe}^{3+}$ catalyst, as was confirmed by subsequent VDG irradiations. The presence of precipitate does not cause any safety concerns; however, it is undesirable, as it may complicate solution transfer and sample collection. Prior to irradiation of the LEU solution, we removed the precipitate from the system by repeated pumping of the solution from the TSV to the verification tank through a high-capacity filter. In addition, an iron catalyst was added to the LEU solution to minimize the likelihood of future precipitation.

The commissioning activities were completed in the manner described previously and all associated work procedures were followed. Despite some technical difficulties that involved failure of electronic components (such as power supply, heater, solenoid valve, and pressure transducer) due to radiation damage caused by irradiations performed in the D-035 glovebox for other programs, all systems performed well, and the solution was moved from the D-035 glovebox into the D-024 hot cell, where the concentration-column operation and LMC process were performed. All necessary samples were collected and analyzed.

Performance of the Gas Handling system pre-checks and interlock verification, linac prechecks and interlock verification, and Supplemental Radiation Monitoring System was successfully completed. Gas Handling procedures were followed as written. Some lessons learned were incorporated into the procedures to improve clarity. The relevant parameters for the experimental systems were confirmed to be as described in the facility SAD and ASE. In short, all acceptance criteria were met. All follow-up items required to be completed prior to the next irradiation were tracked to completion by the ${ }^{99}$ Mo Program Manager.

\subsubsection{Irradiation \#1, 10/1/19}

The first irradiation after the restart was conducted on 10/1/19. For the restart, the time for irradiation was limited to a maximum of 4 hours at high power. Essentially, we were trying to return to the same starting point as Irradiation \#0. Because the plan for the experiment included the processing of the solution immediately after irradiation without significant interruptions, the end of irradiation \#1 was scheduled for $10 \mathrm{pm}$, leaving enough time to prepare for the experiment during the day. 
On the morning of Irradiation \#1, the beam was tuned and prepared for transport to the target. The beam energy spectrum is presented in Figure 3.1.2.1. This spectrum corresponds to a peak energy of $37 \mathrm{MeV}$ at $0.53 \mathrm{~A}$ peak current. After spectrum acquisition, the peak beam current was reduced to $0.42 \mathrm{~A}$, which shifted the beam energy peak to $40 \mathrm{MeV}$. After that, the energy verification beam was placed on the target window at low power, and the beam shape was adjusted to produce a 16.2x15.7-mm FWHM beam spot on the target face (Figure 3.1.2.2).

The irradiation began at $4 \mathrm{pm}$ with $\sim 150 \mathrm{~W}$ of beam power on the target. Over $45 \mathrm{~min}$, the power was increased to $14 \mathrm{~kW}$, where it stayed for the duration of the experiment. Around $7 \mathrm{pm}$, instabilities in the injector current caused beam position changes that led to beam losses in the beamline and a worsening vacuum. This caused $\sim 15$ minutes of interruption in irradiation. At $7: 15 \mathrm{pm}$, irradiation at $\sim 14 \mathrm{~kW}$ was resumed. The maximum beam power used in this experiment was $14.4 \mathrm{~kW}$; the maximum power was limited by hydrogen production and the requirement to maintain hydrogen concentration under $1 \%$ in the irradiation-vessel off-gas. Figure 3.1.2.3 depicts the beam history for the irradiation. Irradiation \#1 was completed at $10 \mathrm{pm}$. After the end of irradiation, the gas-analysis and -collection system was purged to reduce the amount of radioactive gases in the gas-analysis manifold. Processing of the irradiated solution started at $11 \mathrm{pm}$.
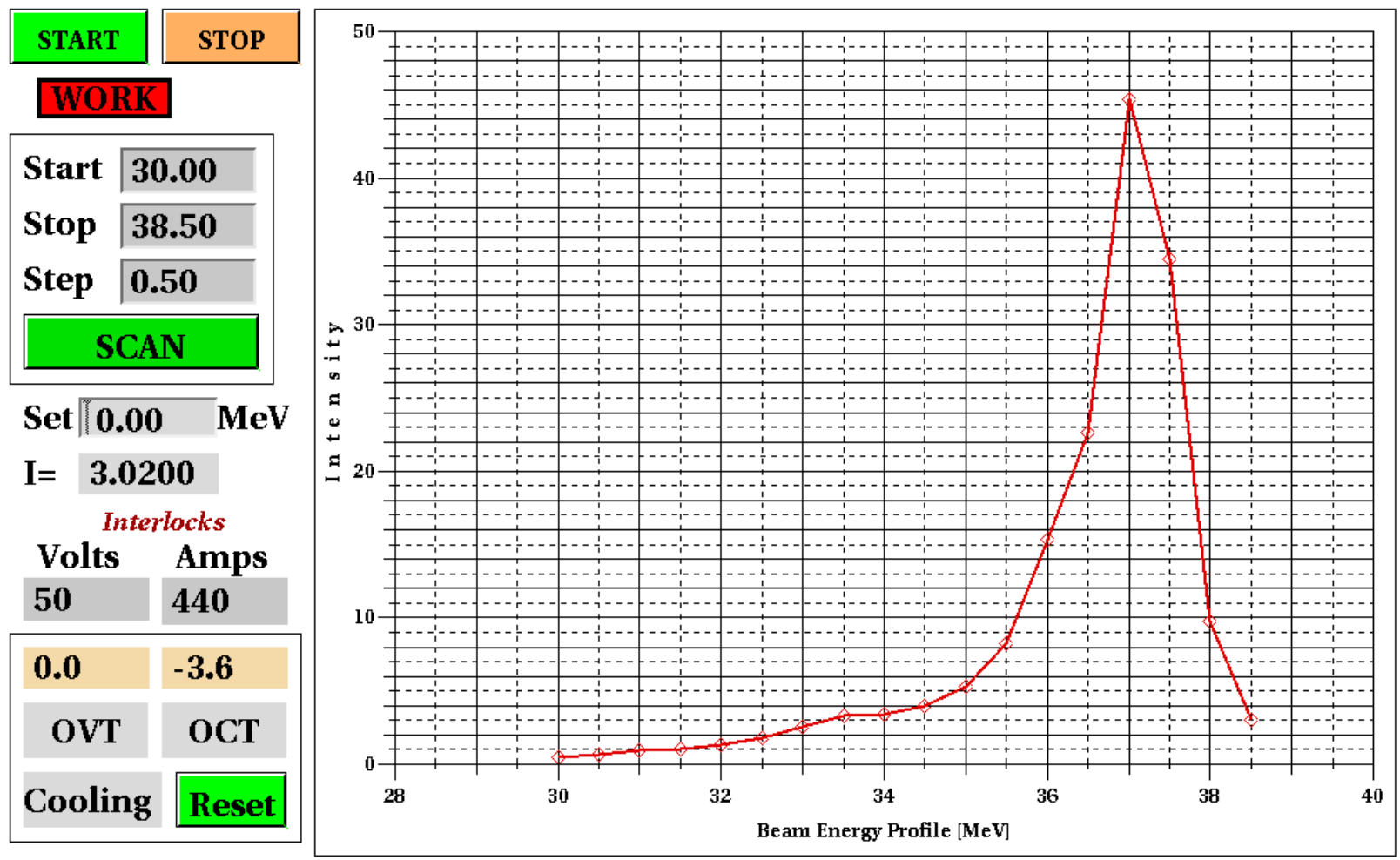

SAVE PRINT I(inj) $=-\mathbf{0 . 6 2} \mathrm{A}$

10-01-2019 12:40

FIGURE 3.1.2.1 Beam-energy spectrum for Irradiation \#1 on 10/1/19. The energy spectrum was recorded at a lower energy than $40 \mathrm{MeV}$ because of spectrometer limitations. After initial tune-up, the beam peak current was reduced to adjust the peak energy to $40 \mathrm{MeV}$. 


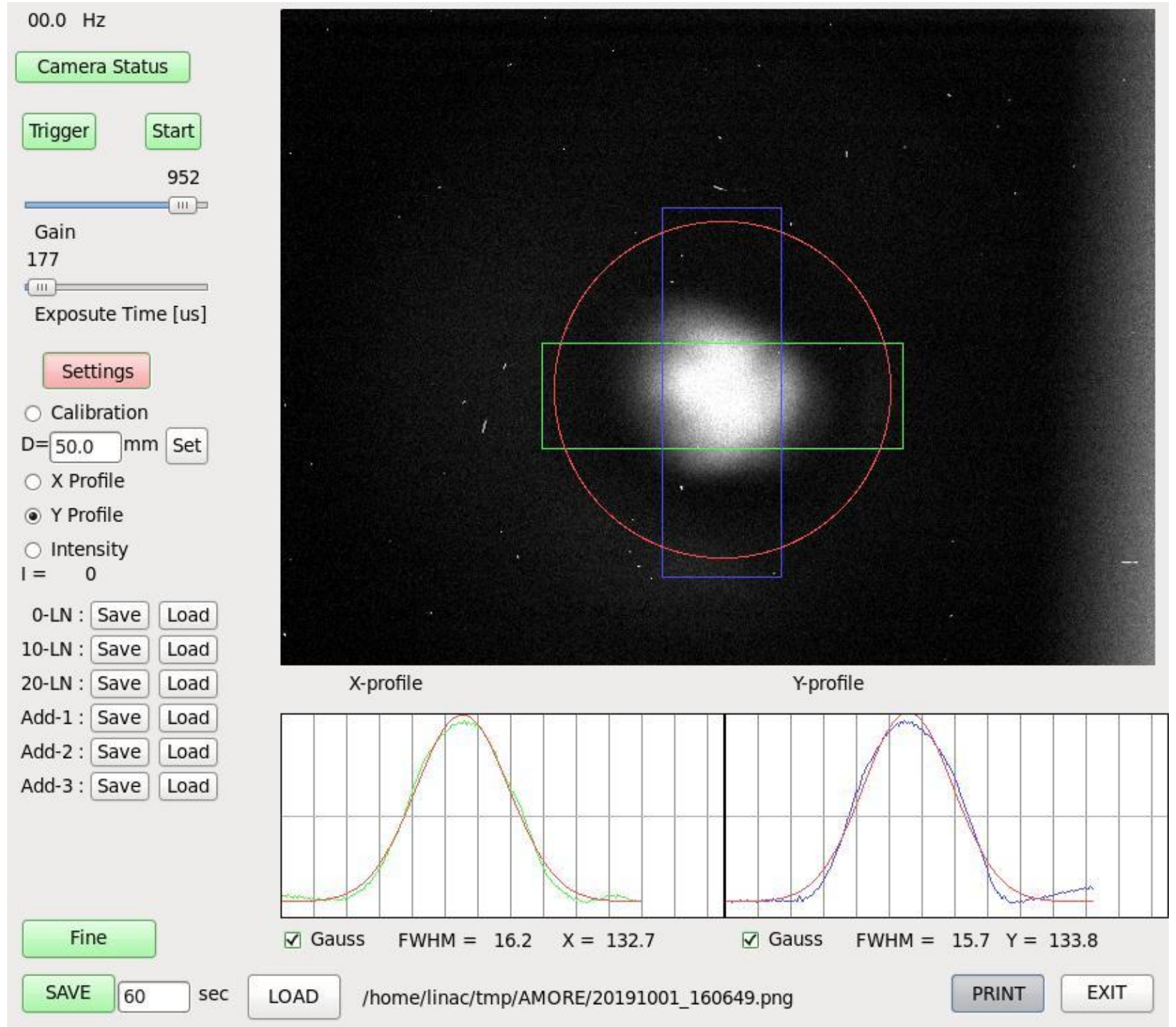

FIGURE 3.1.2.2 Beam profile on the target window for Irradiation \#1. Red circle outlines the target beam window boundary. 


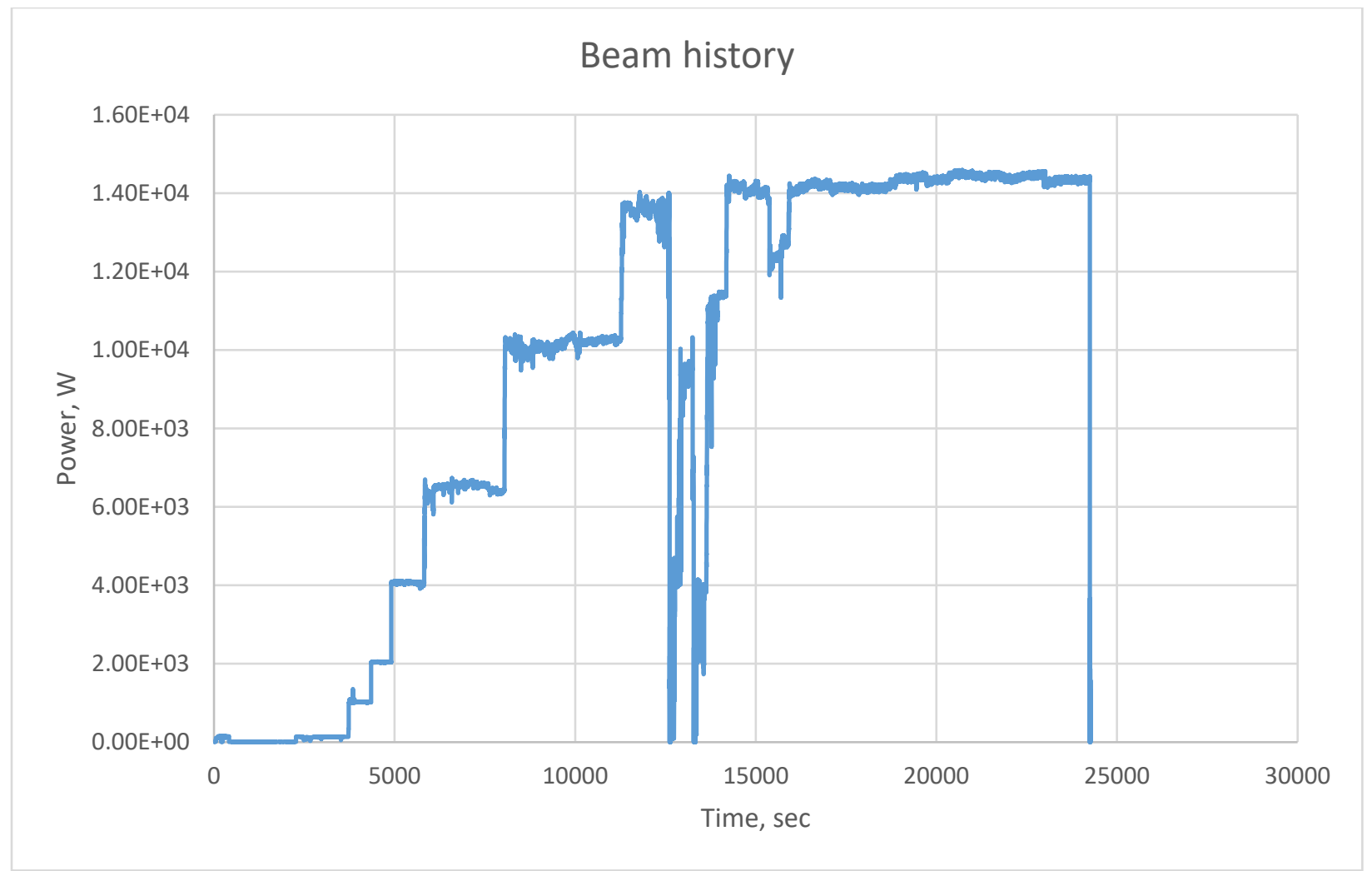

FIGURE 3.1.2.3 Beam history for Irradiation \#1

\subsubsection{Irradiation \#2, 11/11/19}

The second experiment after restart was conducted on 11/11/19. The plan was to irradiate the uranyl sulfate solution for 16 hours at a maximum beam power limited by hydrogen production/recombination. Because the plan for the experiment included the processing of the solution immediately after irradiation without significant interruptions, the end of irradiation was scheduled for 2 am. Initial beam tune-up was conducted on 11/8/19.

On the morning of the irradiation day, the beam was tuned and prepared for transport to the target. The beam energy spectrum is presented in Figure 3.1.3.1. This spectrum corresponds to a peak energy of $37 \mathrm{MeV}$ at $0.57 \mathrm{~A}$ peak current. After spectrum acquisition, the peak beam current was reduced to $0.53 \mathrm{~A}$, which shifted the beam energy peak to $40 \mathrm{MeV}$. After energy verification, the beam was placed on the target window at low power $(175 \mathrm{~W})$ and the beam shape was adjusted to produce a $17.1 \times 13.5-\mathrm{mm}$ FWHM beam spot on the target face (Figure 3.1.3.2). 


\begin{tabular}{|c|c|}
\hline \multicolumn{2}{|l|}{ START } \\
\hline \multicolumn{2}{|c|}{ WORK } \\
\hline \multirow{3}{*}{\multicolumn{2}{|c|}{\begin{tabular}{l|l}
\cline { 2 - 2 } Start & 30.00 \\
Stop & 39.00 \\
Step & $\mathbf{0 . 2 5}$
\end{tabular}}} \\
\hline & \\
\hline & \\
\hline \multicolumn{2}{|c|}{ SCAN } \\
\hline \multirow{3}{*}{\multicolumn{2}{|c|}{$\begin{array}{ll}\text { Set } & 0.00 \\
\mathrm{I}= & \mathbf{- 0 . 0 9 0 0} \\
\text { Interlocks }\end{array}$}} \\
\hline & \\
\hline & \\
\hline Volts & Amps \\
\hline 50 & 440 \\
\hline-0.0 & -2.9 \\
\hline OVT & OCT \\
\hline Cooling & Reset \\
\hline
\end{tabular}

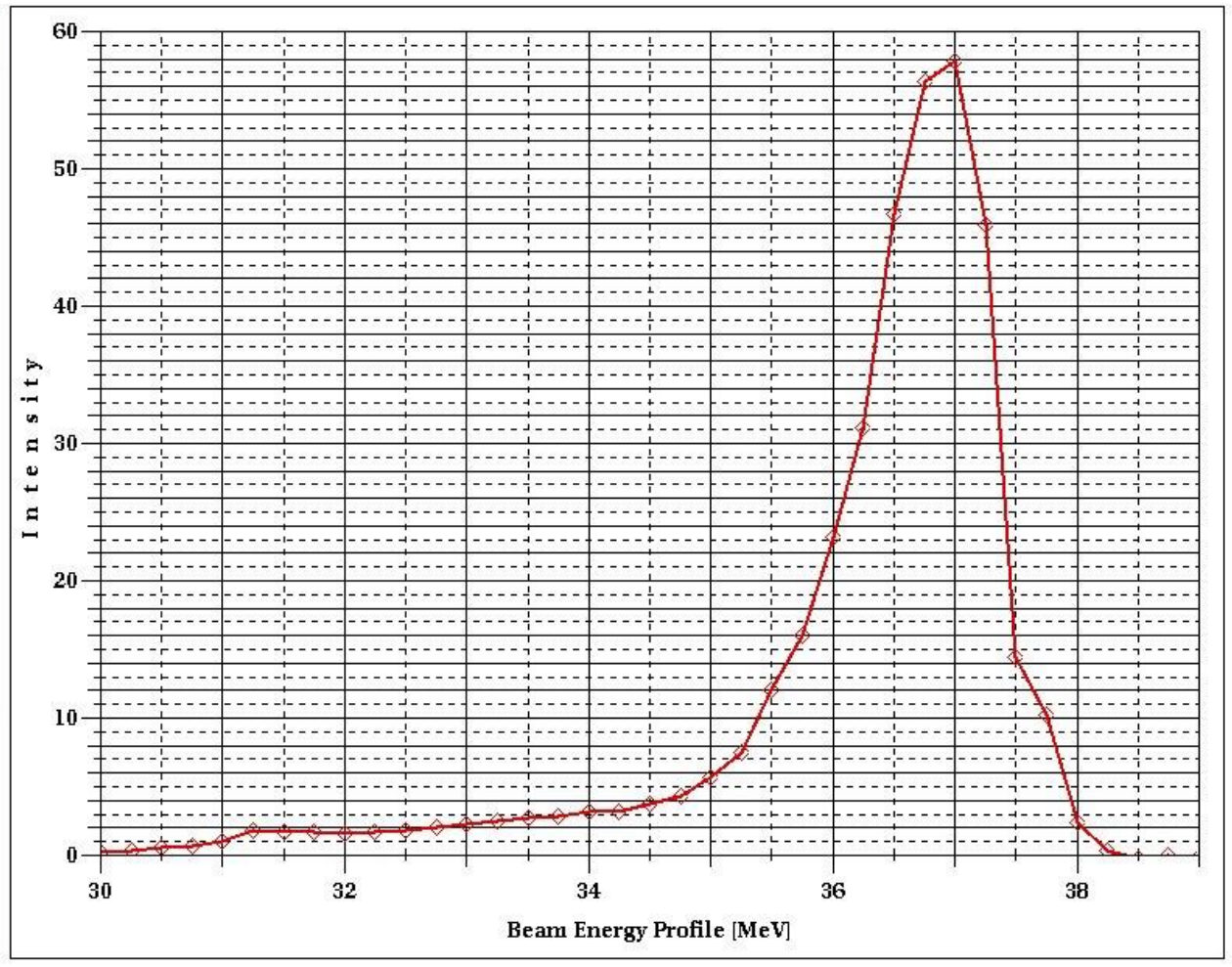

SAVE PRINT I(inj) $=-0.66 \mathrm{~A}$

11-11-2019 09:18

FIGURE 3.1.3.1 Beam energy spectrum for Irradiation \#2 on 11/11/19. The energy spectrum was recorded at a lower energy than $40 \mathrm{MeV}$ because of the thermal limitation of the spectrometer. After the initial tune-up, the beam peak current was reduced to adjust the peak energy to $40 \mathrm{MeV}$. 


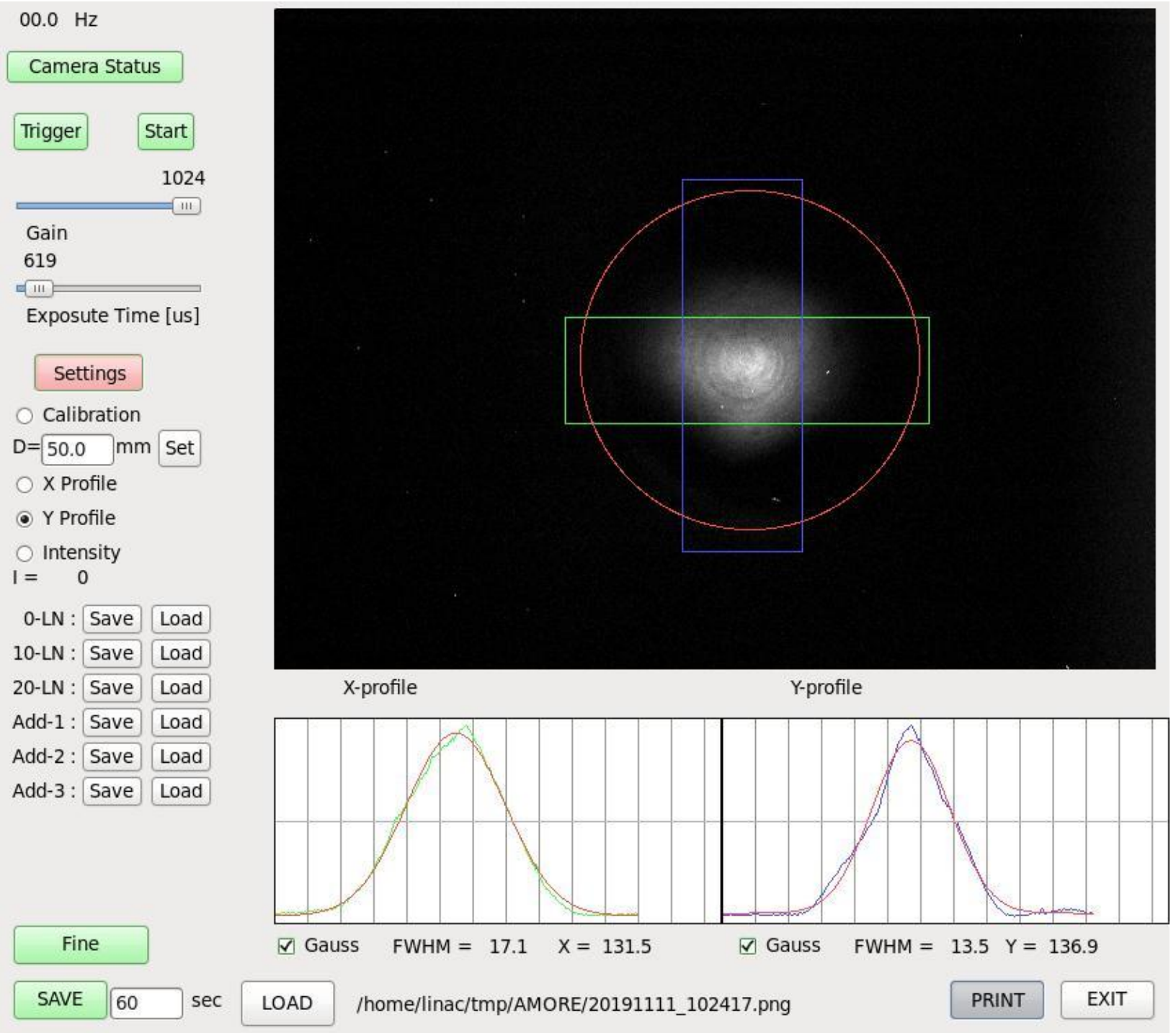

FIGURE 3.1.3.2 Beam profile on the target window for Irradiation \#2. Red circle outlines the target beam window boundary. 
Irradiation started at $10 \mathrm{am}$ at $\sim 175 \mathrm{~W}$ of beam power on the target. Over 1.5 hours, the power was increased to $15 \mathrm{~kW}$. The beam was stopped at 12:11 pm and restarted at 12:15 pm. At $12: 42,16.8 \mathrm{~kW}$ of beam power on the target was achieved. Beam power was maintained at $17.2 \mathrm{~kW}$ for the duration of the irradiation. The maximum beam power used in this experiment was $17.6 \mathrm{~kW}$, limited by hydrogen production and the requirement to maintain hydrogen concentration under 1\%. Figure 3.1.3.3 shows the beam history for the irradiation. Irradiation was finished at $2 \mathrm{am}$ the next day. At 1:55 am, the beam power was shut off, and the gas-analysis and -collection system was purged to reduce the amount of radioactive gas in the gas-analysis manifold. Processing of the irradiated solution started at $3 \mathrm{am}$.

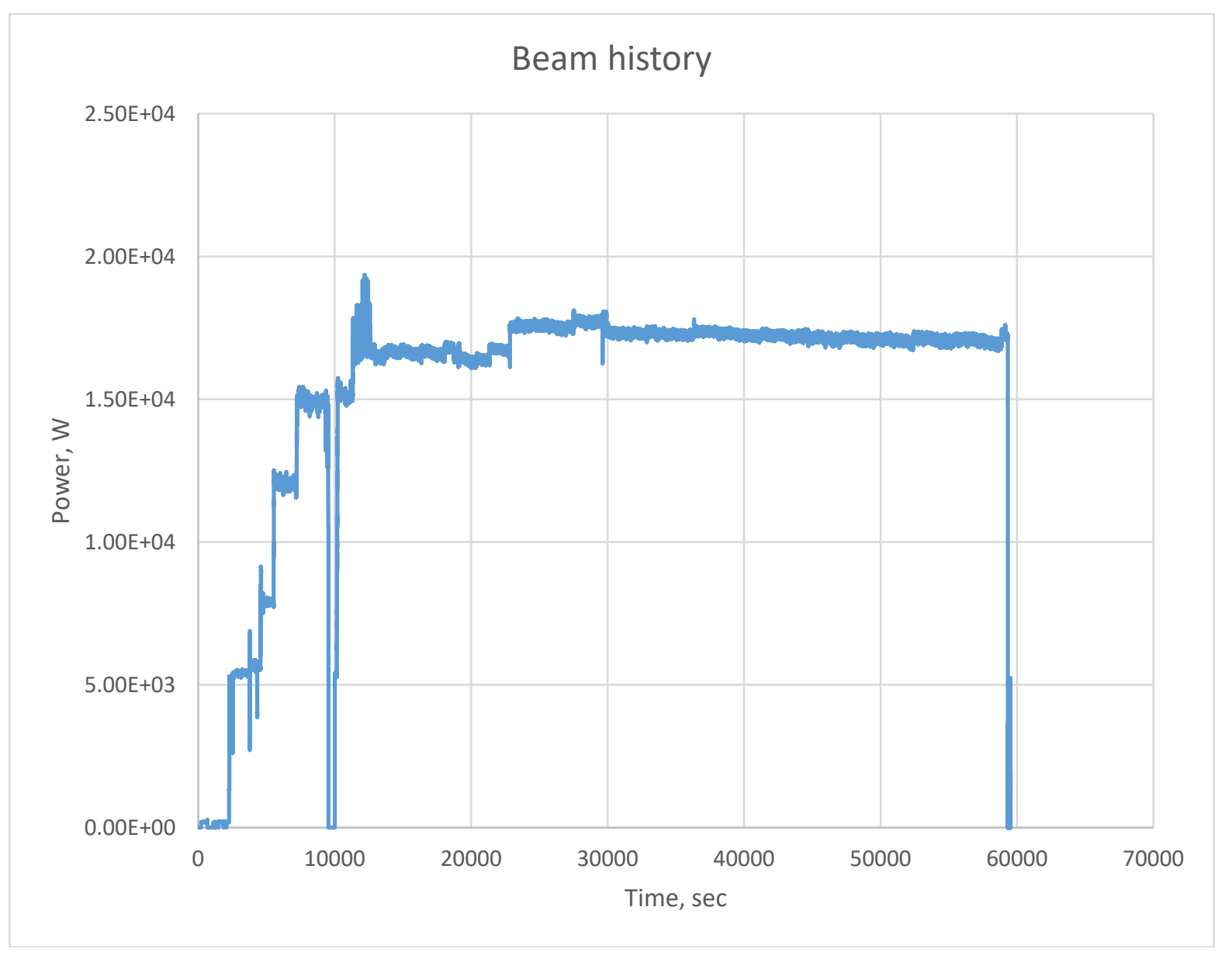

FIGURE 3.1.3.3 Beam history for Irradiation \#2

\subsubsection{Irradiation \#3, 3/2/20}

The third experiment after restart was completed on $3 / 2 / 20$. The plan was to irradiate the uranyl sulfate solution for 24 hours at a maximum beam power limited by hydrogen production/recombination, to produce up to $20 \mathrm{Ci}$ of ${ }^{99} \mathrm{Mo}$. Initial beam tune-up was conducted on February 26 and 28, 2020. 
At 10 am on the irradiation day, the beam was tuned and prepared for transport to the target. The beam energy spectrum is presented in Figure 3.1.4.1. This spectrum corresponds to a peak energy of $38 \mathrm{MeV}$ at $0.56 \mathrm{~A}$ peak current. After spectrum acquisition, the peak beam current was reduced to $0.50 \mathrm{~A}$, which shifted the beam energy peak to $40 \mathrm{MeV}$. After energy verification, the beam was placed on the target window at low power $(175 \mathrm{~W})$, and the beam shape was adjusted to produce a 20x20.5-mm FWHM beam spot on the target face (Figure 3.1.4.2).

The irradiation started at $12 \mathrm{pm}$ with $175 \mathrm{~W}$ of beam power on the target. Over 2 hours, power was increased to $15 \mathrm{~kW}$. Irradiation was stopped at 1:56 pm because of a worsening vacuum in the beamline. Irradiation was restarted at 3:43 pm. Power on the target was gradually increased to $17.5 \mathrm{~kW}$. The maximum beam power used in this experiment was $18 \mathrm{~kW}$, limited by hydrogen production and the requirement to maintaining hydrogen concentration under $1 \%$. Figure 3.1.4.3 shows the beam history for the irradiation. Irradiation was interrupted at 5:28 am the next day because of a trip in the modulator and a worsening vacuum in the beamline. At that point, irradiation could not be continued and had to be canceled. Investigation of the vacuum issues revealed significant scraping of the beam in the beamline that led to the leak in the beamline. No processing of the irradiated solution was attempted because estimated production of ${ }^{99} \mathrm{Mo}$ was too low, and we would not be able to meet minimum activity requirements for the shipment.

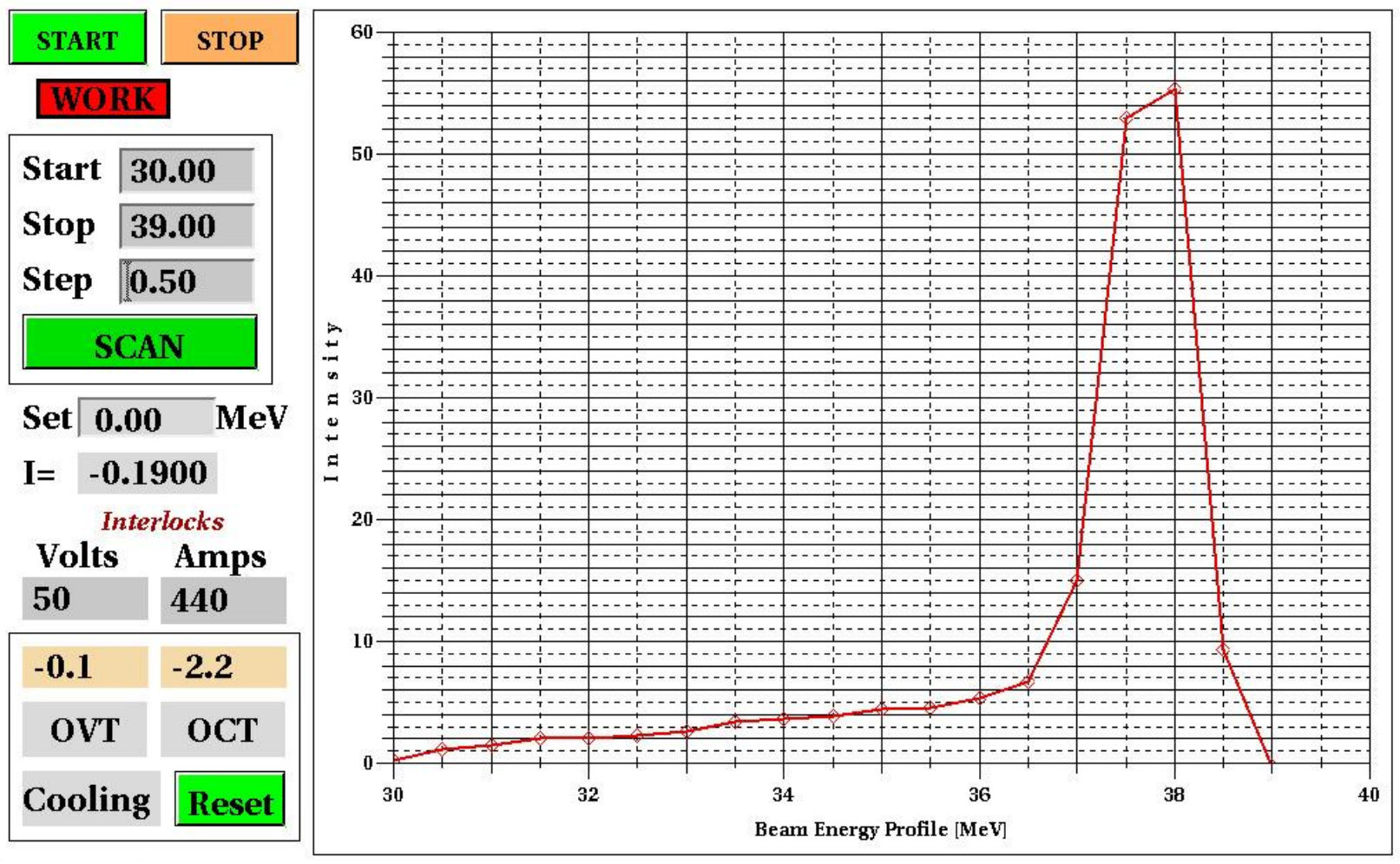

FIGURE 3.1.4.1 Beam energy spectrum for Irradiation \#3 on 3/1/20. The energy spectrum was recorded at a lower energy than $40 \mathrm{MeV}$ because of the thermal limitation of the spectrometer. After initial tune-up, beam peak current was reduced to adjust the peak energy to $40 \mathrm{MeV}$ 


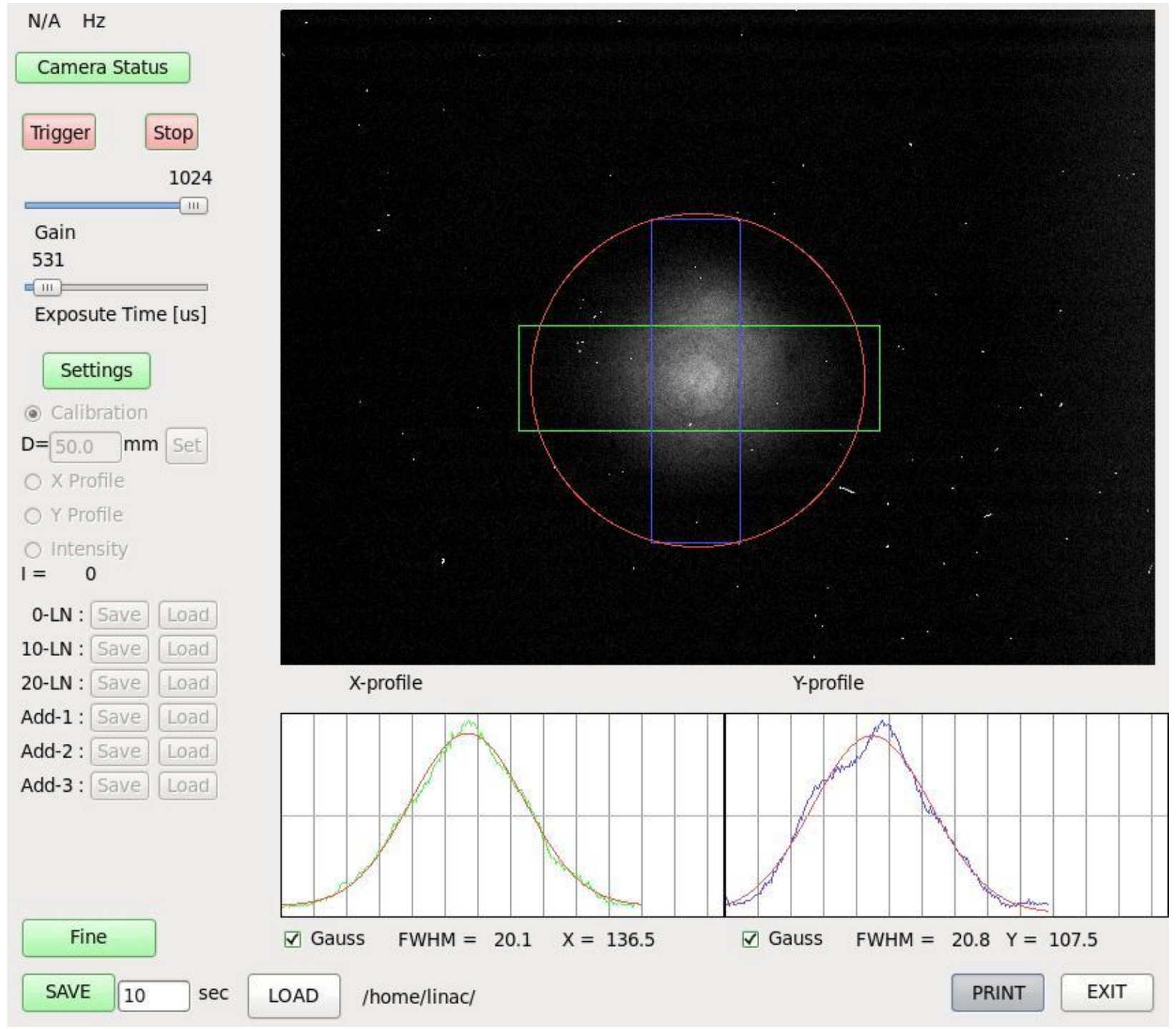

FIGURE 3.1.4.2 Beam profile on the target window for Irradiation \#3. Red circle outlines the target beam window boundary. 


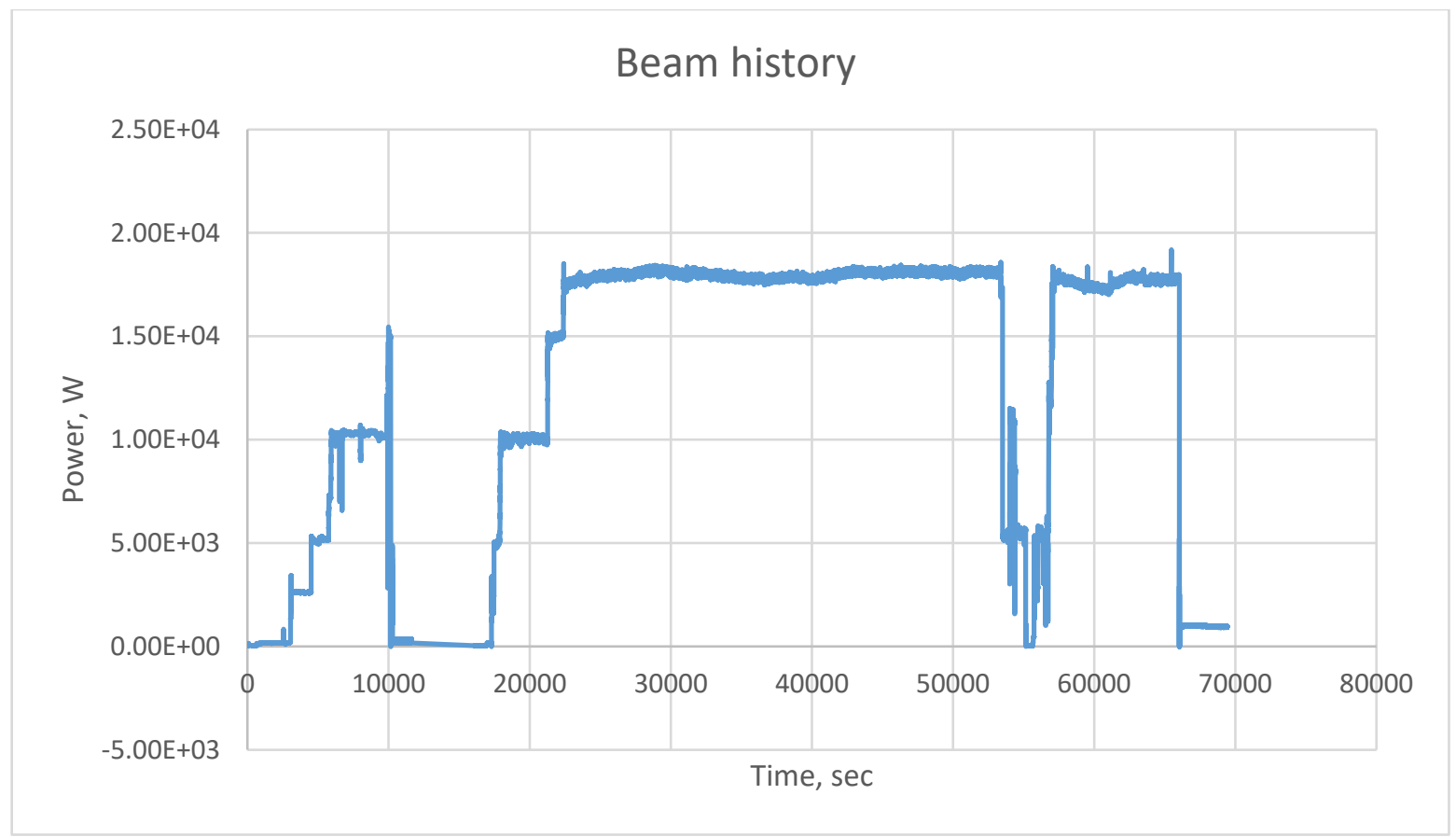

FIGURE 3.1.4.3 Beam history for Irradiation \#3

\subsubsection{Irradiation \#4, 8/30/20}

The fifth experiment after restart was conducted on 8/30/20. The plan was to irradiate uranyl sulfate solution for 10 hours at a maximum beam power limited by hydrogen production/recombination. Initial beam tune-up was conducted on $8 / 26 / 20$. The final tune-up was conducted on $8 / 28 / 20$. The beam energy spectrum is presented in Figure 3.1.5.1. This spectrum corresponds to a peak energy of $36 \mathrm{MeV}$ at $0.64 \mathrm{~A}$ peak current. After spectrum acquisition, the peak beam current was reduced to $0.53 \mathrm{~A}$, shifting the beam energy peak to $40 \mathrm{MeV}$.

Irradiation started at 7 am on 8/30/20. After energy verification, the beam was placed on the target window at low power $(\sim 180 \mathrm{~W})$, and the beam shape was adjusted to produce a 16.1x19.4-mm FWHM beam spot on the target face (Figure 3.1.5.2). Over 1.5 hours, the power was increased to $12.5 \mathrm{~kW}$. Beam power was maintained at $\sim 12.5 \mathrm{~kW}$ for the duration of the irradiation. The maximum beam power used in this experiment was $12.5 \mathrm{~kW}$, limited by hydrogen production and the requirement to maintain hydrogen concentration under $1 \%$. Figure 3.1.5.3 shows the beam history for the irradiation. The irradiation was interrupted at $7 \mathrm{pm}$ because of loss of vacuum in the beamline. The loss of vacuum was caused by a sudden change of the injector current, causing the beam to deviate from the proper trajectory. This deviation caused the beam to strike the wall of the vacuum chamber, leading to a loss of vacuum. At the end of irradiation, the gas-analysis and -collection system was purged to reduce the amount of radioactive gas in the gas-analysis manifold. Processing of the irradiated solution started shortly thereafter. 


\begin{tabular}{|c|c|}
\hline START & STOP \\
\hline \multicolumn{2}{|l|}{ WORK } \\
\hline \multicolumn{2}{|c|}{ Start 30.00} \\
\hline \multicolumn{2}{|c|}{$\begin{array}{l}\text { Stop } \\
\mathbf{3 8 . 0 0}\end{array}$} \\
\hline \multicolumn{2}{|c|}{ Step 0.50} \\
\hline \multicolumn{2}{|c|}{ SCAN } \\
\hline \multicolumn{2}{|l|}{ Set 0.00} \\
\hline \multicolumn{2}{|c|}{$I=3.0200$} \\
\hline InteI & locks \\
\hline Volts & Amps \\
\hline 50 & 440 \\
\hline 0.0 & -3.6 \\
\hline OVT & OCT \\
\hline Cooling & Reset \\
\hline
\end{tabular}

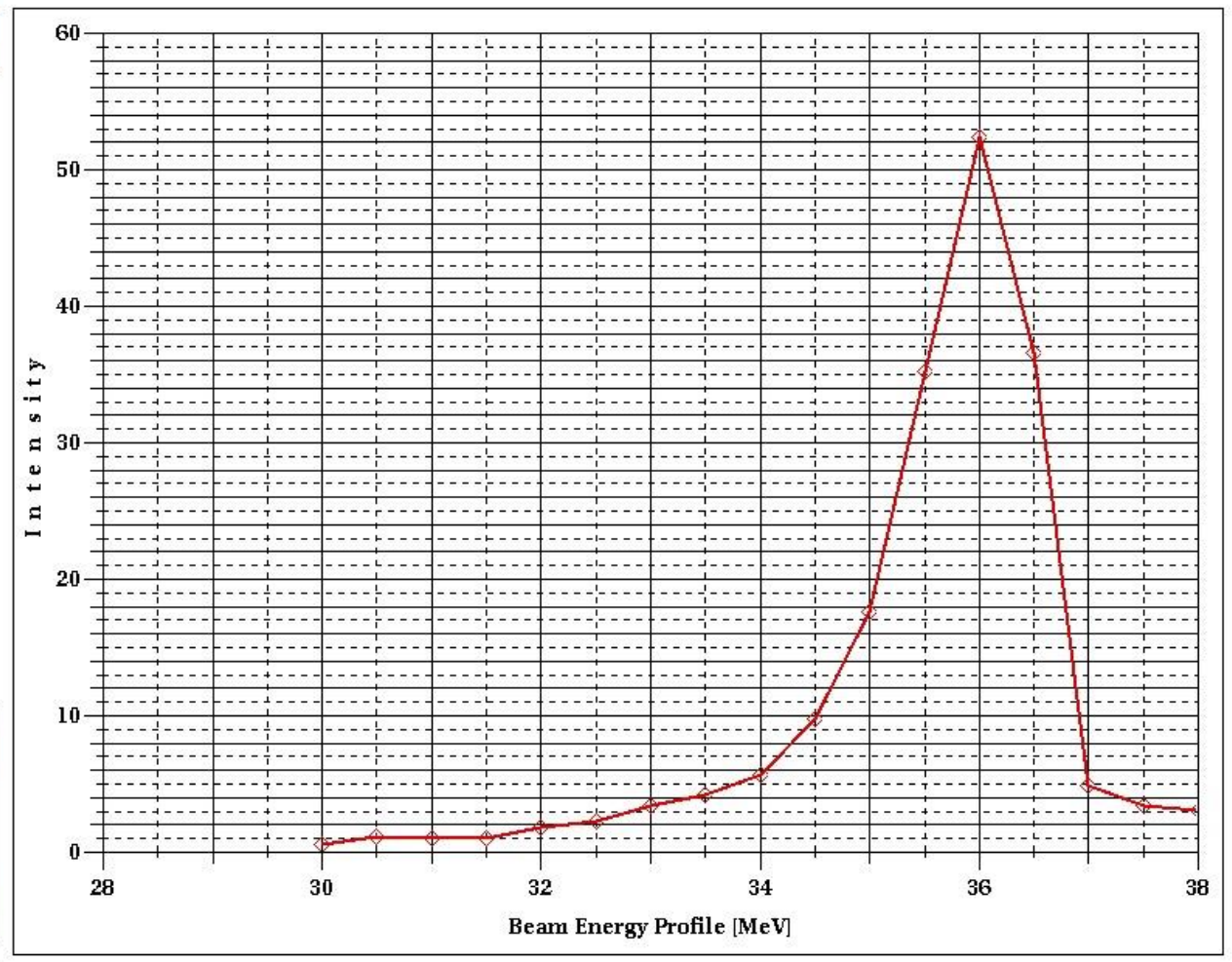

SAVE PRINT I (inj) $=0.00 \quad$ A

08-28-2020 12:03

FIGURE 3.1.5.1 Beam energy spectrum for Irradiation \#4 on 8/30/20. The energy spectrum was recorded on August 28 at a lower energy than $40 \mathrm{MeV}$ because of the thermal limitation of the spectrometer. After initial tune-up, the beam peak current was reduced to adjust the peak energy to $40 \mathrm{MeV}$. 


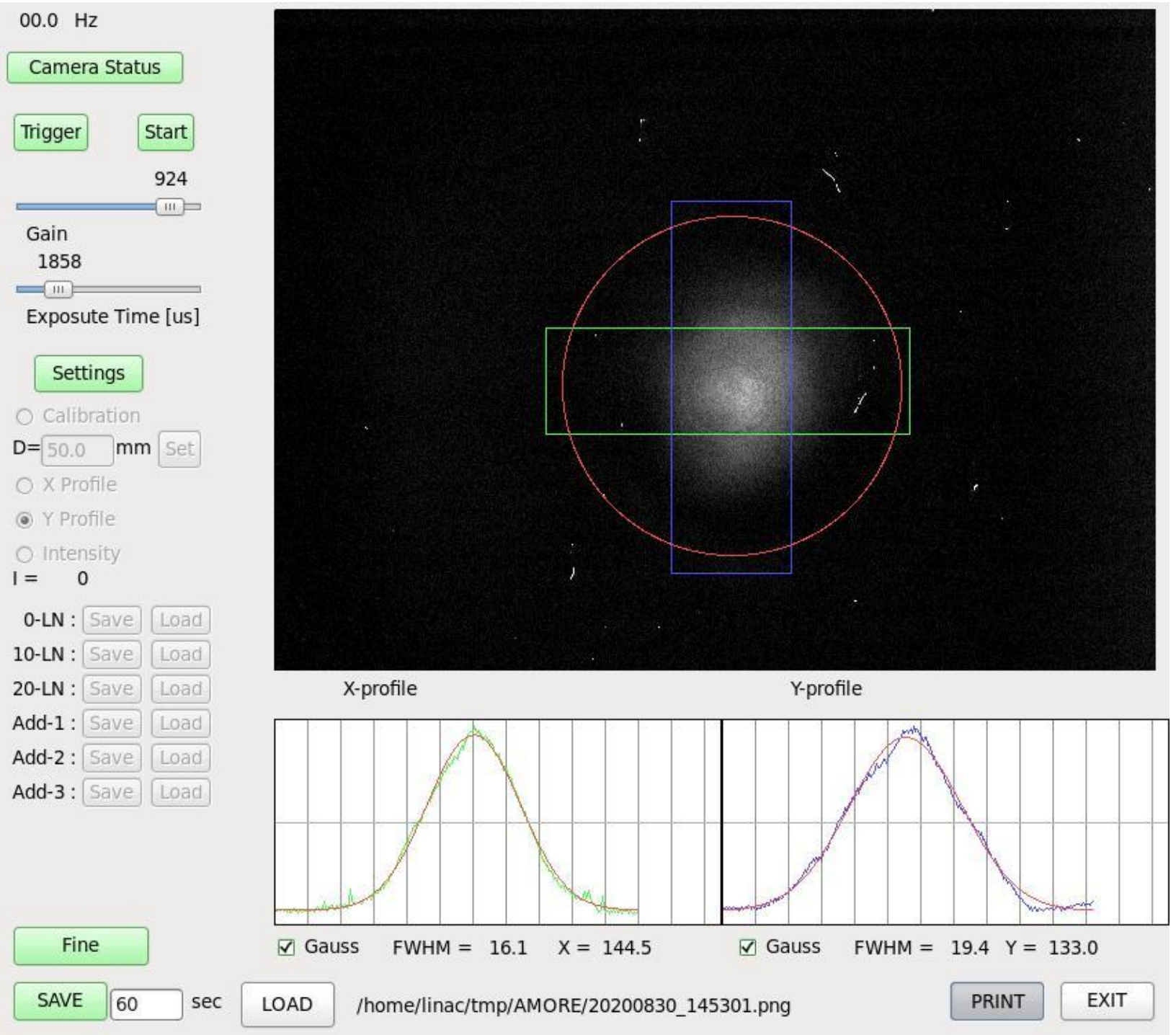

FIGURE 3.1.5.2 Beam profile on the target window for Irradiation \#4. Red circle outlines the target beam window boundary. 


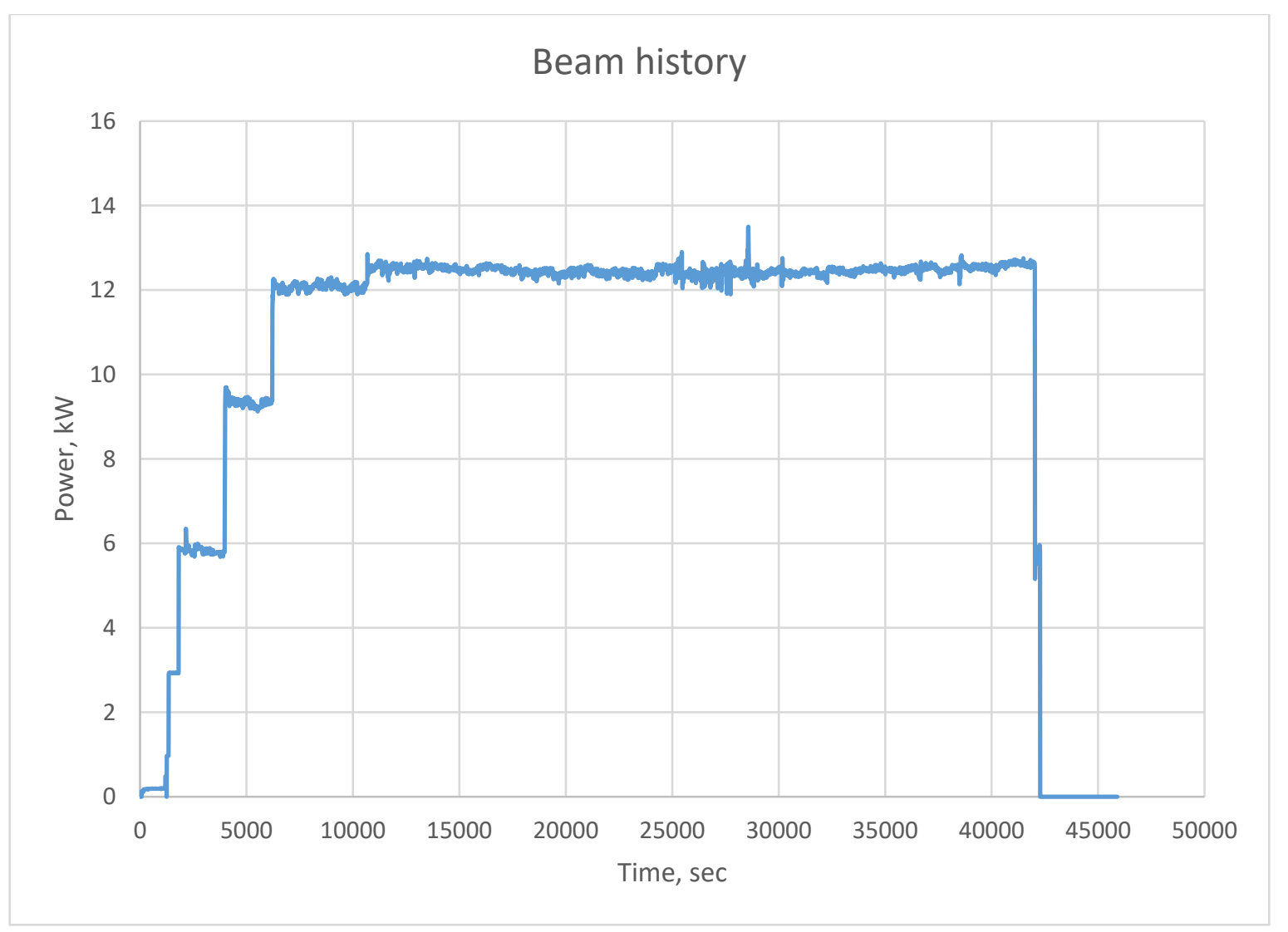

FIGURE 3.1.5.3 Beam history for Irradiation \#4

\subsubsection{Irradiation \#5, 1/18/21}

The last experiment was completed on $1 / 18 / 21$. The plan was to irradiate the uranyl sulfate solution for 24 hours at a maximum beam power limited by hydrogen production/recombination. Initial beam tune-up was conducted on $1 / 15 / 21$. The beam energy spectrum is presented in Figure 3.1.6.1. This spectrum corresponds to a peak energy of $37 \mathrm{MeV}$ at $0.56 \mathrm{~A}$ peak current. After spectrum acquisition, the peak beam current was reduced to $0.46 \mathrm{~A}$, which shifted the beam energy peak to $40 \mathrm{MeV}$.

Irradiation started at 7 am on 1/17/21. The beam was placed on the target window at low power and the beam shape was adjusted to produce an 18.6x21.1-mm FWHM beam spot on the target face (Figure 3.1.6.2). The beam power was gradually increased to $13.5 \mathrm{~kW}$ and maintained at $\sim 12.5 \mathrm{~kW}$ for most of the duration of the irradiation. The maximum beam power used in this experiment was $12.5 \mathrm{~kW}$, limited by hydrogen production and the requirement to maintain hydrogen concentration under $1 \%$. The irradiation was interrupted three times: first, for two hours because of sudden changes in RF parameters of the modulator; and twice more for several minutes each because of interlock protection trips on the RF modulators. The irradiation was finished at 8 am the next day. Figure 3.1.6.3 shows the beam history for the irradiation. At the end of irradiation, the gas-analysis and -collection system was purged to reduce the amount of radioactive gas in the gas-analysis manifold. Processing of the irradiated solution started at $\sim 9 \mathrm{am}$. 


\begin{tabular}{|c|c|}
\hline START & STOP \\
\hline \multicolumn{2}{|c|}{ Standby } \\
\hline \multicolumn{2}{|c|}{ Start 30.00} \\
\hline \multicolumn{2}{|c|}{ Stop 39.50} \\
\hline \multicolumn{2}{|c|}{$\begin{array}{ll}\text { Step } & 0.50\end{array}$} \\
\hline \multicolumn{2}{|c|}{ SCAN } \\
\hline \multirow{3}{*}{\multicolumn{2}{|c|}{$\begin{array}{l}\text { Set } \|_{0.00} \mathrm{MeV} \\
\mathrm{I}=\frac{-\mathbf{0 . 1 4 0 0}}{\text { Interlocks }}\end{array}$}} \\
\hline & \\
\hline & \\
\hline Volts & Amps \\
\hline 50 & 450 \\
\hline-0.2 & -2.9 \\
\hline OVT & OCT \\
\hline Cooling & Reset \\
\hline
\end{tabular}

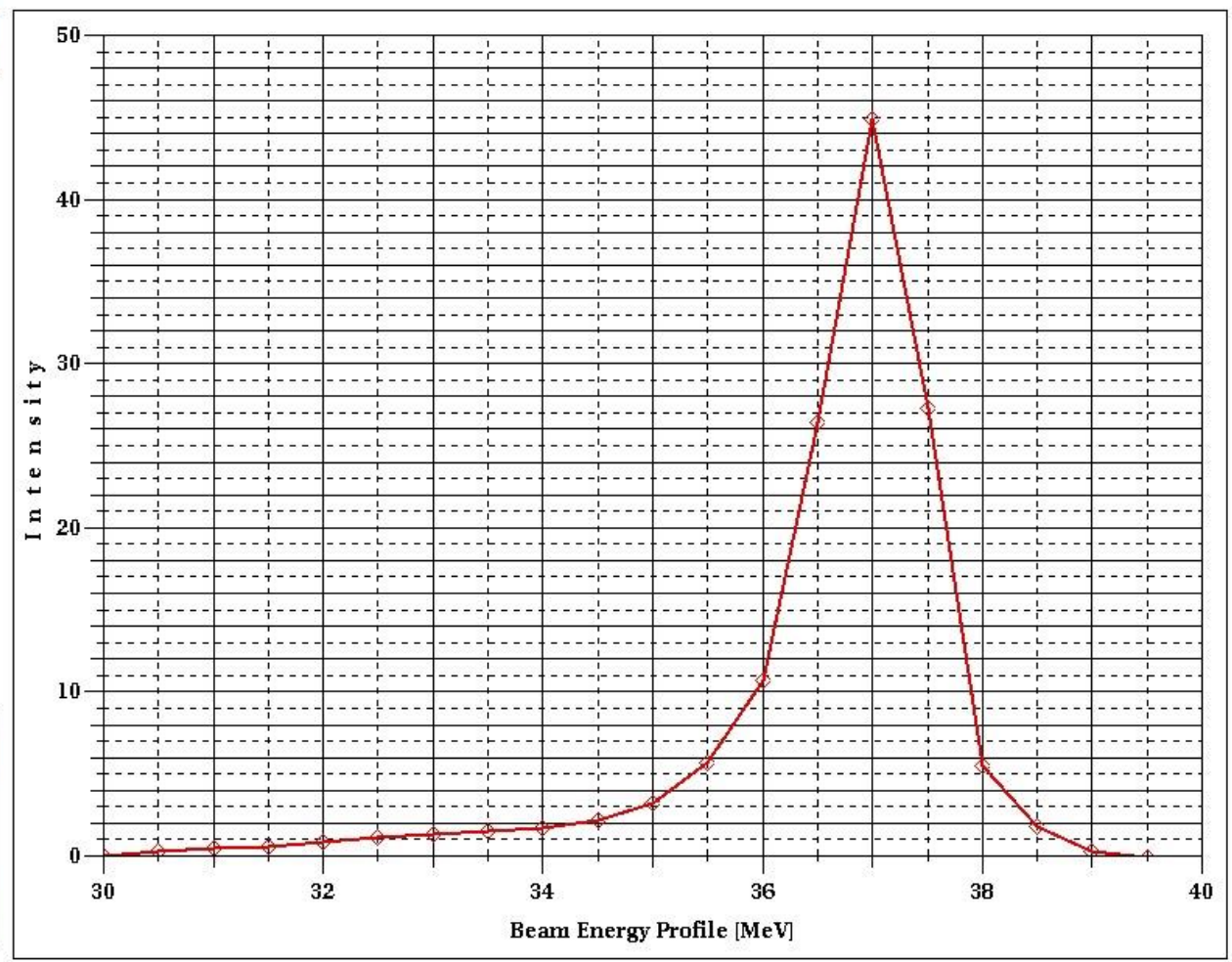

SAVE PRINT I(inj) $=-\mathbf{0 . 0 2} \mathrm{A}$

01-15-2021 10:50

FIGURE 3.1.6.1 Beam energy spectrum for Irradiation \#5 on 1/17/21. The energy spectrum was recorded on January 15 at a lower energy than $40 \mathrm{MeV}$ because of the thermal limitation of the spectrometer. After initial tune-up, the beam peak current was reduced to adjust the peak energy to $40 \mathrm{MeV}$. 


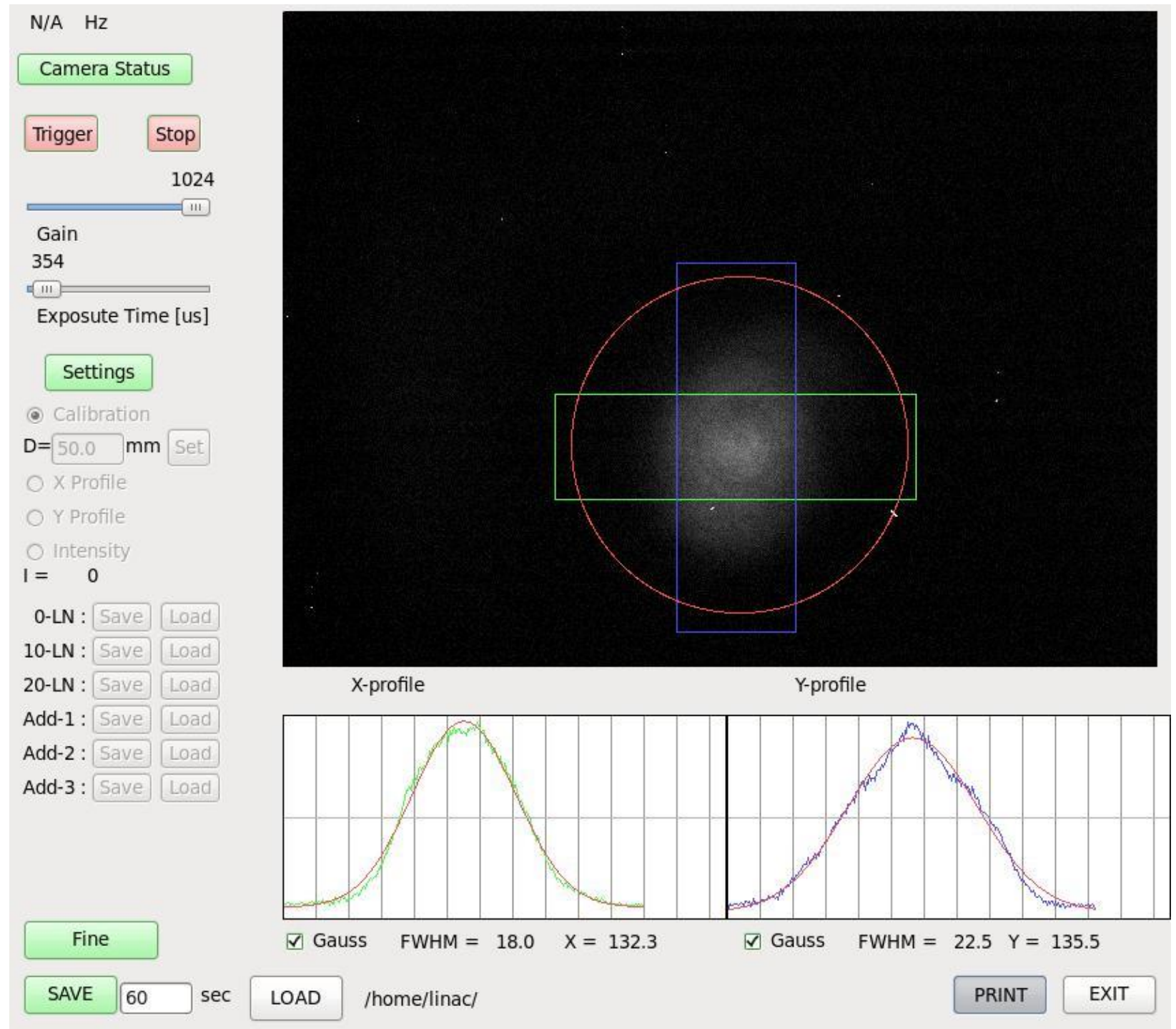

FIGURE 3.1.6.2 Beam profile on the target window for Irradiation \#5. Red circle outlines the target beam window boundary. 


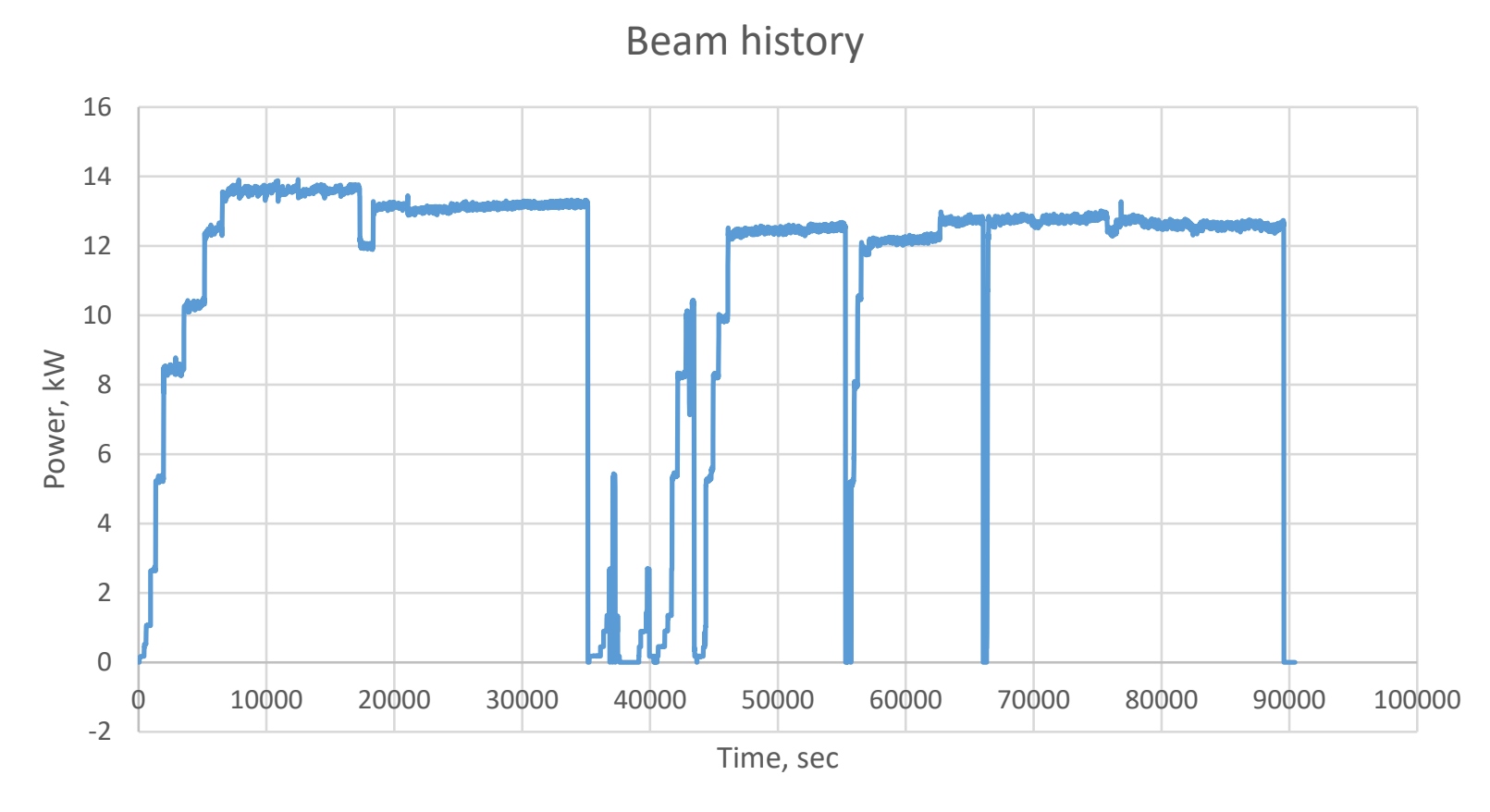

FIGURE 3.1.6.3 Beam history for Irradiation \#5

\subsubsection{Summary and Conclusions}

A total of five irradiations of uranyl sulfate solution were conducted. On two occasions, the irradiations were interrupted because of loss of vacuum in the beamline, due to beam misplacement. Because of the chromatic nature of the beamline (beams with different energies will emerge after the bend at different points and traveling in different directions), the trajectory of the beam was very sensitive to the beam energy and stability of the accelerator parameters. An achromatic transport line (a line where beams will emerge with the same position and direction after the bend regardless of beam energy) would improve the reliability of the irradiations.

All irradiations were limited in maximum beam power delivered to the target because of hydrogen production. This was the only limitation; the target design allowed placement of the full beam power, $20 \mathrm{~kW}$ at $40 \mathrm{MeV}$, on the target.

\subsection{GAS ANALYSIS}

Each experimental section shows data generated by the RGA. The RGA was calibrated by applying a standard of known concentration. Equation (1) was used to generate a Relative Response Factor (RRF) for each analyte. The RRF is based on the ion current of the analyte ( $\left.\mathrm{IC}_{\text {analyte }}\right)$, the concentration of the analyte ([\% Analyte]), the ion current of the internal standard ( $\mathrm{IC}_{\mathrm{is}}$ ), and the concentration of the internal standard ([IS]). During the experiments, the analyte concentration was determined using Equation (2). Helium used to purge the solution vessel 
contained $1.0 \%$ xenon as the internal standard. Since the solution vessel was not purged before experiments, the accuracy of the hydrogen and oxygen values generated by the RGA was subject to error because of the low concentration of internal standard in the vessel headspace. The RGA data were compared to the HYOptima Hydrogen Sensor during each experiment. The hydrogen concentration values generated by the RGA were consistently lower than the sensor data. Since the hydrogen sensor does not suffer from the same error as the RGA, the percent error was calculated for the RGA data when compared to the sensor data, which are assumed to be the true values. The analytical data presented in graphical form for each experiment were generated by the RGA. The percent error for hydrogen is shown in the discussion section for each experiment, where the true value is assumed to be that of the sensor and the error is in the graphical data. Before each experiment, the RGA and hydrogen sensor were calibrated using standards prepared from a primary standard with a certified accuracy of $\pm 2 \%$. The certified standards were procured from a vendor. The instruments were calibrated at the same pressure that occurs during an irradiation. The calibrations were verified with check standards, and the acceptance ranges for percent recovery were $\pm 10 \%$ for the RGA and $\pm 5 \%$ for the Hydrogen Sensor.

Since the analytical instruments were located in an adjacent room outside the irradiation

cell, there is a time delay of about 4 minutes between the actual solution vessel concentration and the analytical data.

$$
\begin{aligned}
& \mathrm{RRF}=\mathrm{IC}_{\text {analyte }} \times[\mathrm{IS}] / \mathrm{IC}_{\mathrm{IS}} \times[\% \text { Analyte }] \\
& {[\% \text { Analyte }]=\mathrm{IC}_{\text {analyte }} \times[\mathrm{IS}] / \mathrm{IC} \text { IS } \times \mathrm{RRF}}
\end{aligned}
$$

\subsubsection{Commissioning Run, No Irradiation, 8/15/19}

The Gas Handling System was commissioned on 8/15/19. System checks were performed and gas composition was monitored in the same way that they would be during actual experiments. The GCS (see Figure 2.2.2.7) functioned as designed. This functioning was tested by adding helium to the system so that the pressure rose at about 60 mbar per minute. The pump in Chamber \#1 started at 961 mbar and evacuated the system to 940 mbar, at which time the pump shut off. This protocol kept the pressure in the experiment between 940 and 961 mbar. Chamber \#2 was filled with the excess gas. As the pressure in Chamber \#2 rose to $1141 \mathrm{mbar}$, the compressor inside that chamber started operating and evacuated the chamber to 1020 mbar. The excess gas was pumped into the collection cylinders, causing a pressure rise of about 2 psig per compressor cycle.

The GCS interlocks were tested for the collection cylinders, Chamber \#2, and Chamber \#1. The function of the interlocks is to disable power to the linac during an experiment. The interlock for the collection cylinders was tested and tripped by setting the trip pressure lower than the pressure that was actually in the cylinders at the time of the test. The Chamber \#2 interlock was tested and tripped by setting the trip pressure lower than the pressure in the chamber at the time of the test. The alarm state for Chamber \#2 also actuates and closes a solenoid valve located between the collection cylinders and Chamber \#2. The pumps in each 
chamber are also disabled. The purpose is twofold: In the event the Chamber \#2 compressor fails, (1) no more gas can be added to the GCS and (2) any leaks through the check valve between the cylinders and Chamber \#2 are stopped, preventing the over-pressurization of Chamber \#2 and release of gas through the rupture disc. The Chamber \#1 interlock was tested and tripped by setting the trip pressure lower than the pressure in the chamber at the time of the test. Alarms were activated in the monitoring control room during each test.

Hydrogen alarms and interlocks were tested by introducing standards into the system. Hydrogen alarms activated with the introduction of a $1 \%$ standard. The hydrogen interlock tripped the linac interlock chain, and an alarm sounded in the monitoring control room, upon introduction of a $2 \%$ standard.

The verification of the alarm for the gas-sampling-pump flow was successfully completed. The verification of alarm and interlock responses for the catalyst pump was successful. The alarm test for solution-vessel pressure was successful. The alarm was tested by setting the alarm value lower than the actual pressure in the vessel. Oxygen introduction and a post-irradiation system purge were tested successfully. A $40 \%$ oxygen-in-helium mix was added at a rate of $50 \mathrm{~mL} / \mathrm{minute}$ to the vessel. The rise in oxygen concentration was observed in the RGA monitoring data over an hour, and the increase was $3.5 \%$.

The checklist tasks for pre-irradiation valve configuration were performed, and minor modifications were made for clarity.

Performance of the Gas Handling System pre-checks and interlocks, linac pre-checks and interlocks, and post-irradiation purge was successfully verified. Gas Handling procedures were followed as written. Some lessons learned were incorporated into a later revision of the procedures to improve clarity. The relevant parameters for the experimental systems were confirmed to be as described in the facility SAD and ASE, and all acceptance criteria were met. The checks described above were performed before each irradiation.

\subsubsection{Irradiation \#1, 10/1/19}

Figure 3.2.2.1 shows gas concentration and linac beam power for Irradiation \#1 on $10 / 1 / 19$. The RGA data presented has a percent error of $22 \%$ and is lower when compared to the sensor data for hydrogen. In this experiment, hydrogen concentration began to rise when the beam energy reached $1.37 \mathrm{kWh}$, whereas the residual concentration of oxygen began to decrease through recombination with hydrogen at the catalyst at $0.34 \mathrm{kWh}$. The dip in hydrogen concentration was due to beam-power fluctuations. What is striking, and was not expected, is that we needed to add oxygen to the solution vessel. The oxygen/helium mixture was added to the system at $120 \mathrm{~min}$, at a rate of $50 \mathrm{~mL} / \mathrm{min}$, to control hydrogen concentration in the vessel. The system was not purged with helium before the irradiation, and oxygen was at about $13 \%$. It was assumed that the residual oxygen would be sufficient to compensate for the initial oxygen deficit that occurred during the initial stage of an irradiation. 


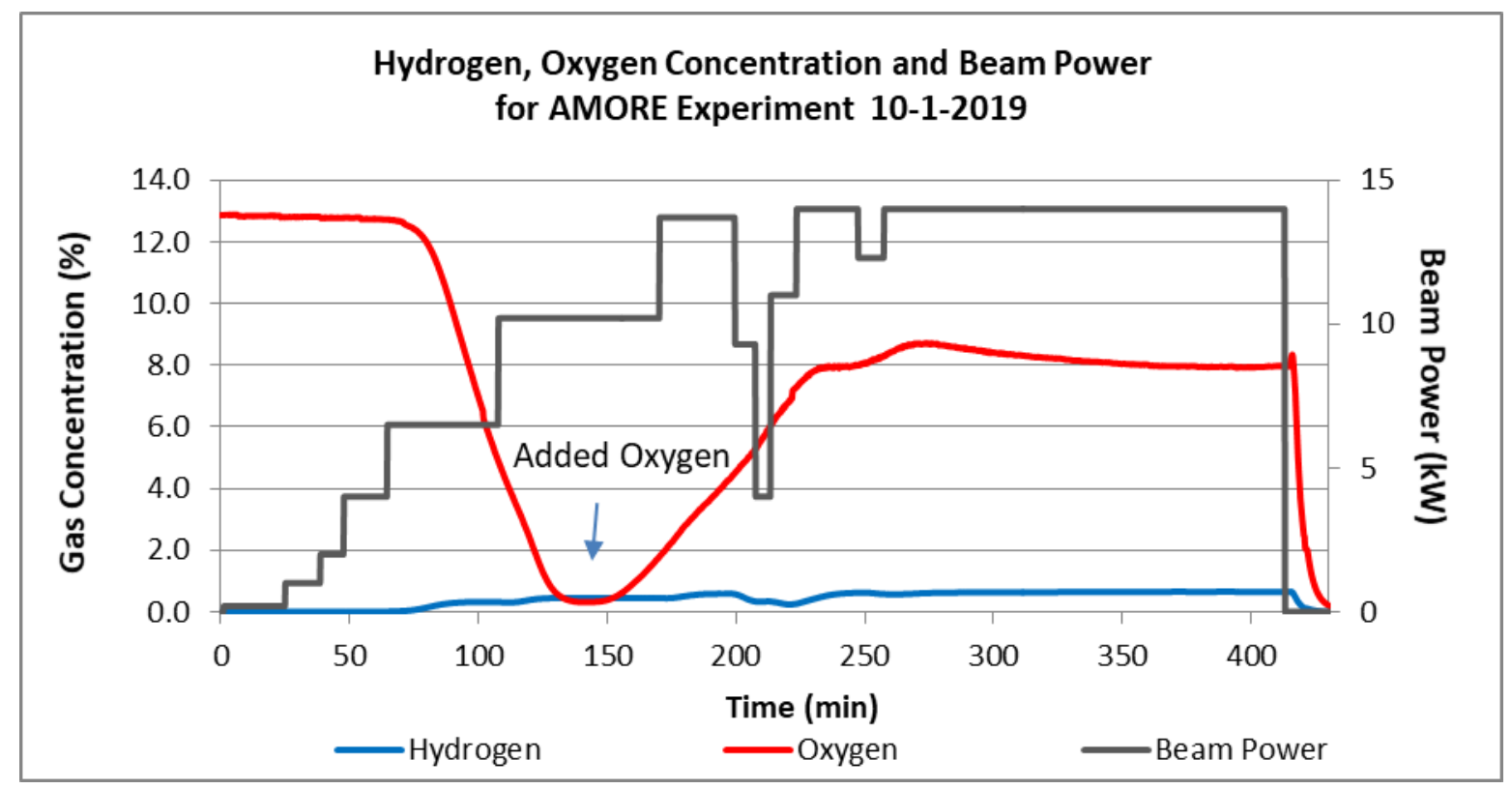

FIGURE 3.2.2.1 Gas concentration and linac beam power for Irradiation \#1 on 10/1/19

Prior to this irradiation, while transferring the uranyl sulfate solution from the vessel during sample collection, uranyl peroxide precipitate was observed in the solution. An attempt was made to remove as much of the precipitate as possible. On 9/9/20, a solution of iron sulfate was added to the solution vessel at $164 \mathrm{ppm}$ in an attempt to mitigate the precipitation[1]. The iron concentration was determined by Inductively Coupled Plasma Mass Spectrometry (ICPMS) analysis on $9 / 23 / 19$. We observed additional precipitate every time the solution was moved, including on $11 / 6 / 19$. On $2 / 13 / 20$, the solution was moved for the first time with no visible precipitate, and none was observed after that.

The reaction of hydrogen peroxide and uranyl sulfate forms a precipitate, uranyl peroxide. In the initial stage of water radiolysis, $\mathrm{H}_{2}$ and $\mathrm{H}_{2} \mathrm{O}_{2}$ are generated. Subsequently, $\mathrm{H}_{2} \mathrm{O}_{2}$ will radiolytically decompose to $\mathrm{O}_{2}$ and $\mathrm{H}_{2} \mathrm{O}$. If $\mathrm{H}_{2} \mathrm{O}_{2}$ reacts with uranyl sulfate to form uranyl peroxide, this will sequester the oxygen that would normally be released into the vessel as a gas. This sequestration can cause an oxygen deficit in the vessel headspace, which was seen here. Even though iron was added before Irradiation \#1, crystals already present in solution could possibly have facilitated further precipitation.

\subsubsection{Irradiation \#2, 11/11/19}

Figure 3.2.3.1 shows gas concentration and linac beam power for Irradiation \#2 on $11 / 11 / 19$. The RGA data presented has a percent error of $19 \%$ and is lower when compared to the sensor data for hydrogen. In this experiment, hydrogen concentration began to rise when beam energy reached $1.40 \mathrm{kWh}$, whereas the residual concentration of oxygen began to decrease through recombination with hydrogen at the catalyst at $0.03 \mathrm{kWh}$. Hydrogen was maintained at a steady concentration throughout the experiment, with a dip when the beam dropped out. Oxygen 
showed a slow, steady decline. No additional oxygen was added during this experiment. When compared to the previous experiment, the decline in oxygen could be indicating that a precipitate is forming but not as rapidly. One possible explanation is that the precipitate that formed from the previous experiment had mostly decomposed as the solution sat in the vessel between experiments. Uranyl peroxide has been shown to decompose radiolytically. Prior to this experiment, uranyl peroxide precipitate was observed in the solution. An attempt was made to remove it. Here, it is possible that the rate of precipitate formation was slower than in Irradiation \#1 because there were fewer crystals in solution to facilitate precipitation.

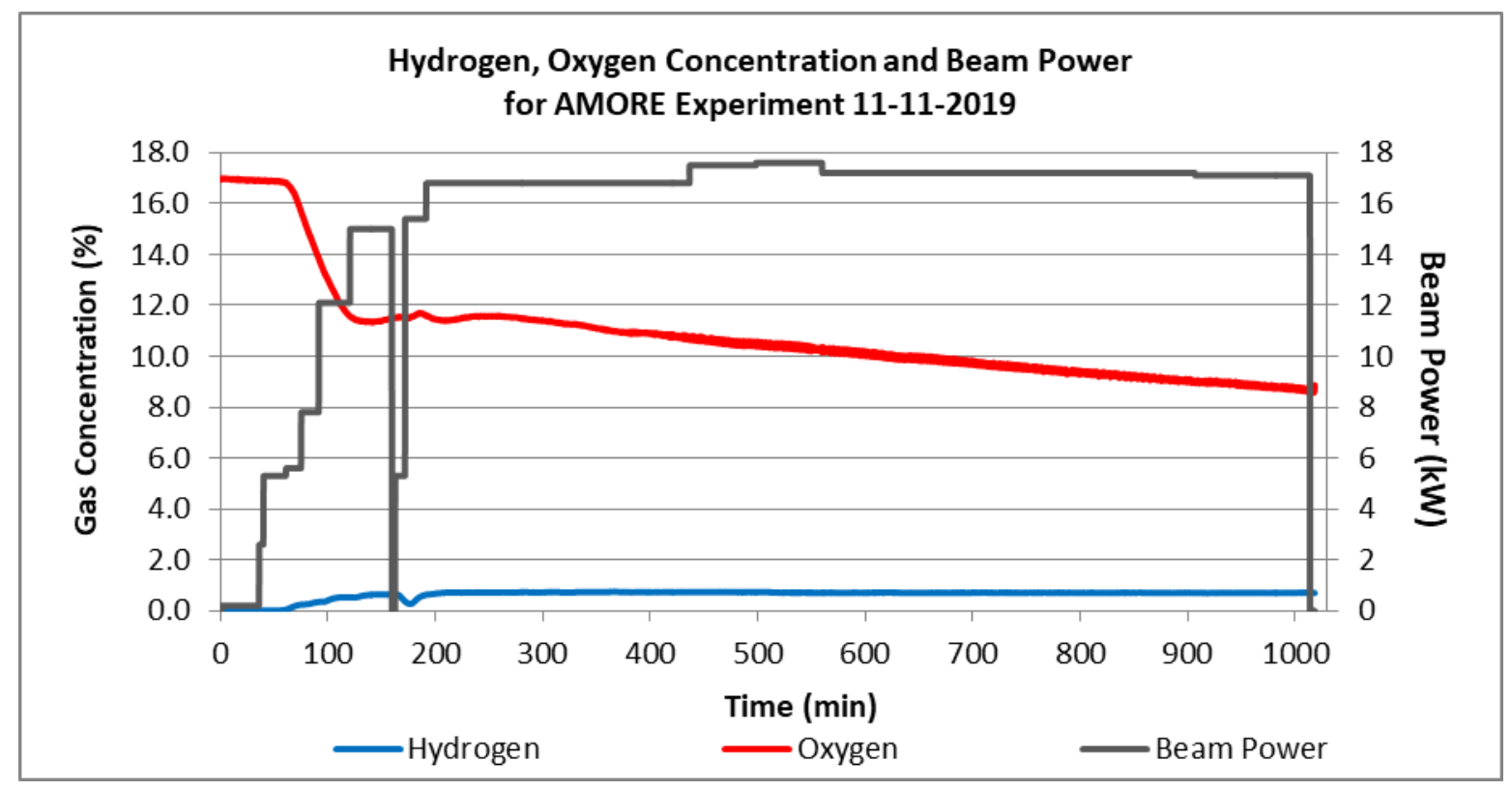

FIGURE 3.2.3.1 Gas concentration and linac beam power for Irradiation \#2 on 11/11/19

\subsubsection{Irradiation \#3, 3/2/20}

Figure 3.2.4.1 shows gas concentration and linac beam power for Irradiation \#3, completed on $3 / 2 / 20$. The RGA data presented have a percent error of $18 \%$ and are lower when compared to the sensor data for hydrogen. In this experiment, hydrogen concentration began to rise when beam energy reached $1.77 \mathrm{kWh}$, whereas the residual concentration of oxygen began to decrease through recombination with hydrogen at the catalyst at $0.81 \mathrm{kWh}$. In this experiment, it appears that oxygen concentration continued to show a downward trend similar to that seen in Irradiation \#2, though not as dramatic. The oxygen decrease for Irradiation \#2 was approximately $0.0037 \%$ per minute, whereas that for Irradiation \#3 was $0.001 \%$ per minute. Each subsequent experiment following the precipitation event became more stable with respect to gas generation. Prior to Irradiation \#3, no visible sign of precipitate was observed. The concentration of iron in the solution was 150 ppm, as determined by ICPMS analysis on 2/28/20. Iron concentration was lower than previously determined because there was a slight dilution of the uranium solution in the vessel due to processing. 


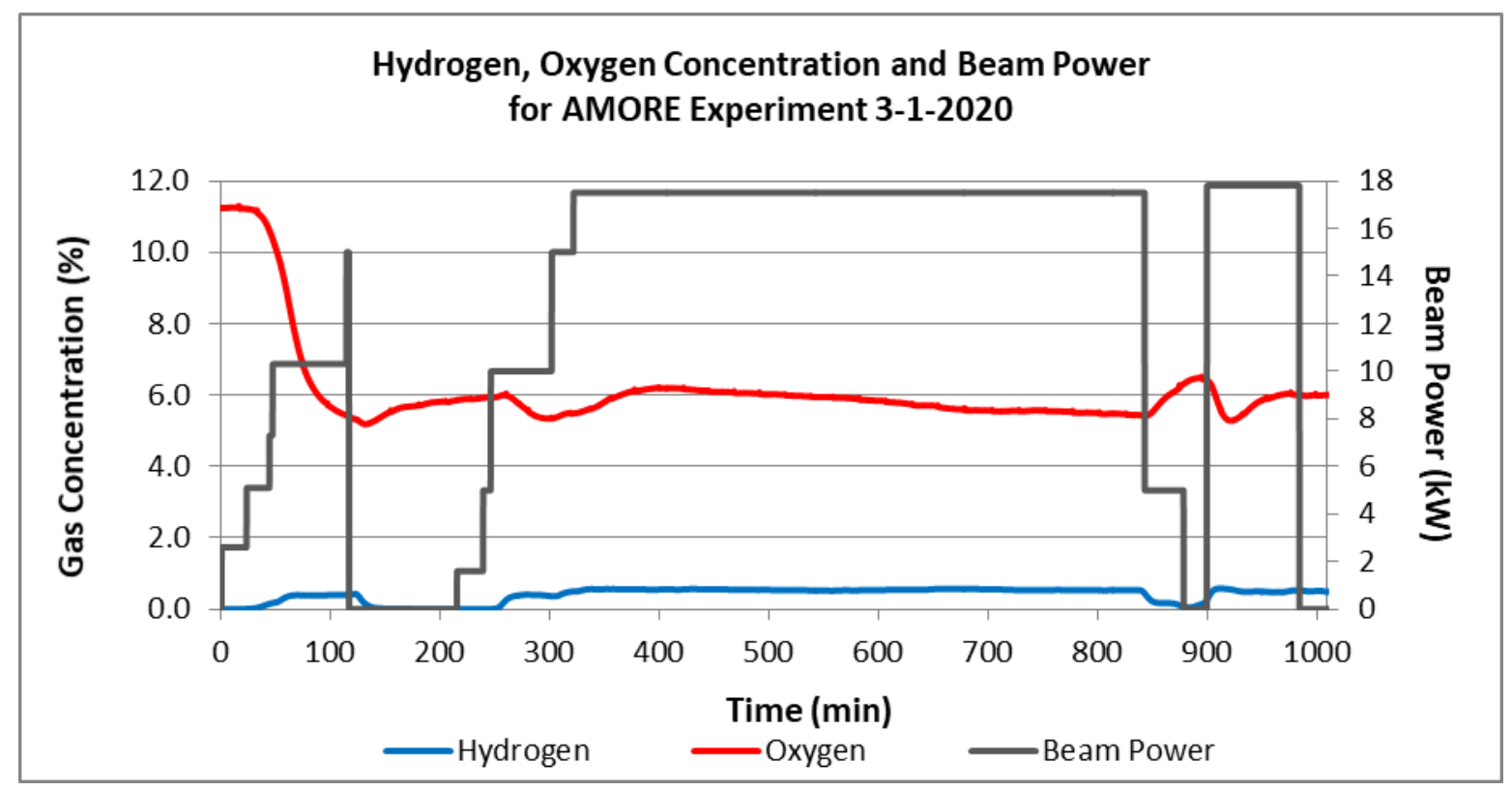

FIGURE 3.2.4.1 Gas concentration and linac beam power for Irradiation \#3 on 3/1/20

\subsubsection{Irradiation \#4, 8/30/20}

Figure 3.2.5.1 shows gas concentration and linac beam power for Irradiation \#4 on $8 / 30 / 20$. The RGA data presented have a percent error of $13 \%$ and are lower when compared to the sensor data for hydrogen. Before this experiment, the KNF Neuberger N186 catalyst pump needed replacement. The new pump was a Senior Aerospace Metal Bellows MB-151. The smaller replacement pump meant a reduction in flow through the catalytic recombiner. The reduced flow required limiting the beam power to about $12.5 \mathrm{~kW}$ in order to keep hydrogen concentration $<1 \%$. In this experiment, the hydrogen concentration began to rise when the beam energy reached $1.22 \mathrm{kWh}$, whereas the residual concentration of oxygen began to decrease through recombination with hydrogen at the catalyst at $1.70 \mathrm{kWh}$. The initial drop in oxygen occurred at a higher beam energy than in the previous experiments. After the initial drop, oxygen showed no deficit throughout the irradiation. A steady-state hydrogen and oxygen concentration was maintained throughout the experiment. This observation indicates a balanced gas generation and catalytic recombination. No precipitate was observed in the solution before or after this experiment. 


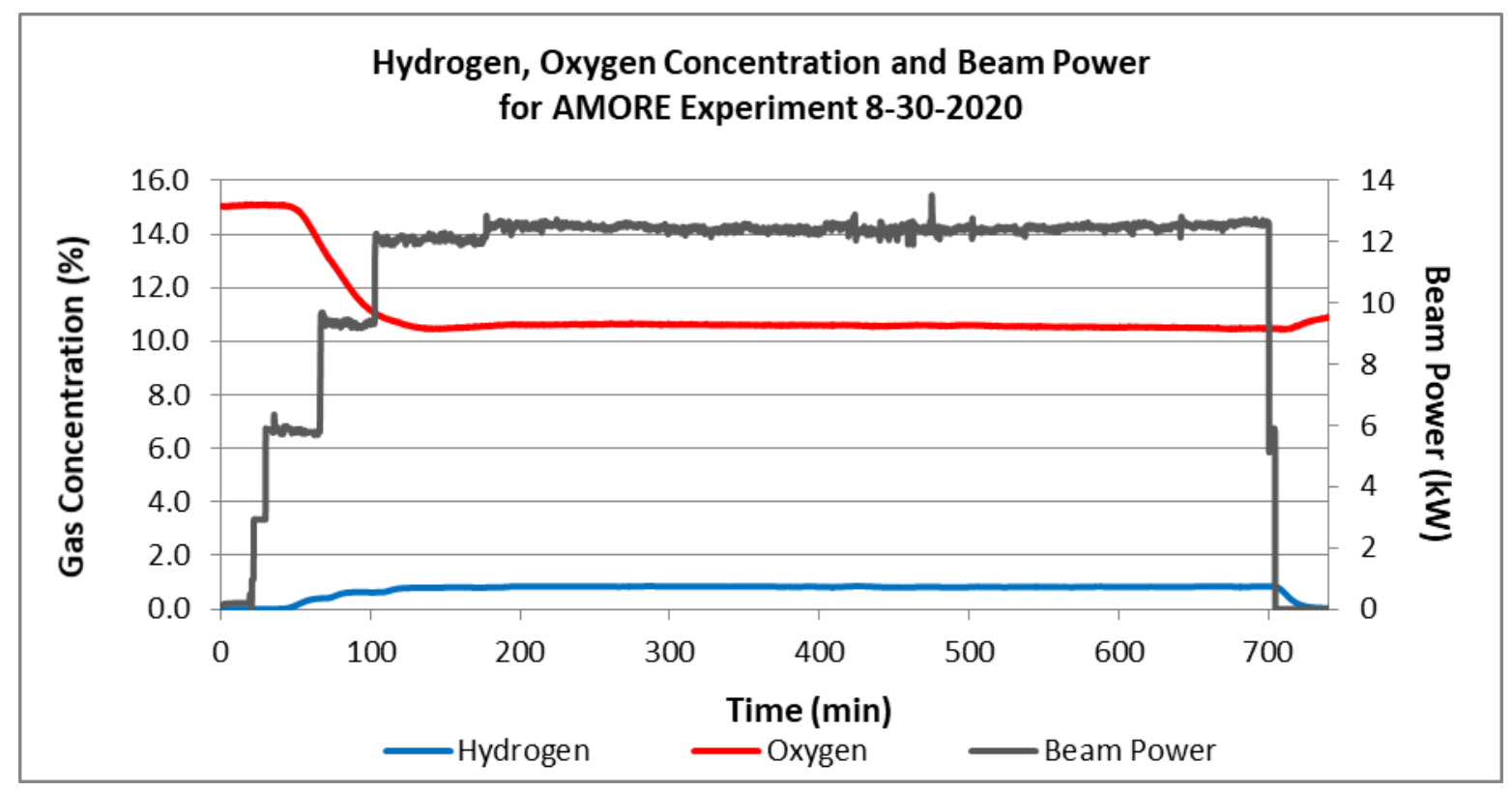

FIGURE 3.2.5.1 Gas concentration and linac beam power for Irradiation \#4 on 8/30/20

\subsubsection{Irradiation \#5, 1/18/21}

Figure 3.2.6.1 shows gas concentration and linac beam power for Irradiation \#5, completed on $1 / 18 / 21$. The RGA data presented have a percent error of $13 \%$ and are lower when compared to the sensor data for hydrogen. In this experiment, hydrogen concentration began to rise when beam energy reached $1.78 \mathrm{kWh}$, whereas the residual concentration of oxygen began to decrease through recombination with hydrogen at the catalyst at $0.14 \mathrm{kWh}$. The concentrations of hydrogen and oxygen remained steady until the beam dropped out. At those times, hydrogen concentration was reduced through recombination at the catalyst, and oxygen increased because it came out of the solution as hydrogen peroxide decomposed. This experiment showed similar characteristics to Irradiation \#4 in that steady-state hydrogen and oxygen concentrations were maintained throughout the experiment. This observation indicates a balanced gas generation and catalytic recombination and no uranyl peroxide precipitation. No precipitate was observed in the solution before or after Irradiation \#5. 


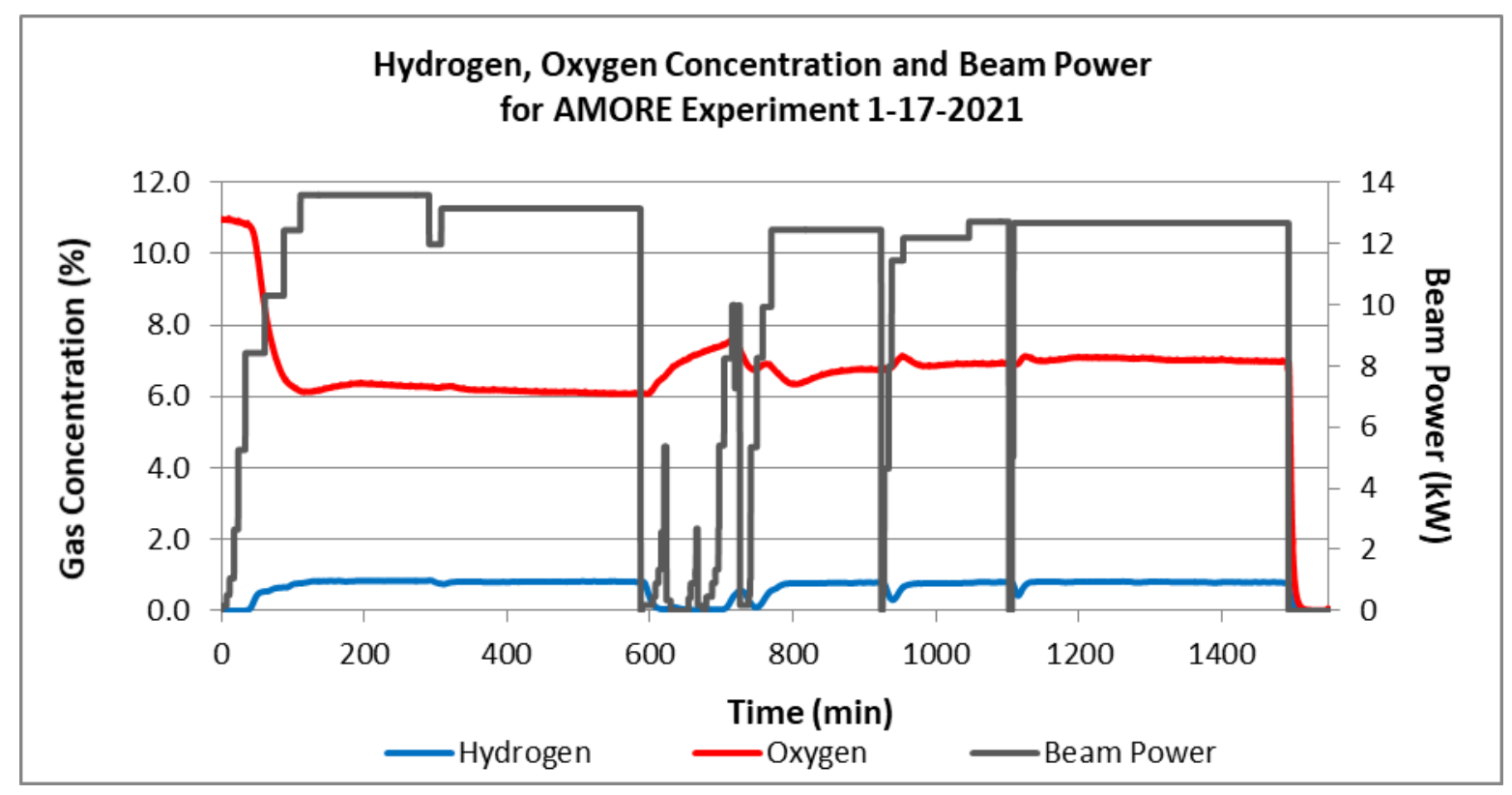

FIGURE 3.2.6.1 Gas concentration and linac beam power for Irradiation \#5 on 1/17/21

\subsubsection{Summary}

Before Irradiation \#1 on 10/1/19, a precipitate (uranyl peroxide) was observed in the uranyl sulfate solution. Iron, as ferrous sulfate, was added to mitigate further precipitation. The concentration of iron was $164 \mathrm{ppm}$ in the solution vessel, as determined by ICPMS analysis on 9/23/19. Later analysis of the solution on 2/28/20 showed $150 \mathrm{ppm}$ iron. We have observed a slight dilution of the uranium solution in the vessel due to processing. The oxygen deficit observed in Irradiation \#1 was indicative of continued precipitation of uranium as uranyl peroxide. Uranyl peroxide crystals already present in solution could possibly have facilitated further precipitation. Hydrogen peroxide is generated by the radiolysis of water and reacts with uranyl sulfate to form uranyl peroxide instead of decomposing to oxygen and water. This reaction sequesters the oxygen that would normally be released into the vessel as a gas. Subsequent experiments showed a much smaller oxygen deficit. The final two experiments Irradiations \#4 and \#5 on 8/30/20 and 1/17/21, showed hydrogen and oxygen concentration stable throughout. A steady-state hydrogen and oxygen concentration was maintained throughout those experiments, indicating balanced gas generation and catalytic recombination.

In previous experiments, we observed that radiolysis can cause the uranyl sulfate solution to directly precipitate during an irradiation, form a precipitate after irradiation (delayed), and radiolytically decompose the precipitate during an irradiation [1,2]. It is possible that after the initial precipitation events that occurred before and during Irradiation \#1 on 10/1/19, uranyl peroxide began to decompose as the solution sat between experiments. During subsequent irradiations, the hydrogen peroxide generated was decomposed rapidly enough by the added iron to prevent further precipitation. No precipitate was observed on 2/13/20 during solution processing and transfer. 
Before Irradiation \#4 on 8/30/20, the catalyst pump was replaced with a new but smaller pump. The smaller pump meant a reduction in flow through the catalytic recombiner. The reduced flow required limiting beam power to about $12.5 \mathrm{~kW}$ in order to keep hydrogen concentration $<1 \%$.

\subsubsection{References}

[1] Youker, A., Kalensky, M., Quigley, K., Brossard, T., Chemerisov, S.D., and Vandegrift, G.F. Uranyl Sulfate Irradiations at the Van de Graaff: A Means to Combat Uranyl Peroxide Precipitation, ANL/NE-17/17, Argonne National Laboratory, 2017.

[2] Silverman, M.D., Watson, G.M., and McDuffie, H.F., Peroxide Decomposition in Aqueous Homogeneous Reactor Fuels, Industrial and Engineering Chemistry 1956; 8:1238-1241.

\subsection{RECOVERY COLUMN}

\subsubsection{Commissioning Run, No Irradiation, 8/14/19}

The commissioning run was carried out by adding a known amount of ${ }^{99} \mathrm{Mo}$ to the target solution and circulating it to simulate an irradiation and exercise LEAF-PROC-024 (see Appendix 17). At the same time, a small $(50 \mathrm{~mL})$ solution of ferrous sulfate dissolved in $0.1 \mathrm{M}$ $\mathrm{H}_{2} \mathrm{SO}_{4}$ was added to the target solution as a catalyst to ensure that any further precipitation of uranyl peroxide would be prevented. The target solution had a mass of $20.795 \mathrm{~kg}$, a concentration of $131.93 \mathrm{~g} \mathrm{U} / \mathrm{L}$, and a density of $1.18357 \mathrm{~g} / \mathrm{mL}$, resulting in a total of $2318 \mathrm{~g} \mathrm{U}$ in a volume of $17.57 \mathrm{~L}$ for this simulated irradiation. During the pre-irradiation checks of the system, it was noted that the balances used for measuring the masses of the verification tank, feed bottles, and effluent bottles were out of calibration. Because this was a simulated irradiation, the commissioning run continued as planned with the understanding that the balances needed to be calibrated before the first real irradiation.

After loading molybdenum into the system, the solution was transferred between the verification tank and the TSV several times to allow for good mixing of the ${ }^{99}$ Mo spike. During mixing of the ${ }^{99}$ Mo spike with the LEU solution, the presence of uranyl peroxide precipitate (later confirmed by powder XRD analysis) was observed in the target solution. This precipitate likely formed during the first Phase II irradiation in March 2018 (prior to the addition of the iron catalyst). Formation of uranyl peroxide under irradiation conditions is not unexpected, and was confirmed during small-scale irradiations of a similar uranyl sulfate solution at the $3 \mathrm{MeV}$ VDG electron accelerator. The formation of uranium precipitate is due to complexation of the uranyl ion with peroxide that forms because of the radiolysis of water. This precipitate did not impede processing during the commissioning run, and the bulk of it was removed prior to the first irradiation (shown in Figure 3.3.1.1). 


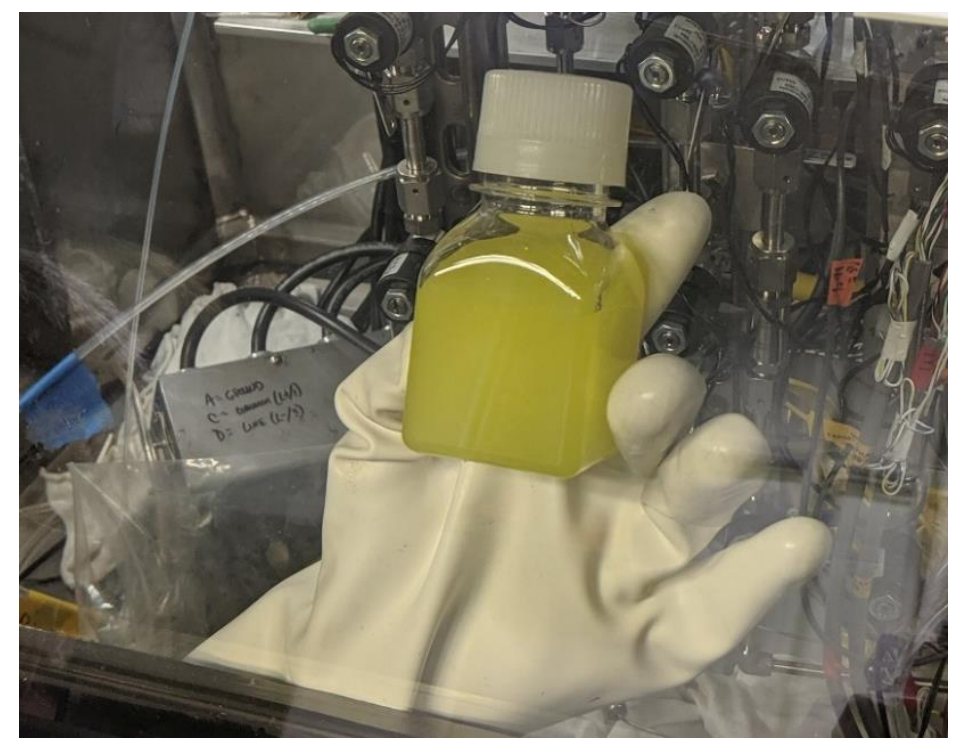

FIGURE 3.3.1.1 Uranyl peroxide precipitate in target solution that was extracted from the system prior to the first irradiation

After mixing, the LEU target solution containing the ${ }^{99}$ Mo spike was loaded onto the column, molybdenum and uranium were eluted from the column in separate fractions, and the solution was sent to and received in the D024 Hot Cell. It was noted that several valves and sensors inside the glovebox were not working, owing to radiation damage or precipitate buildup.

Fourteen samples were collected during the various solution processing steps, divided as shown in Table 3.3.1.1. On the basis of observations from the previous irradiation, there were several sample loops that had to be skipped during sample collection because they were not operable. These included target mixing loop 1, column loading loop 3, and column loading loop 5.

Several issues arose while trying to retrieve the various samples from sample loops. The vacuum pump used to pull solution from the sample loops to the evacuated sample vials became inoperable and had to be replaced. Once replaced, it was found that solution could not be recovered from target mixing loop 3, column loading loops 1, 6, and 7, and column stripping loops 1 and 3. It was still possible to retrieve at least one sample from each processing step, but the number of failures was concerning since they appeared to be the result of uranyl peroxide precipitate clogging the valves used to recover the samples, which could not be easily replaced in the system. 
TABLE 3.3.1.1 Processing samples taken and retrieved during the commissioning run

\begin{tabular}{lcc}
\hline \multicolumn{1}{c}{ Processing Step } & $\begin{array}{c}\text { No. Samples } \\
\text { Taken }\end{array}$ & $\begin{array}{c}\text { No. Samples } \\
\text { Retrieved }\end{array}$ \\
\hline Target Solution Mixing Pre-Irradiation & 1 & \\
Target Solution Mixing During Irradiation & 2 & 1 \\
Column Loading & 2 & 1 \\
Post-Load Acid Wash & 2 & 1 \\
Post-Load $\mathrm{H}_{2} \mathrm{O}$ Wash & 2 & 1 \\
Column Stripping & 3 & 1 \\
Post-Strip $\mathrm{H}_{2} \mathrm{O}$ Wash & 2 & 2 \\
\hline
\end{tabular}

This simulated irradiation was also used to refine LEAF-PROC-24, which was a compilation of the various glovebox processing procedures used in the first irradiation. A lengthy list of revisions was compiled and implemented prior to the next experiment.

Though the target solution was not irradiated, the collected samples were analyzed via gamma spectroscopy to track the efficacy of the ${ }^{99}$ Mo processing steps. The samples recovered from the target mixing ladder showed only $85.9 \%$ of the known ${ }^{99} \mathrm{Mo}$, indicating that a small amount of solution was trapped in the sample retrieval system even after it was cleaned out at the end of the previous processing. The single recovered column stripping sample showed $84.1 \%$ of the added ${ }^{99} \mathrm{Mo}$, indicating that the majority of the ${ }^{99} \mathrm{Mo}$ would be removed from the column in the first $500 \mathrm{~mL}$ of stripping. Following column stripping, roughly $1.3 \%$ of the ${ }^{99} \mathrm{Mo}$ was still present in the system for the Post-Strip $\mathrm{H}_{2} \mathrm{O}$ Wash samples. Table 3.3.1.2 shows the average results.

TABLE 3.3.1.2 Results from gamma analysis of samples recovered during the commissioning run

\begin{tabular}{lccc}
\hline \multicolumn{1}{c}{ Sample } & $\begin{array}{c}\text { Average Total } \\
{ }^{99} \mathrm{Mo}(\mathrm{mCi})\end{array}$ & $\begin{array}{c}\text { Std Dev } \\
(\%)\end{array}$ & $\begin{array}{c}\text { Yield } \\
(\%)\end{array}$ \\
\hline${ }^{99}$ Mo Added & & & \\
Target Mixing & 49.1 & $1.12 \%$ & N/A \\
Column Stripping & 42.2 & $5.70 \%$ & $85.9 \%$ \\
Post-Strip $\mathrm{H}_{2} \mathrm{O}$ Wash & 41.3 & $2.47 \%$ & $84.1 \%$ \\
\hline
\end{tabular}




\subsubsection{Irradiation \#1, 10/1/19}

Referred to as the "Short Irradiation," this run was used to ensure that all the systems would operate as planned during a real irradiation. Prior to this irradiation, the uranyl peroxide precipitate was removed by pumping solution from the TSV into an external bottle until no more precipitate was observed in the stream. After removing as much precipitate as possible, the $\mathrm{U}$ concentration was adjusted with makeup solution. After adjustment, the target solution had a mass of $20.11 \mathrm{~kg}$, concentration of $135.4 \mathrm{~g} \mathrm{U} / \mathrm{L}$, and density of $1.18274 \mathrm{~g} / \mathrm{mL}$, resulting in a total of $2298 \mathrm{~g} \mathrm{U}$ in a volume of $16.97 \mathrm{~L}$ for this irradiation. This was the first irradiation in which LEAF-PROC-024 Rev. 2 was used for preparing the system and processing the solution. This revision removed 32 pages of redundant instructions from the first revision and moved all replacement-part descriptions to the end. Because of the issues with retrieving samples during the commissioning run, samples for this run were re-prioritized. As a result, fewer processing steps were sampled, but more samples were taken from the process steps that were deemed more important. The distribution of samples taken during this irradiation is shown in Table 3.3.2.1. Further issues with sample retrieval occurred, resulting in recovery of only seven of the 18 collected samples. The recovered samples were representative of the target mixing, column loading, and column stripping processing steps.

TABLE 3.3.2.1 Samples taken and retrieved during processing for the first irradiation

\begin{tabular}{lcc}
\hline \multicolumn{1}{c}{ Processing Step } & $\begin{array}{c}\text { No. Samples } \\
\text { Taken }\end{array}$ & $\begin{array}{c}\text { No. Samples } \\
\text { Retrieved }\end{array}$ \\
\hline Target Solution Mixing Pre-Irradiation & 0 & 0 \\
Target Solution Mixing After Irradiation & 3 & 2 \\
Column Loading & 7 & 3 \\
Post-Load Acid Wash & 0 & 0 \\
Post-Load $\mathrm{H}_{2} \mathrm{O}$ Wash & 0 & 0 \\
Column Stripping & 6 & 2 \\
Post-Strip $\mathrm{H}_{2} \mathrm{O}$ Wash & 2 & 0 \\
\hline
\end{tabular}

During processing, the pressure readings measured by the system were observed to increase slowly during column stripping. The filter on the base side of the system was bypassed to remedy this issue. Because bypassing the filter did not relieve the pressure, the sampling loop was also changed in case there had been some buildup of solids during processing. This change also had no effect, but processing was able to be completed before the pressure increased to the point of tripping the system pressure switch. 
Gamma spectroscopy analysis was carried out on the two samples recovered from target mixing, the three recovered from column loading, and the two recovered from column stripping. The average masses of sample counted for target mixing, column loading, and column stripping were $224 \mathrm{mg}, 268 \mathrm{mg}$, and $122 \mathrm{mg}$, respectively. Each sample was counted for one hour on $10 / 4 / 19$, then counted for a second hour roughly three days later (10/7-8) to ensure the accuracy of the results.

Because mixing was not carried out during the irradiation, all target mixing samples reflect the contents of the target solution after irradiation. Both samples were taken in the last 30 min of mixing to ensure that they were representative of the homogenized solution. Unfortunately, the first target mixing sample was diluted by some solution that remained in the sample recovery line after washing the system at the end of the sample recovery run. For this reason, only the results from the second target mixing sample (designated TM-2) are reported in Table 3.3.2.2. The results indicate the presence of ${ }^{97} \mathrm{Nb} /{ }^{97} \mathrm{Zr},{ }^{239} \mathrm{~Np},{ }^{99} \mathrm{Mo},{ }^{103} \mathrm{Ru},{ }^{131} \mathrm{I},{ }^{132} \mathrm{I},{ }^{133} \mathrm{I}$, ${ }^{140} \mathrm{Ba} /{ }^{140} \mathrm{La}$, and ${ }^{143} \mathrm{Ce}$ in the irradiated target with high confidence. Several other isotopes, including ${ }^{105} \mathrm{Rh},{ }^{91} \mathrm{Sr},{ }^{135} \mathrm{I},{ }^{127} \mathrm{Sb},{ }^{125} \mathrm{Sn},{ }^{135} \mathrm{Xe}$, and ${ }^{133} \mathrm{Xe}$ may have been present, but had poor peak shape or high $1 \sigma$ values. The remaining radionuclides were below the minimum detectable activity (MDA) for the solution analyzed.

The recovered column loading samples were taken at 5, 10, and 40 min into the loading phase of this processing run. Relatively little $\mathrm{Zr} / \mathrm{Nb}$ was seen in these samples (designated CL in Table 3.3.2.2), reinforcing the conclusion drawn during the Mini-SHINE experiments that $\mathrm{Zr}$ has a high affinity for the titania column. All samples showed the presence of the same set of radionuclides with high confidence (low $1 \sigma$ uncertainty), including ${ }^{237} \mathrm{U},{ }^{239} \mathrm{~Np},{ }^{103} \mathrm{Ru},{ }^{133} \mathrm{I}$, ${ }^{140} \mathrm{Ba} /{ }^{140} \mathrm{La},{ }^{143} \mathrm{Ce},{ }^{132} \mathrm{Te},{ }^{105} \mathrm{Rh}$, and ${ }^{91} \mathrm{Sr}$. The results indicate that ${ }^{239} \mathrm{~Np},{ }^{143} \mathrm{Ce}$, and ${ }^{91} \mathrm{Sr}$ are not retained at all, as their activities are approximately the same across the target mixing and all column loading samples. Though ${ }^{140} \mathrm{Ba} /{ }^{140} \mathrm{La},{ }^{127} \mathrm{Sb},{ }^{105} \mathrm{Rh},{ }^{132} \mathrm{Te},{ }^{133} \mathrm{I},{ }^{131} \mathrm{I},{ }^{133} \mathrm{Xe}$, and ${ }^{103} \mathrm{Ru}$ are seen in all the loading samples collected, their activities vary enough that it is likely they are retained by the titania column to some extent.

The recovered column stripping samples were taken at 3.5 and 14 min into the stripping phase of this processing run. It is unclear whether the first recovered sample (CS-2, at $3.5 \mathrm{~min}$ ) was still composed of the post-load acid wash solution or if it was mostly solution trapped in the sample recovery line from the previous washing, but it effectively contained no activity. The other column stripping sample (CS-6, at $14 \mathrm{~min}$ ) clearly contained ${ }^{99} \mathrm{Mo},{ }^{103} \mathrm{Ru},{ }^{133} \mathrm{Xe},{ }^{131} \mathrm{I},{ }^{133} \mathrm{I}$, ${ }^{97} \mathrm{Zr},{ }^{135} \mathrm{Xe},{ }^{127} \mathrm{Sb}$, and ${ }^{135} \mathrm{I}$. All remaining isotopes had high $1 \sigma$ uncertainty or were below the MDA. 
TABLE 3.3.2.2 Activities of isotopes in the various samples collected, with 1 $\sigma$ uncertainty, for Irradiation \#1. Activities are listed as total $\mathrm{mCi}$ in the system

\begin{tabular}{|c|c|c|c|c|c|c|c|c|c|c|c|c|}
\hline Radionuclide & TM-2 & $1 \sigma$ & CL-1 & $1 \sigma$ & CL-2 & $1 \sigma$ & CL-4 & $1 \sigma$ & CS-2 & $1 \sigma$ & CS-6 & $1 \sigma$ \\
\hline${ }^{95} \mathrm{Zr}$ & 104 & $2.9 \%$ & 0.85 & $58.1 \%$ & 0.60 & $84.1 \%$ & 0.93 & $81.9 \%$ & 0.0027 & $37.5 \%$ & 0.25 & $33.0 \%$ \\
\hline${ }^{237} \mathrm{U}$ & 31 & $12.8 \%$ & 32 & $9.3 \%$ & 29 & $10.8 \%$ & 26 & $14.7 \%$ & 0.0064 & $67.8 \%$ & 1.1 & $57.8 \%$ \\
\hline${ }^{239} \mathrm{~Np}$ & 4054 & $4.6 \%$ & 4393 & $4.4 \%$ & 3909 & $4.5 \%$ & 4116 & $4.6 \%$ & 0.027 & $61.3 \%$ & 2.0 & $80.8 \%$ \\
\hline${ }^{99} \mathrm{Mo}$ & 2391 & $5.1 \%$ & 76 & $40.5 \%$ & 105 & $53.5 \%$ & 85 & $62.1 \%$ & 0.062 & $76.1 \%$ & 131 & $5.9 \%$ \\
\hline${ }^{103} \mathrm{Ru}$ & 79 & $3.2 \%$ & 13.1 & $5.0 \%$ & 13 & $7.1 \%$ & 43 & $4.1 \%$ & 0.0028 & $31.5 \%$ & 7.5 & $3.1 \%$ \\
\hline${ }^{133} \mathrm{Xe}$ & 195 & $12.9 \%$ & 5.9 & $100.6 \%$ & 15 & $46.4 \%$ & 16 & $36.8 \%$ & 0.0060 & $102.5 \%$ & 88 & $5.8 \%$ \\
\hline${ }^{131} \mathrm{I}$ & 276 & $8.1 \%$ & 17 & $40.7 \%$ & 27 & $29.7 \%$ & 44 & $32.9 \%$ & 0.014 & $74.4 \%$ & 141 & $2.8 \%$ \\
\hline${ }^{133} \mathrm{I}$ & 7317 & $3.0 \%$ & 76 & $7.8 \%$ & 195 & $3.6 \%$ & 227 & $4.0 \%$ & 0.058 & $60.7 \%$ & 3114 & $2.6 \%$ \\
\hline${ }^{136} \mathrm{Cs}$ & $<1.1$ & MDA & $<0.67$ & MDA & 3.2 & $23.5 \%$ & $<0.89$ & MDA & 0.00073 & $53.2 \%$ & 0.023 & $34.3 \%$ \\
\hline${ }^{140} \mathrm{Ba}$ & 493 & $3.1 \%$ & 306 & $2.8 \%$ & 383 & $2.7 \%$ & 489 & $2.7 \%$ & 0.0041 & $89.4 \%$ & 0.33 & $60.5 \%$ \\
\hline${ }^{140} \mathrm{La}$ & 4285 & $3.2 \%$ & 1766 & $3.2 \%$ & 2230 & $3.2 \%$ & 2769 & $3.2 \%$ & 0.00055 & $68.5 \%$ & 0.097 & $32.0 \%$ \\
\hline${ }^{88} \mathrm{Kr}$ & \multicolumn{2}{|c|}{$>12$ half-lives } & \multicolumn{2}{|c|}{$>12$ half-lives } & \multicolumn{2}{|c|}{$>12$ half-lives } & \multicolumn{2}{|c|}{$>12$ half-lives } & \multicolumn{2}{|c|}{$>12$ half-lives } & \multicolumn{2}{|c|}{$>12$ half-lives } \\
\hline${ }^{132} \mathrm{Te}$ & 1716 & $10.3 \%$ & 537 & $10.7 \%$ & 750 & $10.5 \%$ & 1379 & $10.3 \%$ & 0.015 & $110.6 \%$ & 1.5 & $89.6 \%$ \\
\hline${ }^{97} \mathrm{Nb}$ & \multicolumn{2}{|c|}{$>12$ half-lives } & \multicolumn{2}{|c|}{$>12$ half-lives } & \multicolumn{2}{|c|}{$>12$ half-lives } & \multicolumn{2}{|c|}{$>12$ half-lives } & \multicolumn{2}{|c|}{$>12$ half-lives } & \multicolumn{2}{|c|}{$>12$ half-lives } \\
\hline${ }^{105} \mathrm{Rh}$ & 944 & $6.3 \%$ & 841 & $5.1 \%$ & 746 & $5.5 \%$ & 854 & $5.7 \%$ & 0.058 & $72.0 \%$ & 12 & $42.2 \%$ \\
\hline${ }^{125} \mathrm{Sn}$ & $<5.8$ & MDA & 5.8 & $75.9 \%$ & 20 & $38.8 \%$ & 8.2 & $91.6 \%$ & 0.022 & $61.9 \%$ & 2.7 & $33.3 \%$ \\
\hline${ }^{127} \mathrm{Sb}$ & 37.9 & $17.7 \%$ & 13 & $27.1 \%$ & 11 & $29.8 \%$ & 11 & $28.3 \%$ & 0.0047 & $47.2 \%$ & 4.4 & $6.7 \%$ \\
\hline${ }^{91} \mathrm{Sr}$ & 16682 & $3.2 \%$ & 13244 & $2.4 \%$ & 11430 & $2.8 \%$ & 13037 & $3.4 \%$ & $<0.031$ & MDA & 65 & $34.3 \%$ \\
\hline${ }^{135} \mathrm{I}$ & 16042 & $16.2 \%$ & 4521 & $44.8 \%$ & 908 & $92.8 \%$ & $<666$ & MDA & 1.1 & $73.5 \%$ & 8320 & $4.1 \%$ \\
\hline
\end{tabular}




\subsubsection{Irradiation \#2, 11/11/19}

This irradiation was the first full-scale irradiation following the gas release that occurred in early 2018. The target solution was not adjusted prior to this irradiation, but it was sampled to capture any dilution/loss that might have occurred during the previous irradiation and processing. Measurements determined that the target solution had a mass of $19.625 \mathrm{~kg}$, concentration of $133.7 \mathrm{~g}-\mathrm{U} / \mathrm{L}$, and density of $1.17983 \mathrm{~g} / \mathrm{mL}$, resulting in a total of $2224 \mathrm{~g} \mathrm{U}$ in a volume of $16.63 \mathrm{~L}$ for this irradiation. To ensure the best odds of retrieving a sample that was representative of the solution after irradiation, all eight target mixing samples were collected regardless of past failures. All eight column loading samples were also collected during processing, but no other processing steps were sampled.

Several issues arose during solution processing for this irradiation. Neither the acid preheater nor the column heater worked when activated, meaning that the temperature of the solution being loaded onto the column was much lower than $80^{\circ} \mathrm{C}$. The lowest temperature observed at the pre-heater during loading was $19^{\circ} \mathrm{C}$, and on the column was $21^{\circ} \mathrm{C}$. Further, the pressure switch in the system tripped three times during column loading. The issue was mitigated by pumping for a longer time at lower solution velocity to load the column. This issue was likely due to a combination of residual precipitate in the TSV that was dislodged during the previous irradiation processing and an overly packed column.

Despite the number of samples collected, none could be retrieved at the end of processing (see Table 3.3.3.1). Multiple novel strategies were attempted to retrieve the samples after allowing several days for the radiation field to decrease sufficiently. These included replacing the plastic manifold parts, reversing the direction of the pump to relieve pressure on the valves, and manually forcing the solenoid valves open using positive pressure. Though forcing the valves open worked, it required far too much time in close proximity to the sample ladder right after an irradiation, when the dose rates would be much higher. To ensure that at least one high-quality sample could be retrieved from the target mixing step in the future, an alternative sample loop with manual valves was installed in the acid flow meter bypass path.

TABLE 3.3.3.1 Samples taken and retrieved during processing for the second irradiation

\begin{tabular}{lcc}
\hline \multicolumn{1}{c}{ Processing Step } & $\begin{array}{c}\text { No. Samples } \\
\text { Taken }\end{array}$ & $\begin{array}{c}\text { No. Samples } \\
\text { Retrieved }\end{array}$ \\
\hline Target Solution Mixing Pre-Irradiation & 0 & 0 \\
Target Solution Mixing After Irradiation & 8 & 0 \\
Column Loading & 7 & 0 \\
Post-Load Acid Wash & 0 & 0 \\
Post-Load $\mathrm{H}_{2} \mathrm{O}$ Wash & 0 & 0 \\
Column Stripping & 0 & 0 \\
Post-Strip $\mathrm{H}_{2} \mathrm{O}$ Wash & 0 & 0 \\
\hline
\end{tabular}


One additional sample was collected from the target solution after it had been transferred back to the TSV and left sitting for several days, to see which fission products remained in the target solution after processing. While pumping solution from the TSV, a large amount of precipitate was seen in the FEP portion of the acid line. As a result, as much precipitate as possible was removed via the same method that was used previously. It is likely that this precipitate is what caused the overpressure during column loading. After removing the precipitate, the target solution was pumped to the dump tank and sampled via the port at valve V-2001 before being returned to the TSV for storage.

Though no samples were recovered from the sample ladders, one was collected from the target solution after all processing had been completed. This sample was referred to as the processed target solution and was analyzed twice by gamma spectroscopy. The mass of target solution analyzed was $98.2 \mathrm{mg}$. The first analysis counted the sample for $10 \mathrm{~min}$ and was used to determine the detector dead time and ensure that no further dilution needed to occur. The second analysis occurred 2.75 hours later, counted the sample for 3.5 hours, and positively identified ${ }^{95} \mathrm{Zr} /{ }^{95} \mathrm{Nb},{ }^{239} \mathrm{~Np},{ }^{103} \mathrm{Ru},{ }^{132} \mathrm{I},{ }^{133} \mathrm{I},{ }^{140} \mathrm{Ba} /{ }^{140} \mathrm{La},{ }^{143} \mathrm{Ce},{ }^{131 \mathrm{~m}} \mathrm{Xe},{ }^{132} \mathrm{Te}$, and ${ }^{147} \mathrm{Nd}$. The radionuclides ${ }^{237} \mathrm{U},{ }^{137} \mathrm{Cs},{ }^{131} \mathrm{I},{ }^{99 \mathrm{~m}} \mathrm{Tc}$ and ${ }^{105} \mathrm{Rh}$ were also detected with higher uncertainties. The detection of ${ }^{132} \mathrm{Te}$ indicates that ${ }^{132} \mathrm{I}$ was present at one time, and ${ }^{99 \mathrm{~m}} \mathrm{Tc}$ indicates that small amounts of ${ }^{99} \mathrm{Mo}$ were likely present, but at quantities undetectable when using the lower-branching-ratio emission. The remaining radionuclide activities were below their respective MDAs, as indicated in Table 3.3.3.2.

TABLE 3.3.3.2 Activities of isotopes in the processed target solution sample, with $1 \sigma$ uncertainty, for Irradiation \#2. Activities are listed as total $\mathbf{m C i}$ in the system.

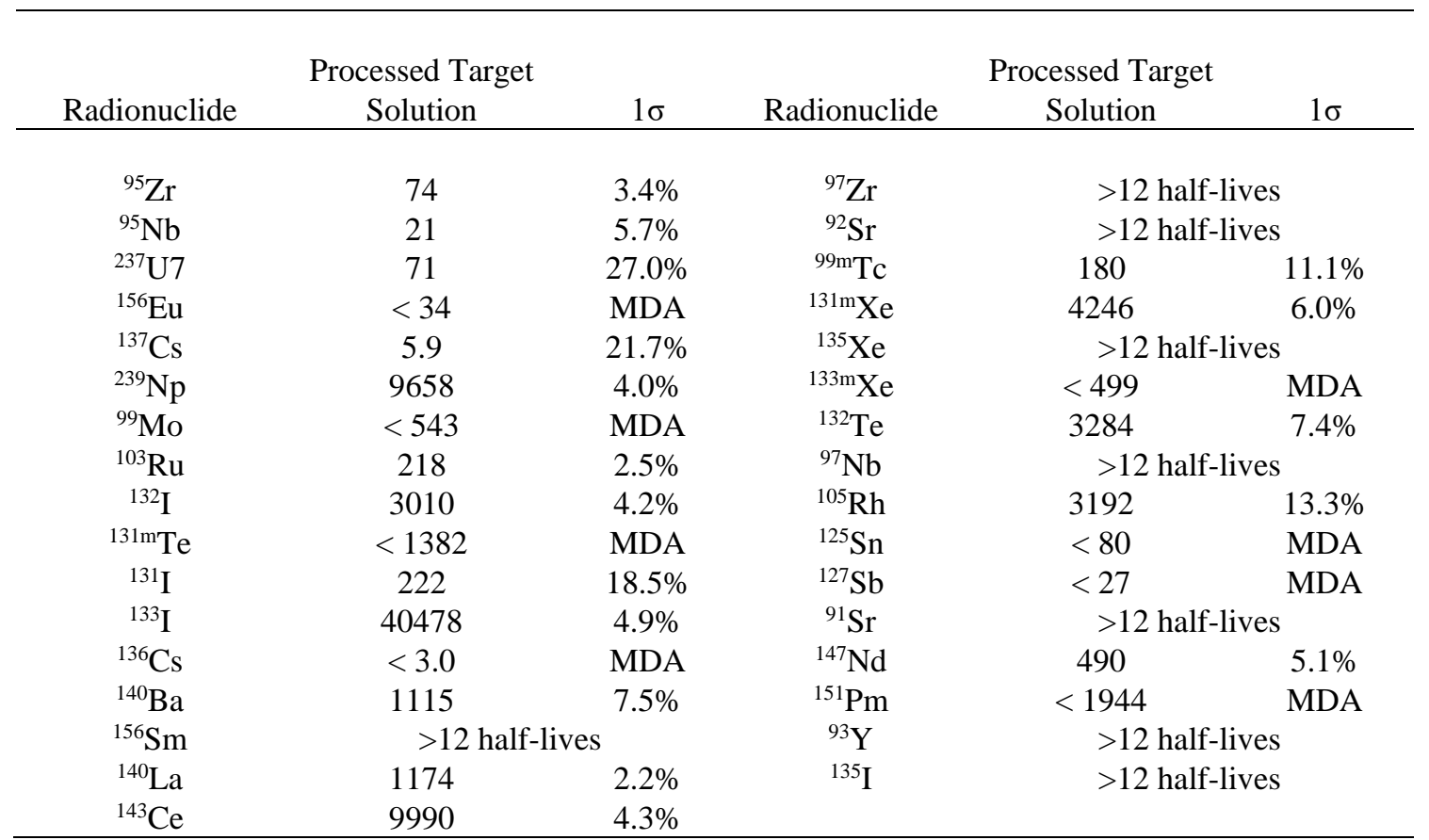




\subsubsection{Irradiation \#3, 3/2/20}

Prior to this irradiation, LEAF-PROC-024 was modified to reflect the addition of the alternate sample loop. In addition, the relays associated with all the system heaters were replaced along with the pressure transducers to ensure they would work, since most had malfunctioned during the previous irradiation processing due to radiation damage. To ensure that the dose rate would not be excessively high while retrieving the sample from the alternate loop, none of the original sample loops in the sample ladders were used. After adjustment and the addition of stable Mo carrier, the target solution had a mass of $20.31 \mathrm{~kg}$, concentration of $140.3 \mathrm{~g}-\mathrm{U} / \mathrm{L}$, and density of $1.19800 \mathrm{~g} / \mathrm{mL}$, resulting in a total of $2378 \mathrm{~g}$ U in a volume of $16.95 \mathrm{~L}$ for this irradiation.

This irradiation was cut short because of a malfunction in the accelerator. The target solution was mixed, but it was determined that the amount of ${ }^{99}$ Mo produced was not sufficient to ship, so the solution was returned to the TSV to monitor hydrogen accumulation while the solution was stored until the next irradiation. Because the processing was aborted during mixing, no samples were taken as part of this irradiation.

\subsubsection{Irradiation \#4, 8/30/20}

The target solution was not adjusted prior to this irradiation, but it was sampled to capture any dilution/loss that might have occurred during the aborted irradiation and mixing from irradiation \#3. Measurements determined that the target solution had a mass of $20.37 \mathrm{~kg}$, concentration of $140.18 \mathrm{~g}-\mathrm{U} / \mathrm{L}$, and density of $1.19802 \mathrm{~g} / \mathrm{mL}$, resulting in a total of $2383 \mathrm{~g} \mathrm{U}$ in a volume of $17.00 \mathrm{~L}$ for this irradiation. As was intended for the previous irradiation, the only sample taken utilized the alternate sample loop. This was the first irradiation where samples were taken from the various effluent bottles to determine which fractions contained which fission products.

The glovebox processing run for this irradiation was carried out without incident or malfunction. After irradiation, the target solution was mixed and loaded onto the column; then molybdenum and uranium were eluted from the column in separate fractions. The ${ }^{99}$ Mo fraction was sent to and received in the D024 Hot Cell.

For this irradiation, one sample, which was representative of the irradiated and mixed target solution, was retrieved from the alternate sample loop for gamma spectroscopy. This sample was serially diluted until the detector dead-time was approximately $5 \%$ when counting at the furthest available distance $(100 \mathrm{~cm})$, which resulted in counting a net mass of $112 \mathrm{mg}$ of the original solution. The target mixing sample was counted three times, for 15.5, 86, and 63.5 hours, with these analyses occurring 4,5 , and 12 days after irradiation, respectively. Table 3.3.5.1 shows the average activities of the various nuclides and their $1 \sigma$ uncertainties calculated in the entire target solution volume from the three replicate analyses. Owing to the delay in counting, ${ }^{92} \mathrm{Sr},{ }^{97} \mathrm{Nb}$, and ${ }^{135} \mathrm{I}$ had undergone $>12$ half-lives of decay prior to the first analysis and could not be quantified. The radionuclides ${ }^{156} \mathrm{Eu},{ }^{136} \mathrm{Cs},{ }^{156} \mathrm{Sm},{ }^{135} \mathrm{Xe},{ }^{133 \mathrm{~m}} \mathrm{Xe}$, and 
${ }^{125} \mathrm{Sn}$ were all below the MDA for all analyses as well. The analysis indicates that roughly $3.5 \mathrm{Ci}$ of ${ }^{99}$ Mo was produced during the irradiation.

TABLE 3.3.5.1 Activities of radionuclides in the target mixing sample, with $1 \sigma$ uncertainty, for Irradiation \#4. Activities are listed as total $\mathbf{m C i}$ in the irradiated target solution.

\begin{tabular}{|c|c|c|c|c|c|}
\hline Radionuclide & Target Mixing & $1 \sigma$ & Radionuclide & Target Mixing & $1 \sigma$ \\
\hline${ }^{95} \mathrm{Zr}$ & 181 & $3.4 \%$ & ${ }^{97} \mathrm{Zr}$ & 7940 & $4.6 \%$ \\
\hline${ }^{95} \mathrm{Nb}$ & 108 & $3.4 \%$ & ${ }^{92} \mathrm{Sr}$ & \multicolumn{2}{|c|}{ >12 Half-lives } \\
\hline${ }^{237} \mathrm{U}$ & 28 & $13.5 \%$ & ${ }^{99 \mathrm{~m}} \mathrm{Tc}$ & 3310 & $6.1 \%$ \\
\hline${ }^{156} \mathrm{Eu}$ & 8.4 & MDA & ${ }^{131 \mathrm{~m}} \mathrm{Xe}$ & 715 & $10.2 \%$ \\
\hline${ }^{137} \mathrm{Cs}$ & 11 & $8.8 \%$ & ${ }^{135} \mathrm{Xe}$ & $<3000$ & MDA \\
\hline${ }^{239} \mathrm{~Np}$ & 5760 & $5.8 \%$ & ${ }^{133 \mathrm{~m}} \mathrm{Xe}$ & $<160$ & MDA \\
\hline${ }^{99} \mathrm{Mo}$ & 3500 & $5.1 \%$ & ${ }^{132} \mathrm{Te}$ & 2758 & $3.6 \%$ \\
\hline${ }^{103} \mathrm{Ru}$ & 172 & $3.7 \%$ & ${ }^{97} \mathrm{Nb}$ & \multicolumn{2}{|c|}{ >12 Half-lives } \\
\hline${ }^{132} \mathrm{I}$ & 2130 & $6.0 \%$ & ${ }^{105} \mathrm{Rh}$ & 18400 & $9.1 \%$ \\
\hline${ }^{131 \mathrm{~m}} \mathrm{Te}$ & 3530 & $20.6 \%$ & ${ }^{125} \mathrm{Sn}$ & $<18$ & MDA \\
\hline${ }^{131} \mathrm{I}$ & 481 & $5.1 \%$ & ${ }^{127} \mathrm{Sb}$ & 61.0 & $14.8 \%$ \\
\hline${ }^{133} I$ & 11650 & $3.0 \%$ & ${ }^{91} \mathrm{Sr}$ & 51000 & $19.1 \%$ \\
\hline${ }^{136} \mathrm{Cs}$ & $<1.0$ & MDA & ${ }^{147} \mathrm{Nd}$ & 221 & $7.1 \%$ \\
\hline${ }^{140} \mathrm{Ba}$ & 797 & $3.7 \%$ & ${ }^{151} \mathrm{Pm}$ & 2230 & $13.7 \%$ \\
\hline${ }^{156} \mathrm{Sm}$ & $<5300$ & MDA & ${ }^{93} \mathrm{Y}$ & 315000 & $15.4 \%$ \\
\hline${ }^{140} \mathrm{La}$ & 916 & $4.1 \%$ & ${ }^{135} \mathrm{I}$ & \multirow{2}{*}{\multicolumn{2}{|c|}{$>12$ Half-lives }} \\
\hline${ }^{143} \mathrm{Ce}$ & 6490 & $7.4 \%$ & & & \\
\hline
\end{tabular}

The various effluent bottles were also sampled after irradiation to determine which radionuclides could be associated with which waste streams. Generally, each sample was counted for a very short time $(\sim 2 \mathrm{~min})$ to determine detector dead time and whether any further dilution was required. This was followed by a longer analysis that ranged from $30 \mathrm{~min}$ to $16 \mathrm{~h}$, dependent on the sample activity. The results from the longer analyses are found in Table 3.3.5.2. The long count for the sample from the post-load water wash bottle was performed on $9 / 10 / 20$, while the base rinse was counted on $9 / 14 / 20$, the acid rinse was counted on $9 / 16 / 20$, and the remaining samples were counted on $9 / 15 / 20$. Because of the $10+$ day delay before analyses were undertaken, several radionuclides had undergone $>12$ half-lives of decay and could not be quantified. These include ${ }^{133} \mathrm{I},{ }^{156} \mathrm{Sm},{ }^{97} \mathrm{Zr} /{ }^{97} \mathrm{Nb},{ }^{92} \mathrm{Sr},{ }^{135} \mathrm{Xe},{ }^{91} \mathrm{Sr},{ }^{93} \mathrm{Y}$, and ${ }^{135} \mathrm{I}$, which have been omitted from Table 3.3.5.1 for brevity. Note also that all activities in Table 3.3.5.2 are in $\boldsymbol{\mu} \boldsymbol{C i}$, while all previous tables have been in $\mathrm{mCi}$. This smaller unit was used because the effluent bottle activities were much lower than the activities of samples from the processing stream. 
TABLE 3.3.5.2 Average activities of radionuclides in the effluent bottle samples, with $1 \sigma$ uncertainty, for Irradiation \#4. Activities are listed as total $\mu \mathrm{Ci}$ in the given effluent bottle.

\begin{tabular}{|c|c|c|c|c|c|c|c|c|c|c|c|c|}
\hline Radionuclide & \begin{tabular}{|c} 
Pre-Load \\
Acid Wash \\
\end{tabular} & $1 \sigma$ & $\begin{array}{l}\text { Post-Load } \\
\text { Acid Wash }\end{array}$ & $1 \sigma$ & $\begin{array}{c}\text { Post-Load } \\
\text { Water Wash } \\
\end{array}$ & $1 \sigma$ & $\begin{array}{c}\text { Post-Strip } \\
\text { Water Wash }\end{array}$ & $1 \sigma$ & Acid Rinse & $1 \sigma$ & Base Rinse & $1 \sigma$ \\
\hline${ }^{95} \mathrm{Zr}$ & $<37$ & MDA & $<42$ & MDA & 41 & $14.1 \%$ & 118 & $4.4 \%$ & 69 & $2.0 \%$ & 0.31 & $14.4 \%$ \\
\hline${ }^{95} \mathrm{Nb}$ & $<24$ & MDA & $<27$ & MDA & 22 & $11.3 \%$ & 45 & $6.9 \%$ & 55 & $2.0 \%$ & 0.41 & $6.7 \%$ \\
\hline${ }^{237} U$ & $<480$ & MDA & $<631$ & MDA & $<80$ & MDA & $<152$ & MDA & $<2.3$ & MDA & $<1.7$ & MDA \\
\hline${ }^{156} \mathrm{Eu}$ & $<469$ & MDA & $<530$ & MDA & $<61$ & MDA & $<52$ & MDA & $<1.4$ & MDA & $<1.0$ & MDA \\
\hline${ }^{137} \mathrm{Cs}$ & 60 & $16.6 \%$ & 48 & $25.3 \%$ & $<9$ & MDA & 7.0 & $26.4 \%$ & 6.2 & $3.4 \%$ & 0.64 & $4.9 \%$ \\
\hline${ }^{239} \mathrm{~Np}$ & 73000 & $14.3 \%$ & 53000 & $25.0 \%$ & 2418 & $24.8 \%$ & $<4582$ & MDA & $<93$ & MDA & $<42$ & MDA \\
\hline${ }^{99} \mathrm{Mo}$ & $<17840$ & MDA & $<24012$ & MDA & $<1420$ & MDA & 14593 & $28.7 \%$ & $<109$ & MDA & 696 & $4.7 \%$ \\
\hline${ }^{103} \mathrm{Ru}$ & 1012 & $3.3 \%$ & 1504 & $3.4 \%$ & 94 & $6.7 \%$ & 3460 & $2.2 \%$ & 23 & $2.3 \%$ & 38 & $2.0 \%$ \\
\hline${ }^{132} \mathrm{I}$ & 15900 & $12.3 \%$ & 67200 & $6.0 \%$ & 10029 & $4.9 \%$ & $<582$ & MDA & 98 & $17.0 \%$ & 24 & $18.8 \%$ \\
\hline${ }^{131 \mathrm{~m}} \mathrm{Te}$ & \multicolumn{2}{|c|}{$>12$ half-lives } & \multicolumn{2}{|c|}{$>12$ half-lives } & $<10294$ & MDA & \multicolumn{2}{|c|}{$>12$ half-lives } & \multicolumn{2}{|c|}{$>12$ half-lives } & 4170 & $21.1 \%$ \\
\hline${ }^{131} \mathrm{I}$ & $<906$ & MDA & $<1276$ & MDA & 466 & $24.6 \%$ & 7714 & $3.0 \%$ & 14 & $21.5 \%$ & 121 & $2.5 \%$ \\
\hline${ }^{136} \mathrm{Cs}$ & $<61$ & MDA & $<67$ & MDA & $<6$ & MDA & $<5$ & MDA & $<0.16$ & MDA & $<0.091$ & MDA \\
\hline${ }^{140} \mathrm{Ba}$ & 5970 & $3.3 \%$ & 4730 & $5.1 \%$ & 184 & $16.3 \%$ & $<30$ & MDA & 28 & $3.6 \%$ & 8.5 & $4.3 \%$ \\
\hline${ }^{140} \mathrm{La}$ & 7060 & $2.6 \%$ & 5320 & $2.8 \%$ & 146 & $4.7 \%$ & $<5$ & MDA & 36 & $2.5 \%$ & 10 & $2.5 \%$ \\
\hline${ }^{143} \mathrm{Ce}$ & $<132558$ & MDA & $<174638$ & MDA & $<2881$ & MDA & $<43953$ & MDA & \multicolumn{2}{|c|}{$>12$ half-lives } & $<350.9$ & MDA \\
\hline${ }^{99 \mathrm{~m}} \mathrm{Tc}$ & $<1299$ & MDA & $<1695$ & MDA & $<96$ & MDA & 16065 & $4.2 \%$ & 33 & $11.6 \%$ & 519 & $3.5 \%$ \\
\hline${ }^{131 \mathrm{~m}} \mathrm{Xe}$ & 23800 & $9.1 \%$ & 17060 & $15.9 \%$ & $<521$ & MDA & $<1525$ & MDA & 48 & $12.2 \%$ & 30 & $22.2 \%$ \\
\hline${ }^{133 \mathrm{~m} X e}$ & $<43559$ & MDA & $<31017$ & MDA & $<1706$ & MDA & $<9063$ & MDA & $<146$ & MDA & $<117$ & MDA \\
\hline${ }^{132} \mathrm{Te}$ & 18090 & $4.2 \%$ & 68039 & $2.5 \%$ & 10444 & $2.3 \%$ & $<217$ & MDA & 110 & $3.6 \%$ & 27 & $6.1 \%$ \\
\hline${ }^{105} \mathrm{Rh}$ & 14110000 & $24.6 \%$ & 3660020 & $18.1 \%$ & $<16995$ & MDA & $<212223$ & MDA & 30289 & $13.5 \%$ & $<1733$ & MDA \\
\hline${ }^{125} \mathrm{Sn}$ & $<1284$ & MDA & $<1582$ & MDA & $<158$ & MDA & $<169$ & MDA & $<3.7$ & MDA & $<2.4$ & MDA \\
\hline${ }^{127} \mathrm{Sb}$ & $<1240$ & MDA & $<1838$ & MDA & $<170$ & MDA & 2348 & $11.8 \%$ & $<7.1$ & MDA & 78 & $4.7 \%$ \\
\hline${ }^{147} \mathrm{Nd}$ & 2080 & $6.9 \%$ & 585 & $21.7 \%$ & $<27$ & MDA & $<38$ & MDA & 6.1 & $7.8 \%$ & $<0.63$ & MDA \\
\hline${ }^{151} \mathrm{Pm}$ & \multicolumn{2}{|c|}{$>12$ half-lives } & \multicolumn{2}{|c|}{$>12$ half-lives } & $<13319$ & MDA & \multicolumn{2}{|c|}{$>12$ half-lives } & \multicolumn{2}{|c|}{$>12$ half-lives } & \multicolumn{2}{|c|}{$>12$ half-lives } \\
\hline
\end{tabular}


Generally, it was found that ${ }^{95} \mathrm{Zr} /{ }^{95} \mathrm{Nb}$ were only present in samples from effluent bottles following the rinsing of the column with water, in line with the conclusion that $\mathrm{Zr}$ is well retained by the titania column under acidic conditions. Also found only in the water rinse samples was ${ }^{127} \mathrm{Sb}$, indicating that it too was retained under acidic conditions. The radionuclides ${ }^{103} \mathrm{Ru},{ }^{132} \mathrm{Te} /{ }^{132} \mathrm{I},{ }^{137} \mathrm{Cs}$, and ${ }^{140} \mathrm{Ba} /{ }^{140} \mathrm{La}$ were either found in all effluent bottles or the MDA reported was higher than the activities in the various other samples. It was observed that ${ }^{147} \mathrm{Nd}$ and ${ }^{105} \mathrm{Rh}$ were only found in acid process effluent bottles, indicating that they are not retained by the column at all. The post-strip water wash is the only effluent to contain significant amounts of ${ }^{99} \mathrm{Mo} /{ }^{99 m} \mathrm{Tc}$, though some is also present in the base rinse sample. This is unsurprising, since a small portion of the strip solution is directed towards the post-strip water wash during processing, and this step is followed immediately by one in which the lines are flushed out to the base rinse bottle.

\subsubsection{Irradiation \#5, 1/18/21}

After adjustment and the addition of stable Mo carrier, the target solution had a mass of $21.18 \mathrm{~kg}$, concentration of $144.8 \mathrm{~g}-\mathrm{U} / \mathrm{L}$, and density of $1.20863 \mathrm{~g} / \mathrm{mL}$, resulting in a total of $2537 \mathrm{~g} \mathrm{U}$ in a volume of $17.52 \mathrm{~L}$ for this irradiation. As with the previous irradiation, the only sample taken during processing utilized the alternate sample loop. One sample was also retrieved from each of the effluent bottles. An additional sample of the target solution was retrieved using valve V-2001 so that a comparison could be made between the freshly irradiated target solution and the processed target solution. The glovebox processing run for this irradiation was carried out without incident or malfunction. After irradiation, the target solution was mixed and loaded onto the column; then molybdenum and uranium were eluted from the column in separate fractions. The ${ }^{99}$ Mo fraction was sent to and received in the D024 Hot Cell.

The target mixing sample was counted for 2,4 , and $64 \mathrm{~h}$, while the post-processing sample was counted for 1 and $18 \mathrm{~h}$. After a short count to determine dead time, effluent bottle samples were counted at least once for $1 \mathrm{~h}$, with low-activity samples recounted for longer times to improve counting statistics.

Unfortunately, delays in sample preparation meant that none of the samples could be analyzed until 10 days after the irradiation. As a result, 10 of the nuclides of interest ${ }^{133} \mathrm{I},{ }^{131 \mathrm{~m}} \mathrm{Te}$, ${ }^{156} \mathrm{Sm},{ }^{97} \mathrm{Zr} /{ }^{97} \mathrm{Nb},{ }^{92} \mathrm{Sr},{ }^{135} \mathrm{Xe},{ }^{91} \mathrm{Sr},{ }^{93} \mathrm{Y}$, and $\left.{ }^{135} \mathrm{I}\right)$ had decayed for $>12$ half-lives and did not provide usable data for any of the collected samples. For brevity, these have been removed from Table 3.3.6.1 and Table 3.3.6.2, below. Analysis indicates that roughly 5.8 Ci of ${ }^{99} \mathrm{Mo}$ was created during the irradiation, though nearly $1 \mathrm{Ci}$ of that was still in the target solution after processing. Regardless of how much was left in solution, $5.8 \mathrm{Ci}$ is far below the intended 10-Ci production run, and it is unclear why this occurred. 
TABLE 3.3.6.1 Average activities of the various radionuclides in the irradiated target and processed target solutions, with $1 \sigma$ uncertainty, for Irradiation \#5. Activities are $\mathbf{m C i}$ present in the total target solution, which had roughly the same mass before and after irradiation.

\begin{tabular}{|c|c|c|c|c|}
\hline Radionuclide & $\begin{array}{c}\text { Irradiated Target } \\
\text { Average }\end{array}$ & $\mathbf{1 \sigma}$ & $\begin{array}{c}\text { Post-Processing } \\
\text { Average }\end{array}$ & $\mathbf{1 \sigma}$ \\
\hline${ }^{95} \mathrm{Zr}$ & 293 & $3.8 \%$ & 53 & $3.0 \%$ \\
\hline${ }^{95} \mathrm{Nb}$ & 99 & $4.8 \%$ & 20 & $3.4 \%$ \\
\hline${ }^{237} \mathrm{U}$ & 104 & $17.5 \%$ & 49 & $8.9 \%$ \\
\hline${ }^{156} \mathrm{Eu}$ & $<29$ & MDA & $<5$ & MDA \\
\hline${ }^{137} \mathrm{Cs}$ & 9.0 & $12.8 \%$ & 2.6 & $8.0 \%$ \\
\hline${ }^{239} \mathrm{~Np}$ & 9342 & $7.6 \%$ & 4727 & $5.4 \%$ \\
\hline${ }^{99} \mathrm{Mo}$ & 5787 & $11.7 \%$ & 1037 & $20.1 \%$ \\
\hline${ }^{103} \mathrm{Ru}$ & 203 & $3.8 \%$ & 51 & $2.8 \%$ \\
\hline${ }^{132} \mathrm{I}$ & 3253 & $6.0 \%$ & 1277 & $5.5 \%$ \\
\hline${ }^{131} \mathrm{I}$ & 957 & $3.4 \%$ & 138 & $3.6 \%$ \\
\hline${ }^{136} \mathrm{Cs}$ & 1.1 & $11.6 \%$ & 0.7 & $11.8 \%$ \\
\hline${ }^{140} \mathrm{Ba}$ & 1308 & $4.4 \%$ & 447 & $2.9 \%$ \\
\hline${ }^{140} \mathrm{La}$ & 84 & $2.5 \%$ & 27 & $7.9 \%$ \\
\hline${ }^{143} \mathrm{Ce}$ & 9697 & $22.3 \%$ & $>12$ half-lives \\
\hline${ }^{99 \mathrm{~m}} \mathrm{Tc}$ & 6106 & $6.3 \%$ & 1138 & $5.3 \%$ \\
\hline${ }^{131 \mathrm{~m}} \mathrm{Xe}$ & 82 & $9.9 \%$ & 34 & $14.4 \%$ \\
\hline${ }^{133 \mathrm{~m}} \mathrm{Xe}$ & $<480$ & MDA & $<380$ & MDA \\
\hline${ }^{132} \mathrm{Te}$ & 7993 & $11.8 \%$ & 3081 & $13.7 \%$ \\
\hline${ }^{105} \mathrm{Rh}$ & 11549 & $33.5 \%$ & 17910 & $17.0 \%$ \\
\hline${ }^{125} \mathrm{Sn}$ & $<104$ & MDA & $<19$ & MDA \\
\hline${ }^{127} \mathrm{Sb}$ & 203 & $16.4 \%$ & 61 & $17.6 \%$ \\
\hline${ }^{147} \mathrm{Nd}$ & 507 & $7.2 \%$ & 255 & $5.5 \%$ \\
\hline
\end{tabular}

When retrieving samples from the effluent bottles, it was elected to not collect samples from the acid rinse, pre-load acid wash, and base rinse bottles, since these effectively contain the same nuclides as the post-processing, post-load acid wash, and post-strip water wash bottles, respectively. Unfortunately, the post-strip water wash bottle did not have enough solution to sample, so no representative sample of the base side of the system was recovered. The radionuclide activities of the remaining post-load acid wash and post-load water wash effluent bottles are shown in Table 3.3.6.2. Though the activity was lower, the results obtained for the post-load acid and post-load water washes are similar to those obtained after the previous irradiation, reinforcing the conclusions stated above. 
TABLE 3.3.6.2 Activities of radionuclides found in the effluent bottle samples, with $1 \sigma$ uncertainty, for Irradiation \#5. Activities are listed as total $\mu \mathrm{Ci}$ in the given effluent bottle.

\begin{tabular}{|c|c|c|c|c|}
\hline Radionuclide & $\begin{array}{c}\text { Post-Load Acid } \\
\text { Wash }\end{array}$ & $\mathbf{1 \sigma}$ & $\begin{array}{c}\text { Post-Load } \\
\text { Water Wash }\end{array}$ & $\mathbf{1 \sigma}$ \\
\hline${ }^{95} \mathrm{Zr}$ & $<14$ & MDA & $<5.7$ & MDA \\
\hline${ }^{95} \mathrm{Nb}$ & $<10$ & MDA & $<3.0$ & MDA \\
\hline${ }^{237} \mathrm{U}$ & $<250$ & MDA & $<80$ & MDA \\
\hline${ }^{156} \mathrm{Eu}$ & $<209$ & MDA & $<51$ & MDA \\
\hline${ }^{137} \mathrm{Cs}$ & 19 & $15.0 \%$ & $<3.7$ & MDA \\
\hline${ }^{239} \mathrm{~Np}$ & 32475 & $25.7 \%$ & $<2960$ & MDA \\
\hline${ }^{99} \mathrm{Mo}$ & $<14876$ & MDA & $<3615$ & MDA \\
\hline${ }^{103} \mathrm{Ru}$ & 630 & $28.1 \%$ & 124 & $3.5 \%$ \\
\hline${ }^{132} \mathrm{I}$ & 78114 & $3.5 \%$ & 34833 & $3.6 \%$ \\
\hline${ }^{131} \mathrm{I}$ & 2320 & $9.1 \%$ & 1663 & $6.7 \%$ \\
\hline${ }^{136} \mathrm{Cs}$ & $<22$ & MDA & $<6$ & MDA \\
\hline${ }^{140} \mathrm{Ba}$ & 5474 & $10.5 \%$ & $<300$ & MDA \\
\hline${ }^{140} \mathrm{La}$ & 179 & $25.7 \%$ & $<25$ & MDA \\
\hline${ }^{99 m} \mathrm{Tc}$ & $<1115$ & MDA & $<264$ & MDA \\
\hline${ }^{131 \mathrm{~m}} \mathrm{Xe}$ & $<1526$ & MDA & $<472$ & MDA \\
\hline${ }^{133 \mathrm{~m}} \mathrm{Xe}$ & $<29315$ & MDA & $<6580$ & MDA \\
\hline${ }^{132} \mathrm{Te}$ & 360480 & $12.5 \%$ & 101714 & $9.4 \%$ \\
\hline${ }^{105} \mathrm{Rh}$ & 235389 & $25.2 \%$ & $<55817$ & MDA \\
\hline${ }^{125} \mathrm{Sn}$ & $<529$ & MDA & $<91$ & MDA \\
\hline${ }^{127} \mathrm{Sb}$ & $<715$ & MDA & $<170$ & MDA \\
\hline${ }^{147} \mathrm{Nd}$ & 900 & $9.1 \%$ & 51 & $25.2 \%$ \\
\hline
\end{tabular}

\subsection{HOT CELL PURIFICATION PROCESS}

\subsubsection{Concentration Column}

\subsubsection{Commissioning run $(8 / 4 / 19)$}

The solution from the recovery glovebox was transferred via a transfer line to the D-024 hot cell for volume reduction and further purification using the D-024 hot-cell operations procedure. After the concentration-column operation, the LMC process was performed to produce the final ${ }^{99}$ Mo product. ${ }^{99}$ Mo samples were collected at a variety of process steps and analyzed. 
The concentration-column procedure was performed by following LEAF-PROC-011 (Appendix 27). Minor adjustments were made to the procedure following this experiment to streamline the process and to allow for the researchers to ensure that the operation was completed within the scope of the procedure. During the commissioning run, a thermocouple failed during pre-checks, and, therefore, that particular heating element was not used during this process. The thermocouple was investigated, and a loose wire was found at the connection inside the D-024 hot cell. This wire was repaired before the next experiment.

The solution-transfer and system-interface steps were performed as expected. The concentration column performed as expected. ${ }^{99}$ Mo was successfully loaded on the column; the column was washed; and ${ }^{99}$ Mo was eluted for final processing by the LMC process.

Tables 3.4.1.1.1 and 3.4.1.1.2 summarize the results for samples from the concentration-column process.

TABLE 3.4.1.1.1 Activities detected in the concentration-column fractions, decay corrected to the addition of the spike solution during the commissioning run

8/14/19 Commissioning Run

\begin{tabular}{cccccc}
\hline & & & & & \\
& $\begin{array}{c}\text { Feed, } \\
\mathrm{mCi}\end{array}$ & $\begin{array}{c}\mathrm{HNO}_{3} \\
\mathrm{Wash}, \\
\mathrm{mCi}\end{array}$ & $\begin{array}{c}\mathrm{H}_{2} \mathrm{O} \\
\text { Wash, } \\
\mathrm{mCi}\end{array}$ & $\begin{array}{c}{ }^{99} \mathrm{Mo} \\
\text { Waste, } \\
\text { Product, } \\
(1 \mathrm{~s}, \%)\end{array}$ & $\begin{array}{c}\mathrm{mCi} \\
(1 \mathrm{~s}, \%)\end{array}$ \\
$\begin{array}{cccccc}(1 \mathrm{~s}, \%) \\
(1 \mathrm{~s}, \%)\end{array}$ & $(1 \mathrm{~s}, \%)$ \\
\hline Radionuclide & & & & & \\
${ }^{99} \mathrm{Mo}$ & 43.8 & 0.05 & 0.074 & 0.33 & 16.4 \\
& $(2)$ & $(1.2)$ & $(7.4)$ & $(0.1)$ & $(4.6)$ \\
\hline
\end{tabular}

TABLE 3.4.1.1.2 Relative distribution of ${ }^{99} \mathrm{Mo}$ in various fractions of the concentration column during the commissioning run

8/14/19 Commissioning Run

\begin{tabular}{cccccc}
\hline & & & & & \\
Radionuclide & $\begin{array}{c}\mathrm{HNO}_{3} \\
\text { Wash }\end{array}$ & $\begin{array}{c}\mathrm{H}_{2} \mathrm{O} \\
\text { Wash }\end{array}$ & Waste & Product & $\begin{array}{c}\text { Total } \\
\text { Recovery }\end{array}$ \\
\hline${ }^{99} \mathrm{Mo}$ & $0.1 \%$ & $0.2 \%$ & $0.8 \%$ & $37.4 \%$ & $38.5 \%$ \\
\hline
\end{tabular}

Because of the low recovery of ${ }^{99}$ Mo product from the concentration column during the commissioning run, the elution volume and time were increased in the procedure for subsequent experiments. 


\subsubsection{Irradiation \#1 (10/01/19)}

During the processing after the first irradiation, the concentration column ran smoothly, with no issues observed. The increase of the elution volume and time led to ${ }^{99}$ Mo recovery greater than $100 \%$ from the concentration column. This result was also observed during the Phase I experiments and is attributed to an inaccurate reading from the balance determining the final weight of the feed solution delivered from the glovebox team. The balance was inaccurate for several reasons, including the following: the floor of the D-024 hot cell is not completely level, and the 3-L flask has multiple liquid transfer lines, gas collection lines, and $\mathrm{pH}$ probes connected to it. Owing to these connections, slight shifts of equipment or lines can impact the mass reading of the received solution. However, the concentration column still performed as expected and delivered a high recovery yield during this experiment.

Using gamma spectroscopy, we tracked the ${ }^{99}$ Mo product as well as fission-produced radionuclides through the process. These data showed that major fission radionuclides travel with the product through the concentration-column process. Ruthenium-103, ${ }^{131}$ I, and ${ }^{133}$ / had approximately $25 \%$ of their initial activity carried with the product, while ${ }^{95} \mathrm{Zr},{ }^{105} \mathrm{Rh}$ and ${ }^{127} \mathrm{Sb}$ all had values above $50 \%$. This indicates that there was retention of these radionuclides on the column. We believe that the remainder of the activity was carried through in the solution effluent, since it was not detected in any of the other samples. It was not possible to sample the effluent during this experiment to verify this conclusion.

The calculations of the relative distribution of isotopes in Tables 3.4.1.2.1 and 3.4.1.2.2 used the initial feed sample as the means to standardize the samples.

TABLE 3.4.1.2.1 Activities detected in the concentration column fractions, decay corrected to EOB during the first irradiation

\begin{tabular}{|c|c|c|c|c|c|}
\hline Radionuclide & $\begin{array}{c}\text { Feed, } \\
\mathrm{mCi} \\
(1 \mathrm{~s}, \%)\end{array}$ & $\begin{array}{c}\mathrm{HNO}_{3} \\
\text { Wash, } \\
\mathrm{mCi} \\
(1 \mathrm{~s}, \%) \\
\end{array}$ & $\begin{array}{c}\mathrm{H}_{2} \mathrm{O} \\
\text { Wash, } \\
\mathrm{mCi} \\
(1 \mathrm{~s}, \%) \\
\end{array}$ & $\begin{array}{c}\text { Waste, } \\
\mathrm{mCi} \\
(1 \mathrm{~s}, \%)\end{array}$ & $\begin{array}{c}{ }^{99} \mathrm{Mo} \\
\text { Product, } \\
\mathrm{mCi} \\
(1 \mathrm{~s}, \%) \\
\end{array}$ \\
\hline${ }^{95} \mathrm{Zr}$ & $\begin{array}{c}2.41 \mathrm{E}-01 \\
(20.1)\end{array}$ & $\begin{array}{c}1.76 \mathrm{E}-04 \\
(44.2)\end{array}$ & $\begin{array}{c}2.46 \mathrm{E}-04 \\
(54.9)\end{array}$ & $\begin{array}{c}2.35 \mathrm{E}-03 \\
(29.6)\end{array}$ & $\begin{array}{c}1.72 \mathrm{E}-01 \\
(24.1)\end{array}$ \\
\hline${ }^{99} \mathrm{Mo}$ & $\begin{array}{c}2.08 \mathrm{E}+03 \\
(2.2)\end{array}$ & $\begin{array}{c}6.16 \mathrm{E}-01 \\
(4.6)\end{array}$ & $\begin{array}{c}2.01 \mathrm{E}-02 \\
(71.5)\end{array}$ & $\begin{array}{c}1.04 \mathrm{E}+01 \\
(2.3)\end{array}$ & $\begin{array}{c}2.24 \mathrm{E}+03 \\
(2.1)\end{array}$ \\
\hline${ }^{103} \mathrm{Ru}$ & $\begin{array}{c}6.85 \mathrm{E}+00 \\
(2.8)\end{array}$ & $\begin{array}{c}\text { 7.54E-02 } \\
(2.1)\end{array}$ & $\begin{array}{c}2.58 \mathrm{E}-02 \\
(2.2)\end{array}$ & $\begin{array}{c}8.09 \mathrm{E}-02 \\
(2.4)\end{array}$ & $\begin{array}{c}1.71 \mathrm{E}+00 \\
(3.9)\end{array}$ \\
\hline${ }^{131} \mathbf{I}$ & $\begin{array}{c}1.02 \mathrm{E}+02 \\
(2.3)\end{array}$ & $\begin{array}{c}2.12 \mathrm{E}+00 \\
(1.8)\end{array}$ & $\begin{array}{c}1.24 \mathrm{E}+00 \\
(1.9)\end{array}$ & $\begin{array}{c}2.19 \mathrm{E}+00 \\
(1.9)\end{array}$ & $\begin{array}{c}2.82 \mathrm{E}+01 \\
(2.7)\end{array}$ \\
\hline${ }^{133} \mathrm{I}$ & $\begin{array}{c}2.40 \mathrm{E}+03 \\
(2.4)\end{array}$ & $\begin{array}{c}4.77 \mathrm{E}+01 \\
(1.9)\end{array}$ & $\begin{array}{c}2.82 \mathrm{E}+01 \\
(1.9)\end{array}$ & $\begin{array}{c}4.93 \mathrm{E}+01 \\
(1.9)\end{array}$ & $\begin{array}{c}6.21 \mathrm{E}+02 \\
(2.1)\end{array}$ \\
\hline${ }^{105} \mathrm{Rh}$ & $\begin{array}{c}2.18 \mathrm{E}+01 \\
(35.9)\end{array}$ & $\begin{array}{c}3.06 \mathrm{E}-01 \\
(15.4)\end{array}$ & $\begin{array}{c}1.53 \mathrm{E}-01 \\
(26.9)\end{array}$ & $\begin{array}{c}1.19 \mathrm{E}-01 \\
(40.7)\end{array}$ & $\begin{array}{c}1.19 \mathrm{E}+01 \\
(26.8)\end{array}$ \\
\hline${ }^{127} \mathrm{Sb}$ & $\begin{array}{c}1.41 \mathrm{E}+00 \\
(18.9)\end{array}$ & $\begin{array}{c}1.06 \mathrm{E}-03 \\
(54.9)\end{array}$ & $\begin{array}{c}8.54 \mathrm{E}-04 \\
(48.4)\end{array}$ & $\begin{array}{c}8.51 \mathrm{E}-02 \\
(4.0)\end{array}$ & $\begin{array}{c}2.21 \mathrm{E}+00 \\
(10.0)\end{array}$ \\
\hline
\end{tabular}


TABLE 3.4.1.2.2 Distribution of activity of various isotopes in the fractions collected from the concentration column during the first irradiation

\begin{tabular}{cccccc}
\multicolumn{7}{c}{ 10/01/19 Irradiation \#1 } & & & & \\
Radionuclide & $\begin{array}{c}\mathrm{HNO}_{3} \\
\text { Wash }\end{array}$ & $\begin{array}{c}\mathrm{H}_{2} \mathrm{O} \\
\text { Wash }\end{array}$ & Waste & $\begin{array}{c}{ }^{99} \mathrm{Mo} \\
\text { Product }\end{array}$ & $\begin{array}{c}\text { Total } \\
\text { Recovery }\end{array}$ \\
\hline & & & & & \\
${ }^{95} \mathrm{Zr}$ & $0.1 \%$ & $0.1 \%$ & $1.0 \%$ & $71.3 \%$ & $72.4 \%$ \\
${ }^{99} \mathrm{Mo}$ & $0.0 \%$ & $0.0 \%$ & $0.5 \%$ & $107.8 \%$ & $108.3 \%$ \\
${ }^{103} \mathrm{Ru}$ & $1.1 \%$ & $0.4 \%$ & $1.2 \%$ & $25.0 \%$ & $27.7 \%$ \\
${ }^{131} \mathrm{I}$ & $2.1 \%$ & $1.2 \%$ & $2.1 \%$ & $27.5 \%$ & $33.0 \%$ \\
${ }^{133} \mathrm{I}$ & $2.0 \%$ & $1.2 \%$ & $2.1 \%$ & $25.9 \%$ & $31.1 \%$ \\
${ }^{105} \mathrm{Rh}$ & $1.4 \%$ & $0.7 \%$ & $0.5 \%$ & $54.8 \%$ & $57.4 \%$ \\
${ }^{127} \mathrm{Sb}$ & $0.1 \%$ & $0.1 \%$ & $6.0 \%$ & $156.7 \%$ & $162.9 \%$ \\
\hline
\end{tabular}

\subsubsection{Irradiation \#2 (11/11/19)}

The processing after the second irradiation was performed, and no issues were observed during the concentration-column operation. While processing the solution, it was observed that the solution received from the primary recovery glovebox was cloudy. During Phase I experiments, a cloudy solution typically resulted in sub-optimal recovery from the concentration column. During acidification, the precipitate dissolved, but the concentration-column recovery of the ${ }^{99}$ Mo product suffered, with only $65 \%$ recovery. In an attempt to avoid poor recovery, the collection time for the product was increased to collect more volume during this step. Analysis of the collected fractions showed a lower recovery of the ${ }^{99}$ Mo product than in the previous experiment, and the iodine isotopes showed less retention on the column and a lower overall recovery as well. Conversely, nearly all ${ }^{95} \mathrm{Zr},{ }^{103} \mathrm{Ru},{ }^{105} \mathrm{Rh}$ and ${ }^{127} \mathrm{Sb}$ were recovered in the ${ }^{99} \mathrm{Mo}$ product during this experiment. Many of the percent recoveries were similar to the ${ }^{99} \mathrm{Mo}$ recovery, but when the recovery was above $100 \%$, we attribute this result to either an inaccurate weight of the initial feed solution or incomplete mixing of the sample.

Once the concentration column was loaded and eluted, dose rates were taken from the 5-L storage vessels underneath the D-024 hot cell. The shielding in place for this experiment was shown to be adequate, but additional shielding was needed before the full irradiation experiment. To achieve ALARA and to maintain a safe working environment, it was decided to install an effluent holding bottle inside the D-024 hot cell. This bottle was designed to hold the solution during its initial decay before being transferred underneath the hot cell for long-term storage. Installation of the effluent bottle inside the hot cell also allowed for sampling of the effluent solution during subsequent experiments. This sampling was made possible by use of a three-way valve that was connected to a line that went to the bottom of the holding bottle. An empty syringe was attached to the sampling line on the three-way valve and the valve was turned from receiving to sampling. The syringe was then filled and the sample solution was transferred to a scintillation vial. Tables 3.4.1.3.1 and 3.4.1.3.2 summarize the results for samples from the concentration-column process. 
TABLE 3.4.1.3.1 Activities detected in the concentration-column fractions, decay corrected to EOB during the second irradiation

\begin{tabular}{|c|c|c|c|c|c|}
\hline Radionuclide & $\begin{array}{c}\text { Feed, } \\
\mathrm{mCi} \\
(1 \mathrm{~s}, \%)\end{array}$ & $\begin{array}{c}\mathrm{HNO}_{3} \\
\text { Wash, } \\
\mathrm{mCi} \\
(1 \mathrm{~s}, \%) \\
\end{array}$ & $\begin{array}{c}\mathrm{H}_{2} \mathrm{O} \\
\text { Wash, } \\
\mathrm{mCi} \\
(1 \mathrm{~s}, \%) \\
\end{array}$ & $\begin{array}{c}\text { Waste, } \\
\mathrm{mCi} \\
(1 \mathrm{~s}, \%)\end{array}$ & $\begin{array}{c}{ }^{99} \mathrm{Mo} \\
\text { Product, } \\
\text { mCi } \\
(1 \mathrm{~s}, \%) \\
\end{array}$ \\
\hline${ }^{95} \mathrm{Zr}$ & $\begin{array}{c}2.79 \mathrm{E}+00 \\
(21.5)\end{array}$ & $2.48 \mathrm{E}-03$ & 7.18E-04 & $\begin{array}{c}7.86 \mathrm{E}-03 \\
(18.0)\end{array}$ & $\begin{array}{c}1.81 \mathrm{E}+00 \\
(6.1)\end{array}$ \\
\hline${ }^{99} \mathrm{Mo}$ & $\begin{array}{c}9.74 \mathrm{E}+03 \\
(2.1)\end{array}$ & $2.95 \mathrm{E}-01$ & $1.02 \mathrm{E}-01$ & $\begin{array}{c}4.90 \mathrm{E}+00 \\
(5.5)\end{array}$ & $\begin{array}{c}6.16 \mathrm{E}+03 \\
(2.2)\end{array}$ \\
\hline${ }^{103} \mathrm{Ru}$ & $\begin{array}{c}4.04 \mathrm{E}+00 \\
(18.7)\end{array}$ & $\begin{array}{c}1.80 \mathrm{E}-02 \\
(6.7)\end{array}$ & $\begin{array}{c}4.08 \mathrm{E}-03 \\
(8.8)\end{array}$ & $3.88 \mathrm{E}-03$ & $\begin{array}{c}1.66 \mathrm{E}+00 \\
(6.5)\end{array}$ \\
\hline${ }^{131} \mathrm{I}$ & $\begin{array}{c}7.17 \mathrm{E}+02 \\
(3.0)\end{array}$ & $\begin{array}{c}2.70 \mathrm{E}+00 \\
(2.6)\end{array}$ & $\begin{array}{c}1.62 \mathrm{E}+00 \\
(2.2)\end{array}$ & $\begin{array}{c}5.11 \mathrm{E}+00 \\
(2.4)\end{array}$ & $\begin{array}{c}1.59 \mathrm{E}+01 \\
(12.2)\end{array}$ \\
\hline${ }^{133} \mathrm{I}$ & $\begin{array}{c}1.39 \mathrm{E}+04 \\
(2.1)\end{array}$ & $\begin{array}{c}6.67 \mathrm{E}+01 \\
(2.1)\end{array}$ & $\begin{array}{c}4.20 \mathrm{E}+01 \\
(1.9)\end{array}$ & $\begin{array}{c}1.24 \mathrm{E}+02 \\
(2.1)\end{array}$ & $\begin{array}{c}4.85 \mathrm{E}+02 \\
\quad(11.1)\end{array}$ \\
\hline${ }^{105} \mathrm{Rh}$ & $8.97 \mathrm{E}+01$ & $3.88 \mathrm{E}-01$ & $\begin{array}{c}2.62 \mathrm{E}-01 \\
(25.3)\end{array}$ & $6.24 \mathrm{E}-01$ & $2.88 \mathrm{E}+01$ \\
\hline${ }^{127} \mathrm{Sb}$ & $\begin{array}{c}6.45 \mathrm{E}+00 \\
(21.7)\end{array}$ & $1.03 \mathrm{E}-02$ & $3.23 \mathrm{E}-03$ & 5.73E-02 & $\begin{array}{c}7.83 \mathrm{E}+00 \\
(9.6)\end{array}$ \\
\hline
\end{tabular}

TABLE 3.4.1.3.2 Distribution of activity of various isotopes in the fractions collected from the concentration column during the second irradiation

\begin{tabular}{cccccc}
\multicolumn{1}{c}{ 11/11/19 Irradiation \#2 } & & & & \\
Radionuclide & $\begin{array}{c}\mathrm{HNO}_{3} \\
\text { Wash }\end{array}$ & $\begin{array}{c}\mathrm{H}_{2} \mathrm{O} \\
\text { Wash }\end{array}$ & Waste & $\begin{array}{c}{ }^{99} \mathrm{Mo} \\
\text { Product }\end{array}$ & $\begin{array}{c}\text { Total } \\
\text { Recovery }\end{array}$ \\
\hline & & & & & \\
${ }^{95} \mathrm{Zr}$ & $0.1 \%$ & $0.0 \%$ & $0.3 \%$ & $65.1 \%$ & $65.5 \%$ \\
${ }^{99} \mathrm{Mo}$ & $0.0 \%$ & $0.0 \%$ & $0.1 \%$ & $63.2 \%$ & $63.3 \%$ \\
${ }^{103} \mathrm{Ru}$ & $0.4 \%$ & $0.1 \%$ & $0.1 \%$ & $41.2 \%$ & $41.8 \%$ \\
${ }^{131} \mathrm{I}$ & $0.4 \%$ & $0.2 \%$ & $0.7 \%$ & $2.2 \%$ & $3.5 \%$ \\
${ }^{133} \mathrm{I}$ & $0.5 \%$ & $0.3 \%$ & $0.9 \%$ & $3.5 \%$ & $5.2 \%$ \\
${ }^{105} \mathrm{Rh}$ & $0.4 \%$ & $0.3 \%$ & $0.7 \%$ & $32.1 \%$ & $33.5 \%$ \\
${ }^{127} \mathrm{Sb}$ & $0.2 \%$ & $0.1 \%$ & $0.9 \%$ & $121.5 \%$ & $122.6 \%$ \\
\hline
\end{tabular}




\subsubsection{Irradiation \#3 (03/02/20)}

The third irradiation did not have any chemical processing associated with it, so there are no additional concentration column data from that experiment.

\subsubsection{Irradiation \#4 (08/30/20)}

The fourth irradiation performed was planned to be a complete run with full chemical processing. The steps leading up to the concentration column operated as expected, but during the water wash of the concentration column, a leak was detected, coming from behind the valve board. The experiment was paused to determine the cause of the leak. The source of the leak was not visible, since it was located behind the board. Also, an elevated dose rate was measured on the silver zeolite filter that was connected to the hot-cell exhaust. To avoid further contaminating the inside of the hot cell and increasing the size of the spill, the fourth experiment was stopped. For this reason, samples for gamma spectroscopy are incomplete in Table 3.4.1.5.1.

TABLE 3.4.1.5.1 Activities detected in the concentration column fractions, decay corrected to EOB during the fourth irradiation

\begin{tabular}{|c|c|c|c|c|c|c|}
\hline Radionuclide & $\begin{array}{c}\text { Feed, } \\
\mathrm{mCi} \\
(1 \mathrm{~s}, \%) \\
\end{array}$ & $\begin{array}{c}\text { Eluent, } \\
\mathrm{mCi} \\
(1 \mathrm{~s}, \%) \\
\end{array}$ & $\begin{array}{c}\mathrm{HNO}_{3} \\
\text { Wash, } \\
\mathrm{mCi} \\
(1 \mathrm{~s}, \%) \\
\end{array}$ & $\begin{array}{c}\mathrm{H}_{2} \mathrm{O} \\
\text { Wash, } \\
\mathrm{mCi} \\
(1 \mathrm{~s}, \%) \\
\end{array}$ & $\begin{array}{c}\text { Waste, } \\
\text { mCi } \\
(1 \mathrm{~s}, \%) \\
\end{array}$ & $\begin{array}{c}{ }^{99} \mathrm{Mo} \\
\text { Product, } \\
\text { mCi } \\
(1 \mathrm{~s}, \%) \\
\end{array}$ \\
\hline${ }^{95} \mathrm{Zr}$ & $\begin{array}{c}1.06 \mathrm{E}+00 \\
(13.0)\end{array}$ & $3.40 \mathrm{E}-03$ & $2.76 \mathrm{E}-04$ & & & \\
\hline${ }^{99} \mathrm{Mo}$ & $\begin{array}{c}4.06 \mathrm{E}+03 \\
(0.7)\end{array}$ & $2.94 \mathrm{E}+02$ & $4.51 \mathrm{E}+00$ & & & \\
\hline${ }^{103} \mathrm{Ru}$ & $\begin{array}{c}6.62 \mathrm{E}+01 \\
(0.2)\end{array}$ & $\begin{array}{c}4.72 \mathrm{E}+01 \\
(0.1)\end{array}$ & $1.17 \mathrm{E}+00$ & & & \\
\hline${ }^{131} \mathrm{I}$ & $\begin{array}{c}4.43 \mathrm{E}+02 \\
(3.7)\end{array}$ & $\begin{array}{c}\text { 7.03E+01 } \\
(0.3)\end{array}$ & $5.59 \mathrm{E}+00$ & & & \\
\hline $\begin{array}{c}{ }^{133} \mathrm{I} \\
{ }^{105} \mathrm{Rh}\end{array}$ & & & & & & \\
\hline${ }^{127} \mathrm{Sb}$ & $2.93 \mathrm{E}+01$ & $1.15 \mathrm{E}+00$ & $9.08 \mathrm{E}-02$ & & & \\
\hline
\end{tabular}


A modified procedure for eluting the concentration column and transferring the solution to the 5-L holding vessels below the hot cell was prepared and presented to a safety review committee. The procedure was approved and followed. The concentration column was eluted and all lines were rinsed into the effluent holding container inside the hot cell. The resulting solution was then transferred to the 5-L holding containers below the D-024 hot cell. These efforts sufficiently reduced the radiation field inside the hot cell for a manned entry to begin troubleshooting and repairs. The inside of the D-024 hot cell was also decontaminated by manipulators with paper towels and water. Smears were taken and counted after the cleaning process until the contamination levels inside the hot cell were low enough for entry.

During the hot-cell troubleshooting and repairs, the water line connected to a multi-way valve was found to be damaged. The line that leaked had developed a spiral crack as a result of embrittlement from a combination of time and the heat and radioactivity applied to it since its installation years earlier. As a corrective action, all lines inside the hot cell were inspected and replaced. A provision was also put into the procedure for replacement of all lines in the system at least every two years, to avoid similar complications in the future.

The gamma results from the acid wash were typical and displayed minimal amounts of activity. It was exciting to finally have results from an effluent sample. The results showed some breakthrough of the ${ }^{99} \mathrm{Mo}$ at $7.2 \%$, as well as low levels of ${ }^{95} \mathrm{Zr}$ and ${ }^{127} \mathrm{Sb}$. The ${ }^{131} \mathrm{I}$ in the sample accounted for $16 \%$ of the ${ }^{131}$ I activity when compared to the feed sample. This finding helped confirm our theory that the iodine isotopes are present through the entire processing. The ${ }^{103} \mathrm{Ru}$ had just over $70 \%$ of its activity accounted for in this sample, which means that the majority of this radionuclide is not retained on a titania column and passes with the effluent. The abbreviated results from this experiment can be found in Table 3.4.1.5.2. It should be noted that because of the contamination inside the hot cell and the commensurate corrective actions, these samples were not retrieved and counted in a timely manner, so much of the data was lost to decay during this period.

TABLE 3.4.1.5.2 Distribution of activity of various isotopes in the fractions collected from the concentration column during the fourth irradiation

\begin{tabular}{cccc}
$8 / 30 / 20$ Irradiation \#4 & & \\
\hline & & & \\
Radionuclide & Eluent & $\begin{array}{c}\mathrm{HNO}_{3} \\
\text { Wash }\end{array}$ & $\begin{array}{c}\text { Total } \\
\text { Recovery }\end{array}$ \\
\hline & & & \\
${ }^{95} \mathrm{Zr}$ & $0.3 \%$ & $0.0 \%$ & $0.3 \%$ \\
${ }^{99} \mathrm{Mo}$ & $7.2 \%$ & $0.1 \%$ & $7.3 \%$ \\
${ }^{103} \mathrm{Ru}$ & $71.3 \%$ & $1.8 \%$ & $73.0 \%$ \\
${ }^{131} \mathrm{I}$ & $15.9 \%$ & $1.3 \%$ & $17.1 \%$ \\
${ }^{127} \mathrm{Sb}$ & $3.9 \%$ & $0.3 \%$ & $4.2 \%$ \\
\hline
\end{tabular}




\subsubsection{Irradiation \#5 (01/18/21)}

The final experiment was performed, and the concentration-column operations ran smoothly. No issues were observed during the experiment. Gamma spectroscopy data showed that approximately $9 \mathrm{Ci}$ of ${ }^{99} \mathrm{Mo}$ were received from the primary recovery glovebox in the 3-L flask. From the $9 \mathrm{Ci}$, only about $2 \mathrm{Ci}$ of ${ }^{99} \mathrm{Mo}$ was recovered, which was less than $25 \%$ of the product. The samples from the rinse solutions showed little activity and the effluent sample only contained $5 \%$ of the total product. It is worth noting that nearly all the ${ }^{103} \mathrm{Ru},{ }^{131} \mathrm{I}$, and ${ }^{105} \mathrm{Rh}$ were observed to be in the eluent sample. The percentages of these radionuclides recovered from the product sample also match closely with what was observed during Irradiation \#1. The ${ }^{95} \mathrm{Zr},{ }^{105} \mathrm{Rh}$ and ${ }^{127} \mathrm{Sb}$ all had lower levels of activity detected in the product sample, and while nearly all the ${ }^{105} \mathrm{Rh}$ was accounted for with the eluent sample, the ${ }^{95} \mathrm{Zr}$ and ${ }^{127} \mathrm{Sb}$ had poor overall recoveries, with $44 \%$ and $15 \%$, respectively. Tables 3.4.1.6.1 and 3.4.1.6.2 also show an extremely low recovery of ${ }^{133}$. The likely reason was overlapping gamma lines, which resulted in inaccurate results for this radionuclide; it should be assumed that the ${ }^{133}$ I behaves the same as the ${ }^{131} \mathrm{I}$.

TABLE 3.4.1.6.1 Activities detected in the concentration column fractions, decay corrected to EOB during the fifth irradiation

\begin{tabular}{|c|c|c|c|c|c|}
\hline Radionuclide & $\begin{array}{c}\text { Feed, } \\
\mathrm{mCi} \\
(1 \mathrm{~s}, \%)\end{array}$ & $\begin{array}{c}\text { Eluent, } \\
\mathrm{mCi} \\
(1 \mathrm{~s}, \%)\end{array}$ & $\begin{array}{c}\mathrm{HNO}_{3} \\
\text { Wash, } \\
\mathrm{mCi} \\
(1 \mathrm{~s}, \%) \\
\end{array}$ & $\begin{array}{c}\mathrm{H}_{2} \mathrm{O} \\
\text { Wash, } \\
\mathrm{mCi} \\
(1 \mathrm{~s}, \%) \\
\end{array}$ & $\begin{array}{c}{ }^{99} \mathrm{Mo} \\
\text { Product, } \\
\text { mCi } \\
(1 \mathrm{~s}, \%)\end{array}$ \\
\hline${ }^{95} \mathrm{Zr}$ & $\begin{array}{c}5.00 \mathrm{E}-01 \\
(5.1)\end{array}$ & $\begin{array}{c}1.31 \mathrm{E}-01 \\
(3.4)\end{array}$ & $9.56 \mathrm{E}-04$ & $3.14 \mathrm{E}-04$ & $\begin{array}{c}8.57 \mathrm{E}-02 \\
(16.2)\end{array}$ \\
\hline${ }^{99} \mathrm{Mo}$ & $\begin{array}{c}8.92 \mathrm{E}+03 \\
(2.8)\end{array}$ & $\begin{array}{c}4.80 \mathrm{E}+02 \\
(3.0)\end{array}$ & $\begin{array}{c}4.43 \mathrm{E}-01 \\
(18.2)\end{array}$ & 9.83E-01 & $\begin{array}{c}2.05 E+03 \\
(2.7)\end{array}$ \\
\hline${ }^{103} \mathrm{Ru}$ & $\begin{array}{c}6.43 \mathrm{E}+01 \\
(2.8)\end{array}$ & $\begin{array}{c}6.26 \mathrm{E}+01 \\
(2.1)\end{array}$ & $\begin{array}{c}7.88 \mathrm{E}-01 \\
(2.2)\end{array}$ & $\begin{array}{c}5.94 \mathrm{E}-01 \\
(2.2)\end{array}$ & $\begin{array}{c}1.64 \mathrm{E}+01 \\
(2.1)\end{array}$ \\
\hline${ }^{131} \mathrm{I}$ & $\begin{array}{c}2.49 \mathrm{E}+02 \\
(7.5)\end{array}$ & $\begin{array}{c}3.15 \mathrm{E}+02 \\
(1.9)\end{array}$ & $\begin{array}{c}2.83 \mathrm{E}+00 \\
(2.2)\end{array}$ & $\begin{array}{c}4.34 \mathrm{E}+00 \\
(2.1)\end{array}$ & $\begin{array}{c}5.60 \mathrm{E}+01 \\
(2.3)\end{array}$ \\
\hline${ }^{133} I$ & $\begin{array}{c}9.32 \mathrm{E}+03 \\
(1.9)\end{array}$ & & $\begin{array}{c}5.08 \mathrm{E}+01 \\
(2.6)\end{array}$ & & $\begin{array}{c}5.36 \mathrm{E}+02 \\
(2.5)\end{array}$ \\
\hline${ }^{105} \mathrm{Rh}$ & $\begin{array}{c}1.54 \mathrm{E}+02 \\
(10.2)\end{array}$ & $2.33 \mathrm{E}+02$ & $\begin{array}{c}1.20 \mathrm{E}+00 \\
(15.3)\end{array}$ & $1.01 \mathrm{E}+01$ & \\
\hline${ }^{127} \mathrm{Sb}$ & $1.26 \mathrm{E}+00$ & $1.76 \mathrm{E}-01$ & $4.58 \mathrm{E}-03$ & $7.35 \mathrm{E}-03$ & \\
\hline
\end{tabular}


TABLE 3.4.1.6.2 Distribution of activity of various isotopes in the fractions collected from the concentration column during the fifth irradiation

\begin{tabular}{cccccc}
\multicolumn{1}{l}{$1 / 18 / 21$ Irradiation \#5 } & & & & & \\
Radionuclide & Eluent & $\begin{array}{c}\mathrm{HNO}_{3} \\
\text { Wash }\end{array}$ & $\begin{array}{c}\mathrm{H}_{2} \mathrm{O} \\
\text { Wash }\end{array}$ & $\begin{array}{c}{ }^{99} \mathrm{Mo} \\
\text { Product }\end{array}$ & $\begin{array}{c}\text { Total } \\
\text { Recovery }\end{array}$ \\
\hline & & & & & \\
${ }^{95} \mathrm{Zr}$ & $26.3 \%$ & $0.2 \%$ & $0.1 \%$ & $17.1 \%$ & $43.7 \%$ \\
${ }^{99} \mathrm{Mo}$ & $5.4 \%$ & $0.0 \%$ & $0.0 \%$ & $23.0 \%$ & $28.4 \%$ \\
${ }^{103} \mathrm{Ru}$ & $97.4 \%$ & $1.2 \%$ & $0.9 \%$ & $25.5 \%$ & $125.0 \%$ \\
${ }^{131} \mathrm{I}$ & $126.5 \%$ & $1.1 \%$ & $1.7 \%$ & $22.5 \%$ & $151.8 \%$ \\
${ }^{133} \mathrm{I}$ & $0.0 \%$ & $0.5 \%$ & $0.0 \%$ & $5.7 \%$ & $6.3 \%$ \\
${ }^{105} \mathrm{Rh}$ & $150.7 \%$ & $0.8 \%$ & $6.5 \%$ & $0.0 \%$ & $158.0 \%$ \\
${ }^{127} \mathrm{Sb}$ & $14.0 \%$ & $0.4 \%$ & $0.6 \%$ & $0.0 \%$ & $14.9 \%$ \\
\hline
\end{tabular}

A second elution was performed on the concentration column with $50 \mathrm{~mL}$ of $1 \mathrm{M} \mathrm{NaOH}$, but no additional ${ }^{99}$ Mo product was removed during the second elution. While we cannot definitively say what the problem was with the concentration column, we suggest one of the following possibilities: First, the column could have formed channels between the times of packing and the concentration column operations, which would lead to inefficient elution and loss of product. There could also have been a sampling error during the process, where either a more concentrated sample was taken from the initial feed solution or a more dilute sample was taken from the ${ }^{99}$ Mo product sample. Incomplete mixing of the sampled solutions would cause this problem, and inhomogeneity of the solutions would greatly skew the results. There is also the possibility that the ${ }^{99} \mathrm{Mo}$ was fixed to the concentration column and unable to be eluted.

Each of the concentration-column operations during the irradiations was unique and presented its own challenges. A leak led to incomplete data during the fourth experiment. The eluent solution was unable to be sampled and analyzed until the fourth and fifth irradiation, so there were only two data points. Because of the wide spread of data and incomplete data points, it was difficult to find any overall trends from these experiments. For the first few experiments, we can speculate that the remainder of the activity was present in the eluent solution, but we do not have verified results and we have completed only one full experiment with the eluent sample present; therefore, it is not possible to draw any meaningful conclusions from these data. We are confident that the column washes do not remove significant amounts of activity. The product sample typically had at least a quarter of the other major radionuclides eluted with it, which illustrates retention of other nuclides on the titania column and underlines the fact that the modified Cintichem process is essential to purifying the ${ }^{99}$ Mo product. 


\subsubsection{LEU Modified Cintichem Process}

\subsubsection{Commissioning Run 08/04/19}

The LMC process was performed by following LEAF-PROC-011 (Appendix 27). However, the ruthenium and rhodium carriers were not used during this operation because they were not present in the ${ }^{99} \mathrm{Mo}$ spike solution. At the beginning of LMC operations, it was determined that the vacuum pump could not pull the Mo solution through a $0.3-\mu \mathrm{m}$ filter during the iodine precipitation step. This issue was corrected by pre-wetting the filter inside the hot cell and reinstalling the filter into the filtration assembly. Gamma-counting results for LMC processing are shown in Table 3.4.2.1.1. The Mo-containing feed to the LMC process is designated RF-1; RF-2 is the solution after iodine precipitation; RFW is the filtrate from the $\mathrm{ABO}$ precipitation step; and 1-B is the Mo product following purification. During the filtration of Mo-ABO precipitate, delayed formation of precipitate was observed in the RFW bottle. This delay is likely due to the limited solubility of excess $\mathrm{ABO}$ under acidic conditions, which causes precipitation of ABO. On the basis of the results shown in Table 3.4.2.1.1, Mo losses in the RFW bottle represent only $0.12 \%$ of the total ${ }^{99} \mathrm{Mo}$ activity, confirming the fact that precipitate formed in the RFW bottle did not contain a significant amount of Mo. Recovery of ${ }^{99}$ Mo from the LMC process was $93.6 \%$, which is very good.

TABLE 3.4.2.1.1 Activities detected in LMC fractions

\begin{tabular}{lccc}
\hline \multicolumn{1}{c}{ Sample } & Average, $\mathrm{mCi}$ & $1 \sigma, \mathrm{mCi}$ & $1 \sigma, \%$ \\
\hline & & & \\
RF-1 & 16.2 & 0.737 & $2.0 \%$ \\
RFW & 0.0201 & 0.00079 & $2.0 \%$ \\
1-B & 15.1 & 0.808 & $2.0 \%$ \\
\hline
\end{tabular}

\subsubsection{Irradiation \#1 (10/01/19)}

The LMC operations after this short irradiation went as planned, without any issues. Photographs in Figure 3.4.2.2.1 show the major separation steps: a) initial iodine precipitation; b) precipitation and filtration of Mo-ABO precipitate; c) dissolution of Mo-ABO precipitate with heating using a heat gun; and d) final purification using iodine precipitation and the combination column (Ag/C, HZO, AC).

Major contaminants found entering the LMC process from the concentration column were ${ }^{103} \mathrm{Ru},{ }^{125} \mathrm{Sn},{ }^{127} \mathrm{Sb}$, and iodine isotopes. The presence of ${ }^{133} \mathrm{Xe}$ (the daughter of ${ }^{133} \mathrm{I}$ ) was also detected (Table 3.4.2.2.1). 


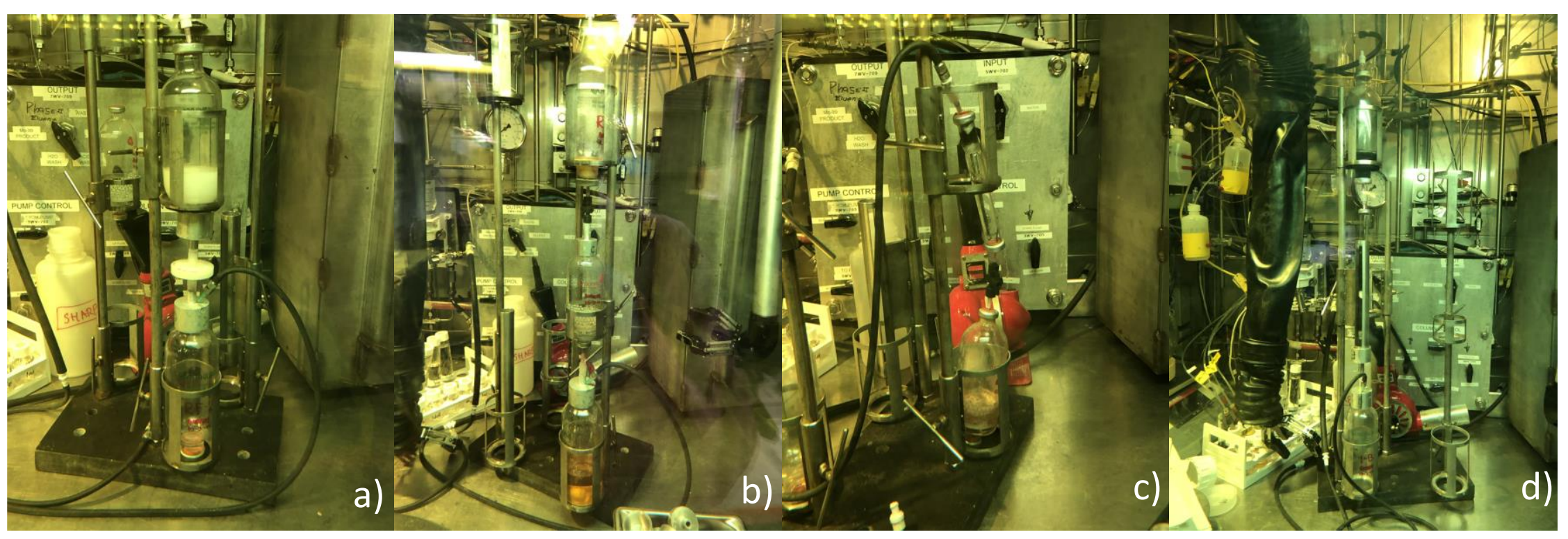

FIGURE 3.4.2.2.1 Photographs of major separation steps of LMC process: a) initial iodine precipitation; b) precipitation and filtration of Mo-ABO precipitate; c) dissolution of Mo-ABO precipitate with heating using heat gun; d) final purification using iodine precipitation and the combination column $(\mathrm{Ag} / \mathrm{C}, \mathrm{HZO}, \mathrm{CC})$ 
TABLE 3.4.2.2.1 Activities detected in LMC fractions calculated at EOB

\begin{tabular}{ccccccc}
\hline & \multicolumn{7}{c}{$\mathrm{A}, \mathrm{mCi}$} \\
& \multicolumn{7}{c}{$1 \sigma$} & $\mathrm{RFW}$ & $1 \sigma \mathrm{a}$ & 1 -B product & $1 \sigma$ \\
\hline & $\mathrm{RF}-1$ & & & & \\
& & & & & \\
${ }^{95} \mathrm{Zr}$ & $1.72 \mathrm{E}-01$ & $24.1 \%$ & $1.22 \mathrm{E}-01$ & $6.5 \%$ & & \\
${ }^{99} \mathrm{Mo}$ & $2.24 \mathrm{E}+03$ & $2.10 \%$ & $5.02 \mathrm{E}+00$ & $5.50 \%$ & $2.12 \mathrm{E}+03$ & $2.10 \%$ \\
${ }^{103} \mathrm{Ru}$ & $1.71 \mathrm{E}+00$ & $3.90 \%$ & $1.37 \mathrm{E}+00$ & $1.90 \%$ & & \\
${ }^{133} \mathrm{Xe}$ & $2.17 \mathrm{E}+01$ & $5.00 \%$ & $8.26 \mathrm{E}+00$ & $4.20 \%$ & & \\
${ }^{131} \mathrm{I}$ & $2.82 \mathrm{E}+01$ & $2.70 \%$ & $1.22 \mathrm{E}+01$ & $2.10 \%$ & \\
${ }^{133} \mathrm{I}$ & $6.21 \mathrm{E}+02$ & $2.10 \%$ & $2.76 \mathrm{E}+02$ & $1.90 \%$ & \\
${ }^{105} \mathrm{Rh}$ & $1.19 \mathrm{E}+01$ & $26.8 \%$ & $1.28 \mathrm{E}+00$ & $13.6 \%$ & \\
${ }^{125} \mathrm{Sn}$ & $1.59 \mathrm{E}+00$ & $30.7 \%$ & $8.29 \mathrm{E}-01$ & $4.50 \%$ & \\
${ }^{127} \mathrm{Sb}$ & $2.21 \mathrm{E}+00$ & $10.0 \%$ & $2.15 \mathrm{E}+00$ & $3.10 \%$ & & \\
\hline
\end{tabular}

Major radionuclides detected in the RFW fraction were ${ }^{95} \mathrm{Zr},{ }^{103} \mathrm{Ru},{ }^{125} \mathrm{Sn},{ }^{127} \mathrm{Sb}$, iodine isotopes, and ${ }^{133} \mathrm{Xe}$ (Table 3.4.2.2.2). Results suggest that $\sim 44 \%$ of iodine was still present after initial precipitation, which could indicate the presence of iodate, which is more difficult to remove by precipitation because of the relatively high solubility of $\mathrm{AgIO}_{3}$ and the slow isotopic exchange between iodide and iodate. The majority of $\mathrm{Ru}, \mathrm{Zr}$, and $\mathrm{Sb}$ was found in the RFW fraction. Approximately $0.2 \%$ of the ${ }^{99}$ Mo was found in the RFW, indicating that Mo is effectively removed by precipitation with $\mathrm{ABO}$. No other gamma-emitting radionuclides were detected in the ${ }^{99}$ Mo product. Recovery of ${ }^{99}$ Mo in the LMC process was $\sim 95 \%$, which is very good. The total recovery of ${ }^{99}$ Mo after all purification steps was $\sim 88 \%$.

TABLE 3.4.2.2.2 Distribution of various fission products and Mo in the RFW and ${ }^{99}$ Mo product as part of the LMC process

\begin{tabular}{ccc}
\hline & \multicolumn{2}{c}{$\begin{array}{c}\text { Fraction of Radioisotope } \\
\text { Activity in the LMC Feed }\end{array}$} \\
\cline { 2 - 3 } & & \\
& RFW & 1-B product \\
\hline & & \\
${ }^{95} \mathrm{Zr}$ & $70.76 \%$ & \\
${ }^{99} \mathrm{Mo}$ & $0.22 \%$ & $94.7 \%$ \\
${ }^{103} \mathrm{Ru}$ & $79.92 \%$ & \\
${ }^{133} \mathrm{Xe}$ & $38.12 \%$ & \\
${ }^{131} \mathrm{I}$ & $43.23 \%$ & \\
${ }^{133} \mathrm{I}$ & $44.38 \%$ & \\
${ }^{105} \mathrm{Rh}$ & $10.71 \%$ & \\
${ }^{125} \mathrm{Sn}$ & $52.27 \%$ \\
${ }^{127} \mathrm{Sb}$ & $97.21 \%$ \\
\hline
\end{tabular}




\subsubsection{Irradiation \#2 (11/11/19)}

The LMC process followed the concentration column without any issues or spills. However, it was observed that the radiation dose rate on the silver-zeolite filter that is connected to the exhaust from the processing hot cell continuously increased during the LMC process. This increase is most likely due to the presence of radioiodine in the hot-cell atmosphere, which could be released during needle removal after solution transfers between septa bottles. No dose was detected past the silver-zeolite filter, indicating capture of iodine on the filter. Recovery yield for ${ }^{99}$ Mo from the LMC process was $78.3 \%$, which is slightly below an average of $\sim 80 \%$ usually achieved in the LMC process. No ${ }^{99} \mathrm{Mo}$ was detected in the RFW bottle. Results can be seen in Table 3.4.2.3.1.

\section{TABLE 3.4.2.3.1 Activities detected in LMC fractions calculated at EOB}

\begin{tabular}{ccccccc}
\hline & \multicolumn{7}{c}{$\mathrm{A}, \mathrm{mCi}$} \\
\cline { 2 - 7 } & $\mathrm{RF}-1$ & $1 \sigma$ & $\mathrm{RFW}$ & $1 \sigma$ & 1 -B Product & $1 \sigma$ \\
\hline & & & & & \\
${ }^{95} \mathrm{Zr}$ & $1.81 \mathrm{E}+00$ & $6.1 \%$ & $7.38 \mathrm{E}-02$ & $2.7 \%$ & & \\
${ }^{95} \mathrm{Nb}$ & $2.35 \mathrm{E}+00$ & $4.4 \%$ & $4.66 \mathrm{E}-02$ & $2.7 \%$ & & \\
${ }^{137} \mathrm{Cs}$ & $2.59 \mathrm{E}+00$ & $4.4 \%$ & $2.07 \mathrm{E}-02$ & $4.5 \%$ & & \\
${ }^{99} \mathrm{Mo}$ & $6.34 \mathrm{E}+03$ & $2.2 \%$ & & & & \\
${ }^{103} \mathrm{Ru}$ & $1.66 \mathrm{E}+00$ & $6.5 \%$ & $8.51 \mathrm{E}-02$ & $2.5 \%$ & & \\
${ }^{131} \mathrm{I}$ & $1.59 \mathrm{E}+01$ & $12.2 \%$ & $5.56 \mathrm{E}+01$ & $2.1 \%$ & & \\
${ }^{133} \mathrm{I}$ & $4.85 \mathrm{E}+02$ & $11.1 \%$ & & & & \\
${ }^{125} \mathrm{Sn}$ & & & $3.52 \mathrm{E}+00$ & $4.3 \%$ & & \\
${ }^{127} \mathrm{Sb}$ & $7.83 \mathrm{E}+00$ & $9.60 \%$ & $6.02 \mathrm{E}+00$ & $3.9 \%$ & & \\
\hline
\end{tabular}

Radionuclides present in the RFW fraction were dominated by the presence of ${ }^{131} \mathrm{I}$. As in previous irradiations, $\mathrm{Zr}, \mathrm{Nb}, \mathrm{Ru}$ and $\mathrm{Sb}$ were present in the RFW fraction; however, their relative fractions compared to starting activities in the RF-1 bottle indicate that the solution in the RFW bottle might not have been mixed properly before an aliquot was taken for gamma counting (Table 3.4.2.3.2). 
TABLE 3.4.2.3.2 Distribution of various

fission products and Mo in the RFW and

${ }^{99}$ Mo product as part of the LMC process

\begin{tabular}{ccc}
\hline & \multicolumn{2}{c}{$\begin{array}{c}\text { Fraction of Radioisotope } \\
\text { Activity in the LMC Feed }\end{array}$} \\
\cline { 2 - 2 } & RFW & 1-B product \\
\hline & & \\
${ }^{95} \mathrm{Zr}$ & $4.08 \%$ & \\
${ }^{95} \mathrm{Nb}$ & $1.98 \%$ & \\
${ }^{137} \mathrm{Cs}$ & $0.80 \%$ & \\
${ }^{99} \mathrm{Mo}$ & $0.00 \%$ & $76.0 \%$ \\
${ }^{103} \mathrm{Ru}$ & $5.13 \%$ & \\
${ }^{131} \mathrm{I}$ & $350 \%$ & \\
${ }^{127} \mathrm{Sb}$ & $76.9 \%$ & \\
\hline
\end{tabular}

No ${ }^{99}$ Mo was detected in the RFW bottle after the ABO precipitation step; however, a small amount of residual Mo-ABO precipitate was visible in the RF-2 bottle. As shown previously [1], some excess of $\mathrm{KMnO}_{4}$ added before precipitation of Mo with $\mathrm{ABO}$ might affect the recovery yield of Mo in the precipitation step. This effect is likely due to some oxidation of $\mathrm{ABO}$ by permanganate, which leads to formation of hard-to-work-with precipitate. The recovery of Mo in the LMC process was $\sim 76.0 \%$, and the total recovery of ${ }^{99} \mathrm{Mo}$ after all purification steps was $\sim 49 \%$. Although the recovery from the LMC process was slightly below the expected recovery $(\sim 80 \%)$, the low overall recovery yield of ${ }^{99}$ Mo could be attributed mainly to the presence of cloudiness in the solution received from the primary recovery column, which negatively affected recovery yield on the concentration column.

\subsubsection{Irradiation \#3 (3/02/20)}

No chemical processing was performed after the third irradiation.

\subsubsection{Irradiation \#4 (8/30/20)}

A leak occurred in the multiway valve during the concentration-column processing inside the hot cell, and the experiment was paused to determine the reason for the leak. Therefore, the LMC process was not performed.

\subsubsection{Irradiation \#5 (1/18/21)}

Table 3.4.2.6.1 shows the distribution of activities in the LMC fractions. About 23-26\% of the iodine was found in the RFW fraction, again suggesting the presence of iodate. The composition of other fission products present in the RFW is similar to that observed in previous 
irradiations. Data indicate that the majority of ${ }^{103} \mathrm{Ru},{ }^{137} \mathrm{Cs}$ and ${ }^{125} \mathrm{Sn}$ partitioned into the RFW fraction, as expected; $93.9 \%$ of the ${ }^{99}$ Mo entering the LMC process was recovered. Details are presented in Tables 3.4.2.6.1 and 3.4.2.6.2.

TABLE 3.4.2.6.1 Activities detected in LMC fractions calculated at EOB

\begin{tabular}{ccccccc}
\hline & \multicolumn{6}{c}{$\mathrm{A}, \mathrm{mCi}$} \\
\cline { 2 - 7 } & $\mathrm{RF}-1$ & $1 \sigma$ & $\mathrm{RFW}$ & $1 \sigma$ & $1-\mathrm{B}$ Product & $1 \sigma$ \\
\hline & & & $1.82 \mathrm{E}-01$ & $2.70 \%$ & & \\
${ }^{95} \mathrm{Zr}$ & & & $2.71 \mathrm{E}-02$ & $6.80 \%$ & & \\
${ }^{95} \mathrm{Nb}$ & & & & \\
${ }^{137} \mathrm{Cs}$ & $1.07 \mathrm{E}-01$ & $4.80 \%$ & $7.47 \mathrm{E}-02$ & $5.30 \%$ & & \\
${ }^{99} \mathrm{Mo}$ & $2.05 \mathrm{E}+03$ & $2.70 \%$ & $4.04 \mathrm{E}+00$ & $5.40 \%$ & $1.92 \mathrm{E}+03$ & $2.76 \%$ \\
${ }^{103} \mathrm{Ru}$ & $1.64 \mathrm{E}+01$ & $2.10 \%$ & $2.47 \mathrm{E}+01$ & $2.10 \%$ & & \\
${ }^{131} \mathrm{I}$ & $5.60 \mathrm{E}+01$ & $2.30 \%$ & $1.48 \mathrm{E}+01$ & $2.20 \%$ & & \\
${ }^{133} \mathrm{I}$ & $1.07 \mathrm{E}+03$ & $2.50 \%$ & $2.48 \mathrm{E}+02$ & $3.30 \%$ & & \\
${ }^{105} \mathrm{Rh}$ & & & $2.09 \mathrm{E}+01$ & $4.00 \%$ & & \\
${ }^{125} \mathrm{Sn}$ & $1.46 \mathrm{E}+00$ & $15.90 \%$ & $1.22 \mathrm{E}+00$ & $7.40 \%$ & & \\
\hline
\end{tabular}

TABLE 3.4.2.6.2 Distribution of various fission products and Mo in the RFW and ${ }^{99}$ Mo product as part of the LMC process

\begin{tabular}{ccc}
\hline & \multicolumn{2}{c}{$\begin{array}{c}\text { Fraction of Radioisotope } \\
\text { Activity in the LMC Feed }\end{array}$} \\
\cline { 2 - 3 } & RFW & 1-B Product \\
\hline & & \\
${ }^{137} \mathrm{Cs}$ & $69.8 \%$ & \\
${ }^{99} \mathrm{Mo}$ & $0.20 \%$ & $93.9 \%$ \\
${ }^{103} \mathrm{Ru}$ & $151 \%$ & \\
${ }^{131} \mathrm{I}$ & $26.5 \%$ & \\
${ }^{133} \mathrm{I}$ & $23.1 \%$ & \\
${ }^{125} \mathrm{Sn}$ & $83.6 \%$ & \\
\hline
\end{tabular}

Aliquots of ${ }^{99}$ Mo product from the LMC process were then used to detect quantities of minor fission products present in the product, using thiocyanate extraction (Figure 3.4.2.6.1) to selectively remove ${ }^{99} \mathrm{Mo}$, and chloroform extraction to selectively separate iodine.

Radionuclides identified in the ${ }^{99}$ Mo product after thiocyanate extraction and gamma counting for 57,000 s and a dead time of $0.43 \%$ are listed in Table 3.4.2.6.3, together with the activity of ${ }^{131}$ I determined after selective extraction by chloroform. The iodine sample was counted for $78,000 \mathrm{~s}$ with a dead time of $0.79 \%$. From the gamma analysis, it was determined that the ${ }^{99}$ Mo product met radionuclide purity specifications. 


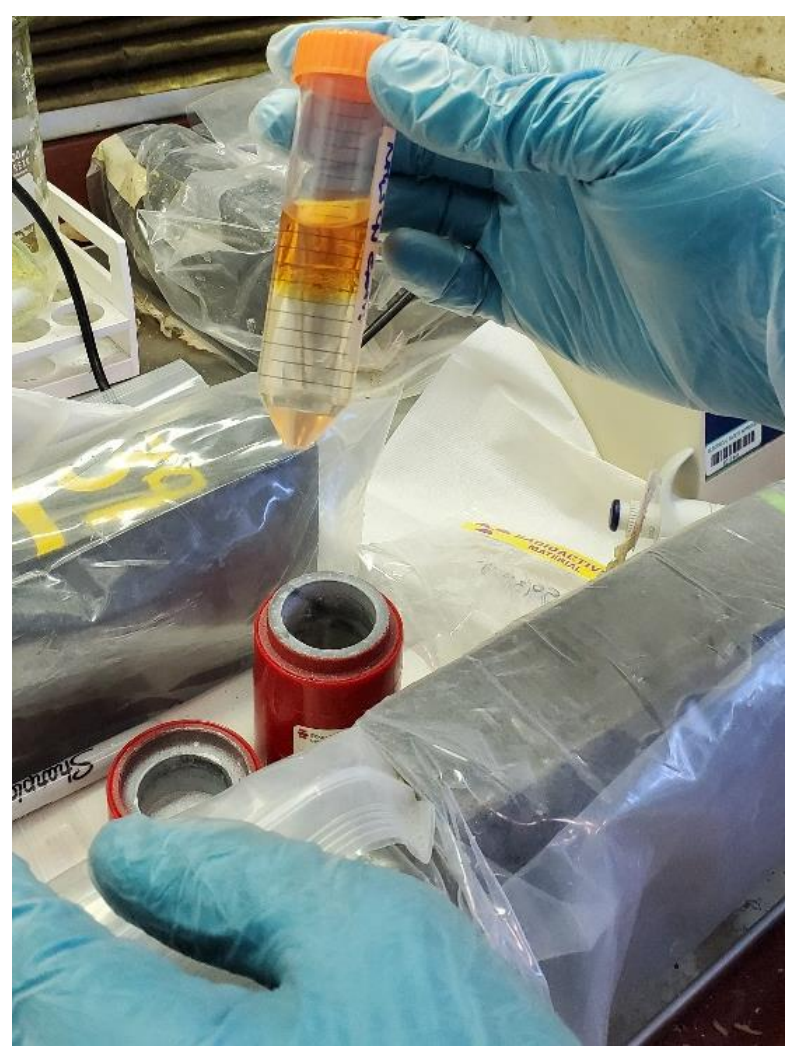

FIGURE 3.4.2.6.1 Thiocyanate extraction

TABLE 3.4.2.6.3 Radionuclidic purity in ${ }^{99} \mathrm{Mo}$ product solution from the LMC process calculated at 36 hours after EOB

\begin{tabular}{|c|c|c|c|c|}
\hline \multirow[b]{2}{*}{$\begin{array}{l}\text { Radionuclides } \\
\text { in 1B Product }\end{array}$} & \multicolumn{2}{|c|}{$\begin{array}{c}\text { Fraction of Radioisotope } \\
\text { Activity in the LMC } \\
\text { Feed, mCi }\end{array}$} & \multicolumn{2}{|c|}{$\begin{array}{c}\text { Ratio of Isotope Activity to } \\
{ }^{99} \text { Mo Activity }\end{array}$} \\
\hline & $\begin{array}{c}36 \mathrm{~h} \text { after } \\
\text { EOB }\end{array}$ & $1 \sigma$ & $\mathrm{X} /{ }^{99} \mathrm{Mo}$ & $\begin{array}{l}\text { Specs } \\
\mathrm{X} /{ }^{99} \mathrm{Mo}\end{array}$ \\
\hline${ }^{99} \mathrm{Mo}$ & $1.34 \mathrm{E}+03$ & $2.00 \%$ & & \\
\hline${ }^{137} \mathrm{Cs}$ & $1.42 \mathrm{E}-03$ & $4.40 \%$ & $1.06 \mathrm{E}-06$ & \\
\hline${ }^{103} \mathrm{Ru}$ & $4.65 \mathrm{E}-02$ & $2.07 \%$ & $3.47 \mathrm{E}-05$ & $5.00 \mathrm{E}-05$ \\
\hline${ }^{131} \mathrm{I}$ & $6.09 \mathrm{E}-02$ & $2.72 \%$ & $4.54 \mathrm{E}-05$ & $5.00 \mathrm{E}-05$ \\
\hline
\end{tabular}

\subsubsection{References}

[1] Bettinardi, D.J., and Tkac, P., Recovery of Mo in LEU-Modified Cintichem Process at Elevated Mo Concentrations, ANL/CFCT-19/15, Argonne National Laboratory, September 2019. 


\subsection{MONTE CARLO CALCULATIONS}

\subsubsection{Scope of the Work}

This work was done to support the AMORE experiment at Argonne National Laboratory and aimed to estimate isotope accumulation in uranyl sulfate solution using Monte Carlo codes. Calculated data were compared with experimental results from the 10/1/19 irradiation to verify the model.

\subsubsection{Simulation Procedure and Experimental Assembly}

The experimental assembly consisted of a water-cooled target converter made of DU, a vessel with uranyl sulfate solution, and a water reflector (see the present work, Section 2, for details). MCNP and FLUKA Monte Carlo transport codes [1,2] were used for isotope yields and radiation energy deposition calculations. As a source of primary particles, a 40-MeV Gaussian electron beam with a 16-mm FWHM was used. The beam axis coincided with the converter's axis.

MCNP [1] was used to predict isotope burnup in regions of the AMORE setup containing fissionable material. The simulations involved the use of the criticality subroutine KCODE and the burnup subroutine CINDER 90. The KCODE subroutine performs iterative calculations to calculate the effective neutron multiplication factor and $\mathrm{K}_{\text {eff. }}$ The burnup subroutine CINDER 90 was used to simulate the production of fission-product isotopes and actinides in the solution. This subroutine tracks up to 3400 isotopes. The final data on isotope composition were calculated by considering isotope decay, burnup, and production rates in a neutron field. A 63-energy-group approximation was used in CINDER by default.

To the author's knowledge, there is no verified Monte Carlo code for burnup studies of subcritical systems driven by an external source. MCNP cannot be used directly for burnup studies of subcritical systems. But it is possible to bypass this limitation by splitting the study into two steps (Figure 3.5.2.1). First, the neutron spectrum and total number of fissions in the fissionable material are calculated. Second, the calculated neutron spectrum is used as an external source for burnup studies, and results are normalized on total number of fissions. 
Stage 1: Input:

(FLUKA) 1. Primary particles: Electron beam

Output:

1. Radiation energy deposition.

2. Neutron energy spectrum generated by the irradiated target.

3. Fission events collection: number of fissions; fission fragments' $A / Z$ range; fission energy.

Stage 2: Input:

(MCNP) 1. Primary particles: Neutron energy spectrum (from stage 1).

2. Fission power (for normalization).

3. Irradiation beam power profile (used in BURN MCNP card).

Output:

Isotope composition in uranyl sulfate solution

\section{FIGURE 3.5.2.1 Simulation procedure}

The energy deposition profile in the target region and a 3D map of deposited energy are shown in Figure 3.5.2.2. The converter absorbs almost all electromagnetic energy; only 3.0\% of energy escapes the system. 

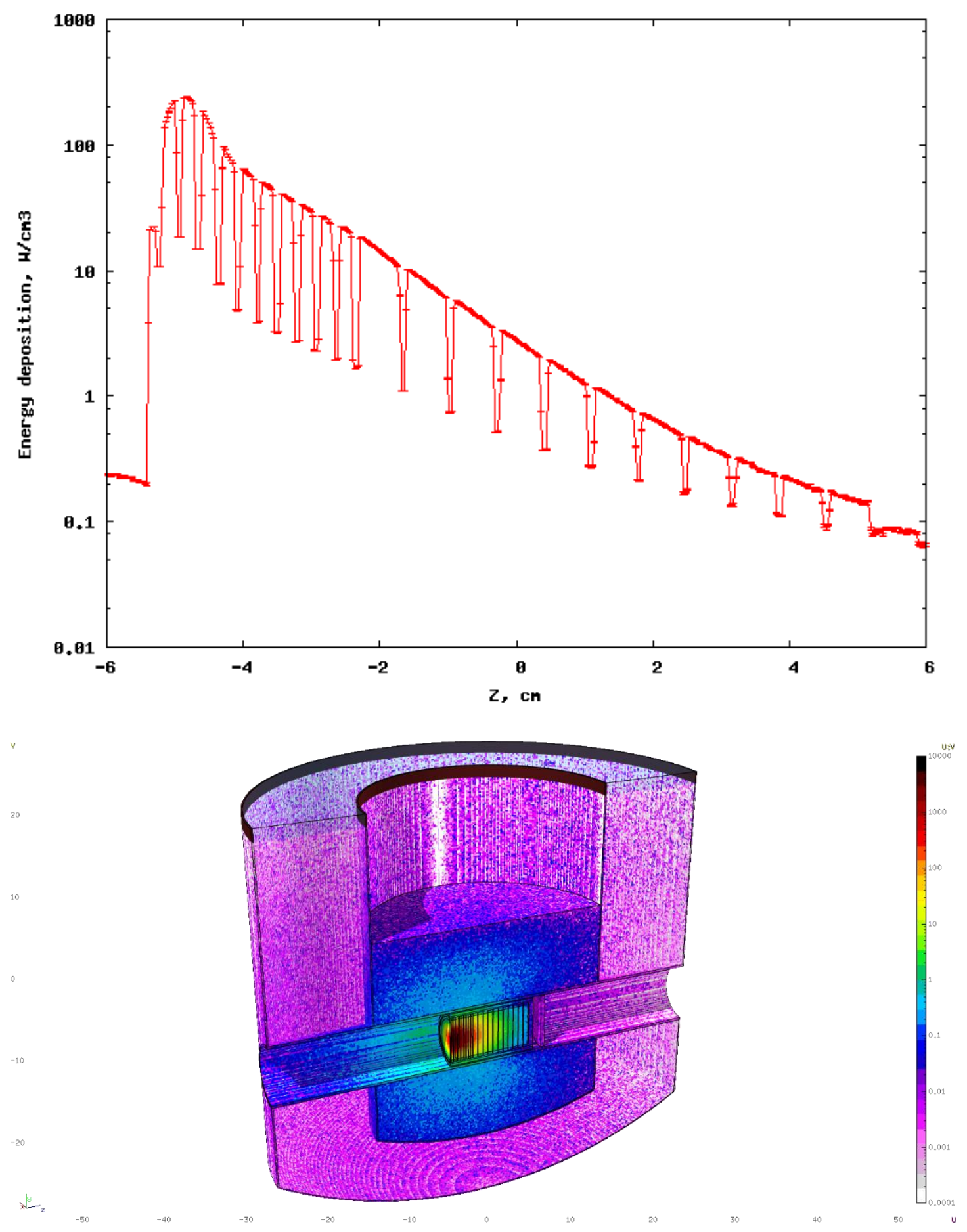

FIGURE 3.5.2.2 Radiation energy deposition: (top) energy deposition profile along the beam axis (averaged over the X,Y directions) in the target; (bottom) 3D map of energy deposition 


\subsubsection{Isotope Accumulation and Burnup Studies}

The solution contains $135 \mathrm{~g}$ of uranium per liter. Uranium enrichment is $20 \%$. The total fission rate in uranyl sulfate solution is $\sim 1.99 \times 10^{-2}$ fission/primary electron. The yield of ${ }^{99} \mathrm{Mo}$ was calculated as a sum of fission yields of isotopic chains, as shown in Figure 3.5.3.1. Decay mode and ${ }^{99}$ Mo yield were taken from Reference [3] and are shown on the diagram. The percentage of ${ }^{99} \mathrm{Mo}$ fission was calculated as $6.14 \%$, very close to the table value for the thermal neutron spectrum [4].

To verify the Monte Carlo model, we considered irradiation with the maximal amount of experimental data on fission fragments and fission-power gamma measurements.

The irradiation was completed on 10/1/19. The irradiation beam power profile (Figure 3.5.3.2) was provided by the linac operators and used for fission-power profile parameters in the BURN card of MCNP input. Total irradiation time was about 5.7 hours, with average power on the target $10.8 \mathrm{~kW}$ (14.4 max.). Total energy delivered to the target was $62 \mathrm{kWh}$. Average fission power calculated in the solution was $0.89 \mathrm{~kW}$ and total fission energy was $5.07 \mathrm{kWh}$. The total fission energy calculated experimentally from fission-fragment activities was $4.34 \mathrm{kWh}$.

Activities of fission fragments calculated at EOB are given in Table 3.5.3.1. The systematic difference between simulated and experimental values is in the range of 10-15\% for most isotopes from MCNP Tier 3 and can be explained by a systematic error in the fission-power normalization factor and uncertainties of the simulation method.

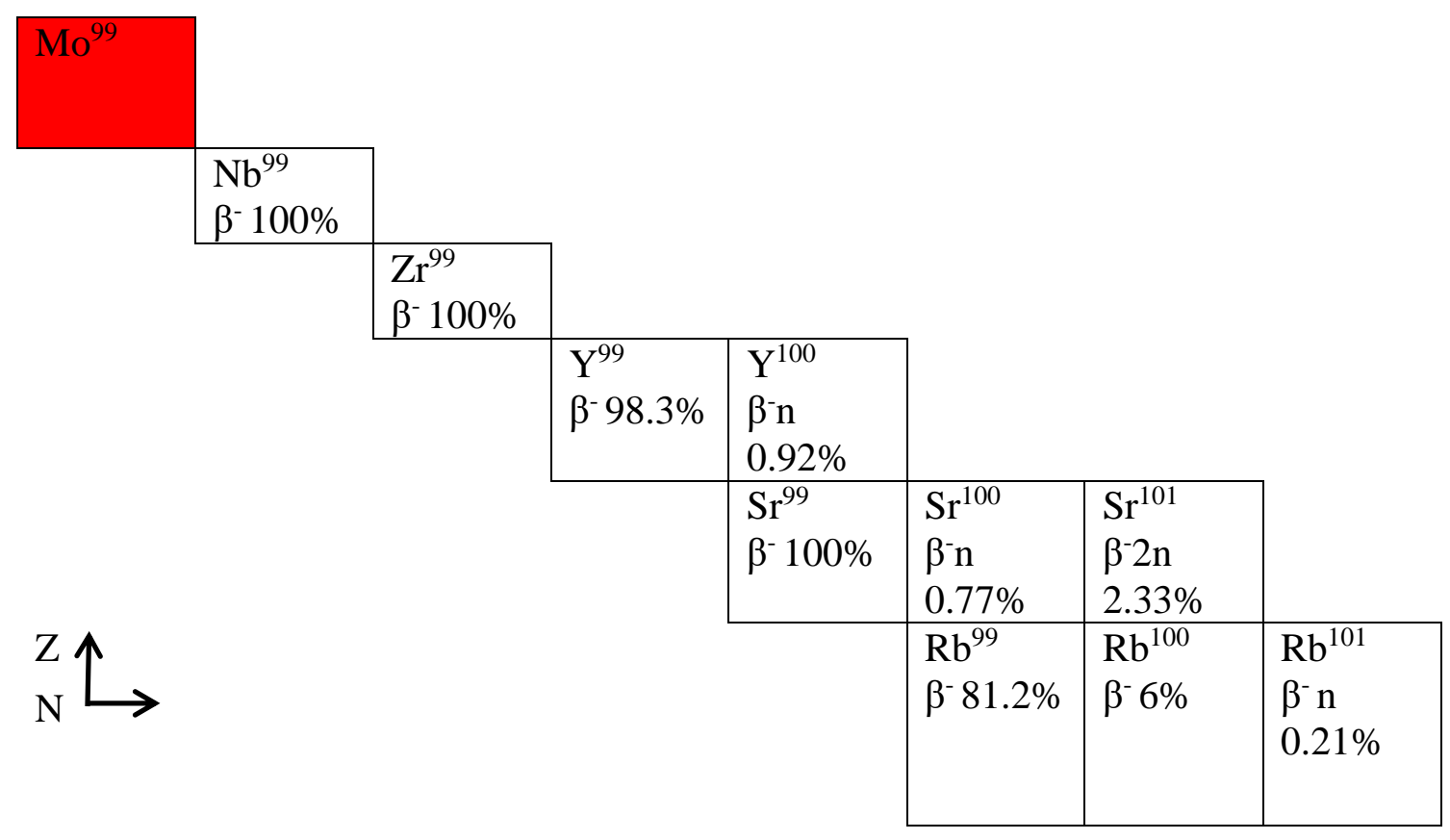

FIGURE 3.5.3.1 ${ }^{99}$ Mo parent nuclides 
The systematic error includes the following factors, all of which can potentially be reduced to zero:

1. Beam power measurement errors on the target caused by linac measurementsystem calibrations;

2. Underestimation of neutron leaks from the system due to Monte Carlo geometry simplifications; and

3. Simplification of neutron-source parameters: energy-spectrum and spatialdistribution averaging.

Uncertainties in fission-energy determination in the solution are caused by the errors of fission-product yield cross-sections (CINDER 90) and by the calculation approach when only neutron interactions are considered. Although the simulation method we used does not take interactions with photons (photo-fissions and burnup in photo-induced reactions) into account, it gives values close to realistic ones, since most of the interactions in fissionable materials are induced by neutrons.

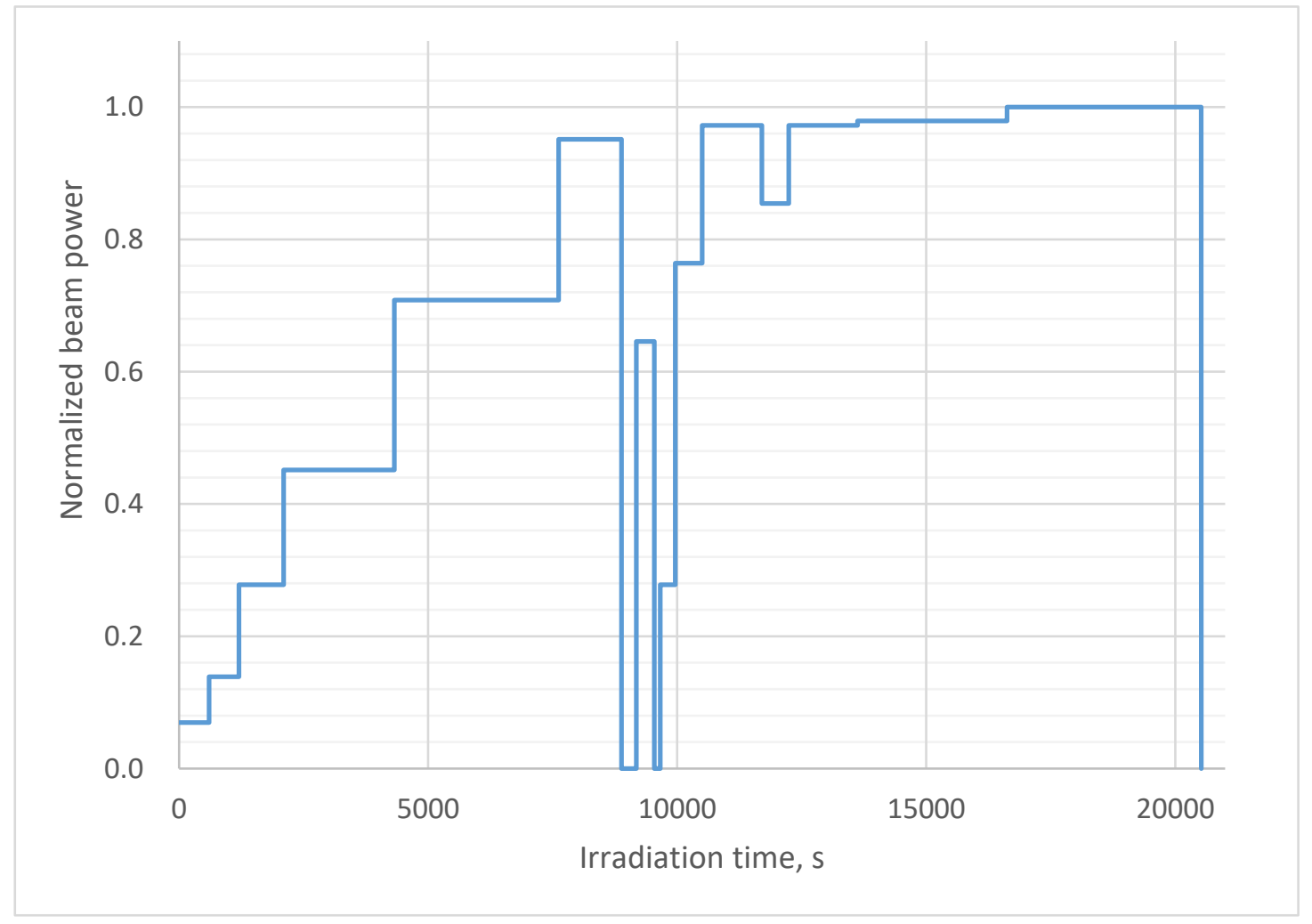

FIGURE 3.5.3.2 Irradiation beam-power profile normalized on maximum value 


\section{TABLE 3.5.3.1 Activities at EOB}

\begin{tabular}{cccccc}
\hline & \multicolumn{2}{c}{ Calculation } & & \multicolumn{2}{c}{ Experiment } \\
\cline { 2 - 3 } \cline { 5 - 6 } Radionuclide & Activity, Ci & ${ }^{99}$ Mo Ratio & & Activity, Ci & ${ }^{99}$ Mo Ratio \\
\hline${ }^{95} \mathrm{Zr}$ & 0.116 & 0.0453 & & 0.104 & 0.044 \\
${ }^{99} \mathrm{Mo}$ & 2.561 & 1.0000 & & 2.39 & 1 \\
${ }^{103} \mathrm{Ru}$ & 0.091 & 0.0357 & & 0.00789 & 0.003 \\
${ }^{131} \mathrm{I}$ & 0.296 & 0.1157 & & 0.276 & 0.115 \\
${ }^{135} \mathrm{Xe}$ & 4.793 & 1.8711 & & - & - \\
${ }^{137} \mathrm{Cs}$ & $8.06 \mathrm{E}-4$ & 0.0003 & & 0.00314 & 0.0013 \\
${ }^{140} \mathrm{Ba}$ & 0.577 & 0.2252 & & 0.493 & 0.206 \\
\hline
\end{tabular}

\subsubsection{References}

[1] Los Alamos National Laboratory, A General Monte Carlo N-Particle (MCNP) Transport Code, https://mcnp.lanl.gov/.

[2] Böhlen, T.T., et al., The FLUKA Code: Developments and Challenges for High Energy and Medical Applications, Nuclear Data Sheets 120, 211-214 (2014).

[3] Live Chart of Nuclides, https://www-nds.iaea.org/relnsd/vcharthtml/VChartHTML.html.

[4] IAEA Physics and Nuclear Data Sections, WIMS Library Update Project, https://wwwnds.iaea.org/wimsd/fpyield.htm. 


\section{SUMMARY}

After the first Phase II irradiation of the uranyl sulfate target solution (March 2018), an elevated background reading occurred at the linac facility during concentration-column processing inside the hot cell. A small amount of fission gases from the hot-cell facility's stack was recirculated into the building, causing elevated readings on the radiation detectors within the linac facility. The experiment was put into safe conditions, and no further processing or sample collection was performed. A new process was developed for screening the experimental work against the linac facility safety bases; a configuration management program was developed; the facility exhaust stack was extended to prevent the possibility of recirculation; and the SAD and ASE were modified to include additional descriptions of the experiment and additional credited controls. In accordance with the corrective actions identified, several modifications were implemented, and modified operational procedures were developed. Phase II experiments were then restarted by performing a commissioning run using ${ }^{99} \mathrm{Mo}$ tracer without irradiation of the target solution. During preparations for the commissioning run, it was discovered that the TSV contained a precipitate that was later identified by X-ray diffraction to be uranyl peroxide. The precipitate was due to formation of uranyl peroxide during or after the first Phase II irradiation in March 2018. An attempt was made to remove the precipitate from the system, but it was not completely successful.

Gas analysis data from the first irradiation after restart (October 2019) indicated that some formation of the uranyl peroxide precipitate continued despite addition of a Fe $e^{2+} / \mathrm{Fe}^{3+}$ catalyst prior to the irradiation. This formation was demonstrated by a high consumption of oxygen during the irradiation, which led to the necessity of actively adding oxygen into the system to ensure recombination of radiation-produced hydrogen gas. A similar trend was also observed during the second irradiation (November 2019), with a steady decrease in oxygen concentration during the irradiation. However, it appeared as if uranyl peroxide precipitate formation was slower compared to the previous irradiation, and no additional oxygen was added into the system. The third irradiation (March 2020) still showed some indication of uranyl peroxide formation, but at a much slower rate. No indication of uranyl peroxide formation was observed during the $4^{\text {th }}$ and $5^{\text {th }}$ irradiations.

From the irradiation perspective, on some occasions, irradiations were interrupted because of loss of the vacuum in the beamline caused by beam displacement. Because of the chromatic nature of the beamline bend (beams with different energies will emerge after the bend at different points and traveling in different directions), the beam position inside the beamline is very sensitive to beam energy and stability of accelerator parameters. An achromatic transport line (a line where beams will emerge with the same position and direction after the bend regardless of beam energy) would improve the reliability of the irradiations. All irradiations were limited in maximum beam power delivered to the target because of radiolytic hydrogen production. If the $\mathrm{H}_{2}$ concentration reached $1 \%$, beam power had to be decreased by $50 \%$, and once the $\mathrm{H}_{2}$ concentration reached $2 \%$, the beam would turn off. For this reason, the beam power was kept as high as possible without letting the $\mathrm{H}_{2}$ concentration reach $1 \%$. This was the only limitation since the target design allowed placement of the full beam power $(20 \mathrm{~kW}$ at $40 \mathrm{MeV})$ on the target. 
Recovery-column data indicate that $\mathrm{Np}, \mathrm{Sr}$, and Ce were not retained by the column at all and followed $\mathrm{U}$ through the column during loading. Several elements, including $\mathrm{Ba}, \mathrm{La}, \mathrm{Sb}, \mathrm{Rh}$, and $\mathrm{Ru}$, were only slightly retained by the column, evidenced by their presence at lower concentrations in samples taken during column loading. The ${ }^{99}$ Mo product sent to the hot cell contained large quantities of I isotopes and their Xe daughter products, and low levels of $\mathrm{Ru}, \mathrm{Zr}$, and $\mathrm{Sb}$. The contents of effluent bottles associated with the various column loading and stripping steps generally supported the trends identified by the in-line sample loops. Over the course of the 6 irradiations, there were several equipment malfunctions, the most impactful of which involved the sample retrieval solenoid valves, the column and liquid line heaters, and the pressure sensors. It is likely that the retrieval solenoid valves became clogged with precipitate over time because of their angled orientation. This idea is supported by the fact that none of the valves in the processing lines malfunctioned over the course of the experimental effort. The heaters and pressure sensors malfunctioned because of accumulated radiation damage to their control relays and the sensors themselves, respectively, even though they were shielded. These pieces will have to be replaced periodically when used in the commercial production system.

Major radionuclides identified in the feed to the concentration column besides ${ }^{99}$ Mo were ${ }^{95} \mathrm{Zr},{ }^{103} \mathrm{Ru},{ }^{105} \mathrm{Rh},{ }^{127} \mathrm{Sb}$ and I isotopes. All these radionuclides were also found in the ${ }^{99}$ Mo strip from the concentration column. Typically, more than $70 \%$ of iodine activity was removed during the concentration-column processing. The ${ }^{99}$ Mo recovery yields from the concentration column were significantly lower than expected in four of the five runs. It appears that the main reason for incomplete ${ }^{99}$ Mo recovery was an insufficient elution volume to effectively strip ${ }^{99}$ Mo from the column. The content of the effluent seems to be dominated by the presence of ${ }^{103} \mathrm{Ru},{ }^{105} \mathrm{Rh}$ and iodine isotopes. Small quantities of ${ }^{95} \mathrm{Zr}$ and ${ }^{127} \mathrm{Sb}$ were also found in concentration-column effluent.

Results from the LMC process after an initial iodide precipitation step showed the presence of iodine isotopes in the bottle containing RFW (consisting of filtrate waste solution from the primary molybdenum purification step), indicating the presence of iodate species, which are more difficult to remove by precipitation as AgI because of the relatively high solubility of $\mathrm{AgIO}_{3}$ and the slow isotopic exchange between iodide and iodate. It was further found that the majority of ${ }^{103} \mathrm{Ru},{ }^{95} \mathrm{Zr}$ and ${ }^{127} \mathrm{Sb}$ partitioned into the RFW. Typical ${ }^{99}$ Mo recovery obtained in the LMC was $\sim 94 \%$, although in one instance yield was found to be $76 \%$. Lower than expected ${ }^{99} \mathrm{Mo}$ yield could be due to a slight over-titration with $\mathrm{KMnO}_{4}$, which is known to negatively impact Mo yield during Mo precipitation with ABO. Radionuclidic purity of the ${ }^{99} \mathrm{Mo}$ product was checked after the final irradiation, and only small amounts of ${ }^{137} \mathrm{Cs},{ }^{103} \mathrm{Ru},{ }^{125} \mathrm{Sn}$ and ${ }^{131}$ I were detected. Activities of these radionuclides were low, and the ${ }^{99}$ Mo product met radionuclide purity specifications. 


\section{APPENDIX 1}

Calculation Note NE-EO-2015-05: "Thermal/Hydraulic Analysis of DU Target for miniSHINE/MIPS" 
Calculation No.: NE-EO-2015-05

Rev. 1

Page

1

of

14

CALCULATION COVER SHEET

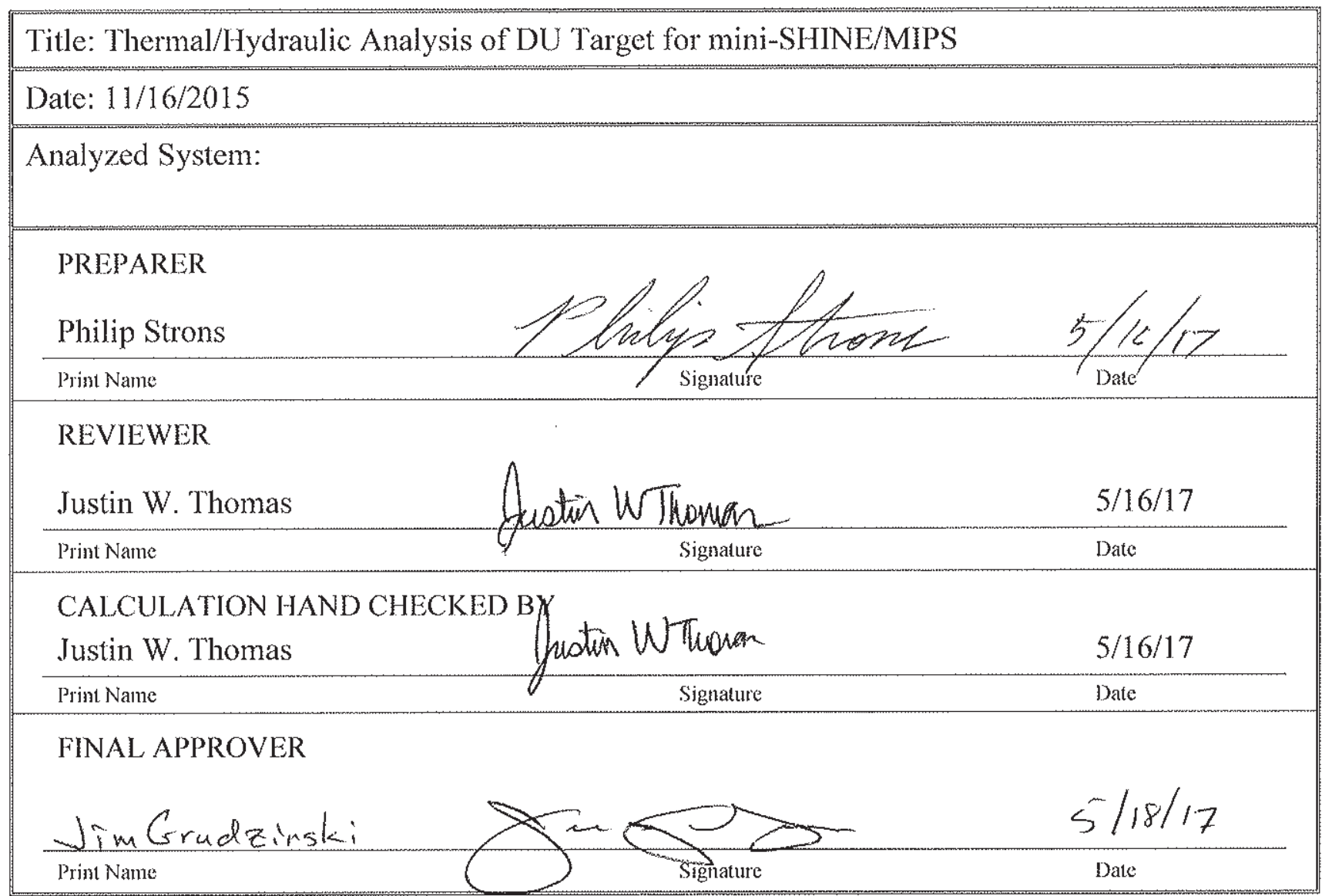

134 


\begin{tabular}{|lllllll|}
\hline \hline Calculation No.: & NE-EO-2015-05 & Rev. 1 & Page & 2 & of & 14 \\
\hline
\end{tabular}

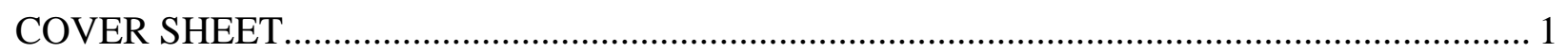

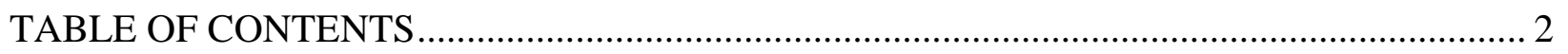

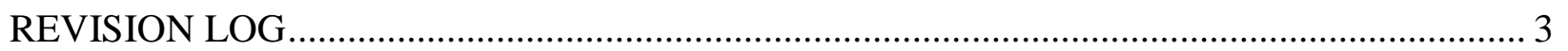

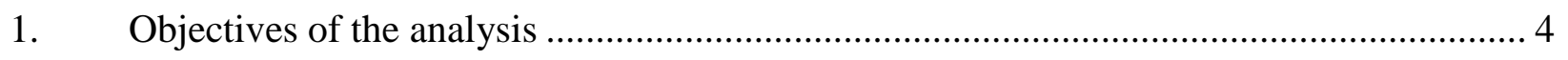

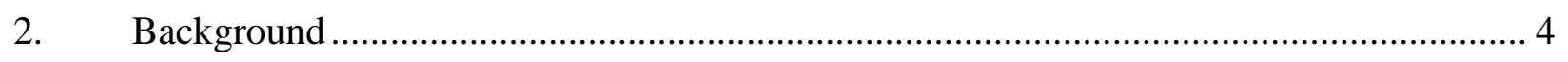

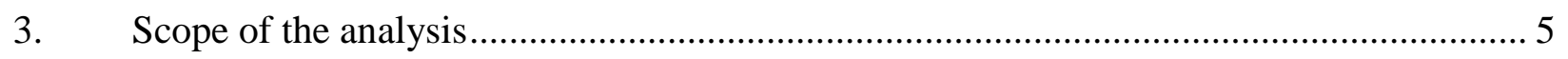

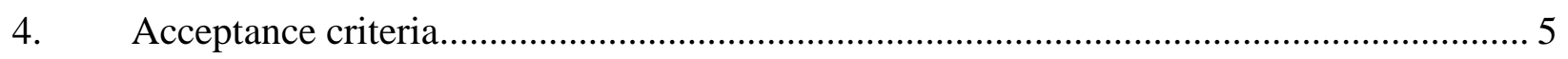

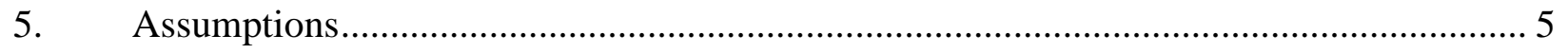

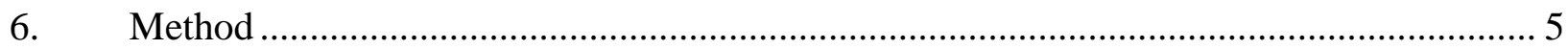

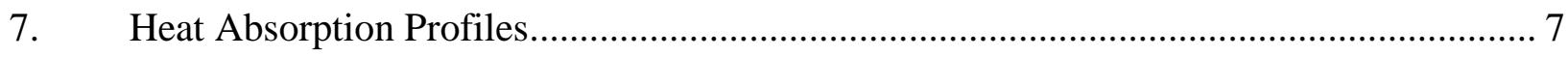

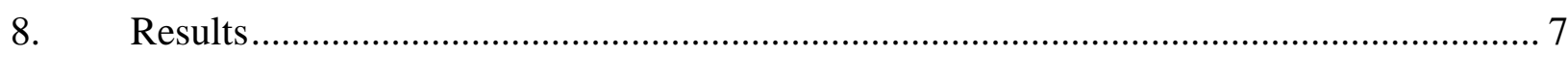

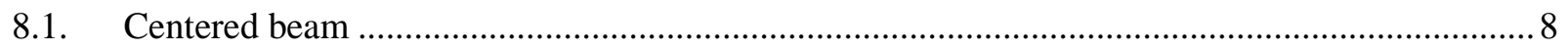

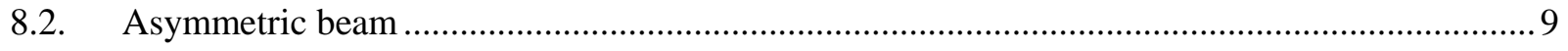

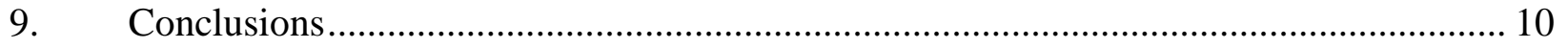

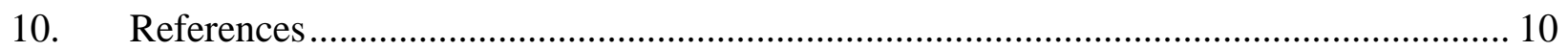

\section{APPENDICES}

Appendix 1 - General Checking Criteria Sheet....................................................................... 11

Appendix 2 - Energy Balance Check ................................................................................ 13 


\begin{tabular}{|lllllll|}
\hline \hline Calculation No.: & NE-EO-2015-05 & Rev. 1 & Page & 3 & of & 14 \\
\hline \hline
\end{tabular}

\section{REVISION LOG}

\begin{tabular}{||l|l|l||}
\hline \hline REVISION & CHANGES & DATE \\
\hline 0 & Initial Release & $11 / 18 / 2015$ \\
\hline 1 & Updates following results of flow tests & $3 / 8 / 2017$ \\
\hline & & \\
\hline & & \\
\hline
\end{tabular}




\section{Objectives of the analysis}

Determine via thermal/hydraulic analysis whether the DU target disks remain below safe temperature limits to prevent boiling of the water coolant and/or damage to the target disks. The data presented in this report is for guidance in operating the electron beam.

\section{Background}

The mini-SHINE/MIPS experiments at Argonne National Laboratory's Low Energy Accelerator Facility (LEAF) examine the possibility of Mo-99 production in a uranyl-sulfate solution. The purpose of this particular experiment, which uses the window, is to examine gas generation in the fuel solution and how it affects the reaction. This window acts both as a vacuum window and a cooling channel wall. The window design utilizes a cylindrical shape to minimize the wall thickness while maintaining sufficient stiffness.

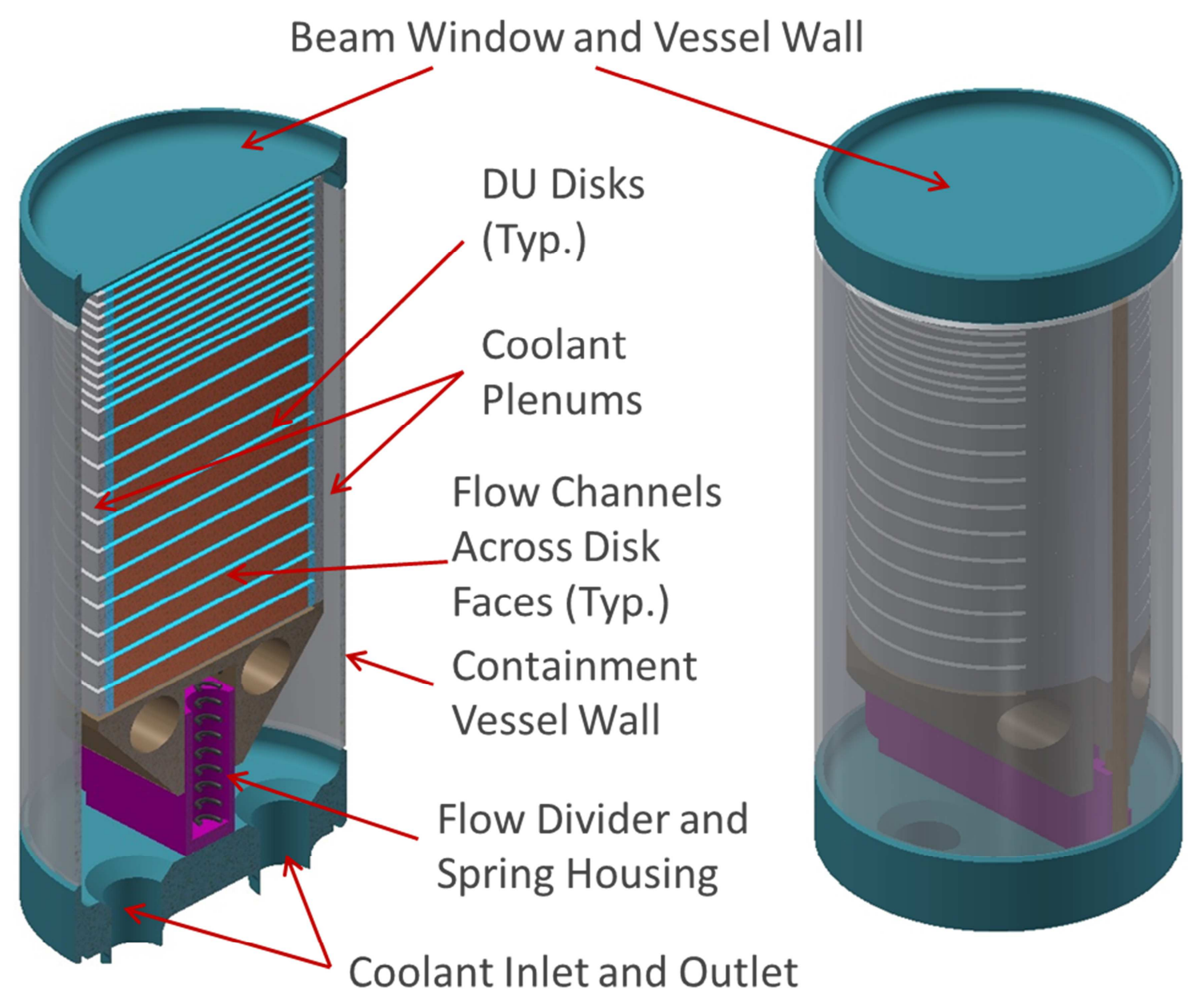

Figure 1 On the left is a cross-sectional view of the DU target assembly. The DU Disks are the subject of the analysis presented in this report. The incident electron beam is coming in from the top of the figure. On the right is a full model representation of the DU target assembly. 


\section{Scope of the analysis}

This calculation note includes thermal/hydraulic analysis of a DU target disk only, and does not examine any of the surrounding parts of the overall target assembly. The water coolant system is merely represented by a constant volumetric flow.

\section{Acceptance criteria}

The temperature limits for the DU target disks are as follows:

- To prevent grain growth and clad fatigue stress, the center of the disks must not exceed $300{ }^{\circ} \mathrm{C}$.

- To prevent boiling of the water coolant, the surface of the disks must not exceed the saturation temperatures of $126{ }^{\circ} \mathrm{C}$ at a coolant flow rate of $4.1 \mathrm{gpm}$ or $134{ }^{\circ} \mathrm{C}$ at a flow rate of $5.0 \mathrm{gpm}$. (Saturation temperatures were provided based on data from the analysis in NE-CALC-2015-03 Revision 1.)

A $15 \%$ margin to boiling is assumed to be a reasonable uncertainty factor based on engineering judgment considering: a 5\% error in analysis (noting that flow testing were performed on the actual target assembly and ANSYS CFX is a well validate thermal hydraulic computer code); a 5\% allowance for the flow switch beam trip (considers a trip of $2 \mathrm{gpm}$ below the operating flow rate); $5 \%$ for beam power and width uncertainty (note that surface temperature is linear with beam power, however it is to the square of the beam width).

\section{Assumptions}

1. Heat is removed from the target disks through the water coolant only.

2. Flow rates: 5 gpm or $4.1 \mathrm{gpm}$ per channel based on data collected during flow tests.

3. Volumetric heating of the disks is assumed to be a Gaussian distribution of the incident electron beam based calculation by CSE Division for total heat absorbed per disk.

4. A $0.25 \mathrm{~mm}$ layer of Zircaloy cladding is included between the uranium and the water.

5. The disk materials are assumed to be isotropic and homogeneous with the following thermal properties:

\begin{tabular}{|c|c|c|}
\hline \multicolumn{3}{|c|}{ Material Thermal Properties } \\
\hline & Uranium & Zircaloy \\
\hline $\begin{array}{c}\text { Thermal conductivity: } \mathrm{k} \\
(\mathrm{W} / \mathrm{m}-\mathrm{K})\end{array}$ & 28 & 25 \\
\hline
\end{tabular}

\section{Method}

Geometry was created in ANSYS Design Modeler [1] based on drawing number R07844. Symmetry was utilized, which can be seen in the result plots of Figures 5 and 9. The geometry of the model includes a disk of depleted uranium sandwiched between two layers of Zircaloy clad, which is cooled by two channels of water. The coolant water geometry includes an inlet plenum and an outlet plenum; each assigned a constant mass flow rate. Symmetry was utilized for all analyses except for the case of the offcenter beam. Meshing of the coolant channel geometry (See Figure 3) includes inflation along the surface that interfaces with the Zircaloy clad. A k- $\varepsilon$ turbulence model was used, and in CFX, Scalable Wall Functions are used for all turbulence models based on the $\varepsilon$-equation [2]. Note that both the scalable wall function and the automatic wall treatment can be run on arbitrarily fine meshes. The inlet turbulence was defined at $5 \%$ intensity and a viscosity ratio $\left(\mu_{\mathrm{t}} / \mu\right)$ equal to 10 . The mesh was refined until the change in 


\begin{tabular}{|llllllll|}
\hline Calculation No.: & NE-EO-2015-05 & Rev. & 1 & Page & 6 & of & 14 \\
\hline
\end{tabular}

results for pressure drop across the channel was less than $1 \%$. The range of near-wall y+ values for the final mesh used was from 17 to 20 .
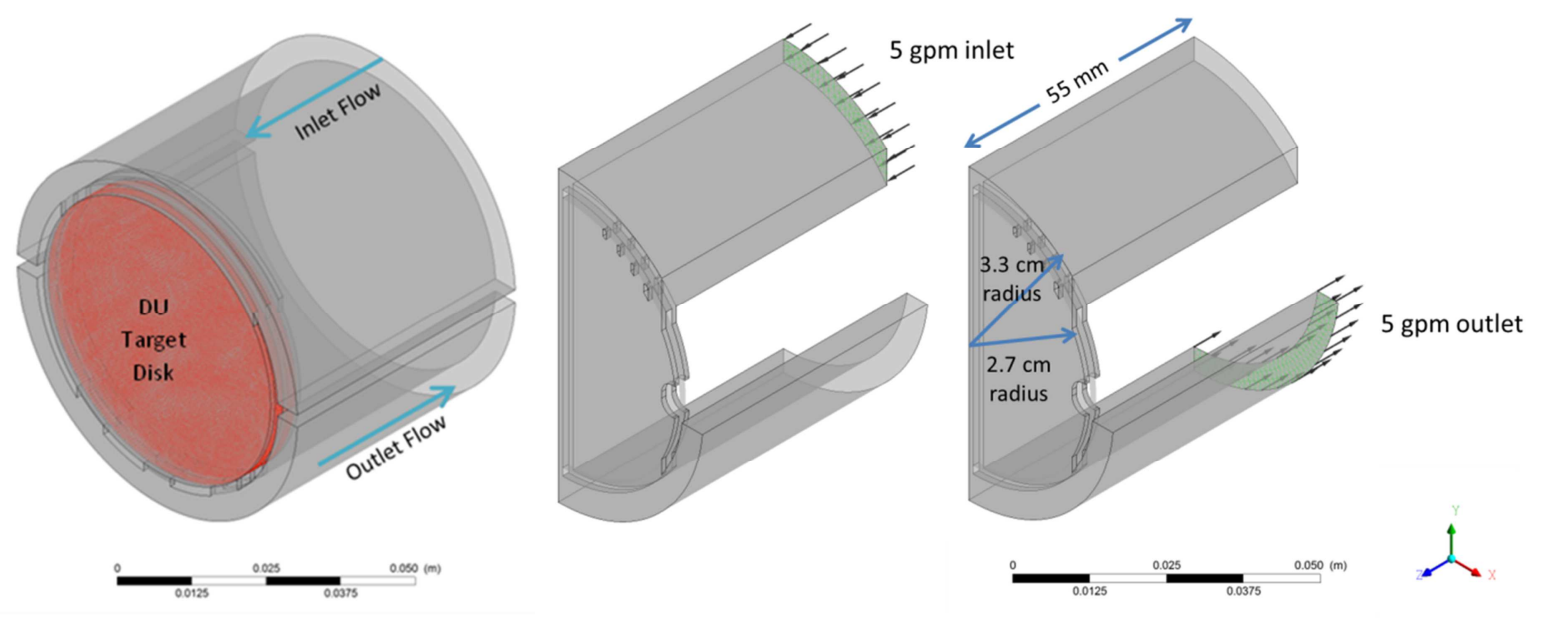

Figure 2. Simplified geometric model of a single DU target disk used in the CFD analysis. The disk is highlighted in red with the inlet plenum above and the outlet plenum below.
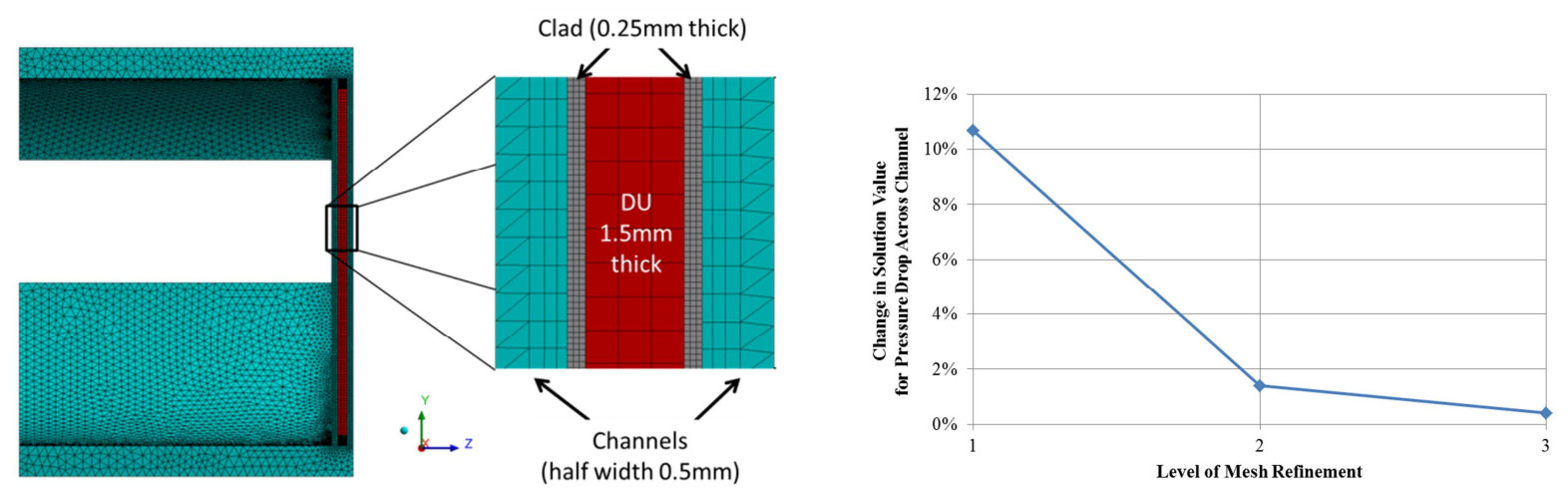

Figure 3 Meshing of the CFD model, showing the depleted uranium in red, the cladding in gray, and the water coolant in cyan. The mesh was refined until the change in results was $<1 \%$.

The thermal/hydraulic analysis of the DU target disks is performed using ANSYS CFX [1]. A total of seven different volumetric heat generation profiles are used based on characteristics given for the incident electron beam. The profiles include three different beam widths for each of two different electron beam energies of $35 \mathrm{MeV}$ and $40 \mathrm{MeV}$ with two different coolant flow rates of $4.1 \mathrm{gpm}$ or $5.0 \mathrm{gpm}$. Each profile was run for three different beam powers for a total of 36 cases, as well as an additional case to examine the effects of an off-center beam profile. 


\begin{tabular}{|llllllll|}
\hline Calculation No.: & NE-EO-2015-05 & Rev. & 1 & Page & 7 & of & 14 \\
\hline
\end{tabular}

Table 1 Summary of Analysis Cases

\begin{tabular}{|c|c|c|c|c|c|c|c|c|c|c|c|c|}
\hline Electron Beam Energy [MeV] & \multicolumn{6}{|c|}{35} & \multicolumn{6}{|c|}{40} \\
\hline Total Absorbed Power $[\mathrm{kW}]$ & \multicolumn{6}{|c|}{4.06} & \multicolumn{6}{|c|}{2.30} \\
\hline Coolant Flow Rate [gpm] & \multicolumn{3}{|c|}{4.1} & \multicolumn{3}{|c|}{5.0} & \multicolumn{3}{|c|}{4.1} & \multicolumn{3}{|c|}{5.0} \\
\hline Beam Width $[\mathrm{mm}]$ & 16 & 18 & 20 & 16 & 18 & 20 & 16 & 18 & 20 & 14 & 16 & 18 \\
\hline
\end{tabular}

\section{Heat Absorption Profiles}

The overall configuration of the DU target assembly is shown above in Figure 1, with the water coolant coming in through a plenum on one side, flowing across the face of the target disks, and exiting through the plenum on the other side. The CFD model only examines the hottest disk for each electron energy level. The values in the plot are based on a $20 \mathrm{~kW}$ incident electron beam, and the total heat for Disk 2 at $35 \mathrm{MeV}$ is $4.06 \mathrm{~kW}$ and for Disk 3 at $40 \mathrm{MeV}$ is $2.3 \mathrm{~kW}$.

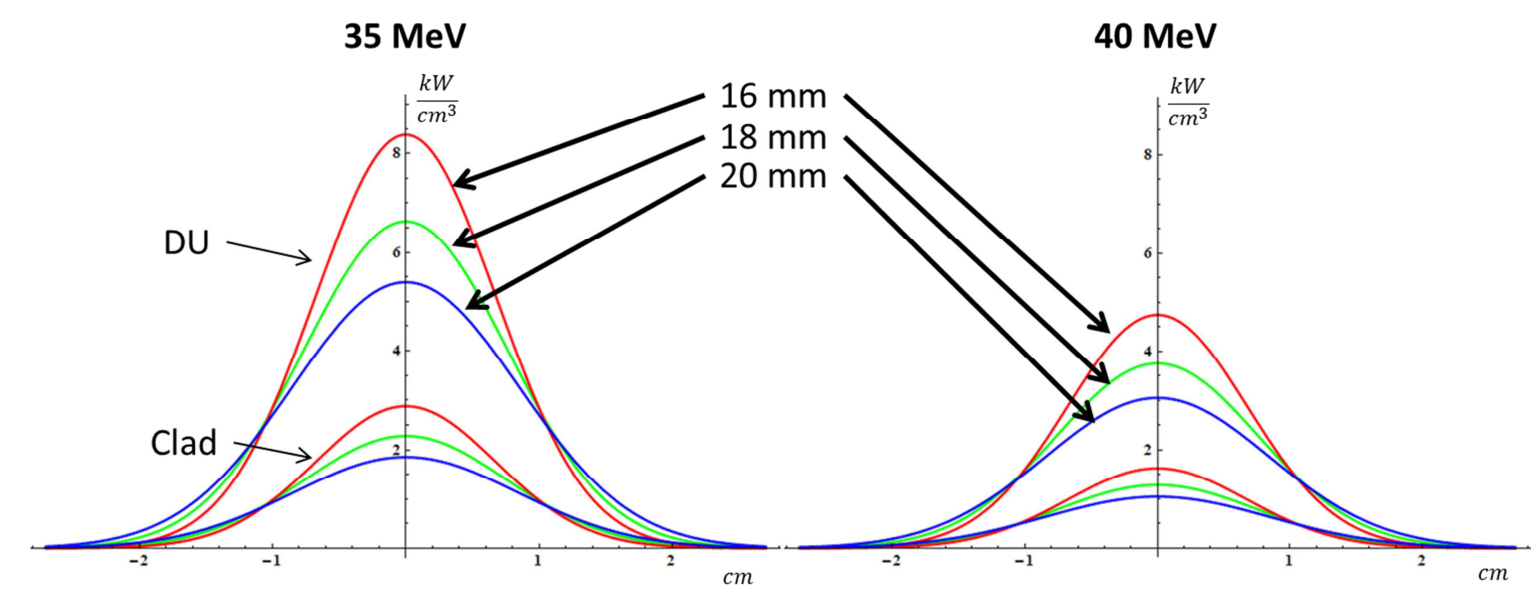

Figure 4. Power absorption profiles for the hottest disk assuming $20 \mathrm{~kW}$ of beam power at two different electron beam energies, 35 and $40 \mathrm{MeV}$. The power absorption is calculated separately for the depleted uranium and the Zircaloy clad, using a Gaussian distribution with FWHM beam sizes of 16, 18, and $20 \mathrm{~mm}$. Total power absorbed at $35 \mathrm{MeV}$ is $4.06 \mathrm{~kW}$, and total power absorbed at $40 \mathrm{MeV}$ is $2.30 \mathrm{~kW}$.

The distributions of the volumetric heat generation were calculated in Mathematica 8 [2] to obtain a Gaussian distribution based on the FWHM size of the beam and the total power. Shown above, in Figure 4, are six separate Gaussian distributions calculated for use in the analysis. A seventh distribution, not shown in the figure, was used for a beam width of $14 \mathrm{~mm}$ for $40 \mathrm{MeV}$ with $5.0 \mathrm{gpm}$ flow rate.

\section{Results}

The same model setup was used throughout all steady-state analyses. Only the volumetric heat generation profile and coolant flow rate were modified for each case. For the case of the off-center beam, a full model was used with the same conditions as for the case with an $18 \mathrm{~mm}$ wide beam at $35 \mathrm{MeV}$ and 10 $\mathrm{kW}$ with a flow rate of $4.1 \mathrm{gpm}$. The center of the beam was placed on a new coordinate system offset from the center by $1 / 4$ of the target disk radius. 


\begin{tabular}{|llllllll|}
\hline Calculation No.: & NE-EO-2015-05 & Rev. & 1 & Page & 8 & of & 14 \\
\hline
\end{tabular}

\subsection{Centered beam}

Using the heat generation profiles described in Section 7, three values of beam power were selected to produce maximum surface temperature plots for each combination of electron beam energy level and coolant flow rate previously summarized in Table 1 . Contour plots of the temperatures at the clad surface and the center of the DU disk are shown in Figure 5 for an $18 \mathrm{~mm}$ beam at $35 \mathrm{MeV}$ and $10 \mathrm{~kW}$ with a flow rate of $4.1 \mathrm{gpm}$. In all cases, the maximum surface temperature of the Zircaloy clad was the limiting factor, and not the maximum temperature of the DU disk. Figures Figure 6 and Figure 7 summarize the effects of beam width and power for a given electron beam energy and coolant flow rate. Each plot includes a dashed horizontal line that represents the limiting saturation temperature associated with the flow rate.

Clad Surface Temperature

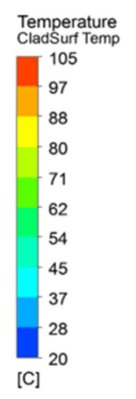

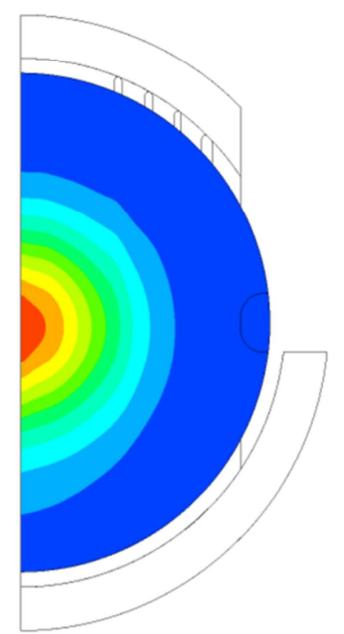

DU Center Temperature
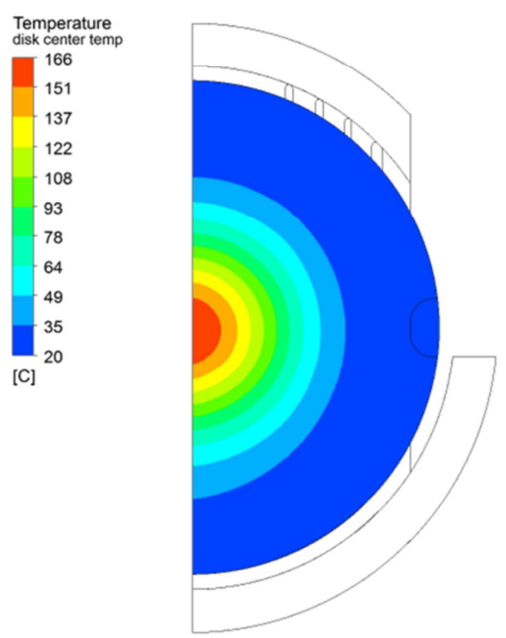

Figure 5. Steady-state temperature results, assuming a $10 \mathrm{~kW}$ beam at $35 \mathrm{MeV}$ with a width of $18 \mathrm{~mm}$ FWHM and a coolant flow rate of $4.1 \mathrm{gpm}$. The plot of the left shows surface temperature distribution of the Zircaloy clad material, and the right plot shows the temperature distribution at the center of the DU target disk.
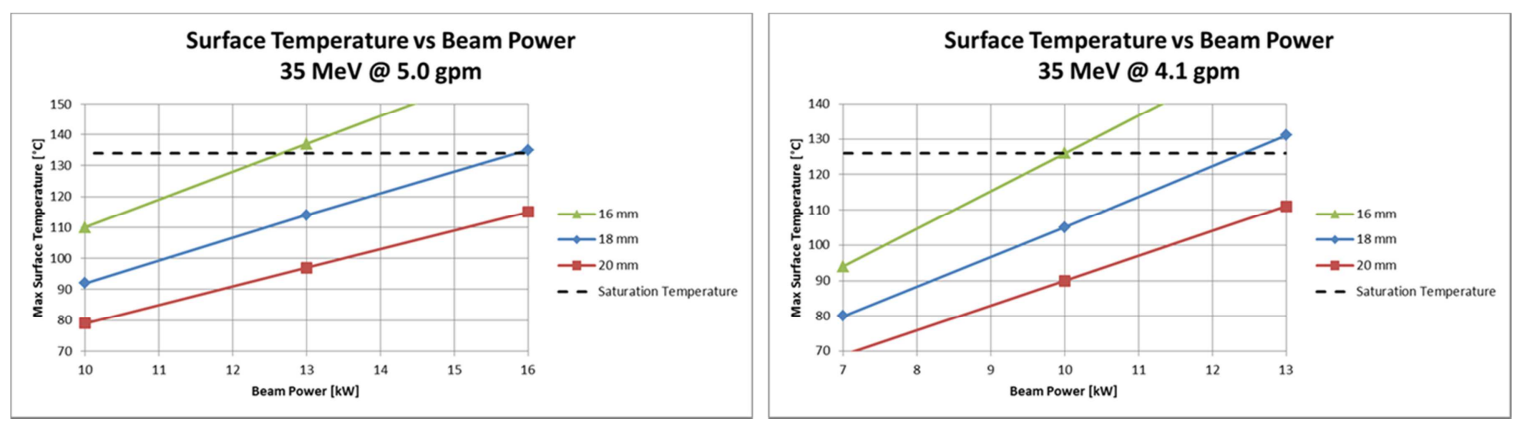

Figure 6 Maximum surface temperatures for $35 \mathrm{MeV}$ for given beam size and coolant flow rate as a function of beam power. With a beam size ranging from 16 to $20 \mathrm{~mm}$, the maximum beam power needs to be limited to prevent boiling at the surface of the target disk. 


\begin{tabular}{|llllllll|}
\hline Calculation No.: & NE-EO-2015-05 & Rev. & 1 & Page & 9 & of & 14 \\
\hline
\end{tabular}
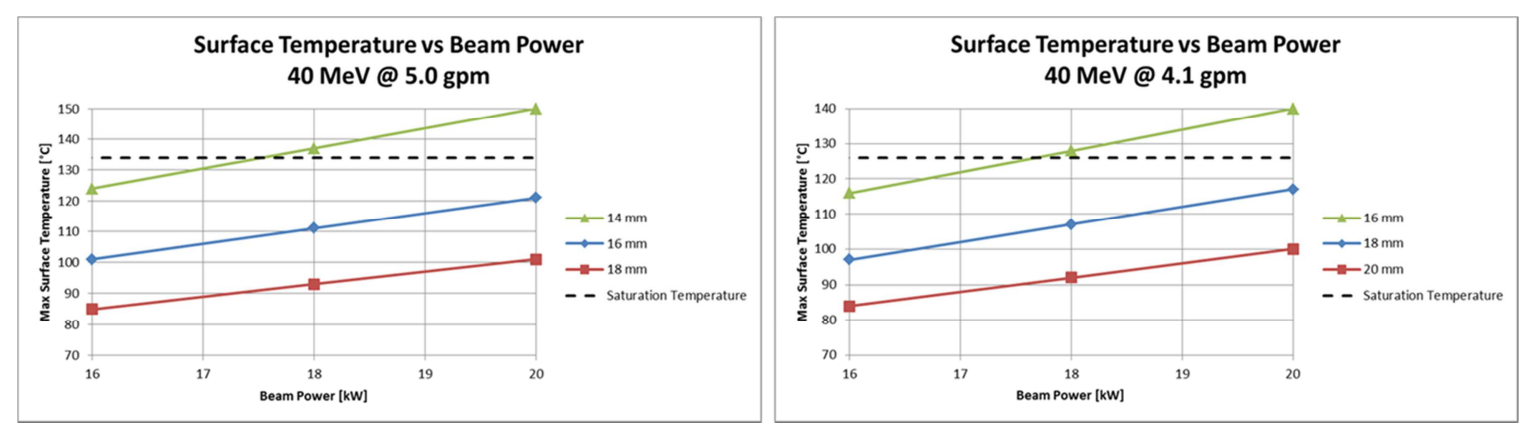

Figure 7 Maximum surface temperatures for $40 \mathrm{MeV}$ for given beam size and coolant flow rate as a function of beam power. Given the right beam width, a maximum beam power of $20 \mathrm{~kW}$ is achievable.

\subsection{Asymmetric beam}

One potential accident scenario could occur in the instance of asymmetric heating of the target. Using the same Gaussian distribution of heat generation for an $18 \mathrm{~mm}$ beam at $35 \mathrm{MeV}$, the beam is shifted away from the center of the target disk by $1 / 4$ of the disk radius (See Figure 8). However, this deviation from off axis has no significant impact on the target disk temperature (See Figure 9).

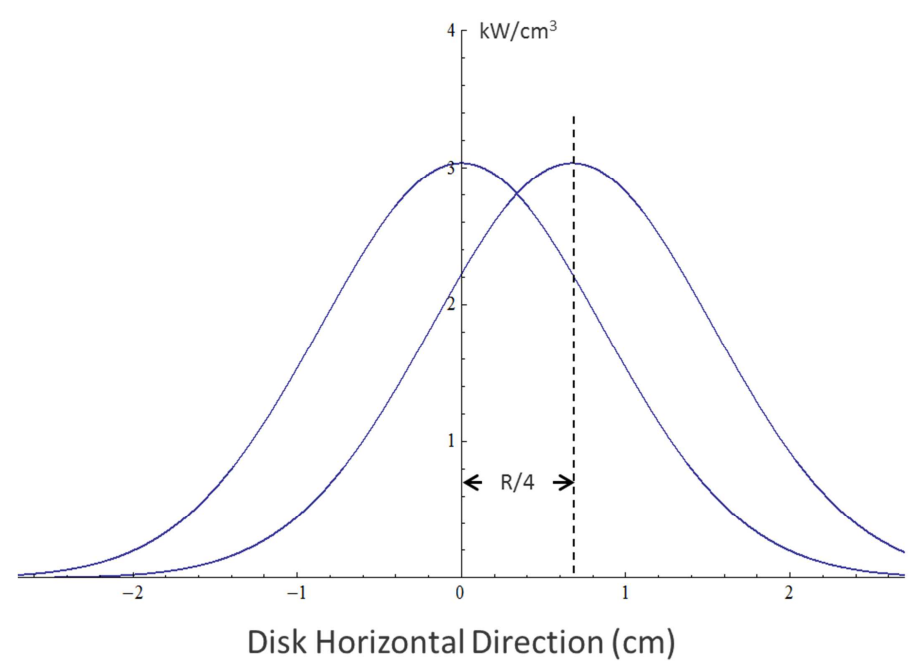

Figure 8. An illustration depicting an asymmetric beam profile based on a typical centered profile with the peak of the distribution moved away from the center by $1 / 4$ of the disk radius. 


\begin{tabular}{|llllllll|}
\hline Calculation No.: & NE-EO-2015-05 & Rev. 1 & Page & 10 & of & 14 \\
\hline
\end{tabular}
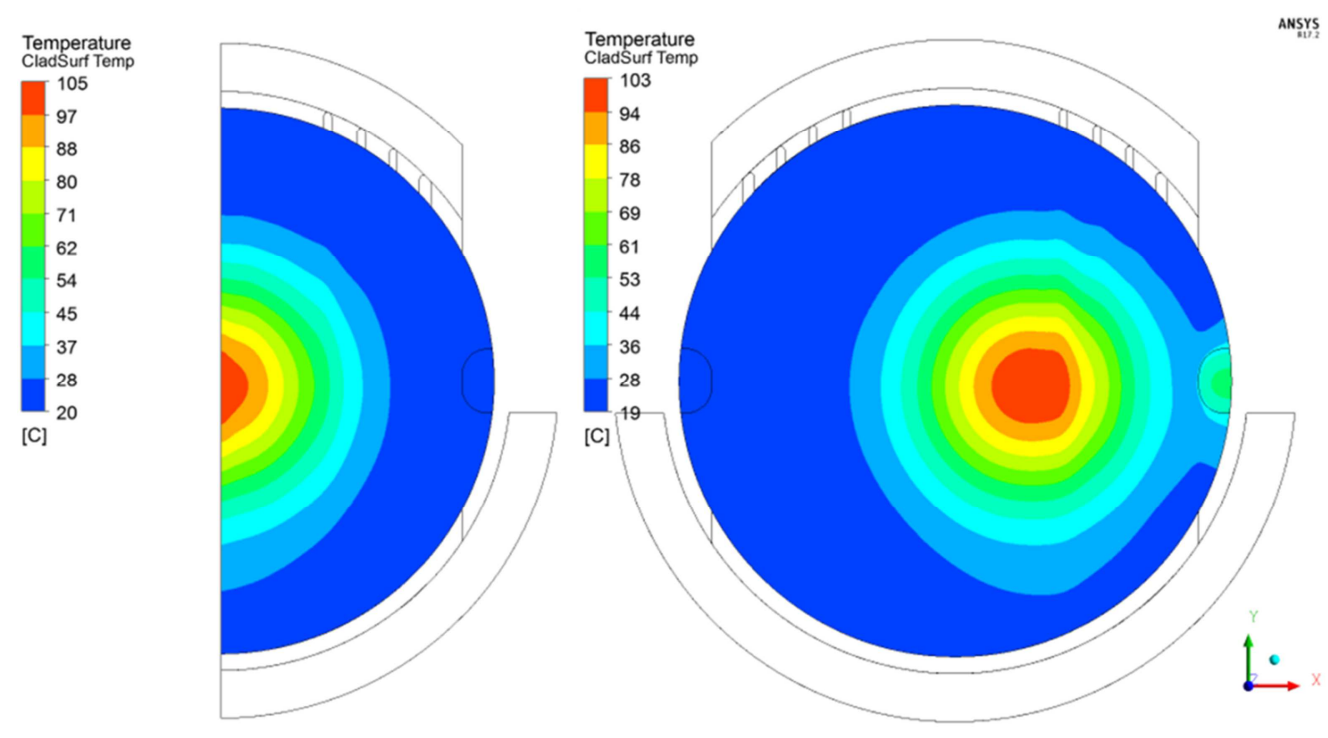

Figure 9. Temperature results from both a symmetric and asymmetric beam. All other conditions of the model remain the same. The asymmetric profile shows little difference in temperature compared to the normal operating conditions.

\section{Conclusions}

- Plots have been presented to show the of maximum surface temperature to be expected for a given beam size and beam power

- At $40 \mathrm{MeV}$, a $16 \mathrm{~mm}$ wide beam with $20 \mathrm{~kW}$ of power will remain below the saturation temperature provided that the water coolant is supplied at a rate of $5 \mathrm{gpm}$.

- Asymmetric beam: does not cause problematic temperature distribution, temperatures still within limits

\section{References}

1. ANSYS. Vers. 17.2. Canonsburg, PA: ANSYS, Inc., 2016. Computer software.

2. CFX Theory Guide, Section 2.8.1.1. Scalable Wall Functions, Canonsburg, PA: ANSYS, Inc., 2016.

3. Wolfram Mathematica. Vers. 8. Champaign, IL: Wolfram Research, Inc., 2011. Computer software. 


\begin{tabular}{|llllllll|}
\hline Calculation No.: & NE-EO-2015-05 & Rev. 1 & Page & 11 & of & 14 \\
\hline
\end{tabular}

APPENDIX

GENERAL CHECKING CRITERIA SHEET

\begin{tabular}{|c|c|c|c|c|}
\hline CALCULATION CHECKLIST & Yes & No & N/A & Comments \\
\hline $\begin{array}{l}\text { 1. Are analytical methods } \\
\text { appropriate? }\end{array}$ & $\bigotimes$ & & & \\
\hline 2. Are assumptions appropriate? & & & & \\
\hline 3. Is the calculation complete? & $\bigotimes$ & & & \\
\hline $\begin{array}{l}\text { 4. Are formulas appropriately } \\
\text { referenced? }\end{array}$ & $\bigotimes$ & & & \\
\hline 5. Are the input data appropriate? & $\bigotimes$ & & & \\
\hline $\begin{array}{l}\text { 6. Was utilized software appropriate } \\
\text { for the task? }\end{array}$ & $\bigotimes$ & & & \\
\hline $\begin{array}{l}\text { 7. Were software input/initial } \\
\text { conditions/properties/boundary } \\
\text { conditions appropriate? }\end{array}$ & $\bigotimes$ & & & Yes, but see comments below. \\
\hline 8. Are the results reasonable? & $\bigotimes$ & & & \\
\hline
\end{tabular}


Calculation No.: NE-EO-2015-05 Rev. $1 \quad$ Page 12 of 14

APPENDIX

GENERAL CHECKING CRITERIA SHEET

\begin{tabular}{|c|c|c|}
\hline \multicolumn{3}{|c|}{ ADDITIONAL COMMENTS } \\
\hline Number & Comment & Resolution \\
\hline 1. & $\begin{array}{l}\text { Since this effort is focused on heat transfer } \\
\text { (rather than flow), I would suggest using the } \\
\text { peak clad temperature as the criterion for } \\
\text { evaluating mesh, i.e. to replace pressure drop } \\
\text { in Fig. } 3 \text {. I would define a } \theta \text { as follows: } \\
\theta=\left(\mathrm{T}_{\text {clad }}-\mathrm{T}_{\text {clad, res }}\right) /\left(\mathrm{T}_{\text {clad }}-\mathrm{T}_{\text {inlel }}\right) \\
\text { where } \\
\mathrm{T}_{\text {clad }}=\text { predicted peak cladding temperature } \\
\text { for this (coarse) mesh } \\
\mathrm{T}_{\text {clad,ref }}=\text { predicted peak cladding } \\
\text { temperature for the finest mesh } \\
\mathrm{T}_{\text {inlet }}=\text { inlet coolant temperature } \\
\text { And then compare } \theta \text { for different meshes, } \\
\text { and plot similarly to Fig. } 3 \text {. }\end{array}$ & $\begin{array}{l}\text { Athough Fig } 3 \text { shows } \\
\text { presinge drop, the themal } \\
\text { solution converged before } \\
\text { the pressure drop solution. }\end{array}$ \\
\hline 2. & $\begin{array}{l}\text { Wall functions (in STAR-CCM+ anyway) } \\
\text { perform best for } 30<y+<100 \text {, whereas near- } \\
\text { wall turbulence models require } y+\sim 1 \text {. Wall } \\
\text { functions have been known to produce } \\
\text { inaccurate results for the in-between range, } \\
\text { say } 5<y+<20 \text {. I am hoping that the } y+\sim 30 \\
\text { range was tested during your mesh } \\
\text { convergence study and shown to not be } \\
\text { important for your case. }\end{array}$ & $\begin{array}{l}\text { yt values greater than } \\
30 \text { did nod have different } \\
\text { thermal results than the } \\
y+\text { values in the final } \\
\text { solution }\end{array}$ \\
\hline 3. & & \\
\hline 4. & & \\
\hline 5. & & \\
\hline 6. & & \\
\hline 7. & & \\
\hline 8. & & \\
\hline 9. & & \\
\hline 10. & & \\
\hline
\end{tabular}


ENERGY BALANCE CHECK

\begin{tabular}{|c|c|c|}
\hline \multicolumn{2}{|c|}{ INPUTS } & OUTPUT \\
\hline Half Symmetric Power $[\mathrm{kW}]$ & Flow Rate $[\mathrm{kg} / \mathbf{s}]$ & $\Delta \mathbf{T}\left[{ }^{\circ} \mathbf{C}\right]$ from outlet average $\mathbf{~}$ \\
\hline \multirow{2}{*}{$4.06 / 2=2.03$} & 0.258 & 1.9 \\
\cline { 2 - 3 } & 0.315 & 1.5 \\
\hline \multirow{2}{*}{$2.30 / 2=1.15$} & 0.258 & 1.1 \\
\cline { 2 - 3 } & 0.315 & 0.9 \\
\hline
\end{tabular}




\section{APPENDIX 2}

Calculation Note NE-CALC-2015, ver. 1: "Thermal-Hydraulic Analysis of the Stoppage of Coolant Flow" 
Calculation No.: NE-CALC-2015-69-v1

Rev. 0

Page

of

10

CALCULATION COVER SHEET

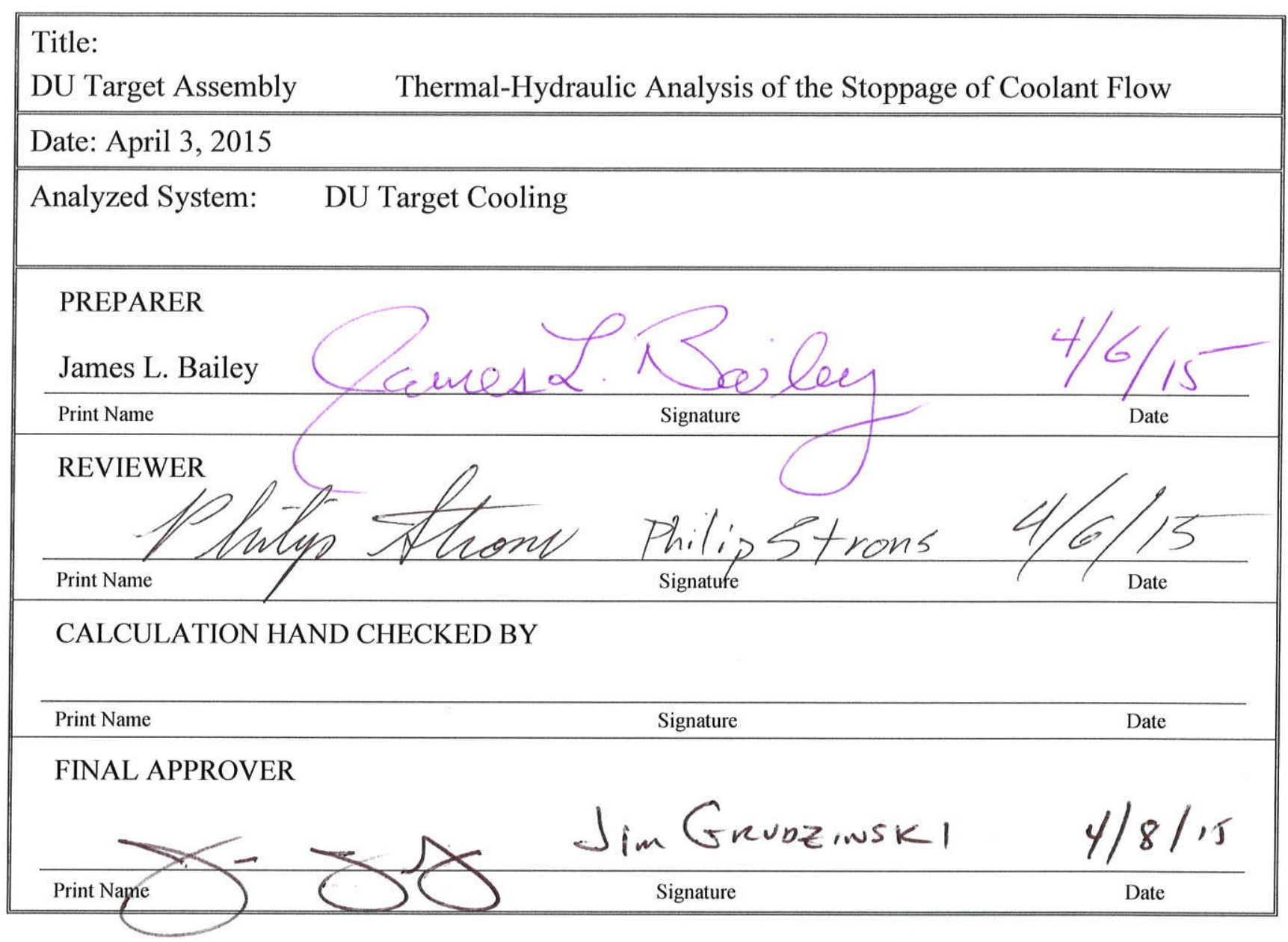

149 
COVER SHEET

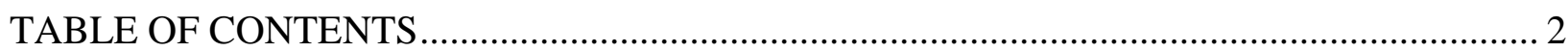

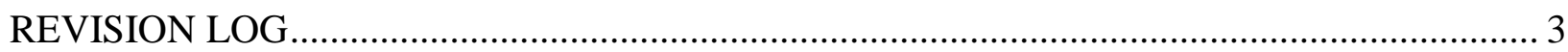

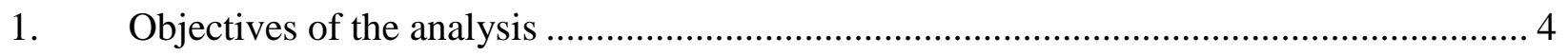

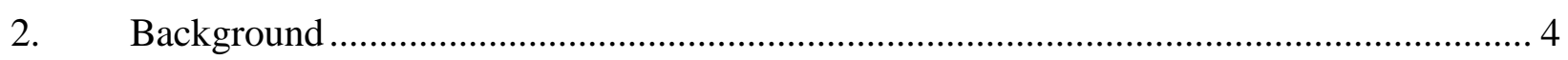

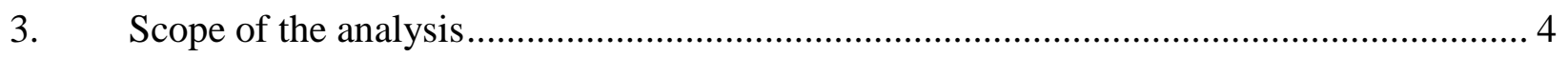

4. Acceptance criteria.............................................................................................. 4

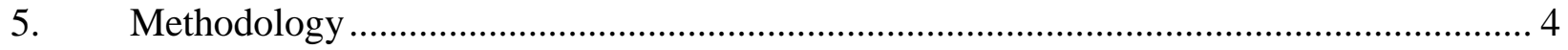

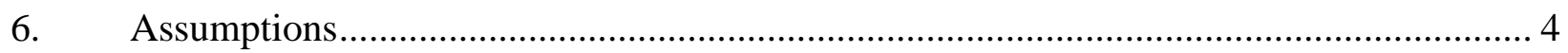

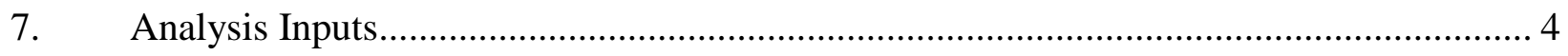

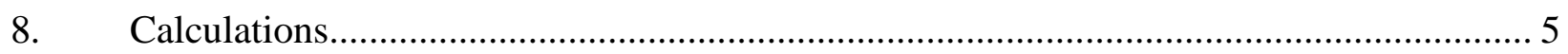

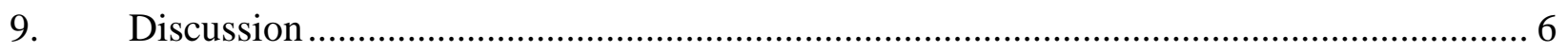

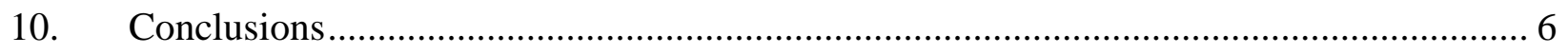

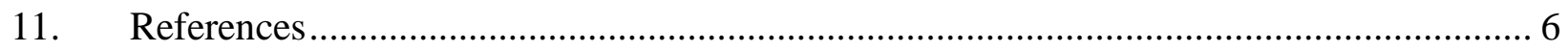

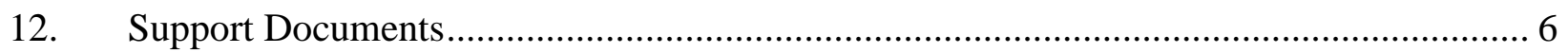

\section{APPENDICES}

Appendix 1 - General Checking Criteria Sheet ...... 
REVISION LOG

\begin{tabular}{||c|c|c||}
\hline REVISION & CHANGES & DATE \\
\hline 0 & Initial Release & \\
\hline & & \\
\hline & & \\
\hline & & \\
\hline
\end{tabular}




\section{Objectives of the analysis}

The object of the analysis is to determine the maximum temperature that the coolant will reach due to decay heat in the uranium disks under the off normal occurrence of a stoppage of coolant flow through the target.

\section{Background}

Upon a stoppage of coolant flow through the cooling system a flow switch initiates the beam trip and shuts down the LINAC. However, the decay heat generated in the uranium continues to heat the coolant in the target. Under the worst case scenario, the total heat generated in the target immediately after shut down is $13.5 \mathrm{~W}$ and then decays to less than a $1 \mathrm{~W}$ after 4 hours. The target vessel is located in the tank sleeve and hence, is surrounded by a small air gap that essentially causes an insulated boundary there. Assuming these conservative conditions the maximum water coolant is calculated.

\section{Scope of the analysis}

The thermal hydraulic behavior of the target coolant under this off normal occurrence is to be determined.

The determination of heat generation rate is not part of this analysis.

\section{Acceptance criteria}

Acceptance criterion is based on avoidance of coolant boiling in the target after the stoppage of flow.

\section{Methodology}

Hand calculations using Excel are used calculate the required transient heat balances.

\section{Assumptions}

The vessel housing is insulated from its surrounding.

The residual heat in the target disks at beam shut down is negligible.

The heat capacity of the disks, vessel and inner stainless steel parts is conservatively neglected.

Only the heat capacity of the coolant water is considered.

Linear interpolation of the decay heat input curve between time steps is sufficiently accurate.

The rate of decay (time constant) for all the disks is the same as for disk 2.

Based on the thermal hydraulic analysis for the target cooling system a maximum temperature of the coolant water entering the target is $90^{\circ} \mathrm{F}$.

\section{Analysis Inputs}

Geometry is per drawing "DU Target DU Disk Assembly + Weldment” Drg. \# R07844, and associated subassemblies and parts.

Decay heat in each disk immediately after shut down is as indicated in reference 1 . 
Transient decay of disk 2 is as indicated in reference 2.

Total heat generation in the target below $1 \mathrm{~W}$ is considered negligible.

\section{Calculations}

This calculation is intended to determine the final maximum temperature of the coolant water and target disks after the stoppage of flow. It is assumed that all the decay heat is stored in the coolant water that remains in the target assembly after the stoppage of flow. Only the heat capacity of the water and the corresponding temperature rise is considered in the heat balance calculation.

Reference the Excel table below. Columns A and B are decay curve information for disk 2 that is taken directly from reference 1 . Total heat generation in the disks immediately after shut down is stored in column E1. This value is calculated by summing the heat generation in all the disks as indicated in reference 2. Column D7 and below are the calculated total heat generation decay that includes all the disks. The values for each time step are calculated by dividing the heat generation in disk 2 at any time by the heat generation in disk 2 at time 0 and then multiplying that ratio by the total heat generation in all the disks at time 0 . The implied assumption for this calculation is that the heat generation decay rate is the same for all the disks. Excel equation for this calculation is [=B7/\$B $\$ 7 * \$ E \$ 1]$. Column E7 and below simply converts the values in D from SI units to English. Column F8 and below assumes a linear interpolation over each time step from column $\mathrm{E}$ and then multiplies the value by the time between time steps. Excel equation for this calculation $[=(\mathrm{E} 7+\mathrm{E} 8) / 2 *(\mathrm{~A} 8-\mathrm{A} 7)]$. Column $\mathrm{G} 8$ and below is the running sum of column F. Excel equation for this calculation [=F8+G7]. The temperature rise of the coolant water and (and disks) for each time step is calculated in Column H8 and below. This calculation conservatively assumes that all the heat calculated in column $\mathrm{G}$ is stored in the coolant water as sensible heat and considers only the density, heat capacity and volume of the water. Excel equation for this calculation $[=\mathrm{G} 8 /(\$ \mathrm{E} \$ 2 * \$ \mathrm{E} \$ 3 * \$ H \$ 2)]$ The water volume has been estimated from the reference drawings. Water properties assume near ambient pressure and temperature. The final temperature differential of $56.6^{\circ} \mathrm{F}$ is indicated in column H30. If an ambient starting temperature of $90^{\circ} \mathrm{F}$ is assumed the final temperature of the water is $\left[90+57=147^{\circ} \mathrm{F}\right]$. This is assuming that the decay heat of less than $1 \mathrm{~W}$ after $240 \mathrm{~min} ., 4$ hours, is negligible.

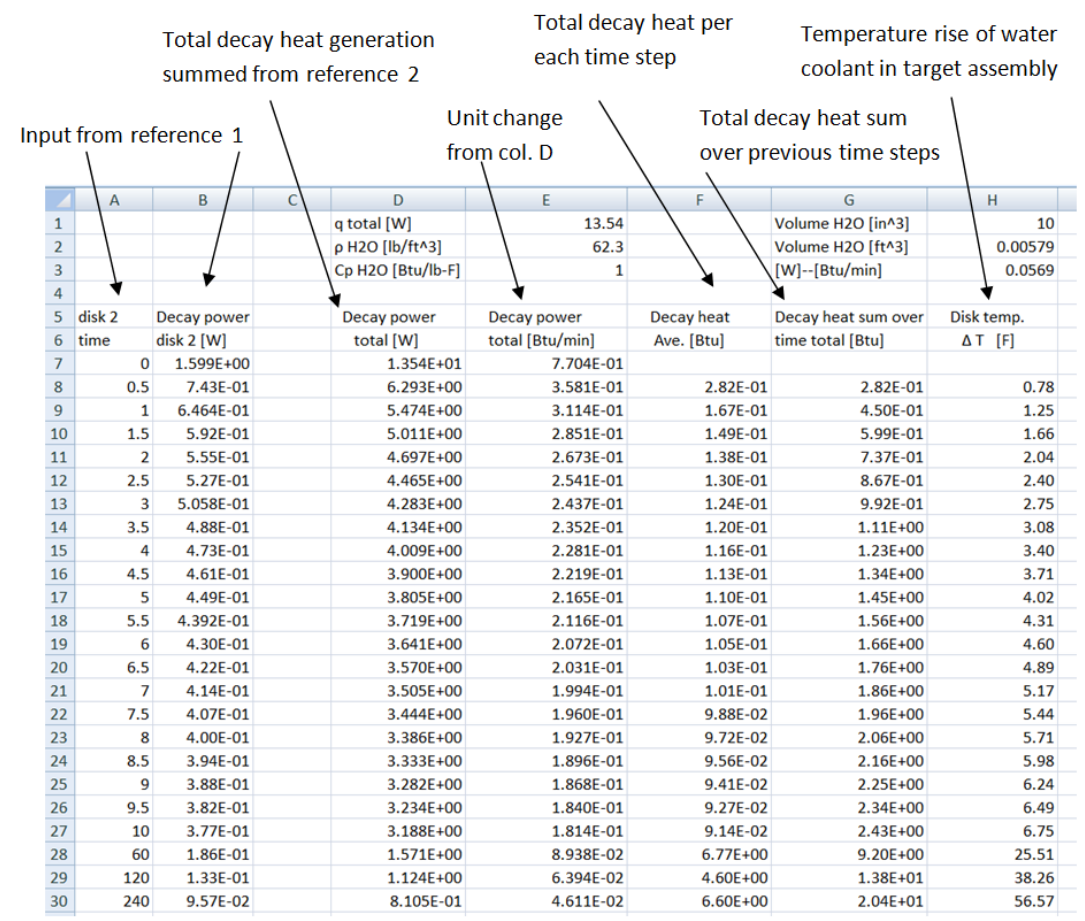




\section{Discussion}

The assumption that the residual heat in the target disks immediately after shut beam is negligible is based on the reasoning that the water flow has a coast down period after the beam shut down is tripped, and as a result, there is a period of time that there is still flow through the target after beam shut down, which is of the order of several seconds. Further, the transient thermal calculations indicate that the response of the disks (cool down) is less 1 second. Hence, there is sufficient time for the disks to cool to ambient temperature before complete stoppage of flow. Also, it should be noted that the heat capacity of the stainless steel internal parts and housing have conservatively not been considered.

The assumption that the decay rate for the entire disk assembly is approximately the same as for disk 2 is per verbal discussions with Brad Micklich.

\section{Conclusions}

Based on the above analysis it is concluded that upon an off normal occurrence of the stoppage of water coolant flow through the target, the temperature of all target components will remain below their maximum allowable design temperatures.

\section{References}

1. Email from Micklich, Bradley J. to Bailey, James L., Chemerisov, Sergey D. dated January 22, 2015, Subject: DU Target SAD comments.

2. Email from Micklich, Bradley J. to Chemerisov, Sergey D. dated November 25, 2014, Subject: decay heat.

\section{Support Documents}

Reference 1 and 2 attached.

Reference Target Assembly drawing attached

\section{Reference 1}

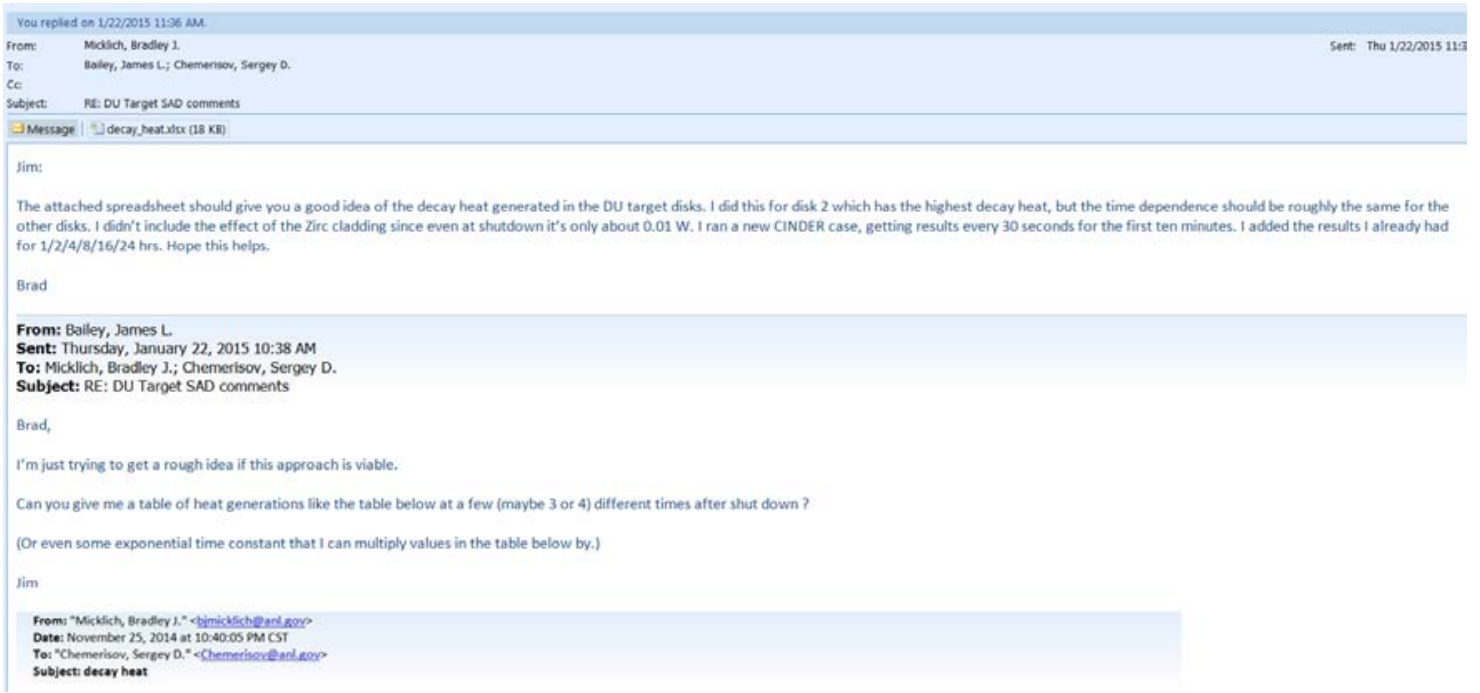


Attachment for reference 1 email

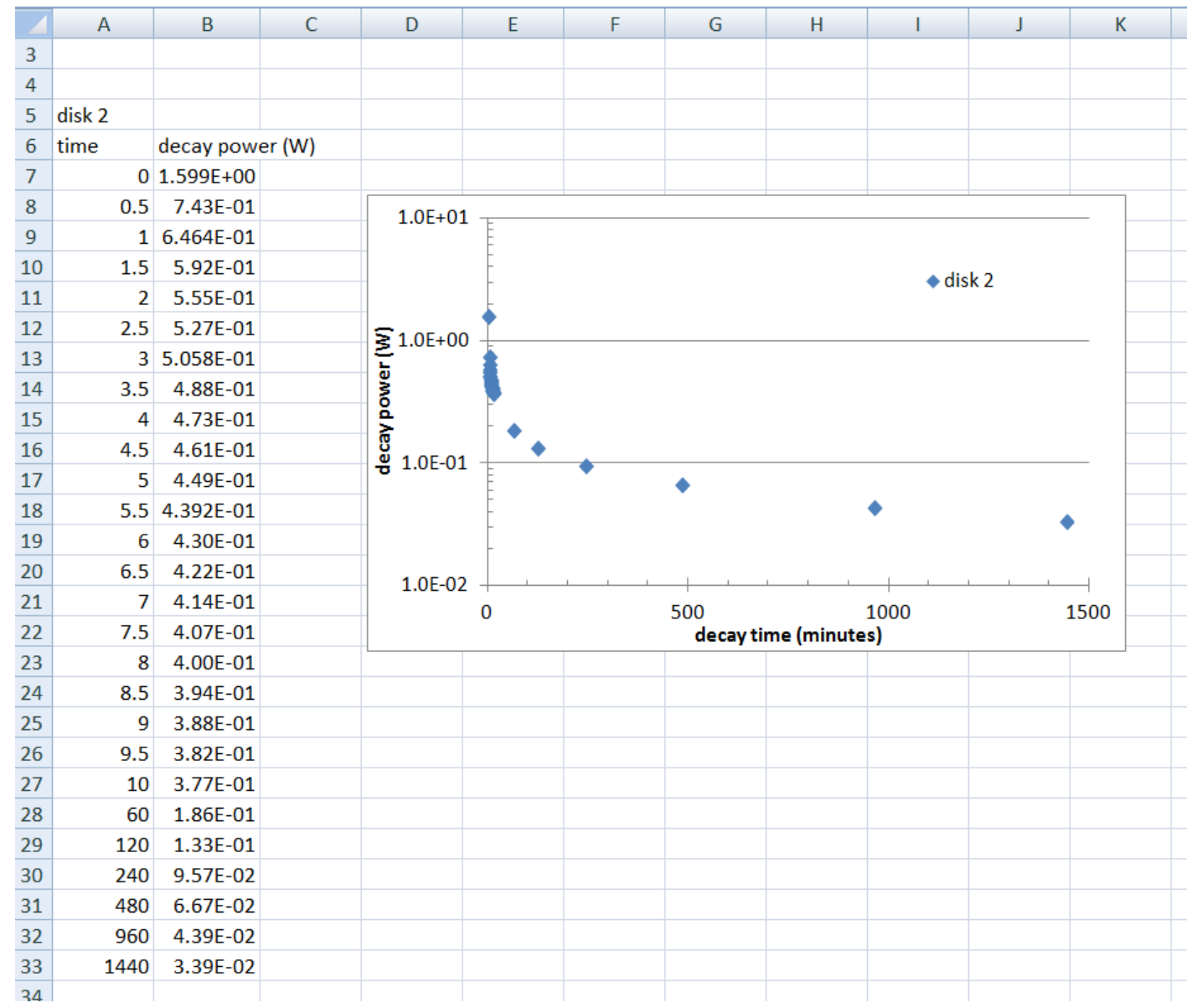

\section{Reference 2}

From: "Micklich, Bradley J." <bjimicklich@anl.gov> Date: November 25, 2014 at 10:40:05 PM CST

To: "Chemerisov, Sergey D." <Chemerisov@anl.gov>

Subject: decay heat

Sergey:

The decay heat in the solution at shutdown after the 5 th irradiation is about $123 \mathrm{~W}$. Here are the decay heats (in watts) in the individual DU disks at shutdown after the 5 th irradiation. Hope this is what you need. Brad

9. $655 \mathrm{E}-001$

$1.599 \mathrm{E}+000$

$1.466 \mathrm{E}+000$

$1.232 \mathrm{E}+000$
$1.010 \mathrm{E}+000$

$1.010 \mathrm{E}+000$

$7.036 \mathrm{E}-001$

5. $945 \mathrm{E}-001$

$5.0378-001$

4. $221 \mathrm{E}-001$

7. $049 \mathrm{E}-001$

4. $994 \mathrm{E}-001$

3. $805 \mathrm{E}-001$

$3.004 \mathrm{E}-001$

$2.173 \mathrm{E}-001$

$1.892 \mathrm{E}-001$

$1.683 \mathrm{E}-001$

$1.683 \mathrm{E}-001$

$1.515 \mathrm{E}-001$

1. $201 \mathrm{E}-001$ 
Reference Target Assembly Drawing

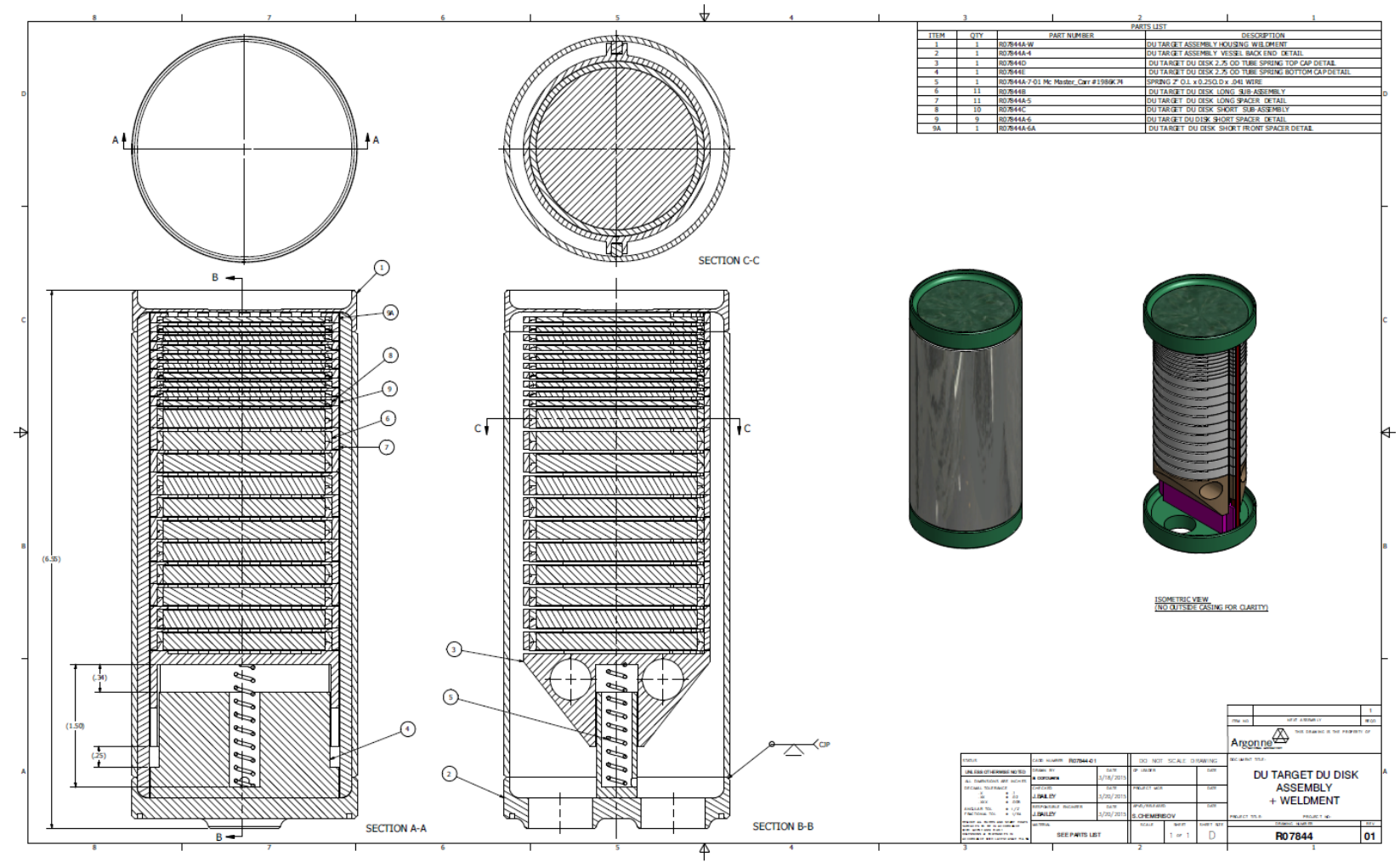




\begin{tabular}{|lllllll|}
\hline Calculation No.: & NE-CALC-2015-69-v1 & Rev. & 0 & Page & 9 & of 10 \\
\hline
\end{tabular}

\section{APPENDIX 1 \\ GENERAL CHECKING CRITERIA SHEET}

\begin{tabular}{|c|c|c|c|c|}
\hline CALCUL ATION CHECKI IST & Yes & No & N/A & Comments \\
\hline $\begin{array}{l}\text { 1. Are analytical methods } \\
\text { appropriate? }\end{array}$ & & & & \\
\hline 2. Are assumptions appropriate? & 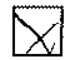 & & & \\
\hline 3. Is the calculation complete? & & & & \\
\hline $\begin{array}{l}\text { 4. Are formulas appropriately } \\
\text { referenced? }\end{array}$ & & & & \\
\hline 5. Are the input data appropriate? & & & & \\
\hline $\begin{array}{l}\text { 6. Was utilized software appropriate } \\
\text { for the task? }\end{array}$ & 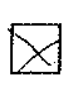 & & & \\
\hline $\begin{array}{l}\text { 7. Were software input/initial } \\
\text { conditions/properties/boundary } \\
\text { conditions appropriate? }\end{array}$ & & & & \\
\hline 8. Are the results reasonable? & ג & & & \\
\hline
\end{tabular}


APPENDIX 1

GENERAL CHECKING CRITERIA SHEET

\begin{tabular}{|c|l|l|}
\hline \multicolumn{3}{|c|}{ ADDITIONAL COMMENTS } \\
\hline Number & Comment & Resolution \\
\hline 1. & & \\
\hline 2. & & \\
\hline 3. & & \\
\hline 4. & & \\
\hline 5. & & \\
\hline 6. & & \\
\hline 7. & & \\
\hline 8. & & \\
\hline 9. & & \\
\hline 10. & & \\
\hline
\end{tabular}




\section{APPENDIX 3}

Calculation Note NE-CALC-2015-03: "Thermal-Hydraulic Analysis of the Overall Performance of the DU Target Cooling System" 


\begin{tabular}{|lllllll|}
\hline Calculation No.: & NE-CALC-2015-03 & Rev. 1 & Page & 1 & of & 12 \\
\hline
\end{tabular}

\section{CALCULATION COVER SHEET}

Title:

Thermal-Hydraulic Analysis of the Overall Performance of the DU

Target Cooling System

Date: August 3, 2015

Analyzed Systems: DU Target Cooling and DU Target Assembly

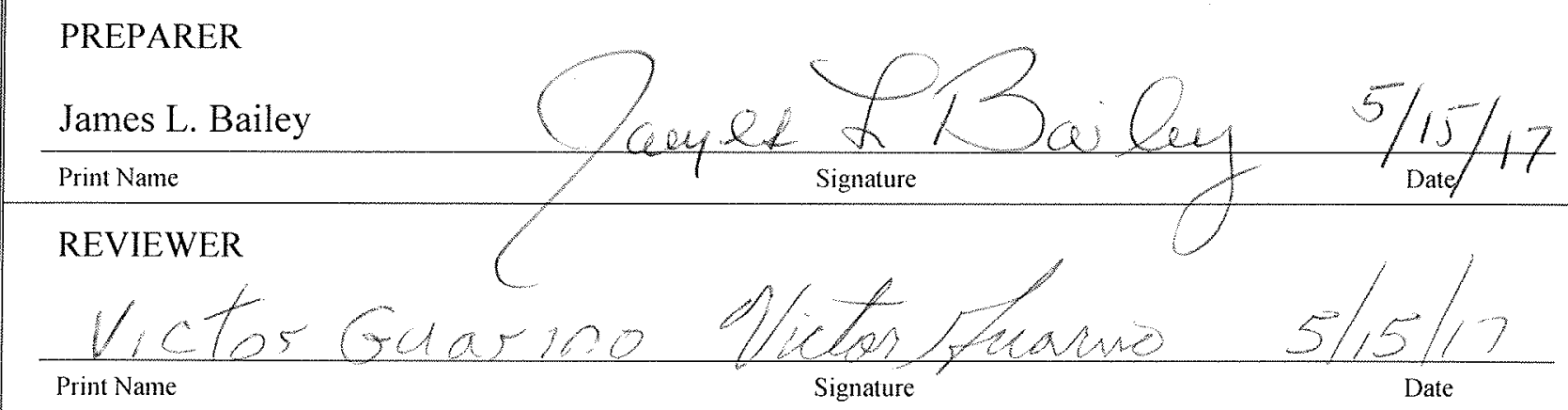

CALCULATION HAND CHECKED BY

Print Name

Signature

Date

FINAL APPROVER

Print Name


COVER SHEET

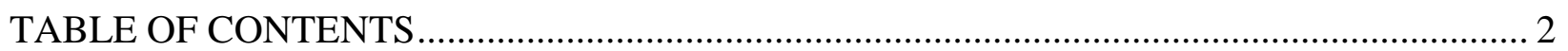

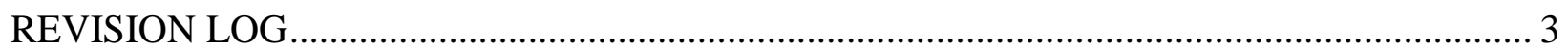

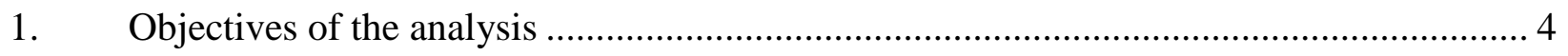

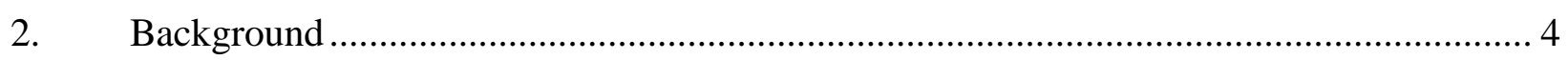

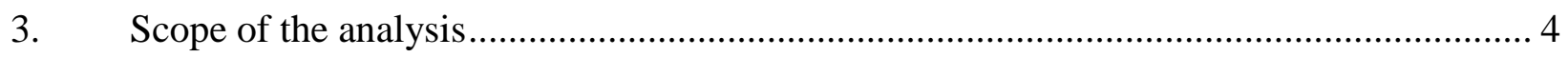

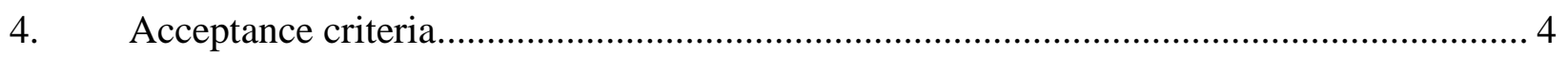

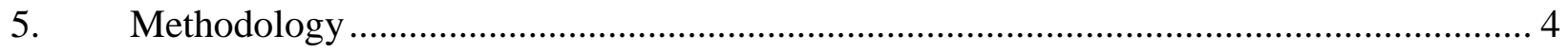

6. Analysis Assumptions and Inputs ......................................................................... 5

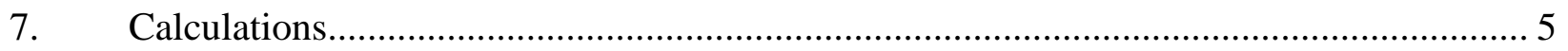

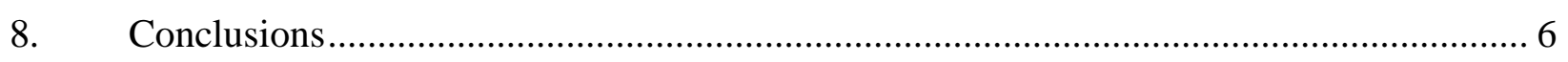

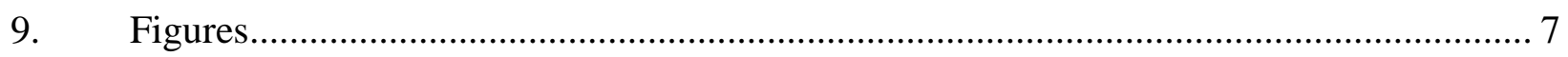

\section{APPENDICES}

Appendix 1 - General Checking Criteria Sheet ..... 
REVISION LOG

\begin{tabular}{||c|c|c||}
\hline \hline REVISION & CHANGES & DATE \\
\hline 0 & Initial Release & March 2, 2017 \\
\hline 1 & $\begin{array}{c}\text { Revised analyses based on flow tests and } \\
\text { analyzed system considering a larger pump }\end{array}$ & \\
\hline & & \\
\hline & & \\
\hline
\end{tabular}




\section{Objectives of the analysis}

The object of the analysis is to verify that the coolant flow distribution through the target assembly is adequate to provide the cooling that is required to maintain the design temperatures for the target disks during normal operation.

\section{Background}

During normal operation heat is generated within the zircaloy-4 clad depleted uranium target disks due to impingement of the electron beam on the target. The target disks are cooled by DI water flowing between the disks (Refer to Figure 1). The coolant supply and return are connected to the existing DU target cooling system that provides the required heat exchanger, pump, strainer and DI unit (Refer to Figure 2).

\section{Scope of the analysis}

The thermal hydraulic behavior of the target assembly and associated cooling system under normal operation is to be determined.

The determination of heat generation rate is not part of this analysis and is considered input to this analysis. (Reference 1)

The local heat transfer analyses (ANSYS CFX) at the target disks are not part of this calculation note and is presented in reference 1 . Only the results from these analyses are used in this note.

The hydraulic performance through the DU target assembly is not part of this calculation note and was determined by flow tests on the actual target as reported in reference 8 . The flow resistances as determined by these test were input to this calculation note.

Two separate analyses are performed. The system is first analyzed considering a smaller capacity pump and then the system is reanalyzed considering a larger pump. The results for the larger pump are noted in brackets following the results for the smaller pump.

\section{Acceptance criteria}

Acceptance criterion is based on avoidance of coolant boiling in the target and allowable maximum material temperature of the uranium. (I.e. surface temperature of the disks to be below saturation temperature at the operating pressure and below $572^{\circ} \mathrm{F}\left(300^{\circ}\right)$ within the disks. (Criteria is based on Reference 1 and thermal hydraulic flow stability in the channels, I.e. avoidance of channel boiling)

\section{Methodology}

ANSYS CFX is used to determine the thermal hydraulic performance locally at the critical disk locations in the target assembly. (Reference 1)

Flow characteristics as determined from the hydraulic tests on the DU Target Assembly (Reference 8) are used as input to the AFT FATHOM overall thermal hydraulic model.

FATHOM's library of piping components and manufactures' data are used to model the cooling system with the exception of the DU Target Assembly. 


\section{Analysis Assumptions and Inputs}

Geometry of the target assembly is per drawing “DU Target DU Disk Assembly + Weldment” Drg. \# R07844, and associated subassemblies and parts. (Figure 1)

Cooling system is per the P/I in Figure 2

Heat generation is per Reference 1

The heat generation in the vessel and inner stainless steel parts negligible.

The vessel housing and cooling system tubing and components are insulated from their surroundings.

The performance of the existing Haskris chiller (Currently located on the service floor in Building 211) is as indicated in the email from the vendor. (Reference 2)

The performance of the pumps is as indicated on the manufacturer's pump curves (Reference 3)

The performance of the plate heat exchanger is as indicated on the vendors quote (Reference 4)

The pressure differential of the particle filter is as indicated in the email from the vendor (Reference 5)

Pressure losses through the valves, pipe and tube are calculated using data from the AFT FATHOM Version 7.0 library (Note: In general this data is in good agreement with that presented in the reference "Fluid Mechanics", $3^{\text {rd }}$ Edition, R.C.Binder.)

The expansion tank is vented to atmospheric pressure

Flow through the system will be manually balanced at start up and under normal operation the flow will remain essentially constant without feedback control.

The temperature of the system will be controlled by the constant temperature of the coolant out of the Haskris chiller $\left(55^{\circ} \mathrm{F}\right)$. As a result, the temperature of the coolant in the primary system will be allowed to vary dependent upon the heat load up to a maximum inlet temperature of $67^{\circ} \mathrm{F}$ at the DU Target. (I.e. maximum heat load condition, $16 \mathrm{~kW}$ )

Coolant fluid is DI water

\section{Calculations}

First, thermal hydraulic analyses were performed on the three critical disks (\#2, \#4, and \#15) using ANSYS CFX. These disks were selected based on the total heat generation in the disk (highest) and the total coolant flow over the faces of the disks (lowest). These conditions result in the worst cases when considering the above acceptance criteria (maximum allowable temperatures). The three worst case disks are noted here. The CFX model included both the heat conduction within the disks and the convective cooling at the surface. The hydraulic performance was performed considering a 5.8psid [9.0psid] from inlet plenum to outlet plenum at the flow rates indicated below and as determined from the flow tests that were performed on the actual target (Reference 8). The flows were balanced between disks by varying the size of the flow control orifices on the spacers (Refer to Figure 1). The pressure drop through the target inlet and outlet plenums was found to be negligible and hence, the pressure differential across each disk face was the same. As a result only three different orifice sizes were required in order to balance the flows. (4.1gpm [5.0gpm] for disks \#1 to \#3, 1.75gpm [2.21gpm] for disks \#4 to \#14, and 1.00gpm [1.33gpm] for disks \#15 to \#22) (Refer to Reference 1 for the details of this analysis). As a result of these flow conditions at the disks the total flow through the target is 42gpm [53gpm] with the corresponding 
pressure loss of 5.8psi [9.0psi]. These conditions were found to be compatible with the existing cooling system by iteration between the CFX and FATHOM models. The total internal heat generation for the critical disk thermal analyses was obtained from reference 1 . The target disks are as shown on the design drawing R0744B and $\mathrm{C}$ and are depleted uranium with 0.010in. Zircaloy-4 clad on the faces. The CFX thermal hydraulic analyses for the target disks summarized in Figure 3 plot are discussed in detail in the report noted in Reference 1.

The commercial computer code AFT FATHOM, Version 7.0, was used to model the overall target cooling system. The AFT FATHOM computer model is shown in Figure 3. The pipe and junction numbers used in the output are referenced on the model shown in Figure 3. The DU target's internal flow configuration is modeled at the top half of the diagram. The beam direction is horizontal from right to left. The flow across the face of each disk is simulated by a parallel channel with an equivalent flow resistance as obtained from the flow tests in Reference 8. This resistance is modeled as an orifice at the channel inlet and a rectangular duct with an average length. These channels are connected to the inlet and outlet plenums shown above and below the parallel channels. The flow resistances in the plenums are also modeled. The expansion and contraction losses for the coolant supply and return at the back of the target assembly are modeled at the upper left of the diagram. Also, the total heat generation from all the disks are modeled thermally as a single $16 \mathrm{~kW}$ input at the back of the target. The support cooling system in the associated enclosure and expansion tank are shown in the lower half of the diagram. The throttle valve at the discharge of the pump is full for both pump analyses. The valve in the DI bypass, J51 is 85 degrees closed in order to reduce the flow to approximately 1.0gpm [1.5gpm]. All other valves are full open. The valves are standard ball valves from the FATHOM Database. Tubing and fittings are stainless steel 16BWG and are also from the FATHOM Database.

The pump performance curve was input as shown in the appendix using the manufacturer's data for the 53/8” [6-5/8”] impeller as indicated in reference 4.

The Particle Filter pressure resistance curve shown in reference 5 was determined from a single point for a clean filter from the vendor's data (I.e. 0.7psi at 50gpm). In order to develop the complete resistance curve the flow through the strainer was assumed turbulent, and therefore, the pressure drop was assumed proportional to the velocity squared.

The hydraulic performance curve of the heat exchanger, HX-1, was input using the manufacturer's data indicated in reference 4 (I.e. 10psi at 50gpm). In order to develop the complete resistance curve the flow through the heat exchanger was assumed turbulent, and therefore, the pressure drop was assumed proportional to the velocity squared.

The thermal performance of the heat exchanger, HX-1, was input from the manufacturer's data as indicated in reference 4.

The expansion tank is modeled by expansion and contraction losses (I.e. area changes, J44 and J45) and tubes, P105 and P106. The tubes have the same dimensions as the actual expansion tank with J47 maintaining atmospheric pressure at J46.

The input to the components in the FATHOM model is shown in reference 6.

\section{Conclusions}

The FATHOM results are shown in reference 7. Volume flow rate through the DU Target is indicated as 42gpm [53gpm]. Noting that the throttle valve at the discharge of the pump is set at full open indicates that the system is operating at full flow required capacity. Also, the temperature of the coolant entering the target is indicated as 67F (Inlet to J87) at the target heat load of $16 \mathrm{~kW}$. Full heat load is only allowable for the high flow rate provided by the larger pump. Further, considering the values shown in the FATHOM results for the tubes and fitting, all flow velocities and pressure drops are within accepted design practices.

The maximum hydrostatic pressure in the system is determined by the maximum pressure at zero flow as indicated by the pump curve in the appendix. Therefore, assuming that the pressure in the expansion tank 
is always at atmospheric pressure the maximum operating pressure of the system is 53psig [72psig]. Also, the maximum operating pressure on the target beam window is 26psig [33psig]. This pressure value can be used for the structural design of the beam window.

\section{9. $\quad$ Figures}

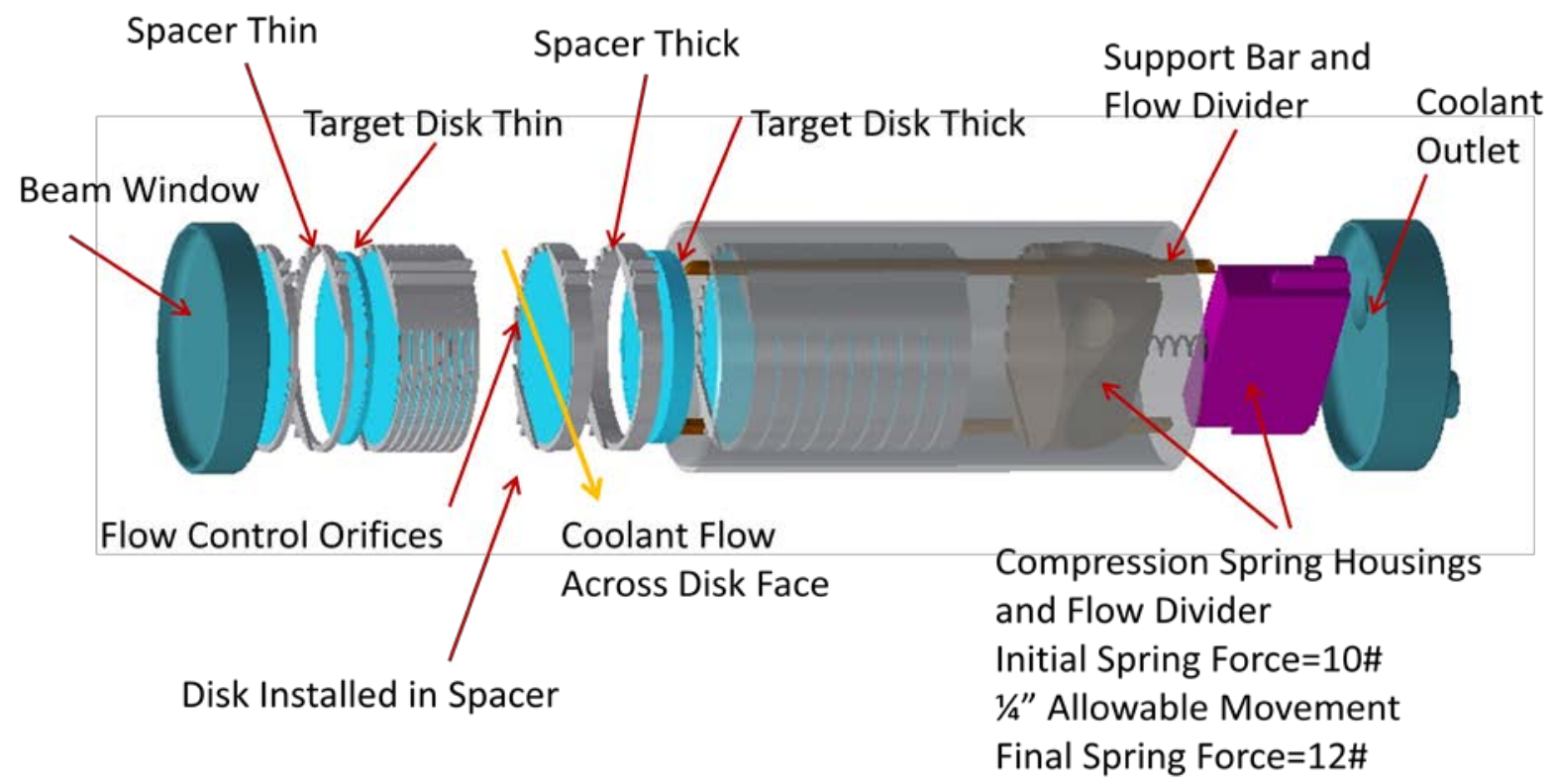




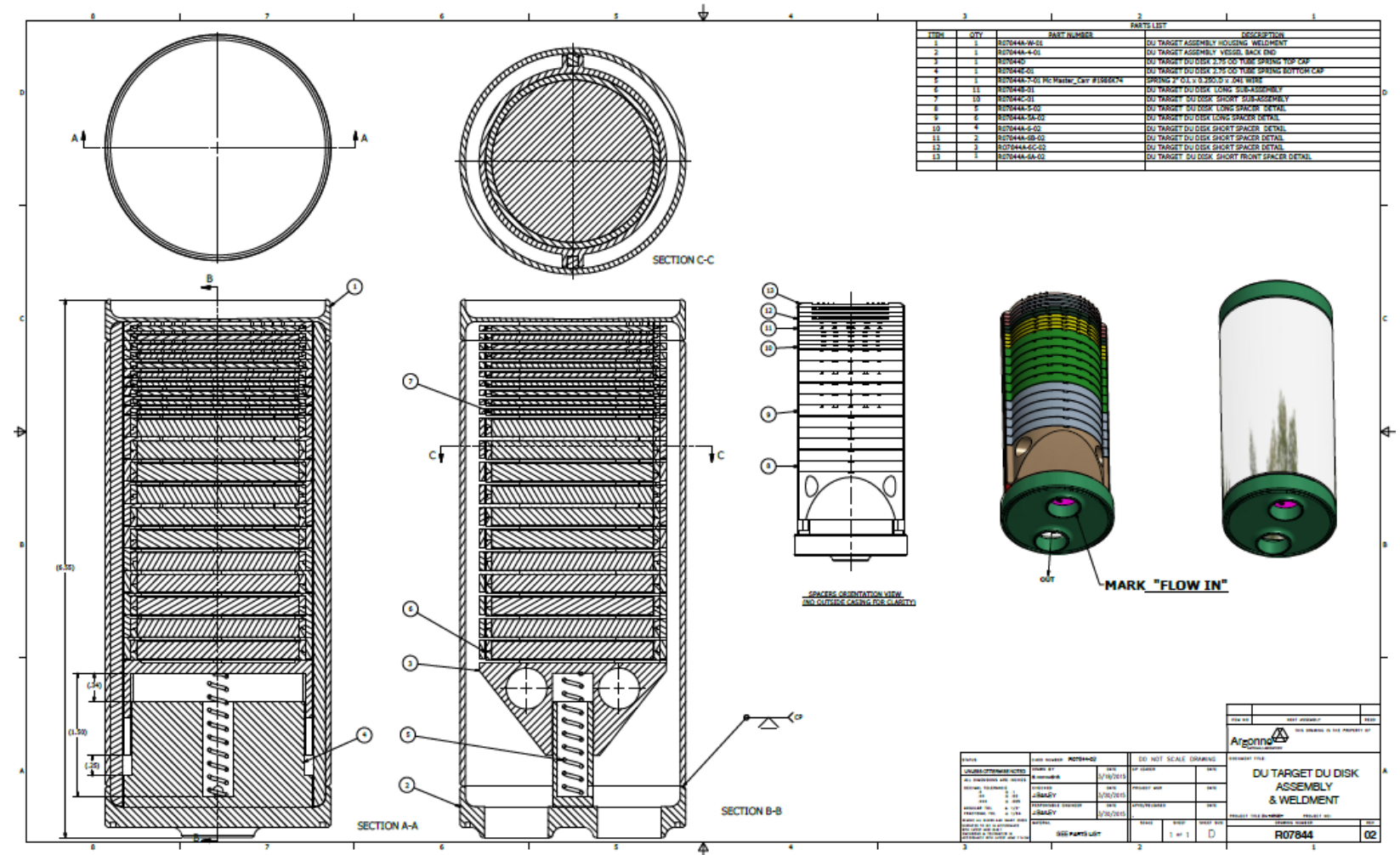

Figure 1 DU Target Assembly 


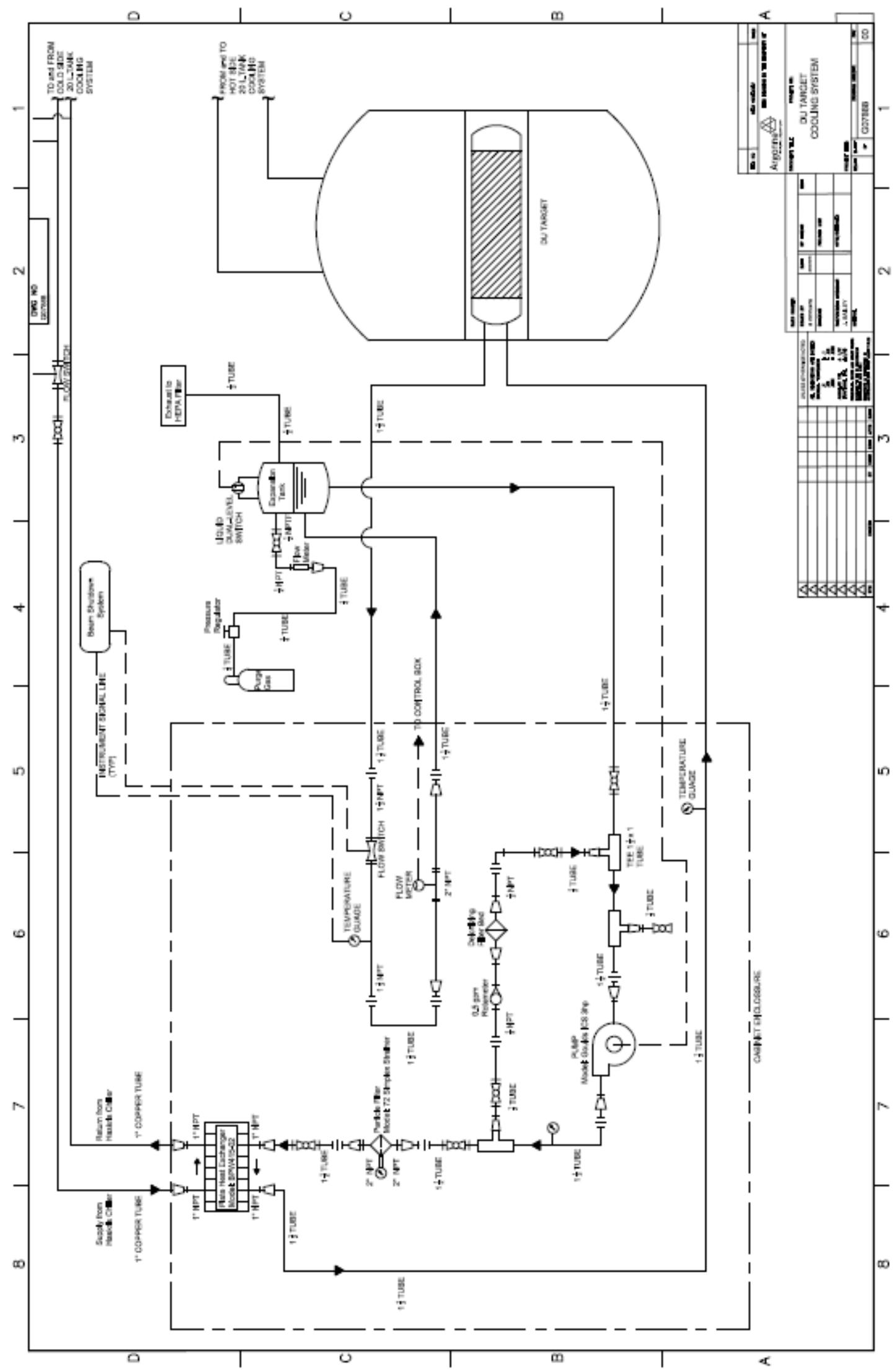

Figure 2 Cooling System P/I 


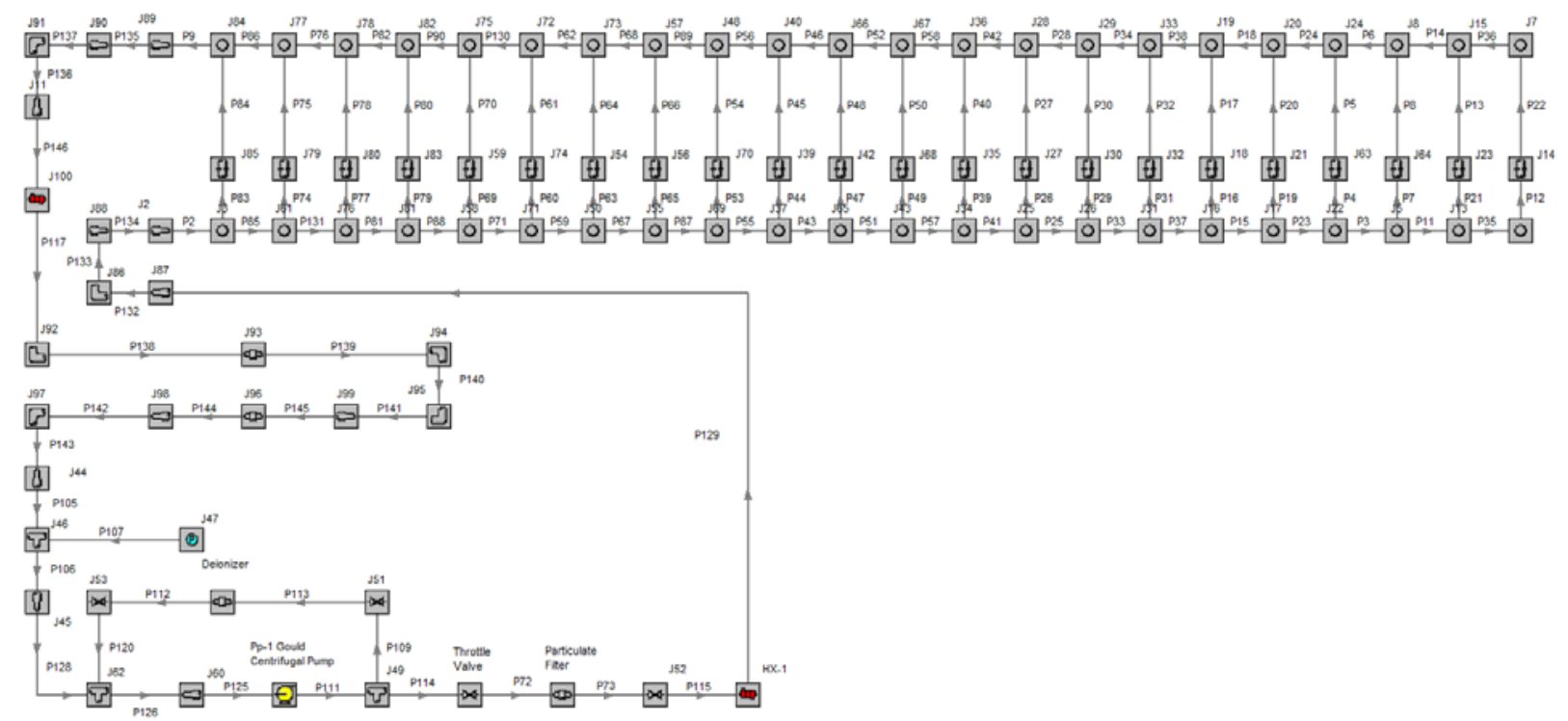

Figure 3 FATHOM Model 
Calculation No.: $\quad$ NE-CALC-2015-03 $\quad$ Rev. $1 \quad$ Page 11 of 12

APPENDIX 1

GENERAL CHECKING CRITERIA SHEET

\begin{tabular}{|c|c|c|c|c|}
\hline CALCULATION CHECKLIST & Yes & No & N/A & Comments \\
\hline $\begin{array}{l}\text { 1. Are analytical methods } \\
\text { appropriate? }\end{array}$ & & & & \\
\hline 2. Are assumptions appropriate? & $\triangle$ & & & \\
\hline 3. Is the calculation complete? & 7 & & & \\
\hline $\begin{array}{l}\text { 4. Are formulas appropriately } \\
\text { referenced? }\end{array}$ & & & & \\
\hline 5. Are the input data appropriate? & $\square$ & & & \\
\hline $\begin{array}{l}\text { 6. Was utilized software appropriate } \\
\text { for the task? }\end{array}$ & & & & \\
\hline $\begin{array}{l}\text { Were software input/initial } \\
\text { conditions/properties/boundary } \\
\text { conditions appropriate? }\end{array}$ & & & & \\
\hline 8. Are the results reasonable? & 7 & & & \\
\hline
\end{tabular}




$\begin{array}{ll}\text { From: } & \text { Bailey, James L. } \\ \text { To: } & \text { Guarino, Victor } \\ \text { Cc: } & \text { Grudzinski, James J.; Strons, Philip S. } \\ \text { Subject: } & \text { Response to comments NE-003 } \\ \text { Date: } & \text { Tuesday, April 25, 2017 11:45:33 AM } \\ \text { Attachments: } & \text { NE-CALC-2015-03 Revised-2.docx }\end{array}$

Vic,

See below for my response to your comments from NE003.

Jim Bailey

Engineer

Nuclear Engineering Division

Argonne National Laboratory

In reference to comments for NE-003:

Comment; "Several places it is stated that turbulent flow is assumed. The pipe and flow rates are known so why assume and why not just calculate Re?"

Reply; I have found two places in the report were "assumed turbulent" is stated:

1. "In order to develop the complete resistance curve the flow through the strainer was assumed turbulent, and therefore, the pressure drop was assumed proportional to the velocity squared."

The flow condition through a mesh or screen is uncertain, particularly near the entrance where flow is not fully developed. Here Re\# is rather useless. E.g. flow through a HEPA filter goes laminar. The assumption of turbulence was used because it is conservative considering pressure drop. Note that the manufacturer has provided a test data point near the design flow that is used for development of the resistance curve. Also, the pressure drop is small compared to the overall system resistance.

2. "In order to develop the complete resistance curve the flow through the heat exchanger was assumed turbulent, and therefore, the pressure drop was assumed proportional to the velocity squared."

The heat exchanger is a standard catalog item with performance specified at only the design condition. The internal design of the exchanger is not known, and therefore, a Re\# cannot be calculated. Further, it is almost a certainty that the flow in the exchanger is turbulent in order to provide a reasonable heat transfer coefficient. Here again, the single design point given by the manufacturer was used to develop the full resistance curve.

Comment; "I think it should be made clearer that the flow rates have been confirmed by tests." 
Reply; There are three places in this Calc Note that state that the flow rates have been confirmed by tests:

1. Section 3; "The hydraulic performance through the DU target assembly is not part of this calculation note and was determined by flow tests on the actual target as reported in reference 8 . The flow resistances as determined by these test were input to this calculation note."

2. Section 5; "Flow characteristics as determined from the hydraulic tests on the DU Target Assembly (Reference 8) are used as input to the AFT FATHOM overall thermal hydraulic model."

3. Section 7; "The hydraulic performance was performed considering a 5.8psid [9.0psid] from inlet plenum to outlet plenum at the flow rates indicated below and as determined from the flow tests that were performed on the actual target (Reference 8)."

Also, Reference 8 is the complete flow test report and is provided in the appendix.

I believe this to be enough clarity.

Also, I have corrected an error in the numbering of the Figures. Attached is the revised Calc Note. 
Thermal-Hydraulic Analysis of the Overall Performance of the DU Target Cooling System

Appendix 2

Reference 1

Calculation Note: NE-CALC-2015-05

Reference 2

Email from the chiller manufacturer verifying performance characteristics

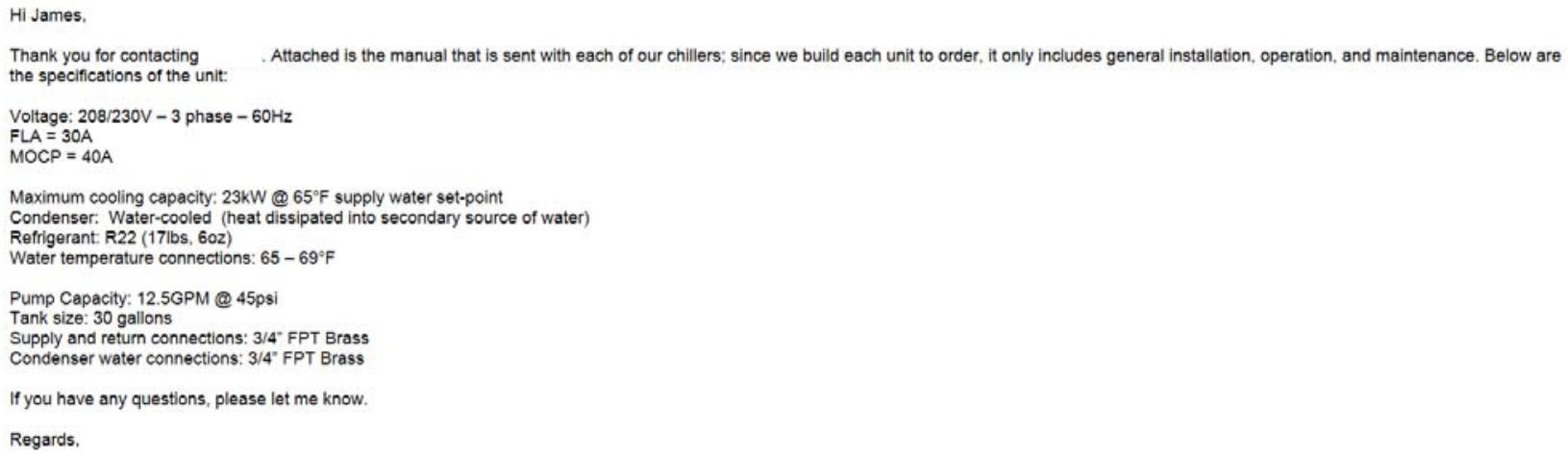




\section{Reference 3}

\section{Gould pump information (Small pump)}

Good afternoon Jim,

Please see the quotation below per your request. If you have any questions or need any additional assistance please let me know!

Reference Quotation Number: UQ0414308

QTY(1) 1SS1H4A0, Goulds model ICS

- $1 \times 1-1 / 4-5$

- $316 \mathrm{SS}$

- 3HP, 3500RPM

- $60 \mathrm{~Hz} / 1 \mathrm{PH} / \mathrm{TEFC}$

- 5-3/8" Impeller Diameter

- Carbon/Silicon Carbide/Viton Mechanical Seal

- Your net price each:

- Typically ships in about 7-10 Days

*Prices quoted are net*

${ }^{*}$ Quotation is valid for 30 Days*

*Freight: Pre-Pay \& Add or Collect, FOB: Shipping Point*

* standard terms \& conditions apply* 


\section{(Large pump)}

Attn: ACCOUNTS PAYABLE

Requested By: Jim Bailey

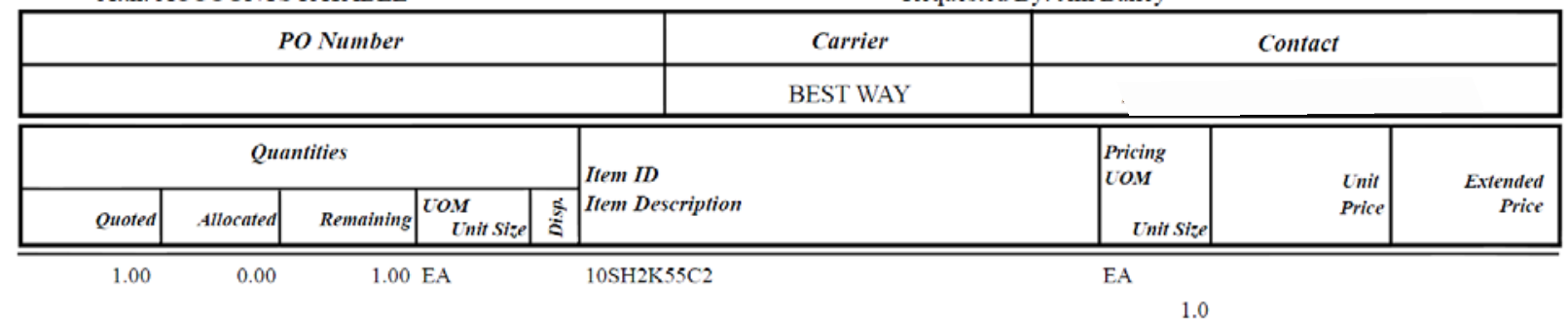




\section{Reference 4}

Plate heat exchanger information

Quotation No. SA 2014-4-29-10:04

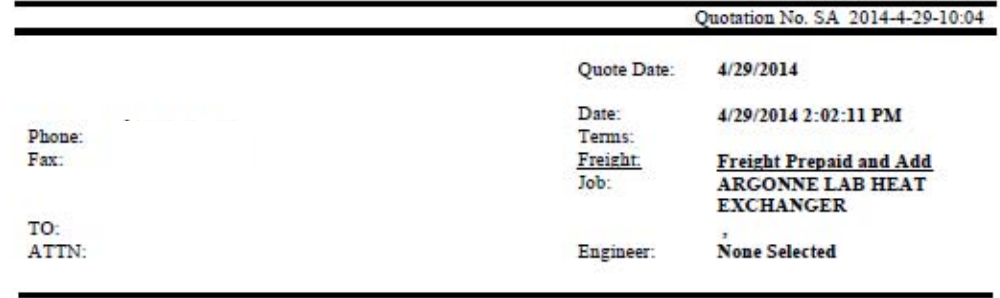

We are pleased to quote you on the following equipment for the above job subject to approval. Quantities listed are not guaranteed and should be verified. Prices will be adjusted accordingly. This quotation is subject to change without notice and void after 30 days unless otherwise stated below. All Contracts or Orders are subject to acceptance by the Company and are contingent upon nonoccurrence of strikes or other delays beyond their control. In addition to prices named herein, you are to pay any applicable sales taxes.

\begin{tabular}{c|l|c|c|c}
\hline Qty & Description \& Tag & $\begin{array}{c}\text { Wt } \\
\text { (lbs) }\end{array}$ & $\begin{array}{c}\text { Net } \\
\text { Price } \\
\text { Ea. }\end{array}$ & $\begin{array}{c}\text { Total Net } \\
\text { Price }\end{array}$ \\
1 & $\begin{array}{l}\text { BRAZED PLATE HEAT } \\
\text { EXCHANGERS }\end{array}$ & $\begin{array}{l}\text { B\&G Model - BPDW415 - 92 Plate Heat } \\
\text { Exchanger - Consisting of a Brazed Pack Unit with } \\
\text { 92 Plates. Thermal Plates are SA240 S31603 X } \\
\text { 0.0157 in. the This unit has the following } \\
\text { connections: Port-1: 1" NPT Male Thread, Port-2: } \\
\text { 1" NPT Male Thread, Port-3: 1" NPT Male Thread, } \\
\text { Port-4: 1" NPT Male Thread, Working Pressure: } \\
\text { 435 psig, Mounting Options: ASME CODE: NO. } \\
\text { Hot Side: 50 GPM of Water from 67 F to 65 F } \\
\text { with 10 psi pressure drop; Cold Side: 10 GPM of } \\
\text { Water from 55 F to 65 F. }\end{array}$ & & \\
$\begin{array}{l}\text { Total BRAZED PLATE HEAT } \\
\text { EXCHANGERS }\end{array}$ & & & \\
\end{tabular}




\section{Reference 5}

\section{Particle filter information 2" Model 72 Simplex}

Jim,

Understand your question , but I decided years ago to standardize their $1 / 2$ " through $2^{\prime \prime}$ sizes around a 600 ANSI class to cover most steam

applications. There is no lower pressure version offered, and the initial request was for the $Y$-strainer so the model 85 was quoted, however, we can certainly go to a basket strainer , model 72 Simplex that would have a $0.7 \mathrm{psi}$ initial clean drop at $50 \mathrm{gpm}$ fitted with a 60 mesh screen. Reason is that a 2 " model 72 Simplex basket has about $51 \mathrm{sq}$. in. of gross screen area whereas the $Y$-strainer has 30.4 . The model 72 is also easier for maintenance. Only drawback is that it's not standardly offered with socket weld connections.

I'm assuming the pipe run is horizontal, necessary for the model 72.

The part \# for the 316 SS 2" NPT \#72 is ST0720200T2C...... 200 PSIG @ 100 deg. F, 2" NPT, Viton seal and includes one 316 SS 60 mesh lined basket................\$ 991.00 net each, same 3 to 5 day lead time to ship.

Support data attached. The appropriate curve for the 72 is at the bottom half of page one of the 'Curves' attachment. 


\section{Reference 6}

\section{Input to the components in the FATHOM Model}

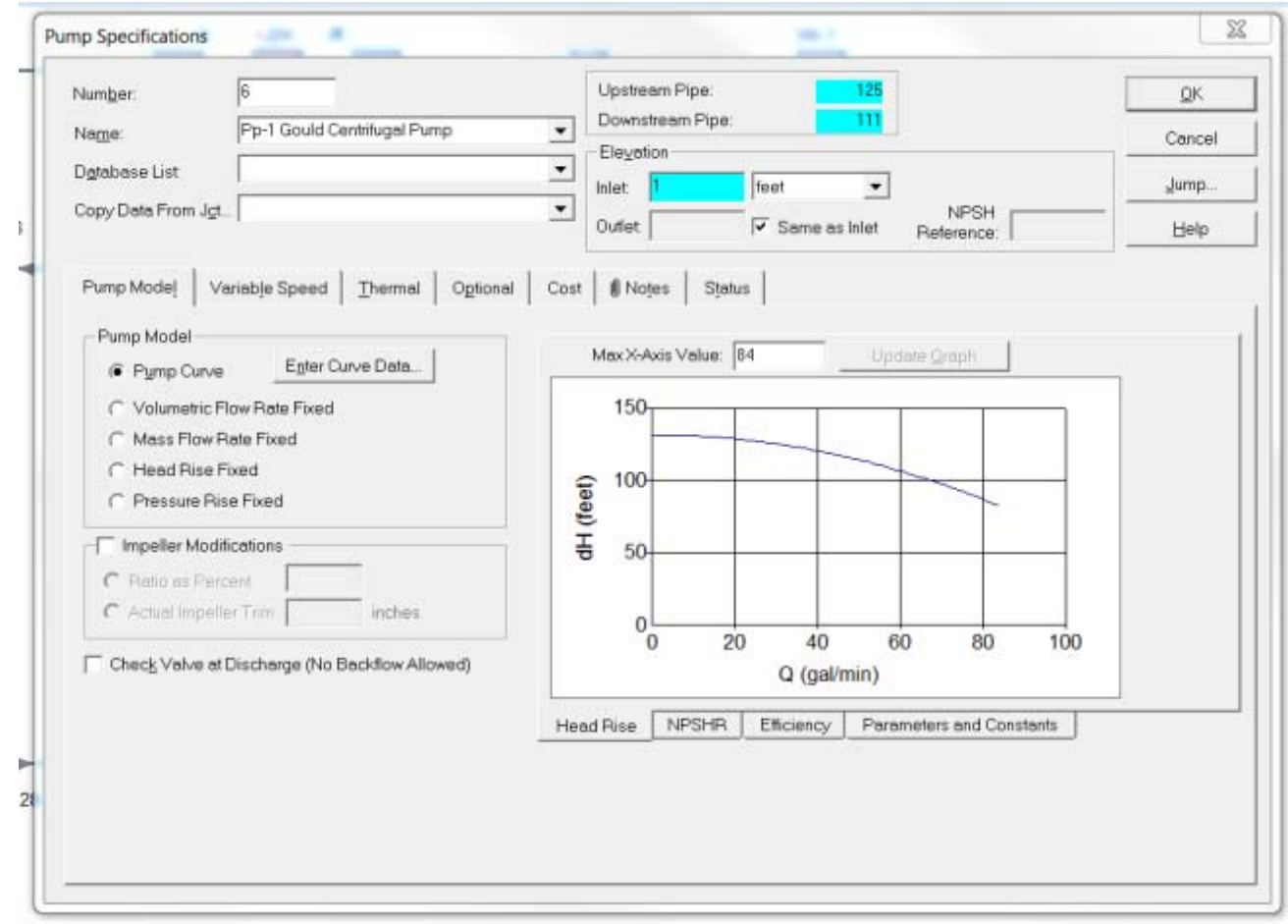

Pump curve

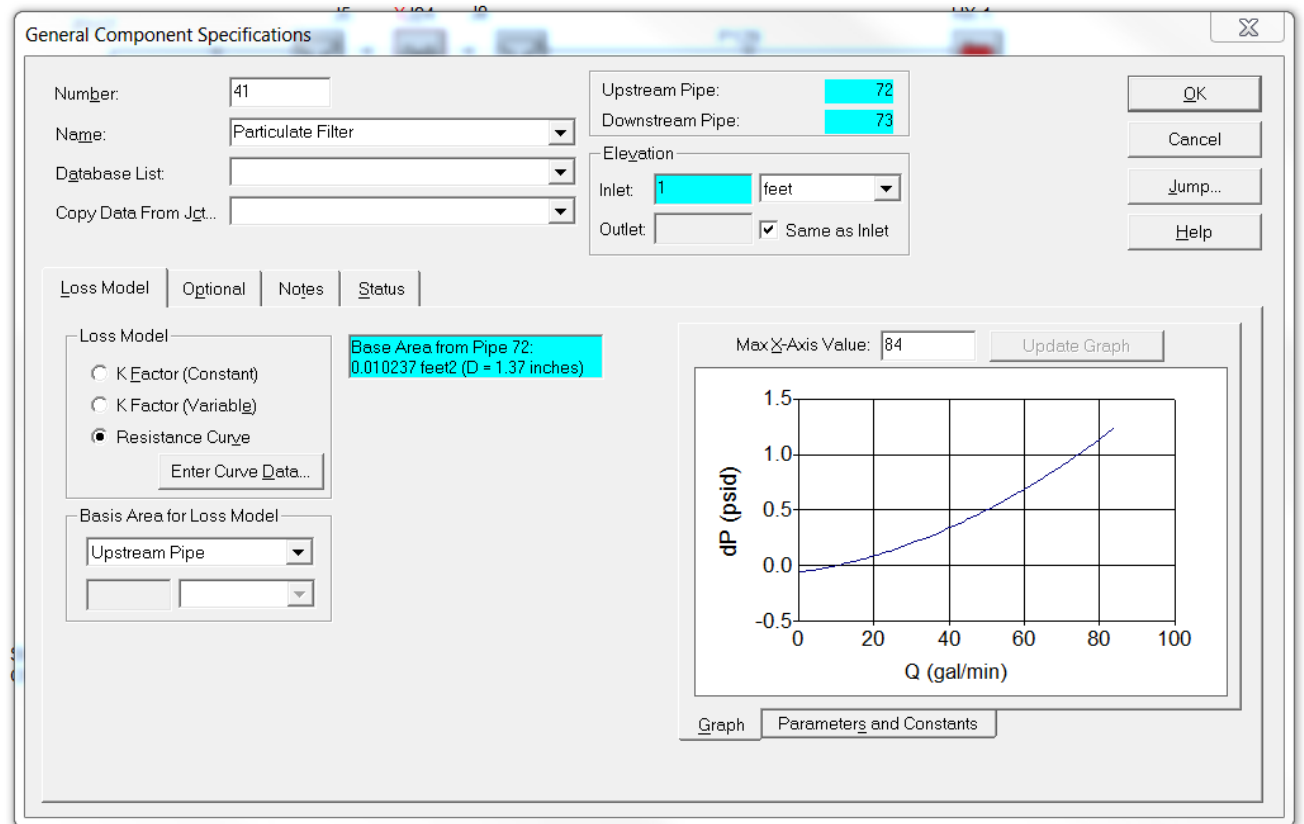

Pressure drop curve for the Particle Filter 


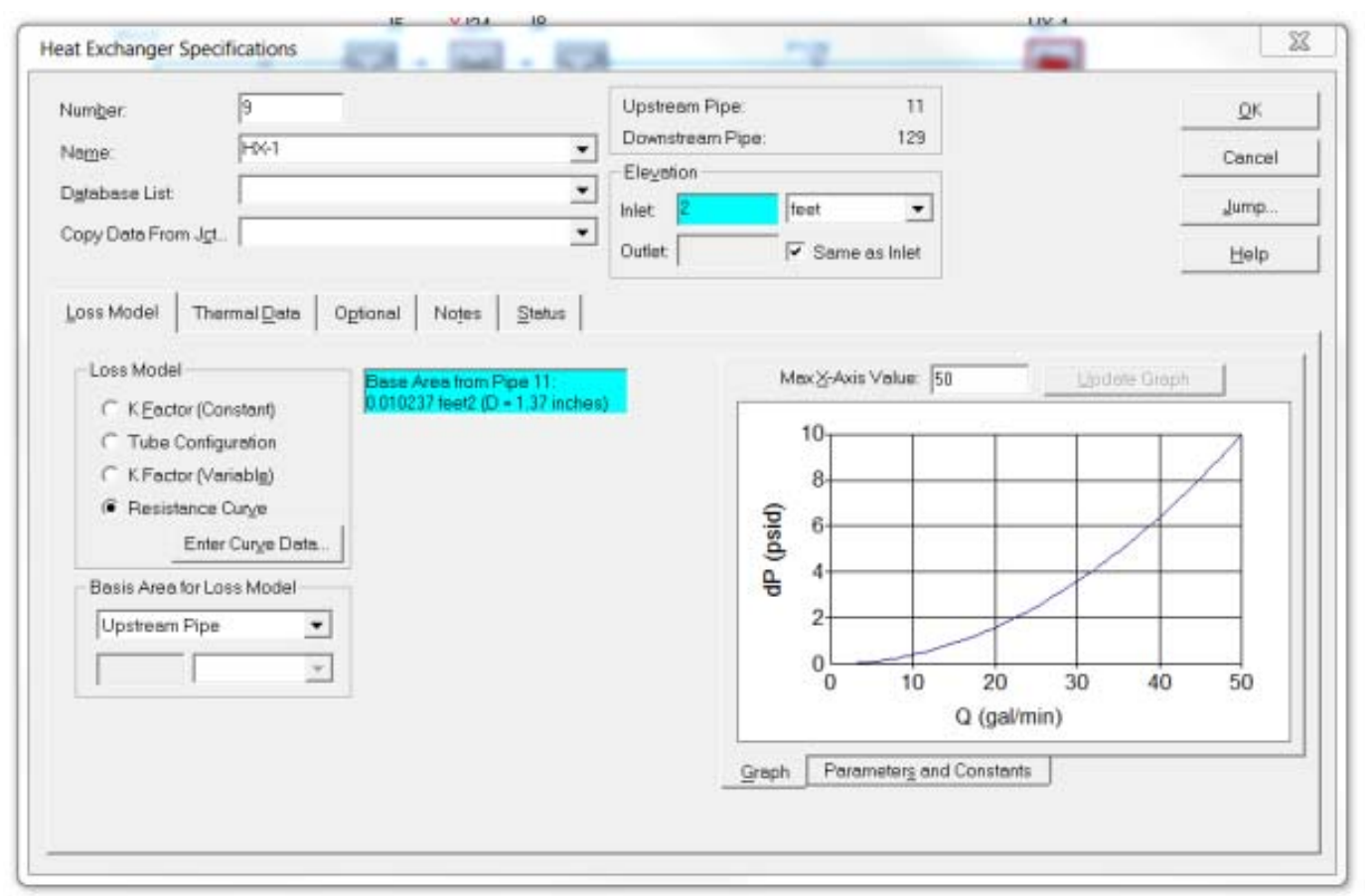

Heat Exchanger Hydraulic Performance Curve

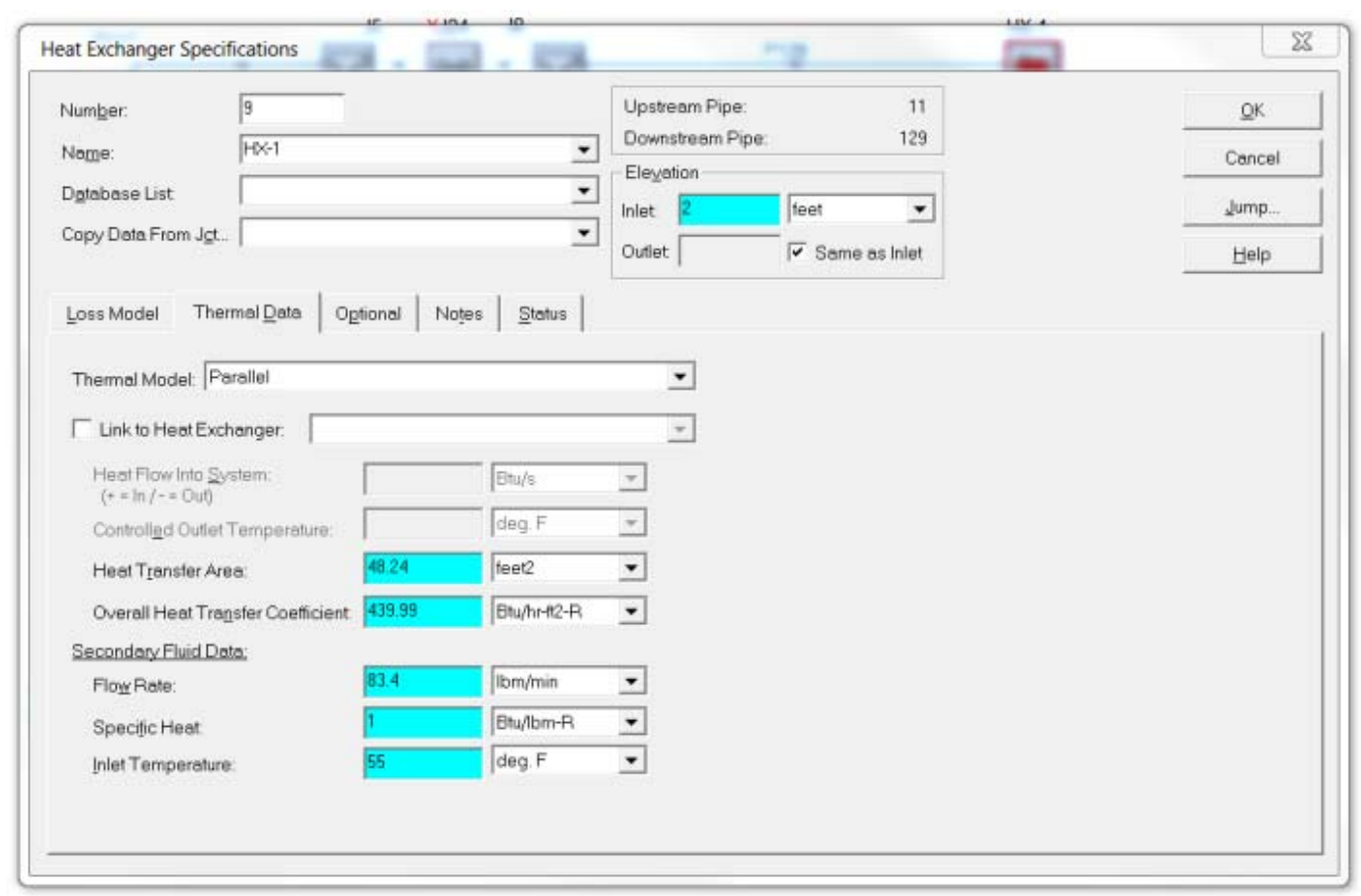

Heat Exchanger Thermal Performance 


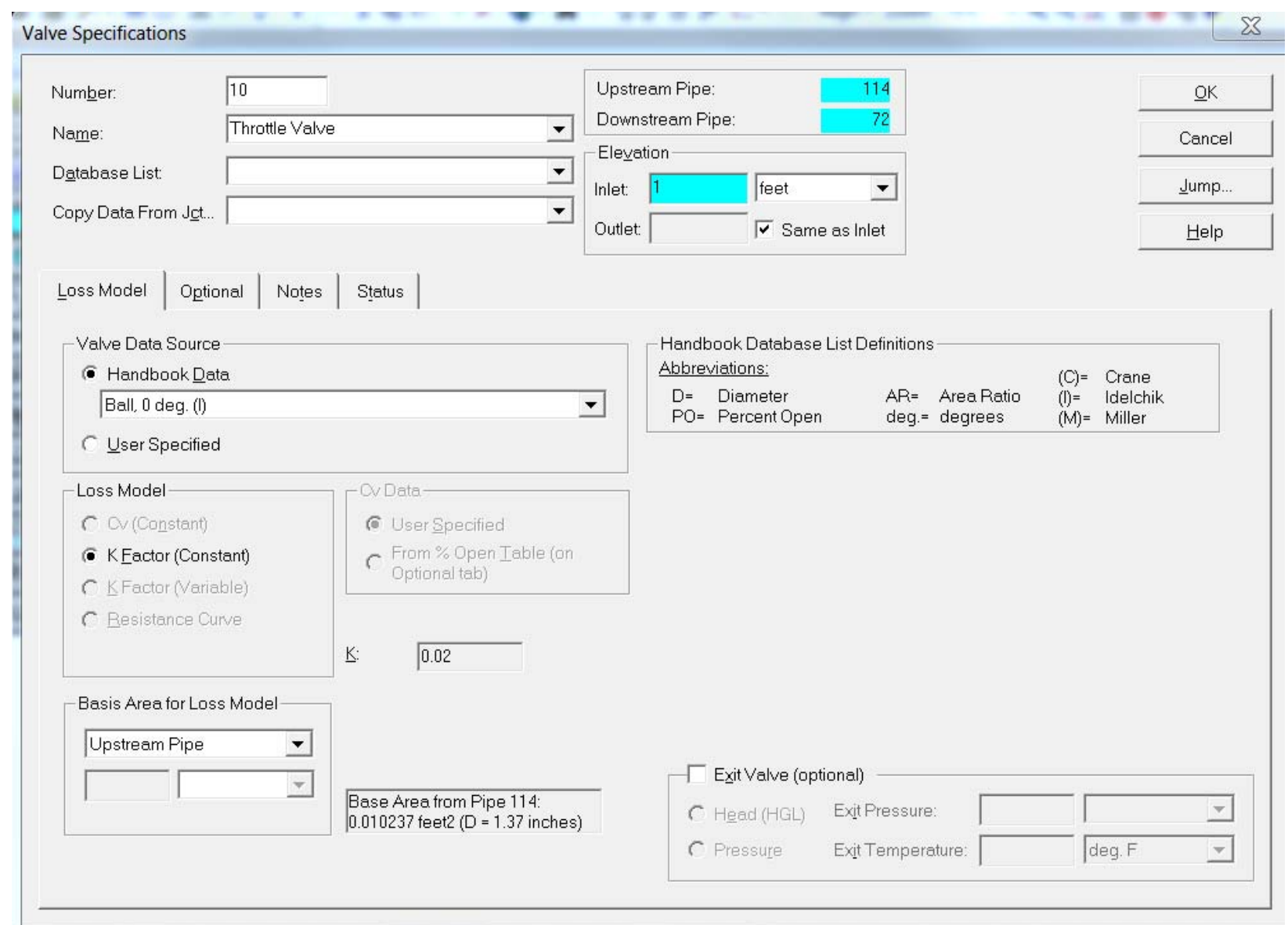

Throttle Valve at Discharge of Pump

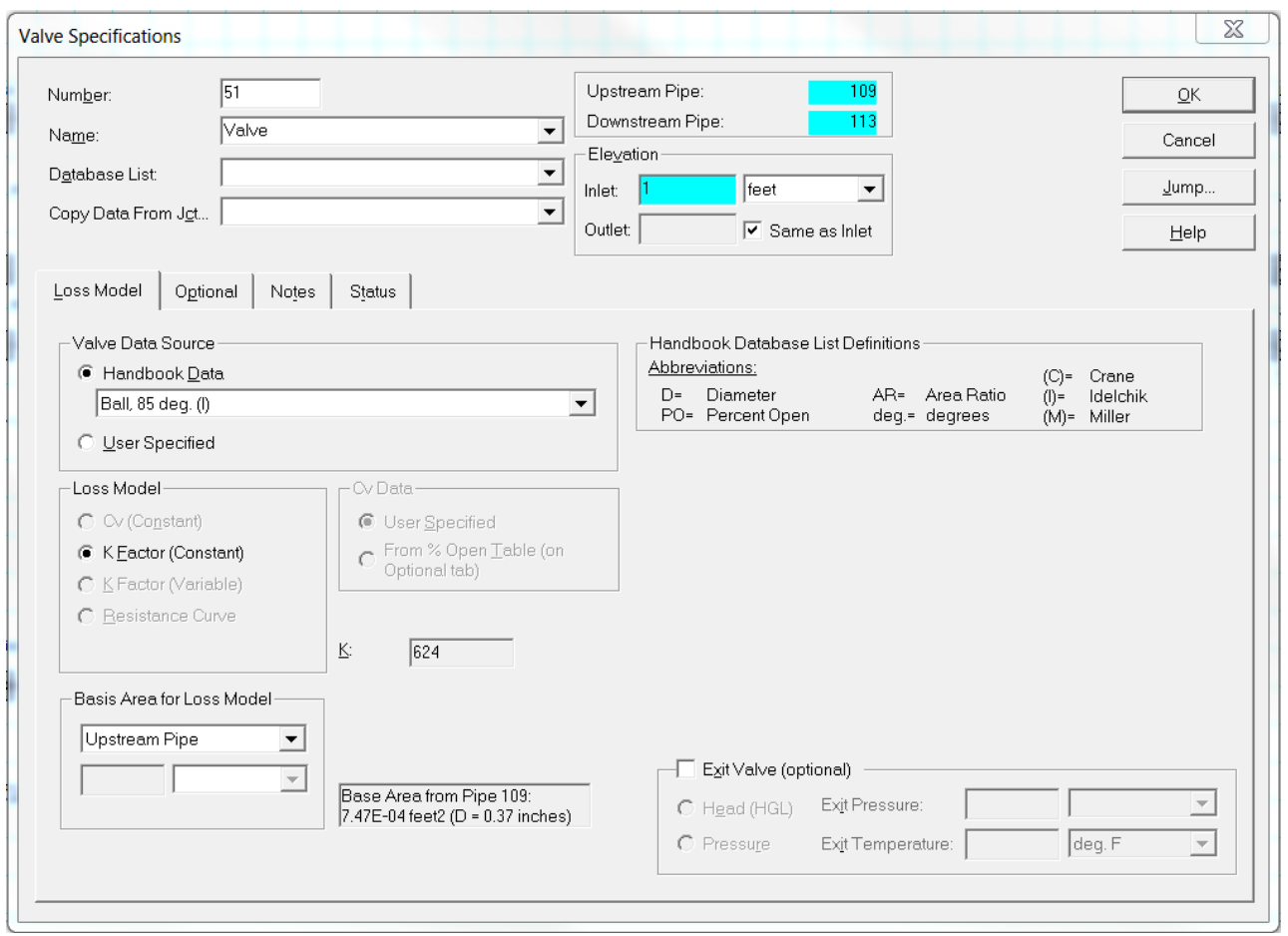

Valve in the DI Bypass Line for Throttling Flow through the DI Unit 


\section{Reference 7}

Results from the FATHOM Model (Small pump) (Note that the input for tube size and lengths are also noted here)

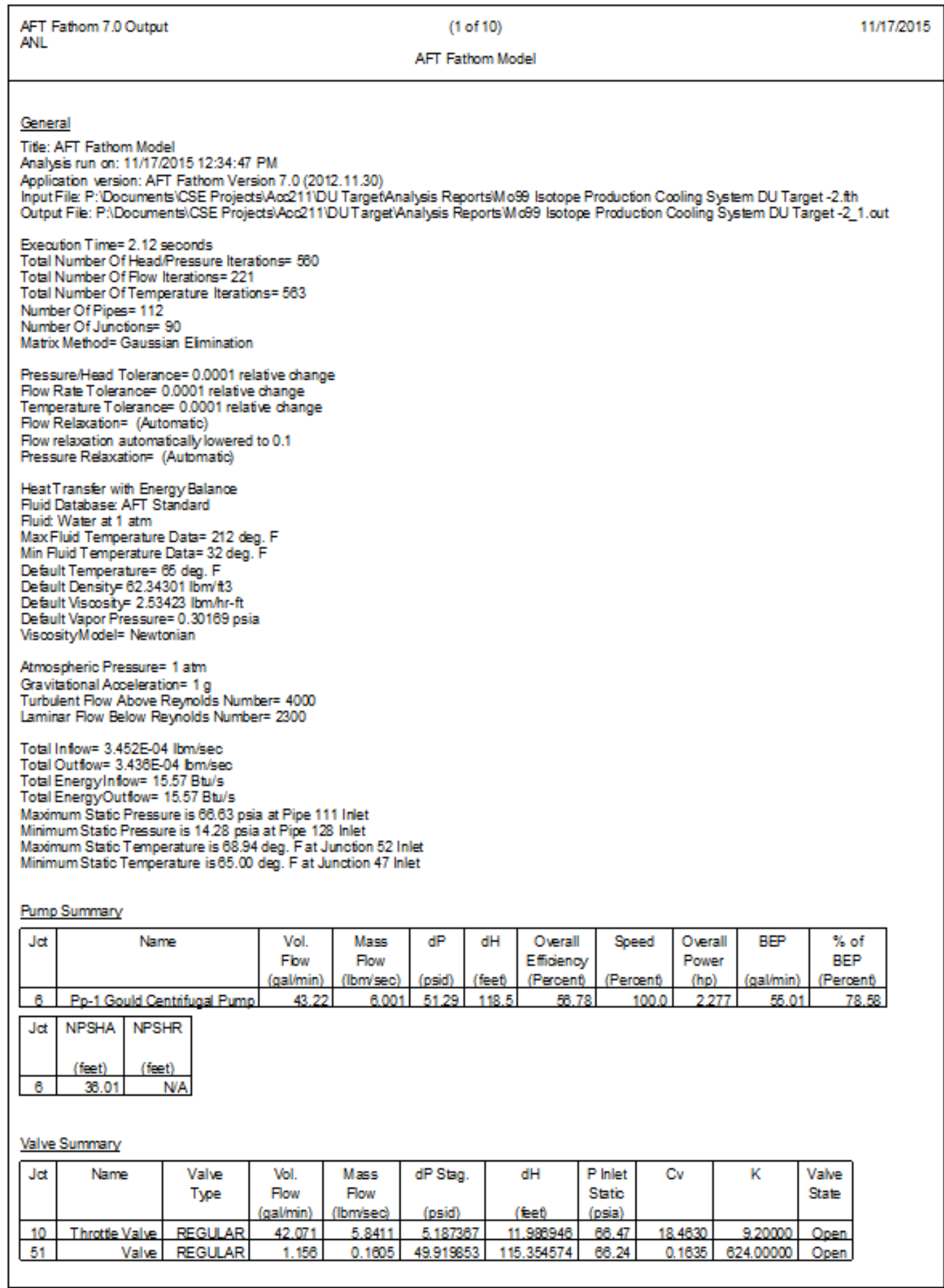


AFT Fathom Model

\begin{tabular}{|c|r|r|r|r|r|r|r|r|r|r|}
\hline Jat & Name & $\begin{array}{c}\text { Valve } \\
\text { Type }\end{array}$ & $\begin{array}{c}\text { Vol. } \\
\text { Flow } \\
\text { (galimin) }\end{array}$ & $\begin{array}{c}\text { Mass } \\
\text { Flow } \\
\text { (lbmised) }\end{array}$ & dP Stag. & dH & $\begin{array}{c}\text { P Inlet } \\
\text { Static } \\
\text { (psid) }\end{array}$ & Cv & $\begin{array}{c}\text { K feed } \\
\text { (psia) }\end{array}$ & $\begin{array}{c}\text { Valve } \\
\text { State }\end{array}$ \\
\hline 52 & Valve & REGULAR & 42.071 & 5.8411 & 0.011277 & 0.028059 & 60.73 & 395.9863 & 0.02000 & Open \\
\hline 53 & Valve & REGULAR & 1.156 & 0.1605 & 0.001600 & 0.003697 & 16.13 & 28.8830 & 0.02000 & Open \\
\hline
\end{tabular}

Heat Exchanger Summary

\begin{tabular}{|c|c|c|c|c|c|c|c|c|c|c|c|}
\hline Jat & Name & $\begin{array}{c}\text { Vol } \\
\text { Flow } \\
\text { (galimin) }\end{array}$ & $\begin{array}{c}\text { Mass } \\
\text { Flow } \\
\text { (lbm/sec) }\end{array}$ & $\begin{array}{c}d P \\
\text { (psid) }\end{array}$ & $\begin{array}{l}\mathrm{dH} \\
\text { (feet) }\end{array}$ & $\begin{array}{c}\text { dT } \\
\text { Loss } \\
\text { (deg. F) }\end{array}$ & $\begin{array}{l}\text { Heat } \\
\text { Rate In } \\
\text { (Btu/s) }\end{array}$ & $\begin{array}{c}\text { T } \\
\text { Inlet } \\
\text { (deg. F ) }\end{array}$ & $\begin{array}{c}\text { T } \\
\text { Outlet } \\
\text { (deg. F) }\end{array}$ & $\begin{array}{l}\text { T 2nd } \\
\text { Inlet } \\
\text { (deg. F) }\end{array}$ & $\begin{array}{l}\text { T 2nd } \\
\text { Outet } \\
\text { (deq. F) }\end{array}$ \\
\hline 9 & & & 5.841 & & 18.36 & & & & & 55.00 & 68.20 \\
\hline 92 & Heat Generafion & 42.06 & 5.841 & 0.000 & 0.00 & -2598 & 15.18 & 6633 & 68.93 & $N / A$ & 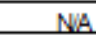 \\
\hline
\end{tabular}

Pipe Output Table

\begin{tabular}{|c|c|c|c|c|c|c|c|c|c|c|}
\hline Pipe & Name & $\begin{array}{l}\text { Vol } \\
\text { Flow Rate } \\
\text { (galimin) }\end{array}$ & $\begin{array}{l}\text { Vebcity } \\
\text { (Eetsec) }\end{array}$ & $\begin{array}{l}\text { dP Stag. } \\
\text { Total } \\
\text { (psid) }\end{array}$ & $\begin{array}{c}\text { dP Static } \\
\text { Total } \\
\text { (psid) }\end{array}$ & $\begin{array}{c}\text { P Static In } \\
\text { (psig) }\end{array}$ & $\begin{array}{c}\text { P Stafc Out } \\
\text { (psig) }\end{array}$ & $\begin{array}{l}\text { P Stag. In } \\
\text { (psig) }\end{array}$ & $\begin{array}{c}\text { P Stzg. } \\
\text { Out } \\
\text { (psig) }\end{array}$ & $\begin{array}{c}\text { T } \\
\text { Inlet } \\
\text { (deg, F) }\end{array}$ \\
\hline 2 & Pipe & 42.058 & 22.04849 & 0.02150172 & 0.02150172 & 20.49501419 & 20.47351456 & 23.76532745 & 23.7438 & 68.27 \\
\hline 4 & Pipe & 1.710 & 0.89628 & 0.00004868 & 0.00004868 & 23.21108246 & 2321103688 & 2321648788 & 23.2164 & 66.31 \\
\hline 5 & Pipe & 1.710 & 7.52124 & 0.39702490 & 0.39702490 & 17.91522598 & 17.51819992 & 1829577255 & 17.8987 & 68.31 \\
\hline 6 & Pipe & 14.857 & 7.78876 & 0.00377939 & 0.00377939 & 17.49442873 & 17.49064636 & 17.90252686 & 17.8987 & 66.32 \\
\hline 8 & Pipe & 4.851 & 2133848 & 2.45297241 & 2.45297241 & 17.59873962 & 15.14576721 & 20.68180 .801 & 18.2088 & 68.31 \\
\hline 9 & Pipe & 42.058 & 22.04882 & 0.02138883 & 0.02138883 & 14.10114479 & 14.07975578 & 17.37147522 & 17.3501 & 66.33 \\
\hline 11 & Pipe & 10.007 & 5.24593 & 0.00188894 & 0.00188694 & 23.02758408 & 23.02569580 & 23.21271133 & 23.2108 & 66.31 \\
\hline 12 & Pipe & 5.003 & 2.62282 & 0.00050047 & 0.00050047 & 23.16398239 & 23.16348267 & 23.21025848 & 23.2098 & 66.31 \\
\hline 13 & Pipe & 5.004 & 22.01215 & 2.59189963 & 2.59189963 & 17.23678589 & 14.64488002 & 20.49631500 & 17.9044 & 68.31 \\
\hline 14 & Pipe & 10.007 & 5.24593 & 0.00188802 & 0.00188692 & 17.71928787 & 17.71739960 & 17.90441513 & 17.9025 & 66.32 \\
\hline 18 & Pipe & 18.272 & 9.57888 & 0.00544846 & 0.00544846 & 17.27691841 & 17.27147102 & 17.89418504 & 17.8887 & 66.32 \\
\hline 19 & Pipe & 1.705 & 0.89383 & 0.00004865 & 0.00004855 & 23.21569443 & 23.21564865 & 23.22106934 & 23.2210 & 68.31 \\
\hline 20 & Pipe & 1.705 & 7.50071 & 0.39516491 & 0.39516491 & 17.94870377 & 17.55353928 & 18.32717896 & 17.9320 & 68.31 \\
\hline 21 & Pipe & 5.004 & 311 & 0.00050067 & 0.00050057 & 23.16453552 & 3580 & 23.21082306 & 23.2103 & 66.31 \\
\hline 22 & Pipe & 5.003 & 22.00967 & 2.59143424 & 2.59143424 & 17.23756790 & 14.64613342 & 20.49638078 & 17.9049 & 66.31 \\
\hline 23 & Pipe & 16.587 & 868503 & 0.00458131 & 0.00458131 & 22.71364212 & 22,70906067 & 23.22106934 & 23.2165 & 66.31 \\
\hline 24 & Pipe & 16.567 & 8.68504 & 0.00458115 & 0.00458115 & 17.39131927 & 17.38673782 & 17.89874649 & 17.8942 & 66.32 \\
\hline 25 & Pipe & 23.399 & 12.26854 & 0.00845415 & 0.00845415 & 22.23652267 & 22.22806931 & 23.24874115 & 23.2403 & 66.30 \\
\hline 26 & Pipe & 1.714 & 0.89851 & 0,00004881 & 0.00004881 & 23.24330902 & 23.24326324 & 23.24874115 & 23.2487 & 66.30 \\
\hline 27 & Pipe & 1.714 & 7.53997 & 0.39874700 & 0.39874700 & 17.92104340 & 17.52229309 & 18.30348969 & 17.9047 & 66.30 \\
\hline 28 & Pipe & 23.399 & 12.26657 & 0.00845357 & 0.00846357 & 16.86273003 & 16.85427066 & 17.87496041 & 17.8665 & 66.32 \\
\hline 37 & Pipe & 19.979 & 10.47382 & 0.00638385 & 0.00838385 & 22.49495897 & 22,48857498 & 23.23290253 & 23.2285 & 68.30 \\
\hline 38 & Pipe & 19.979 & 10.47364 & 0.00638331 & 0.00638331 & 17.15077209 & 17.14439011 & 17.88871765 & 17.8823 & 66.32 \\
\hline 39 & Pipe & 1.717 & 0.90013 & 0.00004890 & 0.00004890 & 23.25288010 & 23.25283061 & 23.25833130 & 23.2583 & 66.30 \\
\hline 40 & Pipe & 1.717 & 7.55353 & 0.39998824 & 0.39998624 & 17.91145325 & 1751146898 & 18.29527684 & 17.8963 & 68.30 \\
\hline 41 & Pipe & 25.113 & 13.16505 & 0.00958904 & 0.00958904 & 22.09239197 & 22.08280182 & 23.25833130 & 23.2487 & 66.30 \\
\hline
\end{tabular}




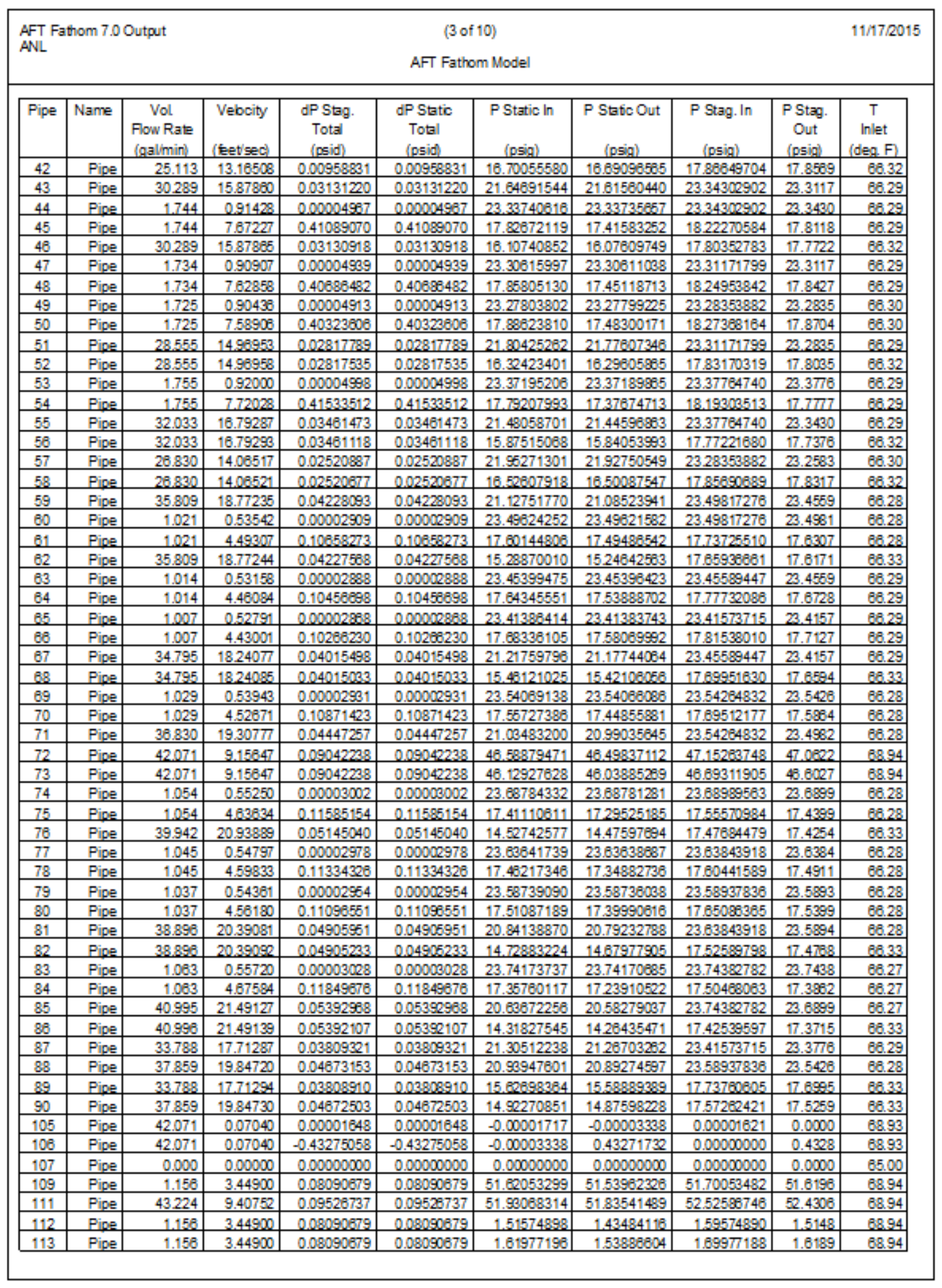




\begin{tabular}{|lcr}
\hline $\begin{array}{l}\text { AFT Fathom } 7.0 \text { Output } \\
\text { ANL }\end{array}$ & $(4$ of 10$)$ & $11 / 17 / 2015$ \\
& AFT Fathom Model &
\end{tabular}

\begin{tabular}{|c|c|c|c|c|c|c|c|c|c|c|}
\hline Pipe & Name & $\begin{array}{c}\text { Vol } \\
\text { Flow Rate } \\
\text { (galimin) }\end{array}$ & $\begin{array}{l}\text { Vebcity } \\
\text { (Eetisec) }\end{array}$ & $\begin{array}{c}\text { dP Stag. } \\
\text { Total } \\
\text { (psid) }\end{array}$ & $\begin{array}{c}\text { dP Static } \\
\text { Total } \\
\text { (psid) }\end{array}$ & $\begin{array}{c}\text { P Static In } \\
\text { (psig) }\end{array}$ & $\begin{array}{c}\text { P Static Out } \\
\text { (psig) }\end{array}$ & $\begin{array}{c}\text { P Stag. In } \\
\text { (psig) }\end{array}$ & $\begin{array}{l}\text { P Stag. } \\
\text { Out } \\
\text { (psig) }\end{array}$ & $\begin{array}{c}\mathrm{T} \\
\text { Inlet } \\
\text { (deg. F) }\end{array}$ \\
\hline 114 & Pipe & 42.071 & 9.15647 & 0.09042238 & 0.09042238 & 51.86858096 & 51.77616501 & 52.43042374 & 52.3400 & 68.94 \\
\hline 115 & Pipe & 42.071 & 9.15647 & 0.52317375 & 0.52317375 & 46.02757645 & 45.50440216 & 46.59141922 & 48.0682 & 68.94 \\
\hline 117 & Pipe & 42.071 & 9.15648 & 5.30923080 & 5.30923080 & 5.30060959 & -0.00862026 & 5.86445236 & 0.5562 & 68.93 \\
\hline 120 & Pipe & 1.156 & 3.44900 & 0.08189785 & 0.08189765 & 1.43324089 & 1.35134315 & 1.51324081 & 1.4313 & 68.94 \\
\hline 125 & Pipe & 43.224 & 9.27167 & 0.10084618 & 0.10084618 & 0.75816154 & 0.65731525 & 1.33628273 & 1.2354 & 68.94 \\
\hline 126 & Pipe & 43.224 & 9.40752 & 0.09493705 & 0.09493705 & 0.83815685 & 0.74122047 & 1.43134308 & 1.3384 & 68.94 \\
\hline 128 & Pipe & 42.071 & 9.15648 & -1.27888775 & -1.27888775 & -0.41138744 & 0.86749935 & 0.15245628 & 1.4313 & 68.93 \\
\hline 129 & Pipe & 42.058 & 9.15375 & 1.86873355 & 1.86873355 & 38.42479706 & 36.55806351 & 38.98847580 & 37.1217 & 66.27 \\
\hline 130 & Pipe & 36.830 & 19.30788 & 0.04446871 & 0.04448671 & 15.10926437 & 15.08479845 & 17.61709213 & 17.5726 & 68.33 \\
\hline 131 & Pipe & 39.941 & $20.938 \pi$ & 0.05145827 & 0.05145827 & 20.74049377 & 20.68903732 & 23.68998563 & 23.6384 & 68.28 \\
\hline 132 & Pipe & 42.058 & 19.86438 & 1.18063545 & 1.18063545 & 34.17090607 & 32.99026871 & 36.82539388 & 35.6448 & 68.27 \\
\hline 133 & Pipe & 42.058 & 19.88438 & 4.72254181 & 4.72254181 & 31.73947525 & 27.01693344 & 34.39396286 & 29.6714 & 68.27 \\
\hline 134 & Pipe & 42.058 & 28.45761 & 0.11757526 & 0.11757526 & 23.88290405 & 23.76533127 & 2933079529 & 29.2132 & 86.27 \\
\hline 135 & Pipe & 42.058 & 28.45777 & 0.11755820 & 0.11756820 & 8.63183403 & 8.51427460 & 14.07975578 & 13.9622 & 66.33 \\
\hline 136 & Pipe & 42.058 & 19.86447 & 1.18045437 & 1.18045437 & 5.00078773 & 3.82033348 & 7.65529060 & 6.4748 & 66.33 \\
\hline 137 & Pipe & 42.058 & 19.86447 & 4.72181749 & 4.72181749 & 10.97340775 & 6.25159073 & 13.62791061 & 8.9081 & 68.33 \\
\hline 138 & Pipe & 42.058 & 9.15381 & 0.09108785 & 0.09108785 & 5.39183998 & 5.300777171 & 5.95552083 & 5.8845 & 66.33 \\
\hline
\end{tabular}

\begin{tabular}{|c|c|c|c|c|}
\hline Pipe & $\begin{array}{c}\text { T } \\
\text { Outet } \\
\text { (deg. F) }\end{array}$ & $f$ & $\begin{array}{l}\text { Length } \\
\text { (feet) }\end{array}$ & $\begin{array}{l}\text { Hyd. } \\
\text { Diameter } \\
\text { (nches) }\end{array}$ \\
\hline 2 & 6627 & 0.02124 & 0.008333 & 0.32467 \\
\hline 3 & 68.31 & 0.02861 & 0.009417 & 0.32467 \\
\hline 4 & 66.31 & 0.02925 & 0.008333 & 0.32467 \\
\hline 5 & 66.31 & 0.03985 & 0.166687 & 0.07641 \\
\hline 6 & 68.32 & 0.02861 & 0.009417 & 0.32467 \\
\hline 7 & 68.31 & 0.03541 & 0.008333 & 0.32467 \\
\hline 8 & 66.31 & 0.03059 & 0.166687 & 0.07641 \\
\hline 9 & 66.33 & 0.02123 & 0.008333 & 0.32467 \\
\hline 11 & 66.31 & 0.02929 & 0.009417 & 0.32467 \\
\hline 12 & 66.31 & 0.03511 & 0.008333 & 0.32467 \\
\hline 13 & 66.31 & 0.03038 & 0.166687 & 0.07641 \\
\hline 14 & 68.32 & 0.02929 & 0.009417 & 0.32467 \\
\hline 15 & 66.31 & 0.02536 & 0.009417 & 0.32467 \\
\hline 16 & 66.31 & 0.02930 & 0.008333 & 0.32467 \\
\hline 17 & 66.31 & 0.03987 & 0.166867 & 0.07641 \\
\hline 18 & 66.32 & 0.02536 & 0.009417 & 0.32467 \\
\hline 19 & 66.31 & 0.02933 & 0.008333 & 0.32467 \\
\hline 20 & 66.31 & 0.03988 & 0.166667 & 0.07641 \\
\hline 21 & 6831 & 0.03511 & 0.008333 & 0.32467 \\
\hline 22 & 66.31 & 0.03038 & 0.166667 & 0.07641 \\
\hline 23 & 66.31 & 0.02594 & 0.009417 & 0.32467 \\
\hline 24 & 6632 & 0.02594 & 0.009417 & 0.32467 \\
\hline 25 & 66.30 & 0.02400 & 0.009417 & 0.32467 \\
\hline 28 & 68.30 & 0.02918 & 0.008333 & 0.32467 \\
\hline 27 & 66.30 & 0.03983 & 0.166667 & 0.07641 \\
\hline 28 & 6632 & 0.02400 & 0.009417 & 0.32467 \\
\hline 29 & 66.30 & 0.02922 & 0.008333 & 0.32467 \\
\hline 30 & 66.30 & 0.03984 & 0.166667 & 0.07641 \\
\hline 31 & 6630 & 0.02926 & 0.008333 & 0.32467 \\
\hline 32 & 66.30 & 0.03986 & 0.166667 & 0.07641 \\
\hline 33 & 66.30 & 0.02440 & 0.009417 & 0.32467 \\
\hline 34 & 66.32 & 0.02440 & 0.009417 & 0.32467 \\
\hline 35 & 68.31 & 0.03511 & 0.009417 & 0.32467 \\
\hline 36 & 66.31 & 0.03511 & 0.009417 & 0.32467 \\
\hline 37 & 66.30 & 0.02485 & 0.009417 & 0.32467 \\
\hline 38 & 68.32 & 0.02485 & 0.009417 & 0.32467 \\
\hline
\end{tabular}




\begin{tabular}{|lcr|}
\hline $\begin{array}{l}\text { AFT Fathom 7.0 Output } \\
\text { ANL }\end{array}$ & $\begin{array}{c}(5 \text { of } 10) \\
\text { AFT Fathom Model }\end{array}$ & $11 / 17 / 2015$ \\
\hline
\end{tabular}

\begin{tabular}{|c|c|c|c|c|}
\hline Pipe & $\begin{array}{c}T \\
\text { Outet } \\
\text { (deg. } F\end{array}$ & $f$ & $\begin{array}{l}\text { Length } \\
\text { (feet) }\end{array}$ & $\begin{array}{c}\text { Hyd. } \\
\text { Diameter } \\
\text { (nches) }\end{array}$ \\
\hline 39 & 66.30 & 0.02913 & 0.008333 & 0.32467 \\
\hline 40 & 68.30 & 0.03981 & 0.168887 & 0.07641 \\
\hline 41 & 6630 & 0.02383 & 0.009417 & 0.32467 \\
\hline 42 & 68.32 & 0.02383 & 0.009417 & 0.32467 \\
\hline 43 & 68.29 & 0.02270 & 0.022000 & 0.32467 \\
\hline 44 & 68.29 & 0.02868 & 0.008333 & 0.32467 \\
\hline 45 & 68.29 & 0.03964 & 0.108687 & 0.07641 \\
\hline 46 & 66.32 & 0.02270 & 0.022000 & 0.32467 \\
\hline 47 & 68.29 & 0.02884 & 0.008333 & 0.32467 \\
\hline 48 & 68.29 & 0.03970 & 0.168887 & 0.07641 \\
\hline 49 & 68.30 & 0.02899 & 0.008333 & 0.32467 \\
\hline 50 & 68.30 & 0.03976 & 0.166687 & 0.07641 \\
\hline 51 & 68.29 & 0.02299 & 0.022000 & 0.32467 \\
\hline 52 & 66.32 & 0.02299 & 0.022000 & 0.32467 \\
\hline 53 & 68.29 & 0.02850 & 0.008333 & 0.32467 \\
\hline 54 & 68.29 & 0.03957 & 0.168887 & 0.07641 \\
\hline 55 & 68.29 & 0.02244 & 0.022000 & 0.32467 \\
\hline 56 & 68.32 & 0.02244 & 0.022000 & 0.32467 \\
\hline 57 & 66.30 & 0.02330 & 0.022000 & 0.32467 \\
\hline 58 & 86.32 & 0.02329 & 0.022000 & 0.32467 \\
\hline 59 & 68.28 & 0.02193 & 0.022000 & 0.32467 \\
\hline 60 & 68.28 & 0.04898 & 0.008333 & 0.32467 \\
\hline 61 & 68.28 & 0.02996 & 0.168687 & 0.07641 \\
\hline 62 & 68.33 & 0.02193 & 0.022000 & 0.32467 \\
\hline 63 & 68.29 & 0.04933 & 0.008333 & 0.32467 \\
\hline 64 & 66.29 & 0.02982 & 0.168887 & 0.07641 \\
\hline 85 & 68.29 & 0.04967 & 0.008333 & 0.32467 \\
\hline 66 & 68.29 & 0.02968 & 0.168687 & 0.07641 \\
\hline 67 & 68.29 & 0.02208 & 0.022000 & 0.32467 \\
\hline 68 & 68.33 & 0.02206 & 0.022000 & 0.32467 \\
\hline 69 & 68.28 & 0.04861 & 0.008333 & 0.32487 \\
\hline 70 & 68.28 & 0.03010 & 0.168887 & 0.07641 \\
\hline 71 & 68.28 & 0.02181 & 0.022000 & 0.32467 \\
\hline 72 & 68.94 & 0.01831 & 10000000 & 1.37000 \\
\hline 73 & 68.94 & 0.01831 & 1.000000 & 1.37000 \\
\hline 74 & 68.28 & 0.04747 & 0.008333 & 0.32467 \\
\hline 75 & 68.28 & 0.03058 & 0.168687 & 0.07641 \\
\hline 78 & 68.33 & 0.02145 & 0.022000 & 0.32467 \\
\hline 77 & 68.28 & 0.04786 & 0.008333 & 0.32487 \\
\hline 78 & 68.28 & 0.03041 & 0.168887 & 0.07641 \\
\hline 79 & 68.28 & 0.04824 & 0.008333 & 0.32467 \\
\hline 80 & 66.28 & 0.03025 & 0.168867 & 0.07641 \\
\hline 81 & 68.28 & 0.02157 & 0.022000 & 0.32467 \\
\hline 82 & 68.33 & 0.02157 & 0.022000 & 0.32467 \\
\hline 83 & 68.27 & 0.04707 & 0.008333 & 0.32467 \\
\hline 84 & 86.27 & 0.03075 & 0.168867 & 0.07641 \\
\hline 85 & 68.27 & 0.02135 & 0.022000 & 0.32467 \\
\hline 86 & 68.33 & 0.02134 & 0.022000 & 0.32467 \\
\hline 87 & 68.29 & 0.02222 & 0.022000 & 0.32467 \\
\hline 88 & 68.28 & 0.02169 & 0.022000 & 0.32467 \\
\hline 89 & 66.33 & 0.02219 & 0.022000 & 0.32467 \\
\hline 90 & 68.33 & 0.02168 & 0.022000 & 0.32467 \\
\hline 10.5 & 68.93 & 0.03225 & 0.250000 & 15.62400 \\
\hline 106 & 68.93 & 0.03225 & 1.250000 & 15.62400 \\
\hline 107 & 85.00 & 0.00000 & 1.000000 & 0.43000 \\
\hline 109 & 68.94 & 0.03118 & 1.000000 & 0.37000 \\
\hline
\end{tabular}


AFT Fathom Model

\begin{tabular}{|c|c|c|c|c|}
\hline Pipe & $\begin{array}{c}\text { T } \\
\text { Outet } \\
\text { (deg. F) }\end{array}$ & f & Length & $\begin{array}{c}\text { Hyd. } \\
\text { Diameter } \\
\text { (feet) }\end{array}$ \\
\hline 111 & 68.94 & 0.01821 & 1.000000 & 1.37000 \\
\hline 112 & 68.94 & 0.03118 & 1.000000 & 0.37000 \\
\hline 113 & 68.94 & 0.03118 & 1.000000 & 0.37000 \\
\hline 114 & 68.94 & 0.01831 & 1.000000 & 1.37000 \\
\hline 115 & 68.94 & 0.01831 & 1.000000 & 1.37000 \\
\hline 117 & 68.93 & 0.01831 & 30.000000 & 1.37000 \\
\hline 120 & 68.94 & 0.03118 & 1.000000 & 0.37000 \\
\hline 125 & 68.94 & 0.02006 & 1.000000 & 1.38000 \\
\hline 126 & 68.94 & 0.01821 & 1.000000 & 1.37000 \\
\hline 128 & 68.93 & 0.01831 & 5.000000 & 1.37000 \\
\hline 129 & 68.27 & 0.01845 & 30.000000 & 1.37000 \\
\hline 130 & 68.33 & 0.02181 & 0.022000 & 0.32467 \\
\hline 131 & 68.28 & 0.02146 & 0.022000 & 0.32467 \\
\hline 132 & 68.27 & 0.01723 & 2.000000 & 0.93000 \\
\hline 133 & 68.27 & 0.01723 & 8.000000 & 0.93000 \\
\hline 134 & 68.27 & 0.01677 & 0.083333 & 0.77700 \\
\hline 135 & 68.33 & 0.01677 & 0.083333 & 0.77700 \\
\hline 136 & 68.33 & 0.01723 & 2.000000 & 0.93000 \\
\hline 137 & 68.33 & 0.01723 & 8.000000 & 0.93000 \\
\hline 138 & 68.33 & 0.01844 & 1.000000 & 1.37000 \\
\hline
\end{tabular}

All Junction Table

\begin{tabular}{|c|c|c|c|c|c|c|c|c|c|}
\hline Jdt & Name & $\begin{array}{c}\text { Vol Flow } \\
\text { Rate Thru Jct } \\
\text { (gal/min) }\end{array}$ & $\begin{array}{l}\text { dP Stag. Total } \\
\text { (psid) }\end{array}$ & $\begin{array}{l}\text { dP Stafic } \\
\text { Total } \\
\text { (psid) }\end{array}$ & $\begin{array}{c}\text { P Stabe } \\
\text { In } \\
\text { (psia) } \\
\end{array}$ & $\begin{array}{c}\text { P Static } \\
\text { Out } \\
\text { (psia) }\end{array}$ & $\begin{array}{c}\text { P Stag. } \\
\text { In } \\
\text { (psia) }\end{array}$ & $\begin{array}{c}\text { P Stzg. } \\
\text { Out } \\
\text { (psia) }\end{array}$ & $\begin{array}{c}\text { T } \\
\text { Inlet } \\
\text { (deg. F) }\end{array}$ \\
\hline 2 & Area Change & 42.068 & 5.4478931 & 3.2703140 & 38.46 & 35.19 & 43.91 & 38.46 & 68.27 \\
\hline 3 & Branch & NA & 0.0000000 & 0.0000000 & 38.99 & 36.99 & 38.44 & 38.44 & 66.27 \\
\hline 4 & Branch & 5.003 & 0.0000000 & 0.0000000 & 37.86 & 37.86 & 37.91 & 37.91 & 66.31 \\
\hline 5 & Branch & NA & 0.0000000 & 0.0000000 & 37.73 & 37.73 & 37.91 & 37.91 & 6831 \\
\hline 6 & Pp-1 Gould Centrifugal Pump & 43.224 & -51.2904282 & -51.2733650 & 15.35 & 68.63 & 15.93 & 6722 & 68.94 \\
\hline 7 & Branch & 5.003 & 0.0000000 & -3.2125158 & 29.34 & 32.55 & 32.60 & 32.60 & 66.31 \\
\hline 8 & Branch & NA & See Mult Losses & NA & 31.71 & 31.71 & 32.60 & 32.60 & 66.32 \\
\hline 9 & $H X-1$ & 42.071 & 7.0797696 & 7.0796022 & 60.20 & 53.12 & 60.76 & 53.68 & 68.94 \\
\hline 10 & Throttle Valve & 42.071 & 5.1873674 & 5.1873674 & 66.47 & 61.28 & 67.04 & 61.85 & 68.94 \\
\hline 11 & Area Change & 42.068 & 0.5193158 & -1.5715067 & 18.52 & 20.09 & 21.17 & 20.65 & 66.33 \\
\hline 13 & Branch & NA & 0.0000000 & 0.0000000 & 37.82 & 37.82 & 37.91 & 37.91 & 6831 \\
\hline 14 & Orifce & 5.003 & 2.7133958 & 5.9259119 & 37.86 & 31.93 & 37.91 & 35.19 & 66.31 \\
\hline 15 & Branch & NA & 0.0000000 & 0.0000000 & 31.93 & 31.93 & 32.60 & 32.60 & 68.31 \\
\hline 16 & Branch & NA & 0.0000000 & 0.0000000 & 37.59 & 37.59 & 37.92 & 37.92 & 66.30 \\
\hline 17 & Branch & NA & 0.0000000 & 0.0000000 & 37.64 & 37.64 & 37.92 & 37.92 & 68.31 \\
\hline 18 & Orifoe & 1.707 & 4.9039555 & 5.2778249 & 37.92 & 32.64 & 37.92 & 33.02 & 68.31 \\
\hline 19 & Branch & NA & See Mult Losses & NA & 32.02 & 32.02 & 32.58 & 32.58 & 66.32 \\
\hline 20 & Branch & NA & See Mult Logses & NA & 32,09 & 32,09 & 32.59 & 32.59 & 66.32 \\
\hline 21 & Orifice & 1.705 & 4.8938427 & 5.2689415 & 37.91 & 32.64 & 37.92 & 33.02 & 66.31 \\
\hline 22 & Branch & NA & 0.0000000 & 0.0000000 & 37.69 & 37.69 & 37.91 & 37.91 & 66.31 \\
\hline 23 & Orifice & 5.004 & 2.7140083 & 5.9272490 & 37.86 & 31.93 & 37.91 & 35.19 & 66.31 \\
\hline 24 & Branch & NA & 0.0000000 & 0.0000000 & 32.16 & 32.16 & 32.59 & 32.59 & 66.32 \\
\hline 25 & Branch & NA & 0.0000000 & 0.0000000 & 37.43 & 37.43 & 37.94 & 37.94 & 68.30 \\
\hline 26 & Branch & NA & 0.0000000 & 0.0000000 & 37.49 & 37.49 & 37.94 & 37.94 & 66.30 \\
\hline 27 & Orifice & 1.714 & 4.9452057 & 5.3222198 & 37.94 & 32.62 & 37.94 & 33.00 & 6830 \\
\hline 28 & Branch & NA & See Mult Losses & NA & 31.75 & 31.75 & 32.56 & 32.56 & 66.32 \\
\hline 29 & Branch & NA & See Mult Losses & NA & 31.84 & 31.84 & 32.57 & 32.57 & 66.32 \\
\hline 30 & Orifice & 1.711 & 4.9296125 & 5.3063308 & 37.93 & 32.63 & 37.94 & 33.01 & 66.30 \\
\hline 31 & Branch & NA & 0.0000000 & 0.0000000 & 37.54 & 37.54 & 37.93 & 37.93 & 66.30 \\
\hline 32 & Orifice & 1.709 & 4.9158039 & 5.2905769 & 37.92 & 32.63 & 37.93 & 33.01 & 66.30 \\
\hline
\end{tabular}




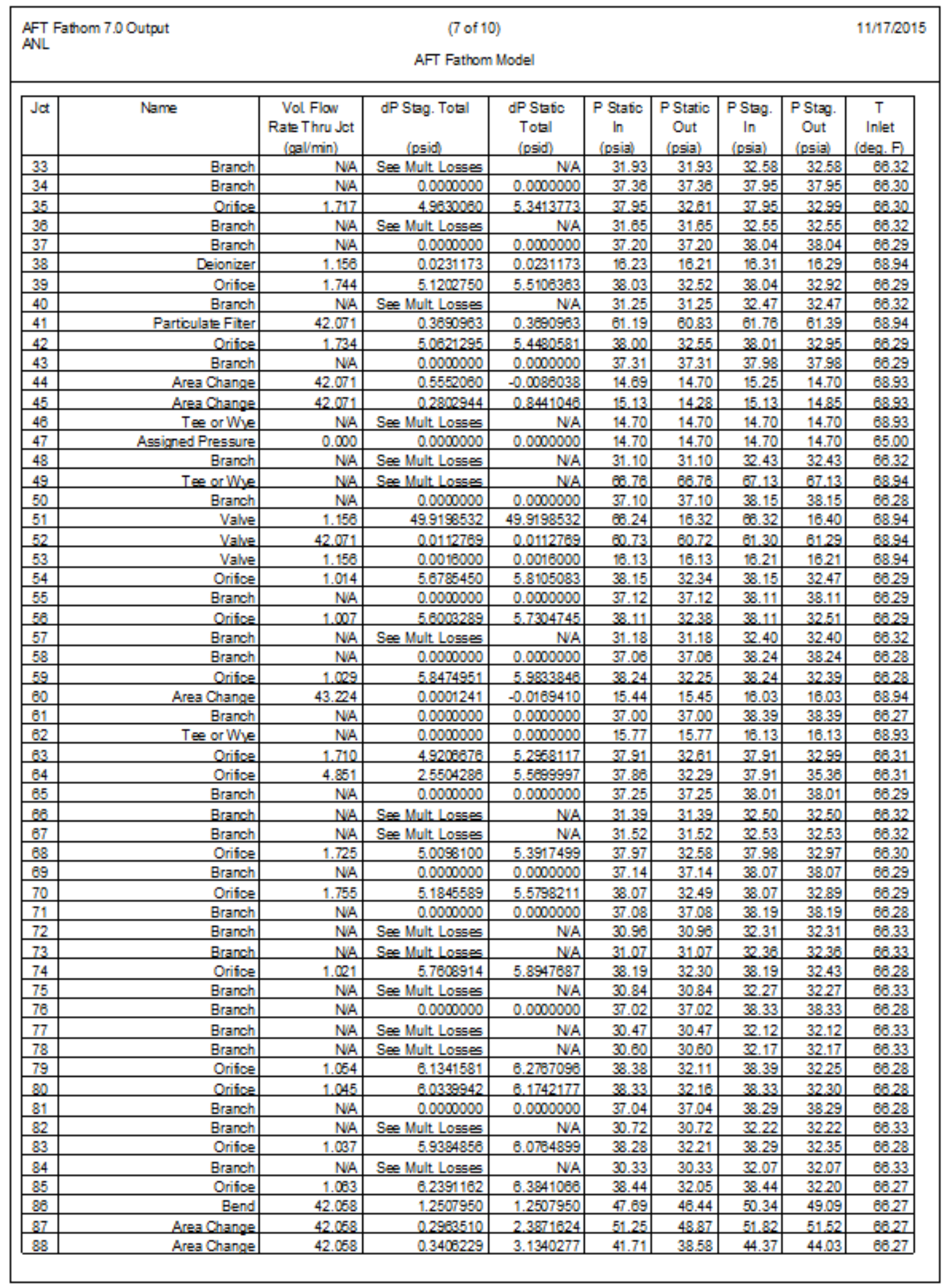




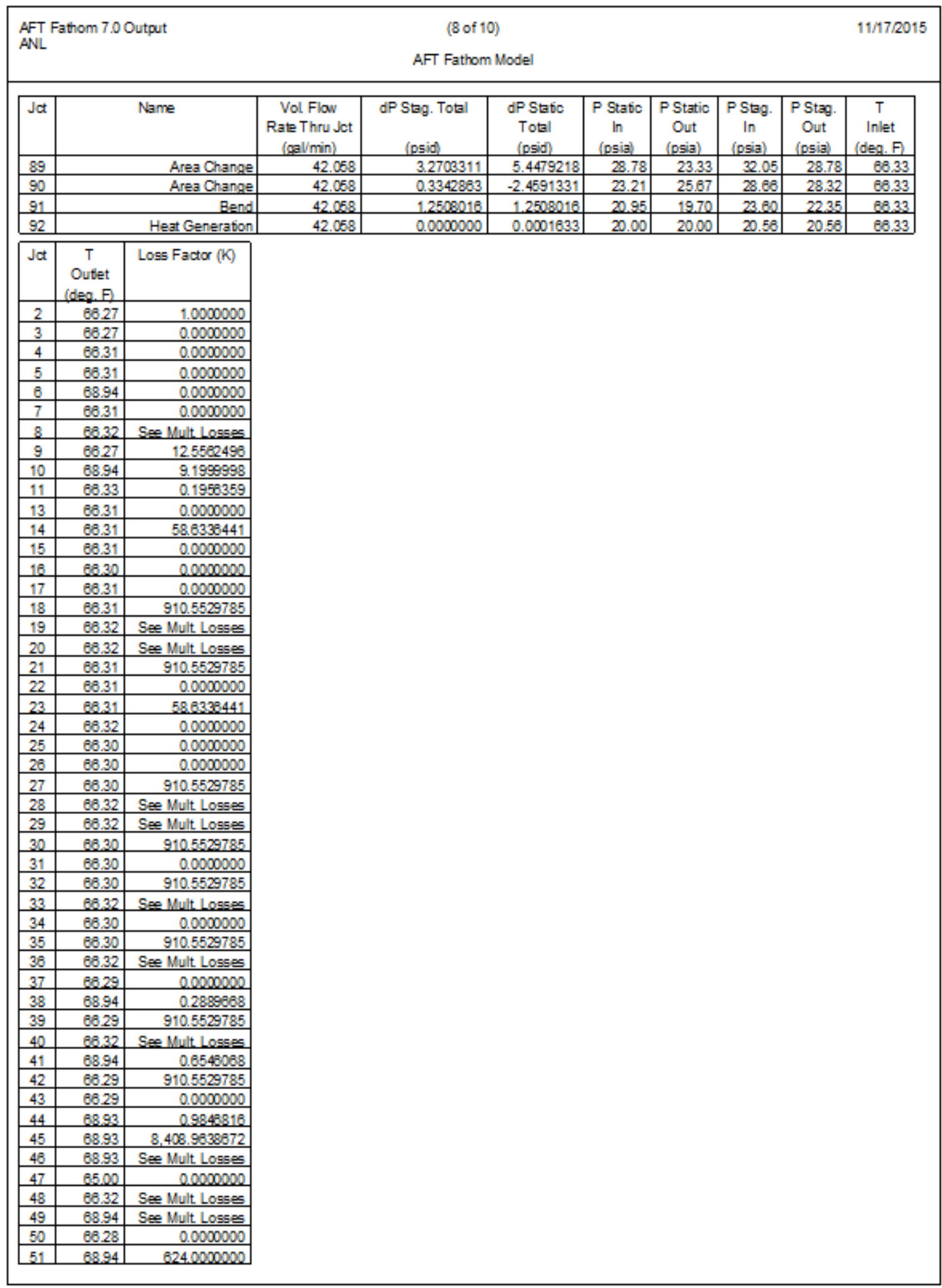




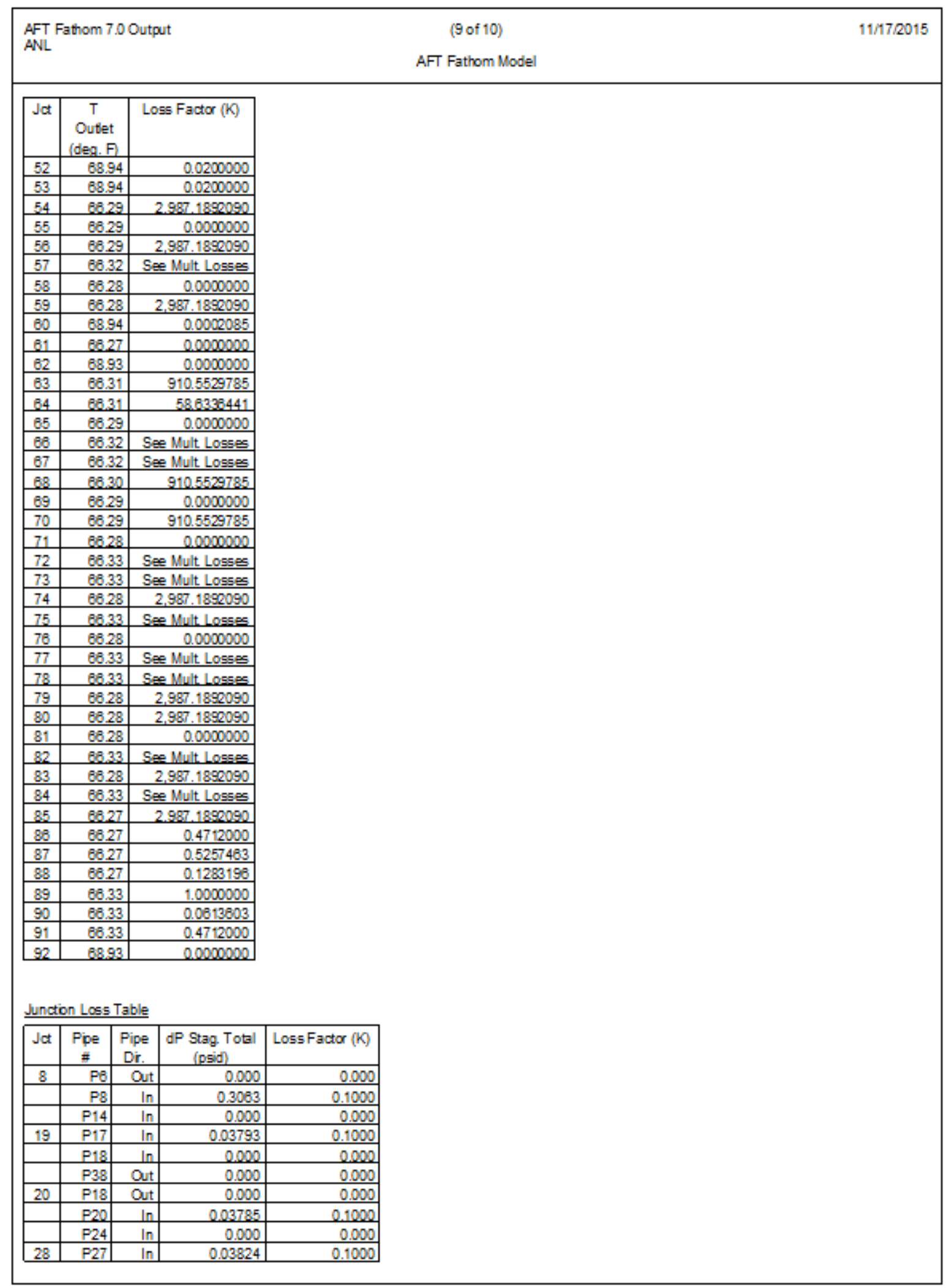




\begin{tabular}{|lrr|}
\hline $\begin{array}{l}\text { AFT Fathom 7.0 Output } \\
\text { ANL }\end{array}$ & $(10$ of 10$)$ & $11 / 17 / 2015$ \\
& AFT Fathom Model &
\end{tabular}

\begin{tabular}{|c|c|c|c|c|}
\hline Jat & $\begin{array}{c}\text { Pipe } \\
\equiv\end{array}$ & $\begin{array}{l}\text { Pipe } \\
\text { Dir. }\end{array}$ & $\begin{array}{c}\text { dP Stag. Total } \\
\text { (psid) }\end{array}$ & Loss Factor (K) \\
\hline & P28 & $\ln$ & 0.000 & 0.000 \\
\hline & $\mathrm{P} 42$ & Out & 0.000 & 0.000 \\
\hline \multirow[t]{3}{*}{29} & P28 & Out & 0.000 & 0.000 \\
\hline & $P 30$ & $\ln$ & 0.03812 & 0.1000 \\
\hline & P34 & $\ln$ & 0.000 & 0.000 \\
\hline \multirow[t]{3}{*}{33} & $P 32$ & $\ln$ & 0.03802 & 0.1000 \\
\hline & P34 & Out & 0.000 & 0.000 \\
\hline & P38 & $\ln$ & 0.000 & 0.000 \\
\hline \multirow[t]{3}{*}{36} & $P 40$ & $\ln$ & 0.03838 & 0.1000 \\
\hline & $\mathrm{P} 42$ & $\ln$ & 0.000 & 0.000 \\
\hline & P58 & Qut & 0.000 & 0,000 \\
\hline \multirow[t]{3}{*}{40} & P45 & $\ln$ & 0.03960 & 0.1000 \\
\hline & P46 & In & 0.000 & 0.000 \\
\hline & P56 & Qut & 0,000 & 0,000 \\
\hline \multirow[t]{3}{*}{46} & P106 & Out & 1.333E-11 & 4.000E- -07 \\
\hline & P105 & $\ln$ & 0.000 & 0.000 \\
\hline & P107 & $\ln$ & 0.000 & 0.000 \\
\hline \multirow[t]{3}{*}{48} & P54 & $\ln$ & 0.04010 & 0.1000 \\
\hline & P56 & $\ln$ & 0.000 & 0.000 \\
\hline & P89 & Out & 0.000 & 0.000 \\
\hline \multirow[t]{3}{*}{49} & P114 & Qut & $1.702 \mathrm{E}-04$ & 3.019E-04 \\
\hline & P111 & $\ln$ & 0.000 & 0.000 \\
\hline & P109 & Out & 0.7301 & 9.126 \\
\hline \multirow[t]{3}{*}{57} & $P 86$ & $\ln$ & 0.01320 & 0.1000 \\
\hline & P88 & Qut & 0.000 & 0.000 \\
\hline & P89 & $\ln$ & 0.000 & 0.000 \\
\hline \multirow[t]{3}{*}{68} & $\mathrm{P} 46$ & Out & 0.000 & 0.000 \\
\hline & P48. & $\ln$ & 0.03915 & 0.1000 \\
\hline & P52 & $\ln$ & 0.000 & 0.000 \\
\hline \multirow[t]{3}{*}{67} & P50 & $\ln$ & 0.03874 & 0.1000 \\
\hline & P52 & Out & 0.000 & 0.000 \\
\hline & P58 & $\ln$ & 0.000 & 0,000 \\
\hline \multirow[t]{3}{*}{72} & P81 & $\ln$ & 0.01358 & 0.1000 \\
\hline & $P 82$ & $\ln$ & 0.000 & 0.000 \\
\hline & $\mathrm{P} 130$ & Qut & 0,000 & 0.000 \\
\hline \multirow[t]{3}{*}{73} & $P 62$ & Out & 0.000 & 0.000 \\
\hline & $P 64$ & $\ln$ & 0.01339 & 0.1000 \\
\hline & $P 88$ & $\ln$ & 0.000 & 0.000 \\
\hline \multirow[t]{3}{*}{75} & P70 & $\ln$ & 0.01378 & 0.1000 \\
\hline & $\mathrm{P} 90$ & Out & 0.000 & 0.000 \\
\hline & $\mathrm{P} 130$ & $\ln$ & 0.000 & 0.000 \\
\hline \multirow[t]{3}{*}{77} & P75 & $\ln$ & 0.01446 & 0.1000 \\
\hline & P76 & $\ln$ & 0.000 & 0.000 \\
\hline & P86 & Out & 0.000 & 0.000 \\
\hline \multirow[t]{3}{*}{78} & P76 & Out & 0.000 & 0.000 \\
\hline & P78 & $\ln$ & 0.01422 & 0.1000 \\
\hline & P82 & $\ln$ & 0.000 & 0.000 \\
\hline \multirow[t]{3}{*}{82} & P8D & $\operatorname{In}$ & 0.01400 & 0.1000 \\
\hline & P82 & Qut & 0.000 & 0.000 \\
\hline & $\mathrm{P90}$ & $\ln$ & 0.000 & 0.000 \\
\hline \multirow[t]{3}{*}{84} & $\mathrm{P9}$ & Out & 0.000 & 0.000 \\
\hline & P84 & In & 0.01471 & 0.1000 \\
\hline & $\mathrm{P} 86$ & $\ln$ & 0.000 & 0.000 \\
\hline
\end{tabular}


Large pump

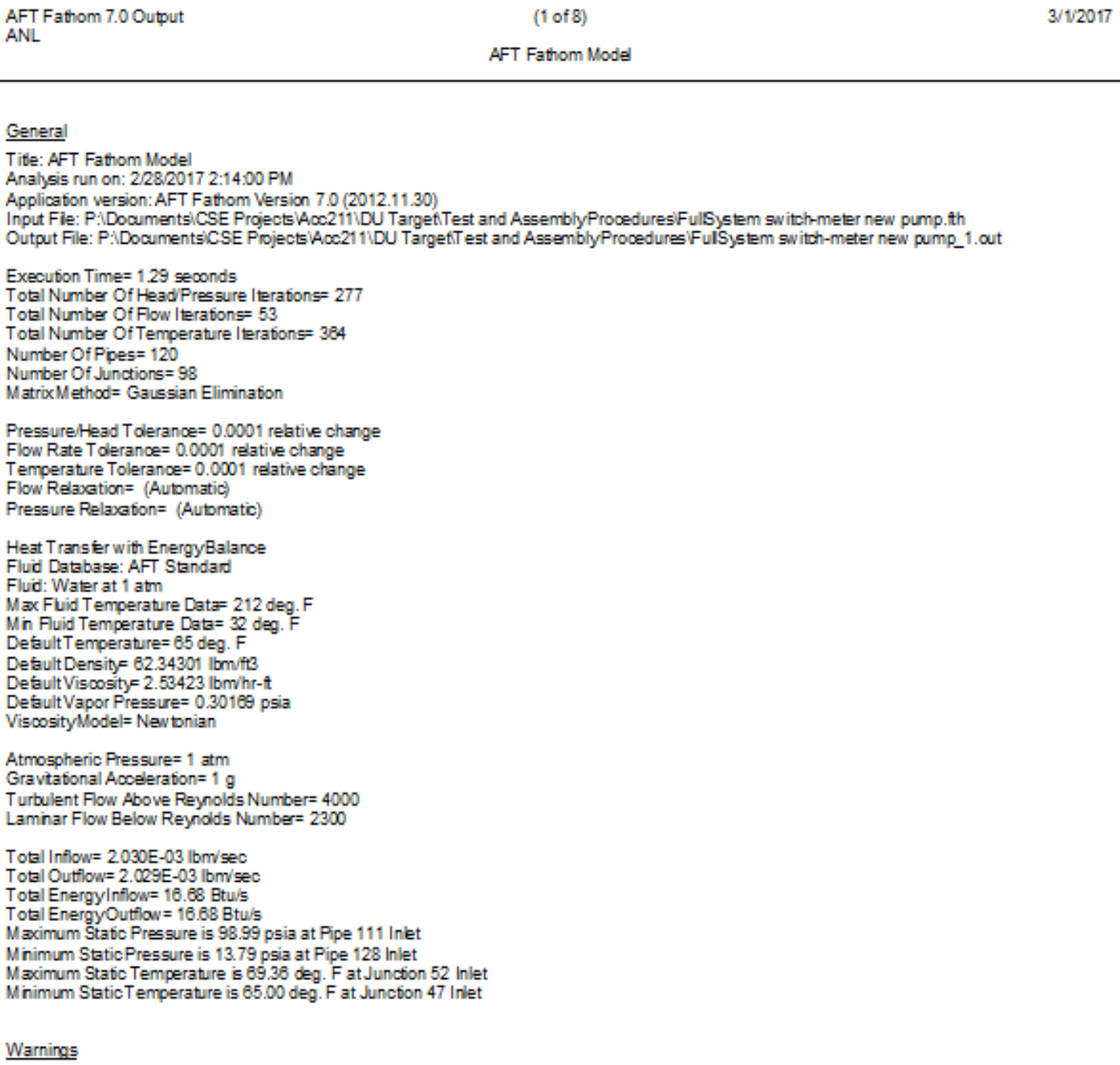

ANL

AFT Fathom Mode

General

Tite: AFT Fathom Mode

Anahsis run on: 2/28/2017 2:14:00 PM

Application version: AFT Fathom Version $7.0(2012.11 .30)$

Input Fie: P:DocumentsiCSE Project Acc211 DU Targetitest and AssemblyProceduresiFulSystem switch-meter new pump.th

Output File: P:DocumentsiC SE Project Acc21 11DU TargetTest and AssemblyProcedures'FulSystem switch-meter new pump_1. out

Execution Time= 1.29 seconds

Total Number Of Head Pressure Iterations= 277

Total Number Of Flow Iteration $5=53$

Total Number Of Temperature Iterafions $=384$

Number Of Pipes $=120$

Number Of Junctions $=98$

Matrix Method= Gaussian Elimination.

Pressure/Head T derance $=0.0001$ relative change

Flow Rate Tolerance $=0.0001$ relative change

Temperature Tolerance $=0.0001$ relative change

Flow Relaxation $=$ (Automatic)

Pressure Relaxation $=($ Automatic $)$

Heat Transfer with Energy Balance

Flud Database: AFT Standard

Flud: Water at $1 \mathrm{~atm}$

Max Fuid Temperature Data $212 \mathrm{deg}$. F

Min Fuid Temperature Data $=32 \mathrm{deg}$. F

Default Temperature $=65 \mathrm{deg}$. F

Default Density $=62.34301 \mathrm{lbm} / \mathrm{ft} 3$

Default Viscosity $=2.53423 \mathrm{lbm} / \mathrm{hr}-\mathrm{t}$

Default Vapor Pressure= 0.30168 psia

ViscositylModel= New tonian

Atmospheric Pressure $=1 \mathrm{~atm}$

Gravtational Acceleration $=10$

Turbulent Flow Above Reynolds Number $=4000$

Laminar Flow Below Reynolds Number= 2300

Total Inflow $=2.030 \mathrm{E}-03 \mathrm{lbm} / \mathrm{sec}$

Total Outtlow $=2.029 \mathrm{E}-03 \mathrm{lbm} / \mathrm{sec}$

Total Energy/nflow $=16.68$ Btu/s

Total EnergyOutflaw $=16.68 \mathrm{Btu} / \mathrm{s}$

Maximum Stafc Pressure is 98.99 psia at Pipe 111 Inkt

Minimum StaticPressure is 13.79 psia at Pipe 128 Inlet

Maximum Stafic Temperature is $69.36 \mathrm{deg}$. F at Junction 52 Inlet

Minimum StaticTemperature is $65.00 \mathrm{deg}$. F at Junction 47 Inlet

Warnings

- WARNING* HGL, EGL and head loss results maynot be meaningful for variable dersitysystems.

Pump Summary

\begin{tabular}{|c|c|c|c|c|c|c|c|c|c|c|}
\hline Jct & Name & $\begin{array}{c}\text { Vol. } \\
\text { Flow } \\
\text { (gal/min) }\end{array}$ & $\begin{array}{c}\text { Mass } \\
\text { Flow } \\
\text { (lbm/sec) }\end{array}$ & $\begin{array}{c}\mathrm{dP} \\
\text { (psid) }\end{array}$ & $\begin{array}{c}\mathrm{dH} \\
\text { (feet) }\end{array}$ & $\begin{array}{c}\text { Overall } \\
\text { Efficiency } \\
\text { Percent) }\end{array}$ & $\begin{array}{c}\text { Speed } \\
\text { (Percent) }\end{array}$ & $\begin{array}{c}\text { Overall } \\
\text { Power } \\
\text { (hp) }\end{array}$ & $\begin{array}{c}\text { BEP } \\
\text { (gal/min) }\end{array}$ & $\begin{array}{c}\% \text { of } \\
\text { (PEP } \\
\text { (Percent }\end{array}$ \\
\hline 6 & Pp-1 Gould Centrifugal Pump & 54.43 & 7.556 & 84.50 & 195.3 & 30.89 & 100.0 & 8.685 & 215.6 & 25.25 \\
\hline
\end{tabular}

\begin{tabular}{|c|c|c|}
\hline Jat & NPSHA & NPSHR \\
(Eef) & (Eet) \\
\hline 6 & 34.85 & NA \\
\hline
\end{tabular}

Valve Summary 
AFT Fathom Model

\begin{tabular}{|c|c|c|c|c|c|c|c|c|c|c|}
\hline Jdt & Name & $\begin{array}{l}\text { Valve } \\
\text { Type }\end{array}$ & $\begin{array}{c}\text { Vol } \\
\text { Fbw } \\
\text { (gal/min) }\end{array}$ & $\begin{array}{c}\text { Mass } \\
\text { Flow } \\
\text { (lbmised }\end{array}$ & $\begin{array}{l}\text { dP Stag. } \\
\text { (psid) }\end{array}$ & $\begin{array}{c}\mathrm{dH} \\
\text { (feet) }\end{array}$ & $\begin{array}{l}\text { P Inlet } \\
\text { Stafic } \\
\text { (psia) }\end{array}$ & $\mathrm{Cr}$ & $\mathrm{K}$ & $\begin{array}{l}\text { Valve } \\
\text { State }\end{array}$ \\
\hline 10 & Throttle Valve & & 52.933 & 7.3488 & 8.211288 & 18.975554 & 98.76 & & & Q Den \\
\hline 51 & Valve & REGULAR & 1.485 & 0.2081 & 82.363846 & 190.335493 & 98.37 & 0.1635 & 624.00000 & Open \\
\hline 52 & Value & REGULAR & 52.933 & 7.3488 & 0.017851 & 0.041251 & 89.73 & 3959883 & 0.02000 & Ooen \\
\hline 53 & Valve & REGULAR & 1.485 & 0.2061 & 0.002640 & 0.006100 & 15.73 & 28.8830 & 0.02000 & Ope \\
\hline
\end{tabular}

Heat Exchanger Summary

\begin{tabular}{|c|c|c|c|c|c|c|c|c|c|c|c|}
\hline Jct & Name & $\begin{array}{c}\text { Vol. } \\
\text { Flow } \\
\text { (galimin) }\end{array}$ & $\begin{array}{c}\text { Mass } \\
\text { Flow } \\
\text { (lbm/sec) }\end{array}$ & $\begin{array}{c}d P \\
\text { (psid) }\end{array}$ & $\begin{array}{c}\mathrm{dH} \\
\text { (feet) } \\
\end{array}$ & $\begin{array}{c}\text { dT } \\
\text { Loss } \\
\text { (deg. F) }\end{array}$ & $\begin{array}{l}\text { Heat } \\
\text { Rate In } \\
\text { (Bty/s) }\end{array}$ & $\begin{array}{c}\text { T } \\
\text { Inlet } \\
\text { (deg. F) }\end{array}$ & $\begin{array}{c}\text { T } \\
\text { Outlet } \\
\text { (deg. F) }\end{array}$ & $\begin{array}{l}\text { T2nd } \\
\text { Inlet } \\
\text { (deg. F) }\end{array}$ & $\begin{array}{l}\text { T 2nd } \\
\text { Outet } \\
\text { (deg. F) }\end{array}$ \\
\hline 9 & $H X-1$ & 52.93 & 7340 & 11.21 & 25.90 & 2.270 & -16.68 & 69.38 & 67.09 & 55.00 & 67.00 \\
\hline 100 & Heat Exchanger & 52.92 & 7.349 & 0.00 & 0.00 & -2.065 & 15.18 & 67.28 & 69.34 & $\mathrm{~N} / \mathrm{A}$ & NA \\
\hline
\end{tabular}

\section{Pipe Outout Table}

\begin{tabular}{|c|c|c|c|c|c|c|c|c|c|c|}
\hline Pipe & Name & $\begin{array}{c}\text { Vol. } \\
\text { Fbow Rate } \\
\text { (gal/min) }\end{array}$ & $\begin{array}{l}\text { Velocity } \\
\text { (Eet/gec) }\end{array}$ & $\begin{array}{c}\text { P Stafic } \\
\text { Max } \\
\text { (psig) }\end{array}$ & $\begin{array}{c}\text { P Static } \\
\text { Min } \\
\text { (psig) }\end{array}$ & $\begin{array}{c}\text { Eevation } \\
\text { Inlet } \\
\text { (feet) }\end{array}$ & $\begin{array}{c}\text { Elevation } \\
\text { Outet } \\
\text { (feet) }\end{array}$ & $\begin{array}{c}\text { dP Stag. } \\
\text { Total } \\
\text { (psid) }\end{array}$ & $\begin{array}{c}\text { dP Static } \\
\text { Total } \\
\text { (psid) }\end{array}$ & $\begin{array}{c}\text { dP } \\
\text { Gravty } \\
\text { (psid) }\end{array}$ \\
\hline 2 & Pipe & 52919 & 27.7421 & 34.9533157 & 34.9222832 & 0.000 & 0.000 & 0.03103379 & 0.03103379 & 0,0000 \\
\hline 3 & Pipe & 15.420 & 8.0838 & 38.8692207 & 38.8652000 & 0.000 & 0.000 & 0.00402333 & 0.00402333 & 0.0000 \\
\hline 4 & Pipe & 5.143 & 2.6963 & 39.2598763 & 39.2590536 & 0.000 & 0.000 & 0.00052308 & 0.00052308 & 0.0000 \\
\hline 5 & Pipe & 5.143 & 22.6265 & 32.9972115 & 30.2840843 & 0.000 & 0.000 & 2.71312714 & 2.71312714 & 0.0000 \\
\hline 6 & Pipe & 15.420 & 8.0838 & 29.8485413 & 29.8445168 & 0.000 & 0.000 & 0.00402310 & 0.00402310 & 0.0000 \\
\hline 7 & Pipe & 5.141 & 2.6951 & 39.2559013 & 39.2553787 & 0.000 & 0.000 & 0.00052265 & 0.00052265 & 0.0000 \\
\hline 8 & Pipe & 5.141 & 22.6162 & 32.9989700 & 30.2881012 & 0.000 & 0.000 & 2.71088621 & 2.71086621 & 0.0000 \\
\hline 9 & Pipe & 52920 & 27.7425 & 24.3169479 & 24.2848375 & 0.000 & 0.000 & 0.03230901 & 0.03230901 & 0.0000 \\
\hline 11 & Pipe & 10.279 & 5.3887 & 39.1094322 & 39.1074600 & 0.000 & 0.000 & 0.00197130 & 0.00197130 & 0.0000 \\
\hline 12 & Pipe & 5.139 & 2.6943 & 39.2533684 & 39.2528458 & 0.000 & 0.000 & 0.00052238 & 0.00052238 & 0.0000 \\
\hline 13 & Pipe & 5.140 & 22.6112 & 32.9998093 & 30.2900696 & 0.000 & 0.000 & 2.70973730 & 2.70973730 & 0.0000 \\
\hline 14 & Pipe & 10.279 & 5.3887 & 30.0947418 & 30.0927734 & 0.000 & 0.000 & 0.00197123 & 0.00197123 & 0.0000 \\
\hline 15 & Pipe & 22722 & 11.9115 & 38.3691025 & 38.3611031 & 0.000 & 0.000 & 0.00800003 & 0.008000003 & 0.0000 \\
\hline 16 & Pipe & 2.160 & & 39.3148537 & 39.3147736 & 0.000 & 0.000 & 0.00008354 & 0.00008354 & 0.0000 \\
\hline 17 & Pipe & 2180 & 95031 & 30.8611908 & 30.2698825 & 0.000 & 0.000 & 0.59180957 & 0.59180957 & 0.0000 \\
\hline 18 & Pipe & 22.722 & 11.9115 & 29.3230019 & 29.3150024 & 0.000 & 0.000 & 0.00799918 & 0.00799918 & 0.0000 \\
\hline 19 & Pipe & 2.158 & 1.1314 & 39.3068696 & 39.3067856 & 0.000 & 0.000 & 0.00008334 & 0.00008334 & 0.0000 \\
\hline 20 & Pipe & 2.158 & 9.4946 & 30.86828232 & 30.2773 .819 & 0.000 & 0.000 & 0.590 .88171 & 0.59088171 & 0.0000 \\
\hline 21 & Pipe & 5.140 & 2.6945 & 39.2539520 & 39.2534294 & 0.000 & 0.000 & 0.00052244 & 0.00 & 0.0000 \\
\hline 22 & Pipe & 5.139 & 22.6096 & 33.0000725 & 30.2900352 & 0.000 & 0.000 & 2.71003842 & & \\
\hline 23 & Pipe & 20.563 & 10.7801 & 38.5337944 & 38.5270958 & 0.000 & 0.000 & 0.00689902 & 0.00689902 & 0.0000 \\
\hline 24 & Pipe & 20.563 & 10.7801 & 295023918 & 29.4956932 & 0.000 & 0.000 & 0.00869852 & & 0.0000 \\
\hline 25 & Pipe & 29.209 & 15.3124 & 37.7791596 & 37.7606359 & 0.000 & 0.000 & 0.01252464 & 1252464 & 0.0000 \\
\hline 28 & Pipe & 2.168 & 1.1366 & 39.3476334 & 39.3475494 & 0.000 & 0.000 & 0.00008433 & 0.00008433 & 0.0000 \\
\hline 27 & Pipe & 2168 & 95378 & 30.8321496 & 30.2366456 & 0.000 & 0.000 & 0.59580215 & 0.59560215 & 0.0000 \\
\hline 28 & Pipe & 29.209 & 15.3125 & 28.6718903 & 28.6593704 & 0.000 & 0.000 & 0.01252249 & 0.01252249 & 0.0000 \\
\hline 29 & Pipe & 2.165 & 1.1350 & 39.3351326 & 39.3350487 & 0.000 & 0.000 & 0.00008403 & 0.00008403 & 0.0000 \\
\hline 30 & Pipe & 2.165 & 9.5245 & 30.8432274 & 30.2490692 & 0.000 & 0.000 & 0.59415793 & 0.59415793 & 0.0000 \\
\hline 31 & Pipe & 2162 & 1.1336 & 39.3242416 & 39.3241577 & 0.000 & 0.000 & 0.00008378 & 0.00008376 & 0.0000 \\
\hline 32 & Pipe & 2.162 & 9.5130 & 30.8528786 & 30.2599792 & 0.000 & 0.000 & 0.59289789 & 0.59289789 & 0.0000 \\
\hline 33 & Pipe & 27.044 & 14.1775 & 37.9917717 & 37.9808578 & 0.000 & 0.000 & 0.01091360 & 0.01091360 & 0.0000 \\
\hline 34 & Pipe & 27.044 & 14.1775 & 28.9079437 & 28.8970299 & 0.000 & 0.000 & 0.01091192 & 0.01091192 & 0.0000 \\
\hline 35 & Pipe & 5.139 & 2.6943 & 39.2539597 & 39.25336884 & 0.000 & 0.000 & 0.00059029 & 0.00059029 & 0.0000 \\
\hline 36 & Pipe & 5.139 & 2.6943 & 30.2412415 & 30.2412071 & 0.000 & 0.000 & 0.00003700 & 0.000003700 & 0.0000 \\
\hline 37 & Pipe & 24.882 & 13.0439 & 38.1884232 & 38.1790161 & 0.000 & 0.000 & 0.00940517 & 0.00940517 & 0.0000 \\
\hline 38 & Pipe & 24.882 & 13.0439 & 29.1249123 & 29.1156090 & 0.000 & 0.000 & 0.00940396 & 0.00940396 & 0.0000 \\
\hline 39 & Pipe & 2.171 & 1.1384 & 39.3618469 & 39.3617592 & 0.000 & 0.000 & 0.00008468 & 0.00008468 & 0.0000 \\
\hline
\end{tabular}




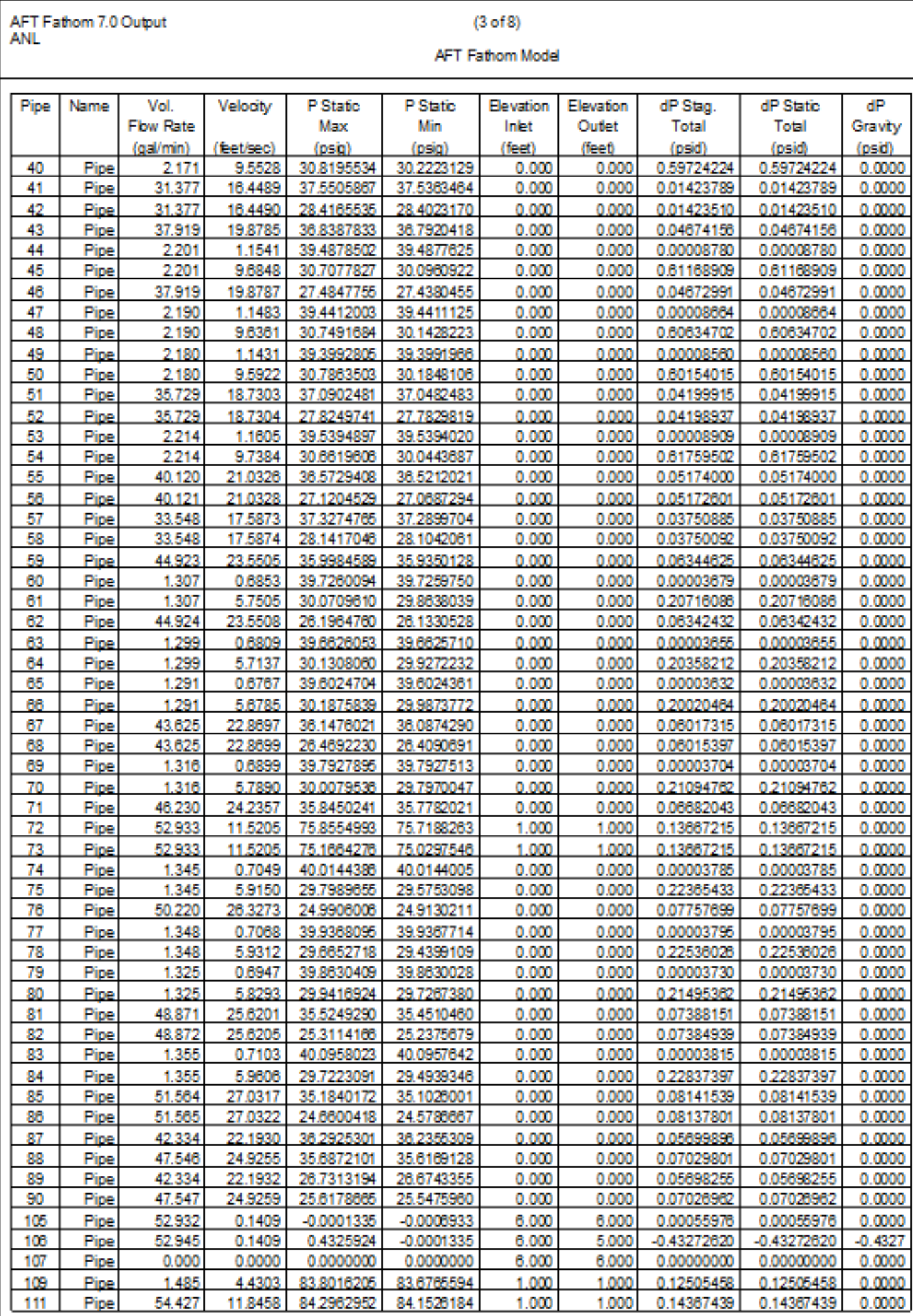


AFT Fathom Model

\begin{tabular}{|c|c|c|c|c|c|c|c|c|c|c|}
\hline Pipe & Name & $\begin{array}{c}\text { Vol. } \\
\text { Fbow Rate } \\
\text { (gal/min) }\end{array}$ & $\begin{array}{l}\text { Velocity } \\
\text { (Eet/sec) }\end{array}$ & $\begin{array}{c}\text { P Stafo } \\
\text { Max } \\
\text { (psiq) }\end{array}$ & $\begin{array}{c}\text { P Static } \\
\text { Min } \\
\text { (psig) }\end{array}$ & $\begin{array}{c}\text { Eevation } \\
\text { Inket } \\
\text { (feet) }\end{array}$ & $\begin{array}{c}\text { Elevation } \\
\text { Outet } \\
\text { (feet }\end{array}$ & $\begin{array}{c}\text { dP Stag. } \\
\text { Total } \\
\text { (psid) }\end{array}$ & $\begin{array}{c}\text { dP Static } \\
\text { Total } \\
\text { (psid) }\end{array}$ & $\begin{array}{l}d P \\
\text { Gravty } \\
\text { (psid) }\end{array}$ \\
\hline 112 & Pipe & 1.485 & 4.4303 & 1.1579696 & 1.0329142 & 1.000 & 1.000 & 0.12505458 & 0.12506458 & 0.0000 \\
\hline 113 & Pipe & 1.485 & 4.4303 & 1.3127174 & 1.1876631 & 1.000 & 1.000 & 0.12505458 & 0.12506458 & 0.0000 \\
\hline 114 & Pipe & 52.933 & 11.5205 & 84.2034607 & 84.0687877 & 1.000 & 1.000 & 0.13867215 & 0.13867215 & 0.0000 \\
\hline 115 & Pipe & 52.933 & 11.5205 & 75.0119019 & 74.4425049 & 1.000 & 2.000 & 0.56940198 & 0.56940198 & 0.4327 \\
\hline 117 & Pipe & 52.932 & 11.5204 & 10.8014338 & 4.9701614 & 0.000 & 4.000 & 5.83127117 & 5.83127117 & 1.7309 \\
\hline 120 & Pipe & 1.485 & 4.4303 & 1.0302744 & 0.9061832 & 1.000 & 1.000 & 0.12409154 & 0.12409154 & 0.0000 \\
\hline 125 & Pipe & 54.427 & 11.6747 & -0.0223379 & -0.1773243 & 1.000 & 1.000 & 0.15498601 & 0.15498601 & 0.0000 \\
\hline 126 & Pipe & 54.427 & 11.8458 & 0.0945339 & -0.0491972 & 1.000 & 1.000 & 0.14373083 & 0.14373083 & 0.0000 \\
\hline 128 & Pipe & 52.945 & 11.5233 & 0.1452131 & -0.9028184 & 5.000 & 1.000 & -1.04783118 & -1.04783118 & -1.7309 \\
\hline 129 & Pipe & 52919 & 11.5175 & 63.2353249 & 59.9760003 & 2.000 & 0.000 & 3.25896899 & 325896992 & -0.8657 \\
\hline 130 & Pipe & 46.231 & 24.2360 & 25.9127808 & 25.8458854 & 0.000 & 0.000 & 0.06679527 & 0.06679527 & 0.0000 \\
\hline 131 & Pipe & 50.219 & 26.3289 & 35.3555794 & 35.2779694 & 0.000 & 0.000 & 0.07761186 & 0.07761186 & 0.0000 \\
\hline 132 & Pipe & 52.919 & 24.9939 & 56.1974831 & $54,40,80009$ & 0.000 & 0.000 & 1.78948557 & 1.78948557 & 0.0000 \\
\hline 133 & Pipe & 52.919 & 24.9939 & 52.4279900 & 45.2700424 & 0.000 & 0.000 & 7.15794230 & 7.15794230 & 0.0000 \\
\hline 134 & Pipe & 52.919 & 35.8062 & 40.3088722 & 40.1302299 & 0.000 & 0.000 & 0.178840 .89 & 0.17884089 & 0.0000 \\
\hline 135 & Pipe & 52.920 & 35.8068 & 15.6804919 & 15.4819280 & 0.000 & 0.000 & 0.17858525 & 0.17858525 & 0.0000 \\
\hline 136 & Pipe & 52.920 & 24.9944 & 10.2400208 & 8.4513416 & 0.000 & 0,000 & 1.78867829 & 1.78887829 & 0.0000 \\
\hline 137 & Pipe & 52.920 & 24.9944 & 19.3747711 & 12.2200584 & 0.000 & 0.000 & 7.15471315 & 7.15471315 & 0.0000 \\
\hline 138 & Pipe & 52.932 & 11.5204 & 4.5880196 & 4.3126821 & 4.000 & 4.000 & 0.27335658 & 0.27335656 & 0.0000 \\
\hline 139 & Pipe & 52.932 & 115204 & 3.1919348 & 3.0552559 & 4.000 & 4.000 & 0.13687828 & 0.13687828 & 0.0000 \\
\hline 140 & Pipe & 52.932 & 11.5204 & 2.6711140 & 2.6597233 & 4.000 & 4.000 & 0.01138986 & 0.01138986 & 0.0000 \\
\hline 141 & Pipe & 52.932 & 11.5204 & 2.2755814 & 2.2072430 & 4.000 & 4.000 & 0.06833913 & 0.06833913 & 0.0000 \\
\hline 142 & Pipe & 52.932 & 11.5204 & 1.6715584 & 1.6373882 & 4.000 & 4.000 & 0.03416957 & 0.03418957 & 0.0000 \\
\hline 143 & Pipe & 52932 & 11.5204 & 1.2532454 & -0.0222511 & 4.000 & 6.000 & 127549848 & 1.27549648 & 0.8655 \\
\hline 144 & Pipe & 52.932 & 5.0609 & 2.4608040 & 2.4440937 & 4.000 & 4.000 & 0.01671079 & 0.01671079 & 0.0000 \\
\hline 145 & Pipe & 52.932 & 5.0609 & 2.8322964 & 2.7988758 & 4.000 & 4.000 & 0.03342158 & 0.03342158 & 0.0000 \\
\hline 148 & Pipel & 52.920 & 11.5177 & 10.9390602 & 10.8018415 & 0.000 & 0.000 & 0.13741986 & 0.13741986 & 0.0000 \\
\hline
\end{tabular}

\begin{tabular}{|c|c|c|c|c|c|c|c|c|}
\hline Pipe & $\mathrm{dH}$ & P Static In & $\begin{array}{c}\text { P Sta6c } \\
\text { Out } \\
\text { (psig) }\end{array}$ & P Stag. In & $\begin{array}{c}\text { P Stag. } \\
\text { Out } \\
\text { (psig) }\end{array}$ & $\begin{array}{c}\text { T } \\
\text { Inlet } \\
\text { (degig) }\end{array}$ & $\begin{array}{c}\text { T } \\
\text { Outlet } \\
\text { (deg. F) }\end{array}$ & f f
\end{tabular}

\begin{tabular}{|c|c|c|c|c|c|c|c|c|}
\hline & & & & & & & & \\
\hline 2 & 0.07169785 & 34.9533157 & 34.9222832 & 40.1302299 & 40.0992 & 67.09 & 67.09 & 0.02027 \\
\hline 3 & 0.00929628 & 38.8692207 & 38.8652000 & 39.3087807 & 39.3048 & 67.20 & 67.20 & 0.02830 \\
\hline 4 & 0.00120848 & 39.2588763 & 39.2593538 & 39.3087807 & 39.3083 & 67.20 & 67.20 & 0.03473 \\
\hline 5 & 6.26825113 & 32.9972115 & 30.2840843 & 36.4409065 & 33.7278 & 67.20 & 67.20 & 0.03011 \\
\hline 6 & 0.00929475 & 29.8485413 & 29.8445108 & 30.2881012 & 30.2841 & 67.22 & 67.22 & 0.02630 \\
\hline 7 & 0.00120751 & 39.2569013 & 39.2553787 & 39.3047600 & 39.3042 & 67.20 & 67.20 & 0.03473 \\
\hline 8 & 6.26303085 & 32.9989700 & 30.2881012 & 36.4396103 & 33.7286 & 67.20 & 67.20 & 0.03011 \\
\hline 9 & 0.07464552 & 24.3189479 & 24.2846375 & 29.4939308 & 29.4816 & 67.28 & 67.28 & 0.02028 \\
\hline 11 & 0.00455438 & 39.1094322 & 39.1074600 & 39.30476000 & 39.3028 & 67.20 & 67.20 & 0.02900 \\
\hline 12 & 0.001 & 39.2533 & 39.25 & 39.3021965 & 39.3017 & 67.21 & 67.21 & 0.03473 \\
\hline 13 & 6.26042855 & 32.9998093 & 30.2900696 & 36.4388161 & 33.7291 & 67.21 & 67.21 & 0.03011 \\
\hline 14 & 0.00455423 & 30.0947418 & 30.0927734 & 30.2900696 & 30.2881 & 67.21 & 67.21 & 0.02900 \\
\hline 15 & 0.018 & 38.369 & 38.3811031 & 39.3234787 & 39.3155 & 67.19 & 67.19 & 0.02409 \\
\hline 16 & 0.00019300 & 39.314 & 39.31 & 39.323 & 39.3234 & 67.19 & 67.19 & 0.03144 \\
\hline 17 & 1.36728058 & 30.8611908 & 30.2693825 & 31.4686508 & 30.8768 & 67.19 & 67.19 & 0.03726 \\
\hline 18 & 0.01849088 & 29.3230019 & 293150024 & 30.2773781 & 30.2694 & 87.22 & 67.22 & 0.02408 \\
\hline 19 & 0.00019256 & 39.3068695 & 39.3067856 & 39.3154793 & 39.3154 & 67.19 & 67.19 & 0.03142 \\
\hline 20 & 1.38513788 & 30.80822632 & 30.2773819 & 31.4748399 & 30.8838 & 67.19 & 67.19 & 0.03727 \\
\hline 21 & 0.00120702 & 39.2539520 & 39.2534294 & 39.3027878 & 39.3023 & 67.21 & 67.21 & 0.03473 \\
\hline 22 & 6.28112225 & 33.0000725 & 30.2900352 & 36.4388139 & 33.7286 & 67.21 & 67.21 & 0.03011 \\
\hline 23 & 0.01547701 & 38.5337944 & 38.5270958 & 39.3154793 & 39.3088 & 67.19 & 67.19 & 0.02462 \\
\hline 24 & 0.01547590 & 29.5023918 & 29.4956932 & 30.2840767 & 30.2774 & 67.22 & 67.22 & 0.02462 \\
\hline 25 & 0.02893811 & 37.7791595 & 37.7686389 & 39.3563232 & 39.3438 & 67.17 & 67.17 & 0.02282 \\
\hline 26 & 0.00019484 & 39.3476334 & 39.3475494 & 39.35603232 & 39.3562 & 67.17 & 67.17 & 0.03151 \\
\hline 27 & 1.37604046 & 30.8321495 & 30.2365456 & 31.4440498 & 30.8484 & 67.17 & 67.17 & 0.03723 \\
\hline 28 & 0.02893133 & 28.6718903 & 28.6593704 & 30.2490816 & 30.2385 & 67.23 & 67.23 & 0.02281 \\
\hline
\end{tabular}




\begin{tabular}{|lcr|}
\hline $\begin{array}{l}\text { AFT Fathom 7.0 Output } \\
\text { ANL }\end{array}$ & $(5$ of 8$)$ & 3/1/2017 \\
& AFT Fathom Model
\end{tabular}

\begin{tabular}{|c|c|c|c|c|c|c|c|c|}
\hline Pipe & (feet) & P Static In & $\begin{array}{c}\text { P Static } \\
\text { Out } \\
\text { (psig) } \\
\end{array}$ & $\begin{array}{l}\text { P Stag. In } \\
\text { (psig) }\end{array}$ & $\begin{array}{l}\text { P Stag. } \\
\text { Out } \\
\text { (psig) }\end{array}$ & $\begin{array}{c}T \\
\text { Inlet } \\
\text { (deg. } F \text { ) }\end{array}$ & $\begin{array}{c}T \\
\text { Outlet } \\
\text { (deg. F) }\end{array}$ & $f$ \\
\hline 29 & 0.00019414 & 39.3351326 & 39.3350487 & 39.3437996 & 39.3437 & 67.18 & 67.18 & 0.03148 \\
\hline 30 & 1.37270467 & 30.8432274 & 30.2490692 & 31.4534340 & 30.8693 & 67.18 & 67.18 & 0.03724 \\
\hline 31 & 0,00019852 & 39.3242416 & $3932415 \pi$ & 39.3328857 & 39.3328 & 67,18 & 67.18 & 0.03146 \\
\hline 32 & 1.36979412 & 30.8528788 & 30.2599792 & 31.4616089 & 30.8687 & 67.18 & 67.18 & 0.03726 \\
\hline 33 & 0.02521409 & 37.9917717 & 37.9808578 & 39.3437996 & 39.3329 & 67.18 & 67.18 & 0.02319 \\
\hline 34 & 0.02521034 & 28.9079437 & 28.8970299 & 30.2599754 & 30.2491 & 67.23 & 67.23 & 0.02319 \\
\hline 35 & 0.00136377 & 39.2539597 & 39.2533694 & 39.3027878 & 39.3022 & 67.21 & 67.21 & 0.03473 \\
\hline 36 & 0.000008548 & 30.2412071 & 30.2412415 & 30.29003 .52 & 30.2901 & 67.21 & 67.21 & 0.03473 \\
\hline 37 & 0.02172911 & 38.1884232 & 38.1790161 & 39.3328857 & 39.3235 & 67.18 & 67.18 & 0.02381 \\
\hline 38 & 0.02172642 & 29.1249123 & 29.1155090 & 30.2698787 & 30.2600 & 67.23 & 67.23 & 0.02381 \\
\hline 39 & 0.00019664 & 39.3618469 & 39.3617592 & 39.3706635 & 39.3705 & 67.17 & 67.17 & 0.03154 \\
\hline 40 & 1.37982860 & 30.8195534 & 23129 & 31.4333801 & 30.8361 & 67.17 & 67.17 & 0.03722 \\
\hline 41 & 0.03289427 & 37.5505887 & 37.5363484 & 39.3706835 & 39.3563 & 67.17 & 67.17 & 0.02248 \\
\hline 42 & 0.03288807 & 28.41 & 28.4023170 & 30.2366417 & 30.2223 & 67.23 & 67.23 & 0.02247 \\
\hline 43 & 0.10798837 & 36.8387833 & 36.7920418 & 39.4968109 & 39.4501 & 67.15 & 67.15 & 0.02163 \\
\hline 44 & 0.00020284 & 39.4878502 & 39.4877625 & 39.4968109 & 39.4967 & 67.15 & 67.15 & 0.03182 \\
\hline 45 & 1.41320002 & 30.7077827 & 30.0980922 & 31.3386955 & 30.7270 & 67.15 & 67.15 & 0.03708 \\
\hline 46 & 255 & 27.4 & 80456 & 8146 & 30.0961 & 67.24 & 67.24 & 0.02162 \\
\hline 47 & 0.00020017 & 39.4 & 1125 & 00694 & 39.4500 & 67.15 & 67.15 & 0.03171 \\
\hline 48 & 1.400886196 & 30.7 & 8223 & 77564 & 30.7874 & 67.15 & 67.15 & 0.03713 \\
\hline 49 & 0.00019777 & 39.3992805 & 91968 & 0696 & 39.4080 & 67.18 & 67.16 & 0.03162 \\
\hline 50 & 8749 & 30.7 & 8108 & 2544 & 0.8037 & 67.18 & 67.16 & 0.03718 \\
\hline 51 & 191 & 37.0902481 & 2483 & 00694 & 39.4081 & 67.15 & 67.15 & 0.02189 \\
\hline 52 & 0.097 & 27.8249741 & 9819 & 48068 & 30.1428 & 67.24 & 67.24 & 0.02188 \\
\hline 53 & & 1897 & 4020 & 6497 & & 67.14 & 67.14 & 0.03193 \\
\hline 54 & 1.42684862 & 30.6619606 & 30.0443687 & 31.2998772 & 30.6823 & 67.14 & 67.14 & 0.03703 \\
\hline 55 & & 36.5729408 & 36.5212021 & 39.5485497 & 39.4968 & 67.14 & 67.14 & 0.02138 \\
\hline 56 & 627 & 27 & 7294 & & .0444 & 7.24 & 67.24 & 0.02138 \\
\hline 57 & 0.08885790 & 4765 & 9704 & 0896 & 39.3706 & 67.18 & 67.16 & 0.02217 \\
\hline 58 & 0.0888 & 28.1 & 28.1042061 & 30.2223053 & 30.1848 & 67.24 & 67.24 & 0.02217 \\
\hline 59 & 0.14858132 & 35.9984589 & 35.9350128 & 39.7291679 & 39.6857 & 67,12 & 67.12 & 0.02092 \\
\hline 60 & 0.00008499 & 39.7260094 & 39.7259750 & 39.7291679 & 39.7291 & 67.12 & 67.12 & 0.03781 \\
\hline 61 & 0850 & 30.0 & 8039 & 3960 & 0.0862 & 67.12 & 7.12 & 0.03574 \\
\hline 62 & 289 & 28. & 2528 & 2232 & 2.8838 & 67.25 & 67.25 & 0.02091 \\
\hline 63 & 08444 & 2053 & 5710 & 7219 & 39.6657 & 67.13 & 67.13 & 0.03805 \\
\hline 64 & 4075 & 30.1 & 2232 & 4028 & 30.1468 & 67.13 & 67.13 & 0.03558 \\
\hline 65 & 0.00008391 & 39.6024704 & 39.6024361 & 39.6055489 & 39.6055 & 67.14 & 67.14 & 0.03829 \\
\hline 68 & & 30.1875839 & 29.9873772 & 30.4044876 & 30.2043 & 67.14 & 67.14 & 0.03542 \\
\hline 67 & & & & & & .13 & 13 & 2104 \\
\hline 68 & 97893 & 92230 & 900991 & 73772 & 29.9272 & 67.24 & 67.24 & 0.02103 \\
\hline 69 & 008657 & 39.7927895 & 7513 & 9900 & 39.7960 & 67.12 & 67.12 & 0.03758 \\
\hline 70 & 5888 & 79538 & 70047 & 23794 & 30.0224 & 67.12 & 67.12 & \\
\hline 71 & 0.15437689 & 35.8450241 & 35.7782021 & 39.7959900 & 39.7292 & 67.12 & 67.12 & 0.02080 \\
\hline 72 & 0.31583714 & 75.8554993 & 75.7188283 & 76.7480316 & 76.6114 & 69.38 & 69.38 & 0.01748 \\
\hline 73 & & & & & & 39.36 & 38 & 0.01748 \\
\hline 74 & 008745 & & & 7803 & 40.0177 & 67.11 & 67.11 & 0.03677 \\
\hline 75 & 0.51671268 & 29.7989655 & 29.5753098 & 30.0343132 & 29.8107 & 67.11 & 67.11 & 0.03646 \\
\hline 76 & 0.17923089 & 24.99060008 & 24.9130211 & 29.6528854 & 29.5753 & 67.28 & 67.28 & 0.02046 \\
\hline 77 & 0.00008768 & 39.9388095 & 39.9367714 & 39.9401703 & 39.9401 & 67.11 & 67.11 & 0.03687 \\
\hline 78 & 0.52085397 & 29.6652718 & 29.4399109 & 29.9019089 & 29.6765 & 67.11 & 67.11 & 0.03853 \\
\hline 79 & 0.00008817 & 39.8630409 & 39.8630028 & 39.8682872 & 39.8682 & 67.11 & 67.11 & 0.03731 \\
\hline 80 & 0.49681172 & 29.9416924 & & & 29.9653 & 67.11 & 67.11 & \\
\hline 81 & 0.17068981 & 35.5249290 & 35.4510460 & 39.9401703 & 39.8683 & 67.11 & 67.11 & 0.02058 \\
\hline 82 & 0.17081860 & 25.3114168 & 25.2375879 & 29.7267342 & 29.6629 & 67.27 & 67.27 & 0.02057 \\
\hline 83 & 0,00008813 & 40.0968023 & 40,0957642 & 40.0991974 & 40.0992 & 67.10 & 67.10 & 0.03649 \\
\hline 84 & 0.52761623 & 29.7223091 & 29.4939348 & 29.9612961 & 29.7329 & 67.10 & 67.10 & 0.03686 \\
\hline
\end{tabular}




\begin{tabular}{|c|c|c|c|c|c|c|c|c|}
\hline \multicolumn{3}{|c|}{$\begin{array}{l}\text { AFT Fathom } 7.0 \text { Output } \\
\text { ANL }\end{array}$} & \multicolumn{6}{|c|}{ ( 6 of 8$)$} \\
\hline Pipe & $\begin{array}{c}\mathrm{dH} \\
\text { (feet) }\end{array}$ & $\begin{array}{l}\text { P Static In } \\
\text { (psig) }\end{array}$ & $\begin{array}{c}\text { P Static } \\
\text { Out } \\
\text { (psig) }\end{array}$ & $\begin{array}{l}\text { P Stag. In } \\
\text { (psig) }\end{array}$ & $\begin{array}{l}\text { P Stag. } \\
\text { Out } \\
\text { (psiq) }\end{array}$ & $\begin{array}{c}T \\
\text { Inlet } \\
\text { (deg. } F)\end{array}$ & $\begin{array}{c}T \\
\text { Outlet } \\
\text { (deg. } F \text { ) }\end{array}$ & $f$ \\
\hline 85 & 0.18809635 & 35.1840172 & 35.1026001 & 40.0991974 & 40.0178 & 67.10 & 67.10 & 0.02037 \\
\hline 86 & 0.18801260 & 24.66000418 & 24.5786087 & 29.5753059 & 29.4939 & 67.28 & 67.28 & 0.02036 \\
\hline 87 & 0.13168816 & 38.2925301 & 38.2355309 & 39.6056489 & 39.5485 & 67.14 & 67.14 & 0.02116 \\
\hline 88 & 0.16241092 & 35.6872101 & 35.6169128 & 39.8682872 & 39.7960 & 67.11 & 67.11 & 0.02069 \\
\hline 89 & 0.13164978 & 26.7313194 & 26.6743356 & 30.0443811 & 29.9874 & 67.24 & 67.24 & 0.02115 \\
\hline 90 & 0.16234797 & 25.6178685 & 25.5475960 & 29.7970047 & 29.7267 & 67.26 & 67.26 & 0.02068 \\
\hline 105 & 0.00129855 & -0.0000933 & -0.0001335 & -0.0006598 & 0.0000 & 69.34 & 69.34 & 0.02856 \\
\hline 108 & 0.00001087 & -0.0001335 & 0.4325924 & 0.0000000 & 0.4327 & 69.34 & 69.34 & 0.02856 \\
\hline 107 & 0.00000000 & 0.0000000 & 0.00000000 & 0.0000000 & 0.0000 & 65.00 & 65.00 & 0.00000 \\
\hline 109 & 0.28898997 & 83.8016205 & 83.6785594 & 83.9330090 & 83.8086 & 69.36 & 69.38 & 0.02921 \\
\hline 111 & 0.33201824 & 84.2962952 & 84.1526184 & 85.2399445 & 85.0963 & 69.35 & 69.35 & 0.01739 \\
\hline 112 & 0.28898997 & 1.1579695 & 1.0329142 & 1.2899628 & 1.1849 & 69.38 & 69.38 & 0.02921 \\
\hline 113 & 0.28898997 & 1.3127174 & 1.1870831 & 1.4447117 & 1.3197 & 69.38 & 69.38 & 0.02921 \\
\hline 114 & 0.31583714 & 84.2034607 & 84.0687877 & 85.0959930 & 84.9693 & 69.38 & 69.38 & 0.01748 \\
\hline 115 & 0.31583714 & 75.0119019 & 74.4425049 & 75.9044342 & 75.3350 & 69.38 & 69.38 & 0.01748 \\
\hline 117 & 9.47551856 & 10.8014336 & 4.9701614 & 11.6939564 & 5.8627 & 69.34 & 69.34 & 0.01748 \\
\hline 120 & 0.28678446 & 1.0302744 & 0.9081832 & 1.1622877 & 1,0382 & 69.38 & 69.36 & 0.02921 \\
\hline 125 & 0.35815835 & -0.0223379 & -0.1773243 & 0.8942490 & 0.7393 & 69.35 & 69.35 & 0.01945 \\
\hline 128 & 0.33214868 & 0.0945339 & -0.0491972 & 1.0381765 & 0.8944 & 69.35 & 69.35 & 0.01739 \\
\hline 128 & 1.57856130 & -0.9026184 & 0.1452131 & -0.0098550 & 1.0382 & 69.34 & 69.34 & 0.01748 \\
\hline 129 & 9.52924120 & 63.2353249 & 59.9763603 & 64.1278245 & 60.8687 & 67.09 & 67.09 & 0.01759 \\
\hline 130 & 0.15432084 & 25.9127808 & 25.8459854 & 29.8638000 & 29.7970 & 67.25 & 67.25 & 0.02079 \\
\hline 131 & 0.17930809 & 35.3565794 & 35.2779694 & 40.0177803 & 39.9402 & 67.11 & 67.11 & 0.02047 \\
\hline 132 & 4.134278003 & 56.1974831 & 54.4080000 & 60.3996399 & 58.6101 & 67.09 & 67.09 & 0.01650 \\
\hline 133 & 16.53710411 & 52.4279900 & 45.2700424 & 56.6300468 & 49.4721 & 67.09 & 67.09 & 0.01650 \\
\hline 134 & 0.41271679 & 40.3088722 & 40.1302299 & 48.9328957 & 48.7543 & 67.09 & 67.09 & 0.01610 \\
\hline 135 & 0.41255043 & 15.6604919 & 15.4819260 & 24.2848375 & 24.1081 & 67.28 & 67.28 & 0.01609 \\
\hline 138 & 4.13249495 & 10.2400208 & 8.4513416 & 14.4421387 & 12.6635 & 67.28 & 67.28 & 0.01649 \\
\hline 137 & 16.52997982 & 19.3747711 & 12.2200584 & 23.5768890 & 16.4222 & 67.28 & 67.28 & 0.01649 \\
\hline 138 & 0.63170116 & 4.5860195 & 4.3126621 & 5.4785423 & 5.2052 & 69.34 & 69.34 & 0.01748 \\
\hline 139 & 0.31585058 & 3.1919346 & 3.0552559 & 4.0844574 & 3.9478 & 69.34 & 69.34 & 0.01748 \\
\hline 140 & 0.02832088 & 2.6711140 & 2.8597233 & 3.5638388 & 3.5622 & 69.34 & 69.34 & 0.01748 \\
\hline 141 & 0.15792527 & 2.2755814 & 2.2072430 & 3.1681042 & 3.0998 & 69.34 & 69.34 & 0.01748 \\
\hline 142 & 0.07896284 & 1.6715584 & 1.8373882 & 2.5640812 & 2.5299 & 69.34 & 69.34 & 0.01748 \\
\hline 143 & 0.94755167 & 1.2532454 & -0.02222511 & 2.1457691 & 0.8703 & 69.34 & 69.34 & 0.01748 \\
\hline 144 & 0.03881706 & 2.4608040 & 2.4440937 & 2.8330471 & 2.6163 & 69.34 & 69.34 & 0.02005 \\
\hline 145 & 0.07723412 & 2.8322964 & 2.7988758 & 3.0045395 & 2.9711 & 69.34 & 69.34 & 0.02005 \\
\hline 148 & 0.31748968 & 10.9390602 & 10.8016415 & 11.8313751 & 11.6940 & 67.28 & 67.28 & 0.01758 \\
\hline
\end{tabular}

All Junction Table

\begin{tabular}{|c|c|c|c|c|c|c|c|c|}
\hline Jet & Name & $\begin{array}{l}\text { P Statc In } \\
\text { (psig) }\end{array}$ & $\begin{array}{c}\text { P Stafic Out } \\
\text { (psig) } \\
\end{array}$ & $\begin{array}{l}\text { Vol. Flow } \\
\text { Rate Thru Jot } \\
\text { (galimin) }\end{array}$ & $\begin{array}{c}T \\
\text { Inlet } \\
\text { (deg. } F \text { ) }\end{array}$ & $\begin{array}{c}\mathrm{T} \\
\text { Outet } \\
\text { (deg. F) }\end{array}$ & $\begin{array}{c}\text { P Stag. } \\
\text { In } \\
\text { (psig) }\end{array}$ & $\begin{array}{c}\text { P Stag. Out } \\
\text { (psig) }\end{array}$ \\
\hline 2 & Area Change & 40.13022995 & 34.95331965 & 52.919 & 67.09 & 67.09 & 48.7543 & 40.1302299 \\
\hline 3 & Branch & 37.79774857 & 37.79774867 & NA & 67.09 & 67.09 & 40.0992 & 40.0991974 \\
\hline 4 & Branch & 39.25336838 & 39.25336838 & 5.139 & 67.21 & 67.21 & 39.3022 & 39.3021985 \\
\hline 5 & Branch & 39.10983885 & 39.10933685 & $\mathrm{NA}$ & 67.20 & 67.20 & 39.3048 & 39.3047000 \\
\hline 6 & Pp-1 Gould Centrifugal Pump & -0.17732430 & 84.29629517 & 54.427 & 69.35 & 69.35 & 0.7393 & 85.2399445 \\
\hline 7 & Branch & 30.29003525 & 30.24120712 & 5.139 & 67.21 & 67.21 & 33.7286 & 30.2900352 \\
\hline 8 & Branch & 2931430435 & 2931430435 & NA & 67.21 & 6721 & 30.2881 & 30.2881012 \\
\hline 9 & $H X-1$ & 74.44250488 & 63.235324898 & 52.933 & 69.36 & 67.09 & 75.3350 & 64.1276245 \\
\hline 10 & Throtte Valve & 84.06678772 & 75.855499927 & 52.933 & 69.36 & 69.36 & 84.9693 & 76.7480316 \\
\hline 11 & Ara Change & 8.45134163 & 10.93906021 & 52.920 & 67.28 & 67.28 & 12.6635 & 11.8313751 \\
\hline 13 & Branch & 39.21594238 & 39.21594238 & NA & 67.20 & 67.20 & 39.3028 & 39.3027878 \\
\hline 14 & Orifice & 39.25284576 & 33.00007248 & 5.139 & 67.21 & 67.21 & 39.3017 & 38.4386139 \\
\hline
\end{tabular}




\begin{tabular}{|c|c|c|c|c|c|c|c|c|c|}
\hline \multirow{2}{*}{\multicolumn{2}{|c|}{$\begin{array}{l}\text { AFT Fathom } 7.0 \text { Output } \\
\text { ANL }\end{array}$}} & \multicolumn{3}{|r|}{$(7 \circ$ of 8$)$} & & & & & \multirow[t]{2}{*}{$3 / 1 / 2017$} \\
\hline & & \multicolumn{4}{|c|}{ AFT Fathom Model } & & & & \\
\hline Jet & Name & $\begin{array}{c}\text { P Static In } \\
\text { (psig) }\end{array}$ & $\begin{array}{c}\text { P Stafic Out } \\
\text { (psig) }\end{array}$ & \begin{tabular}{c|} 
Vol. Flow \\
Rate Thru Jot \\
(galimin)
\end{tabular} & \begin{tabular}{|c|}
$T$ \\
Inlet \\
(deg. F)
\end{tabular} & $\begin{array}{c} \\
\text { Outet } \\
\text { (deg. F) }\end{array}$ & $\begin{array}{c}\text { P Stag. } \\
\text { In } \\
\text { (psig) }\end{array}$ & $\begin{array}{c}\text { P Stag. Out } \\
\text { (psig) }\end{array}$ & \\
\hline 15 & Branch & 29.58581707 & 29.58581707 & \begin{tabular}{|r|} 
NA \\
\end{tabular} & 67.21 & \begin{tabular}{|l|}
67.21 \\
\end{tabular} & 30.2901 & 30.2900696 & \\
\hline 16 & Branch & 38.81470490 & 38.81470490 & NA & 67.18 & 67.18 & 39.3235 & 39.3234787 & \\
\hline 17 & Branch & 38.89120102 & 38.89120102 & $N A$ & 67.19 & 67.19 & 39.3155 & 39.3154793 & \\
\hline 18 & Orifice & 39.31477356 & 30.86119080 & 2.160 & 67.19 & 67.19 & 39.3234 & 31.4888508 & \\
\hline 19 & Branch & 29.38167953 & 29.38167963 & $\mathrm{NA}$ & 67.22 & 67.22 & 30.2694 & 30.2693787 & \\
\hline 20 & Branch & 29.50287628 & 29.50287028 & NA & 67.22 & 67.22 & 30.2774 & 30.2773781 & \\
\hline 21 & Orifice & 39.30678558 & 30.88826324 & 2.158 & 67.19 & 67.19 & 39.3154 & 31.4746399 & \\
\hline 22 & Branch & 38.96128082 & 38.96128082 & $\mathrm{NA}$ & 67.19 & 67.19 & 39.3088 & 39.3087807 & \\
\hline 23 & Orifice & 39.25342941 & 32.99980927 & 5.140 & 67.21 & 67.21 & 39.3023 & 38.4388161 & \\
\hline 24 & Branch & 28.99702454 & 28.99702454 & $\mathrm{NA}$ & 87.21 & 6721 & 30.2841 & 30.2840767 & \\
\hline 25 & Branch & 38.54724503 & 38.54724503 & NA & 67.17 & 67.17 & 39.3563 & 39.3663232 & \\
\hline 28 & Branch & 38.64285442 & 38.64285442 & $\mathrm{NA}$ & 67.17 & 67.17 & 39.3438 & 39.3437996 & \\
\hline 27 & Orifice & 39.34754944 & 30.83214961 & 2108 & 87.17 & 67.17 & 39.3562 & 31.4440498 & \\
\hline 28 & Branch & 28.96141434 & 28.96141434 & $\mathrm{NA}$ & 67.23 & 67.23 & 30.2385 & 30.2385417 & \\
\hline 29 & Branch & 29.11109543 & 29.11109543 & NA & 67.22 & 67.22 & 30.2491 & 30.2490616 & \\
\hline 30 & Orifice & 39.33504888 & 30.84322739 & 2.165 & 67.18 & 67.18 & 39.3437 & 31.4534340 & \\
\hline 31 & Branch & 38.73182878 & 38.73182878 & $\mathrm{NA}$ & 67.18 & 67.18 & 39.3329 & 39.3328857 & \\
\hline 32 & Orifice & 39.32415771 & 30.85287857 & 2.162 & 67.18 & 67.18 & 39.3328 & 31.4816089 & \\
\hline 33 & Branch & 29.25112915 & 29.25112915 & NA & 67.22 & 67.22 & 30.2600 & 30.2599754 & \\
\hline 34 & Branch & 38.44562912 & 38,44562912 & NA & 67.16 & 67.16 & 39.3706 & 39.3705835 & \\
\hline 35 & Orifice & 39.36175919 & 30.81955338 & 2.171 & 67.17 & 67.17 & 39.3705 & 31.4333801 & \\
\hline 36 & Branch & 28.80186081 & 28.80188081 & $\mathrm{NA}$ & 67.23 & 67.23 & 30.2223 & 30.2223053 & \\
\hline 37 & Branch & 38.17398834 & 38.173988834 & NA & 67.14 & 67.14 & 39.4968 & 39.4968109 & \\
\hline 38 & Deionizer & 1.18786308 & 1.15796947 & 1.485 & 69.36 & 69.38 & 1.3197 & 1.2899628 & \\
\hline 39 & Orifice & 39.48776245 & 30.70778275 & 2.201 & 67.15 & 67.15 & 39.4967 & 31.3386955 & \\
\hline 40 & Branch & 28.18226624 & 28.18228624 & $\mathrm{NA}$ & 67.23 & 6723 & 30.0961 & 30.0960846 & \\
\hline 41 & Parficulate Filter & 75.71882629 & 75.10842781 & 52.933 & 69.38 & 69.38 & 76.6114 & 76.0689800 & \\
\hline 42 & Orifoe & 39.44111252 & 30.74916440 & 2.190 & 67.15 & 67.15 & 39.4500 & 31.3737564 & \\
\hline 43 & Branch & 38.35898590 & 38.35898590 & NA & 67.15 & 67.15 & 39.4081 & 39.4080696 & \\
\hline 44 & Area Change & -0.02225113 & -0.00069332 & 52.932 & 69.34 & 69.34 & 0.8703 & -0.0005598 & \\
\hline 45 & Ara Change & 0.43259239 & -0.90281841 & 52945 & 69.34 & 69.34 & 0.4327 & -20096550 & \\
\hline 46 & Tee or We & -0.00005913 & -0.00005913 & NA & 69.34 & 69.34 & 0.0000 & 0.0000000 & \\
\hline 47 & Assigned Pressure & 0.00000000 & 0.00000000 & 0.000 & 85.00 & 85.00 & 0.0000 & 0.0000000 & \\
\hline 48 & Branch & 27.94720459 & 2794720459 & $\mathrm{NA}$ & 67.23 & 67.23 & 30.0444 & 30.0443811 & \\
\hline 49 & Tee or We & 84.51898193 & 84.51898193 & NA & 69.35 & 69.35 & 85.0963 & 85.0962877 & \\
\hline 50 & Branch & 38.00719833 & 38.00719833 & NA & 67.12 & 67.12 & 39.0857 & 39.0857219 & \\
\hline 51 & Valve & 83.67665945 & 1.31271744 & 1.486 & 69.38 & 69.36 & 83.8086 & 1.4447117 & \\
\hline 52 & Valve & 75.02975464 & 75.01190188 & 52.933 & 69.36 & 69.38 & 75.9223 & 75.9044342 & \\
\hline 53 & Valve & 1.03291416 & 1.03027439 & 1.485 & 69.38 & 69.36 & 1.1849 & 1.1622677 & \\
\hline 54 & Orifice & 39.66257095 & 30.13080597 & 1.299 & 67.13 & 67.13 & 39.6857 & 30.3604028 & \\
\hline 55 & Branch & 38.04153442 & 38.04153442 & $\mathrm{NA}$ & 67.13 & 67.13 & 39.6055 & 39.6055489 & \\
\hline 56 & Orifice & 39.60243607 & 30.18758392 & 1.291 & 67.14 & 67.14 & 39.6055 & 30.4044876 & \\
\hline 57 & Branch & 28.06256886 & 28.06258986 & $\mathrm{NA}$ & 67.24 & 67.24 & 29.9874 & 29.9873772 & \\
\hline 58 & Branch & 37.93814850 & 37.93814860 & NA & 67.11 & 67.11 & 39.7960 & 39.7959900 & \\
\hline 59 & Orifice & 39.79275131 & 30.00795384 & 1.316 & 67.12 & 67.12 & 39.7960 & 30.2333794 & \\
\hline 60 & Area Change & -0.04919720 & -0.02233791 & 54.427 & 69.35 & 69.35 & 0.8944 & 0.8942490 & \\
\hline 61 & Branch & 37.83287975 & 37.83287975 & NA & 67.10 & 67.10 & 40.0178 & 40.0177803 & \\
\hline 62 & Teeor Me & 0.46078014 & 0.46078014 & NA & 69.34 & 69.34 & 1.0382 & 1.0381705 & \\
\hline 63 & Orifice & 39.25985364 & 32.99721146 & 5.143 & 67.20 & 67.20 & 39.3083 & 36.4409065 & \\
\hline 64 & Orifice & 39.25537872 & 32.99897003 & 5.141 & 67.20 & 67.20 & 39.3042 & 36.4395103 & \\
\hline 65 & Branch & 38.26841354 & 38.26841354 & NA & 67.15 & 67.15 & 39.4501 & 39.4500694 & \\
\hline 66 & Branch & 28.40269852 & 28.40269962 & NA & 67.23 & 67.23 & 30.1428 & 30.1428146 & \\
\hline 67 & Branch & 28.60906801 & 28.60906001 & NA & 67.23 & 67.23 & 30.1848 & 30.1848068 & \\
\hline 68 & Orifice & 39.39919682 & 30.78635025 & 2.180 & 67.16 & 67.16 & 39.4080 & 31.4052544 & \\
\hline 69 & Branch & 38.07572937 & 38.07572987 & $\mathrm{NA}$ & 67.14 & 67.14 & 39.5485 & 39.5485497 & \\
\hline 70 & Orifice & 39.53940201 & 30.68196080 & 2.214 & 67.14 & 67.14 & 39.5485 & 31.2998772 & \\
\hline
\end{tabular}




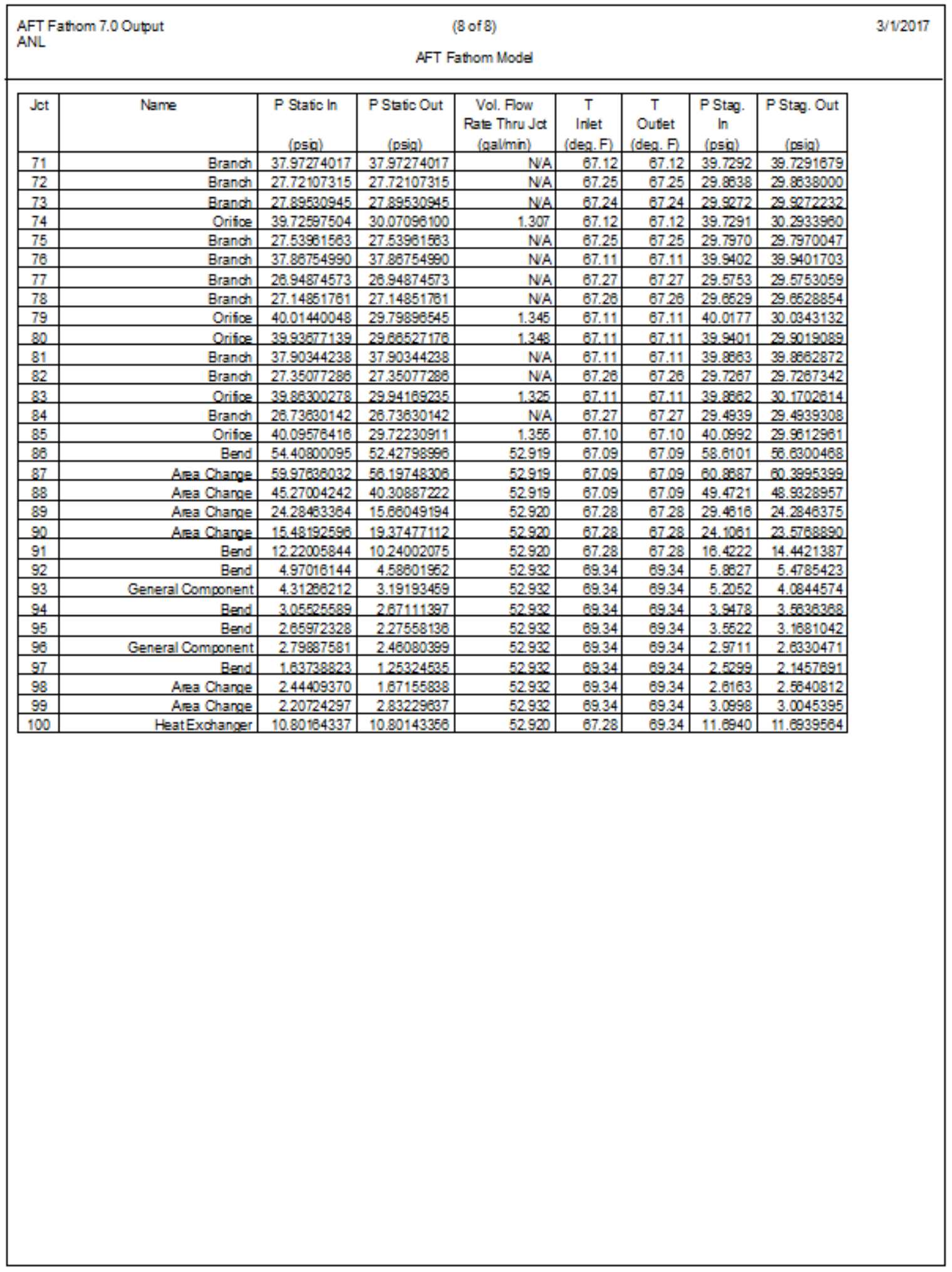




\section{Summary of DU Target Flow Testing}

The flow tests were used to develop the complete FATHOM model by validating each component as it was added to the model. (I.e. Pre-test and then configurations $1-4$ ) as described below. At each step a description the test arrangement is first shown, then the corresponding FATHOM model and finally a plot showing the comparison between the test and model of pressure loss (as determined by the pressure transducers) verse flow. The final flow configuration 4 was then incorporated into the actual model of the cooling system.

\section{$\underline{\text { Test Description Pre-test Configuration }}$}

The test arrangement for the pre-test configuration is shown below. The purpose of this test is to determine the pressure losses inherent in the test system between the pressure taps for the differential pressure gages. Pressure losses in the empty target housing are assumed negligible; however, entrance and exit losses at the back of the housing are significant and will be accounted for.

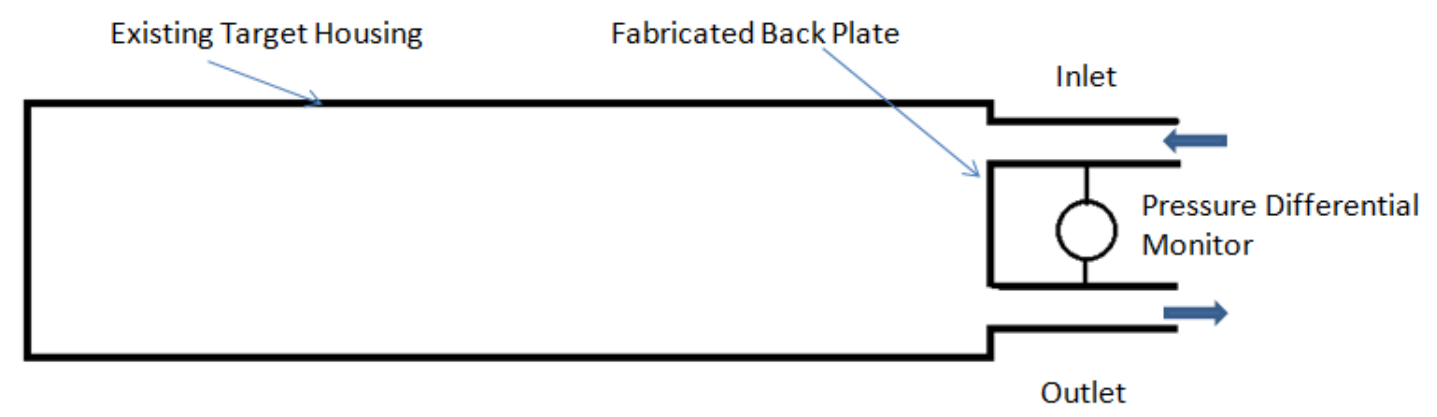

Test Arrangement

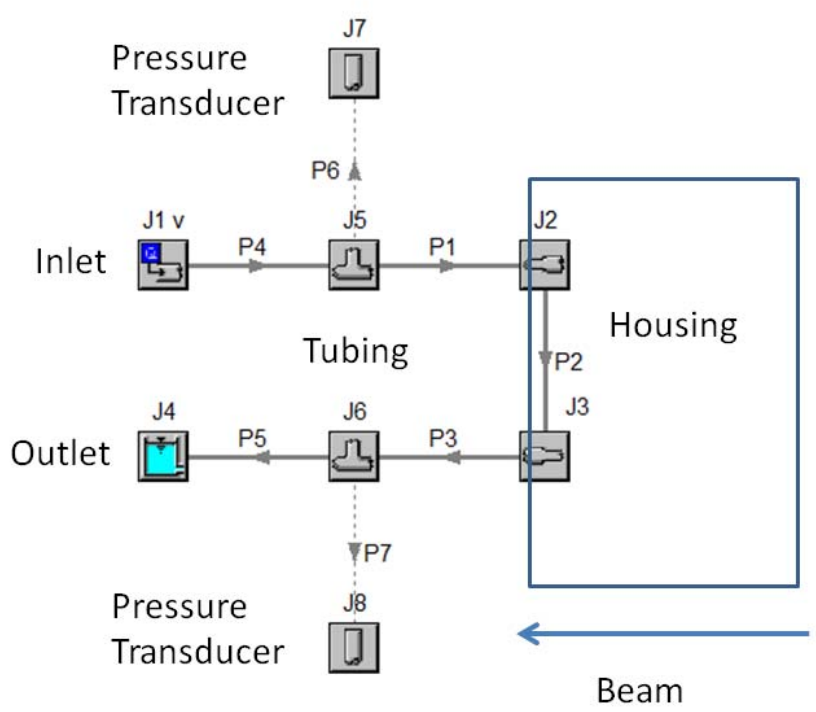

FATHOM Diagram (Note reversed image from Test Arrangement) 


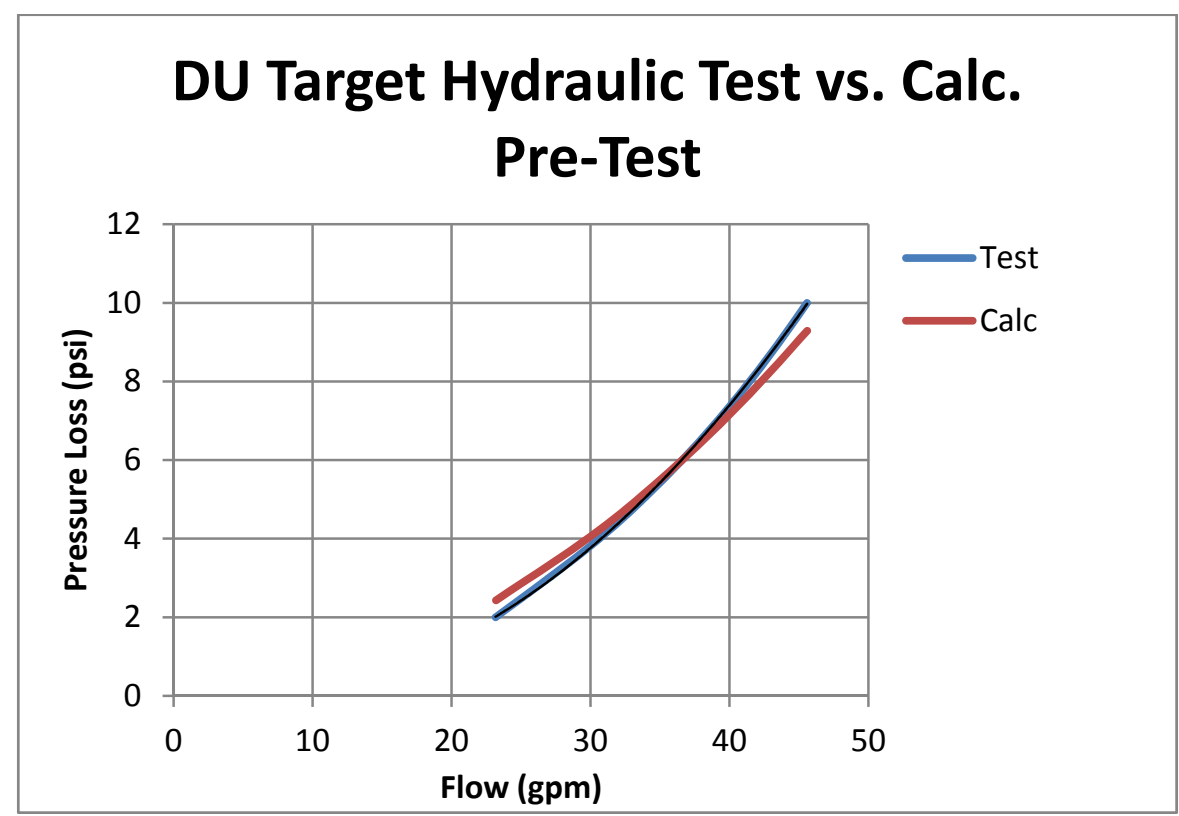

FATHOM vs Test

$\underline{\text { Test Description Configuration } 1}$

The test arrangement for configuration 1 is shown below. The purpose of this test is to validate the pressure losses associated with the flow through the inlet and outlet of the target. This configuration allows for a large flow area within the target housing and, therefore, the pressure loss within the target is assumed negligible relative to those at the inlet and outlet. This test will be used to validate the FATHOM simulation of losses at the target inlet and outlet.

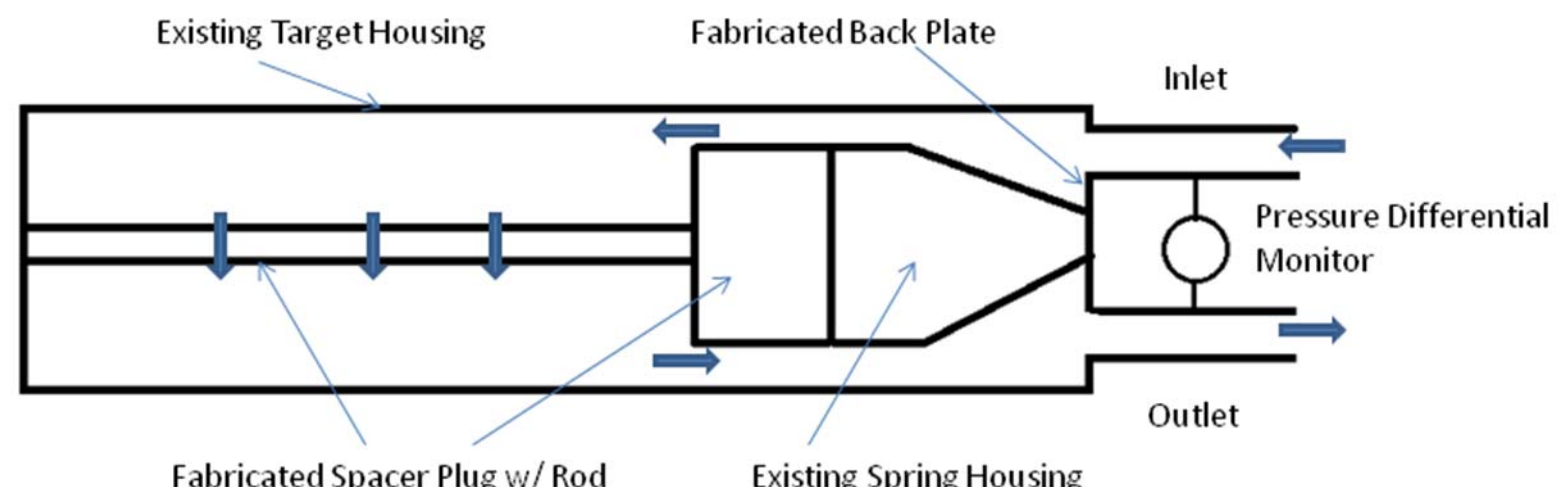

Test Arrangement 


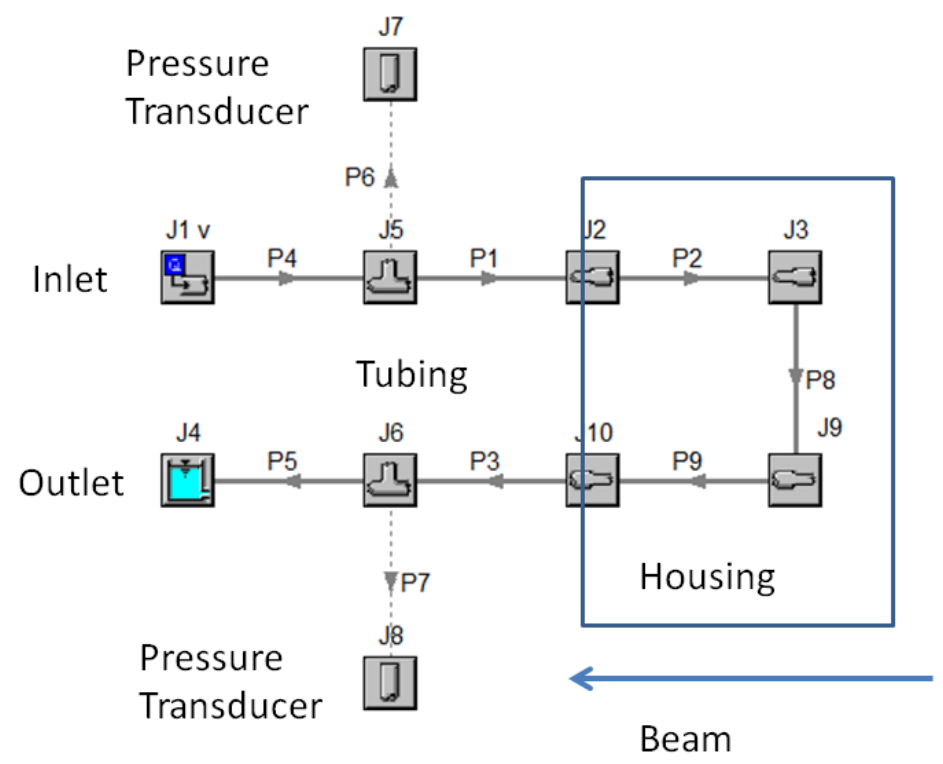

FATHOM Diagram (Note reversed image from Test Arrangement)

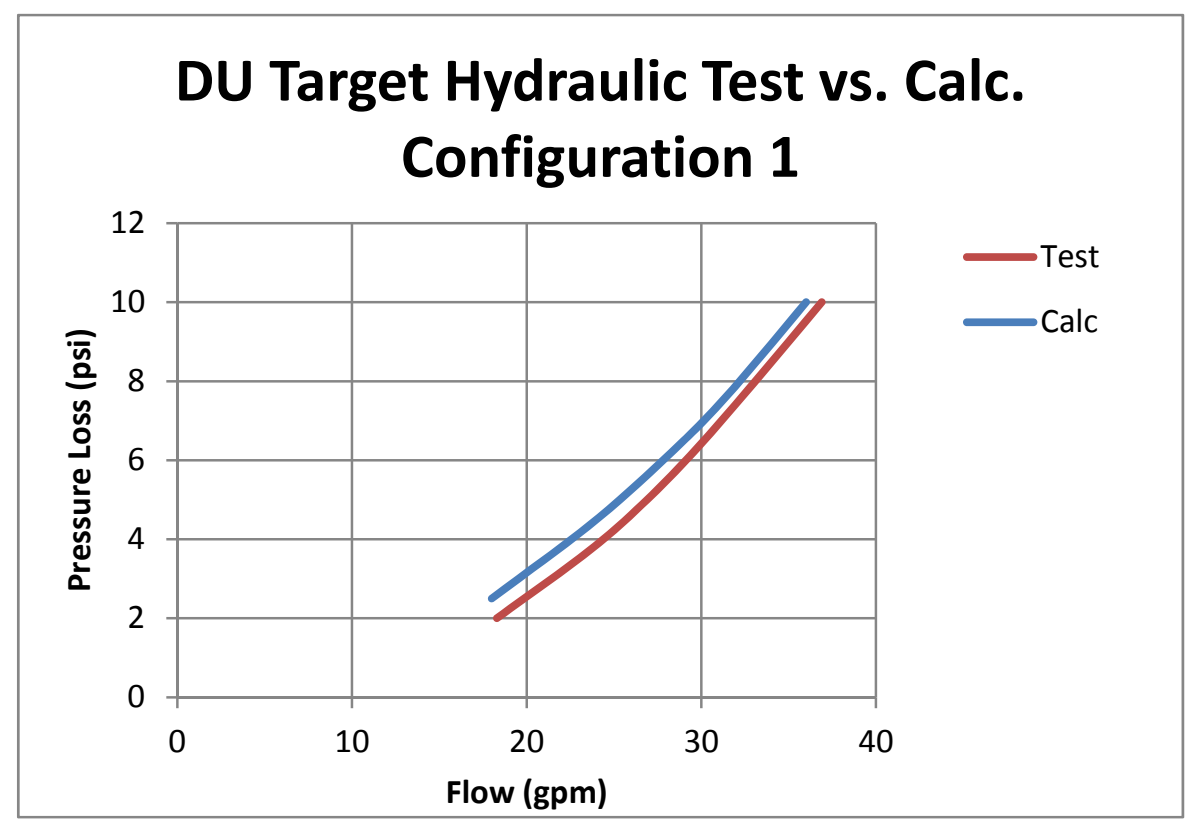

FATHOM vs Test

Test Description Configuration 2

The test arrangement for the full plug is shown below. The purpose of this test is to validate the losses associated with the flow around the sides of the spacers and disks, what will be referred to as 'leakage'. The back plate used for configuration 1 is also used for this test. This configuration allows for a 
closed flow area within the target housing and, therefore, all flow through the target would be due to leakage. This test was used to validate the FATHOM simulation of losses from flow around the disks.

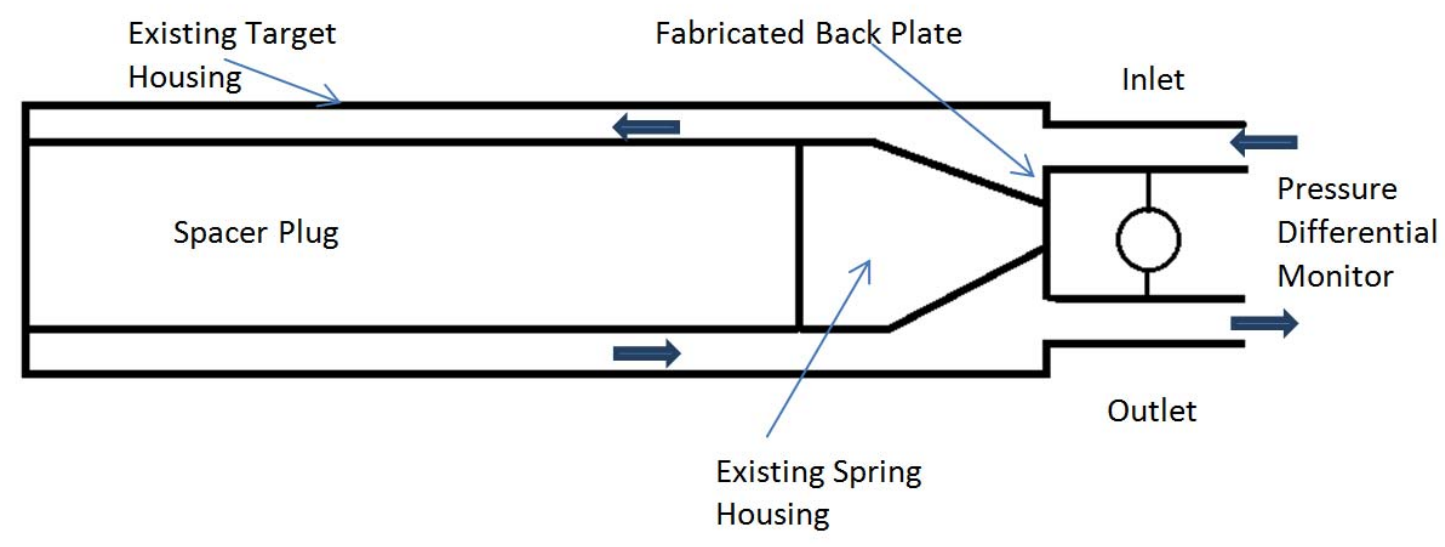

Test Arrangement

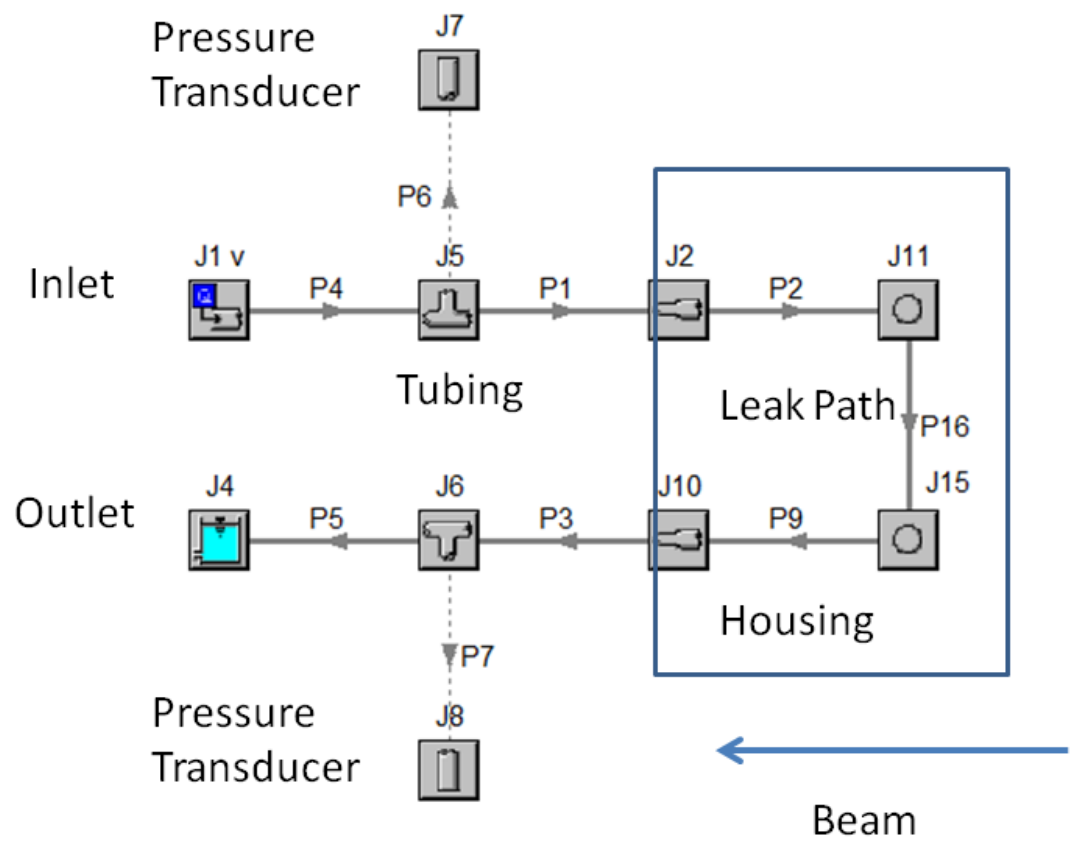

FATHOM Diagram (Note reversed image from Test Arrangement) 


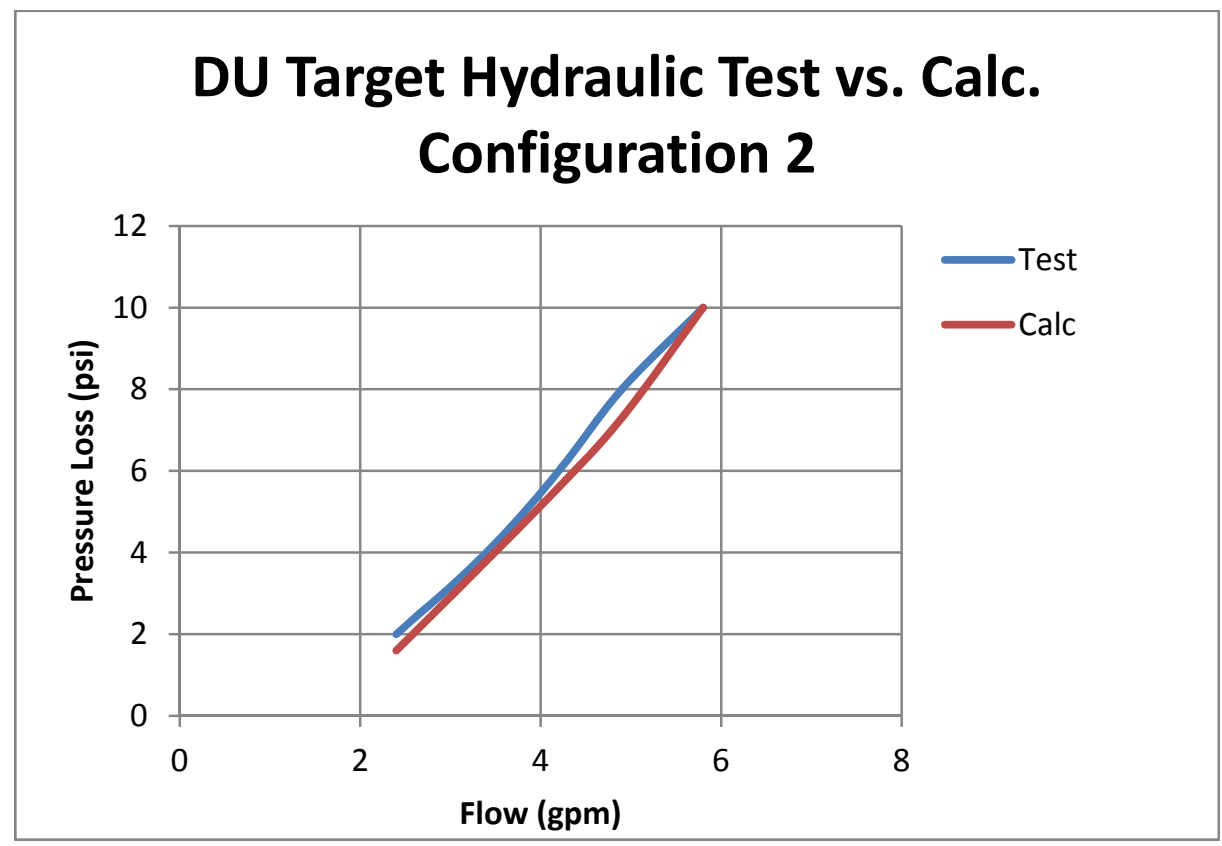

FATHOM vs Test

\section{$\underline{\text { Test Description Configuration } 3}$}

The test arrangement for configuration 3 is shown below. The purpose of this test is to validate the pressure losses associated with the flow across the front 4 disks in the target.

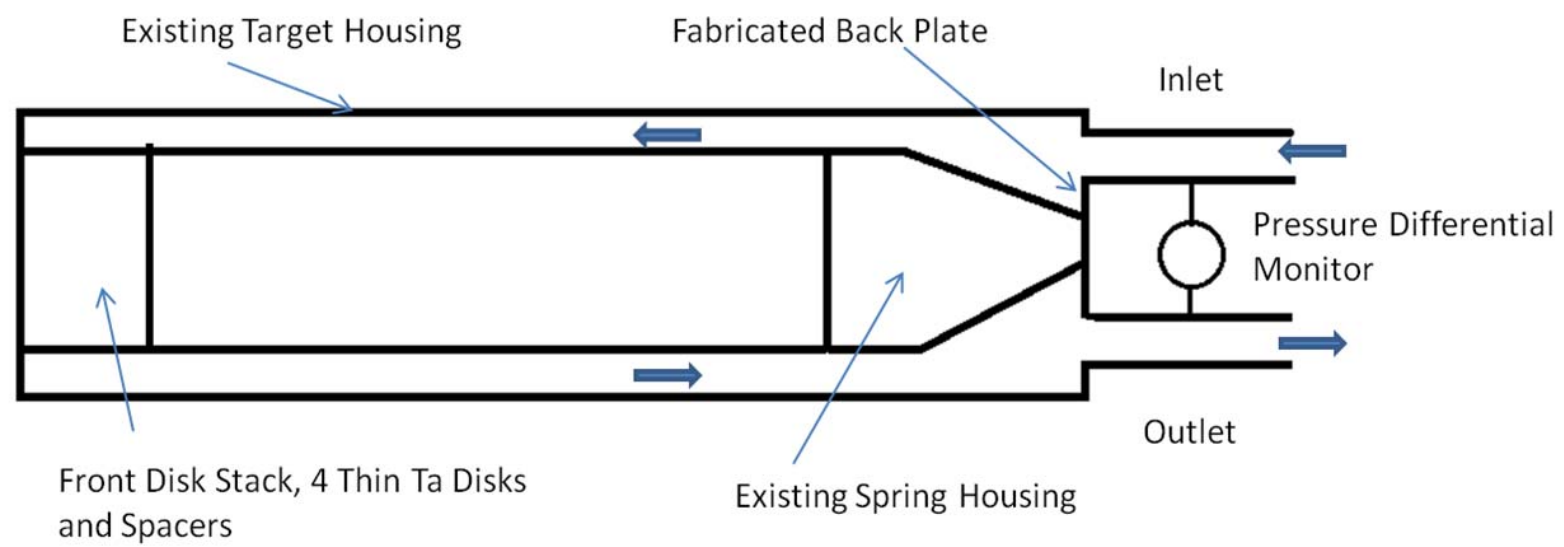

Test Arrangement 


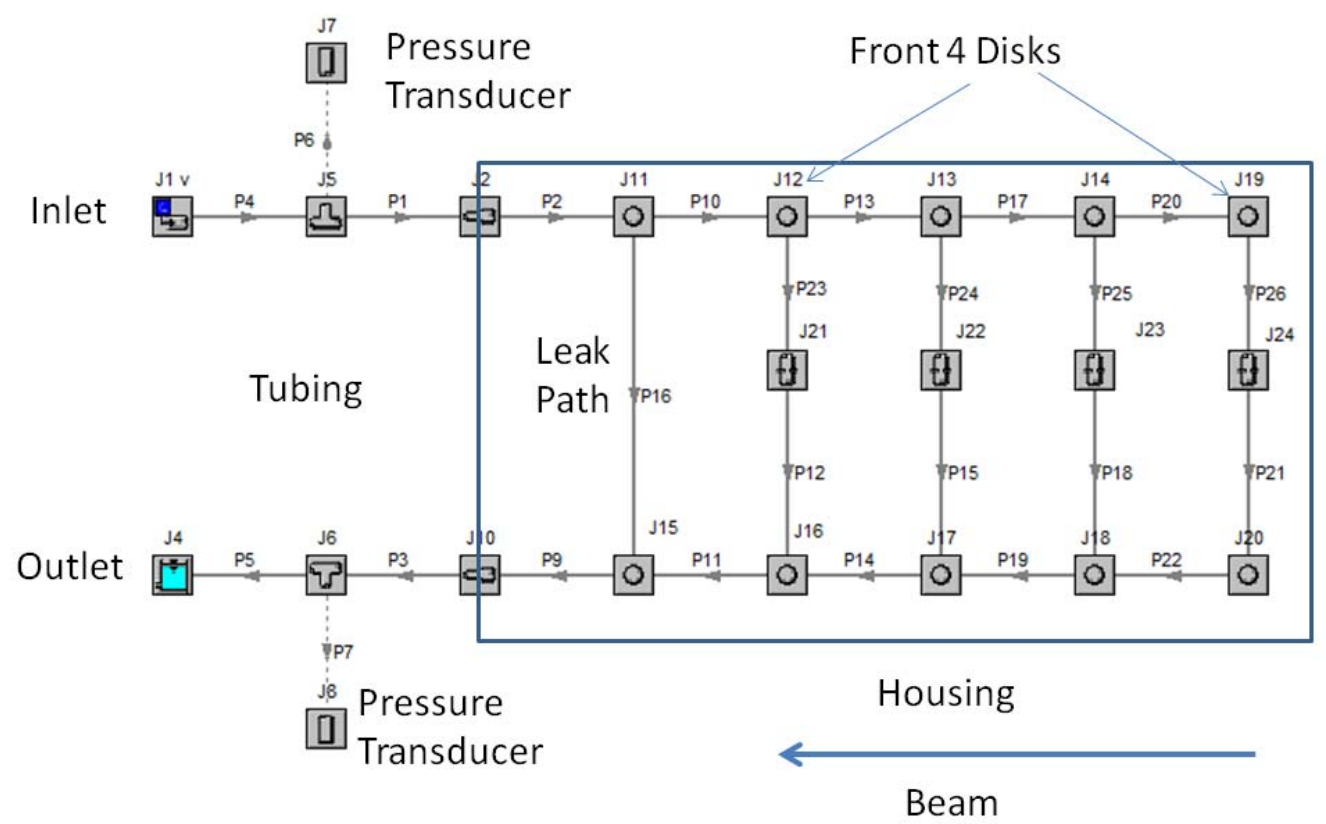

FATHOM Diagram (Note reversed image from Test Arrangement)

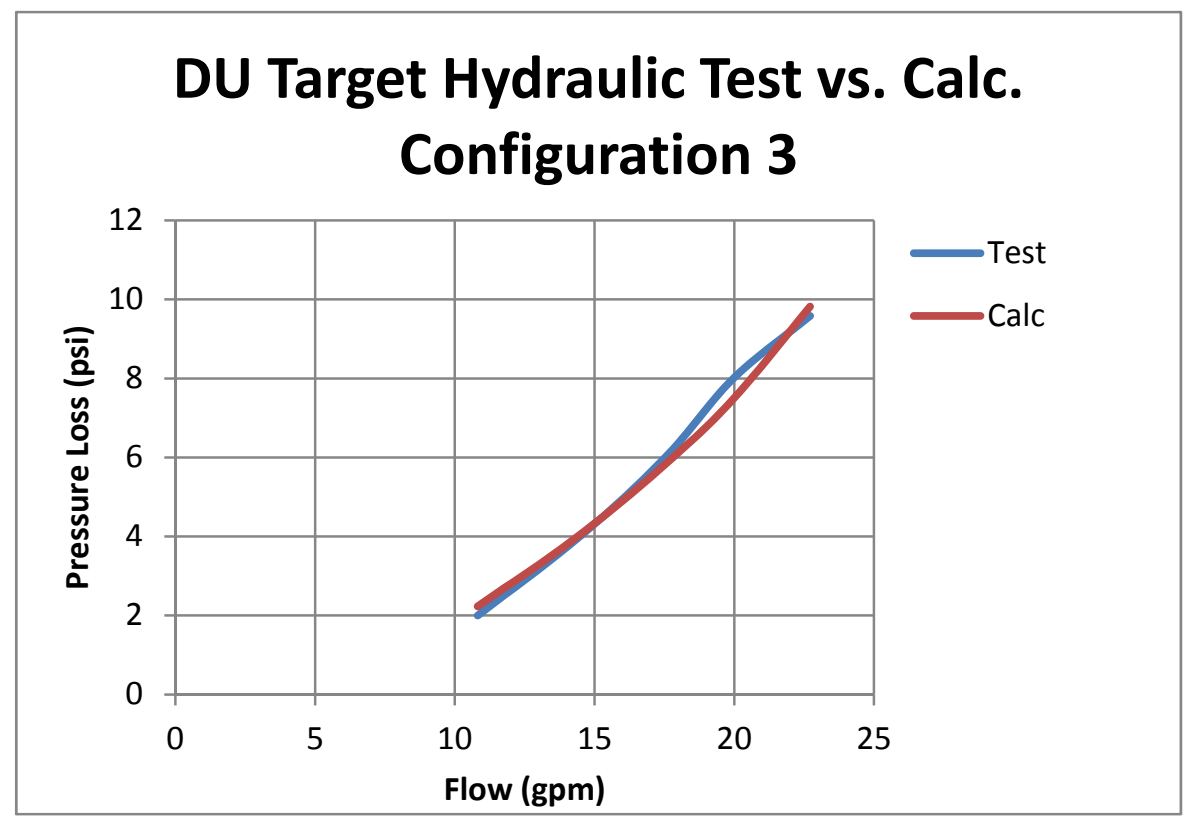

FATHOM vs Test

\section{$\underline{\text { Test Description Configuration } 4}$}

The test arrangement for configuration 4 is shown below. The purpose of this test is to validate the pressure losses associated with the overall flow through the target assembly from entrance to exit as simulated by the FATHOM code. All of the Ta disks and spacers are installed in the target housing along with the spring housing. 


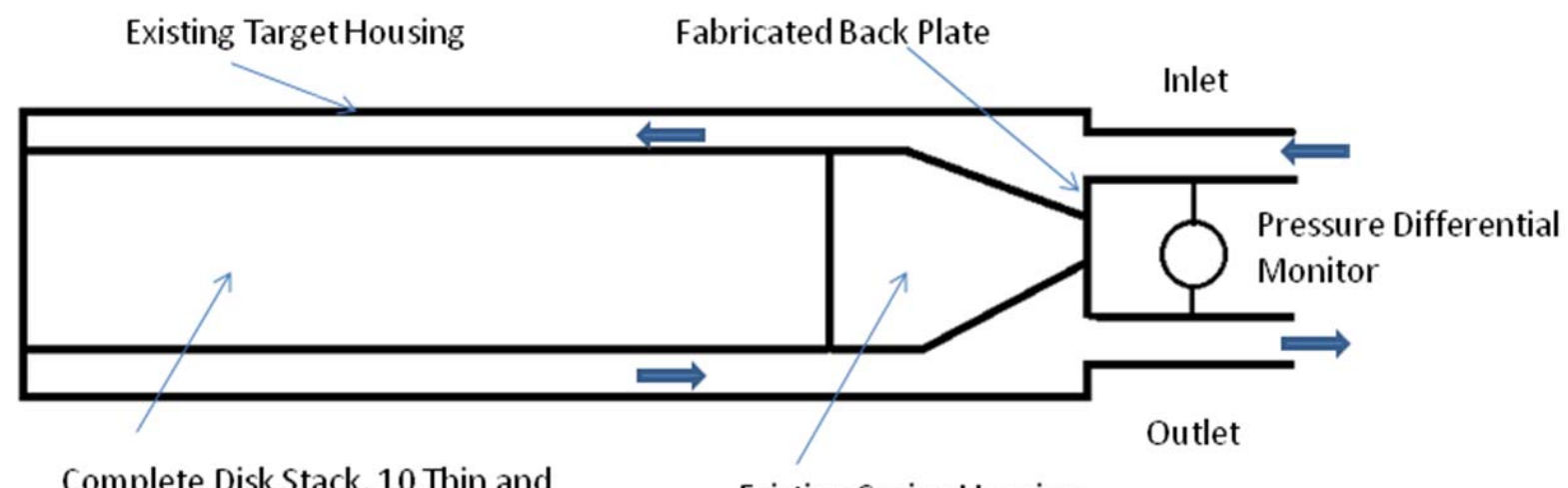

Complete Disk Stack, 10 Thin and

11 thick Ta Disks and Spacers

Existing Spring Housing

Test Arrangement

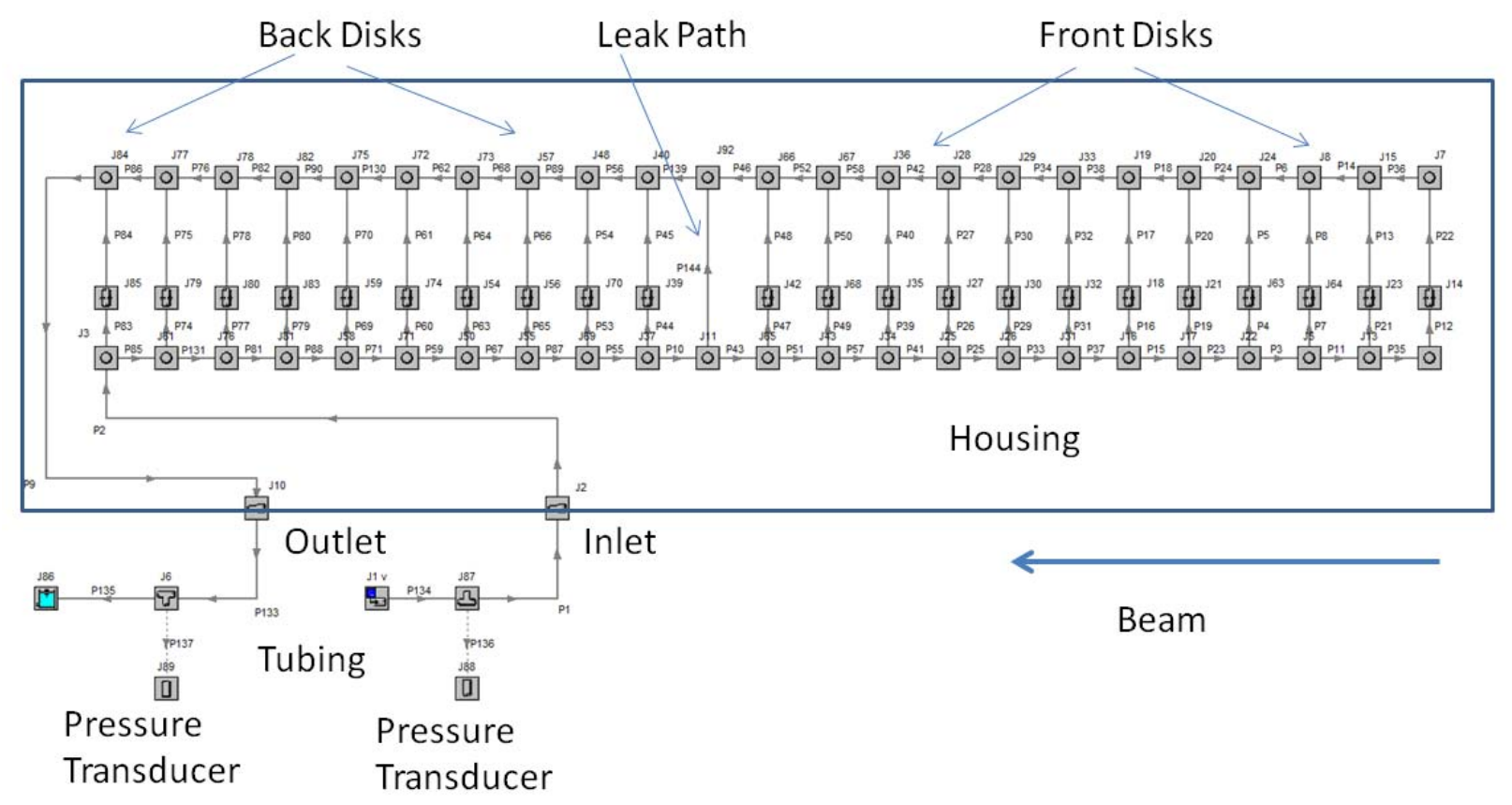

FATHOM Diagram (Note reversed image from Test Arrangement) 


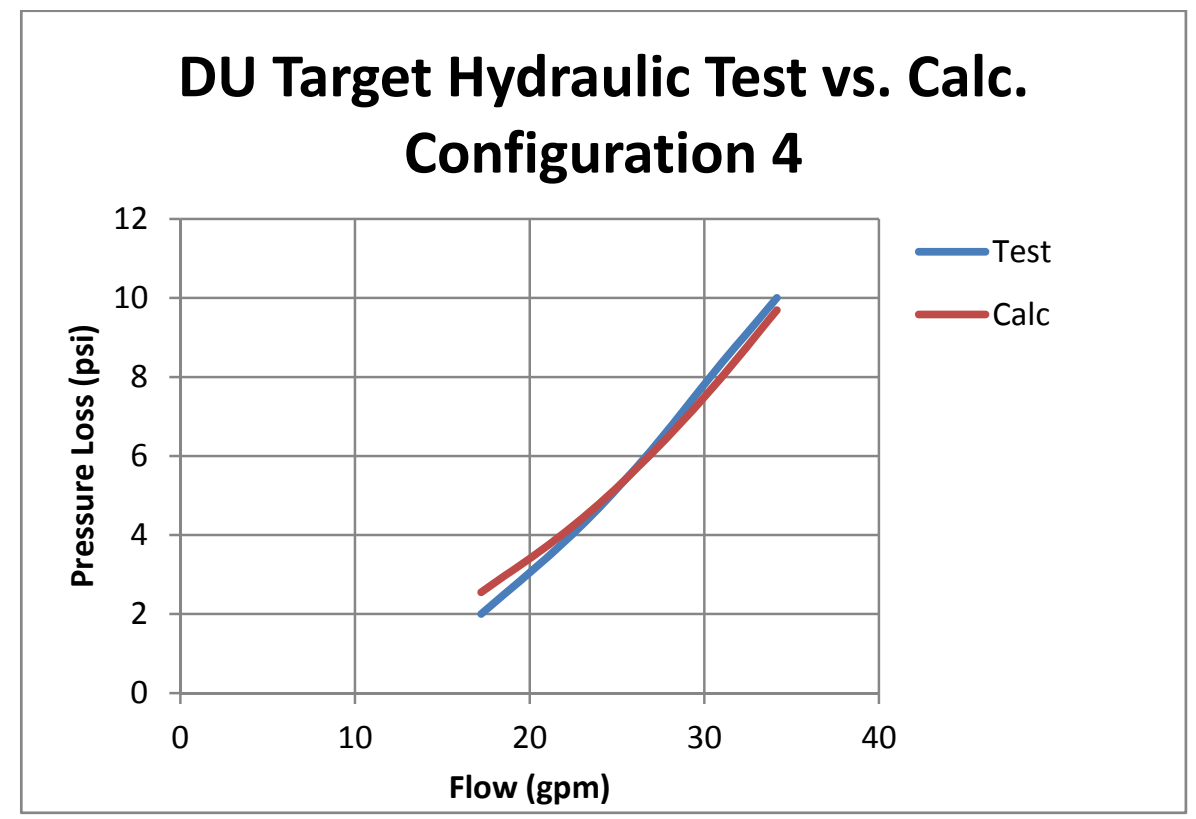

FATHOM vs Test 


\section{APPENDIX 4}

Calculation Note NE-CALC-2015-04: "Evaluation of the Structural Integrity of Zircaloy-4 Clad Containment for the DU Target Disks" 


\section{CALCULATION COVER SHEET}

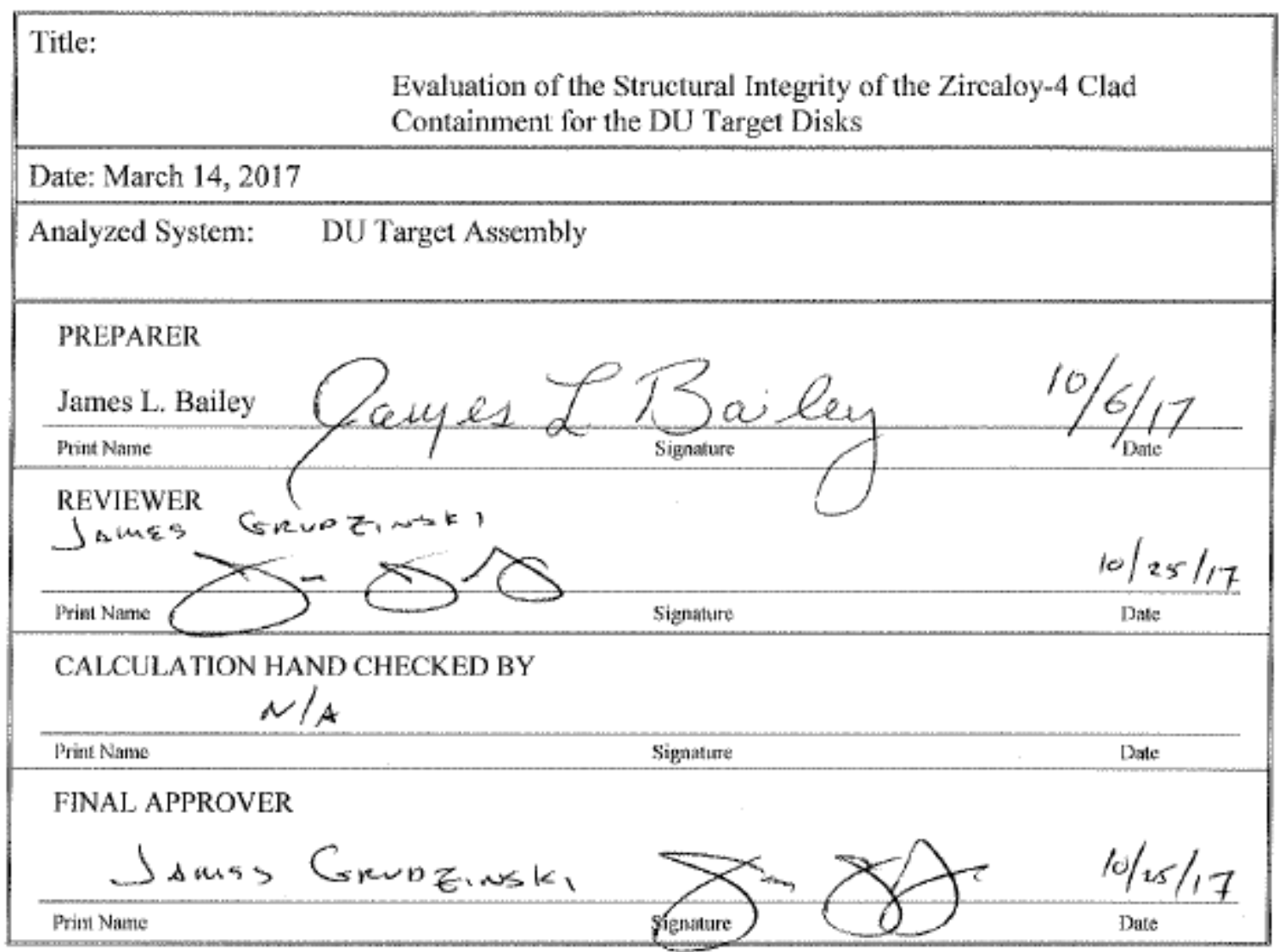


COVER SHEET

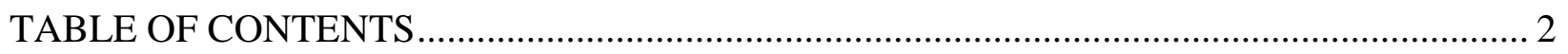

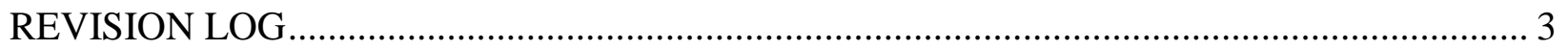

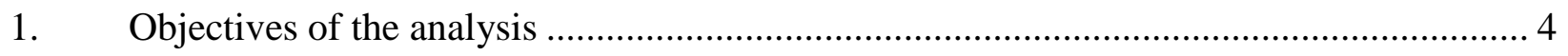

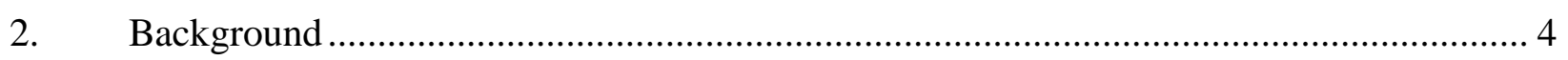

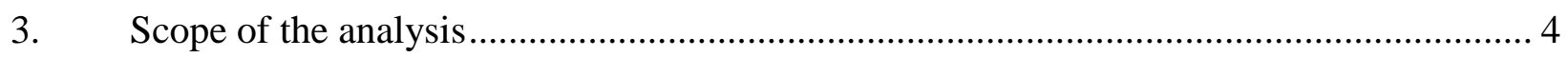

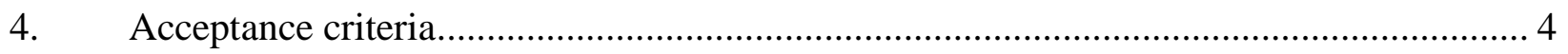

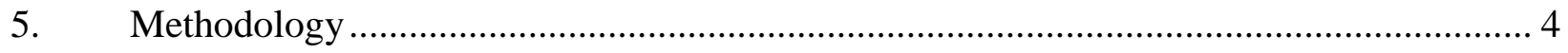

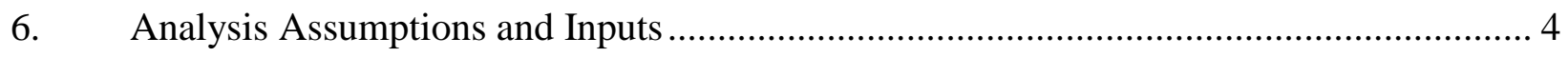

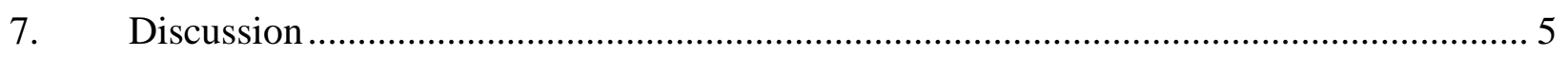

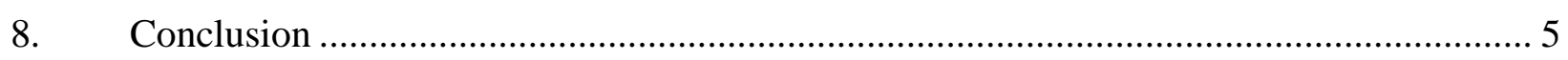

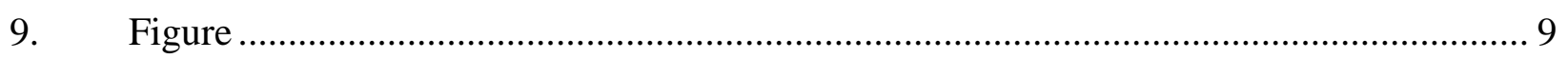

\section{APPENDICES}

Appendix 1 - General Checking Criteria Sheet .....

Appendix 2 - References 
REVISION LOG

\begin{tabular}{||c|c|c||}
\hline REVISION & CHANGES & DATE \\
\hline 0 & Initial Release & March 14,2017 \\
\hline 1 & $\begin{array}{c}\text { Revised analyses based on flow tests and } \\
\text { analyzed system considering a larger pump }\end{array}$ & \\
\hline & & \\
\hline & & \\
\hline
\end{tabular}




\section{Objectives of the analysis}

The object of this note is to verify that the Zircaloy-4 clad on the depleted uranium disks will provide satisfactory containment under normal and off normal operating conditions.

\section{Background}

During normal operation heat is generated within the Zircaloy-4 clad depleted uranium target disks due to impingement of the electron beam on the target. The target disks are cooled by DI water flowing between the disks (Refer to Figure 1). Because of differences in the thermal expansion between the Zircaloy-4 clad and the uranium inner disk, thermal stresses in the clad occur which eventually create cracks in the clad as a result of fatigue failure, that in turn, allows fission products from the uranium to enter the cooling water, thereby, limiting the usable life of the target. Thermal hydraulic analyses, thermal stress analyses, and UT testing has previously been performed for these disks. These references provide the basis for this calculation note.

\section{Scope of the analysis}

This note is intended to verify the structural integrity of Zircaloy-4 clad on the uranium disks during operation using the referenced information.

Essentially, all required analyses and tests have previously been performed as reported in these references. This note summarizes this work and provides a concise evaluation of their results.

\section{Acceptance criteria}

Acceptance criterion is based on a minimum number of allowable operating cycles of the target assembly. A cycle is defined as heat up of the disks from ambient temperature to a steady state condition with the beam at maximum power and then a complete cool down back to ambient temperature. An acceptable minimum number of cycles are 10,000. This requirement includes both normal and off normal conditions.

\section{Methodology}

The thermal hydraulic analysis of the disks is first address using reference 1 . The worst case disk is identified and the thermal parameters are determined. Next, these parameters are used in the thermal stress calculations (reference 2) to determine the stresses and possible fatigue failure limit. Also, a parametric analysis of the effect of un-bonded areas of the clad to uranium is investigated. Lastly, UT test results (reference 3) are used to determine the size and location of possible un-bonded areas of the actual fabricated disks. By comparing the thermal stress calculation results and the UT results an allowable number of cycles are estimated.

\section{Analysis Assumptions and Inputs}

All assumptions are described in the references.

The references provide all inputs to this note.

Supplemental references provide additional information about the determination of the internal heat generation assumed in these reports. References 7, 8 and 9. 


\section{Discussion}

$\underline{\text { Summary and Results for the Thermal/Hydraulic Analysis Note (Reference 1) }}$

The object of this analysis was to determine the operating temperatures of the disks under normal and off normal conditions. The assumed acceptance criteria was to maintain the surface temperature of the worst case disk to below the saturation temperature at the operating pressure to prevent boiling in the coolant channel and to maintain the maximum temperature in the uranium to below $300^{\circ} \mathrm{C}$ to minimize thermal stress in the clad. The commercial computer code ANSYS CFX was used for these analyses. The internal heat generation distribution input for this analysis was a Gaussian profile with total generation obtained from a separate computer analysis. Flow conditions were obtained from the overall hydraulic analysis of the target cooling system and from flow tests performed on the actual target assembly (reference 5). The worst case disk was found to be a thin disk at location 2 and was the only disk analyzed for all cases. The normal and off normal cases were examined. The results of this analysis indicated that the disk temperatures were below the acceptable limit.

\section{Summary and Results for the Thermal/Stress Analysis Note (Reference 2)}

The object of this analysis was to determine the thermal stress in the Zircaloy-4 target clad for the worst case disk and the corresponding fatigue cycle life. The commercial code ANSYS was used for the finite element analysis. A thermal analysis was performed first using a heat generation rate and an effective convective coefficient at the surface of the disk from preliminary thermal hydraulic analysis. Note that the results of this preliminary analysis were found to be in agreement with the thermal convective coefficient and calculated disk temperatures as determined in the subsequent finalized thermal hydraulic report (reference 1), and therefore, assures that these stress results are correct and applicable to the final design.

The normal case for disk 2 was used for all the parametric study. This study evaluated un-bond areas by assuming small insulated circular areas between the clad and the uranium. The diameters of the circular areas were varied as well as their offset distance from the center. The worst case condition was found at the radial center of the disk with the largest area studied (I.e. $2 \mathrm{~mm}$ diameter). The corresponding cladding life was 354,000 cycles. This result is considerably above the minimum acceptable limit of 1,000 cycles.

\section{Summary and Results for the UT Tests (Reference 3)}

The object of the UT tests was to determine the bonding status after final machining of the disks. These tests were performed by the fabricators of the disks (LANL). UT images for both the thin and thick disks are shown in the test reports. The thin disk UT results indicate negligible un-bonded area and essential conclude that these disks are completely bonded. The UT test resolution is noted as $0.125 \mathrm{~mm} \times 0.125 \mathrm{~mm}$ and, as noted in the reference 4 email, any area larger than this size would be show up on the images, hence, it can be concluded that any small un-bonded area are smaller than this resolution. This resolution criterion is to be compared to the $2 \mathrm{~mm}$ diameter un-bonded area evaluated in the thermal stress (reference 2). The UT results for the thicker disks do show notable un-bonded areas; however, because of the negligible heat generation in these thicker back disks their operating temperature is near ambient and thus has negligible thermal stress in the clad.

\section{Conclusion}

Thermal Hydraulic Analysis

The results of the thermal hydraulic analyses (reference 1) are shown in the plots below. Disk surface temperature verse beam power for several beam width are plotted. Figure 1 and 2 plots are considering a 
flow rate for the smaller pump that was analyzed in reference 5. The saturation temperature of $126^{\circ} \mathrm{C}$ at the pressure calculated in the flow channel is also shown. Figure 1 plot indicates that for a beam width of $20 \mathrm{~mm}$ FWHM a beam power of $13 \mathrm{~kW}$ would provide a margin to boiling in the channel of $15 \%$. And, for a beam width of $18 \mathrm{~mm}$ FWHM the beam power is reduced to $10.5 \mathrm{~kW}$ in order to provide a $15 \%$ margin. Figure 2 plot indicates that for a beam width of $18 \mathrm{~mm}$ FWHM a beam power of $18.5 \mathrm{~kW}$ would provide a margin to boiling in the channel of $15 \%$. Note that the electron energy of $40 \mathrm{Mev}$ allows for significantly higher beam power than the $35 \mathrm{Mev}$ energy for the same beam width and flow rate. This result is due to the axial spreading of the heat generation. Figure 3 plot indicates that for a beam width of $18 \mathrm{~mm}$ FWHM a beam power of $18.5 \mathrm{~kW}$ would provide a margin to boiling in the channel of $15 \%$. Figure 3 and 4 plots are considering a flow rate for the larger pump that was analyzed in reference 5. The saturation temperature of $134^{\circ} \mathrm{C}$ at the pressure calculated in the flow channel is also shown. Figure 3 plot indicates that for a beam width of $20 \mathrm{~mm}$ FWHM a beam power of $16 \mathrm{~kW}$ would provide a margin to boiling in the channel of $15 \%$. And, for a beam width of $18 \mathrm{~mm}$ FWHM the beam power is reduced to $13 \mathrm{~kW}$ in order to provide a $15 \%$ margin. Figure 4 plot indicates that for a beam width of $18 \mathrm{~mm}$ FWHM a beam power of $20 \mathrm{~kW}$ would provide a margin to boiling in the channel of greater than $15 \%$.

A $15 \%$ margin to boiling is assumed to be a reasonable uncertainty factor based on engineering judgment considering: a 5\% error in analysis (noting that flow testing were performed on the actual target assembly and ANSYS CFX is a well validate thermal hydraulic computer code); a 5\% allowance for the flow switch beam trip (considers a trip of $2 \mathrm{gpm}$ below the operating flow rate); $5 \%$ for beam power and width uncertainty (note that surface temperature is linear with beam power, however it is to the square of the beam width).

In conclusion, it is recommended that the target systems be operated at the $15 \%$ uncertainty factor as described above.

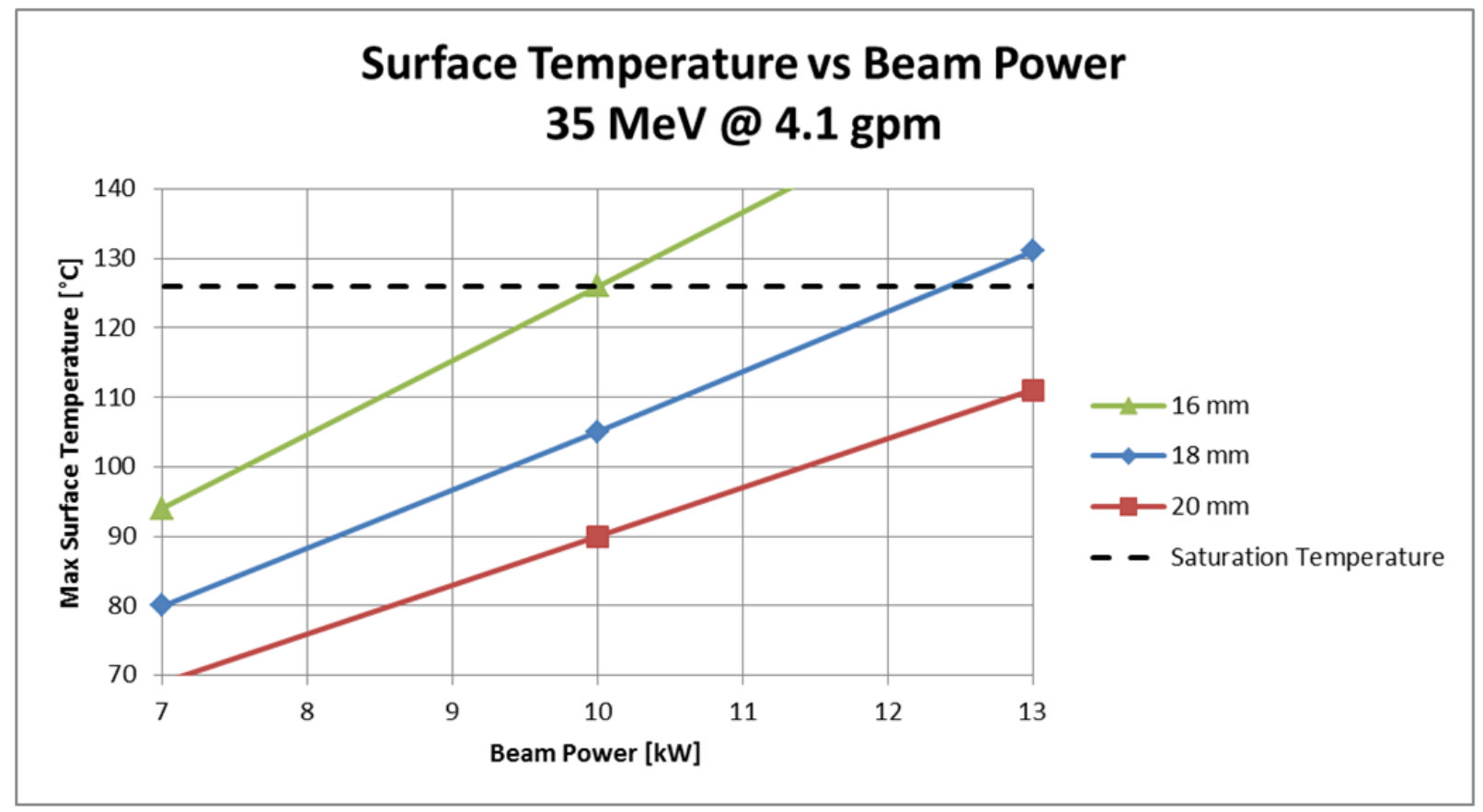

Figure 1 Plot 


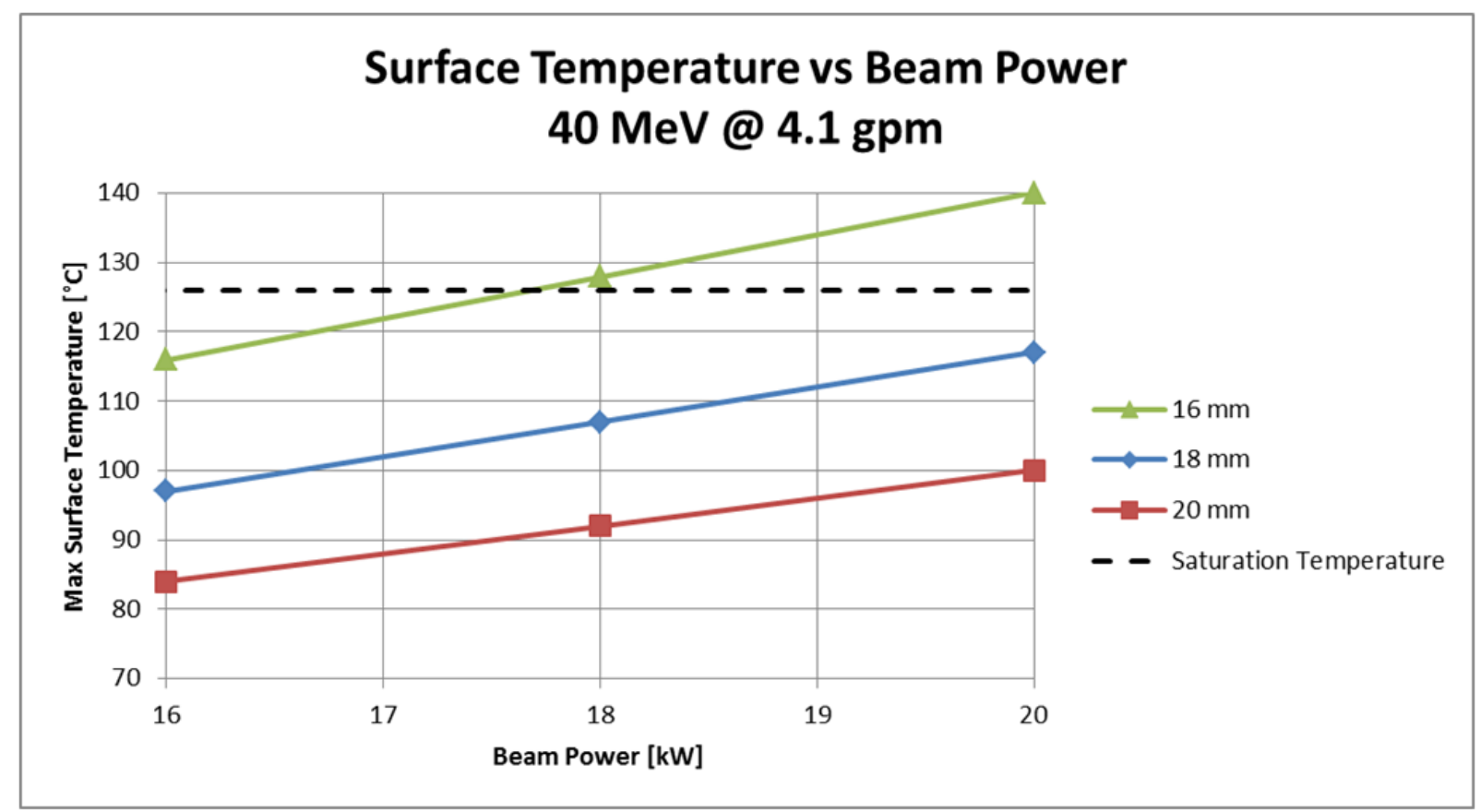

Figure 2 Plot

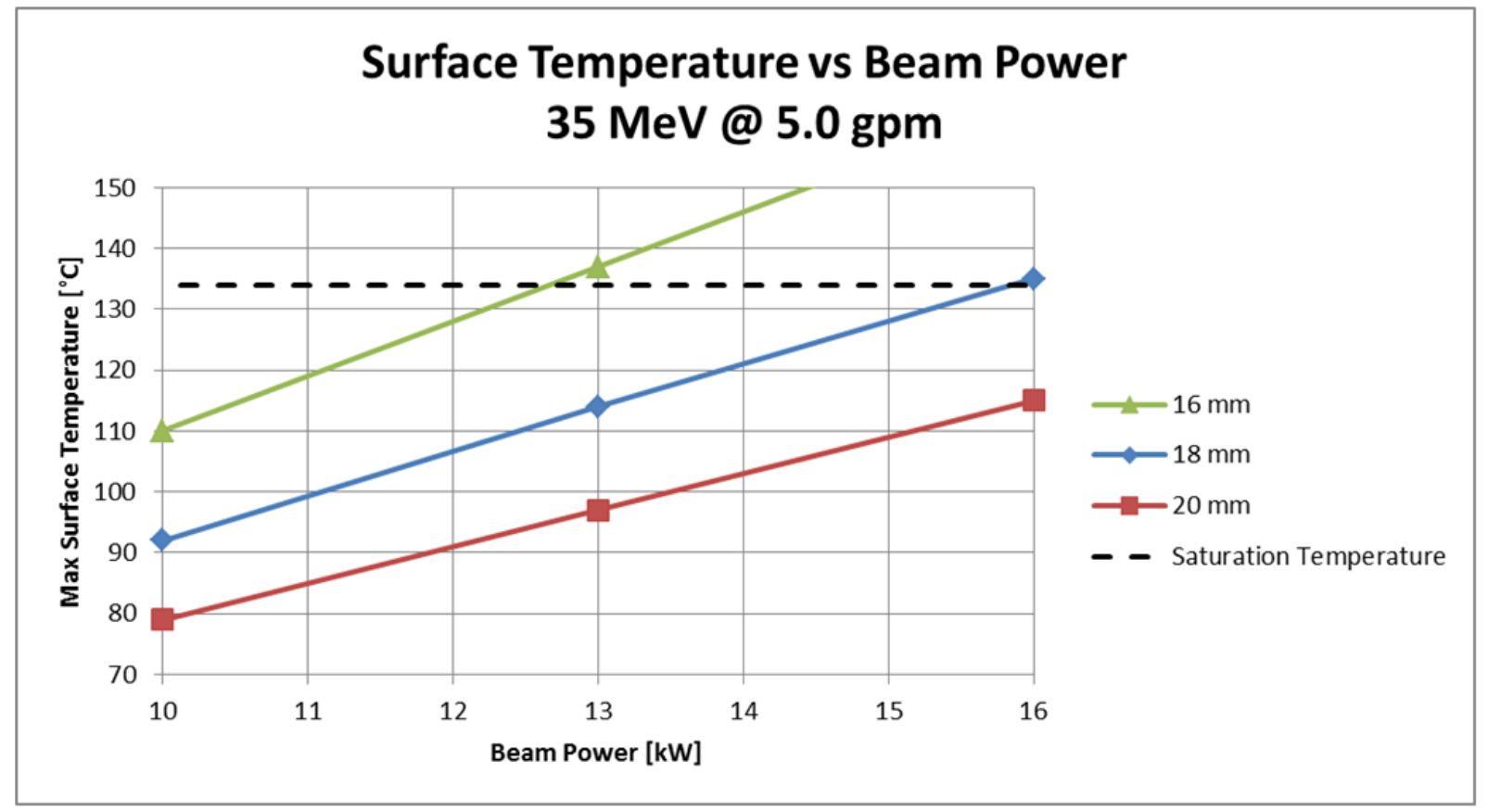

Figure 3 Plot 


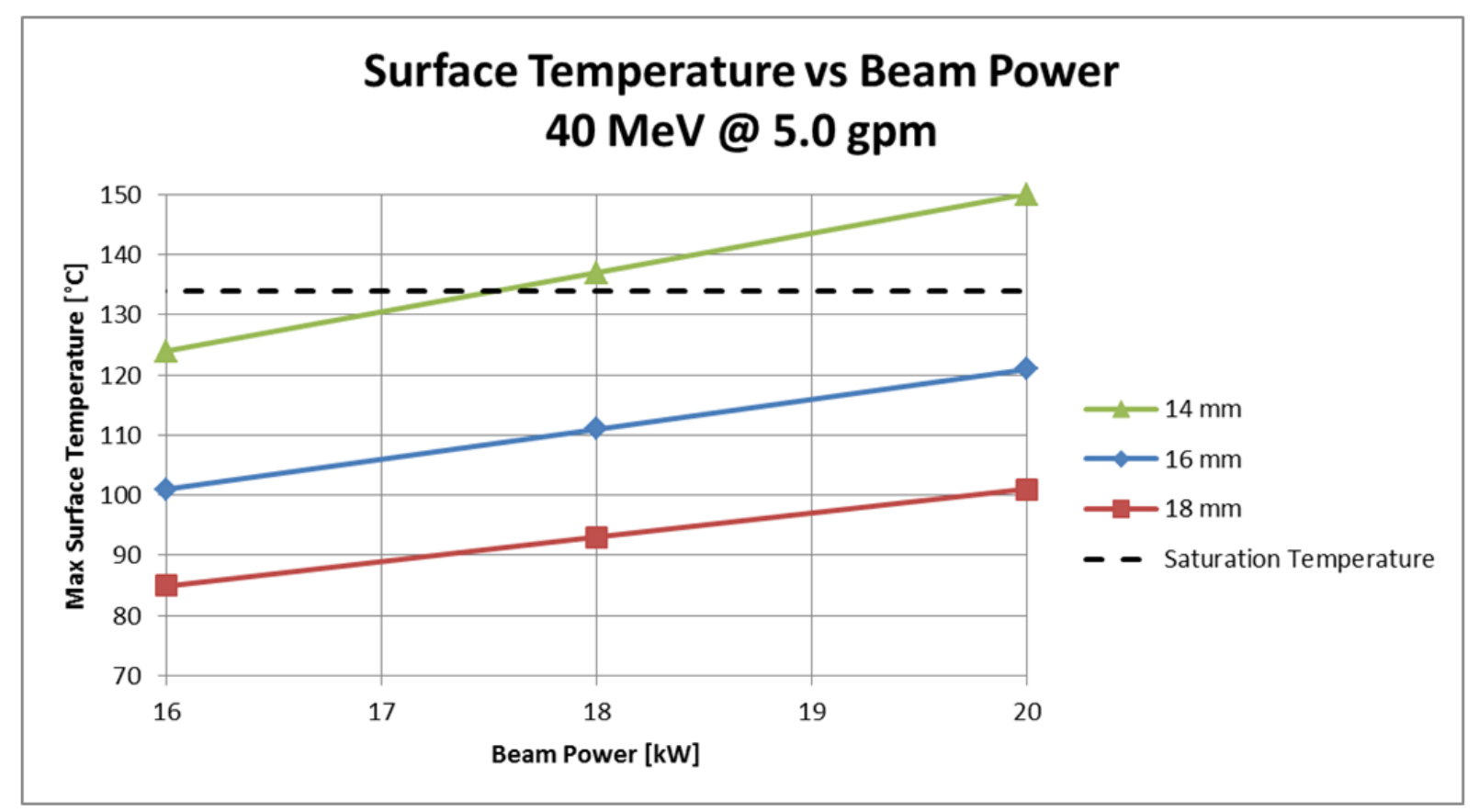

Figure 4 Plot

\section{$\underline{\text { Clad Stress Analysis }}$}

The thermal stress analyses (reference 2) determined that the worst case condition was at the radial center of the disk with the largest area studied (I.e. $2 \mathrm{~mm}$ diameter). The corresponding cladding life was 354 , 000 cycles. This result is considerably above the minimum acceptable limit of 10,000cycles. Further the UT tests for the thin disks (Reference 3) indicated that there was negligible un-bonded areas (I.e. any unbonded areas were smaller than $0.125 \mathrm{~mm} \times 0.125 \mathrm{~mm}$ ) This resolution is below the worst case size studied in the thermal stress analyses ( $2 \mathrm{~mm}$ diameter), hence, the actual cycle life of the disks is expected to be greater than the 354,000 cycles indicated by the analyses which is far greater than the required 10,000 cycles. Because the heat generation in the thicker disks is negligible they were not analyzed.

Also, the off normal occurrence of the stoppage of coolant through the DU Target was analyzed (reference 6). And based on this study, it was concluded that upon an off normal occurrence of the stoppage of water coolant flow through the target, the temperature of all target components will remain below their maximum allowable design temperatures.

Based on the above referenced analyses and tests it is concluded that the disks will provide satisfactory containment under normal and off normal operating conditions. 
9. Figure

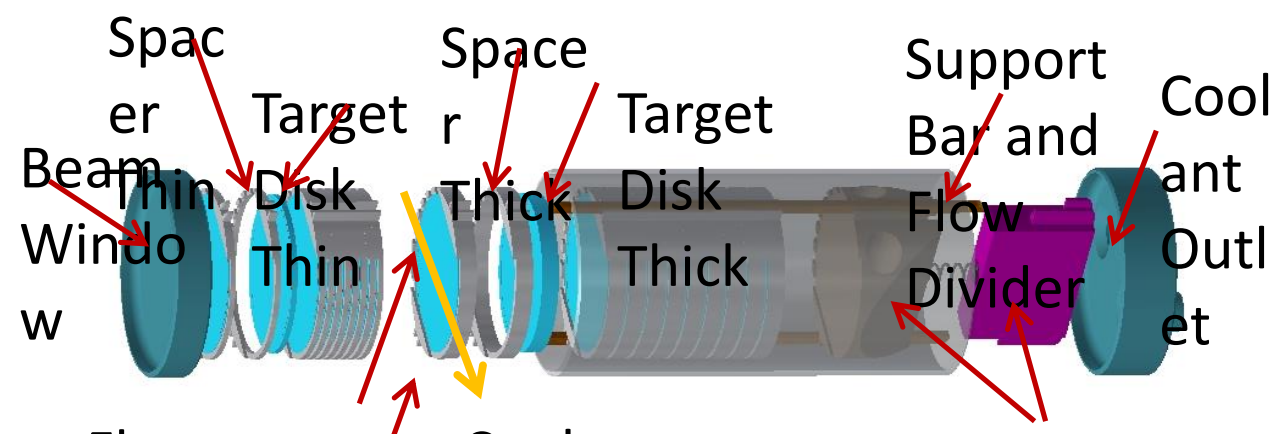

Flow

Control OrPisess Installed in Spacer
Coolant

Flow

Across

Disk

Face
Compression

Spring

Housings and

Flow Divider

Initial Spring

Force=10\#

1/4" Allowable

Movement

Final Spring

Force=12\#

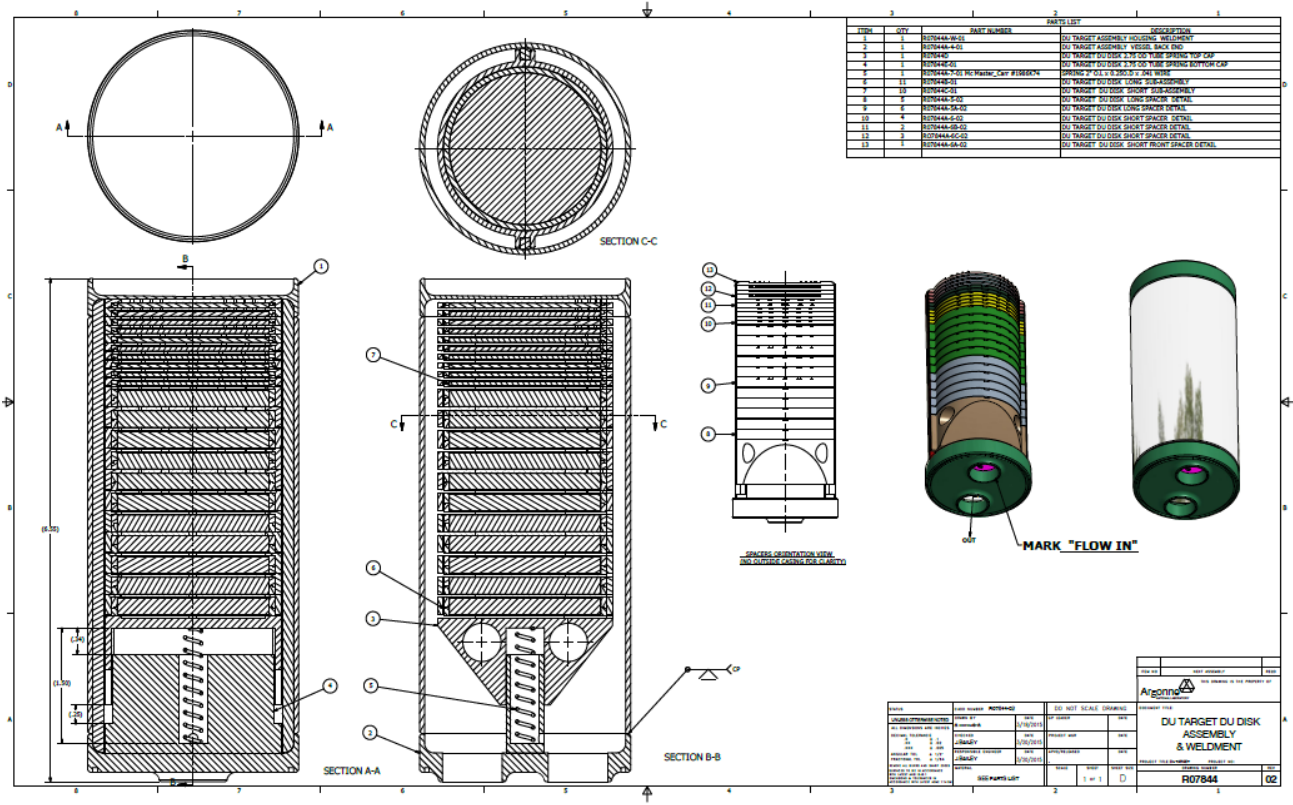

Figure 5 DU Target Assembly 


\section{APPENDIX 1 \\ GENERAL CHECKING CRITERIA SHEET}

\begin{tabular}{|c|c|c|c|c|}
\hline CALCULATION CHECKLIST & Yes & No & N/A & Comments \\
\hline $\begin{array}{l}\text { 1. Are analytical methods } \\
\text { appropriate? }\end{array}$ & $\square$ & $\square$ & $\square$ & \\
\hline 2. Are assumptions appropriate? & $\square$ & $\square$ & $\square$ & \\
\hline 3. Is the calculation complete? & $\square$ & $\square$ & $\square$ & \\
\hline $\begin{array}{l}\text { 4. Are formulas appropriately } \\
\text { referenced? }\end{array}$ & ${ }^{-}$ & L & L & \\
\hline 5. Are the input data appropriate? & $\square$ & $\square$ & $\square$ & \\
\hline $\begin{array}{l}\text { 6. Was utilized software appropriate } \\
\text { for the task? }\end{array}$ & $\square$ & $\square$ & $\square$ & \\
\hline $\begin{array}{l}\text { 7. Were software input/initial } \\
\text { conditions/properties/boundary } \\
\text { conditions appropriate? }\end{array}$ & $\square$ & $\square$ & $\square$ & \\
\hline 8. Are the results reasonable? & $\square$ & $\square$ & $\square$ & \\
\hline
\end{tabular}


APPENDIX 1

GENERAL CHECKING CRITERIA SHEET

\begin{tabular}{|c|l|l|}
\hline \multicolumn{3}{|c|}{ ADDITIONAL COMMENTS } \\
\hline Number & Comment & Resolution \\
\hline 1. & & \\
\hline 2. & & \\
\hline 3. & & \\
\hline 4. & & \\
\hline 5. & & \\
\hline 6. & & \\
\hline 7. & & \\
\hline 8. & & \\
\hline 9. & & \\
\hline 10. & & \\
\hline
\end{tabular}


APPENDIX 2

REFERENCES

Reference 1

Calculation Note: NE-CALC-2015-05 Rev. 1

Reference 2

Calculation Note: NE-CALC-2015-06

Reference 3

Memorandum

Applied Engineering Technologies

AET-6 Nondestructive Testing \& Evaluation

To/MS: Maria Pena, MST-6, x7-4119

From/MS: D. A. Summa, AET-6, MS P915

Phone/Fax 5-1854 / Fax 5-7176

Symbol: SHINE-Thin Post Machining UT-A Disks

Date: 9 March 2015

Ultrasonic Inspection of Thin SHINE Assemblies

Reference 4

Hi Maria-

Pixels for these inspections are $.125 \mathrm{mmx} .125 \mathrm{~mm}(\sim 0.005$ ” $\mathrm{x} 0.005$ ”). For something to be unbonded and show up as bonded, it would have to be considerably smaller than the pixel. How much smaller is a good question, I'm not sure I can give you a definitive answer. (If I had to hazard a guess, I'd say maybe $1 / 4$ of the pixel size? But that's purely just a guess. This is where having that standard with known defects that Don Bucholz wanted to make would be helpful. )The transducer is essentially averaging returns over the spatial area of the pixel. A really small bad area within a single pixel would change the return signal only a little, while a larger area would make for a bigger change. Part of setting the threshold involves looking at the waveform and trying to figure out what the cutoff is. Another thing to consider is that we've looked at these several times with similar results - there is no way we are able to replace/re-align the part exactly to its previous location, meaning that a smallish defect that happened to straddle pixels and thus perhaps not show up in one scan would be unlikely to have the same thing happen in a subsequent scan.

Deb

Maria I. Peña , Ph.D.

Los Alamos National Laboratory

MST-6, MS G770

Phone: 505-667-4119 
Reference 5

Calculation Note: NE-CALC-2015-03

Reference 6

Calculation Note: NE-CALC-2015-69-v1

\section{Supplemental References}

\section{Reference 7}

Power deposition for $35 \mathrm{Mev}$ electron beam

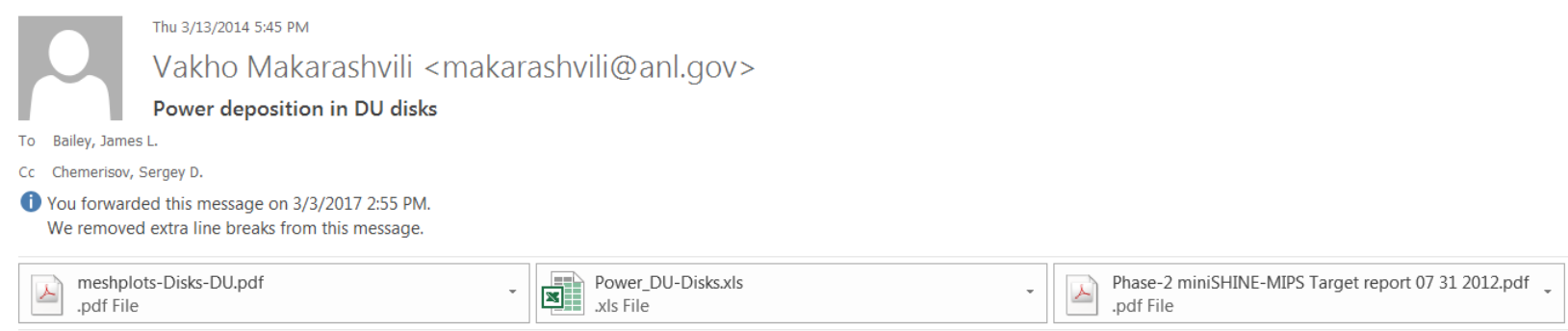

Jim,

Please see the power deposition results in DU disks. The first plot in "meshplots-Disks-DU.pdf" is the power density. The units are [kW/cc] per $1 \mathrm{~kW}$ of beam power. I also included the Excel file which has the total power deposition per disk in kW (per $1 \mathrm{~kW}$ beam).

Figure 5 in the Phase-2 miniSHINE report also shows the power density data but in [W/cc] per kW of beam.

Vakho

Vakho Makarashvili, PhD

Assistant Physicist, CSE Division

Argonne National Laboratory

9700 S. Cass Ave.

Argonne, IL 60439

Tel: $630-252-4538$

Fax: 630-252-5246

E-mail: makarashvili@anl.gov

\section{Reference 8}

Power deposition for $40 \mathrm{Mev}$ electron beam

Text file entitled "Power-data-(r,z)_DU-40Mev" by M. Vakho 


\section{Reference 9}

Email from R. Fischer indicating heat generation density that was used in reference 6.

From: Fischer, Richard

Sent: Thursday, May 25, 2017 2:11 PM

To: Grudzinski, James J.<iig@anl.gov>; Bailey, James L. <ibailey@anl.gov>

Subject: DU Target

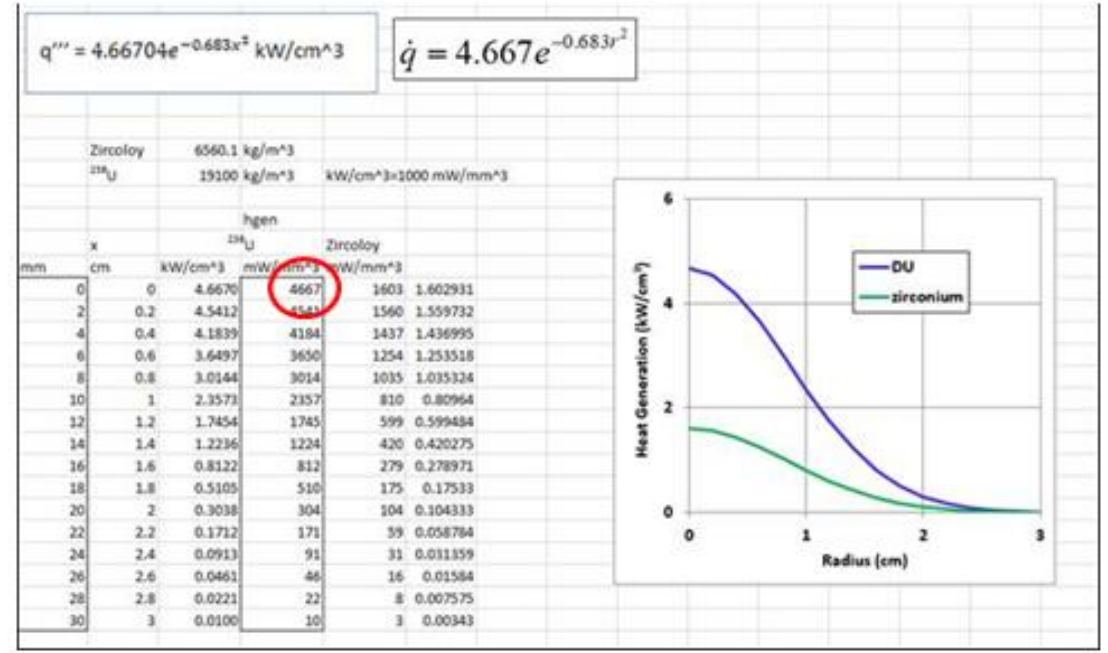

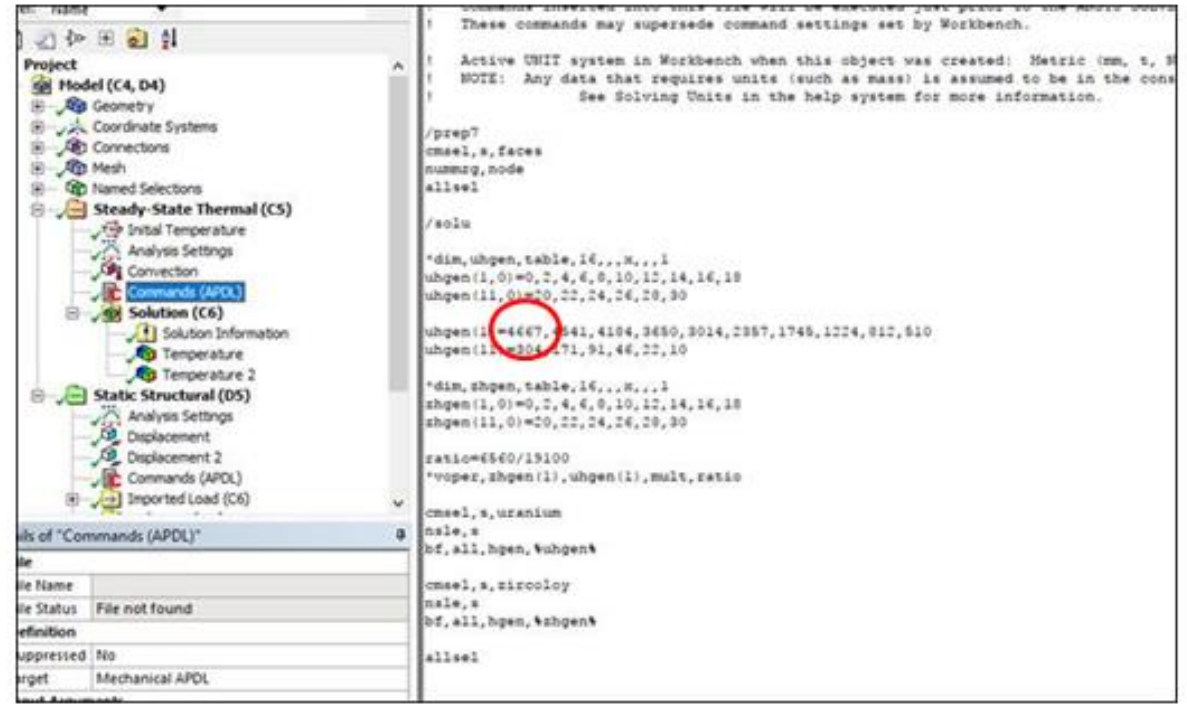




\section{APPENDIX 5}

Calculation Note NE-EO-2014-006: “DU Target Disk Clad Analysis" 
Title: DU Target Disk Clad Analysis

Calculation No.: $\quad$ NE-EO-2014-006

Revision Number:

0

\section{CALCULATION COVER SHEET}

\section{Supersedes Calculation}

Total Number of Attachments:

No.:

Analyzed System: Target Cladding Analysis

Purpose of Revision: Initial Issue

\section{PREPARER}

Richard L. Fischer

Kendere

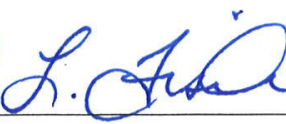

$11 / 10 / 2015$

Print Name

Signature

Date

REVIEWER

SAURIN MAJUMDAR diger $11 / 10 / 15$

VENDOR APPROVER (if vendor-supplied calculation)

n.a.

Print Name

Signature

Date

FINAL APPROVER

Jim Grudzinski

Print Name
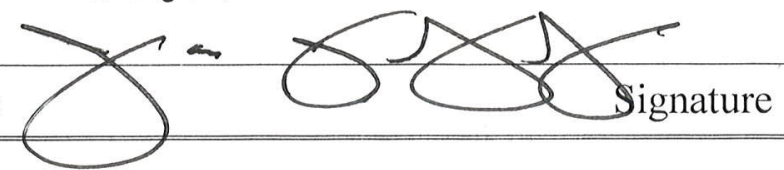

$1 . / 10 / 15$

Date 


\section{TABLE OF CONTENTS}

\section{COVER SHEET 1}

\section{TABLE OF CONTENTS 2}

\section{LIST OF EFFECTIVE PAGES 3}

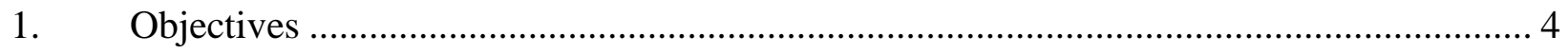

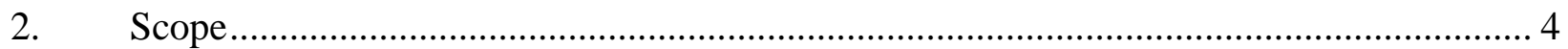

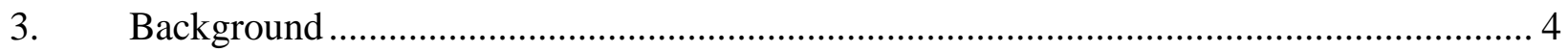

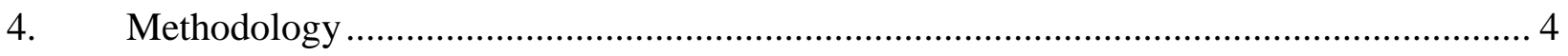

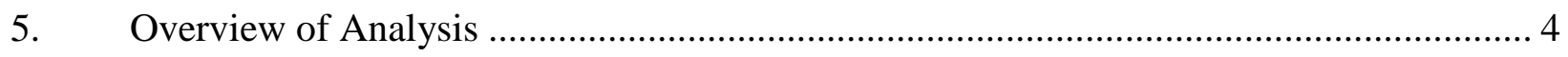

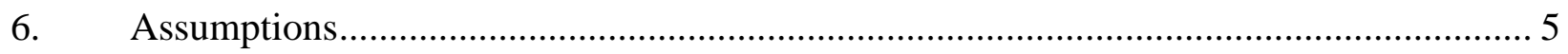

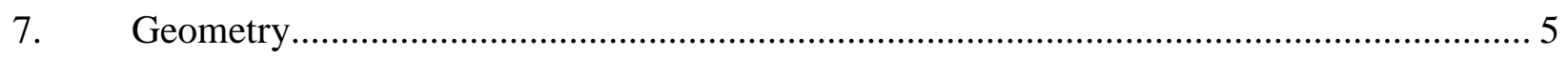

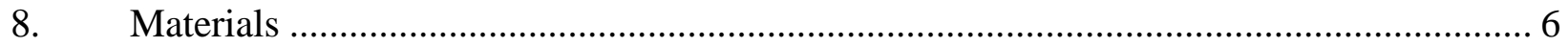

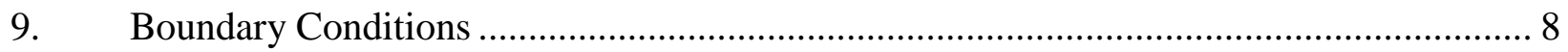

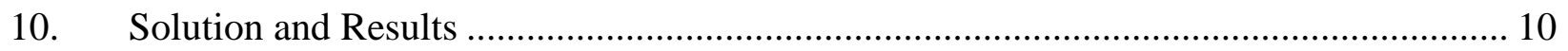

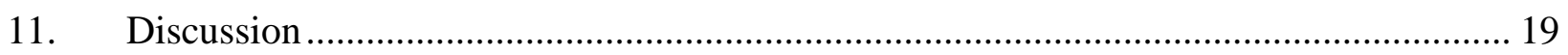

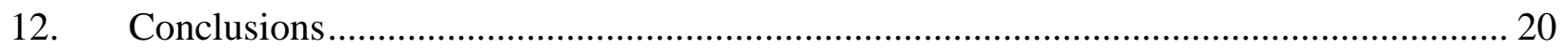

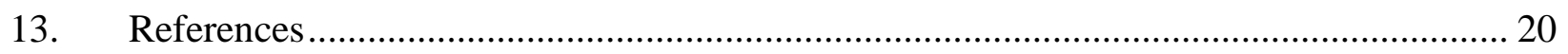

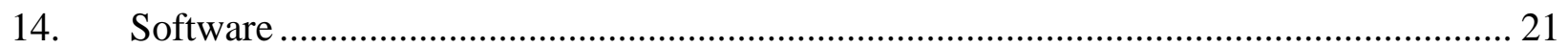

\section{APPENDICES}

Appendix 1 - General Checking Criteria Sheet................................................22

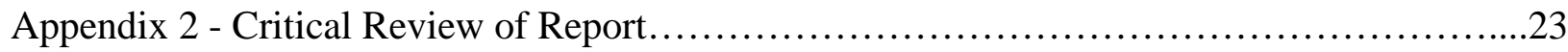




\section{LIST OF EFFECTIVE PAGES}

\begin{tabular}{||l|l||}
\hline \hline Pages & Revision \\
\hline 1 to 22 & 0 \\
\hline \hline
\end{tabular}




\section{Objectives}

The objective of this analysis was to analyze the Zircaloy-4 cladding on the Short Depleted Uranium (DU) Disk Sub-assembly to determine if the presence of bonding flaws constitutes a failure hazard.

\section{Scope}

The scope of this analysis was limited to the Short DU Disk Subassembly used in the DU Target Assembly shown in drawing R07844 dated 2/7/2014.

\section{Background}

The DU disk subassemblies consist of a disk of depleted uranium clad in zirconium alloy. The top and bottom cladding halves are machined from Zircaloy-4 stock. A lump of DU is placed between them and this is heated and compacted in a vacuum. The cladding halves are electron beam welded together and this assembly is then machined to final dimensions. This manufacturing process results in a weld-like bond between the cladding and the DU. Radiographic examination has revealed the prescience of flaws in the bond, which are of random size, shape and location within the disk assembly. There is concern that these flaws will result in localized hot spots due to the lack of heat transfer at these flaws, and that this could lead to higher stress at these locations that could result in fatigue failure. The desired life is thought to be under 1000 cycles.

\section{Methodology}

The short DU disk subassembly was analyzed with the Ansys finite element program. The thermal loads were based on an estimate of heat generated by the specified electron beam, and the cooling parameters were derived from a previous conjugate heat transfer analysis.

\section{Overview of Analysis}

A total of eight load cases were analyzed. These load cases represent a variation in the size and location of a small circular flaw in the bonded interface between the cladding and the depleted uranium. A thermal analysis was performed on a half-symmetry model loaded with a thermal flux representative of the heat generated by the electron beam, and cooled by convection with water running over its outside surface. The resultant temperatures were then applied as a structural temperature load in a structural analysis. The cladding was evaluated for failure by short and long cycle fatigue. 


\section{Assumptions}

This analysis is based on the following assumptions:

1. Material response is constant with time (no effects of aging, corrosion, irradiation, etc.).

2. Materials are isotropic and homogeneous.

3. Residual stresses are not included.

4. The flaw results in a perfectly insulated boundary.

5. The cladding is at nominal 0.010 " thickness, with no variation due to the final machining operation.

\section{Geometry}

Geometry is based on drawing number R07844C, DU Target DU Disk Short Sub-assembly, dated 2/7/2014. A solid model based on the drawing was constructed with the Design Modeler module, and consists of a depleted uranium disk between top and bottom Zircaloy-4 claddings. The geometry represents a uniform nominal configuration after final machining. The circular flaw is created by slicing a disk from the top cladding. All parts except this disk are combined as a multi-body part. This part and the disk are imported into the Workbench environment, where all the nodes on the outside diameter of the disk and the mating surface on the top cladding are merged with a command snippet. This results in a free surface between the bottom of the disk and the top of the uranium. This is shown if Figure 1.

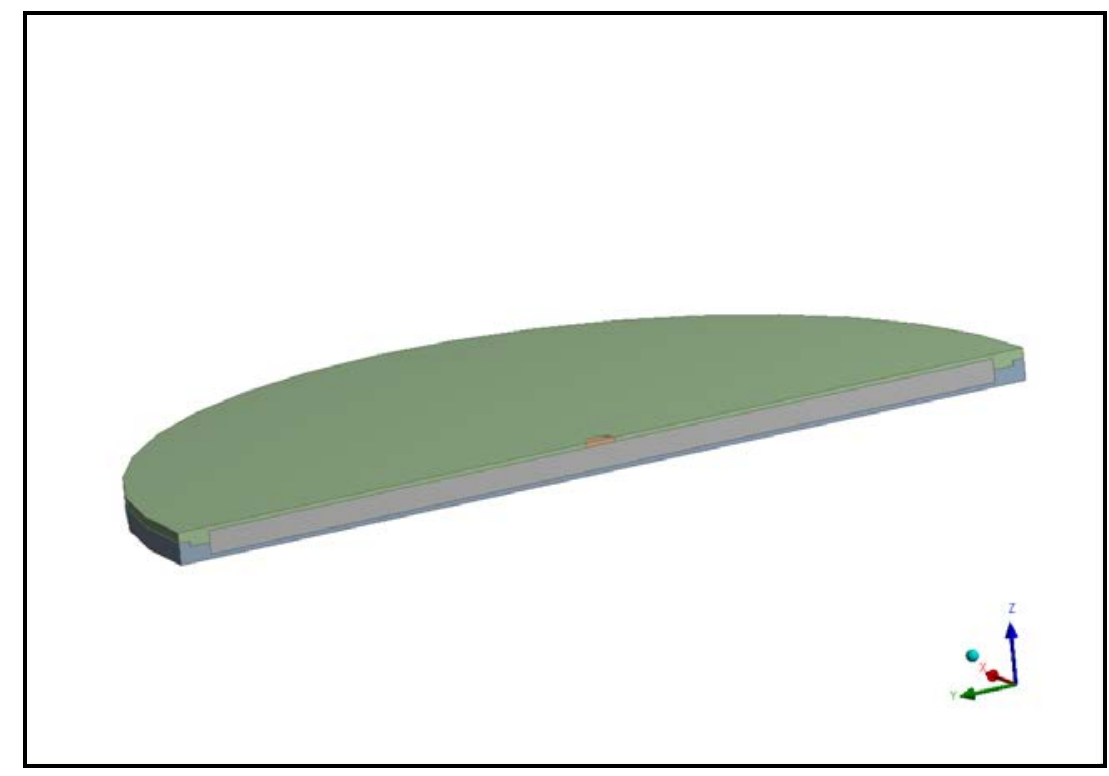

Figure 1

Solid Geometry 
The model was meshed with 173,279 quadratic brick and Tet solid elements, as shown in Figure 2. Sliding contact with a thermal conductance of $1 \mathrm{E}-5 \mathrm{~W} / \mathrm{mm} 2$ was placed on the flaw surface to enforce dimensional continuity. The low thermal conductance was used to thermally insulate the flaw.

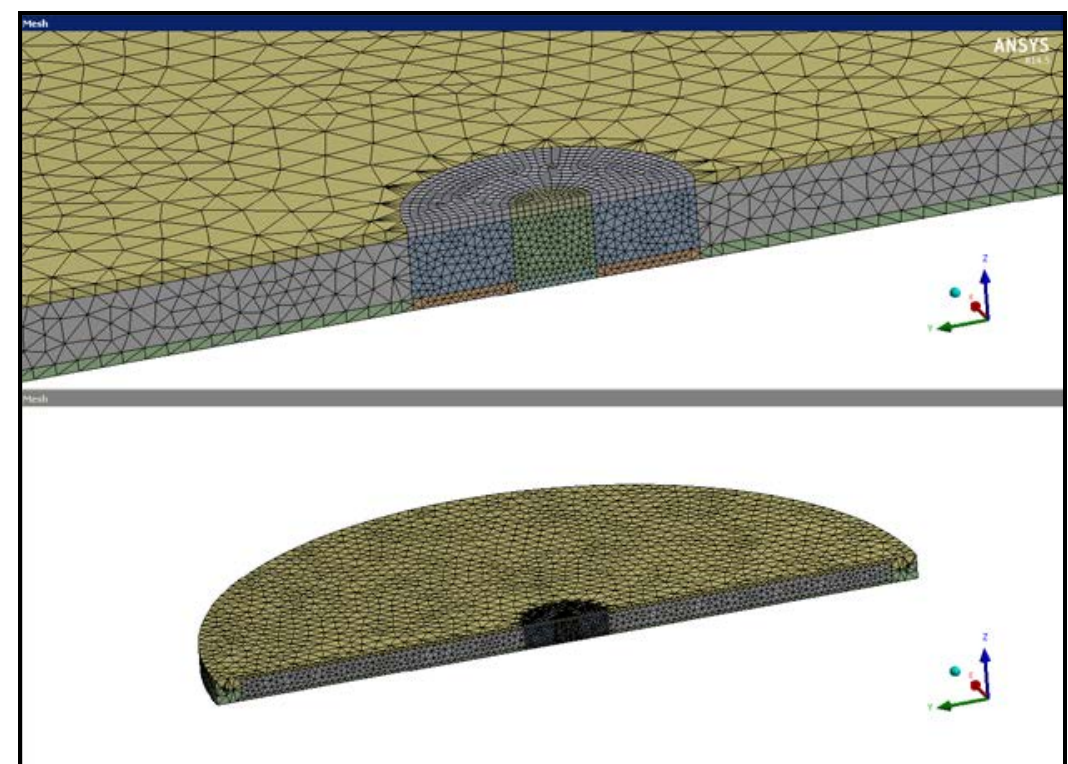

Figure 2

Finite Element Model

\section{Materials}

Material properties for depleted uranium are based on values given for bulk uranium on Wikipedia. Material properties for Zircaloy-4 are based on Ref. 1. Room temperature data is shown in Table 1.

\begin{tabular}{|c|c|c|}
\hline & DU & Zircoloy-4 \\
\hline$\rho\left(\mathrm{kg} / \mathrm{m}^{3}\right)$ & 19100 & 6560 \\
\hline $\mathrm{E}(\mathrm{GPa})$ & 208 & 92.4 \\
\hline Sy (MPa) & - & 381 \\
\hline$v$ & 0.23 & 0.35 \\
\hline$\alpha\left(\mathrm{C}^{-1}\right)$ & $13.9 \mathrm{e}-6$ & $5.59 \mathrm{E}-06$ \\
\hline $\mathrm{k}(\mathrm{W} / \mathrm{m}-\mathrm{K})$ & 27.5 & 21.5 \\
\hline
\end{tabular}

Table 1

Material Properties for DU 
Temperature dependent material data was located for Zircaloy-4 and is show in Figure 3 through Figure 6.

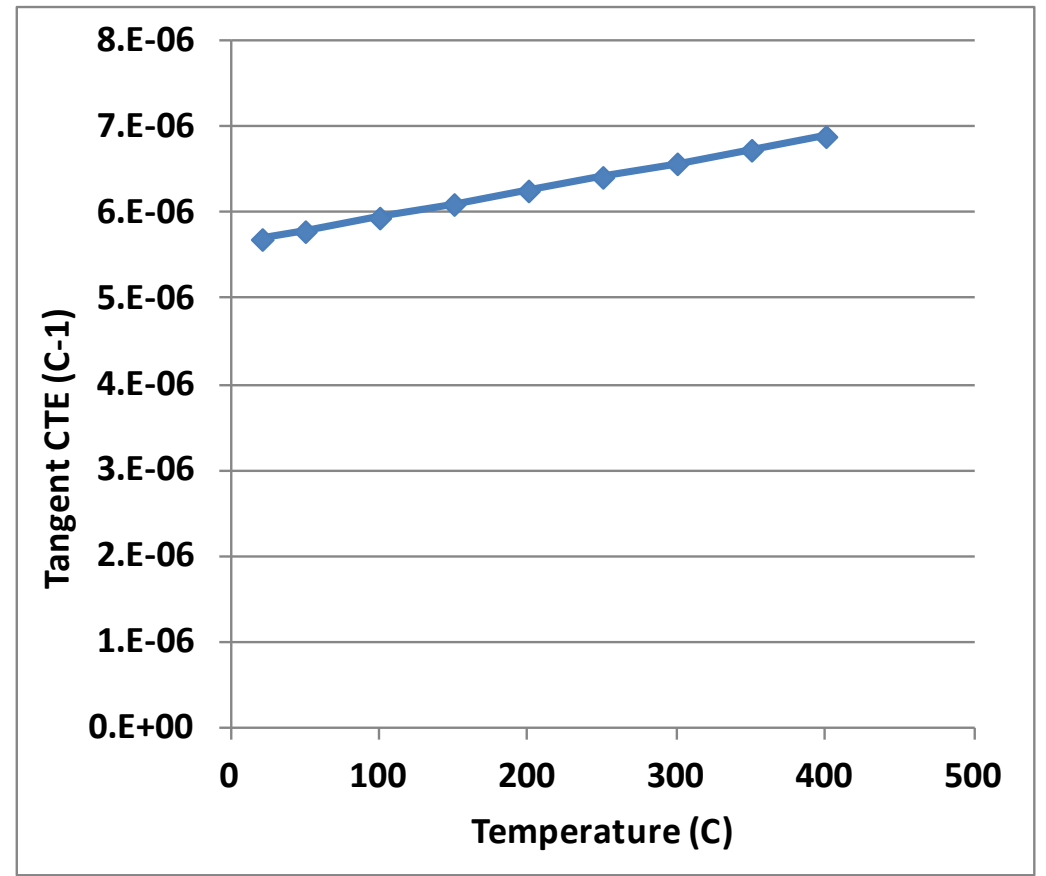

Figure 3

Instantaneous Linear Coefficient of Expansion for Zirconium Alloy from Ref. 1

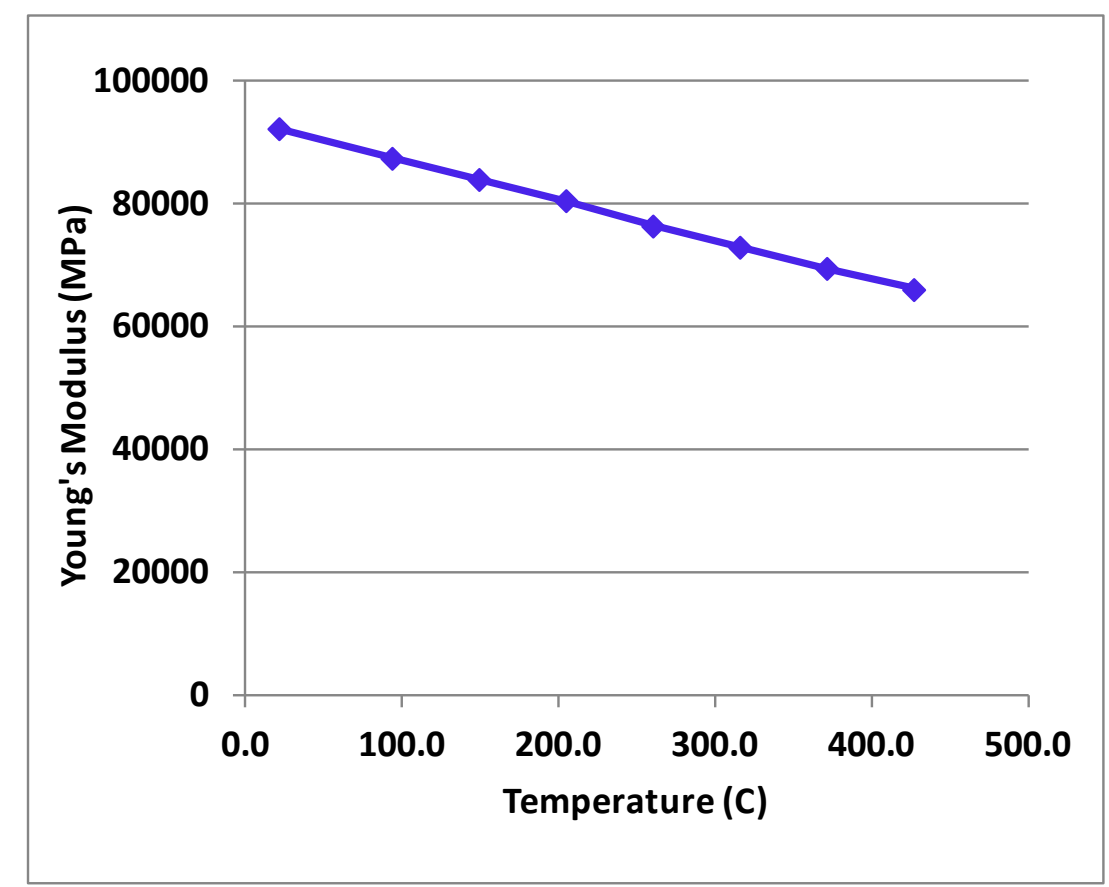

Figure 4

Young’s Modulus for Zirconium Alloy from Ref.1 


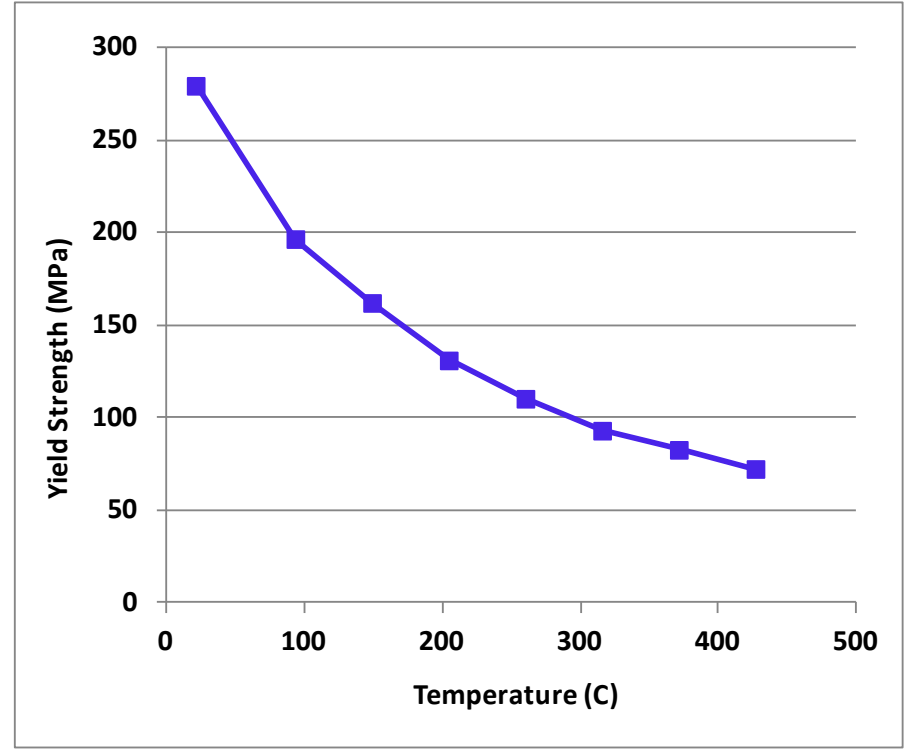

Figure 5

Yield Strength for Zirconium Alloy from Ref. 1

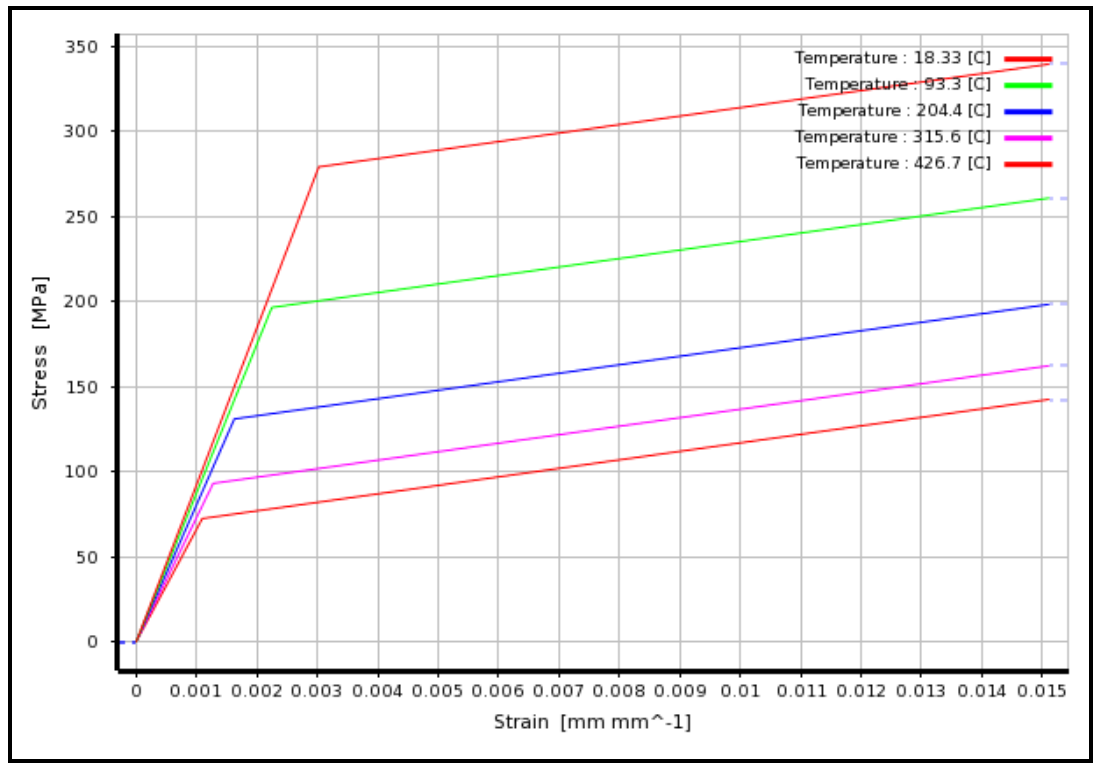

Figure 6

Elastic, Linear Plastic Material Model for Zirconium Alloy

\section{Boundary Conditions}

The internal heat generated in $\mathrm{W} / \mathrm{cm}^{3}$ by the electron beam was based on an estimate of the maximum heat generated in a DU disk by the electron beam. This value was fitted to the following Gaussian distribution that would produce 95\% of that total in the 2" diameter DU disk: 


$$
\dot{q}=4.667 e^{-0.683 r^{2}}
$$

where $r$ is the radius from the beam center. The heat generated in the cladding was found by multiplying this value by the ratio of the density of Zircaloy-4 to the density of DU. These distributions are plotted in Figure 7.

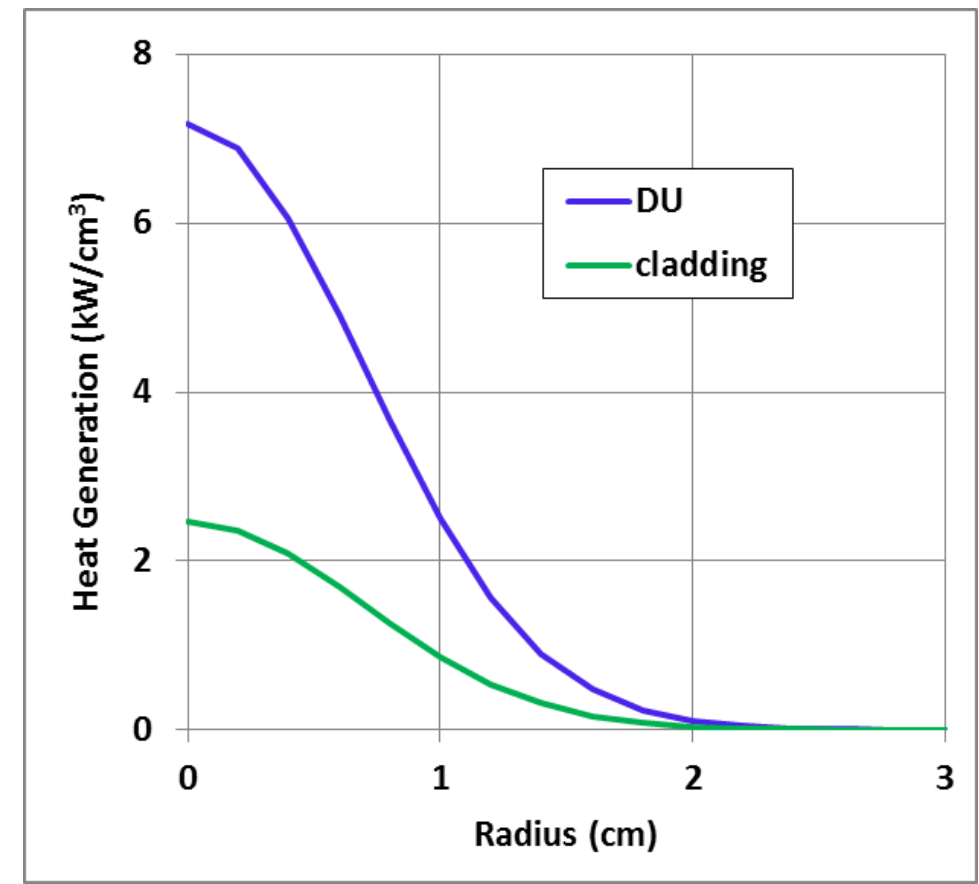

Figure 7

Heat Generation in DU and Cladding

The model was cooled by convection on the top and bottom surfaces of the disk. A film coefficient of $3.93 \mathrm{e}-2 \mathrm{~W} / \mathrm{mm} 2$ was used with a water temperature of $18.33 \mathrm{C}$. The film coefficient value was the average of the calculated values from an unpublished conjugate heat transfer analysis performed by Phil Strons

In the structural analysis, the half symmetry model was restrained as shown in Figure 8. 


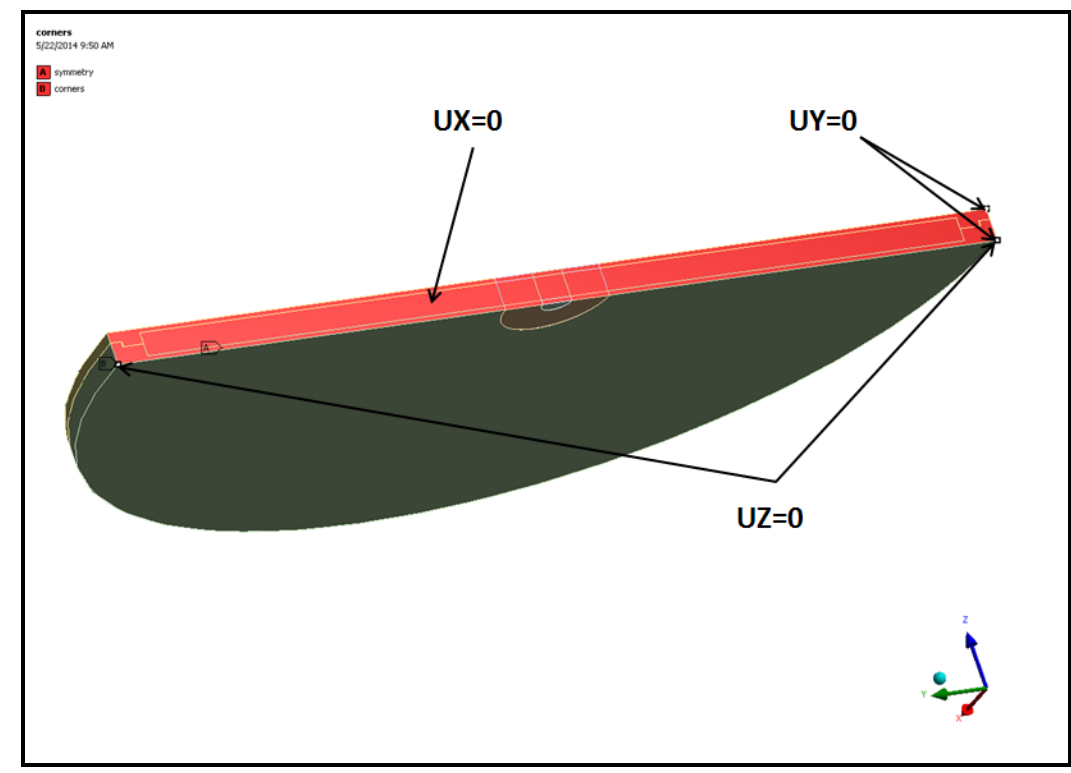

Figure 8

Structural Boundary Conditions

\section{Solution and Results}

The details of the eight load cases are shown in Table 2. Load case \#1 is a baseline run with no flaw. Load Cases \#2 through \#4 include a 1/16" diameter flaw at various distances from the center of the disk. Load cases \#5 through \#8 have flaws of varying diameters in the center of the disk.

\begin{tabular}{|c|c|c|c|c|c|c|c|}
\hline \multirow{2}{*}{$\begin{array}{l}\text { Load } \\
\text { Case }\end{array}$} & \multicolumn{2}{|c|}{ Flaw Parameters } & \multicolumn{2}{|c|}{$\begin{array}{c}\text { Maximum } \\
\text { Temperature (C) }\end{array}$} & \multirow{2}{*}{$\begin{array}{l}\text { Equivilent } \\
\text { Stress } \\
\text { (MPa) }\end{array}$} & \multirow{2}{*}{$\begin{array}{l}\text { Max. Equiv. } \\
\text { Total Strain }\end{array}$} & \multirow{2}{*}{$\begin{array}{c}\text { Cladding } \\
\text { Life } \\
\text { (cycles) }\end{array}$} \\
\hline & Dia. (in) & Offset (in) & DU & Zirconium & & & \\
\hline 1 & 0.0000 & 0.000 & 180.9 & 142.1 & 105.8 & 0.001217 & $3.74 \mathrm{E}+06$ \\
\hline 2 & 0.0625 & 0.000 & 201.6 & 148.5 & 174.2 & 0.002331 & 4. $20 \mathrm{E}+05$ \\
\hline 3 & 0.0625 & 0.093 & 195.0 & 145.2 & 169.2 & 0.002299 & 4.40E+05 \\
\hline 4 & 0.0625 & 0.187 & 180.9 & 142.2 & 157.4 & 0.002117 & $5.81 E+05$ \\
\hline 5 & 0.0469 & 0.000 & 189.9 & 144.9 & 142.4 & 0.001786 & $1.03 E+06$ \\
\hline 6 & 0.0547 & 0.000 & 194.9 & 146.2 & 153.4 & 0.002042 & $6.56 \mathrm{E}+05$ \\
\hline 7 & 0.0703 & 0.000 & 208.2 & 149.9 & 179.1 & 0.002358 & 4.04E+05 \\
\hline 8 & 0.0781 & 0.000 & 215.7 & 152.2 & 185.8 & 0.002452 & $3.54 \mathrm{E}+05$ \\
\hline
\end{tabular}

Table 2

Summary of Results 
All analyses were conducted in the Workbench environment. A steady state thermal analysis was performed on the disk with the heat generation and convection boundary condition described above. The thermal result was transferred to a structural analysis where the nodal temperatures were applied as a structural load. A temperature dependent elastic-linear plastic material model shown in Figure 6 was used for the Zircaloy. A linear elastic material was used for the DU.

Plots of temperature and von Mises stress for load case \#1 is shown in Figure 9 through Figure 11. Maximum temperature was $180.9 \mathrm{C}$ in the DU and $142.15 \mathrm{C}$ in the cladding. The maximum von Mises stress was $105.8 \mathrm{MPa}$. Fatigue was evaluated by comparing the maximum total equivalent strain in the cladding to the fatigue curve shown in Figure 14. This curve is based on Figure 13 from Ref. 2. The raw data was generated for Zircaloy-2, but per Ref. 2, Zircaloy-4 is slightly better than Zircaloy-2, so the use of this data is conservative.

Plots of temperature, stress, strain and cycles to failure as a function of flaw size are shown in Figure 15 through Figure 19. Contour plots of temperature, equivalent stress, and equivalent plastic strain for Load Case \#2 (r=0) are shown in Figure 20 through Figure 24.

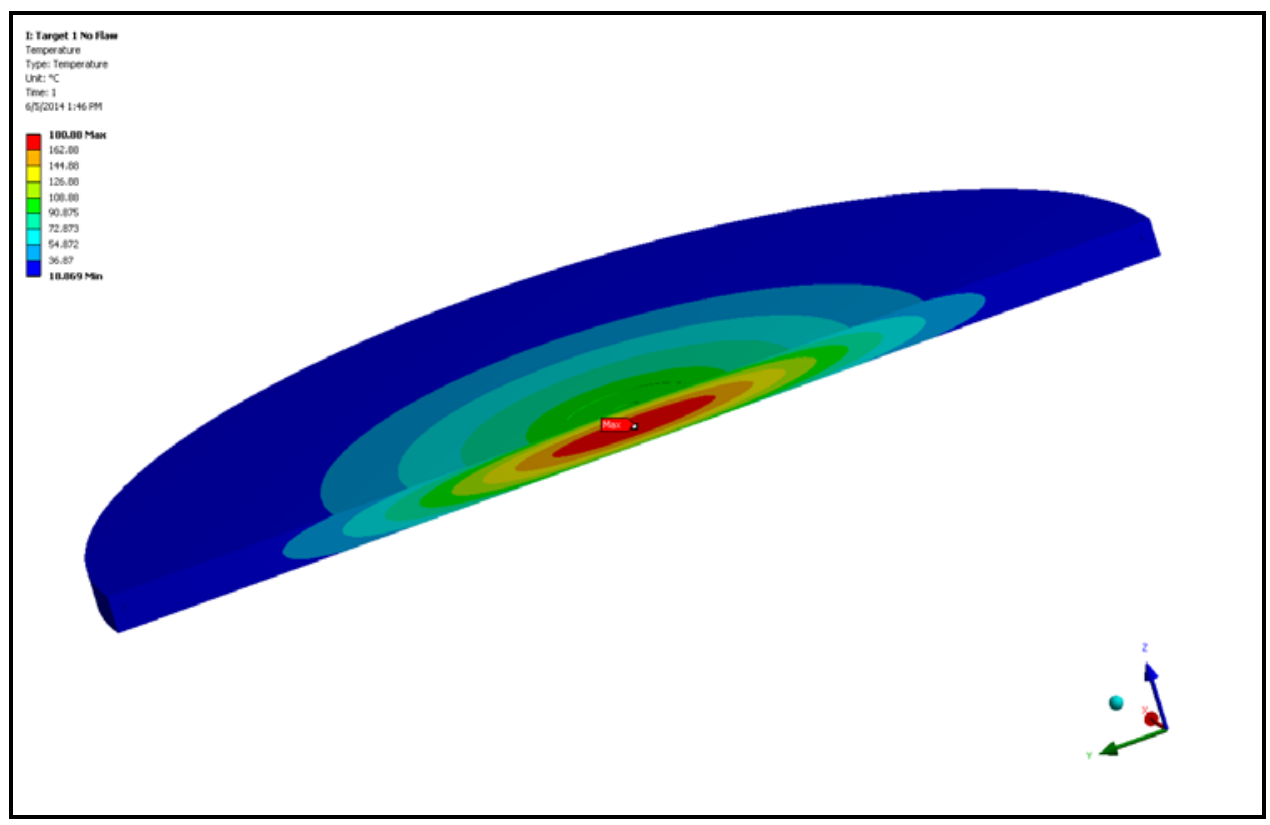

Figure 9

Temperature (C), Load Case 1 


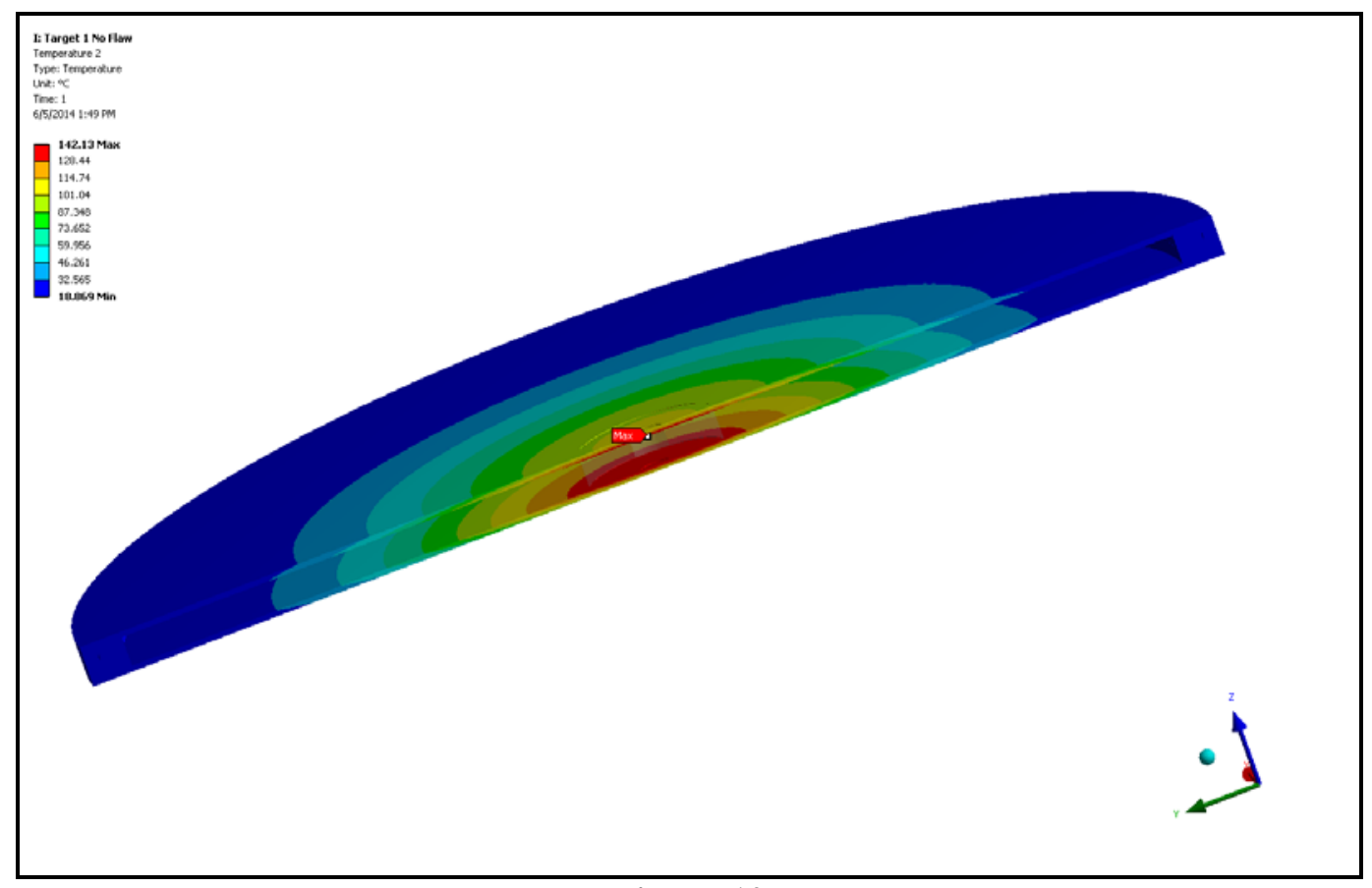

Figure 10

Cladding Temperature, Load Case 1

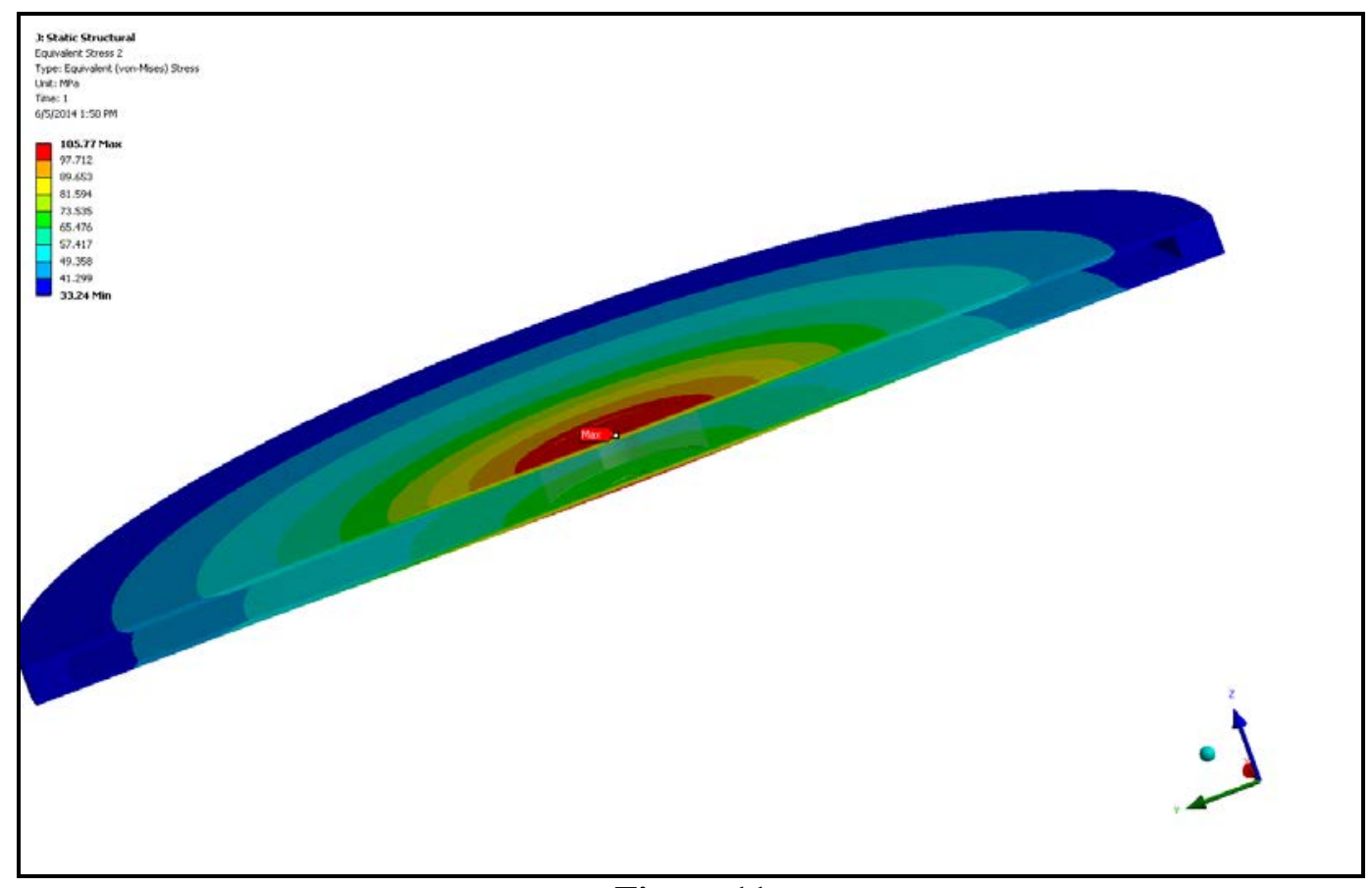

Figure 11

Equivalent Stress in Cladding, Load Case 1

234 


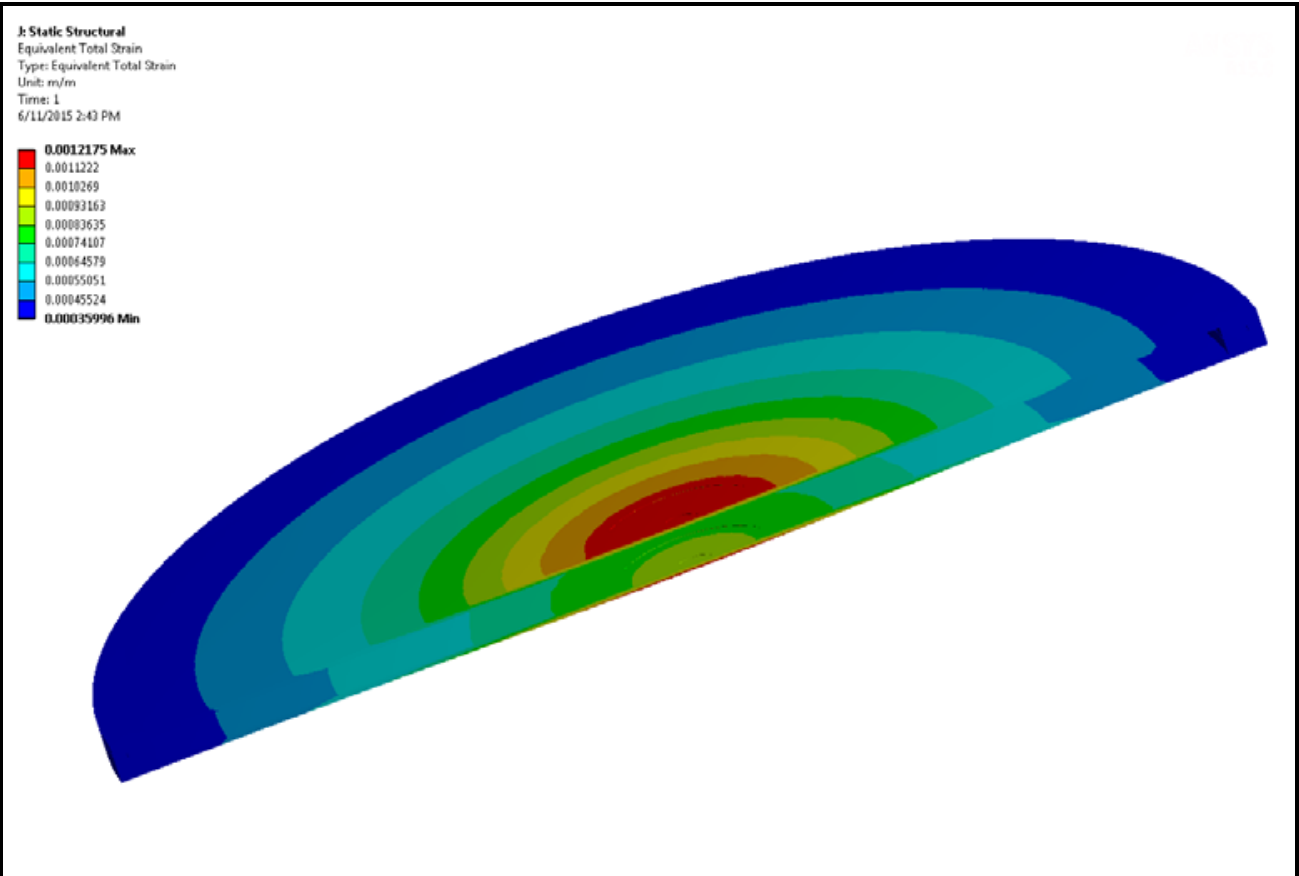

Figure 12

Equivalent Total Strain in Cladding, Load Case 1 


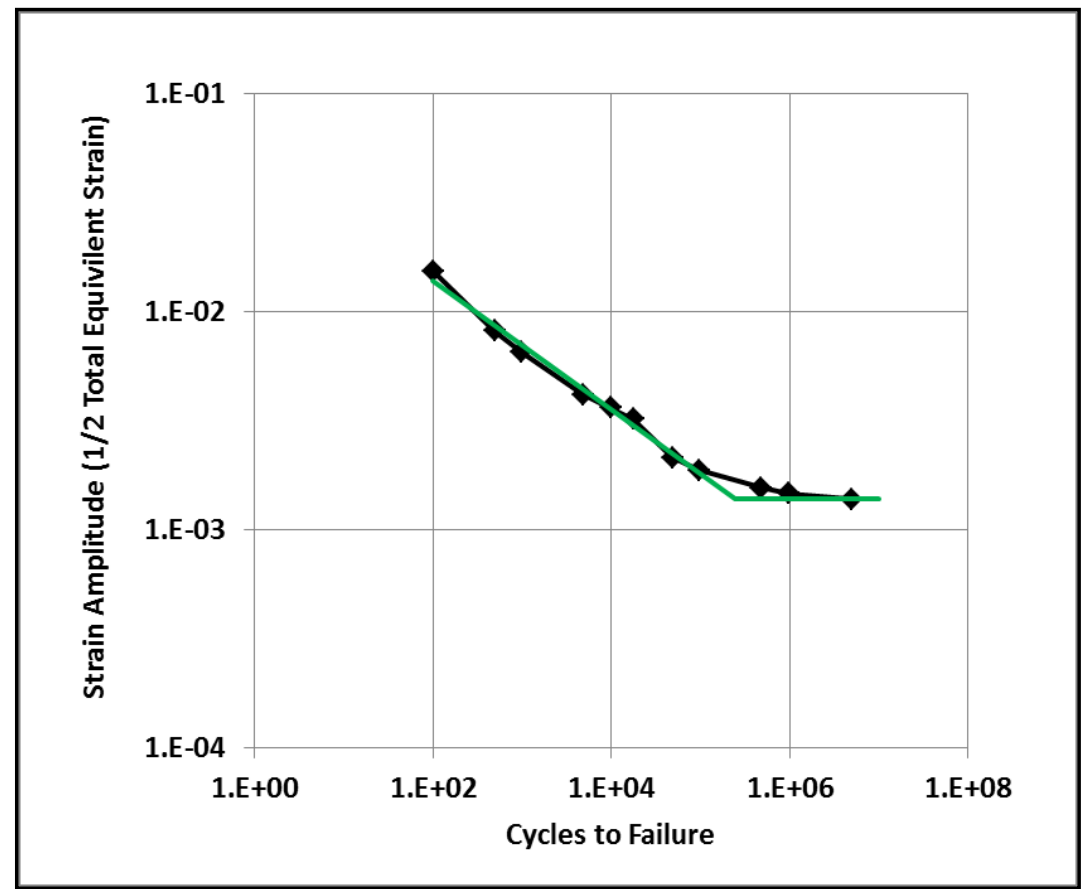

Figure 14

Fatigue Model for Zircaloy-4

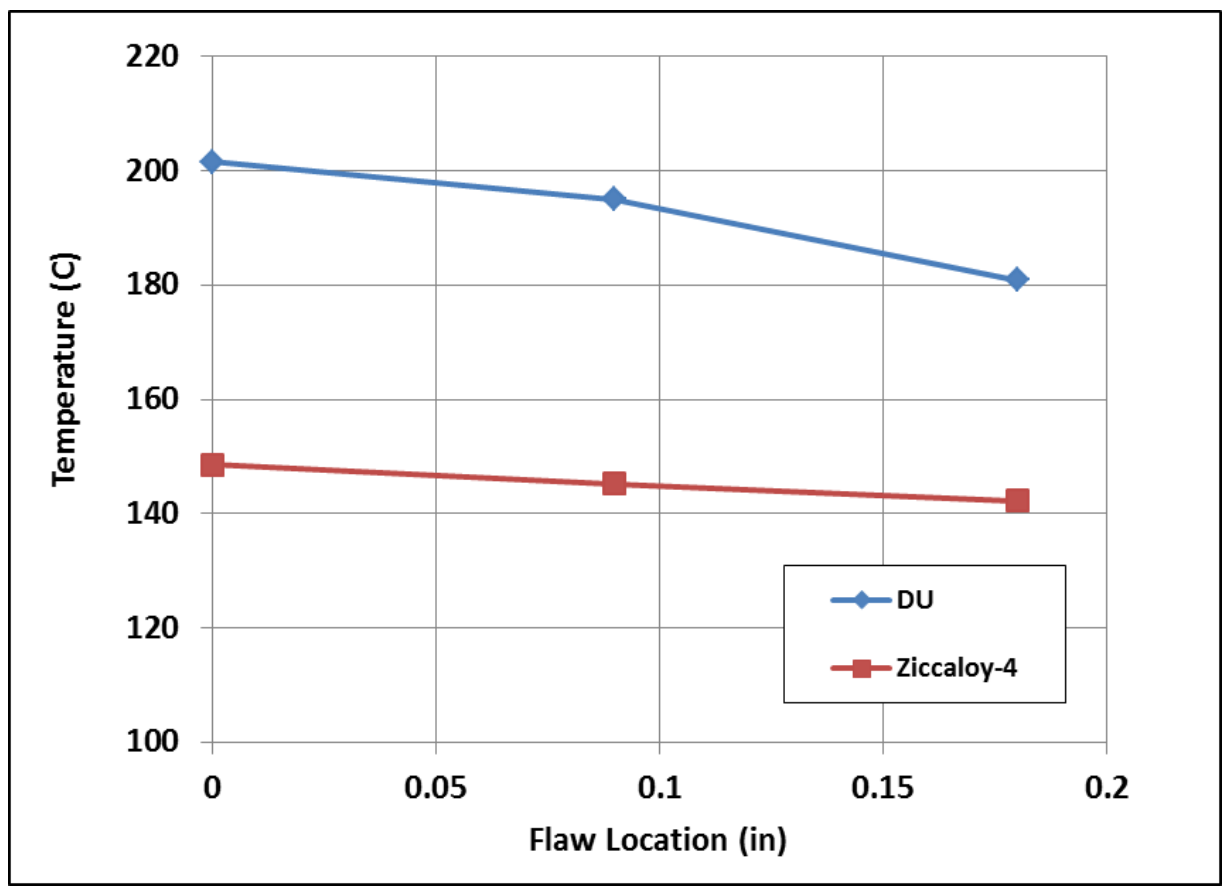

Figure 15

Maximum Temperature vs. Flaw Location 


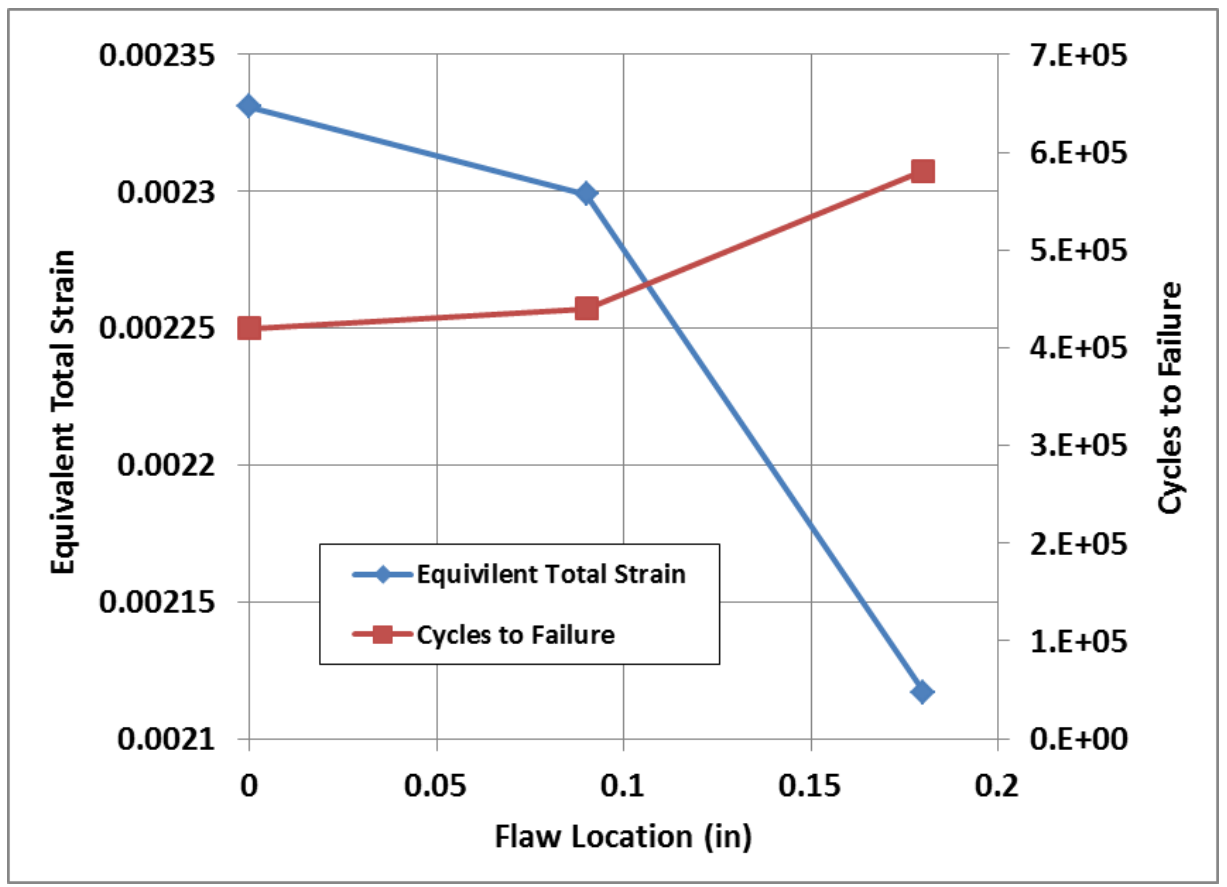

Figure 16

Equivalent Total Strain and Cycles to Failure vs. Flaw Location in Cladding

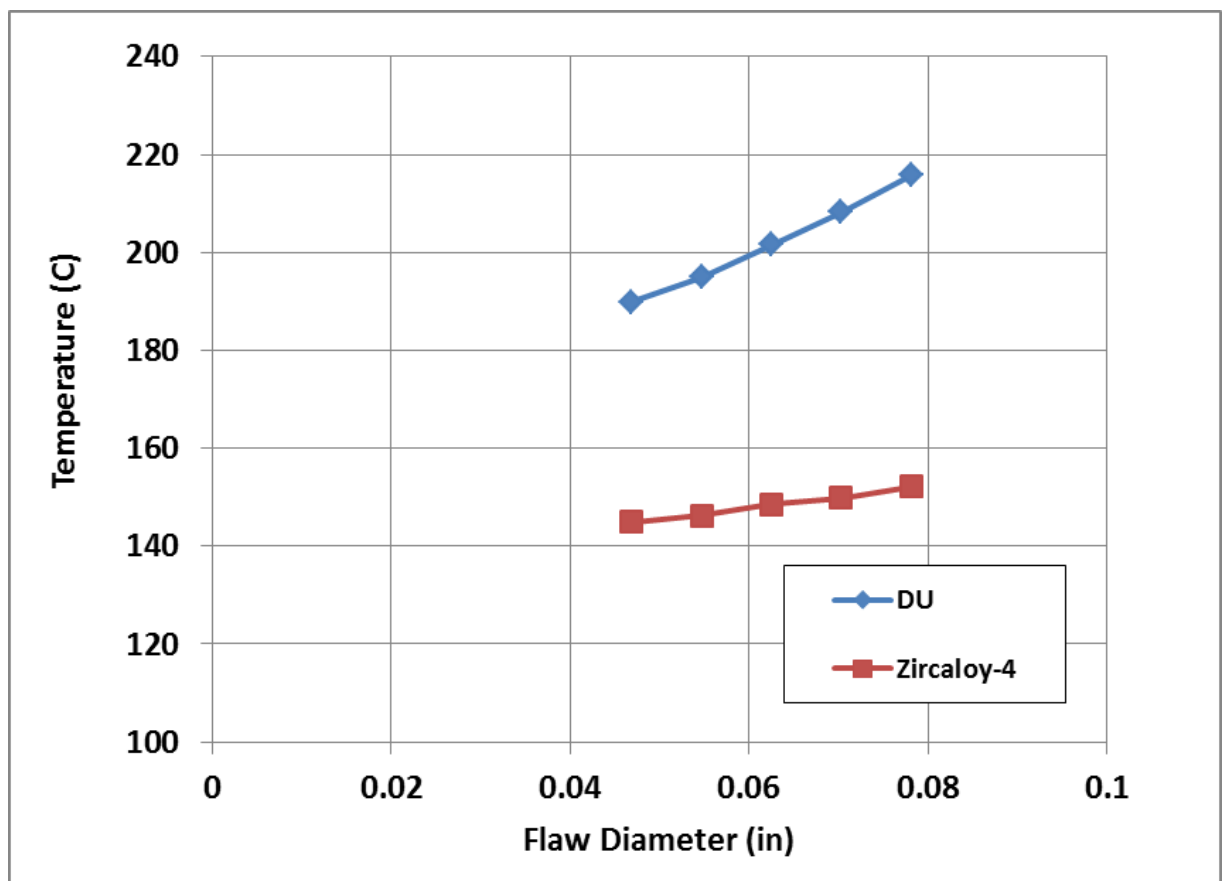

Figure 17

Maximum Temperature vs. Flaw Size 


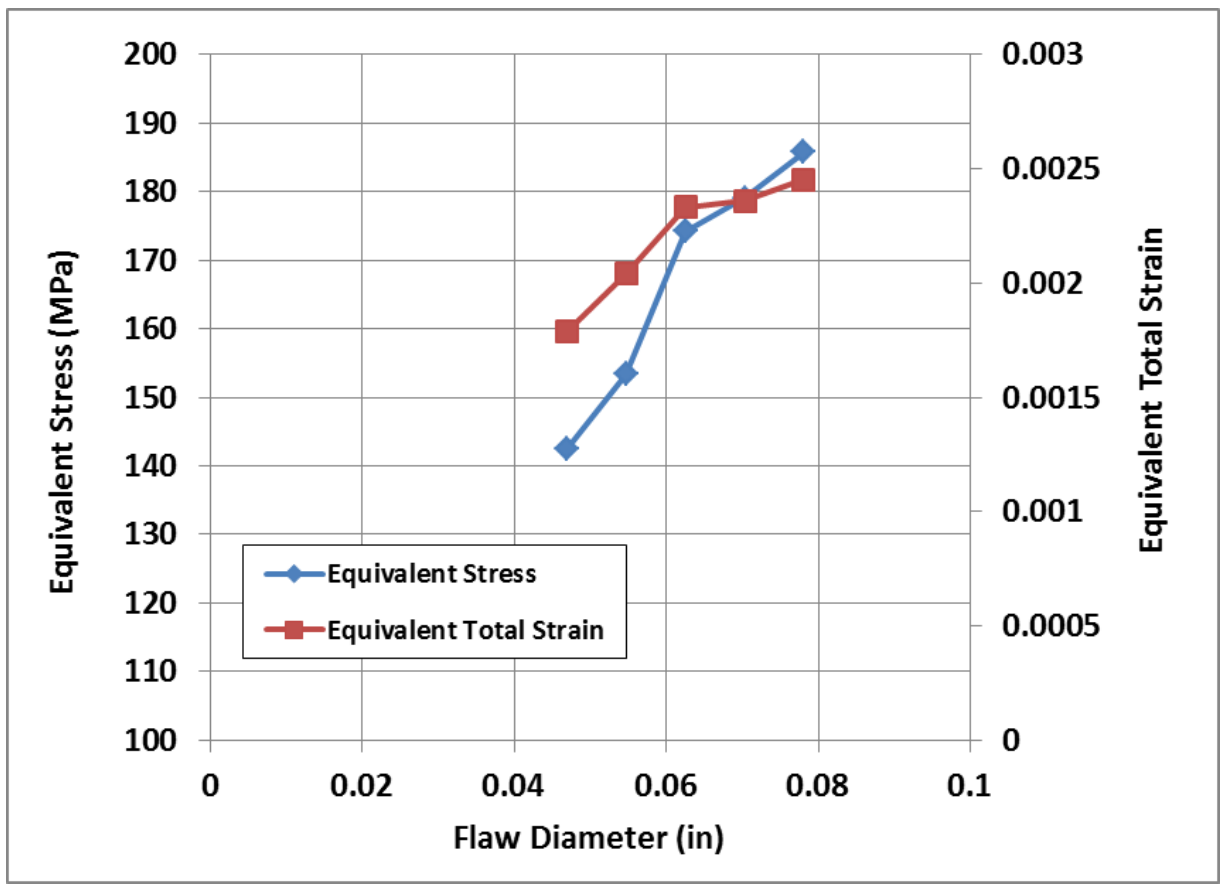

Figure 18

Stress and Strain vs. Flaw Size

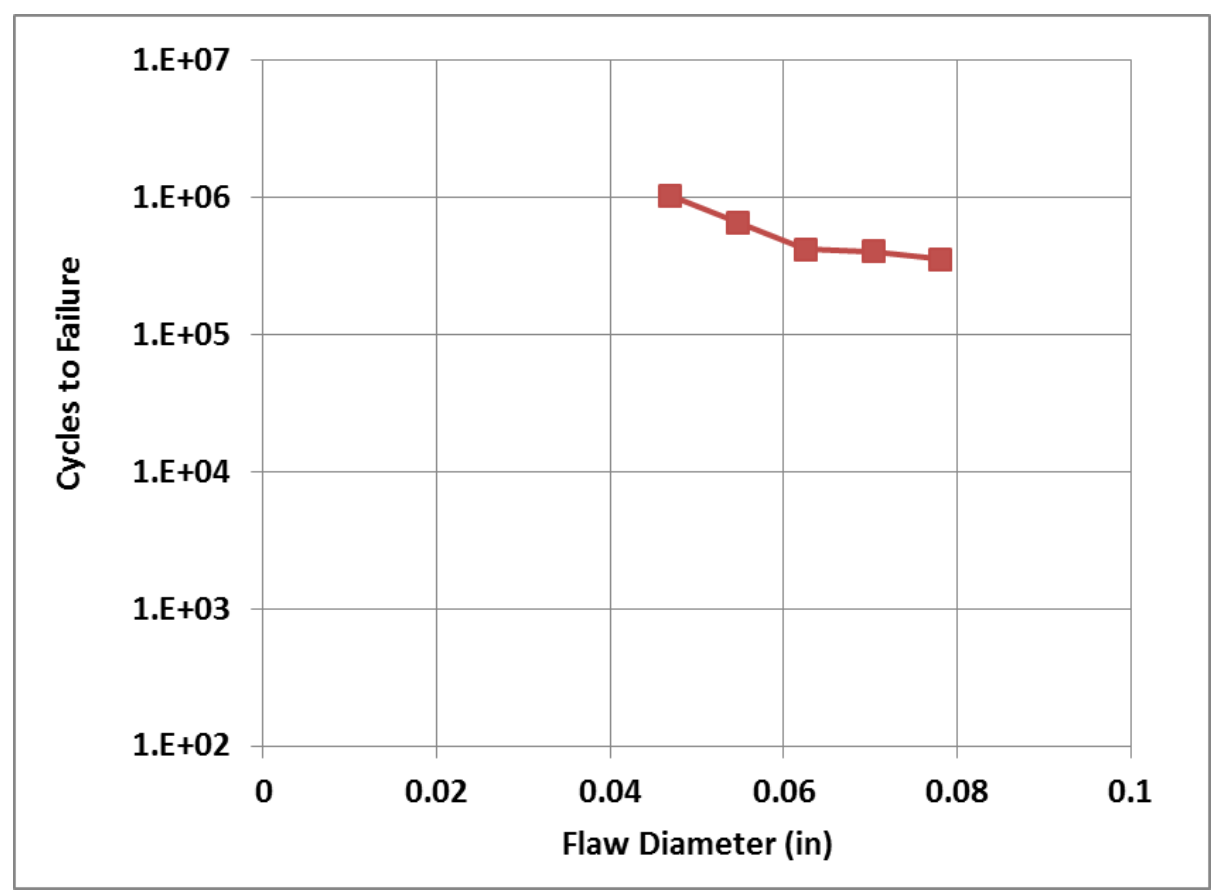

Figure 19

Cycles to Failure vs. Flaw Size 


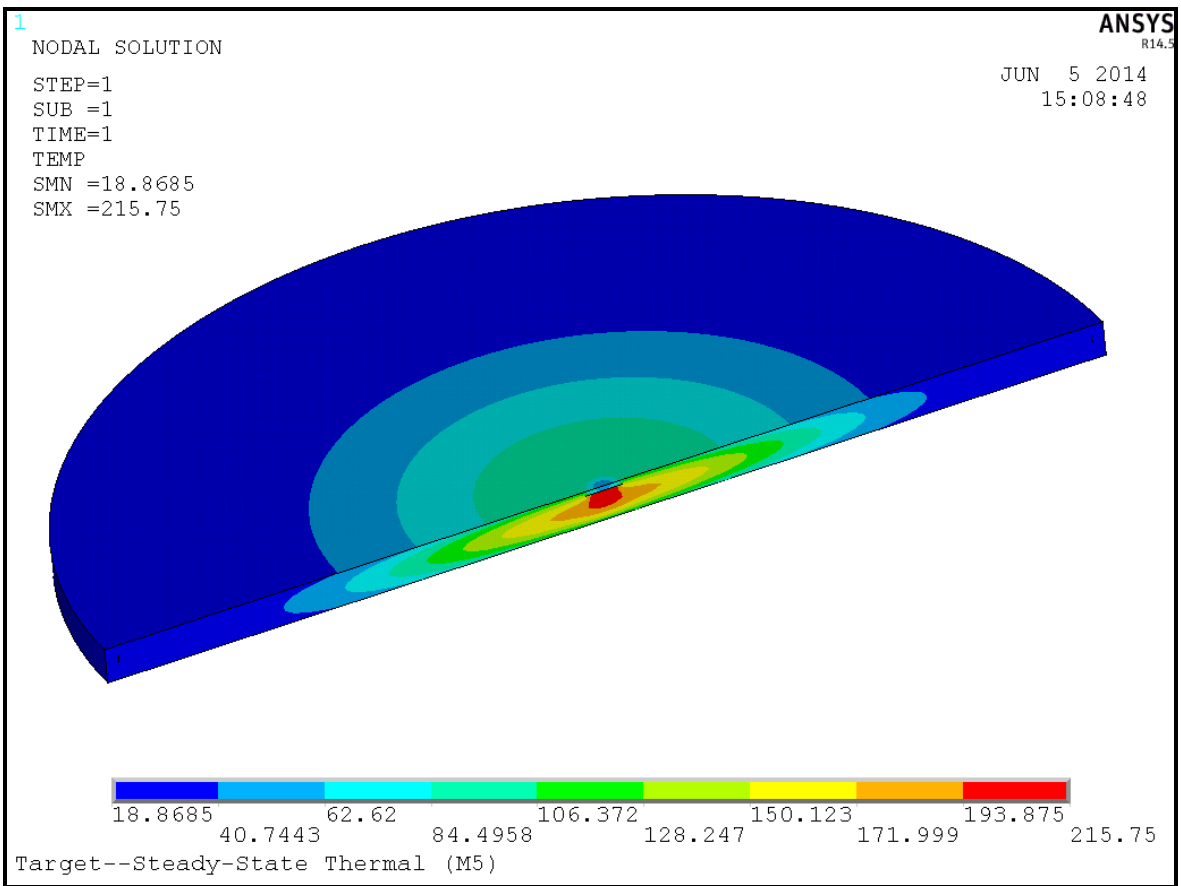

Figure 20

Temperature (C), Load Case 8

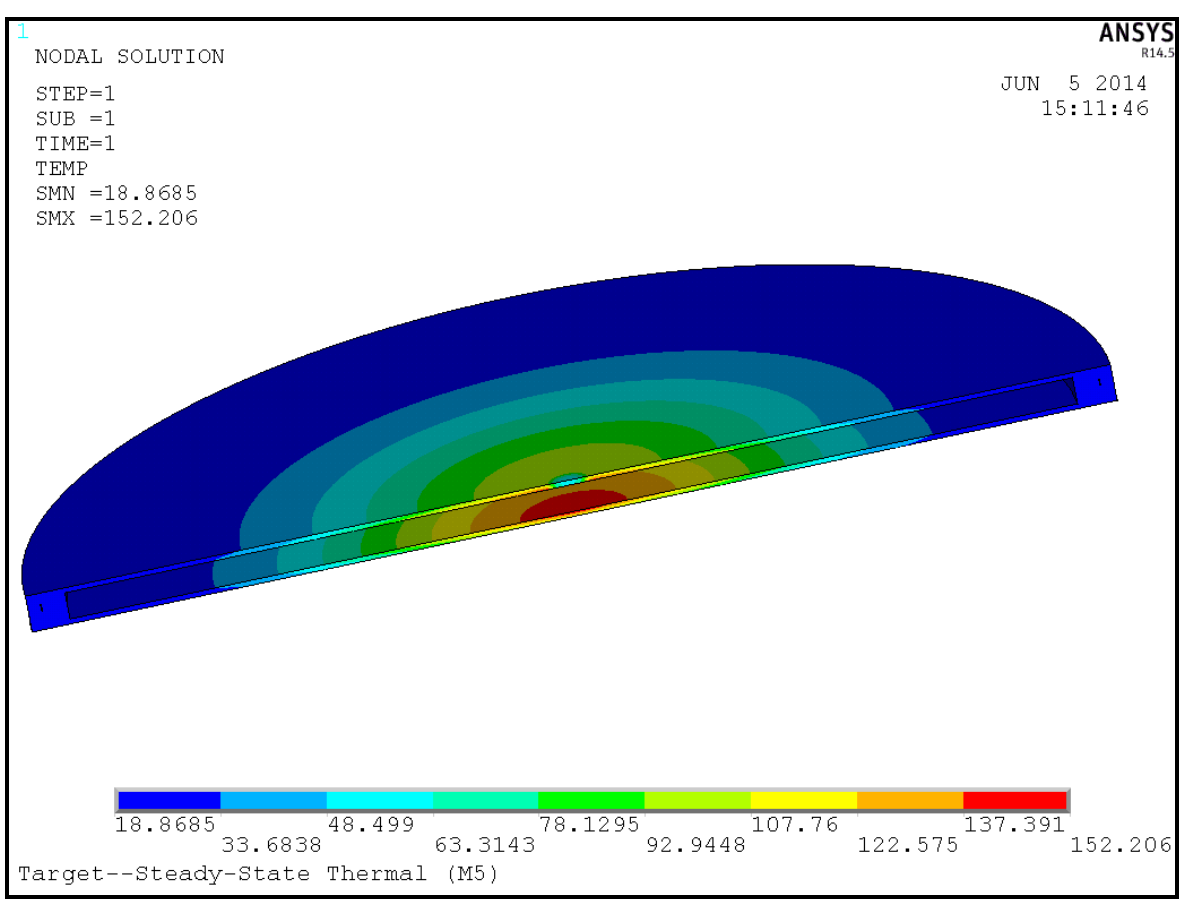

Figure 21

Temperature (C) in Cladding, Load Case 8 


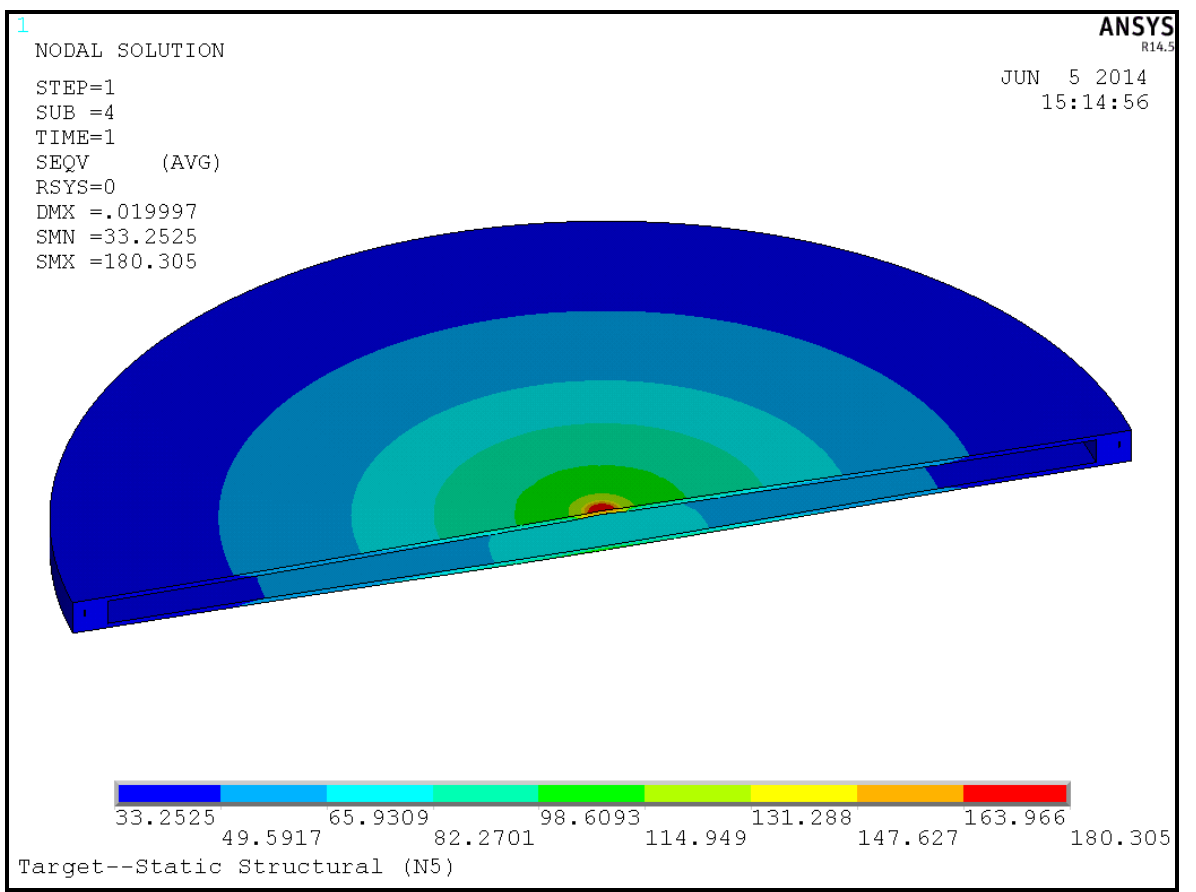

Figure 22

von Mises Stress (MPa) in Cladding, Load Case 8

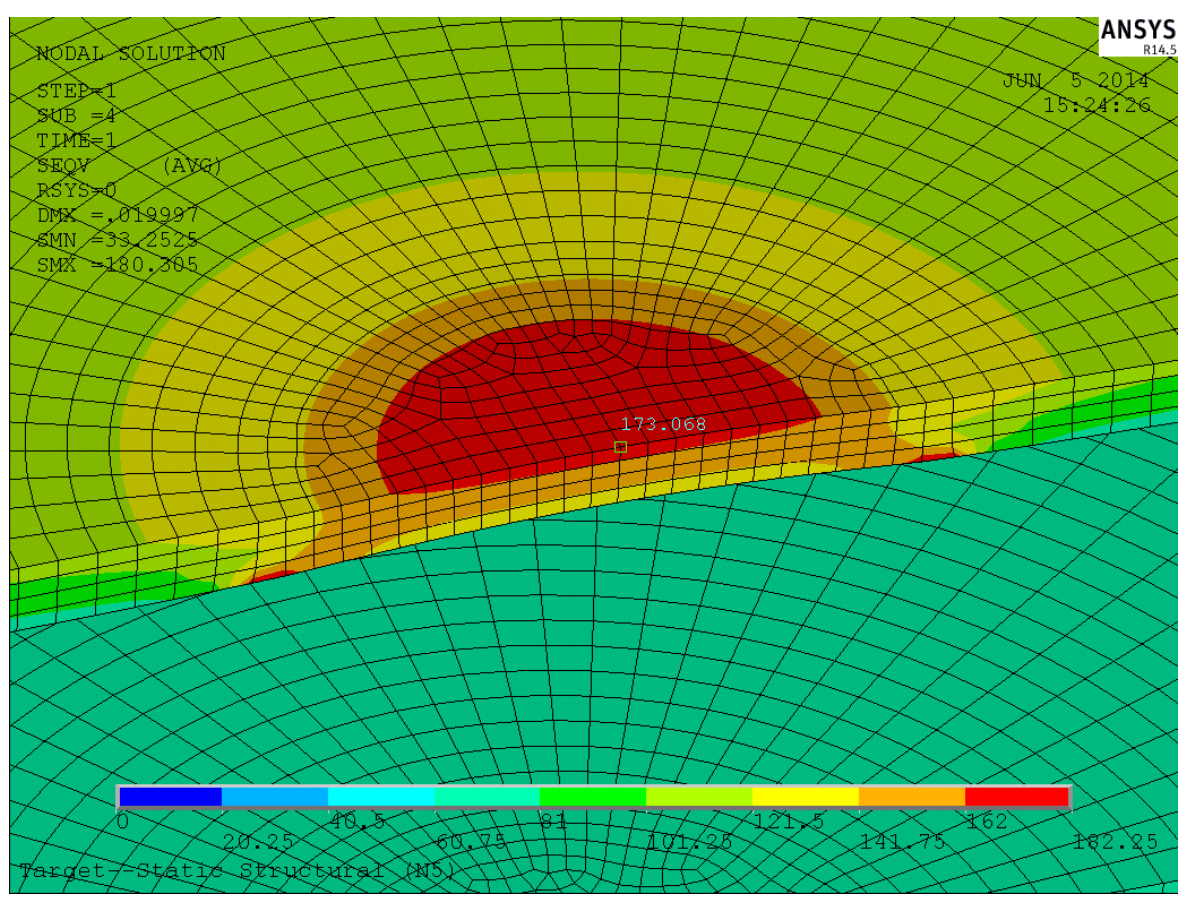

Figure 23

Von Mises Stress (MPa) in Cladding, Load Case 8 


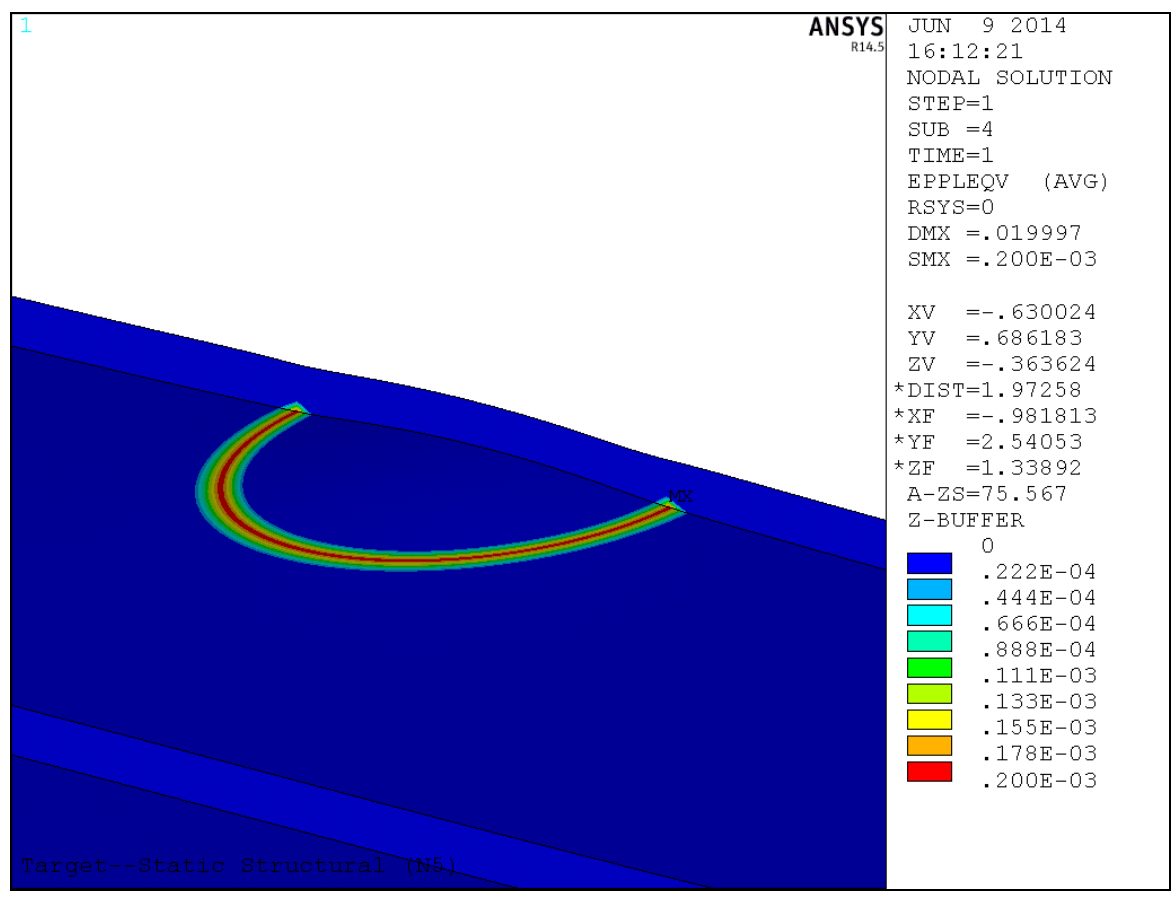

Figure 24

Equivalent Plastic Strain in Cladding, Load Case 8

\section{Discussion}

The highest stress and strain in the cladding is always at the flaw. Per Figure 16, the highest strain value occurs when the flaw is in the center of the disk. Deformation of the disk is driven by thermal expansion of the DU (see Figure 25), and this plus the effects of the flaw result in the highest stress, and therefore the highest fatigue damage, when the flaw is at the center. Flaws of these magnitudes do not seem to be a problem. Note that the lowest cladding life value in Table 2 is two orders of magnitude higher than the desired life of 1000 cycles.

When the flaw is in the middle and the flaw size varies, temperature, stress, plastic strain and fatigue damage all increase with flaw diameter (Figure 17 through Figure 19). The larger the flaw, the larger the insulating effect. This drives up temperature which increases swelling in the DU and reduces the yield strength of the Zircaloy-4. Thus, plastic strain and fatigue damage increase. 


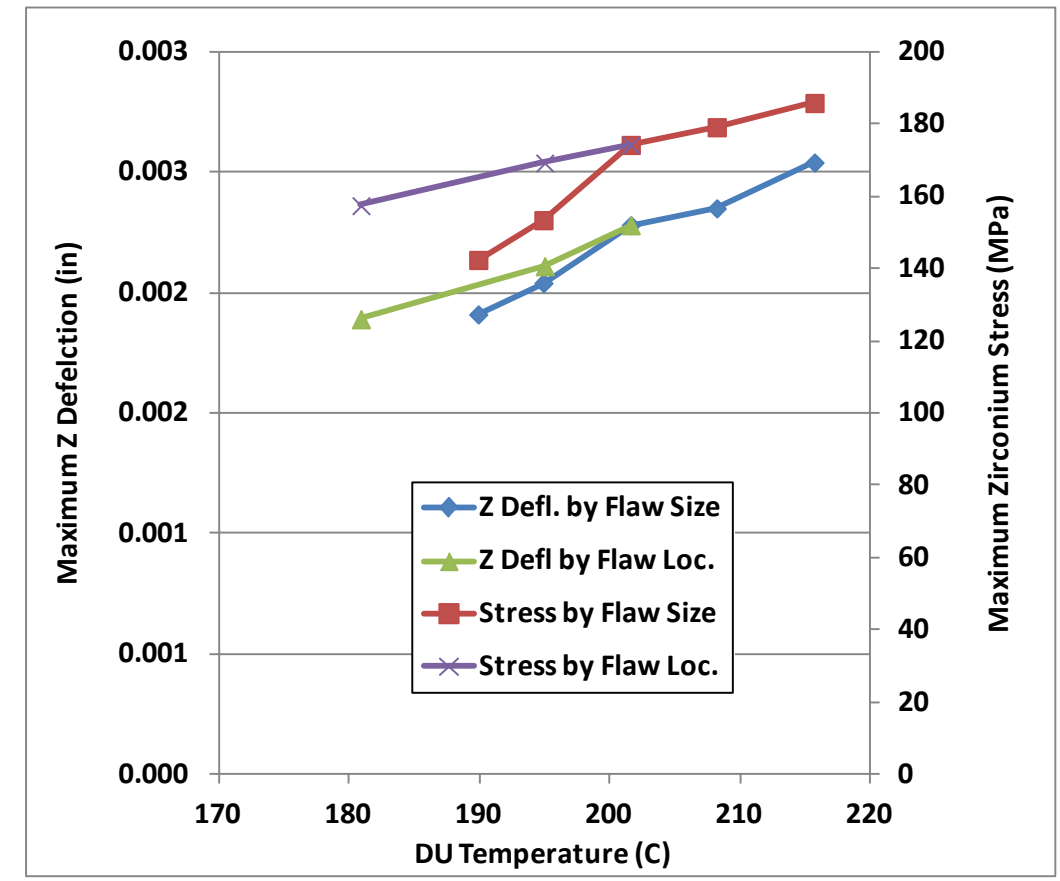

Figure 25

Effect of DU Temperature on Cladding Stress

This analysis was hampered by the inability to locate reliable material data. The materials used to fabricate the disks were identified simply as depleted uranium and Zircaloy-4, without further grade designation, composition, temper, etc. Much of the data also was not clearly identified with grade designation, temper, etc. Furthermore, the lack of quality data over a range of temperatures made it difficult to account for the effect of temperature. These results are believed to be accurate within the limitations imposed by this uncertainty.

\section{Conclusions}

Based on the results of this analysis the following conclusions are drawn:

1. The highest fatigue damage is present for flaws in the center of the disk.

2. Fatigue damage increases with flaw size.

3. The fatigue life of the Zircaloy-4 cladding is at least 1000 cycles.

\section{References}

1. Lemoine, P, and Schmuck, J, Zirconium for Chemical Engineering: Mechanical Properties Useful for Vessel Design, International Meeting on Chemical Engineering and Biotechnology, Frankfurt, June 9-15, 1991

2. O’Donnell, W.J. and Langer, B.F., Fatigue Design Basis for Zircaloy Components, Nuclear Science and Engineering, Col. 20, pgs. 1-12, 1964. 


\section{Software}

- Ansys Mechanical, Version 15.0.7, Build date 4/11/2014, Ansys Inc., Pittsburg, PA.

- Microsoft Windows 7 Enterprise, Service Pack 1, Microsoft Corporation, Redmond WA.

- Microsoft Office Excel Professional Plus2010, (14.0.7015.1000), Microsoft Corporation, Redmond WA. 
APPENDIX 1

GENERAL CHECKING CRITERIA SHEET

\begin{tabular}{|l|l|l|l|l|}
\hline ANALYSIS CHECKLIST & Yes & No & N/A & Comments \\
\hline Are analytical methods appropriate? & $\square$ & $\square$ & $\square$ & \\
\hline Are assumptions appropriate? & $\square$ & $\square$ & $\square$ & \\
\hline Is the analysis complete? & $\square$ & $\square$ & $\square$ & \\
\hline $\begin{array}{l}\text { Is the source of the input geometry } \\
\text { documented? }\end{array}$ & $\square$ & $\square$ & $\square$ & \\
\hline $\begin{array}{l}\text { Is the source of material properties } \\
\text { documented? }\end{array}$ & $\square$ & $\square$ & $\square$ & \\
\hline $\begin{array}{l}\text { Are the boundary conditions clearly } \\
\text { explained? }\end{array}$ & $\square$ & $\square$ & $\square$ & \\
\hline $\begin{array}{l}\text { Was an applicable and valid } \\
\text { computer program used? }\end{array}$ & $\square$ & $\square$ & $\square$ & \\
\hline $\begin{array}{l}\text { Are the conclusions supported by } \\
\text { the results? }\end{array}$ & $\square$ & $\square$ & $\square$ & \\
\hline $\begin{array}{l}\text { Do the results seem reasonable? } \\
\text { (n) }\end{array}$ & $\square$ & $\square$ & $\square$ & \\
\hline
\end{tabular}




\section{APPENDIX 2}

\section{CRITICAL REVIEW OF REPORT}

The report was submitted to Saurin Majumdar, Sr. Mechanical Engineer, who reported his concerns via e-mail. Below are the pertinent excerpts from that e-mail thread.

Saurin Majumdar: I scanned though the report. Figure 12 baffles me. Fatigue involves cyclic stress and creep involves constant stress. How can you plot the two on the same graph and make sense, particularly for tests at various frequencies. First of all what is the ordinate axis? Is this fatigue curve derived from zero to max loading cycles? As it is plotted, the cycle and time correspond only for $1 \mathrm{~Hz}$. As the cyclic rate goes down at high temperature, creep-fatigue interaction increases and the cyclic life a drop. One cannot predict the fatigue curve by integrating the creep damage over the cycles, especially if the fatigue cycling is fully reversed. If that was true we could avoid running fatigue tests at high temperature and do all design based on creep rupture data.

Rick Fischer: My intention was to produce a conservative result. As stated on page 11, when the loading frequency is below $1 \mathrm{~Hz}$, creep dominates and the fatigue curve approaches the creep rupture curve. Cycle rate here is measured in hours, so using the creep rupture curve seemed appropriate. I would have to go back and review the data I found, but I'm guessing this was the only data I had at or above the temperature of the cladding.

Rick Fischer: Also, note in Figure 12 that the data at for $0.1 \mathrm{~Hz}$ is very close to the creep rupture curve. I believe the cycle is the beam is turned on and the target heats to a steady state condition and is held there for an extended time. At the cycle frequency seen by the targets, i.e. $>1$ day, it seems this would be very close to a creep rupture scenario.

Saurin Majumdar: I looked at Kim's paper from where you got Fig. 12. I don't know how this paper got published. He doesn't understand that 1000 cycles at $0.1 \mathrm{~Hz}$ take $10000 \mathrm{~h}$ to complete. In the mean time I found the attached paper by O’Donnell and Langer. However, it is for Zr- 2 .

Rick Fischer: Figure 8 in O'Donnell's paper has a design curve to be used with unirradiated Zircaloy-2, 3, and 4 . On page 4 he states that Zircaloy 4 may be slightly better in fatigue than Zircaloy 2, so the Zircaloy-2 data can be used for Zircaloy 4.

At this point the fatigue calculations were redone using the design curve for unirradiated Zircaloy-2 in O’Donnell's paper, which is now Ref. 2. 


\section{APPENDIX 6}

Calculation Note NE-EO-doc89: "Structural Analysis of Inconel Window for DU Target Assembly for ${ }^{99}$ Mo Production" 
Rev.

0

Page

of

12

\section{CALCULATION COVER SHEET}

Title: Structural Analysis of Inconel Window for DU Target Assembly for Mo99 Production

Date: $4 / 18 / 2017$

Analyzed System:

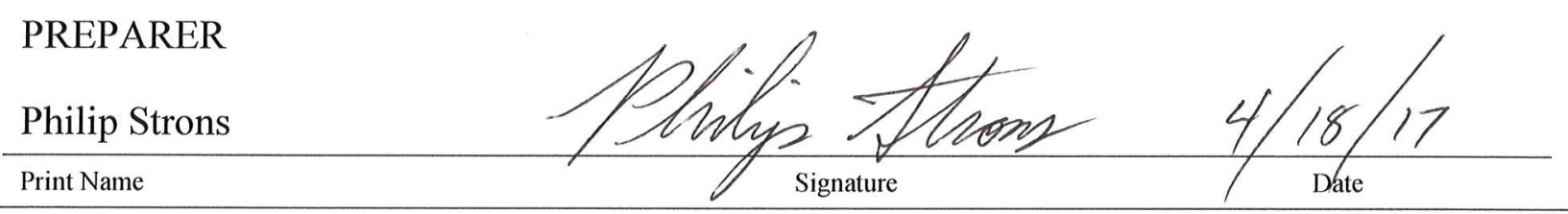

\section{REVIEWER}

Rick Fischer

Print Name
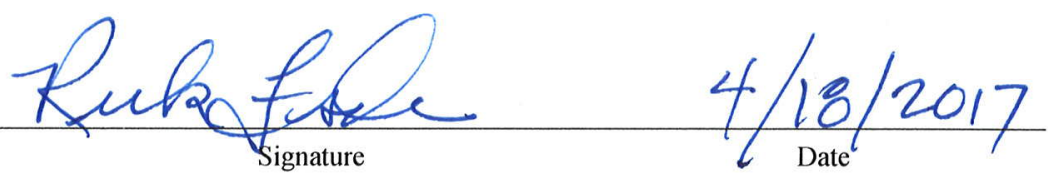

\section{CALCULATION HAND CHECKED BY}

\begin{tabular}{lll}
\hline Print Name & Signature & Date
\end{tabular}

\section{FINAL APPROVER}

James Grudzinski 


\begin{tabular}{|llllllll|}
\hline Calculation No.: & NE-EO-doc-89 & Rev. & 0 & Page & 2 & of & 12 \\
\hline
\end{tabular}

\section{TABLE OF CONTENTS}

\section{TABLE OF CONTENTS 1}

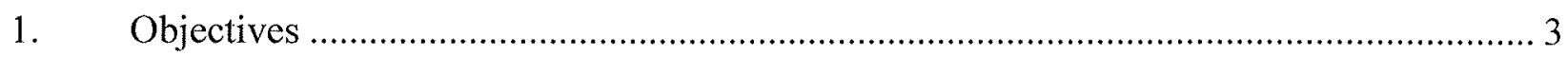

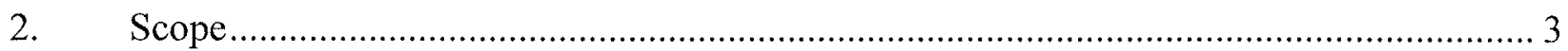

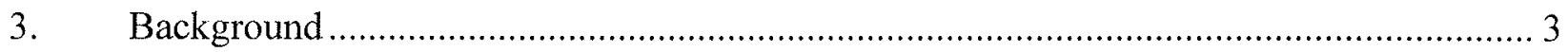

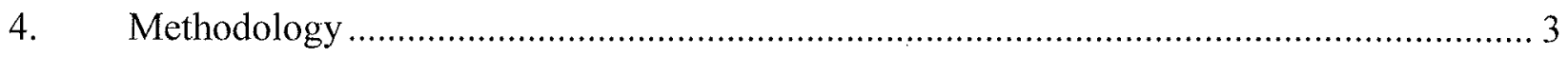

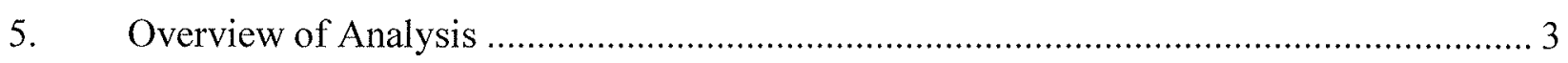

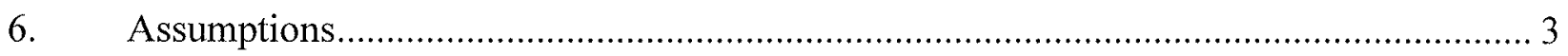

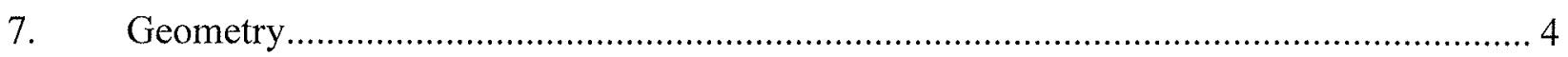

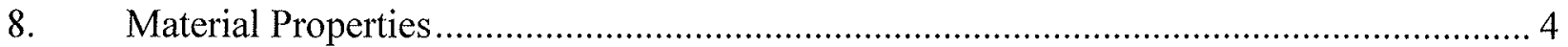

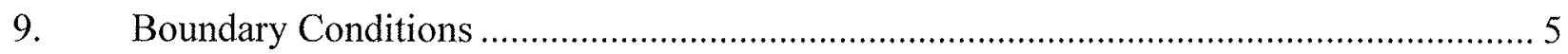

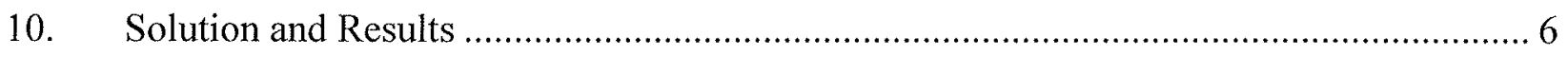

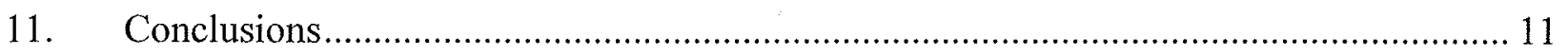

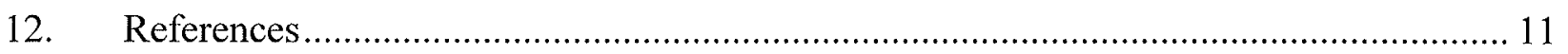

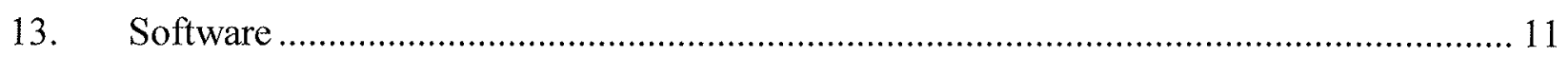




\begin{tabular}{|llllllll}
\hline Calculation No.: & NE-EO-doc-88 & Rev. & 0 & Page & 3 & of & 12
\end{tabular}

\section{Objectives}

The objective of this analysis was to determine compliance of the window for the DU target assembly to be used in the SHINE experiment with ASME BPVC Sect. VIII Div. 2.

\section{Scope}

The scope of this analysis was limited to the Inconel 600 window of the target assembly. The remainder of the target assembly is being built by the vendor in accordance with Sect VIII Div 1 and is not included in this analysis.

\section{Background}

The Inconel window of the DU target assembly provides a containment boundary between the pressurized cooling water within the assembly and the vacuum of the beam tube upstream from the target.

\section{Methodology}

The Design by Analysis method is outlined in Section VIII, Division 2, Part 5 of the ASME Boiler \& Pressure Vessel Code, which utilizes finite element analysis. A model of the window was created with the ANSYS and subjected to pressure, temperature loading, and a spring force, then analyzed to find component stresses. These stresses were then compared to criteria defined per the ASME BPVC.

\section{Overview of Analysis}

\section{A total of five analyses were conducted and are summarized in}

Table 1. The five analysis cases address possible failure criteria defined in the BPVC.

\begin{tabular}{|c|l|l|l|l|}
\hline $\begin{array}{c}\text { Analysis } \\
\text { Case }\end{array}$ & \multicolumn{1}{|c|}{$\begin{array}{c}\text { Failure } \\
\text { Mode }\end{array}$} & \multicolumn{1}{|c|}{ Criteria } & $\begin{array}{c}\text { Analysis } \\
\text { Tool }\end{array}$ & \multicolumn{1}{c|}{$\begin{array}{c}\text { Material } \\
\text { Model }\end{array}$} \\
\hline A & Plastic collapse & Sect VIII, Div 2, Part 5.2.3 & FEA & Elastic Perfectly Plastic \\
\hline B & Local Failure & Sect VIII, Div 2, Part 5.3.2 & FEA & Linear Elastic \\
\hline C & Collapse from buckling & Sect VIII, Div 2, Part 5.4.1 & FEA & Linear Elastic \\
\hline D & Fatigue & Sect VIII, Div 2, Part 3, Annex 3-F & FEA & Linear Elastic \\
\hline E & Ratcheting & Sect VIII, Div 2, Part 5.5.6 & FEA & Linear Elastic \\
\hline
\end{tabular}

Table 1

\section{Analysis Overview}

\section{Assumptions}

This analysis is based on the following assumptions:

1. Loads are steady state or cyclic (no inertial effects are considered).

2. Any loading due to gravity is negligible. 


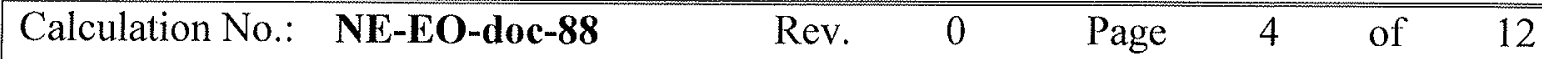

3. The normal operating pressure on the window is 27 psi.

4. The maximum pressure from the cooling system pump is $65 \mathrm{psi}$.

5. Materials are isotropic and homogeneous.

6. Temperature dependent properties are linearly interpolated between data points.

7. Material response is constant with time (no effects of aging, creep, etc).

8. Residual stresses are not included.

\section{Geometry}

The window is a machined part. It is attached to the target assembly by welding to the 316 stainless steel tube with an Inconel filler metal. The finite element model was constructed in ANSYS Design Modeler, referencing Drawing R07844A-3-03 and dated 03/20/2015. A half symmetry solid model was created. This model is shown in Figure 1.

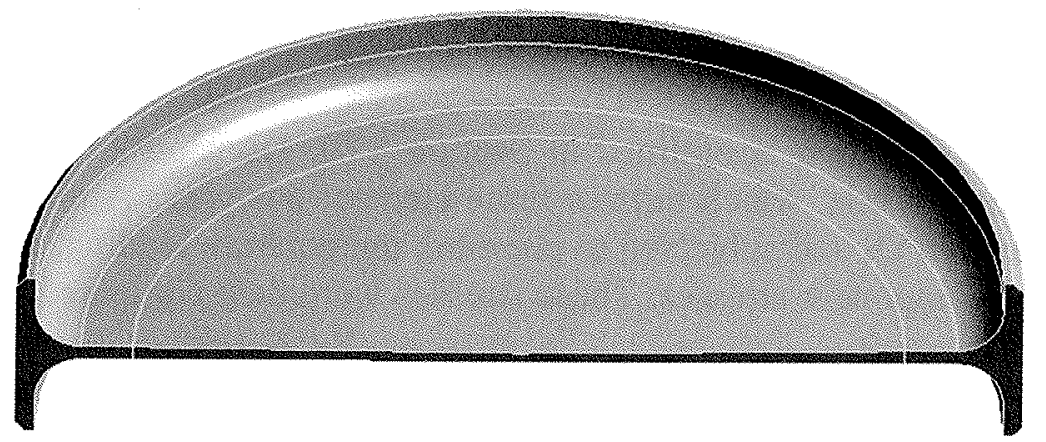

Figure 1

Solid Geometry Model

\section{Material Properties}

The window is fabricated from Inconel 600 with temperature dependent material properties summarized below in

\begin{tabular}{|c|c|c|c|}
\hline \multicolumn{4}{|c|}{ Inconel 600 Material Properties } \\
\hline$\rho\left[1 \mathrm{~b} / \mathrm{in}^{3}\right]$ & & 0.304 & \\
\hline$v[-]$ & & 0.31 & \\
\hline Temperature $\left[{ }^{\circ} \mathbf{F}\right]$ & $70-100$ & 200 & 300 \\
\hline E [psi] & $31.0 \times 10^{6}$ & $30.3 \times 10^{6}$ & $29.9 \times 10^{6}$ \\
\hline Su [psi] & 80,000 & 80,000 & 80,000 \\
\hline Sy [psi] & 35,000 & 32,000 & 31,200 \\
\hline S [psi] & 22,900 & 21,300 & 20,800 \\
\hline $1.5 \mathrm{~S}$ [psi] & 34,350 & 31,950 & 31,200 \\
\hline $4 \mathrm{~S}$ [psi] & 91,600 & 85,200 & 83,200 \\
\hline CTE $\left[\right.$ in $\left./ \mathrm{in} /{ }^{\circ} \mathbf{F}\right]$ & $6.7 \times 10^{-6}$ & $6.9 \times 10^{-6}$ & $7.1 \times 10^{-6}$ \\
\hline
\end{tabular}


Table 2. Values for CTE were obtained from Figure 6.3.2.0 of MIL-HDBK-5J, and all other properties were obtained from the ASME BPVC Electronic Stress Tables.

\begin{tabular}{|c|c|c|c|}
\hline \multicolumn{4}{|c|}{ Inconel 600 Material Properties } \\
\hline$\rho\left[\mathrm{lb}^{\mid} \mathrm{in}^{3}\right]$ & \multicolumn{3}{|c|}{0.304} \\
\hline$v[-]$ & 0.31 \\
\hline Temperature $\left[{ }^{\circ} \mathrm{F}\right]$ & $70-100$ & 200 & 300 \\
\hline $\mathrm{E}[\mathrm{psi}]$ & $31.0 \times 10^{6}$ & $30.3 \times 10^{6}$ & $29.9 \times 10^{6}$ \\
\hline Su [psi] & 80,000 & 80,000 & 80,000 \\
\hline Sy [psi] & 35,000 & 32,000 & 31,200 \\
\hline $\mathrm{S}[\mathrm{psi}]$ & 22,900 & 21,300 & 20,800 \\
\hline $1.5 \mathrm{~S}[\mathrm{psi}]$ & 34,350 & 31,950 & 31,200 \\
\hline $4 \mathrm{~S}[\mathrm{psi}]$ & 91,600 & 85,200 & 83,200 \\
\hline $\mathrm{CTE}\left[\mathrm{in} / \mathrm{in} /{ }^{\circ} \mathrm{F}\right]$ & $6.7 \times 10^{-6}$ & $6.9 \times 10^{-6}$ & $7.1 \times 10^{-6}$ \\
\hline
\end{tabular}

Table 2 Material Properties

\section{Boundary Conditions}

The window is restrained by applying a fixed displacement with respect to the Z-axis applied to the surface that is welded to the target housing and another fixed displacement to the center of the window with respect to the $\mathrm{Y}$-axis, as shown in Figure 2. Including symmetry along the YZ plane provides a kinematic restraint for the window.

Loading comes from multiple sources. The combination of pressurized cooling water on one side of the window and a full vacuum on the other side produces a pressure load. There is also the presence of a small force from a compressed spring within the target assembly. These are applied as shown in Figure 4. The loads described above are applied as two analysis load cases. Analysis load case 1 represents pressurizing the coolant to 80 psi (the maximum absolute pressure of the cooling pump) without thermal loading, load case 2 is the same as load case 1 with the addition of thermal loading, and analysis load case 3 represents normal operating conditions (pressure load of $27 \mathrm{psi}$ ). Thermal loading (Figure 4) is derived from the results of a previous CFD thermal/hydraulic analysis.

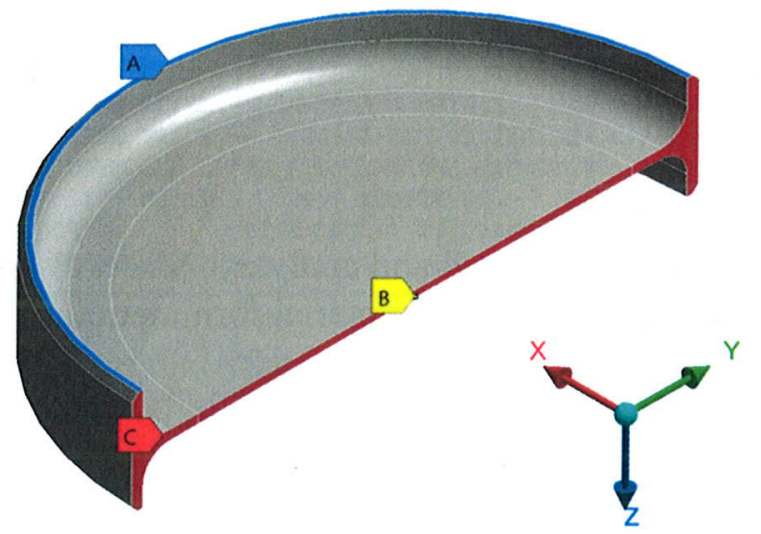

Figure 2 Boundary Conditions 


\begin{tabular}{|llllllll||}
\hline Calculation No.: & NE-EO-doc-88 & Rev. & 0 & Page & 6 & of & 12 \\
\hline
\end{tabular}
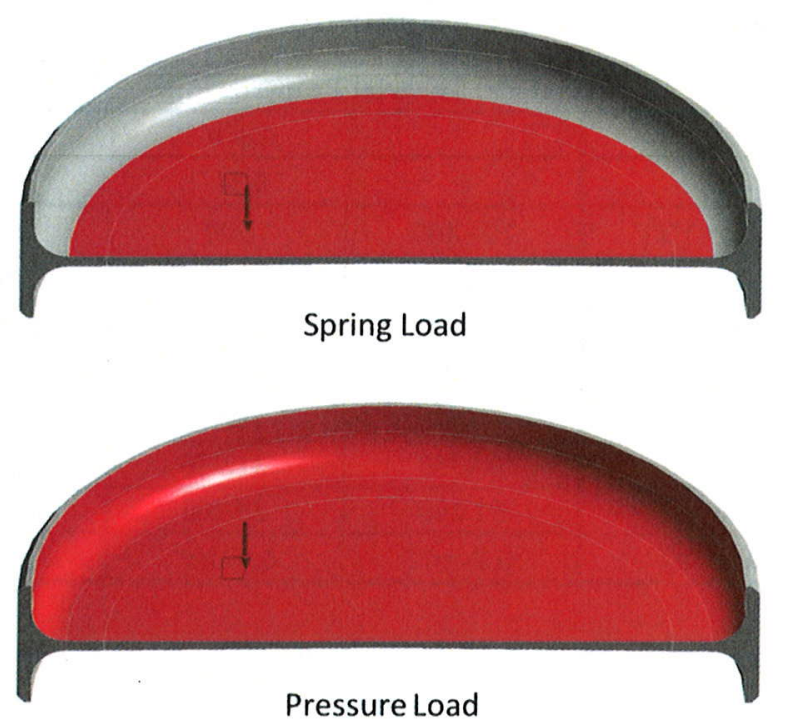

Figure 3 Mechanical Loading

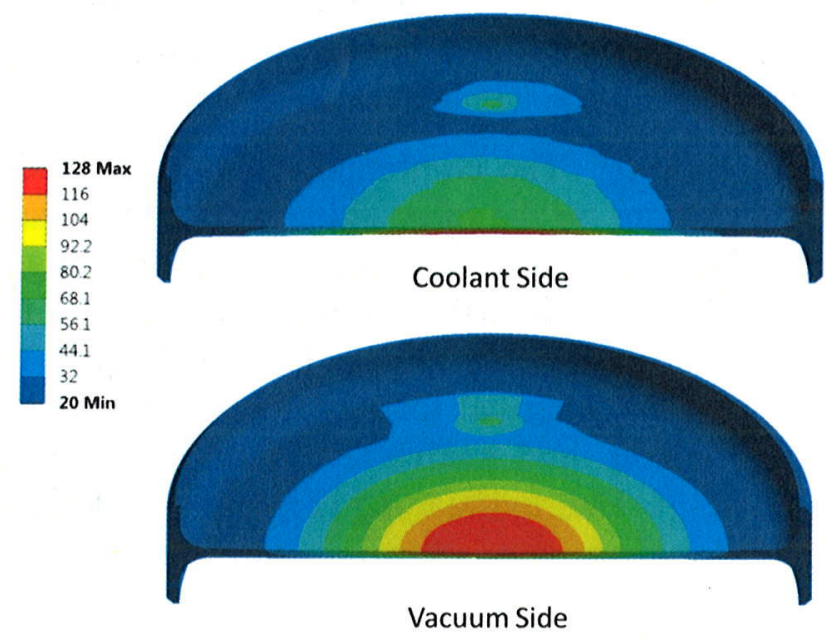

Figure 4 Thermal Loading in degrees Celsius

\section{Solution and Results}

Three finite element models were constructed and analyzed, and are summarized in Table 3. All are based on the same solid model and differ in material formulation, and load factors. 


\begin{tabular}{|llllllll}
\hline Calculation No.: & NE-EO-doc-88 & Rev. & 0 & Page & 7 & of & 12
\end{tabular}

\begin{tabular}{|c|c|c|c|c|c|}
\hline $\begin{array}{c}\text { Finite } \\
\text { Element } \\
\text { Model }\end{array}$ & \multicolumn{2}{|c|}{ Analysis Result } & $\begin{array}{c}\text { Material } \\
\text { Model }\end{array}$ & Load Case & $\begin{array}{c}\text { Load } \\
\text { Factors }\end{array}$ \\
\hline 1 & A & Plastic Collapse & EPP & 1,2 & $1.5,1.3$ \\
\hline 2 & B & Local Failure & LE & 2 & 1 \\
\hline 3 & C & Buckling & LE & 3 & 1 \\
\hline 3 & D & Fatigue & LE & 3 & 1 \\
\hline 2 & E & Ratcheting & LE & 2 & 1 \\
\hline
\end{tabular}

Table 3

Summary of Finite Element Models

\section{A. Protection Against Plastic Collapse}

The limit load method was used to check for plastic collapse. This analysis checks for structural instability due to gross plastic deformation. A load factor (1.5 for load case 1 or 1.3 for load case 2 ) is applied, and structural stability is indicated if the solution converges. This method is outlined at 5.2.3 in Sect VIII, Div 2 of the Code. The load cases, materials and mesh used for this analysis constitute finite element model 1 in Table 3.

The Code specifies that the analysis be run with small displacement theory and an elasticperfectly plastic (EPP) material model. The yield strength defining the plastic limit is specified as $1.5 \mathrm{~S}$. The solid model was meshed with quadratic elements. Ten-node tetrahedral solid elements were used everywhere. Additional refinement was added in areas of the fillets.

Convergence was achieved for Load Case 1, as indicated by the sample from Solution Information shown in Figure 5, indicating compliance with the Code. The requirement for protection against plastic collapse is therefore met.

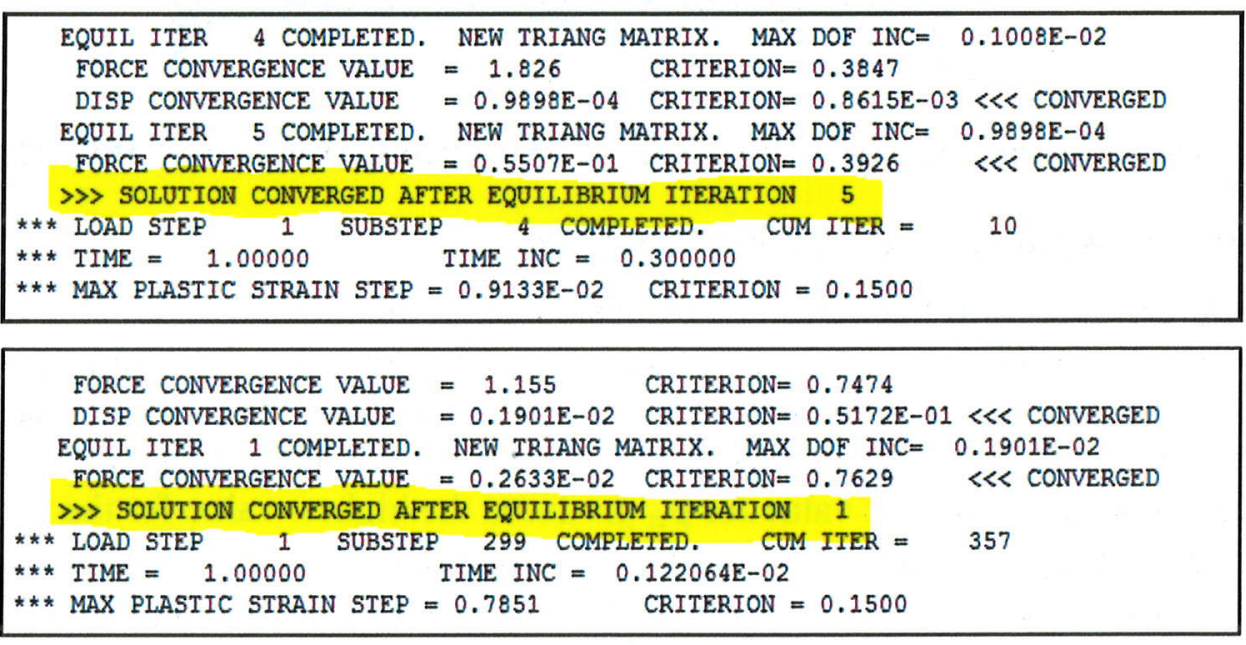

Figure 5

Solution Convergence

\section{B. Protection Against Local Failure}


Protection from local failure was demonstrated with the Elastic Analysis method in 5.3.2 of Sect VIII, Div 2. This method is based on a linear elastic model, and the acceptance criterion is that the sum of the three principal stresses must be less than $4 \mathrm{~S}$.

The finite element model used for the Plastic Collapse analysis was copied and modified to use only a linear elastic material. This is finite element model 2 in Table 3.

Plots of the ratio of the sum of the three principle stresses to $4 \mathrm{~S}$ are shown in Figure 6 . Contour levels have been altered so that any value greater than or equal to 1 , which indicates failure, is shown as red. There are no locations in the model where the sum of the principle stresses exceeds $4 \mathrm{~S}$; therefore, the requirement for protection against local failure is therefore met.

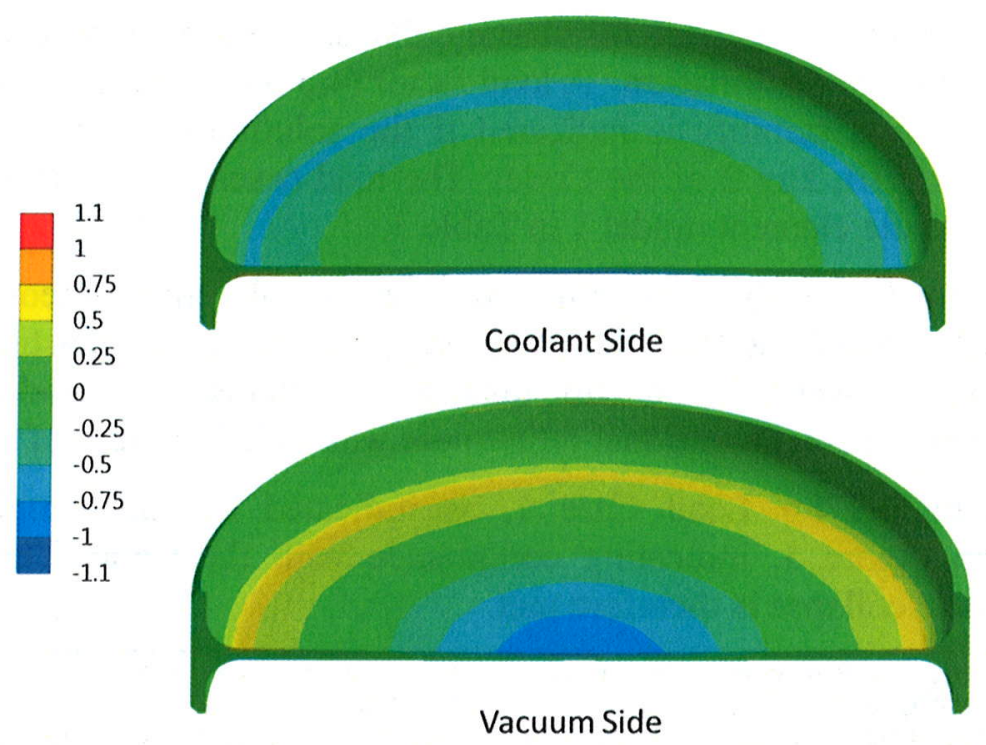

Figure 6 Local Failure, The ratio of the sum of all principal stresses to 4S (83,200 psi).

\section{Protection Against Collapse From Buckling}

Protection from collapse from buckling was evaluated using the method given at 5.4.1.2 in Sect VIII, Div 2, which specifies a linear elastic pre-stressed eigenvalue buckling analysis. The acceptance criterion is that the buckling load factor $\Phi_{b}$ be greater than $2 / \beta_{\mathrm{cr}}$, where $\beta_{\mathrm{cr}}$ is the capacity reduction factor, as the window does not fit within any of the geometry categories listed at 5.4.1.3 in the code. The smallest value for $\beta_{\text {cr }}$ produces the highest value for the buckling load factor, so the smallest appropriate value for $\beta_{\mathrm{cr}}$ must be used to ensure compliance. However, XXX 2.3 states that these capacity reduction factors "account for shape imperfections," and so do not address a shape's resistance to buckling, but rather its sensitivity to manufacturing flaws. Since the flat circular window supported on its edge by a cylinder is less sensitive to geometric flaws than a cylinder under external pressure, the value of $\beta_{\mathrm{cr}}=0.80$ was selected. Thus, $2 / \beta_{\mathrm{cr}}=$ 0.80 for $\mathrm{a} \Phi_{\mathrm{b}}$ of 2.5 . The combination of thermal and mechanical loading results in a value for $\Phi_{\mathrm{b}}$ of 3.5. For thermal loading only, the result is a value for $\Phi_{\mathrm{b}}$ of 3.6. For mechanical loading only, the result is a value for $\Phi_{\mathrm{b}}$ of 66 . Based on these results, it is determined that the requirement of protection against collapse from buckling is met. 


\begin{tabular}{|llllllll}
\hline Calculation No.: & NE-EO-doc-88 & Rev. & 0 & Page & 9 & of & 12
\end{tabular}

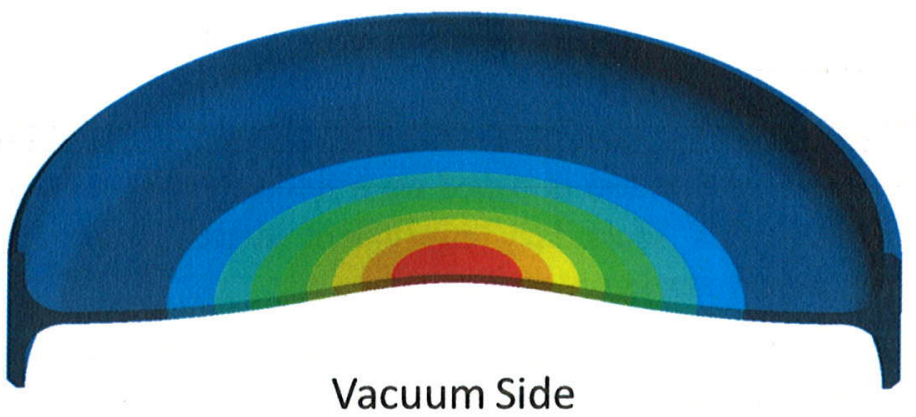

Figure 7

First Mode, $\Phi_{\mathrm{b}}=3.5$, Combined thermal and mechanical loading

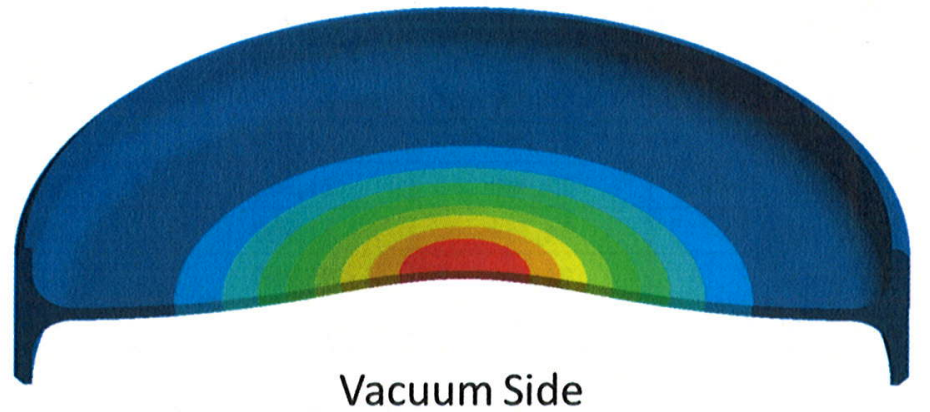

Figure 8 First Mode, $\Phi_{b}=3.6$, Thermal loading only

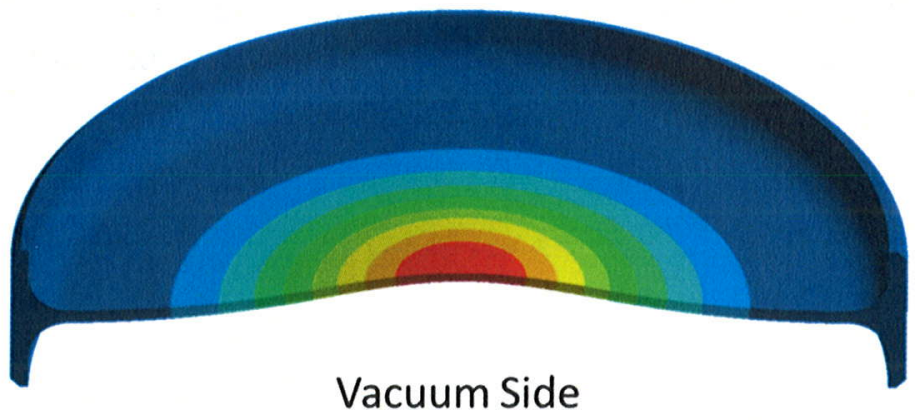

Figure 9 First Mode, $\Phi_{b}=66$, Mechanical loading only

\section{Protection Against Failure from Cyclic Loading}

Protection against failure from cyclic loading (fatigue) was evaluated by calculating the number of allowable cycles in accordance with Sect VIII, Div 2, Part 3, Annex 3-F. The value for stress amplitude was the maximum stress intensity found under normal operating conditions (Load Case 2), which was 28.4 ksi. Using equations 3-F.1, 3-F.2, and 3-F.3 with coefficients for 3-F.2 obtained from Table 3-F.3 resulted in a value of $\sim 22,000$ allowable cycles. 


\begin{tabular}{|llllllll|}
\hline Calculation No.: & NE-EO-doc-88 & Rev. & 0 & Page & 10 & of & 12 \\
\hline
\end{tabular}

\section{E. Ratcheting Assessment}

Protection from Ratcheting was demonstrated with the Elastic Ratcheting Elastic Method in 5.5.6.1 of Sect VIII, Div 2. This method is based on a linear elastic model, and the acceptance criterion is that the primary plus secondary equivalent stress range $\Delta S_{n, k}$ is less than the allowable primary plus secondary stress range $\mathrm{S}_{\mathrm{ps}}$.

Finite element model 2 in Table 3 was used for this assessment. The maximum equivalent stress was taken as $\Delta \mathrm{S}_{\mathrm{n}, \mathrm{k}}$. The value for $\mathrm{S}_{\mathrm{PS}}$ in

\begin{tabular}{|c|c|c|c|}
\hline \multicolumn{4}{|c|}{ Inconel 600 Material Properties } \\
\hline$\rho\left[\mathrm{Ib} / \mathrm{in}^{3}\right]$ & & 0.304 & \\
\hline v I-] & & 0.31 & \\
\hline Temperature $\left[{ }^{\circ} \mathrm{F}\right]$ & $70-100$ & 200 & 300 \\
\hline E [psi] & $31.0 \times 10^{6}$ & $30.3 \times 10^{6}$ & $29.9 \times 10^{6}$ \\
\hline Su [psi] & 80,000 & 80,000 & 80,000 \\
\hline Sy [psi] & 35,000 & 32,000 & 31,200 \\
\hline S [psi] & 22,900 & 21,300 & 20,800 \\
\hline $1.5 \mathrm{~S}$ [psi] & 34,350 & 31,950 & 31,200 \\
\hline $4 \mathrm{~S}$ [psi] & 91,600 & 85,200 & 83,200 \\
\hline CTE $\left[\right.$ in $/$ in $\left./{ }^{\circ} \mathbf{F}\right]$ & $6.7 \times 10^{-6}$ & $6.9 \times 10^{-6}$ & $7.1 \times 10^{-6}$ \\
\hline
\end{tabular}

Table 2 was found using the method given at 5.5.6.1.d in Sect VIII, Div 2, and is essentially the highest of three times the average of S or two times the average of Sy at the highest and lowest temperatures during the operational cycle. Plots of equivalent stress for the Inconel window are shown in Figure 10Figure 10. Contour levels for these plots have been altered so that all values are divided by $S_{P S}$. Failure would be indicated by any contour result greater than 1 and appear as red in the plots. Results show that the requirement for protection from ratcheting is met. 
Rev.

Page

11 of

12

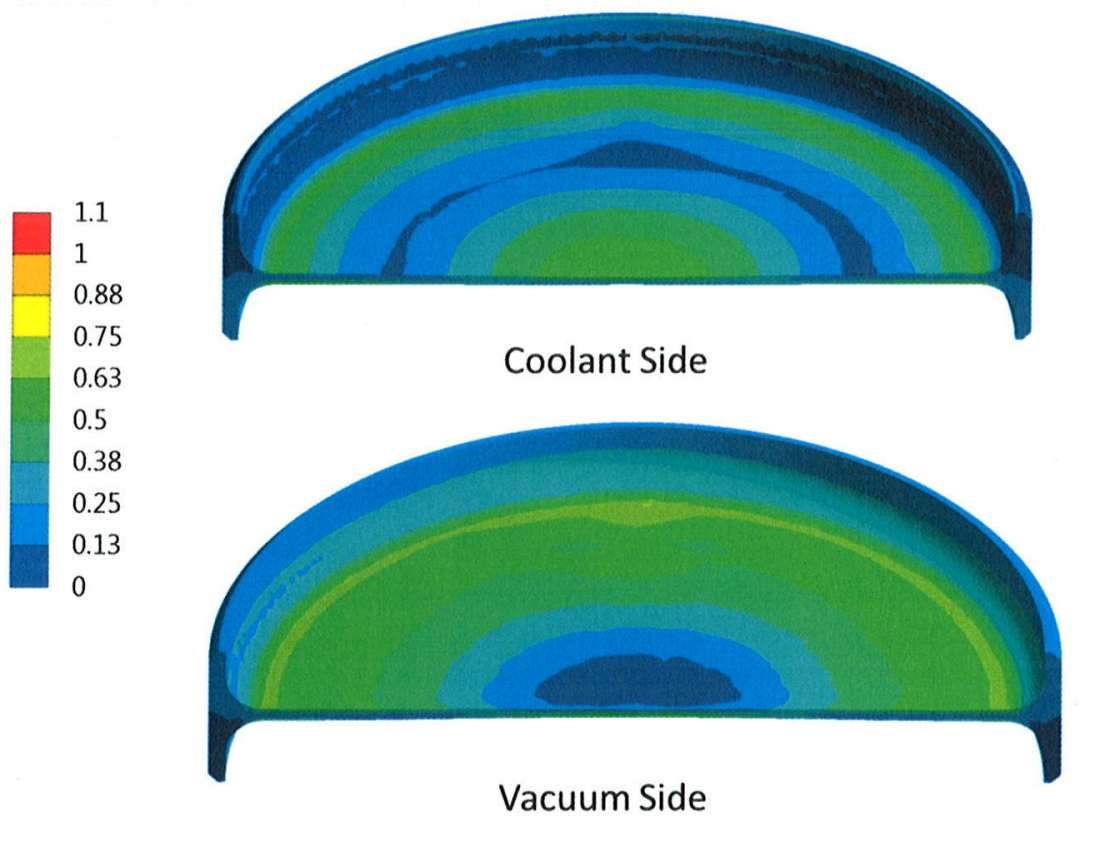

Figure 10 Ratcheting Assessment

\section{Conclusions}

The results of this analysis presented above show that the requirements for Protection Against Plastic Collapse, Protection Against Local Failure, Protection Against Collapse From Buckling, Protection From Ratcheting, Protection Against Failure from Cyclic Loading, per the ASME BPVC, have been met. Based on this, the following conclusions are drawn:

1. The Inconel 600 window for the DU target assembly is in compliance with Sect VIII, Div 2 when subjected to the loads described in this analysis.

2. From the fatigue analysis, the recommended maximum number of cycles for the assembly is 2,000

\section{References}

1. ASME Boiler and Pressure Vessel Code, American Society of Mechanical Engineers, New York, NY 2015.

2. Metallic Materials and Elements for Aerospace Vehicle Structures MIL-HDBK-5J, Department of Defense, 2003

\section{Software}

- ANSYS Mechanical, Version 17.2, Build date 07/27/2016, Ansys Inc, Pittsburg, PA.

- Microsoft Windows 7 Enterprise, Service Pack 1, 2009, Microsoft Corporation, Redmond WA. 


\begin{tabular}{|llllllll|}
\hline Calculation No.: & NE-EO-doc-88 & Rev. & 0 & Page & 12 & of & 12 \\
\hline
\end{tabular}

- Microsoft Office Excel 2010, (14.0.7177.5000), 2010, Microsoft Corporation, Redmond WA. 


\section{APPENDIX 7}

Memo: "Radionuclide inventories and HazCat-3 sum-of-fraction for $35 \mathrm{MeV}$ mini-SHINE irradiations" 


\section{Argonne}

Intra-Laboratory Memo

\section{August 2014}

TO: $\quad$ Sergey Chemerisov

FROM: $\quad$ Brad Micklich

SUBJECT: $\quad$ Radionuclide inventories and HazCat-3 sum-of-fractions for $35 \mathrm{MeV}$ mini-SHINE irradiations

As part of Argonne's support for the mini-SHINE experiments, 20 liters of a uranyl sulfate solution (145 g/liter of uranium enriched to just under $20 \%{ }^{235} \mathrm{U}$ ) will be irradiated in a stainless steel container using neutrons generated by $20 \mathrm{~kW}$ of $35 \mathrm{MeV}$ electrons incident on a depleted uranium (DU) target. The solution tank will be surrounded by a water reflector. A lead shielding box will be used to contain the irradiation experiments. The box has sides of 4 inches of lead on four sides, four inches of lead with a 4-in thick access door on a fifth side, and eight inches of lead with a 14-inch thick viewing window made of leaded glass on the sixth side. The DU target assembly will be capable of being extracted out of the downstream side of the box into lead shielding. This memo describes the calculation of radionuclide inventories inside the box, the solution, and the target assembly.

Radionuclides in the system are produced by both neutron- and photon-induced reactions. The transport calculations were performed using MCNPX, and the transmutation (buildup and decay) calculations were performed with CINDER08. A special version of MCNPX version 2.6.0 was used that not only calculates the energy-dependent neutron flux for the regions of interest but also calculates radionuclide production for neutrons above the maximum energy in the CINDER data libraries, and due to all other particles (e.g., photons), and prints these rates directly in the MCNPX output file.

Calculations were performed for the electron linac running at $35 \mathrm{MeV}, 20 \mathrm{~kW}$ for 19.3 hours (the length of time needed to produce $20 \mathrm{Ci}$ of ${ }^{99} \mathrm{Mo}$ in the 20-liter solution) in each irradiation cycle. The complete irradiation history included four runs of 19.3 hours at 4-week periods, followed by a 5th irradiation. Radionuclide inventories were calculated at shutdown and for decay times out to one year following the final irradiation (as well as at intermediate times during the irradiation history). Figure 1 shows the 20-liter solution tank, and Figure 2 shows the MCNPX model for the shielded box and irradiation experiment. The tanks contain much internal structure which is not included in the MCNPX model. The design of these vessels is not yet complete, and the radionuclide inventory of the system is dominated by that in the uranyl nitrate solution, with a much smaller but still significant contribution from the depleted uranium target assembly. 


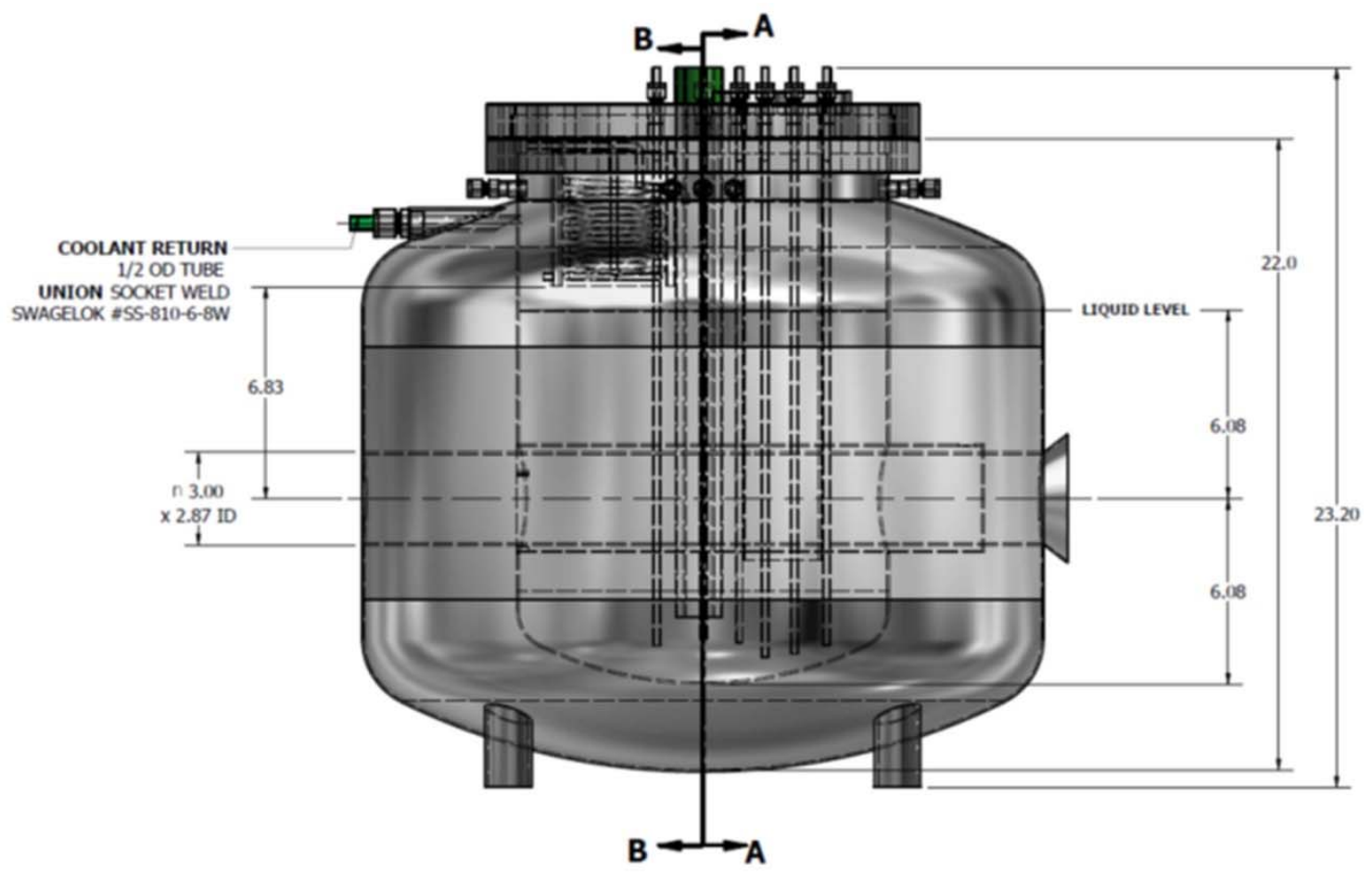

Figure 1. Drawing of the 20-liter uranyl nitrate solution tank surrounded by the reflector tank.
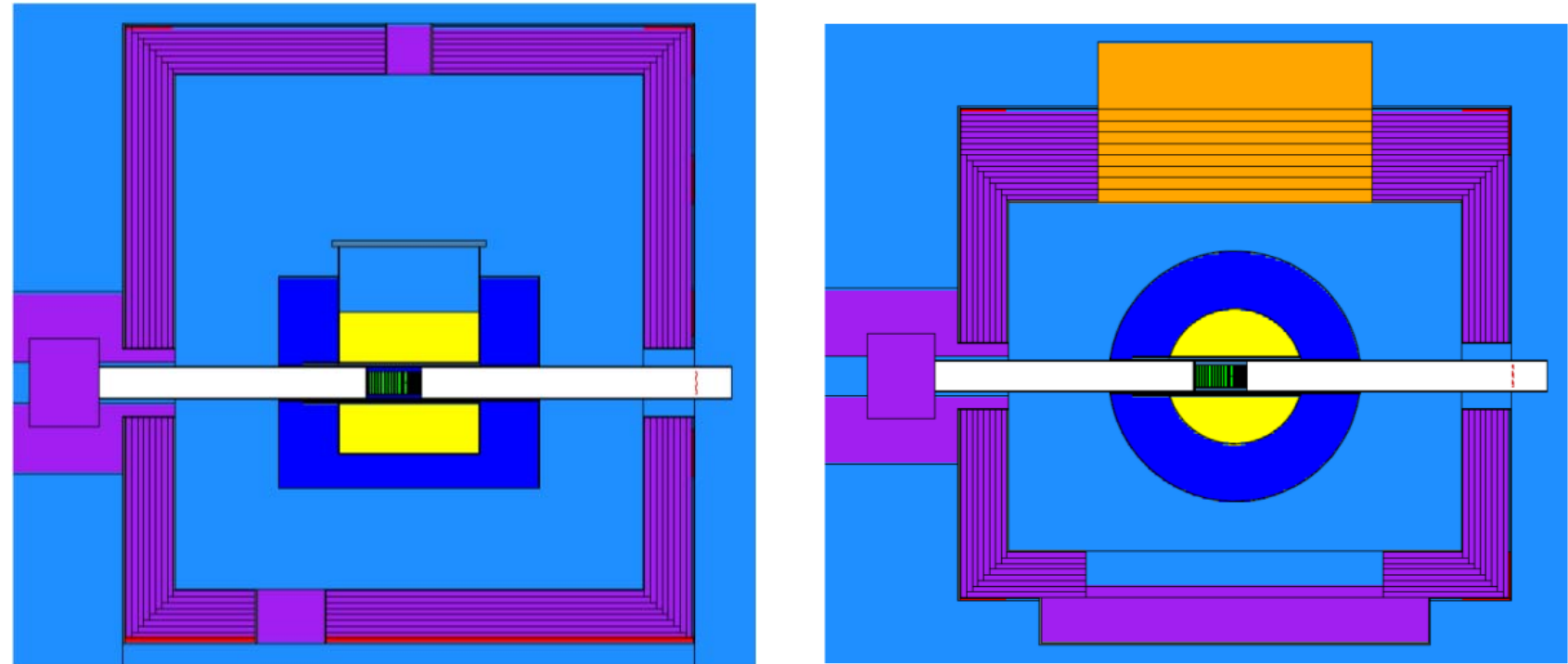

Figure 2. (left) Side view of MCNPX model used for activation calculations. The beam is incident from the right. (right) Top view of the MCNPX model through the beamline. The beam is incident from the right. 


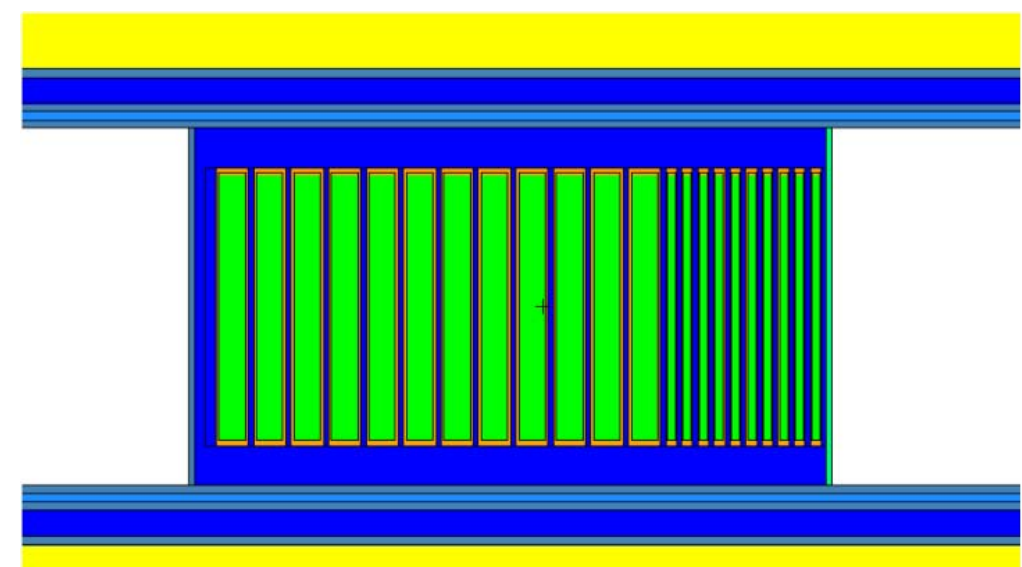

Figure 3. Detailed view of the depleted uranium target assembly from the 20-liter tank model.

First, an optimization study was conducted to find the target position that would maximize the fission rate inside the uranyl nitrate solution. The model for and results of those calculations are shown in Figure 4. The maximum fission rate in the solution occurs when the peak of the neutron source is approximately in the center of the solution tank. This position was used in subsequent calculations for inventories.
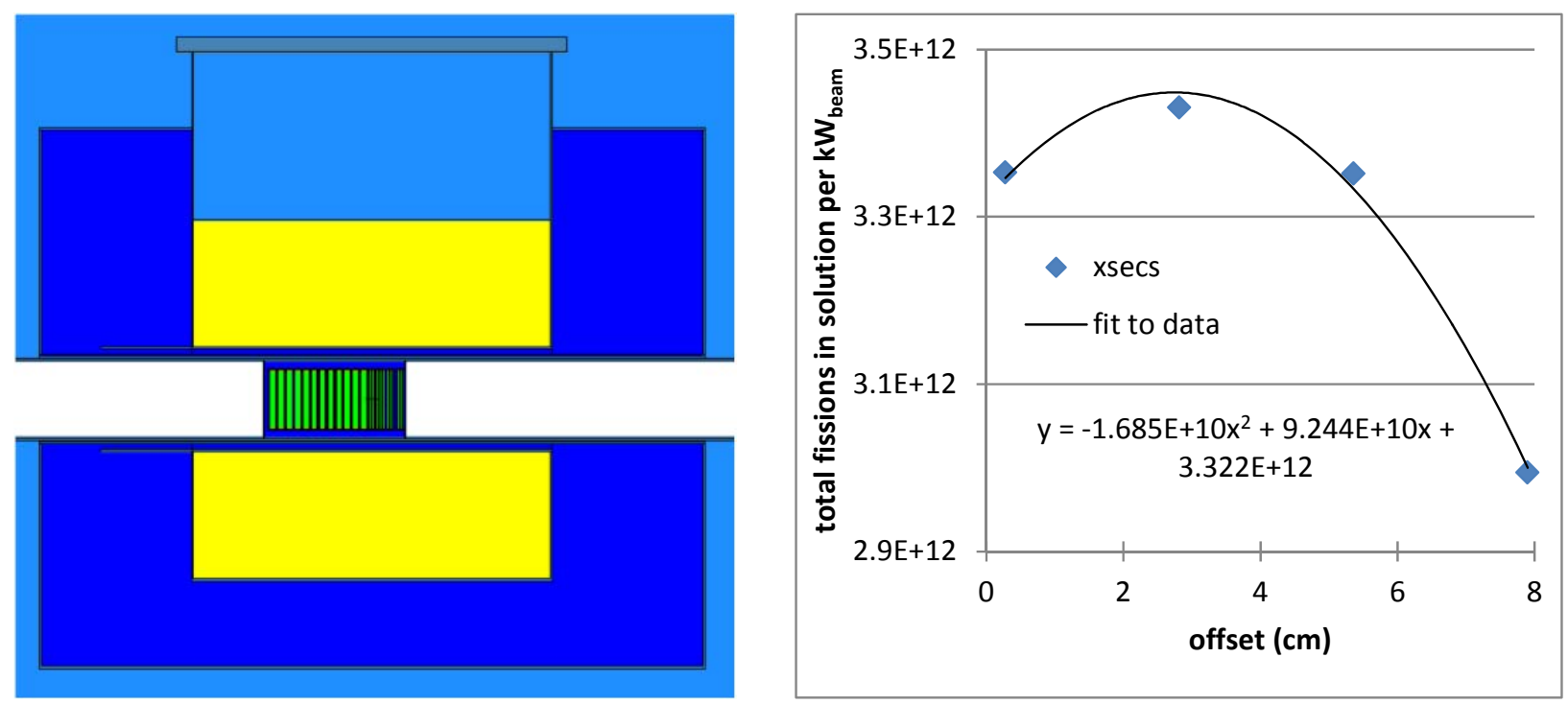

Figure 4. (left) Side view of MCNPX model used for optimizing the source position. The beam is incident from the right. (right) Fission rate in the uranyl sulfate solution as a function of target position. The offset parameter is the difference between the center of the solution container and the front face of the target.

The next step was to determine the running time needed to produce the target quantity (20 Ci) of ${ }^{99}$ Mo. The buildup curve for ${ }^{99}$ Mo under the irradiation conditions described above is shown in Figure 5. These data show that the target quantity is reached after 19.3 hours of irradiation. This irradiation length was used for all irradiations in the campaign. Using CINDER90, the time 
required to reach the desired activity $(20 \mathrm{Ci}$ ) is estimated at $17.3 \mathrm{hrs}$. The difference is due to differences in the ${ }^{235} \mathrm{U}$ thermal fission cross section between the CINDER90 library and the CINDER08 fission-weighted library (see Figure 6). The fission rate calculated using CINDER90 is about $10 \%$ higher than that calculated using CINDER08 for a well-thermalized spectrum, due to an apparently high value for the fission cross section in the $58-67 \mathrm{meV}$ group.

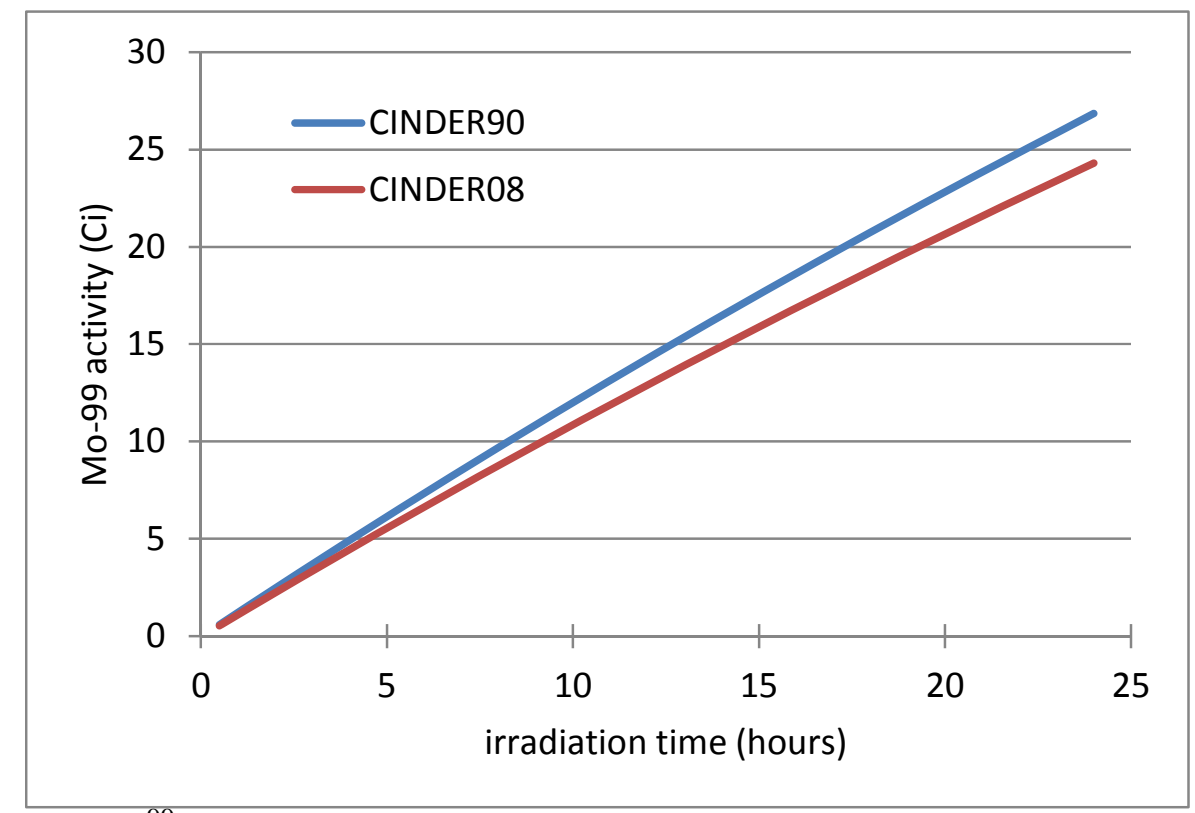

Figure 5. Buildup of ${ }^{99}$ Mo in the 20-liter uranyl nitrate solution.

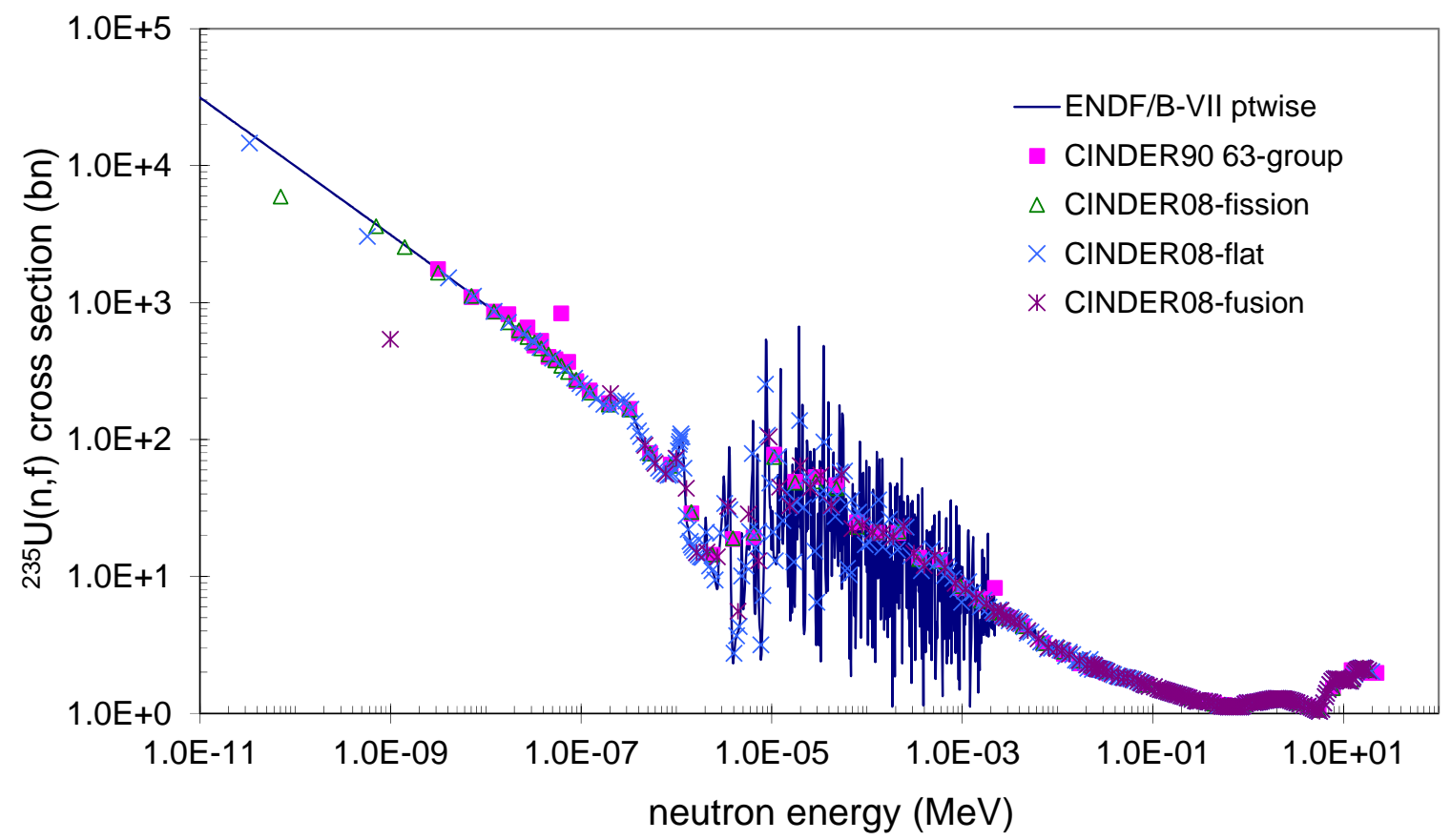

Figure 6. Comparison of the ${ }^{235} \mathrm{U}$ fission cross section in ENDF/B-VII, CINDER90, and CINDER08. 
Figure 7 shows the hazard category 3 (HC-3) sum-of-fractions (SOF) in the entire irradiated volume, as well as in selected subsets, for the complete campaign of five irradiations. The percentage of the SOF for the solution is about $91-92 \%$ of the total. Table 1 lists the top 50 contributors to the sum-of-fractions at shutdown following the fifth irradiation. The contribution of the target varies between 8-9\%, and only a small contribution to SOF comes from the box and vessels. The SOF is also dominated by the fission products ${ }^{131}$ I and ${ }^{133}$ I out to several months following the last irradiation, by which time the entire SOF is only about 0.01 . Figure 8 shows the activity present in the system (in $\mathrm{Ci}$ ) as a function of time. The solution contains about $85 \%$ of the total activity of the system immediately after irradiation stops, declining to around $60 \%$ of the total after about a week of decay, after which the percentage rises again. One year after the last irradiation, the solution contains about $90 \%$ of the activity, with the target assembly accounting for about $8 \%$ and the balance in the shielding box and stainless steel vessels.

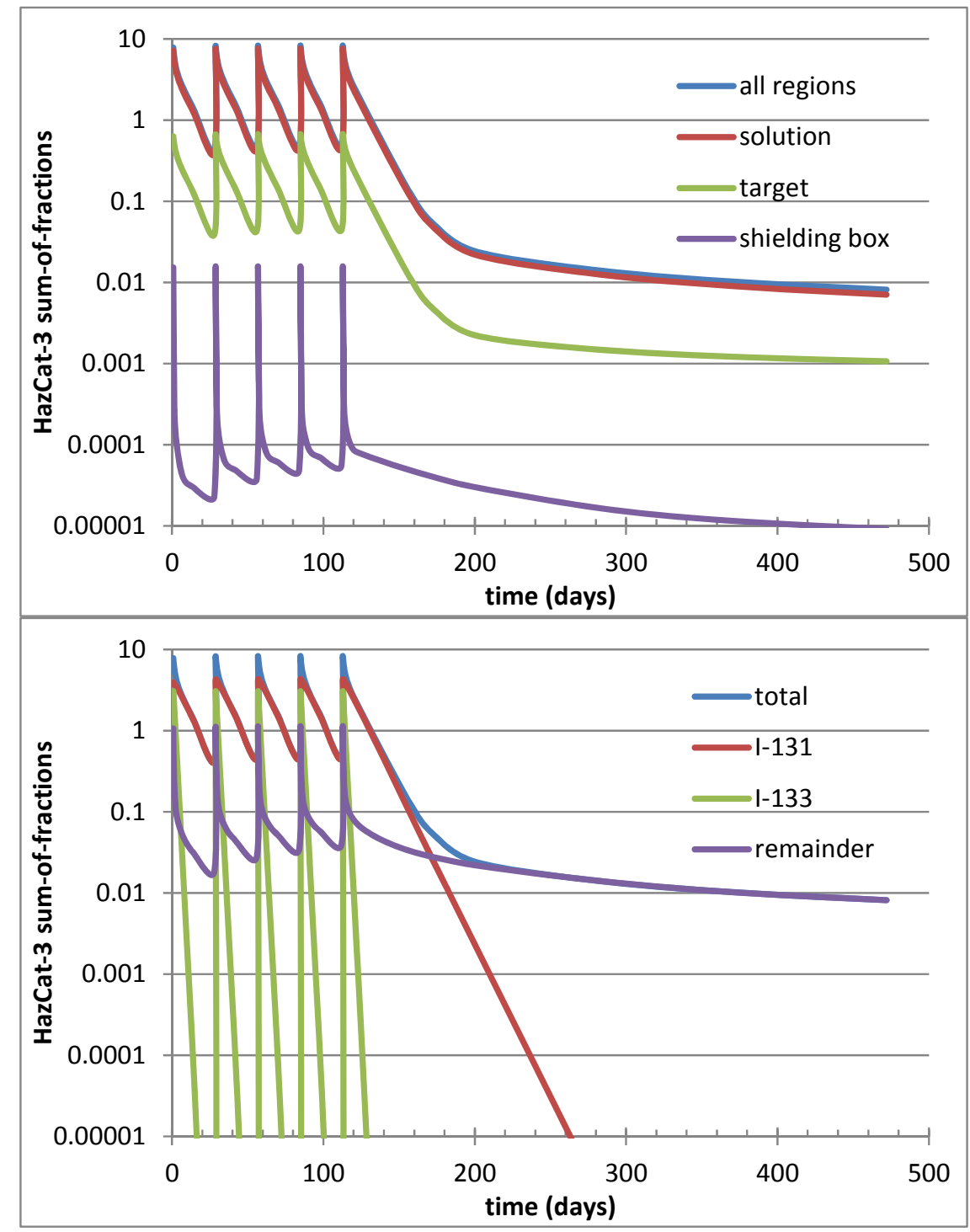

Figure 7. (top) HazCat-3 sum-of-fractions for the entire assembly, the uranyl sulfate solution, the target assembly, and the balance of the system. (bottom) HazCat-3 sum-of-fractions for the entire assembly, the nuclides I-131 and I-133, and the remainder of the nuclides. 
Table 1. Top 50 contributors to the HazCat-3 sum-of-fractions in the uranyl sulfate solution at shutdown following the fifth irradiation.

\begin{tabular}{|c|c|c|c|}
\hline Nuclide & half-life (s) & SOF contribution & Activity (Ci) \\
\hline I 131 & $6.93 \mathrm{E}+05$ & $3.73 \mathrm{E}+00$ & $3.43 E+00$ \\
\hline I 133 & $7.49 \mathrm{E}+04$ & $2.86 \mathrm{E}+00$ & $5.55 \mathrm{E}+01$ \\
\hline I 135 & $2.37 \mathrm{E}+04$ & $2.34 \mathrm{E}-01$ & $9.84 \mathrm{E}+01$ \\
\hline Kr 88 & $1.02 \mathrm{E}+04$ & $1.57 \mathrm{E}-01$ & $6.29 E+01$ \\
\hline Xe138 & $8.45 \mathrm{E}+02$ & $1.42 \mathrm{E}-01$ & $1.13 \mathrm{E}+02$ \\
\hline Sr 91 & $3.47 \mathrm{E}+04$ & 4.97E-02 & $7.86 \mathrm{E}+01$ \\
\hline Kr 87 & $4.58 \mathrm{E}+03$ & 4.65E-02 & $4.65 \mathrm{E}+01$ \\
\hline Zr 97 & $6.03 \mathrm{E}+04$ & 4.33E-02 & $5.97 \mathrm{E}+01$ \\
\hline Sr 92 & $9.58 \mathrm{E}+03$ & 3.13E-02 & $1.06 \mathrm{E}+02$ \\
\hline Xe135 & $3.29 E+04$ & 3.05E-02 & $6.09 \mathrm{E}+01$ \\
\hline I 134 & $3.15 \mathrm{E}+03$ & $2.43 \mathrm{E}-02$ & $1.41 \mathrm{E}+02$ \\
\hline La142 & $5.47 \mathrm{E}+03$ & $2.29 \mathrm{E}-02$ & $1.05 \mathrm{E}+02$ \\
\hline Te132 & $2.77 \mathrm{E}+05$ & $2.06 \mathrm{E}-02$ & $1.24 \mathrm{E}+01$ \\
\hline Cs138 & $2.00 \mathrm{E}+03$ & $1.21 \mathrm{E}-02$ & $1.21 \mathrm{E}+02$ \\
\hline Ba140 & $1.10 \mathrm{E}+06$ & $1.02 \mathrm{E}-02$ & $6.12 \mathrm{E}+00$ \\
\hline Ce143 & $1.19 \mathrm{E}+05$ & $9.26 \mathrm{E}-03$ & $3.52 E+01$ \\
\hline Ce144 & $2.46 \mathrm{E}+07$ & 8.48E-03 & $8.48 \mathrm{E}-01$ \\
\hline Sb128 & $3.24 \mathrm{E}+04$ & 8.24E-03 & $4.61 \mathrm{E}+00$ \\
\hline Sr 89 & $4.37 \mathrm{E}+06$ & 7.30E-03 & $2.48 \mathrm{E}+00$ \\
\hline Y 92 & $1.27 \mathrm{E}+04$ & 7.18E-03 & $1.01 \mathrm{E}+02$ \\
\hline $\mathrm{Xe135m1}$ & $9.17 \mathrm{E}+02$ & 7.14E-03 & $1.29 \mathrm{E}+01$ \\
\hline I 132 & $8.26 \mathrm{E}+03$ & $6.43 \mathrm{E}-03$ & $1.07 \mathrm{E}+01$ \\
\hline Y 91 & $5.06 \mathrm{E}+06$ & 6.39E-03 & $2.30 \mathrm{E}+00$ \\
\hline Np239 & $2.04 \mathrm{E}+05$ & 6.29E-03 & $4.91 E+01$ \\
\hline Te134 & $2.51 \mathrm{E}+03$ & $6.28 \mathrm{E}-03$ & $1.26 \mathrm{E}+02$ \\
\hline Mo 99 & $2.37 \mathrm{E}+05$ & 5.93E-03 & $2.02 \mathrm{E}+01$ \\
\hline La140 & $1.45 \mathrm{E}+05$ & 5.70E-03 & $2.28 \mathrm{E}+00$ \\
\hline $\mathrm{Kr} 85 \mathrm{~m} 1$ & $1.61 \mathrm{E}+04$ & $5.08 \mathrm{E}-03$ & $2.03 \mathrm{E}+01$ \\
\hline Y 93 & $3.66 \mathrm{E}+04$ & 4.73E-03 & $8.32 E+01$ \\
\hline Zr 95 & $5.53 \mathrm{E}+06$ & 4.31E-03 & $3.02 \mathrm{E}+00$ \\
\hline Y 94 & $1.12 \mathrm{E}+03$ & $4.15 \mathrm{E}-03$ & $1.16 \mathrm{E}+02$ \\
\hline Ru105 & $1.60 \mathrm{E}+04$ & 4.15E-03 & $1.66 \mathrm{E}+01$ \\
\hline Sb129 & $1.57 \mathrm{E}+04$ & 3.84E-03 & $8.45 E+00$ \\
\hline Nb 97 & $4.33 \mathrm{E}+03$ & 3.79E-03 & $5.61 \mathrm{E}+01$ \\
\hline P 32 & $1.23 \mathrm{E}+06$ & 3.34E-03 & $4.01 \mathrm{E}-02$ \\
\hline Rb 89 & $9.09 \mathrm{E}+02$ & 3.27E-03 & $8.50 \mathrm{E}+01$ \\
\hline Ce141 & $2.81 \mathrm{E}+06$ & $3.24 \mathrm{E}-03$ & $3.24 \mathrm{E}+00$ \\
\hline Pr143 & $1.17 \mathrm{E}+06$ & 2.17E-03 & $2.25 \mathrm{E}+00$ \\
\hline Sb130 & $2.37 \mathrm{E}+03$ & $1.96 \mathrm{E}-03$ & $1.33 \mathrm{E}+01$ \\
\hline Sb131 & $1.38 \mathrm{E}+03$ & $1.92 \mathrm{E}-03$ & $4.61 \mathrm{E}+01$ \\
\hline Te131m1 & $1.08 \mathrm{E}+05$ & 1.89E-03 & $1.51 \mathrm{E}+00$ \\
\hline Nd147 & $9.49 \mathrm{E}+05$ & $1.86 \mathrm{E}-03$ & $2.38 \mathrm{E}+00$ \\
\hline
\end{tabular}


Table 1. (continued)

\begin{tabular}{|l|c|c|c|}
\hline Nuclide & half-life (s) & SOF contribution & Activity (Ci) \\
\hline Y 95 & $6.18 \mathrm{E}+02$ & $1.85 \mathrm{E}-03$ & $1.15 \mathrm{E}+02$ \\
\hline Nb 95 & $3.02 \mathrm{E}+06$ & $1.84 \mathrm{E}-03$ & $1.77 \mathrm{E}+00$ \\
\hline Sr 90 & $9.09 \mathrm{E}+08$ & $1.72 \mathrm{E}-03$ & $2.75 \mathrm{E}-02$ \\
\hline Y 91m1 & $2.98 \mathrm{E}+03$ & $1.56 \mathrm{E}-03$ & $4.36 \mathrm{E}+01$ \\
\hline Ba142 & $6.36 \mathrm{E}+02$ & $1.52 \mathrm{E}-03$ & $1.04 \mathrm{E}+02$ \\
\hline Ba141 & $1.10 \mathrm{E}+03$ & $1.46 \mathrm{E}-03$ & $1.05 \mathrm{E}+02$ \\
\hline Tc104 & $1.10 \mathrm{E}+03$ & $1.31 \mathrm{E}-03$ & $3.39 \mathrm{E}+01$ \\
\hline Nd149 & $6.22 \mathrm{E}+03$ & $1.25 \mathrm{E}-03$ & $1.95 \mathrm{E}+01$ \\
\hline
\end{tabular}

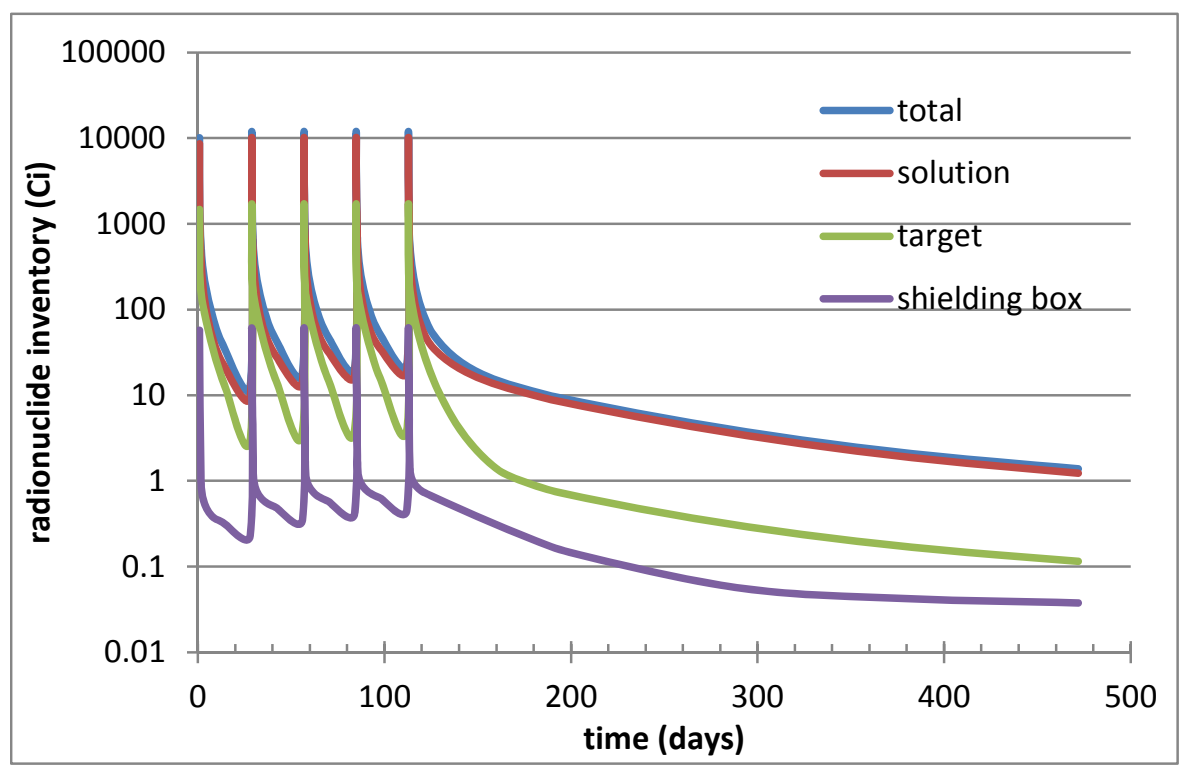

Figure 8. Radionuclide inventory for the entire assembly, the uranyl sulfate solution, the target assembly, and the balance of the system.

The attachments to this report contain printouts of the CINDER08 results. Attachment 1 contains listings by nuclide of the following quantities: total mass ( $\mathrm{kg}$ ), total activity (Ci), total decay power (W), air dilution factor, water dilution factor, total fission power, and fraction of the HazCat-3 threshold. Attachments 2 and 3 contain the same information ranked according to contribution (greatest to least) at the end of the 5th irradiation and 24 hours after the last irradiation ends, respectively. 


\section{APPENDIX 8}

LEAF-PROC-016, Rev. 3: AMORE Gas Handling Alarm and Interlock Checklist 


\section{AMORE Gas Handling Alarm and Interlock Checklist}

Low Energy Accelerator Facility, LEAF-PROC-016, Rev. 3

Approved: SChn Date: $\underline{03.23 .2021}$

Sergey Chemerisov, Manager, IVEM/LEAF

Effective Date: $\underline{03.24 .2021}$ 


\section{Experiment}

\section{Purpose}

Establish a checklist for the gas handling alarms and interlocks in the AMORE experiment.

\section{Scope}

This procedure applies to the following Argonne activities and entities.

\begin{tabular}{ll}
\hline LMS core processes: & Asset Management \\
\hline Organizations: & Experimental Operations and Facilities (EOF) Division \\
\hline Buildings: & 211 \\
\hline Specific locations: & LINAC \\
\hline $\begin{array}{l}\text { Other applicability } \\
\text { factors: }\end{array}$ & None \\
\hline Exclusions: & None \\
\hline USI applicability: & Yes \\
\hline
\end{tabular}

\section{Work Process}

\subsection{Introduction}

The steps in this procedure are used to ensure the safe operation of the AMORE and Mini-AMORE experiments. These steps must be performed before the experiment.

\subsection{Step-by-Step Procedure}

The steps below are mandatory unless noted otherwise. This procedure is to be performed by trained personnel.

\subsubsection{Test the function of the Gas Collection System [ASE 2.5.2.2]}

\begin{tabular}{c|l}
\hline Step & \multicolumn{1}{c}{ Action } \\
\hline 1 & In the LINAC Control Room Chassis \#1, set valve control for "D035” \\
\hline 2 & Open the cylinder and isolation valves on the helium/xenon tank in D032 \\
\hline 3 & Go to the LINAC Cell D035 Gas Distribution Hub Manifold \\
\hline 4 & Close valves V-1 $\square \quad$ V-6 $\square \quad$ V-8 $\square \quad$ V-10 $\square \quad$ V-15 $\square \quad$ V-17 $\square$ \\
\hline 5 & Open Valves V-2 $\square \quad$ V-3 $\square \quad$ V-4 $\square \quad$ V-7 $\square \quad$ V-9 $\square \quad$ V-12 $\square$ \\
\hline 6 & Open the cylinder and isolation valves on the helium/xenon tank in D032 \\
\hline
\end{tabular}




\begin{tabular}{c|l}
\hline Step & \multicolumn{1}{c}{ Action } \\
\hline 7 & $\begin{array}{l}\text { At the Gas Collection System Control Chassis, ensure that the Gas Collection Systems } \\
\text { Chamber \#1 pump and Chamber \#2 compressor function at the designed pressure differential. } \\
\text { [ASE 2.5.2.2] }\end{array}$ \\
\hline
\end{tabular}

\subsubsection{Flush the Analytical Manifold in D024 with Helium}

\begin{tabular}{|c|c|c|}
\hline Step & Action & \\
\hline 1 & Go to the LINAC Cell D035 Gas Distribution Hub Manifold & \\
\hline 2 & Close valves V-4 $\square \quad$ V-7 $\square$ V-9 $\square$ & \\
\hline 3 & SV-3 “ON” & \\
\hline 4 & Go to the D024 Analytical Enclosure & \\
\hline 5 & Attach a $1 \%$ hydrogen standard to the outside calibration port & 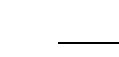 \\
\hline 6 & Close Valves A-1 $\square \quad$ A-2 $\square \quad$ A-10 $\square$ & \\
\hline 7 & $\begin{array}{l}\text { Open valves A-3 } \square \quad \text { A-4 } \square \quad \text { A-5 } \square \quad \text { A-6 } \square \quad \text { A-7 } \square \quad \text { A-8 } \square \quad \text { A-9 } \square \\
\text { A-11 } \square \quad \text { A-12 } \square\end{array}$ & \\
\hline 8 & Turn on the Vacuum Pump & - \\
\hline 9 & Flush the lines for about 1 or 2 minutes & 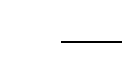 \\
\hline 10 & Close Valve A-7 $\square$ & \\
\hline 11 & When the pressure is $<0.1 \mathrm{mbar}$ approximately, Close valves A-3 $\square$ & A-5 $\square$ \\
\hline
\end{tabular}

\subsubsection{Hydrogen Sensor 1\% Alarm [ASE 3.2.1]}

\begin{tabular}{c|l}
\hline Step & \multicolumn{1}{c}{ Action } \\
\hline 1 & $\begin{array}{l}\text { Open the canister valve to allow standard up to valve A-5. } \\
\text { Then close the canister valve. }\end{array}$ \\
\hline 2 & $\begin{array}{l}\text { Open valve A-5 to introduce the standard into the manifold. Add standard until you reach the } \\
\text { calibration pressure for the instrument. Then close valve A-5. Confirm that the Hydrogen } \\
\text { Alarm is active. The alarm is on Gas Analysis Chassis \#3 (D101 LINAC Control Room). }\end{array}$ \\
\hline
\end{tabular}




\begin{tabular}{c|l}
\hline Step & \multicolumn{1}{|c}{ Action } \\
\hline 3 & $\begin{array}{l}\text { Evacuate the manifold by opening valve A-4. When the pressure } \\
\text { is <0.1mbar approximately, close valve A-4. }\end{array}$ \\
\hline 4 & $\begin{array}{l}\text { Introduce helium to calibration pressure by opening valve A-7. } \\
\text { Close A-7 when complete. }\end{array}$ \\
\hline 5 & Confirm that the alarm is no longer active. \\
\hline
\end{tabular}

\subsubsection{Gas Analyzer \# 1 Hydrogen Interlock Test}

\begin{tabular}{l|l}
\hline Step & \multicolumn{1}{|c}{ Action } \\
\hline 1 & $\begin{array}{l}\text { (Perform these steps only if running the Mini-AMORE Experiment) } \\
\text { Analyzer \#1 should be running analysis template RGA1_Mini-AMORE_ } \\
\text { Analysis.qmt and the inlet valve should be open. Confirm the } \\
\text { interlock is satisfied. }\end{array}$ \\
\hline 2 & ION CURRENT to trip the relay/interlock. \\
\hline 3 & Attach a $2 \%$ hydrogen standard to the outside calibration port. \\
\hline 4 & $\begin{array}{l}\text { Open valves A-3 } \square \quad \text { A-4 } \square \quad \text { A-5 } \square \quad \text { A-6 } \square \quad \text { A-7 } \square \quad \text { A-8 } \square \\
\text { A-9 } \square \quad \text { A-11 } \square \quad \text { A-12 } \square\end{array}$ \\
\hline 5 & Flush the lines with helium. \\
\hline 6 & Close Valve A-7 \\
\hline 7 & $\begin{array}{l}\text { When the pressure is about }<0.1 \text { mbar, Close valves A-4 } \square \quad \text { A-5 } \square \\
\text { A-6 } \square \quad \text { A-8 } \square \quad\end{array}$ \\
\hline 8 & $\begin{array}{l}\text { Open the canister valve to allow standard up to valve A-5. } \\
\text { Then close the canister valve. }\end{array}$ \\
\hline 9 & $\begin{array}{l}\text { Open valve A-5 to introduce the standard into the manifold. } \\
\text { Add standard until you reach the calibration pressure for the instrument. Then close A-5. }\end{array}$ \\
\hline 10 & Confirm that the interlock is no longer satisfied. \\
\hline 11 & $\begin{array}{l}\text { Evacuate the standard from Analyzer \#1 inlet by opening A-4. } \\
\text { Then close A-3 } \square \quad \text { A-4 } \square\end{array}$ \\
\hline 12 & Confirm that the interlock is once again satisfied. \\
\hline
\end{tabular}




\subsubsection{Gas Analyzer \# 2 Hydrogen Interlock Test}

\begin{tabular}{c|l}
\hline Step & \multicolumn{1}{|c}{ Action } \\
\hline 1 & $\begin{array}{l}\text { Analyzer \#2 should be running analysis template RGA\#2_ } \\
\text { AMORE_Analysis.qmt. The inlet valve should be open. } \\
\text { Confirm the RGA interlock is satisfied. }\end{array}$ \\
\hline 2 & ION CURRENT to trip the relay/interlock \\
\hline 3 & Attach a 2\% hydrogen standard. \\
\hline 4 & $\begin{array}{l}\text { Introduce to Analyzer \# 2 by opening valves A-5 \& A-6. Add standard until you reach the } \\
\text { calibration pressure then close A-5. }\end{array}$ \\
\hline 5 & Confirm that the interlock is no longer satisfied. \\
\hline 7 & Evacuate the standard by opening valve A-4. Then close A-4. \\
\hline 8 & Confirm that the Interlock is once again satisfied. \\
\hline
\end{tabular}

\subsubsection{Hydrogen Sensor - Interlock Test [ASE 3.2.1]}

\begin{tabular}{c|l}
\hline Step & \multicolumn{1}{|c}{ Action } \\
\hline 1 & Confirm that the Hydrogen Sensor interlock is satisfied. \\
\hline 2 & Open valve A-8 to the Hydrogen Sensor. \\
\hline 3 & $\begin{array}{l}\text { Introduce the } 2 \% \text { hydrogen standard to Hydrogen Sensor by opening valve A-5. } \\
\text { Add standard to the calibration pressure. Then close A-5. }\end{array}$ \\
\hline 4 & Confirm that the Hydrogen Sensor interlock is no longer satisfied \\
\hline 5 & Confirm that the Hydrogen Alarm in the D101 LINAC Control room is active \\
\hline 6 & Evacuate the standard by opening valve A-4. Then close A-4 \\
\hline 8 & Add helium to about 1010mbar using valve A-7. Then close A-7 \\
\hline 10 & Confirm that the interlock is once again satisfied and the alarm is not active. \\
\hline 11 & Turn off the Vacuum Pump $\square \quad$ Close Valves A-6 $\square \quad$ A-11 $\square$ \\
\hline 12 & Go to the LINAC Cell D035 Gas Distribution Hub Manifold \\
\hline
\end{tabular}




\begin{tabular}{|c|c|c|c|c|c|c|c|}
\hline Step & \multicolumn{7}{|c|}{ Action } \\
\hline 13 & \multicolumn{6}{|c|}{ Close "de-actuate" valve SV-3 $\square$} & \\
\hline 14 & Open valves V-1 $\square$ & $\mathrm{V}-7 \square$ & V-9 $\square$ & $\mathrm{V}-10 \square$ & V-16 $\square$ & $\mathrm{V}-19 \square$ & \\
\hline
\end{tabular}

\subsubsection{Gas Collection System Alarms \& Interlock}

\begin{tabular}{|c|c|}
\hline Step & Action \\
\hline 1 & $\begin{array}{l}\text { Collection Cylinder } \\
\text { In D032, Confirm the LINAC Interlock for the Gas Collection } \\
\text { System is satisfied. }\end{array}$ \\
\hline 2 & $\begin{array}{l}\text { Set the alarm-1 (High) setting on the Collection Cylinder controller to a } \\
\text { value that is less than the one currently displayed. }\end{array}$ \\
\hline 3 & Confirm the alarm on Chassis \#3 in the D-101 LINAC Control Room is active. \\
\hline 4 & Confirm LINAC Interlock for the Gas Collection System is no longer satisfied. \\
\hline 5 & Reset the alarm-1 (High) to its original value (2000 psi). \\
\hline 6 & Confirm the Interlock is once again satisfied and the alarm is no longer active. \\
\hline 7 & $\begin{array}{l}\text { Chamber \#2 } \\
\text { Set the alarm-2 (High) setting on the Chamber \#2 controller to a value that is } \\
\text { less than the one currently displayed. }\end{array}$ \\
\hline 8 & Confirm the alarm on Chassis \#3 in the D-101 LINAC Control Room is active. \\
\hline 9 & Confirm the valve GC-SV-1 on the Gas Collection System has closed. \\
\hline 10 & Confirm LINAC Interlock for the Gas Collection System is no longer satisfied. \\
\hline 11 & Reset the alarm-2 (High) to its original value (1250). \\
\hline 12 & Reset valve GC-SV-1 by pressing the OPEN button. \\
\hline 13 & Confirm the Interlock is once again satisfied and the alarm is no longer active. \\
\hline 14 & $\begin{array}{l}\text { Set the alarm-2 (Low) setting on the Chamber \#2 controller to a value that is } \\
\text { greater than the one currently displayed. }\end{array}$ \\
\hline 15 & Confirm the alarm on Chassis \#3 in the D-101 LINAC Control Room is active. \\
\hline 16 & Confirm the valve GC-SV-1 on the Gas Collection System has closed. \\
\hline 17 & Confirm LINAC Interlock for the Gas Collection System is no longer satisfied. \\
\hline
\end{tabular}




\begin{tabular}{|c|c|}
\hline Step & Action \\
\hline 18 & Reset the alarm-2 (Low) setting to its original value (1000). \\
\hline 19 & Reset valve GC-SV-1 by pressing the OPEN button. \\
\hline 20 & Confirm the Interlock is once again satisfied and the alarm is no longer active. \\
\hline 21 & $\begin{array}{l}\text { Chamber \#1 } \\
\text { Set the alarm-2 (High) setting on the Chamber \#1 controller to a value that is less } \\
\text { than the one currently displayed. }\end{array}$ \\
\hline 22 & Confirm the alarm on Chassis \#3 in the D-101 LINAC Control Room is active. \\
\hline 23 & Confirm LINAC Interlock for the Gas Collection System is no longer satisfied. \\
\hline 24 & Reset the alarm-2 (High) to its original value (990). \\
\hline 25 & Confirm the Interlock is once again satisfied and the alarm is no longer active. \\
\hline
\end{tabular}

\subsubsection{Solution Vessel Pressure Alarm}

\begin{tabular}{c|l}
\hline Step & \multicolumn{1}{|c}{ Action } \\
\hline 1 & On the Gas Analysis Chassis \#1 in D-101, set switch to "Upstairs". \\
\hline 2 & $\begin{array}{l}\text { Set the alarm-2 (High) setting on the Solution Vessel Pressure meter on Chassis \#4 to a value } \\
\text { that is less than the one currently displayed. }\end{array}$ \\
\hline 3 & Confirm the Solution Vessel Pressure Alarm is active. \\
\hline 4 & Set the alarm-2 (High) setting back to its original value (990mbar) \\
\hline 5 & Confirm the Solution Vessel Pressure Alarm is no longer active. \\
\hline
\end{tabular}

\subsubsection{Catalyst Pump Low Flow Alarm and Interlock}

\begin{tabular}{c|l}
\hline Step & \multicolumn{1}{|c}{ Action } \\
\hline 1 & Turn ON the catalyst pump switch on Chassis \#1 \\
\hline 2 & Turn OFF the Catalyst Pump Alarm Bypass on Chassis \#4. \\
\hline 3 & Turn OFF the catalyst pump switch on Chassis \#1 \\
\hline 4 & Confirm the Catalyst Pump Alarm on Chassis \#4 is active \\
\hline 5 & Confirm the Catalyst Pump interlock is not satisfied \\
\hline 6 & Turn ON the catalyst pump switch on Chassis \#1. \\
\hline
\end{tabular}




\begin{tabular}{c|l}
\hline Step & \multicolumn{1}{|c}{ Action } \\
\hline 7 & Confirm the alarm is no longer active. \\
\hline 8 & Confirm the Catalyst Pump interlock is satisfied \\
\hline 9 & Turn ON the Alarm Bypass. \\
\hline 10 & Turn OFF the catalyst pump switch on Chassis \#1 \\
\hline
\end{tabular}

Sampling Pump Low Flow Alarm

\begin{tabular}{c|l}
\hline Step & \multicolumn{1}{|c}{ Action } \\
\hline 1 & Turn OFF the Sampling Pump Alarm Bypass on Chassis \#3. \\
\hline 2 & Confirm the Sampling Pump alarm is active. \\
\hline 3 & Turn ON the Sampling Pump Chassis \#1. \\
\hline 4 & Confirm the alarm is no longer active. \\
\hline 5 & Turn OFF the Sampling Pump. \\
\hline 6 & Turn ON the Sampling Pump Alarm Bypass on Chassis \#3. \\
\hline
\end{tabular}

\section{Records Created by Work Process}

The records listed below must be retained as indicated.

\begin{tabular}{l|c|c|l|l}
\hline $\begin{array}{l}\text { Description of Record } \\
\text { (include form number } \\
\text { if applicable) }\end{array}$ & $\begin{array}{c}\text { Active } \\
\text { Records } \\
\text { Custodian }\end{array}$ & $\begin{array}{c}\text { Active } \\
\text { Records } \\
\text { Retention }\end{array}$ & $\begin{array}{l}\text { Indexing } \\
\text { Method, } \\
\text { Storage } \\
\text { Medium }\end{array}$ & $\begin{array}{l}\text { Federal Retention } \\
\text { Requirements* }\end{array}$ \\
\hline $\begin{array}{l}\text { Completed AMORE Gas } \\
\text { Handling Alarm and }\end{array}$ & $\begin{array}{c}\text { Facility } \\
\text { Manager }\end{array}$ & 3 years & $\begin{array}{l}\text { Index by job } \\
\text { date and name, } \\
\text { store on paper } \\
\text { or } \\
\text { electronically }\end{array}$ & $\begin{array}{l}\text { Destroy 75 years } \\
\text { after the date of the } \\
\text { permit (DOE ADM } \\
18.37)\end{array}$ \\
\hline
\end{tabular}

*If records are maintained in a business information system that is not currently programmed to purge digital records based on age, the records may be retained in that system past the indicated destruction date.

\section{Related Documents}

This procedure implements requirements established by the following safety basis documents:

- LEAF-SAD-100, Linac Safety Assessment Document

- LEAF-ASE-100, Linac Accelerator Safety Envelope 
This procedure implements requirements established by the following Argonne policies and procedures:

- $\quad$ LMS-PROC-188, Accelerator Safety

- EGS-PP-100, Configuration Management Program Plan for Accelerators

\section{Definitions}

None 


\section{About this Procedure}

\begin{tabular}{ll}
\hline Issuing organization: & Low Energy Accelerator Facility \\
\hline Procedure owner: & M. Kalensky \\
\hline Point of contact: & M. Kalensky \\
\hline Review cycle (months): & 36 \\
\hline Date last revised: & 3.18 .2021 \\
\hline Date last reviewed: & 3.23 .2021 \\
\hline
\end{tabular}

\section{Summary of Changes in This Version}

Change to Section 3.2.1 This Section has been revised to "Test the Function of the Gas Collection System [ASE 2.5.2.2]". This created an extra part to this section "3.2", "3.2.9". The previous version contained up to section "3.2.8". There is no longer a section named "Start the flow of helium to D024", instead it has been replace by Section 3.2.2, "Flush the Analytical Manifold in D024 with helium".

New valves were added to the Gas Distribution Hub Manifold "V-15, V-16, V-17, V-18 and V-19" and a new connection was made from valve V-8 to the sampling pump. These changes affect Sections "3.2.1 Test the Function of the Gas Collection System - STEP 4", "3.2.2 Flush the Analytical Manifold with Helium - STEP 9" and "3.2.6 Test the Hydrogen Interlock - STEP 13". 


\section{APPENDIX 9}

LEAF-PROC-017, Rev. 1: Monitoring the Gas Handling System during the AMORE Experiment 


\section{Monitoring the Gas Handling System during the AMORE Experiment}

Low Energy Accelerator Facility, LEAF-PROC-017, Rev. 1

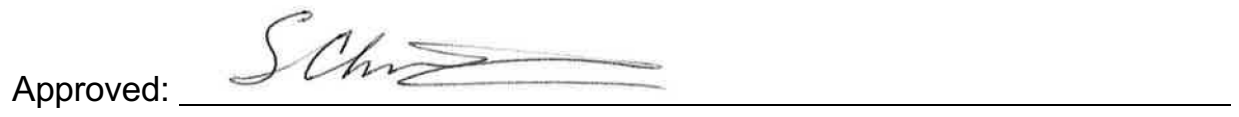

Date: 04.19 .2019

Sergey Chemerisov, Manager, IVEM/LEAF

Effective Date: 04.22 .2019

The current version of this document resides at https://leaf-docdb.ne.anl.gov/cgi-bin/DocumentDatabase.

Printed or electronically downloaded copies may be obsolete. Before using such a copy for work direction, employees must verify that it is current by comparing its revision number to that shown in the on-line version. 


\section{Purpose}

Establish the process for monitoring the Gas Collection System during AMORE experiment.

\section{Scope}

This procedure applies to the following Argonne activities and entities.

\begin{tabular}{ll}
\hline LMS core processes: & Asset Management \\
\hline Organizations: & Experimental Operations and Facilities (EOF) Division \\
\hline Buildings: & 211 \\
\hline Specific locations: & LINAC \\
\hline $\begin{array}{l}\text { Other applicability } \\
\text { factors: }\end{array}$ & None \\
\hline Exclusions: & None \\
\hline USI applicability: & Yes \\
\hline
\end{tabular}

\section{Work Process}

\subsection{Introduction}

The AMORE experiment is equipped with several systems to monitor gas generation and composition.

\subsubsection{Gas Handling System}

The Gas Handling System was designed to monitor the hydrogen concentration in the Target Solution Vessel (TSV) and to minimize the risk of releasing radioactive gases by keeping the TSV and chemical processes subatmospheric.

During the AMORE experiment, hydrogen and oxygen are generated by the radiolysis of water. A Catalyst Pump, located on top of the D035 Hot Cell, circulates the TSV headspace gases through a Pt-Pd Couderite Catalyst, which re-combines the radiolytically generated hydrogen and oxygen. There is also a Sampling Pump, located in the Gas Distribution Hub, which circulates the headspace gas of the TSV to the D024 Analytical Enclosure, where it is analyzed by a H2 Scan HY-OPTIMA 2700 hydrogen sensor (Hydrogen Sensor) and a Pfeiffer Omni-Star Residual Gas Analysis System (Analyzer \#2). The following paragraphs discuss these four components of the Gas Handling System in more detail.

The Catalyst Pump is monitored using the Gas Hub Pressure on Chassis \#4. The Catalyst Pump causes a pressure decrease, as read on transducer PT-1. The decrease in pressure is a positive indication that the Catalyst Pump is in operation. The process controller on Chassis \#4 is interlocked with LINAC power.

The Sampling Pump flow is monitored using a flow meter. In the event that the pump flow becomes too low, an alarm will sound on Chassis \#3 in the LINAC Control Room.

Analyzer \#2 also provides continuous monitoring of the gas composition of the TSV headspace. It has a mass selective detector. Analyzer \#2 is interlocked with the LINAC accelerator power. If hydrogen concentration of the TSV reaches $2 \%$, the LINAC will automatically shut down. The Analyzer monitors the ion current of mass 2

The current version of this document resides at $\mathrm{https}$ ://leaf-docdb.ne.anl.gov/cgi-bin/DocumentDatabase.

Printed or electronically downloaded copies may be obsolete. Before using such a copy for work direction, employees must verify that it is current by comparing its revision number to that shown in the on-line version. 
for hydrogen and mass 32 for oxygen. When the instrument is calibrated, the ion current for hydrogen at $2 \%$ is logged. That value is entered into the analysis template RGA-2_AMORE_ANALYSIS.

The Hydrogen Sensor provides continuous monitoring of the hydrogen concentration of the TSV. It has two alarm states. The first alarm state is at $1 \%$ hydrogen concentration. At this point, an alarm sounds on Chassis \#3 in the LINAC Control Room, warning the operators that hydrogen has reached $1 \%$. The second alarm state is interlocked with LINAC accelerator power. In the event hydrogen concentration reaches $2 \%$, the LINAC is automatically shut down. [ASE 3.2.1]

\subsubsection{Gas Collection System}

The Gas Collection System is connected to all parts of the experiment through the Gas Distribution Hub and directly to the D024 Hot Cell. The Gas Collection System stores radioactive gases generated from the fissioning of uranium during the experiment in Collection Cylinders. It also serves to keep the TSV and the various chemical processing sub-atmospheric. It is interlocked with the LINAC main power. If the pressure in any part of the experiment rises to 900 mbar, the interlock will shut down the LINAC.

Tables at the end of this document provide a quick reference to settings, actions to take, and descriptions of various parts of the experimental system. Gas Handling Chassis in the LINAC control room is used to control the operation of solenoid valves, pumps, and additions of oxygen and helium.

\subsubsection{Mini-AMORE Experiment}

The Phase II Mini-AMORE experiment is monitored using another Pfeiffer Omni-Star Gas Analysis System (Analyzer \#1). This instrument monitors gases generated during the Mini-AMORE experiment. Through a series of tubing, Analyzer \#1 is attached to a capsule containing $2 \mathrm{~mL}$ of uranium sulfate solution. Helium flows into the capsule, sweeping headspace gas from the capsule. Analyzer \#1 samples the gas stream and monitors the hydrogen and oxygen concentration. The outlet of the gas stream flows into the Gas Collection System. Analyzer \#1 is interlocked with the LINAC accelerator power supply such that if the hydrogen concentration reaches $2 \%$, the LINAC is automatically shut down. The value the Analyzer monitors is the ion current of mass 2 for hydrogen and mass 32 for oxygen. When the instrument is recalibrated, the ion current for hydrogen at $2 \%$ is logged. That value is entered into the analysis template RGA-1_Mini_AMORE_ANALYSIS. The administrative limit for hydrogen in this experiment is $1 \%$. If hydrogen concentration gets to $1 \%$, inform the LINAC operator to reduce power. To reduce the concentration of hydrogen in the capsule, increase the helium flow though the capsule.

The current version of this document resides at https://leaf-docdb.ne.anl.gov/cgi-bin/DocumentDatabase. Printed or electronically downloaded copies may be obsolete. Before using such a copy for work direction, employees must verify that it is current by comparing its revision number to that shown in the on-line version. 


\subsection{Step-by-Step Procedure}

The steps below are mandatory unless noted otherwise. This procedure is to be performed by trained personnel.

\subsubsection{Log Entries}

\begin{tabular}{|c|c|}
\hline Step & Action \\
\hline 1 & $\begin{array}{l}\text { In the Gas Analysis Logbook, log in: } \\
\text { - Date } \\
\text { - Experiment Title } \\
\text { - } \text { Gas Analyzer Templates (Methods) } \\
\text { The Analyzers should be started and running the analysis templates. Pre-experiment checks } \\
\text { and interlock/alarm checks should already be complete. }\end{array}$ \\
\hline 2 & $\begin{array}{l}\text { Monitor Gas Constituents: } \\
\text { The experimenter should monitor the gas constituents continually by observing concentration } \\
\text { displayed on the Analyzer computers and the H2Scan hydrogen sensor displays. }\end{array}$ \\
\hline 3 & $\begin{array}{l}\text { During the experiment: } \\
\text { 3.1 Use the notebook to log: } \\
\text { - Log actions such as adding oxygen, purging with helium, increasing LINAC power } \\
\text { - Log problems or interesting occurrences } \\
\text { - Log the time and duration. Add notes for more information. } \\
3.2 \text { Be mindful of the hydrogen concentration in the AMORE Target Solution Vessel } \\
\text { (Analyzer \#2 and Hydrogen Sensor) and in the Mini-AMORE Experiment (Analyzer } \\
\text { \#1). } \\
\text { - The administrative limit for this experiment is } 1 \% \text { hydrogen. } \\
\text { - If the hydrogen concentration reaches } 1 \% \text {, the LINAC operator needs to be notified } \\
\text { and the beam power should be reduced. [ASE 3.2.1] }\end{array}$ \\
\hline 4 & $\begin{array}{l}\text { - For the AMORE experiment, add oxygen to the Solution Vessel to reduce the hydrogen } \\
\text { concentration. } \\
\text { For the Mini-AMORE Experiment, increase the helium flow to reduce the hydrogen } \\
\text { concentration. If the hydrogen concentration reaches } 2 \% \text { the experiment needs to be shut } \\
\text { down. }\end{array}$ \\
\hline
\end{tabular}

The current version of this document resides at https:/leaf-docdb.ne.anl.gov/cgi-bin/DocumentDatabase. Printed or electronically downloaded copies may be obsolete. Before using such a copy for work direction, employees must verify that it is current by comparing its revision number to that shown in the on-line version. 


\begin{tabular}{c|l}
\hline Step & \\
\hline 5 & Monitor alarms for: \\
& $\bullet \quad$ Gas Collection System \\
& $\bullet \quad$ Sampling Pump flow, \\
& $\bullet \quad$ Catalyst Pump pressure, and \\
& SEE TABLE 1, ALARM CONDITIONS, for actions that need to be taken in the event of an \\
& alarm state. \\
\hline
\end{tabular}

\subsubsection{Adding Oxygen to Reduce the Concentration of Hydrogen}

\begin{tabular}{|c|c|}
\hline Step & Action \\
\hline 1 & $\begin{array}{l}\text { A } 60 / 40 \text { helium/oxygen mixture is added to the Target Solution Vessel. It is best to add } \\
\text { oxygen well before hydrogen reaches } 1 \% \text {. There is a three-minute delay from the time you } \\
\text { begin adding oxygen till a reduction in hydrogen is observed. } \\
\text { When hydrogen is approximately } 0.3 \% \text {, it is time to add oxygen: } \\
1.1 \quad \text { Actuate (open) SV-5 on Chassis \#1 } \\
1.2 \quad \text { Use the oxygen flow control knob on Chassis } \# 2 \text { to set the desired gas flow. }\end{array}$ \\
\hline 2 & $\begin{array}{l}\text { When a sufficient amount of gas is delivered: } \\
2.1 \quad \text { Close SV-5 } \\
2.2 \quad \begin{array}{l}\text { Stop the gas flow using the Stop Flow switch or by turning down the control knob } \\
\text { on chassis } \# 2\end{array}\end{array}$ \\
\hline
\end{tabular}

\subsubsection{Increasing the Helium Flow to Reduce Hydrogen Concentration in the Mini-AMORE Experiment}

\begin{tabular}{c|l}
\hline Step & \multicolumn{1}{c}{ Action } \\
\hline 1 & $\begin{array}{l}\text { Use the Mini-AMORE Flow Control knob on Chassis \#2 to increase the flow of helium } \\
\text { through the capsule. Turn the knob to the right and observe the Flow readout. Observe } \\
\text { Analyzer \#1 data. Adjust flow until Hydrogen concentration is below 1\%. }\end{array}$ \\
\hline
\end{tabular}

The current version of this document resides at https://leaf-docdb.ne.anl.gov/cgi-bin/DocumentDatabase.

Printed or electronically downloaded copies may be obsolete. Before using such a copy for work direction, employees must verify that it is current by comparing its revision number to that shown in the on-line version. 


\subsubsection{Post Irradiation}

\begin{tabular}{|c|c|c|}
\hline Step & & Action \\
\hline \multirow[t]{5}{*}{1} & \multirow{2}{*}{\multicolumn{2}{|c|}{$\begin{array}{l}\text { Add Helium to Reduce Radiation Levels in D } 024 \\
\text { It will be necessary to decrease radiation levels in D024 to allow access. This is done by } \\
\text { purging the Analytical Manifold with helium. SV-1 will isolate the Vessel and SV-3 allows } \\
\text { helium into the Analytical Manifold. }\end{array}$}} \\
\hline & & \\
\hline & & Put the Catalyst Pump and Sampling Pump alarms in bypass mode. \\
\hline & & Actuate (close) SV-1 \\
\hline & & Actuate (open) SV-3 \\
\hline \multirow[t]{6}{*}{2} & \multicolumn{2}{|c|}{ When radiation in D024 reaches acceptable levels } \\
\hline & & De-actuate (close) SV-3 \\
\hline & 2.2 & Turn off the Gas Sampling Pump \\
\hline & 2.3 & Actuate SV-6 (close) \\
\hline & 2.4 & Stop the flow of helium to the Mini-AMORE capsule. \\
\hline & 2.5 & $\begin{array}{l}\text { Close the valves on the three cylinders and their isolation valves in D032. Oxygen } \\
\text { and the two helium/xenon cylinders. }\end{array}$ \\
\hline \multirow[t]{4}{*}{3} & \multicolumn{2}{|c|}{ Put the System in Standby Mode (usually the next day) } \\
\hline & 3.1 & De-actuate (open) SV-1 and SV-6 \\
\hline & 3.2 & On the Analytical Manifold, close valves A-1, A-2, A-7, A-8, A-9 and A-10. \\
\hline & & Turn off the catalyst heater. \\
\hline \multirow[t]{6}{*}{4} & \multicolumn{2}{|r|}{ Save Analyzer Data (Perform when no more data needs to be collected) } \\
\hline & & Stop the analysis template \\
\hline & 4.2 & Close the inlet valve \\
\hline & & Turn off the filaments and SEM \\
\hline & & Save the data file \\
\hline & & Copy Data Files to the GTRI shared drive. This will ensure the data is backed up. \\
\hline
\end{tabular}

The current version of this document resides at https://leaf-docdb.ne.anl.gov/cgi-bin/DocumentDatabase. Printed or electronically downloaded copies may be obsolete. Before using such a copy for work direction, employees must verify that it is current by comparing its revision number to that shown in the on-line version. 


\subsection{Reference Tables (see Exhibit A)}

Table 1. Alarm and Interlock Conditions and Action: Gives a brief description of the alarms and interlocks that may occur during an experiment and the actions that need to be taken by the operator.

Table 2. Hub Manifold Solenoid Valves: Gives a brief description of the solenoid valves on the Hub Manifold in the Gas Distribution Hub Enclosure in D035. The solenoid valves are remotely actuated from Chassis \#1.

Table 3. Switches and Controls on Gas Handling Chassis: Located in the LINAC Control Room: Gives a brief description of the function of other switches and control knobs on the Gas Handling Chassis. Included are oxygen and mini-AMORE, flow control and ON/OFF switches for the Catalyst and Sampling pumps.

Table 4. Hub Manifold Manual Valves: Located in the Gas Distribution Hub Enclosure (D035): Gives a brief description of the manually operated valve located on the Hub Manifold.

Table 5. Gas Collection System Valves: Located in the Gas Collection Enclosure in (D035): Gives a brief description of the function of valves in the Gas Collection System.

Table 6. Dump Tank Valves: Located in D035 beneath the D035 Hot Cell: Gives a brief description of the function of valves on the Dump Tank.

Table 7. Gas Collection System Chassis Settings: Located in (D032) shows Control settings to actuate pumps, alarms and interlocks in the Gas Collection System.

Table 8. Analytical Manifold Valves: located in the D024 Analytical Enclosure, it gives a brief description of the manually operated valves.

\section{Records Created by Work Process}

The records listed below must be retained as indicated.

\begin{tabular}{l|c|c|l|l}
\hline $\begin{array}{l}\text { Description of Record } \\
\text { (include form number if } \\
\text { applicable) }\end{array}$ & $\begin{array}{c}\text { Active } \\
\text { Records } \\
\text { Custodian }\end{array}$ & $\begin{array}{c}\text { Active } \\
\text { Records } \\
\text { Retention }\end{array}$ & $\begin{array}{l}\text { Indexing Method, } \\
\text { Storage Medium }\end{array}$ & $\begin{array}{l}\text { Federal Retention } \\
\text { Requirements* }\end{array}$ \\
\hline Lab notebook & $\begin{array}{c}\text { Facility } \\
\text { Manager }\end{array}$ & 3 years & $\begin{array}{l}\text { Index by job date } \\
\text { and name, store on } \\
\text { paper or } \\
\text { electronically }\end{array}$ & $\begin{array}{l}\text { Destroy 75 years after } \\
\text { the date of the permit } \\
\text { (DOE ADM 18.37) }\end{array}$ \\
\hline
\end{tabular}

*If records are maintained in a business information system that is not currently programmed to purge digital records based on age, the records may be retained in that system past the indicated destruction date.

\section{Related Documents}

This procedure implements requirements established by the following safety basis documents:

- LEAF-SAD-100, Linac Safety Assessment Document

- LEAF-ASE-100, Linac Accelerator Safety Envelope

This procedure implements requirements established by the following Argonne policies and procedures:

- LMS-PROC-188, Accelerator Safety

- EGS-PP-100, Configuration Management Program Plan for Accelerator

The current version of this document resides at https:/leaf-docdb.ne.anl.gov/cgi-bin/DocumentDatabase. Printed or electronically downloaded copies may be obsolete. Before using such a copy for work direction, employees must verify that it is current by comparing its revision number to that shown in the on-line version. 


\section{Definitions}

None

\section{$7 \quad$ About this Procedure}

\begin{tabular}{ll}
\hline Issuing organization: & Low Energy Accelerator Facility \\
\hline Procedure owner: & M. Kalensky \\
\hline Point of contact: & M. Kalensky \\
\hline Review cycle (months): & 36 \\
\hline Date last revised: & 04.19 .2019 \\
\hline Date last reviewed: & 04.22 .2019 \\
\hline
\end{tabular}

\section{Summary of Changes in This Version}

Initial release.

Rev. 1. Addition of the references to the ASE controlled parameters.

The current version of this document resides at https://leaf-docdb.ne.anl.gov/cgi-bin/DocumentDatabase. Printed or electronically downloaded copies may be obsolete. Before using such a copy for work direction, employees must verify that it is current by comparing its revision number to that shown in the on-line version. 


\section{Exhibit A: Reference Tables}

TABLE 1. Alarm and Interlock Conditions and Action

\begin{tabular}{|l|l|}
\hline \multicolumn{1}{|c|}{ Condition } & \multicolumn{1}{c|}{ Action } \\
\hline $\begin{array}{l}\text { AMORE Hydrogen concentration 1\% } \\
\text { (Alarm on Chassis \#3) [ASE 3.2.1] }\end{array}$ & $\begin{array}{l}\text { Warn LINAC Operator to reduce beam power. Add } \\
\text { oxygen to re-combine the excess hydrogen to }<1 \% .\end{array}$ \\
\hline $\begin{array}{l}\text { AMORE Hydrogen Concentration 2\% } \\
\text { (Alarm on Chassis \#3) [ASE 3.2.1] }\end{array}$ & $\begin{array}{l}\text { Interlocked. Warn LINAC Operator to Stop the } \\
\text { Experiment if Interlock fails. Add oxygen to re-combine } \\
\text { the excess hydrogen. }\end{array}$ \\
\hline $\begin{array}{l}\text { Gas Collection Alarm. } \\
\text { (Alarm on Chassis \#3) }\end{array}$ & $\begin{array}{l}\text { Interlocked. Warn LINAC Operator to Stop the } \\
\text { experiment. It indicates that the pressure in chambers } 1, \\
\text { 2 or the Collection Cylinder is too high. Monitored on } \\
\text { LabView. }\end{array}$ \\
\hline $\begin{array}{l}\text { Sampling Pump Alarm } \\
\text { (Alarm on Chassis \#3) [ASE 3.2.1] }\end{array}$ & $\begin{array}{l}\text { Warn LINAC Operator to STOP the experiment. This } \\
\text { indicates a problem with the Gas Sampling Pump. }\end{array}$ \\
\hline $\begin{array}{l}\text { Catalyst Pump Alarm } \\
\text { (Alarm on Chassis \#4) }\end{array}$ & $\begin{array}{l}\text { Warn LINAC Operator. STOP the experiment. This is } \\
\text { interlocked }\end{array}$ \\
\hline $\begin{array}{l}\text { Solution Vessel Pressure } \\
\text { (Alarm on Chassis \#4) }\end{array}$ & $\begin{array}{l}\text { Warn LINAC Operator. Shut Down the experiment. } \\
\text { This may be an indication of a Gas Collection System } \\
\text { problem. This is Interlocked. }\end{array}$ \\
\hline
\end{tabular}

The current version of this document resides at https://leaf-docdb.ne.anl.gov/cgi-bin/DocumentDatabase. Printed or electronically downloaded copies may be obsolete. Before using such a copy for work direction, employees must verify that it is current by comparing its revision number to that shown in the on-line version. 
TABLE 2. Hub Manifold Solenoid Valves - Located in the Gas Distribution Hub Enclosure (D035) (Controls on Chassis 1) \& (Gas Distribution Hub Enclosure)

\begin{tabular}{|c|c|c|}
\hline Valve & Description & Function \\
\hline SV-1 & Gas Sampling Isolation & $\begin{array}{l}\text { Normally Open }- \text { Allows the gas stream from the Target } \\
\text { Solution Vessel into the Hub Manifold. Purge the Analytical } \\
\text { Manifold in D024 post irradiation by actuating SV-3 and SV- } \\
\text { 1. }\end{array}$ \\
\hline SV-2 & Sampling Pump Bypass & $\begin{array}{l}\text { Normally Closed }- \text { Should the sampling pump fail, the } \\
\text { Analytical Manifold in D024 can be purged by actuating SV- } \\
1, \text { SV-2 and SV-3. }\end{array}$ \\
\hline SV-3 & $\begin{array}{l}\text { Isolation Valve/Helium } \\
\text { Purge }\end{array}$ & $\begin{array}{l}\text { Normally Closed }- \text { Isolates the Gas Sample Path from the } \\
\text { helium purge line during an experiment. Open it to purge the } \\
\text { Analytical Manifold for calibration and pre-run. You can } \\
\text { purge the Analytical Manifold post irradiation by actuating } \\
\text { SV-3 and SV-1. }\end{array}$ \\
\hline SV-4 & Helium purge & $\begin{array}{l}\text { Normally Closed } \\
\text { Vessel. }\end{array}$ \\
\hline SV-5 & Oxygen Addition & $\begin{array}{l}\text { Normally Closed }- \text { Open it to add oxygen to the Target } \\
\text { Solution Vessel to reduce hydrogen concentration. Use in } \\
\text { conjunction with the Oxygen Flow potentiometer on Chassis } \\
2 \text {. }\end{array}$ \\
\hline SV-6 & $\begin{array}{l}\text { Sampling Pump } \\
\text { Isolation }\end{array}$ & $\frac{\text { Normally Open }}{\text { Solution Vessel }}-$ Isolates the Sampling Pump from the \\
\hline SV-7 & Gas Sampling Isolation & $\begin{array}{l}\text { Normally Open }- \text { Allows the analytical gas stream from the } \\
\text { Solution Vessel into the Condenser. Close when removing } \\
\text { condensate from the condenser. }\end{array}$ \\
\hline
\end{tabular}

The current version of this document resides at https://leaf-docdb.ne.anl.gov/cgi-bin/DocumentDatabase. Printed or electronically downloaded copies may be obsolete. Before using such a copy for work direction, employees must verify that it is current by comparing its revision number to that shown in the on-line version. 
TABLE 3. Switches and Controls on Gas Handling Chassis - Located in the LINAC Control Room

\begin{tabular}{|c|c|c|}
\hline Switch & Description & Function \\
\hline $\begin{array}{l}\text { Catalyst Pump } \\
\text { (Chassis 1) }\end{array}$ & $\begin{array}{l}\text { Catalyst Pump Switch } \\
\text { ON/OFF }\end{array}$ & $\begin{array}{l}\text { Turns ON and OFF the Catalyst Pump that } \\
\text { circulates Target Solution Vessel headspace gas } \\
\text { through the catalyst to recombine hydrogen and } \\
\text { oxygen. This should be kept ON during an } \\
\text { AMORE irradiation. }\end{array}$ \\
\hline $\begin{array}{l}\text { Sampling Pump } \\
\text { (Chassis 1) }\end{array}$ & $\begin{array}{l}\text { Sampling Pump Switch } \\
\text { ON/OFF }\end{array}$ & $\begin{array}{l}\text { Turns ON and OFF the Sampling Pump that } \\
\text { circulates the Target Solution Vessel headspace gas } \\
\text { to the Analytical Manifold in D024. This should be } \\
\text { kept ON during an AMORE irradiation. }\end{array}$ \\
\hline $\begin{array}{l}\text { Oxygen Flow } \\
\text { Control } \\
\text { (Chassis 2) }\end{array}$ & $\begin{array}{l}\text { Potentiometer which } \\
\text { remotely adjusts the flow } \\
\text { controller on the oxygen } \\
\text { cylinder }\end{array}$ & $\begin{array}{l}\text { Regulates Oxygen flow to the Target Solution } \\
\text { Vessel to reduce the level of hydrogen. Turn right } \\
\text { to increase flow. The meter will read out in } \\
\mathrm{mL} / \mathrm{min} \text {. The maximum flow is } 50 \mathrm{~mL} / \mathrm{min} \text {. Use in } \\
\text { conjunction with SV-5 on chassis } 1 .\end{array}$ \\
\hline $\begin{array}{l}\text { Oxygen OFF } \\
\text { (Chassis 2) }\end{array}$ & $\begin{array}{l}\text { Internal valve on the } \\
\text { oxygen mass flow } \\
\text { controller }\end{array}$ & $\begin{array}{l}\text { Stops the flow of oxygen without using the } \\
\text { potentiometer. A flow setting can be maintained } \\
\text { even when no gas is being added. SV-5 needs to be } \\
\text { closed to use this function. }\end{array}$ \\
\hline $\begin{array}{l}\text { Mini-AMORE } \\
\text { Flow Control } \\
\text { (Chassis 2) }\end{array}$ & $\begin{array}{l}\text { Potentiometer which } \\
\text { remotely adjusts the flow } \\
\text { controller on the Mini- } \\
\text { AMORE helium cylinder }\end{array}$ & $\begin{array}{l}\text { Regulates the helium flow in the mini-AMORE } \\
\text { experiment. Turn right to increase flow }\end{array}$ \\
\hline
\end{tabular}

The current version of this document resides at https://leaf-docdb.ne.anl.gov/cgi-bin/DocumentDatabase. Printed or electronically downloaded copies may be obsolete. Before using such a copy for work direction, employees must verify that it is current by comparing its revision number to that shown in the on-line version. 
TABLE 4. Hub Manifold Manual Valves - Located in the Gas Distribution Hub Enclosure (D035)

\begin{tabular}{|c|c|c|}
\hline Valve & Description & Function \\
\hline V-1 & Condenser Isolation & Isolates the Condenser from the Hub Manifold \\
\hline $\begin{array}{l}V-2 \& \\
V-10\end{array}$ & $\begin{array}{l}\text { Analytical Manifold } \\
\text { Isolation }\end{array}$ & $\begin{array}{l}\text { Closing V-2 \& V-10 isolate the Analytical Manifold in D024 } \\
\text { from the Hub Manifold. }\end{array}$ \\
\hline V-3 & Helium Isolation Valve & $\begin{array}{l}\text { Allows helium in to the Hub Manifold for purging the Solution } \\
\text { Vessel, Dump Tank and the Analytical Manifold. }\end{array}$ \\
\hline V-4 & Main Isolation & $\begin{array}{l}\text { Isolates sampling and purge connections from the Gas } \\
\text { Collection System connections on the Hub Manifold. }\end{array}$ \\
\hline V-5 & Vacuum Inlet & Opens the manifold to Vacuum Pump inlet. \\
\hline V-6 & $\begin{array}{l}\text { Target Solution Vessel } \\
\text { Head Space Isolation }\end{array}$ & $\begin{array}{l}\text { Isolates the headspace of the Solution Vessel from the Gas } \\
\text { Collection System and Vacuum Pump. Opening V-6, V-7 \& V- } \\
9 \text { opens the Target Solution Vessel headspace to the Gas } \\
\text { Collection System. }\end{array}$ \\
\hline V-7 & $\begin{array}{l}\text { Target Solution Vessel } \\
\text { Head Space Isolation }\end{array}$ & $\begin{array}{l}\text { Opening V-6, V-7 \& V-9 opens the Target Solution Vessel } \\
\text { headspace to the Gas Collection System. }\end{array}$ \\
\hline V-8 & $\begin{array}{l}\text { Vacuum Exhaust to Gas } \\
\text { Collection }\end{array}$ & Opens the vacuum pump exhaust to the Gas Collection System \\
\hline V-9 & Gas Collection Isolation & Isolates the Hub Manifold from the Gas Collection System \\
\hline V-10 & $\begin{array}{l}\text { Analytical Manifold } \\
\text { Isolation }\end{array}$ & $\begin{array}{l}\text { Closing V-2 \& V-10 isolate the Analytical Manifold in D024 } \\
\text { from the Hub Manifold. (Attached to the Sampling Pump) }\end{array}$ \\
\hline V-11 & $\begin{array}{l}\text { Primary Recovery } \\
\text { Glovebox Isolation }\end{array}$ & $\begin{array}{l}\text { Isolates the Primary Recovery Glovebox from the Gas } \\
\text { Collection System }\end{array}$ \\
\hline V-12 & $\begin{array}{l}\text { Analyzer Exhaust } \\
\text { Isolation }\end{array}$ & Isolates the Analyzer Exhaust from the Gas Collection System \\
\hline V-13 & $\begin{array}{l}\text { Condensate Collection } \\
\text { Isolation }\end{array}$ & $\begin{array}{l}\text { Isolates the Condenser from the condensate collection canisters } \\
\text { valve. }\end{array}$ \\
\hline V-14 & $\begin{array}{l}\text { Condensate Collection } \\
\text { Canister valve }\end{array}$ & Valve on the Condensate Collection Canister \\
\hline
\end{tabular}

The current version of this document resides at https:/leaf-docdb.ne.anl.gov/cgi-bin/DocumentDatabase.

Printed or electronically downloaded copies may be obsolete. Before using such a copy for work direction, employees must verify that it is current by comparing its revision number to that shown in the on-line version. 
TABLE 5. Gas Collection System Valves - Located in the Gas Collection System Enclosure (D035)

\begin{tabular}{|c|c|c|}
\hline Valve & Description & Function \\
\hline GC-1 & $\begin{array}{l}\text { D024 Hot Cell } \\
\text { Isolation }\end{array}$ & Isolates the Gas Collection System from the D024 Hot Cell \\
\hline GC-2 & $\begin{array}{l}\text { Open Only For Non- } \\
\text { Rad Experiments }\end{array}$ & $\begin{array}{l}\text { This valve can be open during the Commissioning Tests. When } \\
\text { sulfuric acid solution is in the Vessel. Keep valves GC-3 and } \\
\text { GC- } 4 \text { closed to protect the zeolite cartridge from moisture. }\end{array}$ \\
\hline $\begin{array}{c}\text { GC-3 and } \\
\text { GC-4 }\end{array}$ & $\begin{array}{l}\text { Silver Zeolite } \\
\text { Isolation valves }\end{array}$ & $\begin{array}{l}\text { Open during AMORE Experiments. Valve GC-2 is kept } \\
\text { closed. }\end{array}$ \\
\hline $\begin{array}{l}\text { GC-5 and } \\
\text { GC-6 }\end{array}$ & $\begin{array}{l}\text { Condensate Drain } \\
\text { Valves }\end{array}$ & Open when draining condensate \\
\hline GC-7 & Port & Port for maintenance. Capped \\
\hline CSV-1 & $\begin{array}{l}\text { High Pressure } \\
\text { Isolation }\end{array}$ & $\begin{array}{l}\text { Part of the interlocks. Closed when the Gas Collection } \\
\text { interlock is tripped }\end{array}$ \\
\hline
\end{tabular}

TABLE 6. Dump Tank Valves - Located beneath the D035 Hot Cell

\begin{tabular}{|l|l|l|}
\hline \multicolumn{1}{|c|}{ Valve } & \multicolumn{1}{|c|}{ Description } & \multicolumn{1}{c|}{ Function } \\
\hline $\begin{array}{l}\text { D-1A \& } \\
\text { D-1B }\end{array}$ & $\begin{array}{l}\text { Pick-up line to Primary } \\
\text { Recovery Glovebox }\end{array}$ & $\begin{array}{l}\text { Siphons solution from the bottom of the Dump Tank to } \\
\text { transport it to the Primary Recovery Glovebox. }\end{array}$ \\
\hline $\begin{array}{l}\text { D-2A \& } \\
\text { D-2B }\end{array}$ & $\begin{array}{l}\text { Return Line from } \\
\text { Primary Recovery } \\
\text { Glovebox }\end{array}$ & $\begin{array}{l}\text { Returns solution to the top of the Tank from the Primary } \\
\text { Recovery Glovebox }\end{array}$ \\
\hline $\begin{array}{l}\text { D-3A \& } \\
\text { D-3B }\end{array}$ & $\begin{array}{l}\text { Dump Tank headspace } \\
\text { gas to Gas Distribution } \\
\text { Hub Manifold }\end{array}$ & $\begin{array}{l}\text { Allows for gas displacement when solution is entering the } \\
\text { Dump Tank }\end{array}$ \\
\hline
\end{tabular}

TABLE 7. Gas Collection System Chassis Control Settings - Located in D032

\begin{tabular}{|l|c|c|c|}
\hline \multicolumn{1}{|c|}{ Chamber } & Set point -1 & Alarm-2 High & Dead Band \\
\hline Chamber \#1 & 900 to $960 \mathrm{mbar}$ & $990 \mathrm{mbar}$ & $10 \mathrm{mbar}$ \\
\hline Chamber \#2 & $1030 \mathrm{mbar}$ & $1300 \mathrm{mbar}$ & $110 \mathrm{mbar}$ \\
\hline Collection Cylinders & $2000 \mathrm{psig}$ & $2000 \mathrm{psig}$ & N/A \\
\hline
\end{tabular}

The current version of this document resides at https:/leaf-docdb.ne.anl.gov/cgi-bin/DocumentDatabase.

Printed or electronically downloaded copies may be obsolete. Before using such a copy for work direction, employees must verify that it is current by comparing its revision number to that shown in the on-line version. 
TABLE 8. Analytical Manifold Valves - Located in the D024 Analytical Enclosure

\begin{tabular}{|c|c|c|}
\hline Valve & Description & Function \\
\hline A-1 & Mini-AMORE isolation & Isolates Mini-AMORE from the manifold \\
\hline A-2 & $\begin{array}{l}\text { Gas Collection System } \\
\text { Isolation }\end{array}$ & $\begin{array}{l}\text { Close to isolate the manifold and Mini-AMORE from Gas } \\
\text { Collection System }\end{array}$ \\
\hline A-3 & Analyzer \#1 Isolation & Isolates Analyzer \#1 from the manifold \\
\hline A-4 & Vacuum Inlet & Opens the manifold to vacuum. \\
\hline A-5 & Calibration isolation & Isolate calibration gas \\
\hline A-6 & Analyzer \#2 Isolation & Isolates Analyzer \#2 from the manifold \\
\hline A-7 & Hub Manifold isolation & $\begin{array}{l}\text { Isolates the Hub Manifold in the D- } 035 \text { Gas Distribution Hub } \\
\text { Enclosure from the Analytical Manifold }\end{array}$ \\
\hline A-8 & $\begin{array}{l}\text { Analyzer \#2 Hydrogen } \\
\text { Sensor isolation }\end{array}$ & Isolates Analyzer \#2 from the Hydrogen Sensor \\
\hline $\begin{array}{l}\text { A-9 and } \\
\text { A-10 }\end{array}$ & $\begin{array}{l}\text { Hydrogen Sensor } \\
\text { Isolation }\end{array}$ & Isolates the Hydrogen Sensor for removal \\
\hline A-11 & Vacuum Exhaust & $\begin{array}{l}\text { Isolates the Vacuum Pump exhaust from the Gas Collection } \\
\text { System }\end{array}$ \\
\hline A-12 & Calibration Gas & Allows calibration gas up to A-5 \\
\hline
\end{tabular}

The current version of this document resides at https://leaf-docdb.ne.anl.gov/cgi-bin/DocumentDatabase. Printed or electronically downloaded copies may be obsolete. Before using such a copy for work direction, employees must verify that it is current by comparing its revision number to that shown in the on-line version. 


\section{APPENDIX 10}

LEAF-PROC-018, Rev.3: AMORE Gas Handling Pre-Run Checklist 


\section{AMORE Gas Handling Pre-Run Checklist}

Low Energy Accelerator Facility, LEAF-PROC-018, Rev.3

Approved: SChn Date: $\underline{03.23 .2021}$

Sergey Chemerisov, Manager, IVEM/LEAF

Effective Date: $\underline{03.24 .2021}$ 


\section{Experiment}

\section{NOTE: A second person should verify the steps on this checklist}

\section{Purpose}

Establish a pre-run checklist for gas handling in the AMORE experiment.

\section{Scope}

This procedure applies to the following Argonne activities and entities.

\begin{tabular}{ll}
\hline LMS core processes: & Asset Management \\
\hline Organizations: & Experimental Operations and Facilities (EOF) Division \\
\hline Buildings: & 211 \\
\hline Specific locations: & LINAC \\
\hline $\begin{array}{l}\text { Other applicability } \\
\text { factors: }\end{array}$ & None \\
\hline Exclusions: & None \\
\hline USI applicability: & Yes \\
\hline
\end{tabular}

\section{Work Process}

\subsection{Introduction}

This procedure established the order in which pre-run checks of the AMORE gas handling system shall be performed.

\subsection{Step-by-Step Procedure}

The steps below are mandatory unless noted otherwise. This procedure is to be performed by trained personnel.

\subsubsection{Gas Collection System Chassis Settings in D032}

\begin{tabular}{c|lcc}
\hline Step & \multicolumn{3}{|c}{ Action } \\
\hline 1 & Collection Cylinders should be less than 1600psi. & Initials & Verified \\
\hline 2 & $\begin{array}{l}\text { Chamber \#1: Controller on far right - Set Point-1: between 900 and 960 mbar } \\
\text { Alarm-2 High setting: 990 mbar } \\
\text { Dead-Band 10mbar. }\end{array}$ & \\
\hline
\end{tabular}




\begin{tabular}{c|ll}
\hline Step & \multicolumn{1}{|c}{ Action } \\
\hline 3 & $\begin{array}{l}\text { Chamber \#2: Controller in the middle - Set Point-1: 1020mbar } \\
\text { Alarm-2 Low: 1000mbar } \\
\text { Alarm-2 High: 1250mbar } \\
\text { Dead-Band: } 100 \text { mbar. }\end{array}$ \\
\hline 4 & $\begin{array}{l}\text { Collection Cylinders: Controller on the left - } \\
\text { Alarm-2 High: 2000 psi } \\
\text { Alarm-1 High: } 2000 \text { psi. }\end{array}$ \\
\hline 5 & $\begin{array}{l}\text { RESET the Catalyst heater. Set to } 130^{\circ} \mathrm{C} . \\
\text { Turn the dial to the second mark. }\end{array}$ \\
\hline
\end{tabular}

\subsubsection{Gas Distribution Hub Manifold in D035 LINAC Cell [ASE2.5.2.1]}

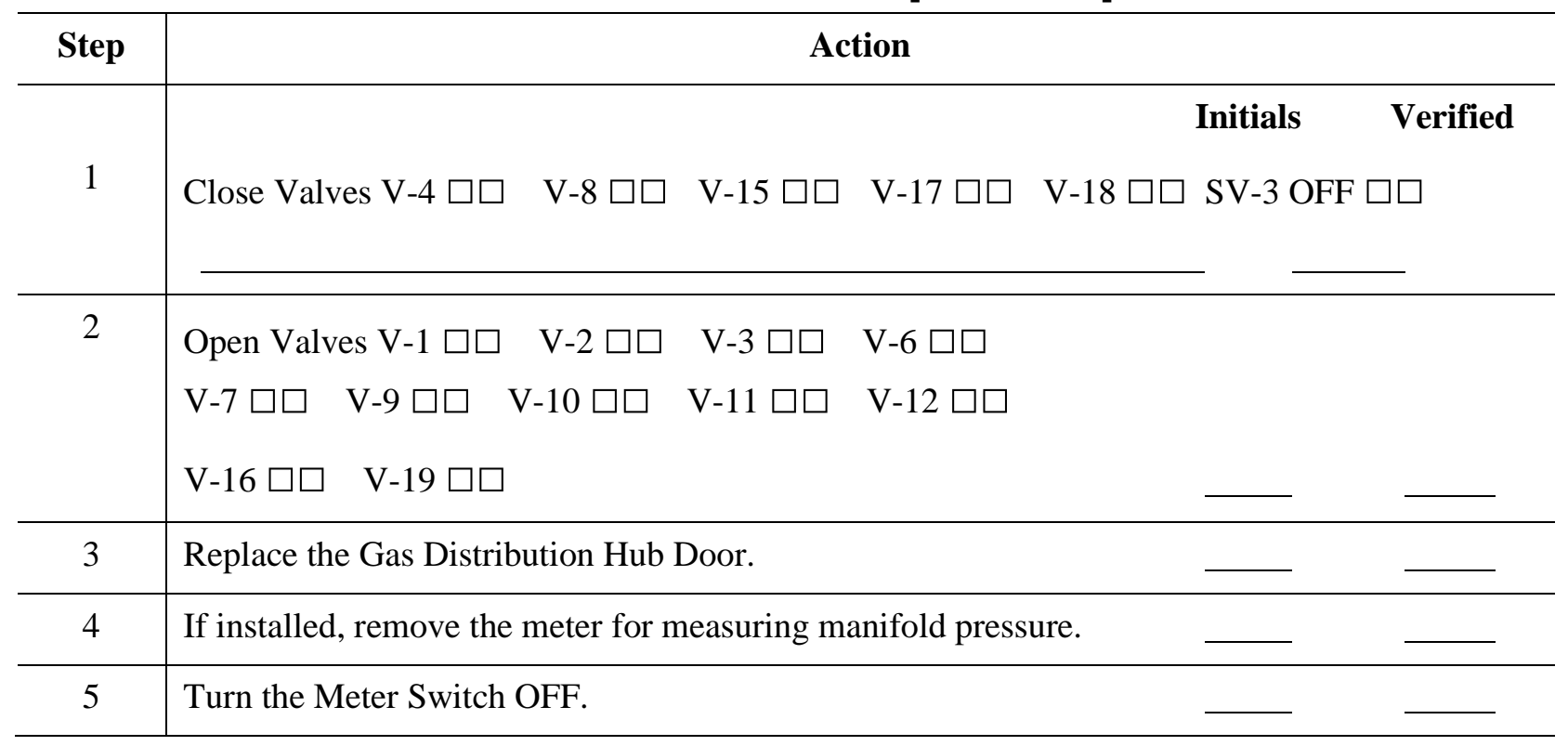

3.2.3 Dump Tank in D035 LINAC CELL [ASE2.5.2.1]

\begin{tabular}{c|ccccc}
\hline Step & \multicolumn{5}{|c}{ Action } \\
\hline & & & & Initials & Verified \\
1 & Open all valves D-1A $\square \square$ & D-2A $\square \square$ & D-3A $\square \square$ & & \\
\hline
\end{tabular}


3.2.4 Gas Collection Enclosure D035 LINAC Cell

\begin{tabular}{c|lcc}
\hline Step & \multicolumn{3}{|c}{ Action } \\
\hline \\
1 & Verify the presence of a backflow orifice [ASE 2.5.2.3] & Initials & Verified \\
\hline 2 & Open valve GC-1 [ASE2.5.2.1] & - & - \\
\hline
\end{tabular}

\subsubsection{D024 Analytical Enclosure [ASE2.5.2.1]}

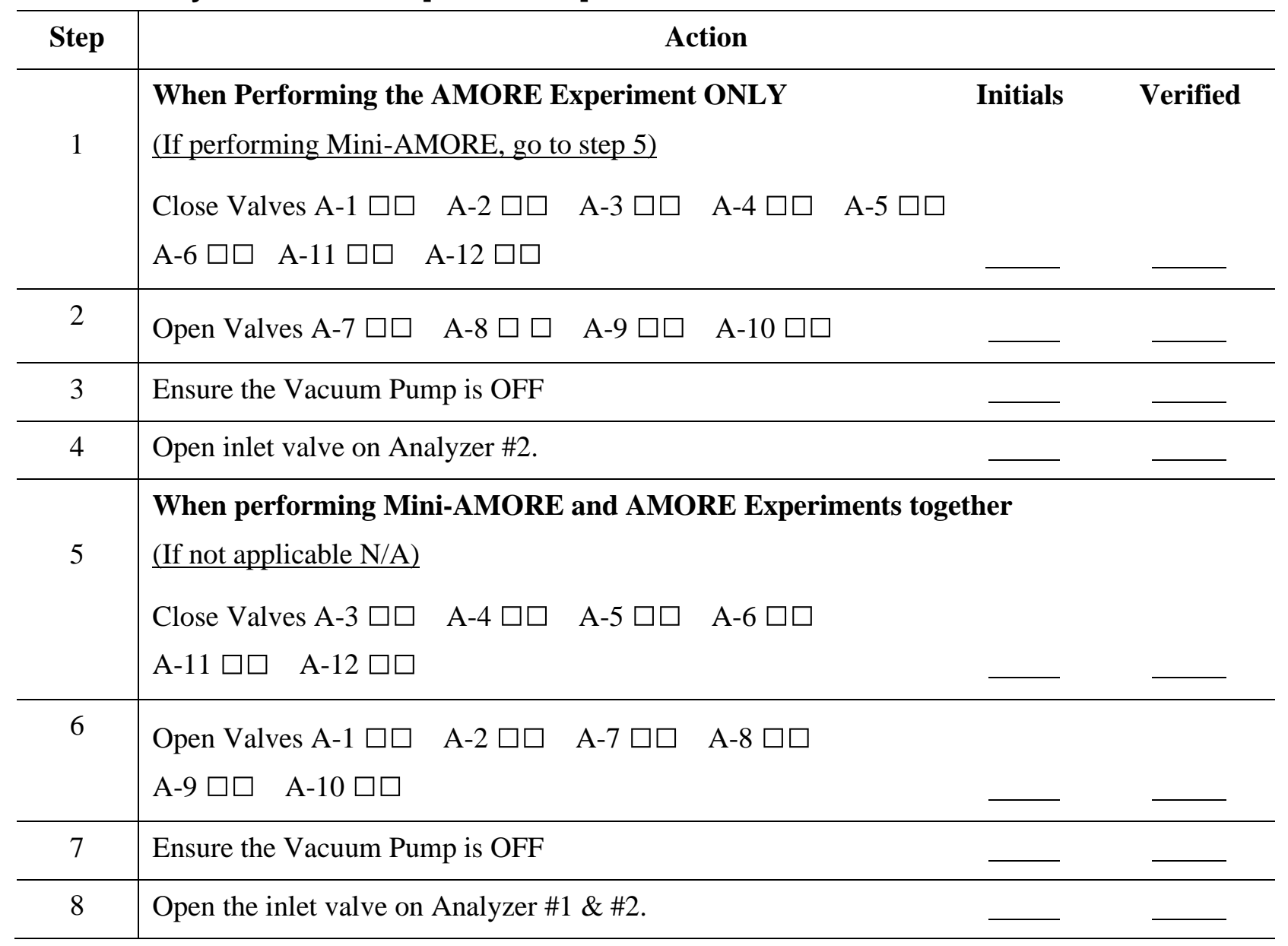


3.2.6 Gas Cylinders in D032 [ASE2.5.2.1]

\begin{tabular}{c|lll}
\hline Step & \multicolumn{3}{|c}{ Action } \\
\hline 1 & Open Isolation and cylinder valves on the Oxygen Tank. & Initials & Verified \\
\hline 2 & Open Isolation and cylinder valves on Helium/Xenon Tank. & \\
\hline 3 & $\begin{array}{l}\text { Open Isolation and cylinder valves on Mini-AMORE } \\
\text { Helium/Xenon Tank when preforming that experiment. (If not applicable "N/A) }\end{array}$ & \\
\hline
\end{tabular}

3.2.7 Cell \# 2

\begin{tabular}{c|ccc}
\hline Step & \multicolumn{3}{|c}{ Action } \\
\\
\hline 1 & Chiller ON [ASE2.5.2.1] & Initials & Verified \\
& & & \\
\hline
\end{tabular}

\subsubsection{Gas Analysis Chassis in the D-101 LINAC Control Room}

\begin{tabular}{c|lcc}
\hline Step & \multicolumn{3}{|c}{ Action } \\
\hline 1 & Catalyst Pump ON & Initials & Verified \\
\hline 2 & Sampling Pump ON & - & - \\
\hline 3 & Catalyst Pump Alarm Bypass OFF & - & - \\
\hline 4 & Sampling Pump Alarm Bypass OFF & \\
\hline 5 & Solution Vessel Pressure alarm high setting 990mbar & \\
\hline 6 & Catalyst Pump Pressure alarm high setting < Gas Collection System & \\
\hline
\end{tabular}

\section{Records Created by Work Process}

The records listed below must be retained as indicated.

Description of Record (include form number if applicable)
Active

Records

Custodia

n

Active
Records
Retentio
$\quad$ n

Indexing

Method, Storage

Medium
Federal Retention

Requirements* 


\begin{tabular}{l|c|c|l|l}
\hline $\begin{array}{l}\text { Description of Record } \\
\text { (include form number } \\
\text { if applicable) }\end{array}$ & $\begin{array}{c}\text { Active } \\
\text { Records } \\
\text { Custodia } \\
\mathbf{n}\end{array}$ & $\begin{array}{c}\text { Active } \\
\text { Records } \\
\text { Retentio } \\
\mathbf{n}\end{array}$ & $\begin{array}{l}\text { Indexing } \\
\text { Method, Storage } \\
\text { Medium }\end{array}$ & $\begin{array}{l}\text { Federal Retention } \\
\text { Requirements* }\end{array}$ \\
\hline $\begin{array}{l}\text { Completed LEAF-PROC- } \\
018\end{array}$ & $\begin{array}{c}\text { Facility } \\
\text { Manager }\end{array}$ & 3 years & $\begin{array}{l}\text { Index by job date } \\
\text { and name, store on } \\
\text { paper or } \\
\text { electronically }\end{array}$ & $\begin{array}{l}\text { Destroy 75 years } \\
\text { after the date of the } \\
\text { permit (DOE ADM } \\
18.37)\end{array}$ \\
\hline
\end{tabular}

*If records are maintained in a business information system that is not currently programmed to purge digital records based on age, the records may be retained in that system past the indicated destruction date.

\section{$5 \quad$ Related Documents}

This procedure implements requirements established by the following safety basis documents:

- LEAF-SAD-100, Linac Safety Assessment Document

- LEAF-ASE-100, Linac Accelerator Safety Envelope

This procedure implements requirements established by the following Argonne policies and procedures:

- LMS-PROC-188, Accelerator Safety

- EGS-PP-100, Configuration Management Program Plan for Accelerators

\section{Definitions}

None

7 About this Procedure

\begin{tabular}{ll}
\hline Issuing organization: & Low Energy Accelerator Facility \\
\hline Procedure owner: & M. Kalensky \\
\hline Point of contact: & M. Kalensky \\
\hline Review cycle (months): & 36 \\
\hline Date last revised: & 3.18 .2020 \\
\hline Date last reviewed: & 3.23 .2020 \\
\hline
\end{tabular}

\section{Summary of Changes in This Version}

Change in Section 3.2.2: Step 1 "Turn off the Pump" has been removed since the "Pump" is no longer in use. There are only 5 Steps in this version so they have been re-numbered.

In Section 3.2.2: Step 1: "Close Valves" V-15, V-17 and V-18 have been added. "Close Valves" V-5 has been deleted. (This reflects changes made to the manifold to accommodate new experiments) 
In Section 3.2.2: Step 2: "Open Valves" V-16 and V-19 has been added. . (This reflects changes made to the manifold to accommodate new experiments) 


\section{APPENDIX 11}

LEAF-PROC-020, Rev. 2: Maintenance and Leak Testing in Catalyst Pump Enclosure 


\section{Maintenance and Leak Testing in Catalyst Pump Enclosure}

Low Energy Accelerator Facility, LEAF-PROC-020, Rev. 2

Approved:

Date:

Sergey Chemerisov, Manager, IVEM/LEAF

Effective Date: using is current by comparing the revision number as printed copies can be obsolete. 


\section{Purpose}

Establish the process for ...

\section{Scope}

This procedure applies to the following Argonne activities and entities.

\begin{tabular}{ll}
\hline LMS core processes: & Asset Management \\
\hline Organizations: & Experimental Operations and Facilities (EOF) Division \\
\hline Buildings: & 211 \\
\hline Specific locations: & LINAC \\
\hline $\begin{array}{l}\text { Other applicability } \\
\text { factors: }\end{array}$ & None \\
\hline Exclusions: & None \\
\hline USI applicability: & Yes \\
\hline
\end{tabular}

\section{Work Process}

\subsection{Introduction}

The following applies to maintenance and replacement of parts in the Catalyst Pump Enclosure

\subsection{Step-by-Step Procedure}

The steps below are mandatory unless noted otherwise. This procedure is to be performed by <leave blank>.

NOTE: *Do Not apply a vacuum of $<800$ mbar to the system.

NOTE: *Do Not apply a pressure of $>1345$ mbar to the system.

NOTE: *Do not set the Setpoint 1 of Chamber \#1 on the Gas Collection System to $<800$ mbar.

\subsubsection{Purging and Maintenance}

\begin{tabular}{c|l}
\hline Step & \multicolumn{1}{|c}{ Action } \\
\hline 1 & $\begin{array}{l}\text { Ensure that solution from the Solution Vessel has been transferred to the Dump Tank or } \\
\text { Verification Tank. }\end{array}$ \\
\hline 2 & $\begin{array}{l}\text { In the LINAC Control Room, turn off the Catalyst Pump and Sampling Pump. Set Chassis } \\
\# 1 \text { for control in D-035 (downstairs). }\end{array}$ \\
\hline 3 & In D-032, open the helium cylinder and isolation valves. \\
\hline 4 & In the Gas Collection System Enclosure, Close valve GC-1. \\
\hline 5 & On the Dump Tank, close valves D-1A, D2A and D-3A to isolate it. \\
\hline 6 & $\begin{array}{l}\text { On the Gas Collection System Chassis for Chamber \#1 control, set the Setpoint-1 to 940 } \\
\text { mbar. Then set the Dead-band to 10mbar. }\end{array}$ \\
\hline
\end{tabular}

The current version of this procedure resides in the Argonne Document Center. Verify that the copy of the procedure you are using is current by comparing the revision number as printed copies can be obsolete. 


\begin{tabular}{c|l}
\hline Step & \multicolumn{1}{c}{ Action } \\
\hline 7 & Install the pressure meter and turn on the switch \\
\hline 8 & On the Hub Manifold (see Figure 1), open valve V-3 to allow helium into the manifold. \\
\hline 9 & $\begin{array}{l}\text { Close valves to isolate the various parts of the AMORE system. Close V-2 and V-10 to } \\
\text { isolate the Analytical Manifold in D-024. Close V-11 to isolate the Primary Recovery } \\
\text { Glovebox. }\end{array}$ \\
\hline 10 & Open valves V-1, V-6, V-7, V-9 and V-19. \\
\hline 11 & Actuate SV-3 to purge with helium for about 5 minutes. \\
\hline 12 & De-actuate SV-3 and allow the Gas Collection System to evacuate. \\
\hline 13 & Perform maintenance. \\
\hline
\end{tabular}

\subsubsection{Leak Test}

\begin{tabular}{c|l}
\hline Step & \multicolumn{1}{c}{ Action } \\
\hline 1 & On the Hub Manifold, Close V-6 to isolate the Gas Collection System. \\
\hline 2 & $\begin{array}{l}\text { Pressurize the Catalyst Pump fittings to 1100 mbar by actuating SV-3 (open). Close when } \\
\text { complete. }\end{array}$ \\
\hline 3 & Use the Leak Detector sniffer to test fittings. \\
\hline 4 & When complete, on the Hub Manifold, open valve V-6. \\
\hline 5 & Close valve V-3 \\
\hline 6 & $\begin{array}{l}\text { Reset the settings on the Gas Collection System Chassis controller for Chamber \#1 Setpoint- } \\
1 \text { anywhere from 900 to 960mbar and Dead-band to 10. }\end{array}$ \\
\hline
\end{tabular}




\section{Records Created by Work Process}

The records listed below must be retained as indicated.

\begin{tabular}{l|c|c|l|l}
\hline $\begin{array}{l}\text { Description of Record } \\
\text { (include form number if } \\
\text { applicable) }\end{array}$ & $\begin{array}{c}\text { Active } \\
\text { Records } \\
\text { Custodian }\end{array}$ & $\begin{array}{c}\text { Active } \\
\text { Records } \\
\text { Retention }\end{array}$ & $\begin{array}{l}\text { Indexing Method, } \\
\text { Storage Medium }\end{array}$ & $\begin{array}{l}\text { Federal Retention } \\
\text { Requirements* }\end{array}$ \\
\hline$<\ldots .>$ & $\begin{array}{c}\text { Facility } \\
\text { Manager }\end{array}$ & 3 years & $\begin{array}{l}\text { Index by job date } \\
\text { and name, store on } \\
\text { paper or } \\
\text { electronically }\end{array}$ & $\begin{array}{l}\text { Destroy 75 years after } \\
\text { the date of the permit } \\
\text { (DOE ADM 18.37) }\end{array}$ \\
\hline
\end{tabular}

*If records are maintained in a business information system that is not currently programmed to purge digital records based on age, the records may be retained in that system past the indicated destruction date.

\section{$5 \quad$ Related Documents}

This procedure implements requirements established by the following safety basis documents:

- LEAF-SAD-100, Linac Safety Assessment Document

- LEAF-ASE-100, Linac Accelerator Safety Envelope

This procedure implements requirements established by the following Argonne policies and procedures:

- LMS-PROC-188, Accelerator Safety

- EGS-PP-100, Configuration Management Program Plan for Accelerators

\section{Definitions}

None

\section{$7 \quad$ About this Procedure}

\begin{tabular}{ll}
\hline Issuing organization: & Low Energy Accelerator Facility \\
\hline Procedure owner: & S. Chemerisov \\
\hline Point of contact: & S. Chemerisov \\
\hline Review cycle (months): & 36 \\
\hline Date last revised: & TBD \\
\hline Date last reviewed: & TBD \\
\hline
\end{tabular}

\section{Summary of Changes in This Version}

Section 3.2.1 Step 7 Setpoint-1 Changed from 900 to 940 mbar and Then Dead-band from 50 to 10mbar.

The current version of this procedure resides in the Argonne Document Center. Verify that the copy of the procedure you are using is current by comparing the revision number as printed copies can be obsolete. 


\section{Exhibit A: Figure}

Figure 1: Diagram of the Hub Manifold in the Gas Distribution Hub Enclosure.

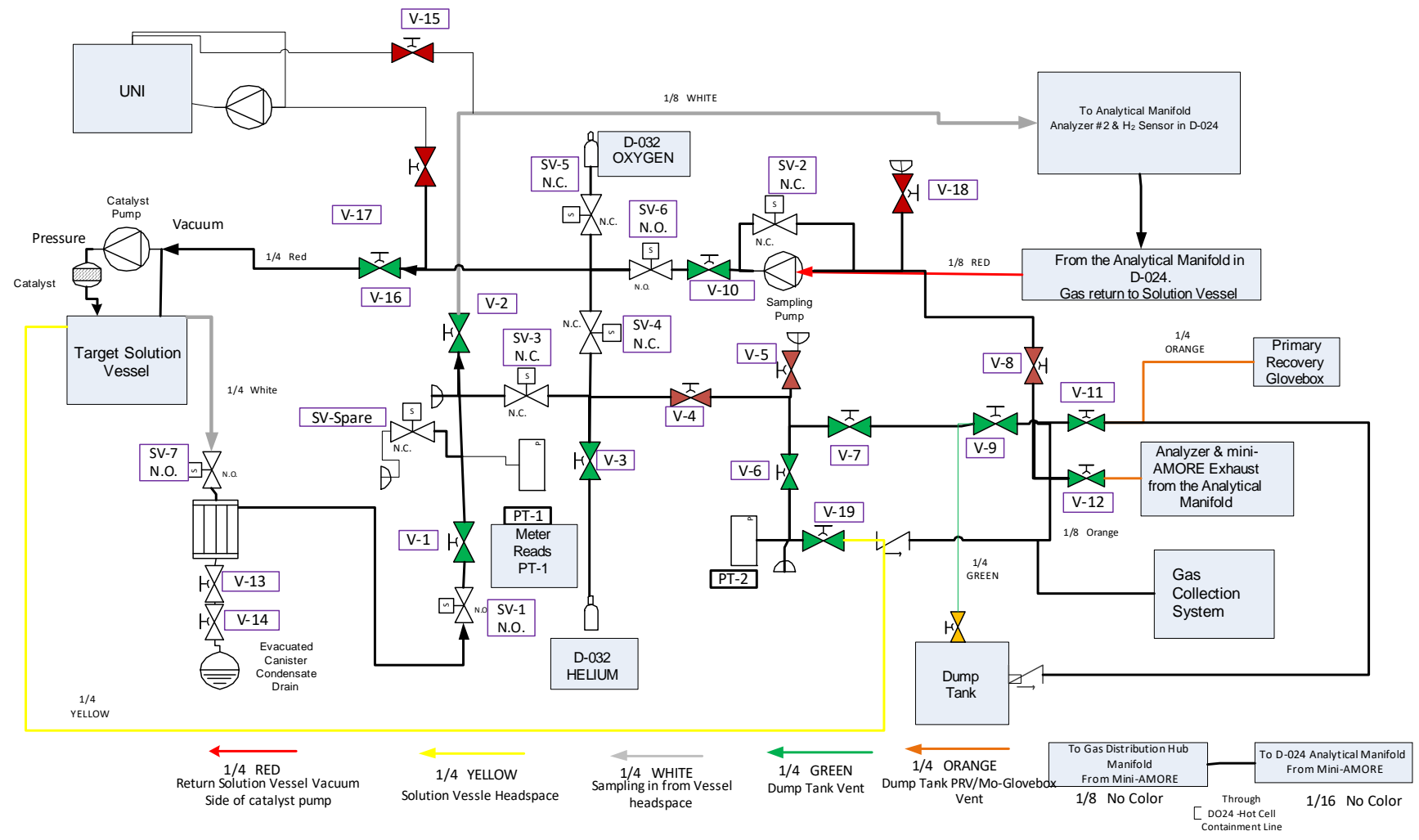

The current version of this procedure resides in the Argonne Document Center. Verify that the copy of the procedure you are using is current by comparing the revision number as printed copies can be obsolete. 


\section{Exhibit B: Tables}

TABLE 1. Hub Manifold Manual Valves - Located in the Gas Distribution Hub Enclosure (D-035)

\begin{tabular}{|c|c|c|}
\hline Valve & Description & Function \\
\hline V-1 & Condenser Isolation & Isolates the Condenser from the Hub Manifold \\
\hline $\begin{array}{l}\mathrm{V}-2 \text { and } \\
\mathrm{V}-10\end{array}$ & $\begin{array}{l}\text { Analytical Manifold } \\
\text { Isolation }\end{array}$ & $\begin{array}{l}\text { Closing V-2 and V-10 isolate the Analytical Manifold in D- } \\
024 \text { from the Hub Manifold. }\end{array}$ \\
\hline V-3 & Helium Isolation Valve & $\begin{array}{l}\text { Allows helium in to the Hub Manifold for purging the Target } \\
\text { Solution Vessel, Dump Tank and the Analytical Manifold. }\end{array}$ \\
\hline V-4 & Main Isolation & $\begin{array}{l}\text { Isolates sampling and purge connections from the Gas } \\
\text { Collection System connections on the Hub Manifold. }\end{array}$ \\
\hline V-5 & Vacuum Inlet & Opens the manifold to Vacuum Pump inlet. \\
\hline V-6 & $\begin{array}{l}\text { Target Solution Vessel } \\
\text { Head Space Isolation }\end{array}$ & $\begin{array}{l}\text { Isolates the headspace of the Solution Vessel from the Gas } \\
\text { Collection System and Vacuum Pump. Opening V-6, V-7 and } \\
\text { V-9 opens the Solution Vessel headspace to the Gas } \\
\text { Collection System. }\end{array}$ \\
\hline V-7 & $\begin{array}{l}\text { Target Solution Vessel } \\
\text { Head Space Isolation }\end{array}$ & $\begin{array}{l}\text { Opening V-6, V-7, and V-9 opens the Solution Vessel } \\
\text { headspace to the Gas Collection System. }\end{array}$ \\
\hline V-8 & $\begin{array}{l}\text { Vacuum Exhaust to Gas } \\
\text { Collection }\end{array}$ & $\begin{array}{l}\text { Opens the vacuum pump exhaust to the Gas Collection } \\
\text { System }\end{array}$ \\
\hline V-9 & Gas Collection Isolation & Isolates the Hub Manifold from the Gas Collection System \\
\hline V-10 & $\begin{array}{l}\text { Analytical Manifold } \\
\text { Isolation }\end{array}$ & $\begin{array}{l}\text { Closing V-2 and V-10 isolate the Analytical Manifold in D- } \\
024 \text { from the Hub Manifold. (Attached to the Sampling } \\
\text { Pump) }\end{array}$ \\
\hline V-11 & $\begin{array}{l}\text { Primary Recovery } \\
\text { Glovebox Isolation }\end{array}$ & $\begin{array}{l}\text { Isolates the Primary Recovery Glovebox from the Gas } \\
\text { Collection System }\end{array}$ \\
\hline V-12 & $\begin{array}{l}\text { Analyzer Exhaust } \\
\text { Isolation }\end{array}$ & $\begin{array}{l}\text { Isolates the Analyzer Exhaust from the Gas Collection } \\
\text { System }\end{array}$ \\
\hline V-13 & $\begin{array}{l}\text { Condensate Collection } \\
\text { Isolation }\end{array}$ & $\begin{array}{l}\text { Isolates the Condenser from the condensate collection } \\
\text { canisters valve. }\end{array}$ \\
\hline V-14 & $\begin{array}{l}\text { Condensate Collection } \\
\text { Canister valve }\end{array}$ & Valve on the Condensate Collection Canister \\
\hline V-15 & UNI to Analysis & UNI to D024 Analytical Hub \\
\hline V-16 & Return to TSV & Return to TSV from D024 Analytical \\
\hline V-17 & UNI return & UNI return from D024 Analytical Hub \\
\hline V-18 & Spare (Capped) & \\
\hline
\end{tabular}

The current version of this procedure resides in the Argonne Document Center. Verify that the copy of the procedure you are using is current by comparing the revision number as printed copies can be obsolete. 


\begin{tabular}{l|l|l} 
V-19 & $\begin{array}{l}\text { Vessel Headspace to Gas } \\
\text { Collection }\end{array}$ & Vessel to Gas Collection \\
\hline
\end{tabular}


TABLE 2. Hub Manifold Solenoid Valves - Located in the Gas Distribution Hub Enclosure (D035) (Controls on Chassis 1)

\begin{tabular}{|c|c|c|}
\hline Valve & Description & Function \\
\hline SV-1 & Gas Sampling Isolation & $\begin{array}{l}\text { Normally Open - Allows the gas stream from the Solution } \\
\text { Vessel into the Hub Manifold. Purge the Analytical Manifold } \\
\text { in D- } 024 \text { post irradiation by actuating SV-3 and SV-1. }\end{array}$ \\
\hline SV-2 & Sampling Pump Bypass & $\begin{array}{l}\text { Normally Closed }- \text { Should the sampling pump fail, the } \\
\text { Analytical Manifold in D-024 can be purged by actuating SV- } \\
1, \text { SV-2 and SV-3. }\end{array}$ \\
\hline SV-3 & $\begin{array}{l}\text { Isolation Valve/Helium } \\
\text { Purge }\end{array}$ & $\begin{array}{l}\text { Normally Closed - Isolates the Gas Sample Path from the } \\
\text { helium purge line during an experiment. Open it to purge the } \\
\text { Analytical Manifold for calibration and pre-run. You can } \\
\text { purge the Analytical Manifold post irradiation by actuating } \\
\text { SV-3 and SV-7. }\end{array}$ \\
\hline SV-4 & Helium purge & 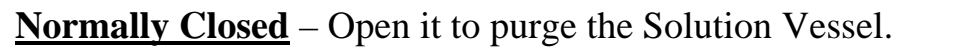 \\
\hline SV-5 & Oxygen Addition & $\begin{array}{l}\text { Normally Closed }- \text { Open it to add oxygen to the Solution } \\
\text { Vessel to reduce hydrogen concentration. Use in conjunction } \\
\text { with the Oxygen Flow potentiometer on Chassis } 2 \text {. }\end{array}$ \\
\hline SV-6 & $\begin{array}{l}\text { Sampling Pump } \\
\text { Isolation }\end{array}$ & $\frac{\text { Normally Open }}{\text { Solution Vessel }}-$ Isolates the Sampling Pump from the \\
\hline SV-7 & Gas Sampling Isolation & $\begin{array}{l}\text { Normally Open }- \text { Allows the analytical gas stream from the } \\
\text { Solution Vessel into the Condenser. }\end{array}$ \\
\hline
\end{tabular}

TABLE 3. Dump Tank Valves - Located beneath the D-035 Hot Cell

\begin{tabular}{|c|l|l|}
\hline Valve & \multicolumn{1}{|c|}{ Description } & \multicolumn{1}{c|}{ Function } \\
\hline $\begin{array}{c}\text { D-1A } \\
\text { and D-1B }\end{array}$ & $\begin{array}{l}\text { Pick-up line to Primary } \\
\text { Recovery Glovebox }\end{array}$ & $\begin{array}{l}\text { Siphons solution from the bottom of the Dump Tank to } \\
\text { transport it to the Primary Recovery Glovebox. }\end{array}$ \\
\hline $\begin{array}{c}\text { D-2A } \\
\text { and D-2B }\end{array}$ & $\begin{array}{l}\text { Return Line from } \\
\text { Primary Recovery } \\
\text { Glovebox }\end{array}$ & $\begin{array}{l}\text { Returns solution to the top of the DumpTank from the } \\
\text { Primary Recovery Glovebox }\end{array}$ \\
\hline $\begin{array}{c}\text { D-3A } \\
\text { and D-3B }\end{array}$ & $\begin{array}{l}\text { Dump Tank headspace } \\
\text { gas to Gas Distribution } \\
\text { Hub Manifold }\end{array}$ & $\begin{array}{l}\text { Allows for gas displacement when solution is entering the } \\
\text { Dump Tank }\end{array}$ \\
\hline
\end{tabular}

The current version of this procedure resides in the Argonne Document Center. Verify that the copy of the procedure you are using is current by comparing the revision number as printed copies can be obsolete. 


\section{APPENDIX 12}

LEAF-PROC-021, Rev. 2: Maintenance and Leak Testing in D-024 Analytical Enclosure 


\section{Maintenance and Leak Testing in D-024 Analytical Enclosure}

Low Energy Accelerator Facility, LEAF-PROC-021, Rev. 2

Approved:

Date:

Sergey Chemerisov, Manager, IVEM/LEAF

Effective Date:

The current version of this procedure resides in the Argonne Document Center. Verify that the copy of the procedure you are using is current by comparing the revision number as printed copies can be obsolete. 


\section{Purpose}

Establish the process for maintenance and leak testing of the D-024 Analytical Enclosure

\section{Scope}

This procedure applies to the following Argonne activities and entities.

\begin{tabular}{ll}
\hline LMS core processes: & Asset Management \\
\hline Organizations: & Experimental Operations and Facilities (EOF) Division \\
\hline Buildings: & 211 \\
\hline Specific locations: & LINAC \\
\hline $\begin{array}{l}\text { Other applicability } \\
\text { factors: }\end{array}$ & None \\
\hline Exclusions: & None \\
\hline USI applicability: & Yes \\
\hline
\end{tabular}

\section{Work Process}

\subsection{Introduction}

The following applies to maintenance of equipment located in the D024 Analytical Enclosure

\subsection{Step-by-Step Procedure}

The steps below are mandatory unless noted otherwise. This procedure is to be performed by qualified personel.

NOTE: *Do Not apply a vacuum of $<800$ mbar to the system.

NOTE: *Do Not apply a pressure of $>\mathbf{1 3 4 5} \mathbf{m b a r}$ to the system.

NOTE: *Do not set the Setpoint 1 of Chamber \#1 on the Gas Collection System to $<800$ mbar.

\subsubsection{Purging and Maintenance}

\begin{tabular}{c|l}
\hline Step & \multicolumn{1}{c}{ Action } \\
\hline 1 & $\begin{array}{l}\text { In the LINAC Control Room, turn off the Catalyst Pump and Sampling Pump. Set Chassis } \\
\# 1 \text { for control in D-035 (downstairs). }\end{array}$ \\
\hline 2 & $\begin{array}{l}\text { In D-032, open the helium cylinder and isolation valves. The regulator should be set to less } \\
\text { than 5psig. }\end{array}$ \\
\hline 3 & Turn off both Analyzers. \\
\hline 4 & On the Gas Collection System, Close GC-1. \\
\hline 6 & $\begin{array}{l}\text { On the Dump Tank, close valves D-1A, D-2A and D-3A to isolate it. } \\
900 \text { mbar. Then set the Dead-band to 50 mbar. }\end{array}$ \\
\hline
\end{tabular}




\begin{tabular}{c|l}
\hline Step & \multicolumn{1}{c}{ Action } \\
\hline 7 & On the Gas Distribution Hub Enclosure install the pressure meter and turn on the switch. \\
\hline 8 & On the Hub Manifold (see Figure 1), open valve V-3 to allow helium into the manifold \\
\hline 9 & Close V-1, V-4, V-6, V-8, V-10 and V-11. \\
\hline 10 & Actuate SV-3 (Open) to allow helium to the Analytical Manifold in D024 \\
\hline 11 & $\begin{array}{l}\text { On the Analytical Manifold in the D-024 Analytical Enclosure (See Figure 2), valve A-7 } \\
\text { allows helium into the manifold. } \\
\text { Close valves A-1, A-4, A-5, A-11, and A-12. Purge the manifold by opening A-2, A-3, A-6, } \\
\text { A-7, A-8, A-9, and A-10. } \\
\text { Purge for about a minute then close valve A-7. }\end{array}$ \\
\hline 12 & \begin{tabular}{l} 
Allow the manifold to evacuate. Perform maintenance on valves or devices. \\
\hline
\end{tabular}
\end{tabular}

\subsubsection{Leak Test}

\begin{tabular}{c|l}
\hline Step & \multicolumn{1}{c}{ Action } \\
\hline 1 & On the Gas Distribution Hub Manifold in D035, Close valve V-12. \\
\hline 2 & $\begin{array}{l}\text { On the D024 Analytical manifold, close valve A-10. Open A-7 to pressurize the manifold to } \\
\text { about 1100 mbar as read on the manometer. Close A-7 when complete. }\end{array}$ \\
\hline 3 & Use the Leak Detector sniffer to check fittings. \\
\hline 4 & $\begin{array}{l}\text { When complete, on the Gas Distribution Hub Manifold open V-12 to relieve manifold } \\
\text { pressure then open V-6. De-actuate (close) SV-3 and close V-3. }\end{array}$ \\
\hline 5 & On the D024 Analytical Manifold, close valves A-2 and A-3. \\
\hline 7 & $\begin{array}{l}\text { Reset the settings on the Gas Collection System Controller (Chamber \#1 settings Setpoint-1 } \\
\text { anywhere from 900 to 960 mbar and Dead-band to 10). }\end{array}$ \\
\hline 8 & Restart the Analyzers. \\
\hline
\end{tabular}

\section{Records Created by Work Process}

The records listed below must be retained as indicated.

\begin{tabular}{l|c|c|l|l}
\hline $\begin{array}{l}\text { Description of Record } \\
\text { (include form number if } \\
\text { applicable) }\end{array}$ & $\begin{array}{c}\text { Active } \\
\text { Records } \\
\text { Custodian }\end{array}$ & $\begin{array}{c}\text { Active } \\
\text { Records } \\
\text { Retention }\end{array}$ & $\begin{array}{l}\text { Indexing Method, } \\
\text { Storage Medium }\end{array}$ & $\begin{array}{l}\text { Federal Retention } \\
\text { Requirements* }\end{array}$ \\
\hline$<\ldots .>$ & Facility & 3 years & $\begin{array}{l}\text { Index by job date } \\
\text { and name, store on }\end{array}$ & $\begin{array}{l}\text { Destroy 75 years after } \\
\text { the date of the permit }\end{array}$ \\
\hline
\end{tabular}




\begin{tabular}{l|c|l|l|l}
\hline $\begin{array}{l}\text { Description of Record } \\
\text { (include form number if } \\
\text { applicable) }\end{array}$ & $\begin{array}{c}\text { Active } \\
\text { Records } \\
\text { Custodian }\end{array}$ & $\begin{array}{c}\text { Active } \\
\text { Records } \\
\text { Retention }\end{array}$ & $\begin{array}{l}\text { Indexing Method, } \\
\text { Storage Medium }\end{array}$ & $\begin{array}{l}\text { Federal Retention } \\
\text { Requirements* }\end{array}$ \\
\hline & Manager & $\begin{array}{l}\text { paper or } \\
\text { electronically }\end{array}$ & (DOE ADM 18.37) \\
\hline
\end{tabular}

*If records are maintained in a business information system that is not currently programmed to purge digital records based on age, the records may be retained in that system past the indicated destruction date.

\section{Related Documents}

This procedure implements requirements established by the following safety basis documents:

- LEAF-SAD-100, Linac Safety Assessment Document

- LEAF-ASE-100, Linac Accelerator Safety Envelope

This procedure implements requirements established by the following Argonne policies and procedures:

- $\quad$ LMS-PROC-188, Accelerator Safety

- $\quad$ EGS-PP-100, Configuration Management Program Plan for Accelerators

\section{Definitions}

None

\section{$7 \quad$ About this Procedure}

\begin{tabular}{ll}
\hline Issuing organization: & Low Energy Accelerator Facility \\
\hline Procedure owner: & S. Chemerisov \\
\hline Point of contact: & S. Chemerisov \\
\hline Review cycle (months): & 36 \\
\hline Date last revised: & TBD \\
\hline Date last reviewed: & TBD \\
\hline
\end{tabular}

\section{Summary of Changes in This Version}

Section 3.2.1 Step 1 "Ensure that solution from the Solution Vessel has been transferred to the Dump Tank or Verification Tank.” This has been removed because it is unnecessary.

Section 3.2.1 Step 7 Setpoint-1 change from 850 to 900 mbar. Then set the Dead-band from 100 to 50 mbar.

Section 3.2.2 Has been edited because the previous versions steps were too complex and unnecessary.

Exhibit A Figure has been updated.

Exhibit B Table 1 has been updated. New valves V-15, V-16, V-17, V-18 \& V-19. 


\section{LEAF-PROC-021}

\section{Exhibit A: Figures}

\section{Figure 1. Diagram of the Hub Manifold.}

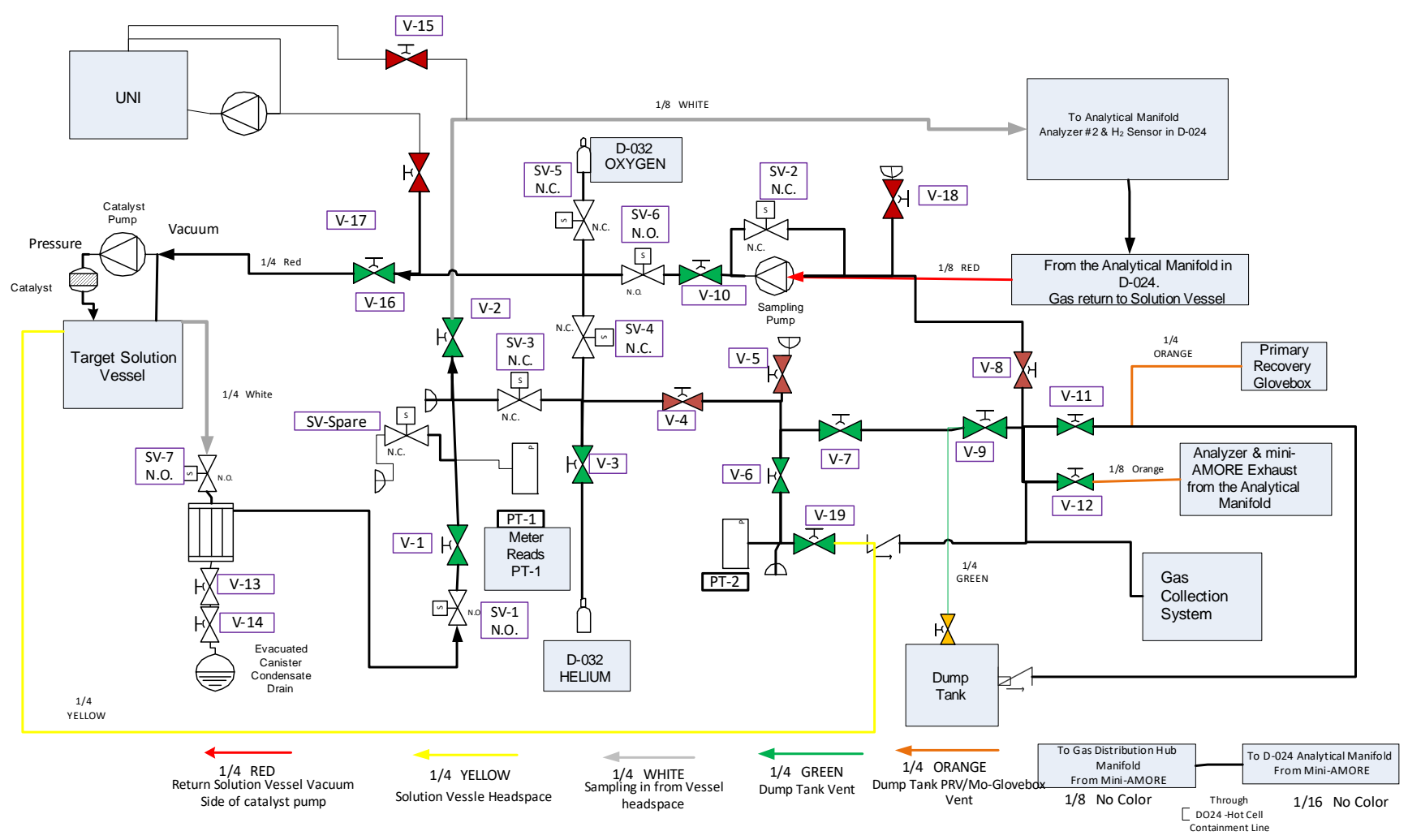

The current version of this procedure resides in the Argonne Document Center. Verify that the copy of the procedure you are using is current by comparing the revision number as printed copies can be obsolete. 


\section{Figure 2. Diagram of the D-024 Analytical Manifold.}

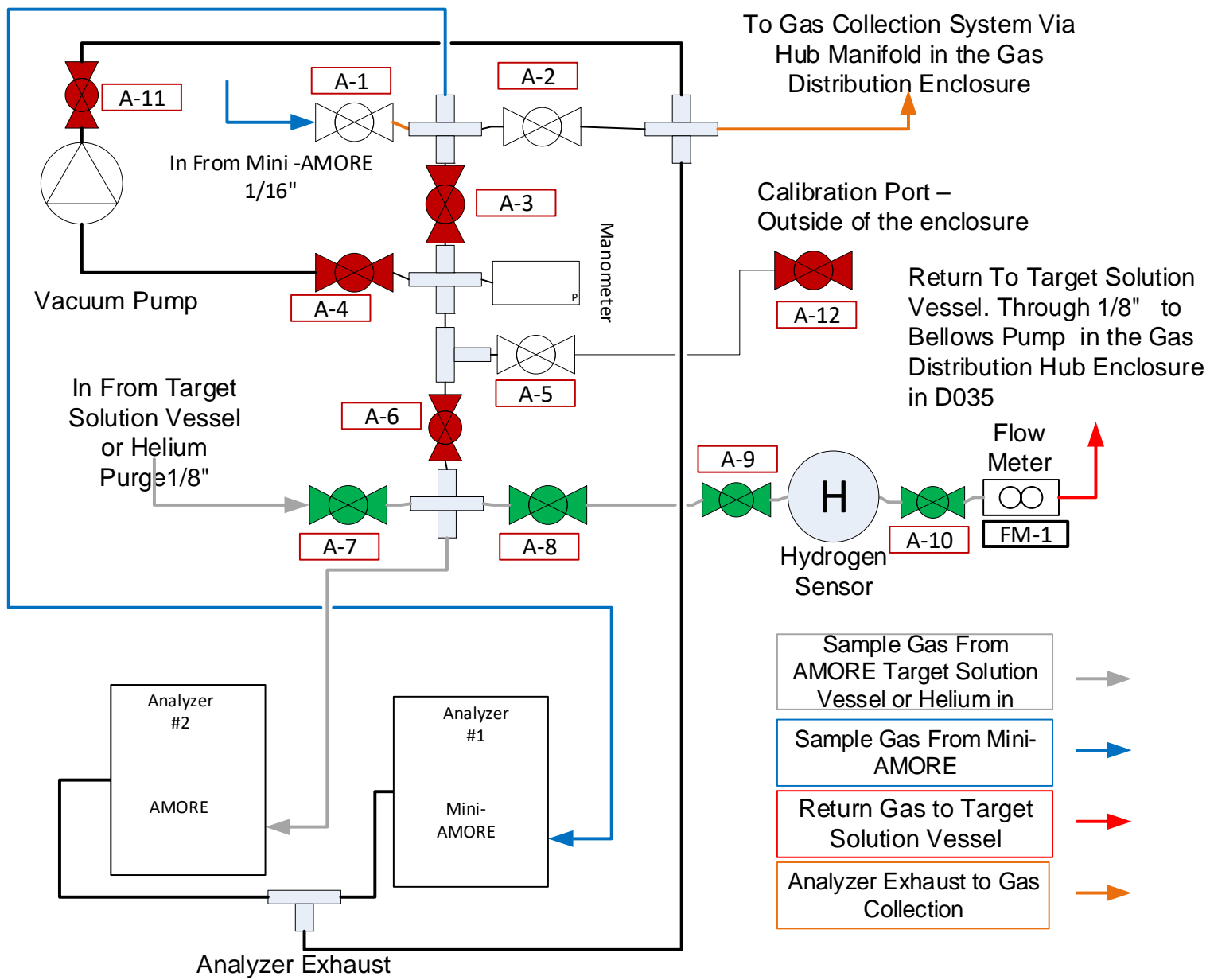

\section{Exhibit B: Tables}

TABLE 1. Hub Manifold Manual Valves - Located in the D-035 Gas Distribution Hub Enclosure

\begin{tabular}{|c|l|l|}
\hline Valve & \multicolumn{1}{|c|}{ Description } & \multicolumn{1}{c|}{ Function } \\
\hline V-1 & Condenser Isolation & Isolates the Condenser from the Hub Manifold \\
\hline $\begin{array}{c}\text { V-2 and } \\
\text { V-10 }\end{array}$ & $\begin{array}{l}\text { Analytical Manifold } \\
\text { Isolation }\end{array}$ & $\begin{array}{l}\text { Closing V-2 and V-10 isolate the Analytical Manifold in D- } \\
\text { 024 from the Hub Manifold. }\end{array}$ \\
\hline V-3 & Helium Isolation Valve & $\begin{array}{l}\text { Allows helium in to the Hub Manifold for purging the Target } \\
\text { Solution Vessel, Dump Tank and the Analytical Manifold. }\end{array}$ \\
\hline V-4 & Main Isolation & $\begin{array}{l}\text { Isolates sampling and purge connections from the Gas } \\
\text { Collection System connections on the Hub Manifold. }\end{array}$ \\
\hline V-5 & Vacuum Inlet & Opens the manifold to Vacuum Pump inlet. \\
\hline V-6 & $\begin{array}{l}\text { Target Solution Vessel } \\
\text { Head Space Isolation }\end{array}$ & $\begin{array}{l}\text { Isolates the headspace of the Solution Vessel from the Gas } \\
\text { Collection System and Vacuum Pump. Opening V-6, V-7 and }\end{array}$ \\
\hline
\end{tabular}

The current version of this procedure resides in the Argonne Document Center. Verify that the copy of the procedure you are using is current by comparing the revision number as printed copies can be obsolete. 


\begin{tabular}{|c|c|c|}
\hline & & $\begin{array}{l}\text { V-9 opens the Solution Vessel headspace to the Gas } \\
\text { Collection System. }\end{array}$ \\
\hline V-7 & $\begin{array}{l}\text { Target Solution Vessel } \\
\text { Head Space Isolation }\end{array}$ & $\begin{array}{l}\text { Opening V-6, V-7, and V-9 opens the Solution Vessel } \\
\text { headspace to the Gas Collection System. }\end{array}$ \\
\hline V-8 & $\begin{array}{l}\text { Vacuum Exhaust to Gas } \\
\text { Collection }\end{array}$ & $\begin{array}{l}\text { Opens the vacuum pump exhaust to the Gas Collection } \\
\text { System }\end{array}$ \\
\hline V-9 & Gas Collection Isolation & Isolates the Hub Manifold from the Gas Collection System \\
\hline V-10 & $\begin{array}{l}\text { Analytical Manifold } \\
\text { Isolation }\end{array}$ & $\begin{array}{l}\text { Closing V-2 and V-10 isolate the Analytical Manifold in D- } \\
024 \text { from the Hub Manifold. (Attached to the Sampling } \\
\text { Pump) }\end{array}$ \\
\hline V-11 & $\begin{array}{l}\text { Primary Recovery } \\
\text { Glovebox Isolation }\end{array}$ & $\begin{array}{l}\text { Isolates the Primary Recovery Glovebox from the Gas } \\
\text { Collection System }\end{array}$ \\
\hline V-12 & $\begin{array}{l}\text { Analyzer Exhaust } \\
\text { Isolation }\end{array}$ & $\begin{array}{l}\text { Isolates the Analyzer Exhaust from the Gas Collection } \\
\text { System }\end{array}$ \\
\hline V-13 & $\begin{array}{l}\text { Condensate Collection } \\
\text { Isolation }\end{array}$ & $\begin{array}{l}\text { Isolates the Condenser from the condensate collection } \\
\text { canisters valve. }\end{array}$ \\
\hline V-14 & $\begin{array}{l}\text { Condensate Collection } \\
\text { Canister valve }\end{array}$ & Valve on the Condensate Collection Canister \\
\hline V-15 & UNI to Analysis & UNI gas to D024 Analytical Manifold \\
\hline V-16 & Return to TSV & TVS return from D024 Analytical Manifold \\
\hline V-17 & Return to UNI & UNI gas return from D024 Analytical Manifold \\
\hline V-18 & Spare (Capped) & \\
\hline V-19 & $\begin{array}{l}\text { TSV Headspace to Gas } \\
\text { Collection }\end{array}$ & TSV Headspace to Gas Collection \\
\hline
\end{tabular}


TABLE 2. Hub Manifold Solenoid Valves - Located in the D-035 Gas Distribution Hub Enclosure - (Controls on Chassis 1)

\begin{tabular}{|c|l|l|}
\hline Valve & \multicolumn{1}{|c|}{ Description } & \multicolumn{1}{c|}{ Function } \\
\hline SV-1 & Gas Sampling Isolation & $\begin{array}{l}\text { Normally Open }- \text { Allows the gas stream from the Target } \\
\text { Solution Vessel into the Hub Manifold. Purge the Analytical } \\
\text { Manifold in D-024 post irradiation by actuating SV-3 and } \\
\text { SV-1. }\end{array}$ \\
\hline SV-2 & Sampling Pump Bypass & $\begin{array}{l}\text { Normally Closed }- \text { Should the sampling pump fail, the } \\
\text { Analytical Manifold in D-024 can be purged by actuating } \\
\text { SV-1, SV-2, and SV-3. }\end{array}$ \\
\hline SV-3 & $\begin{array}{l}\text { Isolation Valve/Helium } \\
\text { Purge }\end{array}$ & $\begin{array}{l}\frac{\text { Normally Closed }- \text { Isolates the Gas Sample Path from the }}{\text { helium purge line during an experiment. Open it to purge the }} \\
\text { Analytical Manifold for calibration and pre-run. You can } \\
\text { purge the Analytical Manifold post irradiation by actuating } \\
\text { SV-3 and SV-7. }\end{array}$ \\
\hline SV-4 & Helium purge & $\begin{array}{l}\text { Normally Closed }- \text { Open it to purge the Target Solution } \\
\text { Vessel. }\end{array}$ \\
\hline SV-5 & Oxygen Addition & $\begin{array}{l}\text { Normally Closed }- \text { Open it to add oxygen to the Solution } \\
\text { Vessel to reduce hydrogen concentration. Use in conjunction } \\
\text { with the Oxygen Flow potentiometer on Chassis 2. }\end{array}$ \\
\hline SV-6 & Sampling Pump Isolation & $\begin{array}{l}\text { Normally Open }- \text { Isolates the Sampling Pump from the } \\
\text { Solution Vessel }\end{array}$ \\
\hline
\end{tabular}


TABLE 3. Analytical Manifold Valves - Located in the D-024 Analytical Enclosure

\begin{tabular}{|c|c|c|}
\hline Valve & Description & Function \\
\hline A-1 & Mini-AMORE isolation & Isolates Mini-AMORE from the manifold \\
\hline A-2 & $\begin{array}{l}\text { Gas Collection System } \\
\text { Isolation }\end{array}$ & $\begin{array}{l}\text { Close to isolate the manifold and Mini-AMORE from Gas } \\
\text { Collection System }\end{array}$ \\
\hline A-3 & Analyzer \#1 Isolation & Isolates Analyzer \#1 from the manifold \\
\hline A-4 & Vacuum Inlet & Opens the manifold to vacuum. \\
\hline A-5 & Calibration isolation & Isolate calibration gas \\
\hline A-6 & Analyzer \#2 Isolation & Isolates Analyzer \#2 from the manifold \\
\hline A-7 & Hub Manifold isolation & $\begin{array}{l}\text { Isolates the Hub Manifold in the D-035 Gas Distribution Hub } \\
\text { Enclosure from the Analytical Manifold }\end{array}$ \\
\hline A-8 & $\begin{array}{l}\text { Analyzer \#2 Hydrogen } \\
\text { Sensor isolation }\end{array}$ & Isolates Analyzer \#2 from the Hydrogen Sensor \\
\hline $\begin{array}{l}\text { A-9 and } \\
\text { A-10 }\end{array}$ & $\begin{array}{l}\text { Hydrogen Sensor } \\
\text { Isolation }\end{array}$ & Isolates the Hydrogen Sensor for removal \\
\hline A-11 & Vacuum Exhaust & $\begin{array}{l}\text { Isolates the Vacuum Pump exhaust from the Gas Collection } \\
\text { System }\end{array}$ \\
\hline A-12 & Calibration Gas & Allows calibration gas up to A-5 \\
\hline
\end{tabular}




\section{APPENDIX 13}

LEAF-PROC-022, Rev. 2: Maintenance and Leak Testing in D-035 Gas Distribution Hub Enclosure 


\section{Maintenance and Leak Testing in D-035 Gas Distribution Hub Enclosure}

Low Energy Accelerator Facility, LEAF-PROC-022, Rev. 2

Approved:

Date:

Sergey Chemerisov, Manager, IVEM/LEAF

Effective Date:

The current version of this procedure resides in the Argonne Document Center. Verify that the copy of the procedure you are using is current by comparing the revision number as printed copies can be obsolete. 


\section{Purpose}

Establish the process for maintenance and leak testing in the D-035 Gas Distribution Hub enclosure.

\section{Scope}

This procedure applies to the following Argonne activities and entities.

\begin{tabular}{ll}
\hline LMS core processes: & Asset Management \\
\hline Organizations: & Experimental Operations and Facilities (EOF) Division \\
\hline Buildings: & 211 \\
\hline Specific locations: & LINAC \\
\hline $\begin{array}{l}\text { Other applicability } \\
\text { factors: }\end{array}$ & None \\
\hline Exclusions: & None \\
\hline USI applicability: & Yes \\
\hline
\end{tabular}

\section{Work Process}

\subsection{Introduction}

The following pertains to equipment removal and installation in the Gas Distribution Hub enclosure.

\subsection{Step-by-Step Procedure}

The steps below are mandatory unless noted otherwise. This procedure is to be performed by qualified.

NOTE: *Do Not apply a vacuum of $<800$ mbar to the system.

NOTE: *Do Not apply a pressure of >1345 mbar to the system.

NOTE: *Do not set the Setpoint 1 of Chamber \#1 on the Gas Collection System to $<800$ mbar.

\subsubsection{Purge the Hub Manifold and perform maintenance}

See Exhibit A, Hub Manifold Diagram; and Exhibit B, Table 1, Hub Manifold Manual Valves; Table 2, Hub Manifold Solenoid Valves; and Table 3. Dump Tank Valves.

\begin{tabular}{c|l}
\hline Step & \multicolumn{1}{|c}{ Action } \\
\hline 1 & $\begin{array}{l}\text { In the LINAC Control Room, turn off the Catalyst Pump and Sampling Pump. Set } \\
\text { Chassis \#1 for control in D-035 (downstairs). }\end{array}$ \\
\hline 2 & $\begin{array}{l}\text { In D-032, open the helium cylinder and isolation valves. The regulator should be set to less } \\
\text { than 5 psig. }\end{array}$ \\
\hline 3 & Turn off both Analyzers. \\
\hline 4 & On the Gas Collection System, Close GC-1. \\
\hline 5 & On the Dump Tank, close valves D-1A, D-2A and D-3A to isolate the Dump Tank. \\
\hline
\end{tabular}

The current version of this procedure resides in the Argonne Document Center. Verify that the copy of the procedure you are using is current by comparing the revision number as printed copies can be obsolete. 


\begin{tabular}{c|l}
\hline Step & \multicolumn{1}{c}{ Action } \\
\hline 6 & $\begin{array}{l}\text { On the Gas Collection System Chassis for Chamber \#1 control, set the Setpoint-1 to } \\
900 \text { mbar. Then, set the Dead-band to 50 mbar. }\end{array}$ \\
\hline 7 & $\begin{array}{l}\text { On the D-035 Gas Distribution Hub Enclosure, install the pressure meter and turn on the } \\
\text { switch. }\end{array}$ \\
\hline 8 & On the Hub Manifold (see Figure 1), open valve V-3 to allow helium into the manifold. \\
\hline 9 & Close valves V-2 V-11, V-12, V-15, V-16, V-17, V-19 and SV-7 (actuate) \\
\hline 10 & Open valves V-1, V-4, V-6, V-7, V-8, V-9 and V-10 \\
\hline 11 & To purge, open V-3 and actuate SV-3. Purge for about a minute. \\
\hline 12 & Close valve V-3 and allow the manifold to evacuate. \\
\hline 13 & Perform Maintenance
\end{tabular}

\subsubsection{Leak Test}

\begin{tabular}{c|l}
\hline Step & \multicolumn{1}{|c}{ Action } \\
\hline 1 & Close valves V-8 and V-9. \\
\hline 2 & Open valve SV-4 (actuate). \\
\hline 3 & Pressurize to about 1100 mbar by opening V-3. Close V-3 when complete. \\
\hline 4 & Use the Leak Detector sniffer to test. \\
\hline 5 & When complete, open valves V-11, V-12, V-16 and V-19. \\
\hline 6 & Close valves V-4, V-8 and de-actuate (close) SV-7, SV-4 and SV-3 \\
\hline 7 & $\begin{array}{l}\text { Reset the settings on the Gas Collection System Chassis control for Chamber \#1 (Setpoint-1 } \\
\text { anywhere from 900 to 940 mbar and Dead-band to 10). }\end{array}$ \\
\hline 8 & Restart the Analyzers. \\
\hline
\end{tabular}

\section{Records Created by Work Process}

The records listed below must be retained as indicated.

\begin{tabular}{l|c|c|l|l}
\hline $\begin{array}{l}\text { Description of Record } \\
\text { (include form number if } \\
\text { applicable) }\end{array}$ & $\begin{array}{c}\text { Active } \\
\text { Records } \\
\text { Custodian }\end{array}$ & $\begin{array}{c}\text { Active } \\
\text { Records } \\
\text { Retention }\end{array}$ & $\begin{array}{l}\text { Indexing Method, } \\
\text { Storage Medium }\end{array}$ & $\begin{array}{l}\text { Federal Retention } \\
\text { Requirements* }\end{array}$ \\
\hline <enter if needed> & $\begin{array}{c}\text { Facility } \\
\text { Manager }\end{array}$ & 3 years & $\begin{array}{l}\text { Index by job date } \\
\text { and name, store on } \\
\text { paper or } \\
\text { electronically }\end{array}$ & $\begin{array}{l}\text { Destroy 75 years after } \\
\text { the date of the permit } \\
\text { (DOE ADM 18.37) }\end{array}$ \\
\hline
\end{tabular}

The current version of this procedure resides in the Argonne Document Center. Verify that the copy of the procedure you are using is current by comparing the revision number as printed copies can be obsolete. 
*If records are maintained in a business information system that is not currently programmed to purge digital records based on age, the records may be retained in that system past the indicated destruction date.

\section{Related Documents}

This procedure implements requirements established by the following safety basis documents:

- LEAF-SAD-100, Linac Safety Assessment Document

- LEAF-ASE-100, Linac Accelerator Safety Envelope

This procedure implements requirements established by the following Argonne policies and procedures:

- LMS-PROC-188, Accelerator Safety

- EGS-PP-100, Configuration Management Program Plan for Accelerators

\section{Definitions}

None

\section{$7 \quad$ About this Procedure}

\begin{tabular}{ll}
\hline Issuing organization: & Low Energy Accelerator Facility \\
\hline Procedure owner: & S. Chemerisov \\
\hline Point of contact: & S. Chemerisov \\
\hline Review cycle (months): & 36 \\
\hline Date last revised: & TBD \\
\hline Date last reviewed: & TBD \\
\hline
\end{tabular}

\section{Summary of Changes in This Version}

Section 3.2.1 Step 1 "Ensure that solution from the Target Solution Vessel has been transferred to the Dump Tank or Verification Tank.” Has been removed since it is unnecessary.

Section 3.2.1 Step 7 set the Setpoint-1 changed from 850 to 900 mbar. Then, set the Dead-band changed from 100 to 50 mbar.

Section 3.2.2 Has been changed to reflect new valves and reduce complexity.

Exhibit A Hub Manifold Diagram has been updated to show new valves.

Exhibit B Table 1 Hub Manifold Manual Valves has been updated to show new valves.

The current version of this procedure resides in the Argonne Document Center. Verify that the copy of the procedure you are using is current by comparing the revision number as printed copies can be obsolete. 


\section{Exhibit A: Diagram of the Hub Manifold}

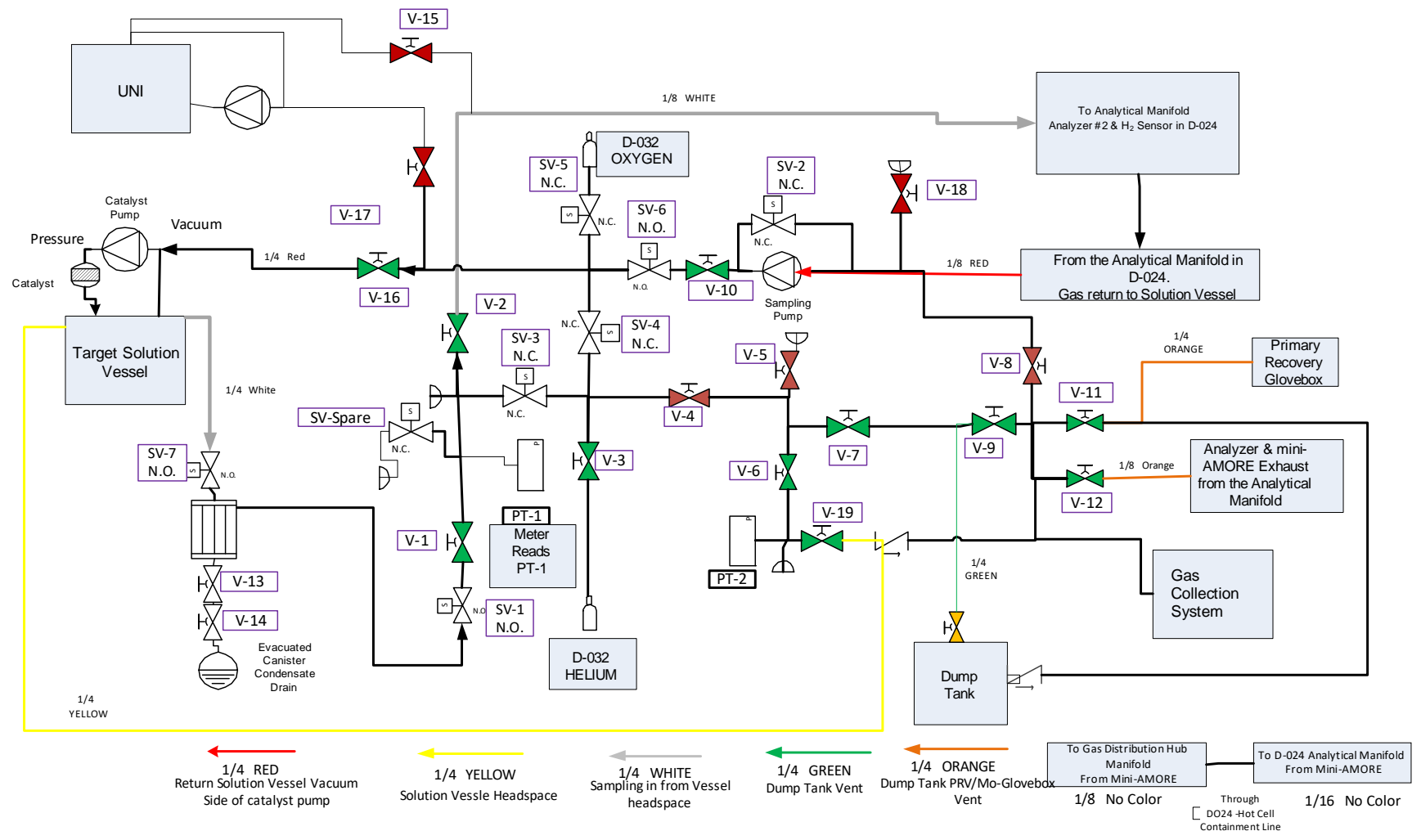

The current version of this procedure resides in the Argonne Document Center. Verify that the copy of the procedure you are using is current by comparing the revision number as printed copies can be obsolete. 


\section{Exhibit B: Tables}

TABLE 1. Hub Manifold Manual Valves - Located in the D-035 Gas Distribution Hub Enclosure

\begin{tabular}{|c|c|c|}
\hline Valve & Description & Function \\
\hline V-1 & Condenser Isolation & Isolates the Condenser from the Hub Manifold \\
\hline $\begin{array}{l}\mathrm{V}-2 \text { and } \\
\mathrm{V}-10\end{array}$ & $\begin{array}{l}\text { Analytical Manifold } \\
\text { Isolation }\end{array}$ & $\begin{array}{l}\text { Closing V-2 and V-10 isolate the Analytical Manifold in D- } \\
024 \text { from the Hub Manifold. }\end{array}$ \\
\hline V-3 & Helium Isolation Valve & $\begin{array}{l}\text { Allows helium in to the Hub Manifold for purging the Target } \\
\text { Solution Vessel, Dump Tank and the Analytical Manifold. }\end{array}$ \\
\hline V-4 & Main Isolation & $\begin{array}{l}\text { Isolates sampling and purge connections from the Gas } \\
\text { Collection System connections on the Hub Manifold. }\end{array}$ \\
\hline V-5 & Vacuum Inlet & Opens the manifold to Vacuum Pump inlet. \\
\hline V-6 & $\begin{array}{l}\text { Target Solution Vessel } \\
\text { Head Space Isolation }\end{array}$ & $\begin{array}{l}\text { Isolates the headspace of the Solution Vessel from the Gas } \\
\text { Collection System and Vacuum Pump. Opening V-6, V-7 and } \\
\text { V-9 opens the Solution Vessel headspace to the Gas } \\
\text { Collection System. }\end{array}$ \\
\hline V-7 & $\begin{array}{l}\text { Target Solution Vessel } \\
\text { Head Space Isolation }\end{array}$ & $\begin{array}{l}\text { Opening V-6, V-7, and V-9 opens the Solution Vessel } \\
\text { headspace to the Gas Collection System. }\end{array}$ \\
\hline V-8 & $\begin{array}{l}\text { Vacuum Exhaust to Gas } \\
\text { Collection }\end{array}$ & $\begin{array}{l}\text { Opens the vacuum pump exhaust to the Gas Collection } \\
\text { System }\end{array}$ \\
\hline V-9 & Gas Collection Isolation & Isolates the Hub Manifold from the Gas Collection System \\
\hline V-10 & $\begin{array}{l}\text { Analytical Manifold } \\
\text { Isolation }\end{array}$ & $\begin{array}{l}\text { Closing V-2 and V-10 isolate the Analytical Manifold in D- } \\
024 \text { from the Hub Manifold. (Attached to the Sampling } \\
\text { Pump) }\end{array}$ \\
\hline V-11 & $\begin{array}{l}\text { Primary Recovery } \\
\text { Glovebox Isolation }\end{array}$ & $\begin{array}{l}\text { Isolates the Primary Recovery Glovebox from the Gas } \\
\text { Collection System }\end{array}$ \\
\hline V-12 & $\begin{array}{l}\text { Analyzer Exhaust } \\
\text { Isolation }\end{array}$ & $\begin{array}{l}\text { Isolates the Analyzer Exhaust from the Gas Collection } \\
\text { System }\end{array}$ \\
\hline V-13 & $\begin{array}{l}\text { Condensate Collection } \\
\text { Isolation }\end{array}$ & $\begin{array}{l}\text { Isolates the Condenser from the condensate collection } \\
\text { canisters valve. }\end{array}$ \\
\hline V-14 & $\begin{array}{l}\text { Condensate Collection } \\
\text { Canister valve }\end{array}$ & Valve on the Condensate Collection Canister \\
\hline V-15 & UNI to Analysis & UNI to Analysis \\
\hline V-16 & Return to TSV & Return to TSV from analytical \\
\hline V-17 & Return to UNI & Return to UNI from analytical \\
\hline V-18 & Spare (capped) & \\
\hline
\end{tabular}

The current version of this procedure resides in the Argonne Document Center. Verify that the copy of the procedure you are using is current by comparing the revision number as printed copies can be obsolete. 


\begin{tabular}{|l|l|l|} 
V-19 & $\begin{array}{l}\text { TSV Headspace to Gas } \\
\text { Collection }\end{array}$ & TSV Headspace to Gas Collection \\
\hline
\end{tabular}


TABLE 2. Hub Manifold Solenoid Valves - Located in the D-035 Gas Distribution Hub Enclosure - (Controls on Chassis 1)

\begin{tabular}{|c|c|c|}
\hline Valve & Description & Function \\
\hline SV-1 & Gas Sampling Isolation & $\begin{array}{l}\text { Normally Open }- \text { Allows the gas stream from the Target } \\
\text { Solution Vessel into the Hub Manifold. Purge the Analytical } \\
\text { Manifold in D- } 024 \text { post irradiation by actuating SV-3 and } \\
\text { SV- } 1 \text {. }\end{array}$ \\
\hline SV-2 & Sampling Pump Bypass & $\begin{array}{l}\text { Normally Closed }- \text { Should the sampling pump fail, the } \\
\text { Analytical Manifold in D-024 can be purged by actuating SV- } \\
1 \text {, SV-2 and SV-3. }\end{array}$ \\
\hline SV-3 & $\begin{array}{l}\text { Isolation Valve/Helium } \\
\text { Purge }\end{array}$ & $\begin{array}{l}\text { Normally Closed - Isolates the Gas Sample Path from the } \\
\text { helium purge line during an experiment. Open it to purge the } \\
\text { Analytical Manifold for calibration and pre-run. You can } \\
\text { purge the Analytical Manifold post irradiation by actuating } \\
\text { SV-3 and SV-7. }\end{array}$ \\
\hline SV-4 & Helium purge & $\begin{array}{l}\text { Normally Closed - Open it to purge the Target Solution } \\
\text { Vessel. }\end{array}$ \\
\hline SV-5 & Oxygen Addition & $\begin{array}{l}\text { Normally Closed }- \text { Open it to add oxygen to the Target } \\
\text { Solution Vessel to reduce hydrogen concentration. Use in } \\
\text { conjunction with the Oxygen Flow potentiometer on } \\
\text { Chassis } 2 \text {. }\end{array}$ \\
\hline SV-6 & $\begin{array}{l}\text { Sampling Pump } \\
\text { Isolation }\end{array}$ & $\begin{array}{l}\text { Normally Open - Isolates the Sampling Pump from the } \\
\text { Target Solution Vessel }\end{array}$ \\
\hline SV-7 & Gas Sampling Isolation & $\begin{array}{l}\text { Normally Open }- \text { Allows the analytical gas stream from the } \\
\text { Target Solution Vessel into the Condenser. }\end{array}$ \\
\hline
\end{tabular}

TABLE 3. Dump Tank Valves - Located beneath the D035 Hot Cell

\begin{tabular}{|c|l|l|}
\hline Valve & \multicolumn{1}{|c|}{ Description } & \multicolumn{1}{c|}{ Function } \\
\hline $\begin{array}{c}\text { D-1A } \\
\text { and D- } \\
\text { 1B }\end{array}$ & $\begin{array}{l}\text { Pick-up line to Primary } \\
\text { Recovery Glovebox }\end{array}$ & $\begin{array}{l}\text { Siphons solution from the bottom of the Dump Tank to } \\
\text { transport it to the Primary Recovery Glovebox. }\end{array}$ \\
\hline $\begin{array}{c}\text { D-2A } \\
\text { and D- } \\
\text { 2B }\end{array}$ & $\begin{array}{l}\text { Return Line from } \\
\text { Primary Recovery } \\
\text { Glovebox }\end{array}$ & $\begin{array}{l}\text { Returns solution to the top of the DumpTank from the } \\
\text { Primary Recovery Glovebox }\end{array}$ \\
\hline $\begin{array}{c}\text { D-3A } \\
\text { and D- } \\
\text { 3B }\end{array}$ & $\begin{array}{l}\text { Dump Tank headspace } \\
\text { gas to Gas Distribution } \\
\text { Hub Manifold }\end{array}$ & $\begin{array}{l}\text { Allows for gas displacement when solution is entering the } \\
\text { Dump Tank }\end{array}$ \\
\hline
\end{tabular}

The current version of this procedure resides in the Argonne Document Center. Verify that the copy of the procedure you are using is current by comparing the revision number as printed copies can be obsolete. 


\section{APPENDIX 14}

LEAF-PROC-023, Rev. 2: Maintenance and Leak Testing of the Gas Collection System Enclosure 


\section{Maintenance and Leak Testing of the Gas Collection System Enclosure}

Low Energy Accelerator Facility, LEAF-PROC-023, Rev. 2

Approved: SChn Date: $03.23 .2021 \ldots$

Sergey Chemerisov, Manager, IVEM/LEAF

Effective Date: 03.24 .2021 


\section{Purpose}

Establish the process for maintenance and leak testing of the Gas Collection System Enclosure.

\section{Scope}

This procedure applies to the following Argonne activities and entities.

\begin{tabular}{ll}
\hline LMS core processes: & Asset Management \\
\hline Organizations: & Experimental Operations and Facilities (EOF) Division \\
\hline Buildings: & 211 \\
\hline Specific locations: & LINAC \\
\hline $\begin{array}{l}\text { Other applicability } \\
\text { factors: }\end{array}$ & None \\
\hline Exclusions: & None \\
\hline USI applicability: & Yes \\
\hline
\end{tabular}

\section{Work Process}

\subsection{Introduction}

The following pertains to maintenance activities on equipment in the Gas Collection System Enclosure.

\subsection{Step-by-Step Procedure}

The steps below are mandatory unless noted otherwise. This procedure is to be performed by qualified personnel.

NOTE: *Do Not apply a vacuum of $<800$ mbar to the system.

NOTE: *Do Not apply a pressure of $>1340$ mbar to the system.

NOTE: *Do not set the Setpoint 1 of Chamber \#1 on the Gas Collection System to $<800$ mbar.

\subsubsection{Purging and Maintenance}

\begin{tabular}{c|l}
\hline Step & \multicolumn{1}{|c}{ Action } \\
\hline 1 & $\begin{array}{l}\text { Inform LINAC Facility and AMORE personnel that the Gas Collection System will be out of } \\
\text { service. }\end{array}$ \\
\hline 2 & $\begin{array}{l}\text { In the LINAC Control Room, turn off the Catalyst Pump and Sampling Pump. Set } \\
\text { Chassis \#1 for control in D-035 (downstairs). }\end{array}$ \\
\hline 3 & Turn off both Analyzers. \\
\hline 4 & On the Gas Collection System Enclosure, Close GC-1. \\
\hline 5 & On the Dump Tank, close valves D-1A, D-2A and D-3A to isolate the tank. \\
\hline 6 & On the Hub Manifold, open valve V-3, V-4, V-7 and V-9 to allow helium to the System. \\
\hline
\end{tabular}




\begin{tabular}{c|l}
\hline Step & \multicolumn{1}{c}{ Action } \\
\hline 8 & $\begin{array}{l}\text { Close valves to isolate the various parts of the AMORE system. Close V-1, V-16 and V-19 } \\
\text { to isolate the Target Solution Vessel. Close V-2 and V-8 to isolate Analytical Manifold and } \\
\text { UNI. Close V-11 to isolate the Primary Recovery Glovebox. Close V-12 to isolate the } \\
\text { Analyzer Exhaust. }\end{array}$ \\
\hline 9 & $\begin{array}{l}\text { On the Gas Collection System Chassis for Chamber \#1 control, set the Setpoint-1 to } \\
940 \text { mbar and Dead-band to 10 mbar. }\end{array}$ \\
\hline 10 & $\begin{array}{l}\text { Open the helium cylinder and isolation valves to purge the Gas Collection System. Allow } \\
\text { Chamber \#2 to cycle at least three times. }\end{array}$ \\
\hline 11 & $\begin{array}{l}\text { Close the Helium Cylinder and Isolation Valves to stop the purge. Allow the system to } \\
\text { evacuate. Close GC-SV-1. Turn OFF the Gas Collection System by pushing in the red } \\
\text { button below the controllers. Close the valves on the Collection Cylinder. }\end{array}$ \\
\hline
\end{tabular}

\subsubsection{Leak Test Chamber \#1 inside Enclosure and attached to the system}

\begin{tabular}{c|l}
\hline Step & \multicolumn{1}{|c}{ Action } \\
\hline 1 & On the Chamber \#1 controller, set the Setpoint-1 to 1100 mbar. Set Alarm-2 to 1200 mbar. \\
\hline 2 & Open the helium cylinder and isolation valves to add pressure to Chamber \#1 to 1050mbar. \\
\hline 3 & Close the helium cylinder and isolation valves. \\
\hline 4 & Use the Leak Detector sniffer to test Chamber \#1 fittings. \\
\hline
\end{tabular}

\subsubsection{Leak Test Chamber \#2 inside Enclosure and attached to the system}

\begin{tabular}{c|l}
\hline Step & \multicolumn{1}{c}{ Action } \\
\hline 1 & Open a Collection Cylinder \\
\hline 2 & $\begin{array}{l}\text { On the Gas Collection System Chassis for Chamber \#1 controller, set Setpoint-1 to } \\
950 \text { mbar. Then, set the Dead-band to } 10 \text { mbar. Start the Gas collection System by pulling } \\
\text { out the red button. Open GC-SV-1 }\end{array}$ \\
\hline 3 & Open the helium cylinder and isolation valves in D032. \\
\hline 4 & Let Chamber \#1 cycle at least once. Then close the helium cylinder and isolation valves. \\
\hline 5 & Use the Leak Detector sniffer to test Chamber \#2 fittings. \\
\hline
\end{tabular}




\subsubsection{Leak Test the Collection Cylinders}

\begin{tabular}{c|l}
\hline Step & \multicolumn{1}{|c}{ Action } \\
\hline 1 & Briefly open one of the collection cylinders to pressurize the fittings. \\
\hline 2 & Use the Leak Detector sniffer to test Collection Cylinder fittings. \\
\hline
\end{tabular}

\subsubsection{Leak testing either chamber outside of the Enclosure and detached from the system}

\begin{tabular}{c|l}
\hline Step & \multicolumn{1}{|c}{ Action } \\
\hline 1 & $\begin{array}{l}\text { Attach a 5 psig PRV 37 SCFM and a 0.01" orifice to a helium cylinder and regulator. Set the } \\
\text { regulator to 1 psig. }\end{array}$ \\
\hline 2 & Attach the cylinder to the Chamber and pressurize to 1 psig, \\
\hline 3 & Use the Leak Detector sniffer to test Chambers fittings and gasket. \\
\hline 4 & When complete, release the gas into the Gas Collection Enclosure up the vent. \\
\hline
\end{tabular}

\subsubsection{Restart the Gas Collection System}

\begin{tabular}{c|l}
\hline Step & \multicolumn{1}{|c}{ Action } \\
\hline 1 & $\begin{array}{l}\text { In D-035 Gas Distribution Hub Manifold, close valve V-3 and V-4. Open valves V-1, V-6, } \\
\text { V-12 and V-19 }\end{array}$ \\
\hline 2 & In the Gas Collection Enclosure Open a Collection Cylinder \\
\hline 3 & In D-032 close the helium cylinder and isolation valves. \\
\hline 4 & $\begin{array}{l}\text { On the Gas Collection System Chassis for Chamber \#1 control, set the Setpoint-1 to between } \\
900-950 \text { mbar. Then, set the Dead-band to 10 mbar. Turn on the Gas Collection System by } \\
\text { pulling out the red button. Open GC-SV-1. }\end{array}$ \\
\hline 5 & Restart the Gas Analyzers \\
\hline
\end{tabular}

\subsubsection{Monthly Check Valve Test GC-CK-2 (Between Collection Cylinders and Chamber \#2)}

\begin{tabular}{c|l}
\hline Step & \multicolumn{1}{|c}{ Action } \\
\hline 1 & Turn off the Gas Analyzers \\
\hline 2 & $\begin{array}{l}\text { On the D035 Hub Manifold Close valves V-1, V-4, V-8, V-9, V-11, V-12, V-15, V-16 and } \\
\text { V-17. }\end{array}$ \\
\hline 3 & In the Gas Collection Enclosure, Close both Collection Cylinder valves. \\
\hline 4 & Briefly open and then close the valve on the cylinder with the highest pressure. \\
\hline 5 & Observe the pressure drop in the collection cylinders for at least an hour \\
\hline
\end{tabular}




\begin{tabular}{c|l}
\hline Step & \multicolumn{1}{|c}{ Action } \\
\hline 6 & Record the pressure drop in the logbook. \\
\hline 7 & Acceptance criteria for Check Valve GC-CK-2 is (Pressure Drop $<12$ psig/hour) \\
\hline 8 & If the acceptance criteria is not met, the Check Valve should be replaced. \\
\hline
\end{tabular}

\subsubsection{Monthly Check Valve Test GC-CK-1 (Between Chamber \#1 and Chamber \#2)}

\begin{tabular}{c|l}
\hline Step & \multicolumn{1}{|c}{ Action } \\
\hline 1 & Turn off the Gas Analyzers \\
\hline 2 & $\begin{array}{l}\text { On the D035 Hub Manifold Close valves V-1, V-4, V-8, V-9, V-11, V-12, V-15, V-16 and } \\
\text { V-17. }\end{array}$ \\
\hline 3 & In the Gas Collection Enclosure, Close both Collection Cylinder valves. \\
\hline 4 & Observe the pressure rise in Chamber \#1 for at least an hour \\
\hline 5 & Record the pressure rise in the logbook. \\
\hline 6 & Acceptance criteria for Check Valve GC-CK-1 is (Pressure Rise < 4.9 mbar/hour) \\
\hline 7 & If the acceptance criteria is not met, the Check Valve should be replaced. \\
\hline
\end{tabular}

\subsubsection{Monthly test of the UPS Battery Backup}

\begin{tabular}{c|l}
\hline Step & \multicolumn{1}{c}{ Action } \\
\hline 1 & $\begin{array}{l}\text { In D032 locate the Battery Backup that the controllers for the Gas Collection System are } \\
\text { plugged into. }\end{array}$ \\
\hline 2 & Unplug the Battery Backup from the line voltage (wall outlet) \\
\hline 3 & Wait 5 minutes. \\
\hline 4 & $\begin{array}{l}\text { Ensure that the Controllers for the Gas Collection System stay active. Record the available } \\
\text { battery time and percent battery life in the logbook. }\end{array}$ \\
\hline 5 & Acceptance criteria for the battery life should be $>50 \%$ and Controllers stay active. \\
\hline 6 & If the acceptance criteria is not met, the battery or the UPS should be replaced. \\
\hline
\end{tabular}


3.2.10 Response to Gas Collection System Alarms. After the initial assessment and actions, see Exhibit B: Table 2. Alarm Investigation

\begin{tabular}{c|l}
\hline Step & \multicolumn{1}{c}{ Action } \\
\hline 1 & Assess which alarm is active, Chamber \#1, Chamber \#2 or Collection Cylinders \\
\hline 2 & $\begin{array}{l}\text { If "Collection Cylinders". This indicates that the "in use" Cylinder is Full. The action is to } \\
\text { close the full cylinder and switch to a new Cylinder. }\end{array}$ \\
\hline 3 & $\begin{array}{l}\text { If "Chamber \#1 or Chamber \#2" } \\
\text { Isolate the Gas Collection System from gas sources. In the Gas Collection Enclosure, close } \\
\text { GC-1. In the D024 Analytical Enclosure, turn of the Gas Analyzers. In D032, Close all } \\
\text { cylinder and isolation valves. On the D035 Gas Hub Manifold, Close valves V-1, V-4, V-8, } \\
\text { V-9, V-11, V-12, V-15, V-16 and V-17. } \\
\text { On the Gas Collection System Chassis Close GC-SV-1. } \\
\text { In the Gas Collection System Enclosure, close the Collection Cylinder Valves. }\end{array}$ \\
\hline
\end{tabular}

\section{Records Created by Work Process}

The records listed below must be retained as indicated.

\begin{tabular}{l|c|c|l|l}
\hline $\begin{array}{l}\text { Description of Record } \\
\text { include form number if } \\
\text { applicable) }\end{array}$ & $\begin{array}{c}\text { Active } \\
\text { Records } \\
\text { Custodian }\end{array}$ & $\begin{array}{c}\text { Active } \\
\text { Records } \\
\text { Retention }\end{array}$ & $\begin{array}{l}\text { Indexing Method, } \\
\text { Storage Medium }\end{array}$ & $\begin{array}{l}\text { Federal Retention } \\
\text { Requirements* }\end{array}$ \\
\hline$<\ldots>$ & $\begin{array}{c}\text { Facility } \\
\text { Manager }\end{array}$ & 3 years & $\begin{array}{l}\text { Index by job date } \\
\text { and name, store on } \\
\text { paper or } \\
\text { electronically }\end{array}$ & $\begin{array}{l}\text { Destroy 75 years after } \\
\text { the date of the permit } \\
\text { (DOE ADM 18.37) }\end{array}$ \\
\hline
\end{tabular}

*If records are maintained in a business information system that is not currently programmed to purge digital records based on age, the records may be retained in that system past the indicated destruction date.

\section{Related Documents}

This procedure implements requirements established by the following safety basis documents:

- LEAF-SAD-100, Linac Safety Assessment Document

- LEAF-ASE-100, Linac Accelerator Safety Envelope

This procedure implements requirements established by the following Argonne policies and procedures:

- $\quad$ LMS-PROC-188, Accelerator Safety

- EGS-PP-100, Configuration Management Program Plan for Accelerators

\section{Definitions}

None 


\section{About this Procedure}

\begin{tabular}{ll}
\hline Issuing organization: & Low Energy Accelerator Facility \\
\hline Procedure owner: & S. Chemerisov \\
\hline Point of contact: & S. Chemerisov \\
\hline Review cycle (months): & 36 \\
\hline Date last revised: & 3.18 .2021 \\
\hline Date last reviewed: & 3.23 .2021 \\
\hline
\end{tabular}

\section{Summary of Changes in This Version}

Inclusion of section 3.2.7 to test the leak rate of Check Valve GC-CK-2

Inclusion of section 3.2.8 to test the leak rate of Check Valve GC-CK-1

Inclusion of section 3.2.9 Response to Alarms

Update of Exhibit A Figure 2 Gas Collection System

Added Exhibit B: Table 2. Alarm Investigation 


\section{Exhibit A: Figures}

Figure 1. Diagram of the Hub Manifold in the D-035 Gas Distribution Hub Enclosure.

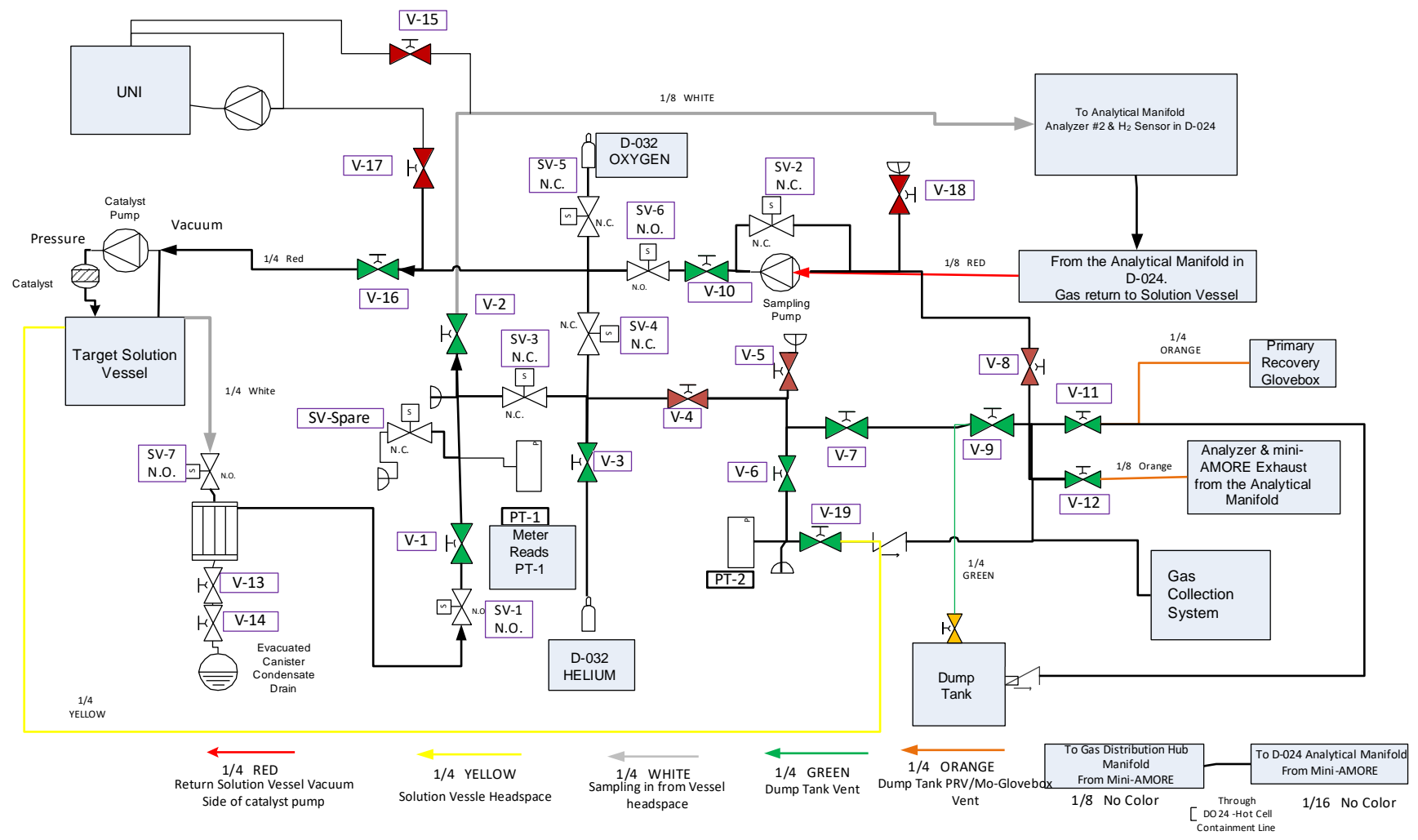


Figure 2. Diagram of the Gas Collection System

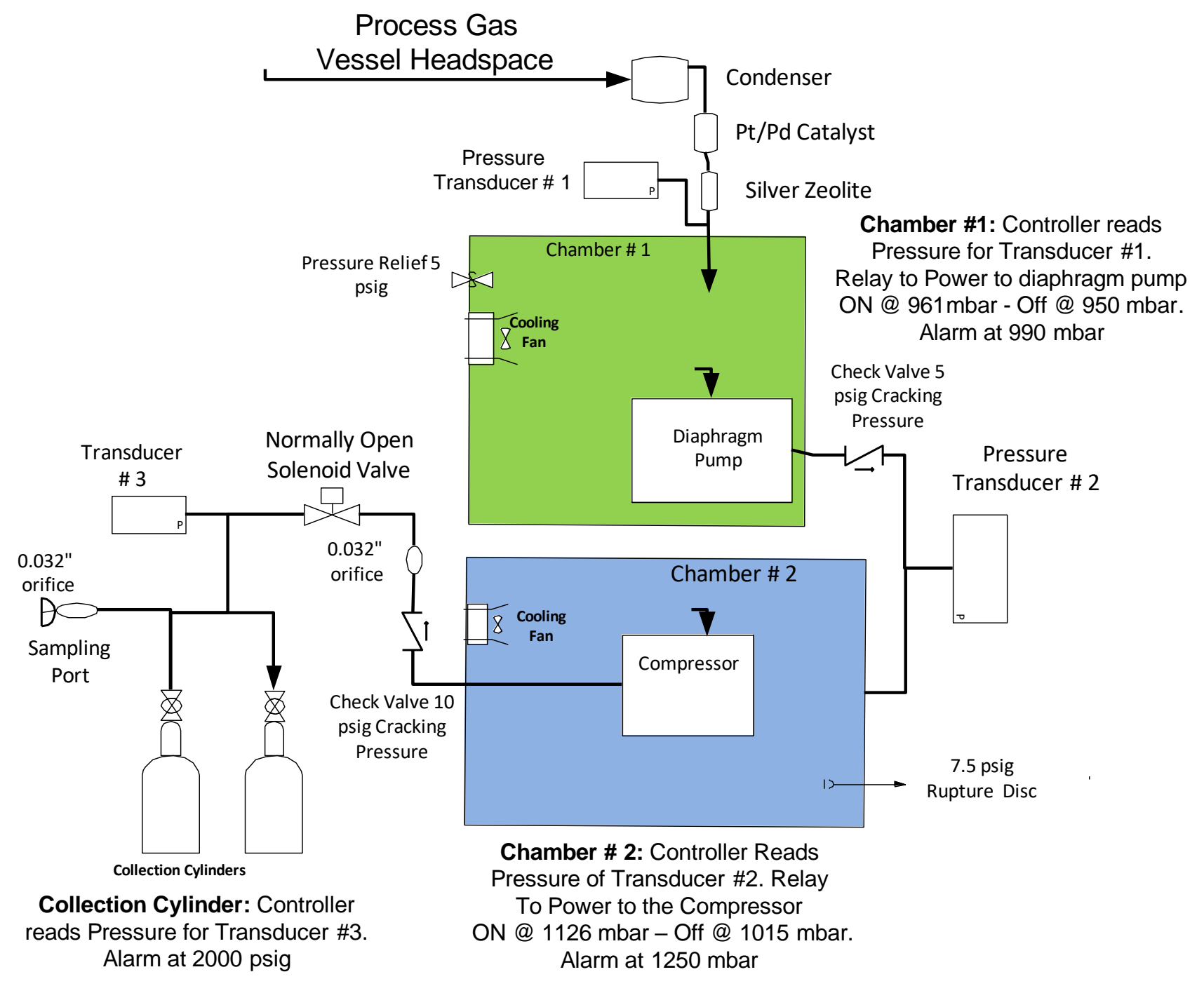




\section{Exhibit B: Tables}

TABLE 1. Hub Manifold Manual Valves - Located in the D-035 Gas Distribution Hub Enclosure

\begin{tabular}{|c|c|c|}
\hline Valve & Description & Function \\
\hline V-1 & Condenser Isolation & Isolates the Condenser from the Hub Manifold \\
\hline $\begin{array}{l}\text { V-2 and } \\
\text { V-10 }\end{array}$ & $\begin{array}{l}\text { Analytical Manifold } \\
\text { Isolation }\end{array}$ & $\begin{array}{l}\text { Closing V-2 and V-10 isolate the Analytical Manifold in D- } \\
024 \text { from the Hub Manifold. }\end{array}$ \\
\hline V-3 & Helium Isolation Valve & $\begin{array}{l}\text { Allows helium in to the Hub Manifold for purging the Target } \\
\text { Solution Vessel, Dump Tank and the Analytical Manifold. }\end{array}$ \\
\hline $\mathrm{V}-4$ & Main Isolation & $\begin{array}{l}\text { Isolates sampling and purge connections from the Gas } \\
\text { Collection System connections on the Hub Manifold. }\end{array}$ \\
\hline V-5 & Vacuum Inlet & Opens the manifold to Vacuum Pump inlet. \\
\hline V-6 & $\begin{array}{l}\text { Target Solution Vessel } \\
\text { Head Space Isolation }\end{array}$ & $\begin{array}{l}\text { Isolates the headspace of the Solution Vessel from the Gas } \\
\text { Collection System and Vacuum Pump. Opening V-6, V-7 and } \\
\text { V-9 opens the Solution Vessel headspace to the Gas } \\
\text { Collection System. }\end{array}$ \\
\hline V-7 & $\begin{array}{l}\text { Target Solution Vessel } \\
\text { Head Space Isolation }\end{array}$ & $\begin{array}{l}\text { Opening V-6, V-7, and V-9 opens the Solution Vessel } \\
\text { headspace to the Gas Collection System. }\end{array}$ \\
\hline V-8 & $\begin{array}{l}\text { Vacuum Exhaust to Gas } \\
\text { Collection }\end{array}$ & $\begin{array}{l}\text { Opens the vacuum pump exhaust to the Gas Collection } \\
\text { System }\end{array}$ \\
\hline V-9 & Gas Collection Isolation & Isolates the Hub Manifold from the Gas Collection System \\
\hline $\mathrm{V}-\mathbf{1 0}$ & $\begin{array}{l}\text { Analytical Manifold } \\
\text { Isolation }\end{array}$ & $\begin{array}{l}\text { Closing V-2 and V-10 isolate the Analytical Manifold in D- } \\
024 \text { from the Hub Manifold. (Attached to the Sampling } \\
\text { Pump) }\end{array}$ \\
\hline V-11 & $\begin{array}{l}\text { Primary Recovery } \\
\text { Glovebox Isolation }\end{array}$ & $\begin{array}{l}\text { Isolates the Primary Recovery Glovebox from the Gas } \\
\text { Collection System }\end{array}$ \\
\hline V-12 & $\begin{array}{l}\text { Analyzer Exhaust } \\
\text { Isolation }\end{array}$ & $\begin{array}{l}\text { Isolates the Analyzer Exhaust from the Gas Collection } \\
\text { System }\end{array}$ \\
\hline V-13 & $\begin{array}{l}\text { Condensate Collection } \\
\text { Isolation }\end{array}$ & $\begin{array}{l}\text { Isolates the Condenser from the condensate collection } \\
\text { canisters valve. }\end{array}$ \\
\hline V-14 & $\begin{array}{l}\text { Condensate Collection } \\
\text { Canister valve }\end{array}$ & Valve on the Condensate Collection Canister \\
\hline V-15 & UNI to Analysis & UNI to Gas Analysis \\
\hline V-16 & Return to TSV & Analytical gas returns to TSV \\
\hline V-17 & UNI Return & Analytical gas returns to UNI \\
\hline$V-18$ & Spare (capped) & \\
\hline
\end{tabular}




\begin{tabular}{|l|l|l|l} 
V-19 & $\begin{array}{l}\text { TSV Headspace to Gas } \\
\text { Collection }\end{array}$ & TSV Headspace to Gas Collection \\
\hline
\end{tabular}

TABLE 2. Alarm Investigation

\begin{tabular}{|c|l|l|}
\hline Chamber \#1 Alarm & Chamber \#2 Alarm & Possible Items to Investigate \\
\hline High & None & Chamber \#1 Pump - Check Valve GC-CK-1 \\
\hline High & Low (see also below) & Chamber \#2 Compressor - Check Valves \\
\hline High & High & Chamber \#2 Compressor - Check Valves \\
\hline None & High & Chamber \#2 Compressor - Check Valve GC-CK-2 \\
\hline High (>1350mbar) & & \begin{tabular}{l} 
Possible of Gas Release through Chamber \#1 PRV \\
\hline
\end{tabular} \\
& Low & $\begin{array}{l}\text { Possible of Gas Release through Chamber \#2 Rupture } \\
\text { Disc }\end{array}$ \\
\hline
\end{tabular}


TABLE 2. Hub Manifold Solenoid Valves - Located in the D-035 Gas Distribution Hub Enclosure - (Controls on Chassis 1)

\begin{tabular}{|c|l|l|}
\hline Valve & \multicolumn{1}{|c|}{ Description } & \multicolumn{1}{c|}{ Function } \\
\hline SV-1 & Gas Sampling Isolation & $\begin{array}{l}\text { Normally Open - Allows the gas stream from the Target } \\
\text { Solution Vessel into the Hub Manifold. Purge the Analytical } \\
\text { Manifold in D-024 post irradiation by actuating SV-3 and } \\
\text { SV-1. }\end{array}$ \\
\hline SV-2 & Sampling Pump Bypass & $\begin{array}{l}\text { Normally Closed - Should the sampling pump fail, the } \\
\text { Analytical Manifold in D-024 can be purged by actuating SV- } \\
1, \text { SV-2 and SV-3. }\end{array}$ \\
\hline SV-3 & $\begin{array}{l}\text { Isolation Valve/Helium } \\
\text { Purge }\end{array}$ & $\begin{array}{l}\text { Normally Closed - Isolates the Gas Sample Path from the } \\
\text { helium purge line during an experiment. Open it to purge the } \\
\text { Analytical Manifold for calibration and pre-run. You can } \\
\text { purge the Analytical Manifold post irradiation by actuating } \\
\text { SV-3 and SV-7. }\end{array}$ \\
\hline SV-4 & Helium purge & $\begin{array}{l}\text { Normally Closed }- \text { Open it to purge the Target Solution } \\
\text { Vessel. }\end{array}$ \\
\hline SV-5 & Oxygen Addition & $\begin{array}{l}\text { Normally Closed }- \text { Open it to add oxygen to the Target } \\
\text { Solution Vessel to reduce hydrogen concentration. Use in } \\
\text { conjunction with the Oxygen Flow potentiometer on } \\
\text { Chassis 2. }\end{array}$ \\
\hline SV-6 & $\begin{array}{l}\text { Sampling Pump } \\
\text { Isolation }\end{array}$ & $\begin{array}{l}\text { Gas Sampling Isolation } \\
\text { Normally Open - Isolates the Sampling Pump from the } \\
\text { Target Solution Vessel }\end{array}$ \\
\hline $\begin{array}{l}\text { Normally Open - Allows the analytical gas stream from the } \\
\text { Target Solution Vessel into the Condenser. }\end{array}$ \\
\hline
\end{tabular}

TABLE 3. Dump Tank Valves - Located beneath the D035 Hot Cell

\begin{tabular}{|c|l|l|}
\hline Valve & \multicolumn{1}{|c|}{ Description } & \multicolumn{1}{c|}{ Function } \\
\hline $\begin{array}{c}\text { D-1A and } \\
\text { D-1B }\end{array}$ & $\begin{array}{l}\text { Pick-up line to } \\
\text { Primary Recovery } \\
\text { Glovebox }\end{array}$ & $\begin{array}{l}\text { Siphons solution from the bottom of the Dump Tank to } \\
\text { transport it to the Primary Recovery Glovebox. }\end{array}$ \\
\hline $\begin{array}{c}\text { D-2A and } \\
\text { D-2B }\end{array}$ & $\begin{array}{l}\text { Return Line from } \\
\text { Primary Recovery } \\
\text { Glovebox }\end{array}$ & $\begin{array}{l}\text { Returns solution to the top of the Tank from the Primary } \\
\text { Recovery Glovebox }\end{array}$ \\
\hline $\begin{array}{c}\text { D-3A and } \\
\text { D-3B }\end{array}$ & $\begin{array}{l}\text { Dump Tank headspace } \\
\text { gas to Gas } \\
\text { Distribution Hub } \\
\text { Manifold }\end{array}$ & $\begin{array}{l}\text { Allows for gas displacement when solution is entering the } \\
\text { Dump Tank }\end{array}$ \\
\hline
\end{tabular}


TABLE 4. Gas Collection System Valves - Located in the Gas Collection System Enclosure (D035)

\begin{tabular}{|c|c|c|}
\hline Valve & Description & Function \\
\hline GC-1 & $\begin{array}{l}\text { D024 Hot Cell } \\
\text { Isolation }\end{array}$ & Isolates the Gas Collection System from the D024 Hot Cell \\
\hline GC-2 & $\begin{array}{l}\text { Open Only For Non- } \\
\text { Rad Experiments }\end{array}$ & $\begin{array}{l}\text { This valve can be open during the Commissioning Tests. When } \\
\text { sulfuric acid solution is in the Vessel. Keep valves GC- } 3 \text { and } \\
\text { GC- } 4 \text { closed to protect the zeolite cartridge from moisture. }\end{array}$ \\
\hline $\begin{array}{l}\text { GC-3 and } \\
\text { GC-4 }\end{array}$ & $\begin{array}{l}\text { Silver Zeolite } \\
\text { Isolation valves }\end{array}$ & $\begin{array}{l}\text { Open during AMORE Experiments. Valve GC-2 is kept } \\
\text { closed. }\end{array}$ \\
\hline $\begin{array}{l}\text { GC-5 and } \\
\text { GC-6 }\end{array}$ & $\begin{array}{l}\text { Condensate Drain } \\
\text { Valves }\end{array}$ & Open when draining condensate \\
\hline GC-7 & Port & Port for maintenance. Capped \\
\hline CSV-1 & $\begin{array}{l}\text { High Pressure } \\
\text { Isolation }\end{array}$ & $\begin{array}{l}\text { Part of the interlocks. Closed when the Gas Collection } \\
\text { interlock is tripped }\end{array}$ \\
\hline
\end{tabular}




\section{APPENDIX 15}

Sampling Gas from AMORE Collection Cylinders 


\section{Sampling Gas from AMORE Collection Cylinders}

\section{Scope Summary}

The procedure describes the steps necessary to sample gas from the AMORE Collection Cylinders. The sample is taken to determine the amount of radioactive gases that are present in the cylinders to report for release. This is done after most of the radioactive gases have significantly decayed (60 days post irradiation). This procedure occurs in the Gas Collection Enclosure in D035 of the LINAC Facility. Other activities that require operation of the Gas Collection System must be suspended.

The Gas Collection System is isolated from the various parts of the AMORE system. Then a sampling Assembly is attached to the sampling port. The Sample Cylinder is filled and removed. The Gas Collection System is re-started. The sample is analyzed by gamma spectroscopy. The gamma results are reported to QAS.

\subsection{Procedure}

2.1 Shutdown the Gas Analyzers in the D024 Analytical Enclosure.

2.2 On the Gas Distribution Hub Manifold, close valves V-1, V-5, V-6, V-7, V-8, V-9, V-10, V11 and $V-12$. This will isolate the Gas Collection System from other parts of the AMORE Experiment.

2.3 In the Gas Collection System, close valve GC-1 (to isolate the D024 Hot Cell) and close both Collection Cylinder Valves

2.4 On the Gas Collection Control Chassis in D032, Close GC-SV 1. This isolates Chamber 2 from the High Pressure Cylinders. Then turn off the Gas Collection System by pushing the Red Button.

2.5 Using the glove port on the Gas Collection Enclosure, remove the cap from the Gas Sampling Port. A small amount of gas will be released. Wait for a minute so the Ventilation system evacuates the gas from the enclosure.

2.6 Attach a 0.025inch Orifice and a Sampling Cylinder to a dual stage regulator to make a Sampling Assembly. Close all valves on the assembly and close the regulator valve. (Figure 1)

2.7 Attach the Sampling Assembly to the Sampling Port on the Gas Collection System. (Figure 2)

2.8 Open the valve on the Collection Cylinder that is being sampled.

2.9 Open valve \#1. The first gauge on the regulator should rise to the pressure in the Collection Cylinder.

2.10 Set the regulator output to about 150 psig.

2.11 Open Valve \#2 and the Sample Valve. This will fill the Cylinder.

2.12 Close all valves on the Sampling Assembly.

2.13 Close the valve on the Collection Cylinder. 
2.14 Close the door on the enclosure. Break the connection between Sample Valve and the Orifice. Leave the Cylinder attached. Have a Health Physics Tech survey that connection.

2.15 Remove the Sample Cylinder leaving the Orifice attached to the Regulator. Cap the Sample Valve.

2.16 Remove the Regulator and cap the Sampling Port.

2.17 Open the Collection Cylinder valve.

2.18 Re-start the Gas Collection System by pulling out the Red Button.

2.19 Open GC-SV-1.

2.20 On the Gas Distribution Hub Manifold open valves V-6, V-7, V-9 and V-11.

2.21 The gas sample is analyze using gamma spectroscopy.

Figure 1. Sampling Assembly

\section{Sampling Assembly}

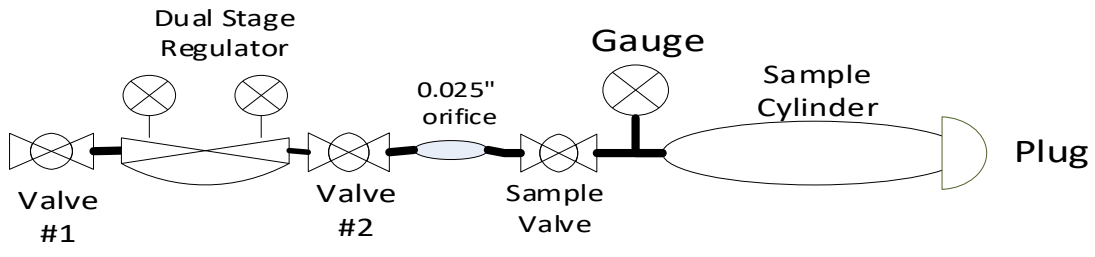

Figure 1. Attachment to Sampling Port in Gas Collection System

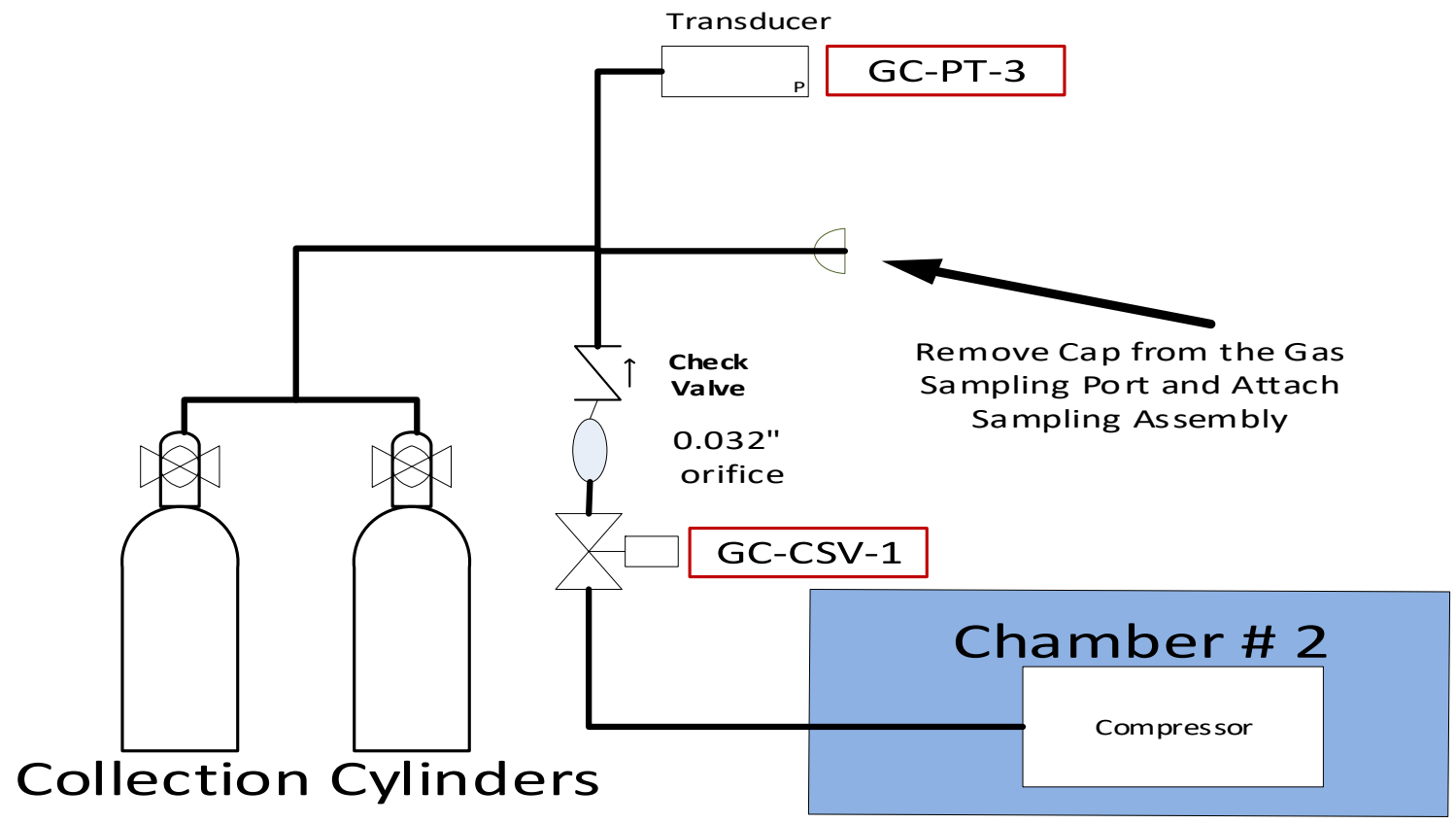




\section{APPENDIX 16}

\section{Release of Gas from AMORE Collection Cylinders}




\section{Release of Gas from AMORE Collection Cylinders}

\section{Scope Summary}

The procedure describes the steps necessary to release gas from the AMORE Collection Cylinders. This procedure is performed only after approval to release the gas has been given by the QAS division. This activity occurs in the Gas Collection Enclosure in D035 of the LINAC Facility. Other activities that require operation of the Gas Collection System must be suspended.

First, the Gas Collection System is isolated from the various parts of the AMORE system. Then a Release Assembly is attached to the sampling port. The gas in the Collection Cylinders is slowly released into the D035 Process Ventilation. The Gas Collection System is re-started.

\subsection{Procedure}

2.1 Shutdown the Gas Analyzers in the D024 Analytical Enclosure.

2.2 On the Gas Distribution Hub Manifold, close valves V-1, V-5, V-6, V-7, V-8, V-9, V-11 and V12. This will isolate the Gas Collection System from other parts of the AMORE Experiment.

2.3 In the Gas Collection System Enclosure, close both Collection Cylinder Valves and close valve GC-1 (to isolate the D024 Hot Cell)

2.4 On the Gas Collection Control Chassis in D032, Close GC-SV 1. This isolates Chamber 2 from the High Pressure Cylinders. Then turn off the Gas Collection System by pushing the Red Button.

2.5 Using the glove port on the Gas Collection Enclosure, remove the cap from the Gas Sampling Port. A small amount of gas will be released. Wait for a minute so the Ventilation system evacuates the gas from the enclosure.

2.6 Attach a 0.025inch Orifice, Hepa-filter and Exhaust Tube to a dual stage regulator to make the Release Assembly. Close all valves on the assembly and close the regulator valve. See Figure 1.

2.7 Attach the assembly to the Sampling Port and run Exhaust Tubing from the output of the filter up into the ventilation duct.

2.8 Open the valve on the Collection Cylinder.

2.9 Open valve \#1. The first gauge on the regulator should rise to the pressure in the Cylinder.

2.10 Set the regulator output to 10 psig.

2.11 Slowly Open Valve \#2 to begin releasing gas.

2.12 Close the door on the enclosure. The cylinder will take several hours to empty. The pressure in the cylinder can be monitored on the Gas Collection System Control Chassis in D032.

2.13 When the Cylinder is empty, HP Tech should survey the enclosure.

2.14 Close the valve on the Collection Cylinder. Remove the assembly and cap the port.

2.15 Re Open the Collection Cylinder valve. 
Page 2 of 2

2.16 Re-start the Gas Collection System by pulling out the Red Button.

2.17 Open GC-SV-1.

2.18 On the Gas Distribution Hub Manifold Open valve V-6, V-7, V-9 and V-11.

Figure 1. Release Assembly

\section{Release Assembly}

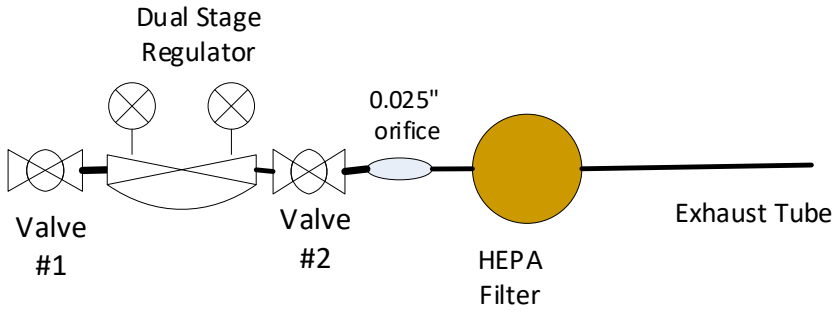

Attach 0.025" Orifice, Hepa-Filter and Exhaust Tube to a Dual Stage Regulator to form the Release Assembly

Figure 2. Release Assembly Attachment onto Gas Collection System

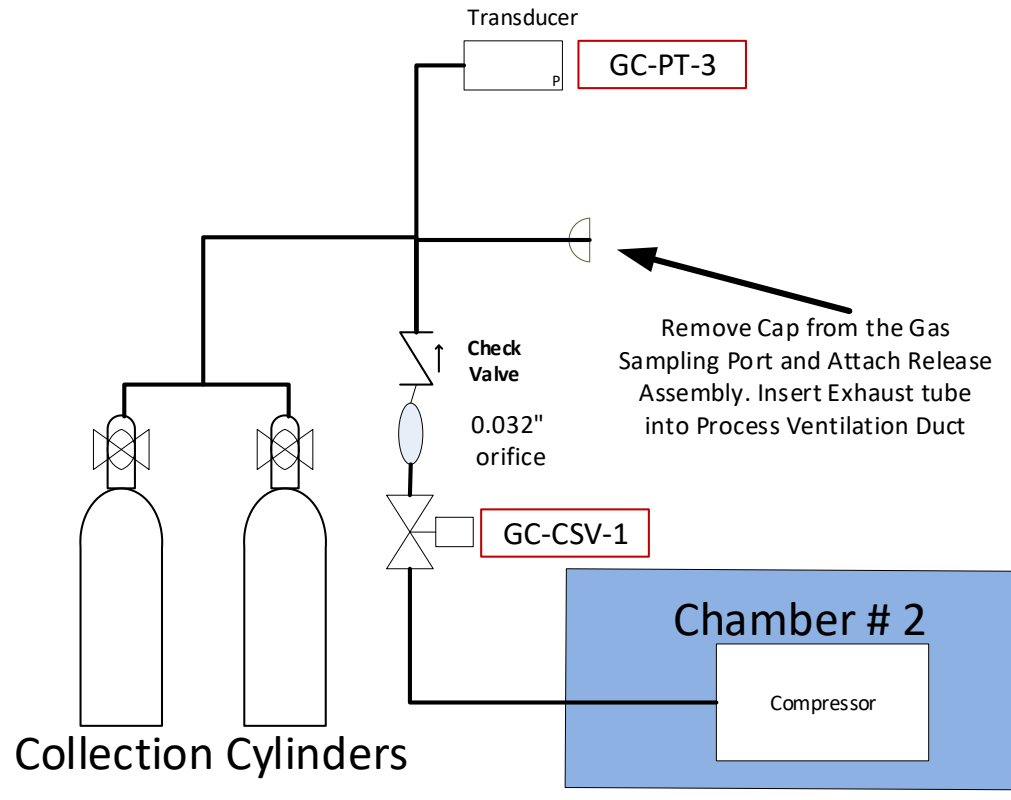

D035 Process Ventilation

Duct inside the Gas

Collection Enclosure
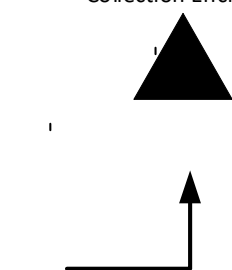

Insert Exhaust Tube into Duct 


\section{APPENDIX 17}

LEAF-PROC-024, Rev. 3: ${ }^{99}$ Mo PHASE II Production Tests - LabVIEW ${ }^{99}$ Mo Remote Recovery Data Acquisition and Control System: Complete Operations Abridged Version 
${ }^{99}$ Mo PHASE II Production Tests - LabVIEW ${ }^{99}$ Mo Remote Recovery Data Acquisition and Control System: Complete Operations Abridged Version

Low Energy Accelerator Facility, LEAF-PROC-024, Rev. 3

Approved: SChn Date: 02.25 .2020

Sergey Chemerisov, Manager, IVEM/LEAF

Effective Date: 02.26 .2020 


\section{Purpose}

Provide complete instructions for glovebox operations for the AMORE experiment in the LEAF facility, including the LabVIEW ${ }^{99}$ Mo Remote Recovery Data Acquisition and Control System.

\section{Scope}

This procedure applies to the following Argonne activities and entities.

\begin{tabular}{ll}
\hline LMS core processes: & Asset Management \\
\hline Organizations: & Experimental Operations and Facilities (EOF) Division \\
\hline Buildings: & 211 \\
\hline Specific locations: & LINAC \\
\hline $\begin{array}{l}\text { Other applicability } \\
\text { factors: }\end{array}$ & None \\
\hline Exclusions: & None \\
\hline USI applicability: & Yes \\
\hline
\end{tabular}

\section{Work Process}

\subsection{Introduction}

This document combines previous releases of six different work instructions, as listed in Table 1, below. The work instructions are to be used in the order given here:

TABLE 1 Work Instructions in this Procedure

\begin{tabular}{|c|c|c|c|}
\hline Section & Steps & Work Instruction Name & Previous Release File Name \\
\hline 3.2 .1 & $1-7$ & $\begin{array}{l}\text { Production Feeds Analysis and Process } \\
\text { Conditions Summary Sheet }\end{array}$ & $\begin{array}{l}\text { 194-v3_AMORE_Glovebox__ } \\
\text { FeedsAnalysisProcessCondsSummary } \\
\text { Sheet_Rev3.docx }\end{array}$ \\
\hline 3.2 .1 & $8-24$ & $\begin{array}{l}\text { Installation, Operation, and Removal of } \\
\text { Verification Tank }\end{array}$ & $\begin{array}{l}\text { 199-v3_AMORE_Glovebox_-__Rev3.docx } \\
\text { InstallOpsRemovalVerifTank_Rem }\end{array}$ \\
\hline 3.2 .3 & $25-33$ & $\begin{array}{l}\text { Installation of Feed Bottles, Effluent Cart, } \\
\text { Recovery Column, and Priming of Non- } \\
\underline{\text { Rad Feed Lines }}\end{array}$ & $\begin{array}{l}\text { 197-v3_AMORE_Glovebox__ } \\
\text { InstFeedsEffluCartColumnPrimeLines_ } \\
\text { Rev3.docx) }\end{array}$ \\
\hline 3.2 .4 & $34-69$ & Solution Irradiation and 99Mo Recovery & $\begin{array}{l}\text { 195-v4_AMORE_Glovebox_ } \\
\text { SolnIrradMo99RecoveryOps_Rev4.docx }\end{array}$ \\
\hline 3.2 .5 & $70-78$ & 99Mo Recovery Sample Retrieval & $\begin{array}{l}\text { 219-v4_AMORE_Glovebox_- } \\
\text { Mo99RecoverySampRetriev_Rev4.docx }\end{array}$ \\
\hline 3.2 .6 & $79-92$ & $\begin{array}{l}\text { Washout of 99Mo Recovery System and } \\
\text { Sample Retrieval Subsystems }\end{array}$ & $\begin{array}{l}\text { 196-v3_AMORE_Glovebox__ } \\
\text { Mo99RecoverySystemWashout_ } \\
\text { Rev3.docx }\end{array}$ \\
\hline
\end{tabular}

The steps in each section have been formatted to be followed in the sequential style presented. The sequential numbering of the steps is used to transcribe information between steps. 
When printing this document for use in operations, the user must:

- PRINT PAGES IN COLOR

- Hole punch ALL pages and secure in a binder (e.g. 3-ring binder or 3/4 in. binding comb) before each experiment.

\subsection{Step-by-Step Procedure}

The parameters found in step 7 MUST be determined and verified to be within ASE control limits prior to irradiation. All system interface steps (17, 38, 39, 66, 82, and 85) MUST be completed as written. All remaining steps in this procedure are considered work instructions and may be modified to complete the processing steps as long as modifications are documented. This procedure is to be performed by Workers appointed to the AMORE ${ }^{99}$ Mo Recovery Operations Team.

\section{IMPORTANT NOTES:}

- $\quad$ VCR connections with SS gaskets require a 1/8 turn past finger tight

- ALWAYS use a new VCR gasket when making a connection

- When installing/uninstalling/moving shielded containers (verification tank, effluent cart, etc.) ALWAYS wear steel toed shoes

\subsubsection{Production Feeds Analysis and Process Conditions Summary Sheet (PFA-PCS Sheet)}

LabVIEW - Lab Notebook No.: Pages:

\begin{tabular}{|c|c|}
\hline Step & Action \\
\hline \multirow[t]{10}{*}{1.} & Samples To Be Collected \\
\hline & 1.1. Number of Target Solution Mixing samples pre-irradiation: \\
\hline & 1.2. Number of Target Solution Mixing samples during irradiation: \\
\hline & 1.3. Number of Pre-Load Acid Wash samples: ___ OR $\square$ Skipped \\
\hline & 1.4. Number of Column Loading samples: \\
\hline & 1.5. Number of Post-Load Acid Wash samples: \\
\hline & 1.6. Number of Post-Load H2O Wash samples: \\
\hline & 1.7. Number of Post-Load $\mathrm{NaOH}$ Wash samples: __ OR $\square$ Skipped \\
\hline & 1.8. Number of Column Stripping samples: \\
\hline & 1.9. Number of Post-Strip H2O Wash samples: \\
\hline \multirow[t]{4}{*}{2.} & Select a Column Stripping Path \\
\hline & 2.1. $\square$ To transfer cask \\
\hline & $\underline{\mathbf{O R}}$ \\
\hline & 2.2. $\square$ To Mo99 processing cell (D024 Hot Cell) \\
\hline \multirow[t]{2}{*}{3.} & Fresh Acid - Used for the following processing steps \\
\hline & 3.1. Leak checking the column \\
\hline
\end{tabular}




\begin{tabular}{|c|c|}
\hline Step & $\begin{array}{r}\text { Action } \\
\end{array}$ \\
\hline & A. Volume to be used: \\
\hline & i. Default $=5 \min . \times 167 \mathrm{~mL} / \min =835 \mathrm{~mL}$ \\
\hline & ii. Flow rate: _ \\
\hline & 3.2. Pre-Pre-Load Acid Wash (Acid Pre-heater activation) \\
\hline & A. Volume to be used: \\
\hline & i. Default $=5 \mathrm{~min} . \times 84 \mathrm{~mL} / \mathrm{min}=420 \mathrm{~mL}$ \\
\hline & B. Flow rate: $\_\mathrm{mL} / \mathrm{min}$ \\
\hline & 3.3. Pre-Load Acid Wash \\
\hline & A. Volume to be used: \\
\hline & i. Default $=7.5 \mathrm{~min} . \times 167 \mathrm{~mL} / \mathrm{min}=1252 \mathrm{~mL}$ \\
\hline & B. Flow rate: $\_\mathrm{mL} / \mathrm{min}$ \\
\hline & 3.4. Post-Load Acid Wash \\
\hline & A. Volume to be used: \\
\hline & i. Default $=7.5 \mathrm{~min} . \times 167 \mathrm{~mL} / \mathrm{min}=1252 \mathrm{~mL}$ \\
\hline & B. Flow rate: __ $\mathrm{mL} / \mathrm{min}$ \\
\hline & 3.5. Final Acid Wash \\
\hline & A. Volume to be used: \\
\hline & i. Default $=7.5 \mathrm{~min} . \times 84 \mathrm{~mL} / \mathrm{min}=630 \mathrm{~mL}$ \\
\hline & B. Flow rate: __ $\mathrm{mL} / \mathrm{min}$ \\
\hline & 3.6. Loop rinsing for spike test \\
\hline & A. Volume to be used: $\_$mL \\
\hline & i. Default $=7.5 \mathrm{~min} . \mathrm{x} 84 \mathrm{~mL} / \mathrm{min}=630 \mathrm{~mL}$ \\
\hline & B. Flow rate: _ \\
\hline & 3.7. Acid system rinse out \\
\hline & A. Volume to be used: ___ $\mathrm{mL}$ \\
\hline & i. Default $=7.5 \mathrm{~min} . \times 84 \mathrm{~mL} / \mathrm{min}=630 \mathrm{~mL}$ \\
\hline & B. Flow rate: $\_\mathrm{mL} / \mathrm{min}$ \\
\hline & 3.8. Priming lines \\
\hline & A. Volume to be used: __ $\mathrm{mL}$ \\
\hline & B. Flow rate: varies \\
\hline & 3.9. Total Volume to be used: \\
\hline & A. Sum of $[\operatorname{step} 3.1 . A]+[\operatorname{step} 3.2 . A]+[\operatorname{step} 3.3 . A]+[$ step 3.4.A $]+[$ step 3.5.A $]+$ \\
\hline & {$[$ step 3.6.A $]+[$ step 3.7.A $]+[$ step 3.8.A $]=\_m L$} \\
\hline
\end{tabular}




\begin{tabular}{|c|c|}
\hline Step & Action \\
\hline & 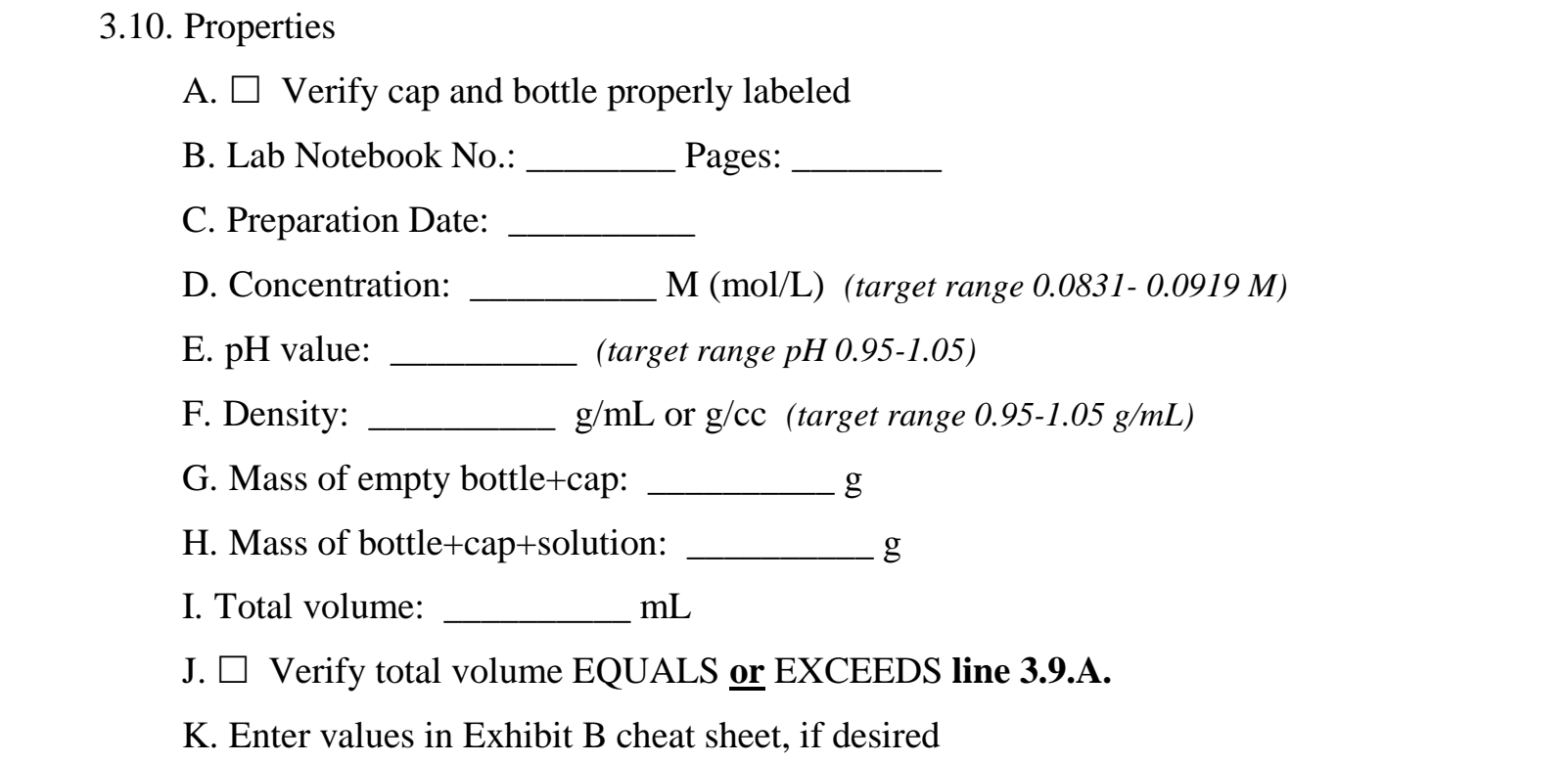 \\
\hline 4. & 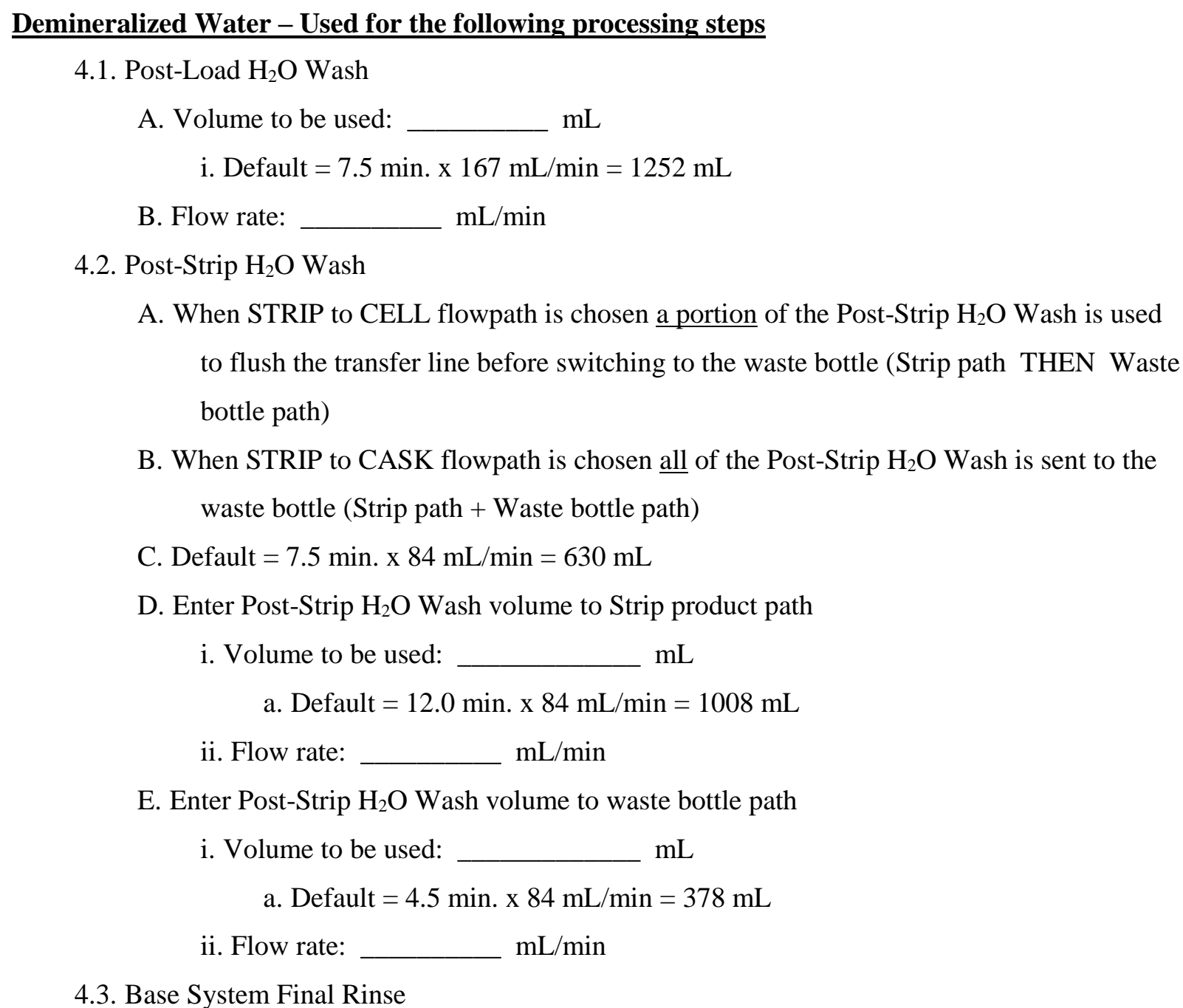 \\
\hline
\end{tabular}




\begin{tabular}{|c|c|}
\hline Step & $\begin{array}{rc}\text { Action } \\
\end{array}$ \\
\hline & 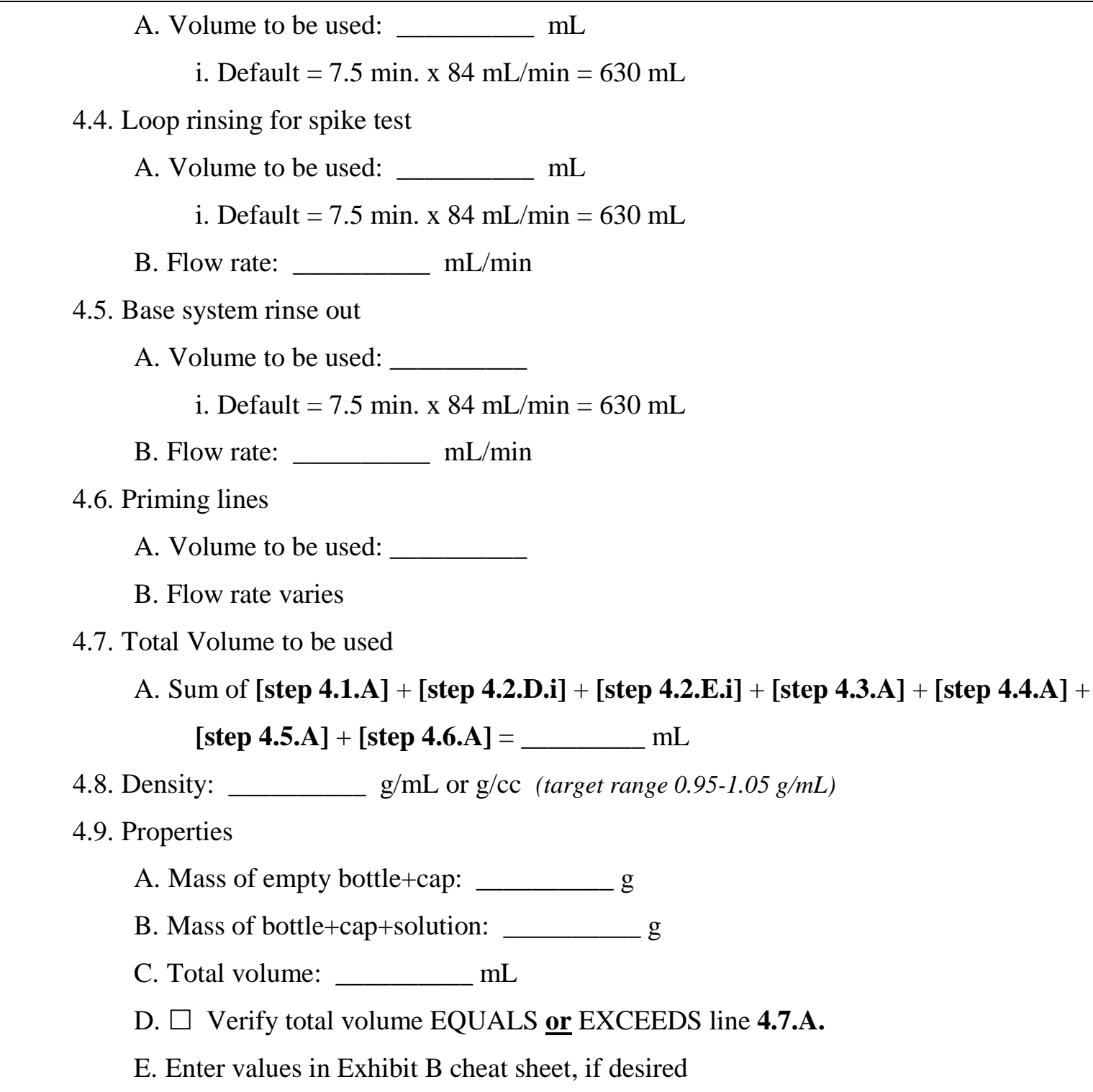 \\
\hline 5. & 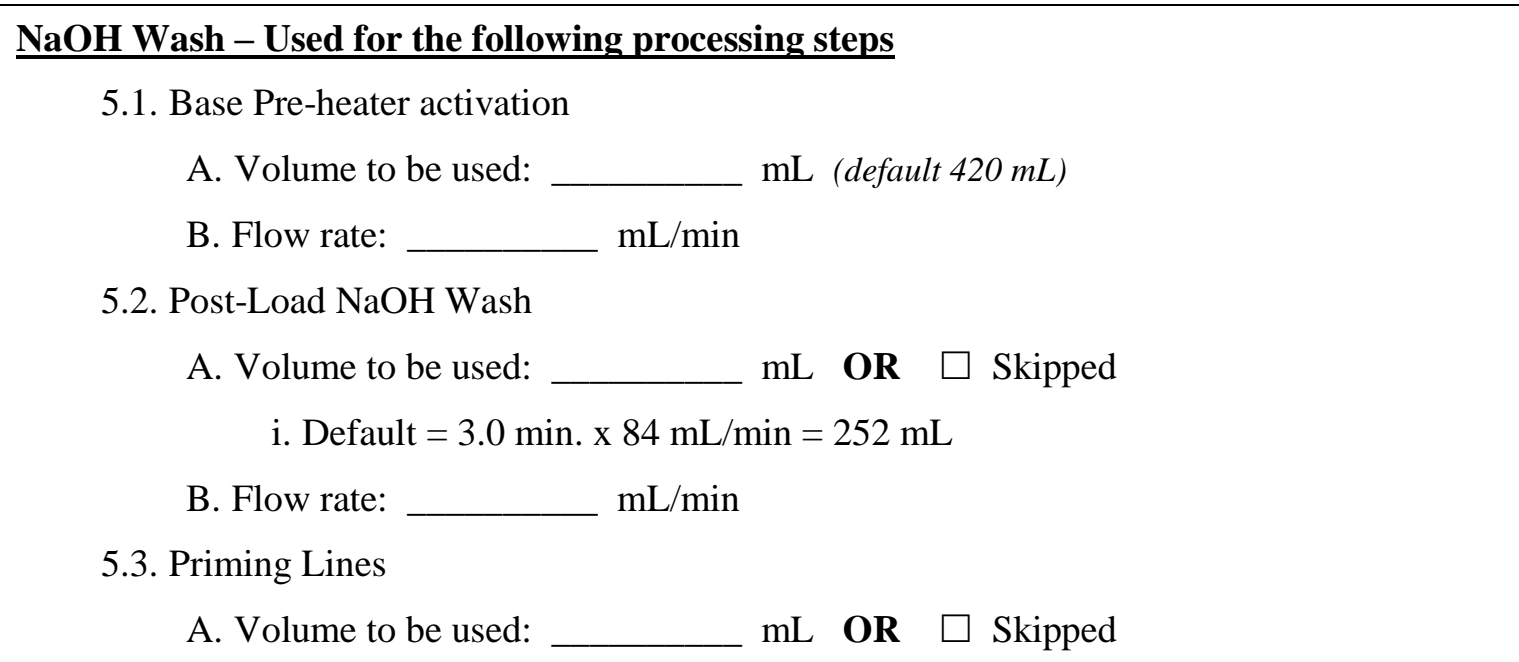 \\
\hline
\end{tabular}




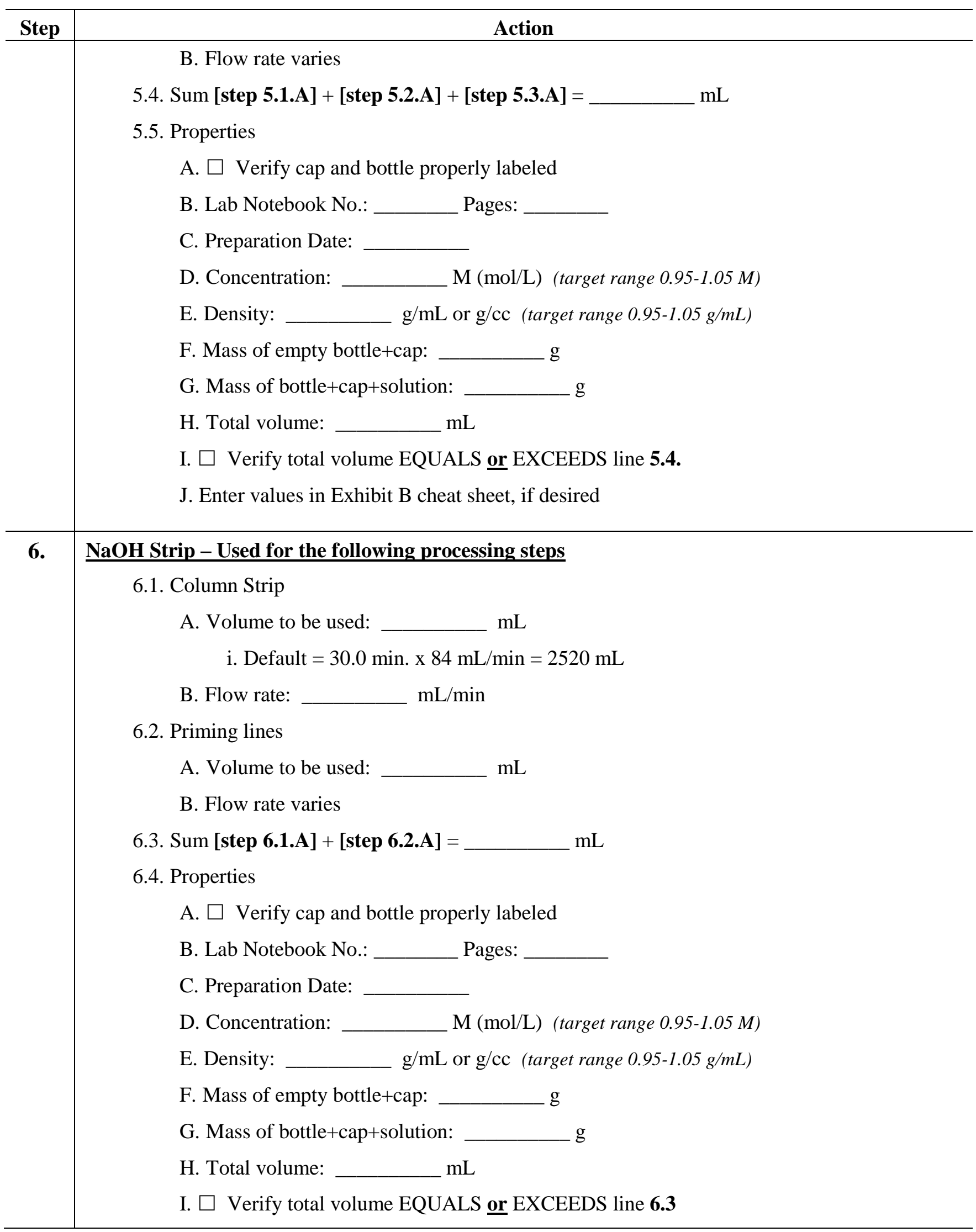




\begin{tabular}{|c|c|}
\hline Step & Action \\
\hline & J. Enter values in Exhibit B cheat sheet, if desired \\
\hline \multirow[t]{32}{*}{7.} & Uranyl Sulfate Target Solution - Used for the following processing steps \\
\hline & 7.1. Target Solution Mixing during irradiation \\
\hline & 7.2. Column loading \\
\hline & A. Volume to be used: \\
\hline & i. Default $=108 \mathrm{~min} . \times 167 \mathrm{~mL} / \mathrm{min}=18036 \mathrm{~mL}$ \\
\hline & B. Flow rate: $\_\mathrm{mL} / \mathrm{min}$ \\
\hline & 7.3. Properties \\
\hline & A. Target solution CURIE No.: \\
\hline & B. SPM No.: \\
\hline & C. Post-irradiation analysis \\
\hline & i. Date of last irradiation: \\
\hline & ii. Lab Notebook No.: _____ Pages: __ \\
\hline & iii. Analysis Date: \\
\hline & iv. Analysis ID: \\
\hline & v. Uranium concentration: ___ \\
\hline & a. Must be measured by ACL using High Precision ICP-OES \\
\hline & b. Concentration limit $145 \mathrm{~g}$ U/L set in [ASE 2.9.1.1] \\
\hline & vi. Acid concentration: ___ $\mathrm{M}(\mathrm{mol} / \mathrm{L})$ (target range $0.0831-0.0919 \mathrm{M})$ \\
\hline & vii. $\mathrm{pH}$ value: __ (target range $\mathrm{pH}$ 0.95-1.05) \\
\hline & viii. Density: __ $\mathrm{g} / \mathrm{mL}$ or $\mathrm{g} / \mathrm{cc}$ (target range $1.14-1.26 \mathrm{~g} / \mathrm{mL}$ ) \\
\hline & ix. Mass in verification tank: _ـ $\mathrm{g}$ (from step 19.26 on p. 24) \\
\hline & x. Calculate volume in verification tank: ___ $\mathrm{mL}($ target range $\leq 18 \mathrm{~L})$ \\
\hline & a. [Line 7.3.C.ix] / [Line 7.3.C.viii] = volume in mL \\
\hline & b. Volume limit of $20 \mathrm{~L}$ set in [ASE 2.9.1.1] \\
\hline & D. Post-adjustment analysis \\
\hline & i. Date of last irradiation: \\
\hline & ii. Lab Notebook No.: _____ Pages: \\
\hline & iii. Analysis Date: \\
\hline & iv. Analysis ID: \\
\hline & v. Uranium concentration: ___ $\mathrm{g} \mathrm{U} / \mathrm{L}$ (target range $\leq 145 \mathrm{~g} \mathrm{U} / \mathrm{L}$ ) \\
\hline & a. Must be measured by ACL using High Precision ICP-OES \\
\hline & b. Concentration limit $145 \mathrm{~g}$ U/L set in [ASE 2.9.1.1] \\
\hline
\end{tabular}




\begin{tabular}{|c|c|}
\hline Step & Action \\
\hline & 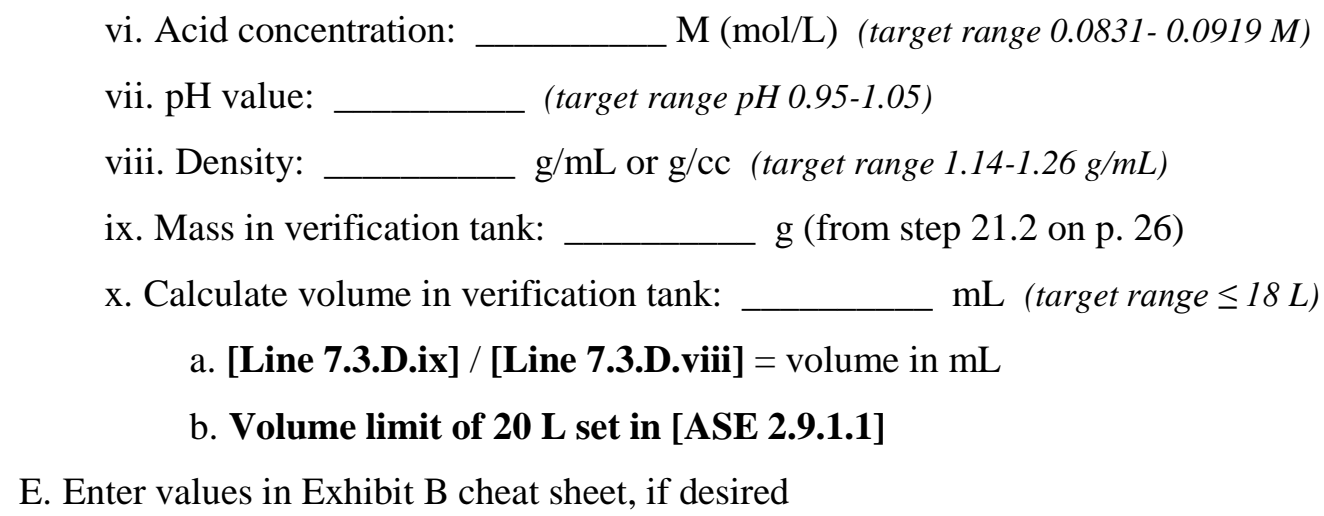 \\
\hline
\end{tabular}




\subsubsection{Installation, Operation, and Removal of Verification Tank}

\begin{tabular}{|c|c|}
\hline Step & Action \\
\hline \multirow[t]{15}{*}{8.} & $\underline{\text { Remove Spent Packed Column from Glovebox Cabinet \#1 (Left Cabinet) }}$ \\
\hline & 8.1. Verify most current RWP signed by workers \\
\hline & A. RWP \#: \\
\hline & 8.2. Open cabinet $\# 1$ door (left most cabinet) \\
\hline & 8.3. Have HP Tech perform pre-job survey smears \\
\hline & $\begin{array}{l}\text { A. Suggested smear locations: interface between lead shielded pig and glove box, exterior } \\
\text { of lead shielded pig, LEFT, RIGHT, and BOTTOM sides of Cabinet \#1 }\end{array}$ \\
\hline & i. DO NOT remove support table under lead shielded pig to perform smears \\
\hline & ii. Smear around support table under lead shielded pig \\
\hline & iii. ONLY smear legs of support table \\
\hline & $\begin{array}{l}\text { iv. DO NOT smear top surface of support table - POTENTIAL PINCH/CRUSH } \\
\text { HAZARD }\end{array}$ \\
\hline & $\begin{array}{l}\text { v. DO NOT smear back wall of Cabinet \#1 - this area can be smeared once the lead } \\
\text { shielded pig has been removed }\end{array}$ \\
\hline & $\begin{array}{l}\text { 8.4. Close cabinet \#1 door and hold for pre-job smears. If HP Tech determines it is required, } \\
\text { assist in reducing contamination levels prior to proceeding to step } 8.5\end{array}$ \\
\hline & $\begin{array}{l}\text { 8.5. Stage the transport collar for the column/pig assembly, ratcheting transport strap, } 4 \text { gallon } \\
\text { trash bag and tape, } 4 \text {-section ramp, and two VCR caps with two NEW VCR gaskets. Stage } \\
\text { crescent wrench and } 3 / 4 \text { in open-ended wrench in glovebox }\end{array}$ \\
\hline & A. Transport collar has two large eye bolts attached \\
\hline & B. DO NOT INSTALL THE RAMP YET \\
\hline
\end{tabular}




\subsection{Inside the glovebox:}

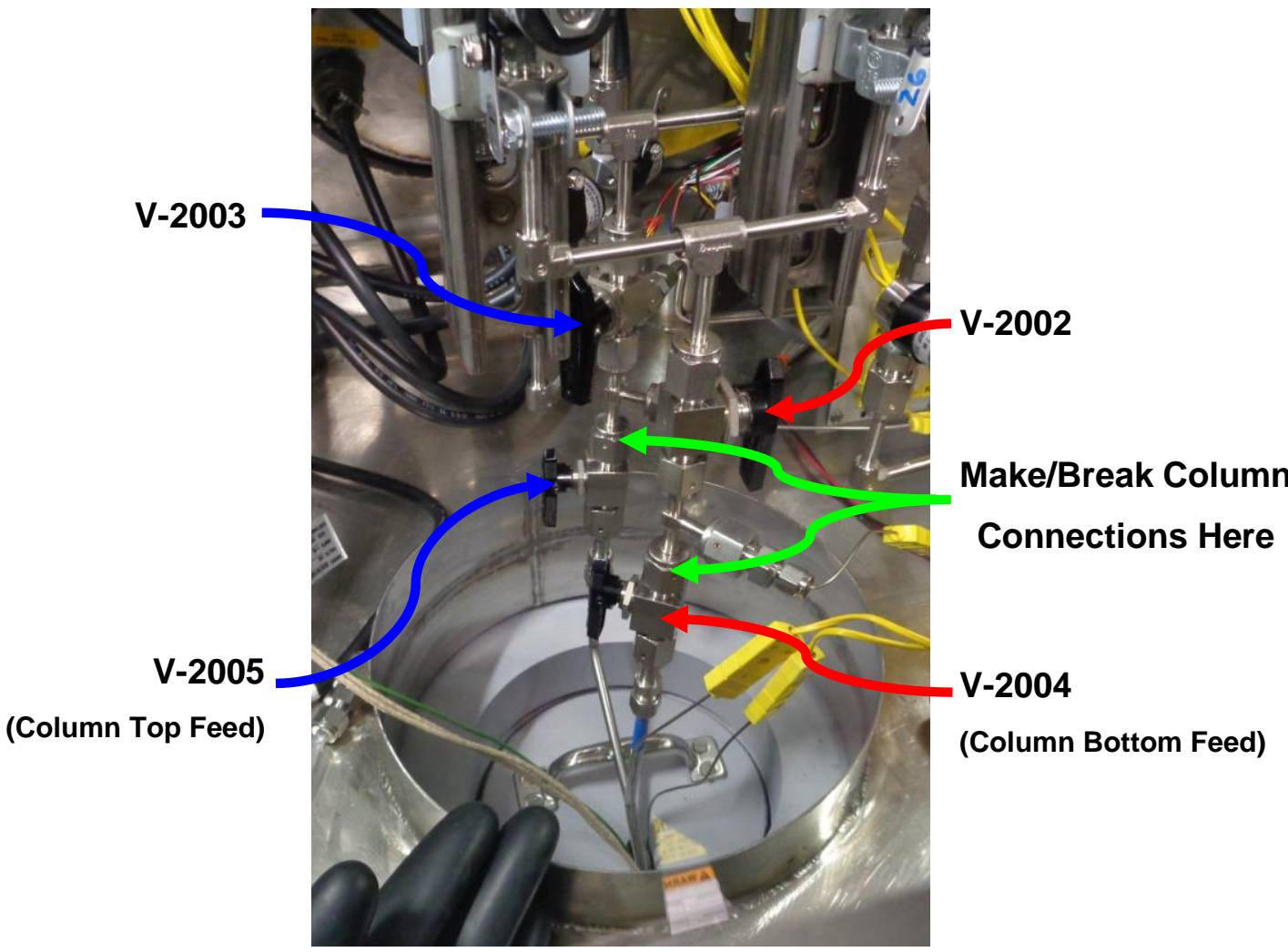

A. Stage column jumper (see Exhibit B for components)

B. Verify that the VCR 2-way system column connection valves (V-2002 \& V-2003) and the VCR 2-way ball valves attached to the column (V-2004 \& V-2005) are closed

C. Prepare paper towels or rags to catch residual liquid trapped between system valve/column valve interfaces

D. Disconnect column valves at the VCR fittings shown by green arrows in figure (above) i. The VCR tees should remain attached to the system valves V-2002 \& V-2003

E. Verify connections are FULLY loosened and FREE

F. Use a CLEAN rag or paper towel (may be wetted with Radiac) to wipe down column valves V-2004 \& V-2005, the lead shielded pig cover and handle, and exposed surfaces of the connection collar

G. Disconnect column heat tape from receptacle and tuck power cable in the interior ring of the connection collar

H. Disconnect cables from Column CONTROL \& OVER-TEMPERATURE thermocouples and tuck cables out of the way, off to the side, away from the column port hole

I. Disconnect leak sensor cable

i. Tuck the end of the leak sensor cable CONNECTED TO THE LEAK SENSOR into the interior ring of the connection collar 


\begin{tabular}{|c|c|}
\hline Step & Action \\
\hline & $\begin{array}{l}\text { ii. Tuck the end of the leak sensor cable CONNECTED TO THE GLOVEBOX } \\
\text { LEAK SENSOR PANEL out of the way, off to the side, away from the column } \\
\text { port hole }\end{array}$ \\
\hline & 8.7. Inside Cabinet \#1 / Outside the Glovebox \\
\hline & A. Open cabinet \#1 door \\
\hline & B. Install 4-section ramp from in front of Cabinet \#1 \\
\hline & C. DO NOT remove support table under lead shielded pig \\
\hline & $\begin{array}{l}\text { D. Roll pig transport cart into place - near support table under lead shielded pig - and raise } \\
\text { platform of pig transport cart EQUAL to height of support table under lead shielded } \\
\text { pig }\end{array}$ \\
\hline & $\begin{array}{l}\text { E. REMOVE support table under lead shielded pig THEN IMMEDIATELY roll pig } \\
\text { transport cart under lead shielded pig and center the cart under the pig }\end{array}$ \\
\hline & $\begin{array}{l}\text { F. Have HP Tech smear TOP surface AND BOTTOM feet of support table once removed } \\
\text { and set the table to the side }\end{array}$ \\
\hline & $\begin{array}{l}\text { G. Ratchet pig transport cart platform until surface is snug against bottom of lead shielded } \\
\text { column pig }\end{array}$ \\
\hline & $\begin{array}{l}\text { H. Chock wheels of pig transport cart to prevent the cart from moving as lead shielded } \\
\text { column pig is detached from glovebox mounting ring }\end{array}$ \\
\hline & I. Inside the glovebox - Verify the following \\
\hline & i. $\square$ Column connection valves are closed \\
\hline & ii. $\square$ Column is freed from system VCR connections \\
\hline & iii. $\square$ Column thermocouples are disconnected \\
\hline & iv. $\square$ Column heat tape plug is disconnected \\
\hline & v. $\square$ Column leak sensor is disconnected \\
\hline & $\begin{array}{l}\text { J. Disengage one toggle clamp from appropriate toggle clamp hook of pig locking ring to } \\
\text { lower the column/pig assembly slightly }\end{array}$ \\
\hline & K. Inside the glovebox - Verify VCR connections are still free \\
\hline & $\begin{array}{l}\text { L. Disengage remaining toggle clamp from appropriate toggle clamp hook of pig locking } \\
\text { ring to fully lower the column/pig assembly onto the cart }\end{array}$ \\
\hline & M. Inside the glovebox - Verify VCR connections are still free \\
\hline & $\begin{array}{l}\text { N. Slowly lower platform of pig transport cart, ensuring cables are free and come down } \\
\text { with the column pig }\end{array}$ \\
\hline & O. Fully lower platform of pig transport cart \\
\hline & $\begin{array}{l}\text { P. IMMEDIATELY attach VCR caps with NEW gaskets to column valves V-2004 \& V- } \\
2005\end{array}$ \\
\hline & $\begin{array}{l}\text { Q. Have HP Tech smear the column valves (V-2004 \& V-2005), column heater power } \\
\text { cable, column thermocouple ends, cable attached to column leak sensor }\end{array}$ \\
\hline & a. DO NOT pull the leak sensor out \\
\hline
\end{tabular}




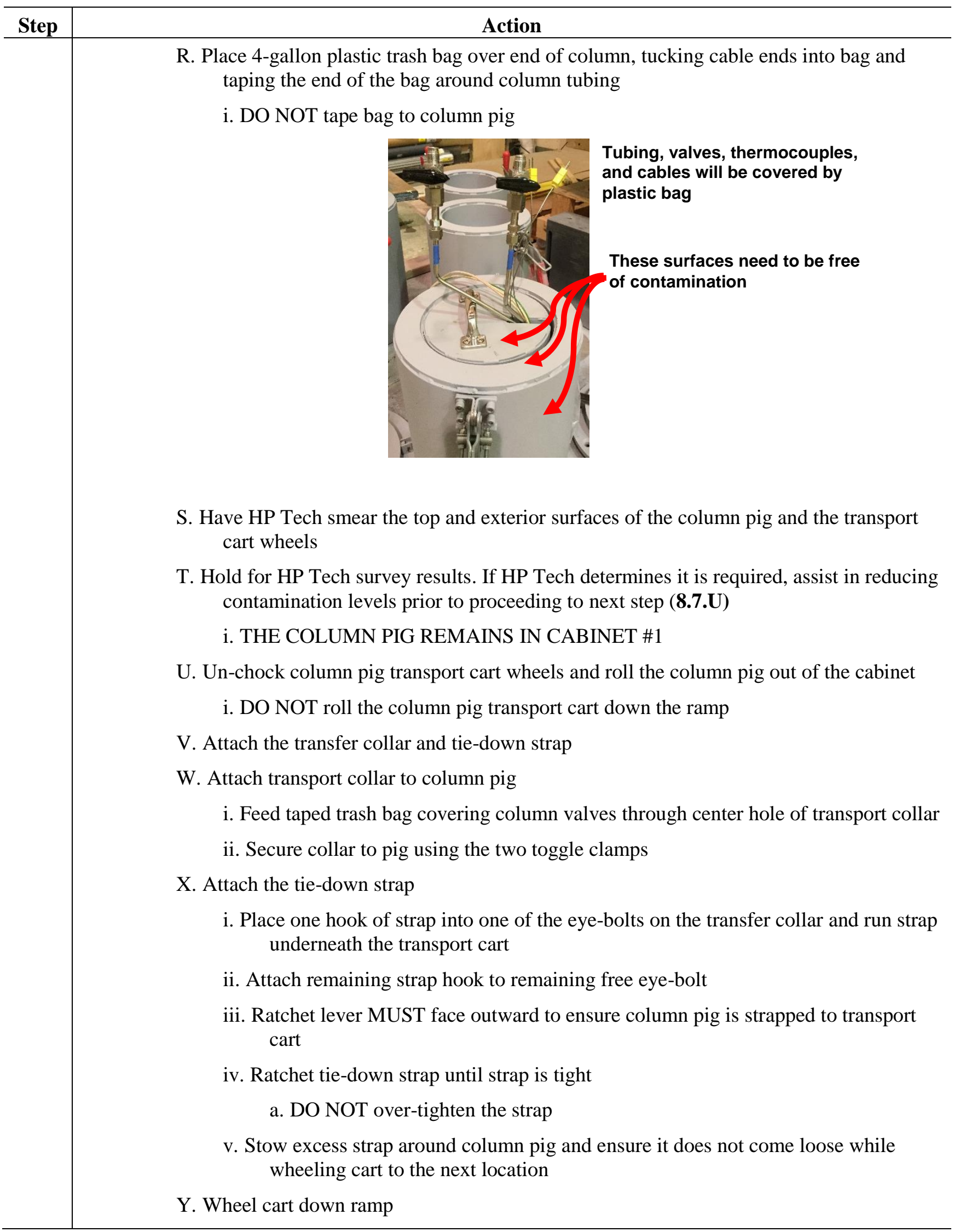




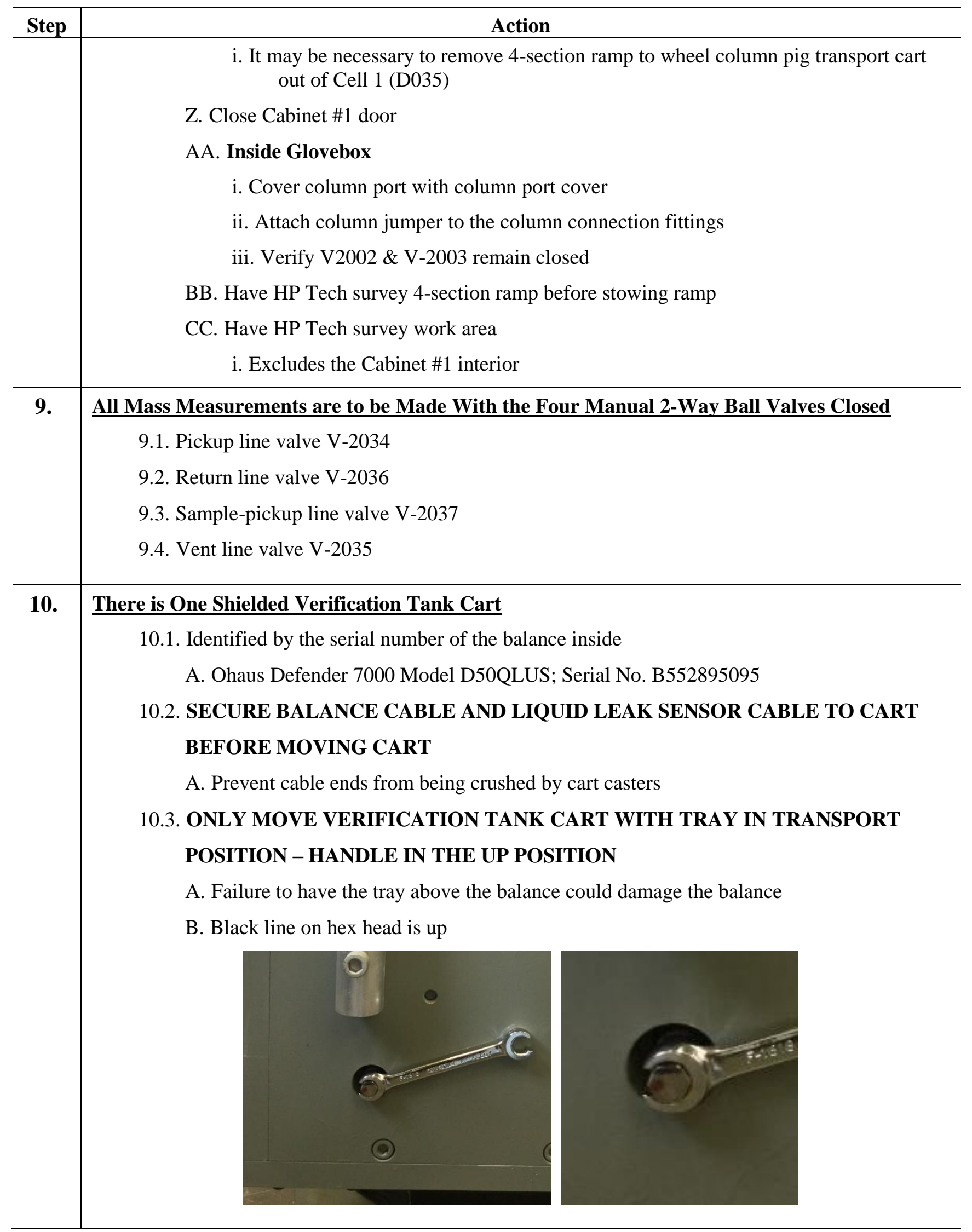




\begin{tabular}{|c|c|}
\hline Step & Action \\
\hline & 10.4. IF NECESSARY ONLY REMOVE THE RIGHT SIDE OF THE SHIELDED \\
\hline & VERIFICATION TANK CART LID \\
\hline & A. Right half of lid weighs over $120 \mathrm{lbs}$. \\
\hline & 10.5. The tank has four $1 / 4$ in liquid lines \\
\hline & A. Pickup line attached at bottom of tank through 1-1/2 in. tri-clamp fitting (see Exhibit B \\
\hline & for list of parts) \\
\hline & i. BALL VALVE HANDLE POINTS AWAY FROM TANK (DIRECTION OF \\
\hline & FLOW FROM TANK) \\
\hline & B. Return line attached to tank cover (see Exhibit B for list of parts) \\
\hline & i. BALL VALVE HANDLE POINTS TOWARD TANK (DIRECTION OF \\
\hline & FLOW INTO TANK) \\
\hline & C. $1 / 8$ in. sample-pickup \& vent lines (see Exhibit B for list of parts) \\
\hline & i. BALL VALVE HANDLE POINTS AWAY FROM TANK (DIRECTION OF \\
\hline & FLOW FROM TANK) \\
\hline & D. Sample-pickup line attached to tank cover \\
\hline & i. BALL VALVE HANDLE POINTS TOWARD TANK (DIRECTION OF \\
\hline & FLOW TO TANK) \\
\hline \multirow[t]{5}{*}{11.} & $\begin{array}{l}\text { Verify Verification Tank Balance is Calibrated (calibration sticker is affixed to Feed Balance } \\
\text { Indicator located on the left side of the LabVIEW control rack) }\end{array}$ \\
\hline & 11.1. Record feed balance calibration date: \\
\hline & A. Next calibration due: \\
\hline & B. If balance is out of calibration have balance calibrated \\
\hline & C. DO NOT PROCEED IF FEED BALANCE IS NOT CALIBRATED \\
\hline
\end{tabular}




\section{Install Verification Tank Cart}

12.1. Open cabinet \#1 (left side) door to full open

12.2. Position the 4-section ramp to roll verification tank cart into cabinet \#1

12.3. $\square$ Verify balance lever in transport positon

12.4. Roll verification tank cart to edge of cabinet \#1

12.5. $\square$ Attach balance cable

A. Connectors operate smoothly when mated to one another correctly - DO NOT FORCE CONNECTORS TOGETHER

12.6. $\square$ Verify balance is read by verification balance indicator

A. May need to turn appropriate balance indicator on

B. After startup if balance is read by indicator the indicator will show 0

i. If no reading double check connection

\section{ii. DO NOT PROCEED UNTIL BALANCE IS READ BY INDICATOR}

12.7. $\square$ Attach liquid leak sensor cable

12.8. Center the four plastic lines on top of verification tank cart lid

12.9. Push the fully connected verification cart into position in cabinet \#1

12.10. Remove the handle from the verification tank cart and store in D032 until verification tank cart is removed

12.11. Zero verification balance indicator by pressing the ON/ZERO soft key

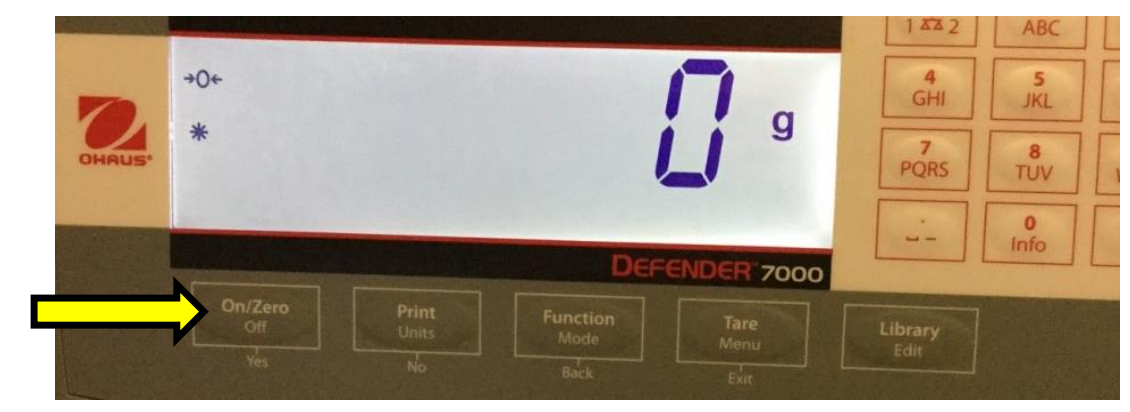

12.12. $\square$ Turn balance transport handle to weigh (down, with red dot on hex head facing up) 


\begin{tabular}{|c|c|}
\hline Step & Action \\
\hline & $\begin{array}{l}\text { 12.13. } \square \text { Verify verification balance is read by balance indicator } \\
\text { A. Should read a positive, non-zero value } \\
\text { 12.14. Remove the 4-section ramp and slowly close cabinet \#1 } \\
\text { A. DO NOT STORE 4-SECTION RAMPS IN CELL } 1 \text { (D035) }\end{array}$ \\
\hline 13. & $\begin{array}{l}\text { Inside the Glovebox - MAKE/BREAK LIQUID CONNECTIONS AT VCR FITTINGS ONLY } \\
\text { 13.1. Identify the feed connection jumper and effluent connection jumper (see Exhibit B items } \\
\text { 4-5) } \\
\text { 13.2. Extend verification vent line jumper from V-0160 to column/tank port hole } \\
\text { 13.3. Attach verification vent line V-2035 to V-0160 vent line jumper (sends verification tank } \\
\text { vent to gas collection system) } \\
\text { 13.4. Attach verification sample-pickup line to thief vial assembly }\end{array}$ \\
\hline 14. & $\begin{array}{l}\text { Checking Column Stripping Transfer Cask Installation } \\
\text { 14.1. Outside the white glovebox in Cell } 1 \text { (D035) } \\
\text { 14.2. } \square \text { Verify column stripping transfer cask is attached to white glovebox } \\
\text { A. Inside the white glovebox in Cell } 1 \text { (D035) } \\
\text { 14.3. } \square \text { Verify both } 2 \text {-way valves for liquid service (on the } 1 / 4 \text { in. line) are open } \\
\text { A. Handles parallel to the long axis of valve body } \\
\text { 14.4. } \square \text { Verify both } 2 \text {-way valves for vent service (on the } 1 / 8 \text { in. line) are open } \\
\text { A. Handles parallel to the long axis of valve body }\end{array}$ \\
\hline 15. & $\begin{array}{l}\text { At the Rack - Verify Sample Retrieval Valves Powered Off } \\
\text { 15.1. Verify Sample Retrieve Valve Power to OFF (switch is down) }\end{array}$ \\
\hline
\end{tabular}




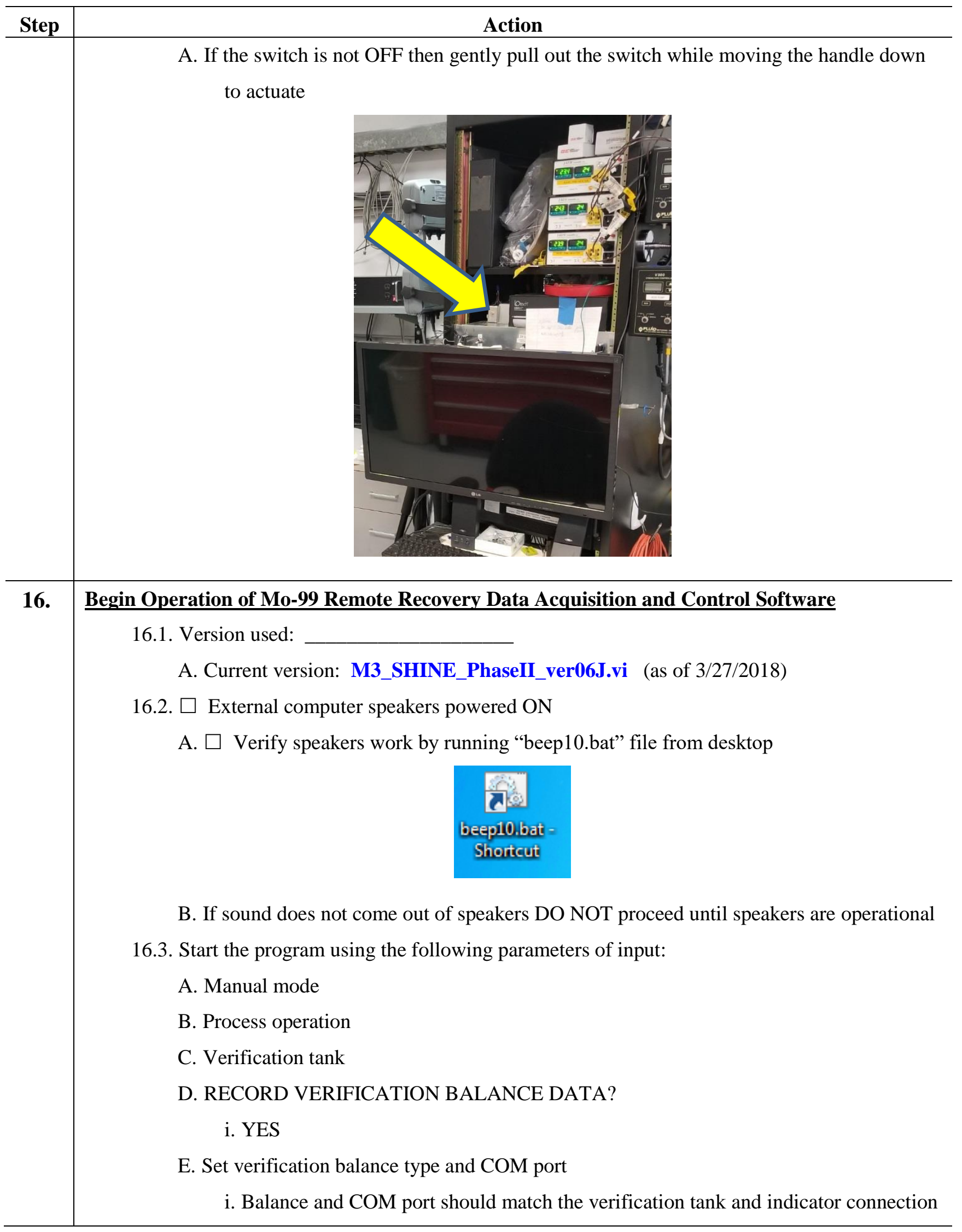




\begin{tabular}{|c|c|}
\hline Step & Action \\
\hline & F. Fresh Acid density $\rightarrow$ as default value $\rightarrow$ press OK \\
\hline & G. Base Wash density $\rightarrow$ as default value $\rightarrow$ press OK \\
\hline & H. Base Strip density $\rightarrow$ as default value $\rightarrow$ press OK \\
\hline & I. Target solution volume \\
\hline & i. Enter last target solution volume \\
\hline & J. Target solution concentration \\
\hline & i. Enter last target solution concentration \\
\hline & K. Target solution density \\
\hline & i. Enter last target solution density \\
\hline & L. Column effluent path \\
\hline & i. To Transfer Cask \\
\hline & M. Pre-Load Acid Wash processing volume $\rightarrow$ as default value $\rightarrow$ press OK \\
\hline & N. Column Loading processing volume $\rightarrow$ as default value $\rightarrow$ press $\mathrm{OK}$ \\
\hline & O. Post-Load Acid Wash processing volume $\rightarrow$ as default value $\rightarrow$ press OK \\
\hline & P. Post-Load Water Wash processing volume $\rightarrow$ as default value $\rightarrow$ press OK \\
\hline & Q. Use the Post-Load NaOH Wash step? $\rightarrow$ NO \\
\hline & R. Column stripping processing volume $\rightarrow$ as default value $\rightarrow$ press $\mathrm{OK}$ \\
\hline & S. Post-Strip Water Wash To Strip product processing volume $\rightarrow$ as default value $\rightarrow$ press \\
\hline & OK \\
\hline & T. Post-Strip Water Wash To Waste processing volume $\rightarrow$ as default value $\rightarrow$ press OK \\
\hline & U. Final Base System Water Wash processing volume $\rightarrow$ as default value $\rightarrow$ press $\mathrm{OK}$ \\
\hline & V. Final Acid System Acid Wash processing volume $\rightarrow$ as default value $\rightarrow$ press OK \\
\hline & W. Record LINAC temperatures? $\rightarrow$ YES \\
\hline & X. Filename prefix: ___ \\
\hline & Y. $\square$ ACID Pump controller powered ON (Rocker switch under front/left of ACID Pump \\
\hline & V300 controller) \\
\hline & Z. $\square$ ACID Pump to $\underline{\text { STOP }}$ (display alternates between OFF and current setting) \\
\hline & AA. $\square$ Verify verification tank balance reading at [Sensors].tab \\
\hline & i. Compare LabVIEW value to value on verification tank balance indicator \\
\hline 17. & Check Manual Dump Tank Valve OPEN \\
\hline
\end{tabular}

17.1. Contact a Gas Analysis/Collection team member 


\begin{tabular}{|c|c|}
\hline Step & Action \\
\hline & $\begin{array}{l}\text { 17.2. At the Dump Tank (211-D035) } \\
\text { A. } \square \text { Verify manual dump tank valve is OPEN } \\
\text { B. Recovery team member } \\
\quad / / \text { Name: } \_ \text {Date: __ Time: } \\
\text { C. Gas Analysis/Collection team member } \\
\quad / / \text { Name: } \_ \text {Dintials: ___ Date: __ Time: } \\
\text { 17.3. Recovery personnel continue to step } \mathbf{0} \text {. }\end{array}$ \\
\hline 18. & 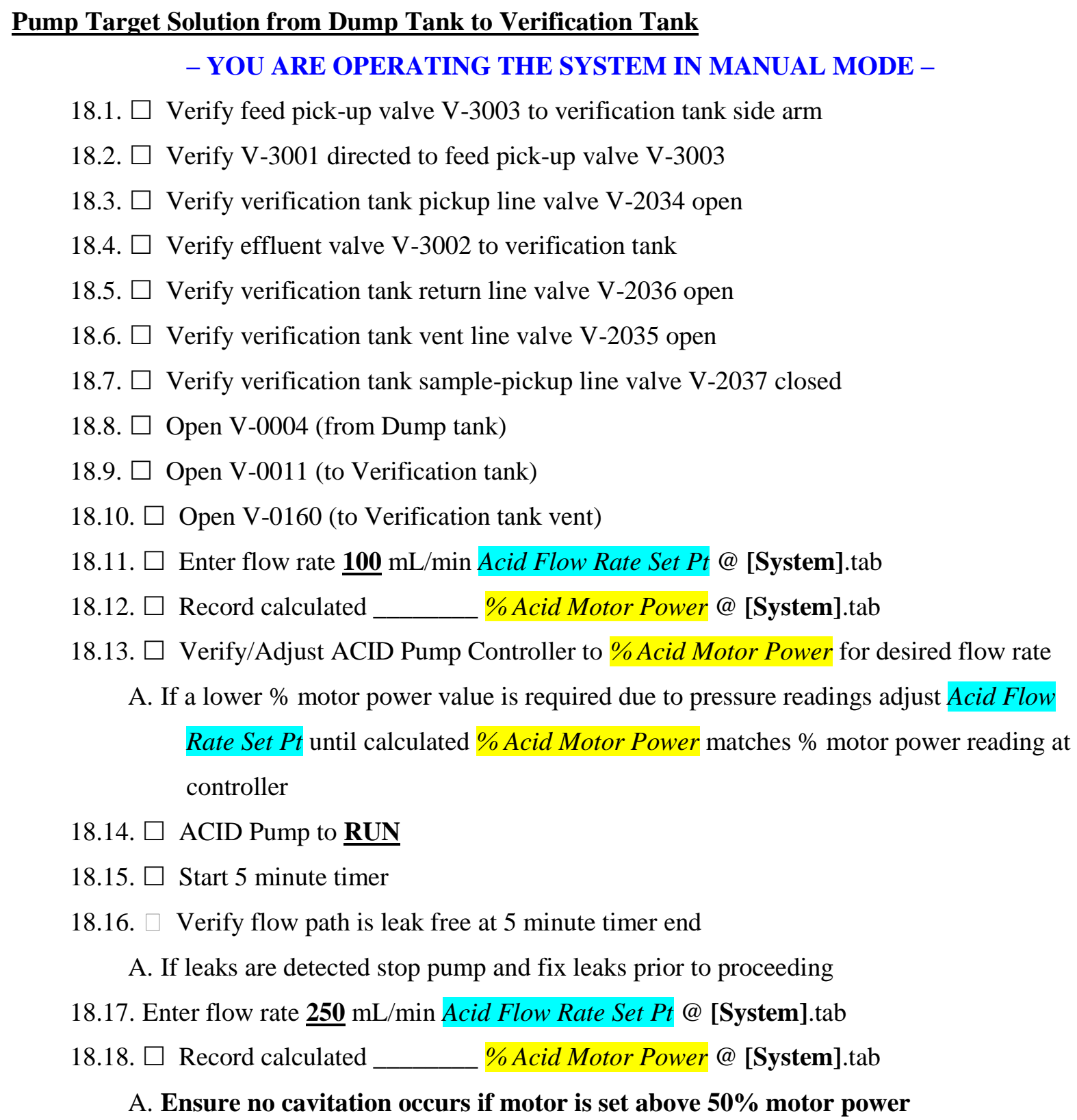 \\
\hline
\end{tabular}




\begin{tabular}{|c|c|}
\hline \multirow[t]{15}{*}{ Step } & Action \\
\hline & 18.19. $\square$ Verify/Adjust ACID Pump Controller to \% Acid Motor Power for desired flow rate \\
\hline & A. If a lower \% motor power value is required due to pressure readings adjust Acid Flow \\
\hline & Rate Set Pt until calculated \% Acid Motor Power matches \% motor power reading at \\
\hline & controller \\
\hline & 18.20. Monitor FEP tubing from V-0011 for target solution \\
\hline & 18.21. $\square$ Once FEP tubing from V-0011 is empty, set ACID Pump to $\underline{\text { STOP }}$ \\
\hline & 18.22. $\square$ Close V-0004 (from Dump tank) \\
\hline & 18.23. $\square$ Close V-0011 (to Verification tank) \\
\hline & 18.24. $\square$ Close V-0160 (to Verification tank vent) \\
\hline & 18.25. $\square$ Close verification tank pickup line valve V-2034 \\
\hline & 18.26. $\square$ Close verification tank return line valve V-2036 closed \\
\hline & 18.27. $\square$ Verify verification tank sample-pickup line valve V-2037 closed \\
\hline & 18.28. Record: verification tank balance indicator: ___ grams \\
\hline & 18.29. Record verification tank balance value at [Sensors].tab: ____ grams \\
\hline \multirow[t]{10}{*}{19.} & $\underline{\text { Retrieve Analytical Sample }}$ \\
\hline & 19.1. $\square$ Verify V-0004 closed (from Dump tank) \\
\hline & 19.2. $\square$ Verify V-0011 closed (to Verification tank) \\
\hline & 19.3. $\square$ Open V-0160 (to Verification tank vent) \\
\hline & 19.4. $\square$ Verify verification tank pickup line valve V-2034 closed \\
\hline & 19.5. $\square$ Verify verification tank return line valve V-2036 closed \\
\hline & 19.6. Attach thief vial assembly to verification tank sample-pickup valve V-2037 \\
\hline & A. Use disposable needles \\
\hline & B. Do not use all metal needles - hubs are made of nickel plated brass \\
\hline & C. Step 24, Alternate Sample Retrieval Configuration, page 29 may be used \\
\hline
\end{tabular}




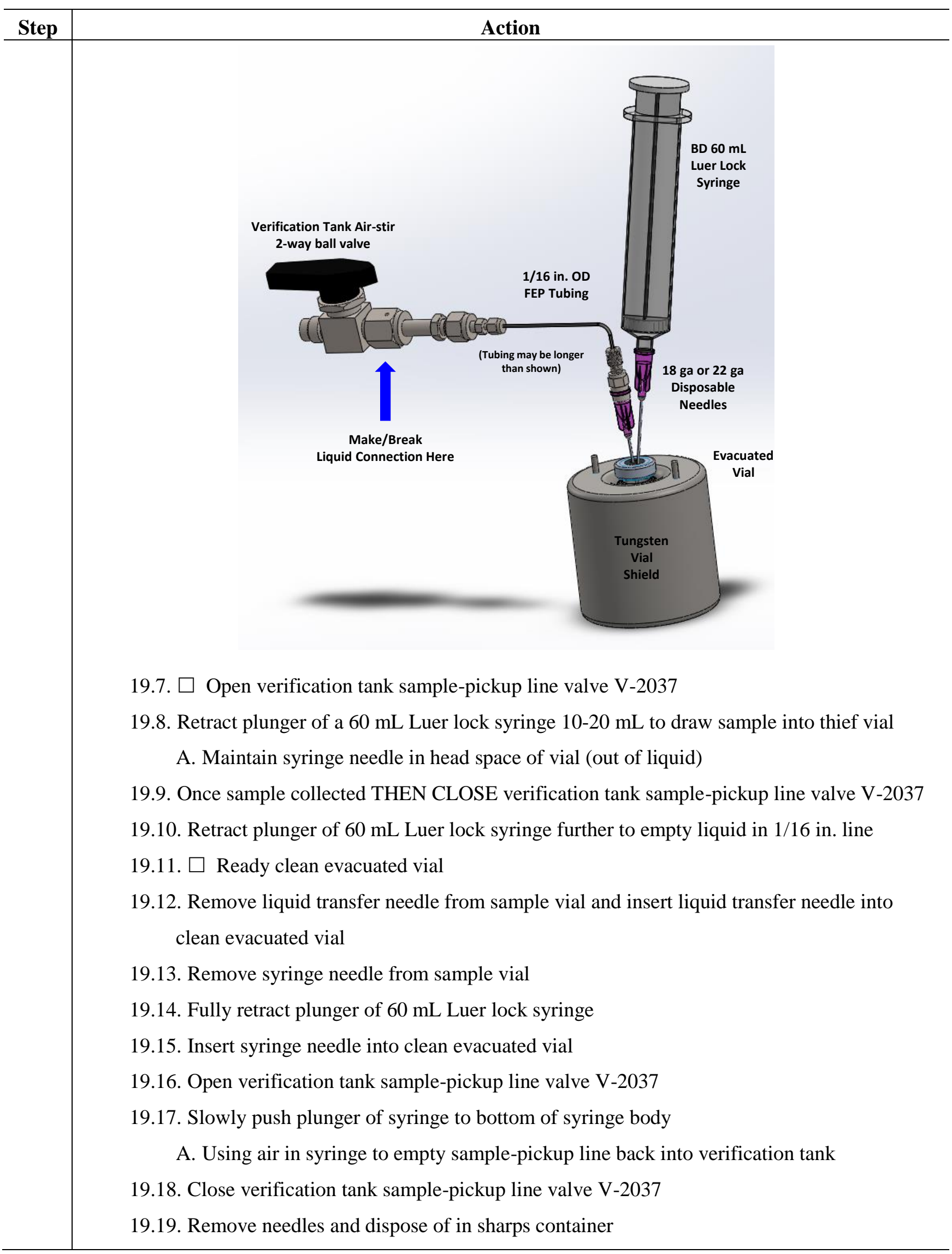




\begin{tabular}{|c|c|}
\hline \multirow[t]{12}{*}{ Step } & Action \\
\hline & 19.20. Disconnect thief vial assembly from verification tank sample-pickup line valve V-2037 \\
\hline & 19.21. $\square$ Verify V-0160 closed (to Verification tank vent) \\
\hline & 19.22. Close verification tank vent line valve V-2035 \\
\hline & 19.23. Record verification tank balance indicator: ____ grams \\
\hline & 19.24. Record verification tank balance value at [Sensors].tab: ___ grams \\
\hline & 19.25. Submit sample for analysis (if from step 21.1, continue to step 21.2, p 26) \\
\hline & 19.26. Enter analysis values at step 7.3.C on page 8 \\
\hline & 19.27. Person In Charge to determine if feed adjustment is required (choose ONE) \\
\hline & A. $\square$ If adjustment is required go to step 20 \\
\hline & $\underline{\mathbf{O R}}$ \\
\hline & B. $\square$ If no adjustment is required go to step 22 \\
\hline \multirow[t]{21}{*}{20.} & Adding Make-up Solution or Mo Spike to Verification Tank \\
\hline & 20.1. Record verification tank balance indicator: ___ grams \\
\hline & 20.2. Record verification tank balance value at [Sensors].tab: ___ grams \\
\hline & 20.3. If only a small volume needs to be added, a syringe may be used to inject the volume \\
\hline & through valve V-2037 using the Alternative Sample Retrieval Configuration (Step 24, \\
\hline & p. 29) \\
\hline & 20.4. Insert tubing from feed valve V-3003 into make-up bottle \\
\hline & 20.5. Turn feed valve V-3003 to bottle side-arm port \\
\hline & 20.6. $\square$ Verify V-3001 directed to feed pick-up valve V-3003 \\
\hline & 20.7. $\square$ Verify verification tank pickup line valve V-2034 open \\
\hline & 20.8. $\square$ Verify verification tank return line valve V-2036 open \\
\hline & 20.9. $\square$ Verify verification tank vent line valve V-2035 open \\
\hline & 20.10. $\square$ Verify verification tank sample-pickup line valve V-2034 closed \\
\hline & 20.11. $\square$ Open V-0003 (from External vessel) \\
\hline & 20.12. $\square$ Open V-0011 (to Verification tank) \\
\hline & 20.13. $\square$ Open V-0160 (to Verification tank vent) \\
\hline & 20.14. $\square$ Enter flow rate $\underline{\mathbf{1 0 0}} \mathrm{mL} / \mathrm{min}$ Acid Flow Rate Set Pt @ [System].tab \\
\hline & 20.15. $\square$ Record calculated ____ \% Acid Motor Power @ [System].tab \\
\hline & 20.16. $\square$ Verify/Adjust ACID Pump Controller to \% Acid Motor Power for desired flow rate \\
\hline & A. If a lower $\%$ motor power value is required due to pressure readings adjust Acid Flow \\
\hline & $\begin{array}{l}\text { Rate Set Pt until calculated \% Acid Motor Power matches \% motor power reading at } \\
\text { controller }\end{array}$ \\
\hline
\end{tabular}




\begin{tabular}{|c|c|}
\hline Step & Action \\
\hline & 20.17. $\square$ ACID Pump to $\underline{\mathbf{R U N}}$ \\
\hline & 20.18. Monitor feed make-up bottle until contents have been pumped into verification tank \\
\hline & 20.19. When feed make-up bottle contents are in system ACID pump to $\underline{\text { STOP }}$ \\
\hline & 20.20. Turn feed valve V-3003 to verification tank side-arm port \\
\hline & 20.21. $\square$ ACID Pump to $\underline{\mathbf{R U N}}$ \\
\hline & A. Using pump to mix contents of verification tank \\
\hline & 20.22. $\square$ Enter flow rate $\underline{\mathbf{3 0 0}} \mathrm{mL} / \mathrm{min}$ Acid Flow Rate Set Pt @ [System].tab \\
\hline & 20.23. $\square$ Record calculated ___ $\quad \%$ Acid Motor Power @ [System].tab \\
\hline & A. Ensure no cavitation occurs if motor is set above $50 \%$ motor power \\
\hline & 20.24. $\square$ Verify/Adjust ACID Pump Controller to \% Acid Motor Power for desired flow rate \\
\hline & 20.25. If a lower $\%$ motor power value is required due to pressure readings adjust Acid Flow \\
\hline & Rate Set Pt until calculated \% Acid Motor Power matches \% motor power reading at \\
\hline & controller \\
\hline & 20.26. NOTE - at $300 \mathrm{~mL} / \mathrm{min}$ it will take 60 minutes to completely circulate $18000 \mathrm{~mL}$ \\
\hline & 20.27. Record start time of mixing: ___ (LabVIEW time) \\
\hline & 20.28. Record mixing duration: ___ minutes \\
\hline & 20.29. Record end time of mixing: ___ (LabVIEW time) \\
\hline & 20.30. After sufficient mixing time ACID pump to $\underline{\text { STOP }}$ \\
\hline & 20.31. Insert tubing from feed valve V-3003 bottle side-arm port into empty bottle \\
\hline & 20.32. Turn feed valve V-3003 to bottle side-arm port \\
\hline & 20.33. $\square$ ACID Pump to $\underline{\mathbf{R U N}}$ \\
\hline & A. Drawing glovebox atmosphere through 3-way ball valve bottle side-arm port \\
\hline & B. Pushing solution from tubing into verification tank \\
\hline & 20.34. Start 3 minute timer \\
\hline & 20.35. At 3 minute timer end ACID pump to $\underline{\mathbf{S T O P}}$ \\
\hline & 20.36. $\square$ Close V-0003 (from External vessel) \\
\hline & 20.37. $\square$ Close V-0011 (to Verification tank) \\
\hline & 20.38. $\square$ Close V-0160 open (to Verification tank vent) \\
\hline & 20.39. $\square$ Close verification tank pickup line valve V-2034 \\
\hline & 20.40. $\square$ Close verification tank return line valve V-2036 \\
\hline & 20.41. $\square$ Close verification tank vent line valve V-2035 \\
\hline & 20.42. $\square$ Verify verification tank sample-pickup line valve V-2034 closed \\
\hline & 20.43. Record verification tank balance indicator: _____ grams \\
\hline
\end{tabular}




\begin{tabular}{|c|c|}
\hline Step & Action \\
\hline & $\begin{array}{l}\text { 20.44. Record verification tank balance value at [Sensors].tab: __ grams } \\
\text { 20.45. Go to step 21, Retrieve Analytical Sample After Addition of Make-up Solution. }\end{array}$ \\
\hline 21. & $\begin{array}{l}\text { Retrieve Analytical Sample After Addition of Make-up Solution or Mo Spike } \\
\text { 21.1. Follow step } 19 \text { for retrieval of analytical sample (page 22), proceeding to step } \mathbf{2 1 . 2} \text { after } \\
\text { step } \mathbf{1 9 . 2 5} \\
\text { 21.2. Enter analysis values at step 7.3.D on page } 8 \\
\text { 21.3. Go to step 22, Return Target Solution to Target Vessel }\end{array}$ \\
\hline 22. & 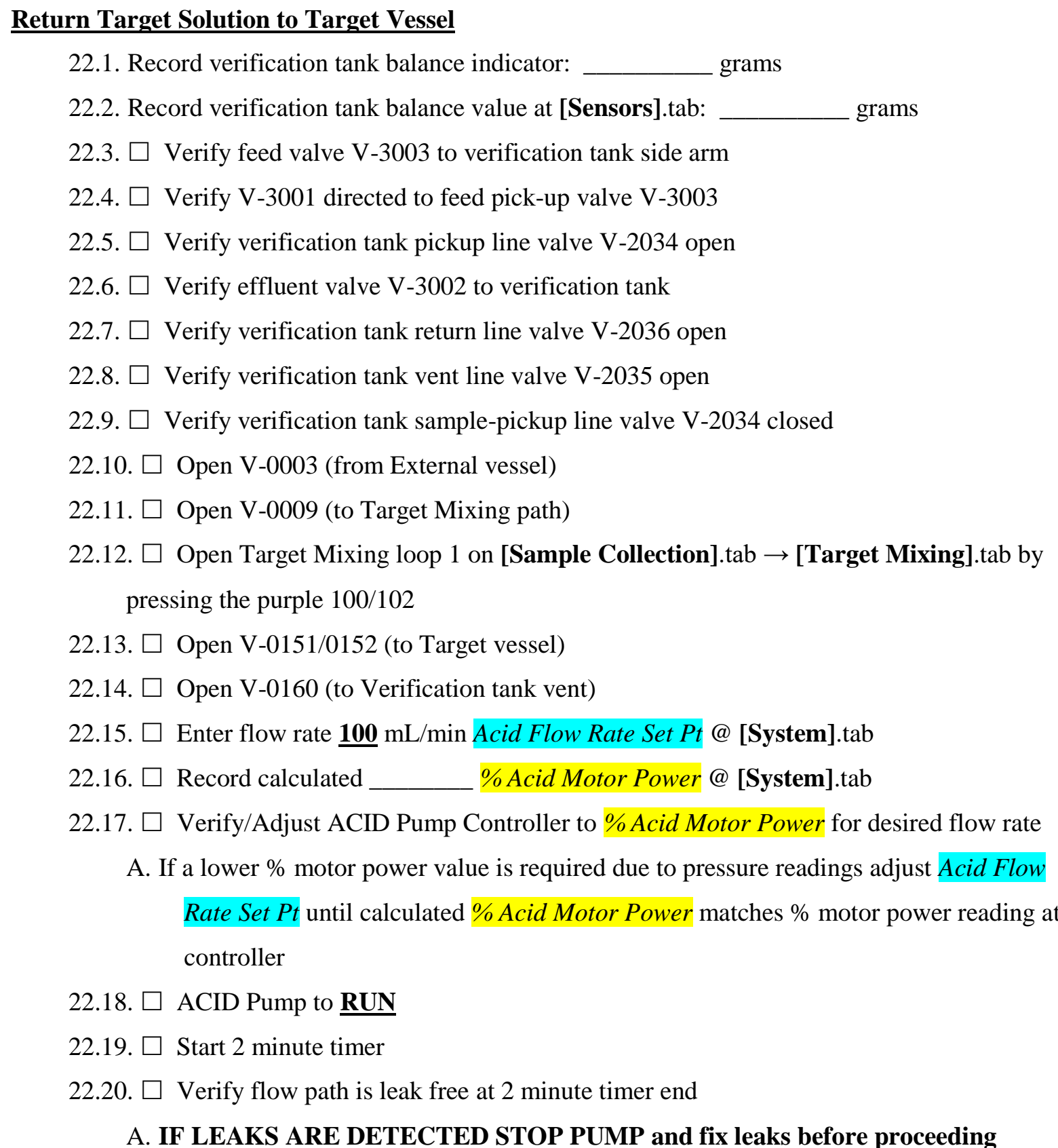 \\
\hline
\end{tabular}




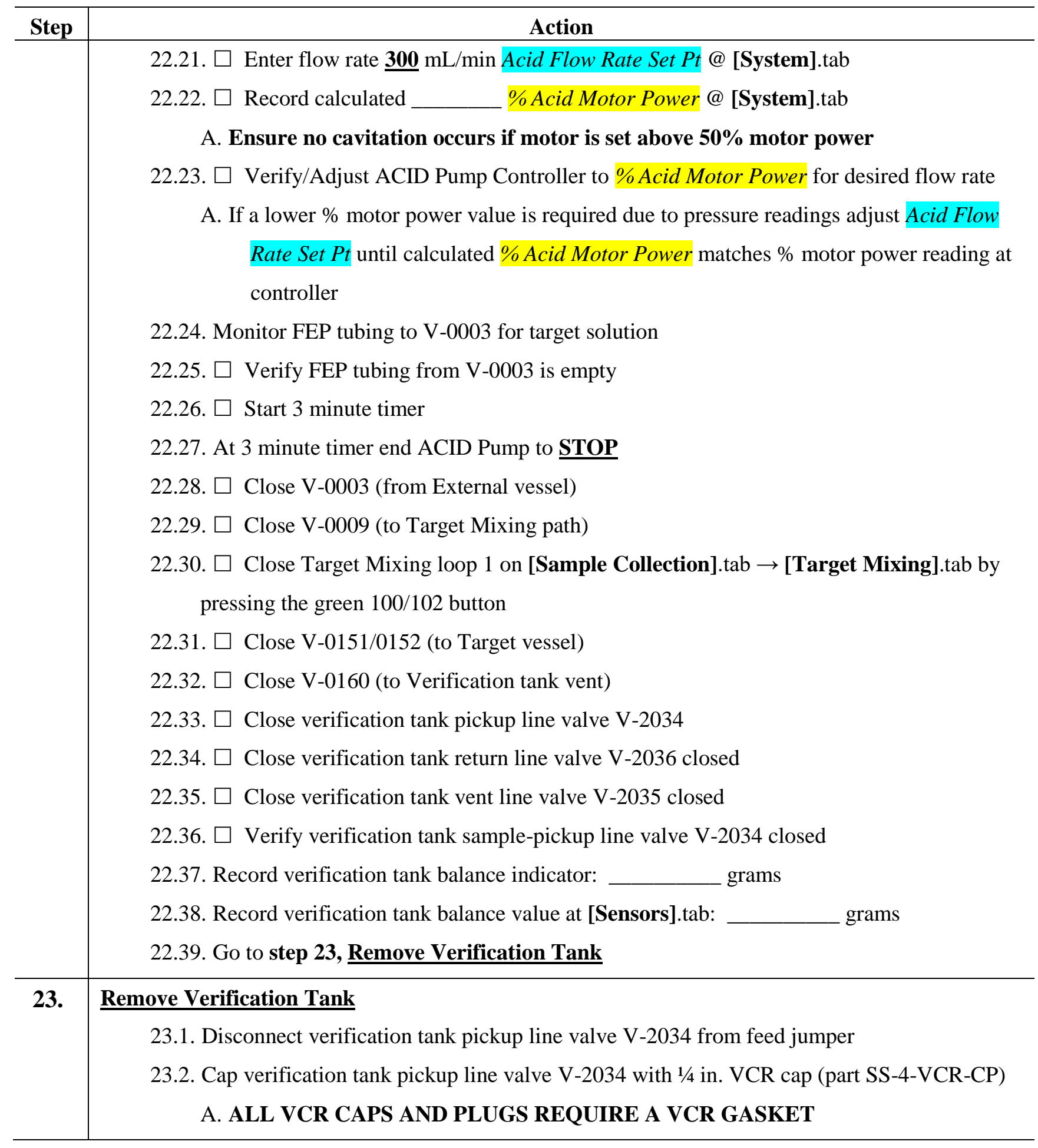




\begin{tabular}{|c|c|}
\hline Step & Action \\
\hline & $\begin{array}{l}\text { 23.7. Disconnect verification tank vent line valve V-2035 from vent line jumper } \\
\text { 23.8. Cap verification tank vent line valve V-2035 with } 1 / 4 \text { in. Swagelok cap (part SS-400-P) } \\
\text { 23.9. Wipe down all four lines and insert ends into zip-lock bag } \\
\text { 23.10. Pass zip-lock bag with ends through column interface port (hole in bottom of glovebox) } \\
\text { so bag is sitting/laying on verification tank cart lid } \\
\text { 23.11. Follow steps } 8.2 \text { through } 8.4 \text { for opening cabinet \#1 } \\
\text { 23.12. Upon cabinet \#1 open } \\
\text { A. Assemble and place } 4 \text {-section ramps for removing verification tank cart } \\
\text { B. Lower and remove leveling jacks } \\
\text { C. Attach handle to verification tank cart } \\
\text { D. Put balance in transport mode } \\
\text { E. Pull verification tank cart out enough to access balance and leak sensor connections } \\
\text { F. Disconnect balance and leak sensor } \\
\text { G. Secure interior balance and leak sensor cables on hook on right side of cabinet \#1 } \\
\text { H. Secure balance and leak sensor cables inside cart to cart handle to avoid being crushed } \\
\text { I. Remove verification tank cart } \\
\text { J. Follow HP guidelines for smearing cabinet \#1, cart, and ramps }\end{array}$ \\
\hline
\end{tabular}




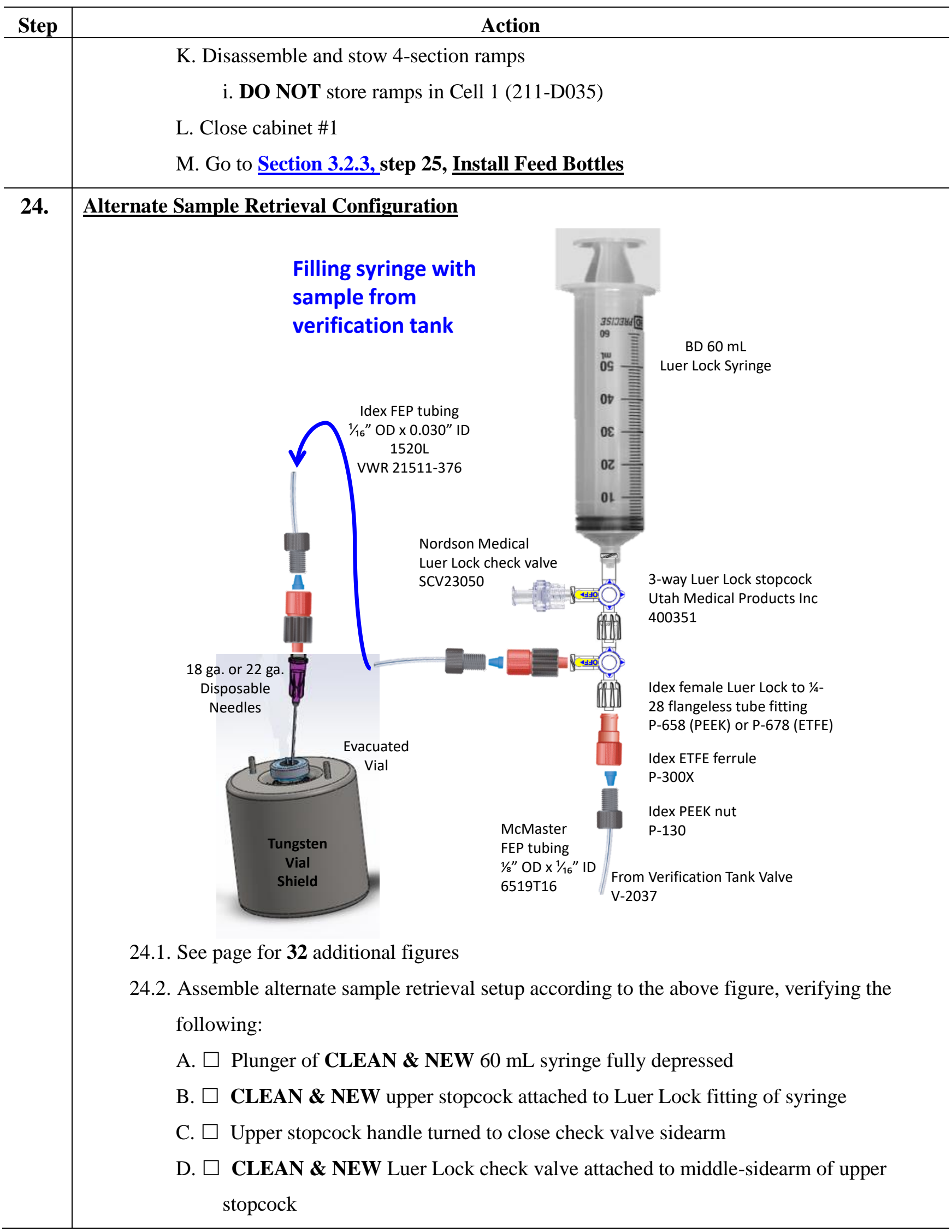




\begin{tabular}{|c|c|}
\hline Step & Action \\
\hline & $\begin{array}{l}\text { E. } \square \text { CLEAN \& NEW lower stopcock attached to upper stopcock and tubing from V- } \\
2037 \text { (labeled "sparge line") }\end{array}$ \\
\hline & F. $\square$ Lower stopcock handle turned to close collection vial sidearm \\
\hline & $\begin{array}{l}\text { G. } \square \text { CLEAN \& NEW } 1 / 16 \text { in. OD FEP tubing attached to middle-sidearm of lower } \\
\text { stopcock }\end{array}$ \\
\hline & H. $\square$ Other end of 1/16 in. tubing attached to CLEAN \& NEW disposable needle \\
\hline & i. DO NOT remove needle shroud at this time \\
\hline & I. $\square$ CLEAN \& NEW pre-evacuated vial inserted into a vial shield (shield may be \\
\hline & TUNGSTEN or STAINLESS STEEL) \\
\hline & 24.3. Detach disposable needle shroud THEN insert disposable needle into pre-evacuated vial \\
\hline & residing in vial shield, saving the needle shroud \\
\hline & 24.4. Attach CLEAN \& NEW $1 / 8$ in. OD FEP tubing to bottom-sidearm of lower stopcock \\
\hline & 24.5. Attach other end of CLEAN \& NEW $1 / 8$ in. OD FEP tubing to $1 / 4$ in. VCR x $1 / 8$ in. \\
\hline & Swagelok fitting assembly (see Exhibit B item 6) \\
\hline & 24.6. Attach adapter $1 / 4$ in. VCR $\times 1 / 8$ in. Swagelok fitting assembly to V-2037 \\
\hline & 24.7. $\square$ Verify upper stopcock handle turned to close check valve sidearm \\
\hline & 24.8. $\square$ Verify lower stopcock handle turned to close collection vial middle-sidearm \\
\hline & 24.9. Open verification tank sample-pickup line valve V-2037 \\
\hline & 24.10. Retract plunger of $60 \mathrm{~mL}$ Luer lock syringe to draw at least $5 \mathrm{~mL}$ target solution (sample) \\
\hline & into syringe for measurements and analysis \\
\hline & A. If no sample is drawn up check all fittings are tight and sealed, return plunger to syringe \\
\hline & bottom, and try again \\
\hline & 24.11. When sufficient sample is in syringe close verification tank sample-pickup line valve V- \\
\hline & 2037 \\
\hline & 24.12. Turn lower stopcock handle to close V-2037 tubing sidearm, directing flow to the sample \\
\hline & collection vial \\
\hline & A. NOTE - sample may start flowing to the vial as the small vacuum in the vial may draw \\
\hline & solution into the vial \\
\hline & 24.13. Slowly push the plunger of the syringe to finish delivering the sample to the vial, \\
\hline & stopping once a slight resistance is felt \\
\hline & A. DO NOT attempt to fully push the plunger to the bottom of the syringe as this may \\
\hline & over-pressurize the vial \\
\hline & 24.14. Turn the upper stopcock handle to the bottom-sidearm \\
\hline
\end{tabular}




Step
A. This opens the syringe to the check valve and closes the path to the vial
24.15. Retract plunger of syringe to draw $20-40 \mathrm{~mL}$ of air into the syringe
24.16. Turn the upper stopcock handle to the middle-sidearm
A. This opens the syringe to the path of the vial and closes the path to the check valve
24.17. Slowly push the syringe plunger to further empty liquid in the $1 / 16$ in. OD FEP line to
the vial
24.18. Turn lower stopcock handle to the middle-sidearm to close the path to the vial
24.19. Turn the upper stopcock handle to the bottom-sidearm
A. This opens the syringe to the check valve and closes the path to the verification tank
24.20. Retract plunger of syringe to draw $20-40$ mL of air into the syringe
24.21. Turn the upper stopcock handle to the middle-sidearm
A. This opens the syringe to the path of the verification tank and closes the path to the
check valve
24.22. Open verification tank sample-pickup line valve V-2037
24.23. Slowly push plunger of syringe to bottom of syringe body using air in syringe to empty
sample pickup line
24.24. Close verification tank sample-pickup line valve V-2037
24.25. Remove needles and dispose of in sharps container
24.26. Go to step 19.22 on page 24




\section{Figures for Alternate Sample Retrieval}

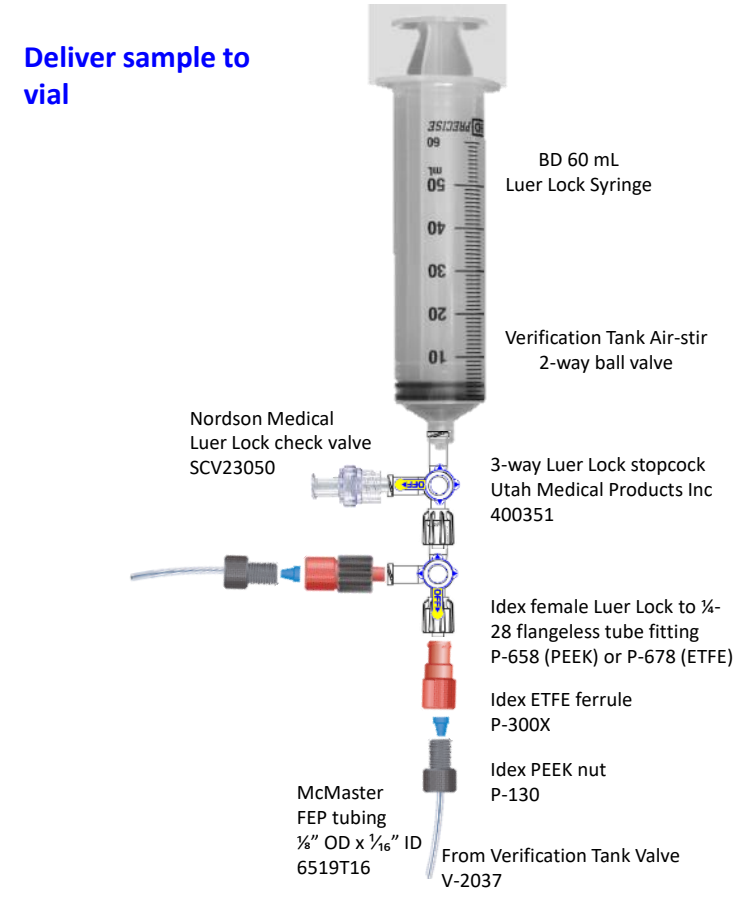

Draw air to empty sample pickup line back to verification tank

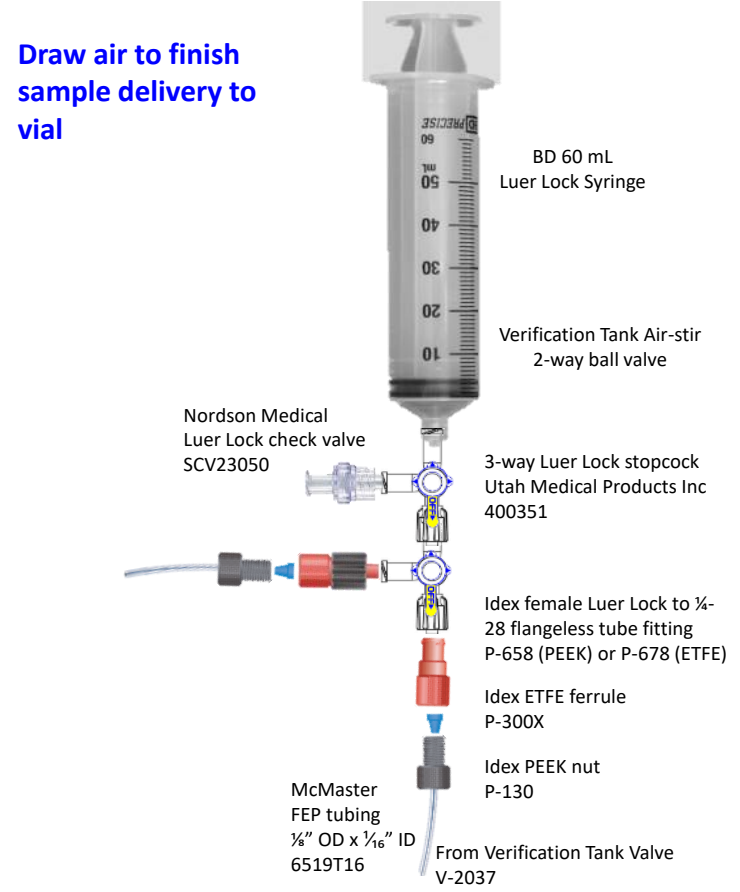




\subsubsection{Installation of Feed Bottles, Effluent Cart, and Recovery Column; and Priming of Non- Rad Feed Lines}

\begin{tabular}{|c|c|}
\hline Step & Action \\
\hline \multirow[t]{26}{*}{25.} & Install Feed Bottles Date: \\
\hline & 25.1. All non-rad feed bottles are to be located in the $\boldsymbol{M I D D L E}$ cabinet (cabinet \#2) \\
\hline & 25.2. Record feed balance calibration date: _____ (calibration sticker is affixed to Feed \\
\hline & Balance Indicator on left side of LabVIEW control rack) \\
\hline & A. Next calibration due: \\
\hline & B. If balance is out of calibration have balance calibrated \\
\hline & C. DO NOT PROCEED IF FEED BALANCE IS NOT CALIBRATED \\
\hline & 25.3. $\square$ Remove secondary tray \\
\hline & 25.4. $\square$ Ensure nothing is touching feed balance \\
\hline & 25.5. $\square$ Tare feed balance \\
\hline & 25.6. $\square$ Verify feed bottle secondary tray is in good condition (no cracks or separation) \\
\hline & A. DO NOT PROCEED WITHOUT A SECONDARY TRAY \\
\hline & $\begin{array}{l}\text { B. If cracks or separations are found replace secondary tray (use those found in Exhibit B or } \\
\text { equivalent) }\end{array}$ \\
\hline & 25.7. $\square$ Center secondary tray on feed balance \\
\hline & $\begin{array}{l}\text { 25.8. Install feed pickup lines into feed bottles (recommended to use the same type of bottle every } \\
\text { time for a given feed bottle) }\end{array}$ \\
\hline & $\begin{array}{l}\text { A. Leave a lid that fits the appropriate feed bottle in the middle cabinet with the feed line } \\
\text { inserted }\end{array}$ \\
\hline & $\begin{array}{l}\text { B. Verify that each feed line is properly inserted into the appropriate feed bottle to the } \\
\text { proper depth }\end{array}$ \\
\hline & $\begin{array}{l}\text { i. Length of inserted tubing should be } 1 / 2 \text { in. shorter than overall height of bottle } \\
\text { (ensures proper pickup of fluid from bottle) }\end{array}$ \\
\hline & ii. Distilled water feed bottle \\
\hline & a. $\square$ Fresh water line from ACID sub-system \\
\hline & b. $\square$ Fresh water line from BASE sub-system \\
\hline & 1. Place this bottle in the center of the balance as it is the largest bottle \\
\hline & iii. Acid feed bottle \\
\hline & a. $\square$ Acid line from ACID sub-system \\
\hline & iv. $\mathrm{NaOH}$ Wash feed bottle \\
\hline & a. $\square \mathrm{NaOH}$ Wash line from BASE sub-system \\
\hline
\end{tabular}




\begin{tabular}{|c|c|}
\hline Step & Action \\
\hline & v. $\mathrm{NaOH}$ Strip feed bottle \\
\hline & a. $\square$ Strip line from BASE sub-system \\
\hline & vi. $\square$ Verify all feed bottles are located within perimeter of balance pan \\
\hline & 25.9. Position lab stand and clamp to help hold liquid transfer lines to keep bottles upright \\
\hline & 25.10. $\square$ Verify leak sensor in place within secondary \\
\hline \multirow[t]{27}{*}{26.} & Install Effluent Bottles Into A Shielded Effluent Bottle Cart Date: \\
\hline & 26.1. There are two shielded effluent bottle carts identified by the serial number of the balance \\
\hline & inside them \\
\hline & A. CART \#1: Ohaus Defender 7000 Model D25QRUS; Serial No. B541541414 \\
\hline & B. CART \#2: Ohaus Defender 7000 Model D25QRUS; Serial No. B541541416 \\
\hline & 26.2. SECURE BALANCE CABLE AND LIQUID LEAK SENSOR CABLE TO CART \\
\hline & BEFORE MOVING CART (prevents cable ends from being crushed by cart casters) \\
\hline & 26.3. ONLY MOVE AN EFFLUENT CART WITH TRAY IN TRANSPORT POSITION - \\
\hline & HANDLE IN THE DOWN POSITION (failure to do so could damage the balance) \\
\hline & 26.4. Record effluent balance calibration date: ___ (calibration sticker is affixed to \\
\hline & appropriate effluent balance indicator located on the left side of LabVIEW control rack) \\
\hline & A. Next calibration due: \\
\hline & B. If balance is out of calibration have balance calibrated \\
\hline & C. DO NOT PROCEED IF FEED BALANCE IS NOT CALIBRATED \\
\hline & 26.5. ONLY REMOVE THE RIGHT SIDE OF THE SHIELDED EFFLUENT BOTTLE \\
\hline & CART LID \\
\hline & A. Right half of lid weighs over $100 \mathrm{lbs}$ and requires hoisting/rigging to remove \\
\hline & B. The left half of the lid has two manifolds attached as well as a secondary tray serving the \\
\hline & valve manifolds \\
\hline & i. Manifold nearest the handle: effluent bottle // glovebox liquid manifold \\
\hline & ii. Manifold furthest from handle: effluent bottle // glovebox vent manifold \\
\hline & 26.6. Replace effluent cart bottles as needed. A list of appropriate bottles with silicone seals is \\
\hline & found in the Exhibit B. ALL EFFLUENT BOTTLES MUST BE GAS TIGHT (prevents \\
\hline & escape of fission gases) \\
\hline & A. Effluent bottles have $1 / 4$ in. Liquid connections and $1 / 8$ in. Vent connections, as described \\
\hline & in the Exhibit B \\
\hline & 26.7. $\square$ Verify liquid leak sensor in plastic secondary inside of cart \\
\hline
\end{tabular}




\begin{tabular}{|c|c|}
\hline Step & Action \\
\hline & 26.8. $\square$ Verify liquid leak sensor in stainless steel secondary on left half of lid \\
\hline \multirow[t]{31}{*}{27.} & Install Shielded Effluent Bottle Cart \\
\hline & 27.1. Open cabinet \#3 (right side) door to full open \\
\hline & 27.2. Position the 4 -section ramp to roll the shielded effluent bottle cart into cabinet \#3 \\
\hline & 27.3. $\square$ Verify balance lever in transport positon \\
\hline & 27.4. Roll shield effluent bottle cart to edge of cabinet \#3 \\
\hline & 27.5. $\square$ Attach balance cable \\
\hline & A. Connectors operate smoothly when mated to one another correctly - DO NOT FORCE \\
\hline & CONNECTORS TOGETHER \\
\hline & 27.6. $\square$ Verify balance is read by appropriate balance indicator (should show a 0 , may require to \\
\hline & be turned on) \\
\hline & A. If no reading double check connection \\
\hline & 27.7. $\square$ Attach liquid leak sensor cable \\
\hline & 27.8. $\square$ Connect 2-way VCR ball valve from vent line to effluent bottle // glovebox vent \\
\hline & manifold connection \\
\hline & 27.9. $\square$ Double check all vent lines are connected and VCR vent connections from bottles are \\
\hline & tight \\
\hline & 27.10. Open 2-way VCR ball valves for: \\
\hline & A. $\square$ Glovebox vent line \\
\hline & B. $\square$ PRE-LOAD ACID WASH vent line \\
\hline & C. $\square$ POST-LOAD ACID WASH vent line \\
\hline & D. $\square$ POST-LOAD $\mathrm{H}_{2} \mathrm{O}$ WASH vent line \\
\hline & E. $\square$ ACID RINSE vent line \\
\hline & F. $\square$ POST-LOAD NaOH WASH vent line \\
\hline & G. $\square$ POST-STRIP $\mathrm{H}_{2} \mathrm{O}$ WASH vent line \\
\hline & H. $\square$ BASE RINSE vent line \\
\hline & $\begin{array}{l}\text { 27.11. It may be necessary to push the effluent cart into cabinet \#3 a little further to make liquid } \\
\text { connections }\end{array}$ \\
\hline & 27.12. Connect 2-way VCR ball valves on the glovebox liquid manifold from: \\
\hline & A. $\square$ PRE-LOAD ACID WASH line to effluent bottle \\
\hline & B. $\square$ POST-LOAD ACID WASH line to effluent bottle \\
\hline & C. $\square$ POST-LOAD $\mathrm{H}_{2} \mathrm{O}$ WASH line to effluent bottle \\
\hline & D. $\square$ ACID RINSE line to effluent bottle \\
\hline
\end{tabular}




\begin{tabular}{|c|c|}
\hline Step & Action \\
\hline & E. $\square$ POST-LOAD NaOH WASH line to effluent bottle \\
\hline & F. $\square$ POST-STRIP $\mathrm{H}_{2} \mathrm{O}$ WASH line to effluent bottle \\
\hline & G. $\square$ BASE RINSE line to effluent bottle \\
\hline & 27.13. $\square$ Double check all liquid lines from bottles are connected and bottle-side VCR liquid \\
\hline & connections are tight \\
\hline & 27.14. $\square$ Double check all liquid lines from glovebox effluent liquid lines are connected and \\
\hline & glovebox-side VCR liquid connections are tight \\
\hline & 27.15. Open BOTH 2-way VCR ball valves for: \\
\hline & A. $\square$ PRE-LOAD ACID WASH liquid line \\
\hline & B. $\square$ POST-LOAD ACID WASH liquid line \\
\hline & C. $\square$ POST-LOAD $\mathrm{H}_{2} \mathrm{O}$ WASH liquid line \\
\hline & D. $\square$ ACID RINSE liquid line \\
\hline & E. $\square$ POST-LOAD NaOH WASH liquid line \\
\hline & F. $\square$ POST-STRIP $\mathrm{H}_{2} \mathrm{O}$ WASH liquid line \\
\hline & G. $\square$ BASE RINSE liquid line \\
\hline & 27.16. Fully push the fully connected effluent cart into cabinet \#3 \\
\hline & 27.17. Remove the handle from the effluent cart and store in the instrument room until the cart \\
\hline & needs to be removed \\
\hline & 27.18. Tare appropriate balance indicator \\
\hline & 27.19. $\square$ Turn balance transport handle to weigh (up) \\
\hline & 27.20. $\square$ Verify balance is read by appropriate balance indicator (should be a positive, non-zero \\
\hline & 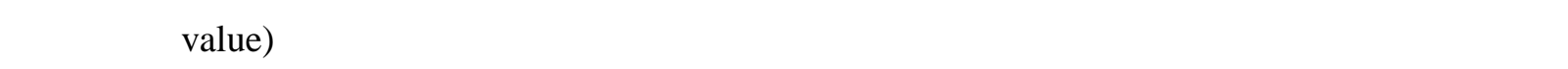 \\
\hline & 27.21. Remove 4-section ramp (DO NOT STORE 3-SECTION RAMPS IN CELL 1) \\
\hline & 27.22. Close cabinet \#3 door slowly \\
\hline \multirow[t]{8}{*}{28.} & Install Packed Recovery Column Date: \\
\hline & 28.1. Place the column in column pig (see figures on following page) \\
\hline & $\begin{array}{l}\text { A. Face pig so that toggle clamps are to the left \& right (toggle clamp plane) with the pig lid } \\
\text { slot facing front (facing user) }\end{array}$ \\
\hline & B. Remove pig lid (if already inserted) \\
\hline & C. Insert column so that column bottom feed line faces user / column top feed line is away \\
\hline & from user \\
\hline & i. Tubing plane is perpendicular to toggle clamp plane \\
\hline & D. Insert pig lid such that tubing, heater connection, and thermocouples fit through slot \\
\hline
\end{tabular}




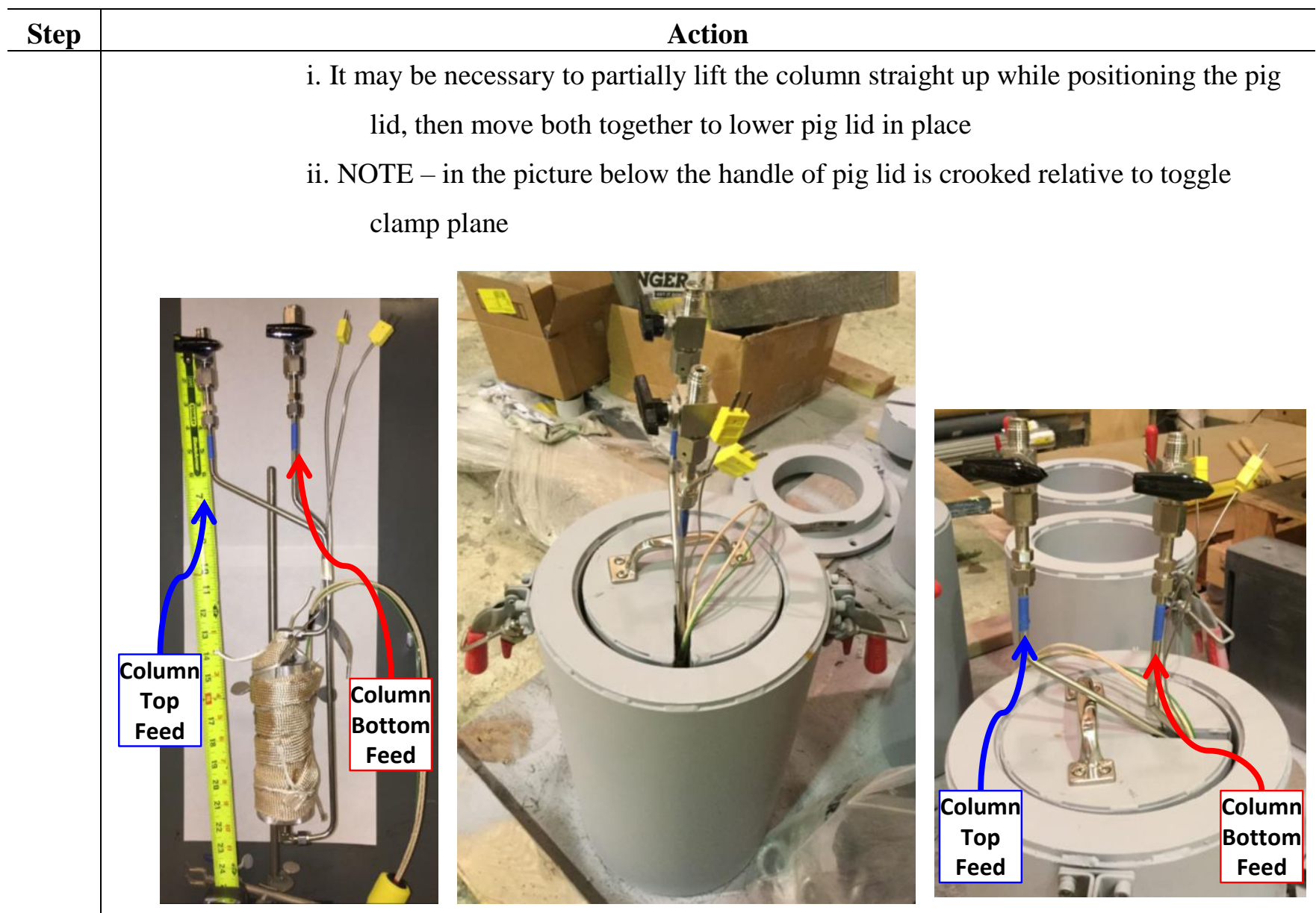

28.2. Secure the column pig to the transport cart using a ratcheting strap

\section{A. COLUMN PIG MUST ALWAYS BE STRAPPED DOWN DURING MOVEMENT OF COLUMN PIG CART}

B. Wind excess strap to cart to prevent the cart from rolling over it and to remove a potential tripping hazard

28.3. Open door to cabinet \#1

28.4. Install 4-section ramp in front of cabinet \#1

28.5. Roll column/pig assembly into cabinet \#1

28.6. The slot in the pig lid faces the front of the glovebox

A. This configuration will orient the toggle clamps on the column/pig assembly left/right

28.7. Remove the ratcheting strap and install the black gasket on the top of the column pig

28.8. Position column/pig assembly under pig locking ring on the top, inside face of cabinet \#1

28.9. $\square$ Verify that toggle clamps on column/pig assembly align with the two toggle clamp hooks on the pig locking ring

28.10. $\square$ Verify that slot of pig lid points to front of glovebox 


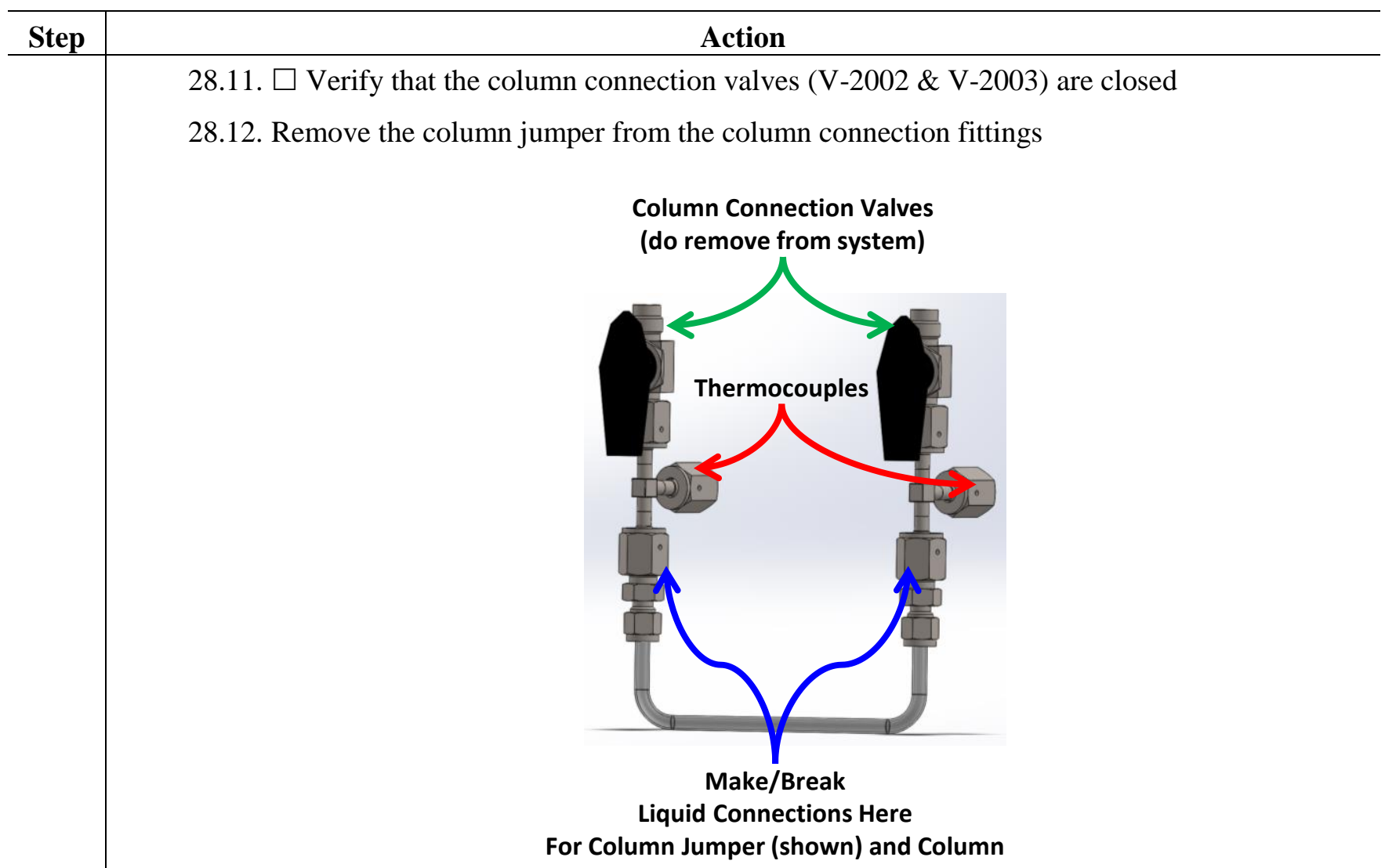

28.13. $\square$ Verify the two VCR 2-way ball valves (V-2004 \& V-2005) attached to the column are closed

28.14. Remove VCR caps from the two VCR 2-way ball valves

28.15. Use a ratcheting wrench to jack the column/pig assembly toward the pig locking ring

28.16. While raising the column/pig assembly pass the column heater cable into the glovebox and align the two VCR 2-way ball valves to their appropriate ports
A. Column top feed is the rear connection
B. Column bottom feed is the front connection

28.17. Make the two VCR connections when the jack is nearly fully extended

A. The column will hang free inside the pig even though the pig is not locked in place yet

28.18. Plug column heater cable into column heater receptacle

28.19. $\square$ Connect column heater control thermocouple

28.20. $\square$ Connect column heater over-temperature thermocouple

28.21. $\square$ Verify thermocouples reading at Column Heater temperature controller at LabVIEW rack

28.22. Secure one toggle clamp to appropriate toggle clamp hook of pig locking ring

A. Clamp will be locked down which slightly lifts the column/pig assembly 


\begin{tabular}{|c|c|}
\hline Step & Action \\
\hline & $\begin{array}{l}\text { 28.24. Remove the pig transport cart and insert the pig table under the column/pig assembly } \\
\text { A. The black rubber gasket at the glovebox/column pig interface may prevent the ability to } \\
\text { remove the transport cart. In this case detach the ratcheting wrench and leave the cart } \\
\text { in the cabinet } \\
\text { 28.25. Check cables are hung on hook inside of cabinet \#1 } \\
\text { 28.26. Remove 4-section ramp from in front of cabinet \#1 } \\
\text { 28.27. Close cabinet \#1 door }\end{array}$ \\
\hline 29. & $\begin{array}{l}\text { Verify Sample Retrieval Valves Powered Off } \\
\text { 29.1. At the rack } \\
\text { A. Verify Sample Retrieve Valve Power to OFF (switch is down) } \\
\text { B. If the switch is not OFF then actuate the switch by gently pulling out while moving the } \\
\text { handle down }\end{array}$ \\
\hline
\end{tabular}




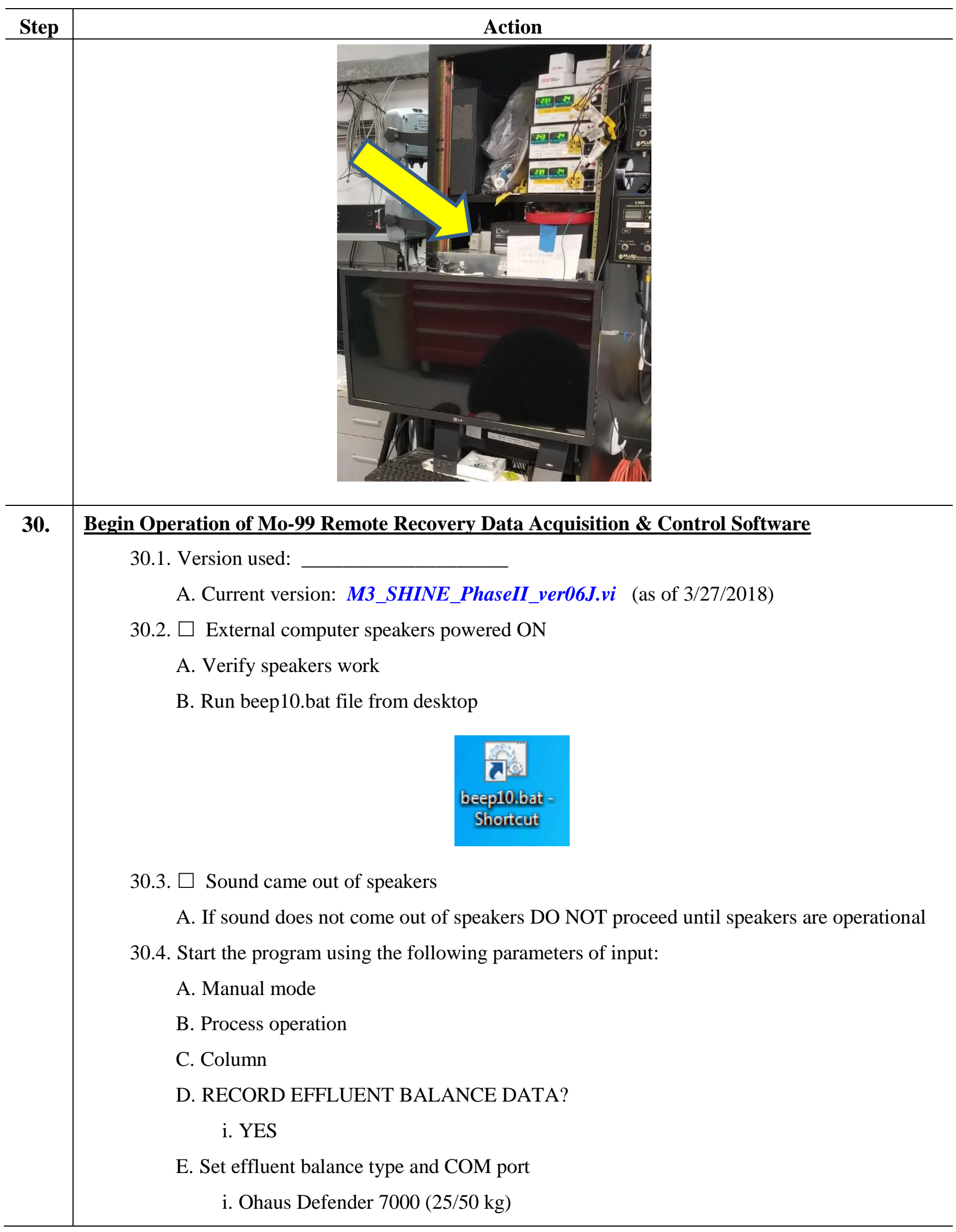




\begin{tabular}{|c|c|}
\hline Step & Action \\
\hline & ii. Pick ONE \\
\hline & a. Effluent cart \#1: ends in 414, set COM 11 \\
\hline & b. Effluent cart \#1: ends in 416, set COM 10 \\
\hline & F. Fresh Acid density $\rightarrow$ as default value $\rightarrow$ press OK \\
\hline & G. Base Wash density $\rightarrow$ as default value $\rightarrow$ press OK \\
\hline & H. Base Strip density $\rightarrow$ as default value $\rightarrow$ press OK \\
\hline & I. Target solution volume \\
\hline & i. Enter last target solution volume \\
\hline & J. Target solution concentration \\
\hline & i. Enter last target solution concentration \\
\hline & K. Target solution density \\
\hline & i. Enter last target solution density \\
\hline & L. Column effluent path \\
\hline & i. To Transfer Cask \\
\hline & M. Pre-Load Acid Wash processing volume $\rightarrow$ as default value $\rightarrow$ press OK \\
\hline & N. Column Loading processing volume $\rightarrow$ as default value $\rightarrow$ press OK \\
\hline & O. Post-Load Acid Wash processing volume $\rightarrow$ as default value $\rightarrow$ press OK \\
\hline & P. Post-Load Water Wash processing volume $\rightarrow$ as default value $\rightarrow$ press OK \\
\hline & Q. Use the Post-Load NaOH Wash step? $\rightarrow$ NO \\
\hline & R. Column stripping processing volume $\rightarrow$ as default value $\rightarrow$ press OK \\
\hline & S. Post-Strip Water Wash To Strip product processing volume $\rightarrow$ as default value $\rightarrow$ press \\
\hline & $\mathrm{OK}$ \\
\hline & T. Post-Strip Water Wash To Waste processing volume $\rightarrow$ as default value $\rightarrow$ press OK \\
\hline & U. Final Base System Water Wash processing volume $\rightarrow$ as default value $\rightarrow$ press OK \\
\hline & V. Final Acid System Acid Wash processing volume $\rightarrow$ as default value $\rightarrow$ press OK \\
\hline & W. Record LINAC temperatures? $\rightarrow$ YES \\
\hline & X. Filename prefix: ___ \\
\hline & Y. $\square$ ACID Pump controller powered ON (rocker switch under front/left of ACID Pump \\
\hline & V300 controller) \\
\hline & Z. $\square$ ACID Pump to $\underline{\text { STOP }}$ (display alternates between OFF and current setting) \\
\hline & AA. $\square$ Verify feed and effluent balances are reading at [Sensors].tab and compare to feed \\
\hline & balance indicator \\
\hline & i. Feed balance \\
\hline
\end{tabular}




\begin{tabular}{|c|c|}
\hline Step & Action \\
\hline & 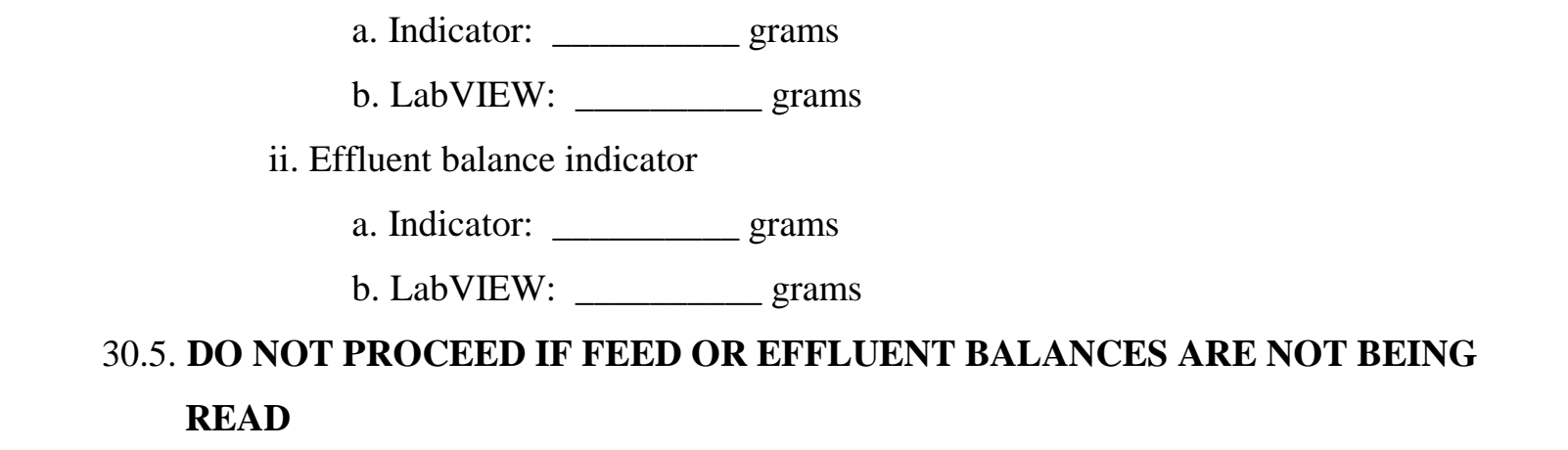 \\
\hline 31. & 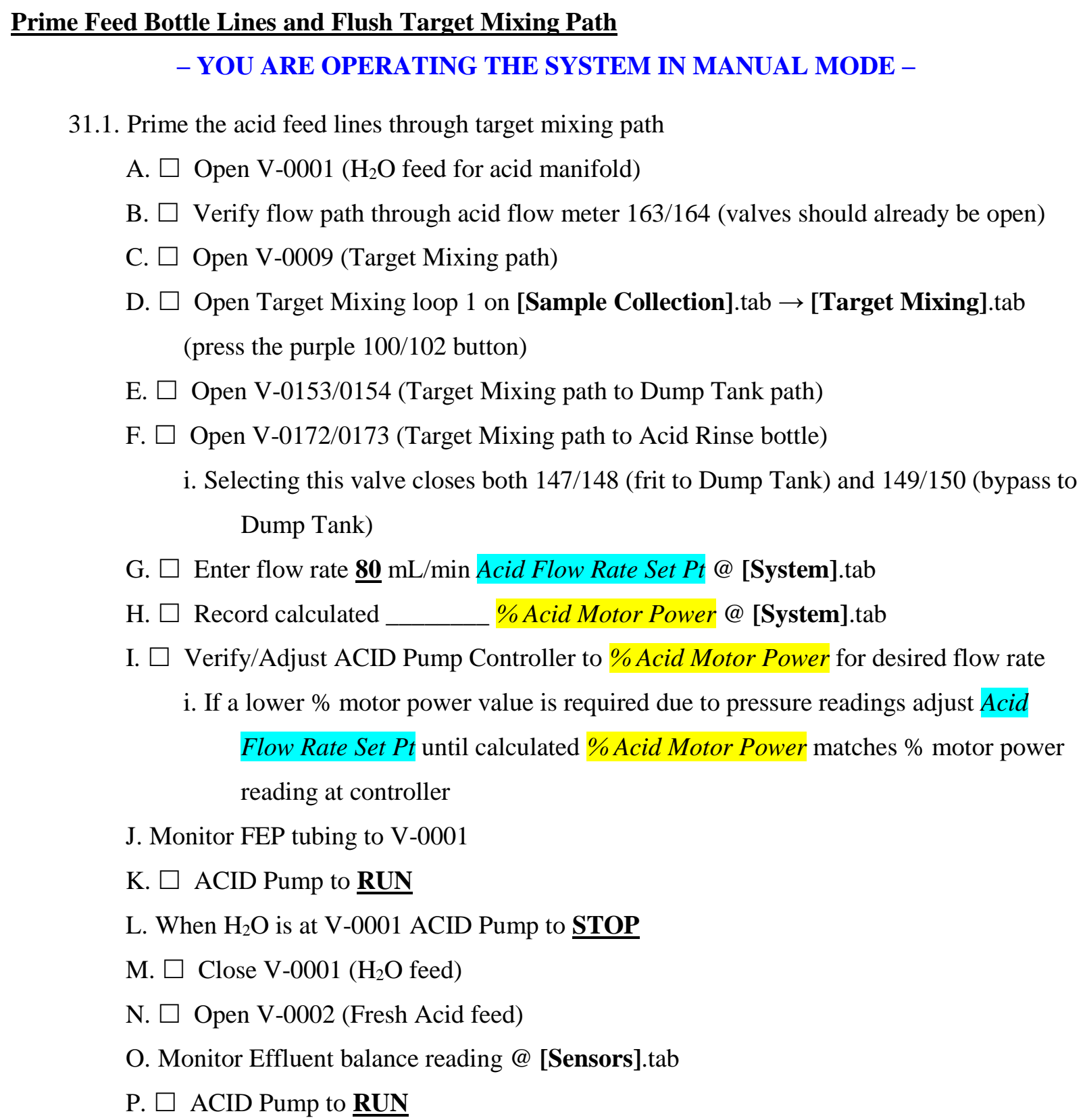 \\
\hline
\end{tabular}




\begin{tabular}{|c|c|}
\hline Step & Action \\
\hline & Q. When Fresh Acid is at Effluent balance ACID Pump to $\underline{\text { STOP }}$ \\
\hline & R. $\square$ Enter flow rate $\underline{\mathbf{3 0 0}} \mathrm{mL} / \mathrm{min}$ Acid Flow Rate Set Pt @ [System].tab \\
\hline & S. $\square$ Record calculated ___ \% Acid Motor Power @ [System].tab \\
\hline & i. Ensure no cavitation occurs if motor is set above $50 \%$ motor power \\
\hline & T. $\square$ Verify/Adjust ACID Pump Controller to \% Acid Motor Power for desired flow rate \\
\hline & i. If a lower \% motor power value is required due to pressure readings adjust Acid \\
\hline & Flow Rate Set Pt until calculated \% Acid Motor Power matches \% motor power \\
\hline & reading at controller \\
\hline & U. $\square$ Prepare 2 minute timer \\
\hline & V. $\square$ ACID Pump to $\underline{\mathbf{R U N}}$ and start timer to flush the path of possible residual uranium \\
\hline & before performing column leak check \\
\hline & W. At 2 minute timer end ACID Pump to $\underline{\text { STOP }}$ \\
\hline & X. $\square$ Verify ACID Pump to $\underline{\text { STOP }}$ \\
\hline & Y. $\square$ Close V-0002 (Fresh Acid feed) \\
\hline & Z. $\square$ Open V-0171 (Surge Vessel gas) \\
\hline & AA. $\square$ Open V-0158 (Surge tank vent) \\
\hline & BB. $\square$ Enter flow rate $\underline{\mathbf{3 0 0}} \mathrm{mL} / \mathrm{min}$ Acid Flow Rate Set Pt @ [System].tab \\
\hline & CC. $\square$ Record calculated____ \% Acid Motor Power @ [System].tab \\
\hline & i. Ensure no cavitation occurs if motor is set above $\mathbf{5 0 \%}$ motor power \\
\hline & DD. $\square$ Verify/Adjust ACID Pump Controller to \% Acid Motor Power for desired flow rate \\
\hline & i. If a lower \% motor power value is required due to pressure readings adjust Acid \\
\hline & Flow Rate Set Pt until calculated \% Acid Motor Power matches \% motor power \\
\hline & reading at controller \\
\hline & EE. Monitor effluent balance [Sensors].tab \\
\hline & FF. $\square$ ACID Pump to $\underline{\mathbf{R U N}}$ to empty the Target Mixing Path of acid rinse solution \\
\hline & GG. At stable effluent balance reading (no increase) ACID Pump to $\underline{\text { STOP }}$ \\
\hline & HH. $\square$ Close V-0171 (Surge Vessel gas) \\
\hline & II. $\square$ Close V-0158 (Surge tank vent) \\
\hline & JJ. $\square$ Close V-0009 (Target Mixing path) \\
\hline & KK. $\square$ Close Target Mixing loop 1 on [Sample Collection].tab $\rightarrow$ [Target Mixing].tab by \\
\hline & pressing the purple $100 / 102$ button \\
\hline & LL. $\square$ Close V-0153/0154 (Target Mixing path to Dump Tank path) \\
\hline & MM. $\square$ Close V-0172/0173 (Target Mixing path to Acid Rinse bottle) \\
\hline
\end{tabular}




\begin{tabular}{|c|c|}
\hline Step & Action \\
\hline & NN. $\square$ ACID Pump controller powered OFF using rocker switch under front/left of ACID \\
\hline & Pump controller \\
\hline & 31.2. Priming base feed lines through column stripping path \\
\hline & A. $\square$ BASE Pump controller powered ON using the rocker switch under front/left of \\
\hline & BASE Pump V300 controller \\
\hline & B. $\square$ Open V-0006 ( $\mathrm{H}_{2} \mathrm{O}$ feed for base manifold $)$ \\
\hline & C. $\square$ Verify flow path through base flow meter $167 / 168$ (valves should already be open) \\
\hline & D. $\square$ Open V-0024/0025 (Base column bypass) using toggle switch at lower left corner of \\
\hline & {$[$ System].tab } \\
\hline & E. $\square$ Verify flow path through base column stripping filter $28 / 29$ (valves should already be \\
\hline & open) \\
\hline & F. $\square$ Open Column Stripping loop 1 on [Sample Collection].tab $\rightarrow$ [Column \\
\hline & Stripping].tab by pressing the purple $66 / 68$ button \\
\hline & G. $\square$ Open V-0134 (Base rinse) \\
\hline & H. $\square$ Enter flow rate $\underline{\mathbf{8 0}} \mathrm{mL} / \mathrm{min}$ Base Flow Rate Set Pt @ [System].tab \\
\hline & I. $\square$ Record calculated ___ \% Base Motor Power @ [System].tab \\
\hline & J. $\square$ Verify/Adjust BASE Pump Controller to \% Base Motor Power for desired flow rate \\
\hline & i. If a lower \% motor power value is required due to pressure readings adjust Base \\
\hline & Flow Rate Set Pt until calculated \% Base Motor Power matches \% motor power \\
\hline & reading at controller \\
\hline & K. Monitor FEP tubing to V-0006 \\
\hline & L. $\square$ BASE Pump to $\underline{\mathbf{R U N}}$ \\
\hline & M. When $\mathrm{H}_{2} \mathrm{O}$ is at V-0006 BASE Pump to $\underline{\text { STOP }}$ \\
\hline & N. $\square$ Close V-0006 ( $\mathrm{H}_{2} \mathrm{O}$ feed for base manifold $)$ \\
\hline & O. Will Post-Load NaOH wash be used? (Pick one) \\
\hline & i. $\square$ NO \\
\hline & a. Skip to step 31.2.P. \\
\hline & ii. $\square$ YES \\
\hline & a. $\square$ Open V-0007 (NaOH wash feed) \\
\hline & b. $\square$ Enter flow rate $\underline{\mathbf{8 0}} \mathrm{mL} / \mathrm{min}$ Base Flow Rate Set Pt @ [System].tab \\
\hline & c. $\square$ Record calculated ___ \% Base Motor Power @ [System].tab \\
\hline & d. $\square$ Verify/Adjust BASE Pump Controller to \% Base Motor Power for desired \\
\hline & flow rate \\
\hline
\end{tabular}




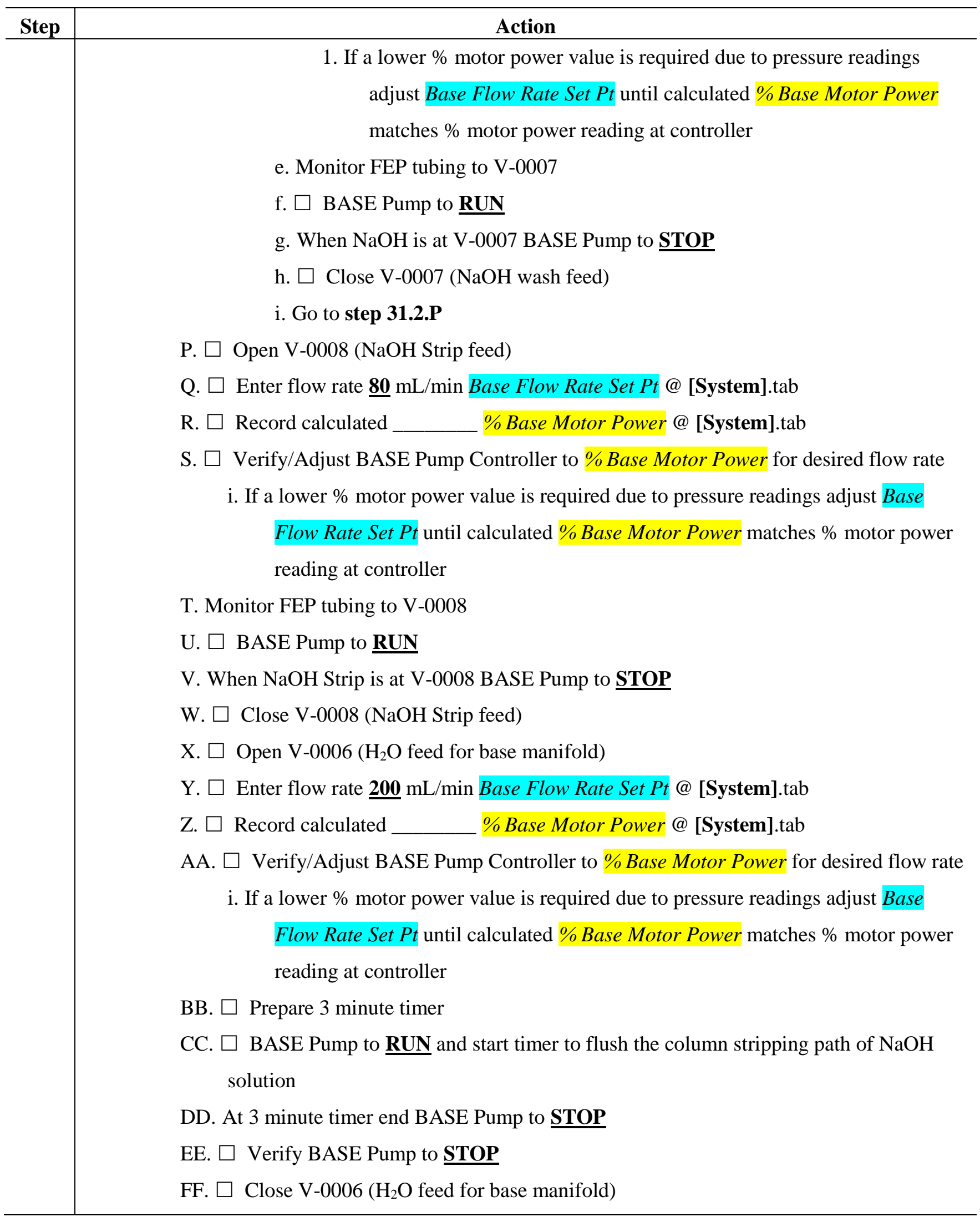




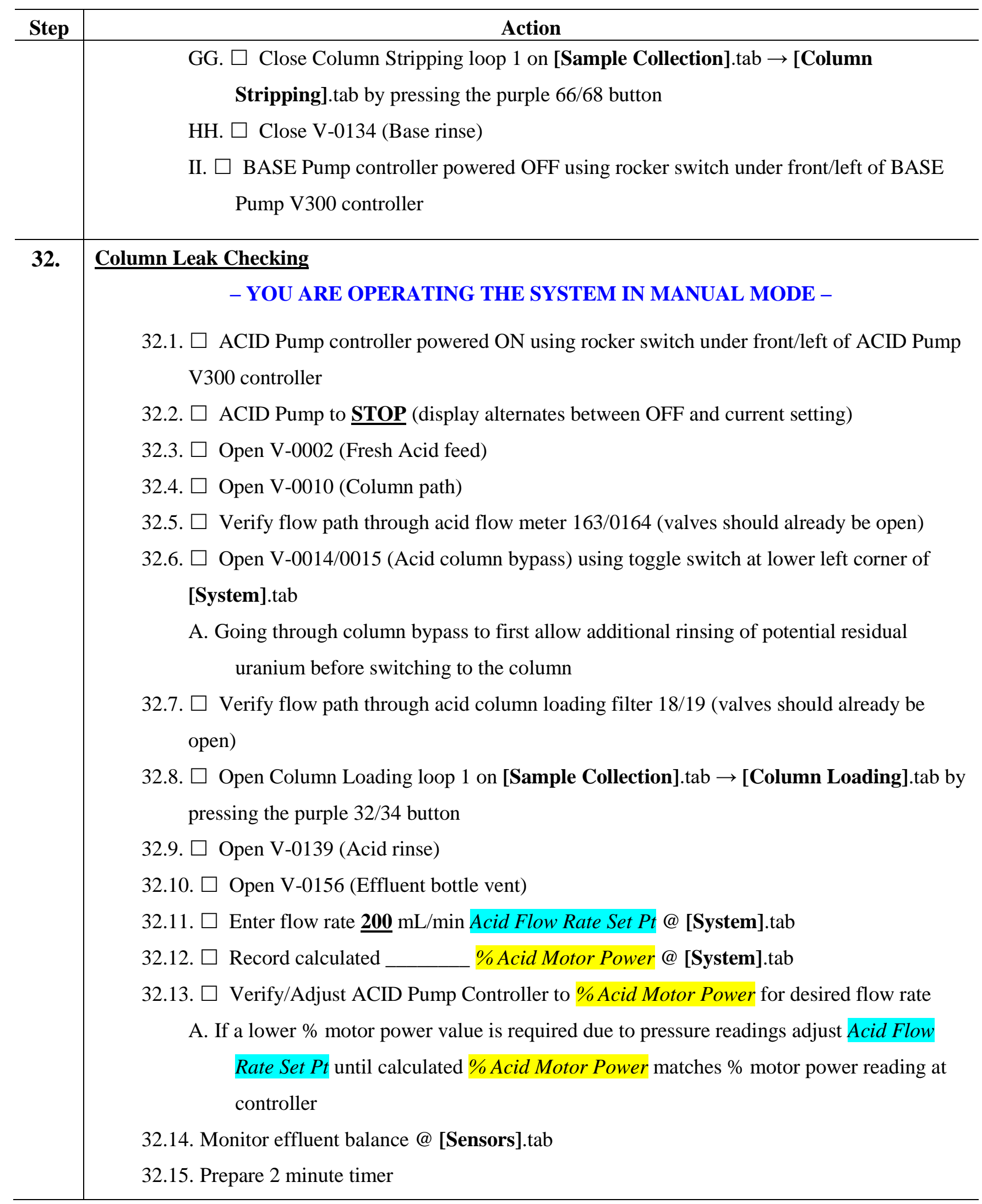




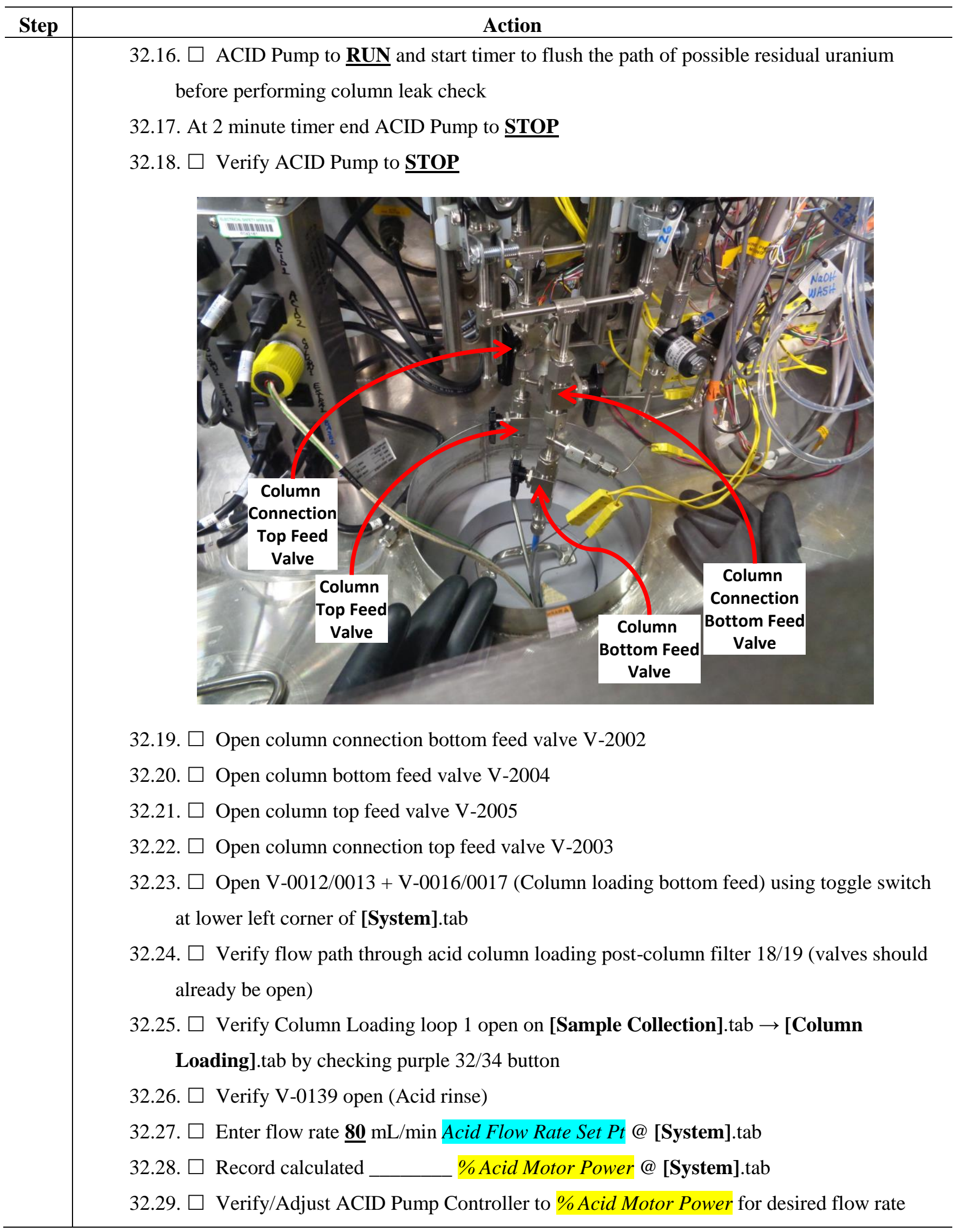




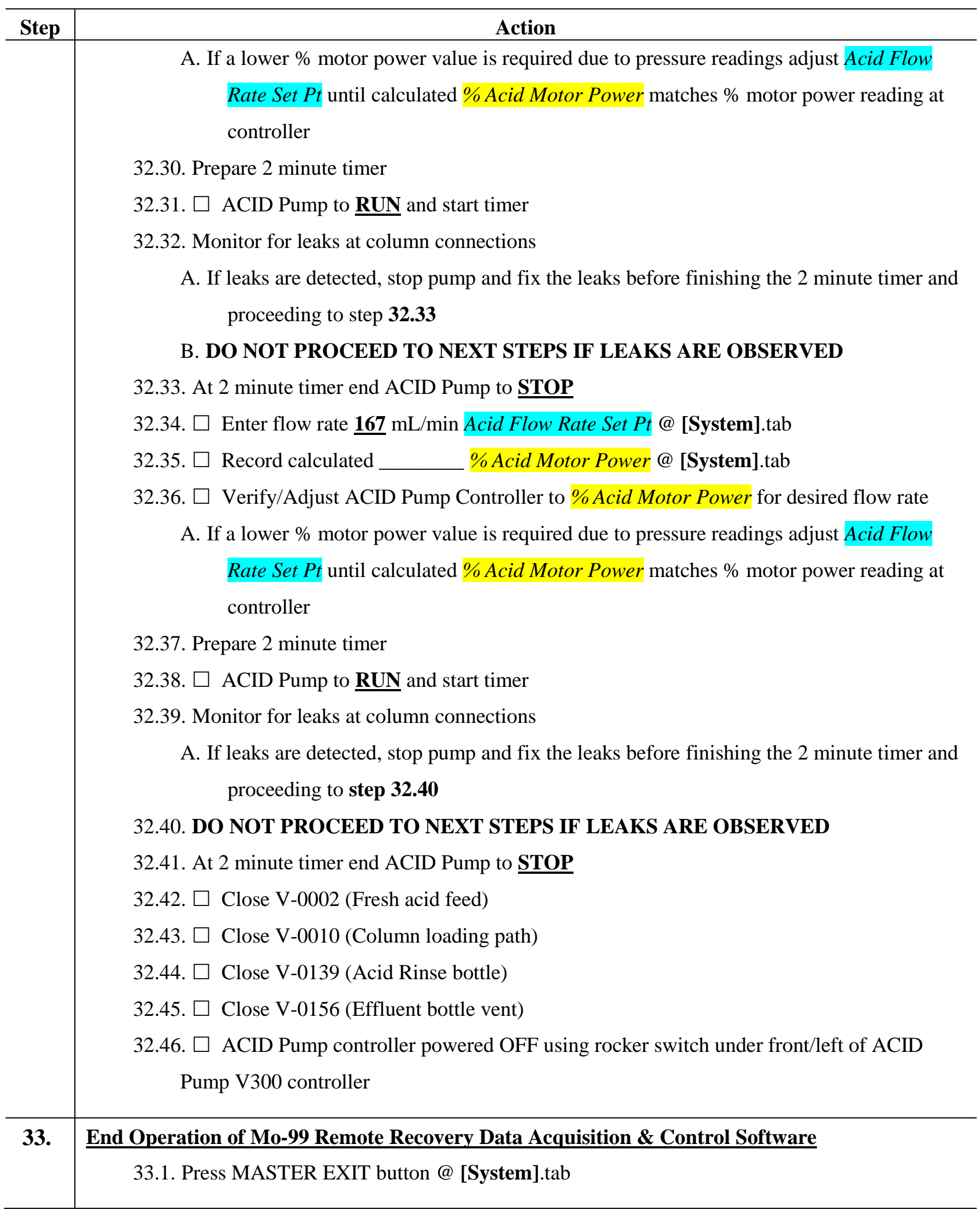




\subsubsection{Solution Irradiation and ${ }^{99}$ Mo Recovery}

Linac - Lab Notebook No.: Pages:

GC/MS-RGA - Lab Notebook No.: Pages:

LabVIEW - Lab Notebook No.: Pages:

\begin{tabular}{|c|c|}
\hline Step & Action \\
\hline 34. & 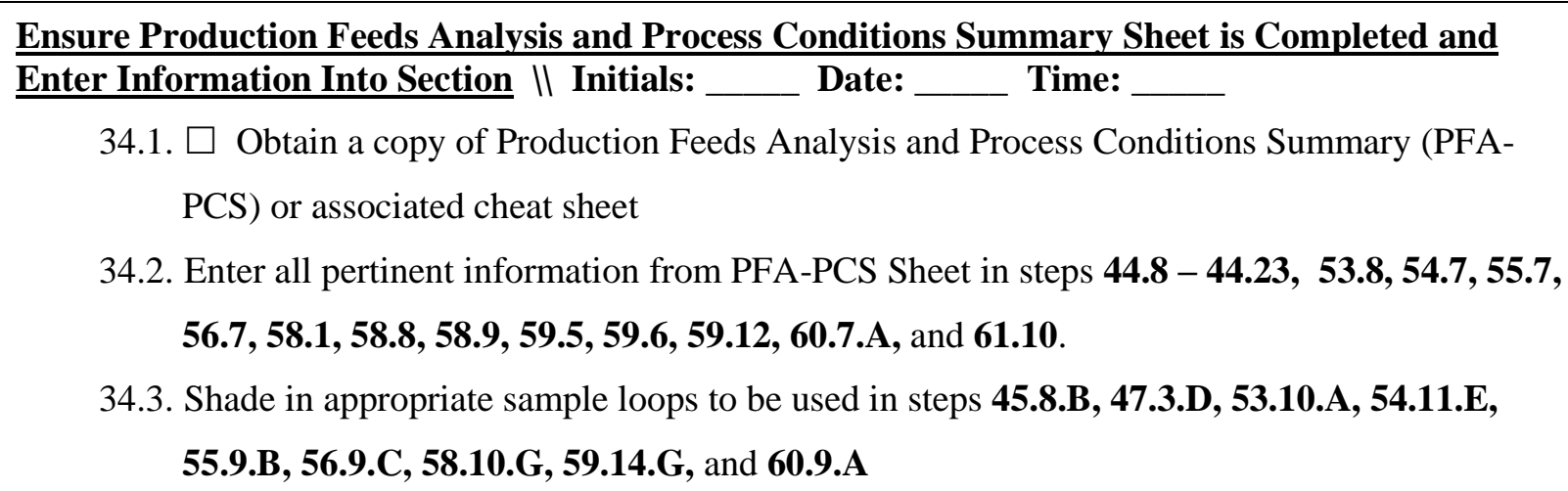 \\
\hline 35. & 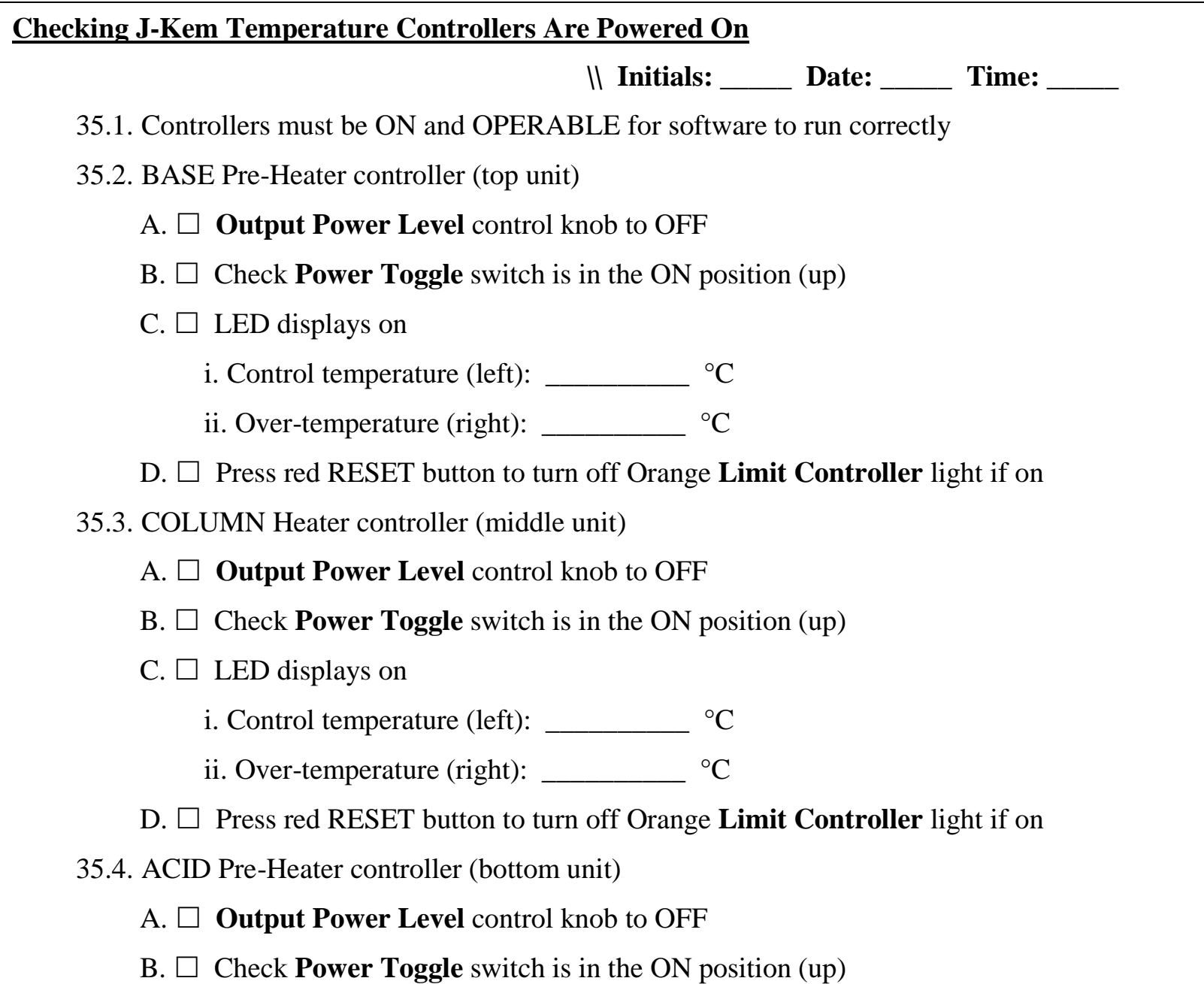 \\
\hline
\end{tabular}




\begin{tabular}{|c|c|}
\hline Step & Action \\
\hline & $\begin{array}{l}\text { C. } \square \text { LED displays on } \\
\text { i. Control temperature (left): } \\
\text { ii. Over-temperature (right): } \\
\text { D. } \square \text { Press red RESET button to turn off Orange Limit Controller light if on } \\
\text { 35.5. If LED display shows alternating "inPt" and "FAiL" } \\
\text { A. Thermocouple path is broken or the thermocouple is bad and must be repaired before } \\
\text { proceeding } \\
\text { B. Record repair in lab notebook, then record readings in appropriate step } \\
\text { 35.6. } \square \text { Turn on electrical box for heaters }\end{array}$ \\
\hline 36. & $\begin{array}{l}\text { Checking Leak Sensors Active } \| \text { Initials: } \_ \text {Date: } \_ \text {Time: } \\
\text { 36.1. Leak sensor panel is located below display panel for load cells } \\
\text { 36.2. } \square \text { Verify leak sensor power panel is on (if not, turn it on) } \\
\text { 36.3. } \square \text { Verify leak sensor audible alarm mute is off } \\
\text { A. The mute audible alarm switch should be in the down position } \\
\text { B. If it is in the up position, gently pull the switch out so that it can be moved into the down } \\
\text { position }\end{array}$ \\
\hline 37. & $\frac{\text { Verify Power To Sampling Valves is OFF }}{\text { 37.1. See figure below, toggle points down }}$ \\
\hline 38. & Checking Manual Dump Tank Valve OPEN \\
\hline
\end{tabular}




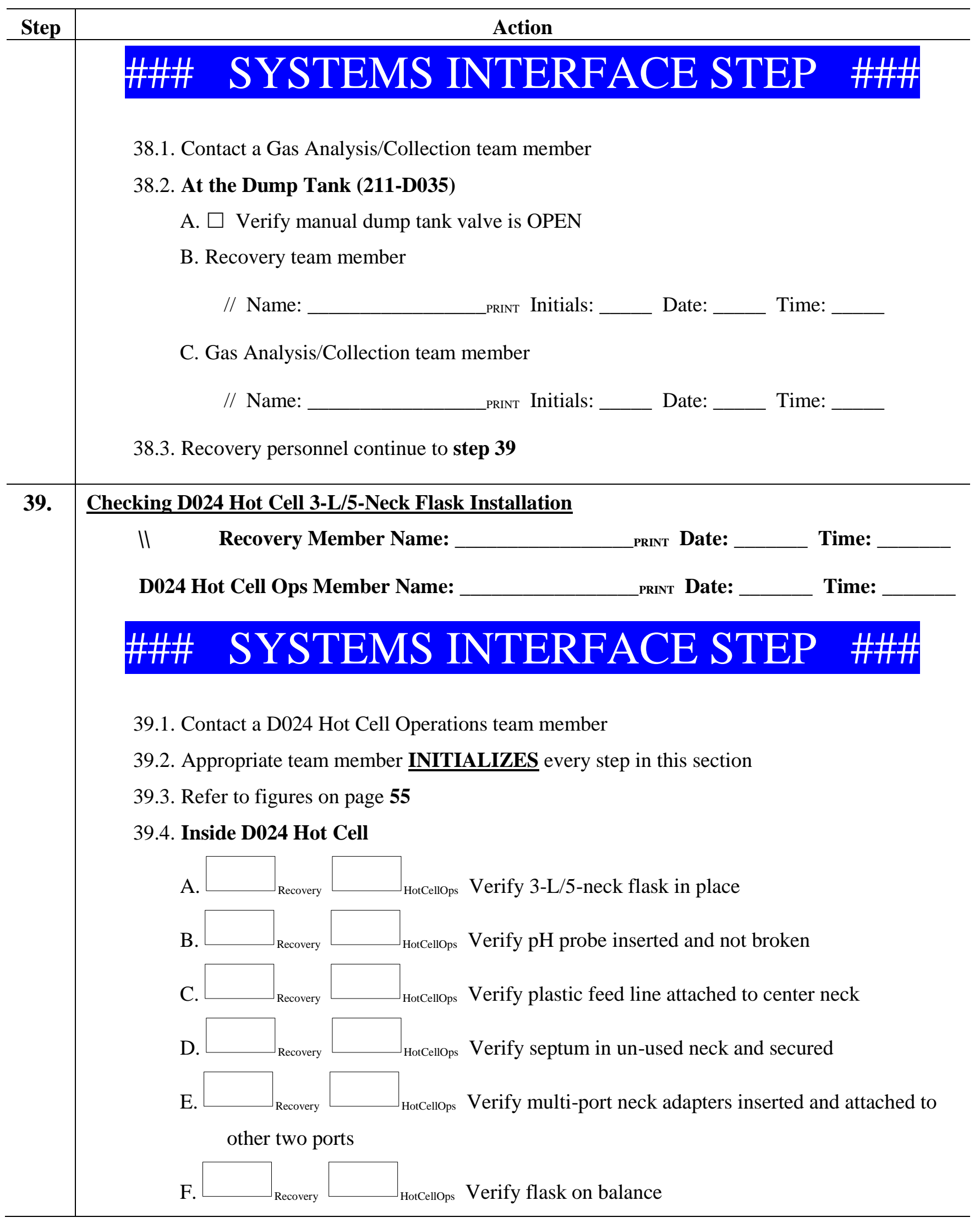




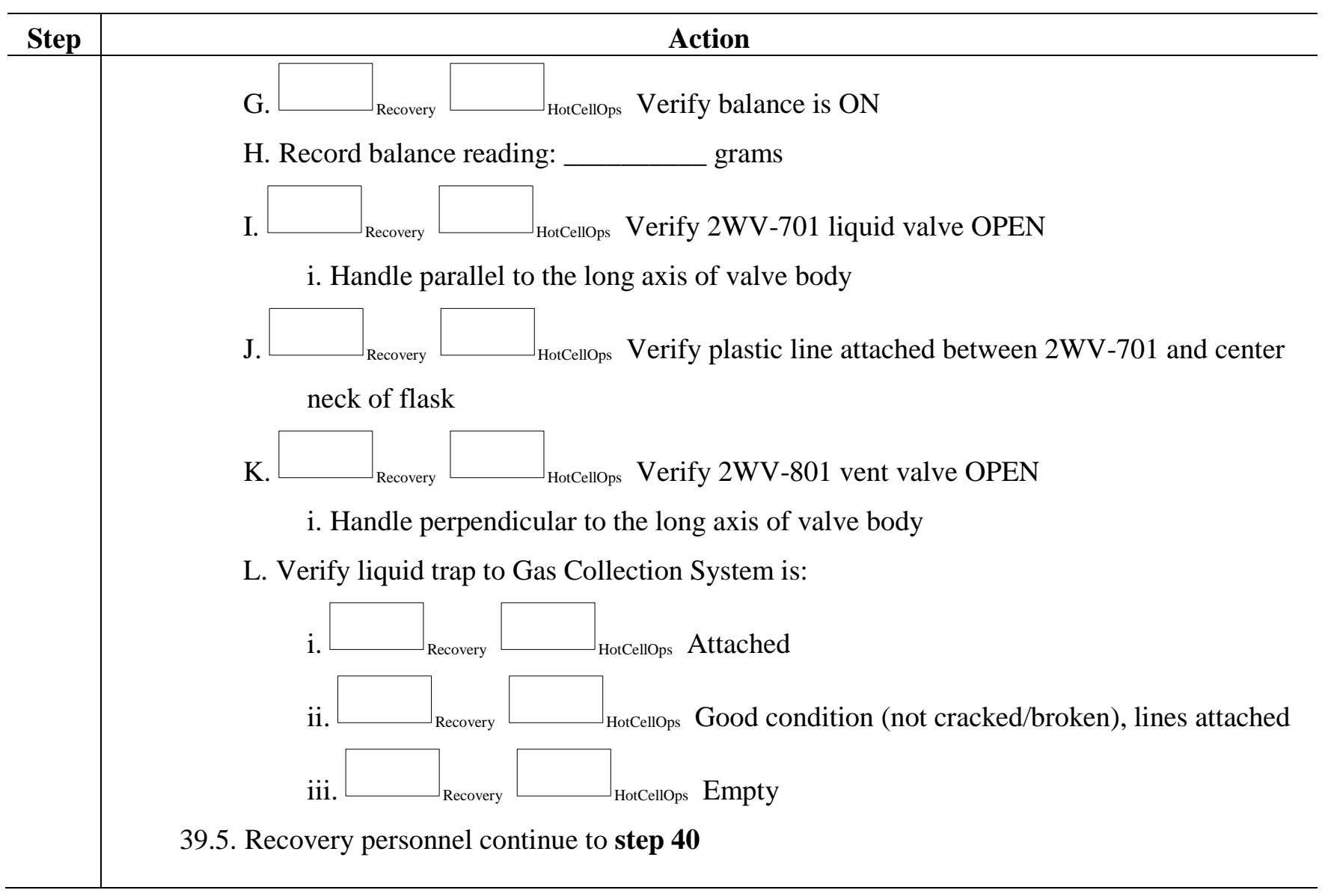


3-L / 5-neck Flask

in D024 Hot Cell
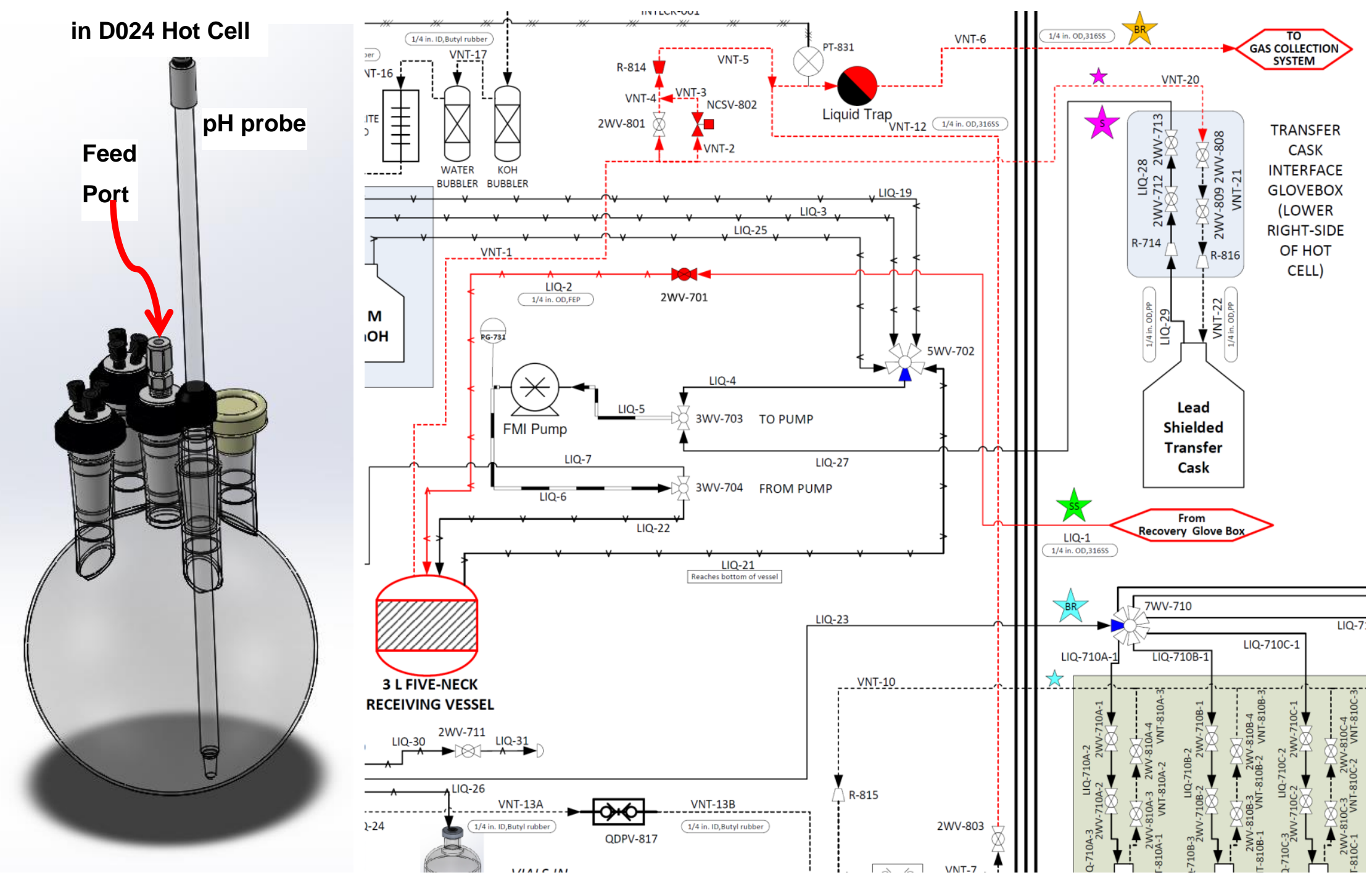


\begin{tabular}{|c|c|}
\hline Step & Action \\
\hline \multirow[t]{17}{*}{40.} & Checking Column Stripping Transfer Cask Installation \\
\hline & II Initials: ___ Date: ___ Time: __ \\
\hline & 40.1. Outside the white Transfer Cask Interface glovebox in Cell 1 (D035) \\
\hline & A. $\square$ Verify column stripping transfer cask is attached to white glovebox \\
\hline & 40.2. Inside the white Transfer Cask Interface glovebox in Cell 1 (D035) \\
\hline & A. $\square$ Verify $1 / 4$ in. liquid line connections secure (segment between V-2007 \& V-2009) \\
\hline & B. $\square$ Verify both 2 -way valves for liquid service (on the $1 / 4 \mathrm{in}$. line) are open \\
\hline & i. Handles parallel to the long axis of valve body \\
\hline & C. $\square$ Verify $1 / 8$ in. liquid line connections secure \\
\hline & i. Segment between V-2006 \& V-2008 \\
\hline & D. $\square$ Verify both 2 -way valves for vent service (on the $1 / 8$ in. line) are open \\
\hline & i. Handles parallel to the long axis of valve body \\
\hline & E. $\square$ Verify Transfer Cask Interface glovebox leak sensor attached \\
\hline & F. $\square$ Verify Transfer Cask leak sensor attached \\
\hline & 40.3. IF Section 3.2.3 (Installation of Feed Bottles, Effluent Cart, and Recovery Column) \\
\hline & was completed directly before irradiation, operators may skip to step 44 , otherwise proceed \\
\hline & to step 41 \\
\hline \multirow[t]{14}{*}{41.} & $\begin{array}{l}\text { Checking Column Installed and Manual Valves Open } \| \text { Initials: ___ Date: } \\
\text { Time: }\end{array}$ \\
\hline & 41.1. In Cabinet \#1 \\
\hline & A. $\square$ Verify column pig in place and latches are locked \\
\hline & B. $\square$ Verify that Mytec support table is under column pig \\
\hline & 41.2. Inside Mo99 Recovery Glovebox \\
\hline & A. $\square$ Verify that all four (4x) manual 2-way valves are open to the column \\
\hline & i. Handles parallel to the long axis of valve body \\
\hline & ii. $\square$ V-2002 \\
\hline & iii. $\square$ V-2004 \\
\hline & iv. $\square$ V-2005 \\
\hline & v. $\square \mathrm{V}-2003$ \\
\hline & B. $\square$ Verify that the liquid pickup line from the Pressure Relief Valve surge tank is \\
\hline & attached to side-arm of V-3001 \\
\hline & C. $\square$ Verify center arm of V-3001 is attached to V-0003 \\
\hline
\end{tabular}




\begin{tabular}{|c|c|}
\hline Step & Action \\
\hline & $\begin{array}{l}\text { D. } \square \text { Verify point of handle of V-3001 points to side-arm attached to pickup line from the } \\
\text { Pressure Relief Valve surge tank }\end{array}$ \\
\hline 42. & $\begin{array}{l}\text { Checking Feed Bottles in Place } \| \text { Initials: } \_ \text {Date: } \_ \text {Time: } \\
\text { 42.1. Perform if step } 25 \text { was completed more than } 1 \text { week ago: In Cabinet \#2 } \\
\text { A. } \square \text { Verify feed bottles are in secondary } \\
\text { B. } \square \text { Verify that pickup tubes are properly inserted into bottles } \\
\text { i. Tubes opening should just touch bottom of feed bottle } \\
\text { C. } \square \text { Verify leak sensor lays flat in secondary tray }\end{array}$ \\
\hline 43. & $\begin{array}{l}\text { Checking Effluent Cart in Place and Manual Valves Open } \\
\text { 43.1. Perform if step } 26 \text { was completed more than } 1 \text { week ago: In Cabinet \#3 } \\
\text { A. Record last three digits of balance serial number: } \\
\text { B. } \square \text { Verify tare handle is in the up READ position } \\
\text { C. Verify BOTH 2-way liquid valves (1/4 in.) open for each process stream (handles parallel } \\
\text { to the long axis of the valve body) } \\
\text { i. Pre-load acid wash } \\
\text { a. } \square \text { V-2012 } \\
\text { b. } \square \text { V-2031 } \\
\text { ii. Post-load acid wash } \\
\text { a. } \square \text { V-2013 } \\
\text { b. } \square \text { V-2029 } \\
\text { iii. Post-load } \mathrm{H}_{2} \mathrm{O} \text { wash } \\
\text { a. } \square \text { V-2014 } \\
\text { b. } \square \text { V-2027 } \\
\text { iv. Acid rinse } \\
\text { a. } \square \text { V-2018 } \\
\text { b. } \square \text { V-2019 } \\
\text { v. Post-load NaOH wash } \\
\text { a. } \square \text { V-2015 } \\
\text { b. } \square \text { V-2025 } \\
\text { vi. Post-strip } \mathrm{H}_{2} \mathrm{O} \text { wash } \\
\text { a. } \square \text { V-2016 } \\
\text { V }\end{array}$ \\
\hline
\end{tabular}




\begin{tabular}{|c|c|}
\hline Step & Action \\
\hline & $\begin{array}{l}\text { b. } \square \text { V-2023 } \\
\text { vii. Base rinse } \\
\text { a. } \square \text { V-2017 } \\
\text { b. } \square \text { V-2021 } \\
\text { D. Verify single 2-way vent valves (1/8 in.) for each process stream and main 2-way vent } \\
\text { valve are open (handles parallel to the long axis of the valve body) } \\
\text { i. } \square \text { V-2032 Pre-load acid wash } \\
\text { ii. } \square \text { V-2030 Post-load acid wash } \\
\text { iii. } \square \text { V-2028 Post-load } \mathrm{H}_{2} \mathrm{O} \text { wash } \\
\text { iv. } \square \text { V-2020 Acid rinse } \\
\text { v. } \square \text { V-2026 Post-load } \mathrm{NaOH} \text { wash } \\
\text { vi. } \square \text { V-2024 Post-strip } \mathrm{H}_{2} \mathrm{O} \text { wash } \\
\text { vii. } \square \text { V-2022 Base rinse } \\
\text { viii. } \square \text { V-2011 Main 2-way vent valve }\end{array}$ \\
\hline 44. & $\begin{array}{l}\text { Begin Operation of Mo-99 Remote Recovery Data Acquisition \& Control Software } \\
\text { 44.1. Version used: } \\
\text { A. Current version: M3_SHINE_PhaseII_ver06J.vi_ (as of 3/27/2018) } \\
\text { 44.2. } \square \text { External computer speakers powered ON } \\
\text { 44.3. Verify speakers work } \\
\text { A. Run beep10.bat file from desktop } \\
\text { B. } \square \text { Sound came out of speakers } \\
\text { i. If sound does not come out of speakers DO NOT proceed until speakers are } \\
\quad \text { operational } \\
\text { 44.4. Start the program } \\
\text { 44.5. Mode (PICK ONLY ONE) } \\
\text { A. } \square \text { Automated }\end{array}$ \\
\hline
\end{tabular}




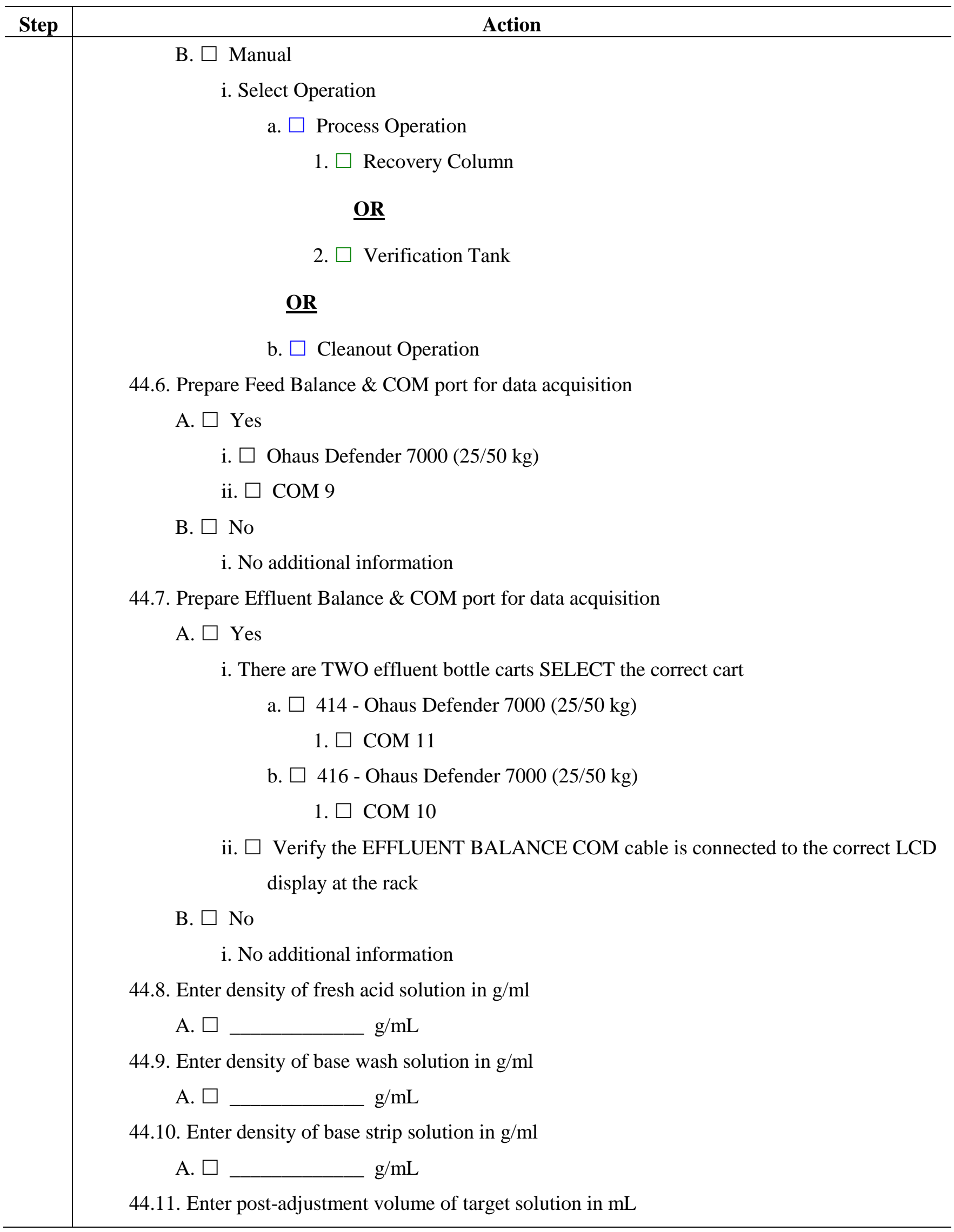




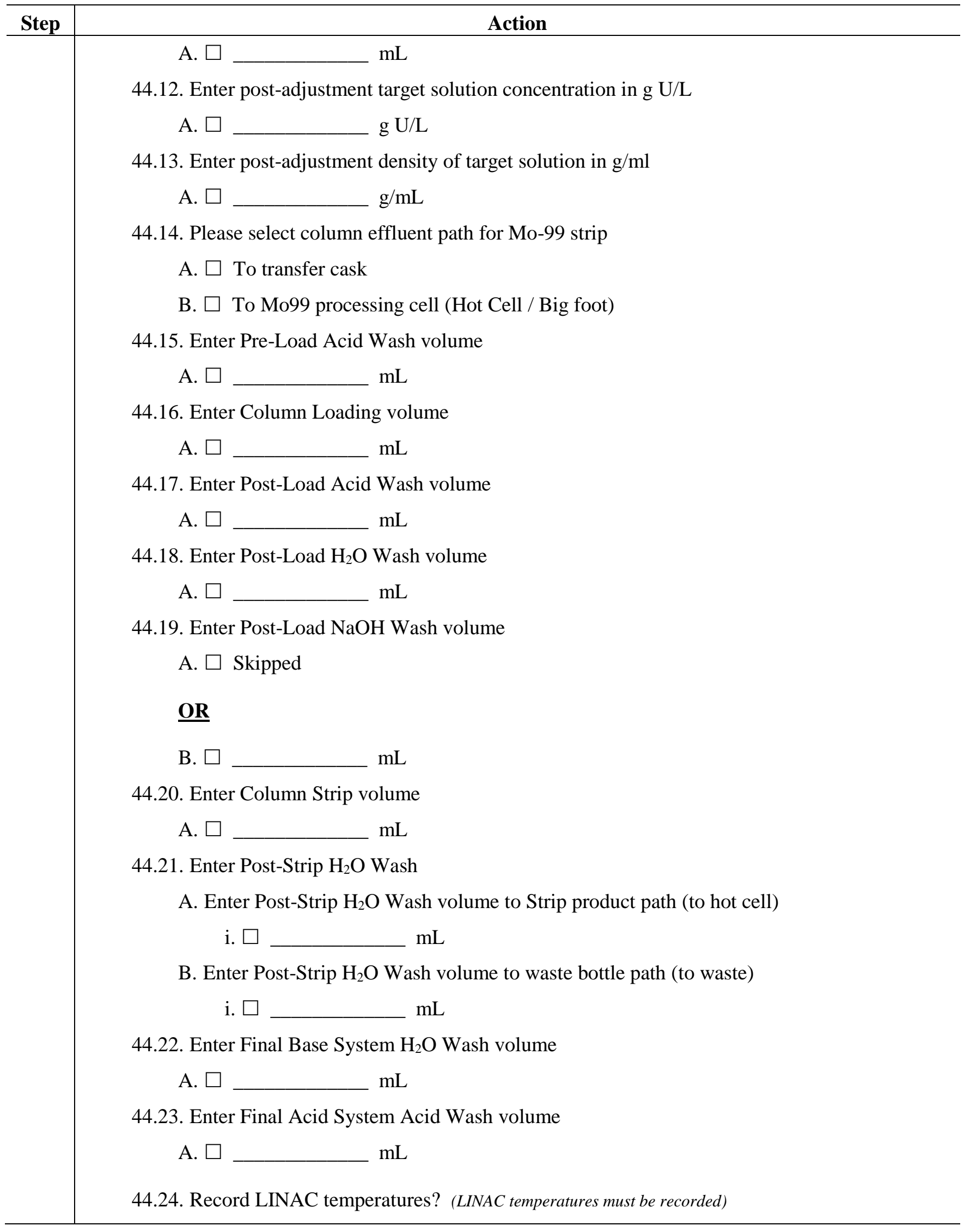


A. $\square$ Yes

44.25. Filename prefix: (see [File Paths].tab $\rightarrow$ File Prefix)

44.26. Verify gas collection system pressure readings

A. Record gas collection system pressure readings from LED displays

i. $0-2000$ mbar gauge $($ Chamber 1$)=$ mbar (RIGHT)

ii. $0-2000$ mbar gauge $($ Chamber 2$)=$ mbar (MIDDLE)

iii. 0-5000 psig gauge $($ High Pressure Cyl. $)=$ psig (LEFT)

B. Record gas collection system pressure readings from LabVIEW displays at

i. 0-2000 mbar gauge $($ Chamber 1$)=$ mbar (RIGHT)

ii. 0-2000 mbar gauge (Chamber 2$)=$ mbar (MIDDLE)

iii. 0-5000 psig gauge $($ High Pressure Cyl. $)=$ psig (LEFT)

44.27. Verify balance readings

A. Record balance readings from balance displays

i. Feed balance: g

ii. Effluent cart balance: g

44.28. $\square$ ACID Pump controller powered ON using rocker switch under front/left of ACID Pump V300 controller

44.29. $\square$ ACID Pump to $\underline{\text { STOP }}$ (display alternates between OFF and current setting)

44.30. $\square$ BASE Pump controller powered OFF using rocker switch under front/left of BASE Pump V300 controller

44.31. $\square$ Verifydial @ Start

44.32. $\square$ Enter desired flow rate mL/min Acid Flow Rate Set Pt @ [System].tab (default this step is $167 \mathrm{~mL} / \mathrm{min}$ )

44.33. $\square$ Record calculated \% Acid MotorPower@ [System].tab

44.34. $\square$ Verify/Adjust ACID Pump Controller to \% Acid Motor Power for desired flow rate A. If a lower \% motor power value is required due to pressure readings adjust Acid Flow Rate Set Pt until calculated \% Acid Motor Power matches \% motor power reading at controller

44.35. Press \{NEXT STEP\}
A. $\square$ Answer OK
B. $\square$ Answer OK
C. $\square$ Press $\{$ NEXT SAMPLE $\}$ on [Sample Collection].tab $\rightarrow$ [Target Mixing].tab 44.36. $\square$ Verify dial @ Target Solution Mixing

A. Path reference information 


\begin{tabular}{|c|c|}
\hline Step & Action \\
\hline & i. Uses ACID path pump \\
\hline & ii. V-0005 - from target vessel \\
\hline & iii. V-0163/0164 - Acid Flow Meter \\
\hline & iv. V-0009 - Mixing Path Sampling Assembly \\
\hline & v. Loop 1 on target solution monitoring sample collection - V-0100/0102 \\
\hline & vi. V-0151/0152 - to target vessel \\
\hline & vii. Valve text: $5>163 / 164>9>100 / 102$ Loop $1>151 / 152>$ Target Vessel \\
\hline & 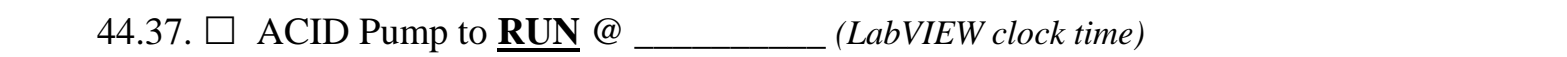 \\
\hline & 44.38. $\square$ Ensure flow path bypasses the flow meter (open valves V-0165/0166) \\
\hline & 44.39. $\square$ Verify flow path is leak free ___ (LabVIEW clock time) \\
\hline & A. If leaks are observed STOP the ACID pump and report leaks to Linac Operator \\
\hline & B. Address leaks \\
\hline & i. If leaks CANNOT be successfully addressed/fixed notify Linac Operator do not \\
\hline & continue this procedure \\
\hline & ii. If leaks are successfully addressed/fixed continue to step 45 \\
\hline \multirow[t]{18}{*}{45.} & Prepare to Report System Ready to Linac Operator \\
\hline & II Initials: ___ Date: ___ Time: \\
\hline & 45.1. Record temperature DU Target Cooling Water in and out before beam is put on target \\
\hline & 45.2. Prepare to collect samples of pre-irradiation target solution \\
\hline & 45.3. $\square$ Enter desired flow rate ___ $\mathrm{mL} / \mathrm{min}$ Acid Flow Rate Set Pt @ [System].tab \\
\hline & (default this step is $300 \mathrm{~mL} / \mathrm{min}$ ) \\
\hline & 45.4. $\square$ Record calculated____ \% Acid Motor Power @ [System].tab \\
\hline & i. Ensure no cavitation occurs if motor is set above $50 \%$ motor power \\
\hline & 45.5. $\square$ Verify/Adjust ACID Pump Controller to \% Acid Motor Power for desired flow rate \\
\hline & A. If a lower $\%$ motor power value is required due to pressure readings adjust Acid Flow \\
\hline & Rate Set Pt until calculated \% Acid Motor Power matches \% motor power reading at \\
\hline & controller \\
\hline & 45.6. $\square$ Verify ACID Pump to $\underline{\mathbf{R U N}}$ \\
\hline & 45.7. $\square$ Verify $\boldsymbol{D U M P}$ TANK manual vent valve is $\boldsymbol{O P E N}$ \\
\hline & 45.8. $\square$ Collect samples of target solution pre-irradiation \\
\hline & A. Press $\{N E X T$ SAMPLE\} on [Sample Collection].tab $\rightarrow$ [Target Mixing].tab \\
\hline & i. Wait a minimum of $90 \mathrm{sec}$. between samples \\
\hline & B. Samples Collected (all times LabVIEW computer time) \\
\hline
\end{tabular}




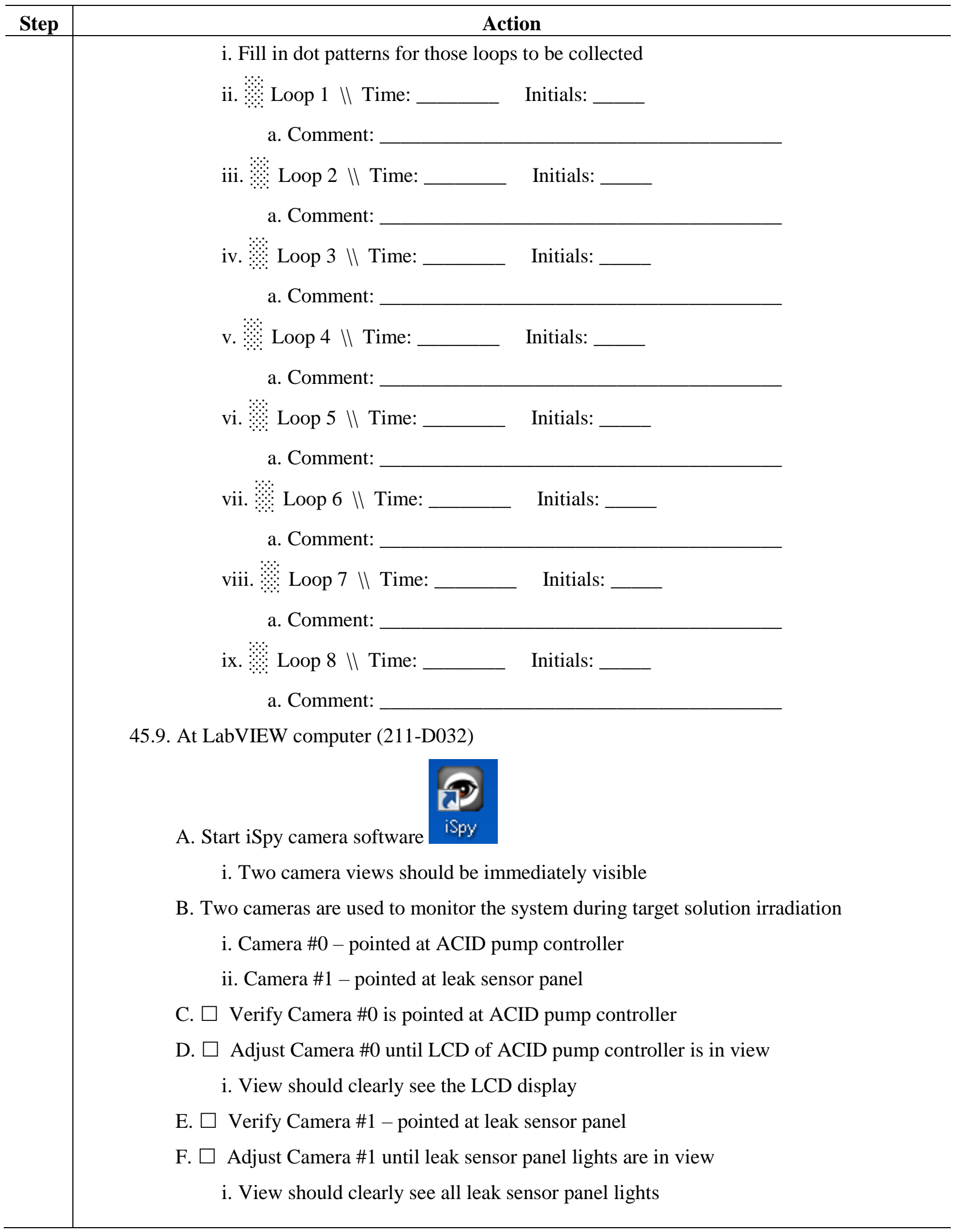




\begin{tabular}{c|c}
\hline Step & Action \\
\hline 46. & $\begin{array}{c}\text { Startup Remote Monitoring Computer (211-B121) and Access Downstairs } \\
\text { 46.1. } \square \text { Use Remote Desktop to access downstairs Mo99 Recovery Process primary computer } \\
\text { 46.2. } \square \text { Verify that Remote Desktop access is working } \\
\text { A. If Remote desktop access is not working properly notify Mo99 Recovery Team Lead } \\
\text { B. DO NOT proceed to line } \mathbf{4 6 . 3} \text { until Remote Desktop access is established and stable } \\
\text { 46.3. } \square \text { Report to Linac Operator that Cell 1 (D035) door ready to be closed }\end{array}$ \\
\hline 47. & $\begin{array}{l}\text { Irradiation Started \& Target Solution Mixing Sample Collection During Irradiation } \\
\text { II Initials: }\end{array}$
\end{tabular}

47.1. Energy
A. All times are LabVIEW times
B. Time 0 (
):
$\mathrm{MeV}$ $\mathrm{kW}$
C. Time ): $\mathrm{MeV}$ $\mathrm{kW}$
D. Time ( ): $\mathrm{MeV}$ $\mathrm{kW}$
E. Time ): $\mathrm{MeV}$ $\mathrm{kW}$
F. Time ): $\mathrm{MeV}$ $\mathrm{kW}$
G. Time ( ): $\mathrm{MeV}$ $\mathrm{kW}$
H. Time ( ): $\mathrm{MeV}$ $\mathrm{kW}$
I. Time ( ): $\mathrm{MeV}$ $\mathrm{kW}$
J. Time ( ): $\mathrm{MeV}$ $\mathrm{kW}$
K. Time END ( ): $\mathrm{MeV}$ $\mathrm{kW}$

47.2. Monitor LINAC thermocouples and pressure for total time of irradiation - report any issues to LINAC operator

47.3. Target Solution Mixing Sample Collection During Irradiation

A. Samples to be collected: $\square$ YES $\square$ NO (if YES fill out information below)

B. Number of samples to collect [ (max. of 8) - (no. of pre-irradiation samples taken,

45.8.B.i) ] =

C. Calculate time between samples $(\mathrm{y})=$ minutes

i. $y=\left(\frac{\text { Target Solution Volume }(\mathrm{mL})}{\text { Flow rate }\left(\frac{\mathrm{mL}}{\mathrm{min}}\right)}\right) /$ No. of samples

ii. Target solution volume: $\mathrm{mL}$

iii. Flow rate: $\mathrm{mL} / \mathrm{min}$

D. Press $\{$ NEXT SAMPLE\} on [Sample Collection].tab $\rightarrow$ [Target Mixing].tab i. Start from empty loop after step 45.8.B 


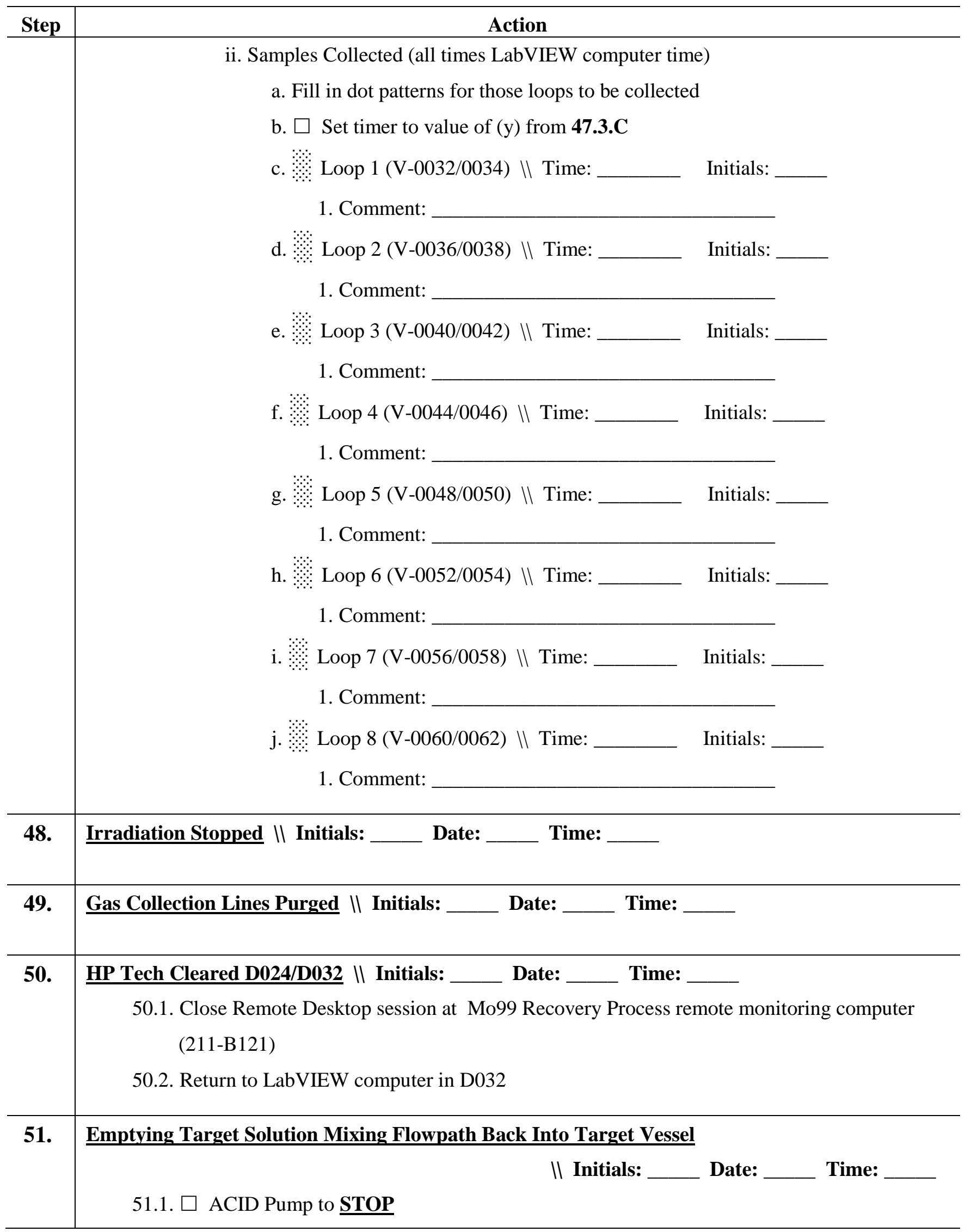




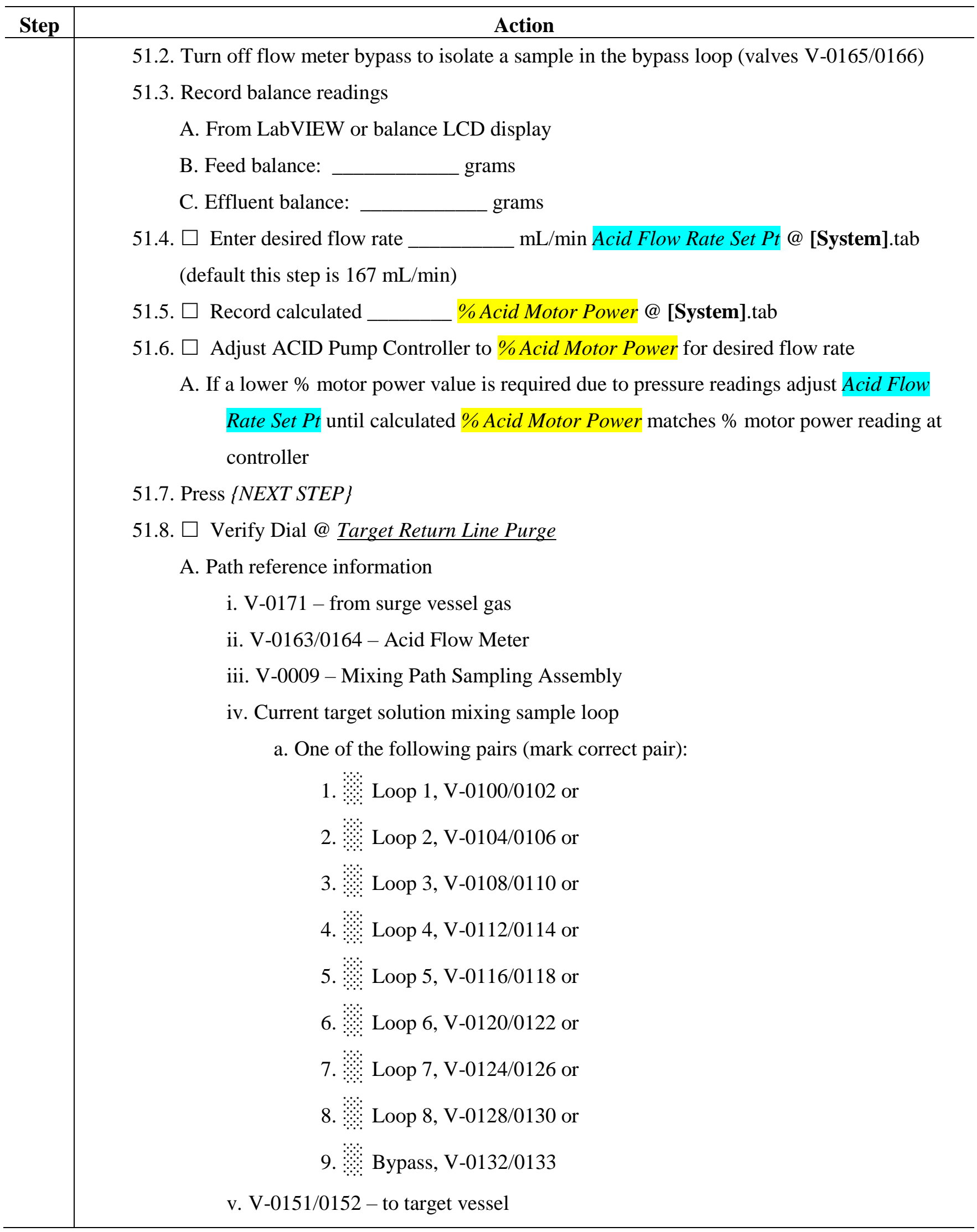




\begin{tabular}{|c|c|}
\hline \multirow[t]{11}{*}{ Step } & Action \\
\hline & vi. Valve text: $171>163 / 164>9>$ \{current target mixing sample loop\} $>151 / 152>$ \\
\hline & Target Vessel \\
\hline & 51.9. $\square$ ACID Pump to $\underline{\mathbf{R U N}}$ \\
\hline & 51.10. $\square$ Hold for approximately 5 minutes \\
\hline & 51.11. $\square$ Hold time ended \\
\hline & 51.12. $\square$ ACID Pump to $\underline{\text { STOP }}$ \\
\hline & 51.13. Record balance readings \\
\hline & A. From LabVIEW or balance LCD display \\
\hline & B. Feed balance: __ grams \\
\hline & C. Effluent balance: ___ grams \\
\hline \multirow[t]{23}{*}{52.} & Pre-Pre-Load Acid Wash $\|$ Initials: ___ Date: ___ Time: \\
\hline & 52.1. Primes lines to column and turns on acid solution pre-heater \\
\hline & 52.2. $\square$ ACID Pump to $\underline{\text { STOP }}$ \\
\hline & 52.3. $\square$ Enter desired flow rate ___ $\mathrm{mL} / \mathrm{min}$ Acid Flow Rate Set Pt @ [System].tab \\
\hline & (default this step is $84 \mathrm{~mL} / \mathrm{min}$ ) \\
\hline & 52.4. $\square$ Record calculated ___ \% Acid Motor Power @ [System].tab \\
\hline & 52.5. $\square$ Verify/Adjust ACID Pump Controller to \% Acid Motor Power for desired flow rate \\
\hline & A. If a lower $\%$ motor power value is required due to pressure readings adjust Acid Flow \\
\hline & Rate Set Pt until calculated \% Acid Motor Power matches \% motor power reading at \\
\hline & controller \\
\hline & 52.6. Press $\{N E X T S T E P\}$ \\
\hline & A. $\square$ Answer OK \\
\hline & B. $\square$ Answer OK \\
\hline & C. $\square$ Answer OK \\
\hline & D. $\square$ Press $\{$ NEXT SAMPLE\} on [Sample Collection].tab $\rightarrow$ [Column Loading].tab \\
\hline & 52.7. Verify Dial @ Pre-Pre-Load Acid Wash \\
\hline & A. Path reference information \\
\hline & i. V-0002 - Fresh Acid \\
\hline & ii. V-0163/0164 - Acid Flow Meter \\
\hline & iii. V-0010 - Acid Column Path \\
\hline & iv. V-0014/0015 - Acid Column Bypass \\
\hline & v. V-0018/0019 - Acid Column Post-Column Filter \\
\hline & vi. Open Loop 1 on column loading sample collection - V-0032/0034 \\
\hline
\end{tabular}




\begin{tabular}{|c|c|}
\hline \multirow[t]{17}{*}{ Step } & Action \\
\hline & vii. V-0139 - Acid Rinse \\
\hline & viii. Valve text: $2>163 / 164>10>14 / 15>18 / 19>32 / 34$ Loop $1>139>$ Pre-Load \\
\hline & Acid Wash \\
\hline & 52.8. ACID Pump to $\underline{\mathbf{R U N}}$ \\
\hline & 52.9. $\square$ Hold for approximately 5 minutes \\
\hline & 52.10. $\square$ Verify effluent balance reading increasing @ [Sensors].tab $\rightarrow$ [Balances].tab \\
\hline & 52.11. Activate $\underline{\text { Acid Pre-Heater }}$ \\
\hline & A. $\square$ Adjust Acid Pre-Heater OUTPUT POWER LEVEL control knob to $300 \mathrm{~mL}-2 \mathrm{~L}$ \\
\hline & setting \\
\hline & B. $\square$ Press red RESET button to turn Acid Pre-Heater orange $\boldsymbol{O V E R}$-TEMP light off \\
\hline & 52.12. $\square$ Hold time ended \\
\hline & 52.13. $\square$ ACID Pump to $\underline{\text { STOP }}$ \\
\hline & 52.14. Record balance readings \\
\hline & A. From LabVIEW or balance LCD display \\
\hline & B. Feed balance: __ grams \\
\hline & C. Effluent balance: ___ grams \\
\hline \multirow[t]{17}{*}{53.} & Pre-Load Acid Wash $\|$ Initials: ___ Date: $\ldots$ Time: \\
\hline & 53.1. $\square$ Verify ACID Pump to $\underline{\text { STOP }}$ \\
\hline & 53.2. $\square$ Enter desired flow rate ___ $\mathrm{mL} / \mathrm{min}$ Acid Flow Rate Set Pt @ [System].tab \\
\hline & (default this step is $167 \mathrm{~mL} / \mathrm{min}$ ) \\
\hline & 53.3. $\square$ Record calculated___ \% Acid Motor Power@ [System].tab \\
\hline & 53.4. $\square$ Verify/Adjust ACID Pump Controller to \% Acid Motor Power for desired flow rate \\
\hline & A. If a lower $\%$ motor power value is required due to pressure readings adjust Acid Flow \\
\hline & Rate Set Pt until calculated \% Acid Motor Power matches \% motor power reading at \\
\hline & controller \\
\hline & 53.5. Press $\{N E X T$ STEP $\}$ \\
\hline & 53.6. Verify Dial @ Pre-Load Acid Wash \\
\hline & A. Path reference information \\
\hline & i. V-0002 - Fresh Acid \\
\hline & ii. V-0163/0164 - Acid Flow Meter \\
\hline & iii. V-0010 - Acid Column Path \\
\hline & iv. V-0012/0013 - Acid Column Bottom Feed \\
\hline & v. V-0016/0017 - Acid column Top Exit \\
\hline
\end{tabular}




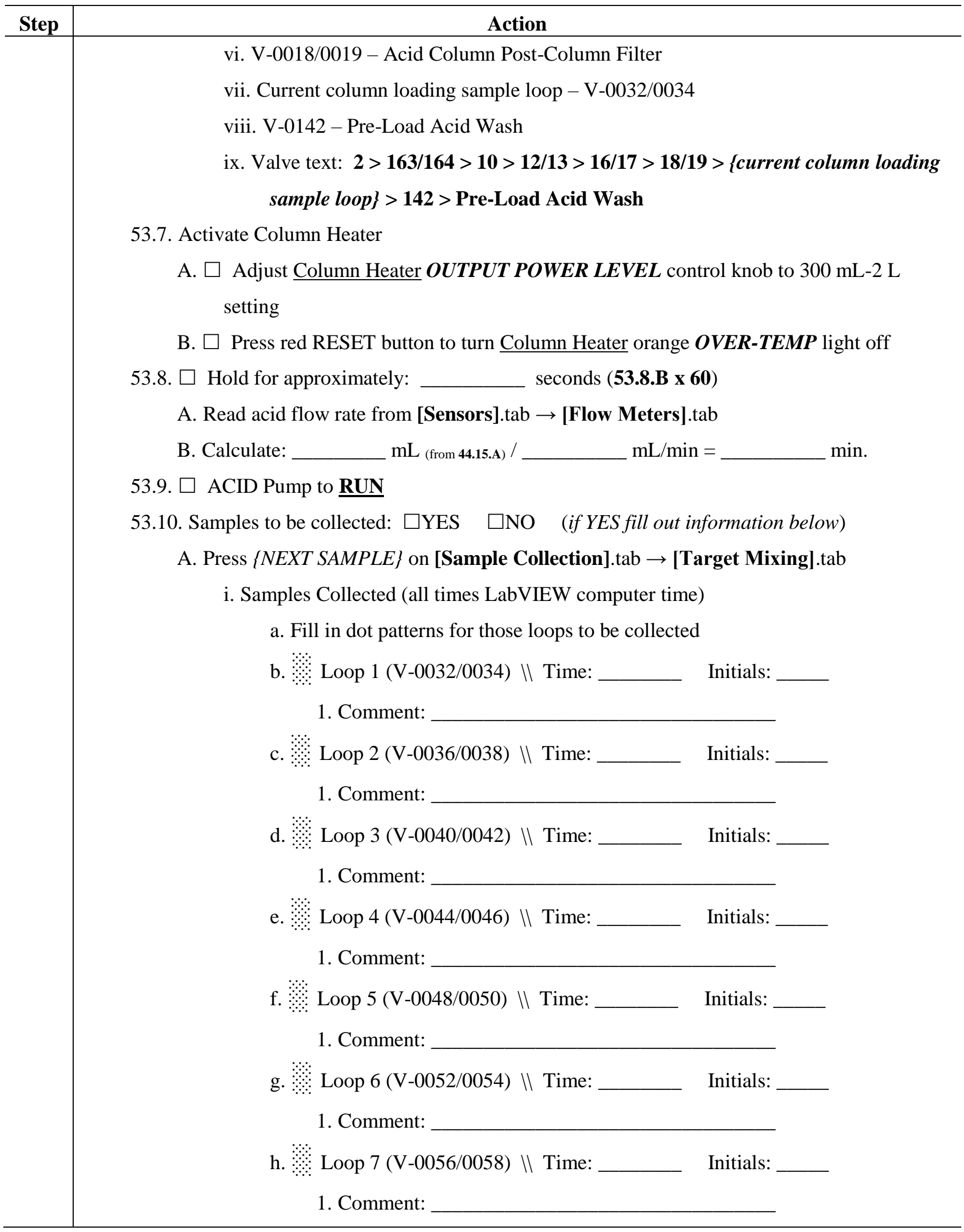




\begin{tabular}{|c|c|}
\hline Step & Action \\
\hline & 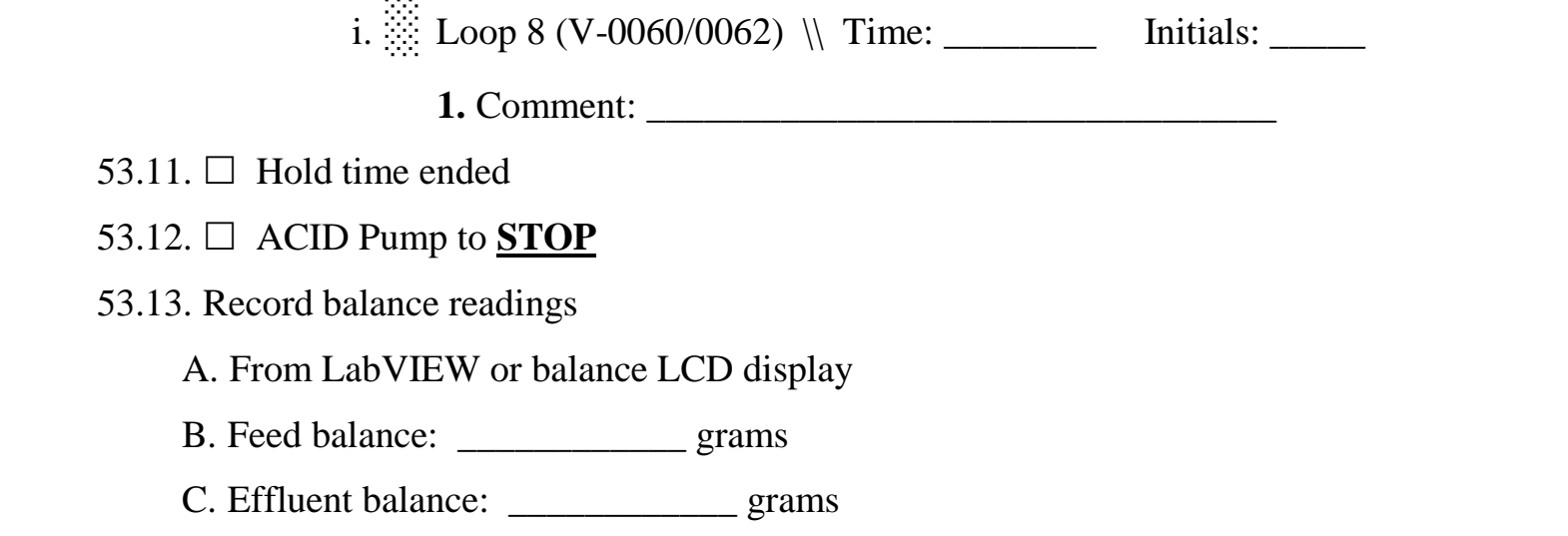 \\
\hline 54. & 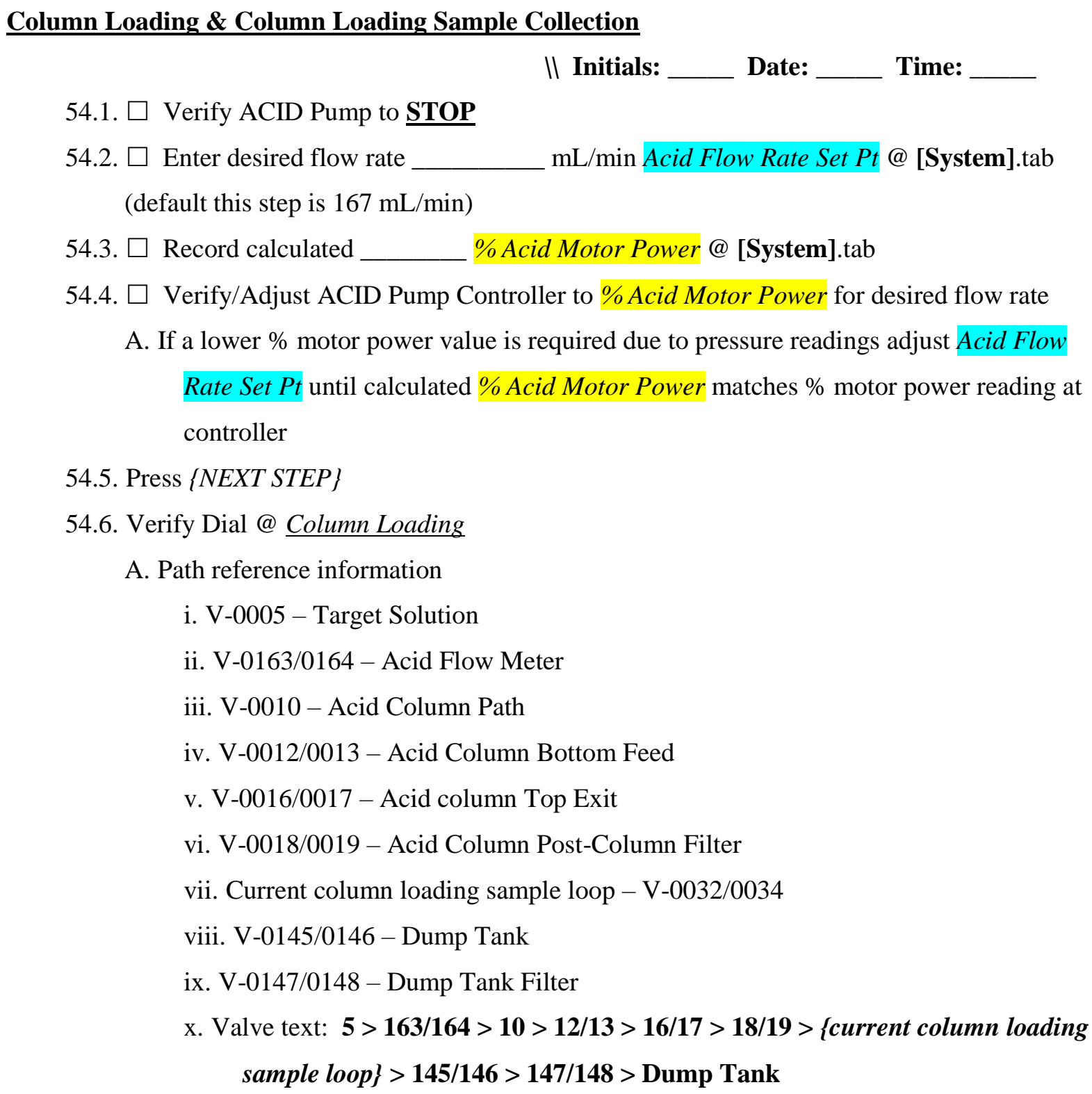 \\
\hline
\end{tabular}




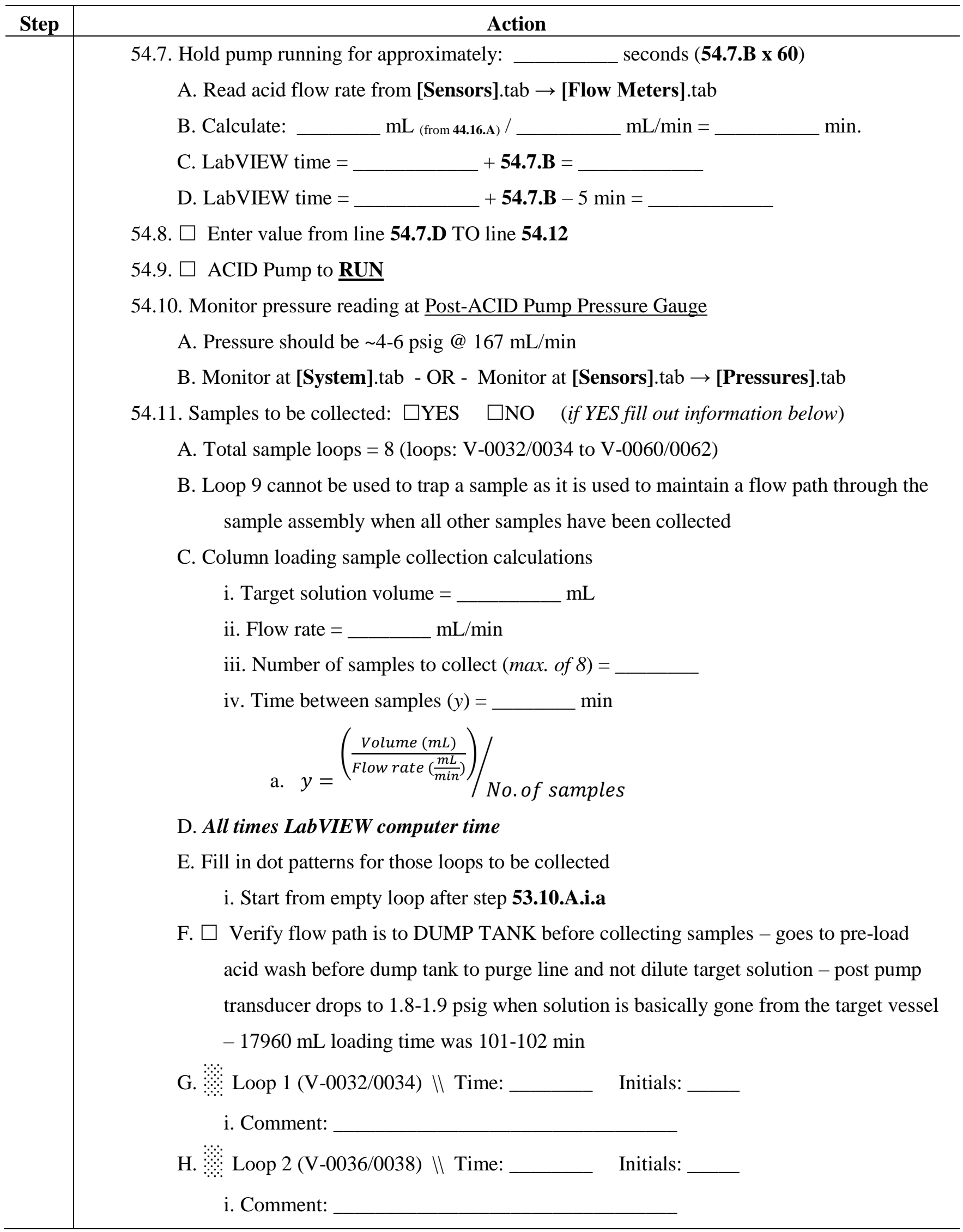




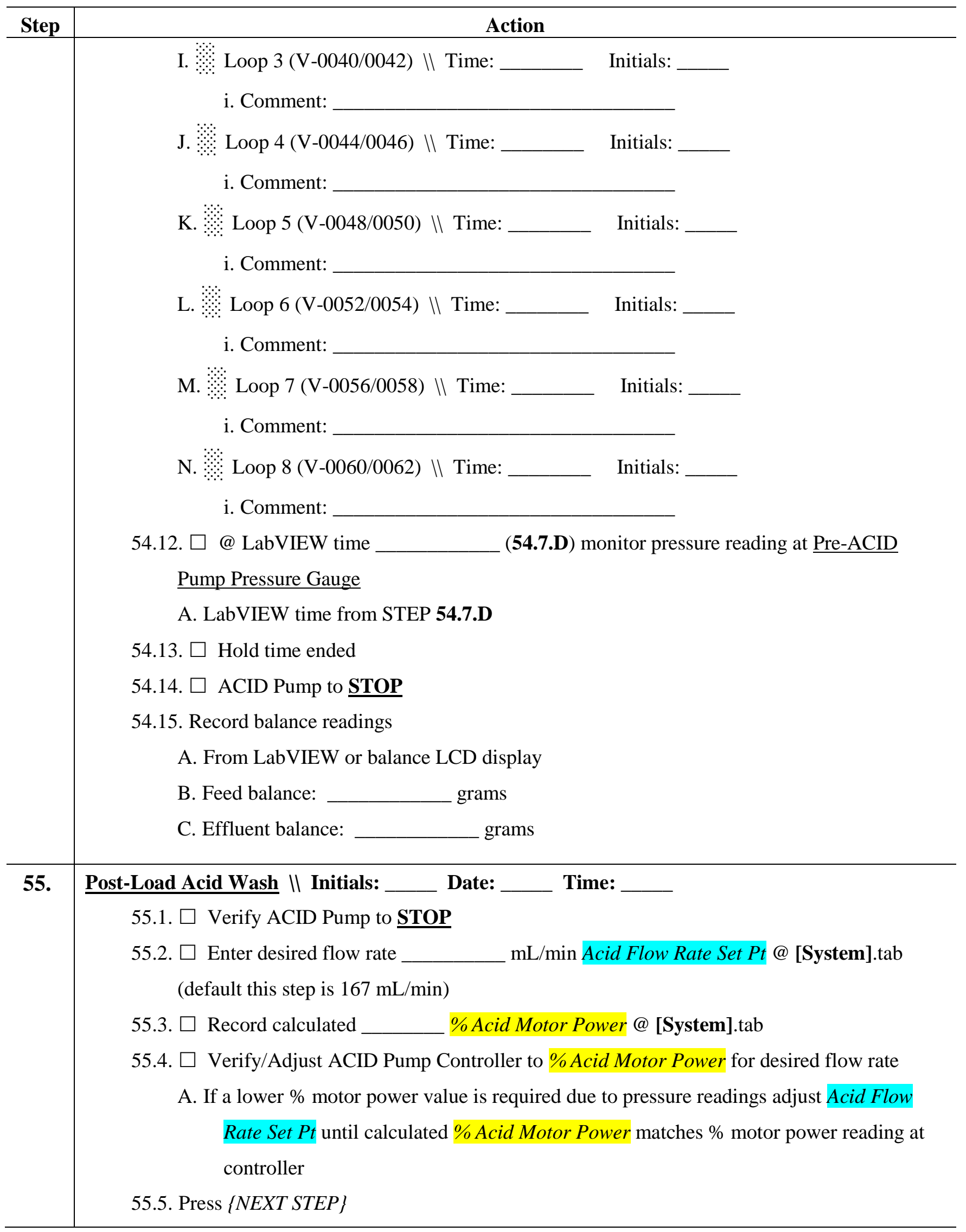




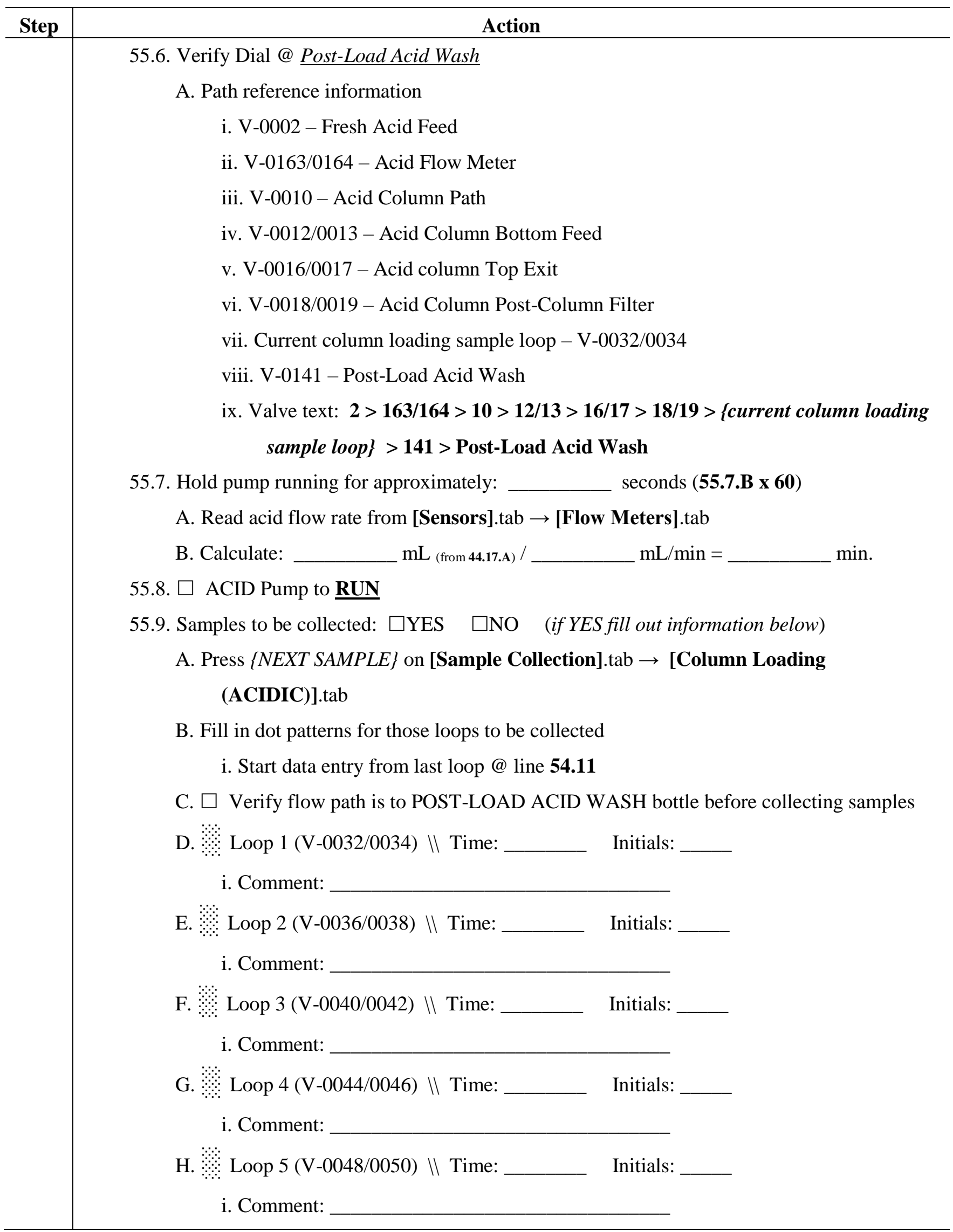




\begin{tabular}{|c|c|}
\hline Step & Action \\
\hline & $\begin{array}{l}\text { I. Loop } 6(\mathrm{~V}-0052 / 0054) \| \text { Time: ___ Initials: } \\
\text { i. Comment: }\end{array}$ \\
\hline & $\begin{array}{l}\text { J. Loop } 7 \text { (V-0056/0058) \ Time: } \\
\text { i. Comment: }\end{array}$ \\
\hline & $\begin{array}{l}\text { K. Loop } 8 \text { (V-0060/0062) \ Time: } \\
\text { i. Comment: }\end{array}$ \\
\hline & 55.10. $\square$ Hold time ended \\
\hline & 55.11. $\square$ ACID Pump to $\underline{\text { STOP }}$ \\
\hline & 55.12. Record balance readings \\
\hline & A. From LabVIEW or balance LCD display \\
\hline & B. Feed balance: __ grams \\
\hline & C. Effluent balance: ___ grams \\
\hline 56. & Post-Load $\mathrm{H}_{2} \mathrm{O}$ Wash $\|$ Initials: ___ Date: $\_$Time: \\
\hline & 56.1. $\square$ Verify ACID Pump to $\underline{\text { STOP }}$ \\
\hline & $\begin{array}{l}\text { 56.2. } \square \text { Enter desired flow rate ___ } \mathrm{mL} / \mathrm{min} \text { Acid Flow Rate Set Pt @ [System].tab } \\
\text { (default this step is } 167 \mathrm{~mL} / \mathrm{min} \text { ) }\end{array}$ \\
\hline & 56.3. $\square$ Record calculated ___ \% Acid Motor Power @ [System].tab \\
\hline & 56.4. $\square$ Verify/Adjust ACID Pump Controller to \% Acid Motor Power for desired flow rate \\
\hline & A. If a lower $\%$ motor power value is required due to pressure readings adjust Acid Flow \\
\hline & Rate Set Pt until calculated \% Acid Motor Power matches \% motor power reading at \\
\hline & controller \\
\hline & 56.5. Press $\{N E X T S T E P\}$ \\
\hline & 56.6. Verify Dial @ Post-Load $\mathrm{H}_{2} \mathrm{O}$ Wash \\
\hline & A. Path reference information \\
\hline & i. V-0001 - Fresh $\mathrm{H}_{2} \mathrm{O}$ \\
\hline & ii. V-0163/0164 - Acid Flow Meter \\
\hline & iii. V-0010 - Acid Column Path \\
\hline & iv. V-0012/0013 - Acid Column Bottom Feed \\
\hline & v. V-0016/0017 - Acid column Top Exit \\
\hline & vi. V-0018/0019 - Acid Column Post-Column Filter \\
\hline & vii. Current column loading sample loop - V-0032/0034 \\
\hline & viii. V-0140 - Post-Load Water Wash \\
\hline
\end{tabular}




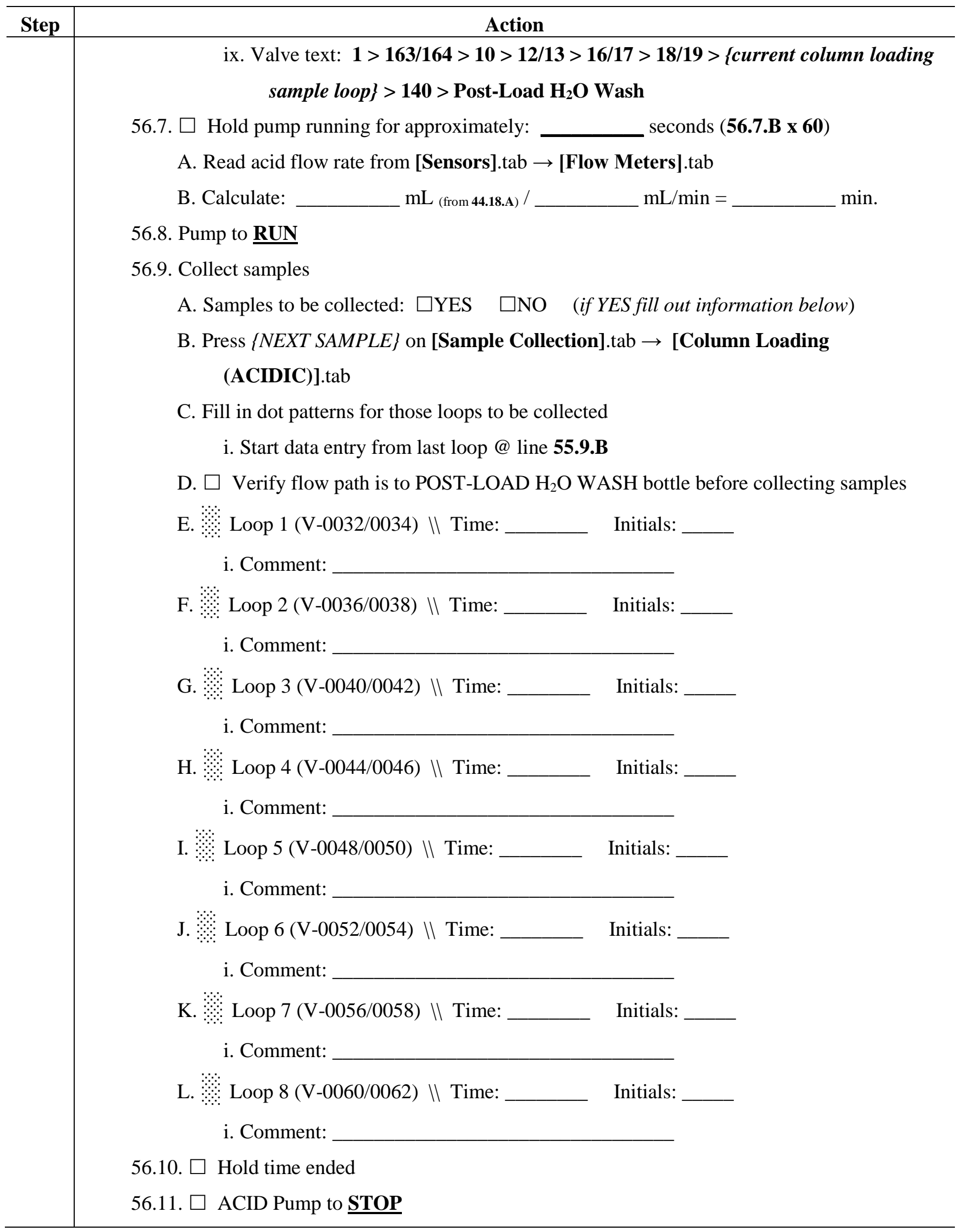




\begin{tabular}{|c|c|}
\hline \multirow[t]{9}{*}{ Step } & Action \\
\hline & 56.12. Record balance readings \\
\hline & A. From LabVIEW or balance LCD display \\
\hline & B. Feed balance: ___ grams \\
\hline & C. Effluent balance: ___ grams \\
\hline & 56.13. $\square$ ACID Pump power to $\underline{\mathbf{O F F}}$ \\
\hline & A. Rocker switch under front/left of ACID Pump V300 controller \\
\hline & 56.14. $\square$ Acid Pre-Heater OUTPUT POWER LEVEL knob from $\underline{300 ~ \mathbf{~ L L}-2 \mathbf{L}} \rightarrow$ TO $\rightarrow$ \\
\hline & $\underline{\text { OFF }}$ \\
\hline \multirow[t]{24}{*}{57.} & 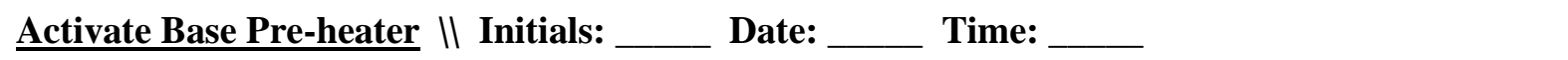 \\
\hline & 57.1. $\square$ BASE Pump controller power to $\underline{\mathbf{O N}}$ using rocker switch under front/left of BASE \\
\hline & Pump V300 controller \\
\hline & 57.2. $\square$ BASE Pump to $\underline{\text { STOP }}$ \\
\hline & 57.3. Record Recovery System balance readings \\
\hline & A. From LabVIEW or balance LCD display \\
\hline & B. Feed balance: __ grams \\
\hline & C. Effluent balance: ___ grams \\
\hline & 57.4. Record 3-L/5-neck flask balance reading (D024 Hot Cell): ____ grams \\
\hline & 57.5. $\square$ Enter desired flow rate ___ $\mathrm{mL} / \mathrm{min}$ Base Flow Rate Set Pt @ [System].tab \\
\hline & (default this step is $84 \mathrm{~mL} / \mathrm{min}$ ) \\
\hline & 57.6. $\square$ Record calculated ___ \% Base Motor Power @ [System].tab \\
\hline & 57.7. $\square$ Verify/Adjust BASE Pump Controller to \% Base Motor Power for desired flow rate \\
\hline & A. If a lower \% motor power value is required due to pressure readings adjust Base Flow \\
\hline & Rate Set Pt until calculated \% Base Motor Power matches \% motor power reading at \\
\hline & controller \\
\hline & 57.8. Press $\{N E X T$ STEP $\}$ \\
\hline & A. $\square$ Answer OK \\
\hline & B. $\square$ Answer OK \\
\hline & C. $\square$ Answer $\mathrm{OK}$ \\
\hline & D. $\square$ Press $\{N E X T$ SAMPLE $\}$ on [Sample Collection].tab $\rightarrow$ [Column Stripping].tab \\
\hline & 57.9. Verify Dial @ Post-Load NaOH Wash \\
\hline & A. Path reference information \\
\hline & i. V-0007 - NaOH Wash \\
\hline
\end{tabular}




\begin{tabular}{|c|c|}
\hline Step & Action \\
\hline & 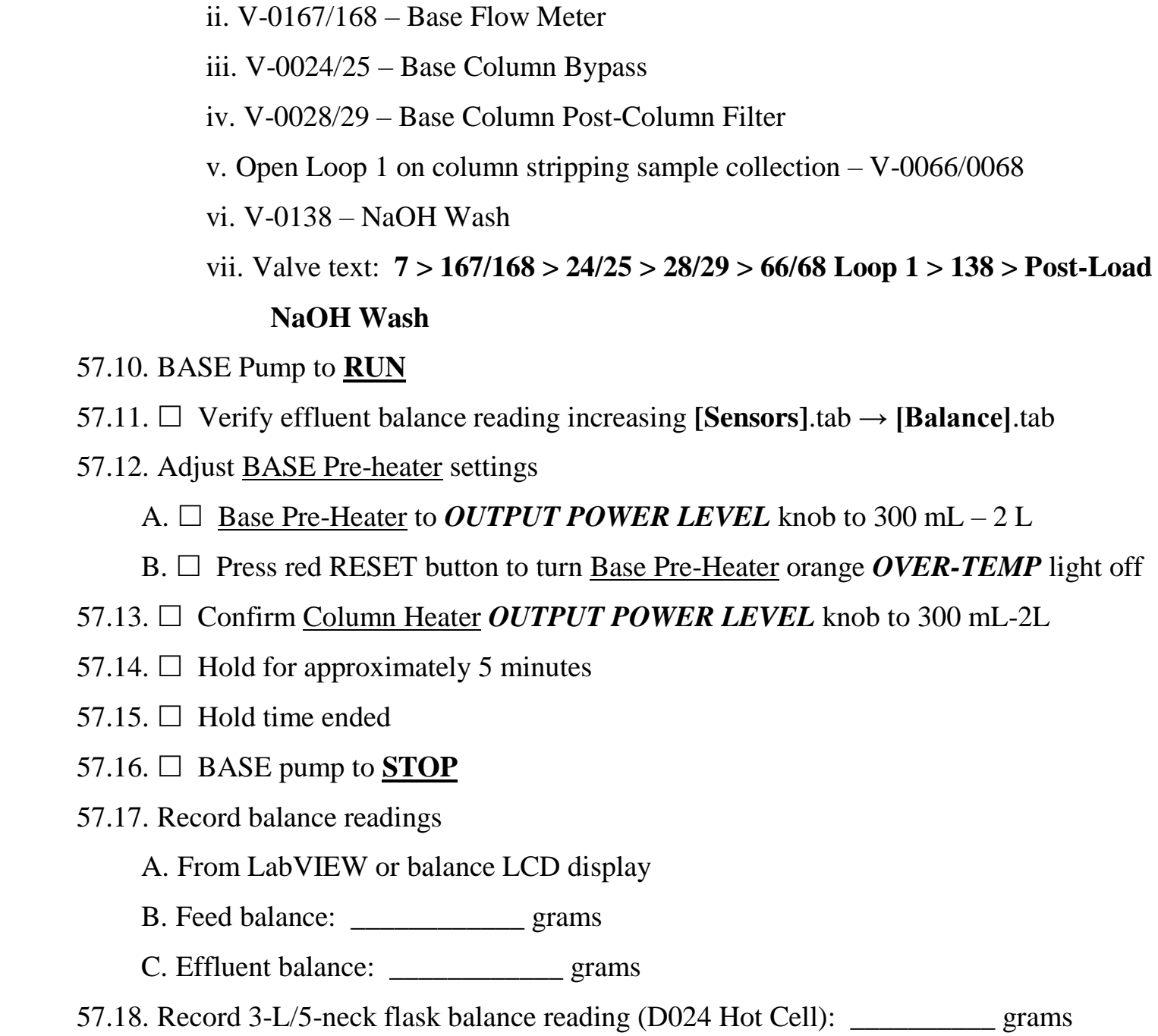 \\
\hline 58. & 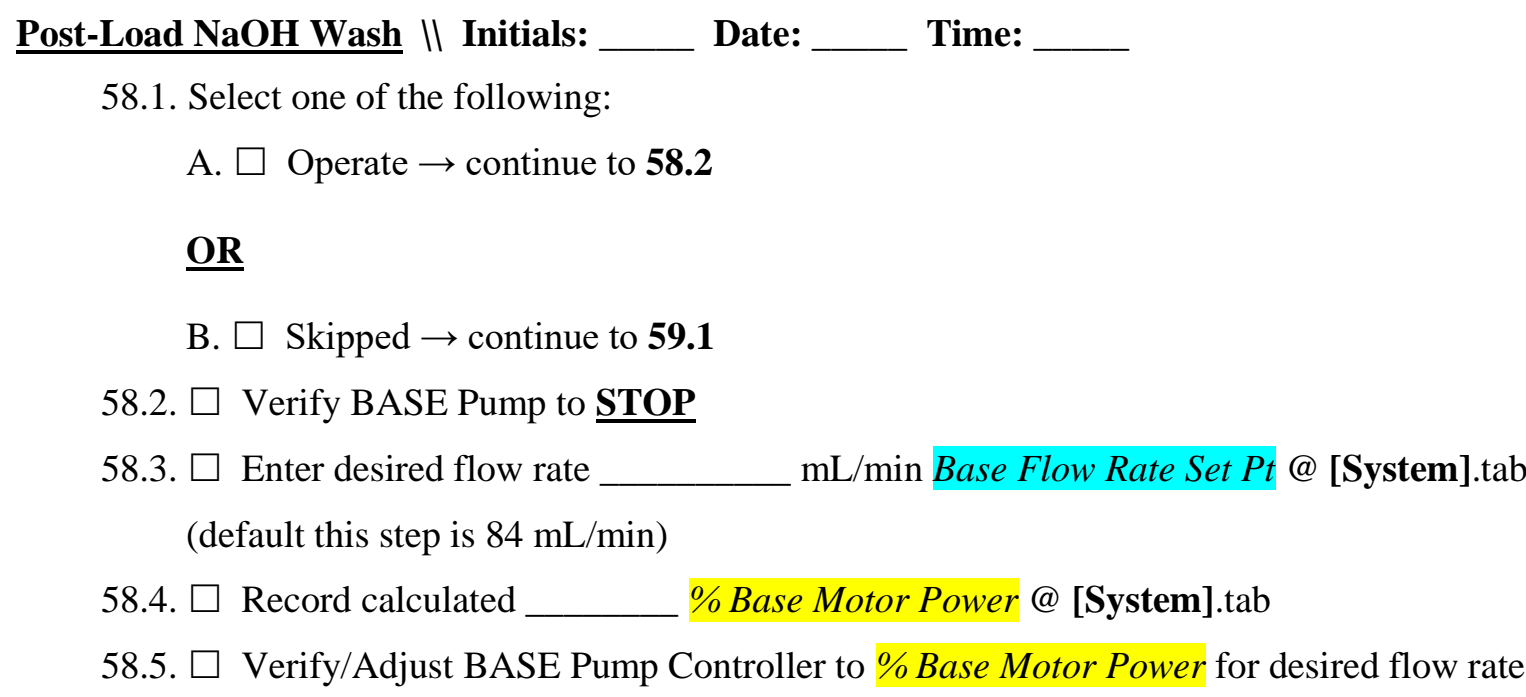 \\
\hline
\end{tabular}




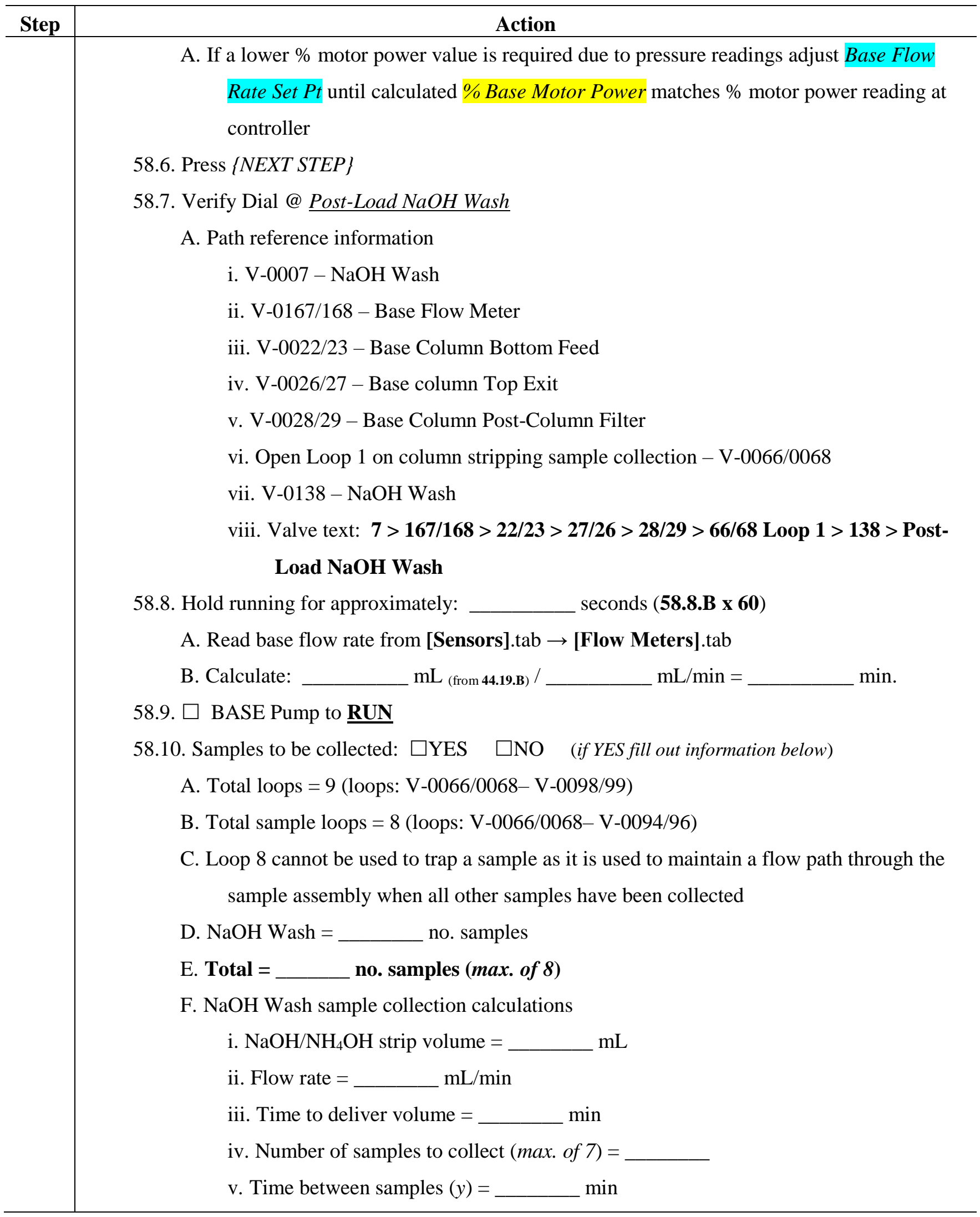




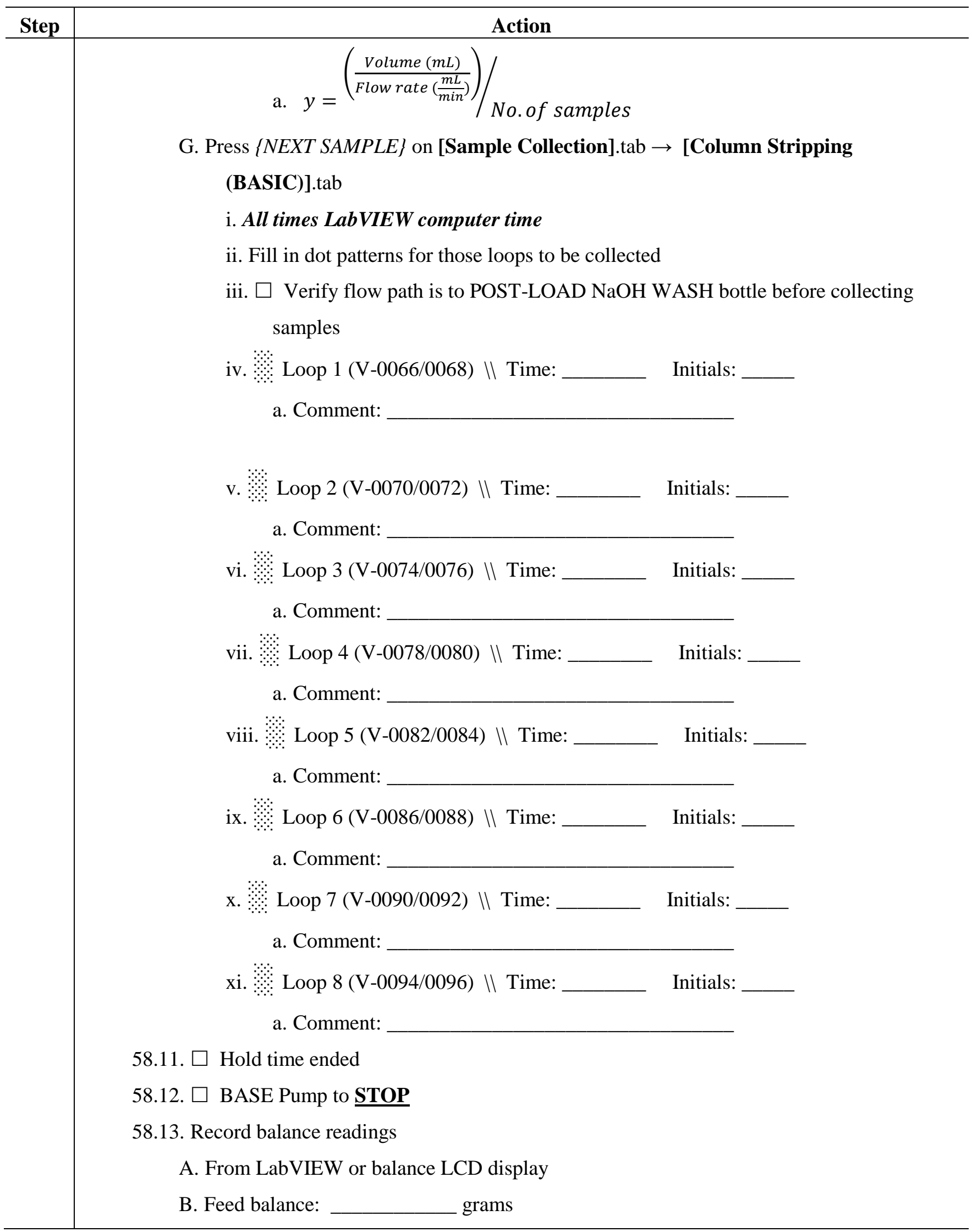




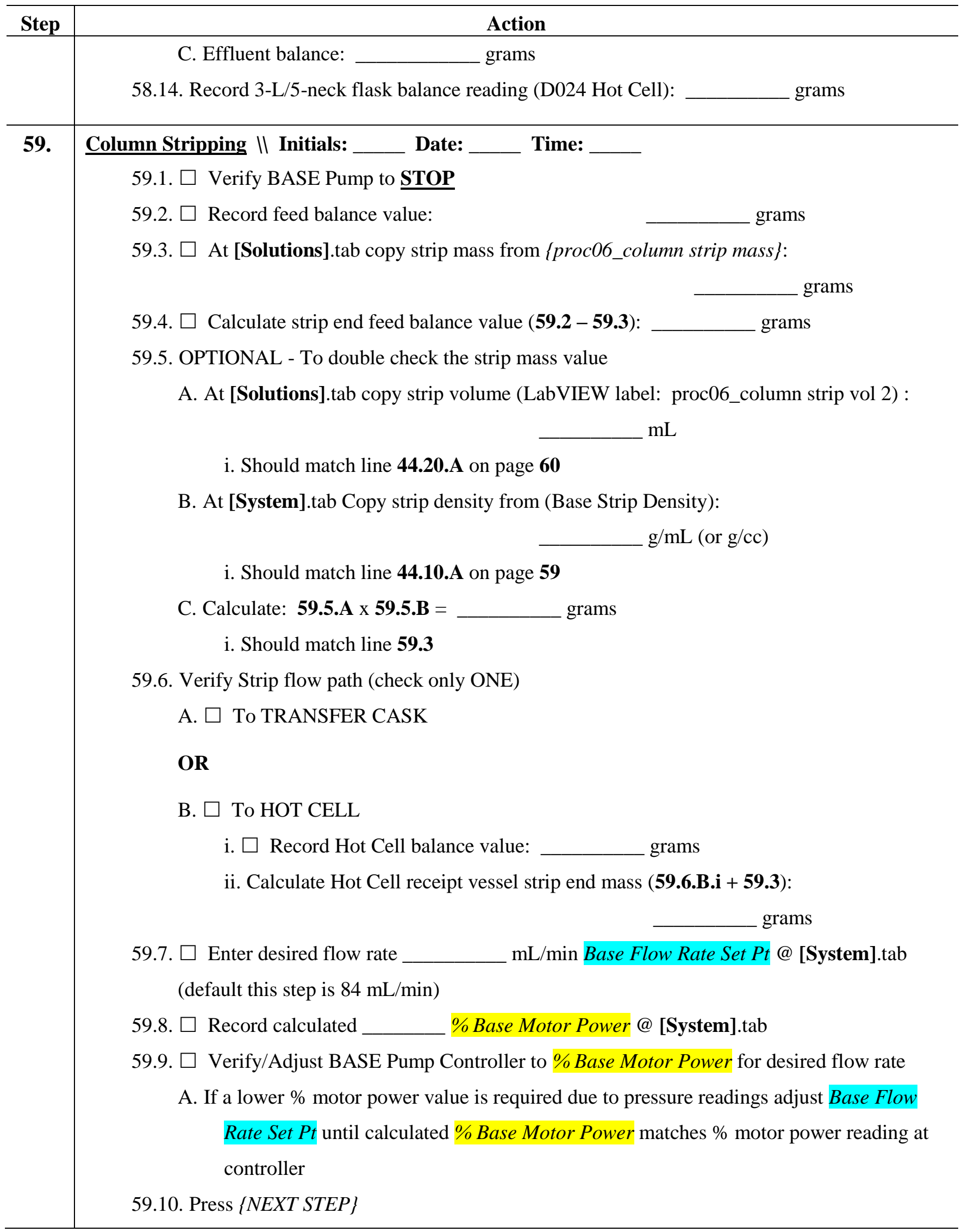




\begin{tabular}{|c|c|}
\hline \multirow[t]{2}{*}{ Step } & Action \\
\hline & 59.11. Verify Dial @ Hydroxide strip \\
\hline & A. Path reference information \\
\hline & i. V-0008 - NaOH Strip \\
\hline & ii. V-0167/168 - Base Flow Meter \\
\hline & iii. V-0022/23 - Base Column Bottom Feed \\
\hline & iv. V-0026/27 - Base column Top Exit \\
\hline & v. V-0028/29 - Base Column Post-Column Filter \\
\hline & vi. Current column stripping sample loop - V-0066/0068 \\
\hline & vii. V-0136 - Strip to Transfer Cask \\
\hline & viii. Valve text: $8>167 / 168>22 / 23>27 / 26>28 / 29>$ \{current column stripping \\
\hline & sample loop\} $>136>$ CASK $($ or HOT CELL) \\
\hline & 59.12. Hold pump running for approximately: ___ seconds $(\mathbf{5 9 . 1 2 . B} \times \mathbf{6 0})$ \\
\hline & A. Read balance base flow rate @ [Sensors].tab $\rightarrow$ [Flow Meters].tab \\
\hline & B. Calculate: __ $\mathrm{mL}_{(\text {from 44.20.A) }} / \ldots \mathrm{mL} / \mathrm{min}=\ldots \min$. \\
\hline & 59.13. $\square$ BASE Pump to $\underline{\mathbf{R U N}}$ \\
\hline & 59.14. Samples to be collected: $\square$ YES $\quad \square$ NO (if YES fill out information below) \\
\hline & A. Total loops $=9$ (loops: V-0066/0068- V-00098/99) \\
\hline & B. Total sample loops $=8$ (loops: V-0066/0068-V-00094/96) \\
\hline & C. Loop 8 cannot be used to trap a sample as it is used to maintain a flow path through the \\
\hline & sample assembly when all other samples have been collected \\
\hline & D. $\mathrm{NaOH}$ Strip $=\ldots$ no. samples \\
\hline & E. Total $=\ldots$ no. samples $(\max$. of 8$)$ \\
\hline & F. $\mathrm{NaOH}$ strip sample collection calculations \\
\hline & i. $\mathrm{NaOH}$ strip volume $=\ldots \mathrm{mL}$ \\
\hline & ii. Flow rate $=\ldots \mathrm{mL} / \mathrm{min}$ \\
\hline & iii. Time to deliver volume $=\ldots \min$ \\
\hline & iv. Number of samples to collect $(\max$. of 8$)=$ \\
\hline & v. Time between samples $(y)=\ldots$ min \\
\hline & $($ Volume $(m L)) /$ \\
\hline & a. $y=\left(\overline{\text { Flow rate }\left(\frac{\mathrm{mL}}{\mathrm{min}}\right)}\right) /$ \\
\hline & T No.of samples \\
\hline & G. Press $\{N E X T$ SAMPLE $\}$ on [Sample Collection].tab $\rightarrow$ [Column Stripping \\
\hline & (BASIC)].tab \\
\hline & i. Start data entry from last loop @ line $\mathbf{5 8 . 9}$ \\
\hline
\end{tabular}




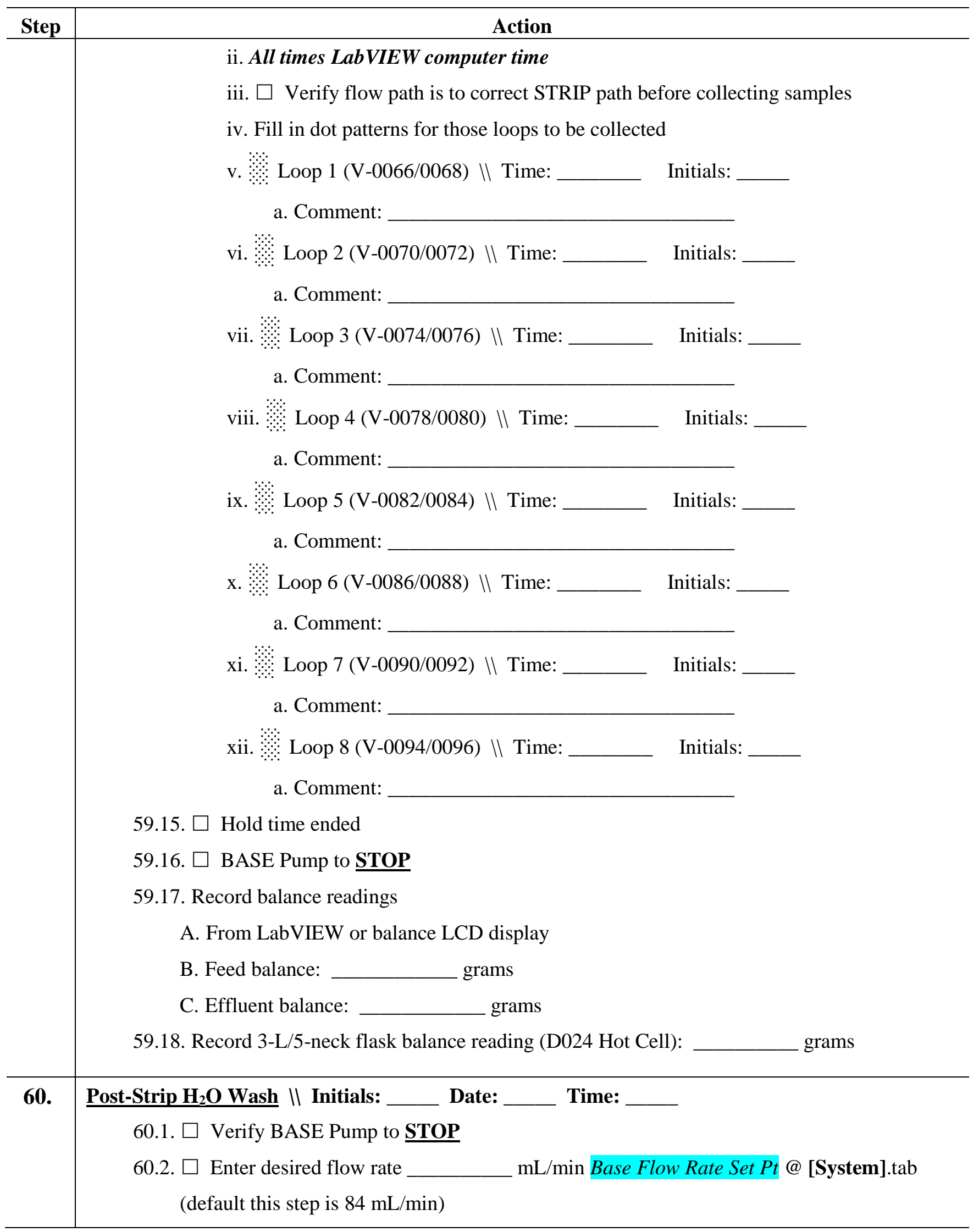




\begin{tabular}{|c|c|}
\hline Step & Action \\
\hline & 60.3. $\square$ Record calculated ___ \% Base Motor Power @ [System].tab \\
\hline & 60.4. $\square$ Verify/Adjust BASE Pump Controller to \% Base Motor Power for desired flow rate \\
\hline & A. If a lower $\%$ motor power value is required due to pressure readings adjust Base Flow \\
\hline & Rate Set Pt until calculated \% Base Motor Power matches $\%$ motor power reading at \\
\hline & controller \\
\hline & 60.5. Press $\{N E X T$ STEP $\}$ \\
\hline & 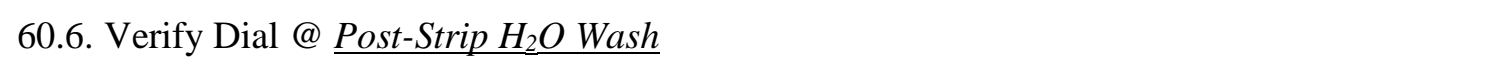 \\
\hline & A. Path reference information \\
\hline & i. V-0006 - Fresh $\mathrm{H}_{2} \mathrm{O}$ \\
\hline & ii. V-0167/168 - Base Flow Meter \\
\hline & iii. V-0022/23 - Base Column Bottom Feed \\
\hline & iv. V-0026/27 - Base column Top Exit \\
\hline & v. V-0028/29 - Base Column Post-Column Filter \\
\hline & vi. Current column loading sample loop - V-0066/0068 \\
\hline & vii. V-0135 - Post-Strip $\mathrm{H}_{2} \mathrm{O}$ Wash \\
\hline & viii. Valve text: $6>167 / 168>22 / 23>27 / 26>28 / 29>$ \{current column stripping \\
\hline & sample loop\} $>135>$ Post-Strip $\mathrm{H}_{2} \mathrm{O}$ Wash \\
\hline & 60.7. HOLD TIME (pick one: $60.7 . A$ OR 60.7.B) \\
\hline & A. $\square$ STRIP to HOT CELL \\
\hline & i. $\square \mathrm{H}_{2} \mathrm{O}$ to HOT CELL hold for approximately: ____ __ minutes \\
\hline & a. Read balance base flow rate @ [Sensors].tab $\rightarrow$ [Flow Meters].tab \\
\hline & b. Calculate: __ $\mathrm{mL}_{(\text {from 44.21.A.i) }} / \ldots \mathrm{mL} / \mathrm{min}=$ \\
\hline & min. \\
\hline & ii. $\square \mathrm{H}_{2} \mathrm{O}$ to WASTE BOTTLE hold for approximately: __ minutes \\
\hline & a. Read base flow rate from [Sensors].tab $\rightarrow$ [Flow Meters].tab \\
\hline & 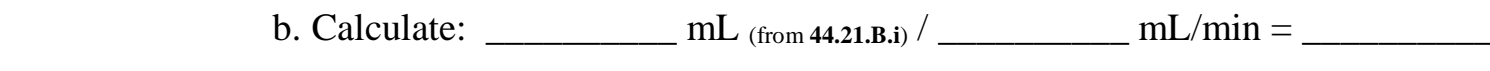 \\
\hline & min. \\
\hline & 1. Portion of $\mathrm{H}_{2} \mathrm{O}$ used to flush transfer line \\
\hline & $\mathbf{O R}$ \\
\hline & B. $\square$ STRIP to TRANSPORT CASK \\
\hline & i. $\square \mathrm{H}_{2} \mathrm{O}$ to WASTE BOTTLE hold for approximately: ___ minutes \\
\hline & a. Read balance base flow rate @ [Sensors].tab $\rightarrow$ [Flow Meters].tab \\
\hline
\end{tabular}




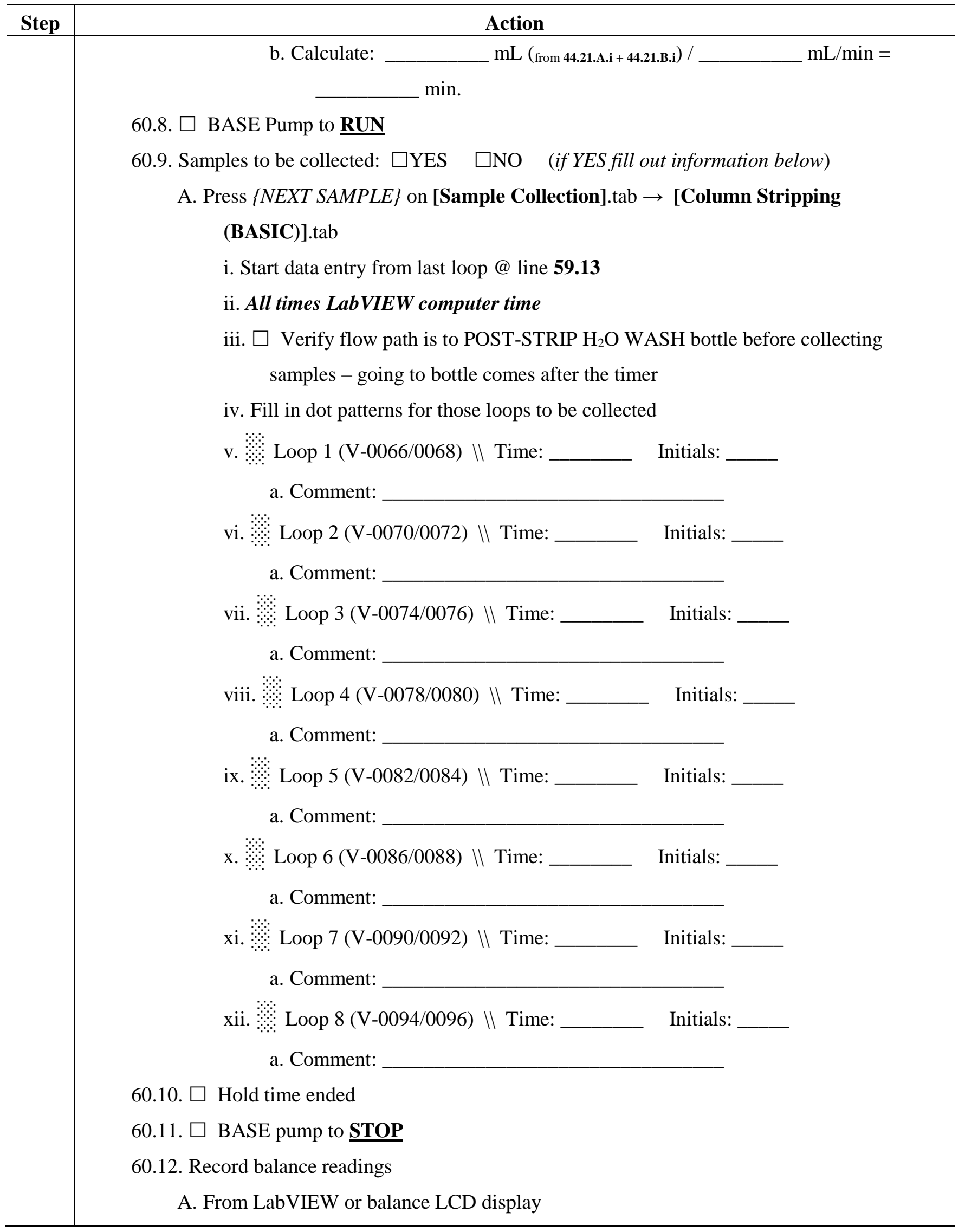




\begin{tabular}{|c|c|}
\hline Step & Action \\
\hline & 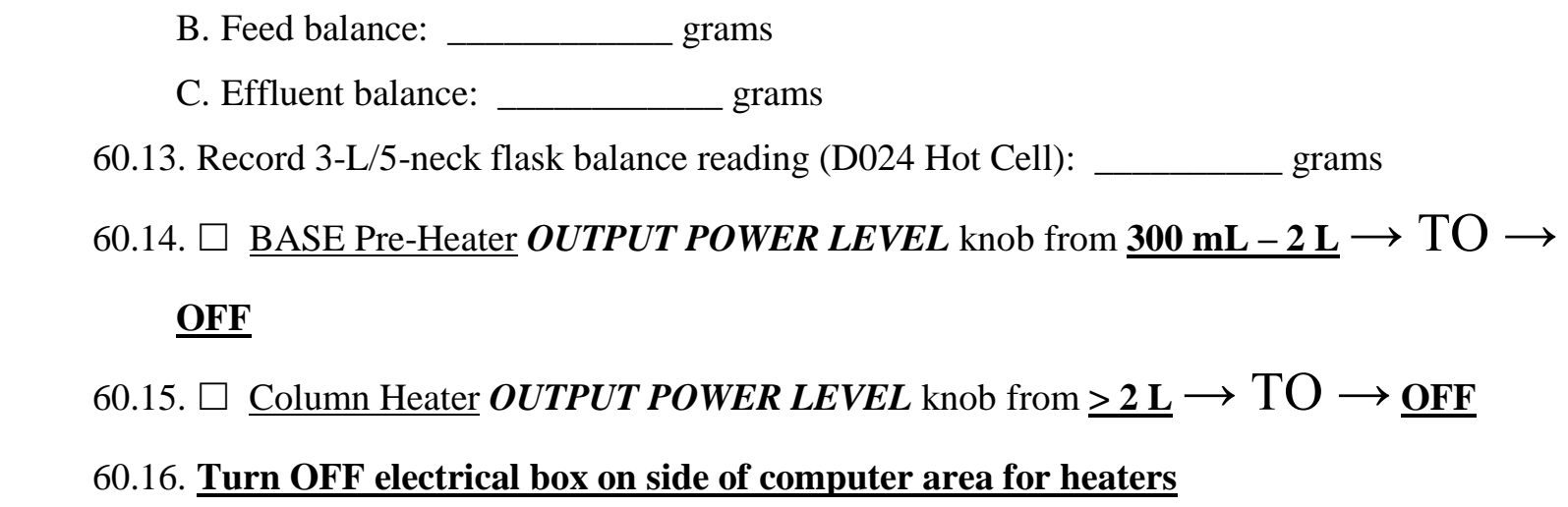 \\
\hline 61. & 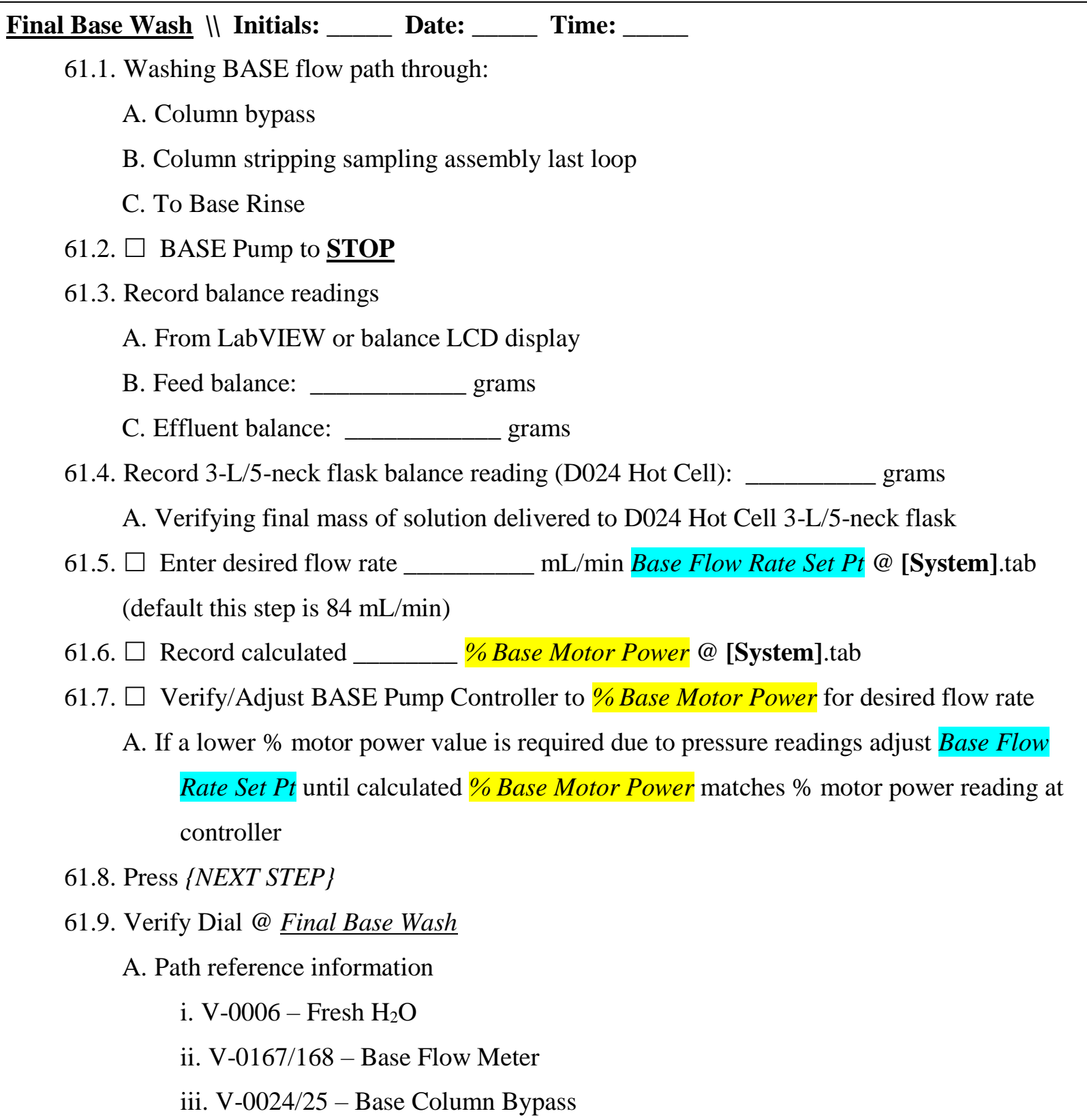 \\
\hline
\end{tabular}




\begin{tabular}{|c|c|}
\hline Step & Action \\
\hline & iv. V-0028/29 - Base Column Post-Column Filter \\
\hline & v. Current column stripping sample loop - V-00098/99 \\
\hline & vi. V-0134 - Base Rinse \\
\hline & vii. Valve text: $6>167 / 168>24 / 25>28 / 29>$ \{ current column stripping sample \\
\hline & loop $\}>134>$ Base Rinse \\
\hline & 61.10. $\square$ Hold pump running for approximately: ___ seconds $(\mathbf{6 1 . 1 0 . B} \times \mathbf{6 0})$ \\
\hline & A. Read base flow rate from [Sensors].tab $\rightarrow$ [Flow Meters].tab \\
\hline & B. Calculate: ___ $\mathrm{mL}($ from 44.22.A) $/ \ldots \mathrm{mL} / \mathrm{min}=\ldots$ min. \\
\hline & 61.11. $\square$ BASE Pump to $\underline{\mathbf{R U N}}$ \\
\hline & 61.12. $\square$ Hold time ended \\
\hline & 61.13. $\square$ BASE Pump to $\underline{\text { STOP }}$ \\
\hline & 61.14. Record balance readings \\
\hline & A. From LabVIEW or balance LCD display \\
\hline & B. Feed balance: __ grams \\
\hline & C. Effluent balance: ___ grams \\
\hline & 61.15. $\square$ BASE Pump controller power OFF using rocker switch under front/left of BASE Pump \\
\hline & V300 controller \\
\hline \multirow[t]{16}{*}{62.} & Final Acid Wash $\|$ Initials: $\_$__ Date: ___ Time: \\
\hline & 62.1. Washing ACID flow path through: \\
\hline & A. Column bypass \\
\hline & B. Column loading sampling assembly last loop \\
\hline & C. To Acid Rinse \\
\hline & 62.2. $\square$ ACID Pump controller power ON using rocker switch under front/left of ACID Pump \\
\hline & V300 controller \\
\hline & 62.3. $\square$ ACID Pump to $\underline{\text { STOP }}$ \\
\hline & 62.4. $\square$ Enter desired flow rate ___ $\mathrm{mL} / \mathrm{min}$ Acid Flow Rate Set Pt @ [System].tab \\
\hline & (default this step is $84 \mathrm{~mL} / \mathrm{min}$ ) \\
\hline & 62.5. $\square$ Record calculated ___ \% Acid Motor Power @ [System].tab \\
\hline & 62.6. $\square$ Verify/Adjust ACID Pump Controller to \% Acid Motor Power for desired flow rate \\
\hline & A. If a lower $\%$ motor power value is required due to pressure readings adjust Acid Flow \\
\hline & Rate Set Pt until calculated \% Acid Motor Power matches \% motor power reading at \\
\hline & controller \\
\hline & 62.7. Press $\{N E X T$ STEP $\}$ \\
\hline
\end{tabular}




\begin{tabular}{|c|c|}
\hline Step & Action \\
\hline & 62.8. Verify Dial @ Final Acid Wash \\
\hline & A. Path reference information \\
\hline & i. V-0002 - Fresh Acid \\
\hline & ii. V-0163/0164 - Acid Flow Meter \\
\hline & iii. V-0014/0015 - Acid Column Bypass \\
\hline & iv. V-0018/0019 - Acid Column Post-Column Filter \\
\hline & v. Current column loading sample loop - V-0064/0065 \\
\hline & vi. V-0139 - Acid Rinse \\
\hline & vii. Valve text: $2>163 / 164>14 / 15>18 / 19>$ \{current column loading sample loop\} \\
\hline & $>139>$ Acid Rinse \\
\hline & 62.9. $\square$ Hold for approximately: ___ seconds $(\mathbf{6 2 . 9 . B} \times \mathbf{6 0})$ \\
\hline & A. Read acid flow rate from [Sensors].tab $\rightarrow$ [Flow Meters].tab \\
\hline & B. Calculate: __ $\mathrm{mL}_{(\text {from 44.23.A) }} / \ldots \mathrm{mL} / \mathrm{min}=\ldots \min$. \\
\hline & 62.10. $\square$ ACID Pump to $\underline{\mathbf{R U N}}$ \\
\hline & 62.11. $\square$ Hold time ended \\
\hline & 62.12. $\square$ ACID Pump to $\underline{\text { STOP }}$ \\
\hline & 62.13. Record balance readings \\
\hline & A. From LabVIEW or balance LCD display \\
\hline & B. Feed balance: __ grams \\
\hline & C. Effluent balance: __ grams \\
\hline & 62.14. $\square$ ACID Pump controller power OFF \\
\hline & A. Rocker switch under front/left of ACID Pump V300 controller \\
\hline \multirow[t]{5}{*}{63.} & Calculate Feeds Delivered and Effluents Received \\
\hline & 63.1. Complete entries in the following table \\
\hline & 63.2. Enter value of balance reading from indicated step \\
\hline & 63.3. Calculate difference and record in space provided \\
\hline & 63.4. Refer to this table when conducting washout work instructions \\
\hline
\end{tabular}




\begin{tabular}{|c|c|c|c|c|c|c|}
\hline & \multicolumn{2}{|c|}{ Feed Balance } & \multicolumn{2}{|c|}{ Effluent Balance } & D024 Balance & Feed Used \\
\hline Emptying Target Path & $51.13 . \mathrm{B}$ & ${ }_{\text {51.3.B }}=$ & 51.13. & ${ }_{\text {51.3.C }}={ }^{\mathrm{x}}, \mathrm{y}$ & Not Applicable & $\begin{array}{l}\text { Acid Feed Used (sum a) } \\
\qquad- \\
\end{array}$ \\
\hline $\begin{array}{l}\text { Pre-Pre Load Acid } \\
\text { Wash }\end{array}$ & $52.14 . \mathrm{B}$ & ${ }_{\text {51.13.B }}={ }^{\mathrm{a}}$ & 52.14. & $\tau_{\text {51.13.C }}=\ldots \mathrm{x}$ & Not Applicable & $\begin{array}{l}\text { Base Wash Used (sum b) } \\
\\
\end{array}$ \\
\hline Pre-Load Acid Wash & 53.13.B & $-\frac{\mathrm{a}}{52.14 . \mathrm{B}}$ & 53.13. & $\overline{52.14 . \mathrm{C}}=$ & Not Applicable & $\begin{array}{l}\text { Base Strip Used (sum c) } \\
\\
\end{array}$ \\
\hline Column Loading & $54.15 . \mathrm{B}$ & $\begin{array}{l} \\
53.13 . \mathrm{B}\end{array}$ & 54.15. & 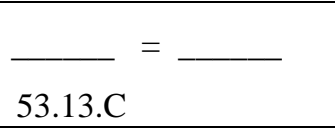 & Not Applicable & $\begin{array}{l}\text { Water Wash Used (sum d) } \\
\qquad \\
\end{array}$ \\
\hline Post-Load Acid Wash & $55.12 . \mathrm{B}$ & $-{ }_{54.15 . \mathrm{B}}={ }^{\mathrm{a}}$ & 55.12. & $\tau_{54.15 . \mathrm{C}}=$ & Not Applicable & \\
\hline Post-Load H2O Wash & $56.12 . \mathrm{B}$ & ${ }_{55.12 . \mathrm{B}}=\mathrm{d}$ & 56.12. & $\tau_{55.12 . \mathrm{C}}=$ & Not Applicable & $\begin{array}{l}\text { Acid Rinse Rec. V. Left } \\
(8000-\text { sum } \mathrm{x})\end{array}$ \\
\hline Base Heater On & $57.17 . \mathrm{B}$ & ${ }_{\text {57.3.B }}={ }^{\mathrm{b}}$ & 57.17. & $\overline{\text { 57.3.C }}=$ & $\frac{}{57.18}-\frac{}{57.4}=$ & $\begin{array}{r}\text { Base Rinse Rec. V. Left } \\
(8000-\text { sum y })\end{array}$ \\
\hline Post-Load NaOH Wash & 58.13.B & ${ }_{57.17 . \mathrm{B}}={ }^{\mathrm{b}}$ & 58.13. & ${ }_{\text {57.17.C }}=$ & $\overline{58.14}^{-} \frac{}{57.18}=$ & \\
\hline Column Strip & $59.17 . \mathrm{B}$ & $\begin{array}{r} \\
\text { 58.13.B }\end{array}$ & 59.17. & ${ }_{\text {58.13.C }}=$ & $\frac{}{59.18}-\frac{}{58.14}=$ & \\
\hline Post-Strip H2O Wash & $60.12 . \mathrm{B}$ & ${ }_{59.17 . B}={ }^{\mathrm{d}}$ & 60.12. & ${ }_{\text {59.17.C }}=$ & $\frac{}{60.13}-\frac{}{59.18}=$ & \\
\hline Base System Wash & 61.14.B & ${ }_{60.12 . \mathrm{B}}=\mathrm{C}^{\mathrm{d}}$ & 61.14. & ${ }_{60.12 . \mathrm{C}}=\varlimsup^{\mathrm{y}}$ & Not Applicable & \\
\hline Acid System Wash & $\overline{62.13 . \mathrm{B}}$ & $\tau_{61.14 . \mathrm{B}}=\amalg^{\mathrm{a}}$ & 62.13. & $\tau_{61.14 . C}=L^{x}$ & Not Applicable & \\
\hline
\end{tabular}




\begin{tabular}{|c|c|}
\hline Step & Action \\
\hline 64. & $\square$ Turn Off the Relays to The Glovebox Heaters \\
\hline 65. & $\begin{array}{l}\square \text { Hang Reminder Sign to LabVIEW Rack to Turn OFF Manual Dump Tank Valve } \\
\text { 65.1. After sign is posted continue to step } 66 \text { (if the manual dump tank valve cannot be accessed } \\
\text { yet, proceed to step 67) }\end{array}$ \\
\hline 66. & 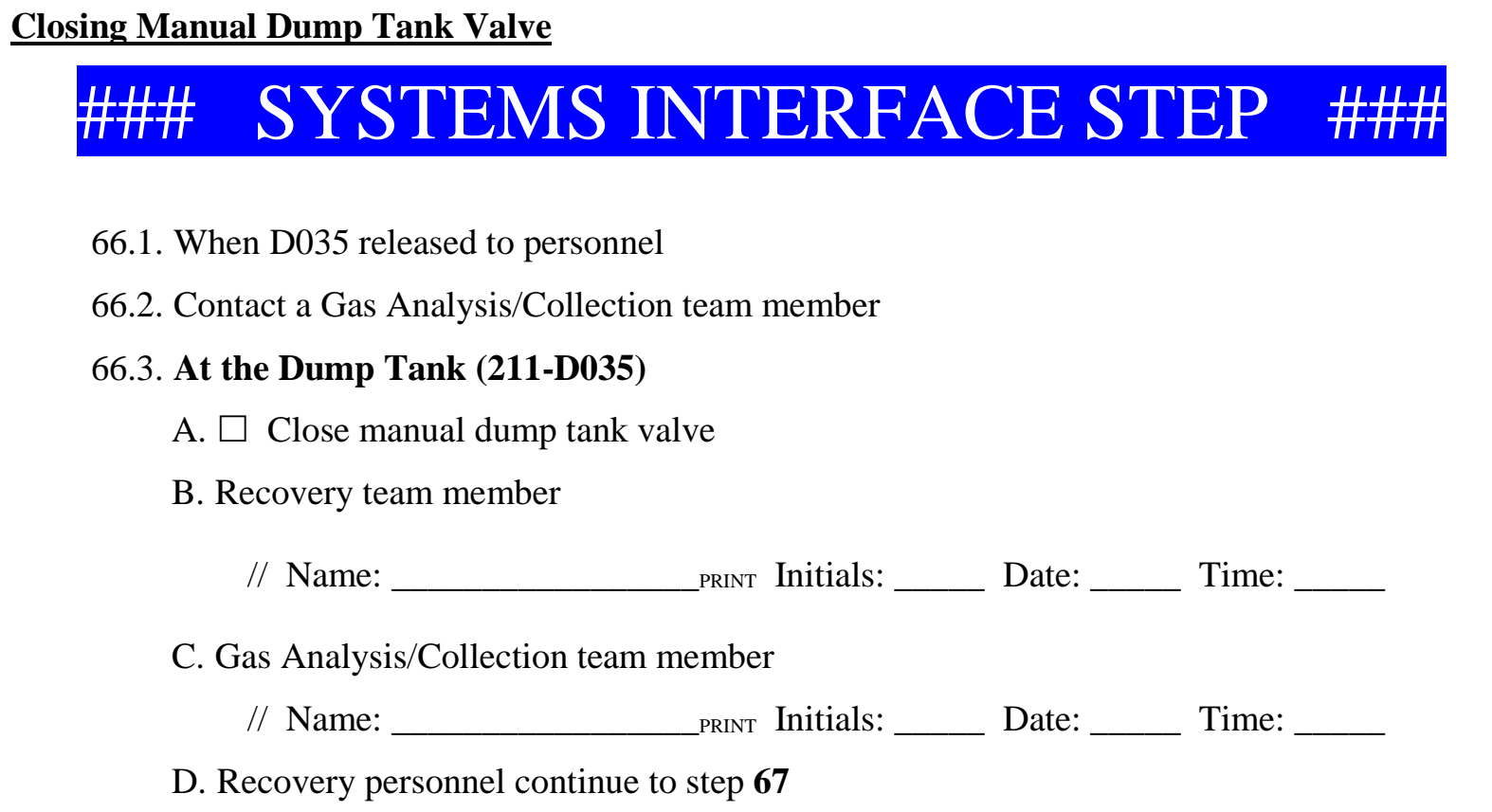 \\
\hline 67. & $\begin{array}{l}\text { End } \text { II Initials: } \_ \text {Date: } \_ \text {Time: } \\
\text { 67.1. } \square \text { Confirm ACID Pump controller powered OFF } \\
\text { 67.2. } \square \text { Confirm BASE Pump controller powered OFF } \\
\text { 67.3. Press \{NEXT STEP\} } \\
\text { 67.4. Verify Dial @ End }\end{array}$ \\
\hline 68. & $\begin{aligned} & \text { End of Run } \| \text { Initials: } \_ \text {Date: } \_ \text {Time: } \_- \\
& \text {68.1. } \square \text { Enter any final comments } \\
& \text { 68.2. } \square \text { Press the MASTER EXIT button at [System].tab } \\
& \text { A. Properly stops Mo99 Remote Recovery Data Acquisition \& Control Software }\end{aligned}$ \\
\hline
\end{tabular}




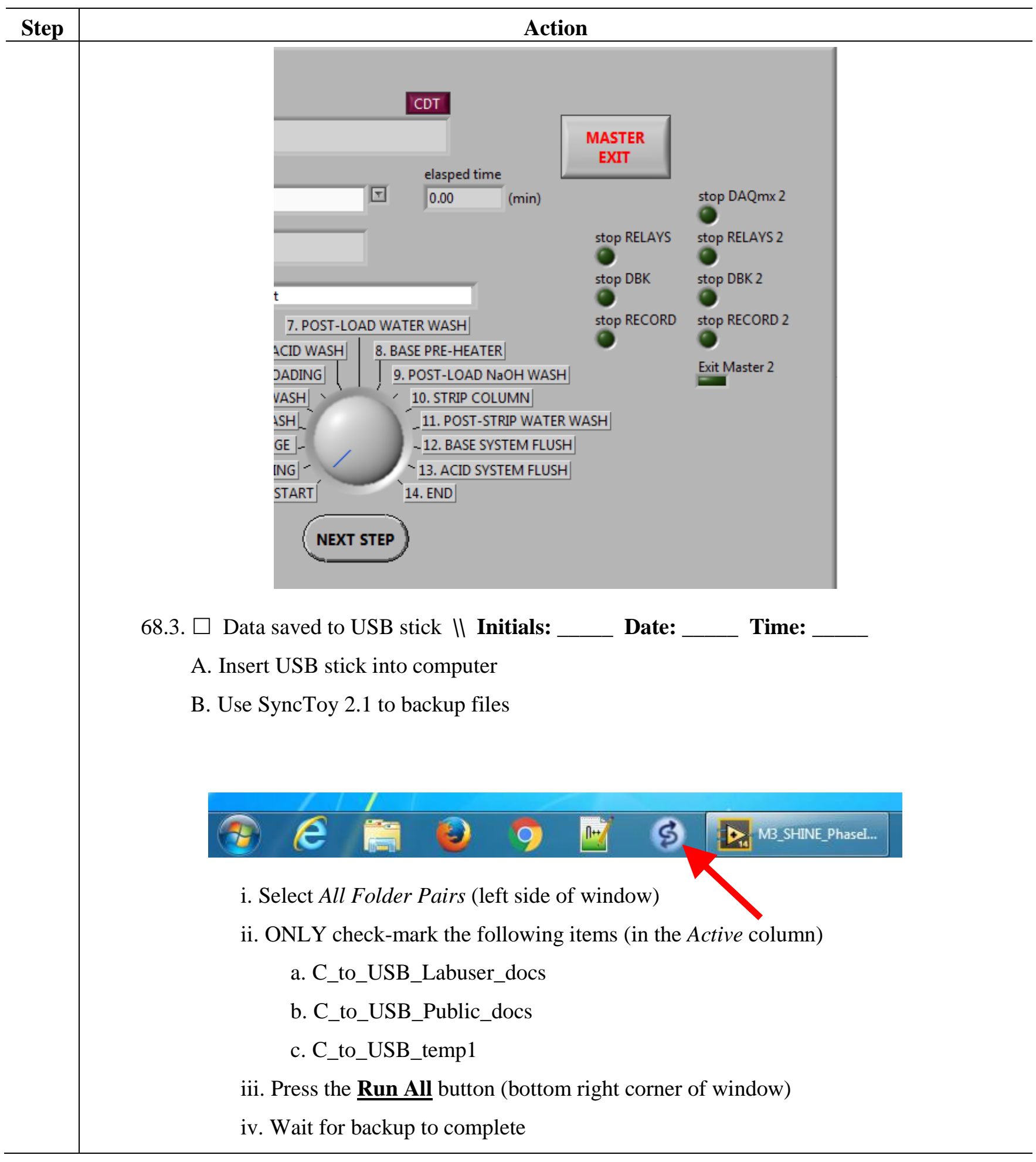




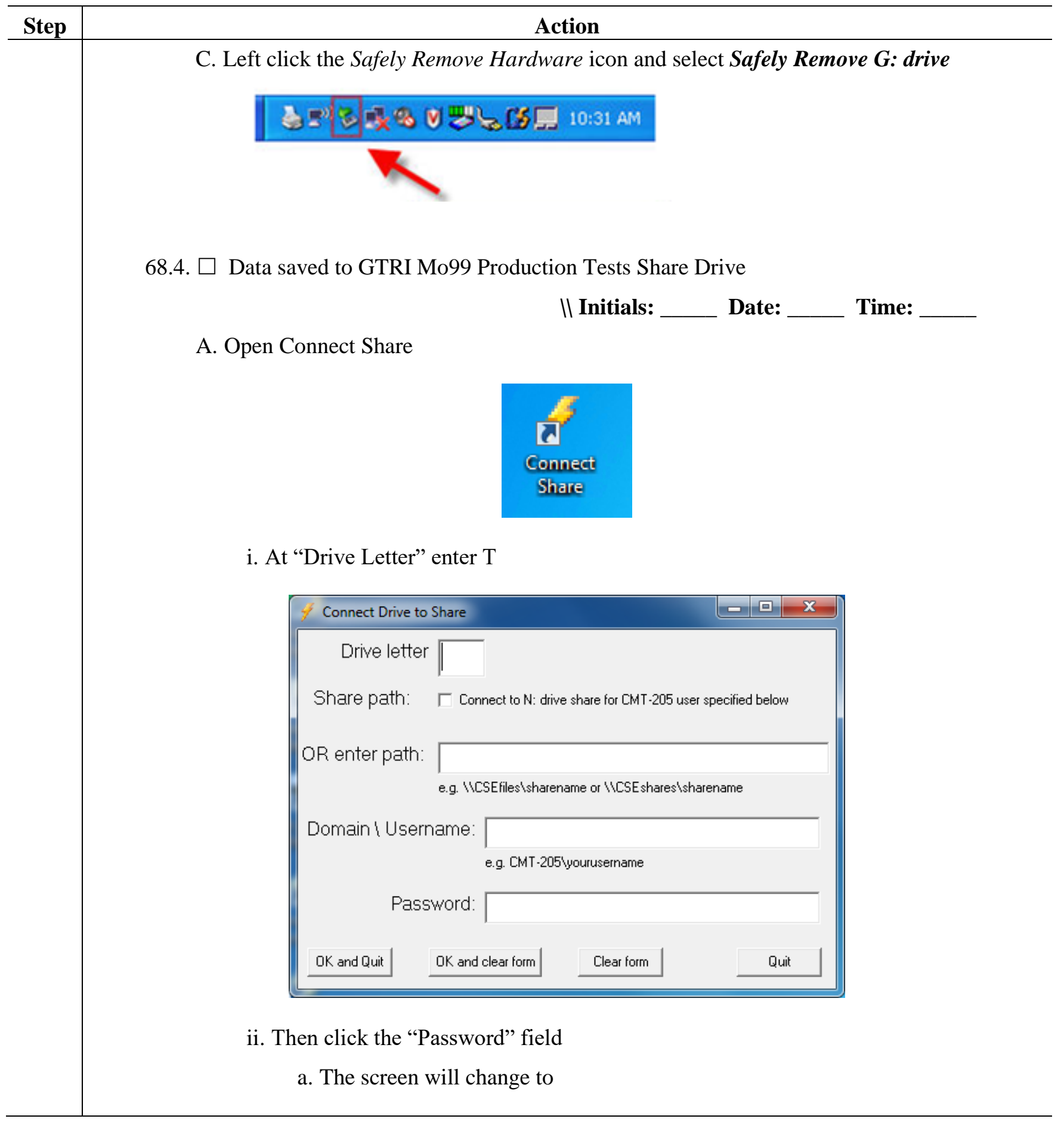




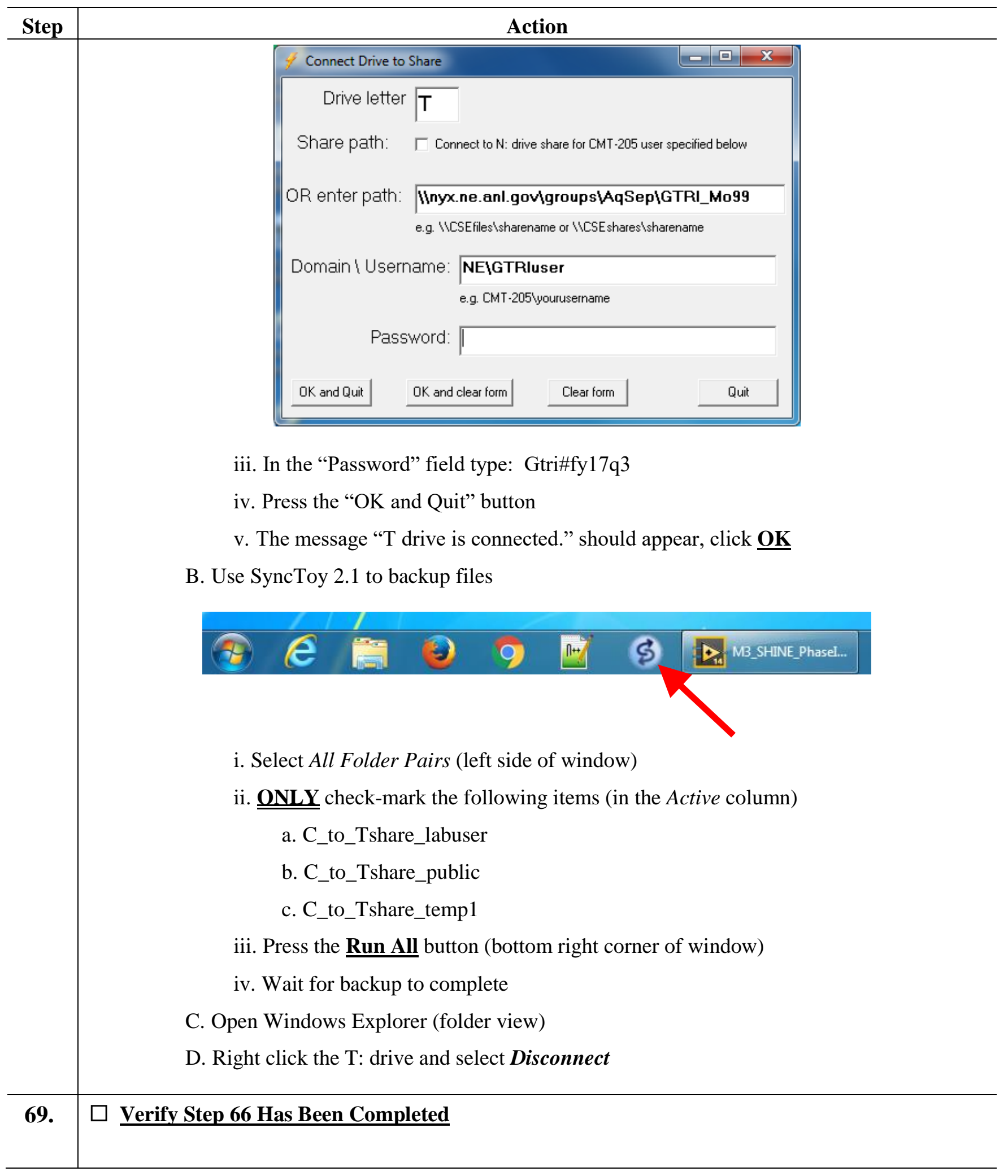




\subsection{5 $\quad{ }^{99}$ Mo Recovery Sample Retrieval}

\begin{tabular}{c|c}
\hline Step & Action \\
\hline 70. & Check Most Current RWP \\
& 70.1. $\square$ Sign most current RWP \\
& 70.2. $\square$ Check most current RWP for PPE requirements Obtain Production Feeds Analysis and \\
& Process Conditions Summary Sheet \\
\hline
\end{tabular}

71. It is Recommended that Two People Retrieve Samples

71.1. Person A collects the samples in the Mo Recovery glovebox

71.2. Person B actuates the solenoid valves via computer and double checks vial labels prior to retrieving each sample

\section{Prepare the Following Items}

72.1. Up to 24 labeled $10 \mathrm{~mL}$ pre-evacuated vials (number should be $\geq \#$ of samples taken). Part number can be found in Exhibit B
A. $\square$ Vials for Target Solution Mixing
B. $\square$ Vials for Column Loading
C. $\square$ Vials for Column Stripping
D. Labels should have the following information
i. RMS number
ii. Lab notebook number and page number(s)
iii. Date of irradiation
iv. Date samples retrieved
v. Process step identification
vi. Approximate solution composition
vii. Valve identification

72.2. $\square$ Twenty-four (24x) vial holders with covers
A. Tungsten holders

i. $\square$ Set of eight for Target Solution samples

B. 304 stainless steel

i. $\square$ Set of eight for Column Loading samples

ii. $\square$ Set of eight for Column Stripping samples 


\begin{tabular}{|c|c|}
\hline \multirow[t]{4}{*}{ Step } & Action \\
\hline & C. ALL HOLDERS MUST HAVE COVERS THAT STAY SEATED WITH THE \\
\hline & TWO PINS ON SHIELD BODY - IF THE PINS ARE BROKEN DO NOT USE \\
\hline & THAT SHIELD BODY \\
\hline \multirow[t]{2}{*}{73.} & Materials at the Ready \\
\hline & 73.1. Parts for the sampling retrieval assemblies can be found in Exhibit B \\
\hline \multirow[t]{21}{*}{74.} & Staging Collection Vials with Vial Shields \\
\hline & 74.1. The following operational checklist uses the Target Solution Sampling Assembly as an \\
\hline & example \\
\hline & 74.2. YOU WILL BE PASSING THROUGH THESE STEPS THREE TIMES \\
\hline & A. First pass (Target Solution samples) mark the black boxes $\square$ \\
\hline & B. Second pass (Column Loading samples) mark the red boxes $\square$ \\
\hline & C. Third pass (Column Stripping samples) mark the blue boxes $\square$ \\
\hline & 74.3. At the Mo Recovery Glovebox \\
\hline & A. Stage eight $(8 \mathrm{x})$ TUNGSTEN shielded vial holders (may not be possible to stage all 24 \\
\hline & vials at one time \\
\hline & i. Use 316 STAINLESS STEEL vial holders for Column Loading and Column \\
\hline & Stripping samples \\
\hline & 74.4. $\square \square \square$ Remove the cover from the vial shield body \\
\hline & 74.5. $\square \square \square$ Insert the individual appropriately numbered concentric needle assembly into \\
\hline & the appropriately numbered individual evacuated vial \\
\hline & 74.6. $\square \square \square$ Leave the vials in the vial holders when inserting the needles to minimize risk \\
\hline & of glove puncture \\
\hline & 74.7. $\square \square \square$ Set the 3-way Luer Lock valves into the following position \\
\hline & A. Top-row: all handles pointing to the right \\
\hline & i. Check valves are only on the top row \\
\hline & B. Bottom row: all handles pointing to the right \\
\hline
\end{tabular}




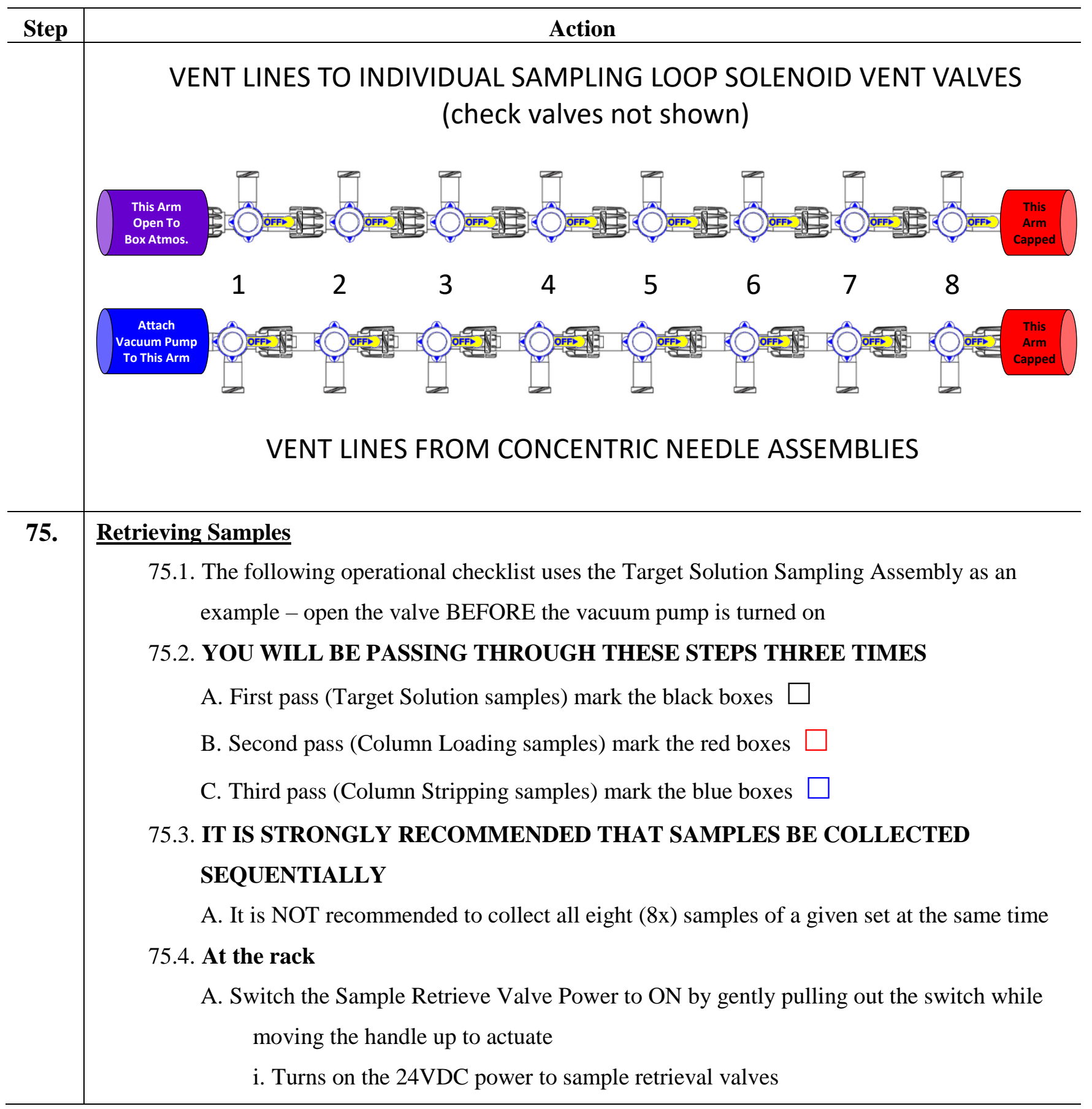




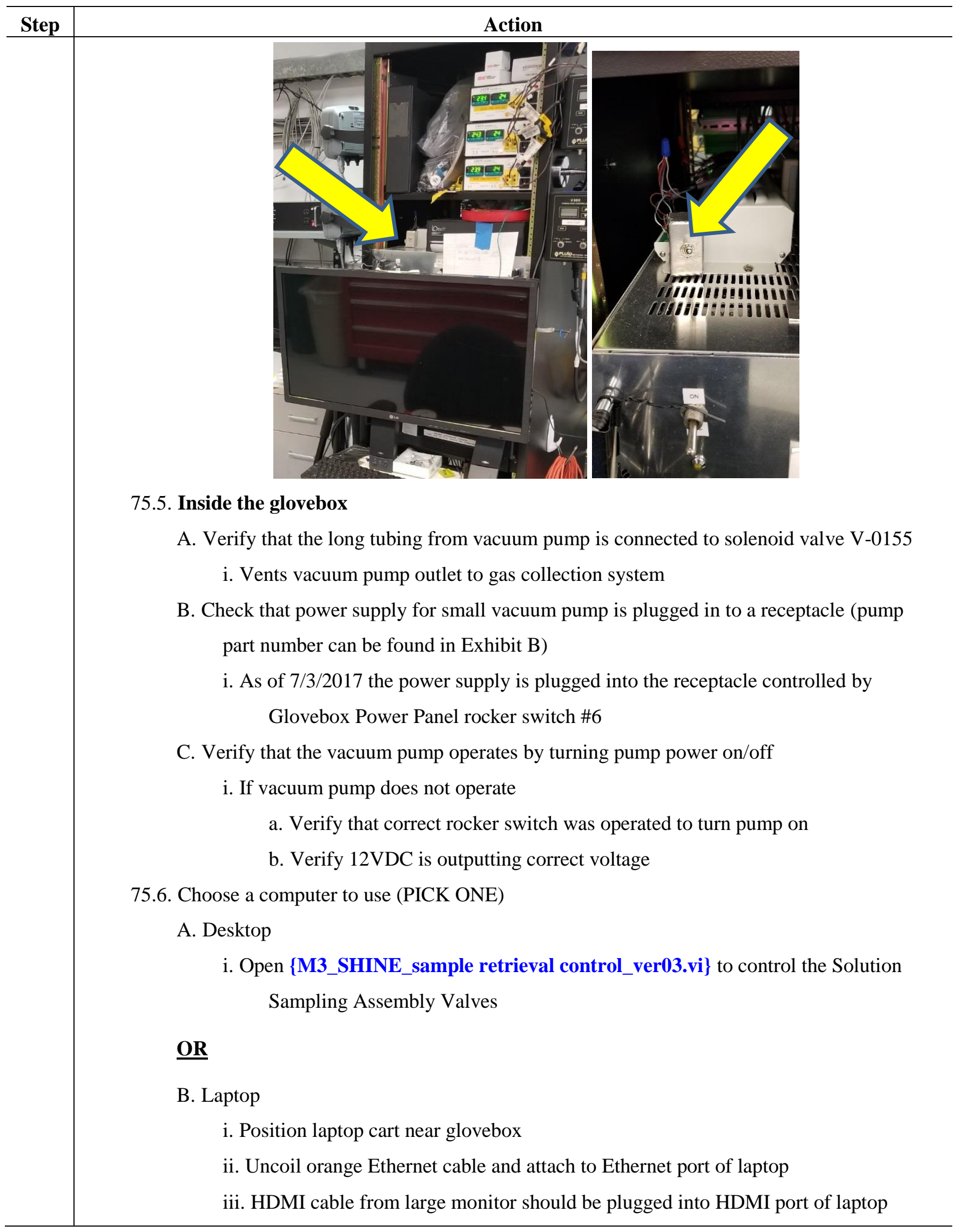




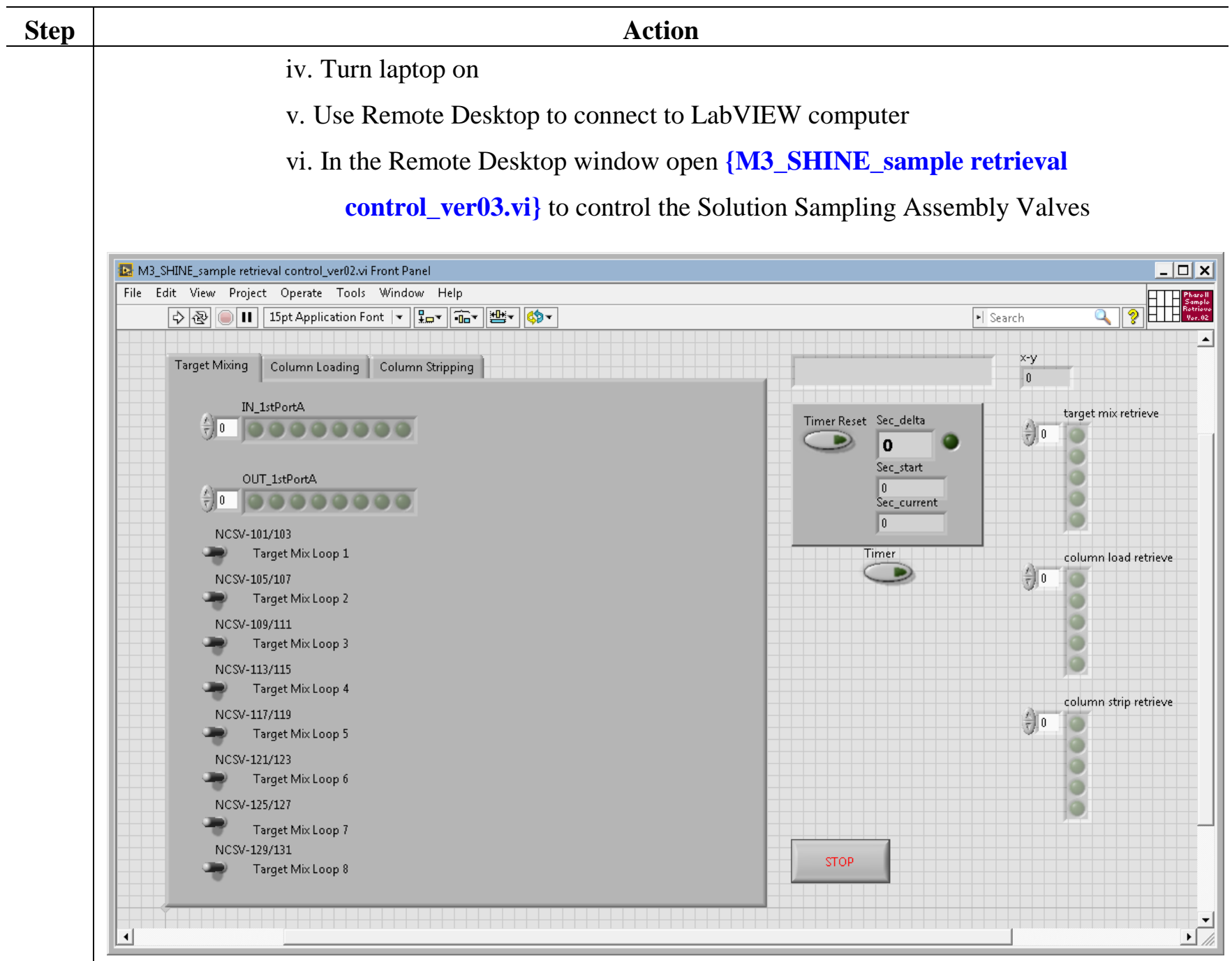

75.7. Press the LabVIEW play button to activate the program

75.8. Select the [Target Mixing] tab

A. Choose the appropriate tab for the samples to be retrieved

75.9. $\square \square \square$ Attach vacuum tube inlet to left side, bottom row of 3-way Luer valve manifold to be collected

75.10. DOSE HAZARD!! - KEEP HANDS AWAY FROM 1/16 in. LIQUID LINE WHILE TRANSFFERING LIQUID

75.11. $\square \square \square$ Open Loop 1 using LabVIEW program BEFORE turning on vacuum pump to ensure liquid can be retrieved

A. This opens the liquid and vent valves associated with a given loop and valve V-0155 to gas collection system

75.12. Watch for liquid in $1 / 16$ in. line while switching vacuum pump power switch \#6 75.13. When liquid appears to have been transferred turn off rocker switch \#6 


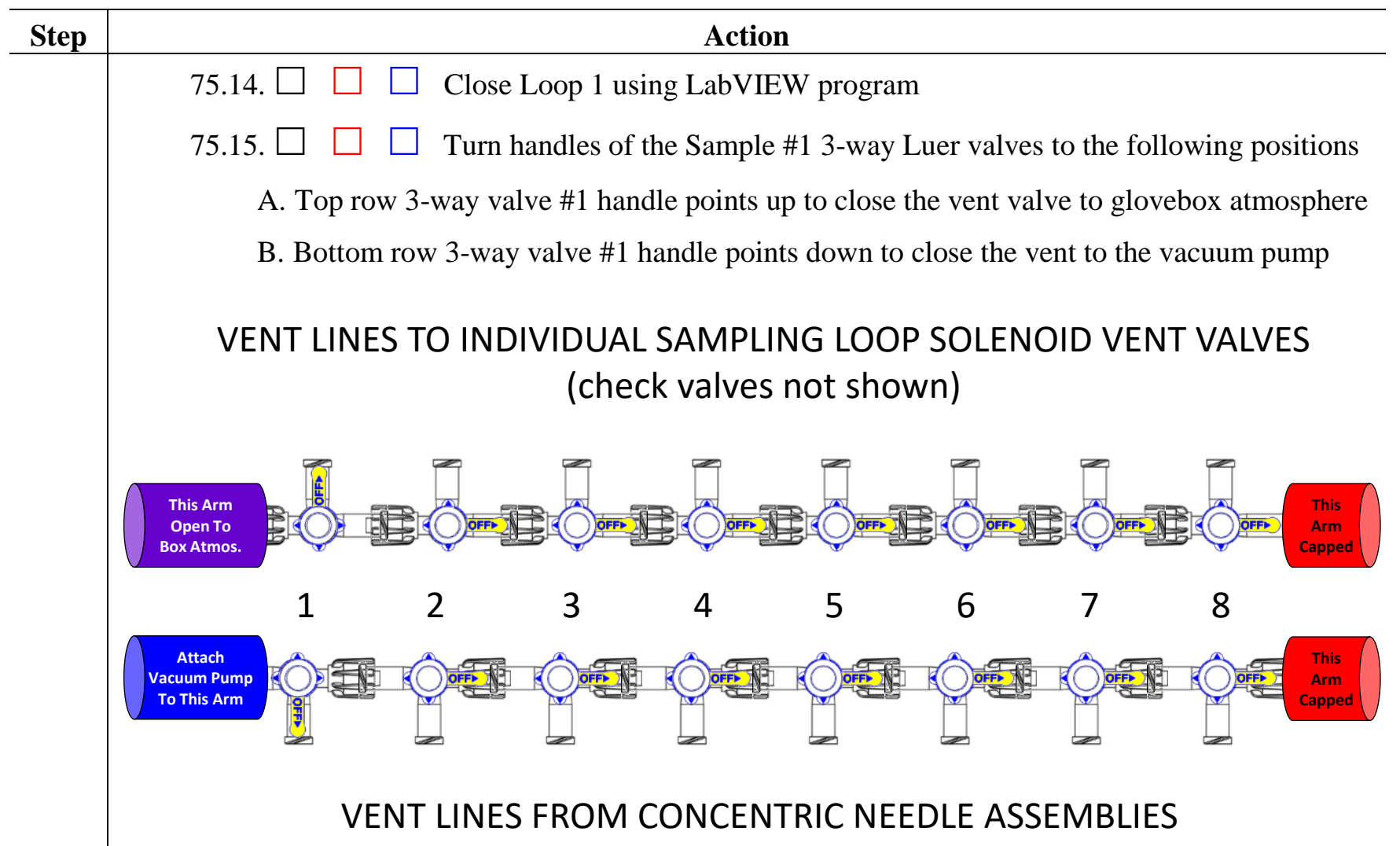

75.16. Repeat steps 75.11 - 75.15.B for each of the loops

A. After collecting Loops 1-7 but before collecting Loop 8 the manifold should look like the following

\section{VENT LINES TO INDIVIDUAL SAMPLING LOOP SOLENOID VENT VALVES (check valves not shown)}
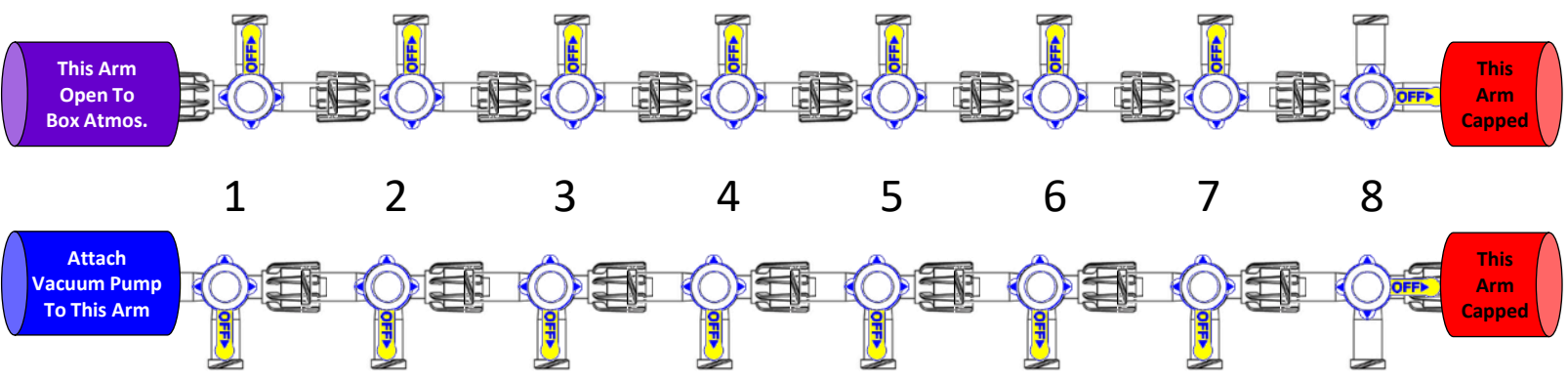

\section{VENT LINES FROM CONCENTRIC NEEDLE ASSEMBLIES}

75.17. After collecting the last sample the 3-way Luer valves for each of the loops collected will be pointing up for the top row and down for the bottom row 


\begin{tabular}{|c|c|}
\hline Step & Action \\
\hline & A. Verify the vacuum pump is turned off at switch \#6 \\
\hline & B. Verify all loops are closed in the LabVIEW program \\
\hline & 75.18. DOSE HAZARD!! — DO NOT PLACE YOUR HAND ON TOP OF ANY OF THE \\
\hline & LOADED VIALS \\
\hline & 75.19. Using long tweezers/forceps to hold vial in vial shield and pull concentric needle assembly \\
\hline & from vial \\
\hline & 75.20. Insert free concentric needle assembly into storage vessel \\
\hline & 75.21. Place vial shield cover on vial shield body \\
\hline & 75.22. Repeat steps $\mathbf{7 5 . 1 8}-\mathbf{7 5 . 2 1}$ for each collected sample \\
\hline & 75.23. Retrieve the sample from the flow meter bypass sample loop \\
\hline & A. Ensure the evacuated sample collection vial and needle setup (Appendix B item 11, but \\
\hline & with $1 / 8$ " lines) is attached to the BOTTOM manual valve \\
\hline & B. Open the bottom manual valve to the sample collection bottle \\
\hline & C. Turn on the small vacuum pump using Glovebox Power Panel rocker \#6 \\
\hline & D. Slowly open the top valve to allow solution to flow out into the vial \\
\hline & E. When the sample has been collected, close BOTH manual valves and turn off the small \\
\hline & vacuum pump \\
\hline & 75.24. DOSE HAZARD!! — ALL SAMPLES SHOULD BE COVERED BEFORE \\
\hline & COLLECTING ANY OTHER SAMPLES OR DOING ANY OTHER WORK TO \\
\hline & MINIMIZE EXPOSURE \\
\hline \multirow[t]{4}{*}{76.} & If Collecting Samples from other Sampling Assemblies \\
\hline & 76.1. Move the vacuum pump inlet tubing to the appropriate left, bottom row 3 -way Luer valve \\
\hline & manifold (see step 75.9) \\
\hline & 76.2. Repeat steps $\mathbf{7 5 . 1 0}-\mathbf{7 5 . 2 4}$. \\
\hline \multirow[t]{2}{*}{77.} & $\underline{\text { Effluent Cart Sample Collection }}$ \\
\hline & $\begin{array}{l}\text { 77.1. Follow the instructions outlined in WCD-EZ "Sampling AMORE Effluent Bottles Using a } \\
\text { Syringe" appended to WCD 56833.1 } \\
\text { 77.2. Proceed to the following step. }\end{array}$ \\
\hline \multirow[t]{2}{*}{78.} & When All Samples Have Been Collected \\
\hline & 78.1. Press [STOP] button in \{M3_SHINE_sample retrieval control_ver03.vi\} \\
\hline
\end{tabular}




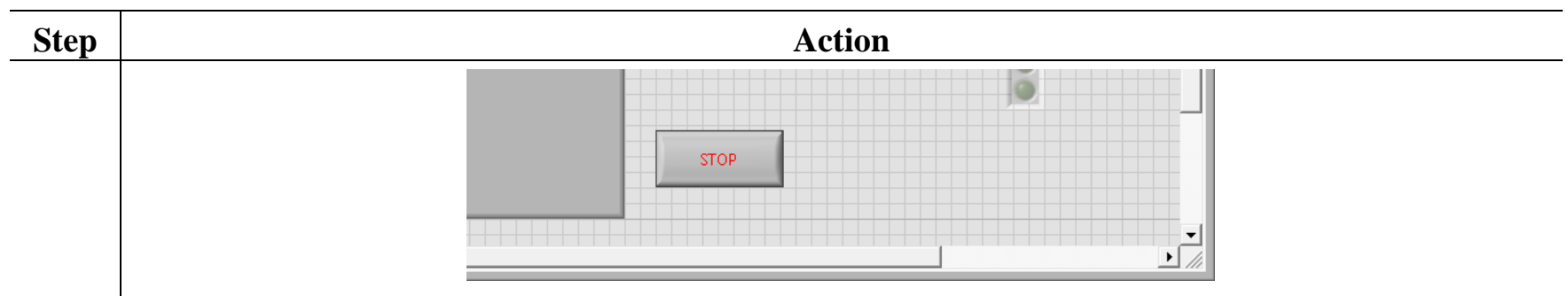

78.2. Close the $\{$ M3_SHINE_sample retrieval control_ver03.vi\} program

\subsection{Close LabVIEW}

78.4. If using the laptop
A. Close the Remote Desktop connection
B. Disconnect the orange Ethernet cable, neatly coil it up and hang it on the lower right side of the main LabVIEW rack
C. Turn off the laptop and roll it back into the Instrument Room (D027) 


\subsubsection{Washout of ${ }^{99} \mathrm{Mo}$ Recovery System and Sample Retrieval Subsystems}

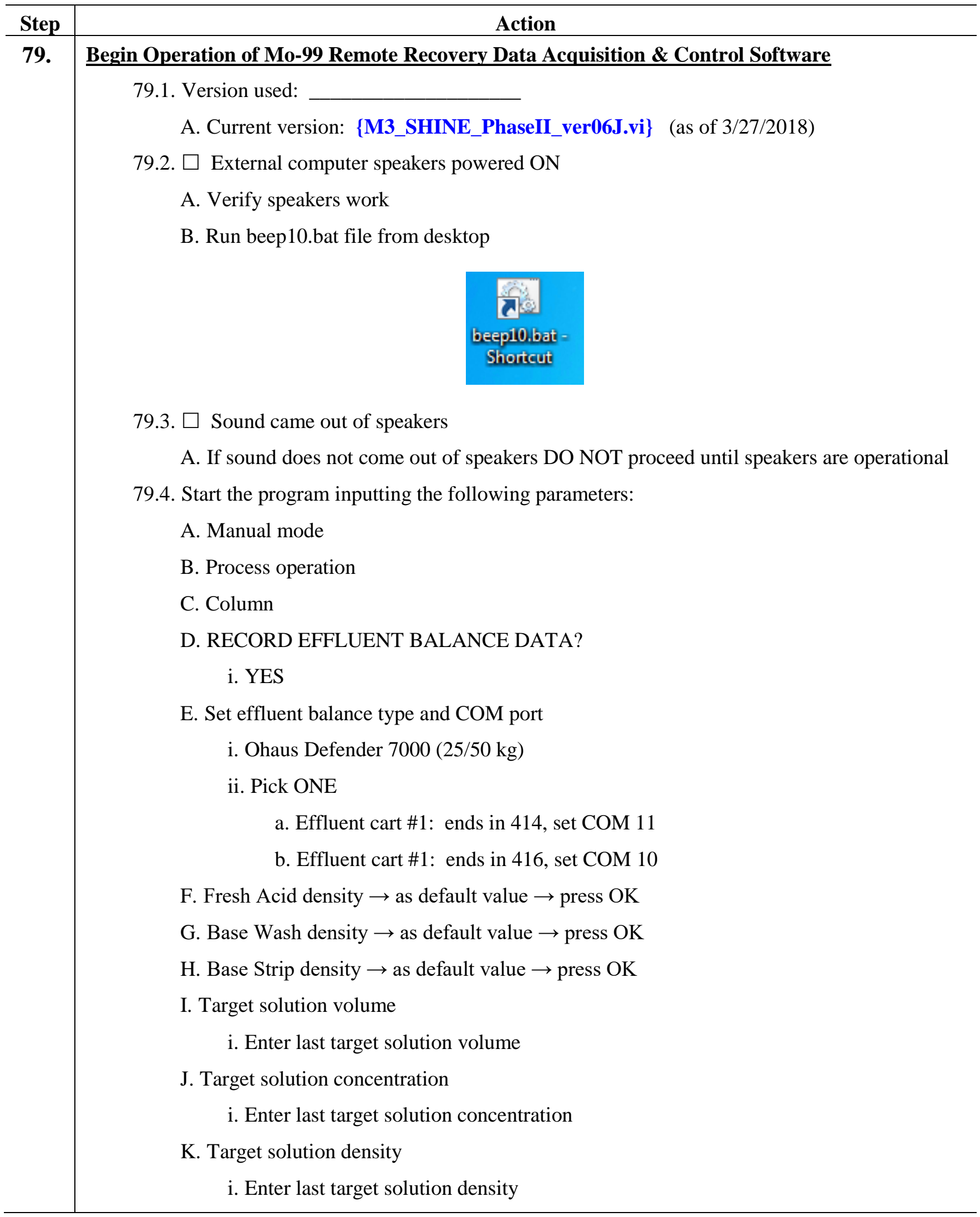




\begin{tabular}{|c|c|}
\hline Step & Action \\
\hline & L. Column effluent path \\
\hline & i. To Transfer Cask \\
\hline & M. Pre-Load Acid Wash processing volume $\rightarrow$ as default value $\rightarrow$ press OK \\
\hline & N. Column Loading processing volume $\rightarrow$ as default value $\rightarrow$ press OK \\
\hline & O. Post-Load Acid Wash processing volume $\rightarrow$ as default value $\rightarrow$ press OK \\
\hline & P. Post-Load Water Wash processing volume $\rightarrow$ as default value $\rightarrow$ press OK \\
\hline & Q. Use the Post-Load NaOH Wash step? $\rightarrow$ NO \\
\hline & R. Column stripping processing volume $\rightarrow$ as default value $\rightarrow$ press $\mathrm{OK}$ \\
\hline & S. Post-Strip Water Wash To Strip product processing volume $\rightarrow$ as default value $\rightarrow$ press \\
\hline & $\mathrm{OK}$ \\
\hline & T. Post-Strip Water Wash To Waste processing volume $\rightarrow$ as default value $\rightarrow$ press OK \\
\hline & U. Final Base System Water Wash processing volume $\rightarrow$ as default value $\rightarrow$ press OK \\
\hline & V. Final Acid System Acid Wash processing volume $\rightarrow$ as default value $\rightarrow$ press OK \\
\hline & W. Record LINAC temperatures? $\rightarrow$ YES \\
\hline & X. Filename prefix: ___ \\
\hline & Y. $\square$ ACID Pump controller powered ON \\
\hline & i. Rocker switch under front/left of ACID Pump V300 controller \\
\hline & Z. $\square$ ACID Pump to $\underline{\text { STOP }}$ \\
\hline & i. Display alternates between OFF and \#\#.\# (current setting) \\
\hline & AA. $\square$ Verify feed and effluent balances are reading at [Sensors].tab \\
\hline & i. Compare LabVIEW value to value on feed balance indicator \\
\hline & a. Indicator: _ grams \\
\hline & b. LabVIEW: ___ grams \\
\hline & ii. Compare LabVIEW value to value on effluent balance indicator \\
\hline & a. Indicator: _ grams \\
\hline & b. LabVIEW: ___ grams \\
\hline & BB. DO NOT PROCEED IF FEED OR EFFLUENT BALANCES ARE NOT BEING \\
\hline & READ \\
\hline \multirow[t]{5}{*}{80.} & Acid System Rinse Out With Acid Solution \\
\hline & 80.1. At feed bottle cabinet \\
\hline & A. $\square$ Verify $>3000 \mathrm{~mL}$ of $\mathrm{pH} 1 \mathrm{H}_{2} \mathrm{SO}_{4}$ is in the Fresh Acid feed bottle $\mathbf{A N D} \geq 3000 \mathrm{~mL}$ \\
\hline & free volume in Acid Rinse effluent bottle (use table on page 88) \\
\hline & i. If sufficient volume, continue to step $\mathbf{8 0 . 2}$, otherwise proceed directly to step $\mathbf{8 1}$ \\
\hline
\end{tabular}




\begin{tabular}{|c|c|}
\hline Step & Action \\
\hline & 80.2. At LabVIEW computer \\
\hline & - YOU ARE OPERATING THE SYSTEM IN MANUAL MODE - \\
\hline & A. $\square$ Open V-0003 (Fresh Acid) \\
\hline & B. $\square$ Verify flow path through acid flow meter $163 / 164$ (valves should already be open) \\
\hline & C. $\square$ Open V-0009 (Target Mixing path) \\
\hline & D. $\square$ Open Target Mixing loop 1 on [Sample Collection].tab $\rightarrow$ [Target Mixing].tab by \\
\hline & pressing the purple $100 / 102$ button \\
\hline & E. $\square$ Open V-0153/0154 (Target Mixing path to Dump Tank path) \\
\hline & F. $\square$ Open V-0172/0173 (Target Mixing path to Acid Rinse bottle) \\
\hline & i. Selecting this valve closes both $147 / 148$ (frit to Dump Tank) and 149/150 (bypass to \\
\hline & Dump Tank) \\
\hline & G. $\square$ Open V-0156 (Effluent bottle vent) \\
\hline & i. V-0156 MUST BE OPEN DURING OPERATIONS - WHEN OPERATIONS \\
\hline & ARE NOT BEING CONDUCTED CLOSE THE VALVE \\
\hline & H. $\square$ Enter flow rate $\underline{\mathbf{1 0 0}} \mathrm{mL} / \mathrm{min}$ Acid Flow Rate Set Pt @ [System].tab \\
\hline & I. $\square$ Record calculated____ \% Acid Motor Power@ [System].tab \\
\hline & J. $\square$ Verify/Adjust ACID Pump Controller to \% Acid Motor Power for desired flow rate \\
\hline & i. If a lower $\%$ motor power value is required due to pressure readings adjust $A$ cid \\
\hline & Flow Rate Set Pt until calculated \% Acid Motor Power matches \% motor power \\
\hline & reading at controller \\
\hline & K. $\square$ Prepare 3 minute timer \\
\hline & L. $\square$ ACID Pump to $\underline{\text { RUN }}$ and start timer \\
\hline & M. At 3 minute timer end ACID Pump to $\underline{\text { STOP }}$ \\
\hline & N. $\square$ Open Target Mixing loop 2 on [Sample Collection].tab $\rightarrow$ [Target Mixing].tab by \\
\hline & pressing the purple $104 / 106$ button \\
\hline & i. The previous loop valves will automatically close when another loop is opened \\
\hline & O. $\square$ Prepare 1 minute 15 second timer ( 75 seconds total) \\
\hline & P. $\square$ ACID Pump to $\underline{\mathbf{R U N}}$ and start timer \\
\hline & Q. At 75 second timer end ACID Pump to $\underline{\text { STOP }}$ \\
\hline & R. Repeat steps 80.2.N - 80.2.Q for each Target Mixing loop, actuating the loop valves \\
\hline & using the following purple buttons on [Sample Collection].tab $\rightarrow$ [Target \\
\hline & Mixing].tab \\
\hline & i. $\square$ Loop 3: $108 / 110$ \\
\hline
\end{tabular}




\begin{tabular}{|c|c|}
\hline Step & Action \\
\hline & ii. $\square$ Loop 4: 112/114 \\
\hline & iii. $\square$ Loop 5: 118/118 \\
\hline & iv. $\square$ Loop 6: 120/122 \\
\hline & v. $\square$ Loop 7: 124/126 \\
\hline & vi. $\square$ Loop 8: 128/130 \\
\hline & vii. $\square$ Loop 9: 132/133 \\
\hline & S. $\square$ Verify ACID Pump to $\underline{\text { STOP }}$ \\
\hline & T. $\square$ Close V-0009 (Target Mixing path) \\
\hline & U. $\square$ Close V-0153/0154 (Target Mixing path to Dump Tank path) \\
\hline & V. $\square$ Close V-0172/0173 (Target Mixing path to Acid Rinse bottle) \\
\hline & W. $\square$ Verify V-0003 open (Fresh acid) \\
\hline & X. $\square$ Verify flow path through acid flow meter $163 / 164$ (valves should already be open) \\
\hline & Y. $\square$ Open V-0010 (Column Loading path) \\
\hline & $\begin{array}{l}\text { Z. } \square \text { Open Column Loading loop } 1 \text { on [Sample Collection].tab } \rightarrow \text { [Column Loading].tab } \\
\text { by pressing the purple 32/34 button }\end{array}$ \\
\hline & AA. $\square$ Open V-0014/0015 (Acid column bypass) using toggle switch at lower left corner \\
\hline & of [System].tab (using column bypass in case column still attached to system) \\
\hline & $\begin{array}{l}\text { BB. } \square \text { Verify flow path through acid column loading filter } 18 / 19 \text { (valves should already be } \\
\text { open) }\end{array}$ \\
\hline & CC. $\square$ Open V-0139 (Acid rinse) \\
\hline & DD. $\square$ Verify V-0156 (Effluent bottle vent) \\
\hline & EE. $\square$ Enter flow rate $\underline{\mathbf{1 0 0}} \mathrm{mL} / \mathrm{min}$ Acid Flow Rate Set Pt @ [System].tab \\
\hline & FF. $\square$ Record calculated ___ \% Acid Motor Power @ [System].tab \\
\hline & GG. $\square$ Verify/Adjust ACID Pump Controller to \% Acid Motor Power for desired flow rate \\
\hline & i. If a lower $\%$ motor power value is required due to pressure readings adjust Acid \\
\hline & Flow Rate Set Pt until calculated \% Acid Motor Power matches \% motor power \\
\hline & reading at controller \\
\hline & HH. $\square$ Prepare 6 minute timer \\
\hline & II. $\square$ ACID Pump to $\underline{\mathbf{R U N}}$ and start timer \\
\hline & JJ. At 6 minute timer end ACID Pump to $\underline{\text { STOP }}$ \\
\hline & KK. $\square$ Open Column Loading loop 2 on [Sample Collection].tab $\rightarrow$ [Column \\
\hline & Loading].tab by pressing the purple $36 / 38$ button \\
\hline & i. The previous loop valves will automatically close when another loop is opened \\
\hline
\end{tabular}




\begin{tabular}{|c|c|}
\hline Step & Action \\
\hline & $\begin{array}{l}\text { LL. } \square \text { Prepare } 75 \text { second timer (1 min: } 15 \mathrm{sec} \text { ) } \\
\text { MM. } \square \text { ACID Pump to } \underline{\mathbf{R U N}} \text { and start timer } \\
\text { NN. At } 75 \text { second timer end ACID Pump to } \underline{\text { STOP }} \\
\text { OO. Repeat steps 80.2.KK - 80.2.NN for each Column Loading loop, actuating the loop } \\
\quad \text { valves using the following purple buttons on [Sample Collection].tab } \rightarrow \text { [Column } \\
\quad \text { Loading].tab } \\
\quad \text { i. } \square \text { Loop 3: } 40 / 42 \\
\quad \text { ii. } \square \text { Loop 4: } 44 / 46 \\
\quad \text { iii. } \square \text { Loop 5: } 48 / 50 \\
\quad \text { iv. } \square \text { Loop 6: } 52 / 54 \\
\quad \text { v. } \square \text { Loop 7: } 56 / 58 \\
\quad \text { vi. } \square \text { Loop 8: 60/62 } \\
\quad \text { vii. } \square \text { Loop 9: 64/65 } \\
\text { PP. } \square \text { Verify ACID Pump to STOP } \\
\text { QQ. } \square \text { Close V-0003 (Fresh acid) } \\
\text { RR. } \square \text { Close V-0010 (Column Loading path) } \\
\text { SS. } \square \text { Close V-0139 (Acid rinse) } \\
\text { TT. } \square \text { Close V-0156 (Effluent bottle vent) } \\
\text { UU. } \square \text { ACID Pump controller powered OFF using rocker switch under front/left of ACID } \\
\quad \text { Pump V300 controller } \\
\text { VV. Proceed to step 81 }\end{array}$ \\
\hline 81. & $\begin{array}{l}\text { Acid System Purge with } 5 \text { psig } \mathbf{N}_{2} \\
\text { 81.1. Outside of recovery glovebox } \\
\text { THE 2-WAY BALL VALVE (V-2038) FOR NITROGEN SERVICE IS TO REMAIN } \\
\text { ATTACHED TO THE GLOVEBOX - DO NOT REMOVE THIS VALVE } \\
\text { A. Verify V-2038 for nitrogen service is closed } \\
\text { B. Attach nitrogen cylinder to V-2038 on right side of recovery glovebox, above white } \\
\quad \text { transfer cask glovebox } \\
\text { C. Set regulator to } 5 \text { psig } \\
\text { D. DO NOT OPEN V-2038 FOR NITROGEN SERVICE } \\
\text { 81.2. Inside of recovery glovebox }\end{array}$ \\
\hline
\end{tabular}




\begin{tabular}{|c|c|}
\hline Step & Action \\
\hline & $\begin{array}{l}\text { A. Verify acid injection port V-2001 closed (valve is after acid pump and before acid flow } \\
\text { meter) }\end{array}$ \\
\hline & B. If present remove needle port guide from acid injection port valve V-2001 \\
\hline & i. Needle port guide is three pieces \\
\hline & a. Needle port guide nut, Septum, White $1 / 4$ in. Teflon one-piece ferrule \\
\hline & C. Verify check valve V-0406 attached to end of $1 / 4$ in. FEP tubing line from V-2038 \\
\hline & i. Prevents potential of glovebox atmosphere exiting V-2038 \\
\hline & D. Attach existing $1 / 4$ in. FEP tubing line to acid injection port valve $V-2001$ via check valve \\
\hline & V-0406 \\
\hline & i. FEP tubing is attached to a stainless steel line that passes across glovebox wall and \\
\hline & ends near the solenoid vent valves manifold \\
\hline & 81.3. Outside of recovery glovebox \\
\hline & A. Open V-2038 for nitrogen service \\
\hline & 81.4. At LabVIEW computer AND inside recovery glovebox \\
\hline & A. $\square$ Verify flow path through acid flow meter $163 / 164$ (valves are already open) \\
\hline & B. $\square$ Open V-0009 (Target Mixing path) \\
\hline & C. $\square$ Open Target Mixing loop 1 on [Sample Collection].tab $\rightarrow$ [Target Mixing].tab by \\
\hline & pressing the purple $100 / 102$ button \\
\hline & D. $\square$ Open V-0153/0154 (Target Mixing path to Dump Tank path) \\
\hline & E. $\square$ Open V-0172/0173 (Target Mixing path to Acid Rinse bottle) \\
\hline & i. Selecting this valve closes both $147 / 148$ (frit to Dump Tank) and 149/150 (bypass to \\
\hline & Dump Tank) \\
\hline & F. $\square$ Open V-0156 (Effluent bottle vent) \\
\hline & i. V-0156 MUST BE OPEN DURING OPERATIONS - WHEN OPERATIONS \\
\hline & ARE NOT BEING CONDUCTED CLOSE THE VALVE \\
\hline & G. Prepare 3 minute timer for loop 1 \\
\hline & H. Open acid injection port valve V-2001 and start timer \\
\hline & I. At 3 minute timer end close injection port valve V-2001 \\
\hline & J. $\square$ Open Target Mixing loop 2 on [Sample Collection].tab $\rightarrow$ [Target Mixing].tab by \\
\hline & pressing the purple $104 / 106$ button \\
\hline & i. The previous loop valves will automatically close when another loop is opened \\
\hline & K. Prepare 1 minute timer \\
\hline & L. Open acid injection port valve V-2001 and start timer \\
\hline
\end{tabular}




\begin{tabular}{|c|c|}
\hline Step & Action \\
\hline & M. At 1 minute timer timer end close injection port valve V-2001 \\
\hline & N. Repeat steps 81.4.J - 81.4.M for each Target Mixing loop, actuating the loop valves \\
\hline & using the following purple buttons on [Sample Collection].tab $\rightarrow$ [Target \\
\hline & Mixing].tab \\
\hline & i. $\square$ Loop 3: $108 / 110$ \\
\hline & ii. $\square$ Loop 4: $112 / 114$ \\
\hline & iii. $\square$ Loop 5: 118/118 \\
\hline & iv. $\square$ Loop 6: 120/122 \\
\hline & v. $\square$ Loop 7: 124/126 \\
\hline & vi. $\square$ Loop 8: $128 / 130$ \\
\hline & vii. $\square$ Loop 9: 132/133 \\
\hline & O. $\square$ Verify injection port valve V-2001 is closed \\
\hline & P. $\square$ Close V-0009 (Target Mixing path) \\
\hline & Q. $\square$ Close V-0153/0154 (Target Mixing path to Dump Tank path) \\
\hline & R. $\square$ Close V-0172/0173 (Target Mixing path to Acid Rinse bottle) \\
\hline & S. $\square$ Verify flow path through acid flow meter $163 / 164$ (valves already open) \\
\hline & T. $\square$ Open V-0010 (Column Loading path) \\
\hline & $\begin{array}{c}\text { U. } \square \text { Open Column Loading loop } 1 \text { on [Sample Collection].tab } \rightarrow \text { [Column Loading].tab } \\
\text { by pressing the purple 32/34 }\end{array}$ \\
\hline & V. $\square$ Open V-0014/0015 (Acid column bypass) using toggle switch at lower left corner of \\
\hline & [System].tab (using column bypass in case column still attached to system) \\
\hline & W. $\square$ Verify flow path through acid column loading filter 18/19 (valves already open) \\
\hline & X. $\square$ Open V-0139 (Acid rinse) \\
\hline & Y. $\square$ Verify V-0156 (Effluent bottle vent) \\
\hline & Z. Prepare 3 minute timer for loop 1 \\
\hline & AA. Open acid injection port valve V-2001 and start timer \\
\hline & BB. At 3 minute timer end close injection port valve $\mathrm{V}-2001$ \\
\hline & CC. $\square$ Open Column Loading loop 2 on [Sample Collection].tab $\rightarrow$ [Column \\
\hline & Loading].tab by pressing the purple $36 / 38$ button \\
\hline & i. The previous loop valves will automatically close when another loop is opened \\
\hline & DD. Prepare 1 minute timer timer \\
\hline & EE. Open acid injection port valve V-2001 and start timer \\
\hline & FF. At 1 minute timer timer end close injection port valve V-2001 \\
\hline
\end{tabular}




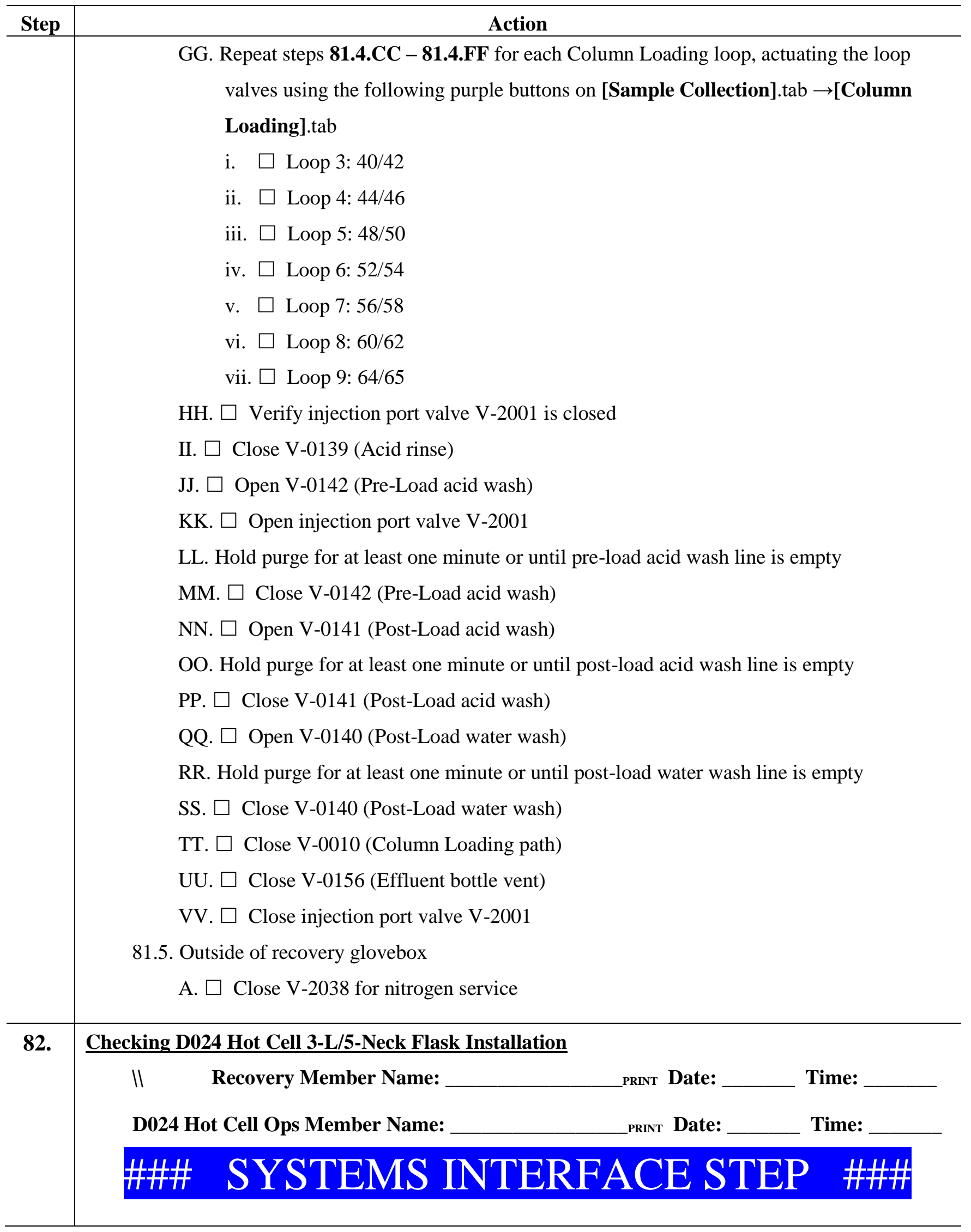




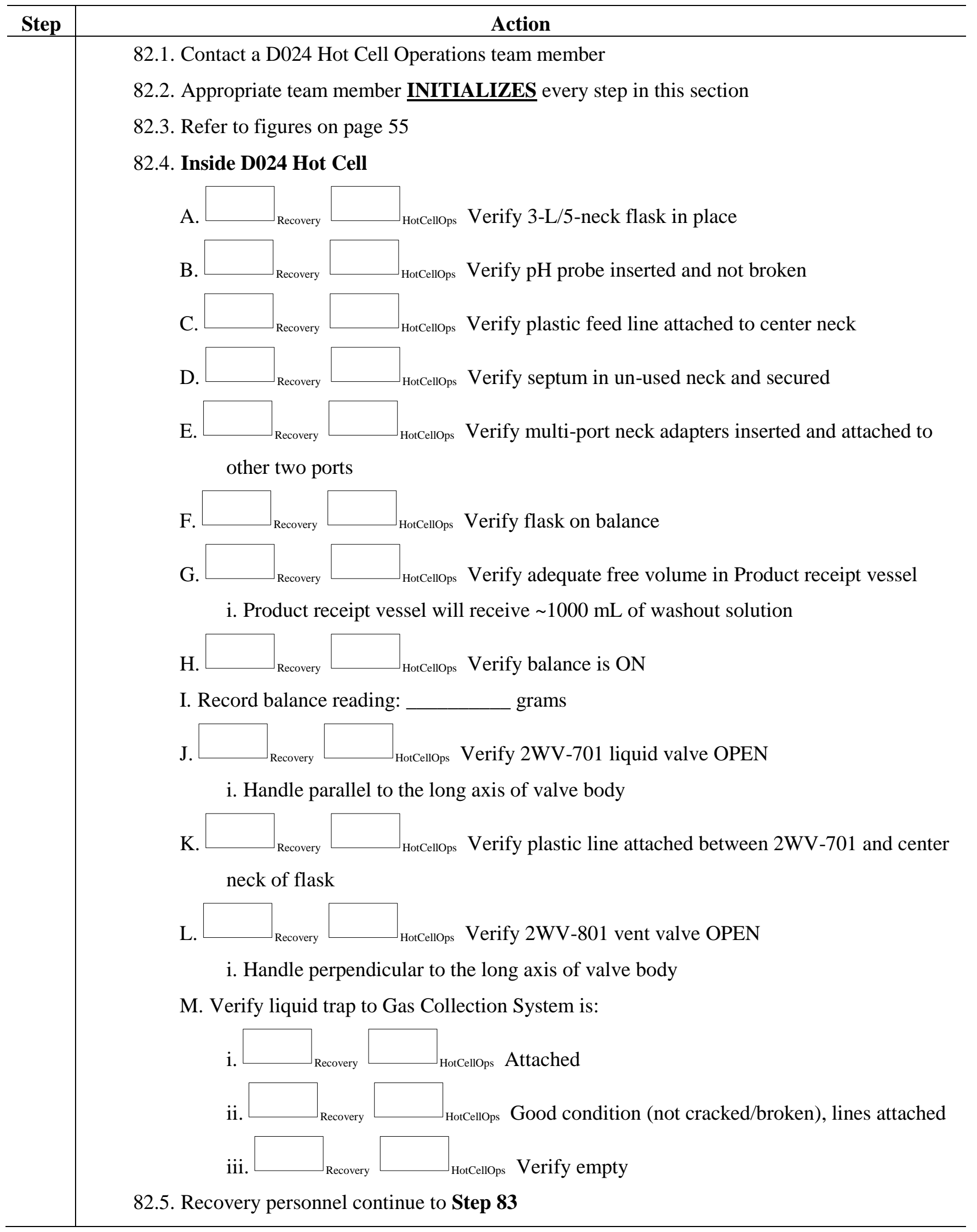




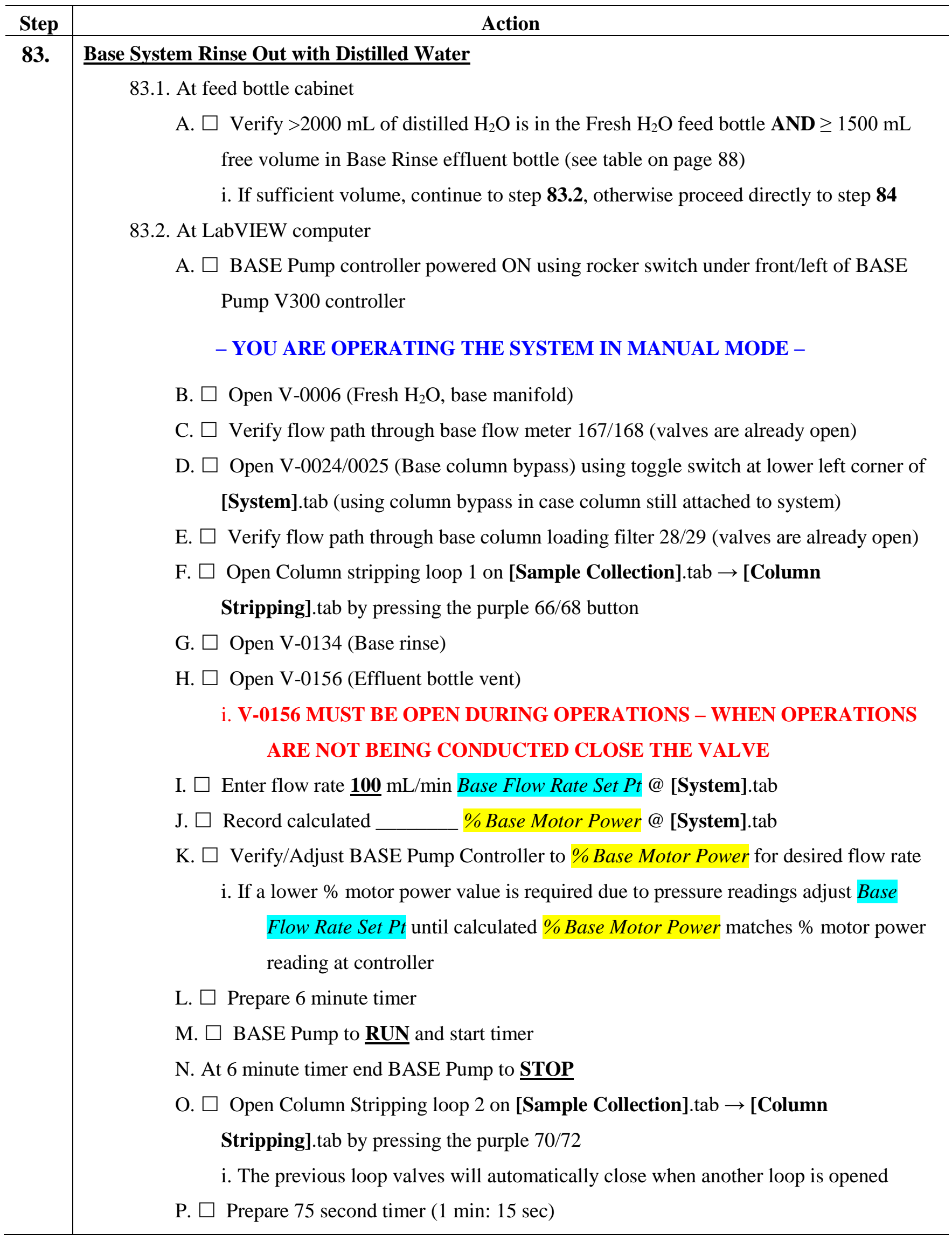




\begin{tabular}{|c|c|}
\hline Step & Action \\
\hline & Q. $\square$ BASE Pump to $\underline{\mathbf{R U N}}$ and start timer \\
\hline & R. At 75 second timer end BASE Pump to $\underline{\text { STOP }}$ \\
\hline & S. Repeat steps 83.2.O - 83.2.R for each Column Stripping loop, actuating the loop valves \\
\hline & using the following purple buttons on [Sample Collection].tab $\rightarrow$ [Column \\
\hline & Stripping].tab \\
\hline & i. $\square$ Loop 3: $74 / 76$ \\
\hline & ii. $\square$ Loop 4: $78 / 80$ \\
\hline & iii. $\square$ Loop 5: 82/84 \\
\hline & iv. $\square$ Loop 6: $86 / 88$ \\
\hline & v. $\square$ Loop 7: 90/92 \\
\hline & vi. $\square$ Loop 8: 94/96 \\
\hline & vii. $\square$ Loop 9: 98/99 \\
\hline & T. $\square$ Verify BASE Pump to $\underline{\text { STOP }}$ \\
\hline & U. $\square$ Close V-0134 (Base rinse) \\
\hline & V. $\square$ Close V-0156 (Effluent bottle vent) \\
\hline & 83.3. Washout of ${ }^{99} \mathrm{Mo}$ product transfer line to Bigfoot \\
\hline & A. $\square$ Open V-0137 (Product to Hot Cell) \\
\hline & B. $\square$ Open V-0159 (Cell Receipt Vessel vent) \\
\hline & C. $\square$ Prepare 5 minute timer \\
\hline & D. $\square$ BASE Pump to $\underline{\mathbf{R U N}}$ and start timer \\
\hline & E. At 5 minute timer end BASE Pump to $\underline{\mathbf{S T O P}}$ \\
\hline & F. $\square$ Close V-0006 (Fresh $\mathrm{H}_{2} \mathrm{O}$, base feed manifold) \\
\hline & G. $\square$ Close V-0137 (Product to Hot Cell) \\
\hline & H. $\square$ Close V-0159 (Cell Receipt Vessel vent) \\
\hline & I. $\square$ BASE Pump controller powered OFF using rocker switch under front/left of BASE \\
\hline & Pump V300 controller \\
\hline & J. Proceed to step 84 \\
\hline \multirow[t]{5}{*}{84.} & Base System Purge with 5 psig $\mathrm{N}_{2}$ \\
\hline & 84.1. Outside of recovery glovebox \\
\hline & THE V-2038 for nitrogen service IS TO REMAIN ATTACHED TO THE GLOVEBOX - DO \\
\hline & NOT REMOVE THIS VALVE \\
\hline & A. Verify V-2038 for nitrogen service is closed \\
\hline
\end{tabular}




\begin{tabular}{|c|c|}
\hline Step & Action \\
\hline & B. Verify $\mathrm{N}_{2}$ tank attached and set to 5 psig \\
\hline & i. If not attached see step $\mathbf{8 1 . 1}$ then return to this step \\
\hline & C. DO NOT OPEN V-2038 for nitrogen service \\
\hline & 84.2. Inside of recovery glovebox \\
\hline & A. Verify base system purge port valve V-2033 closed (valve is after base pump and before \\
\hline & base flow meter) \\
\hline & B. If present remove needle port guide from base system purge port valve V-2033 \\
\hline & i. Needle port guide is three pieces \\
\hline & a. Needle port guide nut, Septum, and White $1 / 4$ in. Teflon one-piece ferrule \\
\hline & C. Verify check valve V-0406 attached to end of $1 / 4$ in. FEP tubing line from V-2038 \\
\hline & i. Prevents potential of glovebox atmosphere exiting V-2038 \\
\hline & D. Attach existing $1 / 4$ in. FEP tubing line to base system purge port valve V-2033 via check \\
\hline & valve V-0406 \\
\hline & i. FEP tubing is attached to a stainless steel line that passes across glovebox wall and \\
\hline & ends near the solenoid vent valves manifold \\
\hline & 84.3. Outside of recovery glovebox \\
\hline & A. Open V-2038 for nitrogen service \\
\hline & 84.4. At LabVIEW computer AND inside recovery glovebox \\
\hline & A. $\square$ Verify flow path through base flow meter $167 / 168$ (valves are already open) \\
\hline & B. $\square$ Open V-0024/0025 (Base column bypass) using toggle switch at lower left corner of \\
\hline & [System].tab (using column bypass in case column still attached to system) \\
\hline & C. $\square$ Verify flow path through base column loading filter 28/29 (valves are already open) \\
\hline & D. $\square$ Open Column stripping loop 1 on [Sample Collection].tab $\rightarrow$ [Column \\
\hline & Stripping].tab by pressing the purple $66 / 68$ button \\
\hline & E. $\square$ Open V-0134 (Base rinse) \\
\hline & F. $\square$ Open V-0156 (Effluent bottle vent) \\
\hline & i. V-0156 MUST BE OPEN DURING OPERATIONS - WHEN OPERATIONS \\
\hline & ARE NOT BEING CONDUCTED CLOSE THE VALVE \\
\hline & G. Prepare 3 minute timer for loop 1 \\
\hline & H. Open base system purge port valve V-2033 and start timer \\
\hline & I. At 3 minute timer end close base system purge port valve V-2033 \\
\hline & J. $\square$ Open Column Stripping loop 2 on [Sample Collection].tab $\rightarrow$ [Column \\
\hline & Stripping].tab by pressing the purple $70 / 72$ button \\
\hline
\end{tabular}




\begin{tabular}{|c|c|}
\hline Step & Action \\
\hline & i. The previous loop valves will automatically close when another loop is opened \\
\hline & K. Prepare 1 minute timer \\
\hline & L. Open base system purge port valve V-2033 and start timer \\
\hline & M. At 1 minute timer end close base system purge port valve V-2033 \\
\hline & N. Repeat steps 84.4.J - 84.4.M for each Column Stripping loop, actuating the loop valves \\
\hline & using the following purple buttons on [Sample Collection].tab $\rightarrow$ [Column \\
\hline & Stripping].tab \\
\hline & i. $\square$ Loop 3: $74 / 76$ \\
\hline & ii. $\square$ Loop 4: $78 / 80$ \\
\hline & iii. $\square$ Loop 5: 82/84 \\
\hline & iv. $\square$ Loop 6: 86/88 \\
\hline & v. $\square$ Loop 7: 90/92 \\
\hline & vi. $\square$ Loop 8: 94/96 \\
\hline & vii. $\square$ Loop 9: 98/99 \\
\hline & O. $\square$ Verify base system purge port valve V-2033 is closed \\
\hline & P. $\square$ Close V-0134 (Base rinse) \\
\hline & Q. $\square$ Open V-0135 (post-strip water wash) \\
\hline & R. $\square$ Open base system purge port valve V-2033 \\
\hline & S. Leave purge for at least one minute to flush out post strip water wash line \\
\hline & T. $\square$ Close V-0135 (post-strip water wash) \\
\hline & U. $\square$ Close V-0156 (Effluent bottle vent) \\
\hline & V. $\square$ Close base system purge port valve V-2033 \\
\hline & 84.5. Purge out of ${ }^{99} \mathrm{Mo}$ product transfer line to Bigfoot \\
\hline & A. $\square$ Open V-0137 (Product to Hot Cell) \\
\hline & B. $\square$ Open V-0159 (Cell Receipt Vessel vent) \\
\hline & C. $\square$ Prepare 1 minute timer \\
\hline & D. Open base system purge port valve V-2033 and start timer \\
\hline & E. At 1 minute timer end close base system purge port valve V-2033 \\
\hline & F. $\square$ Close V-0137 (Product to Hot Cell) \\
\hline & G. $\square$ Close V-0159 (Cell Receipt Vessel vent) \\
\hline & 84.6. Outside of recovery glovebox \\
\hline & A. $\square$ Close V-2038 for nitrogen service \\
\hline 85. & Report RecoveryOps Done with D024 Hot Cell 3-L/5-Neck Flask \\
\hline
\end{tabular}




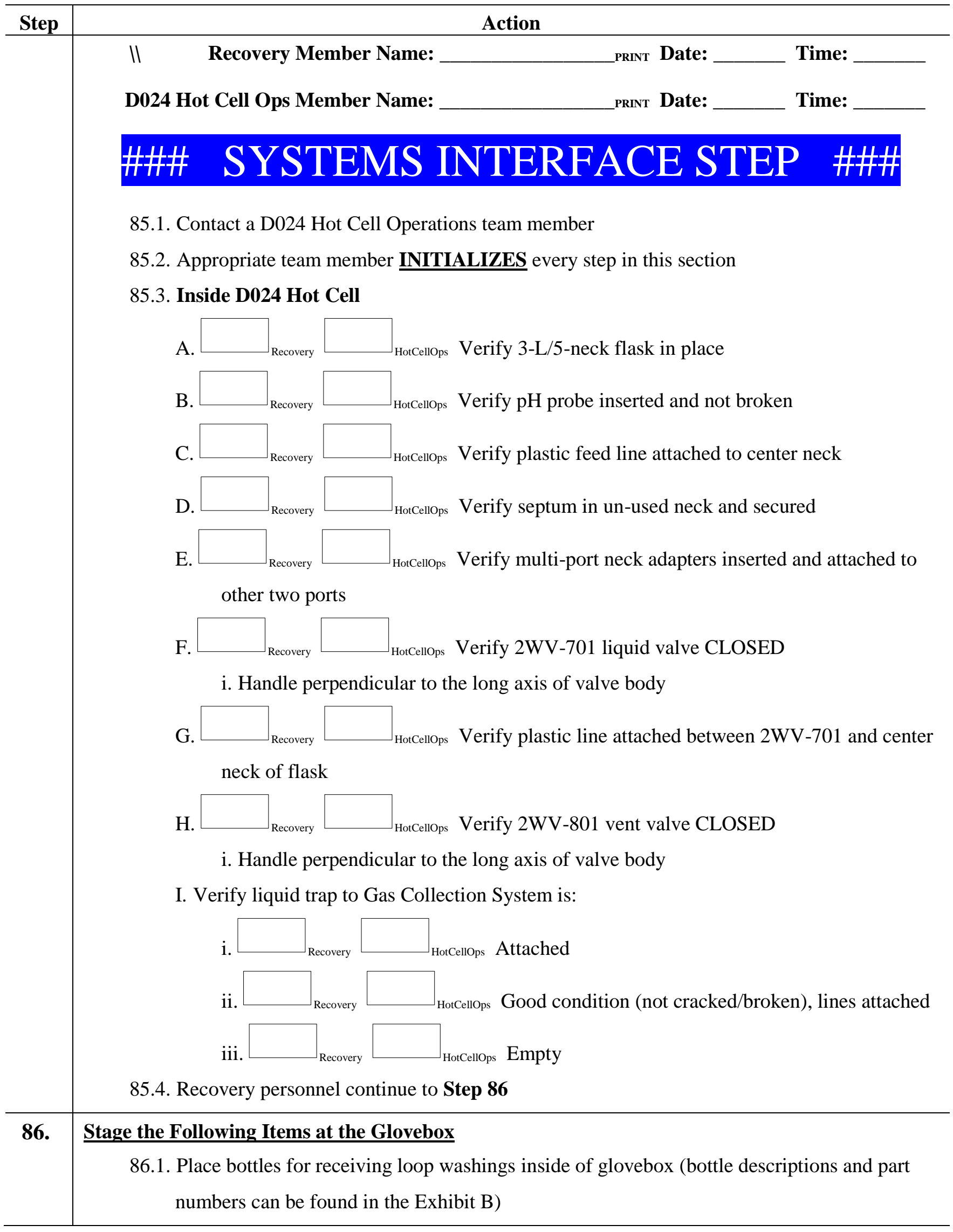




\begin{tabular}{|c|c|}
\hline Step & Action \\
\hline & A. Three bottles are required \\
\hline & i. One for target mixing washes \\
\hline & ii. One for column loading washes \\
\hline & iii. One for column stripping washes \\
\hline & a. Insert 18 gauge disposable needle into each bottle to vent purge $\mathrm{N}_{2}$ into \\
\hline & glovebox \\
\hline & iv. DO NOT COMBINE TARGET MIXING AND COLUMN LOADING \\
\hline & a. Having separate bottles will keep the needles as their respective sets \\
\hline & B. NOTE - for very first time through these washout steps you may want to consider using \\
\hline & two $60 \mathrm{~mL}$ bottles for each individual retrieval needle washout. This would allow for \\
\hline & analysis to verify that amounts coming out of second pass through the procedure are \\
\hline & very low and would help determine if a third pass is required. Once this information is \\
\hline & known then washings of the eight retrieval needles for a given set can be combined \\
\hline & into a $125 \mathrm{~mL}$ bottle. \\
\hline & 86.2. Ensure there is enough solution for wash out in feed bottles in middle cabinet (cabinet \#2) \\
\hline & A. Deionized water feed bottle contains $10 \mathrm{~L}$ of deionized water \\
\hline & B. Fresh Acid Feed bottle of contains $4 \mathrm{~L}$ of $\mathrm{pH} 1 \mathrm{H}_{2} \mathrm{SO}_{4}$ \\
\hline & 86.3. Ensure there is enough empty space to receive wash solution in effluent bottles (cabinet \#3) \\
\hline & A. Effluent bottle 10 L empty $\rightarrow$ to Acid Rinse line (from valve \#139) \\
\hline & B. Effluent bottle 10 L empty $\rightarrow$ to Base Rinse line (from valve \#134) \\
\hline & 86.4. If insufficient empty volume in the effluent bottles perform the following, otherwise \\
\hline & continue to step $\mathbf{8 6 . 5}$ \\
\hline & A. Remove effluent bottle cart following Steps 91 and 92 \\
\hline & B. Install new Acid Rinse and Base Rinse bottles in cabinet \#3, making the following \\
\hline & connections: \\
\hline & i. $\square$ Acid Rinse Bottle connected to Acid Rinse line (from valve \#139 via 2-way ball \\
\hline & valve \#2018) \\
\hline & ii. $\square$ Acid Rinse Bottle connected to Acid Rinse vent line (to 2-way ball valve \#2020) \\
\hline & iii. $\square$ Base Rinse Bottle connected to Base Rinse line (from valve \#134 via 2-way ball \\
\hline & valve \#2017) \\
\hline & iv. $\square$ Base Rinse Bottle connected to Base Rinse vent line (to 2-way ball valve \#2022) \\
\hline & $\begin{array}{l}\text { 86.5. } \square \text { Verify all effluent cart (or newly installed effluent bottle) liquid lines are connected and } \\
\text { open }\end{array}$ \\
\hline
\end{tabular}




\begin{tabular}{|c|c|}
\hline Step & Action \\
\hline & $\begin{array}{l}\text { 86.6. } \square \text { Verify all effluent cart (or newly installed effluent bottle )vent lines are connected and } \\
\text { open }\end{array}$ \\
\hline \multirow[t]{29}{*}{87.} & Washout of Acid Sample Loops \\
\hline & - YOU ARE OPERATING THE SYSTEM IN MANUAL MODE - \\
\hline & 87.1. Outside of recovery glovebox \\
\hline & THE V-2038 for nitrogen service IS TO REMAIN ATTACHED TO THE GLOVEBOX - DO \\
\hline & NOT REMOVE THIS VALVE \\
\hline & A. Verify V-2038 for nitrogen service is closed \\
\hline & B. Attach nitrogen cylinder to V-2038 on right side of recovery glovebox, above white \\
\hline & transfer cask glovebox \\
\hline & C. Set regulator to 5 psig \\
\hline & D. DO NOT OPEN V-2038 for nitrogen service \\
\hline & 87.2. Inside of recovery glovebox \\
\hline & A. Verify acid injection port valve V-2001 closed (valve is after acid pump and before acid \\
\hline & flow meter) \\
\hline & B. If present remove needle port guide from acid injection port valve V-2001 \\
\hline & i. Needle port guide is three pieces \\
\hline & a. Needle port guide nut, Septum, and White $1 / 4$ in. Teflon one-piece ferrule \\
\hline & C. Verify check valve V-0406 attached to end of $1 / 4$ in. FEP tubing line from V-2038 \\
\hline & i. Prevents potential of glovebox atmosphere exiting V-2038 \\
\hline & D. Attach existing $1 / 4$ in. FEP tubing line to acid injection port valve $\mathrm{V}-2001$ via check valve \\
\hline & V-0406 \\
\hline & i. FEP tubing is attached to a stainless steel line that passes across glovebox wall and \\
\hline & ends near the solenoid vent valves manifold \\
\hline & 87.3. Outside of recovery glovebox \\
\hline & A. Open V-2038 for nitrogen service \\
\hline & 87.4. YOU WILL BE PASSING THROUGH THESE STEPS AT LEAST TWICE \\
\hline & A. First pass mark the black boxes $\square$ \\
\hline & B. Second pass mark the red boxes $\square$ \\
\hline & C. Third pass mark the blue boxes $\square$ \\
\hline & D. Fourth pass (if required) mark the green boxes $\square$ \\
\hline
\end{tabular}




\begin{tabular}{|c|c|}
\hline \multirow[t]{2}{*}{ Step } & Action \\
\hline & i. NOTE - only steps 87.6.A through 87.6.EEE have the green boxes \\
\hline & 87.5. At LabVIEW computer AND inside recovery glovebox \\
\hline & 87.6. Removing residual liquid from purged sample loops \\
\hline & A. $\square \square \square \square$ Verify flow path through acid flow meter V-0163/0164 (valves are \\
\hline & open) \\
\hline & B. $\square \square \square \square$ Open V-0009 (Target Mixing path) \\
\hline & C. $\square \square \square \square$ Open Target Mixing loop 1 on [Sample Collection].tab $\rightarrow$ [Target \\
\hline & Mixing].tab by pressing the purple $100 / 102$ button \\
\hline & D. $\square \square \square \square$ Open V-0153/0154 (Target Mixing path to Dump Tank path) \\
\hline & E. $\square \square \square \square$ Open V-0172/0173 (Target Mixing path to Acid Rinse bottle) \\
\hline & i. Selecting this valve closes both $147 / 148$ (frit to Dump Tank) and 149/150 (bypass to \\
\hline & Dump Tank) \\
\hline & F. $\square \square \square \square$ Open V-0156 (Effluent bottle vent) \\
\hline & i. V-0156 MUST BE OPEN DURING OPERATIONS - WHEN OPERATIONS \\
\hline & ARE NOT BEING CONDUCTED CLOSE THE VALVE \\
\hline & G. $\square \square \square \square$ Insert needle for Target Mixing loop 1into $60 \mathrm{~mL}$ square PETG bottle \\
\hline & with septum closure \\
\hline & H. $\square \square \square \square$ Open Target Mixing loop 1 retrieval valves on [Sample \\
\hline & Collection].tab $\rightarrow$ [Target Mixing].tab by pressing the purple 101/103 button \\
\hline & I. $\square \square \square \square$ Close V-0153/0154 (Target Mixing path to Dump Tank path) \\
\hline & J. $\square \square \square \square$ Prepare 10 second timer \\
\hline & K. Open acid injection port valve V-2001 and start timer (pre and post-acid pump pressures \\
\hline & will rise) \\
\hline & L. At 10 second timer end close injection port valve V-2001 \\
\hline & M. $\square \square \square \square$ Close Target Mixing loop 1 retrieval valves on [Sample \\
\hline & Collection].tab $\rightarrow$ [Target Mixing].tab by pressing the green 101/103 button \\
\hline & N. $\square \square \square \square$ Open V-0153/0154 (Target Mixing path to Dump Tank path) \\
\hline & O. $\square \square \square \square$ Remove and stow needle for Target Mixing loop 1 \\
\hline & P. $\square \square \square \square$ Open Target Mixing loop 2 on [Sample Collection].tab $\rightarrow$ [Target \\
\hline & Mixing].tab by pressing the purple $104 / 106$ \\
\hline & i. The previous loop valves will automatically close when another loop is opened \\
\hline
\end{tabular}




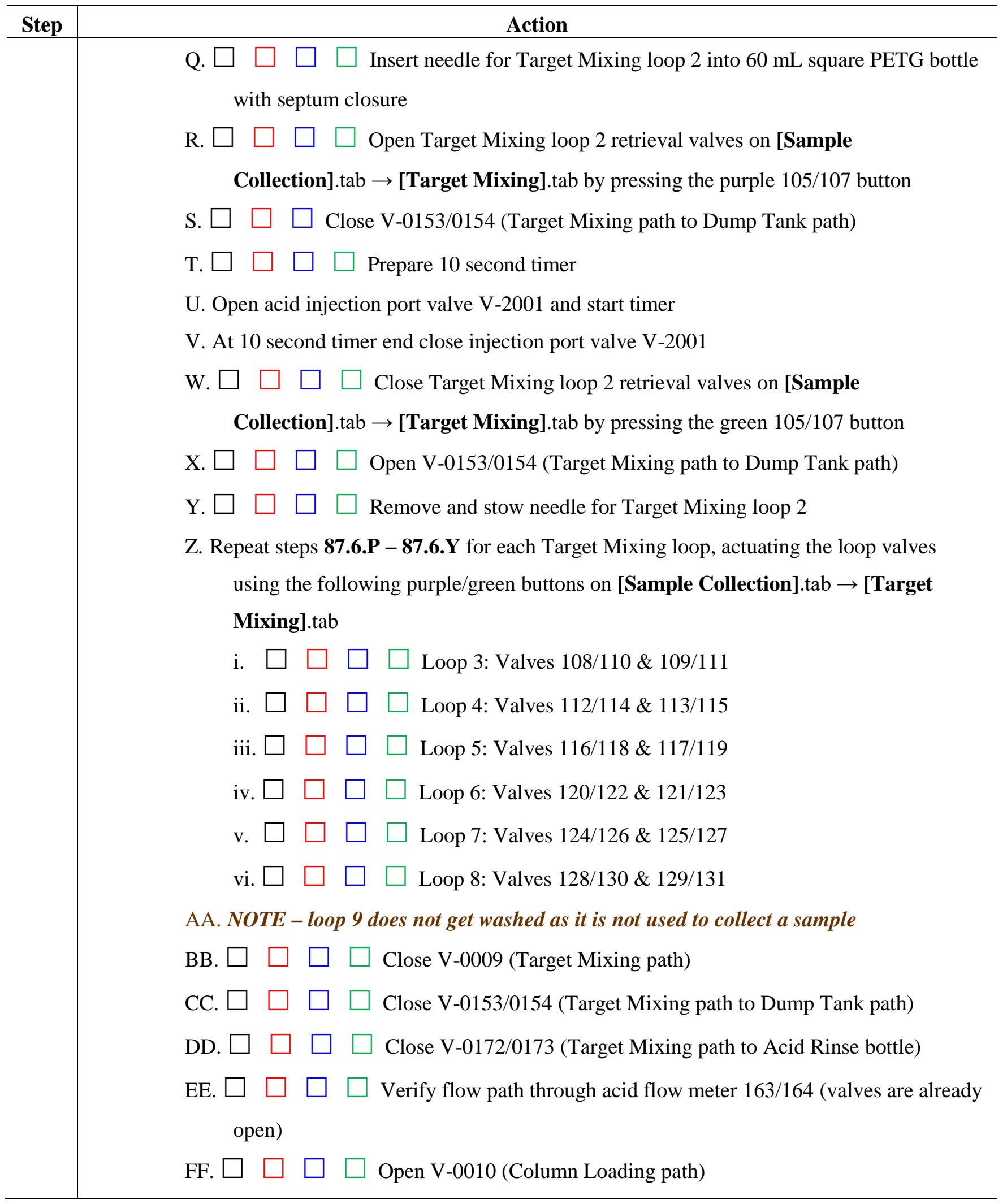




\begin{tabular}{|c|c|}
\hline Step & Action \\
\hline & $\begin{array}{l}\text { GG. } \square \square \square \text { Open V-0014/0015 (Acid column bypass) using toggle switch at } \\
\text { lower left corner of [System].tab (using column bypass in case column still attached to } \\
\text { system) }\end{array}$ \\
\hline & $\begin{array}{l}\text { HH. } \square \square \square \square \text { Verify flow path through acid column loading filter 18/19 (valves } \\
\text { already open) }\end{array}$ \\
\hline & $\begin{array}{l}\text { II. } \square \square \square \square \text { Open Column Loading loop } 1 \text { on [Sample Collection].tab } \rightarrow \\
\text { [Column Loading].tab by pressing the purple 32/34 button }\end{array}$ \\
\hline & JJ. $\square \square \square \square$ Open V-0139 (Acid rinse) \\
\hline & KK. $\square \square \square \square$ Verify V-0156 open (Effluent bottle vent) \\
\hline & i. V-0156 MUST BE OPEN DURING OPERATIONS - WHEN OPERATIONS \\
\hline & ARE NOT BEING CONDUCTED CLOSE THE VALVE \\
\hline & $\begin{array}{l}\text { LL. } \square \square \square \square \text { Insert needle for Column Loading loop 1into } 60 \mathrm{~mL} \text { square PETG } \\
\text { bottle with septum closure }\end{array}$ \\
\hline & MM. $\square \square \square \square$ Open Column Loading loop 1 retrieval valves on [Sample \\
\hline & Collection].tab $\rightarrow$ [Column Loading].tab by pressing the purple 33/35 button \\
\hline & NN. $\square \square \square \square$ Close V-0139 (Acid rinse bottle) \\
\hline & OO. $\square \square \square \square$ Prepare 10 second timer \\
\hline & PP. Open acid injection port valve V-2001 and start timer (pre- and post-acid pump \\
\hline & pressures will rise) \\
\hline & QQ. At 10 second timer end close injection port valve V-2001 \\
\hline & RR. $\square \square \square \square$ Close Column Loading loop 1 retrieval valves on [Sample \\
\hline & Collection].tab $\rightarrow$ [Target Mixing].tab by pressing the green $33 / 35$ button \\
\hline & SS. $\square \square \square \square$ Open V-0139 (Acid rinse bottle) \\
\hline & TT. $\square \square \square \square$ Remove and stow needle for Column Loading loop 1 \\
\hline & UU. $\square \square \square \square$ Open Column Loading loop 2 on [Sample Collection].tab $\rightarrow$ \\
\hline & [Column Loading].tab by pressing the purple $36 / 38$ button \\
\hline & i. The previous loop valves will automatically close when another loop is opened \\
\hline & $\begin{array}{l}\text { VV. } \square \square \square \square \text { Insert needle for Column Loading loop } 2 \text { into } 60 \mathrm{~mL} \text { square PETG } \\
\text { bottle with septum closure }\end{array}$ \\
\hline & WW. $\square \square \square \square$ Open Column Loading loop 2 retrieval valves on [Sample \\
\hline & Collection].tab $\rightarrow$ [Column Loading].tab by pressing the purple $37 / 39$ button \\
\hline
\end{tabular}




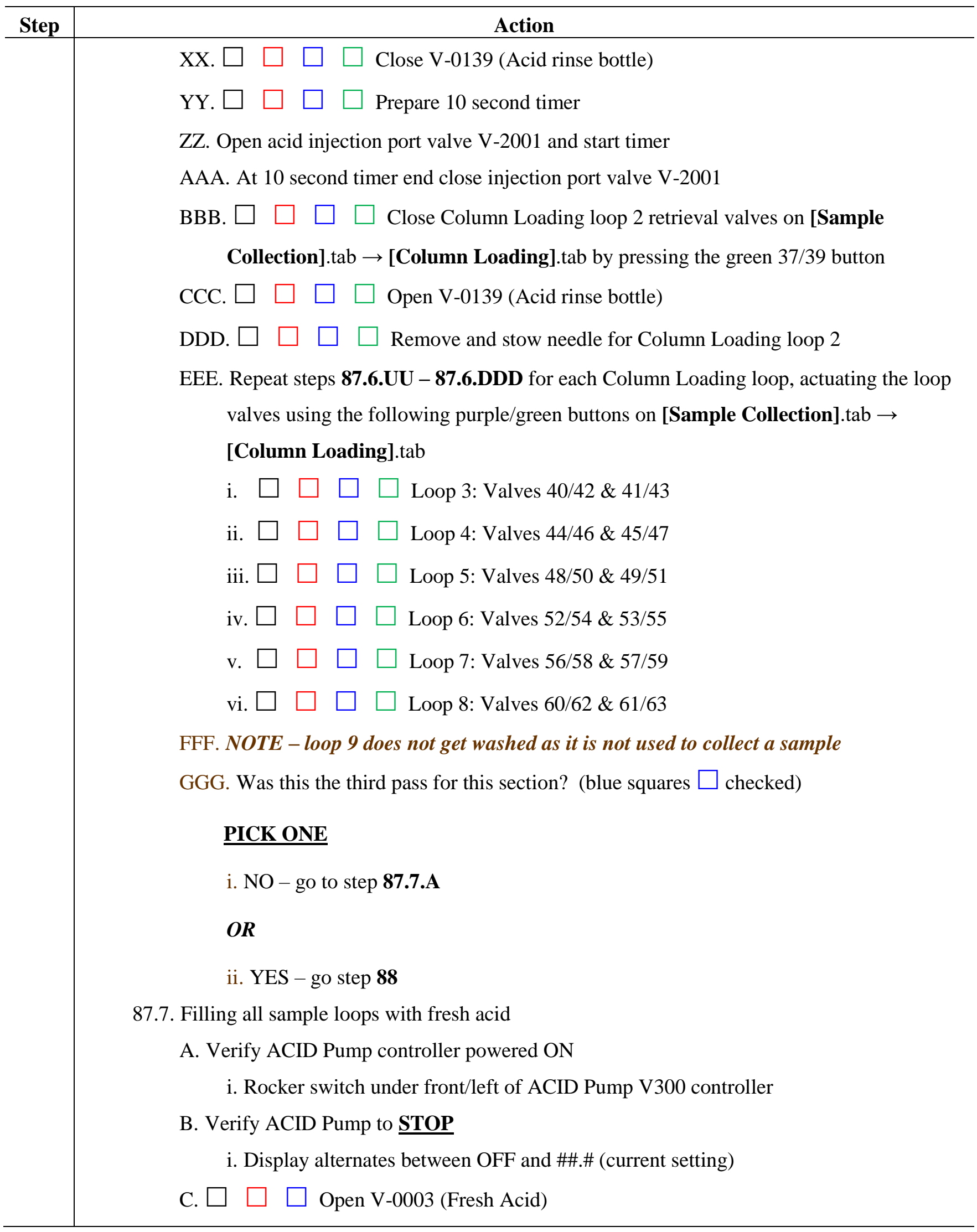




\begin{tabular}{|c|c|}
\hline Step & Action \\
\hline & $\begin{array}{l}\text { D. } \square \square \square \text { Verify flow path through acid flow meter 163/164 (valves are already } \\
\text { open) }\end{array}$ \\
\hline & E. $\square \square \square$ Open V-0009 (Target Mixing path) \\
\hline & F. $\square \square \square$ Open Target Mixing loop 1 on [Sample Collection].tab $\rightarrow$ [Target \\
\hline & Mixing].tab by pressing the purple $100 / 102$ button \\
\hline & G. $\square \square \square$ Open V-0153/0154 (Target Mixing path to Dump Tank path) \\
\hline & H. $\square \square \square$ Open V-0172/0173 (Target Mixing path to Acid Rinse bottle) \\
\hline & i. Selecting this valve closes both $147 / 148$ (frit to Dump Tank) and 149/150 (bypass to \\
\hline & Dump Tank) \\
\hline & I. $\square \square \square$ Open V-0156 (Effluent bottle vent) \\
\hline & i. V-0156 MUST BE OPEN DURING OPERATIONS - WHEN OPERATIONS \\
\hline & ARE NOT BEING CONDUCTED CLOSE THE VALVE \\
\hline & J. $\square \square \square$ Enter flow rate $\underline{\mathbf{1 0 0}} \mathrm{mL} / \mathrm{min}$ Acid Flow Rate Set Pt @ [System].tab \\
\hline & K. $\square \square \square$ Record calculated___ \% Acid Motor Power @ [System].tab \\
\hline & L. $\square \square \square$ Verify/Adjust ACID Pump Controller to \% Acid Motor Power for desired \\
\hline & flow rate \\
\hline & i. If a lower $\%$ motor power value is required due to pressure readings adjust Acid \\
\hline & Flow Rate Set Pt until calculated \% Acid Motor Power matches \% motor power \\
\hline & reading at controller \\
\hline & M. $\square \square \square$ Prepare 3 minute timer \\
\hline & N. $\square \square \square$ ACID Pump to $\underline{\mathbf{R U N}}$ and start timer \\
\hline & O. At 3 minute timer end ACID Pump to $\underline{\text { STOP }}$ \\
\hline & P. $\square \square \square$ Open Target Mixing loop 2 on [Sample Collection].tab $\rightarrow$ [Target \\
\hline & Mixing].tab by pressing the purple $104 / 106$ button \\
\hline & i. The previous loop valves will automatically close when another loop is opened \\
\hline & Q. $\square \square \square$ Prepare 1 minute 15 second timer ( 75 seconds total) \\
\hline & R. $\square \square \square$ ACID Pump to $\underline{\mathbf{R U N}}$ and start timer \\
\hline & S. At 75 second timer end ACID Pump to $\underline{\text { STOP }}$ \\
\hline & T. Repeat steps 87.7.P - 87.7.S for each Target Mixing loop, actuating the loop valves \\
\hline & using the following purple buttons on [Sample Collection].tab $\rightarrow$ [Target \\
\hline & Mixing].tab \\
\hline
\end{tabular}




\begin{tabular}{|c|c|}
\hline Step & Action \\
\hline & i. $\square \square \square$ Loop 3: Valves $108 / 110$ \\
\hline & ii. $\square \square \square$ Loop 4: Valves $112 / 114$ \\
\hline & iii. $\square \square \square$ Loop 5: Valves 116/118 \\
\hline & iv. $\square \square \square$ Loop 6: Valves 120/122 \\
\hline & v. $\square \square \square$ Loop 7: Valves 124/126 \\
\hline & vi. $\square \square \square$ Loop 8: Valves 128/130 \\
\hline & U. $\square \square \square$ Verify ACID Pump to $\underline{\text { STOP }}$ \\
\hline & V. NOTE - loop 9 does not get washed as it is not used to collect a sample \\
\hline & W. $\square \square \square$ Close V-0009 (Target Mixing path) \\
\hline & X. $\square \square \square$ Close V-0153/0154 (Target Mixing path to Dump Tank path) \\
\hline & Y. $\square \square \square$ Close V-0172/0173 (Target Mixing path to Acid Rinse bottle) \\
\hline & Z. $\square \square \square$ Verify V-0003 open (Fresh acid) \\
\hline & AA. $\square \square \square$ Verify flow path through acid flow meter 163/164 (valves already open) \\
\hline & BB. $\square \square \square$ Open V-0010 (Column Loading path) \\
\hline & CC. $\square \square \square$ Open V-0014/0015 (Acid column bypass) using toggle switch at lower \\
\hline & $\begin{array}{l}\text { left corner of [System].tab (using column bypass in case column still attached to } \\
\text { system) }\end{array}$ \\
\hline & $\begin{array}{l}\text { DD. } \square \quad \square \square \text { Verify flow path through acid column loading filter 18/19 (valves are } \\
\text { already open) }\end{array}$ \\
\hline & EE. $\square \square \square$ Open Column Loading loop 1 on [Sample Collection].tab $\rightarrow$ [Column \\
\hline & Loading].tab by pressing purple $32 / 34$ button \\
\hline & FF. $\square \square \square$ Open V-0139 (Acid rinse) \\
\hline & GG. $\square \square \square$ Verify V-0156 (Effluent bottle vent) \\
\hline & HH. $\square \square \square$ Enter flow rate $\underline{\mathbf{1 0 0}} \mathbf{m L} / \mathrm{min}$ Acid Flow Rate Set Pt @ [System].tab \\
\hline & II. $\square \square \square$ Record calculated____ \% Acid Motor Power @ [System].tab \\
\hline & JJ. $\square \square \square$ Verify/Adjust ACID Pump Controller to \% Acid Motor Power for desired \\
\hline & flow rate \\
\hline & i. If a lower $\%$ motor power value is required due to pressure readings adjust Acid \\
\hline & Flow Rate Set Pt until calculated \% Acid Motor Power matches \% motor power \\
\hline & reading at controller \\
\hline
\end{tabular}




\begin{tabular}{|c|c|}
\hline Step & Action \\
\hline & KK. $\square \square \square$ Prepare 6 minute timer \\
\hline & LL. $\square \square \square$ ACID Pump to $\underline{\mathbf{R U N}}$ and start timer \\
\hline & MM. At 6 minute timer end ACID Pump to $\underline{\text { STOP }}$ \\
\hline & NN. $\square \square \square$ Open Column Loading loop 2 on [Sample Collection].tab $\rightarrow$ [Column \\
\hline & Loading].tab by pressing the purple $36 / 38$ button \\
\hline & i. The previous loop valves will automatically close when another loop is opened \\
\hline & OO. $\square \square \square$ Prepare 75 second timer (1 min: $15 \mathrm{sec})$ \\
\hline & PP. $\square \square \square$ ACID Pump to $\underline{\mathbf{R U N}}$ and start timer \\
\hline & QQ. At 75 second timer end ACID Pump to $\underline{\text { STOP }}$ \\
\hline & RR. Repeat steps 87.7.NN - 87.7.QQ for each Column Loading loop, actuating the loop \\
\hline & valves using the following purple buttons on [Sample Collection].tab $\rightarrow$ [Column \\
\hline & Loading].tab \\
\hline & i. $\square \square \square$ Loop 3: Valves 40/42 \\
\hline & ii. $\square \square \square$ Loop 4: Valves 44/46 \\
\hline & iii. $\square \square \square$ Loop 5: Valves 48/50 \\
\hline & iv. $\square \square \square$ Loop 6: Valves 52/54 \\
\hline & v. $\square \square \square$ Loop 7: Valves $56 / 58$ \\
\hline & vi. $\square \square \square$ Loop 8: Valves 60/62 \\
\hline & SS. $\square \square \square$ Verify ACID Pump to $\underline{\text { STOP }}$ \\
\hline & TT. NOTE - loop 9 does not get washed as it is not used to collect a sample \\
\hline & 87.8. Purging all liquid from all acid paths \\
\hline & A. Outside of recovery glovebox \\
\hline & i. Verify V-2038 for nitrogen service is closed \\
\hline & ii. Verify regulator set to 5 psig \\
\hline & B. Inside of recovery glovebox \\
\hline & i. Verify acid injection port valve V-2001 closed (valve is after acid pump and before \\
\hline & acid flow meter) \\
\hline & ii. Verify existing $1 / 4$ in. FEP tubing line attached to acid injection port valve V-2001 \\
\hline & a. FEP tubing is attached to stainless steel line that passes across glovebox wall \\
\hline & and ends near the solenoid vent valves manifold \\
\hline & C. Outside of recovery glovebox \\
\hline
\end{tabular}




\begin{tabular}{|c|c|}
\hline Step & Action \\
\hline & i. Verify V-2038 is open for nitrogen servi \\
\hline & D. $\square \square \square$ Verify flow path through acid $\mathrm{f}$ \\
\hline & E. $\square \square \square$ Open V-0009 (Target Mixing p \\
\hline & F. $\square \square \square$ Open Target Mixing loop 1 on $\square$ \\
\hline & Mixing].tab by pressing the purple $100 / 10$ \\
\hline & G. $\square \square \square$ Open V-0153/0154 (Target Mir \\
\hline & H. $\square \square \square$ Open V-0172/0173 (Target Mir \\
\hline & i. Selecting this valve closes both $147 / 148$ \\
\hline & Dump Tank) \\
\hline & I. $\square \square \square$ Open V-0156 (Effluent bottle ve \\
\hline & i. V-0156 MUST BE OPEN DURING O \\
\hline & ARE NOT BEING CONDUCTED \\
\hline & J. $\square \square \square$ Prepare 30 second timer \\
\hline & K. Open acid injection port valve V-2001 and st \\
\hline & L. At 30 second timer end close injection port $v$ \\
\hline & M. $\square \square \square$ Open Target Mixing loop 2 on \\
\hline & Mixing].tab by pressing the purple $104 / 10$ \\
\hline & i. The previous loop valves will automatic \\
\hline & N. $\square \square \square$ Prepare 30 second timer \\
\hline & O. Open acid injection port valve V-2001 and st \\
\hline & P. At 30 second timer end close injection port v \\
\hline & Q. Repeat steps 87.8.M - 87.8.P for each Targe \\
\hline & using the following purple buttons on [Sar \\
\hline & Mixing].tab \\
\hline & i. $\square \square \square$ Loop 3: Valves $108 / 110$ \\
\hline & ii. $\square \square \square$ Loop 4: Valves 112/114 \\
\hline & iii. $\square \square \square$ Loop 5: Valves 116/118 \\
\hline & iv. $\square \square \square$ Loop 6: Valves 120/122 \\
\hline & v. $\square \square \square$ Loop 7: Valves 124/126 \\
\hline & vi. $\square \square \square$ Loop 8: Valves $128 / 130$ \\
\hline
\end{tabular}




\begin{tabular}{|c|c|}
\hline Step & Action \\
\hline & R. $\square \square \square$ Verify injection port valve V-2001 is closed \\
\hline & S. NOTE - loop 9 does not get washed as it is not used to collect a sample \\
\hline & T. $\square \square \square$ Close V-0009 (Target Mixing path) \\
\hline & U. $\square \square \square$ Close V-0153/0154 (Target Mixing path to Dump Tank path) \\
\hline & V. $\square \square \square$ Close V-0172/0173 (Target Mixing path to Acid Rinse bottle) \\
\hline & W. $\square \square \square$ Verify flow path through acid flow meter 163/164 (valves are already \\
\hline & open) \\
\hline & X. $\square \square \square$ Open V-0010 (Column Loading path) \\
\hline & Y. $\square \square \square$ Open V-0014/0015 (Acid column bypass) using toggle switch at lower left \\
\hline & corner of [System].tab (using column bypass in case column still attached to system) \\
\hline & Z. $\square \square \square$ Verify flow path through acid column loading filter $18 / 19$ (valves are \\
\hline & already open) \\
\hline & AA. $\square \square \square$ Open Column Loading loop 1 on [Sample Collection].tab $\rightarrow$ [Column \\
\hline & Loading].tab by pressing the purple $32 / 34$ button \\
\hline & BB. $\square \square \square$ Open V-0139 (Acid rinse) \\
\hline & CC. $\square \square \square$ Verify V-0156 (Effluent bottle vent) \\
\hline & DD. $\square \square \square$ Prepare 30 second timer \\
\hline & EE. Open acid injection port valve V-2001 and start timer \\
\hline & FF. At 30 second timer end close injection port valve V-2001 \\
\hline & GG. $\square \square \square$ Open Column Loading loop 2 on [Sample Collection].tab $\rightarrow$ [Column \\
\hline & Loading].tab by pressing the purple $36 / 38$ button \\
\hline & i. The previous loop valves will automatically close when another loop is opened \\
\hline & HH. $\square \square \square$ Prepare 30 second timer \\
\hline & II. Open acid injection port valve V-2001 and start timer \\
\hline & JJ. At 30 second timer end close injection port valve V-2001 \\
\hline & KK. Repeat steps 87.8.GG - 87.8.JJ for each Column Loading loop, actuating the loop \\
\hline & valves using the following purple buttons on [Sample Collection].tab $\rightarrow$ [Column \\
\hline & Loading].tab \\
\hline & i. $\square \square \square$ Loop 3: Valves 40/42 \\
\hline & ii. $\square \square \square$ Loop 4: Valves 44/46 \\
\hline
\end{tabular}




\begin{tabular}{|c|c|}
\hline Step & Action \\
\hline & $\begin{array}{c}\text { iii. } \square \quad \square \quad \square \text { Loop 5: Valves 48/50 } \\
\text { iv. } \square \quad \square \quad \square \text { Loop 6: Valves 52/54 } \\
\text { v. } \square \quad \square \quad \square \text { Loop 7: Valves 56/58 } \\
\text { vi. } \square \quad \square \quad \square \text { Loop 8: Valves 60/62 } \\
\text { LL. } \square \quad \square \quad \square \quad \text { Verify injection port valve V-2001 is closed } \\
\text { MM. NOTE - loop } 9 \text { does not get washed as it is not used to collect a sample } \\
\text { NN. } \square \quad \square \quad \square \text { Close V-0010 (Column Loading path) } \\
\text { OO. } \square \quad \square \quad \square \text { Close V-0139 (Acid rinse) } \\
\text { PP. } \square \quad \square \quad \square \text { Close V-0156 (Effluent bottle vent) } \\
\text { 87.9. Return to step } 87.6\end{array}$ \\
\hline 88. & $\begin{array}{l}\text { Wash Out of Base Sample Loops } \\
\text { - YOU ARE OPERATING THE SYSTEM IN MANUAL MODE - } \\
\text { 88.1. Outside of recovery glovebox } \\
\text { THE V-2038 for nitrogen service IS TO REMAIN ATTACHED TO THE GLOVEBOX - DO } \\
\text { NOT REMOVE THIS VALVE } \\
\text { A. Verify V-2038 for nitrogen service is closed } \\
\text { B. Verify N } 2 \text { tank attached and set to } 5 \text { psig } \\
\text { i. If not attached see step } 81.1 \text { then return to this step } \\
\text { C. DO NOT OPEN V-2038 for nitrogen service } \\
\text { 88.2. Inside of recovery glovebox } \\
\text { A. Verify base system purge port valve V-2033 closed (valve is after base pump and before } \\
\text { flow meter) } \\
\text { B. If present remove needle port guide from base system purge port valve V-2033 } \\
\text { i. Needle port guide is three pieces } \\
\text { a. Needle port guide nut, Septum, and White } 1 / 4 \text { in. Teflon one-piece ferrule } \\
\text { C. Verify check valve V-0406 attached to end of } 1 / 4 \text { in. FEP tubing line from V-2038 } \\
\text { i. Prevents potential of glovebox atmosphere exiting V-2038 } \\
\text { D. Attach existing } 1 / 4 \text { in. FEP tubing line to base system purge port valve V-2033 via check } \\
\text { valve V-0406 }\end{array}$ \\
\hline
\end{tabular}




\begin{tabular}{|c|c|}
\hline Step & Action \\
\hline & $\begin{array}{l}\text { i. FEP tubing is attached to a stainless steel line that passes across glovebox wall and } \\
\text { ends near the solenoid vent valves manifold }\end{array}$ \\
\hline & 88.3. Outside of recovery glovebox \\
\hline & A. Open V-2038 for nitrogen service \\
\hline & 88.4. YOU WILL BE PASSING THROUGH THESE STEPS AT LEAST TWICE \\
\hline & A. First pass mark the black boxes $\square$ \\
\hline & B. Second pass mark the red boxes $\square$ \\
\hline & C. Third pass mark the blue boxes $\square$ \\
\hline & D. Fourth pass (if required) mark the green boxes $\square$ \\
\hline & i. NOTE - only steps 88.6.A through 88.6.AA have the green boxes \\
\hline & 88.5. At LabVIEW computer AND inside recovery glovebox \\
\hline & 88.6. Removing residual liquid from purged sample loops \\
\hline & $\begin{array}{l}\text { A. } \square \square \square \square \text { Verify flow path through base flow meter } 167 / 168 \text { (valves are already } \\
\text { open) }\end{array}$ \\
\hline & $\begin{array}{l}\text { B. } \square \square \square \square \text { Open V-0024/0025 (Base column bypass) using toggle switch at lower } \\
\text { left corner of [System].tab (using column bypass in case column still attached to } \\
\text { system) }\end{array}$ \\
\hline & $\begin{array}{l}\text { C. } \square \square \square \square \text { Verify flow path through base column loading filter 28/29 (valves are } \\
\text { already open) }\end{array}$ \\
\hline & $\begin{array}{l}\text { D. } \square \square \square \square \text { Open Column Stripping loop } 1 \text { on [Sample Collection].tab } \rightarrow \\
\text { [Column Stripping].tab by pressing the purple } 66 / 68 \text { button }\end{array}$ \\
\hline & E. $\square \square \square \square$ Open V-0134 (Base rinse) \\
\hline & F. $\square \square \square \square$ Open V-0156 (Effluent bottle vent) \\
\hline & i. V-0156 MUST BE OPEN DURING OPERATIONS - WHEN OPERATIONS \\
\hline & ARE NOT BEING CONDUCTED CLOSE THE VALVE \\
\hline & $\begin{array}{l}\text { G. } \square \square \square \square \text { Insert needle for Column Stripping loop 1into } 60 \mathrm{~mL} \text { square PETG } \\
\text { bottle with septum closure }\end{array}$ \\
\hline & H. $\square \square \square \square$ Open Column Stripping loop 1 retrieval valves on [Sample \\
\hline & Collection].tab $\rightarrow$ [Column Stripping].tab by pressing the purple $67 / 69$ button \\
\hline & I. $\square \square \square \square$ Close V-0134 (Base rinse) \\
\hline & J. $\square \square \square \square$ Prepare 10 second timer \\
\hline
\end{tabular}




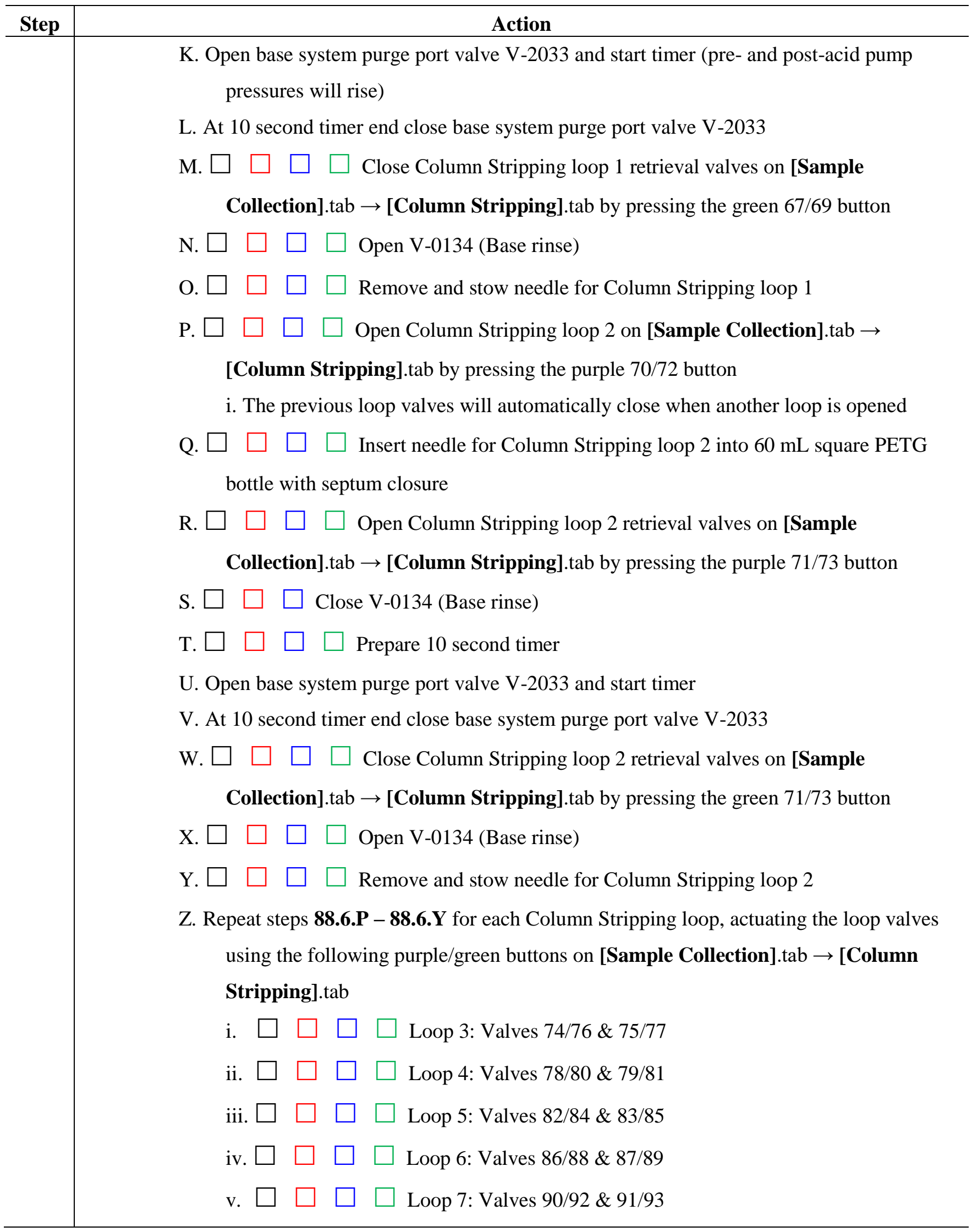




\begin{tabular}{|c|c|}
\hline \multirow[t]{29}{*}{ Step } & Action \\
\hline & vi. $\square \square \square \square$ Loop 8: Valves $94 / 96$ \& 95/97 \\
\hline & AA. $\square \square \square \square$ Verify base system purge port valve V-2033 is closed \\
\hline & BB. NOTE-loop 9 does not get washed as it is not used to collect a sample \\
\hline & CC. Was this the third pass for this section? (blue squares $\square$ checked) \\
\hline & PICK ONE \\
\hline & i. $\mathrm{NO}-$ go to step 88.7 \\
\hline & OR \\
\hline & ii. YES - go step 89 \\
\hline & 88.7. Filling all sample loops with fresh $\mathrm{H}_{2} \mathrm{O}$ \\
\hline & A. Verify BASE Pump controller powered ON (rocker switch under front/left of BASE \\
\hline & Pump V300 controller) \\
\hline & B. $\square \square \square$ Open V-0006 (Fresh $\mathrm{H}_{2} \mathrm{O}$, base manifold) \\
\hline & C. $\square \square \square$ Verify flow path through base flow meter 167/168 (valves are already \\
\hline & open) \\
\hline & D. $\square \square \square$ Open V-0024/0025 (Base column bypass) using toggle switch at lower left \\
\hline & corner of [System].tab (using column bypass in case column still attached to system) \\
\hline & E. $\square \square \square$ Verify flow path through base column loading filter 28/29 (valves are \\
\hline & already open) \\
\hline & F. $\square \square \square$ Open Column stripping loop 1 on [Sample Collection].tab $\rightarrow$ [Column \\
\hline & Stripping].tab by pressing the purple $66 / 68$ button \\
\hline & G. $\square \square \square$ Open V-0134 (Base rinse) \\
\hline & H. $\square \square \square$ Open V-0156 (Effluent bottle vent) \\
\hline & i. V-0156 MUST BE OPEN DURING OPERATIONS - WHEN OPERATIONS \\
\hline & ARE NOT BEING CONDUCTED CLOSE THE VALVE \\
\hline & I. $\square \square \square$ Enter flow rate $\underline{\mathbf{1 0 0}} \mathrm{mL} / \mathrm{min}$ Base Flow Rate Set Pt @ [System].tab \\
\hline & J. $\square \square \square$ Record calculated___ \% Base Motor Power @ [System].tab \\
\hline & K. $\square \square \square$ Verify/Adjust BASE Pump Controller to \% Base Motor Power for desired \\
\hline & flow rate \\
\hline
\end{tabular}




\begin{tabular}{|c|c|}
\hline Step & Action \\
\hline & 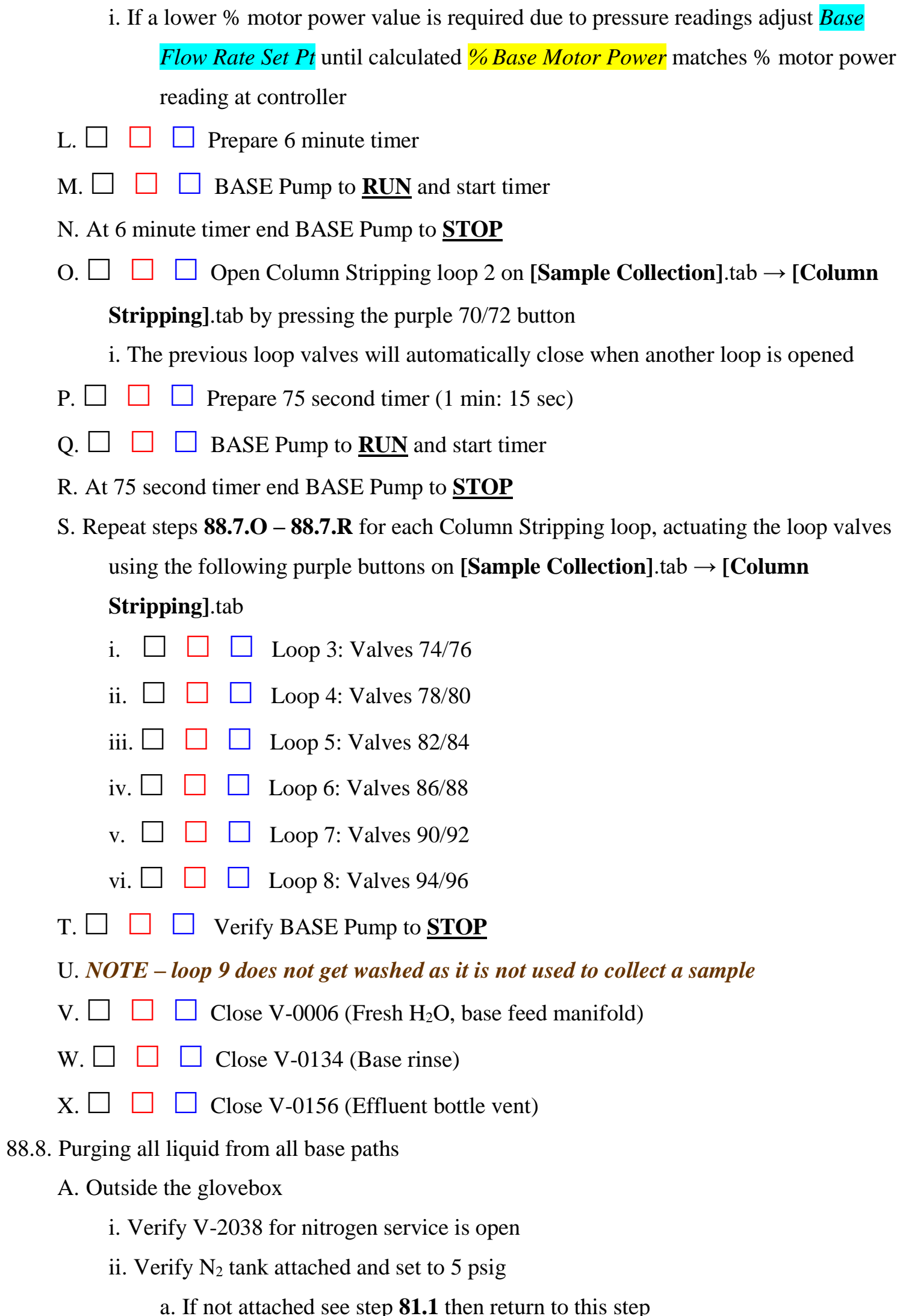 \\
\hline
\end{tabular}




\begin{tabular}{|c|c|}
\hline Step & Action \\
\hline & B. Inside of recovery glovebox \\
\hline & $\begin{array}{l}\text { i. Verify base system purge port valve V-2033 closed (valve is after base pump and } \\
\text { before base flow meter) }\end{array}$ \\
\hline & C. Verify $1 / 4$ in. FEP tubing nitrogen purge line attached to base system purge port valve V- \\
\hline & 2033 \\
\hline & i. FEP tubing is attached to a stainless steel line that passes across glovebox wall and \\
\hline & ends near the solenoid vent valves manifold \\
\hline & D. Outside of recovery glovebox \\
\hline & i. Verify V-2038 for nitrogen service open \\
\hline & E. $\square \square \square$ Verify flow path through base flow meter $167 / 168$ (valves are already \\
\hline & open) \\
\hline & F. $\square \square \square$ Open V-0024/0025 (Base column bypass) using toggle switch at lower left \\
\hline & corner of [System].tab (using column bypass in case column still attached to system) \\
\hline & G. $\square \square \square$ Verify flow path through base column loading filter 28/29 (valves are \\
\hline & already open) \\
\hline & H. $\square \square \square$ Open Column stripping loop 1 on [Sample Collection].tab $\rightarrow$ [Column \\
\hline & Stripping].tab by pressing the purple $66 / 68$ button \\
\hline & I. $\square \square \square$ Open V-0134 (Base rinse) \\
\hline & J. $\square \square \square$ Open V-0156 (Effluent bottle vent) \\
\hline & i. V-0156 MUST BE OPEN DURING OPERATIONS - WHEN OPERATIONS \\
\hline & ARE NOT BEING CONDUCTED CLOSE THE VALVE \\
\hline & K. $\square \square \square$ Prepare 30 second timer \\
\hline & L. Open base system purge port valve V-2033 and start timer \\
\hline & M. At 30 second timer end close base system purge port valve V-2033 \\
\hline & N. $\square$ Open Column Stripping loop 2 on [Sample Collection].tab $\rightarrow$ [Column \\
\hline & Stripping].tab by pressing the purple $70 / 72$ button \\
\hline & i. The previous loop valves will automatically close when another loop is opened \\
\hline & O. $\square \square \square$ Prepare 30 second timer \\
\hline & P. Open base system purge port valve V-2033 and start timer \\
\hline & Q. At 30 second timer end close base system purge port valve V-2033 \\
\hline
\end{tabular}




\begin{tabular}{|c|c|}
\hline Step & Action \\
\hline & $\begin{array}{l}\text { R. Repeat steps } \mathbf{8 8 . 8 . N}-\mathbf{8 8 . 8 . Q} \text { for each Column Stripping loop, actuating the loop valves } \\
\text { using the following purple buttons on [Sample Collection].tab } \rightarrow \text { [Column } \\
\text { Stripping].tab } \\
\text { i. } \square \quad \square \quad \square \text { Loop 3: Valves 74/76 } \\
\text { ii. } \square \quad \square \quad \square \text { Loop 4: Valves 78/80 } \\
\text { iii. } \square \quad \square \quad \square \text { Loop 5: Valves 82/84 } \\
\text { iv. } \square \quad \square \quad \square \text { Loop 6: Valves 86/88 } \\
\text { v. } \square \quad \square \quad \square \text { Loop 7: Valves 90/92 } \\
\text { vi. } \square \quad \square \quad \square \text { Loop 8: Valves 94/96 } \\
\text { S. } \square \quad \square \quad \square \text { Verify base system purge port valve V-2033 is closed } \\
\text { T. NOTE - loop } 9 \text { does not get washed as it is not used to collect a sample } \\
\text { U. } \square \quad \square \quad \square \text { Close V-0134 (Base rinse) } \\
\text { V. } \square \quad \square \quad \square \text { Close V-0156 (Effluent bottle vent) } \\
\text { 88.9. Return to step } 88.6\end{array}$ \\
\hline 89. & $\begin{array}{l}\text { Summary } \\
\text { 89.1. All loops have now been washed and purged with } \mathrm{N}_{2} \\
\text { 89.2. All loops are now ready to receive samples } \\
\text { 89.3. If any acid flow paths are used then repeat steps } \mathbf{8 7 . 6} \text { through } \mathbf{8 7 . 9} \text { for the appropriate loops } \\
\text { 89.4. If any base flow paths are used then repeat steps } \mathbf{8 8 . 6} \text { through } \mathbf{8 8 . 9} \text { for the appropriate loops }\end{array}$ \\
\hline 90. & $\begin{aligned} & \text { End of Run } \text { II Initials: } \_ \text {Date: } \_ \text {Time: } \\
& \text { 90.1. } \square \text { Enter any final comments } \\
& \text { 90.2. } \square \text { Press the MASTER EXIT button at [System].tab } \\
& \text { A. Properly stops Mo99 Remote Recovery Data Acquisition \& Control Software }\end{aligned}$ \\
\hline
\end{tabular}




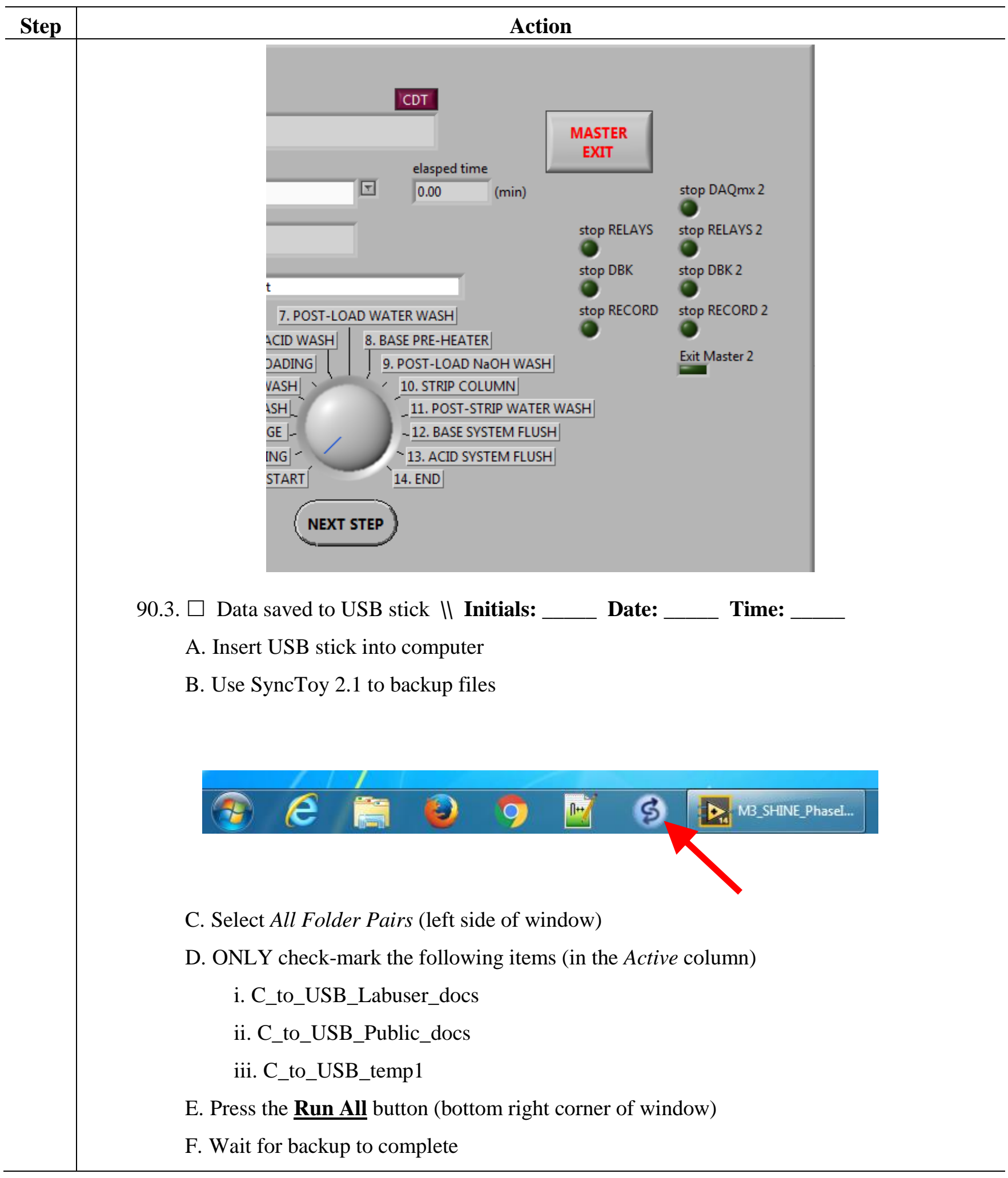




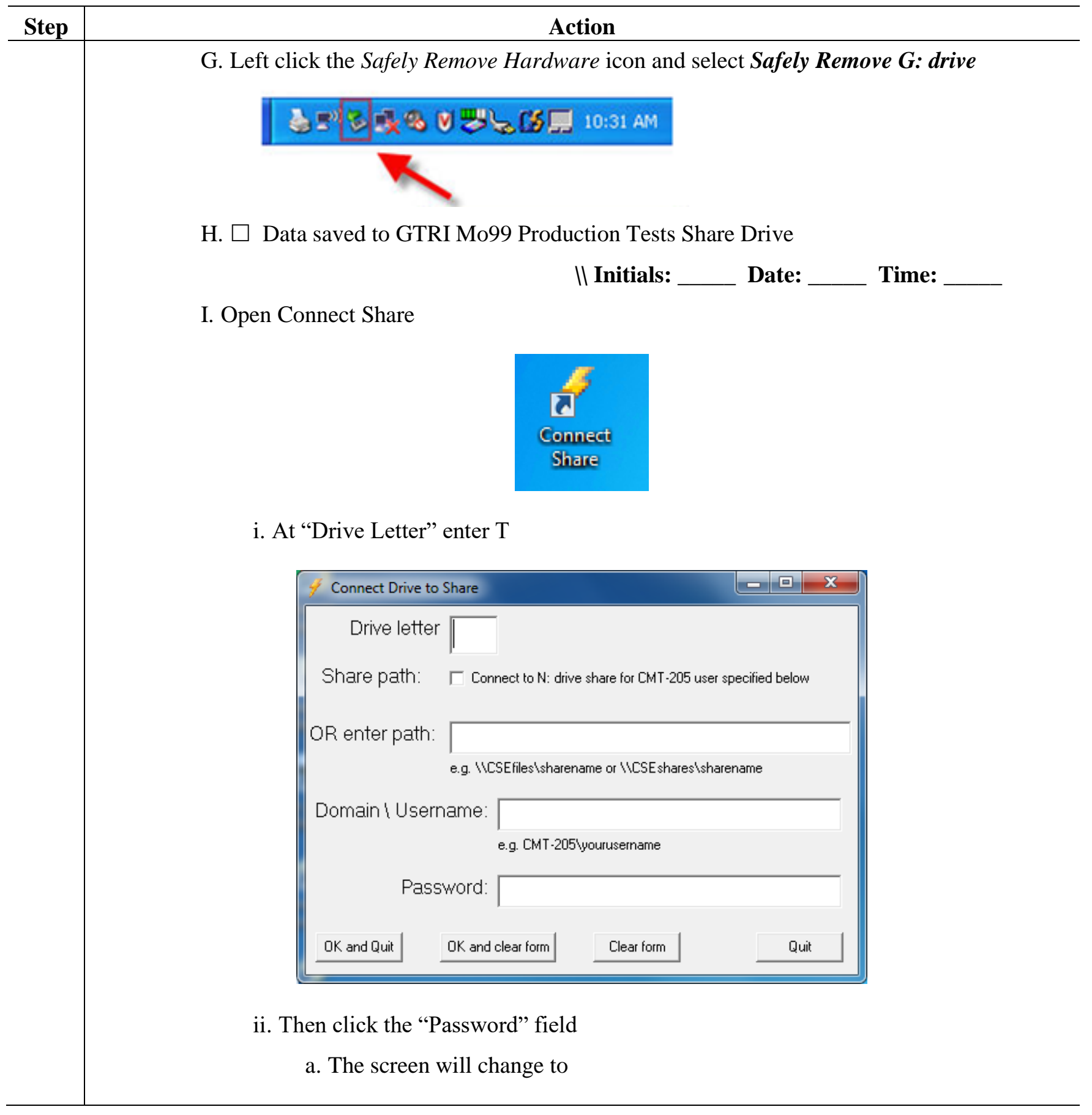




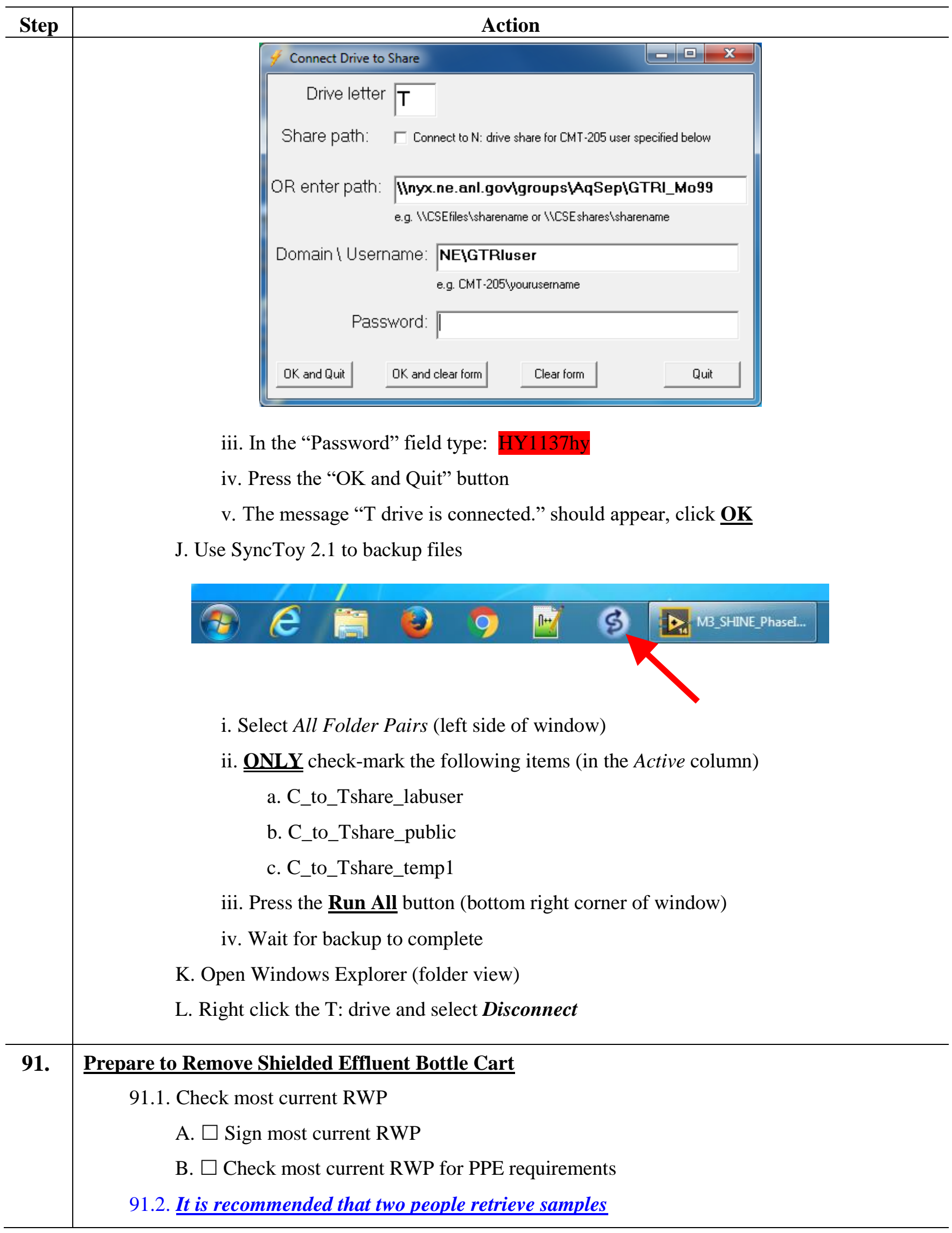




\begin{tabular}{|c|c|}
\hline Step & Action \\
\hline & A. Person A performs the work in cabinet \#3 \\
\hline & B. Person B communicates/marks these instructions to Person A \\
\hline \multirow[t]{19}{*}{92.} & Remove Shielded Effluent Bottle Cart \\
\hline & 92.1. At LabVIEW rack \\
\hline & A. Power off appropriate effluent balance indicator \\
\hline & 92.2. At recovery glovebox \\
\hline & A. Open cabinet \#3 (right side) door to full open \\
\hline & B. Position the 4-section ramps to roll shield effluent bottle cart out of cabinet \#3 \\
\hline & $\begin{array}{l}\text { C. Attach the handle to the effluent cart (handle and handle bolts should have been stored in } \\
\text { the instrument room until effluent cart is removed) }\end{array}$ \\
\hline & $\begin{array}{l}\text { D. Remove jacks used to level shielded effluent bottle cart (stored in cabinet \#3 once } \\
\text { effluent cart is removed) }\end{array}$ \\
\hline & E. $\square$ Verify balance lever in transport positon \\
\hline & $\begin{array}{l}\text { F. It may be necessary to pull the effluent cart out of cabinet \#3 a little to break the liquid } \\
\text { connections }\end{array}$ \\
\hline & 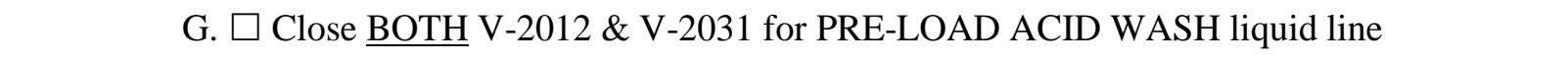 \\
\hline & i. V-2012 is connected to the line to the glovebox \\
\hline & ii. V-2031 is mounted to the manifold panel attached to the cart \\
\hline & H. $\square$ Disconnect V-2012 of PRE-LOAD ACID WASH glovebox line from effluent bottle // \\
\hline & glovebox liquid manifold connection \\
\hline & I. Wipe open end of V-2012 for PRE-LOAD ACID WASH liquid line from glovebox using \\
\hline & a paper towel (may be dampened with Radiac wash) \\
\hline & i. FITTING MUST BE DRY \\
\hline & $\begin{array}{l}\text { J. Install VCR cap on open end V-2012 for PRE-LOAD ACID WASH liquid line from } \\
\text { glovebox }\end{array}$ \\
\hline
\end{tabular}




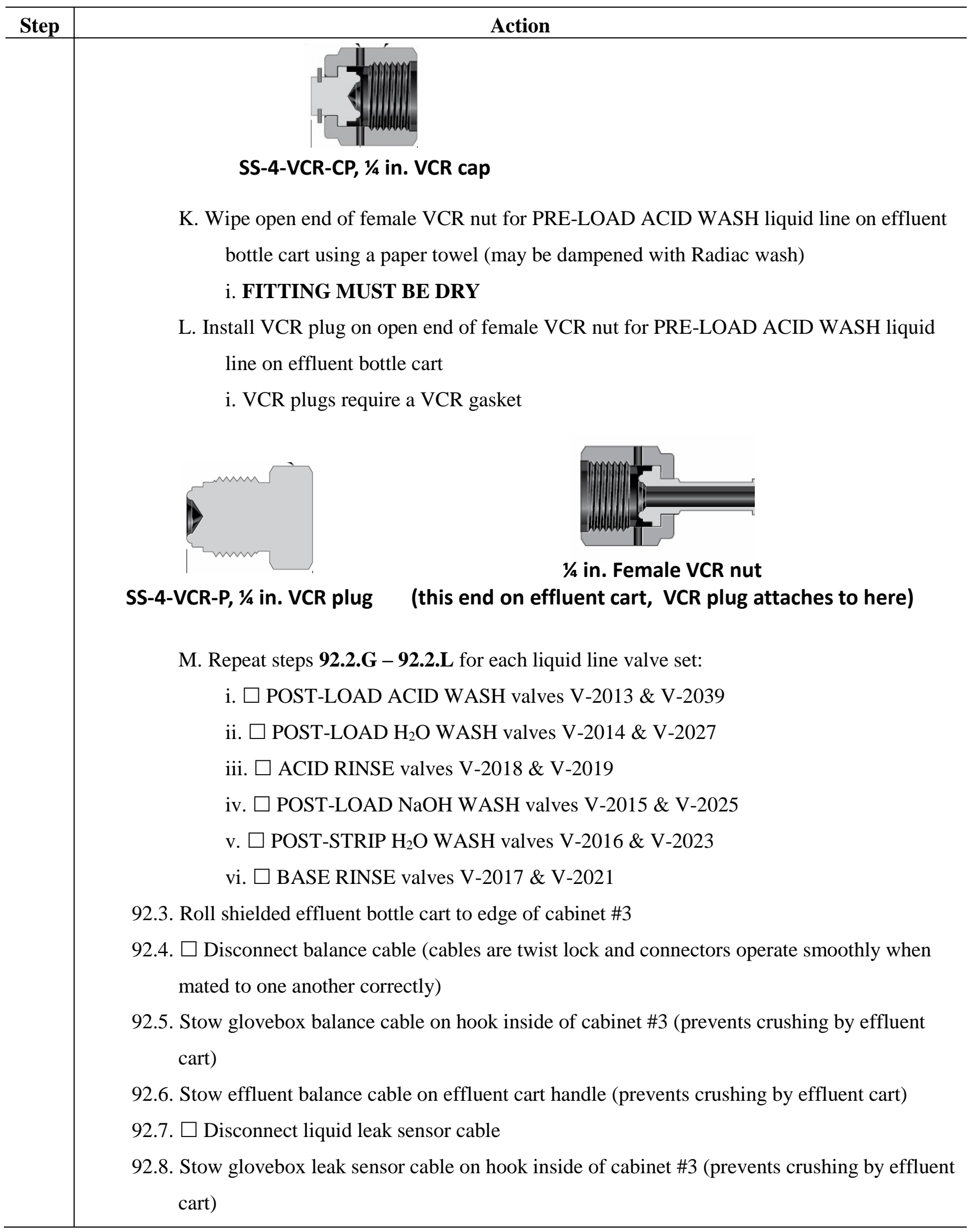




\begin{tabular}{|c|c|}
\hline Step & Action \\
\hline & 92.9. Stow effluent leak sensor cable on effluent cart handle (prevents crushing by effluent cart) \\
\hline & 92.10. $\square$ Close V-2011 for glovebox vent line \\
\hline & 92.11. $\square$ Close V-2032 for PRE-LOAD ACID WASH vent line \\
\hline & 92.12. $\square$ Close V-2030 for POST-LOAD ACID WASH vent line \\
\hline & 92.13. $\square$ Close V-2028 for POST-LOAD $\mathrm{H}_{2} \mathrm{O}$ WASH vent line \\
\hline & 92.14. $\square$ Close V-2020 for ACID RINSE vent line \\
\hline & 92.15. $\square$ Close V-2026 for POST-LOAD NaOH WASH vent line \\
\hline & 92.16. $\square$ Close V-2024 for POST-STRIP $\mathrm{H}_{2} \mathrm{O}$ WASH vent line \\
\hline & 92.17. $\square$ Close V-2022 for BASE RINSE vent line \\
\hline & 92.18. $\square$ Disconnect V-2011 of glovebox vent line from effluent bottle // glovebox vent manifold \\
\hline & connection \\
\hline & 92.19. Wipe open end of V-2011 for vent line from glovebox using a paper towel (may be \\
\hline & dampened with Radiac wash) \\
\hline & A. FITTING MUST BE DRY \\
\hline & 92.20. Install VCR cap on open end of V-2011 vent line from glovebox \\
\hline & A. VCR caps require a VCR gasket \\
\hline & 92.21. Wipe open end of female VCR nut for vent line on effluent bottle cart using a paper towel \\
\hline & (may be dampened with Radiac wash) \\
\hline & A. FITTING MUST BE DRY \\
\hline & 92.22. Install VCR plug on open end of female VCR nut for vent line on effluent bottle cart \\
\hline & A. VCR plugs require a VCR gasket \\
\hline & 92.23. Remove shielded effluent bottle cart from cabinet \#3 \\
\hline & 92.24. Remove 4-section ramps \\
\hline & A. DO NOT STORE 4-SECTION RAMPS IN CELL 1 \\
\hline & Close cabinet \#3 door slowly \\
\hline
\end{tabular}

\section{Records Created by Work Process}

The records listed below must be retained as indicated.

\begin{tabular}{l|c|c|l|l}
\hline $\begin{array}{l}\text { Description of Record } \\
\text { (include form number if } \\
\text { applicable) }\end{array}$ & $\begin{array}{c}\text { Active } \\
\text { Records } \\
\text { Custodian }\end{array}$ & $\begin{array}{c}\text { Active } \\
\text { Records } \\
\text { Retention }\end{array}$ & $\begin{array}{l}\text { Indexing Method, } \\
\text { Storage Medium }\end{array}$ & $\begin{array}{l}\text { Federal Retention } \\
\text { Requirements* }\end{array}$ \\
\hline $\begin{array}{l}\text { LEAF-PROC-024 } \\
\text { sections 3.2.1-3.2.6, data }\end{array}$ & $\begin{array}{c}\text { Facility } \\
\text { Manager }\end{array}$ & 3 years & $\begin{array}{l}\text { Index by job date } \\
\text { and name, store on }\end{array}$ & $\begin{array}{l}\text { Destroy 75 years after } \\
\text { the date of the permit }\end{array}$ \\
\hline
\end{tabular}




\begin{tabular}{l|l|l|l|l}
\hline $\begin{array}{l}\text { Description of Record } \\
\text { (include form number if } \\
\text { applicable) }\end{array}$ & $\begin{array}{c}\text { Active } \\
\text { Records } \\
\text { Custodian }\end{array}$ & $\begin{array}{c}\text { Active } \\
\text { Records } \\
\text { Retention }\end{array}$ & $\begin{array}{l}\text { Indexing Method, } \\
\text { Storage Medium }\end{array}$ & $\begin{array}{l}\text { Federal Retention } \\
\text { Requirements* }\end{array}$ \\
\hline collection pages & & $\begin{array}{l}\text { paper or } \\
\text { electronically }\end{array}$ & (DOE ADM 18.37) \\
\hline
\end{tabular}

*If records are maintained in a business information system that is not currently programmed to purge digital records based on age, the records may be retained in that system past the indicated destruction date.

\section{Exhibit A - Hardware List}

1. Swagelok VCR stainless steel gaskets: SS-4-VCR-2-GR for $1 / 4$ in

2. Verification Tank Lines

a. Pickup line attached at bottom of tank through 1-1/2 in tri-clamp fitting

i. $1 / 4$ in. OD x 0.21 in. ID 316 SS tubing (McMaster 89785K822)

ii. $1 / 4$ in. Swagelok $x 1 / 4$ in. Swagelok union (SS-400-6)

iii. $1 / 4$ in. OD x 3/16 in. ID FEP tubing (McMaster 2129T13)

iv. Stainless steel ferrules can be used with FEP tubing

v. $1 / 4$ in. Swagelok x $1 / 4$ in. VCR female connector (SS-4-WVCR-6-400)

vi. $1 / 4$ in. double VCR male 2-way ball valve (SS-43GVCR4)

b. Return line attached to tank cover

i. $1 / 4$ in. OD x 0.21 in. ID 316SS tubing (McMaster 89785K512)

ii. $1 / 4$ in. Swagelok $x 1 / 4$ in. Swagelok union (SS-400-6)

iii. $1 / 4$ in. OD x 3/16 in. ID FEP tubing (McMaster 2129T13)

iv. $1 / 4$ in. Swagelok $x 1 / 4$ in. VCR female connector (SS-4-WVCR-6-400)

v. $1 / 4$ in. double VCR male 2-way ball valve (SS-43GVCR4)

c. $1 / 8$ in sample pickup \& vent lines

i. Vent line attached to tank cover

ii. $1 / 8$ in. OD x 0.055 in. ID 316SS tubing (McMaster 89785 K511)

iii. $1 / 8$ in. Swagelok $x 1 / 8$ in. Swagelok union (SS-200-6)

iv. $1 / 8$ in. OD FEP tubing (McMaster 2129T11)

v. $1 / 8$ in. Swagelok fittings 2 -way ball valve (SS-41GS2)

d. Sample pickup line attached to tank cover

i. $1 / 8$ in. OD x 0.055 in. ID 316SS tubing (McMaster 89785K511)

ii. $1 / 8$ in. Swagelok $x 1 / 8$ in. Swagelok union (SS-200-6)

iii. $1 / 8$ in. OD FEP tubing (McMaster 2129T11)

iv. $1 / 8$ in. Swagelok fittings 2 -way ball valve (SS-41GS2)

3. Feed connection jumper

a. Feed valve V-3003 (SS-43GXS4)

i. Middle arm to V-3001 
1. $1 / 4$ in. OD x 3/16 in. ID FEP tubing (McMaster 2129T13)

2. $1 / 4 \mathrm{in}$. Swagelok $x 1 / 4 \mathrm{in}$. VCR female connector (SS-4-WVCR-6-400)

a. VCR female end faces away from 3-way valve

3. Middle arm of V-3001 to V-0003

a. $1 / 4$ in. OD x 3/16 in. ID FEP tubing (McMaster 2129T13)

b. $1 / 4$ in. Swagelok $x 1 / 4$ in. VCR female connector (SS-4-WVCR-6-400)

i. VCR female end faces away from 3-way valve

ii. Side arm to Verification tank pickup line V-2034

1. $1 / 4$ in. OD x 3/16 in. ID FEP tubing (McMaster 2129T13)

2. $1 / 4$ in. Swagelok $x 1 / 4$ in. VCR female connector (SS-4-WVCR-6-400)

a. VCR female end faces away from 3-way valve

3. Verification tank pickup 2-way ball valve ends in male VCR

4. VCR female end from feed jumper attaches to free male end of liquid pickup VCR 2-way ball valve

iii. Side arm from feed bottle inside glovebox

1. $1 / 4$ in. OD x 3/16 in. ID FEP tubing (McMaster 2129T13)

4. Effluent connection jumper

a. Effluent valve V-3002 (SS-43GXS4)

i. Middle arm from V-0011

1. $1 / 4$ in. Swagelok $x 1 / 4$ in. VCR female connector (SS-4-WVCR-6-400)

a. VCR female end faces away from 3-way valve and attaches to V-0011

2. $1 / 4$ in. OD x 3/16 in. ID FEP tubing (McMaster 2129T13)

ii. Side arm to Verification tank return V-2036

1. $1 / 4$ in. OD x 3/16 in. ID FEP tubing (McMaster 2129T13)

2. $1 / 4$ in. Swagelok $x 1 / 4$ in. VCR female connector (SS-4-WVCR-6-400)

a. VCR female end faces away from 3-way valve

3. Verification tank return 2-way ball valve ends in male VCR

4. VCR female end from effluent jumper attaches to free male end of liquid pickup VCR 2-way ball valve

iii. Side arm to effluent bottle inside glovebox 
1. $1 / 4$ in. OD x 3/16 in. ID FEP tubing (McMaster 2129T13)

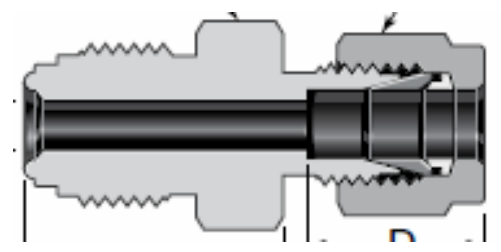

Figure 11/4 in. OD FEP tubing with SS-4-VCR-6-400 attached to each end

5. Alternative Sample Retrieval fitting assembly (p. 30)

a. SS-4-WVCR-6-400, 1/4 in. VCR x 1/4 in. Swagelok nut

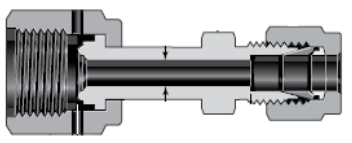

b. SS-200-R-4, 1/8 in. Swagelok nut $x 1 / 4$ in. tubing stub

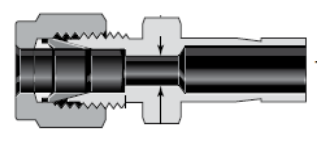

c. 1/8 in. OD FEP tubing from lower stopcock attached to the $1 / 8$ in. Swagelok nut

6. Feed Bottle Secondary Tray

a. McMaster 26775T23

i. Polypropylene Plastic Pan 20-3/8" long, 19" wide, 3-1/4" high

b. McMaster 4141T5

i. Food-Grade Polyethylene Plastic Pan 23" long, 19" wide, 4" high

7. Effluent Bottles with silicone seals

a. McMaster 4322T4

\begin{tabular}{|l|l|l|l|}
\hline \multicolumn{3}{|c|}{ Semi-Clear Polypropylene Plastic Jar 64 oz./1900 ml Capacity, 5" Diameter } \\
\hline Style & M & Mouth OD & 2 3/4" \\
\hline Capacity & 64 oz./1900 mL & Graduated & No \\
\hline Diameter & $5 "$ & Seal Material & Silicone Rubber \\
\hline Height & $83 / 8 "$ & Includes & Lid (threaded) \\
\hline
\end{tabular}

b. McMaster 4322T6

\begin{tabular}{|l|l|l|l|}
\hline \multicolumn{4}{|c|}{ Semi-Clear Polypropylene Plastic Jar 1 Gallon/3775 ml Capacity } \\
\hline Style & M & Mouth OD & $23 / 4 ”$ \\
\hline Capacity & 1 gal./3775 ml & Graduated & No \\
\hline Diameter & $57 / 8 ”$ & Seal Material & Silicone Rubber \\
\hline Height & $111 / 4 "$ & Includes & Lid (threaded) \\
\hline
\end{tabular}

c. McMaster $4322 \mathrm{~T} 7$

\begin{tabular}{|l|l|l|l|}
\hline \multicolumn{4}{|l|}{ Semi-Clear Polypropylene Plastic Jar 2 Gallon/7575 ml Capacity } \\
\hline Style & M & Mouth OD & $23 / 4 "$ \\
\hline Capacity & 2 gal./7575 ml & Graduated & No \\
\hline Diameter & $71 / 2 "$ & Seal Material & Silicone Rubber \\
\hline Height & $131 / 8 ”$ & Includes & Lid (threaded) \\
\hline
\end{tabular}


8. Acid Rinse Bottle (Base Rinse Bottle is same model)

a. McMaster 9884T14

\begin{tabular}{|l|l|l|l|}
\hline \multicolumn{4}{|c|}{ Semi-Clear High-Density Polyethylene Plastic Jug 5 Gallon/18950 ml Capacity } \\
\hline Capacity & 5 gal./18950 ml & Mouth OD & $37 / 8 ”$ \\
\hline Width & $9 "$ & Graduation & $1 / 4$ gal / 1 L \\
\hline Depth & $123 / 4 "$ & Includes & Lid (threaded) \\
\hline Height & $15 / 4 "$ & Handle & Stainless Steel \\
\hline
\end{tabular}

9. Effluent bottle connections

a. 1/4 in. Liquid connections

i. Swagelok SS-400-1-OR

1. $1 / 4$ in. tube $x 7 / 16-20$ straight thread O-Seal

2. Use McMaster 94758A645 18-8 Stainless Steel Flange Nut

ii. $1 / 4$ in. OD x 3/16 in. ID FEP tubing (McMaster 2129T13)

1. $1 / 4$ in. Swagelok $x 1 / 4$ in. VCR female connector (SS-4-WVCR-6-400)

2. $1 / 4$ in. double VCR male 2-way ball valve (SS-43GVCR4)

\section{a. HANDLE POINTS TO BOTTLE (DIRECTION OF FLOW TO BOTTLE)}

b. $1 / 4$ in. VCR female cap (SS-4-VCR-CP)

i. Cap attached to valve when bottle not attached to effluent bottle cart liquid manifold

3. 2-way ball valve from bottle attaches to female VCR nut on inside face of effluent bottle // glovebox liquid manifold

i. Effluent bottle // glovebox liquid manifold is permanently attached to an shielded effluent bottle cart

iii. Effluent lines from glovebox

1. There are 7 effluent lines

a. Acid effluent lines (hexagon tags)

i. Pre-load acid wash

ii. Post-load acid wash

iii. Post-load water wash

iv. Acid rinse

b. Base effluent lines (diamond tags)

i. Post-load $\mathrm{NaOH}$ wash

ii. Post-strip water wash 
iii. Base rinse

2. Terminated with VCR 2-way ball valve (SS-43GVCR4)

\section{a. HANDLE POINTS AWAY FROM GLOVEBOX (DIRECTION OF FLOW TO BOTTLE)}

3. Rotating VCR female union elbow (6LV-4-WVCR-9-DF)

a. Attached to valve outlet

4. $1 / 4$ in. VCR male union (SS-4-VCR-6-DM)

a. Attached to elbow outlet

5. Free end of VCR male union attaches to female VCR fitting at outside face of effluent bottle // glovebox liquid manifold

b. 1/8 in. Vent connections

i. Swagelok SS -200-1-OR

1. $1 / 8$ in. tube $x$ 5/16-24 straight thread O-Seal

a. Use McMaster 93776A451 18-8 Stainless Steel Serrated Flange Locknut

2. 1/8 in. OD FEP tubing (McMaster 2129T11)

3. $1 / 8$ in. tube $x \frac{1 / 4}{4}$ in. stub reducer (SS-200-R-4)

4. $1 / 4$ in. Swagelok $x 1 / 4$ in. VCR female connector (SS-4-WVCR-6-400)

5. $1 / 4$ in. double VCR male 2-way ball valve (SS-43GVCR4)

\section{a. HANDLE POINTS AWAY FROM BOTTLE (DIRECTION OF FLOW TO GAS COLLECTION SYSTEM)}

b. $1 / 4$ in. VCR female cap (SS-4-VCR-CP) attached to valve when bottle not attached to effluent bottle cart liquid manifold

6. 2-way ball valve from bottle attaches to VCR female nut on inside face of effluent bottle // glovebox vent manifold

a. Effluent bottle // glovebox vent manifold is permanently attached to a shielded effluent bottle cart

7. Vent line from glovebox

a. Single vent line comes out of glovebox

i. Line attached to solenoid valve 156 that vents to the gas collection system

b. Terminated with double VCR male 2-way ball valve (SS-43GVCR4)

i. HANDLE POINTS TOWARD GLOVEBOX (DIRECTION OF FLOW TO GAS COLLECTION) 
c. Free end of 2-way valve attaches to female VCR fitting at outside face of effluent bottle // glovebox vent manifold

10. Pre-evacuated vials

a. Hollister-Stier $10 \mathrm{~mL}$ evacuated vial (7519ZA), Fisher Scientific catalog number NC9538328

11. Sampling Retrieval Assemblies

a. There are two $(2 \mathrm{x})$ needles assembled in a concentric fashion

i. The inner 18 gauge needle handles the liquid (1/16 in. OD tubing)

ii. The outer 14 gauge needle handles the venting (1/8 in. OD tubing)

b. Two concentric needle assembly and bottom row 3-way Luer lock valve daisy chain assembly

i. 14 gauge $\mathrm{x} 2$ in. Luer Lock needle (vent to vacuum)

1. Fisher 14-815-404
a. Length (English) Needle 2 in.
b. Diameter (Metric) Outer $2.11 \mathrm{~mm}$
c. Diameter (Metric) Inner $1.6 \mathrm{~mm}$
d. Length (Metric) Needle $51 \mathrm{~mm}$
e. Needle Point Style Sharp, Bevel, Curved
f. Needle Gauge 14 ga.
g. Material (Hub) Nickel Plated Brass
h. Material Stainless steel
i. Hamilton 90014

ii. Connects to double Luer lock male 316SS union

1. McMaster 5194K31

iii. Union connects to bottom arm of female Luer tee

1. Polypropylene Luer adapter tees female

2. Fisher NC0644969

iv. Male Luer quick-turn coupling connects to upper arm of female Luer tee

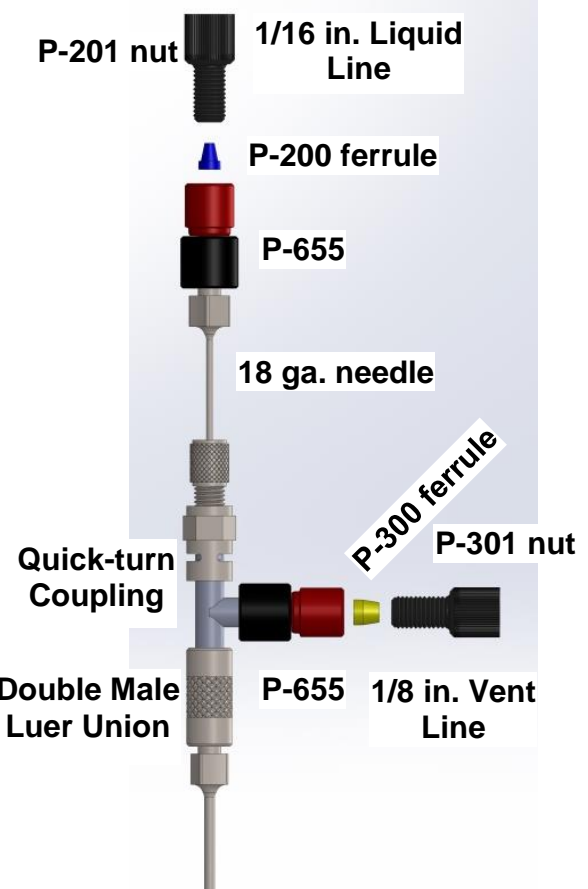

14 ga. needle

1. McMaster 5194K41

v. 18 gauge x 6 in. Luer Lock needle (liquid) inserts through coupling

1. 18 gauge needle inserts through 14 gauge needle

2. 18 gauge needle tip should be slightly below 14 gauge needle tip

vi. Fisher 01-290-25

1. Length (English) Needle 6 in.

2. Length (Metric) Needle $152 \mathrm{~mm}$

3. Needle Point Style Deflected End

4. Needle Gauge 18 ga. 
5. Material (Hub) Micro-Mate ${ }^{\mathrm{TM}}$ Stainless Steel

6. Cadence Science 9860

vii. Male Luer lock x 1/4-28 nut adapter attaches to 18 gauge needle

1. Adapter, Luer (M-lock) to 1/4-28 FB (F) PEEK 1.02 mm (0.040")

a. Fisher 14221-484

b. Upchurch (Idex) P-655

2. Upchurch Scientific ${ }^{\mathrm{TM}}$ Flangeless Nuts: Compatible with 1/4-28, Delrin

a. Upchurch Scientific ${ }^{\text {TM }}$ P301X

b. Fisher 05-700-102

c. $\quad 0.25$ to 28 flat bottom, Flangeless standard knurled head nut

viii. 1/16 in. OD FEP tubing attaches from male Luer lock $x$ 1/4-28 nut adapter to liquid sample solenoid valve of appropriate sampling loop

ix. $\quad 2^{\text {nd }}$ male Luer lock x $1 / 4-28$ nut adapter attaches to side arm of female Luer tee

x. $\quad 1 / 8$ in. OD FEP tubing attaches from $2^{\text {nd }}$ male Luer lock $x$ 1/4-28 nut adapter to side arm of appropriate 3-way valve on bottom row of 3-way valve daisy chain assembly

c. Top row 3-way Luer lock valve daisy chain assembly

i. 1/16 in. OD FEP tubing from appropriate sample loop vent valve to female Luer lock $\mathrm{x}$ 1/4-28 nut adapter

1. Adapter, Luer (F) to 1/4-28 FB (F), PEEK, $1.27 \mathrm{~mm}(0.050 ")$

a. Upchurch (Idex) P-658

b. VWR 14221-486

ii. Female Luer lock x 1/4-28 nut adapter to one-way Luer lock check valve

1. One-way Luer check valve

a. Fisher NC0232677

iii. One-way Luer check valve to double male Luer lock coupling

1. Cole-Parmer Polycarbonate fittings; male lock x male lock, rotating; 10/pack, Non-sterile

a. Cole-Parmer EW-30600-50

b. Fisher NC0580839

d. FEP TUBING .030 in ID x .062 in. OD x $100 \mathrm{ft}$

i. Fisher 05-7011-72

ii. This is the $1 / 16$ in. OD tubing

e. FEP tubing $1 / 8$ in. OD x 3/16 in. ID

i. McMaster 2129T13

f. Clean, dry Becton-Dickinson (B-D) $60 \mathrm{~mL}$ syringe 
i. Used for checking operability of check valves

12. Vacuum pump for sample retrieval

a. McMaster, 4404K29, 12VDC

13. Tweezers for retrieving samples from shields

a. Tweezers, 12 in., McMaster, 7379A24

14. VCR Cap

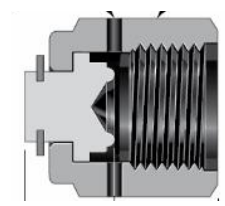

SS-4-VCR-CP, $1 / 4$ in. VCR cap

15. VCR Plug

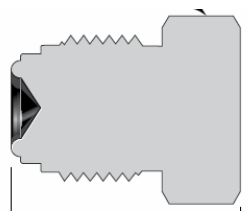

\section{SS-4-VCR-P, $1 / 4$ in. VCR plug}

16. Bottles for receiving loop washings

a. Thermo Scientific ${ }^{\mathrm{TM}}$ Nalgene ${ }^{\mathrm{TM}}$ Square PETG Media Bottles with Septum Closure

i. Use non-glass bottles inside the glovebox

ii. 60 mL: Fisher 03-313-900, Thermo Scientific 3420230060, case of 200

iii. 125 mL: Fisher 03-313-901, Thermo Scientific 3420230125, case of 96

iv. $500 \mathrm{~mL}$ : Fisher 03-313-902, Thermo Scientific 3420230500, case of 40 
6 Exhibit B - PFC-PCS Cheat Sheet

\begin{tabular}{|l|l|l|l|}
\hline \multicolumn{1}{|c|}{ Acid Solution Volumes } & \multicolumn{1}{|c|}{$(\mathbf{m L})$} & \multicolumn{1}{|c|}{ Water Volumes } & \\
\hline Leak Checking Column & & Post-Load Water Wash & \\
\hline Pre-Pre-Load Acid & & Post-Strip Water Wash (cell) & \\
\hline Pre-Load Acid & & Post-Strip Water Wash (waste) & \\
\hline Post Load Acid & & Lase System Final Rinse & \\
\hline Final Acid Wash & & Base System Rinse & \\
\hline Loop Rinsing & & Priming Lines & \\
\hline Acid Rinse Out & & Total Water Required & \\
\hline Line Priming & & & \\
\hline Total Acid Required & & & \\
\hline & & Base Strip Volumes & \\
\hline \multicolumn{1}{|c|}{ Base Wash Volumes } & $(\mathbf{m L})$ & Priming Lines & \\
\hline Base Pre-Heater Start & & Total NaOH Strip Required & \\
\hline Post-Load NaOH Wash & & & \\
\hline Priming Lines & & & \\
\hline Total NaOH Wash Required & & & \\
\hline
\end{tabular}

\begin{tabular}{|l|l|l|l|l|}
\hline \multicolumn{1}{|c|}{ Solution Properties } & Density $(\mathbf{g} / \mathbf{m L})$ & Mass $(\mathbf{g})$ & Volume $(\mathbf{m L})$ & Conc. \\
\hline Acid Solution & & & & \\
\hline Base Wash Solution & & & & \\
\hline Base Strip Solution & & & & \\
\hline Uranyl Sulfate Solution & & & & \\
\hline
\end{tabular}

\begin{tabular}{|c|c|}
\hline \multicolumn{2}{|c|}{ Flow Rate Acid Motor Powers } \\
\hline Flow Rate $(\mathrm{mL} / \mathrm{min})$ & Motor \% \\
\hline 80 & \\
\hline 84 & \\
\hline 100 & \\
\hline 167 & \\
\hline 200 & \\
\hline 300 & \\
\hline \multicolumn{2}{|c|}{ Flow Rate Base Motor Powers } \\
\hline Flow Rate $(\mathrm{mL} / \mathrm{min})$ & Motor $\%$ \\
\hline 84 & \\
\hline
\end{tabular}




\begin{tabular}{|l|l|l|l|l|l|}
\hline \multicolumn{5}{|c|}{ Samples Summary } \\
\hline \multicolumn{2}{|c|}{ Target Mixing } & \multicolumn{2}{c|}{ Column Loading } & \multicolumn{2}{c|}{ Column Stripping } \\
\hline 1. $\square$ Taken & 1. $\square$ Recovered & 1. $\square$ Taken & 1. $\square$ Recovered & 1. $\square$ Taken & 1. $\square$ Recovered \\
\hline 2. $\square$ Taken & 2. $\square$ Recovered & 2. $\square$ Taken & 2. $\square$ Recovered & 2. $\square$ Taken & 2. $\square$ Recovered \\
\hline 3. $\square$ Taken & 3. $\square$ Recovered & 3. $\square$ Taken & 3. $\square$ Recovered & 3. $\square$ Taken & 3. $\square$ Recovered \\
\hline 4. $\square$ Taken & 4. $\square$ Recovered & 4. $\square$ Taken & 4. $\square$ Recovered & 4. $\square$ Taken & 4. $\square$ Recovered \\
\hline 5. $\square$ Taken & 5. $\square$ Recovered & 5. $\square$ Taken & 5. $\square$ Recovered & 5. $\square$ Taken & 5. $\square$ Recovered \\
\hline 6. $\square$ Taken & 6. $\square$ Recovered & 6. $\square$ Taken & 6. $\square$ Recovered & 6. $\square$ Taken & 6. $\square$ Recovered \\
\hline 7. $\square$ Taken & 7. $\square$ Recovered & 7. $\square$ Taken & 7. $\square$ Recovered & 7. $\square$ Taken & 7. $\square$ Recovered \\
\hline 8. $\square$ Taken & 8. $\square$ Recovered & 8. $\square$ Taken & 8. $\square$ Recovered & 8. $\square$ Taken & 8. $\square$ Recovered \\
\hline
\end{tabular}




\section{Related Documents}

None

\section{Definitions}

None

9 About this Procedure

\begin{tabular}{ll}
\hline Issuing organization: & Low Energy Accelerator Facility \\
\hline Procedure owner: & S. Chemerisov \\
\hline Point of contact: & S. Chemerisov \\
\hline Review cycle (months): & 36 \\
\hline Date last revised: & August 23, 2019 \\
\hline Date last reviewed: & August 23, 2019 \\
\hline
\end{tabular}




\section{Summary of Changes in This Version}

Added Flow Rate Base Motor Powers entries and table with samples taken/retrieved to PFC-PCS Cheat Sheet

Removed all instances of recording dump tank load cell mass

Removed step 27.18 (leveling effluent cart) since this is not done

Step 39, changed 2WV-801 specification to OPEN, to match the hot cell team sheet

Added a statement to steps 42 and 43 that if steps 25 and 26 (respectively) have been performed in the last week, these steps may be skipped.

Changed units and associated calculations in steps 53.8, 54.7, 55.7, 56.7, 58.8, 59.12, 61.10, and 62.9 from

minutes to seconds since the countdown timer in the software is also in seconds

Step 59.5C changed formula from 59.5A/59.5B to 59.5A x 59.5B since volume $\mathrm{x}$ density $=$ mass

Removed step 61.15 since the system is already shut off from the hot cell at this point and the balance reading will not change from the beginning to the end of this step. Also changed the associated cell on p. 88 to "not applicable"

Added column to the table on p. 88 to document total acid, base wash, base strip, and water used, as well as volume remaining in acid rinse and base rinse receivers so cabinet does not need to be opened to determine if line and sample loop rinsing can commence

Made the font of the table on p. 88 smaller to make it fit on one page

Added "(if the manual dump tank valve cannot be accessed yet, proceed to step 67)" to step 65

Moved step 80.2.BB to 80.2.Z, where prompted to open the valve

Moved step 81.4.W to 81.4.U, where prompted to open the valve

Step 82 , changed 2WV-801 specification to OPEN, to match the hot cell team sheet

Updated the link in step 85.4

Added step 44.38 to ensure flow path is through the flow meter bypass sample loop

Added step 51.2 to shut off the flow meter bypass loop and isolate the sample during the rest of processing Added step 75.23 describing how to retrieve a sample from the flow meter bypass sample loop

Added step 77 for sampling the effluent cart bottles and updated links in all subsequent steps to be accurate

02/10/2020. Derek McLain, SSS Div. 


\section{Attachment: Mo99 Primary Recovery System - No Specific Path}

Argonne Chemical and Fuel Cycle Technologies (CFC) Division drawing

Drawing Title: AMORE_MO99 RECOVERY PROCESS PHASE 2_REV23.VSD

Revision Date: 2/18/2019

Revised by John F. Krebs

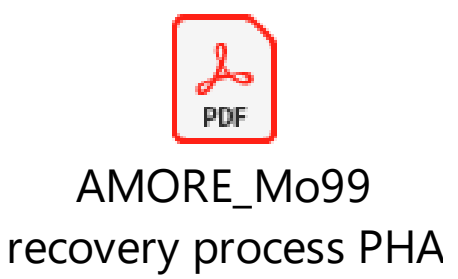




\section{APPENDIX 18}

Resin Washing Procedure 
1) Weigh out approximately $200 \mathrm{~g}$ of dry resin into a $2 \mathrm{~L}$ beaker.

a. Resin: Zirchrom $\mathrm{TiO}_{2}$-Bulk-110(60)

2) Add $1 \mathrm{~L}$ of $0.5 \mathrm{M} \mathrm{H}_{2} \mathrm{SO}_{4}$ to the beaker and place an overhead stirrer into the solution, $1-2 \mathrm{~cm}$ above the bottom of the beaker.

3) Turn on the stirrer and allow to mix for 20 minutes

4) Turn off the stirrer and allow the resin to settle to the bottom of the beaker for approx. 1 min.

5) Decant the used $\mathrm{H}_{2} \mathrm{SO}_{4}$ to waste.

6) Repeat steps 2-5 an additional four times, for a total of five sulfuric acid washes.

7) Add at least $0.5 \mathrm{~L}$ of DI water to the beaker and stir for 5 minutes to rinse the resin.

8) Allow the resin to settle to the bottom of the beaker and decant the water to waste.

9) Use fresh DI water to quantitatively transfer the washed resin to a labeled plastic bottle for storage until needed.

Column Packing Procedure

1) Assemble the bottom half of the column to the specified dimensions and clamp it in a ring stand. The bottom collar should be tightened until the edge is $7 / 8^{\prime \prime}$ from the end of the threading.

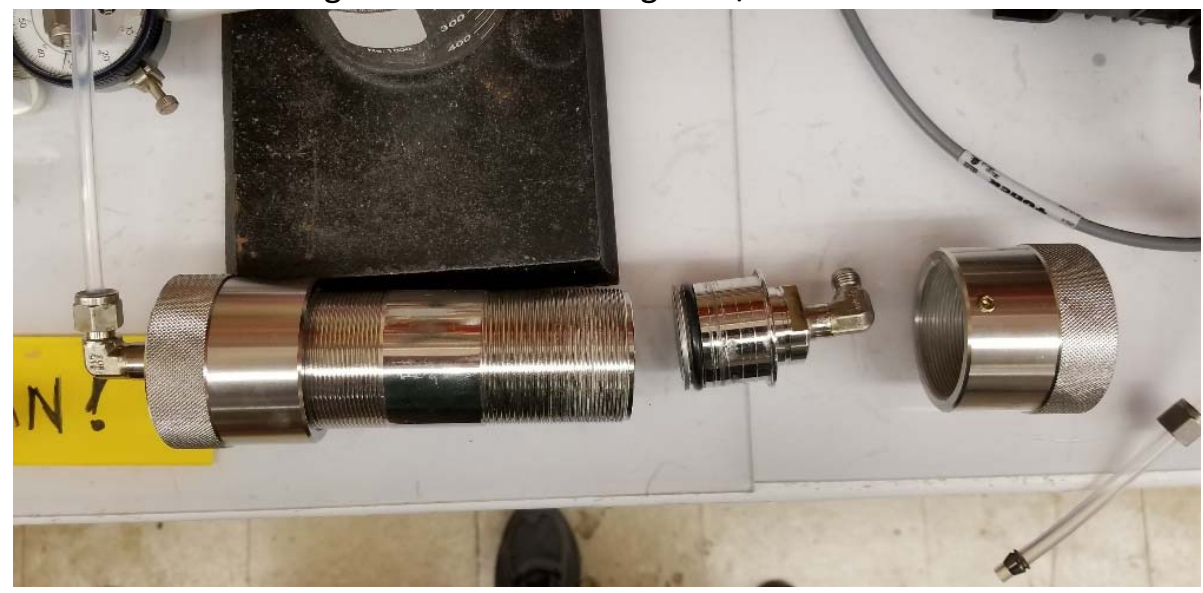

Figure 1: Column with bottom frit and collar in place and connected to tubing. Top frit and collar also included

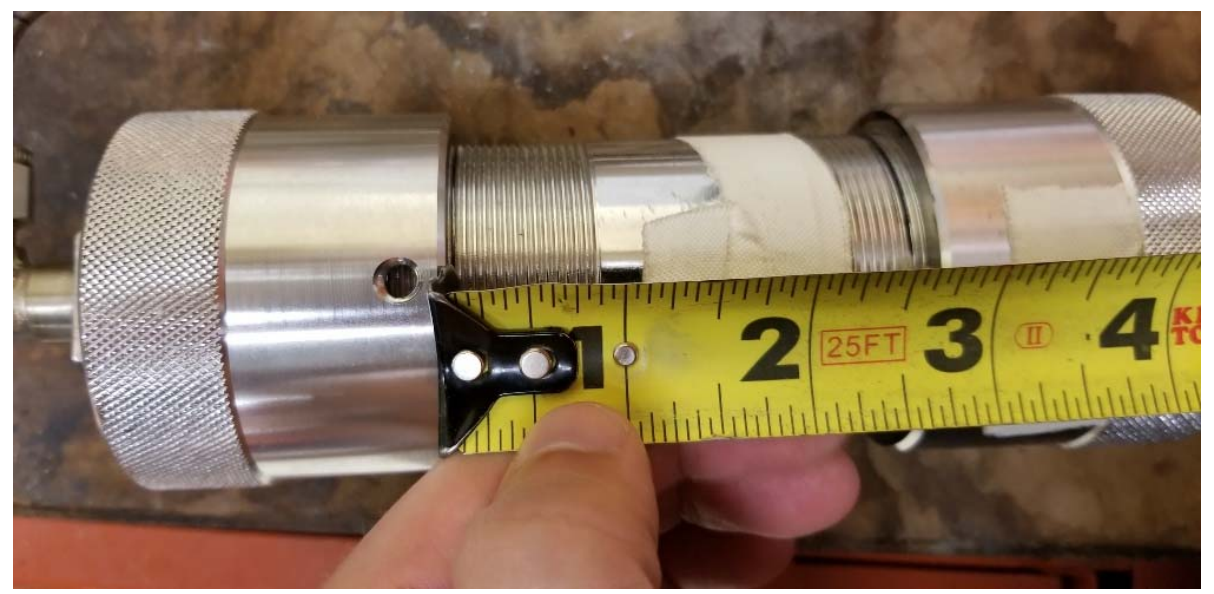

Figure 2: Distance from bottom collar to end of threading 
2) Place a beaker under the column to catch water as it drains through.

3) If desired, place a mark on the inside of the column $4 \frac{1 / 4}{4}$ above the bottom frit using a sharpie. This should be the approximate fill line of the column.

4) Slurry the previously washed titania resin (see above) with water and pour it into the column, allowing excess water to drain out the bottom while keeping a small head of fluid over the resin bed. $200 \mathrm{~g}$ of dry resin should be slightly more than needed.
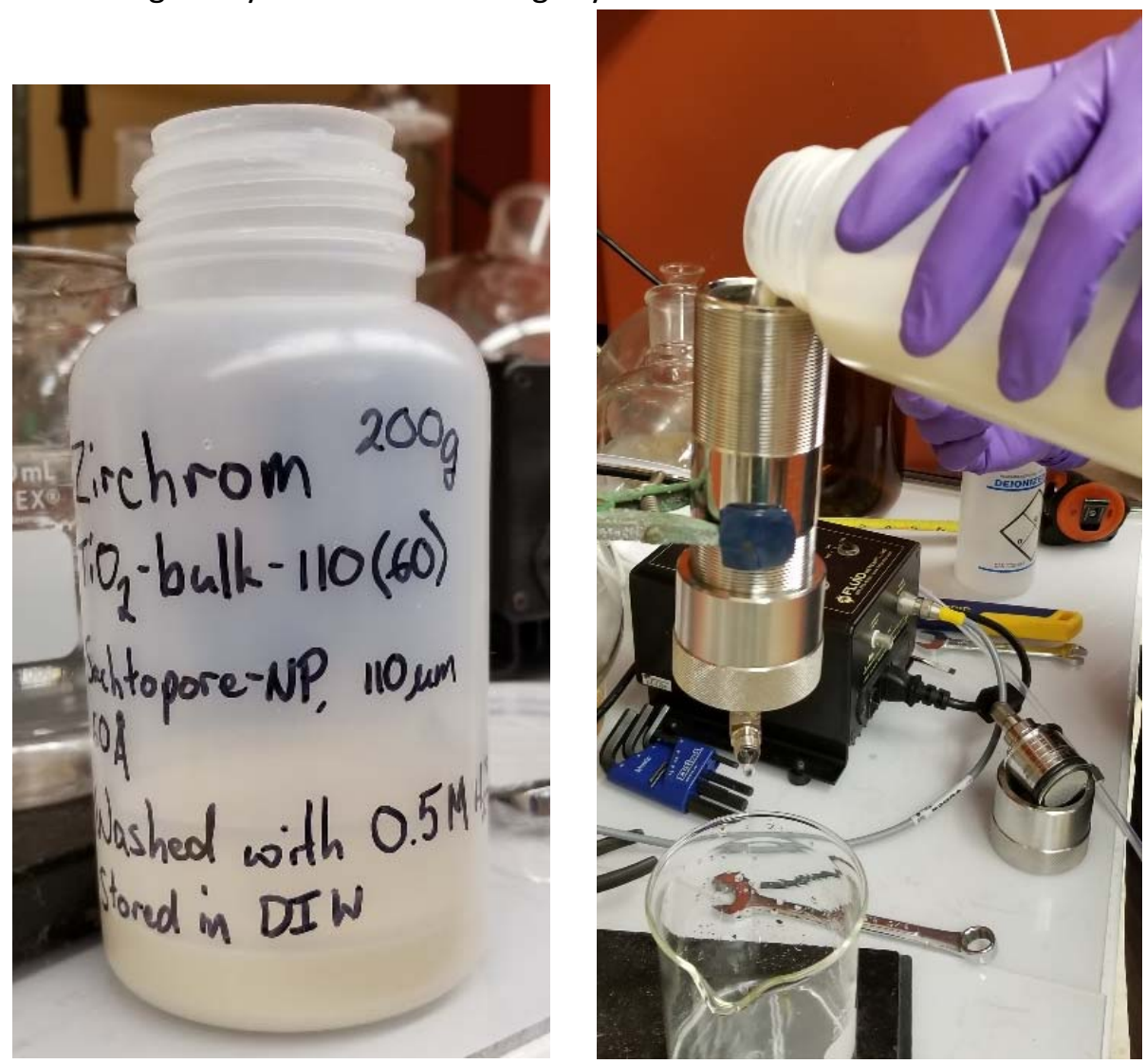

Figure 3: Left) bottle of washed resin (200g) with a small amount of water to slurry with. Right) pouring the slurried resin into the column and allowing the excess water to drip out the bottom into a beaker.

5) After filling to the top of the mark with resin, cap the bottom of the column and install the top frit and collar.

6) Hand tighten the top collar and move it to the vice, being careful not to crush the threads when securing it.

a. Note - When tightening the top frit/collar assembly, water WILL be displaced. To avoid spilling it on the floor, a container should be placed under the column while tightening

7) Finish tightening the collar with a pipe-wrench, making sure the column inlet and outlet are pointing the same direction when the collar is completely tight. The overall length of the column (collar end to collar end) should be 6 3/4". 


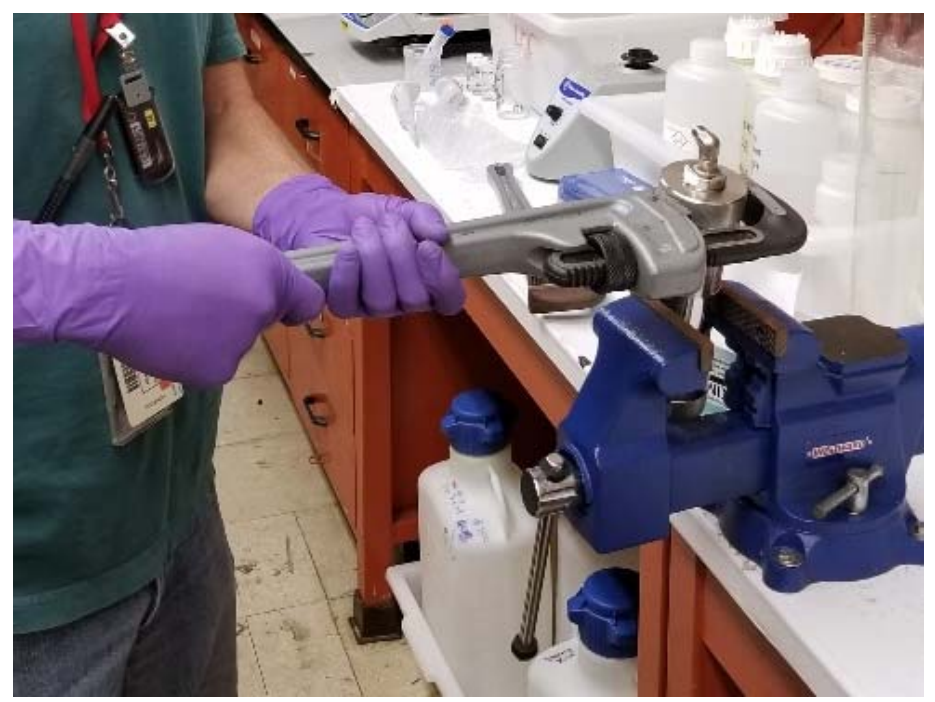

Figure 4: Tightening the top collar using a pipe-wrench and vice. Make sure inlet and outlet point the same direction.

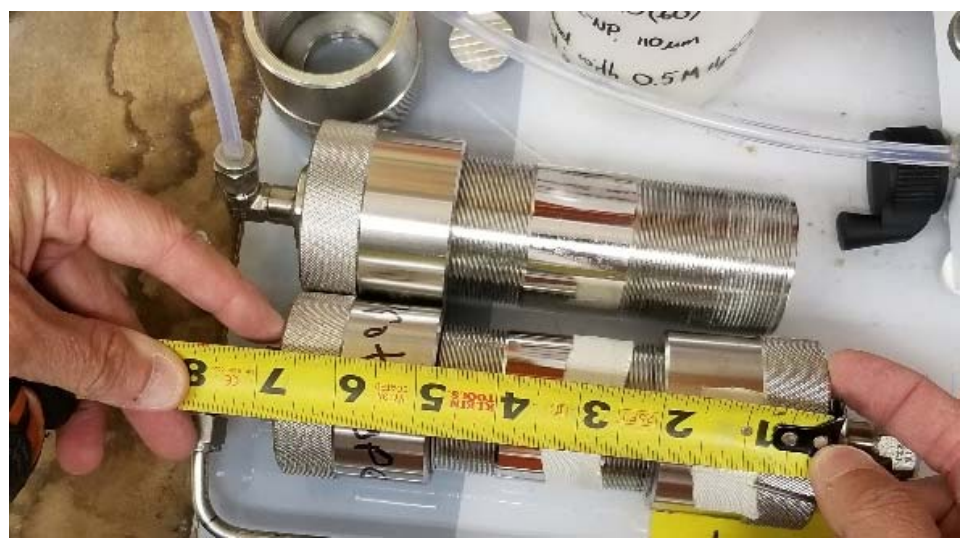

Figure 5: Overall length of the column is $63 / 4$ " once tightened.

8) Prime the FMI (or other equivalent) pump and tubing with water to ensure the column is not dried out at any point during leak testing.

9) Run a line between the pump and column, column and a clean beaker with water, and the beaker and the pump. Dry the outside of the column after assembly. 


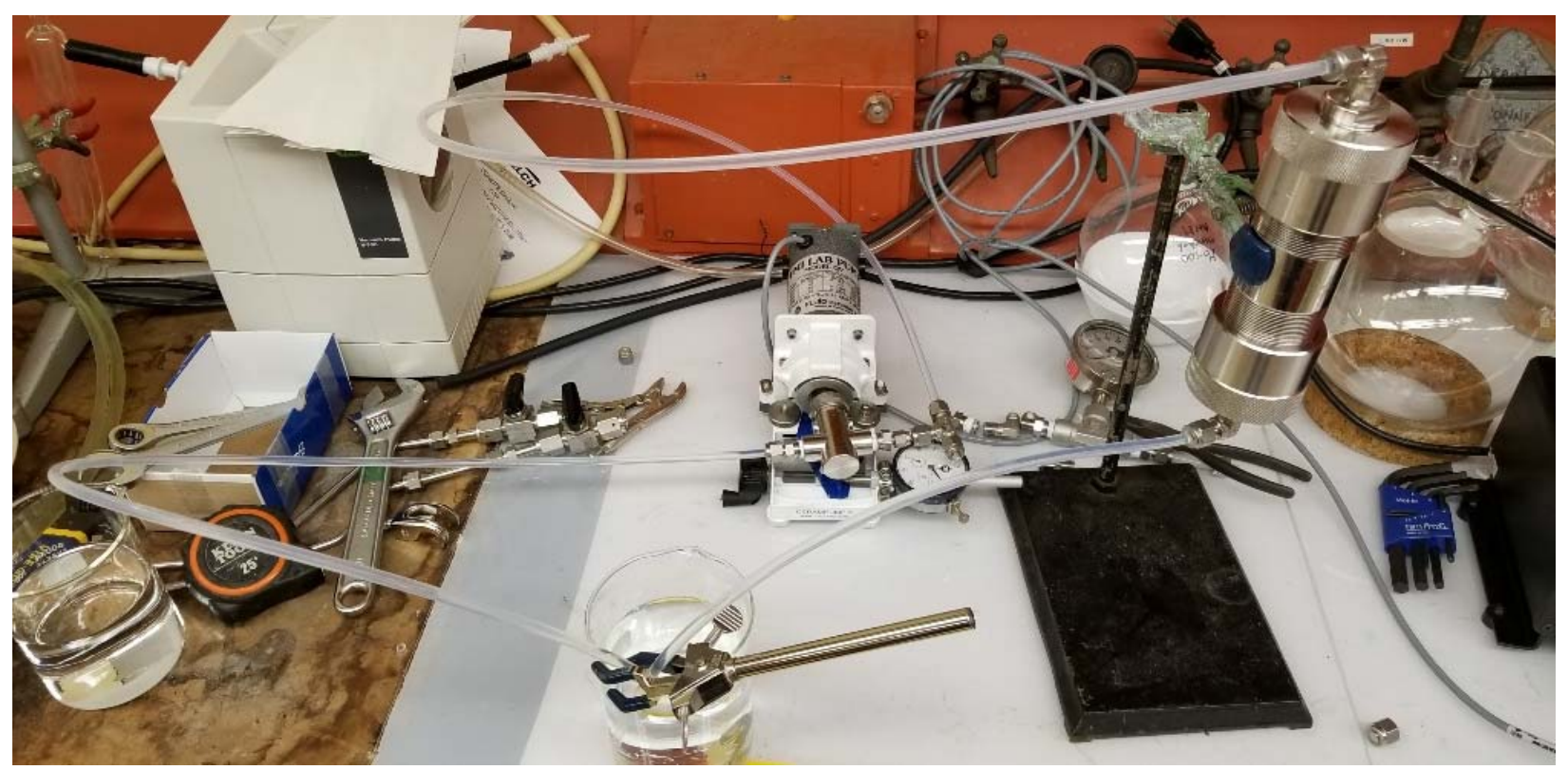

Figure 6: Column/pump/beaker setup and connections

10) Begin circulating water and address any leaks. Small leaks at the swage fittings can be addressed by tightening the fitting.

11) Circulate the water for 1.5-2 hours in each direction, checking for leaks periodically. When finished, also check the beaker to see if any "fines" from the resin have made it through the frit.

a. If fines are found, empty and rinse the beaker and then replace the water and continue circulating until no fines are present. If fines persist, the column will need to be disassembled and reassembled with new frits.

12) Set the pump to circulate water from the top of the column to the bottom and replace the bottom tube line with a pressure gauge.

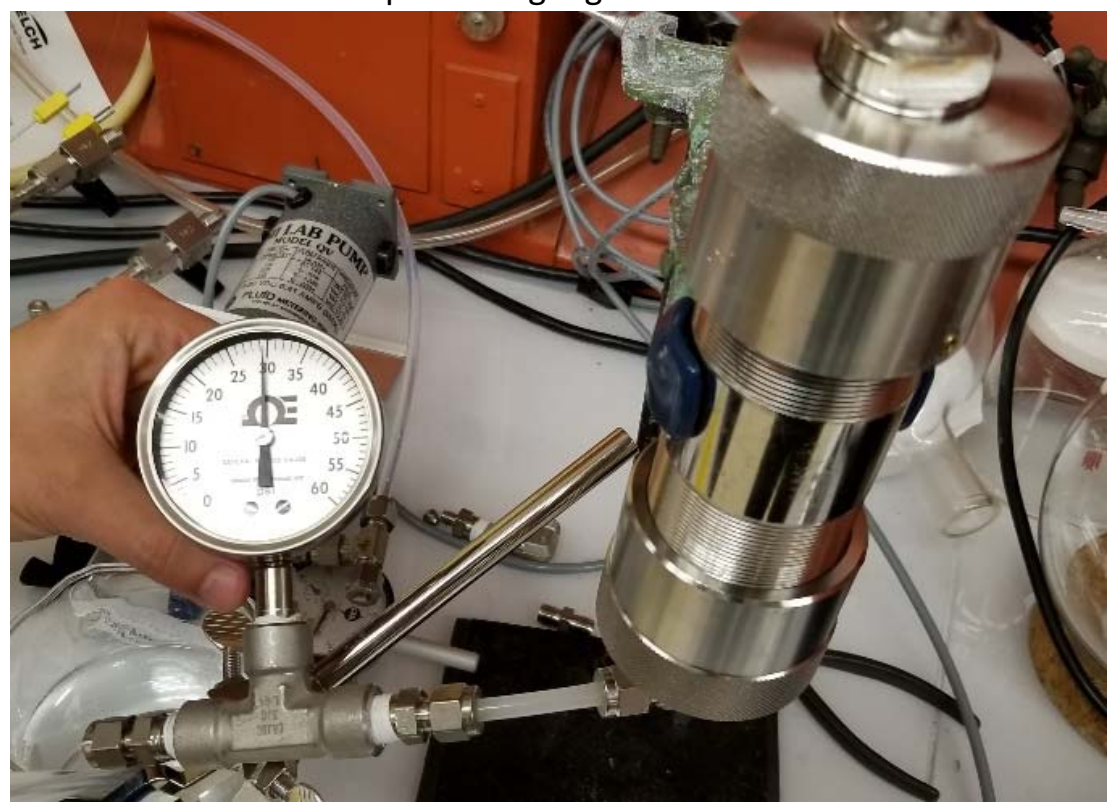

Figure 7: Column with pressure gauge attached 
13) Uncap the pressure gauge and fill with water by pumping it through the column.

14) Cap the pressure gauge and increase pressure to approx. $35 \mathrm{PSI}$, then shut off the pump and check for leaks. A slow pressure decrease is acceptable (0.2 PSI/min), as long as liquid leaks are not found on the column. This is sometimes caused by pressure leaking back through the pump head.

15) Allow the pressurized column to sit for 1-2 hours, checking for leaks periodically.

16) Disconnect the pump from the column to depressurize it and then cap the top column inlet.

17) Disconnect the pressure gauge from the bottom of the column and cap the bottom column outlet.

18) Store the column until the appropriate pipe can be bent and attached to assemble the complete column.

19) Once the pipe is bent and fittings/valves are attached, prime the lines with water and attach to the column.

20) Attach two thermocouples to the assembly. One to either side of the column. The overall height of the column apparatus should be $22 \mathrm{r} / 2$ ". It is better to be slightly over than slightly under.
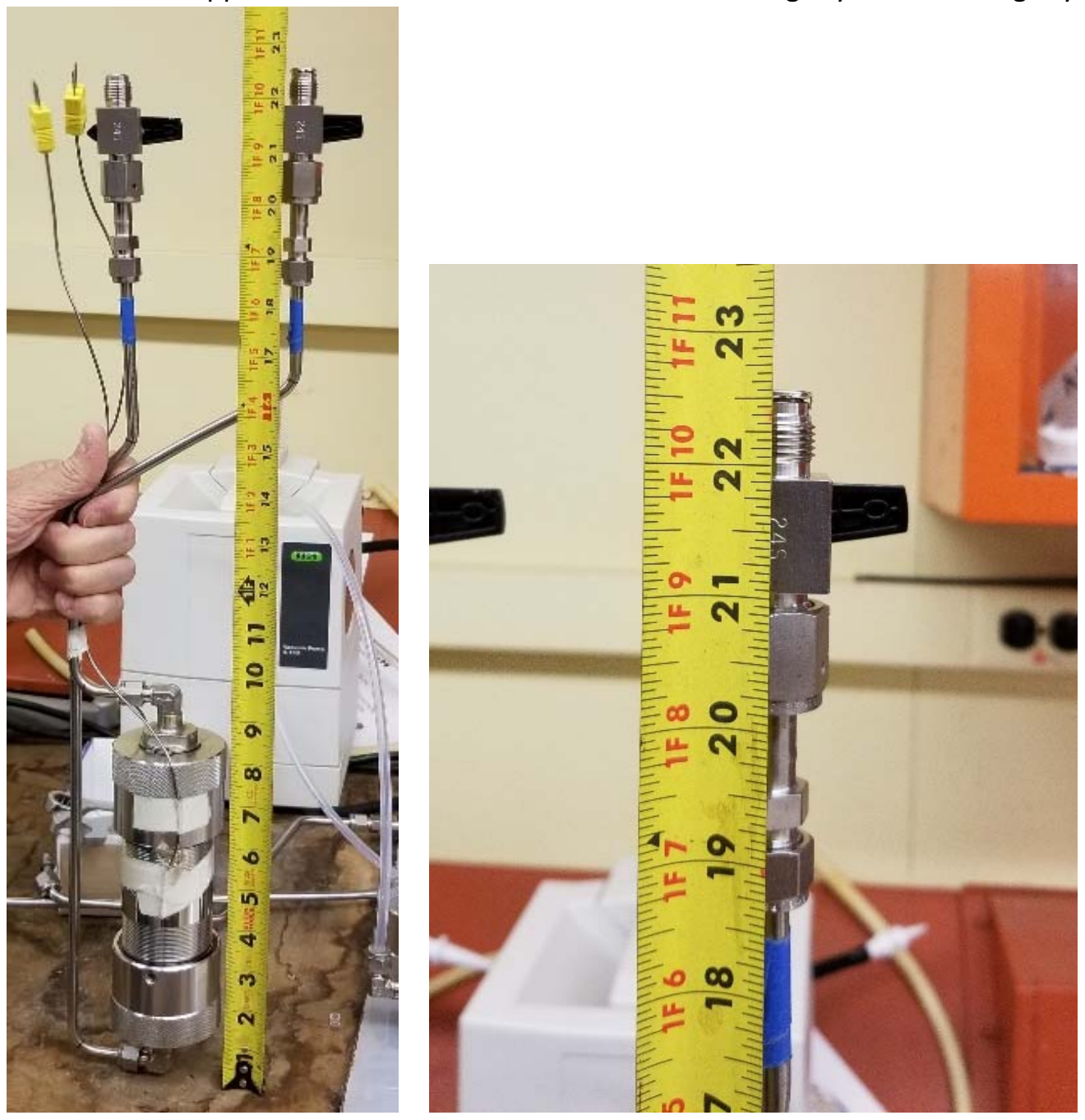

Figure 8: Overall assembly of column, pipes, fittings, and thermocouples with zoom in on overall height of 22 1/2". 


\section{APPENDIX 19}

LEAF-PROC-001, Rev. 1: 20L Tank Cooling System: Initial Startup, Ambient 


\section{L Tank Cooling System: Initial Startup, Ambient}

Low Energy Accelerator Facility, LEAF-PROC-001, Rev. 1

Approved:

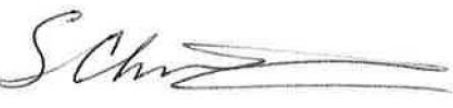

Date: $\underline{09.12 .2019}$

Sergey Chemerisov, Manager, IVEM/LEAF

Effective Date: $\underline{09.16 .2019}$

The current version of this document resides at https://leaf-docdb.ne.anl.gov/cgi-bin/DocumentDatabase. Printed or electronically downloaded copies may be obsolete. Before using such a copy for work direction, employees must verify that it is current by comparing its revision number to that shown in the on-line version. 
The current version of this document resides at https://leaf-docdb.ne.anl.gov/cgi-bin/DocumentDatabase. Printed or electronically downloaded copies may be obsolete. Before using such a copy for work direction, employees must verify that it is current by comparing its revision number to that shown in the on-line version. 


\section{Purpose}

Establish the process for initial startup for the 20-liter (20L) tank cooling system at ambient temperature at the LEAF facility.

\section{Scope}

This procedure applies to the following Argonne activities and entities.

\begin{tabular}{ll}
\hline LMS core processes: & Asset Management \\
\hline Organizations: & Experimental Operations and Facilities (EOF) Division \\
\hline Buildings: & 211 \\
\hline Specific locations: & LINAC \\
\hline $\begin{array}{l}\text { Other applicability } \\
\text { factors: }\end{array}$ & None \\
\hline Exclusions: & None \\
\hline USI applicability: & Yes \\
\hline
\end{tabular}

\section{Work Process}

\subsection{Introduction}

During AMORE irradiation significant energy (up to $5 \mathrm{~kW}$ ) will be deposited in the uranyl sulfate solution. To maintain desired solution temperature in 20C-90C range solution has to be cooled. This is achieved by flowing cooling water through the reflector surrounding the solution volume and through cooling coil placed on top of the solution. Before commencing AMORE irradiation cooling system has to be turned on and operations of the system has to be verified. Procedure outlining steps necessary to perform initial startup is listed below.

\subsection{Step-by-Step Procedure}

The steps below are mandatory unless noted otherwise. All steps must be written into start-up check-list with logging all measurements of temperature, pressure, and flow rate. If at any step the measured value is out of compliance, or the system does not response in proper way (motor won't start, control light not switched on, etc.), immediately stop the process, inform the person in experimental duty, and initiate the troubleshooting process in accordance with proper WCD. This procedure is to be performed by Qualified Operator.

\subsubsection{Actions}

\begin{tabular}{|c|c|}
\hline Step & Action \\
\hline 1 & System in ready condition (full flow through heat exchanger and bypass valve) \\
\hline \multirow[t]{4}{*}{2} & Expansion Tank Float Switches \\
\hline & 2.1 Fill system until both lights go on. \\
\hline & 2.2 Start pump. \\
\hline & 2.3 With pump running, the rest of the system will start to fill, lowering the level in the \\
\hline
\end{tabular}

The current version of this document resides at https://leaf-docdb.ne.anl.gov/cgi-bin/DocumentDatabase. Printed or electronically downloaded copies may be obsolete. Before using such a copy for work direction, employees must verify that it is current by comparing its revision number to that shown in the on-line version. 


\begin{tabular}{|c|c|c|}
\hline \multirow[t]{11}{*}{ Step } & \multicolumn{2}{|r|}{ Action } \\
\hline & & expansion tank; in turn, the bottom light will go off and pump will stop. \\
\hline & 2.4 & $\begin{array}{l}\text { Refill the tank and repeat steps } 1-3 \text { until the system is full and the bottom light stays } \\
\text { on. }\end{array}$ \\
\hline & 2.5 & Start pump and start drain water until bottom light goes off and pump stops. \\
\hline & 2.6 & With pump stopped, add 1 gallon of water to the expansion tank. \\
\hline & 2.7 & Start pump. \\
\hline & 2.8 & Drain system until top light just goes off. \\
\hline & 2.9 & Verify that approximately 1 gallon of water has been drained from the system. \\
\hline & 2.10 & $\begin{array}{l}\text { Refill the tank and repeat steps } 1-3 \text { until the system is full and the bottom light stays } \\
\text { on. }\end{array}$ \\
\hline & 2.11 & Expansion tank level is now set. \\
\hline & 2.12 & Step 2 is completed _____(Initial) \\
\hline \multirow[t]{8}{*}{3} & \multicolumn{2}{|c|}{ Expansion Tank Purge System } \\
\hline & 3.1 & Purge flow rate set at $1.5 \pm 0.2 \mathrm{scfm}$. \\
\hline & 3.2 & Flow Switch interlock for purge \\
\hline & & - Reduce purge flow to $1.0 \pm 0.2 \mathrm{scfm}$ \\
\hline & & - Beam power relay should be deactivated (light on control panel is off). \\
\hline & & - Increase purge flow to $1.5 \pm 0.2 \mathrm{scfm}$ \\
\hline & & - Beam power relay is/should be activated (light on control panel is on). \\
\hline & 3.3 & Step 3is completed ______(Initial) \\
\hline \multirow[t]{5}{*}{4} & \multicolumn{2}{|c|}{ Measurements with Pump Off } \\
\hline & 4.1 & Turn pump off \\
\hline & 4.2 & Pressure sensor reading should be $3.0 \pm 1.0 \mathrm{psi}(4.5 \mathrm{~mA})$ \\
\hline & 4.3 & Differential pressure sensor reading should be $0.0 \pm 0.3 \mathrm{psi}(4.0 \mathrm{~mA})$ \\
\hline & 4.4 & Step 4 is completed ___ (Initial) \\
\hline
\end{tabular}

The current version of this document resides at https://leaf-docdb.ne.anl.gov/cgi-bin/DocumentDatabase. Printed or electronically downloaded copies may be obsolete. Before using such a copy for work direction, employees must verify that it is current by comparing its revision number to that shown in the on-line version. 


\begin{tabular}{|c|c|c|}
\hline Step & & Action \\
\hline \multirow[t]{7}{*}{5} & \multicolumn{2}{|c|}{ Measurements with pump on } \\
\hline & 5.1 & Start pump \\
\hline & 5.2 & Set flow rate to $2.3 \pm 0.5 \mathrm{gpm}$ using the throttle valve. \\
\hline & 5.3 & Adjust flow through DI to $0.25 \pm 0.1 \mathrm{gpm}$ using upstream ball valve. \\
\hline & 5.4 & Pressure sensor reading should be $23.0 \pm 1$ psi. \\
\hline & 5.5 & Differential pressure sensor reading should be $0.4 \pm 0.3 \mathrm{psi}(4.3 \mathrm{~mA})$. \\
\hline & & Step 5 is completed. ___ (Initial) \\
\hline \multirow[t]{10}{*}{6} & \multicolumn{2}{|c|}{ Flow Switch Interlock check } \\
\hline & 6.1 & Reduce the flow rate from $2.3 \pm 0.3 \mathrm{gpm}$ to $2.0 \pm 0.2 \mathrm{gpm}$ using the throttle. \\
\hline & 6.2 & Set the flow switch to open at $2.0 \pm 0.2 \mathrm{gpm}$. \\
\hline & 6.3 & Beam power relay should be deactivated (light on control panel is off). \\
\hline & 6.4 & Increase the flow back to $2.3 \pm 0.3 \mathrm{gpm}$. \\
\hline & 6.5 & Beam power relay should be activated (light on control panel is on). \\
\hline & 6.6 & Reduce the flow to $2.0 \pm 0.2$ gpm to check flow switch setting. \\
\hline & 6.7 & Increase flow to the design flow of $2.3 \pm 0.23 \mathrm{gpm}$. \\
\hline & 6.8 & Beam power relay should be activated (light on control panel is on). \\
\hline & 6.9 & Step 6 is completed. ___ (Initial) \\
\hline \multirow[t]{5}{*}{7} & \multicolumn{2}{|c|}{ Chiller } \\
\hline & 7.1 & $\begin{array}{l}\text { Perform chiller startup steps in accordance with "Chiller Cooling System: Initial and } \\
\text { Routine Startup", LEAF-PROC-004. }\end{array}$ \\
\hline & 7.2 & Start and record temperatures and flow (at chiller). \\
\hline & 7.3 & Adjust flow to $2.5 \pm 0.2 \mathrm{gpm}$; outlet temperature should be $55 \pm 5^{\circ} \mathrm{F}$ \\
\hline & & Step 7 is completed. ___ (Initial) \\
\hline 8 & \multicolumn{2}{|c|}{ Check out is complete } \\
\hline 9 & \multicolumn{2}{|c|}{ Date and sig } \\
\hline
\end{tabular}

The current version of this document resides at https://leaf-docdb.ne.anl.gov/cgi-bin/DocumentDatabase. Printed or electronically downloaded copies may be obsolete. Before using such a copy for work direction, employees must verify that it is current by comparing its revision number to that shown in the on-line version. 


\section{Records Created by Work Process}

The records listed below must be retained as indicated.

\begin{tabular}{l|c|c|l|l}
\hline $\begin{array}{l}\text { Description of Record } \\
\text { (include form number if } \\
\text { applicable) }\end{array}$ & $\begin{array}{c}\text { Active } \\
\text { Records } \\
\text { Custodian }\end{array}$ & $\begin{array}{c}\text { Active } \\
\text { Records } \\
\text { Retention }\end{array}$ & $\begin{array}{l}\text { Indexing Method, } \\
\text { Storage Medium }\end{array}$ & $\begin{array}{l}\text { Federal Retention } \\
\text { Requirements* }\end{array}$ \\
\hline $\begin{array}{l}\text { Completed LEAF-PROC- } \\
001\end{array}$ & $\begin{array}{c}\text { Facility } \\
\text { Manager }\end{array}$ & 3 years & $\begin{array}{l}\text { Index by job date } \\
\text { and name, store on } \\
\text { paper or } \\
\text { electronically }\end{array}$ & $\begin{array}{l}\text { Destroy 75 years after } \\
\text { the date of the permit } \\
\text { (DOE ADM 18.37) }\end{array}$ \\
\hline
\end{tabular}

*If records are maintained in a business information system that is not currently programmed to purge digital records based on age, the records may be retained in that system past the indicated destruction date.

\section{Related Documents}

This procedure implements requirements established by the following safety basis documents:

- LEAF-SAD-100, Linac Safety Assessment Document

- LEAF-ASE-100, Linac Accelerator Safety Envelope

This procedure implements requirements established by the following Argonne policies and procedures:

- LMS-PROC-188, Accelerator Safety

- EGS-PP-100, Configuration Management Program Plan for Accelerators

\section{Definitions}

None

The current version of this document resides at https://leaf-docdb.ne.anl.gov/cgi-bin/DocumentDatabase. Printed or electronically downloaded copies may be obsolete. Before using such a copy for work direction, employees must verify that it is current by comparing its revision number to that shown in the on-line version. 


\section{$7 \quad$ About this Procedure}

\begin{tabular}{ll}
\hline Issuing organization: & Low Energy Accelerator Facility \\
\hline Procedure owner: & S. Chemerisov \\
\hline Point of contact: & S. Chemerisov \\
\hline Review cycle (months): & 36 \\
\hline Date last revised: & 09.05 .2019 \\
\hline Date last reviewed: & 09.12 .2019 \\
\hline
\end{tabular}

\section{Summary of Changes in This Version}

Rev 0: Initial release

Rev 1:

In step 3.2 changed the purge flow from $2.0 \mathrm{scfm}$ to $1.5 \mathrm{scfm}$ to be consistent with required purge flow.

Added additional step prior to step 4.1 to turn pump off before performing the measurements.

Added additional step prior to step 5.1 to turn pump on before performing steps in section 5 .

Changed flow through DI cartridge from 0.35 to 0.25 to reflect real flow rate.

Changed expected pressure sensor reading from 24 to 23 psi to match actual pressure in the system.

In step 6.1 changed the flow rate from $5 \mathrm{gpm}$ to $2.3 \mathrm{gpm}$ and from 4.5 to $2.0 \mathrm{gpm}$ to reflect actual flow through the system. This flow rate is sufficient for solution cooling.

In step 6.4 decreased the flow rate from $5 \mathrm{gpm}$ to $2.3 \mathrm{gpm}$.

In step 6.6 decreased the flow rate from $4.5 \mathrm{gpm}$ to $2.0 \mathrm{gpm}$.

In step 6.7 decrease the flow rate from $5 \mathrm{gpm}$ to $2.3 \mathrm{gpm}$.

The current version of this document resides at https://leaf-docdb.ne.anl.gov/cgi-bin/DocumentDatabase. 


\section{Exhibit A: 20L Cooling System Schematic}

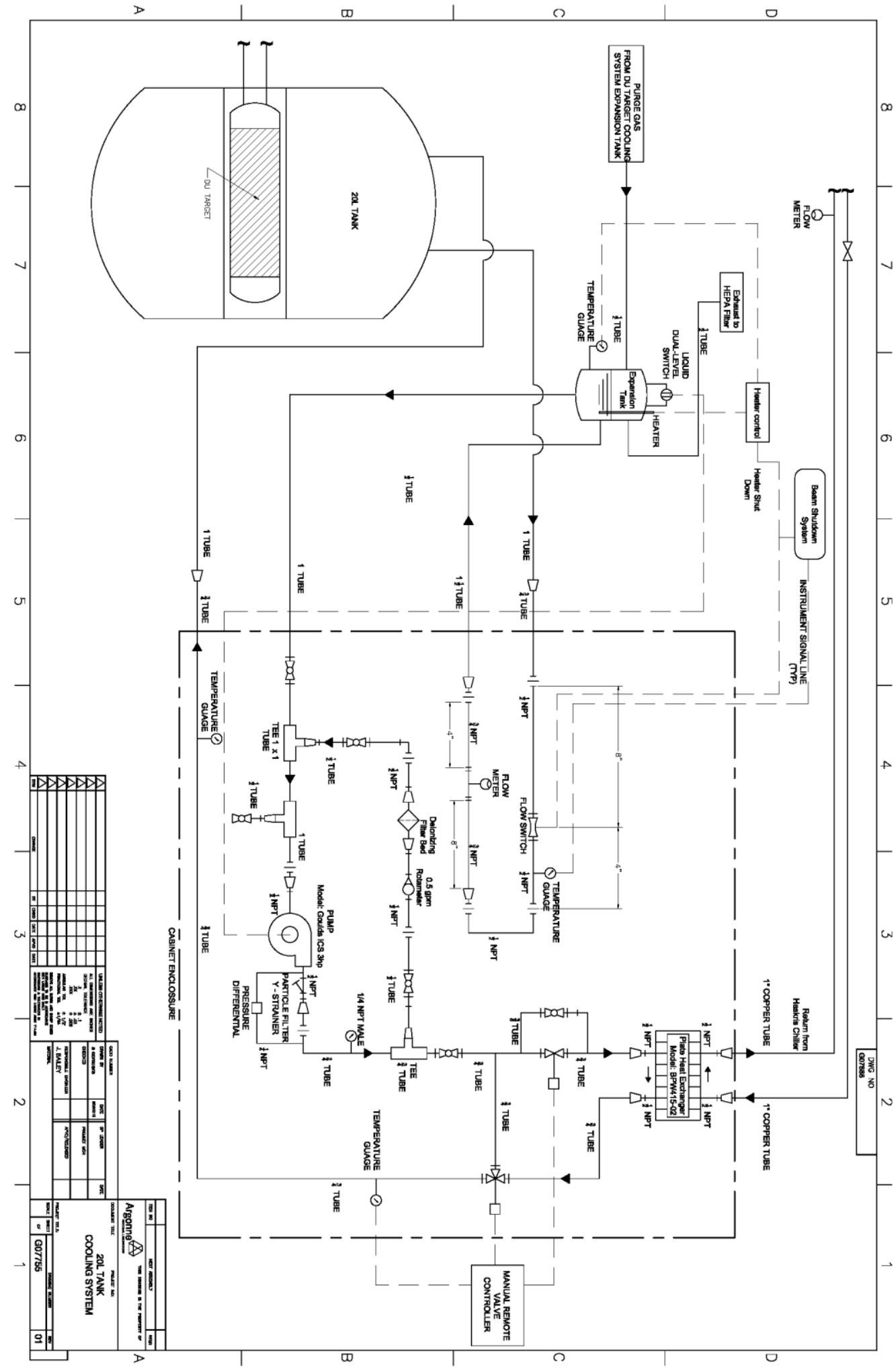

The current version of this document resides at https://leaf-docdb.ne.anl.gov/cgi-bin/DocumentDatabase. Printed or electronically downloaded copies may be obsolete. Before using such a copy for work direction, employees must verify that it is current by comparing its revision number to that shown in the on-line version. 


\section{APPENDIX 20}

LEAF-PROC-002, Rev. 1: 20L Tank Cooling System: Initial Startup, Elevated Temperature 


\section{L Tank Cooling System: Initial Startup, Elevated Temperature}

Low Energy Accelerator Facility, LEAF-PROC-002, Rev. 1

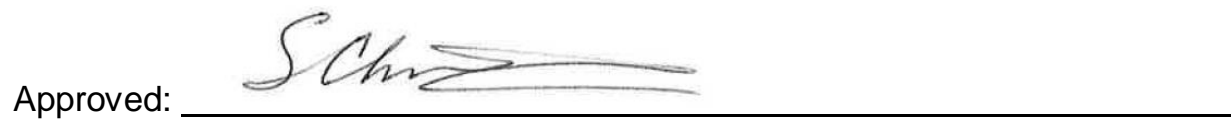

Date: $\underline{09.05 .2019}$

Sergey Chemerisov, Manager, IVEM/LEAF 


\section{Purpose}

Establish the process for initial startup of the 20L Tank Cooling System at the LEAF/Linac facility under elevated temperature conditions.

\section{Scope}

This procedure applies to the following Argonne activities and entities.

\begin{tabular}{ll}
\hline LMS core processes: & Asset Management \\
\hline Organizations: & Experimental Operations and Facilities (EOF) Division \\
\hline Buildings: & 211 \\
\hline Specific locations: & LINAC \\
\hline $\begin{array}{l}\text { Other applicability } \\
\text { factors: }\end{array}$ & None \\
\hline Exclusions: & None \\
\hline USI applicability: & Yes \\
\hline
\end{tabular}

\section{Work Process}

\subsection{Introduction}

During AMORE irradiation significant energy (up to $5 \mathrm{~kW}$ ) will be deposited in the uranyl sulfate solution. To maintain desired solution temperature in $20 \mathrm{C}-90 \mathrm{C}$ range solution has to be cooled. This is achieved by flowing cooling water through the reflector surrounding the solution volume and through cooling coil placed on top of the solution. Before commencing AMORE irradiation cooling system has to be turned on and operations of the system has to be verified. Procedure outlining steps necessary to perform initial startup at elevated temperatures is listed below.

\subsection{Step-by-Step Procedure}

The steps below are mandatory unless noted otherwise. All steps must be written into start-up check-list with logging all measurements of temperature, pressure, and flow rate. If at any step the measured value is out of compliance, or the system does not response in proper way (motor won't start, control light not switched on, etc.), immediately stop the process, inform the person in experimental duty, and initiate the troubleshooting process in accordance with proper WCD. This procedure is to be performed by Qualified Operator.

\subsubsection{Actions}

\begin{tabular}{|c|c|}
\hline Step & Action \\
\hline 1 & System in ready condition (perform LEAF-PROC-001, Initial Startup Steps, Ambient) \\
\hline 2 & $\begin{array}{l}\text { Provide: } \\
\qquad 0-20 \text { psig air supply with regulator and pressure gauge }\end{array}$ \\
\hline 3 & $\begin{array}{l}\text { Calibrate } 3 \text {-Way Valve: } \\
3.1 \quad \text { Pump off. } \\
3.2 \quad \text { Setup the camera to observe valve movement in D-035. }\end{array}$ \\
\hline
\end{tabular}




\begin{tabular}{|c|c|c|}
\hline \multirow[t]{10}{*}{ Step } & \multicolumn{2}{|r|}{ Action } \\
\hline & 3.3 & Put control panel in D-076 in operating mode (i.e., air supply and electric on). \\
\hline & 3.4 & Set the manual setter controller (TC108) to 0. \\
\hline & 3.5 & Increase set point until movement of valve stem actuator occurs. \\
\hline & 3.6 & Record this value (call it low). \\
\hline & 3.7 & Increase setter adjustment until movement of valve actuator stops \\
\hline & 3.8 & Record the setter setting (call it high). \\
\hline & 3.9 & $\begin{array}{l}\text { The "low" to "high" setting is the range that will be used to determine the percent } \\
\text { settings for the } 3 \text {-way valve that are indicated in the table for the elevated } \\
\text { temperature operation } \\
\text { (Note that percent setting as determined here may be reversed from the table). }\end{array}$ \\
\hline & 3.10 & Recheck setter settings against actual valve stem positions. \\
\hline & 3.11 & Step3 is completed. ___ (Initial) \\
\hline \multirow[t]{9}{*}{4} & \multicolumn{2}{|c|}{ Operation: Check 2-Way Valve } \\
\hline & 4.1 & Set 2-position selector switch (SS104) to off position. \\
\hline & 4.2 & Determine and record position of actual valve stem actuator (up or down). \\
\hline & 4.3 & Set 2-position selector switch to on position. \\
\hline & 4.4 & Determine and record position of actual valve stem actuator (up or down). \\
\hline & 4.5 & Remove control air tube from the valve operator. \\
\hline & 4.6 & Determine and record position of actual valve stem actuator (up or down). \\
\hline & This c & leck determines the normally open or closed position of the 2 -Way valve. \\
\hline & & Step 4 is completed. ___ (Initial) \\
\hline \multirow[t]{6}{*}{5} & \multicolumn{2}{|c|}{ Operation Ambient Temperature Test } \\
\hline & 5.1 & Set the 2-Way valve and by pass to the full open position. \\
\hline & 5.2 & Set the 3-Way valve to full flow through the exchanger. \\
\hline & 5.3 & Pump on. \\
\hline & 5.4 & $\begin{array}{l}\text { Record flow rate and pressure (should be about the same as that recorded for the } \\
\text { LEAF-PROC-001. }\end{array}$ \\
\hline & 5.5 & Step 5 is completed. ___ (Initial) \\
\hline
\end{tabular}




\begin{tabular}{|c|c|c|}
\hline Step & & Action \\
\hline \multirow[t]{10}{*}{6} & \multicolumn{2}{|c|}{ Operation Elevated Temperature Test (beam off, pump on) } \\
\hline & & Set 3 -Way valve to $50 \%$ \\
\hline & 6.2 & 2-Way valve and by pass valve open \\
\hline & 6.3 & Set temperature to $80^{\circ} \mathrm{F}$ \\
\hline & 6.4 & Reference table \\
\hline & 6.5 & Record temperature increase of coolant vs. time until steady state of $80^{\circ} \mathrm{F}$ is reached \\
\hline & 6.6 & Record flow rate through $20 \mathrm{~L}$ Tank \\
\hline & 6.7 & $\begin{array}{l}\text { Record chilled water temperature in and out of chiller and flow rate to 20L Tank } \\
\text { cooling system }\end{array}$ \\
\hline & 6.8 & $\begin{array}{l}\text { Repeat steps } 1 \text { through } 7 \text { for set temperatures of } 110^{\circ} \mathrm{F} \text { and } 1130^{\circ} \mathrm{F} \text {, referencing table } \\
\text { for valve settings }\end{array}$ \\
\hline & 6.9 & Step 6 is completed. _____(Initial) \\
\hline \multirow[t]{8}{*}{7} & \multicolumn{2}{|c|}{ Over Temperature Interlock } \\
\hline & 7.1 & Set over temperature beam shutdown to $130^{\circ} \mathrm{F}$ \\
\hline & 7.2 & Increase the water operating temperature to $135^{\circ} \mathrm{F}$ \\
\hline & 7.3 & Beam power relay should be deactivated (light on control panel is off) \\
\hline & 7.4 & Decrease the water operating temperature to $125^{\circ} \mathrm{F}$ \\
\hline & 7.5 & Beam power relay should be activated (light on control panel is on) \\
\hline & 7.6 & Reset over temperature beam shut down to $180^{\circ} \mathrm{F}$ \\
\hline & 7.7 & Step 7 is completed. ___ (Initial) \\
\hline 8 & \multicolumn{2}{|c|}{ Shut systems down } \\
\hline 9 & \multicolumn{2}{|c|}{ Check out is complete } \\
\hline 10 & \multicolumn{2}{|c|}{ Date and sign } \\
\hline
\end{tabular}

\section{$4 \quad$ Records Created by Work Process}

The records listed below must be retained as indicated.

\begin{tabular}{l|c|c|l|l}
\hline $\begin{array}{l}\text { Description of Record } \\
\text { (include form number if } \\
\text { applicable) }\end{array}$ & $\begin{array}{c}\text { Active } \\
\text { Records } \\
\text { Custodian }\end{array}$ & $\begin{array}{c}\text { Active } \\
\text { Records } \\
\text { Retention }\end{array}$ & $\begin{array}{l}\text { Indexing Method, } \\
\text { Storage Medium }\end{array}$ & $\begin{array}{l}\text { Federal Retention } \\
\text { Requirements* }\end{array}$ \\
\hline $\begin{array}{l}\text { Completed LEAF-PROC- } \\
002\end{array}$ & $\begin{array}{c}\text { Facility } \\
\text { Manager }\end{array}$ & 3 years & $\begin{array}{l}\text { Index by job date } \\
\text { and name, store on } \\
\text { paper or } \\
\text { electronically }\end{array}$ & $\begin{array}{l}\text { Destroy 75 years after } \\
\text { the date of the permit } \\
\text { (DOE ADM 18.37) }\end{array}$ \\
\hline
\end{tabular}

*If records are maintained in a business information system that is not currently programmed to purge digital records based on age, the records may be retained in that system past the indicated destruction date. 


\section{Related Documents}

This procedure implements requirements established by the following safety basis documents:

- LEAF-SAD-100, Linac Safety Assessment Document

- LEAF-ASE-100, Linac Accelerator Safety Envelope

This procedure implements requirements established by the following Argonne policies and procedures:

- LMS-PROC-188, Accelerator Safety

- EGS-PP-100, Configuration Management Program Plan for Accelerators

\section{Definitions}

None

\section{$7 \quad$ About this Procedure}

\begin{tabular}{ll}
\hline Issuing organization: & Low Energy Accelerator Facility \\
\hline Procedure owner: & S. Chemerisov \\
\hline Point of contact: & S. Chemerisov \\
\hline Review cycle (months): & 36 \\
\hline Date last revised: & 09.05 .2019 \\
\hline Date last reviewed: & 09.12 .2019 \\
\hline
\end{tabular}

\section{Summary of Changes in This Version}

Initial release

Revision 1:

Section 3 was modified to reflect use of the camera and monitor to calibrate 3-Way valve.

Temperatures settings for calibration points in section 7 reduced to 80,110 , and $130 \mathrm{~F}$ to reduce time necessary to verify system performance.

Reduce interlock check temperature to $130 \mathrm{~F}$ to coincide with highest temperature point in previous step.

Reduce beam shutdown temperature for over temperature protection to $180 \mathrm{~F}$. 


\section{APPENDIX 21}

LEAF-PROC-003, Rev. 0: 20L Tank Cooling System: Routine Startup, Ambient 


\section{L Tank Cooling System: Routine Startup, Ambient}

Low Energy Accelerator Facility, LEAF-PROC-003, Rev. 0

Approved:

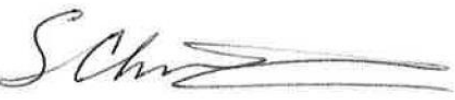

Date: $\underline{09.05 .2019}$

Sergey Chemerisov, Manager, IVEM/LEAF

Effective Date: 09.12 .2019

The current version of this document resides at https://leaf-docdb.ne.anl.gov/cgi-bin/DocumentDatabase. Printed or electronically downloaded copies may be obsolete. Before using such a copy for work direction, employees must verify that it is current by comparing its revision number to that shown in the on-line version. 


\section{Purpose}

Establish the process for starting up the 20L tank cooling system located at the LEAF facility under routine, ambient conditions.

\section{Scope}

This procedure applies to the following Argonne activities and entities.

\begin{tabular}{ll}
\hline LMS core processes: & Asset Management \\
\hline Organizations: & Experimental Operations and Facilities (EOF) Division \\
\hline Buildings: & 211 \\
\hline Specific locations: & LINAC \\
\hline $\begin{array}{l}\text { Other applicability } \\
\text { factors: }\end{array}$ & None \\
\hline Exclusions: & None \\
\hline USI applicability: & Yes \\
\hline
\end{tabular}

\section{Work Process}

\subsection{Introduction}

During AMORE irradiation significant energy (up to $5 \mathrm{~kW}$ ) will be deposited in the uranyl sulfate solution. To maintain desired solution temperature in 20C-90C range solution has to be cooled. This is achieved by flowing cooling water through the reflector surrounding the solution volume and through cooling coil placed on top of the solution. Before commencing AMORE irradiation cooling system has to be turned on and operations of the system has to be verified. Procedure outlining steps necessary to perform those operations is listed below.

\subsection{Step-by-Step Procedure}

The steps below are mandatory unless noted otherwise. All steps must be written into start-up check-list with logging all measurements of temperature, pressure, and flow rate. If at any step the measured value is out of compliance, or the system does not response in proper way (motor won't start, control light not switched on, etc.), immediately stop the process, inform the person in experimental duty, and initiate the troubleshooting process in accordance with proper WCD. This procedure is to be performed by Qualified Operator.

\subsubsection{Actions}

\begin{tabular}{|c|c|c|}
\hline Step & & Action \\
\hline 1 & Sy & in ready condition (full flow through heat exchanger and by pass valve) \\
\hline \multirow[t]{5}{*}{2} & \multicolumn{2}{|c|}{ Expansion Tank Float Switches } \\
\hline & & Fill system until both lights turn on. \\
\hline & 2.2 & Start pump. \\
\hline & & $\begin{array}{l}\text { With pump running, the rest of the system will start to fill, lowering the level in the } \\
\text { expansion tank, and in turn, the bottom light will go off and pump will stop. }\end{array}$ \\
\hline & 2.4 & Refill the tank and repeat steps $1-3$ until the system is full and the bottom light stays \\
\hline
\end{tabular}

The current version of this document resides at https://leaf-docdb.ne.anl.gov/cgi-bin/DocumentDatabase. Printed or electronically downloaded copies may be obsolete. Before using such a copy for work direction, employees must verify that it is current by comparing its revision number to that shown in the on-line version. 


\begin{tabular}{|c|c|c|}
\hline Step & & Action \\
\hline & $\begin{array}{l}2.5 \\
2.6 \\
2.7 \\
2.8 \\
2.9 \\
2.10 \\
2.11 \\
2.12\end{array}$ & $\begin{array}{l}\text { on. } \\
\text { Start pump and drain water until bottom light just goes off and pump stops. } \\
\text { With pump stopped, add } 1 \text { gallon of water to the expansion tank. } \\
\text { Start pump. } \\
\text { Drain system until top light just goes off. } \\
\text { Verify that approximately } 1 \text { gallon of water has been drained from the system. } \\
\text { Refill the tank and repeat steps } 1-3 \text { until the system is full and the bottom light stays } \\
\text { on. } \\
\text { Expansion tank level is now set. } \\
\text { Step } 2 \text { is completed _ Initial) }\end{array}$ \\
\hline 3 & $\begin{array}{l}\text { Expa } \\
3.1 \\
3.2\end{array}$ & $\begin{array}{l}\text { sion Tank Purge System } \\
\text { Purge flow rate set at } 1.5 \pm 0.2 \mathrm{scfm} \text {. } \\
\text { Flow Switch interlock for purge } \\
\text { - } \quad \text { Reduce purge flow to } 1.0 \pm 0.2 \mathrm{scfm} \text {. } \\
\text { - } \quad \text { Beam power relay should be deactivated (light on control panel is off). } \\
\text { - Increase purge flow to } 1.5 \pm 0.2 \mathrm{scfm} \text {. } \\
\text { Step } 3 \text { is completed ___ (Initial) } \\
\quad \text { Note: At initial startup, set flow switch trip at } 1.5 \mathrm{scfm} \text {. }\end{array}$ \\
\hline 4 & $\begin{array}{l}\text { Pum } \\
4.1 \\
4.2 \\
4.3 \\
4.4\end{array}$ & $\begin{array}{l}\text { off measurements } \\
\text { Turn pump off } \\
\text { Pressure sensor reading should be } 3.0 \pm 1.0 \mathrm{psi}(4.5 \mathrm{~mA}) \text {. } \\
\text { Differential pressure sensor reading should be } 0.0 \pm 0.3 \mathrm{psi}(4.0 \mathrm{~mA}) \text {. } \\
\text { Step } 4 \text { is completed }\end{array}$ \\
\hline 5 & $\begin{array}{l}\text { Pum } \\
5.1 \\
5.2 \\
5.3 \\
5.4 \\
5.5\end{array}$ & $\begin{array}{l}\text { on measurements } \\
\text { Start pump } \\
\text { Pressure sensor reading should be } 23.0 \pm 1 \text { psi. } \\
\text { Differential pressure sensor reading should be } 0.4 \pm 0.3 \text { psi }(4.3 \mathrm{~mA}) \text {. } \\
\text { Flow through DI unit should be } 0.25 \pm 0.1 \mathrm{gpm} \\
\text { Step } 5 \text { is completed }\end{array}$ \\
\hline
\end{tabular}




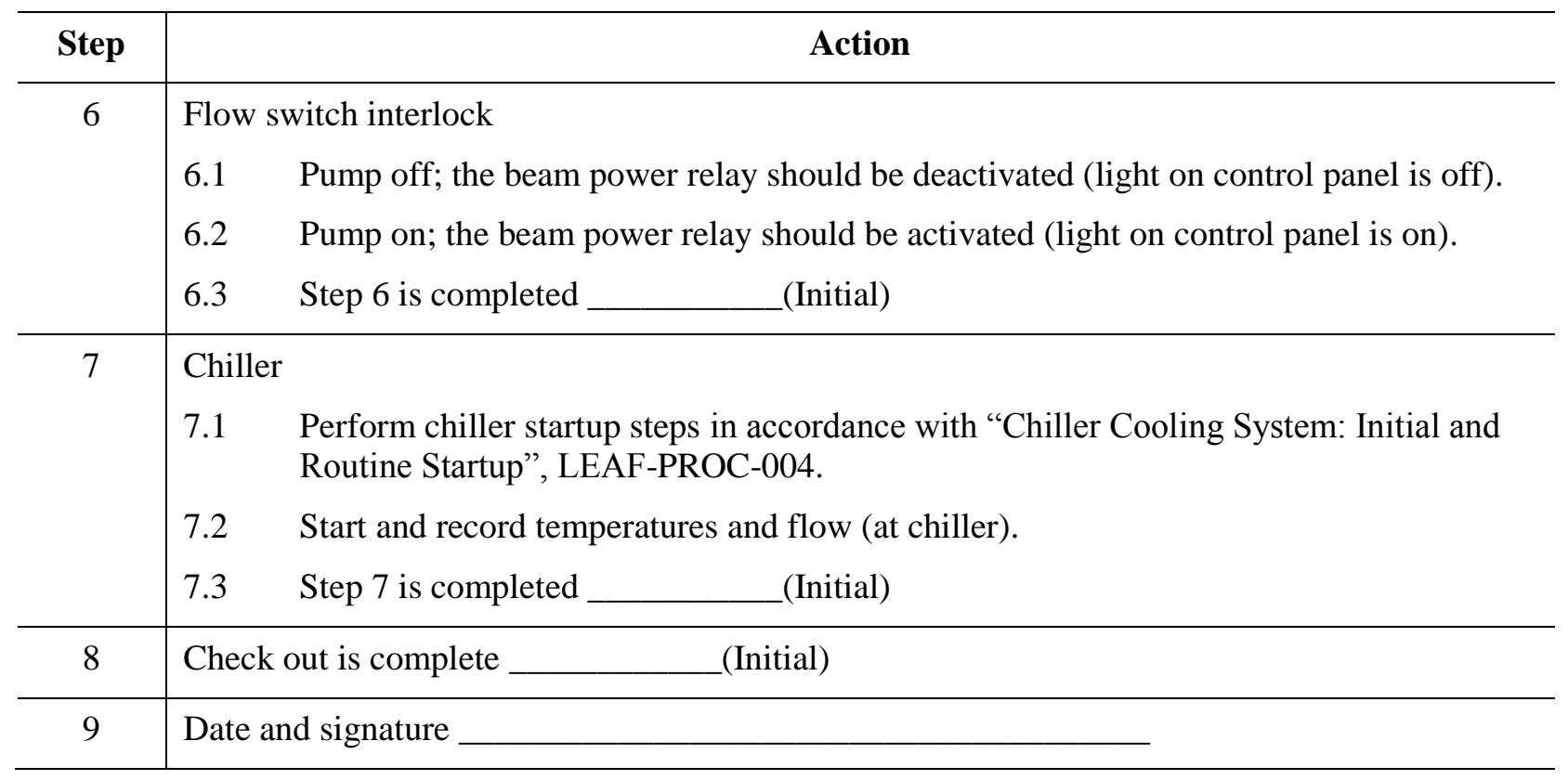

\section{Records Created by Work Process}

The records listed below must be retained as indicated.

\begin{tabular}{l|c|c|l|l}
\hline $\begin{array}{l}\text { Description of Record } \\
\text { (include form number if } \\
\text { applicable) }\end{array}$ & $\begin{array}{c}\text { Active } \\
\text { Records } \\
\text { Custodian }\end{array}$ & $\begin{array}{c}\text { Active } \\
\text { Records } \\
\text { Retention }\end{array}$ & $\begin{array}{l}\text { Indexing Method, } \\
\text { Storage Medium }\end{array}$ & $\begin{array}{l}\text { Federal Retention } \\
\text { Requirements* }\end{array}$ \\
\hline $\begin{array}{l}\text { Completed LEAF-PROC- } \\
003\end{array}$ & $\begin{array}{c}\text { Facility } \\
\text { Manager }\end{array}$ & 3 years & $\begin{array}{l}\text { Index by job date } \\
\text { and name, store on } \\
\text { paper or } \\
\text { electronically }\end{array}$ & $\begin{array}{l}\text { Destroy 75 years after } \\
\text { the date of the permit } \\
\text { (DOE ADM 18.37) }\end{array}$ \\
\hline
\end{tabular}

*If records are maintained in a business information system that is not currently programmed to purge digital records based on age, the records may be retained in that system past the indicated destruction date.

\section{Related Documents}

None

\section{Definitions}

None

\section{About this Procedure}

\begin{tabular}{ll}
\hline Issuing organization: & Low Energy Accelerator Facility \\
\hline Procedure owner: & S. Chemerisov \\
\hline Point of contact: & S. Chemerisov \\
\hline Review cycle (months): & 36 \\
\hline Date last revised: & 09.05 .2019 \\
\hline Date last reviewed: & 09.12 .2019 \\
\hline
\end{tabular}

The current version of this document resides at https://leaf-docdb.ne.anl.gov/cgi-bin/DocumentDatabase. Printed or electronically downloaded copies may be obsolete. Before using such a copy for work direction, employees must verify that it is current by comparing its revision number to that shown in the on-line version. 


\section{Summary of Changes in This Version}

Initial release

Rev1.

In steps 3.1- 3.2 changed the purge flow from $2.0 \mathrm{scfm}$ to $1.5 \mathrm{scfm}$ to be consistent with required purge flow.

Added additional step prior to step 4.1 to turn pump off before performing the measurements.

Added additional step prior to step 5.1 to turn pump on before performing steps in section 5 .

Changed flow through DI cartridge from 0.35 to 0.25 to reflect real flow rate.

Changed expected pressure sensor reading from 24 to 23 psi to match actual pressure in the system. 


\section{Exhibit A: Cooling System P\&ID}

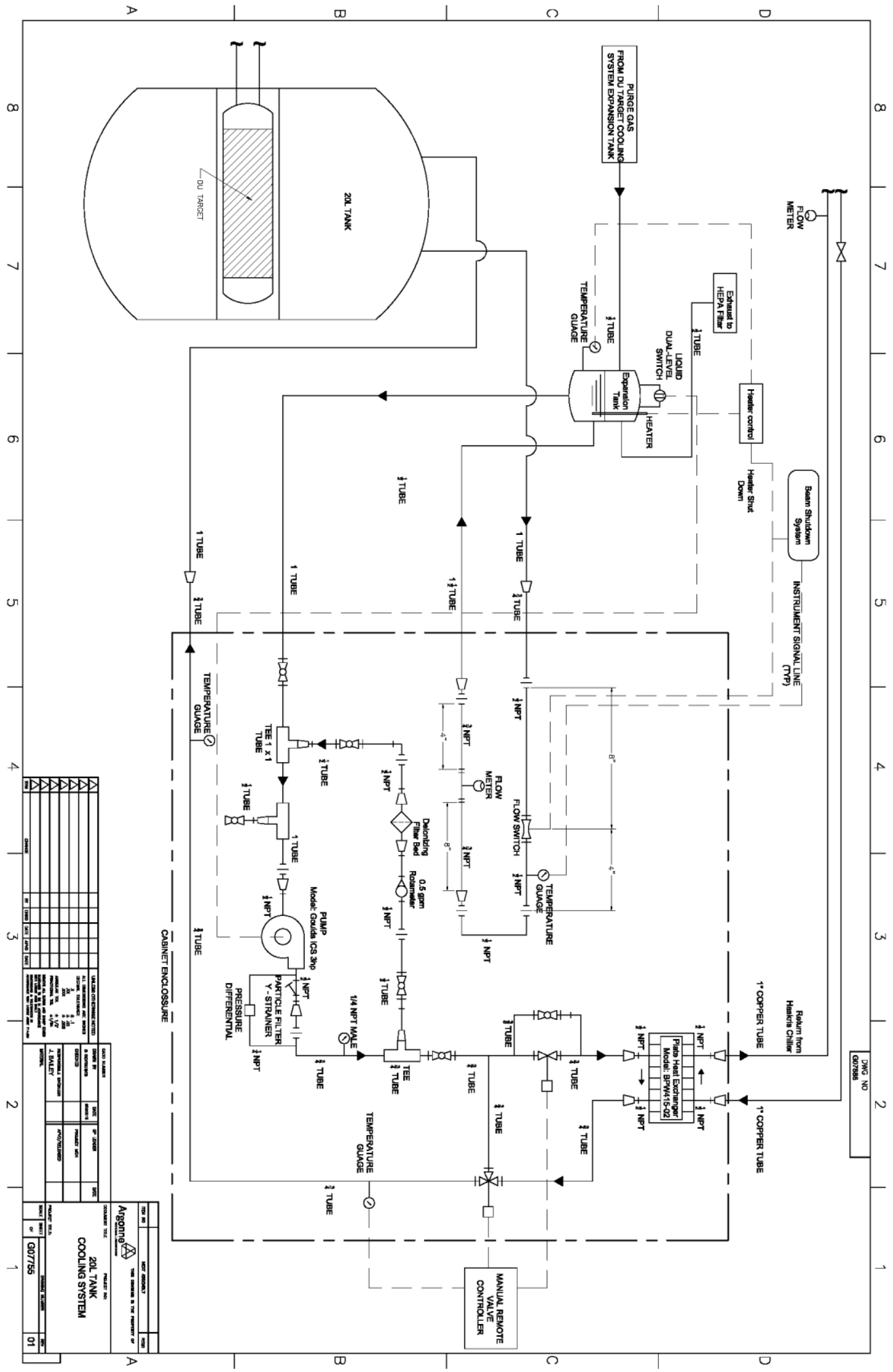

The current version of this document resides at https://leaf-docdb.ne.anl.gov/cgi-bin/DocumentDatabase. Printed or electronically downloaded copies may be obsolete. Before using such a copy for work direction, employees must verify that it is current by comparing its revision number to that shown in the on-line version. 


\section{APPENDIX 22}

LEAF-PROC-004, Rev. 0: Chiller Cooling System: Initial and Routine Startup 


\section{Chiller Cooling System: Initial and Routine Startup}

Low Energy Accelerator Facility, LEAF-PROC-004, Rev. 0

Approved:

Sch

Date: $\quad \underline{03.25 .2019}$

Sergey Chemerisov, Manager, IVEM/LEAF

Effective Date: $\underline{04.01 .2019}$

The current version of this document resides at https://leaf-docdb.ne.anl.gov/cgi-bin/DocumentDatabase. Printed or electronically downloaded copies may be obsolete. Before using such a copy for work direction, employees must verify that it is current by comparing its revision number to that shown in the on-line version. 


\section{Purpose}

Establish the process for initial and routine startup of the chiller cooling system located at the LEAF facility.

\section{Scope}

This procedure applies to the following Argonne activities and entities.

\begin{tabular}{ll}
\hline LMS core processes: & Asset Management \\
\hline Organizations: & Experimental Operations and Facilities (EOF) Division \\
\hline Buildings: & 211 \\
\hline Specific locations: & LINAC \\
\hline $\begin{array}{l}\text { Other applicability } \\
\text { factors: }\end{array}$ & None \\
\hline Exclusions: & None \\
\hline USI applicability: & Yes \\
\hline
\end{tabular}

\section{Work Process}

\subsection{Introduction}

Cooling to the target cooling system and 20L solution cooling system is provided by the chiller unit located in room D-076 in building 211. Operations of the chiller and chiller water level interlock has to be verified prior to the start of AMORE irradiation. These verifications have to be completed at the day of the irradiation.

\subsection{Step-by-Step Procedure}

The steps below are mandatory unless noted otherwise. This procedure is to be performed by Qualified Operator.

\subsubsection{Actions}

\begin{tabular}{c|ll}
\hline Step & \multicolumn{1}{c}{ Action } \\
\hline 1 & System in ready condition \\
\hline 2 & $\begin{array}{l}\text { Reservoir Float Switch } \\
2.1\end{array}$ & $\begin{array}{l}\text { Fill reservoir (to level using float level) using deionized water. } \\
2.2\end{array}$ \\
2.3 & $\begin{array}{l}\text { Lower level sensor to avoid tripping system } \\
\text { Start chiller: }\end{array}$ \\
& $\begin{array}{l}\text { - } \quad \text { Reservoir level will decrease due to filling of chilled water system } \\
\text { With the pump running, and after the reservoir has reached a steady level refill the } \\
\text { reservoir to the full level using the float level with deionized water. } \\
\text { Move the sensor level up and down to assure that it is working smoothly. The water is } \\
\text { below the trip level if the green light on the control box is off; if the water is above the }\end{array}$ \\
\hline
\end{tabular}

The current version of this document resides at https://leaf-docdb.ne.anl.gov/cgi-bin/DocumentDatabase. Printed or electronically downloaded copies may be obsolete. Before using such a copy for work direction, employees must verify that it is current by comparing its revision number to that shown in the on-line version. 


\begin{tabular}{|c|c|c|}
\hline \multirow[t]{8}{*}{ Step } & \multicolumn{2}{|r|}{ Action } \\
\hline & & trip level, the green light will be on. \\
\hline & 2.6 & $\begin{array}{l}\text { Adjust the height of the level switch so that it is just in the deactivated mode (green } \\
\text { light just goes off - starting with the green light on) }\end{array}$ \\
\hline & 2.7 & From this deactivated mode height, lower the float adjustment height down 0.6 " \\
\hline & & - The switch should now be activated - green light on. \\
\hline & 2.8 & $\begin{array}{l}\text { For the initial use of the system, (does not need to be done every time -- this is to } \\
\text { confirm the limit) do the following -- With the pump running, drain system and } \\
\text { capture the water until level switch is deactivated. Note that the chiller pump will go } \\
\text { off when the level is tripped }\end{array}$ \\
\hline & & $\begin{array}{l}\text { - The amount of water drained should be less than } 1.5 \text { gallon. If it is more than } 1.5 \\
\text { gallon, go to step } 2.9 \text { and then repeat at step } 2.5 \text {. Log the change on a tag so that } \\
\text { the next time the level will be set at } 0.3 \text { inches. }\end{array}$ \\
\hline & 2.9 & Refill the reservoir with the water drained out. \\
\hline 3 & \multicolumn{2}{|c|}{ Check out is complete } \\
\hline 4 & \multicolumn{2}{|c|}{ Date and Sign _ } \\
\hline
\end{tabular}

\section{$4 \quad$ Records Created by Work Process}

The records listed below must be retained as indicated.

\begin{tabular}{l|c|c|l|l}
\hline $\begin{array}{l}\text { Description of Record } \\
\text { (include form number if } \\
\text { applicable) }\end{array}$ & $\begin{array}{c}\text { Active } \\
\text { Records } \\
\text { Custodian }\end{array}$ & $\begin{array}{c}\text { Active } \\
\text { Records } \\
\text { Retention }\end{array}$ & $\begin{array}{l}\text { Indexing Method, } \\
\text { Storage Medium }\end{array}$ & $\begin{array}{l}\text { Federal Retention } \\
\text { Requirements* }\end{array}$ \\
\hline $\begin{array}{l}\text { Completed LEAF-PROC- } \\
004\end{array}$ & $\begin{array}{c}\text { Facility } \\
\text { Manager }\end{array}$ & 3 years & $\begin{array}{l}\text { Index by job date } \\
\text { and name, store on } \\
\text { paper or } \\
\text { electronically }\end{array}$ & $\begin{array}{l}\text { Destroy 75 years after } \\
\text { the date of the permit } \\
\text { (DOE ADM 18.37) }\end{array}$ \\
\hline
\end{tabular}

*If records are maintained in a business information system that is not currently programmed to purge digital records based on age, the records may be retained in that system past the indicated destruction date.

\section{Related Documents}

This procedure implements requirements established by the following safety basis documents:

- LEAF-SAD-100, Linac Safety Assessment Document

- LEAF-ASE-100, Linac Accelerator Safety Envelope

This procedure implements requirements established by the following Argonne policies and procedures:

- LMS-PROC-188, Accelerator Safety

- EGS-PP-100, Configuration Management Program Plan for Accelerators

\section{Definitions}

AMORE- Argonne Molybdenum Research Experiment

The current version of this document resides at https://leaf-docdb.ne.anl.gov/cgi-bin/DocumentDatabase. Printed or electronically downloaded copies may be obsolete. Before using such a copy for work direction, employees must verify that it is current by comparing its revision number to that shown in the on-line version. 


\section{About this Procedure}

\begin{tabular}{ll}
\hline Issuing organization: & Low Energy Accelerator Facility \\
\hline Procedure owner: & S. Chemerisov \\
\hline Point of contact: & S. Chemerisov \\
\hline Review cycle (months): & 36 \\
\hline Date last revised: & 03.25 .2019 \\
\hline Date last reviewed: & 03.25 .2019 \\
\hline
\end{tabular}

\section{Summary of Changes in This Version}

Initial release 


\section{APPENDIX 23}

LEAF-PROC-006, Rev. 2: DU Target Cooling System: Initial Startup 


\section{DU Target Cooling System: Initial Startup}

Low Energy Accelerator Facility, LEAF-PROC-006, Rev. 2

Approved: SCh

Sergey Chemerisov, Manager, IVEM/LEAF

Effective Date: $\underline{09.16 .2019}$

The current version of this document resides at https://leaf-docdb.ne.anl.gov/cgi-bin/DocumentDatabase. Printed or electronically downloaded copies may be obsolete. Before using such a copy for work direction, employees must verify that it is current by comparing its revision number to that shown in the on-line version. 


\section{Purpose}

Establish the process for initial startup of the depleted uranium (DU) target cooling system installed at the LEAF facility.

\section{Scope}

This procedure applies to the following Argonne activities and entities.

\begin{tabular}{ll}
\hline LMS core processes: & Asset Management \\
\hline Organizations: & Experimental Operations and Facilities (EOF) Division \\
\hline Buildings: & 211 \\
\hline Specific locations: & LINAC \\
\hline $\begin{array}{l}\text { Other applicability } \\
\text { factors: }\end{array}$ & None \\
\hline Exclusions: & None \\
\hline USI applicability: & Yes \\
\hline
\end{tabular}

\section{Work Process}

\subsection{Introduction}

During AMORE irradiation 20kW of electron beam from accelerator will be placed on the target. Cooling of the target is achieved by flowing cooling water through the target housing and through the spacers between target disks. Before commencing AMORE irradiation cooling system has to be turned on, operations of the system has to be verified and interlocks preventing beam operations has to be checked [ASE2.4.1.1, 2.6.1.1]. Steps necessary to perform those operations are listed below.

\subsection{Step-by-Step Procedure}

The steps below are mandatory unless noted otherwise. All steps must be written into start-up check-list with logging all measurements of temperature, pressure, and flow rate. If at any step the measured value is out of compliance, or the system does not response in proper way (motor won't start, control light not switched on, etc.), immediately stop the process, inform Facility Manager, and initiate the troubleshooting process in accordance with proper WCD. This procedure is to be performed by Qualified Linac Operator.

\subsubsection{Actions}

\begin{tabular}{c|lc}
\hline Step & \multicolumn{1}{|c}{ Action } \\
\hline 1 & System in ready condition & \\
\hline
\end{tabular}

The current version of this document resides at https://leaf-docdb.ne.anl.gov/cgi-bin/DocumentDatabase. Printed or electronically downloaded copies may be obsolete. Before using such a copy for work direction, employees must verify that it is current by comparing its revision number to that shown in the on-line version. 


\begin{tabular}{|c|c|c|}
\hline Step & & Action \\
\hline \multirow[t]{8}{*}{2} & \multicolumn{2}{|c|}{ Expansion Tank Float Switches } \\
\hline & 2.1 & Fill expansion tank until both lights go on. \\
\hline & 2.2 & Start pump. \\
\hline & 2.3 & $\begin{array}{l}\text { With pump running, the rest of the system will start to fill, lowering the level in the } \\
\text { expansion, and in turn the bottom light will go off and pump will stop. }\end{array}$ \\
\hline & 2.4 & $\begin{array}{l}\text { Refill the tank and repeat steps } 1-3 \text { until the system is full and the bottom light stays } \\
\text { on. }\end{array}$ \\
\hline & 2.5 & Start pump and drain water until bottom light goes off and pump stops. \\
\hline & 2.6 & Stop pump and refill system to ready condition (i.e., repeat steps 1-3). \\
\hline & 2.7 & Step 2 is completed ______ (Initial) \\
\hline \multirow[t]{9}{*}{3} & \multicolumn{2}{|c|}{ Expansion Tank Purge System } \\
\hline & 3.1 & Set Purge flow rate to $1.5 \pm 0.2 \mathrm{scfm}$. \\
\hline & 3.2 & Flow Switch interlock for purge: \\
\hline & & - Reduce purge flow to $1.0 \pm 0.2 \mathrm{scfm}$ \\
\hline & & - Adjust flow switch to open at the $1.0 \pm 0.2 \mathrm{scfm}$. \\
\hline & & - Beam power relay should be deactivated (light on control panel is off). \\
\hline & & - Increase purge flow to $1.5 \pm 0.2 \mathrm{scfm}$ \\
\hline & & - Beam power relay is/should be activated (light on control panel is on). \\
\hline & 3.3 & Step 3 is completed ___ (Initial) \\
\hline \multirow[t]{4}{*}{4} & \multicolumn{2}{|c|}{ Pump off } \\
\hline & 4.1 & Pressure sensor reading should be $3.0 \pm 3.0$ psi. \\
\hline & 4.2 & Differential pressure sensor reading should be $0.0 \pm 0.5$ psi. \\
\hline & 4.3 & Step 4 is completed _____ (Initial) \\
\hline \multirow[t]{6}{*}{5} & \multicolumn{2}{|c|}{ Start pump } \\
\hline & 5.1 & Set flow rate to $42.0 \pm 1.0 \mathrm{gpm}$ using the throttle valve [ASE 2.6.1.1]. \\
\hline & 5.2 & Adjust flow through DI to $0.3 \pm 0.1 \mathrm{gpm}$ using upstream ball valve. \\
\hline & 5.3 & Pressure sensor reading should be $50.0 \pm 3.0$ psi. \\
\hline & 5.4 & Differential pressure sensor reading across strainer should be $2.0 \pm 0.5 \mathrm{psi}$. \\
\hline & 5.5 & Step 5 is completed _____ (Initial) \\
\hline
\end{tabular}




\begin{tabular}{|c|c|c|}
\hline Step & & Action \\
\hline \multirow[t]{16}{*}{6} & \multicolumn{2}{|c|}{ Flow switch interlock and thermocouple interlock } \\
\hline & 6.1 & Reduce the flow rate from $42.0 \mathrm{gpm}$ to $40.0 \pm 1.0 \mathrm{gpm}$ using the throttle valve. \\
\hline & 6.2 & Set the flow switch to open at 40.0 $\pm 1.0 \mathrm{gpm}$ [ASE 2.6.1.1]. \\
\hline & 6.3 & Beam power relay should be deactivated (light on control panel is off). \\
\hline & 6.4 & Increase the flow back to $42.0 \pm 1.0 \mathrm{gpm}$ [ASE 2.6.1.1]. \\
\hline & 6.5 & Beam power relay should be activated (light on control panel is on). \\
\hline & 6.6 & Reduce the flow to $40.0 \pm 1.0 \mathrm{gpm}$ to check flow switch setting [ASE 2.6.1.1]. \\
\hline & 6.7 & Increase flow to the design flow of $>42 \pm 1.0 \mathrm{gpm}$ [ASE 2.6.1.1]. \\
\hline & 6.8 & Beam power relay should be activated (light on control panel is on). \\
\hline & 6.9 & $\begin{array}{l}\text { Remove } \mathrm{T} / \mathrm{C} \text { at return line from DU target and insert the sensor in a } 100 \pm 5^{\circ} \mathrm{F} \text { water } \\
\text { bath. }\end{array}$ \\
\hline & 6.10 & $\begin{array}{l}\text { Beam power relay should be deactivated (light on control panel is off) [ASE } \\
\text { 2.6.1.2]. }\end{array}$ \\
\hline & 6.11 & Insert the sensor in an $80 \pm 5^{\circ} \mathrm{F}$ water bath. \\
\hline & 6.12 & Beam power relay should be activated (light on control panel is on) [ASE 2.6.1.2]. \\
\hline & 6.13 & Reinsert $\mathrm{T} / \mathrm{C}$ in return line. \\
\hline & 6.14 & Beam power relay should be activated (light on control panel is on) [ASE 2.6.1.2]. \\
\hline & 6.15 & Step 6 is completed ___ (Initial) \\
\hline \multirow[t]{5}{*}{7} & \multicolumn{2}{|l|}{ Chiller } \\
\hline & 7.1 & $\begin{array}{l}\text { Perform chiller startup steps in accordance with "Chiller Cooling System: Initial and } \\
\text { Routine Startup", LEAF-PROC-004. }\end{array}$ \\
\hline & 7.2 & Start and record temperatures and flow (at chiller). \\
\hline & 7.3 & $\begin{array}{l}\text { Adjust flow to DU target heat exchanger to } 9.0 \pm 0.5 \mathrm{gpm} \text {, outlet temperature should be } \\
55 \pm 5^{\circ} \mathrm{F} \text {. }\end{array}$ \\
\hline & 7.4 & Step 7 is completed ___ (Initial) \\
\hline 8 & \multicolumn{2}{|c|}{ Check out is complete } \\
\hline 9 & \multicolumn{2}{|c|}{ Date and sign } \\
\hline
\end{tabular}

The current version of this document resides at https://leaf-docdb.ne.anl.gov/cgi-bin/DocumentDatabase. Printed or electronically downloaded copies may be obsolete. Before using such a copy for work direction, employees must verify that it is current by comparing its revision number to that shown in the on-line version. 


\section{Records Created by Work Process}

The records listed below must be retained as indicated.

\begin{tabular}{l|c|c|l|l}
\hline $\begin{array}{l}\text { Description of Record } \\
\text { (include form number if } \\
\text { applicable) }\end{array}$ & $\begin{array}{c}\text { Active } \\
\text { Records } \\
\text { Custodian }\end{array}$ & $\begin{array}{c}\text { Active } \\
\text { Records } \\
\text { Retention }\end{array}$ & $\begin{array}{l}\text { Indexing Method, } \\
\text { Storage Medium }\end{array}$ & $\begin{array}{l}\text { Federal Retention } \\
\text { Requirements* }\end{array}$ \\
\hline $\begin{array}{l}\text { Completed LEAF-PROC- } \\
006\end{array}$ & $\begin{array}{c}\text { Facility } \\
\text { Manager }\end{array}$ & 3 years & $\begin{array}{l}\text { Index by job date } \\
\text { and name, store on } \\
\text { paper or } \\
\text { electronically }\end{array}$ & $\begin{array}{l}\text { Destroy 75 years after } \\
\text { the date of the permit } \\
\text { (DOE ADM 18.37) }\end{array}$ \\
\hline
\end{tabular}

*If records are maintained in a business information system that is not currently programmed to purge digital records based on age, the records may be retained in that system past the indicated destruction date.

\section{Related Documents}

This procedure implements requirements established by the following safety basis documents:

- LEAF-SAD-100, Linac Safety Assessment Document

- LEAF-ASE-100, Linac Accelerator Safety Envelope

This procedure implements requirements established by the following Argonne policies and procedures:

- LMS-PROC-188, Accelerator Safety

- EGS-PP-100, Configuration Management Program Plan for Accelerators

\section{Definitions}

None

\section{$7 \quad$ About this Procedure}

\begin{tabular}{ll}
\hline Issuing organization: & Low Energy Accelerator Facility \\
\hline Procedure owner: & S. Chemerisov \\
\hline Point of contact: & S. Chemerisov \\
\hline Review cycle (months): & 36 \\
\hline Date last revised: & 09.06 .2019 \\
\hline Date last reviewed: & 09.12 .2019 \\
\hline
\end{tabular}

\section{Summary of Changes in This Version}

Initial release

Rev. 1. Addition of the references to the ASE controlled parameters.

Rev. 2. Change flow through the DI unit in step 5.2 from $1 \mathrm{gpm}$ to $0.3 \mathrm{gpm}$.

Change the pressure readout for the pressure transducer to $50 \mathrm{psi}$ to reflect real measurements in the system

The current version of this document resides at https://leaf-docdb.ne.anl.gov/cgi-bin/DocumentDatabase. Printed or electronically downloaded copies may be obsolete. Before using such a copy for work direction, employees must verify that it is current by comparing its revision number to that shown in the on-line version. 


\section{Exhibit A: P\&ID of the Target Cooling System}

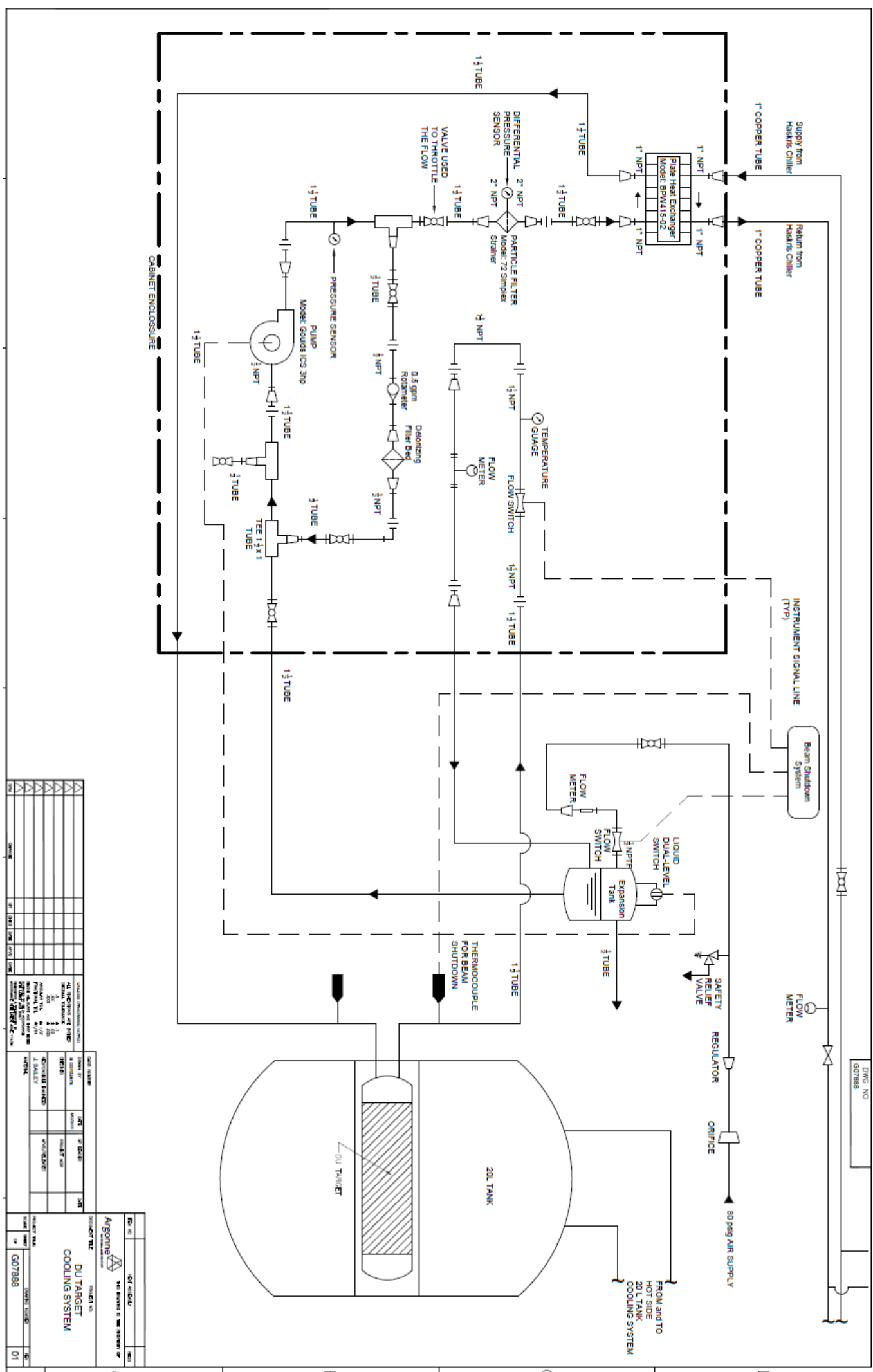

The current version of this document resides at https://leaf-docdb.ne.anl.gov/cgi-bin/DocumentDatabase. Printed or electronically downloaded copies may be obsolete. Before using such a copy for work direction, employees must verify that it is current by comparing its revision number to that shown in the on-line version. 


\section{APPENDIX 24}

LEAF-PROC-007, Rev. 2: DU Target Cooling System: Routine Startup Procedure 


\section{DU Target Cooling System: Routine Startup Procedure}

Low Energy Accelerator Facility, LEAF-PROC-007, Rev. 2

Approved: SChn Date: $\underline{09.18 .2019}$

Sergey Chemerisov, Manager, IVEM/LEAF

Effective Date: 09.19 .2019

The current version of this document resides at https://leaf-docdb.ne.anl.gov/cgi-bin/DocumentDatabase. Printed or electronically downloaded copies may be obsolete. Before using such a copy for work direction, employees must verify that it is current by comparing its revision number to that shown in the on-line version. 


\section{Purpose}

Establish the process for starting up the depleted uranium (DU) target cooling system at the LEAF facility under routine conditions.

\section{Scope}

This procedure applies to the following Argonne activities and entities.

\begin{tabular}{ll}
\hline LMS core processes: & Asset Management \\
\hline Organizations: & Experimental Operations and Facilities (EOF) Division \\
\hline Buildings: & 211 \\
\hline Specific locations: & LINAC \\
\hline $\begin{array}{l}\text { Other applicability } \\
\text { factors: }\end{array}$ & None \\
\hline Exclusions: & None \\
\hline USI applicability: & Yes \\
\hline
\end{tabular}

\section{Work Process}

\subsection{Introduction}

During AMORE irradiation 20kW of electron beam from accelerator will be placed on the target. Cooling of the target is achieved by flowing cooling water through the target housing and through the spacers between target disks. Before commencing AMORE irradiation cooling system has to be turned on, operations of the system has to be verified and interlocks preventing beam operations has to be checked. Steps necessary to perform those operations are listed below. This procedure has to be performed on the day of irradiation.

\subsection{Step-by-Step Procedure}

The steps below are mandatory unless noted otherwise. This procedure is to be performed by Qualified Operator (QLO). QLO should indicate initial each step in this procedure indicating that all required conditions are satisfied.

\subsubsection{Actions}

\begin{tabular}{c|lc}
\hline Step & \multicolumn{1}{|c}{ Action } \\
\hline 1 & System in ready condition & \\
\hline
\end{tabular}

The current version of this document resides at https://leaf-docdb.ne.anl.gov/cgi-bin/DocumentDatabase. Printed or electronically downloaded copies may be obsolete. Before using such a copy for work direction, employees must verify that it is current by comparing its revision number to that shown in the on-line version. 


\begin{tabular}{|c|c|c|}
\hline Step & & Action \\
\hline 2 & $\begin{array}{l}2.1 \\
2.2\end{array}$ & $\begin{array}{l}\text { sion Tank Purge System } \\
\text { Purge flow rate set at } 1.5 \pm 0.2 \mathrm{scfh} \\
\text { Flow Switch interlock for purge } \\
\text { - } \quad \text { Reduce purge flow to } 1.0 \pm 0.2 \mathrm{scfm} \\
\text { - Beam power relay should be deactivated (light on control panel is off) } \\
\text { - Increase purge flow to } 1.5 \pm 0.2 \mathrm{scfm} \\
\text { - Beam power relay is should be activated (light on control panel is on) } \\
\text { Note: At initial startup, set flow switch trip at } 1.5 \pm 0.2 \mathrm{scfm}\end{array}$ \\
\hline 3 & $\begin{array}{l}3.1 \\
3.2\end{array}$ & $\begin{array}{l}\text { Prf } \\
\text { Pressure sensor reading should be } 4.5 \mathrm{ma}(3 \pm 3 \mathrm{psi}) \\
\text { Differential pressure sensor reading should be } 4.0 \mathrm{ma}(0 \pm 0.5 \mathrm{psi})\end{array}$ \\
\hline 4 & $\begin{array}{l}4.1 \\
4.2 \\
4.3 \\
4.4\end{array}$ & $\begin{array}{l}\text { Pressure sensor reading should be } 12.4 \mathrm{ma}(50.0 \pm 3.0 \mathrm{psi}) \\
\text { Differential pressure sensor reading should be } 6.4 \mathrm{ma}(2.0 \pm 0.5 \mathrm{psi}) \\
\text { Flow through DI unit should be } 0.3 \pm 0.1 \mathrm{gpm} \\
\text { Flow though the target is }>42 \mathrm{gpm}\end{array}$ \\
\hline 5 & 5.6 & $\begin{array}{l}\text { witch interlock and thermocouple interlock } \\
\text { Pump off; the beam power relay should be deactivated (light on control panel is off) } \\
\text { [ASE 2.6.1.2] } \\
\text { Pump on; the beam power relay should be activated (light on control panel is on) } \\
\text { [ASE 2.6.1.2] } \\
\text { Remove thermocouple (T/C) at return line from DU target and insert the sensor in a } \\
\text { 32C water bath } \\
\text { Beam power relay should be deactivated (light on control panel is off) } \\
\text { [ASE 2.6.1.2] } \\
\text { Reinsert T/C in return line } \\
\text { Beam power relay should be activated (light on control panel is on) } \\
\text { 2.6.1.2] } \\
\text { Verify the temperature of the water at the exit of the target is }<20 \mathrm{C} \\
\text { 2.6.1.1] }\end{array}$ \\
\hline
\end{tabular}




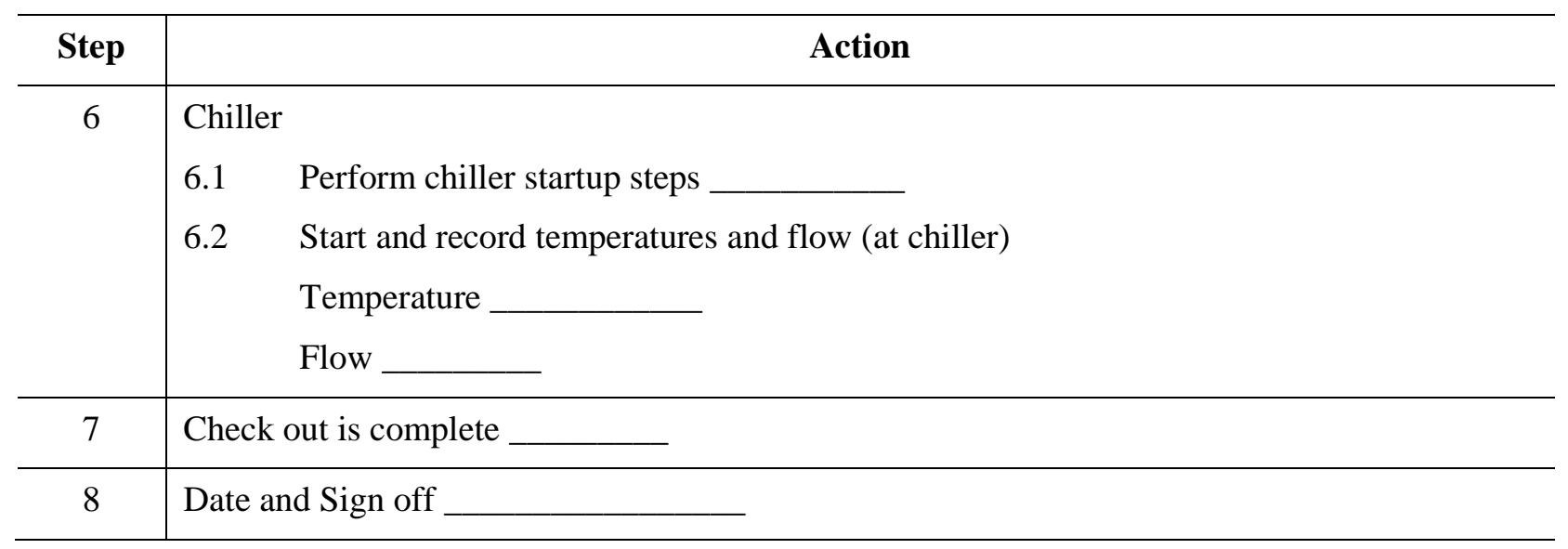

\section{Records Created by Work Process}

The records listed below must be retained as indicated.

\begin{tabular}{l|c|c|l|l}
\hline $\begin{array}{l}\text { Description of Record } \\
\text { (include form number if } \\
\text { applicable) }\end{array}$ & $\begin{array}{c}\text { Active } \\
\text { Records } \\
\text { Custodian }\end{array}$ & $\begin{array}{c}\text { Active } \\
\text { Records } \\
\text { Retention }\end{array}$ & $\begin{array}{l}\text { Indexing Method, } \\
\text { Storage Medium }\end{array}$ & $\begin{array}{l}\text { Federal Retention } \\
\text { Requirements* }\end{array}$ \\
\hline $\begin{array}{l}\text { Completed LEAF-PROC- } \\
007\end{array}$ & $\begin{array}{c}\text { Facility } \\
\text { Manager }\end{array}$ & 3 years & $\begin{array}{l}\text { Index by job date } \\
\text { and name, store on } \\
\text { paper or } \\
\text { electronically }\end{array}$ & $\begin{array}{l}\text { Destroy 75 years after } \\
\text { the date of the permit } \\
\text { (DOE ADM 18.37) }\end{array}$ \\
\hline
\end{tabular}

*If records are maintained in a business information system that is not currently programmed to purge digital records based on age, the records may be retained in that system past the indicated destruction date.

\section{$5 \quad$ Related Documents}

This procedure implements requirements established by the following safety basis documents:

- LEAF-SAD-100, Linac Safety Assessment Document

- LEAF-ASE-100, Linac Accelerator Safety Envelope

This procedure implements requirements established by the following Argonne policies and procedures:

- LMS-PROC-188, Accelerator Safety

- EGS-PP-100, Configuration Management Program Plan for Accelerators

\section{Definitions}

None

The current version of this document resides at https://leaf-docdb.ne.anl.gov/cgi-bin/DocumentDatabase. Printed or electronically downloaded copies may be obsolete. Before using such a copy for work direction, employees must verify that it is current by comparing its revision number to that shown in the on-line version. 


\section{$7 \quad$ About this Procedure}

\begin{tabular}{ll}
\hline Issuing organization: & Low Energy Accelerator Facility \\
\hline Procedure owner: & S. Chemerisov \\
\hline Point of contact: & S. Chemerisov \\
\hline Review cycle (months): & 36 \\
\hline Date last revised: & 09.17 .2019 \\
\hline Date last reviewed: & 09.18 .2019 \\
\hline
\end{tabular}

\section{Summary of Changes in This Version}

Initial release

Rev. 1. Addition of the references to the ASE controlled parameters.

Rev. 2. Changed purge flow rate in step 2.1 and 2.2 from 2.0 schf to 1.5 schf to reflect actual purge flow rate in the system. Changed expected value for pressure transducer from 53.2 psi to $5050 \pm 3$ psi to reflect actual pressure generated by the pump and to be consistent with LEAF-PROC-006 


\section{Exhibit A: P\&ID of the Target Cooling System}

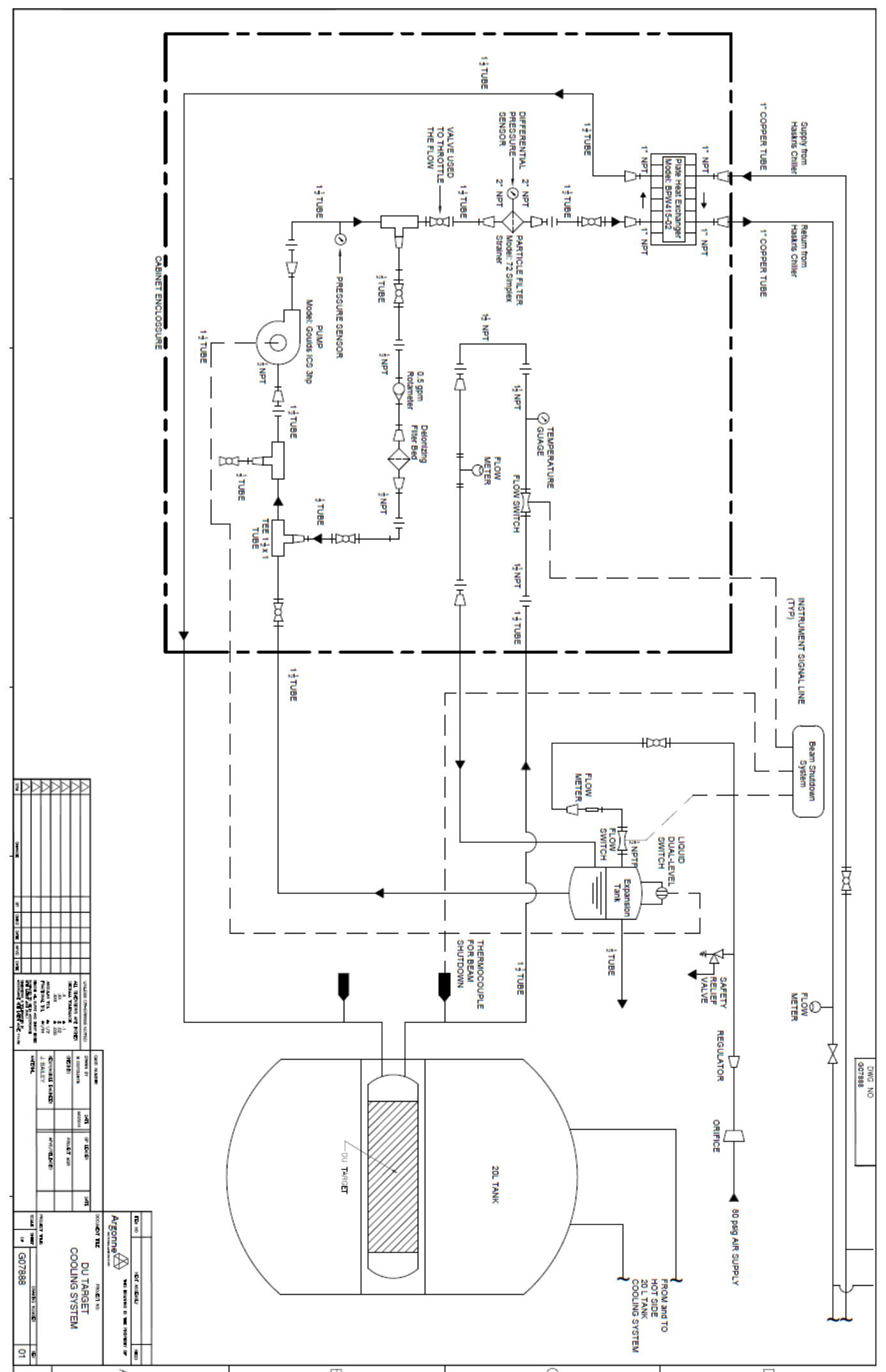

The current version of this document resides at https://leaf-docdb.ne.anl.gov/cgi-bin/DocumentDatabase. Printed or electronically downloaded copies may be obsolete. Before using such a copy for work direction, employees must verify that it is current by comparing its revision number to that shown in the on-line version. 


\section{APPENDIX 25}

LEAF-PROC-027, Rev. 0: LEAF Linac General Operating Procedure 


\section{LEAF Linac General Operating Procedure}

Low Energy Accelerator Facility, LEAF-PROC-027, Rev. 0

Approved: SChn Date: $\underline{03.25 .2019}$

Sergey Chemerisov, Manager, IVEM/LEAF

Effective Date: $\underline{04.01 .2019}$

The current version of this procedure resides at http://inside.anl.gov/documentcenter. Verify that the copy of the procedure you are using is current by comparing the revision number as printed copies can be obsolete. 


\section{Purpose}

Establish the process for operation of the Linac Facility in building 211.

\section{Scope}

This procedure applies to the following Argonne activities and entities.

\begin{tabular}{ll}
\hline LMS core processes: & Asset Management \\
\hline Organizations: & Experimental Operations and Facilities (EOF) Division \\
\hline Buildings: & 211 \\
\hline Specific locations: & LINAC \\
\hline $\begin{array}{l}\text { Other applicability } \\
\text { factors: }\end{array}$ & None \\
\hline Exclusions: & None \\
\hline USI applicability: & Yes \\
\hline
\end{tabular}

\section{Work Process}

\subsection{Introduction}

This document contains the procedures for general operation of the Linac, including start up, normal operation, stand by and shut down.

If any malfunctions occur, which make it impossible to satisfactorily complete any of the procedures; such malfunctions must be corrected before continuing. Due to the complexity of the equipment and the multitude of malfunctions which may occur, no attempt is made to specify repair procedures. All repairs must be made in compliance with applicable safety standards.

\subsection{Step-by-Step Procedure}

The steps below are mandatory unless noted otherwise. This procedure is to be performed by Qualified Linac Operator (QLO).

\subsubsection{Actions}

\begin{tabular}{|c|c|c|}
\hline Step & & Action \\
\hline \multirow[t]{5}{*}{1} & \multicolumn{2}{|c|}{ Set Up Procedure for Linac Operator } \\
\hline & \multicolumn{2}{|r|}{$\begin{array}{l}\text { Follow access procedures in LEAF-PROC-010, Linac Shielding Procedure, Section } .2 \text { when } \\
\text { entering potential beam areas. }\end{array}$} \\
\hline & 1.1 & Determine which beam line the experimenter will use for his run. \\
\hline & 1.2 & $\begin{array}{l}\text { AMORE experimentation shall be limited to five }{ }^{* *} \text { full irradiation runs (full run is any } \\
\text { run where more than } 175 \mathrm{~kW}^{*} \text { hrs delivered to the target). }\end{array}$ \\
\hline & & $\begin{array}{l}\text { AMORE irradiation run shall be limited to an integrated energy deposition of } \leq 700 \mathrm{kw}- \\
\mathrm{hrs} * * \text { without prior approval from DOE-Argonne Site Office (ASO). }\end{array}$ \\
\hline
\end{tabular}

The current version of this procedure resides at http://inside.anl.gov/documentcenter. Verify that the copy of the procedure you are using is current by comparing the revision number as printed copies can be obsolete. 


\begin{tabular}{|c|c|c|c|}
\hline \multirow[t]{8}{*}{ Step } & \multicolumn{3}{|r|}{ Action } \\
\hline & 1.4 & \multirow{2}{*}{\multicolumn{2}{|c|}{$\begin{array}{l}\text { Maximum average beam current in the port located in D-017 (Pit) room is } 200 \mu \mathrm{A}^{* *} \text {. } \\
\text { Maximum average beam current for beam port located in D-035 (Cell 1) is } 200 \mu \mathrm{A} * * \\
\text { for the ports equipped with aluminum window and } 1.5 \mathrm{~mA}^{* *} \text { for water cooled } \\
\text { beryllium window. }\end{array}$}} \\
\hline & 1.5 & & \\
\hline & 1.6 & \multicolumn{2}{|r|}{ Maximum beam energy for any operations o fthe accelerator is limited to $60 \mathrm{MeV}$.** $^{*}$} \\
\hline & 1.7 & \multicolumn{2}{|r|}{$\begin{array}{l}\text { Put quartz over window (if needed) and set up water-cooled beam stopper (if } \\
\text { required). }\end{array}$} \\
\hline & 1.8 & \multicolumn{2}{|c|}{ Hook signal cable to beam stopper and note cable used. } \\
\hline & 1.9 & \multicolumn{2}{|c|}{ Set up TV camera with monitor or OTR-camera, if required. } \\
\hline & 1.10 & \multicolumn{2}{|r|}{$\begin{array}{l}\text { Position Beam Port switch(es) (B.P.) the appropriate position(s). If a B.P. has to be } \\
\text { moved, assure that the Linac exit valve is closed prior to moving B.P. }\end{array}$} \\
\hline \multirow[t]{17}{*}{2} & \multicolumn{3}{|c|}{ Start Up Procedure for Linac Operator } \\
\hline & 2.1 & \multicolumn{2}{|c|}{ In Room D-101 (Control Room): } \\
\hline & & 2.1 .1 & $\begin{array}{l}\text { Log vacuum }(<1 \times 10-7 \text { Torr) (If any vacuum readings are higher than } \\
1 \mathrm{X} 10-7 \text { Torr, check the reason.) }\end{array}$ \\
\hline & & 2.1 .2 & Turn on control power using both the switch and the key. \\
\hline & & \multirow[t]{3}{*}{2.1 .3} & $\begin{array}{l}\text { Run up injector filament voltage (filament needs to be higher than what is } \\
\text { mentioned below in A and B) }\end{array}$ \\
\hline & & & A. $65 \%$ for nsec or picosec work \\
\hline & & & $\begin{array}{l}\text { B. Approximately } 55 \% \text { for } 1.5 \text { A gun. (Try to run gun close to emission limit, } \\
\text { this will give flattest pulse.) }\end{array}$ \\
\hline & & 2.1.4 & $\begin{array}{l}\text { If running nsec or picosec pulse, run short-pulse pulser amplitude control to } \\
\text { zero, so pulser tubes will be in conditioning mode. }\end{array}$ \\
\hline & & 2.1 .5 & Reset trigger generator \\
\hline & 2.2 & \multicolumn{2}{|c|}{ In Room D-117 (Modulator Room): } \\
\hline & & 2.2 .1 & At sub-station, turn on $480 \mathrm{~V}$ disconnect. \\
\hline & & 2.2.2 & $\begin{array}{l}\text { Turn on klystron cooling water pump (check water level in make-up tank is } \\
\text { approx. one-half full). }\end{array}$ \\
\hline & & 2.2 .3 & Turn on modulator cabinet cooling fan. \\
\hline & & 2.2 .4 & Turn on modulator room cooling fans (if weather is warm outside). \\
\hline & & 2.2 .5 & Clear core bias interlocks, if not already clear. \\
\hline & & 2.2 .6 & $\begin{array}{l}\text { Check thyratron control panel (reservoir, filament voltage). If not at marked } \\
\text { meter value, adjust to correct setting. }\end{array}$ \\
\hline & & 2.2.7 & $\begin{array}{l}\text { If pico-sec run, turn on sub-harmonic buncher (SHB) power supply control } \\
\text { voltage and SHB radio frequency (RF) preamplifier. }\end{array}$ \\
\hline
\end{tabular}

The current version of this procedure resides at http://inside.anl.gov/documentcenter. Verify that the copy of the procedure you are using is current by comparing the revision number as printed copies can be obsolete. 


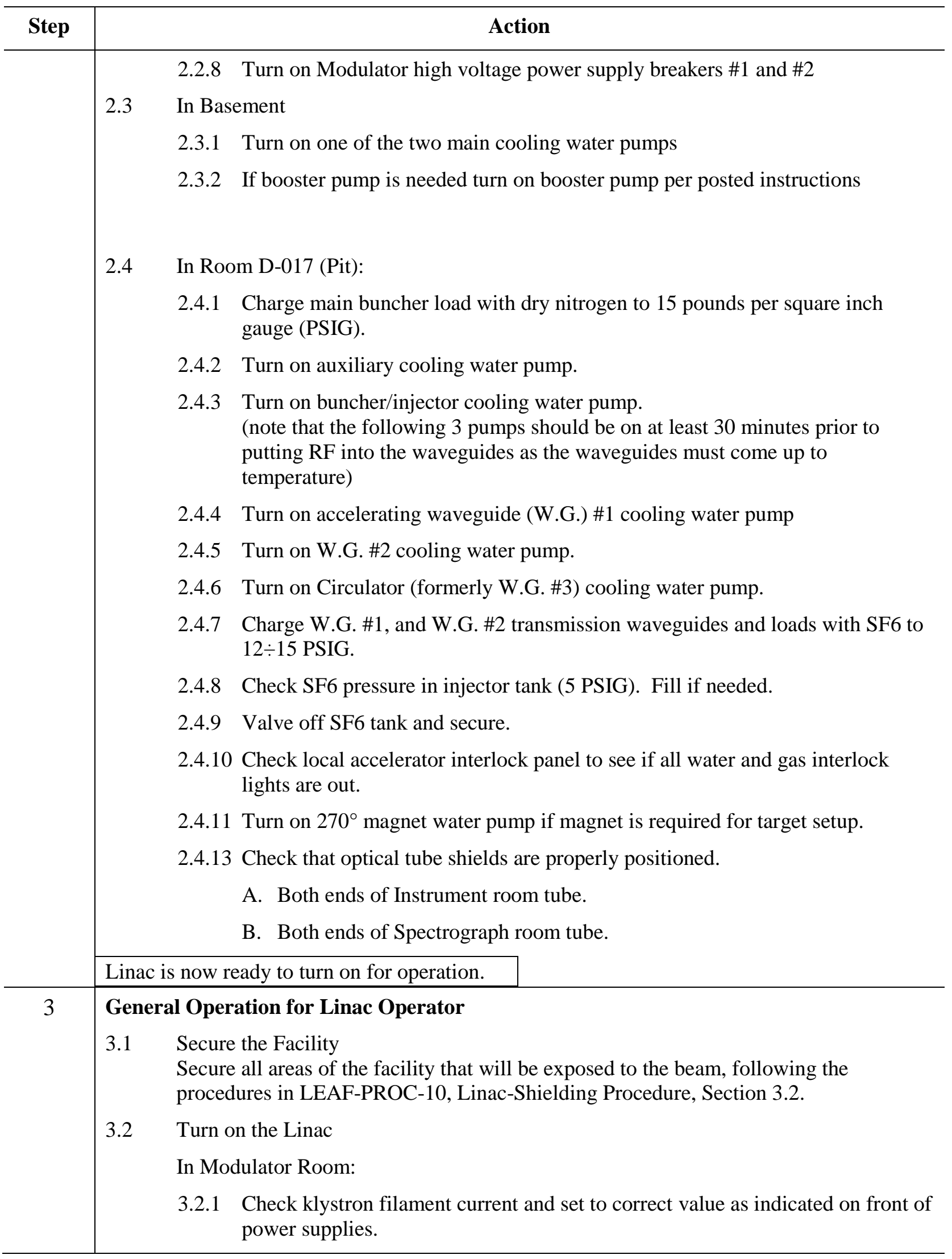

The current version of this procedure resides at http://inside.anl.gov/documentcenter. Verify that the copy of the procedure you are using is current by comparing the revision number as printed copies can be obsolete. 


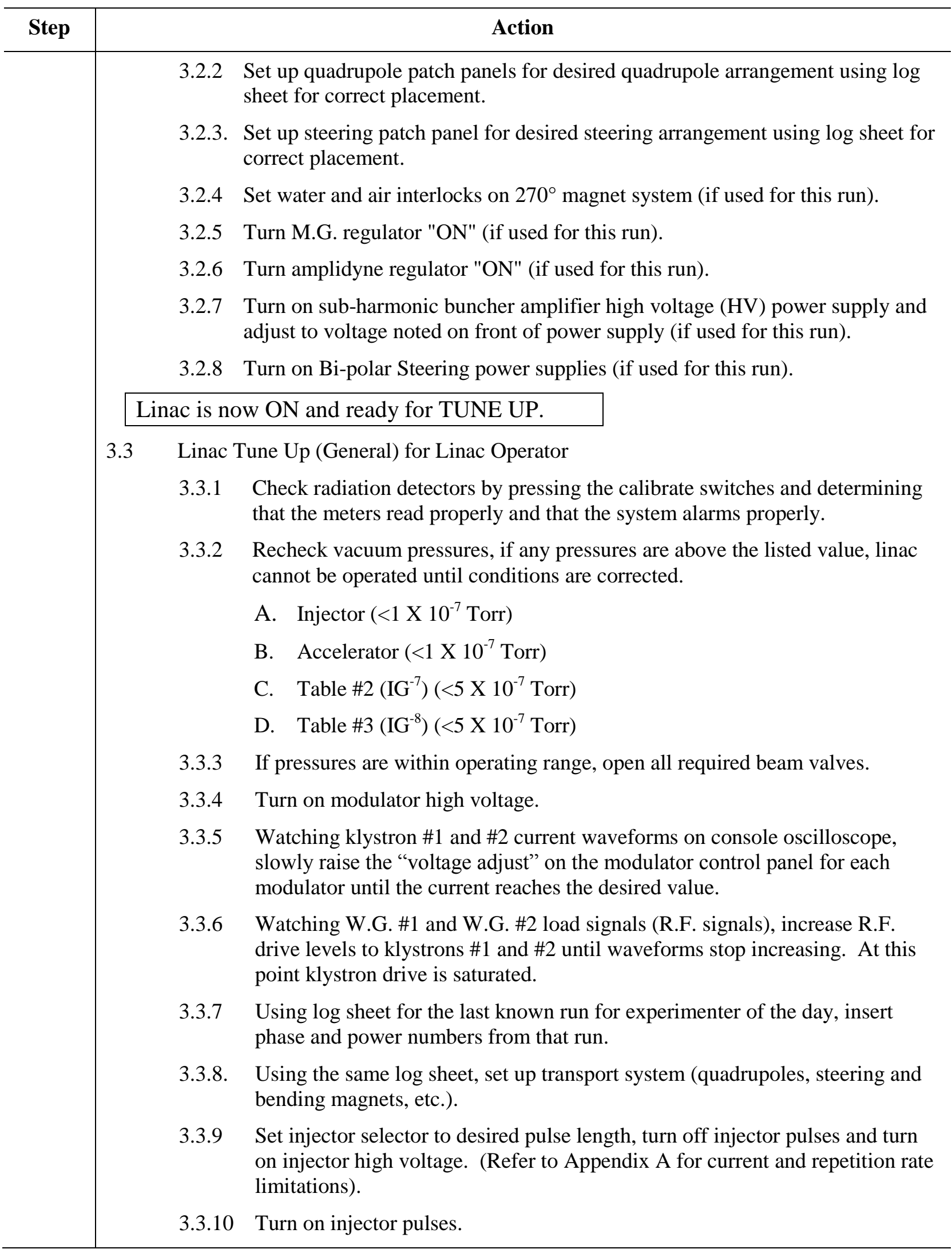

The current version of this procedure resides at http://inside.anl.gov/documentcenter. Verify that the copy of the procedure you are using is current by comparing the revision number as printed copies can be obsolete. 


\begin{tabular}{|c|c|c|}
\hline \multirow[t]{10}{*}{ Step } & \multicolumn{2}{|r|}{ Action } \\
\hline & \multicolumn{2}{|r|}{ 3.3.11 } \\
\hline & & $\begin{array}{l}\text { A. For } \mu \text { sec pulses, watching beam amplitude and shape on scope, raise and } \\
\text { lower RF phases until maximum beam current is obtained for the best pulse } \\
\text { shape. } \\
\text { B. For nano-sec and picosec pulses, watching beam current meter, raise and } \\
\text { lower RF phases until maximum beam current is obtained. }\end{array}$ \\
\hline & & 3.3.12 Adjust lens \#1 and \#2 for maximum beam current. \\
\hline & & $\begin{array}{l}\text { 3.3.13 If a defined energy distribution of the electrons is necessary, an energy } \\
\text { spectrum can be plotted by bending the beam at Table } \# 2-90^{\circ} \text { port. Use the } \\
\text { EPICS program to measure and set the energy. }\end{array}$ \\
\hline & & $\begin{array}{l}\text { 3.3.14 Log the settings of the Linac parameters. (Print out and retain as a record the } \\
\text { Daily Log (Appendix B) generated by the Linac software.) }\end{array}$ \\
\hline & & ac is now ON and ready for use. \\
\hline & 3.4 & Linac Operation for Linac Operator \\
\hline & & $\begin{array}{l}\text { Return the beam to the experimenter's port, shape beam spot to desired shape with } \\
\text { Quadrupoles and steering. }\end{array}$ \\
\hline & & $\begin{array}{l}\text { Linac operator must be present in the control room all the time during beam-on } \\
\text { operations. If one operator have to leave control room for any reason while beam } \\
\text { is on, other operator has to take his place in the control room or Linac has to be } \\
\text { placed in stand-by mode. }\end{array}$ \\
\hline \multirow[t]{8}{*}{4} & \multicolumn{2}{|c|}{ Stand-by Operation for Linac Operator } \\
\hline & \multicolumn{2}{|r|}{ For any long delay in use of the Linac, it should be placed in stand-by as follows: } \\
\hline & 4.1 & Turn off modulator high voltage key switch and remove. \\
\hline & 4.2 & Turn off injector high voltage. \\
\hline & 4.3 & Turn off Helmholtz power supply. \\
\hline & 4.4 & Place short pulser in pulsed diode mode (if used). \\
\hline & 4.5 & Close exit valve from Linac accelerator section \\
\hline & & $\begin{array}{l}\text { NOTE: Potential beam area may be entered during Stand-By Operation. } \\
\text { Vault must be secured before leaving the Stand-By Operation mode. }\end{array}$ \\
\hline \multirow[t]{4}{*}{5} & \multicolumn{2}{|c|}{ Machine Shut Down Instructions for Linac Operator } \\
\hline & 5.1 & $\begin{array}{l}\text { If sub-harmonic buncher is on, run down and turn off high voltage power supply and } \\
\text { RF preamplifier (in modulator room). }\end{array}$ \\
\hline & 5.2 & $\begin{array}{l}\text { Run gun filament voltage to minimum (at control console). Place short pulser in } \\
\text { pulsed diode mode (if used). Turn off Aux. Power switch }\end{array}$ \\
\hline & 5.3 & $\begin{array}{l}\text { If bending magnet is in use, turn off silicon controlled rectifier power supply (SCR), } \\
\text { set generator current to zero and degauss magnet twice, then turn off generator (at } \\
\text { control console). }\end{array}$ \\
\hline
\end{tabular}

The current version of this procedure resides at http://inside.anl.gov/documentcenter. Verify that the copy of the procedure you are using is current by comparing the revision number as printed copies can be obsolete. 


\begin{tabular}{|c|c|c|}
\hline Step & \multicolumn{2}{|r|}{ Action } \\
\hline & 5.4 & Turn off Helmholtz coils and close B.V. 2 and B.V. 5. \\
\hline & 5.5 & Turn off the control power (fire switch at control console). \\
\hline & 5.6 & Turn off the 480 disconnect connector - circuit breaker 2B (in modulator room): \\
\hline & 5.7 & $\begin{array}{l}\text { Turn off the contactors for the EMI power supplies (cabinets on east wall of modulator } \\
\text { room. Check to make sure that the power is off to the capacitor charging supplies in } \\
\text { modulators } 1 \text { and } 2 \text { (lights should be off on power supply.) }\end{array}$ \\
\hline & 5.8 & $\begin{array}{l}\text { Open any of the following which are closed if they are not required to be left closed by } \\
\text { the experimental review documentation: }\end{array}$ \\
\hline & & A. Pit door. \\
\hline & & B. Pit-cell gate \\
\hline & & C. Cell \#1 door. \\
\hline & & D. Cell \#2 gate. \\
\hline & 5.9 & Turn off all unnecessary lighting. \\
\hline
\end{tabular}

** Denotes the ASE controlled parmeters.

\section{$4 \quad$ Records Created by Work Process}

The records listed below must be retained as indicated.

\begin{tabular}{l|l|l|l|l}
\hline $\begin{array}{l}\text { Description of Record } \\
\text { (include form number if } \\
\text { applicable) }\end{array}$ & $\begin{array}{l}\text { Active } \\
\text { Records } \\
\text { Custodian }\end{array}$ & $\begin{array}{l}\text { Active } \\
\text { Records } \\
\text { Retention }\end{array}$ & $\begin{array}{l}\text { Indexing Method, } \\
\text { Storage Medium }\end{array}$ & $\begin{array}{l}\text { Federal Retention } \\
\text { Requirements* }\end{array}$ \\
\hline Linac Daily Log Sheet & $\begin{array}{l}\text { Facility } \\
\text { Manager }\end{array}$ & 3 years & $\begin{array}{l}\text { Index by job date } \\
\text { and name, store on } \\
\text { paper or } \\
\text { electronically }\end{array}$ & $\begin{array}{l}\text { Destroy 75 years after } \\
\text { the date of the permit } \\
\text { (DOE ADM 18.37) }\end{array}$ \\
\hline
\end{tabular}

*If records are maintained in a business information system that is not currently programmed to purge digital records based on age, the records may be retained in that system past the indicated destruction date.

\section{Related Documents}

This procedure implements requirements established by the following safety basis documents:

- LEAF-SAD-100, Linac Safety Assessment Document

- LEAF-ASE-100, Linac Accelerator Safety Envelope

This procedure implements requirements established by the following Argonne policies and procedures:

- LMS-PROC-188, Accelerator Safety

- EGS-PP-100, Configuration Management Program Plan for Accelerators

\section{Definitions}

The current version of this procedure resides at http://inside.anl.gov/documentcenter. Verify that the copy of the procedure you are using is current by comparing the revision number as printed copies can be obsolete. 
None

The current version of this procedure resides at http://inside.anl.gov/documentcenter. Verify that the copy of the procedure you are using is current by comparing the revision number as printed copies can be obsolete. 


\section{$7 \quad$ About this Procedure}

\begin{tabular}{ll}
\hline Issuing organization: & Low Energy Accelerator Facility \\
\hline Procedure owner: & S. Chemerisov \\
\hline Point of contact: & S. Chemerisov \\
\hline Review cycle (months): & 36 \\
\hline Date last revised: & 03.25 .2019 \\
\hline Date last reviewed: & 03.25 .2019 \\
\hline
\end{tabular}

\section{Summary of Changes in This Version}

Initial release. 


\section{Appendix A: Example Linac Daily Log Sheet}

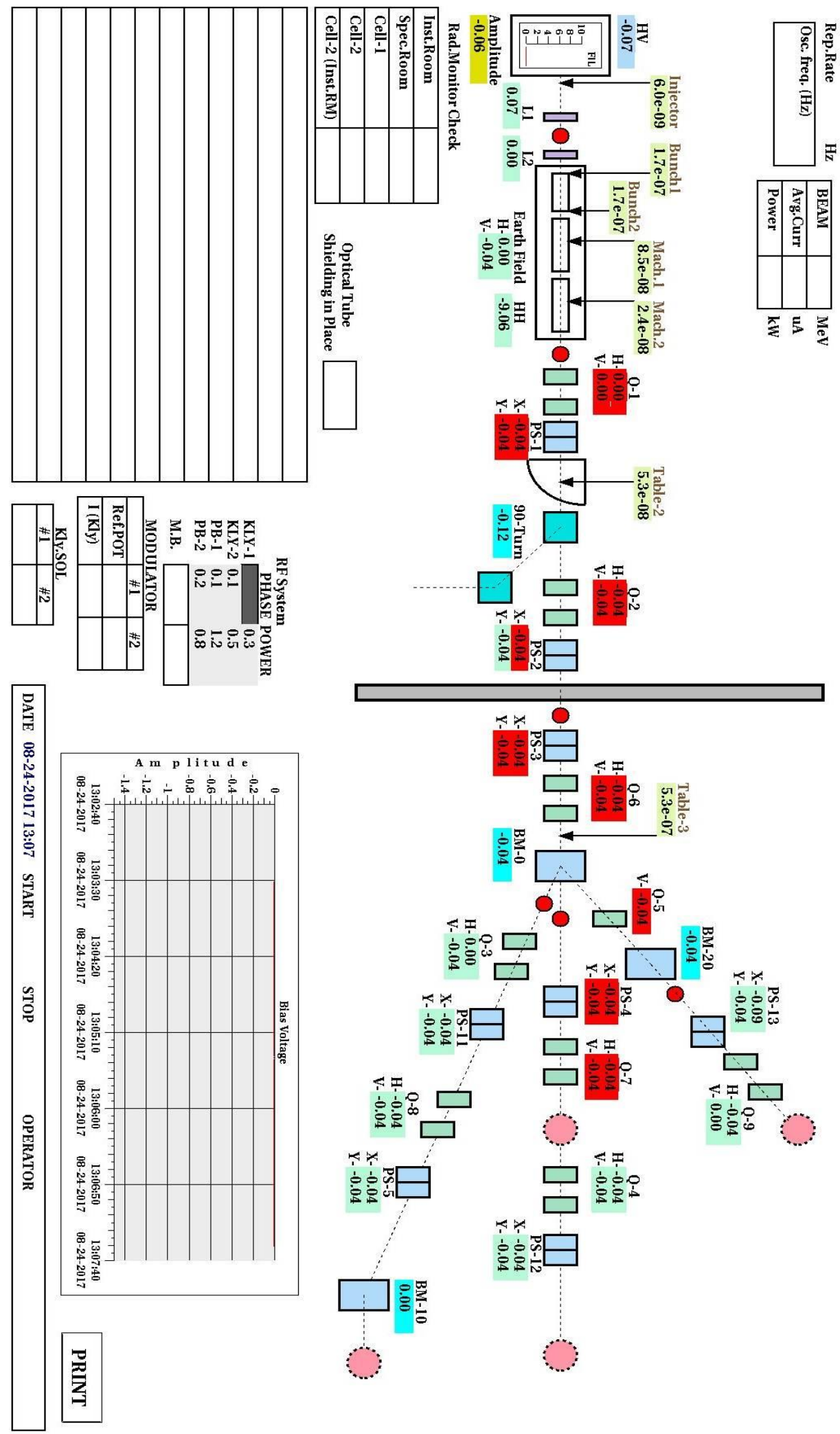




\section{APPENDIX 26}

LEAF-PROC-012, Rev. 0: AMORE Startup Checklist for Beam on Target 


\section{AMORE Startup Checklist for Beam on Target}

Low Energy Accelerator Facility, LEAF-PROC-012, Rev. 0

Approved:

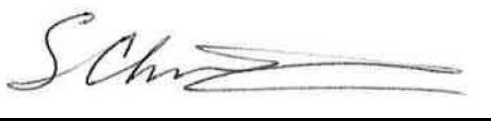

Sergey Chemerisov, Manager, IVEM/LEAF

The current version of this document resides at https://leaf-docdb.ne.anl.gov/cgi-bin/DocumentDatabase. Printed or electronically downloaded copies may be obsolete. Before using such a copy for work direction, employees must verify that it is current by comparing its revision number to that shown in the on-line version. 


\section{Purpose}

Establish the process for verifying all conditions are met to start irradiation for AMORE experiment at the Linac facility.

\section{Scope}

This procedure applies to the following Argonne activities and entities.

\begin{tabular}{ll}
\hline LMS core processes: & Asset Management \\
\hline Organizations: & Experimental Operations and Facilities (EOF) Division \\
\hline Buildings: & 211 \\
\hline Specific locations: & LINAC \\
\hline $\begin{array}{l}\text { Other applicability } \\
\text { factors: }\end{array}$ & None \\
\hline Exclusions: & None \\
\hline USI applicability: & Yes \\
\hline
\end{tabular}

\section{Work Process}

\subsection{Introduction}

In order to put beam on target for AMORE experiments multiple system has to be operational and in proper configuration to perform irradiation. This procedure identifies the step to verify readiness for the beginning of the experiment.

\subsection{Step-by-Step Procedure}

The steps below are mandatory unless noted otherwise. This procedure is to be performed by Linac operator or properly trained facility personnel.

\subsubsection{Actions}

\begin{tabular}{c|ll}
\hline Step & \multicolumn{1}{c}{ Action (Initial) } \\
\hline 1 & DU Target Cooling System \\
& 1.1 & Perform routine startup procedure \\
1.2 & Coolant flow to the target is on \\
& 1.3 & Flowmeter calibration is current \\
& 1.4 & Thermocouple calibration is current \\
& 1.5 & Air purge calibration is current \\
& 1.6 & Verify that stop of the purge gas flow will interrupt interlock chain \\
\hline
\end{tabular}

The current version of this document resides at https://leaf-docdb.ne.anl.gov/cgi-bin/DocumentDatabase. Printed or electronically downloaded copies may be obsolete. Before using such a copy for work direction, employees must verify that it is current by comparing its revision number to that shown in the on-line version. 


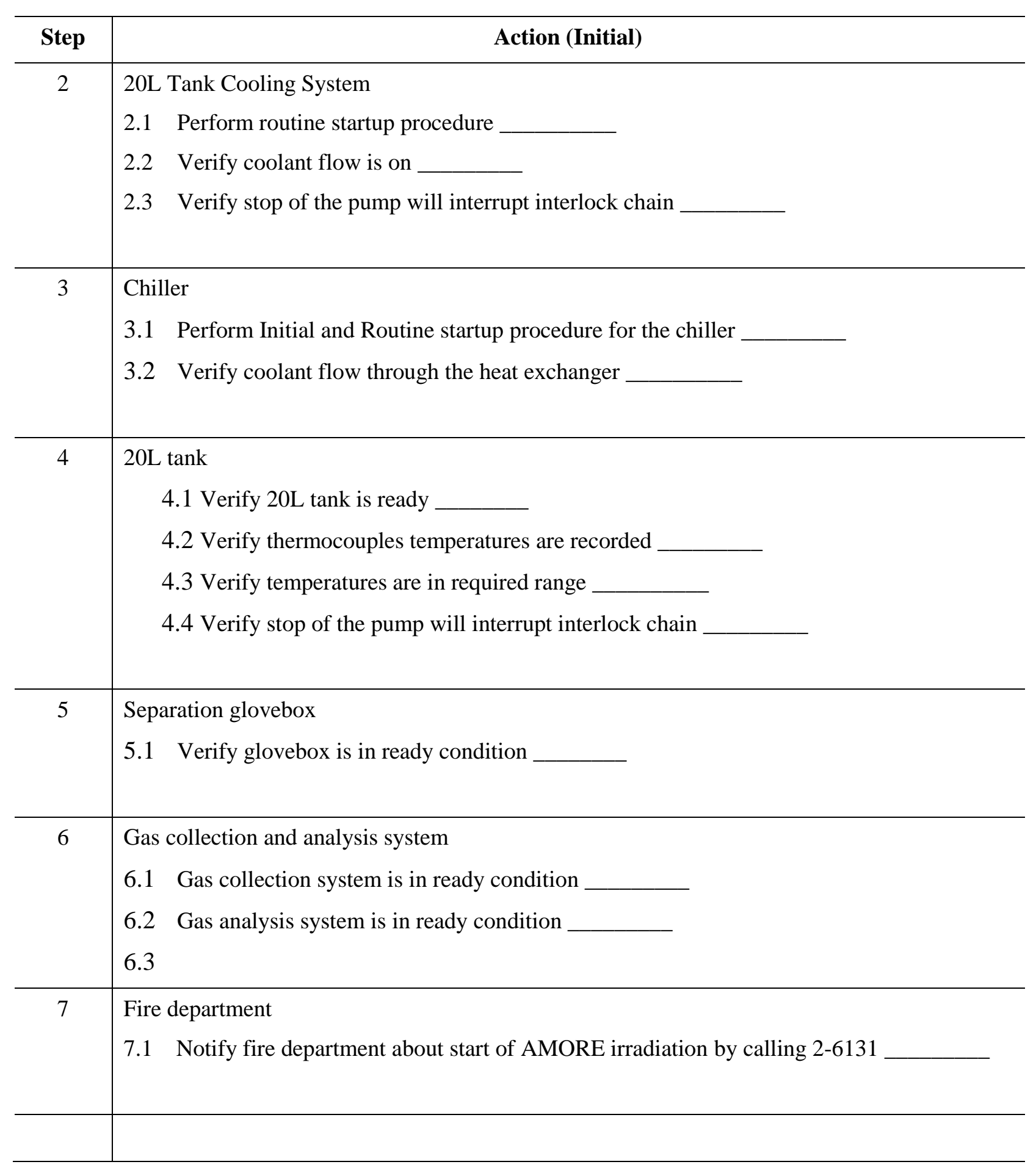

The current version of this document resides at https://leaf-docdb.ne.anl.gov/cgi-bin/DocumentDatabase. Printed or electronically downloaded copies may be obsolete. Before using such a copy for work direction, employees must verify that it is current by comparing its revision number to that shown in the on-line version. 


\section{Records Created by Work Process}

The records listed below must be retained as indicated.

\begin{tabular}{l|c|c|l|l}
\hline $\begin{array}{l}\text { Description of Record } \\
\text { (include form number if } \\
\text { applicable) }\end{array}$ & $\begin{array}{c}\text { Active } \\
\text { Records } \\
\text { Custodian }\end{array}$ & $\begin{array}{c}\text { Active } \\
\text { Records } \\
\text { Retention }\end{array}$ & $\begin{array}{l}\text { Indexing Method, } \\
\text { Storage Medium }\end{array}$ & $\begin{array}{l}\text { Federal Retention } \\
\text { Requirements* }\end{array}$ \\
\hline This completed procedure & $\begin{array}{c}\text { Facility } \\
\text { Manager }\end{array}$ & 3 years & $\begin{array}{l}\text { Index by job date } \\
\text { and name, store on } \\
\text { paper or } \\
\text { electronically }\end{array}$ & $\begin{array}{l}\text { Destroy 75 years after } \\
\text { the date of the permit } \\
\text { (DOE ADM 18.37) }\end{array}$ \\
\hline
\end{tabular}

*If records are maintained in a business information system that is not currently programmed to purge digital records based on age, the records may be retained in that system past the indicated destruction date.

\section{Related Documents}

This procedure implements requirements established by the following safety basis documents:

- LEAF-SAD-100, Linac Safety Assessment Document

- LEAF-ASE-100, Linac Accelerator Safety Envelope

This procedure implements requirements established by the following Argonne policies and procedures:

- LMS-PROC-188, Accelerator Safety

- EGS-PP-100, Configuration Management Program Plan for Accelerators

\section{Definitions}

None

\section{About this Procedure}

\begin{tabular}{ll}
\hline Issuing organization: & Low Energy Accelerator Facility \\
\hline Procedure owner: & S. Chemerisov \\
\hline Point of contact: & S. Chemerisov \\
\hline Review cycle (months): & 36 \\
\hline Date last revised: & 03.20 .2019 \\
\hline Date last reviewed: & \\
\hline
\end{tabular}

\section{Summary of Changes in This Version}

Initial release.

The current version of this document resides at https://leaf-docdb.ne.anl.gov/cgi-bin/DocumentDatabase. Printed or electronically downloaded copies may be obsolete. Before using such a copy for work direction, employees must verify that it is current by comparing its revision number to that shown in the on-line version. 
The current version of this document resides at https://leaf-docdb.ne.anl.gov/cgi-bin/DocumentDatabase. Printed or electronically downloaded copies may be obsolete. Before using such a copy for work direction, employees must verify that it is current by comparing its revision number to that shown in the on-line version. 


\section{APPENDIX 27}

LEAF-PROC-011, Rev. 3: LEAF D-024 Hot Cell. 211/D-024 Hot Cell Operations AMORE 


\section{LEAF D-024 Hot Cell. 211/D-024 Hot Cell Operations AMORE}

Low Energy Accelerator Facility, LEAF-PROC-011, Rev. 3

Approved:

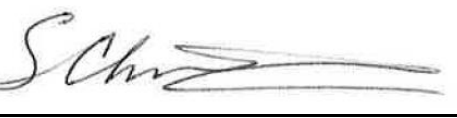

Date: 12.23 .2020

Sergey Chemerisov, Manager, IVEM/LEAF

Effective Date: 01.04.2021 


\section{Purpose}

This procedure provides instructions for performing Argonne Molybdenum Research Experiment (AMORE) Phase II Tests at Building 211 Low Energy Accelerator Facility (LEAF) 211-D024 Hot Cell. The document also includes a Work Aid for operations involving the Concentration Column and LEU Modified Cintichem.

\section{Scope}

This procedure applies to the following Argonne activities and entities.

\begin{tabular}{ll}
\hline LMS core processes: & Asset Management \\
\hline Organizations: & Experimental Operations and Facilities (EOF) Division \\
\hline Buildings: & 211 \\
\hline Specific locations: & LINAC \\
\hline $\begin{array}{l}\text { Other applicability } \\
\text { factors: }\end{array}$ & None \\
\hline Exclusions: & None \\
\hline USI applicability: & Yes \\
\hline
\end{tabular}

\section{Work Process}

\subsection{Introduction}

This document provides instructions for AMORE ${ }^{99}$ Mo Phase II Tests -211-D024 Hot Cell Operations, including a Work Aid for the Concentration Column and LEU Modified Cintichem operations. The flow diagram of the concentration column has been attached to this document and is posted at the job-site.

\subsection{Step-by-Step Procedure}

Sections 3.2.1 through 3.2.2 are mandatory and must be performed exactly as written. Sections 3.2.3 through 3.2.8 are considered guidance and are not required to be performed exactly as written. This procedure is to be performed by trained personnel.

\subsubsection{Concentration Column}

\begin{tabular}{c|l}
\hline Step & \multicolumn{1}{c}{ Action } \\
\hline & $\begin{array}{l}\text { Warning: Steps in this section and the next (3.2.1 and 3.2.2) must be performed exactly } \\
\text { as written. }\end{array}$ \\
\hline 1 & $\begin{array}{l}\text { Follow Sections 3.2.3 through 3.2.6 of this procedure. These sections are meant to provide a } \\
\text { step-by-step operation of the experiment. If deviations from the steps are made, they must be } \\
\text { documented in the associated laboratory notebook and referenced in pen on the printout of this } \\
\text { procedure. }\end{array}$ \\
\hline
\end{tabular}




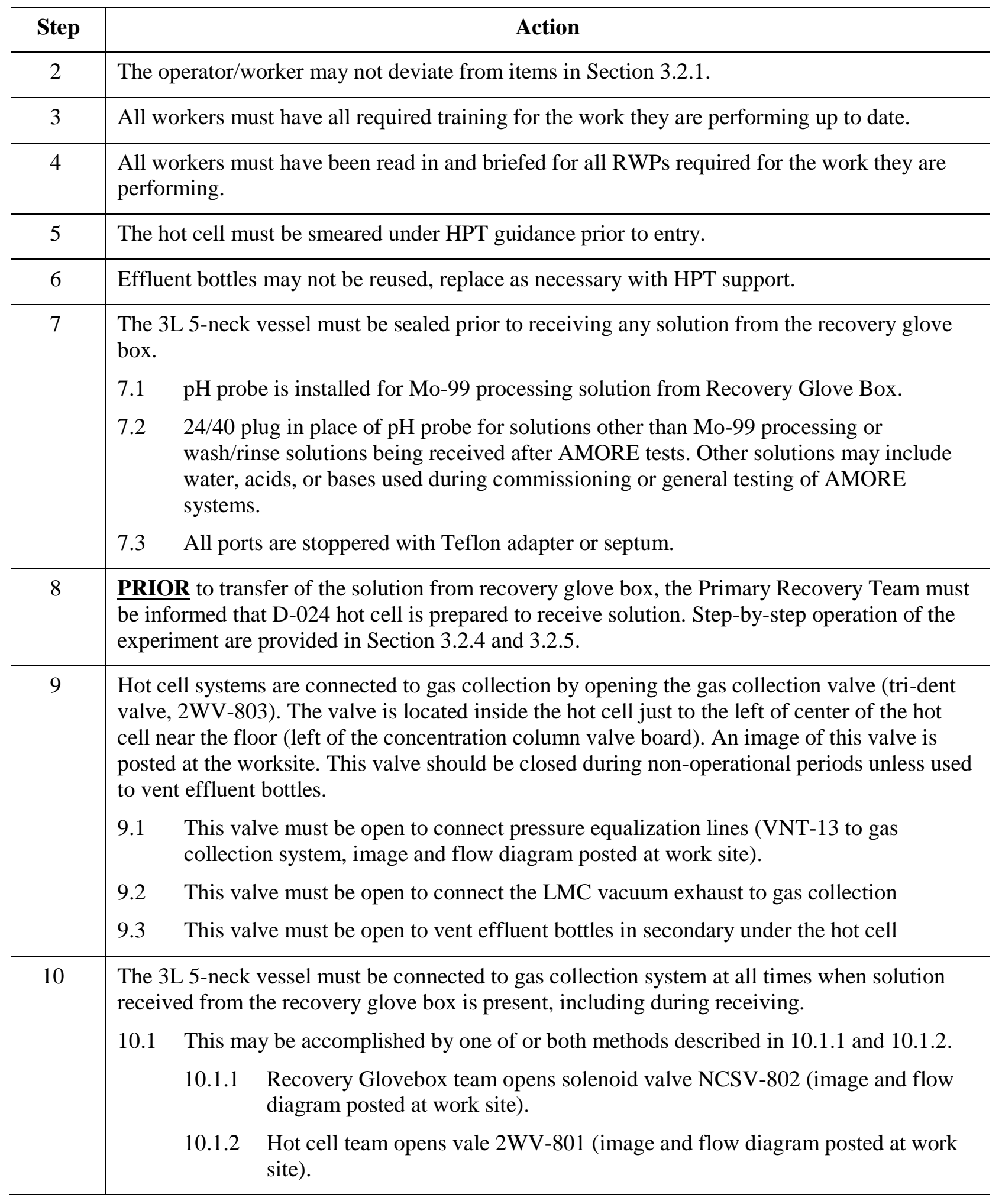




\begin{tabular}{|c|c|}
\hline Step & Action \\
\hline \multirow[t]{4}{*}{11} & $\begin{array}{l}\text { The } 3 \mathrm{~L} 5 \text {-neck vessel is never opened when processed solution from the recovery glove box is } \\
\text { present. }\end{array}$ \\
\hline & 11.1 Never remove the $\mathrm{pH}$ probe when processing solutions are present. \\
\hline & 11.2 Never remove the septum when processing solutions are present. \\
\hline & 11.3 Never remove the Teflon ports when processing solutions are present. \\
\hline 12 & $\begin{array}{l}\text { The } 3 \mathrm{~L} 5 \text {-neck vessel may only be opened when removing the wash solution sent from the } \\
\text { recovery glove box. This solution is only sent after full processing of the irradiated solution } \\
\text { has been completed (after the LEU Modified Cintichem Process). }\end{array}$ \\
\hline 13 & The wash solution must be removed prior to performing the next AMORE process. \\
\hline
\end{tabular}

\section{END OF SEGMENT}




\subsubsection{LEU Modified Cintichem Operations}

\begin{tabular}{|c|c|c|}
\hline \multirow[t]{2}{*}{ Step } & \multicolumn{2}{|r|}{ Action } \\
\hline & \multicolumn{2}{|r|}{ Warning: Steps in this Section (3.2.2) must be performed exactly as written. } \\
\hline 1 & \multicolumn{2}{|r|}{$\begin{array}{l}\text { Follow Sections } 3.2 .7 \text { through } 3.2 .8 \text { of this procedure. These sections are meant to provide a } \\
\text { step-by-step operation of the experiment. If deviations from the steps are made, they must be } \\
\text { documented in the associated laboratory notebook and referenced in pen on the printout of the } \\
\text { procedure. }\end{array}$} \\
\hline 2 & \multicolumn{2}{|r|}{ The operator/worker may not deviate from items in section 3.2.2. } \\
\hline 3 & \multicolumn{2}{|r|}{ All workers must have all required training for the work they are performing up to date. } \\
\hline 4 & \multicolumn{2}{|r|}{$\begin{array}{l}\text { All workers must have been read in and briefed for all RWPs required for the work they are } \\
\text { performing. }\end{array}$} \\
\hline 5 & \multicolumn{2}{|r|}{$\begin{array}{l}\text { The D- } 024 \text { hot cell anti-chamber must be smeared under HPT guidance prior to use of the D- } \\
024 \text { hot cell anti-chamber. }\end{array}$} \\
\hline 6 & \multicolumn{2}{|r|}{$\begin{array}{l}\text { Reagent bottles, sampling syringes, and vials may not be reused. Replace as necessary with } \\
\text { HPT support. }\end{array}$} \\
\hline \multirow[t]{6}{*}{7} & \multicolumn{2}{|r|}{$\begin{array}{l}\text { Hot cell systems are connected to gas collection by opening the gas collection valve (tri-dent } \\
\text { valve, } 2 \mathrm{WV}-803 \text { ). The valve is located inside the hot cell just to the left of center of the hot } \\
\text { cell near the floor (left of the concentration column valve board). An image of this valve is } \\
\text { posted at the worksite. This valve should be closed during non-operational periods unless used } \\
\text { to vent effluent bottles. }\end{array}$} \\
\hline & & $\begin{array}{l}\text { This valve must be open to connect pressure equalization lines (VNT-13 to gas } \\
\text { collection system. Image and flow diagram is posted at work site.) }\end{array}$ \\
\hline & & This valve must be open to connect the LMC vacuum exhaust to gas collection \\
\hline & & This valve must be open to vent effluent bottles in secondary under the hot cell \\
\hline & & $\begin{array}{l}\text { Verify the gas collection valve (tri-dent valve, } 2 \mathrm{WV}-803 \text { ) is OPEN prior performing } \\
\text { LMC process in hot cell. Image and flow diagram is posted at work site. }\end{array}$ \\
\hline & & Date: ___ Time: __ \\
\hline 8 & & $\begin{array}{l}\text { sure that for any operation when solutions are added to LMC bottles, columns, or } \\
\text { e vials, that they are pressure equilibrated. The gas collection line can be used for } \\
\text { are equalization. This prevents pressurization of LMC bottles, columns, and sample vials. }\end{array}$ \\
\hline
\end{tabular}




\begin{tabular}{c|c}
\hline Step & \multicolumn{1}{c}{ Action } \\
\hline 9 & $\begin{array}{l}\text { When LMC processing in hot cell has concluded (including sample collection), close the gas } \\
\text { collection valve (tri-dent valve, 2WV-803). The valve is located inside the hot cell just to the } \\
\text { left of center of the hot cell near the floor (left of the concentration column valve board). An } \\
\text { image of this valve is posted at the worksite. This valve should be closed during non- } \\
\text { operational periods unless used to vent effluent bottles or any LMC bottles. } \\
\text { Verify the gas collection valve (tri-dent valve, 2WV-803) is CLOSED after performing } \\
\text { LMC process in hot cell. Image and flow diagram is posted at work site. } \\
\text { Date: _ Time: }\end{array}$ \\
\hline 10 & $\begin{array}{l}\text { If gas collection valve needs to be left open, provide explanation and notify gas collection } \\
\text { system custodian, Mike Kalensky, at x2-4168. } \\
\text { Explanation: }\end{array}$ \\
\hline
\end{tabular}

END OF SEGMENT 
Note: Sections 3.2.3 through 3.2.8 are considered guidance and are not required to be performed exactly as written.

\subsubsection{Concentration Column}

\begin{tabular}{|c|c|c|}
\hline Step & & Action \\
\hline \multirow[t]{18}{*}{1} & \multicolumn{2}{|r|}{ Prepare D-024 Hot Cell for AMORE operations } \\
\hline & \multirow[t]{6}{*}{1.1} & Number of workers suggested for these Work Aides: \\
\hline & & $\begin{array}{l}\text { a. Minimum for Steps } 1.2 \text { and 1.3, dependent on D-024 hot cell radiological posting } \\
\text { and discussions with Health Physics (HP). }\end{array}$ \\
\hline & & i. Worker 1: \\
\hline & & ii. Worker 2 (optional): \\
\hline & & iii. HPT: \\
\hline & & $\begin{array}{l}\text { b. If respirator is required, a minimum of two personnel and one Health Physics Tech } \\
\text { (HPT) are required. }\end{array}$ \\
\hline & \multirow[t]{3}{*}{1.2} & Confirm $\leq 500,000 \mathrm{dpm}$ removable contamination within D-024 hot cell \\
\hline & & a. D-024 Hot Cell manipulator operator \\
\hline & & b. HP Tech \\
\hline & \multirow[t]{6}{*}{1.3} & Setup of AMORE operations inside D-024 Hot Cell \\
\hline & & a. Entry worker - worker entering D-024 Hot Cell \\
\hline & & b. Watch worker - worker standing watch outside of D-024 Hot Cell \\
\hline & & i. Hands items to entry worker as needed \\
\hline & & ii. Responsible for these work aides and that all steps are checked off \\
\hline & & iii. Documents preparations in lab notebook \\
\hline & 1.4 & Inside-D024 HP Tech monitors activities inside 211-D024 \\
\hline & 1.5 & Outside-D024 HP Tech assists Inside-D024 HP Tech with getting smears counted \\
\hline \multirow[t]{8}{*}{2} & \multicolumn{2}{|c|}{ Verify RWPs are current and workers read-in } \\
\hline & 2.1 & Use https://apps.inside.anl.gov/rwp/permits \\
\hline & 2.2 & RWP suffix -211-004 \\
\hline & & $\begin{array}{l}\text { a. Title: Work in a contamination area without engineering controls (e.g., benchtop, } \\
\text { room) }\end{array}$ \\
\hline & & b. Verify RWP active \\
\hline & & c. Verify personnel identified for assignment in step 1.2.a is read in \\
\hline & & d. Verify personnel identified for assignment in step 1.2.b is read in \\
\hline & 2.3 & RWP suffix $-211-024$ \\
\hline
\end{tabular}




\begin{tabular}{|c|c|c|}
\hline Step & & Action \\
\hline & 2.4 & $\begin{array}{l}\text { a. Title: Transfers in and out of the D-024 Hot cell and shielded glovebox } \\
\text { antechambers (transfer ports) } \\
\text { b. Verify RWP active } \\
\text { c. Verify personnel identified for assignment in step 1.2.a is read in } \\
\text { d. Verify personnel identified for assignment in step 1.2.b is read in } \\
\text { RWP suffix -211-030 } \\
\text { a. Title: Transfer Equipment / Material in or out of D-024 hot cell } \\
\text { b. Verify RWP active } \\
\text { c. Verify personnel identified for assignment in step 1.3.a is read in } \\
\text { d. Verify personnel identified for assignment in step 1.3.b is read in } \\
\text { e. Verify personnel identified for assignment in step } 1.4 \text { is read in } \\
\text { f. Verify personnel identified for assignment in step } 1.5 \text { is read in }\end{array}$ \\
\hline 3 & Veri & $\begin{array}{l}\text { Permit Required Confined Space (PRCS) requirements } \\
\text { Is this permit needed? Verify personnel identified for assignment in step 1.3.a has } \\
\text { completed and is up-to-date on PRCS entry training, ESH113A. } \\
\text { a. D-024 hot cell is classified as a PRCS (211-0D-008) that requires 2-persons for } \\
\text { single person entry } \\
\text { b. Fill out permit for confined space entry (used only for that job and then permit is } \\
\text { terminated) }\end{array}$ \\
\hline 4 & $\begin{array}{l}\text { Con } \\
4.1\end{array}$ & $\begin{array}{l}\text { irm supply of PPE for setup of AMORE Operations inside D-024 Hot Cell } \\
\text { Entry Worker PPE (worker identified in 1.3.a) } \\
\text { a. Training. Verify personnel identified for assignments is up-to-date on the following } \\
\text { training if respirator required (confirm with HP). } \\
\text { - MEDCERT } 114 \text { Respirator Medical Certification } \\
\text { - ESH } 118 \text { Resp. Protection - Air-purifying Respirator } \\
\text { - ESH 118PR Resp. Protection - Air-purifying Respirator Practical Exercise } \\
\text { b. Full respirator or PPE as required by the RWP. } \\
\text { c. Double Tyvek coveralls or PPE as required by the RWP. } \\
\text { - Use one to two sizes larger to allow for reaching/stretching } \\
\text { d. First pair of gloves - Nitrile, long cuff } \\
\text { f. Shocond pair of over-gloves - Nitrile, latex, or other chemical resistant gloves }\end{array}$ \\
\hline
\end{tabular}




\begin{tabular}{|c|c|}
\hline Step & Action \\
\hline & $\begin{array}{l}\text { g. Tape - Vinyl tape } \\
\text { h. Non-permeable gauntlets - Required for working with solutions } \\
\text { Watch worker PPE and Inside-D024 HP Tech } \\
\text { a. Training. Verify personnel identified for assignments has following training up-to- } \\
\text { date } \\
\text { - MEDCERT } 114 \text { Respirator Medical Certification } \\
\text { - ESH } 118 \text { Resp. Protection - Air-purifying Respirator } \\
\text { - ESH } 118 \text { PR Resp. Protection - Air-purifying Respirator Practical Exercise } \\
\text { b. Full respirator or PPE as required by the RWP. } \\
\text { c. Single Tyvek coveralls or PPE as required by the RWP. } \\
\text { d. First pair of gloves - Nitrile, long-cuff } \\
\text { e. Second pair of over-gloves - Nitrile, latex, or other chemical resistant gloves } \\
\text { f. Shoe covers - Orange, rubber } \\
\text { Outside-D024 HP Tech } \\
\text { a. Lab coat } \\
\text { b. First pair of gloves - Nitrile, long-cuff } \\
\text { c. Second pair of over-gloves - Nitrile, latex or other chemical resistant gloves } \\
\text { d. Shoe covers - Orange, rubber }\end{array}$ \\
\hline 5 & \\
\hline 6 & $\begin{array}{l}\text { Use checklist for pre-job brief } \\
\text { - Verify all workers have initialed pre-job brief at conclusion of briefing }\end{array}$ \\
\hline 7 & $\begin{array}{l}\text { Confirm } \leq 500,000 \text { dpm removable contamination within D-024 hot cell } \\
\text { - Work under RWP 211-030 or RWP specified by HP (e.g., RWP 211-004) } \\
\text { - While waiting for results proceed to next step }\end{array}$ \\
\hline 8 & $\begin{array}{l}\text { Outside the D-024 Hot Cell } \\
8.1 \text { Stage lab notebook and pen } \\
\text { a. ANL notebook serial number: } \\
\text { b. Notebook page number(s): } \\
8.2 \text { Stage equipment and chemicals for AMORE operations } \\
\text { 1. Concentration column (see Figure 1). Keep column ends plugged with solid plastic } \\
\text { screw plugs }\end{array}$ \\
\hline
\end{tabular}




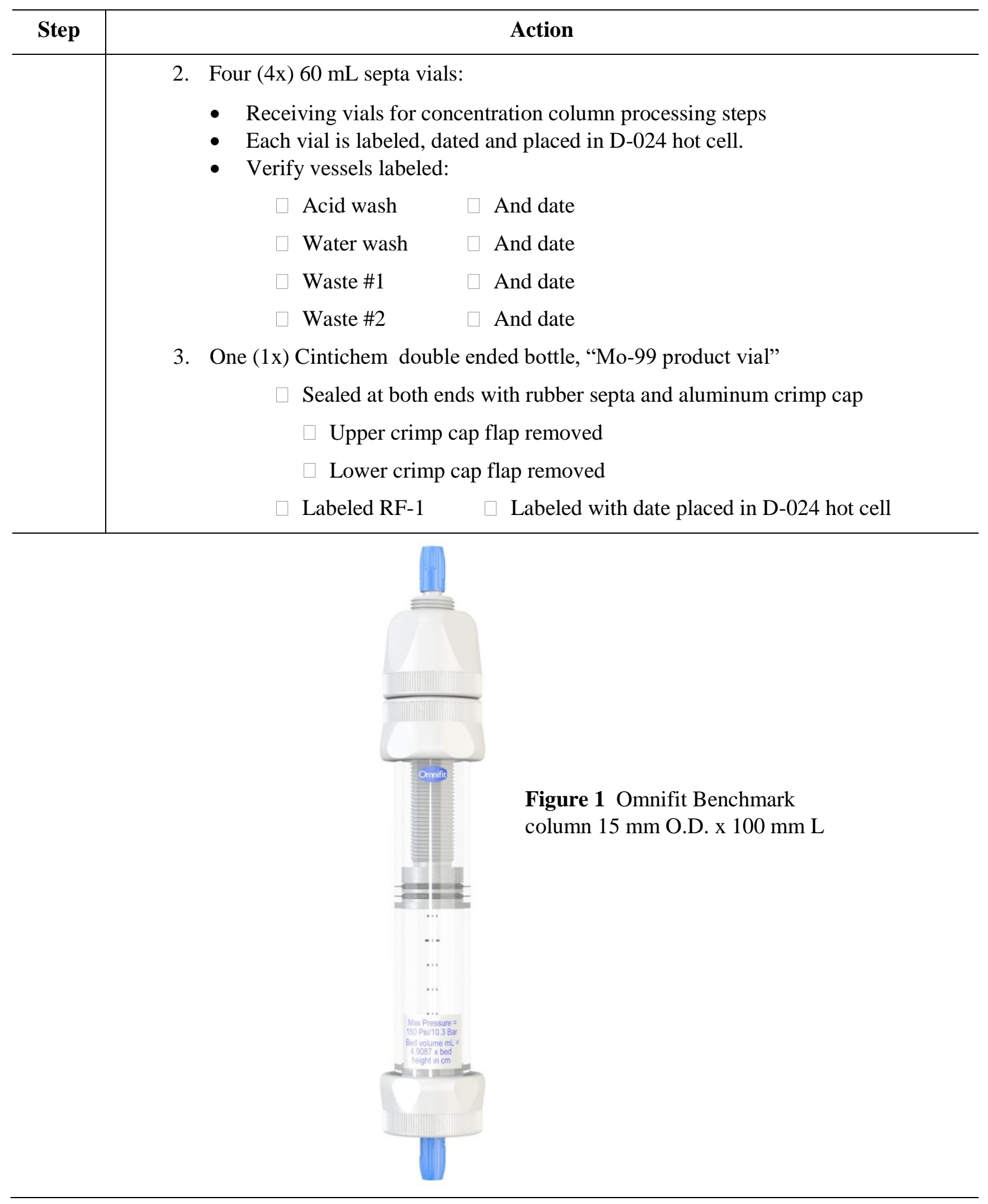




\begin{tabular}{c|cc}
\hline Step & Action \\
\hline 8 & 4. & Five $(5 \mathrm{x}) 20 \mathrm{~mL}$ sampling septa vials \\
(cont.) & $\bullet \quad$ Each vial is labeled, dated and placed in D-024 hot cell \\
& $\bullet \quad$ Verify vials labeled \\
& $\square$ Mo-99 product & $\square$ And date Mass: \\
& $\square$ Acid wash & $\square$ And date Mass: \\
& $\square$ Water wash & $\square$ And date Mass: \\
& $\square$ Feed initial & $\square$ And date Mass: \\
& $\square$ Waste \#2 & $\square$ And date Mass:
\end{tabular}

5. Five (5x) Luer-Lock tip syringes

Concentration column processing solutions:

1. Water reservoir fill

- $\quad$ Syringe size $\mathrm{x} 2=60 \mathrm{~mL}$

- Volume $=120 \mathrm{~mL}$

- Verify syringe labeled

2. $0.01 \mathrm{M} \mathrm{HNO}_{3}$ reservoir fill

- Syringe size $=60 \mathrm{~mL}$

- Volume $=60 \mathrm{~mL}$

- Verify syringe labeled

3. $1 \mathrm{M} \mathrm{NaOH}$ reservoir fill

- $\quad$ Syringe size $=60 \mathrm{~mL}$

- Volume $=60 \mathrm{~mL}$

- Verify syringe labeled

4. $10 \mathrm{M} \mathrm{NaOH}$

- $\quad$ Syringe size $=10 \mathrm{~mL}$

- Volume $=10 \mathrm{~mL}$

- Verify syringe labeled $\quad \square$ And Date

5. Syringe $-30 \mathrm{~mL} \times 1$ in needle

- $30 \mathrm{~mL}$ with Luer-Lock tip syringe

- Load with $20 \mathrm{~mL} 8 \mathrm{M} \mathrm{HNO}_{3}$

- Verify syringe labeled

And Date

6. Bottle of $8 \mathrm{M} \mathrm{HNO}_{3}$

- Bottle \#1 - 140 mL $8 \mathrm{M} \mathrm{HNO}_{3}$

- Verify bottle labeled

7. Six (6x) sampling syringes 


\begin{tabular}{|c|c|}
\hline Step & Action \\
\hline & 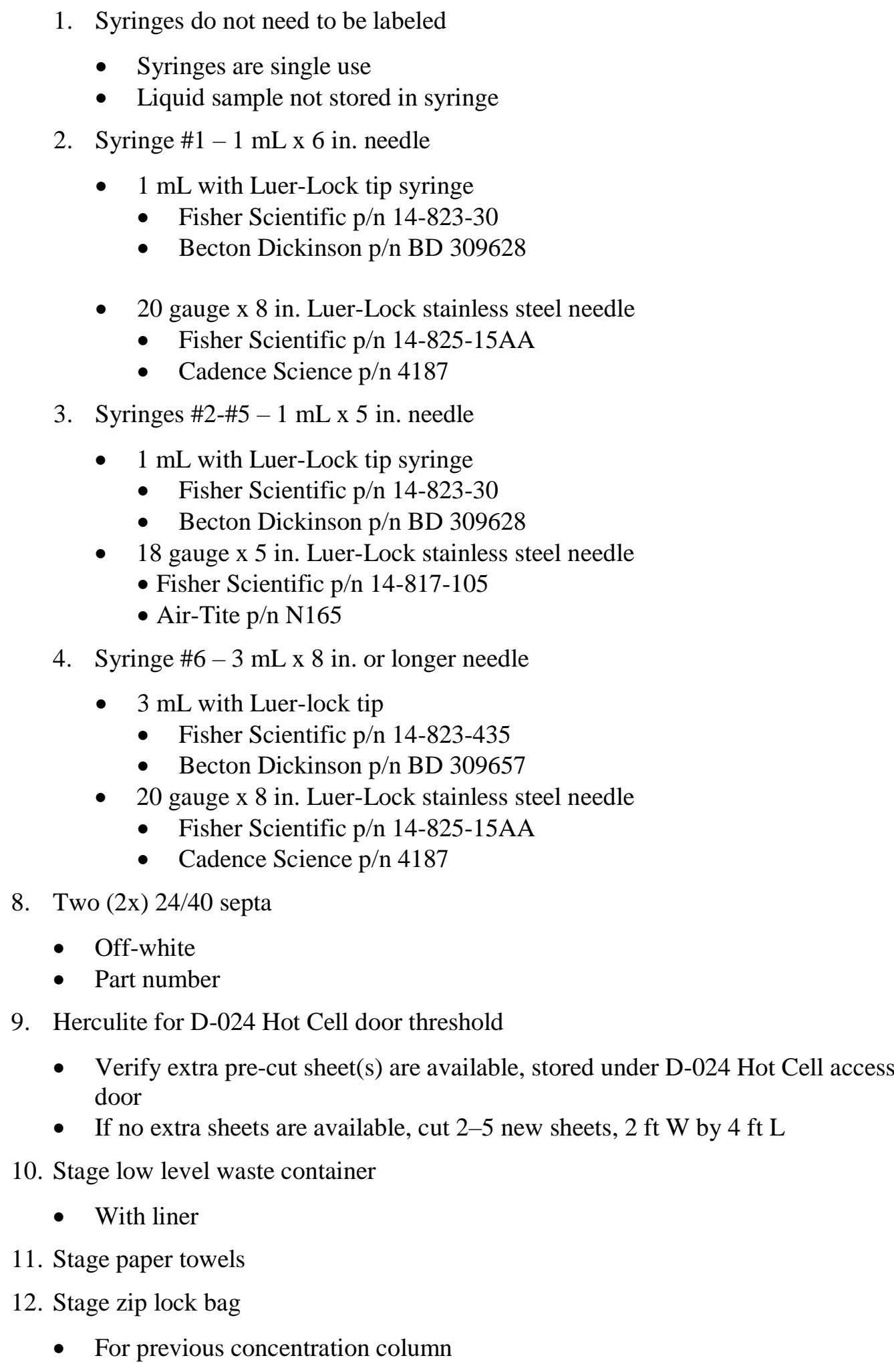 \\
\hline
\end{tabular}




\begin{tabular}{|c|c|}
\hline Step & Action \\
\hline & $\begin{array}{l}\text { 13. One (1x) } 1 \mathrm{~L} \text { bottle with cap } \\
\text { - Receiving vessel for final rinse contents of } 3 \text { L } 5 \text {-neck flask }\end{array}$ \\
\hline 9 & $\begin{array}{l}\text { Clear area below D- } 024 \text { Hot Cell access door } \\
9.1 \text { Remove/re-locate any unnecessary equipment } \\
9.2 \text { Ensure step stool is available }\end{array}$ \\
\hline 10 & Verify step stool is in good working order \\
\hline 11 & If interior smears come back $\leq 500000 \mathrm{dpm}$ proceed to step \#13 \\
\hline 12 & $\begin{array}{l}\text { Open D-024 Hot Cell access door } \\
\text { NOTE: Door is heavy } \\
\text { - Use slow motions when opening/closing } \\
\text { - Use latch on left side of door between wall and door to open } \\
\text { - Use two hands to open door slowly until door rests on bumper - DO NOT bounce } \\
\text { door off of bumper } \\
\text { - Door will maintain open position when full open }\end{array}$ \\
\hline 13 & $\begin{array}{l}\text { At open D-024 Hot Cell access door } \\
13.1 \text { HP performs dose rate surveys } \\
\text { 13.2 HP performs smears } \\
\text { 1. Inner side of door } \\
\text { 2. Door frame } \\
\text { 3. Herculite sheet rolled up inside of D-024 hot cell ledge } \\
\text { 13.3 BEFORE entry into the hot cell, the sheet is surveyed by the HP Tech } \\
\text { 1. Verify Herculite sheet survey completed } \\
\text { 2. Survey comes back with no loose contamination found: } \\
\text { - Proceed with entry into D-024 Hot Cell } \\
\text { - Replace outer gloves frequently, especially if torn or liquid found on } \\
\text { gloves } \\
\text { Roll-up Herculite with containment of contamination inside of roll and } \\
\text { remove from access door threshold and place in low-level waste } \\
\text { receptacle } \\
\text { Survey area under Herculite just removed } \\
\text { 3.a. Survey comes back with no loose contamination found - proceed to } \\
\text { 3.b. Survey comes back with loose contamination found }\end{array}$ \\
\hline
\end{tabular}




\begin{tabular}{|c|c|c|}
\hline \multirow[t]{2}{*}{ Step } & \multicolumn{2}{|r|}{ Action } \\
\hline & \multicolumn{2}{|r|}{$\begin{array}{l}\text { - If within RWP limits, proceed with non-wet cleaning } \\
\text { as described in WCD 55632 Task } 2 \\
\text { - Survey and repeat as necessary to remove loose } \\
\text { contamination and then proceed to 13.4. }\end{array}$} \\
\hline \multirow{18}{*}{14} & \multicolumn{2}{|c|}{ Inside D-024 Hot Cell } \\
\hline & 14.1 & Place four $(4 \mathrm{x}) 60 \mathrm{~mL}$ septa vessels in white rack \\
\hline & 14.2 & Place five (5x) $20 \mathrm{~mL}$ sampling septa vials in white rack \\
\hline & 14.3 & Load $\mathrm{NaOH}$ solution reservoir with $1 \mathrm{M} \mathrm{NaOH}$ from $60 \mathrm{~mL}$ syringe \\
\hline & & $\begin{array}{l}\text { 14.3.1 Pull syringe plunger to help relieve any build-up of pressure within reservoir } \\
\text { while needle engaged with bottle }\end{array}$ \\
\hline & 14.4 & Load $\mathrm{HNO}_{3}$ solution reservoir with $0.01 \mathrm{M} \mathrm{HNO}_{3}$ from $60 \mathrm{~mL}$ syringe \\
\hline & & $\begin{array}{l}\text { 14.4.1 Pull syringe plunger to help relieve any build-up of pressure within reservoir } \\
\text { while needle engaged with bottle }\end{array}$ \\
\hline & 14.5 & Load Water solution reservoir with water from $60 \mathrm{~mL}$ syringe \\
\hline & & $\begin{array}{l}\text { 14.5.1 Pull syringe plunger to help relieve any build-up of pressure within reservoir } \\
\text { while needle engaged with bottle }\end{array}$ \\
\hline & & $\begin{array}{l}\text { 14.5.2 Ensure water reservoir is full. If not full, add water as necessary to fill } \\
\text { reservoir }\end{array}$ \\
\hline & 14.6 & Place sample syringes inside hot cell \\
\hline & & 14.6.1 Preferred position: center of hot cell, in front of valve board \\
\hline & 14.7 & $\begin{array}{l}\text { Inspect solution plastic transfer lines. The solution transfer lines MUST be replaced at } \\
\text { least every two years. Check the date on the log sheet on the front of the D-024 Hot } \\
\text { Cell and verify the last time the lines were replaced. }\end{array}$ \\
\hline & & Date of replacement: \\
\hline & & Today's date: ____ \\
\hline & & $\begin{array}{l}\text { If this date is }>2 \text { years from today's date, replace the lines. If this date is }<2 \text { years } \\
\text { from today's date, proceed with the next step. }\end{array}$ \\
\hline & & 14.7.1 Defects are tubing crimped, brittle, flattened \\
\hline & & 14.7.1.1 From 5-way valve to Waste \#1 $60 \mathrm{~mL}$ vial (liquid effluent line) \\
\hline
\end{tabular}




\begin{tabular}{|c|c|c|}
\hline Step & & Action \\
\hline & & $\begin{array}{l}\text { - Line in good working order } \square \text { date and time of inspection: } \\
\text { Date: } \\
\text { defects replace } \\
\text { - Date of replacement: }\end{array}$ \\
\hline & 14.7.1.2 & From 5-way valve to Acid Wash $60 \mathrm{~mL}$ vial (liquid effluent line) \\
\hline & & $\begin{array}{l}\text { - Line in good working order } \square \text { date and time of inspection: } \\
\text { Date: } \\
\text { defects replace } \\
\text { - Date of replacement: }\end{array}$ \\
\hline & 14.7.1.3 & From 5-way valve to Water Wash $60 \mathrm{~mL}$ vial (liquid effluent line) \\
\hline & & $\begin{array}{l}\text { - Line in good working order } \square \text { date and time of inspection: } \\
\text { Date: } \quad \text { Time:__ If signs of } \\
\text { defects replace } \\
\text { Date of replacement: }\end{array}$ \\
\hline & 14.7.1.4 & $\begin{array}{l}\text { From 5-way valve to RF-1 Cintichem 1-A bottle (liquid effluent } \\
\text { line) }\end{array}$ \\
\hline & & $\begin{array}{l}\text { - Line in good working order } \square \text { date and time of inspection: } \\
\text { Date: } \quad \text { Time:__ If signs of } \\
\text { defects replace } \\
\text { - Date of replacement: }\end{array}$ \\
\hline & 14.7.1.5 & $\begin{array}{l}\text { From } 3 \text {-way valve at FMI pump outlet to } 3 \mathrm{~L} \text { receiving vessel } \\
\text { (liquid bypass line) }\end{array}$ \\
\hline & & $\begin{array}{l}\text { - Line in good working order } \square \text { date and time of inspection: } \\
\text { Date: } \quad \text { Time:___ If signs of } \\
\text { defects replace } \\
\text { - Date of replacement: }\end{array}$ \\
\hline & 14.7.1.6 & $\begin{array}{l}\text { From 3-way valve feed source selector to FMI pump inlet } \\
\text { - Line in good working order } \square \text { date and time of inspection: } \\
\text { Date: } \\
\text { can become brittle } \\
\text { - If signs of defects replace } \\
\text { - Date of replacement: }\end{array}$ \\
\hline & 14.7.1.7 & $\begin{array}{l}\text { From FMI pump outlet to 3-way valve destination selector } \\
\text { - Line in good working order } \square \text { date and time of inspection: } \\
\text { Date: Time:__ PEEK tubing } \\
\text { can become brittle } \\
\text { - If signs of defects replace } \\
\text { - Date of replacement: }\end{array}$ \\
\hline
\end{tabular}




\begin{tabular}{|c|c|c|}
\hline Step & & Action \\
\hline & 14.7.1.8 & $\begin{array}{l}\text { From 3-way valve at FMI pump outlet to } 3 \mathrm{~L} \text { receiving vessel } \\
\text { (liquid bypass line) } \\
\text { - Line in good working order } \square \text { date and time of inspection: } \\
\text { Date: } \\
\text { defects replace } \\
\text { - Date of replacement: }\end{array}$ \\
\hline & 14.7.1.9 & $\begin{array}{l}\text { From 4-way valve at FMI pump outlet to } 3 \mathrm{~L} \text { receiving vessel } \\
\text { (liquid feed line) } \\
\text { - Line in good working order } \square \text { date and time of inspection: } \\
\text { Date: } \\
\text { defects replace } \\
\text { - Date of replacement: }\end{array}$ \\
\hline & 14.7.1.10 & $\begin{array}{l}\text { From 2-way valve at From Recovery Glovebox line to } 3 \mathrm{~L} \text { receiving } \\
\text { vessel (liquid feed line) } \\
\text { - Line in good working order } \square \text { date and time of inspection: } \\
\text { Date: } \\
\text { defects replace } \\
\text { - Date of replacement: }\end{array}$ \\
\hline & 14.7.1.11 & $\begin{array}{l}\text { From } 0.1 \mathrm{M} \mathrm{NaOH} \text { feed bottle to } 4 \text {-way valve (liquid feed line) } \\
\text { - Line in good working order } \square \text { date and time of inspection: } \\
\text { Date: } \\
\text { defects replace } \\
\text { - Dime:__ }\end{array}$ \\
\hline & 14.7.1.12 & $\begin{array}{l}\text { From Water feed bottle to 4-way valve (liquid feed line) } \\
\text { - Line in good working order } \square \text { date and time of inspection: } \\
\text { Date: } \text { dime:__ signs of }^{\text {defects replace }} \text { - Date of replacement: }\end{array}$ \\
\hline & 14.7.1.13 & $\begin{array}{l}\text { From } 1 \mathrm{M} \mathrm{NaOH} \text { feed bottle to 4-way valve (liquid feed line) } \\
\text { - Line in good working order } \square \text { date and time of inspection: } \\
\text { Date: } \\
\text { defects replace } \\
\text { - Dime:__ }\end{array}$ \\
\hline & 14.7.1.14 & $\begin{array}{l}\text { From valved quick-disconnect fitting to needle for concentration } \\
\text { column ops (VQD-24, vent line) }\end{array}$ \\
\hline & & $\begin{array}{l}\text { - Line in good working order } \square \text { date and time of inspection: } \\
\text { Date: } \quad \text { Time:___ If signs of } \\
\text { defects replace }\end{array}$ \\
\hline
\end{tabular}




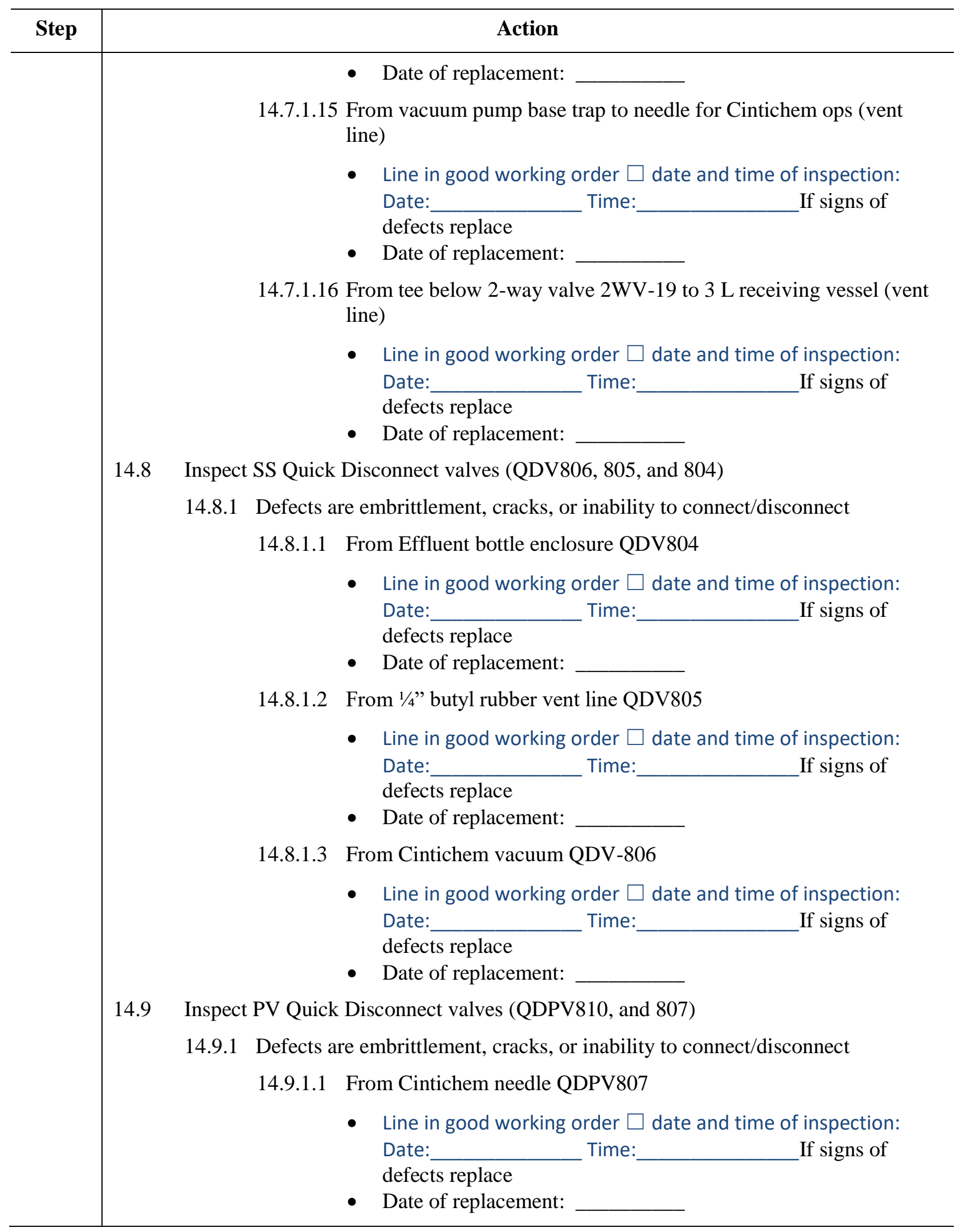




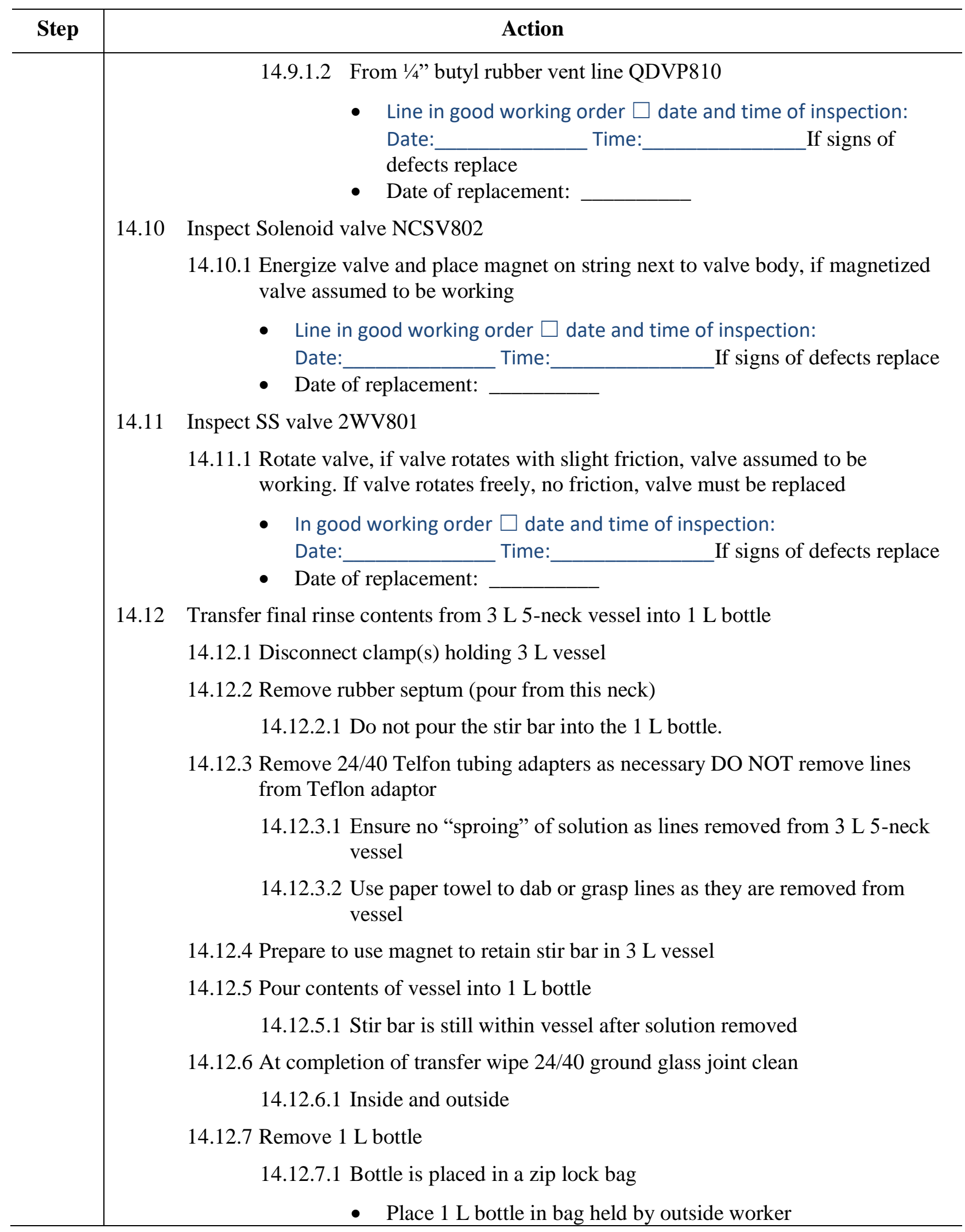




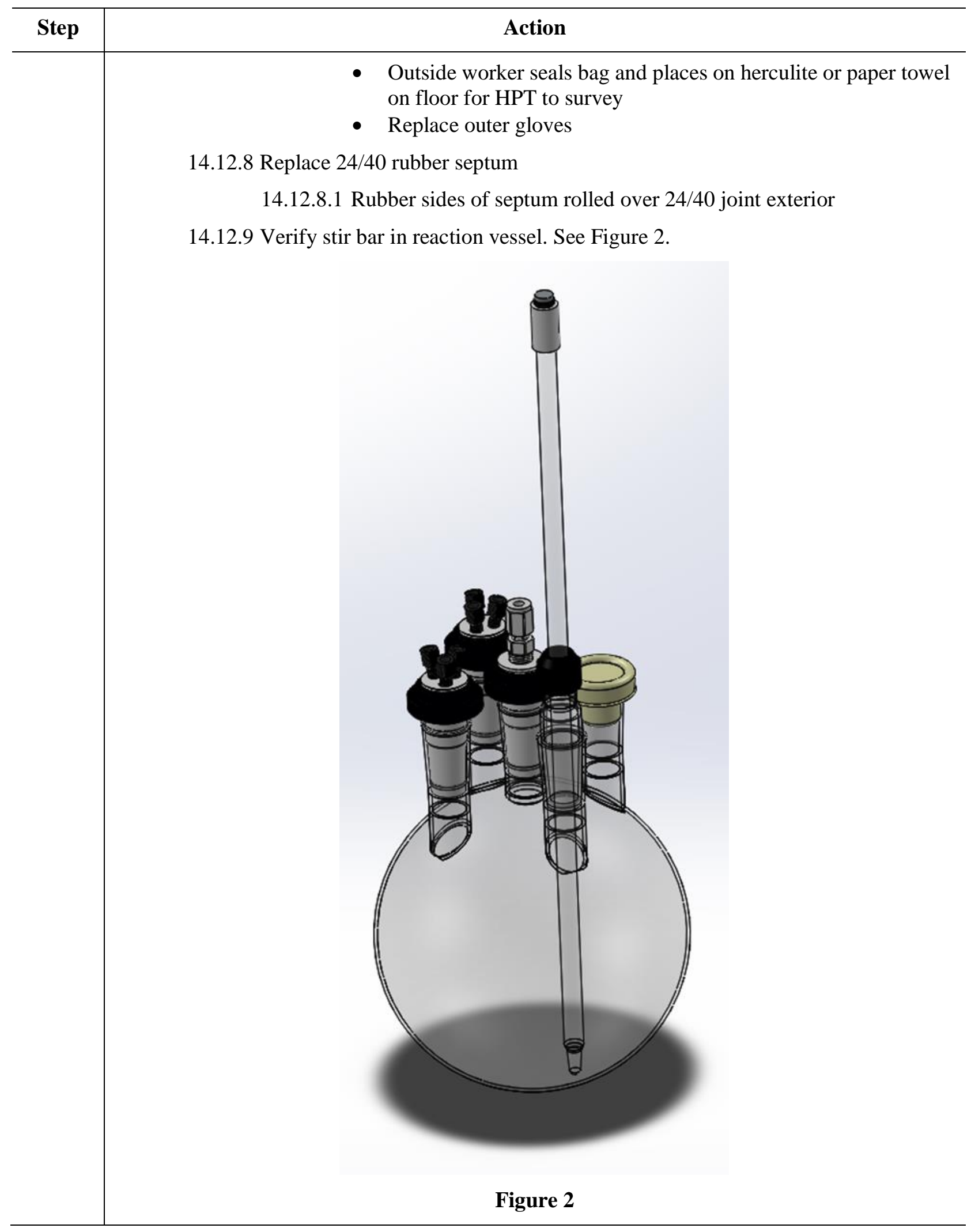




\begin{tabular}{|c|c|c|}
\hline \multirow[t]{14}{*}{ Step } & \multicolumn{2}{|r|}{ Action } \\
\hline & 14.13 & Check and verify operation of balance \\
\hline & 14.14 & Calibrate $\mathrm{pH}$ probe \\
\hline & 14.15 & Wipe down any surfaces as needed \\
\hline & 14.16 & Remove any waste and un-needed items \\
\hline & 14.17 & Replace outer gloves \\
\hline & 14.18 & Exit from interior hot cell \\
\hline & 14.19 & Roll-up Herculite and place inside hot cell on access door threshold \\
\hline & & $\begin{array}{l}\text { 14.19.1 Final diameter of roll should fit on interior ledge of hot cell without spilling } \\
\text { over either edge }\end{array}$ \\
\hline & 14.20 & Replace outer gloves \\
\hline & 14.21 & Entry Worker slowly close door with both hands \\
\hline & & 14.21.1 KEEP HANDS ON OUTER MOST EDGES OF DOOR \\
\hline & & 14.21.2 DO NOT LET DOOR SLAM SHUT \\
\hline & 14.22 & Verify handle is in closed position and is holding door closed \\
\hline 15 & \multicolumn{2}{|c|}{ HP Tech surveys workers and workers exit CA per training } \\
\hline \multirow[t]{6}{*}{16} & \multicolumn{2}{|c|}{ HP Tech down post area } \\
\hline & 16.1 & $\begin{array}{l}\text { Smears are taken of the floor, access door and horizontal surfaces in immediate } \\
\text { vicinity }\end{array}$ \\
\hline & 16.2 & Workers wait in vicinity \\
\hline & & 16.2.1 HP releases respirator \\
\hline & & 16.2.2 HP releases room \\
\hline & & 16.2.3 HP tags waste bags \\
\hline
\end{tabular}

\section{END OF SEGMENT}




\subsubsection{Prepare for Receipt of Primary Recovery Column Strip Solution from Primary Recovery Glovebox}

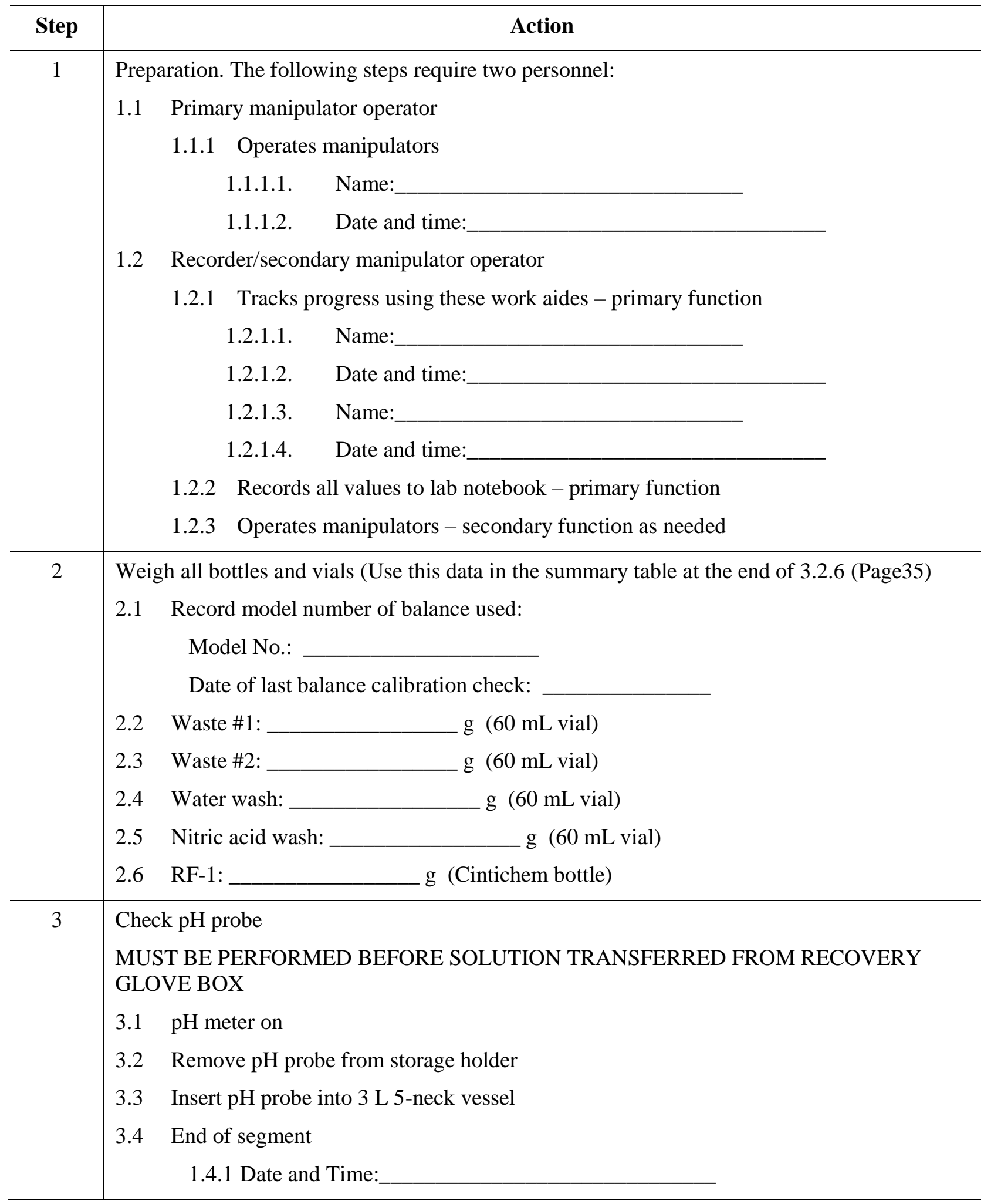


$4 \quad$ Select appropriate storage vessel on solution storage container (below D-024)

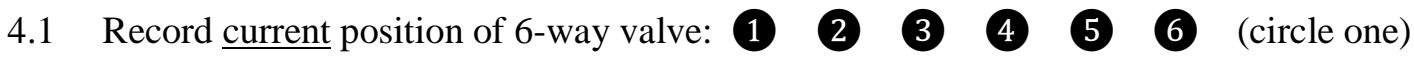

4.2 Calculate next position: (value from step 4.1) $+1=$

4.3 Rotate handle of 6-way valve to next position for current experiment:

$\begin{array}{lllllll}1 & 2 & 3 & 4 & 5 & 6 & \text { (circle one) }\end{array}$

4.4 If bottle position other than the value calculated in 2.4.2 is used, give reason:

4.5 Sign and date log sheet attached to D-024 Hot Cell

4.6 Record value of $10 \mathrm{~kg}$ load cell holding $3 \mathrm{~L}$ 5-neck flask: g

- All tubing is attached

- $\mathrm{pH}$ probe is inserted

- Stir plate in place

4.7 Verify gas collection needle from VNT-13 inserted into Waste \#1 $60 \mathrm{~mL}$ vial

4.8 Open manual 2-way valve solution valve, 2WV-701 (image and flow diagram posted at work site), from Primary Recovery Glovebox line

4.8.1 Rotate valve handle counter-clockwise so that handle points down and is now in vertical position

Checking D024 Hot Cell 3-L/5-Neck Flask Installation for Receipt of Mo-99 solution

II Recovery Member Name: PRINT Date:

Time:

D024 Hot Cell Ops Member Name: PRINT Date:

Time:

\section{\#\#\# SYSTEMS INTERFACE STEP \#\#\#}

4.8.2 Contact a Recovery Glove Box Operation team member

4.8.3 Appropriate team member INITIALIZES every step in this section

\subsubsection{Inside D024 Hot Cell}

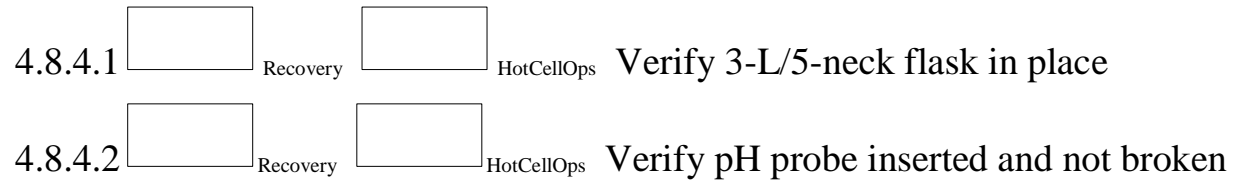




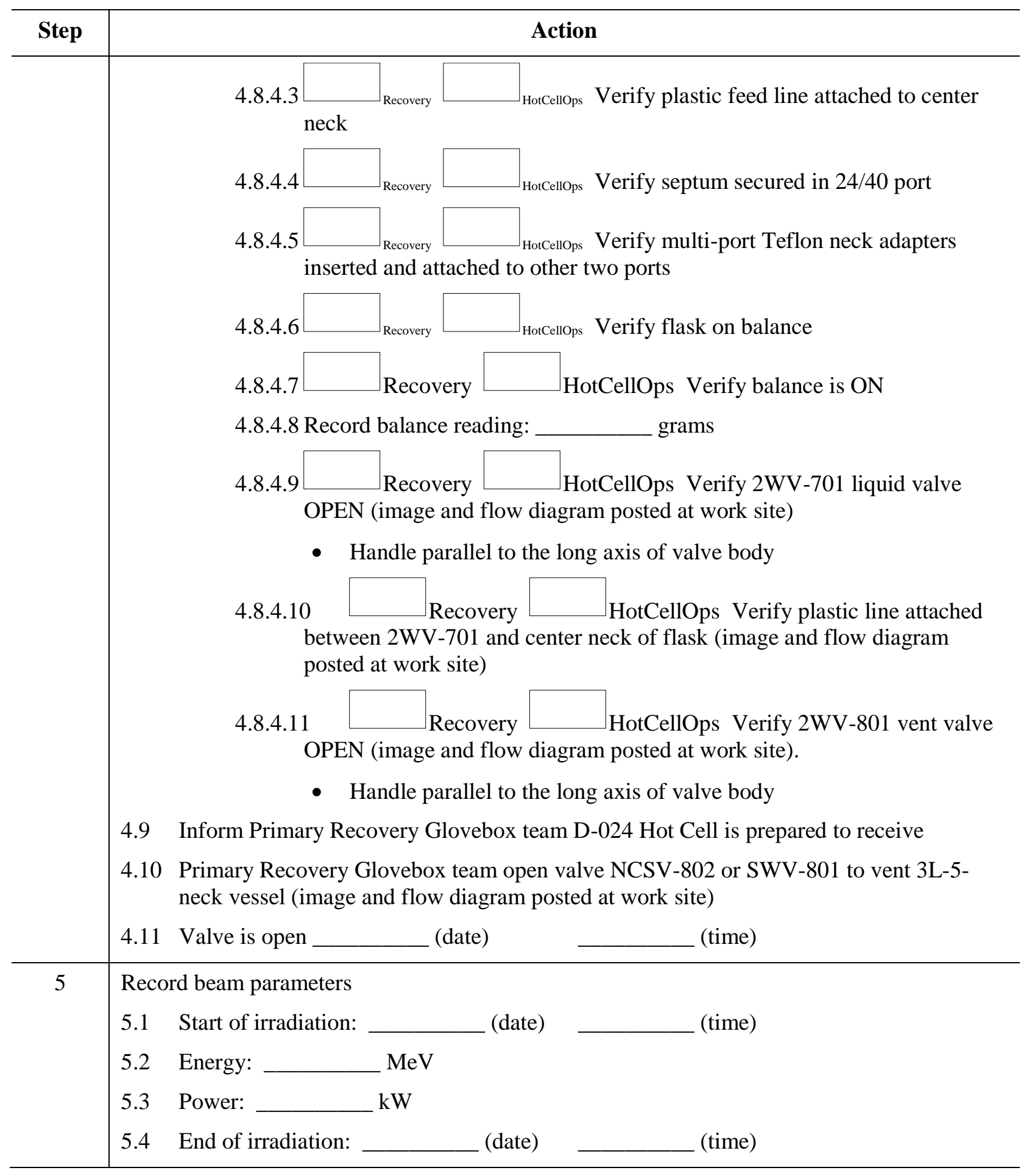




\subsubsection{Receive Primary Recovery Column Strip Solution from Primary Recovery Glovebox}

\begin{tabular}{|c|c|}
\hline Step & Action \\
\hline 1 & $\begin{array}{l}\text { Preparation. The following steps require two personnel: } \\
\text { 1.1 Transfer began: } \\
\text { 1.2 Records dose rates measured by HP Tech into lab notebook } \\
\text { 1.1.1 One of the following personnel } \\
1.1 .2 \quad \text { Primary manipulator operator } \\
\text { - Operates manipulators } \\
\text { 1.1.3 Recorder/secondary manipulator operator } \\
\text { • Tracks progress using these work aides } \\
\text { 1.3 HP Tech } \\
\text { Conducts dose measurements within 211-D024 }\end{array}$ \\
\hline 2 & $\begin{array}{l}\text { Dose rates BEFORE initiation of transfer } \\
2.1 \text { Average dose rate at } 211-\mathrm{D} 024 \text { doorway: } \\
2.2 \text { Average dose rate at } 30 \mathrm{~cm} \text { from transfer line: } \\
\text { 2.3 Average dose rate of lines on tact with lead bricks: }\end{array}$ \\
\hline 3 & $\begin{array}{l}\text { 3.1 Transfer end: } \_ \text {(date) } \quad \text { (time) } \\
3.2 \text { Once solution transfer complete, record value of } 10 \mathrm{~kg} \text { load cell holding } 3 \mathrm{~L} 5 \text {-neck } \\
\text { flask: }\end{array}$ \\
\hline
\end{tabular}

\section{END OF SEGMENT}


3.2.6 Process Primary Recovery Strip Product Through Concentration Column

\begin{tabular}{|c|c|}
\hline Step & Action \\
\hline 1 & Time of operation: ___ (date) __ (time) \\
\hline 2 & $\begin{array}{l}\text { The following steps require two personnel } \\
2.1 \text { Primary manipulator operator } \\
\text { - Operates manipulators } \\
\text { - Name:_- } \\
2.2 \text { Recorder/Secondary manipulator operator } \\
\text { - Tracks progress using these work aides } \\
\text { - Records all values to lab notebook } \\
\text { - Name: } \\
\text { - Name: }\end{array}$ \\
\hline 3 & $\begin{array}{l}\text { Close manual 2-way valve, } 2 \text { WV-701 from Primary Recovery Glovebox line (image and flow } \\
\text { diagram posted at work site) } \\
\text { - Turn valve clockwise so that valve handle is perpendicular to the floor }\end{array}$ \\
\hline 4 & $\begin{array}{l}\text { If not already open, open } 2 \mathrm{WV}-801 \text { to gas collection system } \\
4.1 \text { Vent line From } 3 \mathrm{~L} 5 \text {-neck flask to gas collection system } \\
\text { 4.2 Inform Primary Recovery Glovebox team that the } 3 \text { L 5-neck flask is now manually } \\
\text { open to gas collection system and solenoid valve NCSV-802 can be closed (image and } \\
\text { flow diagram posted at work site) }\end{array}$ \\
\hline
\end{tabular}

$\begin{array}{ll}5 & \text { Fine adjustment of the } \mathrm{pH} \text { of the primary recovery strip product }\end{array}$

5.1 Turn on stir plate

5.2 Set stir rate to

5.3 Adjust $\mathrm{pH}$ of recovery column primary strip product to $\mathrm{pH} 2$ with appropriate solution:

\subsubsection{8 $\mathrm{M} \mathrm{HNO}_{3}$ :}

1.3.1.1. Obtain mass of full syringe: g

1.3.2.10 M NaOH:

1.3.2.1. Obtain mass of full syringe: $\mathrm{g}$

5.4 Insert needle of $30 \mathrm{~mL}$ syringe loaded with solution from 5.3 into septum of $3 \mathrm{~L} 5$-neck vessel

5.4.1 If $\mathrm{pH}>10$ observed, add $5 \mathrm{~mL} 8 \mathrm{M} \mathrm{HNO}_{3}$ and observe change in $\mathrm{pH}$ (If $\mathrm{pH}<1$ observed, add $5 \mathrm{~mL} 10 \mathrm{M} \mathrm{NaOH}$ and observe change in $\mathrm{pH}$ )

5.4.2 Allow $\mathrm{pH}$ to settle

5.4.3 Continue until $\mathrm{pH}<10$ (or $\mathrm{pH}>2$ if using $\mathrm{NaOH}$ ) observed and then proceed if not add another $5 \mathrm{~mL}$ of $8 \mathrm{M} \mathrm{HNO}_{3}$ (or $10 \mathrm{M} \mathrm{NaOH}$ ) 


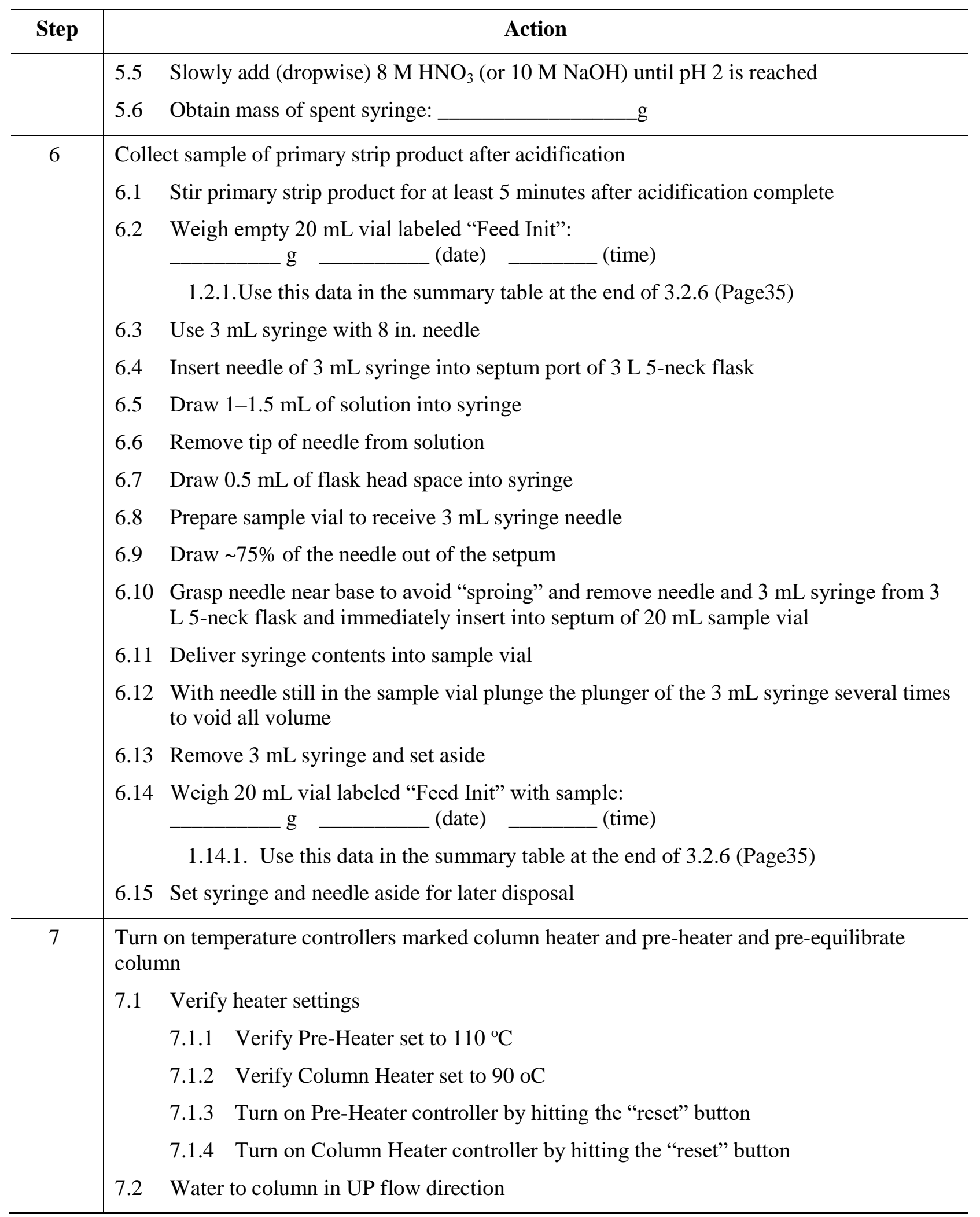




\begin{tabular}{|c|c|c|c|}
\hline \multirow[t]{2}{*}{ Step } & \multicolumn{3}{|r|}{ Action } \\
\hline & & 7.2.1 & $\begin{array}{l}\text { Verify liquid needle from 7-way "Output" valve (7WV-709) inserted into Waste } \\
\# 160 \mathrm{~mL} \text { vial }\end{array}$ \\
\hline & & 7.2 .2 & Verify gas collection needle from VNT-13 inserted into Waste \#1 $60 \mathrm{~mL}$ vial \\
\hline & & 7.2 .3 & Turn 7-way “Output” (7WV-709) valve to Waste \\
\hline & & 7.2 .4 & Turn bottom "Column Control" 3 -way valve (3WV-706) to upflow \\
\hline & & 7.2 .5 & Turn upper "Column Control" 3-way valve (3WV-707) to upflow \\
\hline & & 7.2 .6 & Turn left "Column Control” 3-way valve (3WV-708) to upflow \\
\hline & & 7.2 .7 & Turn right "Column Control" 3-way valve (3WV-705) to upflow \\
\hline & & 7.2 .8 & Turn upper "Pump Control" (inlet) 3-way valve (3WV-703) to COLUMN \\
\hline & & 7.2 .9 & Turn lower "Pump Control" (outlet) 3-way valve (3WV-704) to COLUMN \\
\hline & & 7.2 .10 & Turn feed source "Input" 5-way valve (5WV-702) to water feed bottle "Water" \\
\hline & & 7.2.11 & Open $2 \mathrm{WV}-803$ to gas collection \\
\hline & & 7.2.12 & Verify pump pitch set to ___ micrometer reading \\
\hline & & 7.2 .13 & Calculate pump power setting \\
\hline & & & 7.2.13.1 Desired flow rate $=50 \mathrm{~mL} / \mathrm{min}$ \\
\hline & & & 7.2.13.2 Pitch value $=_{-} \_$in. \\
\hline & & & $\begin{array}{l}\text { 7.2.13.3 }(576 \mathrm{~mL} / \mathrm{min}) \times(\% \text { motor power }) \times(\text { pitch value })=\text { output flow rate } \\
(\mathrm{mL} / \mathrm{min})\end{array}$ \\
\hline & & 7.2.14 & Verify V300 pump controller powered ON \\
\hline & & & 7.2.14.1 Rocker switch at bottom of controller housing \\
\hline & & 7.2 .15 & Verify V300 pump controller to STOP \\
\hline & & & 7.2.15.1 Display alternates between $\%$ motor power setting and the word OFF \\
\hline & & 7.2.16 & Verify V300 pump controller set to ___ $\%$ motor power \\
\hline & & & 7.2.16.1 Recommended $67.2 \%$ \\
\hline & & 7.2.17 & Verify V300 pump controller set to FWD (forward) \\
\hline & & 7.2.18 & Verify V300 pump controller set to MANUAL \\
\hline & & 7.2 .19 & Set timer to 26 seconds $(22 \mathrm{~mL})$ \\
\hline & & 7.2.20 & Press RUN on V300 pump controller and start timer \\
\hline & & 7.2 .21 & At timer end press STOP on V300 pump controller \\
\hline & 7.3 & Water & to column in DOWN flow direction \\
\hline & & 7.3.1 & Turn bottom "Column Control" 3-way valve (3WV-706) to downflow \\
\hline & & 7.3 .2 & Turn upper "Column Control" 3-way valve (3WV-707) to downflow \\
\hline
\end{tabular}




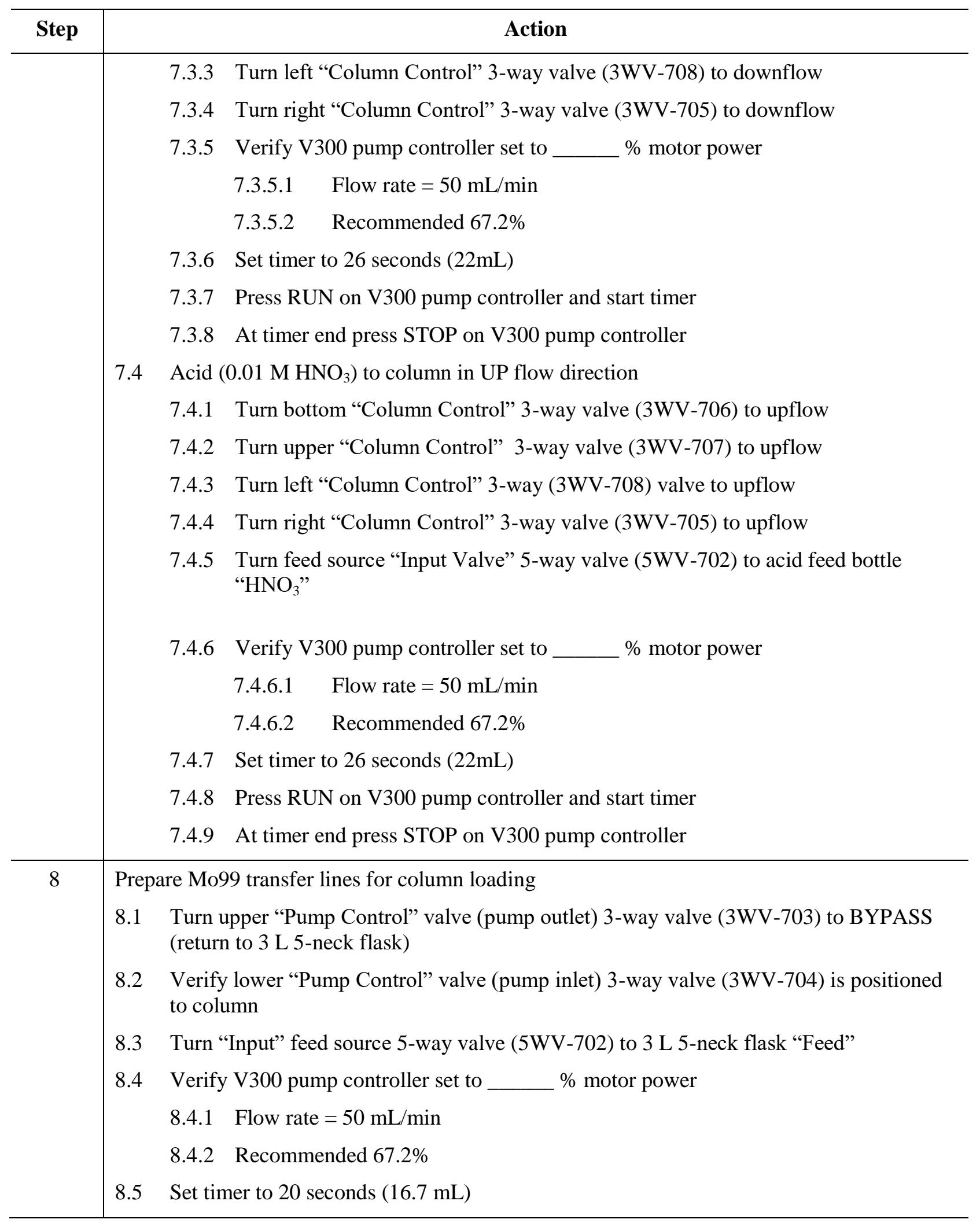




\begin{tabular}{|c|c|c|}
\hline \multirow[t]{3}{*}{ Step } & \multicolumn{2}{|r|}{ Action } \\
\hline & & Press RUN on V300 pump controller and start timer \\
\hline & 8.7 & At timer end press STOP on V300 pump controller \\
\hline \multirow[t]{24}{*}{9} & \multicolumn{2}{|r|}{ Load Mo99 product on to concentration column in UP flow direction } \\
\hline & 9.1 & Verify "Input" 4-way valve turned to 3 L 5-neck flask "Feed" \\
\hline & \multirow[t]{6}{*}{9.2} & $\begin{array}{l}\text { Turn "Output" 7-way valve (7WV-709) to 6-way eluent bottle directing valve "Phase I } \\
\text { Effluent" }\end{array}$ \\
\hline & & 9.2.1.Ensure ball valve of solution line connected to effluent bottle is OPEN \\
\hline & & 9.2.2. Ensure that the black luer-lock valve connected to the effluent bottle is OPEN \\
\hline & & 9.2.3.Ensure that black luer-lock valve for sampling the effluent bottle is CLOSED \\
\hline & & 9.2.4.Ensure that gas collection ball valve connected to the effluent bottle is OPEN \\
\hline & & $\begin{array}{l}\text { 9.2.5. Ensure that the black luer-lock valve connected to the gas collection needle is } \\
\text { CLOSED or is inserted in to one of the } 60 \mathrm{~mL} \text { septum collection vials }\end{array}$ \\
\hline & 9.3 & Verify bottom "Column Control" 3-way valve (3WV-706) to upflow \\
\hline & 9.4 & Verify upper "Column Control" 3-way valve (3WV-707) to upflow \\
\hline & 9.5 & Verify left "Column Control" 3-way valve (3WV-708) to upflow \\
\hline & 9.6 & Verify right "Column Control" 3-way valve (3WV-705) to upflow \\
\hline & 9.7 & Verify upper "Pump Control" (pump outlet) 3-way valve (3WV-703) to "Column" \\
\hline & 9.8 & $\begin{array}{l}\text { Verify lower "Pump Control" (pump inlet) 3-way valve ( } 3 \text { WV-704) to feed source 4- } \\
\text { way valve "Column" }\end{array}$ \\
\hline & 9.9 & Turn "Input" (feed source) 5-way valve (5WV-702) to 3 L 5-neck flask "Feed" \\
\hline & 9.10 & Turn off stirring \\
\hline & \multirow[t]{2}{*}{9.11} & Verify V300 pump controller set to ___ $\%$ motor power \\
\hline & & 9.11.1 Flow rate $=50 \mathrm{~mL} / \mathrm{min}(67.2 \%)$ \\
\hline & 9.12 & Set timer to 50 minutes \\
\hline & 9.13 & Press RUN on V300 pump controller and start timer \\
\hline & 9.14 & Record time pump on: ___ (date) __ (time) \\
\hline & 9.15 & At timer end prepare to tilt vessel to process all of solution \\
\hline & & At air bubbles in flask pickup line press STOP on V300 controller \\
\hline & 9.16 & Record time pump off: ____ (date) ___ (time) \\
\hline \multirow[t]{2}{*}{10} & \multicolumn{2}{|c|}{ Post-load acid wash } \\
\hline & 10.1 & $\begin{array}{l}\text { Verify liquid needle from "Output" 7-way valve (7WV-709) inserted into Acid Wash } 60 \\
\text { mL vial }\end{array}$ \\
\hline
\end{tabular}




\begin{tabular}{|c|c|}
\hline Step & Action \\
\hline & 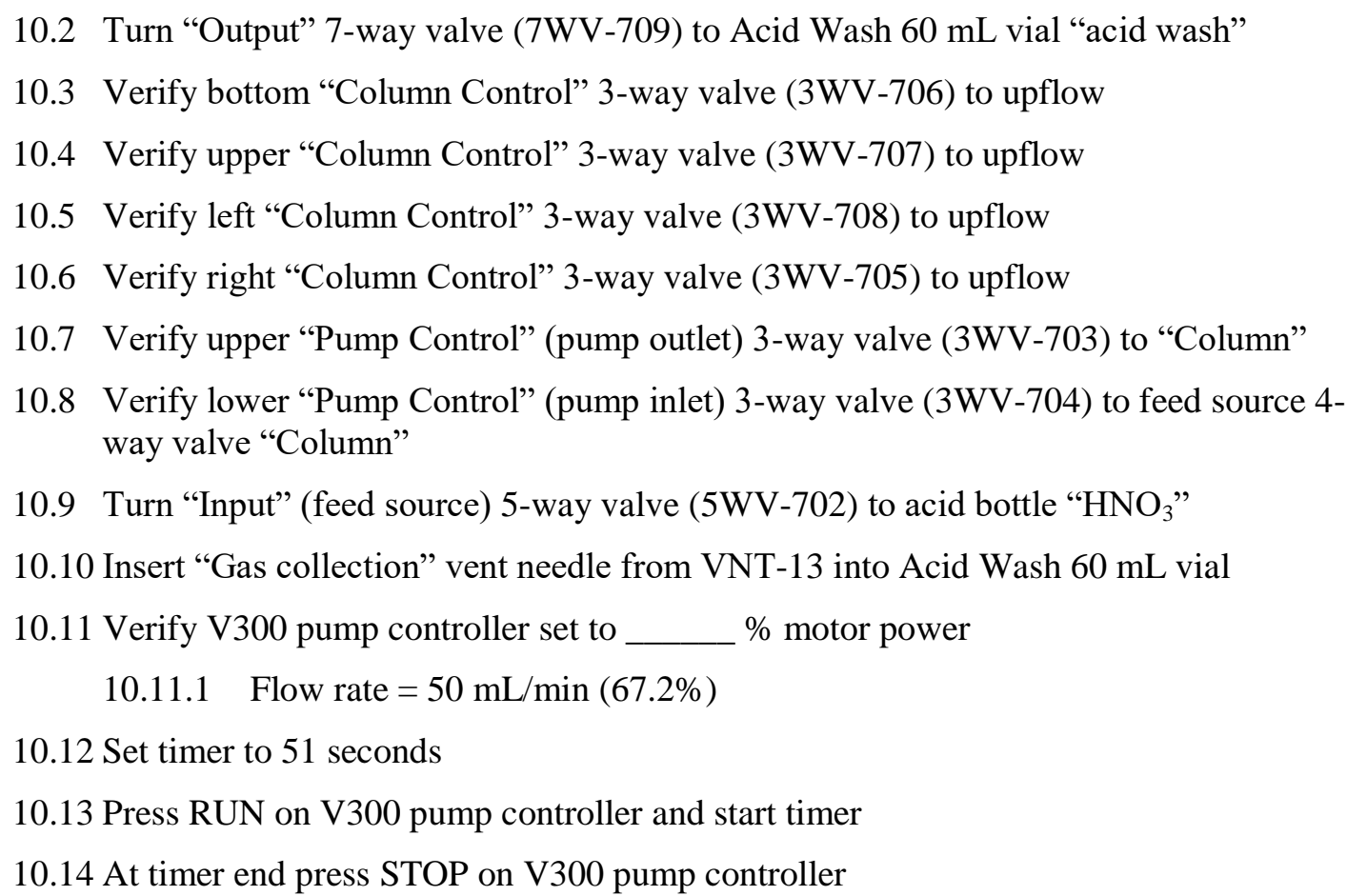 \\
\hline 11 & 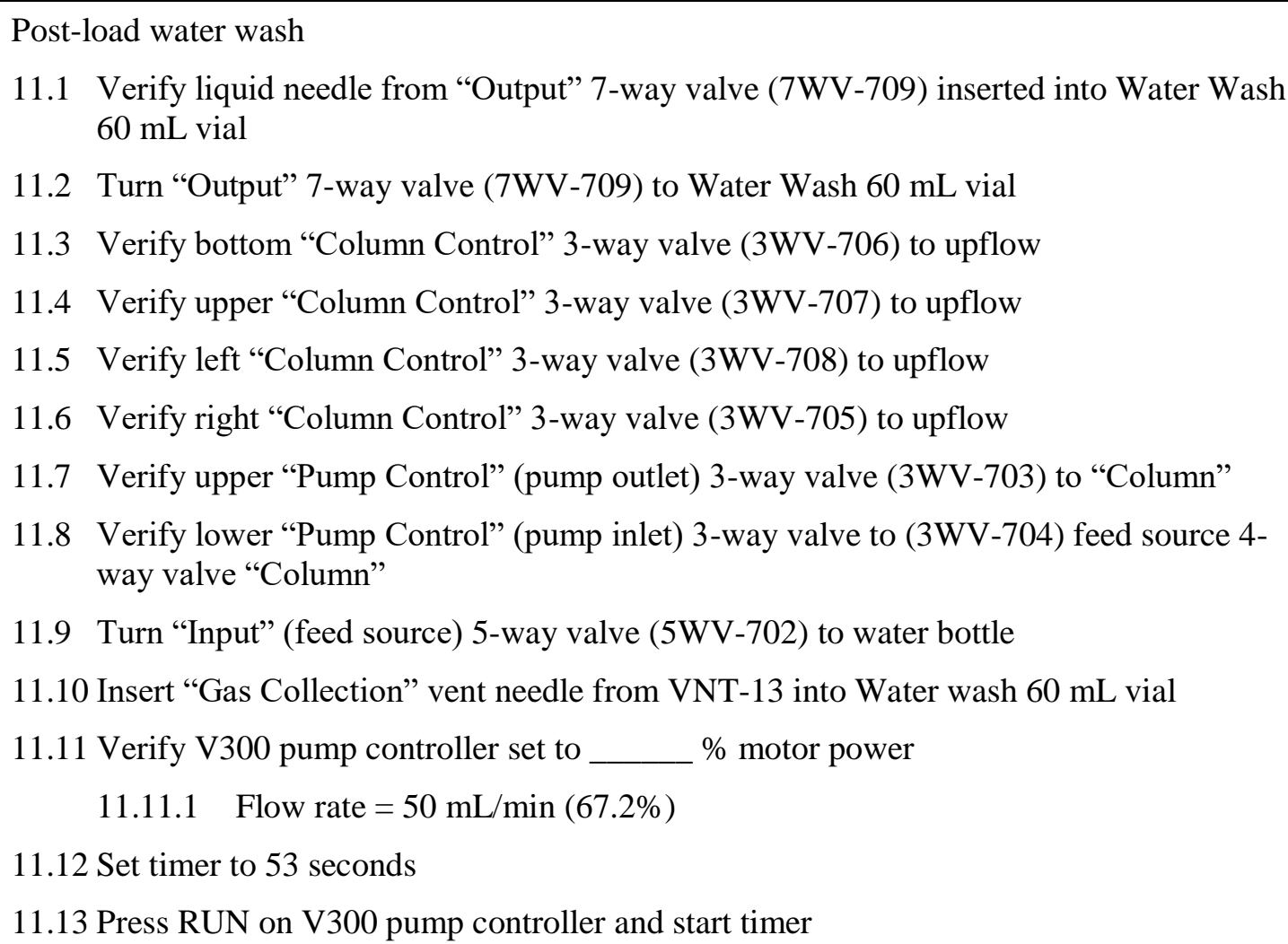 \\
\hline
\end{tabular}




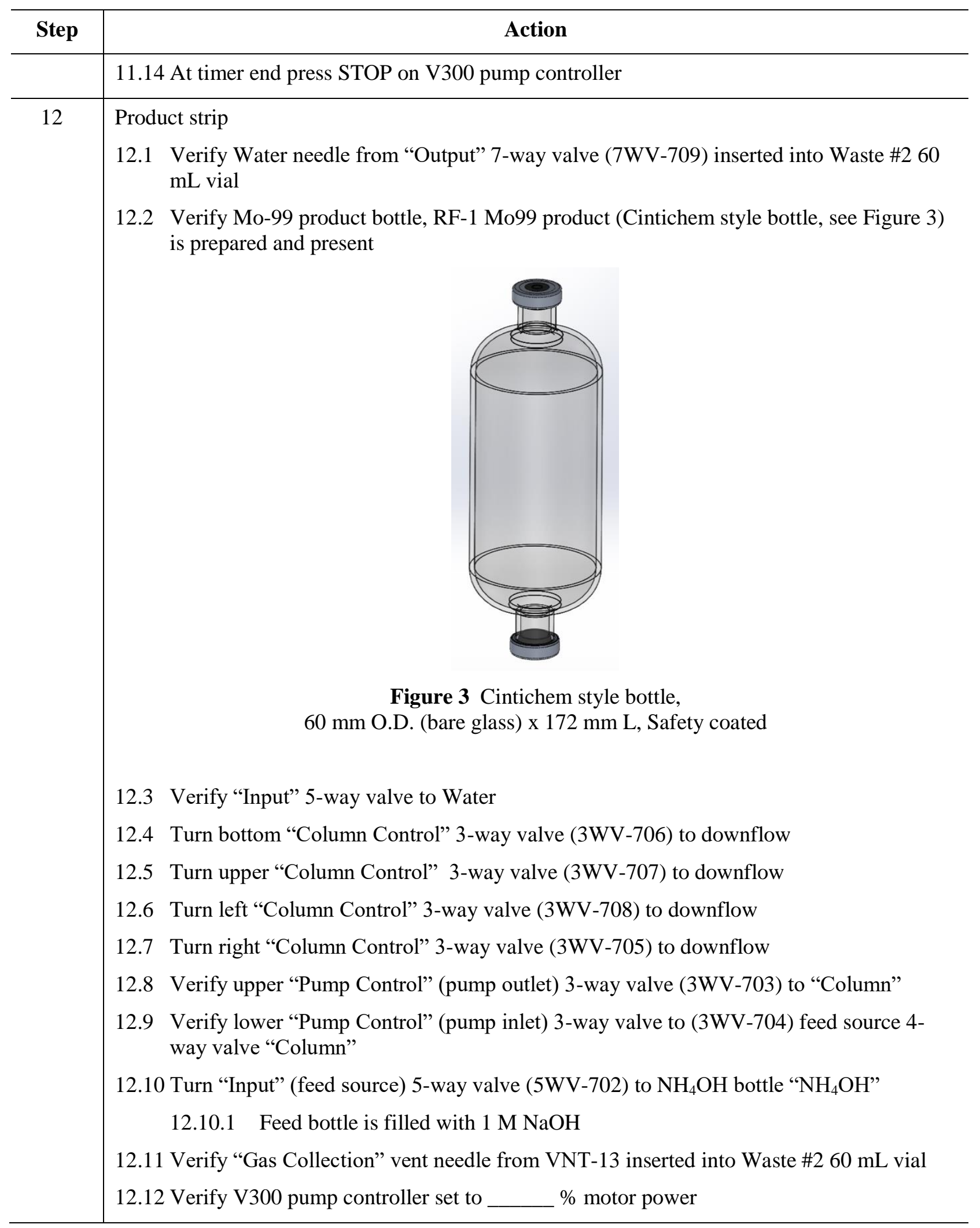




\begin{tabular}{|c|c|}
\hline Step & Action \\
\hline & $\begin{array}{l}\text { 12.12.1 Flow rate }=11 \mathrm{~mL} / \mathrm{min}(14.8 \%) \\
\text { 12.13 Set timer to } 2 \text { minutes ( } 120 \text { seconds) } \\
\text { 12.14 Press RUN on V300 pump controller and start timer } \\
\text { 12.15 At timer end press STOP on V300 pump controller } \\
\text { 12.16 Turn "Output" } 7 \text {-way valve ( } 7 \mathrm{WV}-709) \text { to RF-1 Mo99 product bottle "Product" } \\
\text { 12.17 Insert "Gas Collection" needle from VNT-13 into RF-1 Mo99 product bottle } \\
\text { 12.18 Verify V300 pump controller set to } \\
\text { 12.18.1 Flow rate }=11 \mathrm{~mL} / \mathrm{min}(14.8 \%) \\
\text { 12.19 Set timer to } 6 \text { minutes ( } 360 \text { seconds) } \\
\text { 12.20 Press RUN on V300 pump controller and start timer } \\
\text { 12.21 At timer } 1.36 \text { minutes left } \\
\text { 12.21.1 Verify feed in NaOH feed bottle } \\
\text { 12.21.2 If feed bottle liquid level is too low to reach end of timer press STOP on } \\
\text { 12.22 At timer end press STOP on V300 pump controller }\end{array}$ \\
\hline 13 & $\begin{array}{l}\text { Post-strip water wash } \\
\text { 13.1 Insert Mo-99 needle from "Output" 7-way valve (7WV-709) Waste \#1 valve port into } \\
\text { Waste \#2 } 60 \mathrm{~mL} \text { vial } \\
\text { 13.2 Turn "Output" 7-way valve (7WV-709) to Waste \#2 } 60 \mathrm{~mL} \text { vial } \\
\text { 13.3 Turn bottom "Column Control" 3-way valve (3WV-706) to downflow } \\
\text { 13.4 Turn upper "Column Control" 3-way valve (3WV-707) to downflow } \\
\text { 13.5 Turn left "Column Control" 3-way valve (3WV-708) to downflow } \\
\text { 13.6 Turn right "Column Control" 3-way valve (3WV-705) to downflow } \\
\text { 13.7 Verify upper "Pump Control" (pump outlet) 3-way valve (3WV-703) to "Column" } \\
\text { 13.8 Verify lower "Pump Control" (pump inlet) 3-way valve (3WV-704) to feed source 4- } \\
\text { way valve "Column" } \\
\text { 13.9 Turn "Input" (feed source) 5-way valve (5WV-702) to Water bottle } \\
\text { 13.10 Insert "Gas Collection" vent needle from VNT-13 into Waste \#2 } 60 \mathrm{~mL} \text { vial } \\
\text { 13.11 Adjust V300 pump controller to ___ \% motor power } \\
\text { 13.11.1 Flow rate = 50 mL/min (67.2\%) } \\
\text { 13.12 Set timer to } 1 \text { minute (60 seconds) }\end{array}$ \\
\hline
\end{tabular}




\begin{tabular}{|c|c|}
\hline Step & Action \\
\hline & 13.14 At timer end press STOP on V300 pump controller \\
\hline 14 & Turn off J-Kern controllers \\
\hline 15 & $\begin{array}{l}\text { Weigh RF-1 Mo99 product bottle: ___ } \mathrm{g} \text { (date) __ (time) } \\
\text { Use this data in the summary table at the end of 3.2.6 (Page35) }\end{array}$ \\
\hline 16 & Return RF-1 Mo99 product bottle to vial rack \\
\hline 17 & $\begin{array}{l}\text { Verify that } 2 \mathrm{WV}-019 \text { to gas collection system is still open } \\
\text { 17.1 Vent line From } 3 \text { L 5-neck flask to gas collection system will remain open until system } \\
\text { washed from Primary Recovery Column }\end{array}$ \\
\hline 18 & Leave $\mathrm{pH}$ probe in vessel until next entry into cell \\
\hline 19 & Turn off pump controller \\
\hline 20 & Place gas collection line into RF1 "Mo-99 product bottle" \\
\hline 21 & $\begin{array}{l}\text { Sample solutions } \\
\text { 21.1. Shake all vials with manipulators and obtain mass of each vessel } \\
\text { 21.1.1. Waste \#1:__ 21.1.2. Waste \#2: } \\
\text { 21.1.3. Water Wash: } \\
\text { 21.1.4. Nitric Acid Wash: } \\
\text { 21.1.5. Mo-99 Product (Cintichem Vessel): _ } \\
\text { Use this data in the summary table at the end of 3.2.6 (Page35) } \\
\text { 21.2. Use 1 mL syringes with 6" needles to sample vials. Pull the plunger to } 50 \% \text { of the } \\
\text { syringe shaft. Remove needle from solution while keeping needle within vessel being samples. } \\
\text { Pull plunger to } ~ 80 \% \text { of the syringe shaft. Remove needle from vessel and inject sample into } \\
\text { appropriate sampling vessel. Record mass of sample } \\
\text { 21.2.1 Waste \#2 vessel with sample: } \\
\text { 21.2.2 Water wash vessel with sample: } \\
\text { 21.2.3 Nitric acid wash vessel with sample: } \\
\text { Use this data in the summary table at the end of } 3.2 .6 \text { (Page35) } \\
\text { 21.3. Sample Effluent Bottle } \\
\text { 21.3.1. Ensure effluent bottle is connected to gas collection system } \\
\quad \text { 21.3.1.1. Ensure VQD-014 is connected }\end{array}$ \\
\hline
\end{tabular}




\begin{tabular}{|c|c|}
\hline Step & Action \\
\hline & $\begin{array}{l}\text { 21.3.1.2. Ensure gas collection needle for small bottles is in a septum bottle or } \\
\text { closed }\end{array}$ \\
\hline & 21.3.1.3. Ensure effluent bottle black luer lock valve is OPEN \\
\hline & 21.3.1.4. Ensure effluent bottle ball valve is OPEN \\
\hline & 21.3.2. Connect effluent bottle solution line to syringe \\
\hline & $\begin{array}{l}\text { 21.3.2.1. Ensure that 7WV-709 "OUTPUT" 7-way valve is directed towards } \\
\text { any other output than "Phase I Effluent"- "OUTPUT" valve is NOT } \\
\text { connected to effluent bottle being sampled. }\end{array}$ \\
\hline & 21.3.2.2. Ensure effluent bottle solution ball valve is OPEN \\
\hline & $\begin{array}{l}\text { 21.3.2.3. Connect syringe (suggested } 20-\mathrm{mL} \text { syringe with valve and plunger } \\
\text { fully extended) to black luer lock at "t" connection to effluent bottle. }\end{array}$ \\
\hline & 21.3.3. Mix and collect sample \\
\hline & 21.3.3.1. Open black luer lock connecters \\
\hline & $\begin{array}{l}\text { 21.3.3.2. Depress syringe plunger to force air into the effluent bottle to mix } \\
\text { system (LEAVE } \sim \mathbf{5} \text { ML OF AIR WITHIN THE SYRINGE) }\end{array}$ \\
\hline & $\begin{array}{l}\text { 21.3.3.3. Pull syringe plunger up and down } 3 X \text { to further mix the system } \\
\text { (LEAVE 5 ML OF AIR WITHIN THE SYRINGE) }\end{array}$ \\
\hline & 21.3.3.4. Pull plunger to take sample (suggested $\sim 1-3 \mathrm{~mL}$ ) \\
\hline & 21.3.3.5. Invert syringe so that solution is on plunger side and remaining \\
\hline & $\begin{array}{l}\text { 21.3.3.6. Depress syringe until bubbles are noticed in effluent bottle - OR - } \\
\text { until sample is near top of syringe barrel (the point of this step is to } \\
\text { void the lines and ensure no solution is in disconnect points) }\end{array}$ \\
\hline & 21.3.3.7. CLOSE all solution black luer lock valves $(2 \mathrm{x})$ \\
\hline & $\begin{array}{l}\text { 21.3.3.8. Disconnect the syringe from the effluent bottle system while } \\
\text { maintaining ONE black luer lock valve to the syringe. }\end{array}$ \\
\hline & $\begin{array}{l}\text { 21.3.3.9. Affix a needle to the black luer lock valve still connected to the } \\
\text { syringe. }\end{array}$ \\
\hline & $\begin{array}{l}\text { 21.3.3.10. Inject solution into appropriately marked } 20 \mathrm{~mL} \text { septum vessel and } \\
\text { record mass. }\end{array}$ \\
\hline & 21.2.3.3.10.1 Effluent sample: _____ g (20 mL vial) \\
\hline & Use this data in the summary table at the end of 3.2.6 (Page35) \\
\hline 22 & Notify LMC team of completion \\
\hline & Date:_ Time:__ \\
\hline 23 & $\begin{array}{l}\text { Within one week of the AMORE experiment being completed the plastic lines that radioactive } \\
\text { material transferred through them should be rinsed with water. }\end{array}$ \\
\hline
\end{tabular}




\begin{tabular}{|c|c|}
\hline Step & Action \\
\hline & Water wash pathway of column and acid wash line \\
\hline & $\begin{array}{l}23.1 \text { Verify acid wash needle from "Output" } 7 \text {-way valve (7WV-709) inserted into an empty } \\
60 \mathrm{~mL} \text { vial or collection bottle }\end{array}$ \\
\hline & 23.2 Turn "Output" 7-way valve (7WV-709) to Acid Wash \\
\hline & 23.3 Verify bottom "Column Control" 3-way valve (3WV-706) to upflow \\
\hline & 23.4 Verify upper "Column Control" 3-way valve (3WV-707) to upflow \\
\hline & 23.5 Verify left "Column Control" 3-way valve (3WV-708) to upflow \\
\hline & 23.6 Verify right "Column Control" 3-way valve (3WV-705) to upflow \\
\hline & 23.7 Verify upper "Pump Control" (pump outlet) 3-way valve (3WV-703) to "Column" \\
\hline & $\begin{array}{l}23.8 \text { Verify lower "Pump Control" (pump inlet) 3-way valve to (3WV-704) feed source 4- } \\
\text { way valve "Column" }\end{array}$ \\
\hline & 23.9 Turn "Input" (feed source) 5-way valve (5WV-702) to water bottle \\
\hline & 23.10 Insert "Gas Collection" vent needle from VNT-13 into collection bottle \\
\hline & 23.11 Verify the luer-lock valve on the line is open \\
\hline & 23.12 Verify V300 pump controller is $\mathrm{ON}$ and set to ___ $\%$ motor power \\
\hline & Flow rate $=50 \mathrm{~mL} / \mathrm{min}(67.2 \%)$ \\
\hline & 23.13 Press RUN on V300 pump controller and start timer \\
\hline & 23.14 Observe vial for liquid and rinse line for at least 10 seconds \\
\hline & 23.15 Press STOP on V300 pump controller once rinse is complete \\
\hline & Acid wash line rinse completed: $\square$ Date:__ Time: \\
\hline & Water wash pathway of column and water wash line \\
\hline & $\begin{array}{l}\text { 23.16 Verify water wash needle from "Output" } 7 \text {-way valve (7WV-709) inserted into an } \\
\text { empty } 60 \mathrm{~mL} \text { vial or collection bottle }\end{array}$ \\
\hline & 23.17 Turn "Output" 7-way valve (7WV-709) to Water Wash \\
\hline & 23.18 Verify bottom "Column Control" 3-way valve (3WV-706) to upflow \\
\hline & 23.19 Verify upper "Column Control" 3-way valve (3WV-707) to upflow \\
\hline & 23.20 Verify left "Column Control" 3-way valve (3WV-708) to upflow \\
\hline & 23.21 Verify right "Column Control" 3-way valve (3WV-705) to upflow \\
\hline & 23.22 Verify upper "Pump Control" (pump outlet) 3-way valve (3WV-703) to "Column" \\
\hline & $\begin{array}{l}23.23 \text { Verify lower "Pump Control" (pump inlet) 3-way valve to (3WV-704) feed source } 4 \text { - } \\
\text { way valve "Column" }\end{array}$ \\
\hline & 23.24 Turn "Input" (feed source) 5-way valve (5WV-702) to water bottle \\
\hline
\end{tabular}




\begin{tabular}{|c|c|}
\hline Step & Action \\
\hline & 23.25 Insert "Gas Collection" vent needle from VNT-13 into collection bottle \\
\hline & 23.26 Verify the luer-lock valve on the line is open \\
\hline & 23.27 Verify V300 pump controller is $\mathrm{ON}$ and set to ___ $\%$ motor power \\
\hline & Flow rate $=50 \mathrm{~mL} / \mathrm{min}(67.2 \%)$ \\
\hline & 23.28 Press RUN on V300 pump controller and start timer \\
\hline & 23.29 Observe vial for liquid and rinse line for at least 10 seconds \\
\hline & 23.30 Press STOP on V300 pump controller once rinse is complete \\
\hline & Water wash line rinse completed: $\square$ Date:____ Time: \\
\hline & Water wash pathway of column and Mo99 Product line \\
\hline & $\begin{array}{l}\text { 23.31 Verify Mo99 Product needle from "Output" 7-way valve (7WV-709) inserted into an } \\
\text { empty } 60 \mathrm{~mL} \text { vial or collection bottle }\end{array}$ \\
\hline & 23.32 Turn "Output" 7-way valve (7WV-709) to Mo99 Product \\
\hline & 23.33 Verify bottom "Column Control" 3-way valve (3WV-706) to upflow \\
\hline & 23.34 Verify upper "Column Control" 3-way valve (3WV-707) to upflow \\
\hline & 23.35 Verify left "Column Control" 3-way valve (3WV-708) to upflow \\
\hline & 23.36 Verify right "Column Control" 3-way valve (3WV-705) to upflow \\
\hline & 23.37 Verify upper "Pump Control" (pump outlet) 3-way valve (3WV-703) to "Column" \\
\hline & $\begin{array}{l}23.38 \text { Verify lower "Pump Control" (pump inlet) 3-way valve to (3WV-704) feed source 4- } \\
\text { way valve "Column" }\end{array}$ \\
\hline & 23.39 Turn "Input" (feed source) 5-way valve (5WV-702) to water bottle \\
\hline & 23.40 Insert "Gas Collection" vent needle from VNT-13 into collection bottle \\
\hline & 23.41 Verify the luer-lock valve on the line is open \\
\hline & 23.42 Verify V300 pump controller is $\mathrm{ON}$ and set to ___ $\%$ motor power \\
\hline & Flow rate $=50 \mathrm{~mL} / \mathrm{min}(67.2 \%)$ \\
\hline & 23.43 Press RUN on V300 pump controller and start timer \\
\hline & 23.44 Observe vial for liquid and rinse line for at least 10 seconds \\
\hline & 23.45 Press STOP on V300 pump controller once rinse is complete \\
\hline & Mo99 Product wash line rinse completed: $\square$ Date:___ Time:_ \\
\hline & Water wash pathway of column and waste wash line \\
\hline
\end{tabular}




\begin{tabular}{|c|c|}
\hline Step & Action \\
\hline & $\begin{array}{l}23.46 \text { Verify waste needle from "Output" 7-way valve (7WV-709) inserted into an empty } 60 \\
\text { mL vial or collection bottle }\end{array}$ \\
\hline & 23.47 Turn "Output" 7-way valve (7WV-709) to Waste \\
\hline & 23.48 Verify bottom "Column Control" 3-way valve (3WV-706) to upflow \\
\hline & 23.49 Verify upper "Column Control" 3-way valve (3WV-707) to upflow \\
\hline & 23.50 Verify left "Column Control" 3-way valve (3WV-708) to upflow \\
\hline & 23.51 Verify right "Column Control" 3-way valve (3WV-705) to upflow \\
\hline & 23.52 Verify upper "Pump Control" (pump outlet) 3-way valve (3WV-703) to "Column" \\
\hline & $\begin{array}{l}23.53 \text { Verify lower "Pump Control" (pump inlet) 3-way valve to (3WV-704) feed source 4- } \\
\text { way valve "Column" }\end{array}$ \\
\hline & 23.54 Turn "Input" (feed source) 5-way valve (5WV-702) to water bottle \\
\hline & 23.55 Insert "Gas Collection" vent needle from VNT-13 into collection bottle \\
\hline & 23.56 Verify the luer-lock valve on the line is open \\
\hline & 23.57 Verify V300 pump controller is $\mathrm{ON}$ and set to ___ $\%$ motor power \\
\hline & Flow rate $=50 \mathrm{~mL} / \mathrm{min}(67.2 \%)$ \\
\hline & 23.58 Press RUN on V300 pump controller and start timer \\
\hline & 23.59 Observe vial for liquid and rinse line for at least 10 seconds \\
\hline & 23.60 Press STOP on V300 pump controller once rinse is complete \\
\hline & Waste wash line rinse completed: $\square$ Date:__ Time: \\
\hline \multirow[t]{11}{*}{24} & Rinse Effluent Transfer Line with Water \\
\hline & 24.1 Turn "Output" 7-way valve (7WV-709) to "Phase 1 Effluent" \\
\hline & 24.2 Turn bottom "Column Control" 3-way valve (3WV-706) to downflow \\
\hline & 24.3 Turn upper "Column Control" 3-way valve (3WV-707) to downflow \\
\hline & 24.4 Turn left "Column Control” 3-way valve (3WV-708) to downflow \\
\hline & 24.5 Turn right "Column Control" 3-way valve (3WV-705) to downflow \\
\hline & 24.6 Verify upper "Pump Control" (pump outlet) 3-way valve (3WV-703) to "Column" \\
\hline & $\begin{array}{l}24.7 \text { Verify lower "Pump Control" (pump inlet) 3-way valve (3WV-704) to feed source } 4 \text { - } \\
\text { way valve "Column" }\end{array}$ \\
\hline & 24.8 Turn "Input" (feed source) 5-way valve (5WV-702) to Water bottle \\
\hline & 24.9 Adjust V300 pump controller to ___ \% motor power \\
\hline & Flow rate $=50 \mathrm{~mL} / \mathrm{min}(67.2 \%)$ \\
\hline
\end{tabular}




\begin{tabular}{|c|c|}
\hline Step & Action \\
\hline & $\begin{array}{l}\text { 24.10 Set timer to } 30 \text { seconds } \\
24.11 \text { Press RUN on V300 pump controller and start timer } \\
\text { Stop V300 pump controller after time has elapsed } \\
\text { Effluent line rinse completed: } \square \text { Date:__ Time: }\end{array}$ \\
\hline 25 & 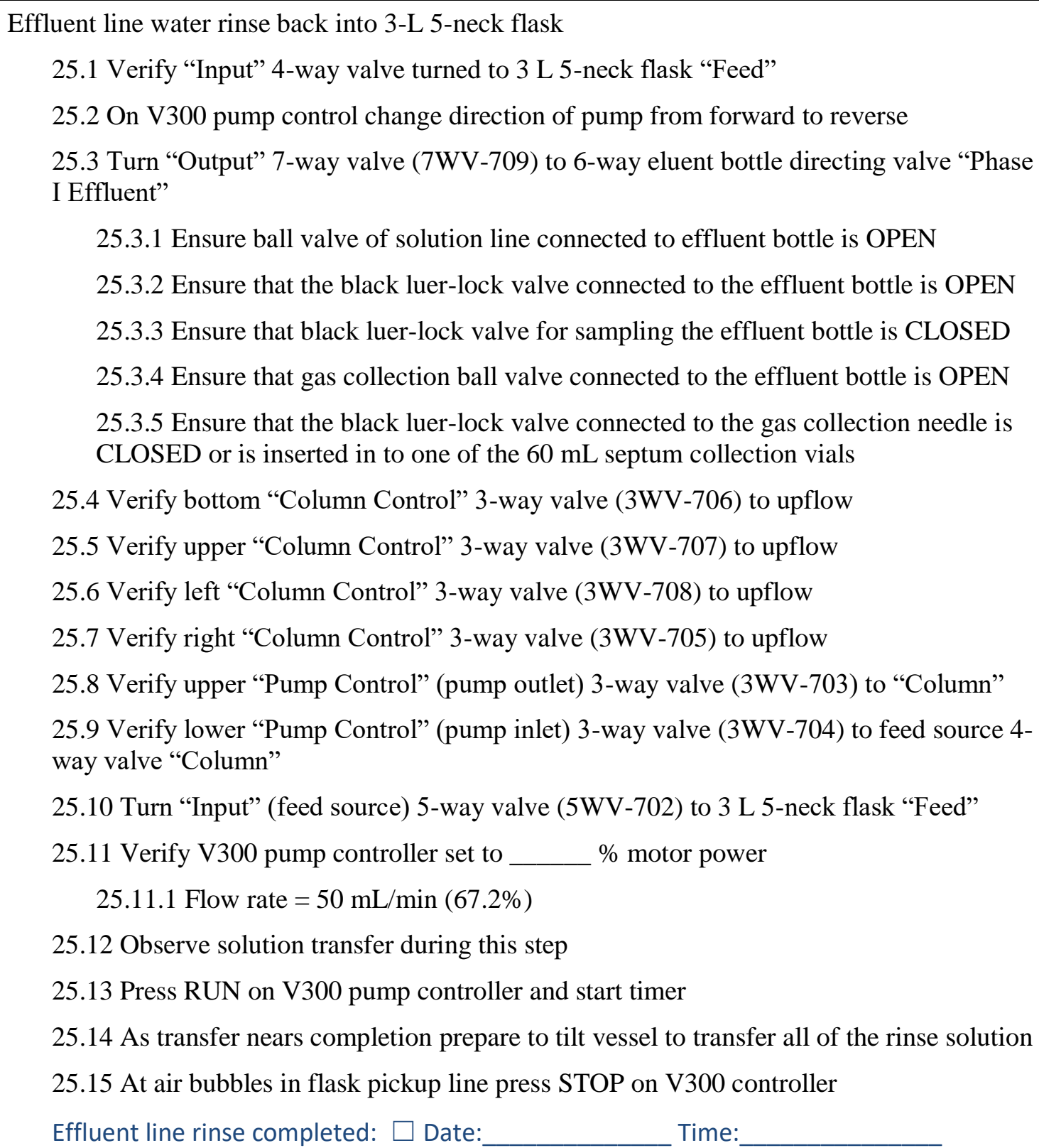 \\
\hline 26 & $\begin{array}{l}\text { Transfer Water Rinse solution to Effluent storage below hot cell } \\
26.1 \text { Verify "Input" 4-way valve turned to } 3 \text { L 5-neck flask "Feed" }\end{array}$ \\
\hline
\end{tabular}




\begin{tabular}{|c|c|}
\hline Step & Action \\
\hline & 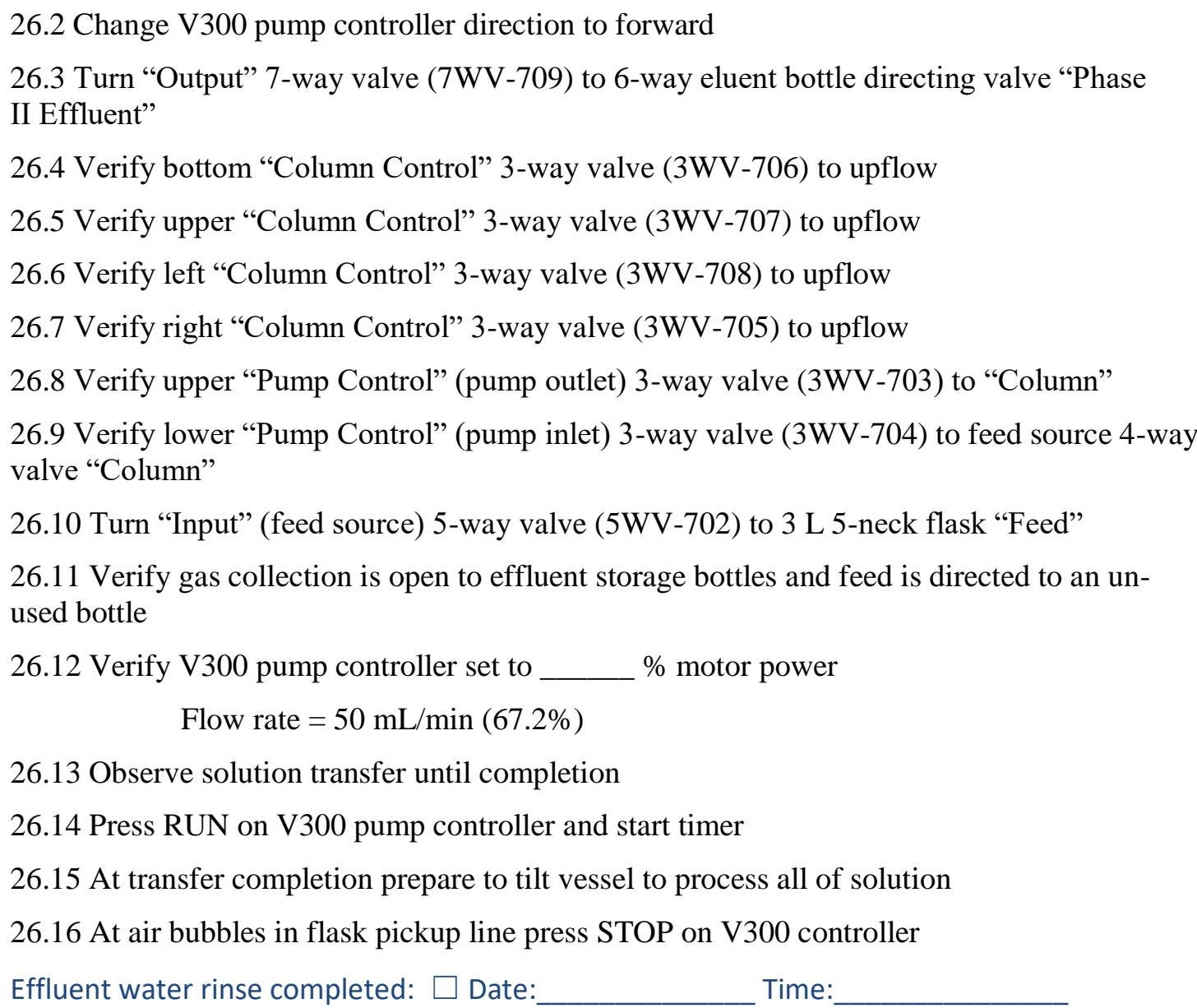 \\
\hline
\end{tabular}




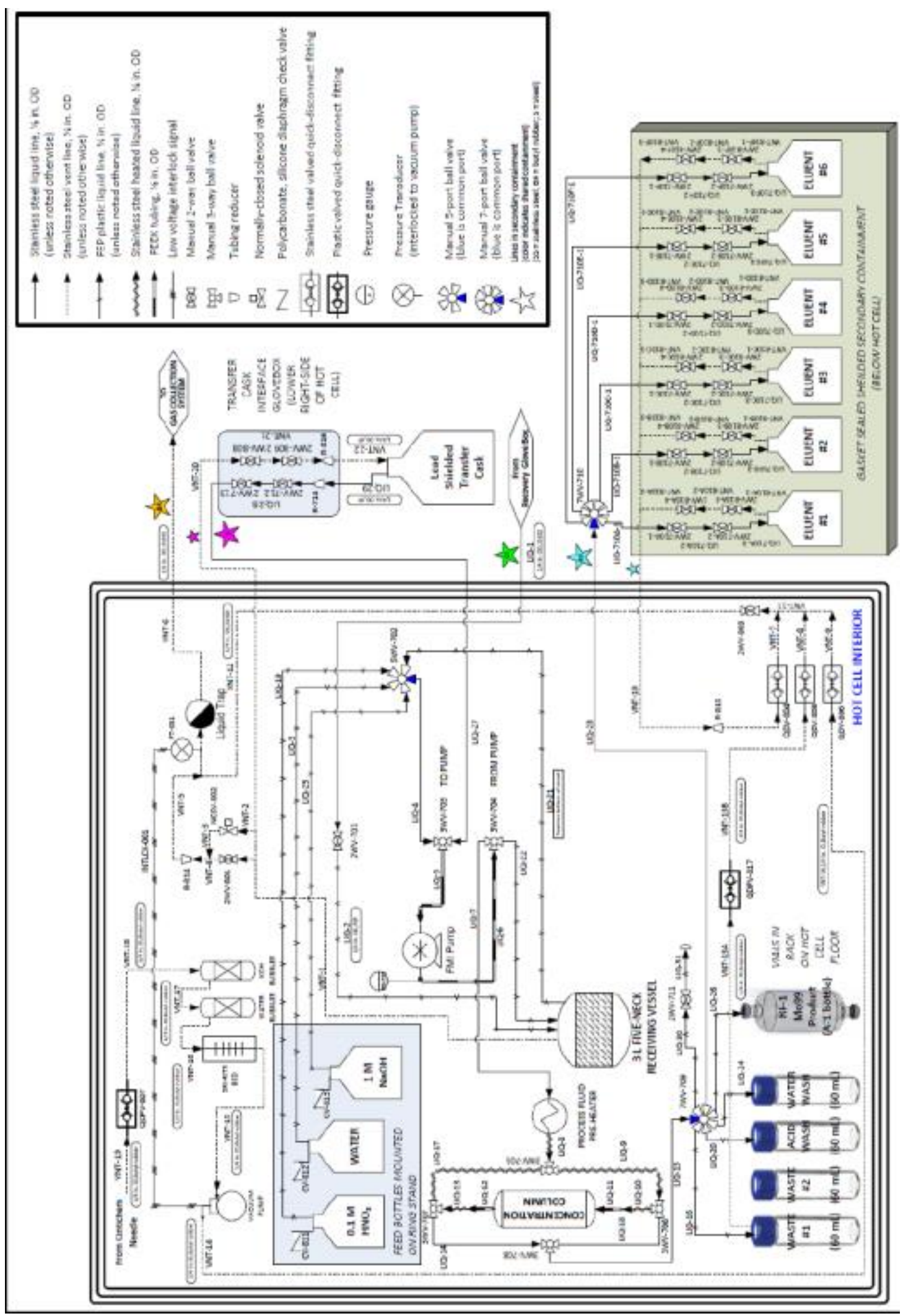


Concentration Column Summary Table

\begin{tabular}{|l|l|l|l|}
\hline & $\begin{array}{l}\text { Mass of Empty } \\
\text { Vessel }\end{array}$ & Mass of Vessel with Solution & Mass of Solution \\
\hline Feed (3L vessel) & & & \\
\hline Waste \#1 & & & \\
\hline Waste \#2 & & & \\
\hline Water Wash & & & \\
\hline Nitric Acid Wash & & & \\
\hline $\begin{array}{l}\text { Mo-99 Product } \\
\text { RF-1 Cintichem } \\
\text { bottle) }\end{array}$ & & & \\
\hline
\end{tabular}

Concentration Column Sample Summary Table

\begin{tabular}{|l|l|l|l|}
\hline & $\begin{array}{l}\text { Mass of Empty } \\
\text { Sampling vessel }\end{array}$ & $\begin{array}{l}\text { Mass of Sampling Vessel } \\
\text { with Sample }\end{array}$ & Mass of sample \\
\hline Feed Initial & & & \\
\hline Waste \#2 & & & \\
\hline Water Wash & & & \\
\hline Nitric Acid Wash & & & \\
\hline Effluent & & & \\
\hline
\end{tabular}

\section{END OF SEGMENT}




\section{PAGE INTENTIONALLY LEFT BLANK}




\subsubsection{Prepare for Cintichem Operations}

\begin{tabular}{|c|c|c|}
\hline Step & & Action \\
\hline \multirow[t]{9}{*}{1} & \multirow[t]{2}{*}{1.1} & $\begin{array}{l}\text { Verify that } 2 \% \text { alpha-benzoin oxime in } 0.4 \mathrm{M} \mathrm{NaOH} \text { has been freshly prepared within } 24 \\
\text { hours as operation is taking place. }\end{array}$ \\
\hline & & $\begin{array}{l}\text { Date: }- \text { Time: } \\
\text { (solution must be }<24 \text { hours old) }\end{array}$ \\
\hline & \multirow[t]{2}{*}{1.2} & Verify that molybdenum carrier solution (10 $\mathrm{mg} \mathrm{Mo} / \mathrm{mL})$ has been freshly prepared. \\
\hline & & $\begin{array}{l}\text { Date: }- \text { Time: } \\
\text { (solution must be }<7 \text { days old) }\end{array}$ \\
\hline & \multirow[t]{2}{*}{1.3} & $\begin{array}{l}\text { OPTIONAL STEP Verify that } \mathrm{Ag} / \mathrm{C} \text { column has been washed with } 0.2 \mathrm{M} \mathrm{NaOH} \text { prior to } \\
\text { using in hot cell. }\end{array}$ \\
\hline & & Washed Date: ____ Time: _____ \\
\hline & \multirow[t]{3}{*}{1.4} & $\begin{array}{l}\text { Verify that combination column }(\mathrm{HZO} / \mathrm{Ag} / \mathrm{C}) \text { has been washed with } 0.2 \mathrm{M} \mathrm{NaOH} \text { and } \\
\mathrm{pH} \text { of effluent was checked and was alkaline prior to using in hot cell. }\end{array}$ \\
\hline & & Washed Date: ____ Time: ____ \\
\hline & & 1.4.1 Verify pH of eluent was alkaline $\square$ \\
\hline \multirow[t]{15}{*}{2} & \multicolumn{2}{|c|}{ Stage the following solutions and glassware: } \\
\hline & \multirow[t]{4}{*}{2.1} & Sampling syringes \\
\hline & & 2.1.1 $1 \mathrm{~mL}$ syringe x 5 " needle with luer lock two-way valve $\square$ \\
\hline & & 2.1.2 $1 \mathrm{~mL}$ syringe x 5 " needle with luer lock two-way valve $\square$ \\
\hline & & 2.1.3 $10 \mathrm{~mL}$ syringe x 5 " needle with luer lock two-way valve $\square$ \\
\hline & \multirow[t]{4}{*}{2.2} & Sampling vials \\
\hline & & 2.2.1 20mL LSC vial with septa for RF1 bottle $\square$ \\
\hline & & 2.2.2 20mL LSC vial with septa for RFW bottle $\square$ \\
\hline & & 2.2.3 20mL LSC vial with septa for 1-B bottle $\square$ \\
\hline & \multirow[t]{6}{*}{2.3} & Cintichem bottles \\
\hline & & 2.3.1 Double-sided bottle labeled RF-2 (plastic coated) $\square$ see Fig. 4 \\
\hline & & 2.3.2 Flat bottom bottle labeled RFW (plastic coated) $\square$ see Fig. 4 \\
\hline & & 2.3.3 51-mm fritted glass column with $\sim 20 \mathrm{~mL}$ of glass beads - pre wet $\square$ see Fig. 4 \\
\hline & & 2.3.4 Double-sided bottle labeled 1-A (plastic coated) $\square$ see Fig. 4 \\
\hline & & 2.3.5 Flat bottom bottle labeled 1-B Mo99 Product (plastic coated) $\square$ see Fig. 4 \\
\hline
\end{tabular}




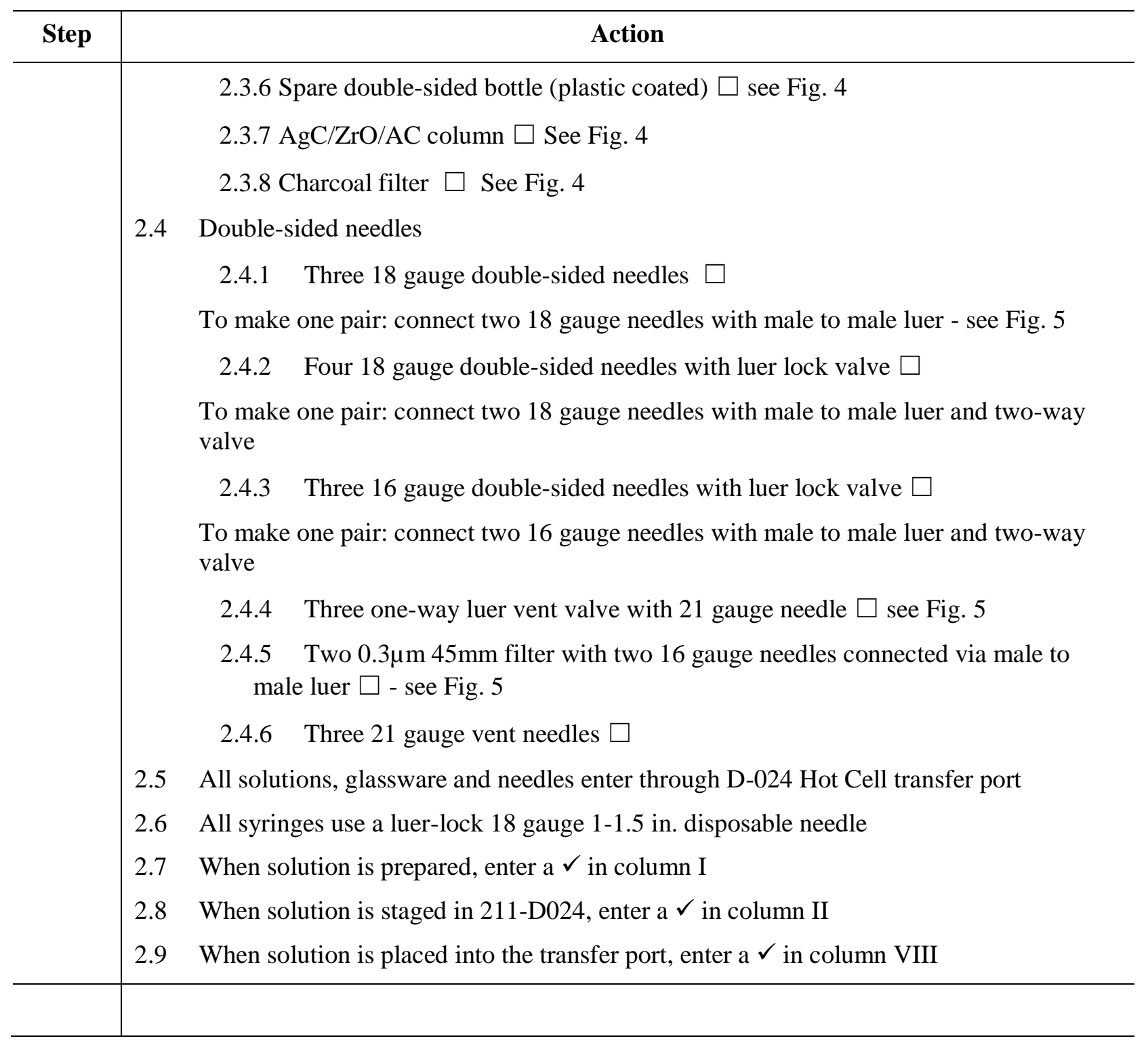




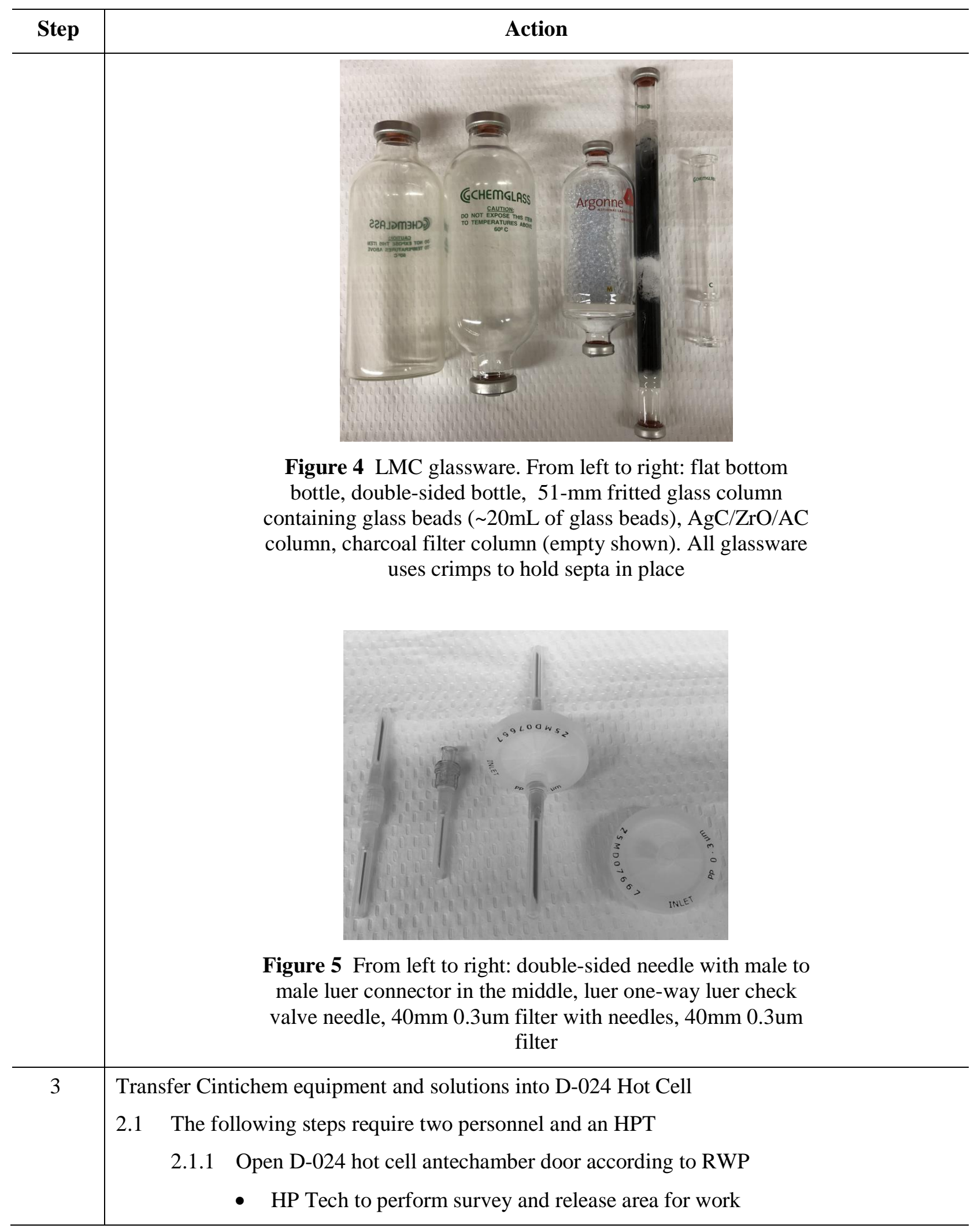




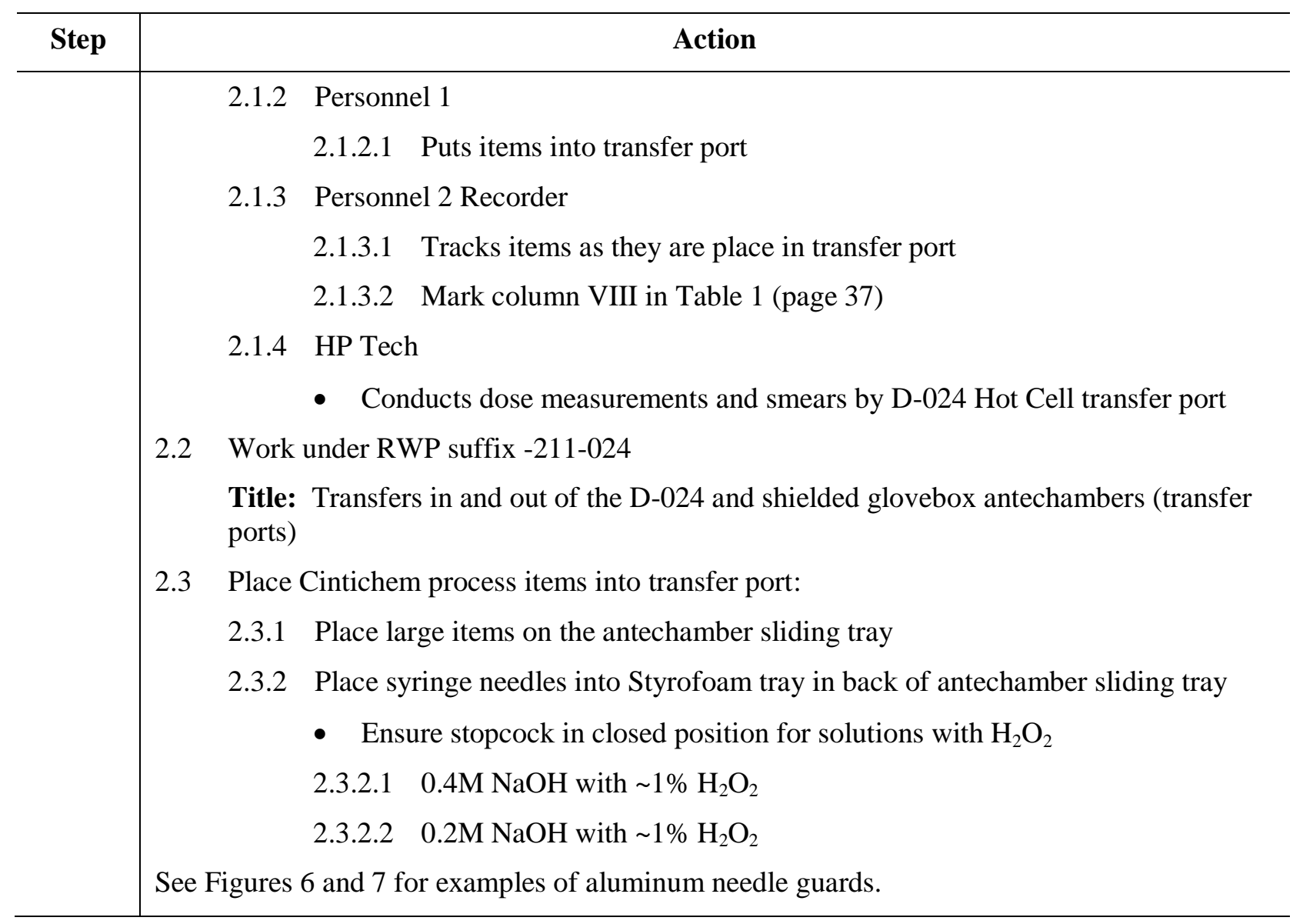




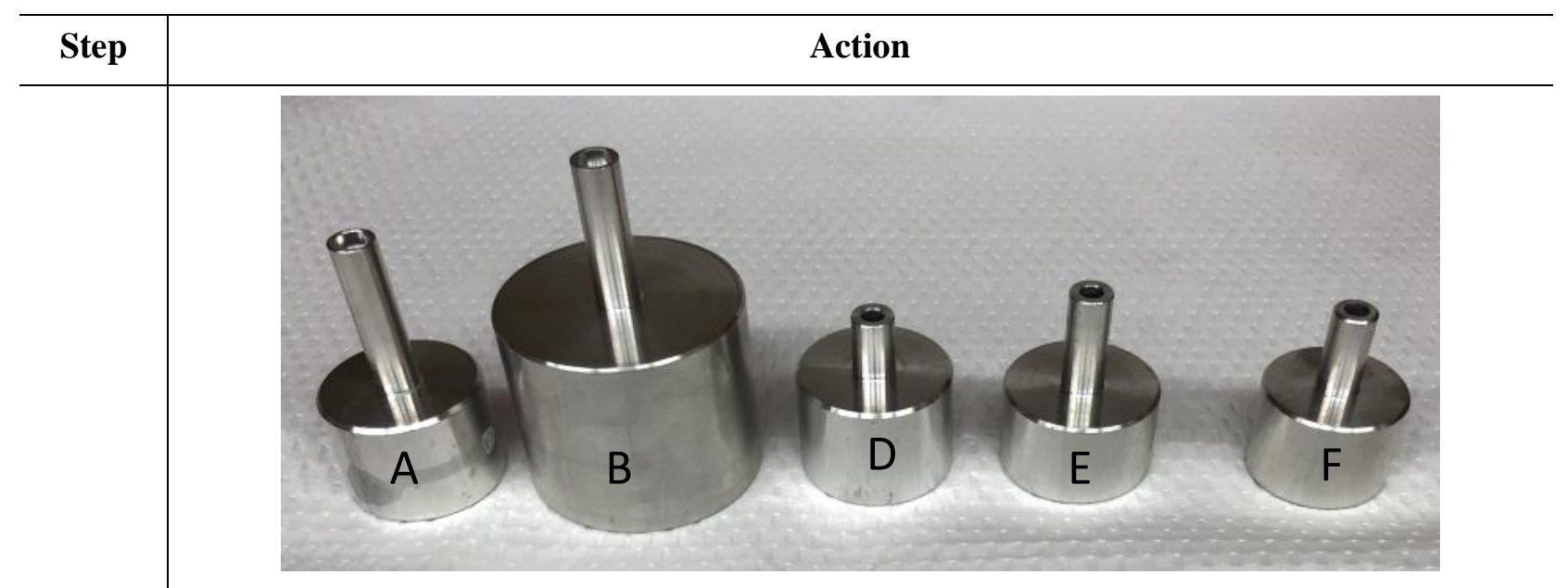

Figure 6 Aluminum Needle Guards (Different Models), Model C Not Shown

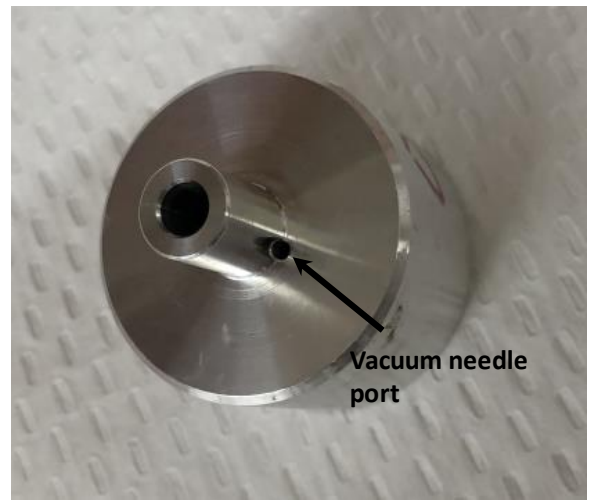

Figure 7 D-Type Aluminum Needle Guard With Side Port for Vacuum Line 
Table 1 Solutions for LEU Modified Cintichem: To Be Passed Into D-024 Hot Cell Via Transfer Port (process steps are reference to Section 3.2.8)

\begin{tabular}{|c|c|c|c|c|c|c|c|}
\hline 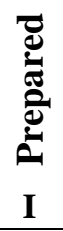 & 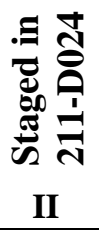 & 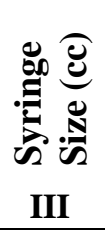 & 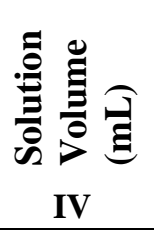 & V & $\begin{array}{c}\text { Purpose } \\
\text { VI } \\
\end{array}$ & 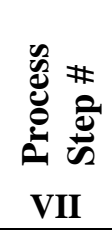 & 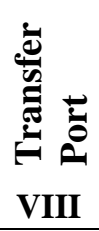 \\
\hline & & 25 & 15 & $10 \mathrm{M} \mathrm{HNO}_{3}$ & Acidification of RF-1 bottle & 6 & \\
\hline & & 5 & 4.0 & $\mathrm{NaI}$ carrier $4.0 \mathrm{~mL}$ at $1 \mathrm{mg} / \mathrm{mL}$ & AgI ppt & 11 & \\
\hline & & 1 & 0.5 & $10 \% \mathrm{AgNO}_{3}$ in $0.1 \mathrm{M} \mathrm{HNO}_{3}$ & AgI ppt & 13 & \\
\hline & & 1 & 1.0 & $1 \mathrm{M} \mathrm{HCl}$ & Aid in ppt. of $\mathrm{NaI} / \mathrm{Cl}$ & 18 & \\
\hline & & 25 & 11 & $4 \mathrm{M} \mathrm{HNO}_{3}$ & Rinse the filter & 33 & \\
\hline & & 1 & 0.5 & Mo carrier $(10 \mathrm{mg} / \mathrm{mL})$ & Mo carrier & 43 & \\
\hline & & 25 & 25 & $2.5 \% \mathrm{KMnO}_{4}$ & Mo oxidation & 44 & \\
\hline & & 2 & 1.5 & $\mathrm{Rh}$ carrier $(8 \mathrm{mg} / \mathrm{mL})$ & Rh carrier & 47 & \\
\hline & & 2 & 2.0 & Ru carrier $(5 \mathrm{mg} / \mathrm{mL})$ & Ru carrier & 50 & \\
\hline & & 25 & 20.0 & $\begin{array}{l}\text { Fresh } 2 \% \text { alpha-benzoin-oxime in } 0.4 \mathrm{M} \mathrm{NaOH} \\
\text { Date: } \\
\text { (solution must be }<24 \text { hours old) }\end{array}$ & Mo ppt & 54 & \\
\hline & & 25 & 20.0 & $0.1 \mathrm{M} \mathrm{HNO}_{3}$ & Rinse & 75 & \\
\hline & & 25 & 20.0 & $0.1 \mathrm{M} \mathrm{HNO}_{3}$ & Rinse-second & 81 & \\
\hline & & 25 & 20.0 & $0.1 \mathrm{M} \mathrm{HNO}_{3}$ & Rinse-third & 87 & \\
\hline & & 10 & 10.0 & $0.1 \mathrm{M} \mathrm{HNO}_{3}$ & Rinse & 101 & \\
\hline & & 10 & 10.0 & $0.1 \mathrm{M} \mathrm{HNO}_{3}$ & Rinse-second & 114 & \\
\hline & & 10 & 10.0 & $0.1 \mathrm{M} \mathrm{HNO}_{3}$ & Rinse-third & 127 & \\
\hline & & 10 & 10.0 & $0.1 \mathrm{M} \mathrm{HNO}_{3}$ & Rinse-fourth & 147 & \\
\hline & & 10 & 10.0 & $0.1 \mathrm{M} \mathrm{HNO}_{3}$ & Rinse-fifth & 167 & \\
\hline & & 10 & 10.0 & $\begin{array}{l}0.4 \mathrm{M} \mathrm{NaOH} \text { with } \sim 1 \% \mathrm{H}_{2} \mathrm{O}_{2} \\
\text { Use polycarbonate } 2 \text {-way valve between needle } \\
\text { and syringe }\end{array}$ & Dissolve ppt & 198 & \\
\hline & & 10 & 10.0 & $\begin{array}{l}0.2 \mathrm{M} \mathrm{NaOH} \text { with } \sim 1 \% \mathrm{H}_{2} \mathrm{O}_{2} \\
\text { Use polycarbonate } 2 \text {-way valve between needle } \\
\text { and syringe }\end{array}$ & Dissolve ppt & 221 & \\
\hline & & 10 & 10.0 & $0.2 \mathrm{M} \mathrm{NaOH}$ & Fritted column rinse & 243 & \\
\hline & & 5 & 4.0 & $1 \mathrm{mg} / \mathrm{mL} \mathrm{NaI}$ & AgI ppt & 256 & \\
\hline & & 1 & 0.5 & $10 \% \mathrm{AgNO}_{3}$ in $0.1 \mathrm{M} \mathrm{HNO}_{3}$ & AgI ppt & 257 & \\
\hline & & 10 & 10.0 & $0.2 \mathrm{M} \mathrm{NaOH}$ & $\mathrm{Ag} / \mathrm{C} / \mathrm{HZO} / \mathrm{AC}$ column rinse & 274 & \\
\hline
\end{tabular}




\section{END OF SEGMENT}


3.2.8 Conduct LEU Modified Cintichem Process

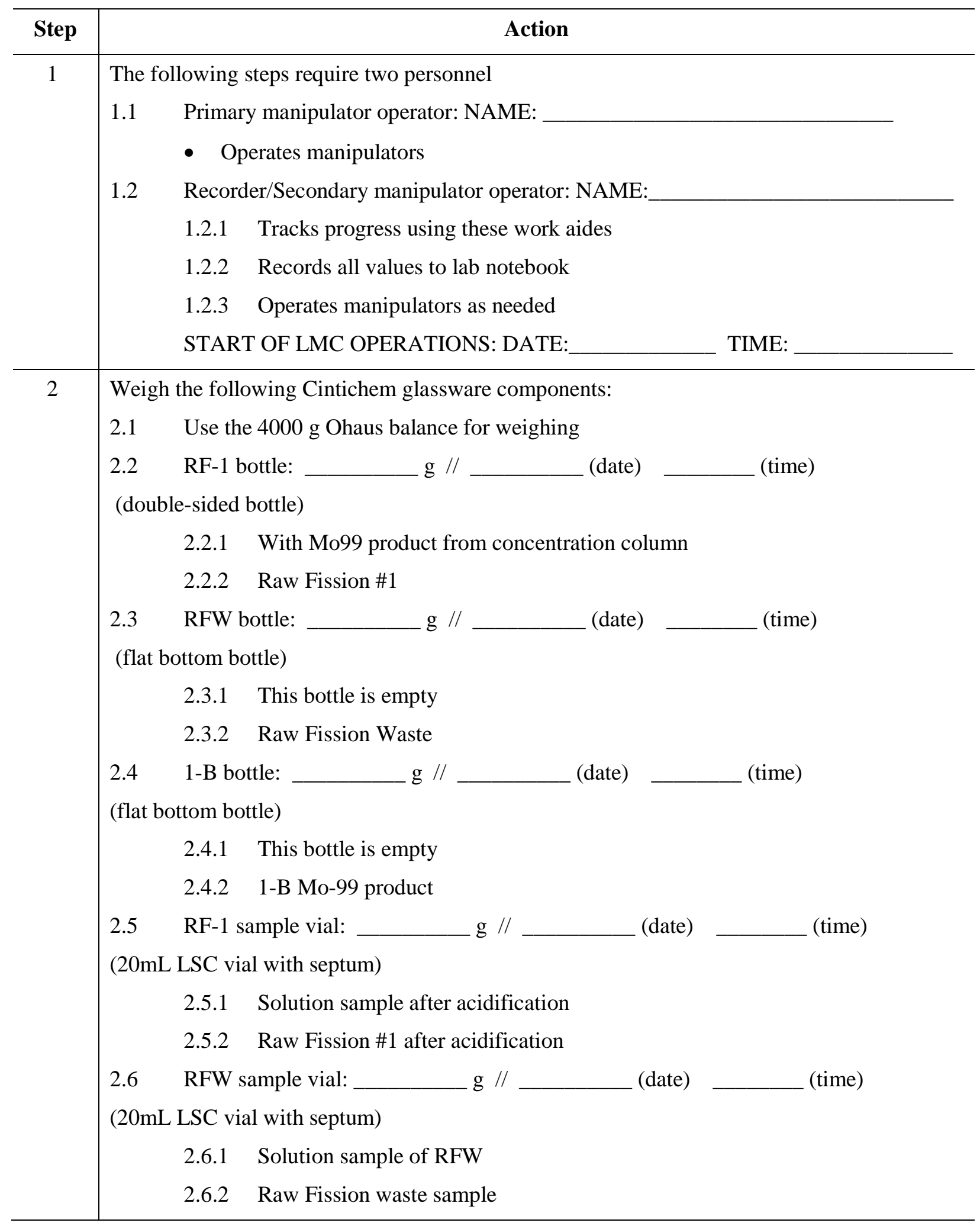




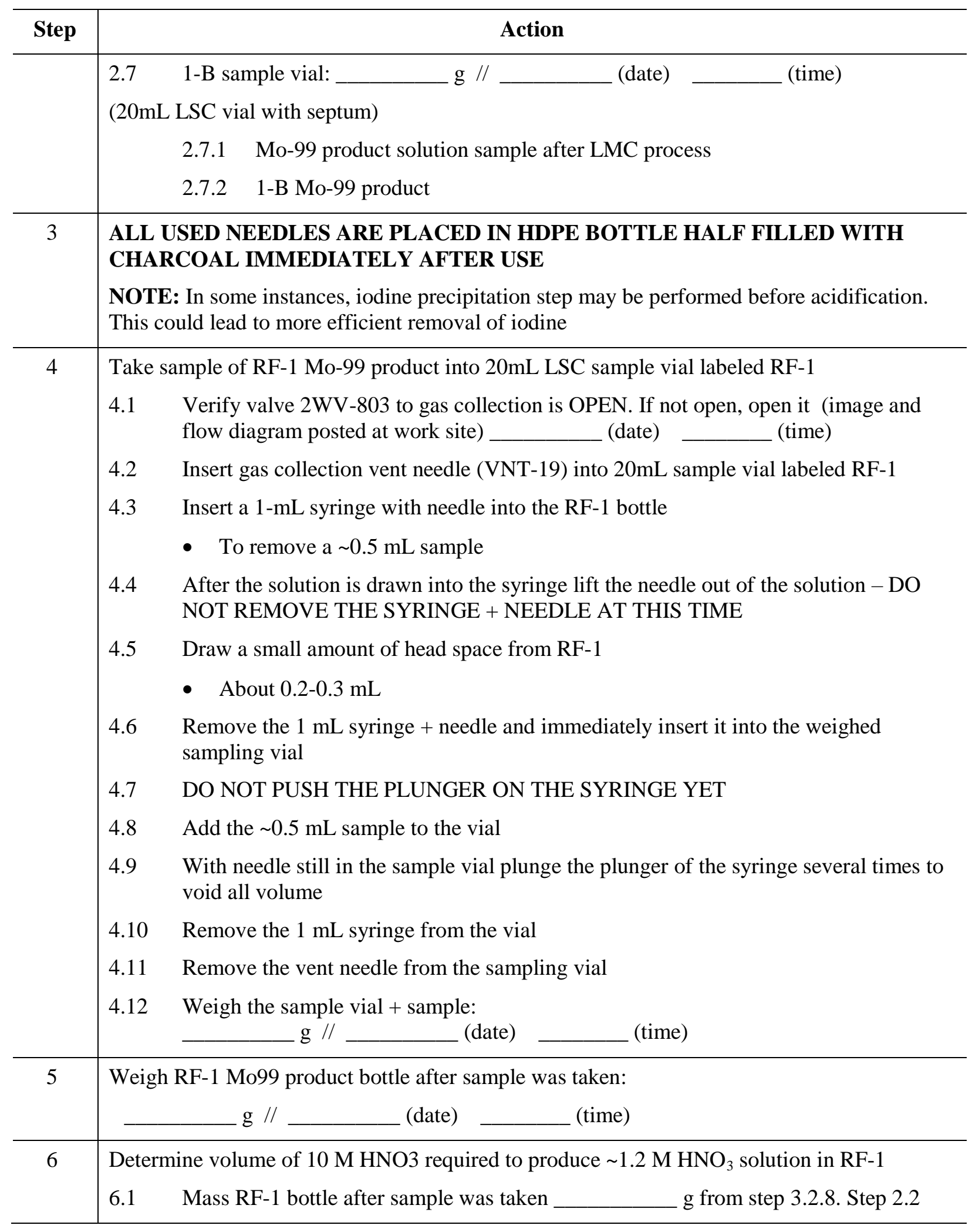




\begin{tabular}{|c|c|c|}
\hline \multirow[t]{4}{*}{ Step } & \multicolumn{2}{|r|}{ Action } \\
\hline & 6.2 & $\begin{array}{l}\text { Mass RF-1 bottle, empty } \_ \text {g obtained during Concentration Column } \\
\text { procedure, 3.2.6. summary table (Page35) }\end{array}$ \\
\hline & 6.3 & Difference $\quad$ g \\
\hline & 6.4 & $\begin{array}{l}0.25 \mathrm{~mL} \text { of } 10 \mathrm{M} \mathrm{HNO}_{3} \text { per } 1 \mathrm{~mL} \text { of product, } 3.2 .8 \text { step } 6.3 \times 0.25= \\
\mathrm{mL} \text { of } 10 \mathrm{M} \mathrm{HNO}_{3}\end{array}$ \\
\hline 7 & \multicolumn{2}{|c|}{ Set 2 minute timer } \\
\hline 8 & \multicolumn{2}{|r|}{ Insert gas collection vacuum needle from $\mathrm{KOH}$ bubbler into RF-1 bottle } \\
\hline 9 & \multicolumn{2}{|c|}{ Turn on vacuum } \\
\hline 10 & \multicolumn{2}{|c|}{ Turn off vacuum } \\
\hline 11 & \multicolumn{2}{|r|}{$\begin{array}{l}\text { Add } 4.0 \mathrm{mg} \text { of NaI carrier }(4.0 \mathrm{~mL} \text { at } 1 \mathrm{mg} / \mathrm{mL}) \text { into the } \mathrm{RF}-1 \text { bottle } \\
\text { // (date) } \\
11.1 \quad \text { Remove syringe after addition } \\
11.2 \quad \text { Contains Mo solution in } \sim 1.2 \mathrm{M} \mathrm{HNO}_{3} \\
11.3 \quad \text { Approximate volume }=\sim 50 \mathrm{~mL} \\
\text { NOTE: In some instances iodine precipitation step may be performed before acidification. } \\
\text { This could lead to more efficient removal of iodine }\end{array}$} \\
\hline 12 & \multicolumn{2}{|c|}{ Shake RF-1 bottle } \\
\hline 13 & \multicolumn{2}{|c|}{$\begin{array}{c}\text { Add } 0.5 \mathrm{~mL} \text { of } 10 \% \mathrm{AgNO}_{3} \text { in } 0.1 \mathrm{M} \mathrm{HNO}_{3} / / \_ \text {(date) } \\
\bullet \quad \text { Remove the syringe after addition }\end{array}$} \\
\hline 14 & \multicolumn{2}{|c|}{$\begin{array}{l}\text { Shake RF-1 bottle } \\
\text { • White precipitate should form }\end{array}$} \\
\hline 15 & \multicolumn{2}{|c|}{ Start timer (2 minutes) } \\
\hline 16 & \multicolumn{2}{|c|}{ At timer end, proceed to next step } \\
\hline 17 & \multicolumn{2}{|c|}{ Set 2 minute timer } \\
\hline 18 & $\begin{array}{l}\text { Add } \\
/ / \\
18.1 \\
18.2\end{array}$ & $\begin{array}{l}\mathrm{mL} \text { of } 1.0 \mathrm{M} \mathrm{HCl} \\
\text { (date) } \\
\text { Additional Precipitate should form } \\
\text { Remove the syringe after addition is complete }\end{array}$ \\
\hline 19 & \multicolumn{2}{|c|}{ Start timer } \\
\hline 20 & \multicolumn{2}{|c|}{ At timer end, proceed to next step } \\
\hline
\end{tabular}




\begin{tabular}{|c|c|}
\hline Step & Action \\
\hline 21 & Remove gas collection vacuum needle (VNT-19) from KOH bubbler from RF-1 \\
\hline 22 & Mount RF-2 bottle into bottom holder \\
\hline 23 & Attach aluminum needle guide type D to RF-2 \\
\hline 24 & $\begin{array}{l}\text { Insert } 16 \text { gauge needle with } 0.3 \mu \mathrm{m} \text { filter and } 16 \text { gauge needle assembly into aluminum needle } \\
\text { guide on RF- } 2 \text { and push through the RF- } 2 \text { septum }\end{array}$ \\
\hline 25 & Place aluminum needle guide type $\mathrm{E}$ (optional) on filter/needle assembly \\
\hline 26 & Adjust upper holder to hold RF-1 bottle \\
\hline 27 & Insert one-way luer check valve needle to RF-1 top septum \\
\hline 28 & $\begin{array}{l}\text { Insert RF-1 bottle onto top needle of filter assembly and press down to puncture the septum of } \\
\text { RF-1 }\end{array}$ \\
\hline 29 & $\begin{array}{l}\text { Insert gas collection vacuum needle (VNT-19) from KOH bubbler into RF-2 bottle through } \\
\text { aluminum needle guide port (see Figure } 4 \text { for reference) } \\
29.1 \text { Turn on vacuum // } \\
29.2 \quad \begin{array}{l}\text { Liquid will be drawn through the filter into RF-2 (example of iodine filtration setup is } \\
\text { shown in Figure 8) }\end{array}\end{array}$ \\
\hline 30 & Wait until all of solution has passed from RF-1 into RF-2 \\
\hline
\end{tabular}




\begin{tabular}{|c|c|}
\hline Step & Action \\
\hline 31 & Turn off vacuum // Check one-way luer valve \\
\hline 32 & Remove RF-1 bottle \\
\hline 33 & $\begin{array}{ll}\text { Add } & 11 \mathrm{~mL} \text { of } 4 \mathrm{M} \mathrm{HNO}_{3} \text { to } \mathrm{RF}-1 \text { bottle } / / \ldots \text { (date) ___ } \\
33.1 & \text { Remove the syringe after addition is complete } \\
33.2 & \text { Rinses the precipitate in RF-1 }\end{array}$ \\
\hline 34 & Insert RF-1 bottle onto top needle and press down to puncture the septum of RF-1 \\
\hline 35 & $\begin{array}{l}\text { Gas collection vacuum needle (VNT-19) from } \mathrm{KOH} \text { bubbler is still connected in RF-2 bottle } \\
\text { through aluminum needle guide point } \\
35.1 \text { Turn on vacuum // (date) } \\
35.2 \text { Liquid will be drawn through the filter into RF-2 }\end{array}$ \\
\hline 36 & Wait until all of solution has passed from RF- 1 through $0.3 \mu \mathrm{m}$ filter into RF-2 \\
\hline 37 & Turn off vacuum // ___ (date) ___ _ (time) \\
\hline
\end{tabular}




\begin{tabular}{|c|c|}
\hline Step & Action \\
\hline 38 & $\begin{array}{l}\text { Remove one-way luer check valve needle from top septum of RF-1 and place into Styrofoam } \\
\text { tray in back of antechamber sliding tray }\end{array}$ \\
\hline 39 & $\begin{array}{l}\text { Remove RF- } 1 \text { from the } 0.3 \mu \mathrm{m} \text { filter assembly } \\
39.1 \quad \text { Set aside for waste }\end{array}$ \\
\hline 40 & $\begin{array}{l}\text { Remove the } 0.3 \mu \mathrm{m} \text { filter assembly from RF- } 2 \\
40.1 \quad \text { Place inside of wide-mouth HDPE bottle and seal } \\
40.2 \quad \text { Set aside for waste }\end{array}$ \\
\hline 41 & Remove gas collection vacuum line (VNT-19) \\
\hline 42 & Remove aluminum needle guide from RF-2 \\
\hline 43 & $\begin{array}{l}\left.\text { Add } 0.5 \mathrm{~mL} \text { of Mo carrier }(10 \mathrm{mg} / \mathrm{mL}) \text { to } \mathrm{RF}-2 / / \ldots \_ \text {(date }\right) \text { ___ (time) } \\
43.1 \quad \text { Remove the syringe after addition is complete }\end{array}$ \\
\hline 44 & $\begin{array}{l}\text { Insert a a gas collection vent needle into RF- } 2 \text { bottle and slowly add } 2.5 \% \mathrm{KMNO}_{4} \text { solution } \\
\text { dropwise to RF-2 until a deep pink color persists for } \sim 30 \text { seconds } \\
\text { // (date) } \\
44.1 \quad \text { This may require up to } 25 \mathrm{~mL} \text { of solution } \\
44.2 \quad \text { Remove the syringe after addition is complete }\end{array}$ \\
\hline 45 & $\begin{array}{l}\text { Add } 1.5 \mathrm{~mL} \text { of } \mathrm{Rh} \text { carrier }(8 \mathrm{mg} / \mathrm{mL}) \text { to } \mathrm{RF}-2 / / \ldots \text { (date }) \text { ___ } \\
45.1 \quad \text { Remove the syringe after addition is complete }\end{array}$ \\
\hline 46 & Remove RF-2 from holder and shake RF-2 \\
\hline 47 & Return RF-2 to holder \\
\hline 48 & Add $2.0 \mathrm{~mL}$ of Ru carrier $(5 \mathrm{mg} / \mathrm{mL})$ to $\mathrm{RF}-2 / / \ldots$ ___ (date) ___ (time) \\
\hline 49 & Remove RF-2 from holder and shake RF-2 \\
\hline 50 & Return RF-2 to holder \\
\hline 51 & Set timer for 1 minute \\
\hline 52 & $\begin{array}{l}\text { Add } 20 \mathrm{~mL} \text { of fresh } 2 \% \text { alpha-benzoin-oxime (in } 0.4 \mathrm{M} \mathrm{NaOH} \text { ) to RF-2 } \\
/ / \text { (date) } \\
52.1 \quad \text { Remove the syringe after addition is complete }\end{array}$ \\
\hline 53 & Remove gas collection vacuum needle (VNT-19) from KOH bubbler from RF-2 \\
\hline 54 & Remove RF-2 from holder and shake RF-2 \\
\hline
\end{tabular}




\begin{tabular}{l|l}
\hline Step & \multicolumn{1}{c}{ Action } \\
\hline 55 & Return RF-2 to holder and start timer for 1 minute \\
\hline 56 & Place RFW in bottom holder of second ring stand \\
\hline 57 & Insert aluminum needle guide type D into RFW bottle \\
\hline 58 & Insert 16 gauge double-sided needle assembly into RFW \\
\hline 59 & Insert aluminum needle guide type C on top of needle assembly (optional) \\
\hline 60 & Position holder for 51mm fritted glass column above double-sided needle \\
\hline 61 & Place 51-mm fritted glass column into upper needle \\
\hline 62 & Place aluminum needle guide type B on top of 51mm fritted glass column (optional) \\
\hline 63 & $\begin{array}{l}\text { Insert 16 gauge double-sided needle assembly into upper septum port of 51-mm fritted glass } \\
\text { column }\end{array}$ \\
\hline 64 & Place aluminum needle guide type C on top of 51mm fritted glass column (optional) \\
\hline 65 & Position top bottle holder above 16 gauge double sided needle assembly \\
\hline 69 & $\begin{array}{l}\text { Insert Gas Collection vacuum needle (VNT-19) from KOH bubbler into RFW bottle through } \\
\text { vacuum line port in D type aluminum needle guide }\end{array}$ \\
\hline 67 & $\begin{array}{l}\text { Insert one-way luer check valve needle into RF-2 upper septum port } \\
69.1 \quad \text { Solution will flow from RF-2 into 51-mm fritted glass column then into RFW }\end{array}$ \\
\hline 6 Place RF-2 bottle into top holder and press down to puncture the septum of RF-2
\end{tabular}




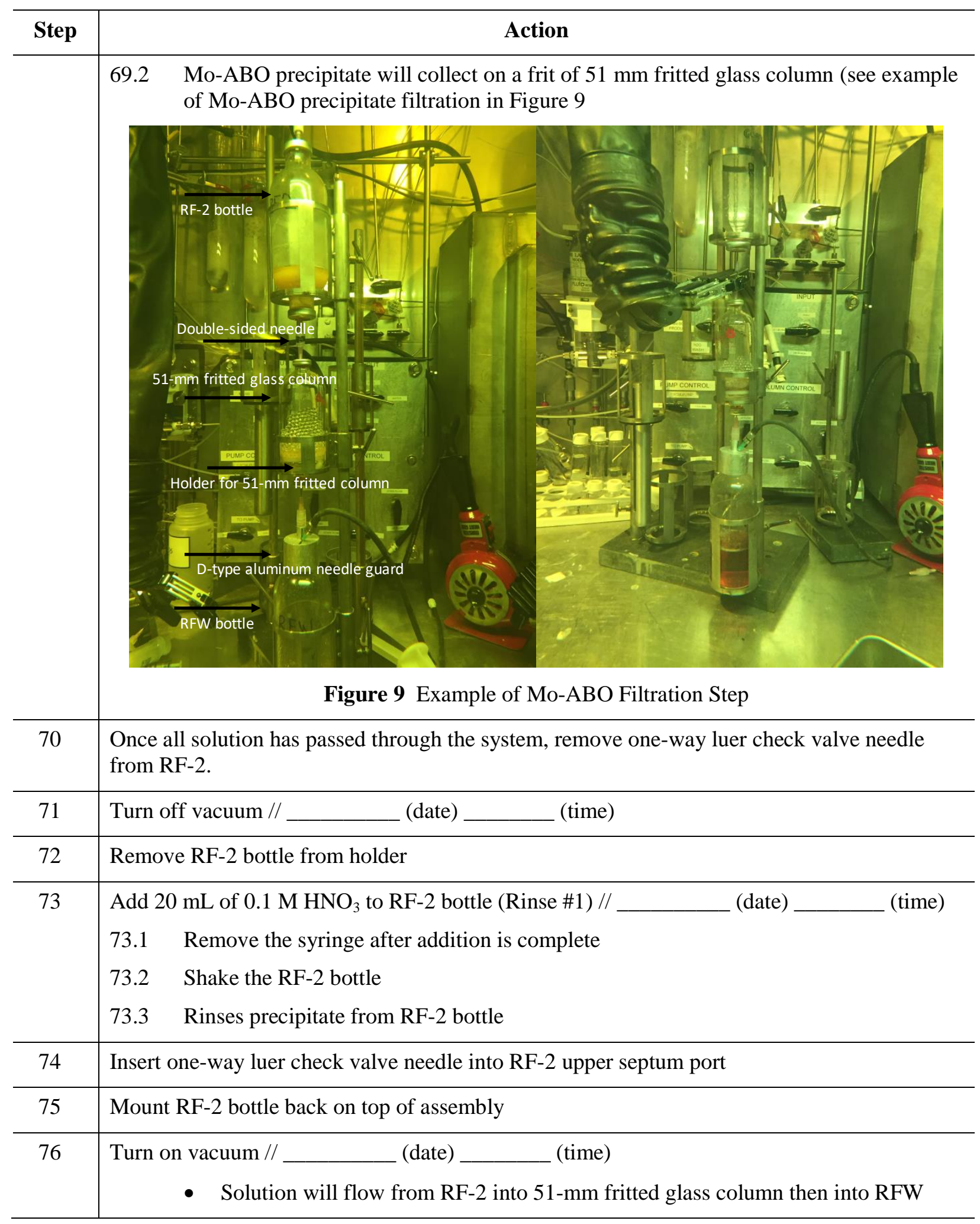




\begin{tabular}{|c|c|}
\hline Step & Action \\
\hline 77 & $\begin{array}{l}\text { Once all solution has passed through the system, remove one-way luer check valve needle } \\
\text { from RF-2 }\end{array}$ \\
\hline 78 & Turn off vacuum // ___ (date) ___ (time) \\
\hline 79 & $\begin{array}{l}\text { Add } 20 \mathrm{~mL} \text { of } 0.1 \mathrm{M} \mathrm{HNO}_{3} \text { to } \mathrm{RF}-2 \text { bottle }(\text { Rinse } \# 2) / / \_ \text {(date) ___ (time) } \\
79.1 \quad \text { Rinses precipitate from RF-2 bottle } \\
79.2 \quad \text { Remove the syringe after addition is complete }\end{array}$ \\
\hline 80 & Insert one-way luer check valve needle into RF-2 upper septum port \\
\hline 81 & Mount RF-2 bottle back on top of assembly \\
\hline 82 & $\begin{array}{l}\text { Turn on vacuum } / / \\
\text { • Solution will flow from RF-2 into } 51-\mathrm{mm} \text { fritted glass column then into RFW }\end{array}$ \\
\hline 83 & $\begin{array}{l}\text { Once all solution has passed through the system, remove one-way luer check valve needle } \\
\text { from RF-2 }\end{array}$ \\
\hline 84 & Turn off vacuum // ___ (date) ___ (time) \\
\hline 85 & $\begin{array}{l}\text { Add } 20 \mathrm{~mL} \text { of } 0.1 \mathrm{M} \mathrm{HNO}_{3} \text { to } \mathrm{RF}-2 \text { bottle (Rinse \#3) //___ (date) ___ (time) } \\
85.1 \quad \text { Rinses precipitate from RF-2 bottle } \\
85.2 \quad \text { Remove the syringe after addition is complete }\end{array}$ \\
\hline 86 & Insert one-way luer check valve needle into RF-2 upper septum port \\
\hline 87 & Mount RF-2 bottle back on top of assembly \\
\hline 88 & $\begin{array}{l}\text { Turn on vacuum } / / \ldots \text { (date) } \_ \text {(time) } \\
\text { • Solution will flow from RF-2 into 51-mm fritted glass column then into RFW }\end{array}$ \\
\hline 89 & Once all solution has passed through the system, turn off vacuum \\
\hline 90 & $\begin{array}{l}\text { Inspect the RF- } 2 \text { bottle for remnants of the precipitate before removing from the holder } \\
\text { 90.1 If any of the alpha-benzoin-oxime precipitate remains in the bottle, repeat Section } \\
\text { 3.2.8, Step 75, above, until the RF- } 2 \text { bottle doesn't contain any removable precipitate } \\
\text { NOTE: Some portion of Mo-ABO precipitate may stick to the wall of bottle and } \\
\text { cannot be easily removed }\end{array}$ \\
\hline & 90.2 If the RF-2 bottle is satisfactorily clean, proceed to next step \\
\hline 91 & $\begin{array}{l}\text { Remove one-way luer check valve needle from RF-2 and store in Styrofoam } \\
\text { 91.1 Remove RF-2 from the top holder and set aside for waste }\end{array}$ \\
\hline
\end{tabular}




\begin{tabular}{|c|c|}
\hline Step & Action \\
\hline 92 & $\begin{array}{l}\text { Remove the } 16 \text { gauge double-sided needle assembly from the top of the } 51 \text {-mm fritted glass } \\
\text { column and dispose in waste }\end{array}$ \\
\hline 93 & $\begin{array}{l}\text { Remove the 51-mm fritted glass column from the holder and place into designated holder } \\
\text { - This column contains Mo-99 }\end{array}$ \\
\hline 94 & $\begin{array}{l}\text { Connect the gas collection vacuum line (VNT-19) into top septum of } 51 \mathrm{~mm} \text { fritted glass } \\
\text { column }\end{array}$ \\
\hline 95 & Turn on vacuum for a few seconds \\
\hline 96 & Turn off vacuum \\
\hline 97 & Remove the gas collection vacuum line from the top septum \\
\hline 98 & Connect the gas collection vacuum line (VNT-19) into top septum of RFW \\
\hline 99 & $\begin{array}{l}\text { Add } 10 \mathrm{~mL} \text { of } 0.1 \mathrm{M} \mathrm{HNO}_{3} \text { to the } 51-\mathrm{mm} \text { fritted glass column into the chamber with glass } \\
\text { beads (chamber with vacuum) (Rinse } \# 1 \text { ) //__time) } \\
99.1 \text { Remove the syringe after addition is complete } \\
99.2 \text { Rinse the precipitate and glass beads }\end{array}$ \\
\hline 100 & Shake the 51-mm fritted glass column \\
\hline 101 & $\begin{array}{l}\text { Return the } 51-\mathrm{mm} \text { fritted glass column to the holder and allow the wash solution to be drawn } \\
\text { down to the RFW bottle }\end{array}$ \\
\hline 102 & Connect one-way luer check valve needle on top of the 51-mm fritted glass column \\
\hline 103 & $\begin{aligned} & \text { Turn on vacuum } / / \text { (date) } \_ \text {(time) } \\
& \bullet \quad \text { Solution will flow from 51-mm fritted glass column into RFW }\end{aligned}$ \\
\hline 104 & $\begin{array}{l}\text { Once all solution has passed through the system, turn off vacuum // ___ (time) }\end{array}$ \\
\hline 105 & Remove the 51-mm fritted glass column from the holder \\
\hline 106 & Remove the one-way luer check valve needle from top of the $51-\mathrm{mm}$ fritted glass column \\
\hline 107 & $\begin{array}{l}\text { Connect the gas collection vacuum line (VNT-19) into top septum of } 51 \mathrm{~mm} \text { fritted glass } \\
\text { column }\end{array}$ \\
\hline 108 & Turn on vacuum for a few seconds \\
\hline 109 & Turn off vacuum // ___ (date) ___ (time) \\
\hline 110 & Remove the gas collection vacuum line (VNT-19) from the top septum \\
\hline 111 & Connect the gas collection vacuum line (VNT-19) into top septum of RFW \\
\hline
\end{tabular}




\begin{tabular}{|c|c|}
\hline Step & Action \\
\hline 112 & $\begin{array}{l}\text { Add } 10 \mathrm{~mL} \text { of } 0.1 \mathrm{M} \mathrm{HNO}_{3} \text { to the } 51-\mathrm{mm} \text { fritted glass column into the chamber with glass } \\
\text { beads (chamber with vacuum) (Rinse } \# 2 \text { ) //_ (tame) } \\
112.1 \text { Remove the syringe after addition is complete } \\
112.2 \text { Rinses the precipitate and glass beads }\end{array}$ \\
\hline 113 & Shake the 51-mm fritted glass column \\
\hline 114 & $\begin{array}{l}\text { Return the } 51-\mathrm{mm} \text { fritted glass column to the holder and allow the wash solution to be drawn } \\
\text { down to the RFW bottle }\end{array}$ \\
\hline 115 & Connect one-way luer check valve needle on top of the 51-mm fritted glass column \\
\hline 116 & $\begin{array}{l}\text { Turn on vacuum } / / \ldots \text { (date) } \_ \text {(time) } \\
\text { • Solution will flow from 51-mm fritted glass column into RFW }\end{array}$ \\
\hline 117 & $\begin{array}{l}\text { Once all solution has passed through the system, turn off vacuum //___ (time) }\end{array}$ \\
\hline 118 & Remove the gas collection vacuum line from the RFW \\
\hline 119 & Remove the 51-mm fritted glass column from the holder \\
\hline 120 & $\begin{array}{l}\text { Equilibrate both chambers of fritted column by connecting one-way luer check valve needle } \\
120.1 \text { No vacuum in both chambers }\end{array}$ \\
\hline 121 & $\begin{array}{l}\text { Flip the bottle upside down and connect the vacuum line above the frit of the } 51-\mathrm{mm} \text { fritted } \\
\text { glass column - this is the chamber not containing glass beads }\end{array}$ \\
\hline 122 & Turn on vacuum \\
\hline 123 & Turn off vacuum \\
\hline 124 & Remove vacuum line \\
\hline 125 & $\begin{array}{l}\text { Add } 10 \mathrm{~mL} \text { of } 0.1 \mathrm{M} \mathrm{HNO}_{3} \text { to the chamber not containing glass beads - chamber with } \\
\text { vacuum (Rinse \#3) // (date)__time) } \\
125.1 \text { Remove the syringe after addition is complete }\end{array}$ \\
\hline 126 & $\begin{array}{l}\text { Insert one-way luer check valve needle to the same chamber to equilibrate pressure (no } \\
\text { vacuum in any of the chambers of the fritted column) }\end{array}$ \\
\hline 127 & Remove one-way luer check valve needle \\
\hline 128 & Flip the bottle, so the $10 \mathrm{~mL}$ of $0.1 \mathrm{M} \mathrm{HNO}_{3}$ is under the frit (normal orientation) \\
\hline 129 & Connect vacuum line above the frit (chamber with glass beads) \\
\hline 130 & Turn on vacuum // ___ (date) _____ (time) \\
\hline
\end{tabular}




\begin{tabular}{|c|c|}
\hline Step & Action \\
\hline 131 & Turn off vacuum // ___ (date) ___ (time) \\
\hline 132 & Remove vacuum line \\
\hline 133 & $\begin{array}{l}\text { Flip the column again, so the } 10 \mathrm{~mL} 0.1 \mathrm{M} \mathrm{HNO}_{3} \text { solution passes through the frit into the } \\
\text { chamber containing glass beads and } \mathrm{Mo}-\mathrm{ABO} \text { precipitate } \\
\text { - This rinses the frit that may contain small particles of Mo-ABO precipitate }\end{array}$ \\
\hline 134 & Shake the 51-mm fritted glass column \\
\hline 135 & $\begin{array}{l}\text { Return the } 51-\mathrm{mm} \text { fritted glass column to the holder and allow the wash solution to be drawn } \\
\text { down to the RFW bottle }\end{array}$ \\
\hline 136 & Connect one-way luer check valve needle on top of the 51-mm fritted glass column \\
\hline 137 & $\begin{aligned} & \text { Turn on vacuum } / / \text { (date) } \_ \text {(time) } \\
& \bullet \quad \text { Solution will flow from 51-mm fritted glass column into RFW }\end{aligned}$ \\
\hline 138 & $\begin{array}{l}\text { Once all solution has passed through the system, turn off vacuum // ___ (time) }\end{array}$ \\
\hline 139 & Remove the 51-mm fritted glass column from the holder \\
\hline 140 & $\begin{array}{l}\text { Equilibrate both chambers of fritted column by connecting one-way luer check valve needle } \\
\text { (no vacuum in both chambers) }\end{array}$ \\
\hline 141 & $\begin{array}{l}\text { Flip the bottle upside down and connect the vacuum line above the frit of the 51-mm fritted } \\
\text { glass column - this is the chamber not containing glass beads }\end{array}$ \\
\hline 142 & Turn on vacuum \\
\hline 143 & Turn off vacuum // ___ (date) ___ (time) \\
\hline 144 & Remove the vacuum line \\
\hline 145 & $\begin{array}{l}\text { Add } 10 \mathrm{~mL} \text { of } 0.1 \mathrm{M} \mathrm{HNO}_{3} \text { to the chamber not containing glass beads - chamber with } \\
\text { vacuum (Rinse \#4) } \\
145.1 \text { Remove the syringe after addition is complete }\end{array}$ \\
\hline 146 & $\begin{array}{l}\text { Insert one-way luer check valve needle to the same chamber to equilibrate pressure (no } \\
\text { vacuum in any of the chambers of the fritted column) }\end{array}$ \\
\hline 147 & Remove one-way luer check valve needle \\
\hline 148 & Flip the bottle, so the $10 \mathrm{~mL}$ of $0.1 \mathrm{M} \mathrm{HNO}_{3}$ is under the frit (normal orientation) \\
\hline 149 & Connect vacuum line above the frit (chamber with glass beads) \\
\hline 150 & Turn on vacuum \\
\hline
\end{tabular}




\begin{tabular}{|c|c|}
\hline Step & Action \\
\hline 151 & Turn off vacuum // ___ (date) ___ (time) \\
\hline 152 & Remove the vacuum line \\
\hline 153 & $\begin{array}{l}\text { Flip the column again, so the } 10 \mathrm{~mL} 0.1 \mathrm{M} \mathrm{HNO}_{3} \text { solution passes through the frit into the } \\
\text { chamber containing glass beads and } \mathrm{Mo}-\mathrm{ABO} \text { precipitate } \\
\text { - This rinses the frit that may contain small particles of Mo-ABO precipitate }\end{array}$ \\
\hline 154 & Shake the 51-mm fritted glass column \\
\hline 155 & $\begin{array}{l}\text { Return the } 51-\mathrm{mm} \text { fritted glass column to the holder and allow the wash solution to be drawn } \\
\text { down to the RFW bottle }\end{array}$ \\
\hline 156 & Connect one-way luer check valve needle on top of the 51-mm fritted glass column \\
\hline 157 & $\begin{aligned} & \text { Turn on vacuum } / / \text { (date) } \_ \text {(time) } \\
& \bullet \quad \text { Solution will flow from 51-mm fritted glass column into RFW }\end{aligned}$ \\
\hline 158 & $\begin{array}{l}\text { Once all solution has passed through the system, turn off vacuum // ___ (time) }\end{array}$ \\
\hline 159 & Remove the 51-mm fritted glass column from the holder \\
\hline 160 & $\begin{array}{l}\text { Equilibrate both chambers of fritted column by connecting one-way luer check valve needle } \\
\text { (no vacuum in both chambers) }\end{array}$ \\
\hline 161 & $\begin{array}{l}\text { Flip the bottle upside down and connect the vacuum line above the frit of the 51-mm fritted } \\
\text { glass column - this is the chamber not containing glass beads }\end{array}$ \\
\hline 162 & Turn on vacuum \\
\hline 163 & Turn off vacuum //____ (date) ____ (time) \\
\hline 164 & Remove vacuum line \\
\hline 165 & $\begin{array}{l}\text { Add } 10 \mathrm{~mL} \text { of } 0.1 \mathrm{M} \mathrm{HNO}_{3} \text { to the chamber not containing glass beads }- \text { chamber with } \\
\text { vacuum (Rinse \#5) // (date) } \\
165.1 \text { Remove the syringe after addition is complete }\end{array}$ \\
\hline 166 & $\begin{array}{l}\text { Insert one-way check luer valve needle to the same chamber to equilibrate pressure (no } \\
\text { vacuum in any of the chambers of the fritted column) }\end{array}$ \\
\hline 167 & Remove one-way luer check valve needle \\
\hline 168 & Flip the bottle, so the $10 \mathrm{~mL}$ of $0.1 \mathrm{M} \mathrm{HNO}_{3}$ is under the frit (normal orientation) \\
\hline 169 & Connect vacuum line (VNT-19) above the frit (chamber with glass beads) \\
\hline 170 & Turn on vacuum \\
\hline
\end{tabular}




\begin{tabular}{|c|c|}
\hline Step & Action \\
\hline 171 & Turn off vacuum //____ (date) ____ (time) \\
\hline 172 & Remove the vacuum line \\
\hline 173 & $\begin{array}{l}\text { Flip the column again, so the } 10 \mathrm{~mL} 0.1 \mathrm{M} \mathrm{HNO}_{3} \text { solution passes through the frit into the } \\
\text { chamber containing glass beads and } \mathrm{Mo}-\mathrm{ABO} \text { precipitate } \\
\text { - This rinses the frit that may contain small particles of Mo-ABO precipitate }\end{array}$ \\
\hline 174 & Shake the 51-mm fritted glass column \\
\hline 175 & $\begin{array}{l}\text { Return the } 51-\mathrm{mm} \text { fritted glass column to the holder and allow the wash solution to be drawn } \\
\text { down to the RFW bottle }\end{array}$ \\
\hline 176 & Connect one-way luer check valve needle on top of the 51-mm fritted glass column \\
\hline 177 & $\begin{array}{l}\text { Turn on vacuum } / / \_ \text {(date) } \_ \text {(time) } \\
\text { • Solution will flow from 51-mm fritted glass column into RFW }\end{array}$ \\
\hline 178 & $\begin{array}{l}\text { Once all solution has passed through the system, turn off vacuum // ___ (time) }\end{array}$ \\
\hline 179 & $\begin{array}{l}\text { Verify that the acid wash appears clear } \\
\text { NOTE: Over-time, some precipitate may form in the RFW bottle. This is due to precipitation } \\
\text { of excess ABO. }\end{array}$ \\
\hline 180 & Remove the 51-mm fritted glass column from the holder \\
\hline 181 & $\begin{array}{l}\text { Save the 51-mm fritted glass column with precipitate } \\
\text { - This contains Mo-99 }\end{array}$ \\
\hline 182 & Remove the double-sided needle from RFW and dispose the needle assembly \\
\hline 183 & Remove the aluminum needle guide from RFW \\
\hline 184 & $\begin{array}{l}\text { Weight the RFW bottle } \\
186.1 \text { Weigh the RFW bottle: ___ g //___ (date)____ (time) }\end{array}$ \\
\hline 185 & Place the RFW into a bottle holder \\
\hline
\end{tabular}




\begin{tabular}{|c|c|}
\hline Step & Action \\
\hline \multirow[t]{14}{*}{186} & Sample the RFW bottle solution \\
\hline & 186.1 Insert a vent needle into $20 \mathrm{~mL}$ sample vial labeled RFW sample \\
\hline & 186.2 Insert a $10-\mathrm{mL}$ syringe into the RFW bottle \\
\hline & 188.2.1 To remove a $\sim 5 \mathrm{~mL}$ sample \\
\hline & $\begin{array}{l}\text { 186.3 After the solution is drawn into the syringe lift the needle out of the solution - DO } \\
\text { NOT REMOVE THE SYRINGE + NEEDLE AT THIS TIME }\end{array}$ \\
\hline & 186.4 Draw a small amount of head space from RFW \\
\hline & 188.4.1 About $0.5 \mathrm{~mL}$ \\
\hline & $\begin{array}{l}\text { 186.5 Remove the } 10 \mathrm{~mL} \text { syringe }+ \text { needle and immediately insert it into the weighed } \\
\text { sampling vial }\end{array}$ \\
\hline & 186.6 DO NOT PUSH THE PLUNGER ON THE SYRINGE YET \\
\hline & 186.7 Add the $\sim 5 \mathrm{~mL}$ sample to the vial \\
\hline & $\begin{array}{l}\text { 186.8 With needle still in the sample vial plunge the plunger of the syringe several times to } \\
\text { void all volume }\end{array}$ \\
\hline & 186.9 Remove the $10 \mathrm{~mL}$ syringe from the vial \\
\hline & 186.10 Remove the vent needle from the sampling vial \\
\hline & $\begin{array}{l}\text { 186.11 Weigh the sample vial + sample: } \\
\text { g } / / \text { (date) ___ (time) }\end{array}$ \\
\hline 187 & Connect vacuum line (VNT-19) into RFW bottle \\
\hline 188 & Turn on vacuum for a few seconds \\
\hline 189 & Turn off vacuum // ___ (date) ___ (time) \\
\hline 190 & Remove vacuum line (VNT-19) from RFW bottle \\
\hline 191 & Store the RFW bottle under vacuum \\
\hline 192 & Insert a new 1-A bottle into the bottom holder \\
\hline 193 & Place aluminum needle guide type D on top of 1-A bottle \\
\hline 194 & $\begin{array}{l}\text { Insert a } 18 \text { gauge double-sided needle assembly with luer valve into the aluminum needle } \\
\text { guide and pierce the top septum port of the } 1 \text {-A bottle }\end{array}$ \\
\hline & 194.1 Leave the valve closed \\
\hline 195 & Insert the 51-mm fritted glass column with precipitate into a holder for dissolution \\
\hline
\end{tabular}




\begin{tabular}{|c|c|}
\hline Step & Action \\
\hline 196 & $\begin{array}{l}\text { Inject } 10 \mathrm{~mL} \text { of } 0.4 \mathrm{M} \mathrm{NaOH} \text { with } \sim 1 \% \mathrm{H}_{2} \mathrm{O}_{2} \text { into the } 51-\mathrm{mm} \text { fritted glass column from } \\
\text { underside of frit//__te } \quad \text { (time) } \\
196.1 \text { Remove the syringe after addition is complete }\end{array}$ \\
\hline 197 & Set an aluminum needle guide type B (optional) on top of the 51-mm fritted glass column \\
\hline 198 & $\begin{array}{l}\text { Insert a } 18 \text { gauge double-sided needle assembly with luer valve into the top septum of the } 51 \text { - } \\
\text { mm fritted glass column } \\
198.1 \text { Leave the valve closed }\end{array}$ \\
\hline 199 & Position short column holder above the needle assembly \\
\hline 200 & $\begin{array}{l}\text { Place the charcoal column into the holder and pierce the septum by pressing down on } \\
\text { charcoal column }\end{array}$ \\
\hline 201 & Insert the Gas Collection vent needle (VNT-13) into a charcoal filter \\
\hline 202 & Open the valve on double-sided needle assembly above $51 \mathrm{~mm}$ fritted column \\
\hline 203 & $\square$ Verify the valve on double-sided needle assembly above $51 \mathrm{~mm}$ fritted column is OPEN \\
\hline 204 & $\begin{array}{l}\text { Heat the } 51-\mathrm{mm} \text { fritted glass column that contains the solution with forced hot air until the } \\
\text { solution begins to boil // (date) } \\
\text { - See Figure } 10 \text { as an example of Mo-ABO dissolution setup. }\end{array}$ \\
\hline 205 & $\begin{array}{l}\text { Remove heat from the 51-mm fritted glass column by turning off heat gun and moving away } \\
\text { into a safe location. } \\
205.1 \text { Record date and time: //_ (date) __ (time) }\end{array}$ \\
\hline
\end{tabular}




\begin{tabular}{|c|c|}
\hline Step & Action \\
\hline 206 & Start timer and wait 5 minutes \\
\hline 207 & At timer end close the luer lock valve on double sided needle assembly. \\
\hline 208 & Slide the charcoal vent higher, so it disconnects from the double sided needle \\
\hline 209 & $\begin{array}{l}\text { Remove the 51-mm fritted glass column from the dissolution holder and place onto the top } \\
\text { needle of the double-sided needle assembly inserted in the 1-A bottle }\end{array}$ \\
\hline 210 & $\begin{array}{l}\text { Insert the gas collection vacuum needle (VNT-19) from KOH bubbler into the 1-A bottle } \\
/ / \text { (date) ___ (time) }\end{array}$ \\
\hline 211 & Connect one-way luer check valve needle on top of the 51-mm fritted glass column \\
\hline 212 & $\begin{array}{l}\text { Turn on vacuum } / / \ldots \text { (date) } \_ \text {(time) } \\
214.1 \text { The solution will be drawn from } 51 \mathrm{~mm} \text { fritted glass column into the 1-A bottle }\end{array}$ \\
\hline 213 & $\begin{array}{l}\text { Once all solution has passed through the system, turn off vacuum //___ (time) }\end{array}$ \\
\hline 214 & $\begin{array}{l}\text { When all solution has been transferred close the valve on double-sided needle assembly above } \\
\text { 1-A bottle }\end{array}$ \\
\hline 215 & Verify the valve on double-sided needle assembly above $51 \mathrm{~mm}$ fritted column is CLOSED \\
\hline
\end{tabular}




\begin{tabular}{|c|c|}
\hline Step & Action \\
\hline 216 & Remove the one-way luer check valve needle from the 51-mm fritted glass column \\
\hline 217 & Remove the 51-mm fritted glass column from the double needle union \\
\hline 218 & Insert the 51-mm fritted glass column with precipitate into a holder for dissolution \\
\hline 219 & $\begin{array}{l}\text { Inject } 10 \mathrm{~mL} \text { of } 0.2 \mathrm{M} \mathrm{NaOH} \text { with } \sim 1 \% \mathrm{H}_{2} \mathrm{O}_{2} \text { into the } 51-\mathrm{mm} \text { fritted glass column from } \\
\text { underside of frit// } \\
219.1 \text { Remove the syringe after addition is complete }\end{array}$ \\
\hline 220 & Set an aluminum needle guide type B (optional) on top of the 51-mm fritted glass column \\
\hline 221 & $\begin{array}{l}\text { Insert a } 18 \text { gauge double-sided needle assembly with luer valve into the top septum of the } 51 \text { - } \\
\text { mm fritted glass column } \\
221.1 \text { Leave the valve closed }\end{array}$ \\
\hline 222 & Position short column holder above the needle assembly \\
\hline 223 & $\begin{array}{l}\text { Place the charcoal column into the holder and pierce the septum by pressing down on } \\
\text { charcoal column }\end{array}$ \\
\hline 224 & Insert the Gas Collection vent needle (VNT-19) into a charcoal filter \\
\hline 225 & Open the valve on double-sided needle assembly above $51 \mathrm{~mm}$ fritted column \\
\hline 226 & Verify the valve on double-sided needle assembly above $51 \mathrm{~mm}$ fritted column is OPEN \\
\hline 227 & $\begin{array}{l}\text { Heat the } 51-\mathrm{mm} \text { fritted glass column that contains the solution with forced hot air until the } \\
\text { solution begins to boil // ___ (date) ___ }\end{array}$ \\
\hline 228 & $\begin{array}{l}\text { Remove heat from the } 51-\mathrm{mm} \text { fritted glass column by turning off heat gun and moving away } \\
\text { into a safe location } / / \ldots \text { (date) ___ _ _ _ _ }\end{array}$ \\
\hline 229 & Start timer and wait 5 minutes \\
\hline 230 & At timer end close the luer lock valve on double sided needle assembly. \\
\hline 231 & Slide the charcoal vent higher, so it disconnects from the double sided needle \\
\hline 232 & $\begin{array}{l}\text { Remove the } 51-\mathrm{mm} \text { fritted glass column from the dissolution holder and place onto the top } \\
\text { needle of the double-sided needle assembly inserted in the 1-A bottle }\end{array}$ \\
\hline 233 & Insert the gas collection vacuum needle (VNT-19) from KOH bubbler into the 1-A bottle \\
\hline 234 & Connect one-way luer check valve needle on top of the 51-mm fritted glass column \\
\hline 235 & $\begin{array}{l}\text { Turn on vacuum } / / \ldots \text { (date) } \_ \text {(time) } \\
235.1 \text { The solution will be drawn from } 51 \mathrm{~mm} \text { fritted glass column into the } 1 \text {-A bottle }\end{array}$ \\
\hline
\end{tabular}




\begin{tabular}{|c|c|}
\hline Step & Action \\
\hline 236 & $\begin{array}{l}\text { Once all solution has passed through the system, turn off vacuum //____ (time) }\end{array}$ \\
\hline 237 & $\begin{array}{l}\text { When all solution has been transferred close the valve on double-sided needle assembly above } \\
\text { 1-A bottle }\end{array}$ \\
\hline 238 & Verify the valve on double-sided needle assembly above $51 \mathrm{~mm}$ fritted column is CLOSED \\
\hline 239 & Remove the one-way luer check valve needle from the 51-mm fritted glass column \\
\hline 240 & Remove the 51-mm fritted glass column from the double-sided needle assembly \\
\hline 241 & $\begin{array}{l}\text { Inject } 10 \mathrm{~mL} \text { of } 0.2 \mathrm{M} \mathrm{NaOH} \text { into the } 51-\mathrm{mm} \text { fritted glass column from underside of frit } \\
/ / \text { (date) } \\
241.1 \quad \text { Rinsing the } 51 \mathrm{~mm} \text { fritted glass column } \\
241.2 \quad \text { Remove the syringe after addition is complete }\end{array}$ \\
\hline 242 & Place the $51 \mathrm{~mm}$ fritted glass column on top of double-sided needle assembly above 1-A bottle \\
\hline 243 & Connect one-way luer check valve needle on top of the 51-mm fritted glass column \\
\hline 244 & Open the valve on double-sided needle assembly above $1 \mathrm{~A}$ bottle \\
\hline 245 & Verify the valve on double-sided needle assembly above 1-A bottle is OPEN \\
\hline 246 & $\begin{array}{l}\text { Turn on vacuum // } \_ \text {(date) } \_ \text {(time) } \\
246.1 \text { The rinse solution will be pulled into the 1-Abottle }\end{array}$ \\
\hline 247 & $\begin{array}{l}\text { Once all the solution has passed through the system, turn off vacuum // ___ } \\
\text { (time) }\end{array}$ \\
\hline 248 & Remove the 51-mm fritted glass column from the double needle union assembly \\
\hline 249 & Remove the double-sided needle assembly from the 1-A bottle \\
\hline 250 & Pull vacuum into 1 -A bottle \\
\hline 251 & Turn off vacuum // ___ (date) ___ (time) \\
\hline 252 & Remove aluminum needle guide from 1-A bottle \\
\hline 253 & Set timer for 5 minutes \\
\hline 254 & $\begin{array}{l}\text { Inject } 4.0 \mathrm{mg} \mathrm{NaI} \text { solution into } 1-\mathrm{A} \text { bottle }(4.0 \mathrm{~mL} \text { of } 1 \mathrm{mg} / \mathrm{mL} \mathrm{NaI}) / / \\
254.1 \quad \text { Shake bottle }\end{array}$ \\
\hline
\end{tabular}




\begin{tabular}{|c|c|}
\hline Step & Action \\
\hline 255 & $\begin{array}{l}\text { Inject } 0.5 \mathrm{~mL} 1010 \% \mathrm{AgNO}_{3} \text { in } 0.1 \mathrm{M} \mathrm{HNO} 3 \text { solution } / / \ldots \text { (date) } \\
\text { (time) } \\
255.1 \text { Shake bottle } \\
255.2 \quad \text { Precipitate will form }\end{array}$ \\
\hline 256 & Start timer and wait 5 minutes \\
\hline 257 & Place 1-B bottle at the bottom of stand \\
\hline 258 & Place aluminum needle guide type D on top of 1-B bottle \\
\hline 259 & $\begin{array}{l}\text { Insert the } 18 \text { gauge double-sided needle assembly into aluminum needle guide on top of 1-B } \\
\text { bottle }\end{array}$ \\
\hline 260 & Place aluminum needle guide type F on top of double sided needle assembly \\
\hline 261 & Position long column holder above double-sided needle assembly \\
\hline 262 & $\begin{array}{l}\text { Place 3-phase column (AgC/ZrO/AC) column on top of assembly, push down to pierce all } \\
\text { septa }\end{array}$ \\
\hline 263 & Place aluminum needle guide type $\mathrm{C}$ on top of 3 -phase column \\
\hline 264 & Insert the 16 gauge double-sided needle assembly into needle guide \\
\hline 265 & Place aluminum needle guide type $\mathrm{E}$ on top of double sided needle assembly \\
\hline 266 & Position top bottle holder above needle \\
\hline 267 & Place one-way luer check valve needle in top septum of 1-A bottle \\
\hline 268 & Place 1-A bottle on top of assembly and push down to pierce all septa \\
\hline 269 & Insert the Gas Collection vacuum needle (VNT-19) from KOH bubbler into 1-B bottle \\
\hline 270 & $\begin{array}{l}\text { Allow gravity flow to move solution through 3-phase column and into 1-B bottle } \\
270.1 \text { Toggle vacuum on/off as needed to initiate flow }\end{array}$ \\
\hline 271 & After all solution has left 1-A bottle, remove 1-A bottle from assembly \\
\hline 272 & 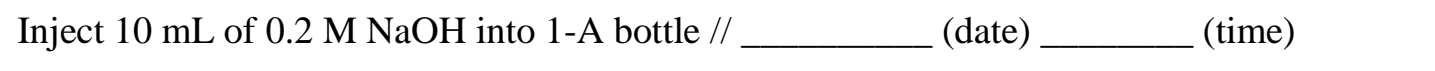 \\
\hline 273 & Shake and replace on top of assembly \\
\hline 274 & $\begin{array}{l}\text { Allow gravity flow to move solution through } 3 \text {-phase column and into 1-B bottle } \\
274.1 \text { Toggle vacuum on/off as needed to initiate flow }\end{array}$ \\
\hline 275 & $\begin{array}{l}\text { After gravity flow completed use vacuum to remove all solution from 3-phase column and } \\
\text { collect solution in 1-B }\end{array}$ \\
\hline
\end{tabular}




\begin{tabular}{|c|c|c|}
\hline Step & & Action \\
\hline 276 & $\begin{array}{l}\text { Disasser } \\
276.1\end{array}$ & $\begin{array}{l}\text { mble the system and dispose 1-A bottle and 3-phase column for waste } \\
\text { Keep the 1-B bottle containing purified Mo-99 product }\end{array}$ \\
\hline 277 & Weigh $\mathrm{t}$ & the 1-B bottle: ___ g // ___ (date) ___ (time) \\
\hline \multirow[t]{16}{*}{278} & \multicolumn{2}{|r|}{ Sample the 1-B bottle solution } \\
\hline & 278.1 & Insert the one-way luer check valve needle into the 1-B bottle \\
\hline & \multicolumn{2}{|c|}{278.2 Insert a 1-mL syringe into the 1-B bottle } \\
\hline & & 278.2.1 To remove a $\sim 0.5 \mathrm{~mL}$ sample \\
\hline & 278.3 & $\begin{array}{l}\text { After the solution is drawn into the syringe lift the needle out of the solution - } \\
\text { NOTE: DO NOT REMOVE THE SYRINGE + NEEDLE AT THIS TIME }\end{array}$ \\
\hline & \multirow[t]{2}{*}{278.4} & Draw a small amount of head space from 1-B bottle \\
\hline & & 278.4.1 About $0.2-0.3 \mathrm{~mL}$ \\
\hline & 278.5 & $\begin{array}{l}\text { Remove the } 1 \mathrm{~mL} \text { syringe }+ \text { needle and immediately insert it into the weighed } \\
\text { sampling vial }\end{array}$ \\
\hline & 278.6 & DO NOT PUSH THE PLUNGER ON THE SYRINGE YET \\
\hline & \multirow[t]{2}{*}{278.7} & Insert the vent needle into sampling vial \\
\hline & & 278.7.1 Ensure the tip of the needle is in the top $25 \%$ of the vial \\
\hline & 278.8 & Add the $\sim 0.5 \mathrm{~mL}$ sample to the vial \\
\hline & 278.9 & $\begin{array}{l}\text { With needle still in the sample vial plunge the plunger of the } 3 \mathrm{~mL} \text { syringe several } \\
\text { times to void all volum }\end{array}$ \\
\hline & \multirow{3}{*}{$\begin{array}{l}278.10 \\
278.11 \\
278.12\end{array}$} & Remove the $1 \mathrm{~mL}$ syringe from the vial \\
\hline & & Remove the vent needle from the sampling vial \\
\hline & & $\begin{array}{l}\text { Weigh the sample vial + sample: } \\
278.12 .1 \_\mathrm{g} / / \ldots \text { (date) ____ (time) }\end{array}$ \\
\hline
\end{tabular}


Table 2. LMC Summary table with bottle masses

\begin{tabular}{|c|r|r|r|}
\hline Bottle & Empty mass, g & Full mass, g & $\begin{array}{c}\text { Mass of } \\
\text { sample, } \mathbf{g}\end{array}$ \\
\hline RF-1 & ,g (3.2.4 step 2.6) & ,g (3.2.8 step 5) & \\
\hline RFW & ,g (3.2.8 step 2.3) & , g (3.2.8 step 184) & \\
\hline 1-B & ,g (3.2.8 step 2.4) & _. g (3.2.8 step 277) & \\
\hline
\end{tabular}

Table 3. LMC Sample Summary Table

\begin{tabular}{|c|r|r|r|}
\hline Sample & Empty mass, g & Full mass, g & $\begin{array}{c}\text { Mass of } \\
\text { sample, } \mathbf{g}\end{array}$ \\
\hline RF-1 & ,g (3.2.8 step 2.5) &, $\mathrm{g}(3.2 .8$ step 4.12) & \\
\hline RFW &, $\mathrm{g}(3.2 .8$ step 2.6) &, $\mathrm{g}(3.2 .8$ step 186.11) & \\
\hline 1-B & ,g (3.2.8 step 2.7) & ,g (3.2.8 step 278.12) & \\
\hline
\end{tabular}

\section{END OF SEGMENT}




\section{PAGE INTENTIONALLY LEFT BLANK}




\subsubsection{Receiving WASH/Rinse Solutions from Recovery Glove Box}

Follow the steps below to receive wash/rinse solutions from Recovery Glove Box.

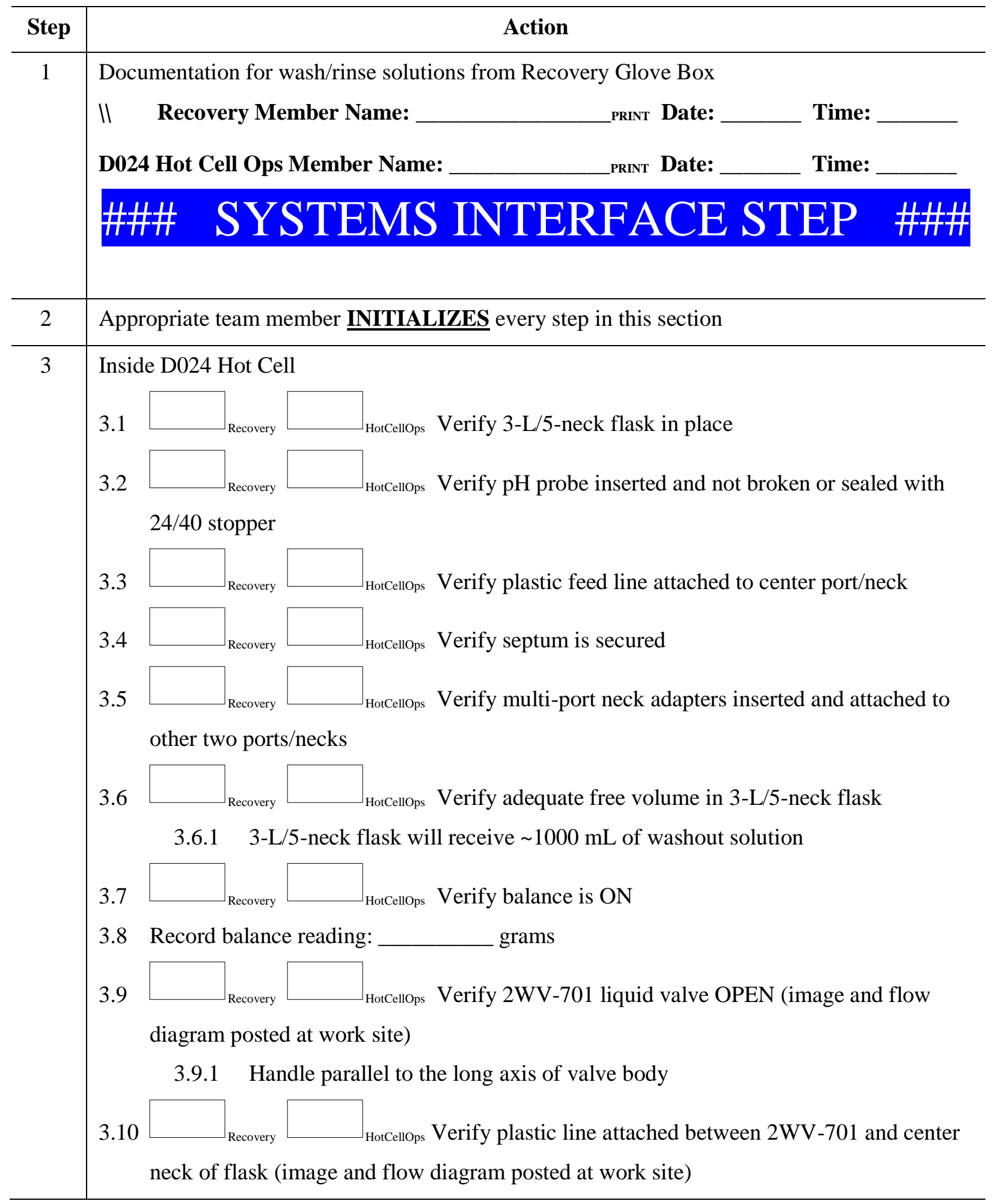




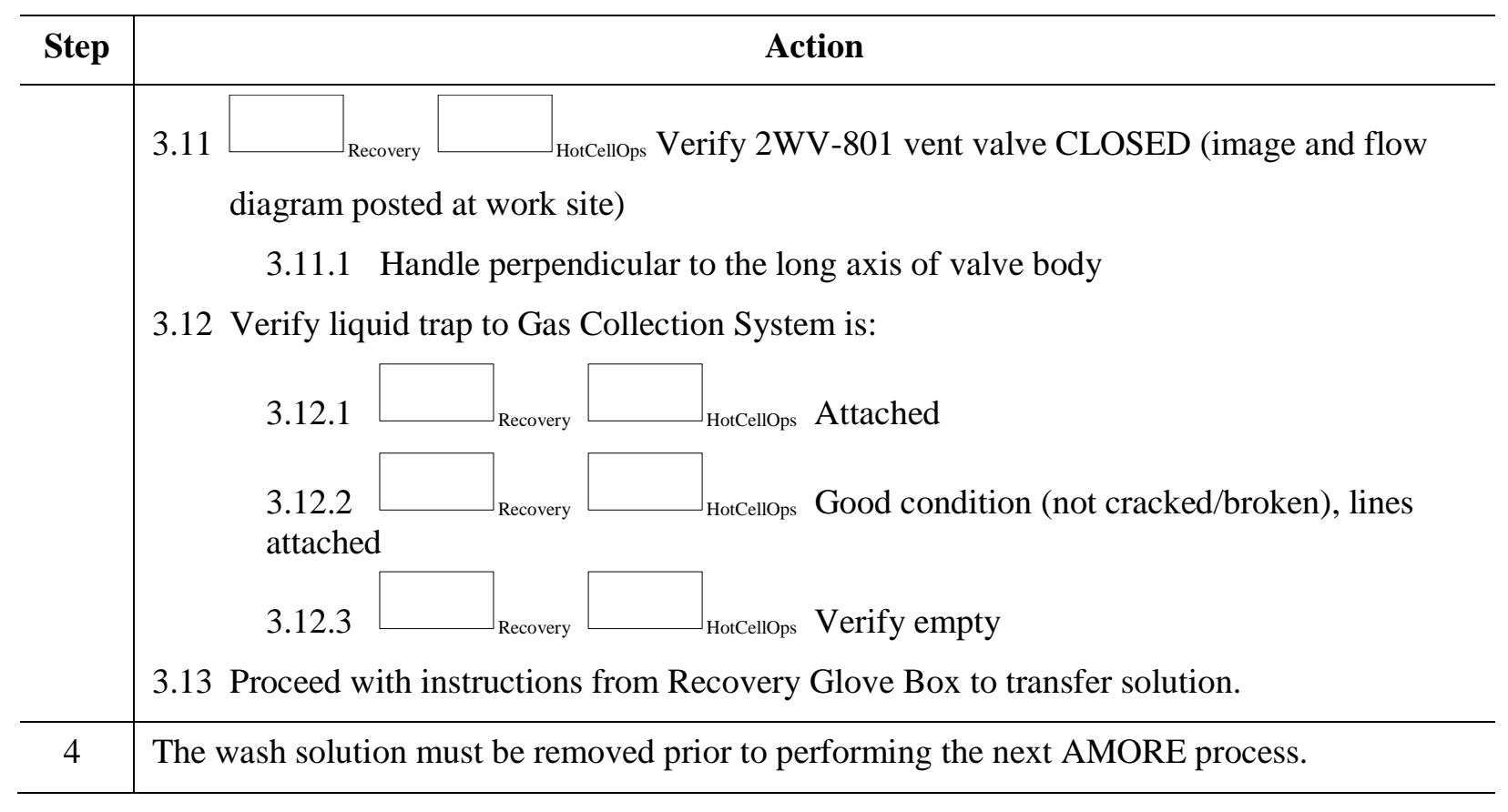




\section{Records Created by Work Process}

The records listed below must be retained as indicated.

\begin{tabular}{l|c|c|l|l}
\hline $\begin{array}{l}\text { Description of Record } \\
\text { (include form number if } \\
\text { applicable) }\end{array}$ & $\begin{array}{c}\text { Active } \\
\text { Records } \\
\text { Custodian }\end{array}$ & $\begin{array}{c}\text { Active } \\
\text { Records } \\
\text { Retention }\end{array}$ & $\begin{array}{l}\text { Indexing Method, } \\
\text { Storage Medium }\end{array}$ & $\begin{array}{l}\text { Federal Retention } \\
\text { Requirements* }\end{array}$ \\
\hline Completed procedure & $\begin{array}{c}\text { Facility } \\
\text { Manager }\end{array}$ & 3 years & $\begin{array}{l}\text { Index by job date } \\
\text { and name, store on } \\
\text { paper or } \\
\text { electronically }\end{array}$ & $\begin{array}{l}\text { Destroy 75 years after } \\
\text { the date of the permit } \\
\text { (DOE ADM 18.37) }\end{array}$ \\
\hline
\end{tabular}

*If records are maintained in a business information system that is not currently programmed to purge digital records based on age, the records may be retained in that system past the indicated destruction date.

\section{Related Documents}

This procedure implements requirements established by the following safety basis documents:

- LEAF-SAD-100, Linac Safety Assessment Document

- LEAF-ASE-100, Linac Accelerator Safety Envelope

This procedure implements requirements established by the following Argonne policies and procedures:

- LMS-PROC-188, Accelerator Safety

- EGS-PP-100, Configuration Management Program Plan for Accelerators

\section{Definitions}

None

\section{About this Procedure}

\begin{tabular}{ll}
\hline Issuing organization: & Low Energy Accelerator Facility \\
\hline Procedure owner: & D. Rotsch \\
\hline Point of contact: & D. Rotsch \\
\hline Review cycle (months): & 36 \\
\hline Date last revised: & 03.25 .2019 \\
\hline Date last reviewed: & 03.25 .2019 \\
\hline
\end{tabular}

\section{Summary of Changes in This Version}

Initial release

LEAF-PROC-011 CHANGES LOG DATE: 8/28/2019

3.2.3. step 1. Action 1.1.a - added

i. Worker 1:

ii. Worker 2 (optional): 
iii. HPT:

3.2.3. step 5 - removed

3.2.3. step 8. Action 5.1 - added "Water reservoir fill"

3.2.3. step 8. Action 5.2 - added " $\mathrm{HNO}_{3}$ reservoir fill"

3.2.3. step 8. Action 5.3 - added " $1 \mathrm{M} \mathrm{NaOH}$ reservoir fill"

3.2.3. step 8. Action 5.5.4 - added “ $\square$ And Date"

3.2.3. step 8. Action 5.5.5 - removed:

- Fisher Scientific p/n \#

- Becton Dickinson p/n BD \#

3.2.3. step 8. Action 5.7.1 - changed "8 in." to "6 in."

3.2.3. step 8. Action 5.7.4 - changed " 8 in." to " 8 in. or longer"

3.2.4 step 1. Action 1.1.1 - added

1.2.3.1. Name:

1.2.3.2. Date and time:

3.2.4 step 1. Action 1.1.2 - added

1.1.1.1. Name:

1.1.1.2. Date and time:

1.1.1.3. Name:

1.1.1.4. Date and time:

3.2.4 step 2. - added "(Use this data in the summary table at the end of 3.2.6"

3.2.4 step 2. Action 2.6 - removed "1-A bottle"

3.2.4 step 3. - added step:

3.6 End of segment

Date and Time:

3.2.4 step 3. Removed:

3.3 Insert probe into $\mathrm{pH} 2$ standard

$3.4 \mathrm{pH}$ value $=$ time: date:

3.2.4 step 4. Action 4.10 - added "or manual valve SWV-801"

3.2.5. step 1 - added

1.1 Transfer began: (date) (time)

3.2.5. step 3 - added

3.1 Transfer end: (date) (time) 
3.2.6 step 2 action 2.1 - added

Name:

3.2.6 step 2 action 2.2 - added

Name:

3.2.6 step 5 Action 5.3 - changed section to

5.3 Adjust $\mathrm{pH}$ of recovery column primary strip product to $\mathrm{pH} 2$ with appropriate solution:

$8 \mathrm{M} \mathrm{HNO}_{3}$ :

Obtain mass of full syringe: g

$10 \mathrm{M} \mathrm{NaOH}$ :

Obtain mass of full syringe: g

3.2.6 step 5 Action 5.4 - added (If $\mathrm{pH}<1$ observed, add $5 \mathrm{~mL} 10 \mathrm{M} \mathrm{NaOH}$ and observe change in $\mathrm{pH}$ )

3.2.6 step 5 Action 5.4.3 - adjusted text "Continue until $\mathrm{pH}<10$ (or $\mathrm{pH}>2$ if using $\mathrm{NaOH}$ ) observed and then proceed if not add another $5 \mathrm{~mL}$ of $8 \mathrm{M} \mathrm{HNO}_{3}$ (or $10 \mathrm{M} \mathrm{NaOH}$ )"

3.2.6 step 5 Action 5.5 - adjusted text "Slowly add (dropwise) $8 \mathrm{M} \mathrm{HNO}_{3}$ (or $10 \mathrm{M} \mathrm{NaOH}$ ) until pH 2 is reached"

3.2.6 step 5 - added Action - "Obtain mass of spent syringe: g"

3.2.6 step 6 action 6.2 - added "(Use this data in the summary table at the end of 3.2.6"

3.2.6 step 6 action 6.14 - added "(Use this data in the summary table at the end of 3.2.6"

Upper "Pump Control" (pump outlet) 3-way valve (3WV-703) to "Column", VALVE 3WV-704 changed to $3 \mathrm{WV}-703$ throughout procedure

Lower "Pump Control" (pump inlet) 3-way valve (3WV-704) to feed source 4-way valve "Column", VALVE $3 \mathrm{WV}-705$ changed to $3 \mathrm{WV}-704$ throughout procedure

3.2.6 Step 12 Action 12.1 - changed text to "Verify Water needle from "Output" 7-way valve (7WV-709) inserted into Waste \#2 $60 \mathrm{~mL}$ vial"

3.2.6 Step 12 Action 12.2 - changed text to "Verify Mo-99 product bottle, RF-1 Mo99 product (Cintichem style bottle, see Figure 3) is prepared and present."

3.2.6 Step 12 Action 12.2 - changed text to "Verify "Input" 5-way valve to Water"

3.2.6 Step 12 Action 12.11 - changed text to "Verify "Gas Collection" vent needle from VNT-13 inserted into Waste \#2 $60 \mathrm{~mL}$ vial"

3.2.6 Step 12 Action 12.11 - changed time from 5 minutes to 6 minutes $(360 \mathrm{sec}$ )

3.2.6 Step 13 Action 13.1 - changed text to "Insert Mo-99 needle from "Output" 7-way valve (7WV-709) Waste \#1 valve port into Waste \#2 $60 \mathrm{~mL}$ vial"

3.2.6 Step 13 Action 13.1 - changed vial from Waste \#1 to Waste \#2

3.2.6 Step 15 - added "Use this data in the summary table at the end of 3.2.6"

Added 3.2.6 step 19 - Turn off pump controller

Added 3.2.6 step 20 - Place gas collection line into RF1 
Added 3.2.6 step 21 - Sample vials

21.1. Shake all vials with manipulators and obtain mass of each vessel

21.1.1. Waste \#1: $\mathrm{g}(60 \mathrm{~mL}$ vial $)$

21.1.2. Waste \#2: $\mathrm{g}(60 \mathrm{~mL}$ vial $)$

21.1.3. Water Wash: $\mathrm{g}(60 \mathrm{~mL}$ vial $)$

21.1.4. Nitric Acid Wash: $\mathrm{g}(60 \mathrm{~mL}$ vial $)$

21.1.5. Mo-99 Product (Cintichem Vessel): $\mathrm{g}(60 \mathrm{~mL}$ vial $)$

Use this data in the summary table at the end of 3.2.6

21.2. Use $1 \mathrm{~mL}$ syringes with 6" needles to sample vials. Pull the plunger to $\sim 50 \%$ of the syringe shaft. Remove needle from solution while keeping needle within vessel being samples. Pull plunger to $\sim 80 \%$ of the syringe shaft. Remove needle from vessel and inject sample into appropriate sampling vessel. Record mass of sample

21.2.1 Waste \#2 vessel with sample: $\mathrm{g}(20 \mathrm{~mL}$ vial $)$

21.2.2 Water wash vessel with sample: g (20 mL vial)

21.2.3 Nitric acid wash vessel with sample: g (20 mL vial)

Use this data in the summary table at the end of 3.2.6

Added 3.2.6 step 22 - Notify LMC team of completion

Date:

Time:

Added Concentration Column Summary Tables

Concentration Column Summary Table

\begin{tabular}{|l|l|l|l|}
\hline & $\begin{array}{l}\text { Mass of Empty } \\
\text { Vessel }\end{array}$ & Mass of Vessel with Solution & Mass of Solution \\
\hline Feed (3L vessel) & & & \\
\hline Waste \#1 & & & \\
\hline Waste \#2 & & & \\
\hline Water Wash & & & \\
\hline Nitric Acid Wash & & & \\
\hline $\begin{array}{l}\text { Mo-99 Product } \\
\text { (Cintichem bottle) }\end{array}$ & & & \\
\hline
\end{tabular}

Concentration Column Sample Summary Table

\begin{tabular}{|l|l|l|l|}
\hline & $\begin{array}{l}\text { Mass of Empty } \\
\text { Sampling vessel }\end{array}$ & $\begin{array}{l}\text { Mass of Sampling Vessel } \\
\text { with Sample }\end{array}$ & Mass of sample \\
\hline
\end{tabular}




\begin{tabular}{|l|l|l|l|}
\hline Feed Initial & & & \\
\hline Waste \#2 & & & \\
\hline Water Wash & & & \\
\hline Nitric Acid Wash & & & \\
\hline
\end{tabular}

\subsubsection{Step 1.3 - "OPTIONAL STEP” was added}

\subsubsection{Step 1.4.1-2.4.6 - check box added}

\subsection{7 steps 2.5-2.12 renumbered}

3.2.7. Steps $2.10-2.11$ are now $2.7-2.9$ check symbol added

3.2.7. Steps 3.2.2 Work under appropriate currently approved RWP (replaced RWP \#)

Table 1 - added line on top of the table to include syringe with 10M HNO3

3.2.8 step 1.1 added NAME: Primary manipulator operator: NAME:

3.2.8 step 1.2 added NAME: Recorder/Secondary manipulator operator: NAME:

After step 3.2.8 step 1.3 added: START OF LMC OPERATIONS: DATE: TIME:

3.2.8 steps 2.2-2.7 added date and time fields

3.2.8 step 4.1 added date and time field

3.2.8 step 4.5 corrected RFW to RF-1

3.2.8 step 6.2 added reference to empty RF-1 bottle weight

3.2.8 step 6.4 corrected step reference to 3.2 .8 step 6.3

3.2.8 step 18 added date and time fields

3.2.8 step 24 changed the description of step to: Insert 16 gauge needle with $0.3 \mu \mathrm{m}$ filter and 16 gauge needle assembly into aluminum needle guide on RF-2 and push through the RF-2 septum

3.2.8 step 29 added date and time fields

3.2.8 step 31 added date and time fields

3.2.8 step 35.1 and 37 added date and time fields

3.2.8 step 44 added: Insert a gas collection vent needle into RF-2 bottle and slowly...

3.2.8 steps $45-46$ removed, old step 47 becomes step 45

3.2.8 step 71 becomes 69 added date and time fields

3.2.8 step 73 becomes 71 added date and time fields

Same edits in old steps added date and time fields - steps: $75,78,80,81,82,84,86,87,90,101,105,106,111$, $113,118,119,127,132,133,139,140,145,153,159,160,165,167,173,179,180,191,198,207,214,215$, $237,238,248,249,253,256,257,274$ 
Table 2 added with summary masses of RF-1, RFW and 1-B bottles

Table 3 added with summary masses of RF-1, RFW and 1-B samples

\section{UPDATES as of $2 / 20 / 2020$}

Section 3.2.6 step 9.2: Changed "Phase II Effluent" to "Phase I Effluent" see text below

Section 3.2.6

9.2 Turn "Output" 7-way valve (7WV-709) to 6-way eluent bottle directing valve "Phase I Effluent"

Section 3.2.6 added the following:

9.2.1. Ensure ball valve of solution line connected to effluent bottle is OPEN

9.2.2. Ensure that the black luer-lock valve connected to the effluent bottle is OPEN

9.2.3. Ensure that black luer-lock valve for sampling the effluent bottle is CLOSED

9.2.4. Ensure that gas collection ball valve connected to the effluent bottle is OPEN

9.2.5. Ensure that the black luer-lock valve connected to the gas collection needle is CLOSED or is inserted in to one of the $60 \mathrm{~mL}$ septum collection vials

21.3. Sample Effluent Bottle

21.3.1. Ensure effluent bottle is connected to gas collection system

21.3.1.1. Ensure VQD-014 is connected

21.3.1.2. Ensure gas collection needle for small bottles is in a septum bottle or closed

21.3.1.3. Ensure effluent bottle black luer lock valve is OPEN

21.3.1.4. Ensure effluent bottle ball valve is OPEN

21.3.2. Connect effluent bottle solution line to syringe

21.3.2.1. Ensure that 7WV-709 "OUTPUT" 7 -way valve is directed towards any other output than "Phase I Effluent" - "OUTPUT" valve is NOT connected to effluent bottle being sampled.

21.3.2.2. Ensure effluent bottle solution ball valve is OPEN

21.3.2.3. Connect syringe (suggested $20-\mathrm{mL}$ syringe with valve and plunger fully extended) to black luer lock at " $t$ " connection to effluent bottle.

21.3.3. Mix and collect sample

21.3.3.1. Open black luer lock connecters

21.3.3.2. Depress syringe plunger to force air into the effluent bottle to mix system (LEAVE $\sim 5$ ML OF AIR WITHIN THE SYRINGE)

21.3.3.3. Pull syringe plunger up and down $3 \mathrm{X}$ to further mix the system (LEAVE $\sim 5$ ML OF AIR WITHIN THE SYRINGE)

21.3.3.4. Pull plunger to take sample (suggested $\sim 1-3 \mathrm{~mL}$ )

21.3.3.5. Invert syringe so that solution is on plunger side and remaining 
21.3.3.6. Depress syringe until bubbles are noticed in effluent bottle - OR - until sample is near top of syringe barrel (the point of this step is to void the lines and ensure no solution is in disconnect points)

21.3.3.7. CLOSE all solution black luer lock valves $(2 \mathrm{x})$

21.3.3.8. Disconnect the syringe from the effluent bottle system while maintaining ONE black luer lock valve to the syringe.

21.3.3.9. Affix a needle to the black luer lock valve still connected to the syringe.

21.3.3.10. Inject solution into appropriately marked $20 \mathrm{~mL}$ septum vessel and record mass.

21.2.3.3.10.1 Effluent sample: g $(20 \mathrm{~mL}$ vial $)$

Updated Table to include effluent sample:

Concentration Column Sample Summary Table

\begin{tabular}{|l|l|l|l|}
\hline & $\begin{array}{l}\text { Mass of Empty } \\
\text { Sampling vessel }\end{array}$ & $\begin{array}{l}\text { Mass of Sampling Vessel } \\
\text { with Sample }\end{array}$ & Mass of sample \\
\hline Feed Initial & & & \\
\hline Waste \#2 & & & \\
\hline Water Wash & & & \\
\hline Nitric Acid Wash & & & \\
\hline Effluent & & & \\
\hline
\end{tabular}

Section 3.2.3.8 Step 8.2.4 updated to include mass of each $20 \mathrm{~mL}$ vessel

4. Five (5x) $20 \mathrm{~mL}$ sampling septa vials

- Each vial is labeled, dated and placed in D-024 hot cell

- Verify vials labeled

$\begin{array}{lll}\text { Mo-99 product } & \square \text { And date } & \text { Mass: } \\ \text { Acid wash } & \square \text { And date Mass: } \\ \text { Water wash } & \square \text { And date Mass: } \\ \text { Feed initial } & \square \text { And date Mass: } \\ \text { Waste \#2 } & \square \text { And date } \text { Mass: }\end{array}$

\section{Updates on 11/2020 to Rev. 3}

Added comment in section 3.2.3 step 14 that the lines must be replaced at least every 2 years.

Section 3.2.6 Step 23-26 were added to include a water rinse of the lines after the AMORE experiment has been completed.

Schematic of the concentration column system added to PROC. 


\section{Argonne}

\section{Experimental Operations and Facilities Division}

Argonne National Laboratory

9700 South Cass Avenue, Bldg. 205

Lemont, IL 60439-4854

www.anl.gov 